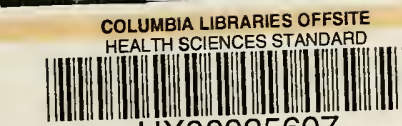

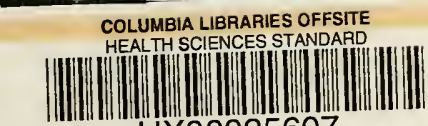 HX00025607
}



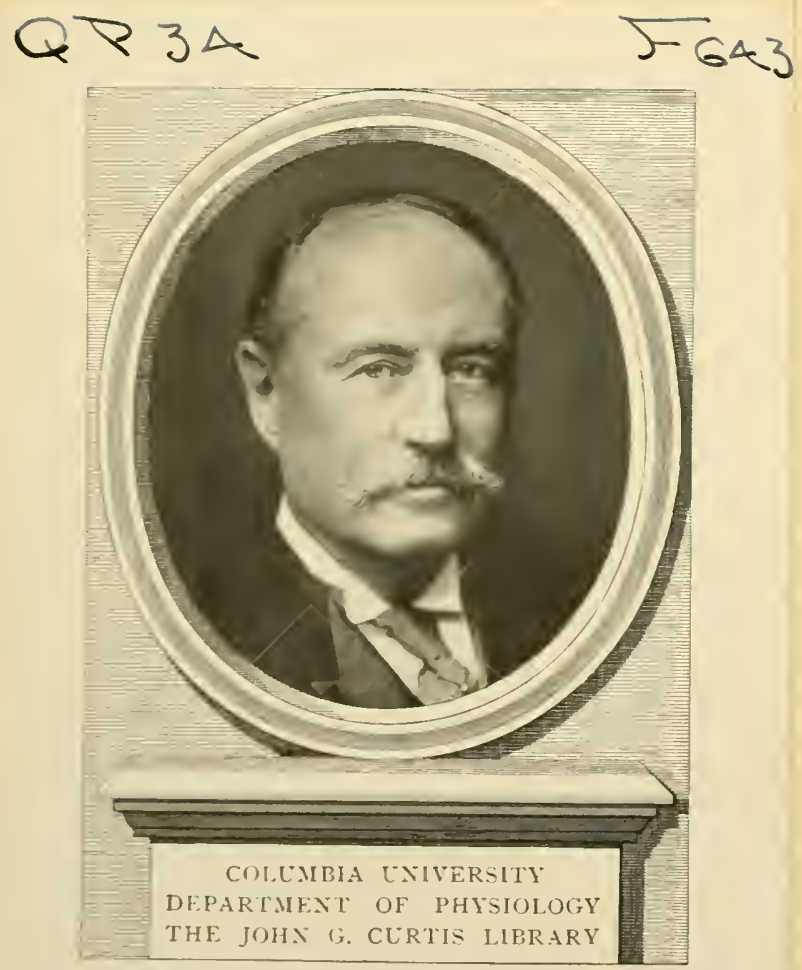
Golm flowits

Qti2d laser. 


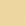


HANDBOOK OF PHYSIOLOGY 


\section{WORKS BY THE SAME AUTHOR}

The Physiology of Man; designed to represent the Existing State of Physiological science as applied to the Functions of the Human Body. Volume I., Introduction; Blood; Circulation; Respiration. I vul., Svo, pp. 500.

The same, Volume II , Alimentation; Digestion; Absorption; Lymph and Chyle. I vol., Sru, Pp. 550.

The same, Volume 1II., Secretion; Excretion; Ductless Glands; Nutrition; Animal Heat; Norements; Voice and Speech. 1 vol., Svo, pp. 520.

The same, Volume IV., The Nervous System. I vol., Sro, pp. 470.

The same, Volume V., Special Senses; Generation. I vol., 8ro, pp. 5 I 7 .

Recherches expérimentales sur une nouvelle fonction du foie, consistant clans la séparation de la cholestérine du sang et son élimination sous forme de stercorine. Paris, Germer Baillière; and New York, D. Appleton \& Company, IS6S. I vol., Svo, pp. I2I. \$0.75.

This work received an "Honorable Mention" with a "Recompense" of I 500 francs from the Institute of France (Acadèmie des Sciences) in I \$69, Concours Montyon (Hédecine et Chirurgie).

On the Physiological Effects of Severe and Protracted Muscular Exercise; with special reference to its Influence upon the Excretion of Nitrogen. 1 $\delta 7$ I. I vol., Svo, cloth, pp. 91. \$1.00.

Manual of Chemical Examination of the Urine in Disease; with brief Directions for the Examination of the most common Varieties of Urinary Calculi. Fifth edition, I 877. I vol., I6mo, cloth, pp. 76. \$I.00.

Collected Essays and Articles on Physiology and Medicine. 2 vols., Sro, pp. 465 and 518 . Set, \$10.00. 


\section{.}



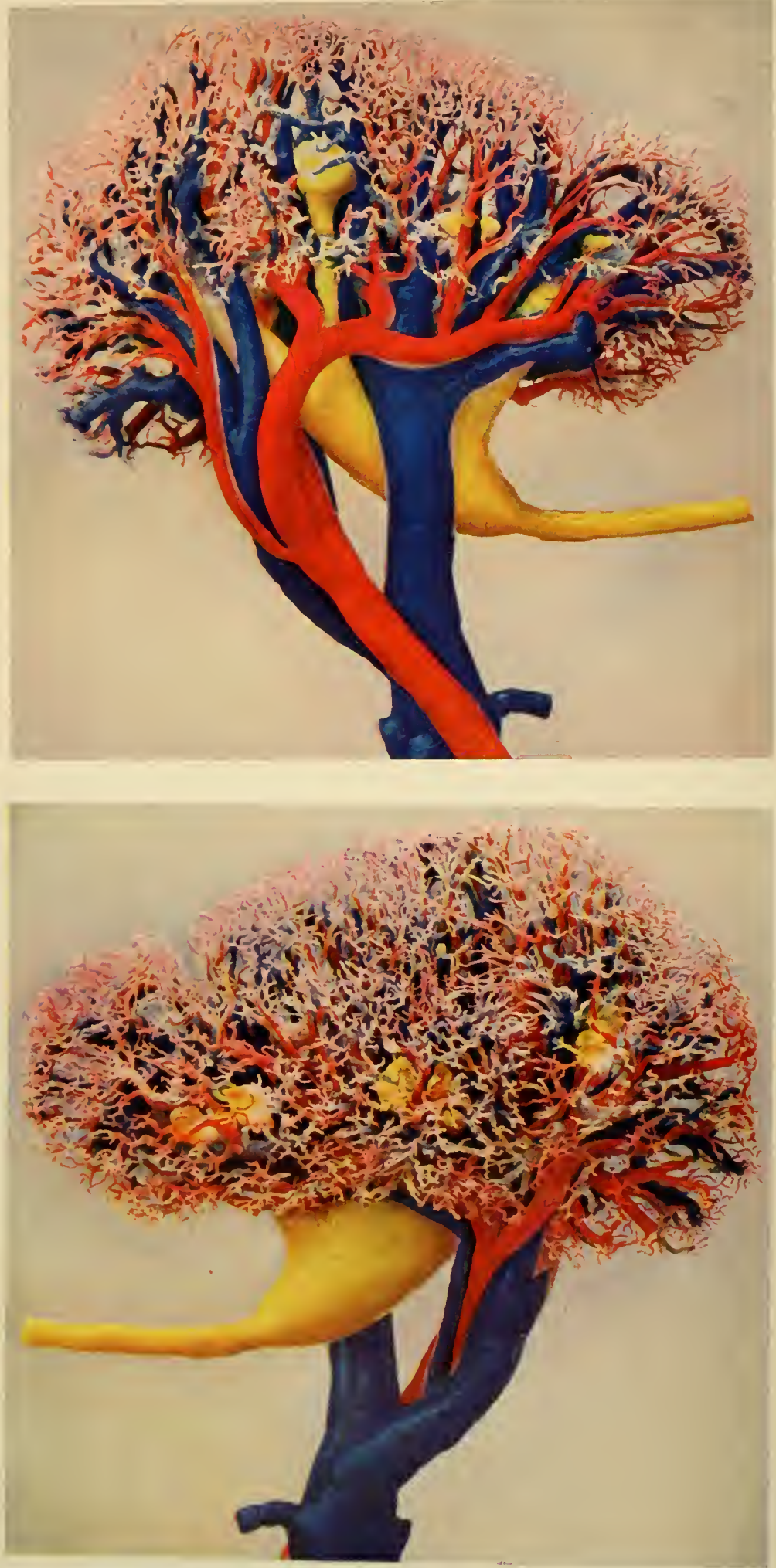

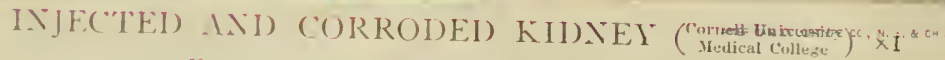


Digitized by the Internet Archive in 2010 with funding from

Open Knowledge Commons (for the Medical Heritage Library project) 



\title{
HANDBOOK OF PHYSIOLOGY
}

\author{
FOR STUDENTS AND PRACTITIONERS \\ OF MIEDICINE
}

BY

AUSTIN FLINT, M.D., LL.D.

PROFESSOR OF PHYSIOLOGY IX THE CORNELL UNIVERSITY MEDICAL COLLEGE; CONSULTING PHYSICIAX TO BELLEVUE HOSPITAL; PRESIDENT OF THE CONSULTING BOARD OF THE MANHATTAY STATE HOSPITAL

FOR THE INSANE; MEMBER OF THE NEW YORK COLNTY MEDICAL ASSOCIATION: FELLOW OF THE NEW YORK STATE MEDICAL ASSOCLATION; MEMBER OF THE AMERICAN MEDICAL ASSOCIATION; HONORARY MEMBER OF THE AMERICAN ACADEMY OF MEDICINE; MEMBER OF THE AMERICAN MEDICO-PSYCHOLOGICAL ASSOCLATION; MEMBER OF THE AMERICAN PHILOSOPHICAL SOCIETY; CORRESPONDENT OF THE ACADEMY OF NATURAL SCIENCES, PHILADELPHIA; FELLOW OF THE AMERICAX ASSOCLATION FOR THE ADVANCEMENT OF SCIENCE; ETC. ETC.

WITH TWO HUVDRED AND FORTY-SEVEN ILLUSTRATIONS IN THE TEXT-INCLUDIVG FOUR IV COLORS AND AN ATLAS OF SIXTEEN COLOR-PLATES, KFCLLDING FORTY-EIGHT ORIGINAL FIGURES TAKEN FROU

X̌eñ 通ark

THE MACMILLAN COMPANY

LONDON : MACMILLAN \& CO., LTD.

1905

All rigbts reserved 


\section{$27-34$ F 643}

COPYRIGHT, 1905,

By THE MACMILLAN COMPANY.

Set up and electrotyped. Published September, 1905. 


\section{PREFACE}

THIS Handbook is the outcome of a desire to present to students a work that may serve to connect pure physiology with the physiology specially useful to physicians; and I have endeavored to adapt it to the curricula of medical schools where the subject is taught in the English language. With this end in view, I undertook the now difficult task of selecting from the vast store of knowledge what I trust may be taken as a fairly symmetrical and comprehensive presentation of human physiology, not too voluminous for students or for ready reference by active practitioners. The subject has been treated from a medical standpoint; not unduly neglecting, it is hoped, pure physiology and biology.

In I875 I published a Text-book of Human Physiology, which was a condensation of a work in five volumes (I866-I874), entitled Physiology of Man. The second and third editions of that work contained such corrections and additions as it was possible to make in the electrotype plates. The book was rewritten for a fourth edition in I888. During the twenty-five years from I 875 to I900, about twentyone thousand copies of the Text-book were distributed by sale. The advances in physiology since I 888 need not be indicated here. Since I 858 I have been actively engaged in teaching physiology to medical students, and since I875 have used my own text-book. About five years ago, however, the book represented my teaching so imperfectly that I felt obliged to use more modern works for class recitations.

This volume represents the instruction in physiology now given at the Cornell University Medical College, somewhat expanded for more extended study and reference. It contains little of the text of former works, except parts relating to descriptive anatomy and established views that have become classic. Few unsettled questions, now under discussion, are considered at length; but I have attempted to present what properly belongs to human physiology in a manner as plain and concise as possible.

Long experience as a teacher of undergraduates has convinced me that students can not be assumed to be sufficiently familiar with the descriptive anatomy and the histology of parts and organs to acquire 
easily a knowledge of their physiology ; and physiological chemistry is too closely connected with physiology to be neglected in a text-book. Again, the ordinary text-books on chemistry and physics contain little information in regard to the modern physical chemistry, ionization, dissociation, osmotic pressure, etc., all of which have important bearings on modern views in regard to physiological processes. While these subjects are by no means exhaustively considered, they are discussed at some length. Embryology might logically precede the study of physiology proper; but the difficulties of this subject are more easily surmounted by advanced than by first-year students, and for that reason it is considered last. Demonstrations, recitations, and laboratory work go hand in hand; but laboratory technique is a subject for practical instruction with the aid of special manuals. Descriptions of laboratory manipulations and illustrations of instruments and apparatus, therefore, are omitted.

I have not thought it desirable to give in extenso minute details of what is called nerve-physiology, and only the general results of such work are presented. Full descriptions of electrical phenomena in nerve and muscle are to be found in laboratory manuals. No considerable account has been taken of mathematical formulæe and calculations involved in certain special studies, such as physiological optics and acoustics. This would assume a knowledge of mathematics on the part of the student which usually does not exist. Still, the special senses are considered quite as fully as seems necessary, except for ophthalmologists and otologists. The same may be said of embryology, a subject too large to be fully presented, except in special treatises. The plan of teaching and the arrangement of subjects do not involve a study of the nervous system in its relations to circulation, respiration, digestion and metabolism until the second year. Consideration of these relations is given mainly in the chapters on the nervous system, and they are briefly mentioned in the first part of the work.

For valuable aid in the important matter of illustration, I am under great obligations to professional friends; but I alone must be held responsible for the text and for the arrangement of subjects.

In the matter of illustration a new departure is made which bids fair to revolutionize this important aid to the study of histology. After more than a year of experimentation, it has been found possible to reproduce, by what is known as the three-color process, stained histological objects, at all magnifications used in such work, as they actually appear under the microscope. This had already been done in 1895I 896 by Dr. Edward Leaming, who photographed the objects, and Mr. Edward Bierstadt, who made gelatin-prints by the three-color process 
of M. Ducos du Hauron; but the cost of reproduction in this way is prohibitory as regards use in ordinary text-books. For several years before $\mathrm{I} 895$, the printing, from ordinary process-plates, of reproductions of colored drawings or lithographs had been accomplished commercially. So far as I know, however, this has not been done before in photomicrography. The difficulties encountered in these efforts were considerable; but they were at last overcome by the skilful and patient coöperation of Dr. Leaming and of the American Colortype Company. Forty-eight illustrations of this kind are in the Atlas at the end of the book. With almost perfect fidelity as regards definition and color, they reproduce the objects used, and they may be studied as one would study with the microscope actual preparations. Of necessity, however, each picture represents a single field focussed for but one plane. The pictures taken from actual objects are supplemented in the Atlas with reproductions, by the same process, of plates selected from the histological atlas of Sobotta. These, though somewhat idealized, are introduced for the reason that they represent difficult objects, and some illustrate in a single page groups of objects that would require many more figures if made from actual specimens. Four figures, showing the first stages of cleavage of the ovum, have been reproduced, without reduction in size, from the original negatives taken by Dr. Leaming for Professor Wilson's Atlas. These are printed in blue, although the original prints were in black and white.

It seemed to me very important to solve the problem of reproducing, at a moderate cost, stained objects in colors, for the reason that photomicrographs in black and white are nearly always inadequate and sometimes misleading. Compare, for example, Fig. I02, p. 464, in the text, with Fig. 4, Plate V, in the Atlas, both showing sections of the pancreas. For useful illustration, indeed, color-figures must be used, in the absence of study of actual objects, if for no other reason than that histological elements are so often differentiated by their affinities for various dyes and can be recognized only in stained preparations. Again, the student seldom sees in his laboratory work the appearances represented in books, even by the best and most costly colored drawings.

The technique employed in the photographic work is given by Dr. Leaming, in a note introductory to the Atlas. The negatives, taken through color-filters, were given, with the objects, to the Colortype Company, and put into the hands of their most intelligent workman, who was instructed first in the use of the microscope. The color-plates were made through a transparent screen with a ruling of one hundred and seventy-five lines to the inch. The etching was done in comparison with the actual objects, which were used as color-guides. This was the 
most difficult part of the process. The printing was done in the same way as in ordinary three-color work. Without the perfect negatives made by Dr. Leaming through absolutely correct color-filters and the very intelligent and skilful work of the Colortype Company, the results obtained would not have been possible, - a fact emphasized by repeated failures during many months of experimentation. The cost of the three-color work has made but an insignificant addition to the price of the book, while, it is believed, the color-illustrations have added greatly to its practical value.

Through the generous interest of the publishers in the manufacture of the book, unusual care was bestowed on the two hundred and fortyseven illustrations in the text. Of this number, one hundred and sixtyfive were selected from three hundred and sixteen that appeared in a previous work. All, however, have been newly reproduced from the originals. Ninety of the one hundred and sixty-five are from Sappey, Bonamy and Beau, and Hirschfeld. These were imported from Paris in I874. They are not only highly illustrative, but are fine examples of engraving on wood, now practically a lost art. Eighty-two figures, including four in colors, are either original or are taken from modern works. The latter could hardly be improved on by new drawings.

Special mention should be made of four figures reduced from photographs in Dalton's Topograplical Anatomy of the Brain, and six figures reduced from photographs in the Atlas of the Karyckinesis and Fertilization of the Oizm, by Professor Edmund B. Wilson, with the coöperation of Dr. Edward Leaming. A small edition of the late Professor Dalton's work was printed in I885. It is now a rare book and little known; but the prints represent the specimens with absolute fidelity, and are quite as useful for purposes of study as actual sections. The prints in Professor WVilson's Atlas are superb and unique. The figures from these works have not before been reproduced.

It may fairly be presumed that a considerable majority of Englishspeaking medical practitioners and students - for whom this work is intended - have been accustomed, by early education and common usage, to English weights and measures. To such readers, the metric system, now commonly used in scientific literature, frequently fails to convey a definite idea without a mental reduction to the familiar standards. For this reason, English weights and measures and the Fahrenheit scale are retained, and metric equivalents are given in parentheses. The micron $\left(\frac{1}{1000}\right.$ of a millimeter, or $2 \frac{1}{5000}$ of an inch), indicated by $\mu$, is frequently employed.

I am much indebted to colleagues and others for aid in preparing illustrations, and extend to them my grateful acknowledgments: 
Dr. James Ewing, Professor of Pathology, and Dr. Jeremiah S. Ferguson, Instructor in Histology, of the Cornell University Medical College, assisted me greatly in the preparation and selection of histological objects. Professor Gage, of Cornell University, furnished the photograph of a human embryo, reproduced in Fig. 226. Dr. Israel Strauss, Instructor in Embryology in the Cornell University Medical College, furnished the sections of chick, reproduced in color in the Atlas. Without such valuable assistance, the illustration would have been more difficult and much less satisfactory.

NEW YoRk,

AUSTIN FLINT.

July, I905. 



\section{CONTENTS}

\section{INTRODUCTION}

The ameba proteus - Protoplasm - The typical animal cell - Karyokinesis (Mitosis) Prophases - Metaphase - Telophases - Amitosis - Ehrlich's side-chain hypothesis - Immunity - Receptors - Passive and active immunity - Cytolysis - Alexins (Complements) - Amboceptors (Immune bodies) - Antibodies - Precipitins - Agglutins

\section{CHAPTER I}

\section{The BLoOD}

Importance of the blood - Quantity of blood - Opacity - Taste - Reaction - Specific gravity - Temperature - Color - Laking of blood - Blood-corpuscles - Red corpuscles - Hemoglobin - Precipitin-test for blood - Development of red corpuscles - Leucocytes - Lymphocytes - Blood-platelets - Plasma and serum - Fibrinogen - Serum-globulin - Serum-albumin - Extractives and salts - Coagulation of the blood - Prothrombin and thrombin - Uses of coagulation

\section{CHAPTER II}

\section{Circulation of the Blood through the Heart}

Discovery of the circulation - Physiological anatomy of the heart - Tricuspid valve Pulmonic valves - Mitral valve - Aortic valves - Movements of the heart - Cardiac cycle - Sounds of the heart - Frequency of the heart's action - Cause of the rhythmical contractions of the heart - Accelerator nerves - Direct inhibition of the heart - Work of the heart.

\section{CHAPTER III}

\section{Circulation of Blood in the Vessels}

Circulation of blood in the arteries - Physiological anatomy of the arteries - Locomotion of the arteries and production of the pulse - Form of the pulse - Pressure of blood in the arteries - Pressure in different arteries - Influence of respiration - Influence of muscular action, etc. - Influence of hemorrhage, etc. - Rapidity of the current of blood in the arteries - Circulation of blood in the capillaries - Physiological anatomy of the capillaries - Pressure of blood in the capillaries - Rapidity of the capillary circulation - Relations of the capillary circulation to respiration - Causes of the capilary circulation - Influence of temperature on the capillary circulation - Circulation of blood in the veins - Structure and properties of the veins - Valves of the veins - Pressure of blood in the veins - Rapidity of the current of blood in the veins - Causes of the venous circulation - Influence of muscular contraction - Influence of aspiration from the thorax - Uses of the valves of the veins - Conditions that impede the venous circulation - Circulation in the cranial carity - Circulation 
in erectile tissues - Derivative circulation - Pulmonary circulation - Circulation in the walls of the heart - Migration and diapedesis - Rapidity of the circulation Phenomena in the circulatory system after death

\section{CHAPTER IV}

\section{RESPIRATORY MOVEMENTS}

Physiological anatomy of the respiratory organs - Movements of respiration - Action of the diaphragm - Action of the muscles which raise the ribs - Scalene muscles Intercostal muscles - Levatores costarum - Auxiliary muscles of inspiration - Expiration - Influence of the elasticity of the pulmonary structure and walls of the chest - Action of muscles in expiration - Internal intercostals - Infracostales - Triangularis sterni-Obliquus externus - Obliquus internus - Types of respiration - Frequency of the respiratory movements - Respiratory sounds - Coughing, sneezing, sighing, yawning, laughing, sobbing and hiccough — Quantity of air changed in the respiratory acts - Diffusion of air in the lungs

\section{CHAPTER V}

\section{Changes which the Air axd the Blood cxdergo in Respiration}

Composition of the air - Consumption of oxygen - Exhalation of carbon dioxide - Influence of age - Influence of sex - Influence of digestion - Influence of diet - Influence of muscular activity - Influence of moisture and temperature - Influence of the season of the year - Relations between the oxygen consumed and the carbon dioxide exhaled - Sources of carbon dioxide in the expired air - Respiratory quotient - Exhalation of watery vapor - Exhalation of ammonia, organic matter etc. - Exhalation of nitrogen - Changes of the biood in respiration - Analysis of the blond for gases - Nitrogen of the blood - Oxygen of the blood - Carbon dioxide of the blood - Respiration by the tissues - Respiratory efforts before birth - Asphyxia .

\section{CHAPTER VI}

\section{Alimentation}

Hunger and thirst - Nitrogenous alimentary substances - Non-nitrogenous alimentary substances - Carbohydrates - Dextrose - Levulose - Galactose - Saccharose Lactose - Maltose - Starch - Glycogen - Cellulose, inosite and gums - The fats : triolein; tripalmitin; tristearin - Saponification - Emulsification - Inorganic alimentary substances - Water - Sodium chloride - Calcium phosophate - Iron Alcohul - Coffee - Tea - Chocolate - Condiments and flavoring articles - The daily ration - Necessity of a varied diet - Meats - Bread - Potatoes - Milk - Eggs

\section{CHAPTER VII}

\section{Mastication, Insalivation and Deglutition}

Physiological anatomy of the organs of mastication - The teeth - Enamel of the teeth - Dentin - Cement - Pulp-cavity — Superior maxillary bones - Inferior maxilla Temporo-maxillary articulation - Muscles of mastication - Saliva - Parotid saliva - Submaxillary saliva - Sublingual saliva - Secretions from the smaller glands of the mouth, tongue and pharynx - Mixed saliva - General properties and compositiun of the saliva - Uses of the saliva - Deglutition - Mechanism of deglutition Protection of the posterior nares during the second period of deglutition - Protection of the opening of the larynx and uses of the epiglottis in deglutition . . . . 163 


\section{CHAPTER VIII}

\section{Gastric Digestion}

Physiological anatomy of the stomach - Peritoneal coat - Mruscular coat - Mucous coat — Glands of the stomach - Closed follicles - Gastric juice - Secretion of gastric

- juice-Quantity of gastric juice - Properties and composition of gastric juice Saline constituents of the gastric juice - Pawlow's experiments on the gastric juice - Action of the gastric juice on meats - Action on albumin, fibrin, casein and gelatin - Action on vegetable nitrogenous substances - Peptones - Action on fats, sugars and amylaceous substances - Duration of stomach digestion - Conditions that influence stomach digestion - Movements of the stomach

\section{CHAPTER IX}

\section{Intestinal Digestion}

Physiological anatomy of the small intestine - Mucous membrane - Intestinal juice Action of the intestinal juice in digestion - Pancreatic juice - Internal secretion by the pancreas-Composition and properties of the pancreatic juice-Action of the pancreatic juice on carbohydrates - Action of the pancreatic juice on proteids Action of the pancreatic juice on fats - Action of bile in digestion - Movements of the small intestine - Physiological anatomy of the large intestine - Ileo-cæcal valve - Peritoneal coat - Muscular coat - Mucous coat - Processes of fermentation in the intestinal canal - Contents of the large intestine - Stercorin - Indol, scatol, phenol, cresol etc. - Movements of the large intestine - Defecation-Gases found in the alimentary canal

\section{CHAPTER $X$}

\section{ABsorption - LyMph AND Chyle}

Absorption by bloodvessels - Absorption by lymphatic and lacteal vessels - Physiological anatomy of the lymphatic and lacteal vessels - Structure of the lymphatic and lacteal vessels - Lymphatic glands - Absorption of proteids by the lacteals - Absorption of sugar and salts by the lacteals - Absorption of water by the lacteals - Absorption by the skin - Absorption by the respiratory surface - Absorption from closed cavities, reservoirs of glands, etc. - Absorption of fats and insoluble substances - Influence of the condition of the blood and of the vessels on absorption-Influence of the nervous system on absorption - Osmosis - Mechanism of the passage of liquids through membranes - Osmotic pressure - Lymph and chyle - Properties and composition of lymph - Corpuscular elements of the lymph - Origin and uses of the lymph - Properties and composition of chyle-Composition of chyle-Microscopical characters of the chyle - Movements of the lymph and chyle .

\section{CHAPTER XI}

\section{SECRETION}

Classification of the secretions - Mechanism of the production of the true secretions Mechanism of the production of the excretions - Influence of the composition and pressure of the blood on secretion - Influence of the nervous system on secretionParalytic secretion by glands - Anatomical classification of glandular organs - Secreting membranes-Follicular glands - Tubular glands - Racemose glands, simple and compound - Ductless, or blood-glands - Secretions and excretions - Synovial membranes and synovia - Mucous membranes and mucus - Mechanism of the secre- 
tion of mucus - Composition and varieties of mucus - General uses of mucus Physiological anatomy of the sebaceous, ceruminous and Meibomian glands - Ordinary sebaceous natter - Smegma of the prepuce and labia minora - Vernix caseosa - Cerumen - Mammary secretion - Mechanism of the secretion of milk - General conditions which modify the lacteal secretion - Properties and composition of milk - Microscopical characters of nilk - Composition of the milk - Colostrum-Lacteal secretion in the newly-born

\section{CHAPTER XII}

\section{Excretion by the Skin}

Physiological anatomy of the skin - Layers of the skin - The corium, or true skin - The epidermis - Physiological anatomy of the nails - Physiological anatomy of the hairs - Roots of the hairs, and hair-follicles - Growth of the hairs - Sudden blanching of the hair - Uses of the hairs - Perspiration - Quantity of cutaneous exhalation Properties and composition of the sweat . . . . . . . .

\section{CHAPTER XIII}

\section{Excretion BY THE KIDNEYS}

Physiological anatomy of the kidneys - Pyramidal substance — Cortical substance — Tubes of the cortical substance - Narrow tubes of Henle - Distribution of bloodvessels in the kidney - Mechanism of the production and discharge of urine - Influence of blood-pressure, the nervous system etc., on the secretion of urine - Physiological anatomy of the urinary passages - Mechanism of the discharge of urine - Properties and composition of the urine - Urea - Origin of urea - Influence of the ingesta on the composition of the urine and on the discharge of nitrogen - Influence of muscular exercise on the discharge of nitrogen - Uric acid and its compounds - Hippuric acid, hippurates and lactates - Calcium oxalate - Xanthin, hypoxanthin, leucin, tyrosin and taurin - Fatty matters - Inorganic constituents of the urine - Chloricles - Sulphates - Phosphates - Water as a product of excretion - Variations in the composition of the urine - Variations with age and sex - Influence of mental exertion - Internal secretion - Work of the kidneys . . . . . . . .

\section{CHAPTER XIV}

\section{Usfes of THE Liver - DuCtless GlaNds}

Physiological anatomy of the liver-Branches of the portal vein, the hepatic artery and the hepatic duct - Interlobular vessels - Structure of a lobule - Arrangement of the bile-ducts in the lobules - Anatomy of the excretory passages - Gall-bladder, cystic and common ducts - Chemistry of the liver - Nerves and lymphatics of the liver Mechanism of the secretion of bile - Quantity of bile - Uses of the bile - Properties and composition of the bile - Biliary salts - Cholesterin and stercorin - Bilirubin Tests for bile - Excretory action of the liver - Origin of cholesterin - Formation of glycogen in the liver - Conditions that influence the quantity of sugar in the blood - Ductless glands and internal secretion - Suprarenal capsules - Cortical substance - Medullary substance - Vessels and nerves - Chemistry of the suprarenal capsules - Addison's disease - The spleen - Fibrous structure - Malpighian bodies - Spleenpulp - Bloodvessels, nerves and lymphatics - Chemical constitution - Variations in volume - Extirpation - Thyroid gland - Structure - Vessels and nerves - Myxœdema - Thymus gland - Pit uitary body and pineal gland - Acromegaly and giantism - Internal secretion by the testes and ovaries . 


\section{CHAPTER XV}

\section{Metabolism - Nutrition - Animal Heat and Force}

Action of glandular cells - Metabolism, anabolism and katabolism - General nutrition Luxus-consumption - Isodynamic values of foods - Animal heat and force - Limits of variations in the normal temperature in man - Variations in different parts of the body - Variations at different periods of life - Variations at different times of the day - Influence of exercise etc., on the heat of the body - Influence of the nervous system on the production of animal heat (heat-centres) - Mechanism of the production of animal heat - Equalization of the animal temperature - Relations of heat to force .

\section{CHAPTER XVI}

\section{MUSCULAR MOVEMENTS}

Amorphous contractile substance and ameboid movements - Ciliary movements - Movements due to elasticity - Elastic tissue- Muscular movements - Contraction of involuntary muscular tissue - Physiological anatomy of the voluntary muscular tissue - Connective tissue - Bloodvessels and lymphatics - Connection of the muscles with the tendons - Chemical composition of the muscles - Physiological properties of the muscles - Elasticity of muscles - Muscular tonicity - Sensibility of the muscles - Muscular contractility and excitability - Muscular contraction - Changes in the form of fibres during contraction - Rigor mortis - Passive organs of locomotion Physiological anatomy of the bones - Lacunæ - Canaliculi - Bone-cells or corpuscles - Marrow of the bones - Periosteum - Physiological anatomy of cartilage —Cartilage-cavities - Cartilage-cells — Elastic cartilage and fibro-cartilage . .

\section{CHAPTER XVII}

\section{VOICE AND SPEECH}

Physiological anatomy of the vocal organs - Muscles of the larynx - Crico-thyroid muscles-Arytenoid muscle - Lateral crico-arytenoid muscles - Thyro-arytenoid muscles - Mechanism of the production of the voice - Movements of the glottis during phonation - Action of the intrinsic muscles of the larynx in phonation - Action of accessory vocal organs - Laryngeal mechanism of the vocal registers - Vocal registers in the male-Vocal registers in the female - Mechanism of speech-Vowels - Consonants - The phonograph and telephone .

\section{CHAPTER XVIII}

\section{Structure and Properties of the Nervous System}

Divisions and structure of the nervous tissue - Medullated nerve-fibres - Non-medullated nerve-fibres - Gelatinous nerve-fibres (fibres of Remak) - Accessory a natomical elements of the nerves - Branching and course of the nerves - Termination of nerves in voluntary muscles - Termination of nerves in glands - Modes of termination of sensory nerves - Corpuscles of Vater, or of Pacini - Tactile corpuscles - End-bulbs - General mode of termination of the sensory nerves - Structure of the nerve-centres - Nerve-cells - Nissl's granules - The neuron - Accessory anatomical elements of the nerve-centres - Degeneration and regeneration of nerves - Motor and sensory nerves - Mode of action of the motor nerves - Associated movements - Mode of action of the sensory nerves - Physiological differences between motor and sensory 
nerves-Nervous excitability and conductivity - Rapidity of nervous conduction Personal equation - Action of electricity on the nerves - Law of contraction Electric current from the exterior to the cut surface of a nerve - Electrotonus, anelectrotonus and catelectrotonus

\section{CHAPTER XIX}

\section{Spinal Nerves - Motor Cranial Nerves}

Spinal nerves - Cranial nerves - Motor oculi communis (third nerve) - Physiological anatomy - Properties and uses of the motor oculi communis - Patheticus, or trochlearis (fourth nerve) - Physiological anatomy - Properties and uses of the patheticus - Motor oculi externus, or abducens (sixth nerve) - Physiological anatomy - Properties and uses of the motor oculi communis - Nerve of mastication (the small, or motor root of the fifth nerve) - Physiological anatomy - Properties and uses of the nerve of mastication - Facial, or nerve of expression (seventh nerve) - Physiological anatomy - General properties of the facial - Uses of branches of the facial given off within the aqueduct of Fallopius - Uses of the chorda tympani - Influence of certain branches of the facial on the movements of the palate and uvula - Uses of the external branches of the facial - Spinal accessory (eleventh nerve) - Physiological anatomy - Properties and uses of the spinal accessory - Uses of the internal branch from the spinal accessory to the pneumogastric - Influence of the internal branch of the spinal accessory on deglutition - Influence of the spinal accessory on the heart - Uses of the external, or muscular branches of the spinal accessory - Sublingual (twelfth nerve) - Physiological anatomy - Properties and uses of the sublingual .

\section{CHAPTER XX}

\section{Trifacial Nerve - PNeumogastric Nerve}

Trifacial (large root of the fifth nerve) - Physiological anatomy - Properties and uses of the trifacial - Immediate effects of division of the trifacial - Remote effects of division of the trifacial - Pneumogastric (tenth nerve) - Physiological anatomy - Properties and uses of the auricular nerves - Properties and uses of the pharyngeal nerves - Properties and uses of the superior laryngeal nerves - Properties and uses of the inferior, or recurrent laryngeal nerves - Properties and uses of the cardiac nerves Depressor nerve - Properties and uses of the pulmonary nerves - Effects of division of the pueumogastrics on respiration - Effects of faradization of the pneumogastrics on respiration - Properties and uses of the œesophageal nerves - Properties and uses of the abdominal nerves - Influence of the pneumogastrics on the liver-Influence of the pneumogastrics on the stomach and intestines - Effects of faradization - Influence of section of the pneumogastrics on the movements of the stomach - Influence of the pneumogastrics on the small intestine . . . . . . . .

\section{CHAPTER XXI}

\section{THE SPINAL CORD}

Membranes of the encephalon and spinal cord-Cephalo-rachidian liquid - Physiological anatomy of the spinal cord - Columns of Türck - Crossed pyramidal tracts - Anterior ground columns - Lateral bundles - Ascending and descending cerebellar fasciculi-Direct cerebellar fasciculi-Columns of Burdach-Columns of GollDirections of nerve-fibres in the cord - General properties of the spinal cord - Relations of the posterior white columns of the cord to muscular coördination Nerve-centres in the spinal cord - Reflex action of the spinal cord - Reflexes in man 


\section{CHAPTER XXII}

\section{The Cerebrum and the Basal Ganglia}

Weights of the encephalon and of certain of its parts - The cerebral hemispheres - Cerebral convolutions - Basal ganglia - Corpora striata, optic thalami and internal capsule - Tubercula quadrigemina - Crura cerebri - Puns Varolii - Directions of fibres in the cerebrum - Fibres connecting the cerebrum with the cerebellum - Fibres connecting the two sides of the brain - Fibres connecting different cerebral convolutions on the same side (association fibres) - Fibres connecting the brain with the spinal cord - Cerebral localization - Motor cortical zone (Rolandic area) - General uses of the cerebrum - Extirpation of the cerebrum - Comparative development of the cerebrum in the lower animals - Development of the cerebrum in different races of men and in different individuals - Facial angle - Pathological observations Reaction-time - Centre for the expressions of ideas in language . . . . 565

\section{CHAPTER XXIII}

\section{The Cerebellum and the Bulb}

The cerebellum - Physiological anatomy - Extirpation of the cerebellum - Pathological observations - The bulb - Physiological anatomy - Uses of the bulb - Nerve-centres in the bulb - Respiratory centre - Vital point (so callea) - Rolling and turning movements following injury of certain parts of the encephalon (forced movements) .

\section{CHAPTER XXIV}

\section{Sympathetic System - Sleep}

Cranial ganglia - Cervical ganglia - Thoracic ganglia - Ganglia in the abdominal and the pelvic cavities - General properties of the sympathetic ganglia and nerves Direct experiments on the sympathetic - Vasomotor centres and nerves - Reflex vasomotor phenomena - Vaso-inhibitory nerves - Trophic centres and nerves Sleep - Dreams - Condition of the brain and nervous system during sleep . .

\section{CHAPTER XXV}

\section{Sense of Touch - Olfaction - Gustation}

Muscular sense - Sense of touch - Appreciation of temperature - Olfaction - Olfactory (first nerve) - Properties and uses of the olfactory nerves - Mechanism of olfaction - Relations of oifaction to the sense of taste - Gustation - Nerves of taste - Chorda tympani-Glosso-pharyngeal (ninth nerve) - General properties of the glossopharyngeal - Relations of the glosso-pharyngeal to gustation - Mechanism of gustation - Physiological anatomy of the organs of taste - Taste-beakers

\section{CHAPTER XXVI}

\section{THE ORGAN OF VISION}

Optic (second nerve) - General properties of the optic nerres - Physiological anatomy of the eyeball - Sclerotic coat - Cornea - Choroid coat - Ciliary processes - Iris Pupillary membrane - Retina - Layer of rods and cones (Jacob's membrane, or bacillar membrane) - Crystalline lens - Suspensory ligament of the lens (zone of Zinn) - Aqueous humor - Vitreous humor - Sumnary of the anatomy of the globe of the eye . 


\section{CHAPTER XXVII}

\section{REFRACTION IN THE EYE-ACCOMMODATION}

Refraction in the eye - Certain laws of refraction, dispersion etc., bearing on the physiology of vision - Refraction by lenses - Spherical monochromatic aberration - Chromatic aberration - Formation of images in the eye - Visual purple and visual yellow and accommodation of the eye for different degrees of illumination - Mechanism of refraction in the eye - Astigmatism - Movements of the iris - Direct action of light on the iris - Accommodation of the eve for vision at different distances - Changes in the crystalline lens in accommodation - Changes in the iris in accommodation Erect impressions produced by images inverted upon the retina - Field of indirect vision - Binocular vision - Corresponding points - The horopter - Duration of luminous impressions (after-images) - Irradiation . . . . . . . . 6

\section{CHAPTER XXVIII}

\section{Movenents of the Eyeball - Parts for Protection of the Eye}

Action of the recti muscles - Action of the oblique muscles - Associated action of the muscles of the eyeball - Centres for vision - Perception of colors - Parts for the protection of the eyeball - Muscles that open and close the eyelids - Conjunctival mucous membrane - The lachrymal apparatus - The tears

\section{CHAPTER XXIX}

\section{Audition}

Auditory (eighth nerve) - General properties of the auditory nerves - Topographical anatomy of the parts essential to the appreciation of sound - The external ear General arrangement of parts composing the middle ear - General arrangement of the bony labyrinth - Physics of sound - P'itch of musical sounds - Nusical scale Quality of musical sounds - Harmonics, or overtones - Harmony - Discords and dissonance - Tones by influence - Uses of different parts of the middle ear - Structure of the membrana tympani-Uses of the membrana tympani-Mechanism of the ossicles of the ear - Physiological anatomy of the internal ear - General arrangement of the membranous labyrinth - Liquids of the labyrinth - Distribution of the nerves in the labyrinth - Organ of Corti-Uses of different parts of the internal ear - Uses of the semicircular canals - Uses of the parts contained in the cochleaCentres for audition . . . . . . . . . . . . .

\section{CHAPTER XXX}

\section{EMBRYOLOGY}

Female organs of generation - The ovaries - Graafian follicles - The parovarium - The uterus - The Fallopian tubes - Structure of the ovum - Discharge of the ovum Passage of ova into the Fallopian tubes - Puberty and menstruation - Changes in the Graafian follicles after their rupture (corpus luteum) - Corpus luteum of pregnancy - Male organs of generation - Interstitial gland of the testis - Vas deferens — Vesiculæ seminales - Prostate - Glands of the urethra - Nale element of generation - Spermatozoids 


\section{CHAPTER XYXI}

\section{Fertilization and Karyokinesis of the OveM}

Fecundation - Naturation of the ovum - Fertilization of the ovum - Mendel's laws of heredity - Superfecundation - Segmentation of the ovum - Gastrulation - Primitive streak - Formation of the membranes - Formation of the amnion - Amniotic liquid - Formation of the umbilical vesicle (yolk-sac) - Formation of the allantois and permanent cherion - Membranæ deciduæ - Formation of the placenta - Uses of the placenta

\section{CHAPTER XXXII}

\section{DevelopMent of the OVuM}

Development of the cavities and layers of the trunk in the chick - Development of the skeleton, muscular system and skin - Notochord - Vertebral column etc. - Development of the nervous system-Development of the digestive system-Development of the respiratory system-Derelopment of the face-Development of the teeth Development of the genito-urinary apparatus - Development of the urinary system - Development of the external organs of generation - Development of the circulatory system - The first, or vitelline circulation - The second, or placental circulation - Deyelopment of the heart - Peculiarities of the foetal circulation - The third, or adult circulation

\section{CHAPTER XXXIII}

\section{Fetal Life - Development after Birth - Death}

Duration of pregnancy - Size, weight and position of the foetus - Multiple pregnancy Cause of the first contractions of the uterus in normal parturition - Involution of the uterus - Meconium - Dextral preëminence - Development after birth - Ages Death 



\section{LIST OF TEXT-ILLUSTRATIONS}

(For list of color-plates, see page $86_{5}$.)

FIG.

I. Ameba proteus (Sedgrick and Wilson)

2. Diagram of a cell (IVilson)

3. Diagram showing the prophases of karyokinesis (Wilson)

4. Diagram of the later phases of karyokinesis (Wilson)

5. Six stages of karyokinesis of the sea-urchin (Wilson)

7. Diagram of a cell with receptors

8. Diagram of a toxic molecule

9. Diagram showing free receptors .

IO. Diagram showing the alexin combined with the receptor . . . . . . II

II. Diagram showing the anticomplement

12. Diagram showing the anti-immune body

13. Blood-corpuscles of the Guinea pig (Sternberg) .

14. Blood-corpuscles of certain of the inferior animals

15. Human blood-corpuscles in rouleaux (Stratford)

I6. Heart in situ (Dalion)

17. Muscular fibres of the auricles (Bonamy and Beau).

18. Heart, anterior view (Bonamy and Beau)

19. Heart, left cavities (Bonamy and Beau)

20. Heart, right cavities (Bonamy and Beau)

2x. Muscular fibres of the ventricles (Bonamy and Beau)

22. Valves of the heart (Bonamy and Beau)

23. Trace of Vierordt

24. Trace of Marey .

25. Small artery and capillaries from the muscular coat of the urinary bladder of a frog (from a photograph taken at the United States Army MTedical MTuseum)

26. Venous radicles uniting to form a small vein, from the muscular coat of the urinary bladder of a frog (from a photograph taken at the United States Army Mledical MIuseum)

27. Migration of leucocytes and diapedesis of red corpuscles (Councilman)

28. Trachea and bronchial tubes (Sappey')

29. Lungs, anterior view (Sappey)

3o. Bronchia and lungs, posterior view (Sappey)

3I. Thorax, anterior view (Sappey).

32. Thorax, posterior view (Sappey').

33. Diaphragm (Sappey) .

34. Section of a molar tooth of man (Sobotta).

35. Muscles of the pharynx (Sappey)

36. Longitudinal fibres of the stomach (Sappey')

37. Fibres seen with the stomach everted (Sappey)

3. Pits in the mucous membrane of the stomach and openings of the glands (Sappey). 
FIG.

39. Glands in the greater pouch of the stomach (Heidenhain)

40. Pyloric glands (Ebstein)

41. Gastric fistula in the case of St. Martin (Beaumont). .

42. Stomach, liver and small intestine (Sappey) . . .

43. Intestinal tubules (Sappey)

44. Axial section of a villus of a dog (Kultschitzky)

45. Patch of Peyer (Sappey)

46. Patch of Peyer seen from its attached surface (Sappey)

47. The pancreas; its direction, relations and two excretory ducts (Sappey)

48. Stomach, pancreas and large intestine (Sappey').

49. Crystals of stercorin

50. Origin of lymphatics (Landois)

51. Lymphatic plexus, showing the endothelium (Belaieff)

52. Superficial lymphatics of the skin of the palmar surface of the finger (Sappey).

53. Deep lymphatics of the skin of the finger (Sappey').

54. Same finger, lateral view, showing lymphatic trunks connected with the superficial network (Sappey)

55. Superficial lymphatics of the arm (Sappey)

56. Superficial lymphatics of the leg (.Sappey).

57. Stumach, intestine and mesentery, with the mesenteric bloodvessels and lacteals (Asellius) .

58. Thoracic duct (Mascagni).

59. Valves of the lymphatics (Sappey)

6o. Lymphatics and lymphatic glands (Mascagni) .

61. Sebaceous glands (Sappey).

62. Meibomian glands of the upper lid (Sappey)

63. Human miik-globules from a healthy woman eight days after delivery (Funke).

64. Colostrum from a healthy lying-in woman twelve hours after delivery (Funke) .

65. Anatomy of the nails (Sappey).

66. Section of a nail (Sappey).

67. Hair and hair-follicle (Sappey).

68. Rout of the hair (Sappey) .

69. Surface of the palm of the hand, a portion of skin about one-half inch ( 2.5 millimeters) square (Sappey')

70. Longitudinal section of the kidney (Sappey)

71. Longitudinal section of the pyramidal substance of the kidney of the fotus (Sappey')

72. Longitudinal section of the cortical substance of the same kidney (Sappey)

73. Section of collecting tubes of the kidney (Author's collection) .

74. Diagram of the structure of the kidney (Landois)

75. Transverse section of a single hepatic lobule (Sappey)

79. Thyroid and thymus glands (Sappey).

8o. Ciliated epithelium (Engelmann)

81. White fibrous tissue; one end of the bundle has been teased to display the component fibrillæ (Piersol).

82. Elastic fibres isolated; from the adventitia of the aorta (Piersol, after Schiefferdecker)

83. Portion of the elastic tissue of the intima of the human aorta; the fibres are so broad and so closely grouped that they constitute an elastic sheet - fenestrated membrane of Henle - (Piersol)

84. Muscular fibres from the urinary bladder of the human subject (Sappey) . 
FIG.

85. Muscular fibres from the aorta of the calf (Sappey).

86. Muscular fibres from the uterus of a woman who died in the ninth month of uterogestation (Sappey)

87. Vascular canals and lacunæ seen in a longitudinal section of the humerus (Sappey)

88. Vascular canals and lacunæ seen in a transverse section of the humerus (Sappey) .

89. Vertical section of diarthrodial cartilage (Sappey) . . . . . . . 433

90. Vertical section of the human larynx, showing the vocal chords (Sappey) . . 436

91. Posterior view of the muscles of the larynx (Sappey) . . . . . . . 437

92. Lateral view of the muscles of the larynx (Sappey) . . . . . . . . 438

93. Glottis seen with the laryngoscope during the emission of high-pitched sounds (Le Bon) . . . . . . . . . . . . . . 440

94. Appearance of the vocal chords in the production of the chest-voice (Mandl) $\quad 446$

95. Appearances of the vocal chords in the production of the falsetto voice (Mills) $\quad 447$

96. Medullated nerve-fibres (Piersol) . . . . . . . . . . 458

97. Gold-stained axis-cylinder (Piersol) . . . . . . . . . . . . . 459

98. Nodes of Ranvier and lines of Fromann (Ranvier). . . . . . . 460

99. Fibres of Remak (Robin) . . . . . . . . . . . 460

100. Branching of a nerve in the abdominal muscle of a mouse (Sobotta) . . . 462

I01. Motor end-plates from a muscle of a lizard (Sobotta) : . . . . . 463

I02. Transverse section of a corpuscle of Vater (Author's collection) . . . . 464

I03. Longitudinal section of a corpuscle of Vater (Sappey) . . . . . . 465

I04. Tactile corpuscle from the human corium (Böhm and Devidoff) . . . . 465

105. Corpuscle of Krause from the human conjunctiva (Dogiel) . . . . . 466

I06. Papillæ of the skin of the palm of the hand (Sappey) . . . . . . . 467

107. Unipolar cell from the Gasserian ganglion (Schwalbe) . . . . . . 468

I08. Unipolar cell with a spiral fibre; bipolar nerve-cell (Landois). . . . . . 469

109. Sympathetic ganglion-cell from man (Key and Retzizes) . . . . . . . 470

I Io. Cell from the electric lobe of the torpedo (Schultze) . . . . . . $47 \mathrm{I}$

1II. Nerve-cells, glia-cells and neuroglia from the spinal cord of the calf (Lavdowsky) * 472

II2. Diagram of a nerve-cell to show Nissl granules $(R \cdot y$ Cajal $) . \quad . \quad . \quad . \quad .472$

II3. Method of testing excitability in electrotonus (Landois) . . . . . . 490

114. Cervical portion of the spinal cord (Hirschfeld) . . . . . . . . . 493

II5. Dorsal portion of the spinal cord (Hirschfeld) . . . . . . . . . 493

I16. Inferior portion of the spinal cord, and cauda equina (Hirschfeld) . . . . 493

117. Roots of the cranial nerves (Hirschfeld) . . . . . . . . . . 495

I18. Distribution of the motor oculi communis (Hirschfeld) . . . . . . . 496

I19. Distribution of the patheticus (Hirschfeld) . . . . . . . . 500

I 20. Distribution of the motor oculi externus (Hirschfeld) . . . . . . . jOI

I2I. Distribution of the small root of the fifth nerve (Hirschfeld) . . . . . 503

122. Superficial branches of the facial and the fifth (Hirschfeld) . . . . . 506

123. Chorda-tympani nerve (Hirschfeld) . . . . . . . . . . 5 Io

124. Spinal accessory nerve (Hirschfeld). . . . . . . . . . . $5^{13}$

125. Distribution of the sublingual nerve $($ Sappey $)$. . . . . . . . . . $5: 9$

126. Principal branches of the large root of the fifth nerve (Robin) . . . . $5^{22}$

127. Ophthalmic division of the fifth (Hirschfeld) . . . . . . . . 523

128. Superior maxillary division of the fifth (Hirschfeld). . . . . . . . 524

129. Inferior maxillary division of the fifth (Hirschfeld) . . . . . . . 525

130. Anastomoses of the pneumogastric (Hirschfeld) . . . . . . . $53 \mathrm{I}$

131. Distribution of the pneumogastric (Hirschfeld) . . . . . . $\quad .533$

132. Transverse section of the spinal cord of a child six months old, at the middle of the lumbar enlargement, treated with potassium auric chloride and uranium nitrate (Gerlach) . 
FIG.

I33. Diagram of the columns of the cord

134. View of the structures displayed on the right side of a median longitudinal section of the brain - semidiagrammatic

I35. Vertical section of the cerebral cortex $(R . y$ Cajal).

136. Cells of the molecular layer of the cerebral cortex (R.y Cajal)

137. Diagrammatic section through the cerebral cortex ( $R \cdot y^{\prime}$ Cajal)

138. Cells with short neurites in the cerebral cortex (R.y Cajal) .

139. Lateral surface of the brain (Dalton)

140. Median section of the brain (Dulton)

I4I. Horizontal section of the brain (Dalton).

142. Vertical section of the brain (Dalton) . . . . . . . . 576

143. Diagrammatic representation of the direction of some of the fibres of the cerebrum (Le Bon)

I44. Motor cortical zone (Exner) .

145. Diagram of a median section of the brain

146. Diagram of certain motor cortical areas.

I47. Cerebellum and bulb (Hirschfeld)

148. Section of a cerebellar lamina perpendicular to its axis (R.y Carjal)

149. Section of a cerebellar lamina parallel to its axis $(R, y$ Cajal $) . \quad . \quad . \quad . \quad 595$

150. Cell of Purkinje (Leaming) . . . . . . . . . . . . 596

151. Anterior view of the bulb $($ Sappey $)$. . . . . . . . . . . 599

152. Floor of the fourth ventricle (Hirschfeld) . . . . . . . . . 600

153. Cervical and thoracic portions of the sympathetic (Sappey') . . . . . 608

I54. Lumbar and sacral portions of the sympathetic $($ Sappey'). . . . . . . 609

155. Map showing the relative distribution of the sensibility to touch, warmth and cold in the paim of the left hand (Golischeider) . . . . . . 630

156. Olfactory ganglion and nerves (Hirschfeld) . . . . . . . . 632

157. Vertical section of the olfactory membrane of an executed criminal - Golgi method (Zimmermann and Sobotti)

158. Glosso-pharyngeal nerve (Sappey)

159. Papillæ of the tongue (Sappey')

160. Medium-sized circumvallate papilla $\left(S_{a t h e y}\right){ }^{\circ} \cdot{ }^{\circ}$

16r. Fungiform, filiform, and hemispherical papillæ (Sappey) . . . . . . 643

162. Transverse section of a taste-bud from a rabbit (Sobotta). . . . . . 645

163. Vertical section of a taste-bud (Sobotta) . . . . . . . . . . 645

164. Optic tracts, conmissure and nerves (Hirschfeld) . . . . . . . 647

165. Diagram of the decussation of fibres at the optic commissure . . . . . 648

166. Choroid coat of the eye $($ Sappey') . . . . . . . . . 65I

167. Ciliary muscle $($ Sappey $)$. . . . . . . . . . . . . . 653

168. Diagram of the retina (Kallius) . . . . . . . . . . 658

r69. Bloodvessels of the retina (Loring) . . . . . . . . . . 660

170. Crystalline lens, anterior view (Babuchin) . . . . . . . . 66r

17I. Section of the crystalline lens (Babuchin) . . . . . . . . . 66r

172. Zone of $\operatorname{Zinn}\left(S_{a f p e y}\right)$. . . . . . . . . . . . . $66_{3}$

173. Diagrammatic section of the eye . . . . . . . . . 665

174. Refraction by convex lenses . . . . . . . . . . . 67I

175. Achrumatic lens . . . . . . . . . . . . . 674

176. Section of the lens etc., showing the mechanism of accommodation (Fick) . . 688

177. Field of vision of the right eye, as projected by the patient on the inner surface of a hemisphere, the pole of which forms the object of regard - semidiagrammatic (Landolt) .

178. Binocular field of vision (Förster) 
I79. Muscles of the eyeball (Sappey)

I80. Diagram illustrating the action of the muscles of the eyeball (Fick) . . . 70 I

rSI. Lachrymal and Meibomian glands (Sappey) . . . . . . . . 708

182. Lachrymal canals, lachrymal sac and nasal canal, opened by their anterior portion (Sappey)

183. The pinna (Sappey).

184. Posterior view of a mold in wax of the cavity of the concha and the external auditory meatus (Sappey).

185. General view of the organ of hearing (Sappey)

186. Ossicles of the tympanum (modified from Riudinger-)

187. The right temporal bone, the petrosal portion removed, showing the ossicles seen from within. From a photograph (Rïdinger) . . . . . .

188. The left bony labyrinth of a new-born child, forward and outward view. From a photograph (Rüdinger)

I89. Resonators of Helmholtz .

190. Right membrana tympani, seen from within. From a photograph and slightly reduced (Rï̈dinger)

191. Diagram of the labyrinth. From a photograph and slightly reduced (K'ïdinger) .

192. Otoliths from various animals (K'̈̈dinger)

193. Section of the first turn of the spinal canal of a cat newly born. Section of the cochlea of a human fœtus at the fourth month. From a photograph and slightly reduced (Rï̈inger) .

194. Distribution of the cochlear nerve in the spiral lamina. The cochlea is from the right side and is seen from its antero-inferior part (Sappey) $\quad$. . . . 747

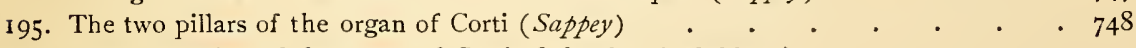

196. Vertical section of the organ of Corti of the dog (Waldeyer) . • . . • . 749

197. Uterus, Fallopian tubes and ovaries, posterior view (Sappey) . . . • • 755

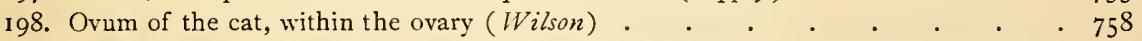

199. Virgin uterus; anterior view; median longitudinal section; transverse longitudinal

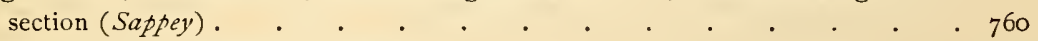

200. Muscular fibres of the uterus $($ Sappey) . . . . . . . . . . . . . . 761

201. Superficial fibres of the anterior surface of the uterus (Liegeois) . . . $\quad$. 762

202. Inner layer of the muscular fibres of the uterus (Liégeois) . . . . . $\quad 7^{63}$

203. Uterine and utero-ovarian veins $($ Sappey') $. \quad . \quad . \quad . \quad . \quad . \quad . \quad .764$

204. Fallopian tube (Williams, after Sappey) . . . . . . . . . . . . 764

205. External erectile organs of the female (Liëgeois) . . . . . . . . $\quad$. 765

206. Deutoplasm-forming ovum from a Graafian follicle of a woman twenty-seven years

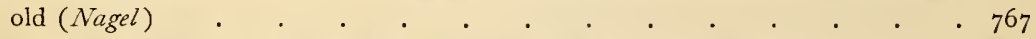

207. Ovum from a Graafian follicle of a woman thirty years old ( Nagel) . . . . $\quad$. 768

208. Primordial ovum, with two germinal vesicles and follicular epithelium; from the

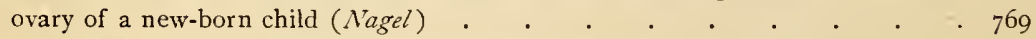

209. Portion of an ovary, showing a corpus luteum of pregnancy (Itilliams) . $\quad . \quad 775$

210. Lobes of the testicle and epididymis (Sappey $)^{\prime}$. . . . . . . . . . 777

211. Interstitial gland of the testicle (Bouin and Ancel). . . . . . . $\quad 779$

212. Vesiculæ seminales, vasa deferentia and ejaculatory ducts (Sappey) . . . . 7 So

213. Spermatozoids, spermatic crystals and leucocytes (Peyer) . . . . . . . $7{ }^{S_{4}}$

214. Human spermatozoids (Retzius and Jensen) . . . . . . . . . $78_{4}$

215. Diagram showing stages of spermatogenesis as seen in different sectors of a seminiferous tubule of a rat (MCM Murrich, after Leuhossek) . . . . . . . . $7 \mathrm{C}_{5}$

216. Germ-nuclei of the thread-worm - ascaris megalocephala (Boveri). . . 790

2I7. Karyokinesis (Boveri) . . . . . . . . . . . . 791

218. Cell-division (IVilson) . 
FIG.

219. Formation of the blastodermic vesicle (van Beneden)

220. Four stages in segmentation of the ovum of a mouse (Sobotta). . . . $\quad 797$

221. Later stages of segmentation of the ovum of a bat (van Beneden) • . . 798

222. Embryonic shicld of a rabbit, showing the primitive streak and the medullary groove above (Kollnann) . . . . . . . . . . . .

223. Transverse section of the embryonic area of a dog's ovum of about fifteen days (Bonnet)

224. Five diagrammatic representations of the formation of membranes in mammalia (Kölliker).

225. Embryo of twenty to twenty-five days (Coste) . . . . . . .

226. Human embryo of the fuurth to the fifth week - prepared by Dr. G. R. Corson, of Savannah, Ga. (Gage).

227. Seventeen days' gravid uterus - from the Anatomical Museum of Johns Hopkins University - embryo drawn relatively too large (IVilliams) . . . . 812

228. Early stage of development of the chick (Seboth and Brïcke). . . . . 817

229. Transverse section near the head (Seboth and Brï̈cke) . . . . . . 817

230. Later stage of development of the chick (Seboth and Brïcke). . . . 818

231. The first six cervical vertebræ of the embryo of a rabbit (Kobin) . . . . 819

232. Human embryo about one month old, showing the large size of the head and upper parts of the body, the twisted form of the spinal column, the rudimentary condition of the upper and lower extremities and the rudimentary tail at the end of the spinal column (Dalton). . . . . . . . . . . 820

233. Development of the nervous system of the chick ( Vagner) . . . . . $\quad 823$

234. Development of the spinal cord and brain of the human subject (Tiedemann) $\quad 824$

235. Fœtal pig, showing a loop of intestine forming an umbilical hernia (Dalton) . $\quad 827$

236. Formation of the bronchial ramifications and of the pulmonary cells (liathke and

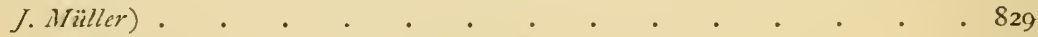

237. Mouth of a human embryo of twenty-five to twenty-eight days (Coste) . . $\quad 830$

238. Mouth of a human embryo of thirty-five days (Coste) . . . . . . 83I

239. Mouth of a human embryo of forty days (Coste) . . . . . . . $83 \mathbf{I}$

240. Temporary and permanent teeth (Sappey) . . . . . . . . 834

24r. Fœtal pig $\frac{5}{8}$ of an inch (16 millimeters) long (Dalton) . . . . . . 836

242. Diagrammatic representation of the genito-urinary system (Henle) . . . $\quad 838$

243. Area vasculosa of a rabbit of ten days (van Beneden and Julin) . . . . 840

244. Area vasculosa of a rabbit of eleven days (van Beneden and Julin) . . . 84r

245. Arterial arches in man and mammals (modified from Rathke) . . . . $\quad$. 844

246. Diagram of the fotal circulation (Kollmann) . . . . . . . . 847

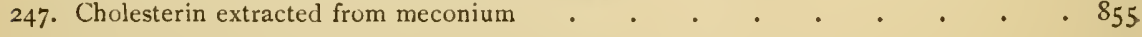




\section{PART I}

INTRODUCTION - THE BLOOD - CIRCULATION - RESPIRATION - ALIMENTATION - DIGESTION-ABSORPTION-SECRETION AND EXCRETION - METABOLISM - NUTRITION ANIMAL HEAT 



\section{INTRODUCTION}

The ameba proteus - Protoplasm - The typical animal cell - Karyokinesis (Mitosis) --

Prophases - Metaphase - Anaphase - Telophases - Amitosis - Ehrlich's side-chain hypothesis - Immunity - Receptors - Passive and active immunity - Cytolysis - Alexins (Complements) - Amboceptors (Immune bodies) - Antibodies - Precipitins - Agglutins.

The Ameba Protens. - It seems natural and logical, as an introduction to human physiology, to begin with the ameba proteus. This is the simplest form of distinct and independent animal existence, and it closely resembles the typical animal cell. This rhizopod is practically

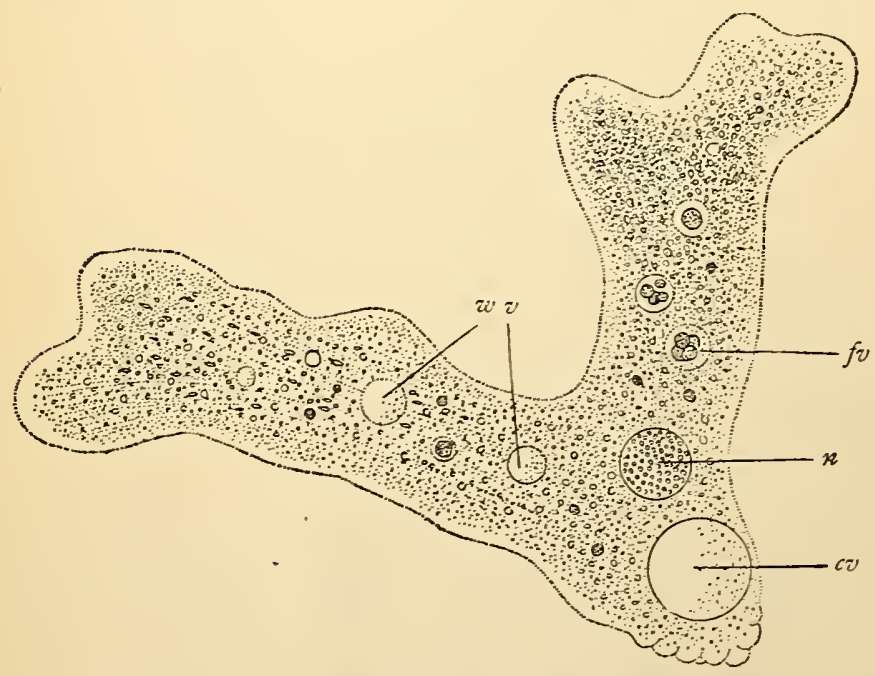

Fig. I. - Ameba proteus, $\times 280$ (Sedgwick and Wilson).

$n$, nucleus; $w v$, water-vacuoles; $c \nu$, contractile vacuole; fo, food-vacuole.

Ectoplasm and pseudopods are not shown in this figure.

a single cell without a distinct investing membrane. It is capable of appropriating nutrient matters and of multiplication. It is little more than a mass of protoplasm, a name derived from the Greek words $\pi \rho \hat{\omega} \tau o s$ (first) and $\pi \lambda a^{\prime} \sigma \mu a$ (form) applied to the substance of many of the cells and tissues of adult animals high in the scale.

The ameba is a mass of contractile matter, with a nucleus, fine and coarse granules, and cavities called vacuoles, some of which are contractile. In addition, there are vacuoles that contain granules of 
nutritive matter, called food-vacuoles, and others called water-vacuoles. The ameba itself is surrounded with protoplasm (ectoplasm), which, by alternate extension and contraction of processes called pseudopods, enables it to move slowly from place to place. These creeping movements are observed in some animal cells and are called ameboid.

Protoplasm. - The various substances known collectively as protoplasm constitute the basis of life, both vegetable and animal. It is composed mainly of carbon, hydrogen, oxygen and nitrogen; but the composition of its molecule is constantly changing, and consequently it has no fixed and definite chemical composition. In this respect it is in a condition known as labile. In studying the structure and composition of animal cells, it will be seen that the general term "protoplasm," aside from the so-called proteids, includes many substances known under different names intended to express, more or less accurately, different properties and relations. The typical animal cell, indeed, is composed of many of these substances called by different names.

The Typical Animal Cell. ${ }^{1}$ - Figure 2 represents diagrammatically an animal cell. This is somewhat more complex in its structure than the ameba. Its basis is a clear protoplasm (hyaloplasm) contained in the meshes of a reticulum (spongioplasm) with minute granules (microsomes), the whole being called cytoplasm. In the cytoplasm are little globules (plastids) and usually, also, dark granules or globules of variable size (metaplasm). The most important structure in the cytoplasm is the "attraction-sphere," containing two little bodies called centrosomes. The cell itself is surrounded with a delicate membrane composed of condensed hyaloplasm. In the cell, near its centre, is a rounded nucleus with a highly refracting nucleolus, the nucleus being surrounded with a membrane and presenting a very fine reticulum formed of "linin" fibres, ${ }^{2}$ that are not seen, however, unless brought out by reagents. The nucleus also contains relatively coarse threads of chromatin, a substance chemically identical with nuclein. The substance of the nucleus is sometimes spoken of as the linin. A collection of chromatin also is observed in the nucleus, called the karyosome. The substance of the nucleus is called karyoplasm, or linin. The cell itself

I The apparent logical necessity of studying the cell as an introduction to human physiology is, perhaps, to be regretted for one reason; and that is a redundancy of nomenclature, always somewhat confusing. Wilson, in his work on The Cell, gives a glossary with two hundred different names that have been applied to parts of the cell and its processes of origin, development and multiplication, only seventeen of which are classed as obsolete. I have striven, however, to minimize this difficulty by adopting, so far as possible, only names that are in common use and to be found in modern works on anatomy.

${ }^{2}$ Linin-elements may be seen in cells fixed with Flemming's solution and stained with picrocarmin. Poljakoff, "Biologie der Zelle," Archiv für mikroscopische Anatomie, Bonn, 1900, Bd. LVI, S. 651 et seq. 
usually presents one or more vacuoles. The uses and properties of these various parts will appear in the study of karyokinesis (mitosis), or indirect cell-division; and the structures just described are observed in what is known as the "resting" cell.

All animal tissues, organs and systems are composed of cells derived originally from a single female cell, the ovum, fertilized by union with a male cell, and are produced by indirect cell-division. "It has been estimated that the number of cells entering into the composition of the body of an adult human being is about $26,500,000,000,000,000$ "

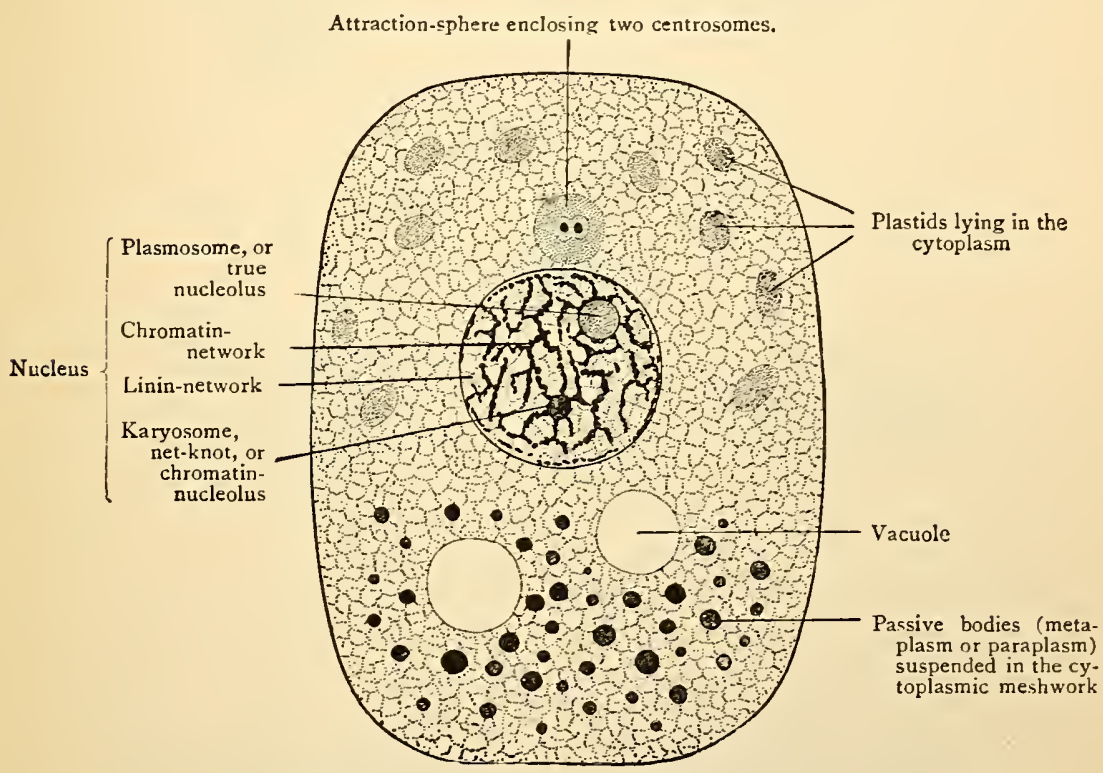

Fig. 2. - Dingram of a Cell (Wilson).

Its basis consists of a thread-work (mitome, or reticulum) composed of minute granules (microsomes) and traversing a transparent ground-substance.

(McMurrich). In the adult the cells appropriate different substances, organic and inorganic, become specialized and are arranged into different tissues and organs possessing varied functions. The one function or property, however, which all cells have in common, is that of assimilating nutritive matters to supply loss by the constant changes characteristic of living structures. The changes that involve the breaking down of living substance are known as katabolic. The process of repair is called anabolism.

\section{KARYOKINESIS (MITOSIS)}

Prophases. - Indirect cell-division begins with a separation of the attraction-sphere into two, each one carrying a centrosome, or polar 
body. The two spheres separate from each other and send out radiating filaments, forming a double star, called the amphiaster. They are connected together, however, by filaments in the form of a spindle
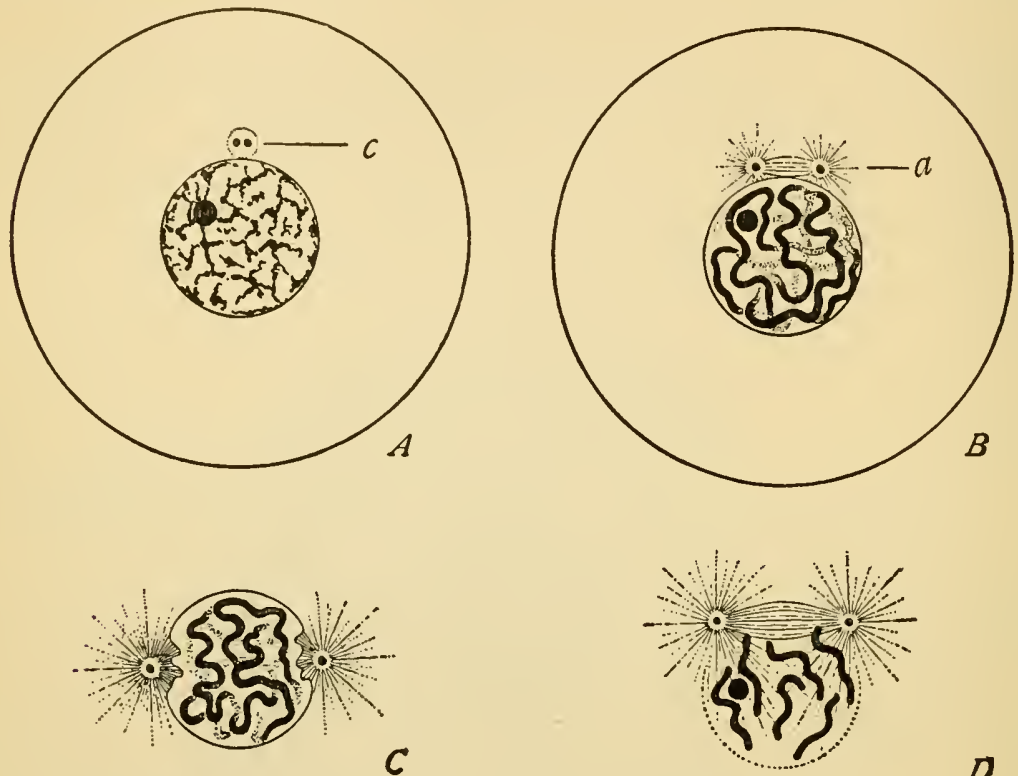

$D$
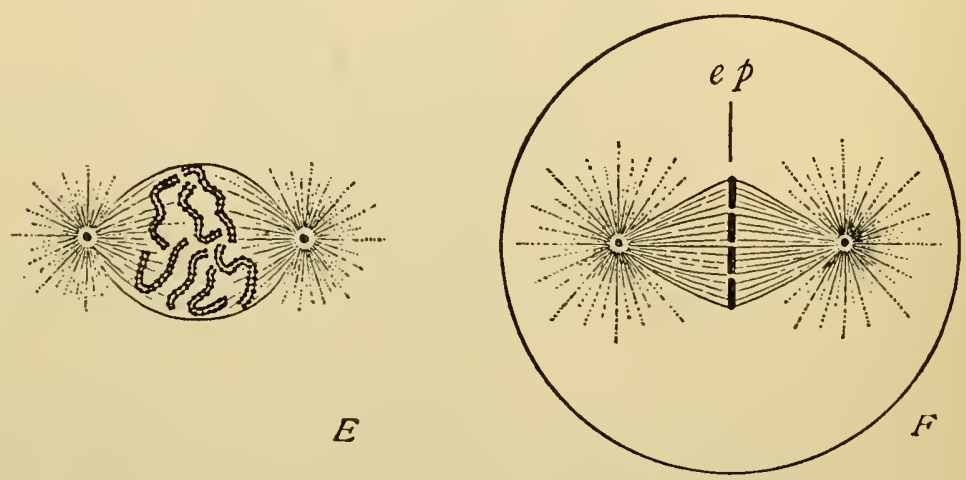

Fig. 3. - Diagram showing the prophases of karyokinesis (Wilson).

$A$, resting-cell : $c$, attraction-sphere containing two centrosomes. $B$, early prophase, the nucleus containing a continuous spirem, the nucleolus still present: $a$, amphiaster. $C$, disappearance of the primary spindle and passage of the centrosomes to opposite poles of the nucleus (the nucleolus has disappeared). $\quad D$, another type of prophase, in which the primary spindle persists. $E$, later prophase, in which a new spindle is formed within the nucleus. $F$, completion of the karyokinetic figure: $e p$, equatorial plane of chromosomes.

(the central spindle), which lies next the nucleus, gradually encroaching upon it. The substance of the amphiaster and the spindle is called archoplasm. As the spindle is formed, the membrane of the nucleus 
disappears and the threads of chromatin become thicker. Sometimes the original spindle remains; but often it disappears and is replaced with a new spindle formed within the nucleus. In many cells the chromatin during the formation of the amphiaster assumes the form of a loose skein between the two attraction-spheres, called the spirem. This is described * by some writers as the spirem stage.

The threads of chromatin now separate into a number of shorter threads called chromosomes, which arrange themselves in the equator of the spindle, forming the equatorial plate. This completes the formation of what is known as the mitosic or karyokinetic figure. The changes just described constitute the prophases of karyokinesis. All embryologists agree that after the division of the chromatic threads the number of resulting chromosomes for each species of plant or animal is fixed and characteristic; and this is true after division of all its cells (Wilson). The number in man is thought to be sixteen; in sharks it is thirty-six; and in ascaris it is four or even two. Wilson gives a list of forty-seven species in which the number has been noted by various investigators. It is a curious fact, also, that the number is always divisible by two. In fertilization, the number in the male and in the female pronucleus is reduced one-half; so that the cleavage-nucleus, which results from the union of the two pronuclei, contains the same number of chromosomes as did the original ovarian nucleus.

Metaphase. - The prophases just described are preparatory to the processes resulting in cell-division, which begin with a splitting of each chromosome lengthwise into two. This is called metakinesis, or the metaphase.

Anaphase.-The arrangement of the chromosomes in the equatorial plane of the spindle is different in different species of animals. In many instances they take the form of the letter $\mathrm{V}$ and are arranged in a cluster with their angles directed inward, so that the group forms a sort of star. With this arrangement, the cell is in what is called the monaster stage, and this stage often comes before the splitting of the threads. Following this, the chromosomes pass one-half each, to the attraction-spheres, around which they are grouped preparatory to the formation of the daughter-nuclei. This is called by some the diaster stage. In this stage, filaments pass between the two masses of chromosomes (interzonal fibres) and form the central spindle. The grouping of the chromosomes around the two attraction-spheres marks the anaphase. It is probable that in most cells the fibres of the central spindle are of new origin and are not derived from the spindle of the amphiaster. Lying in the equatorial plane of the central spindle is a group of granules, constituting the mid-body. 
Some writers describe what is called the dispirem stage, in which the daughter-chromosomes unite into a single thread in the form of a loose skein around each attraction-sphere. In any event, after the formation of the diaster, all begin to divide, entering on what are called telophases.

Telophases. - The changes that follow the dispirem stage are simple, and constitute the final processes of karyokinesis (telophases). Each
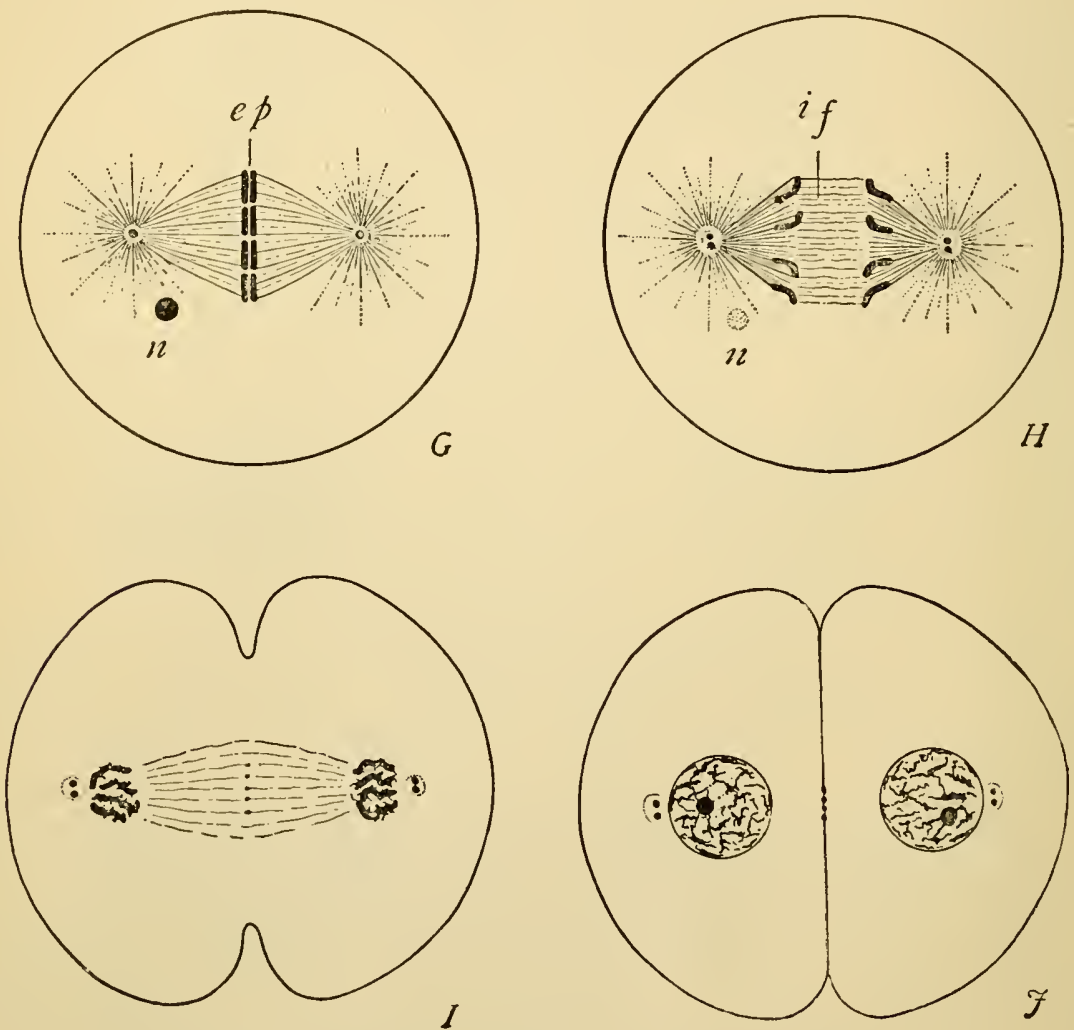

Fig. 4. - Diagram of the later phases of karyokinesis (Wilson).

$G$, metaphase: $e p$, splitting of the chromosomes; $n$, the cast-off nucleolus. $H$, anaphase: if, interzonal fibres, or central spindle, between the diverging daughter-chromosomes. $I$, telophase, showing beginning division of the cell-body, and the mid-body in the equatorial plane of the spindle. $J$, division completed.

collection of chromosomes becomes surrounded with cytoplasm and finally with a membrane; and thus two daughter-nuclei are formed by division of the original nucleus of the parent-cell. The chromosomes now constitute the chromatic substance of the daughter-nuclei, a highly refracting nucleolus is developed and a new attraction-sphere with two centrosomes makes its appearance in the cytoplasm. The parent-cell has become 
A

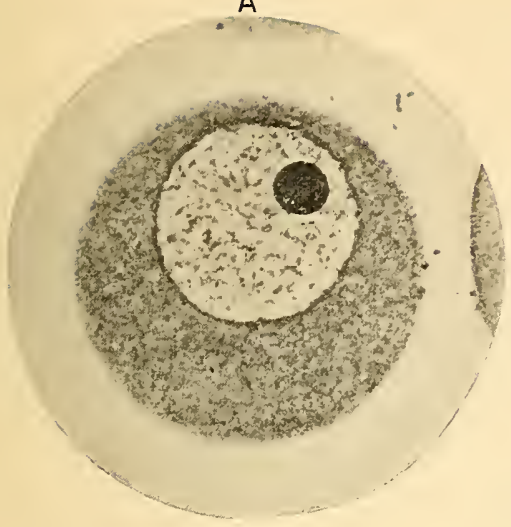

C

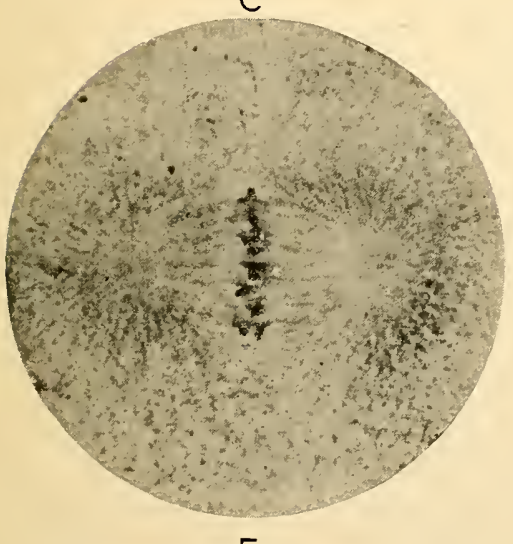

E

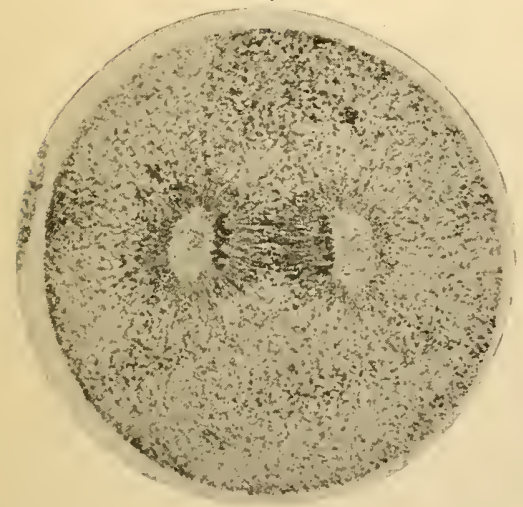

B

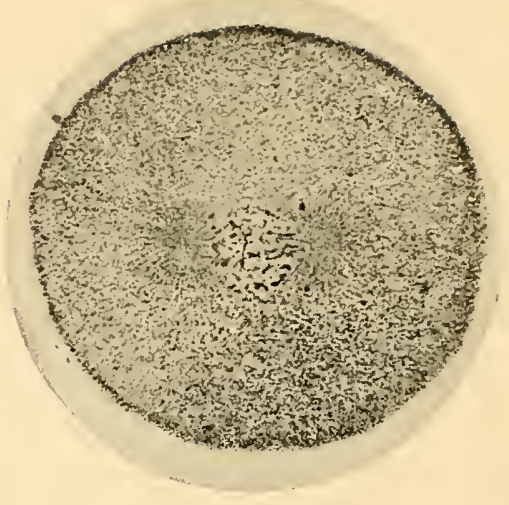

D

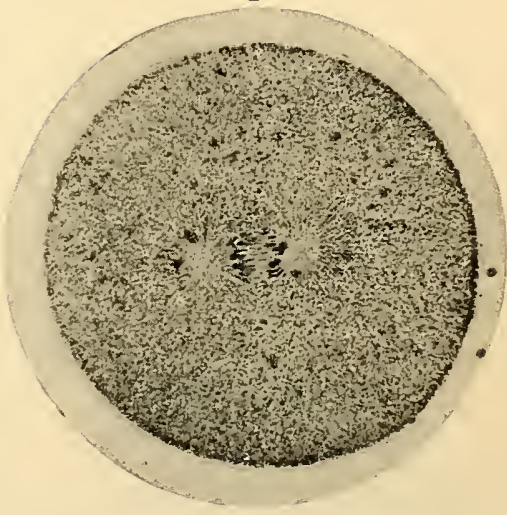

F

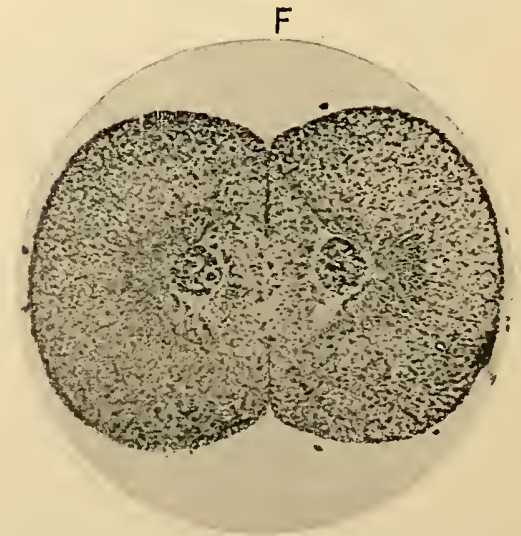

Fig. 5. - Six phases of karyokinesis of the egg of the sea-urchin (Wilson). 
completely divided; and the daughter-cells are themselves capable of karyokinetic division, and so on until the cells thus produced lose this power and die of old age.

It is possible for a single observer to watch the process of karyokinesis through all its phases, which is found to occupy half an hour to three hours; but the different stages are more easily studied in hardened and stained preparations.

Figure 5 is a reproduction, reduced one-half, of plates selected from the superb Atlas of the Fertilization and Karyokinesis of the Ovum, by Professor Edmund B. Wilson, of Columbia University. These are faith$\mathrm{ful}$ reproductions of photographs of the actual processes in the naked egg of the sea-urchin (Toxopneustes variegatus), all magnified in the original 1000 diameters except $C$, which is magnified 3000 diameters.

$A, \times 500$, is the ovarian egg, before maturation, with the germinal vesicle (nucleus) and the germinal spot (nucleolus); $B, \times 500$, shows the formation of the asters and the origin of the spindle (one of the late prophases); $C, \times \mathrm{I} 500$, shows the splitting and separation of the chromosomes (metaphase); $D, \times 500$, shows the beginning divergence of the daughter-chromosomes (early anaphase); $E, \times 500$, shows complete divergence of the daughter-chromosomes (final anaphase) $F, \times 500$,

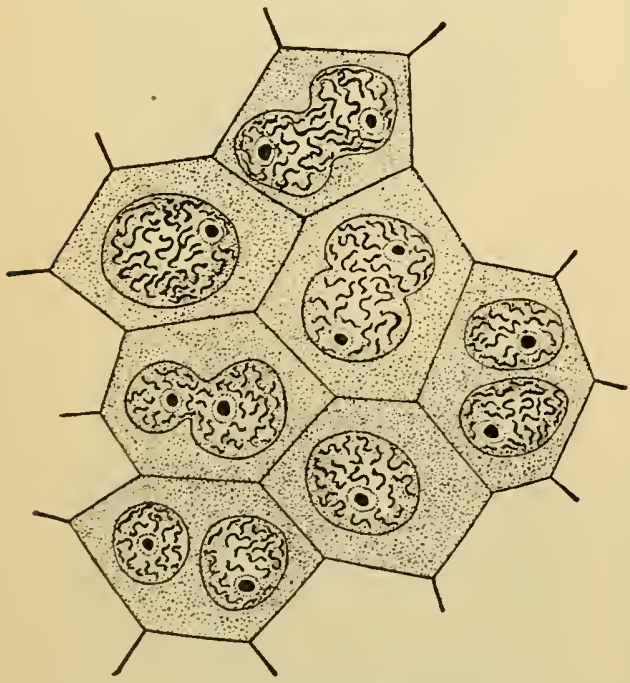

Fig. 6.-Groups of cells with amilotically dividing nuclei (Wilson, after Wheeler).

shows division of the egg nearly completed and re-formation of the daughter-nuclei (telophase).

Amitosis. - Exceptionally, certain cells undergo direct, or amitotic division, there being no formation of chromosomes, spirems or spindles, and the centrosomes taking no part in the process. In amitosis the nuclei enlarge and there is a considerable increase in its chromatin. The nuclei then become constricted and finally divide into two, the cell itself afterward following this division.

The physiological significance of amiosis is obscure; and the process has been the subject of much study and speculation. The chief point of interest, concerning which there is considerable doubt, is the relation of the centrosome and the attraction-sphere to this process; but it is the opinion of most embryologists that the nucleus alone is involved, 
the centrosome being inactive, except, perhaps, in amitosis of spermatids and some leucocytes. At present the most reasonable view of amitosis is that cells dividing in this way are in process of degeneration and are approaching the end of division of any kind.

\section{Ehrlich's Side-chain Hypothesis}

This hypothesis is supposed by some physiologists to account for the processes involved in the nutrition and multiplication of animal cells. It may be diagrammatically illustrated by supposing that the cell sends out from its surface little prominences in the form of a side chain. These hypothetical prominences are called receptors, although their

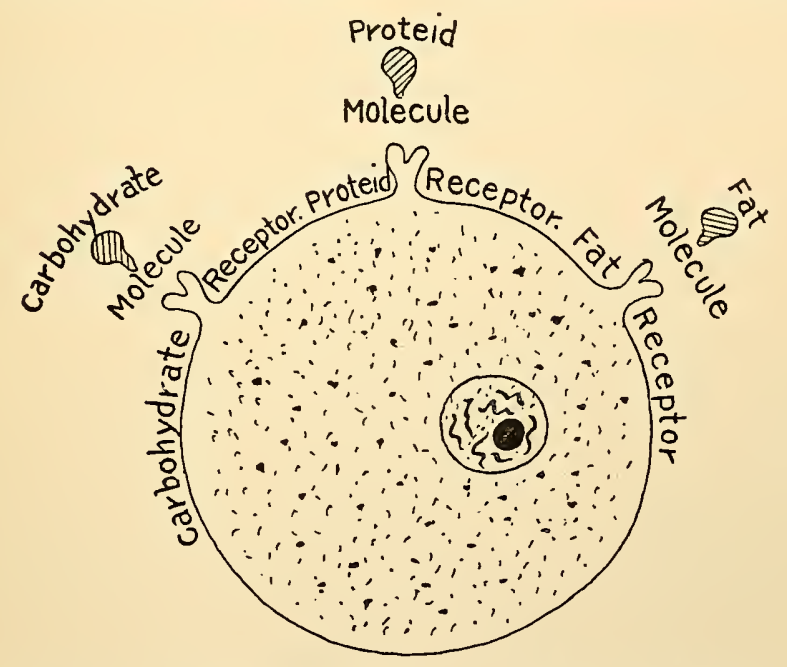

Fig. 7. - Animal cell with three receptors, one each for carbohydrates, proteids and fats.

form has been inferred rather than seen. The different receptors have affinities for special nutrient matters; for example, there are certain receptors that have an affinity for proteid molecules, others for carbohydrates, and others for fats. When the affinity of a special receptor is satisfied by the appropriation of the special molecule with which it is combined, a new receptor is sent out, with the same affinity, and so on until the nutritive requirements of the cell are satisfied. The same process goes on between the carbohydrate molecules and the fat molecules and their appropriate receptors; and thus the cell is nourished.

\section{IMMUNITY}

The side-chain hypothesis leads naturally to a theory of the destruction of cells by the action of toxins; and this, to a theory of 
immunity. A toxic molecule may be assimilated through a special receptor, either damaging or destroying the cell; but if there exist no receptors that have an affinity for a certain toxin, the cell is immune to that special toxic molecule. A toxic molecule is supposed to have two groups of affinities: I. a toxophorous group, which does damage to the cell; 2 . a haptophorous, or combining group, which had an affinity for its special receptor. Figure 8 represents diagram-

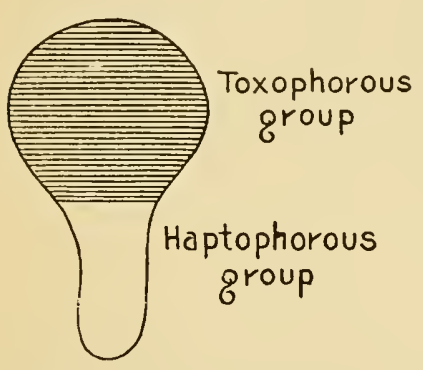

Fig. 8. - Diagram of a toxic molecule. matically a toxic molecule. The shaded portion is the toxophorous group, and the clear portion, the haptophorous group. These toxic molecules may unite with their special receptors and be thrown off by the cell into the blood-current. When this occurs, the toxins do not reach the cell; but their affinities are neutralized and they become innocuous. If, however, the toxic molecules are too abundant to be thus disposed of, they may enter the cell through the appropriate receptors and destroy it. The throwing off of combined receptors and toxins in this way is a protective process, but it is limited.

Receptors. - When cells are attacked by toxins, there is a great over-production of receptors, and many of them are thrown off without having formed a union with toxic molecules. Thus, receptors floating in the blood still have an affinity for special toxins, which they break up and render innocuous. In this way they protect the cells, acting as antitoxins. This process is roughly illustrated in Figure 9.

Passive and Active Immunity. - What is known as passive immunity is secured by introclucing into the body of an animal a serum containing a protective substance that acts as an antitoxin. The antitoxin consists of receptors that have been formed in excess and thrown off into the blood-serum, which thereby becomes antitoxic. These antitoxic receptors combine with and neutralize the toxins. Passive immunity is produced promptly but soon passes away. It is called passive immunity for the reason that the immunizing bodies have been produced by another animal, the animal thus rendered immune being passive and simply receiving the immunizing receptors. Active immunity is a much slower process and is more lasting. In this form of immunity the animal produces its own antitoxin. Receptors unite with toxic molecules, are thrown off into the blood, become innocuous and are eliminated through the emunctories. In addition, new receptors (antitoxins) are produced in large excess (over-production) and are thrown off by the cells into the blood. They thus exist in the blood, ready to neu- 
tralize the special toxins for which they have an affinity. Such an animal is in a condition of active immunity, having itself produced the immu-

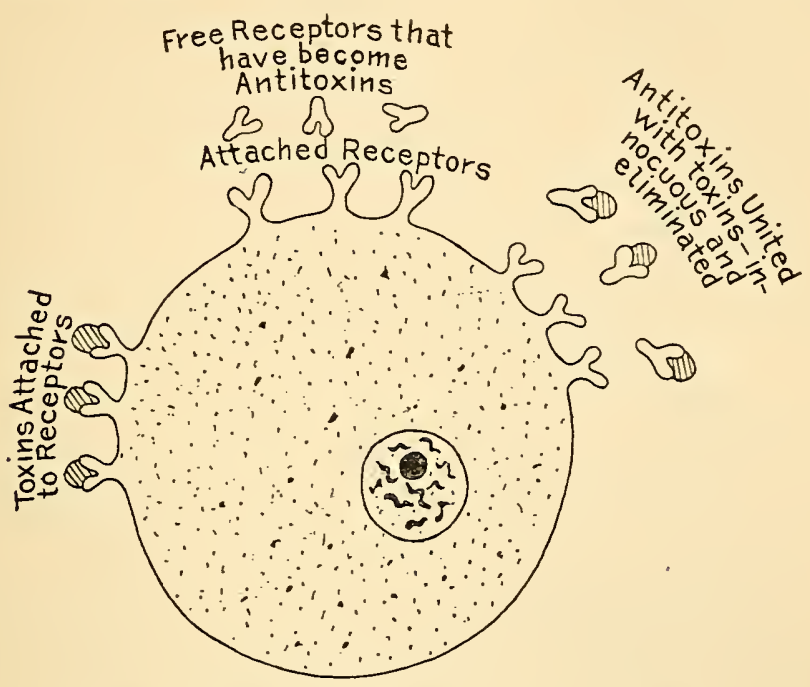

Fig. 9. - Toxins attached to receptors; free receptors (antitoxins); antitoxins united with toxins.

nizing agents; it has a serum, however, that may be introduced into another animal, rendering it passively immune. Both active and passive immunity are antitoxic, as distinguished from bacteriolytic immunity, in which latter, bacteria are dissolved and destroyed.

Cytolysis. - The serum of one species of animal injected into the vessels of an animal of different species may dissolve the red corpuscles of the second animal. This is called laking of blood; and the serum is then said to be lytic. This, however, is not true of the Guinea pig and the rabbit; but the serum of the Guinea pig may be adapted to the blood of the rabbit and rendered lytic for such blood. This is called hemolysis; and the Guinea pig's serum which has been rendered lytic for rabbit's corpuscles is lytic for these and for no other corpuscles.

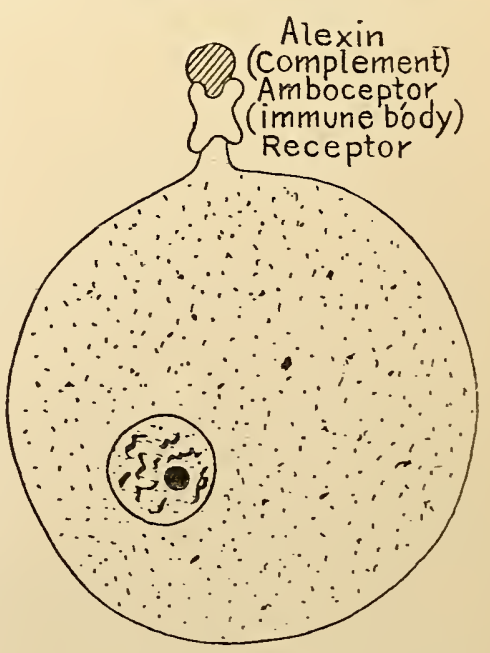

Fig. Io. - Alexin (complement) combined with the receptor through the amboceptor (immune body').

Alexins (Complements). - The lytic properties of the Guinea pig's serum for rabbit's corpuscles may be neutralized by exposing it for half 
an hour to a temperature of $56^{\circ} \mathrm{C}$. (about $133^{\circ} \mathrm{Fahr}$.) ; but the lytic power may be restored by adding fresh serum of the normal Guinea pig. These experimental facts are thought to show that there are two substances in lytic blood, which act together: one of these substances is stable or active at $56^{\circ} \mathrm{C}$., and the other, which exists in normal serum, becomes inert at $56^{\circ} \mathrm{C}$. The stable substance - active at $56^{\circ} \mathrm{C}$. - is the so-called immune body (amboceptor). The unstable substance, inert at $56^{\circ} \mathrm{C}$., is the alexin. The immune body may be obtained free from alexin by the application of heat. The alexin may be obtained, by certain processes, free from the immune body. In cytolysis, however, the

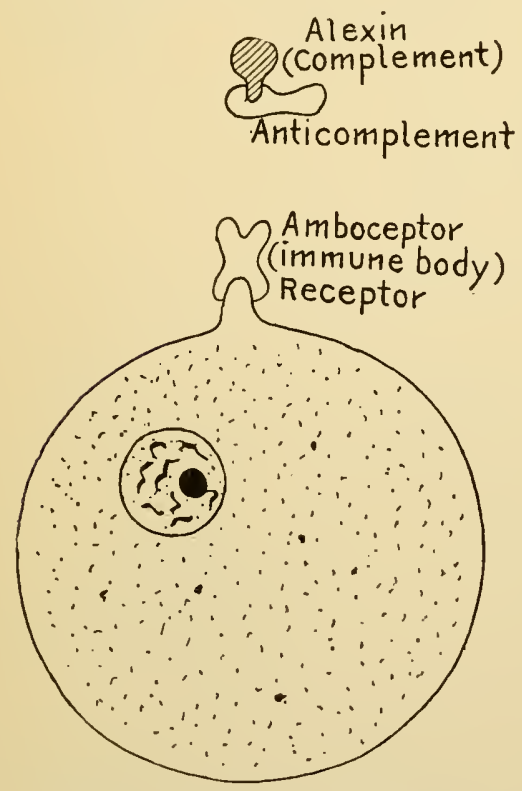

Fig. II. - Anticomplement protecting the receptor by uniting with the complement (alexin).

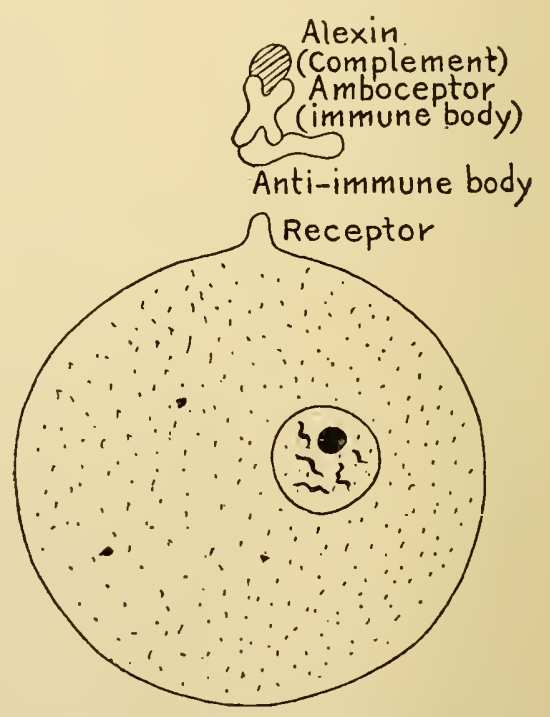

Fig. I2. - Anti-immune body protecting the receptor by uniting with the immune body.

two bodies - alexin (complement) and the immune body - must act together. It is assumed that the alexin cannot combine directly with receptors, but that it can combine with the immune body and through it with the receptors.

Immnne Bodies (Amboceptors). - The immune body has two affinities, one for the complement and one for the receptor; and it is on account of this property that it is called the amboceptor. Figure ro is a rough illustration of this theory. The alexin is often called the complement, and the immune body (amboceptor), the interbody.

Antibodies. - Experiments have shown that in the body of an animal in which serum containing alexins and amboceptors has been 
introduced, the cells produce what are called antibodies, which secure immunity. The antibody (anticomplement) may have a dominating affinity for the complement, unite with it and prevent the union of the complement with the amboceptor; or it may unite, in the same way, with the amboceptor and prevent its union with the receptor, when it is called the anti-immune body. In either event it protects the cell from the alexin.

Figure I I shows the antibody (anticomplement) united with the alexin, preventing a union of the alexin with the amboceptor. Figure I 2 shows the antibody (anti-immune body) united with the amboceptor, preventing a union of the alexin with the receptor.

Precipitins. - It is possible to produce, in an adapted animal, substances known as precipitins; and these have been used as tests for different kinds of blood. The blood-serum of an adapted animal, if added to the blood used in adaption, produces a copious precipitate, which does not occur with other mixtures.

Agglutins. - When the blood of an animal is adapted to the red blood-corpuscles of an animal of a different species by repeated intraperitoneal injections, the blood-serum of the adapted animal becomes agglutinative, not only for the blood-cells, but for other cells as well, belonging to the animal whose blood has been used in the process of adaption; but no agglutination occurs in cells from other sources. This process of agglutination, however, is not protective. 


\section{CHAPTER I}

THE BLOOD

Importance of the blood - Quantity of blood - Opacity - Taste - Reaction - Specific gravity - Temperature - Color - Laking of blood - Blood-corpuscles - Red corpuscles Hemoglobin - Precipitin-test for blood - Development of red corpuscles - Leucocytes - Lymphocytes - Blood-platelets - Plasma and serum - Fibrinogen — Serum-globulin — Serum-albumin - Extractives and salts - Coagulation of the blood - Prothrombin and thrombin - Uses of coagulation.

Importance of the Blood. - With the progress of knowledge and the accumulation of facts in physiology, the importance of the blood in its relation to the processes of animal life becomes more and more thoroughly understood and appreciated. The blood is the most abundant and highly organized of the liquids of the body, providing materials for the re-formation of all parts, without exception, receiving the products of their katabolism and conveying them to the proper organs by which they are removed from the system. These processes require, on the one hand, constant renewal of nutritive constituents, and, on the other, constant purification by the prompt removal of effete matters. Those tissues in which the processes of nutrition are active are supplied with blood by means of vessels; but some, less highly organized, like the epidermis, hair, cartilage, etc., which are called extravascular because they are not penetrated by vessels, are none the less dependent on the blood, as they imbibe nutritive matters from the blood of adjacent parts.

The importance of the blood in the processes of animal life is evident; and in animals in which nutrition is active, death is the immediate result of its abstraction in large quantity. Its importance to life in this regard can readily be demonstrated by experiments on the inferior animals. If, in a small dog, a canula is adapted to a syringe introduced through the right jugular vein into the right side of the heart, and a great part of the blood is suddenly withdrawn, immediate suspension of all the so-called vital processes is the result; and if the blood is afterward returned to the system, the animal is as suddenly revived.

Certain conditions, one of which is diminution of the heart's action after copious hemorrhage, prevent the escape of all the blood from the body, even after division of the largest arteries; but after the arrest of 
the functions, which follows copious discharges of this liquid, life may be restored by injecting into the vessels the same blood or fresh blood from another animal of the same species. This observation, which was first made on the inferior animals, has been applied to the human subject; and it has been ascertained that in patients sinking under hemorrhage, the introduction even of a few ounces of fresh blood may restore the functions for a time and sometimes permanently.

Quantity of Blood. - The determination of the entire quantity of blood contained in the body has long engaged the attention of physiologists, without, however, absolutely definite results. This fact shows the extent of the obstacles to be overcome before the question can be absolutely settled. The chief difficulty is that all the blood is not discharged from the body, even after division of the great arteries, as after decapitation, and no entirely accurate means have been devised for estimating the quantity that remains in the vessels. A process devised by Haldane and Lorrain Smith is open, perhaps, to as few serious objections as any. This method takes account of the hemoglobin, a constant constituent of the red blood-corpuscles. Hemoglobin combines with carbon monoxide in definite proportions, which are about equal to the oxygen capacity of the blood-corpuscles. A man is made to breathe a measured volume of this gas, and the percentage saturation is determined. From this it is easy to calculate the proportion of carbon monoxide which would be required to saturate the hemoglobin contained in the entire mass of blood. It remains only to estimate the quantity of carbon monoxide absorbed, and then it is not difficult to calculate the quantity of this gas that would be required to saturate the entire mass of blood. Such calculations have resulted, from observations on fourteen healthy persons, in the estimate that the entire mass of blood is equal to about one-twentieth part of the weight of the body, which is much less than estimates previously made.

Prolonged abstinence from food has a notable effect in diminishing the quantity of blood, except when large quantities of liquids have been taken; and the reverse is true after the ingestion of food and drink in abundance. The quantity of blood, also, is notably large in persons of plethoric habit. Wrisberg reported the case of a female criminal from whom nearly twenty-one and a half pounds ( 9745 grams) of blood flowed after decapitation. It is not possible to form a definite idea of the quantity of blood during digestion, but it must be considerably greater than in a fasting condition. The percentage of blood that can be drawn from the body without producing death varies greatly. A withdrawal of twenty per cent usually is tolerated; but a loss of thirty per cent is almost invariably fatal. 
Opacity. - The opacity of the blood depends on the fact that it is not a homogeneous liquid, but is composed of plasma and corpuscles, both of which are nearly transparent but which have each a different refractive index. The rays of light, consequently, can not pass through the liquid, and the mixture is opaque.

Odor: - The blood has a faint but characteristic odor. This may be developed so as to be quite distinct, by the addition of a few drops of sulphuric acid, when the odor peculiar to the animal from which the blood has been taken becomes very marked.

Taste. - The taste of the blood is distinctly saline, on account of a number of salts contained in the plasma, and particularly of a considerable proportion - three to four parts per thousand - of sodium chloride.

Reaction. - The reaction of the blood in health is distinctly alkaline ; but it is not alway's easy to demonstrate this, on account of the red color of the corpuscles, which obscures the reaction. If, however, a drop of blood is put upon glazed reddened litmus paper and is then lightly wiped off with a soft sponge, a spot remains that is of a distinctly blue color. The alkaline reaction is due to the presence of sodium carbonate and sodium phosphate in the plasma.

Specific Gravity. - The specific gravity of defibrinated blood is between 1.055 and I.062, varying considerably with conditions of the digestive organs.

Temperature. - The temperature of the blood usually is given as between $98^{\circ}$ and $100^{\circ}$ Fahr. $\left(36.67^{\circ}\right.$ and $37.78^{\circ} \mathrm{C}$.); but experiments have shown that it varies considerably in different vessels, independently of exposure to external refrigerating influences. The blood is warmer in the right than in the left cavities of the heart. With few exceptions, it is warmer in the arteries than in the veins. It is warmer in the portal vein than in the abdominal aorta. It is constantly warmer in the hepatic veins than in the portal vein. The blood is warmest, indeed, in the hepatic veins, where it has a temperature of $101^{\circ}$ to $107^{\circ}$ Fahr. $\left(38.33^{\circ}\right.$ to $41.67^{\circ} \mathrm{C}$.).

Color. - The color of the blood is due to the red corpuscles. In the arterial system the color is uniformly red. In the veins it usually is dark blue but sometimes almost black. The color in the veins, however, is not constant. Many years ago, John Hunter observed, in a case of syncope, that the blood obtained by venesection was bright red. The color of the venous blood depends on the condition of the organ or part from which it is returned. The red color of venous blood was first noticed by Bernard in the renal veins, where it contrasts strongly with the black blood in the ascending rena cava. When the excito-secretory nerve of the submaxillary gland is faradized, the blood coming from the 
gland is bright red. Inasmuch as the red color of the blood depends on the presence of oxygen in the red corpuscles, the loss of oxygen which occurs as the result of the katabolic processes in the gland changes the blood from red to blue; but if the quantity of blood passing through the gland is so great that this change is much diminished, the red color of the arterial blood remains. Although oxygen is replaced by carbon dioxide in molecular katabolism, the quantity of oxygen lost in this process is not sufficient to produce the change from red to blue, on account of the great activity in the local circulation, which occurs during secretion.

Laking of Blood. - When serum from the blood of one animal is added to the blood of another animal of a different species, a curious phenomenon is presented, called the laking of blood. The red corpuscles are broken up and the hemoglobin appears in

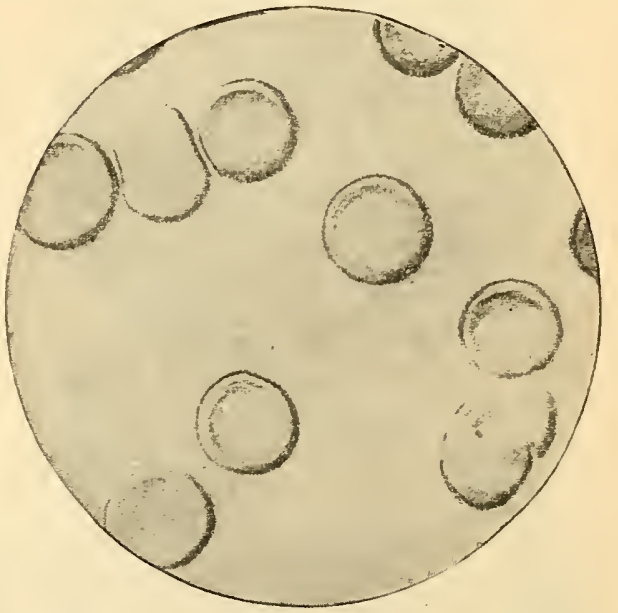

Fig. 13. - Blood of Guinea pig, spread and dried on glass cover, $X \mathrm{I}_{450}$ (Sternberg).

The corpuscles of the Guinea pig have the same form as human corpuscles, but are a little smaller, measuring about $\frac{11}{3600}$ of an inch $(7 \mu)$ in diameter. solution, leaving the filmy remains - called the ghosts of the corpuscles - to settle to the bottom of the vessel. This is merely a solution of the coloring matter, which is freed from the stroma of the corpuscles and gives the peculiar color to the solution. A phenomenon analogous to the laking of blood was observed by Rollett. By alternately freezing and thawing blood, he succeeded in extracting the hemoglobin. When afterward warmed and liquefied, the solution was dark and transparent, with the corpuscles entirely decolorized.

\section{BLOOD-CORPUSCLES}

In I66I, Malpighi, with the imperfect lenses at his command, discovered the blood-corpuscles of the hedgehog. Leeuwenhock saw the human blood-corpuscles in 1673 and described them in a communication to the Philosophical Society in I674. William Hewson discovered the leucocytes about a century later. Neumann, of Königsburg, and Bizzozero, of Turin, described the blood-platelets about I868. Since that time, three varieties of corpuscles have been recognized in human blood. 
Red Corpuscles. - The red blood-corpuscles (erythrocytes) constitute about fifty per cent of the entire mass of blood. (Flint, i863.) Their
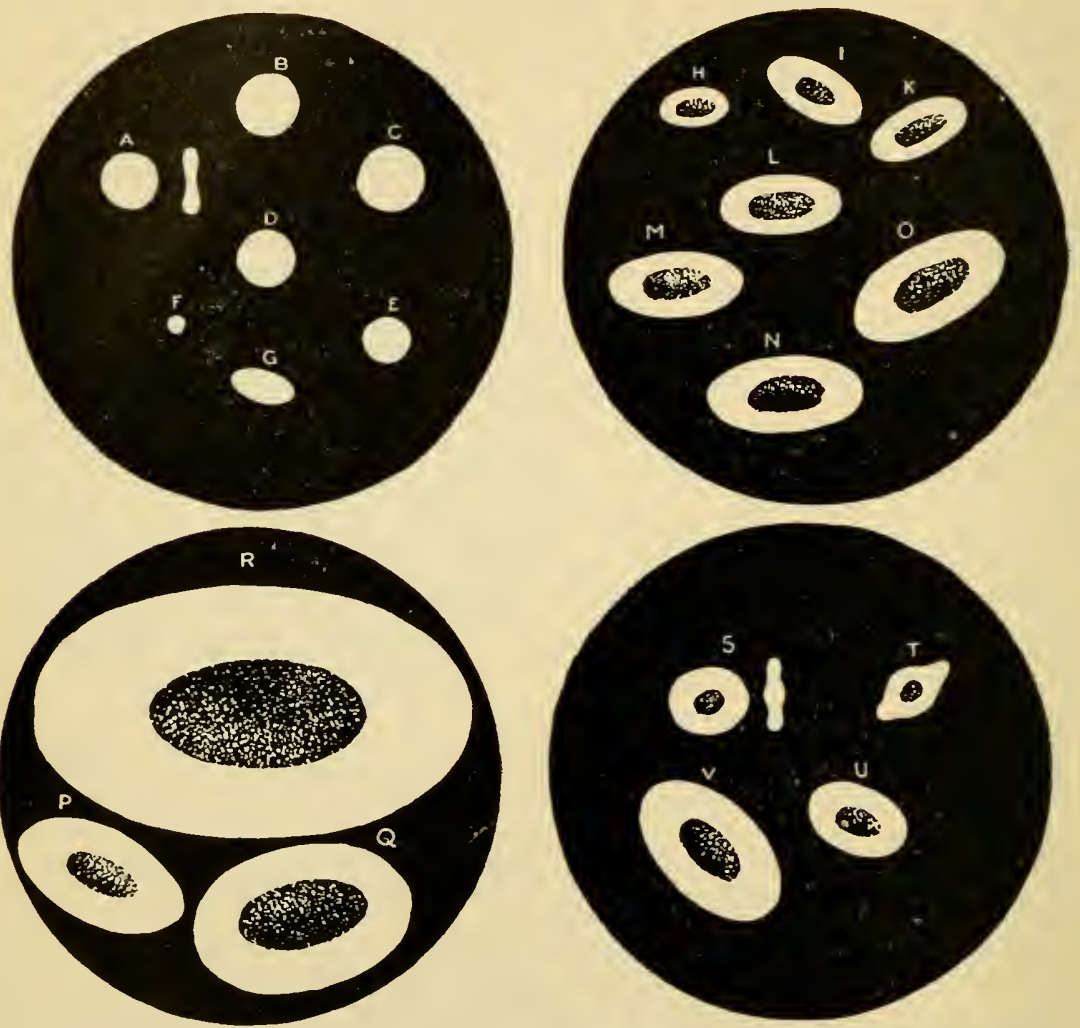

Fig. I4. - Blood-corpuscles from certain of the inferior animals, compared with corpuscles from the human subject, $\times 700$.

The measurements in these figures are taken from Gulliver (1841), and for the elliptical cells the long diameters are given. In the original article by Gulliver about five hundred measurements are given. I published one hundred of these in the Physiology of Man, I866, Vol. I, p. II3. For the convenience of readers, the English measurements have been reduced to microns.
A. Man, $3 \frac{1}{2} \delta \sigma$ inch
$=8.0 \mu$.
B. Whale, 36is inch
$=8.2 \mu$.
C. Elephant, $\frac{1}{2}: \frac{1}{45}$ inch
M. Lizard, 155 inch
$=\mathrm{I} 6.3 \mu$.
$\lambda$. Snake, $1 \frac{2}{2} \div-$ inch $\quad=20.0 \mu$.
$O$. Crocodile, $\frac{1}{12} 3 \overline{1}$ inch $=20.6 \mu$.
$D$. Nouse, $\frac{12}{426}$ inch $\quad=6.0 \mu$.
$E$. Horse, $\frac{1}{6} \sigma a$ inch $\quad=5.5 \mu$.
$F$. Musk-deer, $12 \frac{1}{32}$ inch $=2.0 \mu$.
G. Camel, $3 \frac{1}{2} \frac{1}{3}$ inch $=8.1 \mu$.
$H$. Humming-bird, $\frac{1}{266}$ inch $=9.6 \mu$.
I. Pheasant, $\frac{1}{2} \frac{1}{12} \pi$ inch $=12.0 \mu$.
$K$. Pigeon, $\frac{1}{2} 3^{\frac{1}{2}}$ inch $\quad=\mathrm{I} 1.0 \mu$.
$P$. Toad, 1043 inch $=24.3 \mu$.
Q. Triton, $\frac{1}{14}$ inch $=31.2 \mu$.
$R$. Proteus, $\frac{1}{40}$ inch $=63.5 \mu$.
S. Perch, $\frac{\pi}{2} \frac{1}{61}$ inch $=10.3 \mu$.
T. Pike, $\frac{1}{200}$ inch $=12.7 \mu$.
$U$. Electric eel, $\frac{1}{7 \frac{1}{4}}$ inch $=I_{4} .8 \mu$.
$L$. Ostrich, $\frac{2}{169}$ inch $=15.4 \mu$.
$V$. Shark, $\Sigma_{12}^{2}$ inch $\quad=22.2 \mu$.

form is peculiar and distinctive. Many are circular, and thinner at the central portion than at the periphery, where the stroma is more dense and consistent. 
The corpuscles of cold-blooded animals, birds, and the camelidæ are oval, and with the exception of the camelidæ are nucleated. The corpuscles of the camelidæ are biconvex.

The color of the red corpuscles by transmitted light is pale and faintly yellowish, and is due to hemoglobin, which constitutes about 95.5 per cent of their substance (see Plate II, Fig. 2). Their specific gravity is about 1088 . In the blood of the horse--which coagulates slowly - the corpuscles readily gravitate to the bottom of a vessel, leaving a clear plasma above.

The number of corpuscles in a given volume may be easily calculated by means of an instrument called the hemacytometer. This is an apparatus for counting the corpuscles in a measured space filled with blood properly diluted and the corpuscles evenly distributed in the mixture. The results of modern observers agree remarkably with those of Vierordt (1852), Walker (I854), and Malassez (1872), the general estimate being about $5,000,000$ corpuscles in a cubic millimeter (one millimeter $=\frac{1}{2}$ inch) of blood. Various saline solutions are used in diluting the blood, having a specific gravity of 1020 to I025. The Thoma-Zeiss apparatus is the one most used in chemical observations; and

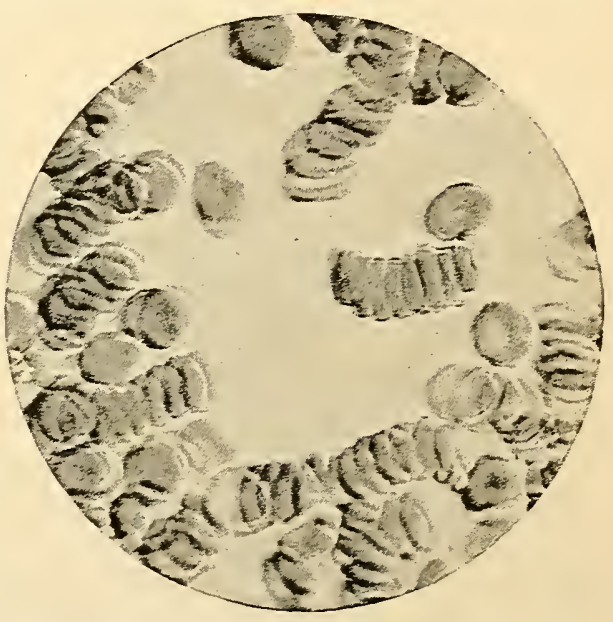

Fig. 15.-Human blood-corpuscles in rouleaux, $\times 840$ (Stratford).

This figure shows fresh corpuscles, most of them arranged in rows. this is merely a convenient modification of the method of Vierordt and Walker and Malassez, which it is unnecessary to describe in detail. The proportion of corpuscles is greater in the veins than in the arteries, the blood of the splenic veins presenting the highest number. In women the corpuscles number about 4,500,000 in a cubic millimeter. The number of corpuscles is gradually increased during fifteen or twenty days' sojourn in high altitudes. At an altitude of about 14,500 feet (4392 meters) the number per cubic millimeter was $8,000,000$ (Viault).

A short time after blood has been drawn from the vessels, the corpuscles usually show a tendency to arrange themselves in rows like rouleaux of coin. This is due to the exudation of a sticky substance which causes them to adhere together by their flat surfaces. Under 
the influence of reagents the corpuscles undergo certain changes in form and appearance. A physiological salt-solution prevents the arrangement in rouleaux; and if a stronger solution is used, the corpuscles shrink and become irregular in form and crenated. In pure water and in dilute alkaline solutions, the red corpuscles dissolve slowly and finally disappear. A two per cent solution of tannic or boric acid decolorizes the corpuscles, and the coloring matter appears in the form of small globules. The stroma of the corpuscles is gradually broken up by the addition of chloroform. Under the influence of a feeble electric current the corpuscles become crenated and covered with little granules. The diameter of the red corpuscles is fairly uniform, measuring about $\frac{1}{3200}$ of an inch $(7 \mu$ to $8 \mu)$. Their thickness at the periphery is about $\frac{1}{12} \frac{1}{000}$ of an inch $(2 \mu)$; but in any given specimen of blood there are a few small immature corpuscles, sometimes called microcytes.

The anatomical structure of the red corpuscles is very simple. The hemoglobin is enclosed in a delicate transparent and elastic filmy network, or stroma, which appears as the "ghosts" of the corpuscles after the coloring matter has been removed, as in the laking of blood. Of the chemistry of the red corpuscles as compared with the chemistry of the plasma, there is little to be said. The organic matter is in the form of what is known as a nucleo-proteid. The principal salts are sodium and potassium chlorides, and in human blood, potassium chloride is the more abundant. Cholesterin is always found associated with the other extractives, including a notable proportion of lecithin.

\section{HeMoglobin}

The molecule of hemoglobin, at least in the blood of the dog, is of very large size, amounting to 16,669 (Jaquet and Hüfner). The empyrical formula is $\mathrm{C}_{758} \mathrm{H}_{1203} \mathrm{~N}_{195} \mathrm{~S}_{3} \mathrm{FeO}_{218}$. There may be obtained from the blood a considerable number of so-called derivatives of hemoglobin. The most important of these is hematin, the detection of which is regarded as one of the most reliable tests for blood. If the dried blood from a suspected stain is boiled with a few drops of glacial acetic acid, on cooling, hemin crystals will appear, formed by the splitting of hemoglobin into hemin, or hemochromogen, and globin. The formula for hematin hydrochlorate (hemin) is $\mathrm{C}_{35} \mathrm{H}_{35} \mathrm{~N}_{4} \mathrm{FeClO}_{4}$ (Mörner). Other derivatives are hematoporphyrin (an iron-free hematin), hematoidin, which closely resembles bilirubin, and compounds of hemoglobin with oxygen, carbon monoxide and nitrous oxide. It is in the form of oxyhemoglobin that uxygen is carried by the blood to the tissues. These 
various derivatives of hemoglobin may be recognized by their absorptionbands in the spectrum. Methemoglobin, formed by adding potassium ferricyanide or amyl nitrite to the blood, contains the same proportion of oxygen as oxyhemoglobin, but in a different form of combination (see Plate II, Fig. 2).

Precipitin-Test for Blood. - A test for blood, which surpasses in delicacy any known test for any known substance, depends on the property of blood-serum, adapted to a certain species of animal, of forming a precipitate when mixed with the blood of the adapted animal. This, it is said, will detect the presence of blood of a given species of animal in the proportion of one part in fifty thousand. The following illustrates the operation of this test: Repeated daily injections of human blood-serum are made into the peritoneal cavity of a rabbit for seven or eight days. In this way the blood of the rabbit becomes adapted to the human blood. A little blood-serum of the rabbit is now to be mixed with a small quantity of a salt-solution of the suspected blood. If the specimen is from the human subject or one of the primates, the mixture will present an abundant precipitate. If there should be no precipitate, it is certain that the specimen does not contain human blood.

\section{Development of the Red Corpuscles}

In the circular area that surrounds the embryo in the earliest stages of development, called the area vasculosa, bodies make their appearance that afterward take on the characters of the red blood-corpuscles. In the process of formation of this area, nucleated mesoblastic cells branch out in various directions to form a sort of network, which is afterward changed into a connected system of vessels. Nucleated bodies then collect in certain parts of this network, which become surrounded with protoplasm and afterward are colored with hemoglobin. At first there are no regular movements in the area vasculosa; but a rudimentary heart is soon formed by a twisting of the great central vessel upon itself, and the currents of the circulation become established. During this time the blood-cells are capable of ameboid movements and undergo multiplication by karyokinesis. They are at first white and nucleated, but soon become colored, the nuclei remaining. These first-formed embryonic corpuscles (erythroblasts) measure $\frac{1}{2500}$ to $\frac{1}{150} \overline{0}$ of an inch ( IO $\mu$ to I6 $\mu$ ) in diameter.

As the liver, spleen, thymus and lymphatic glands are developed, they assume the function of producing red blood-corpuscles, which also multiply by karyokinesis in the substance of these organs. In the fœtus 
the marrow of all the bones is red; but in the adult, red marrow exists in the cancellated structure of the flat bones only. It has been shown that the red marrow is the most efficient agent in the formation of corpuscles. In the adult the corpuscles are developed largely in the marrow of the ribs. In this situation, large pale cells make their appearance, which soon become partly colored with hemoglobin. The protoplasm, thus colored, afterward separates into smaller bodies, cupshaped at first, which eventually take on the characters of the red corpuscles. Mingled with these - which may be called mature corpuscles - are smaller bodies, containing hemoglobin, that are capable of ameboid movements. It is probable that red corpuscles are produced from similar cells developed in the liver, spleen, thymus and lymphatic glands, at least in adult animals (see Plate I, showing cells in bonemarrow).

\section{LeUCocytes AND Lymphocytes}

In addition to the red blood-corpuscles, at least five varieties of white corpuscles have been studied and described in the human blood. The proportion of these corpuscles to the red varies in different persons and at different times of the day. It is usually as one to five hundred, or about ten thousand in a cubic millimeter. These bodies, which were first described by Hensen, are white, globular and nucleated.

Lcucocytes. - The first variety of leucocytes constitutes about seventy-two per cent of the total number. These are described under the name of large polynuclear neutrophiles. They often contain two or more nuclei, and their cytoplasm presents granules that have an affinity for neutral aniline dyes - hence their name. They probably are produced largely in the red marrow of bones. They are capable of ameboid movements and of incorporating in their substance and destroying foreign particles. For this reason, as well as on account of their great number in the blood, they are regarded as more important than other bodies of a similar nature. It is thought possible that these leucocytes are capable of protecting the organism against pathogenic bacteria, acting as phagocytes. They are considerably larger than the red corpuscles, measuring about $\frac{1}{2500}$ of an inch $($ I $\mu$ ) in diameter.

The second variety is known as large mononuclear leucocytes. These also are larger than the red blood-corpuscles. They may be polynuclear leucocytes in a transitional condition, the nucleus finally assuming the forms noted in the first variety. The protoplasm surrounding the single nucleus in these bodies is clear, and this variety has received the name of myelocytes. They probably are developed 
in the cancellated structure of the bones. They constitute about three per cent of the total number of leucocytes.

The third variety is called eosinophiles, on account of their affinity for acid dyes, which strongly color the large granules contained in their protoplasm. They also probably are derived from the red marrow of the bones. They constitute one or two per cent of the total number of leucocytes.

In addition to the three varieties of leucocytes just described, a fourth variety, called basophilic, is sometimes observed. These have an affinity for basic aniline dyes.

These four varieties of leucocytes - probably by virtue of their ameboid movements - when they lie for a considerable time in contact with the walls of the bloodvessels are capable of passing through without solution. of continuity in the vessels themselves. They thus frequently pass out into inflammatory foci. They creep about in the tissues and are then called migratory or wandering cells.

Lymphocytes. - The fifth variety is known under the name of lymphocytes. They exist in the form of a rather small rounded nucleus surrounded with clear cytoplasm. They constitute about twenty-three per cent of the total number of white corpuscles.

The five varieties of white corpuscles are produced chiefly in the red marrow, but they also appear de novo in the substance of the lymphadenoid tissues, the spleen, thymus and the lymphatic glands. Treated with water or acetic acid, they swell and their nuclei become more prominent. They are dissolved in dilute alkalis. Although they undoubtedly are developed in the red marrow, the spleen, thymus and lymphatic glands - situations in which the red corpuscles first make their appearance - the idea that they are finally developed into red corpuscles, which at one time obtained, has been abandoned (see Plate I).

\section{Blood-Platelets}

In addition to the leucocytes, little homogeneous, ovoid, grayish, flattened bodies $\overline{1} \frac{1}{000}$ to $\frac{1}{100 \overline{0}}$ of an inch $(1.5$ to $2.5 \mu)$ in diameter, have been observed and described under the name of blood-platelets (Bizzozero). These bodies exist in the blood in the proportion of one to about fifteen to twenty red disks, or 180,000 to 600,000 in a cubic millimeter. Their origin is somewhat obscure, but it is thought that their chief source is from the red corpuscles, which extrude a globular matter having reactions like the nucleo-proteids. Their function is equally obscure; but it is probable that they are concerned in some way in the production of prothrombin and are instrumental in bringing about coagulation of the blood (see Plate II, Fig. I). 
In addition to the structures in the blood already described, the plasma often contains minute fatty granules, especially during digestion, such as are found in large quantity in chyle and in smaller quantity in lymph. The lymph, like the blood, is composed of plasma and corpuscles, the latter being chiefly in the form of lymphocytes; and the lymph also, like the blood, is capable of coagulation. Blood-platelets, however, have not been observed in lymph.

\section{Plasia and Serum}

Before coagulation, the blood is composed of a relatively clear plasma holding the corpuscles in suspension. After coagulation, the clot, enclosing the corpuscles, separates and leaves the serum, which is the plasma less the constituents that form fibrin. The plasma is slightly yellowish, alkaline and of a specific gravity of 1026 to 1029 . It contains proteids, extractives (including fats) and inorganic salts. The proteids consist of fibrinogen, paraglobulin (serum-globulin) and serumalbumin; and their proportion is about eight parts per thousand.

Fibrinogcn. - Fibrinogen $\left(\mathrm{C}_{108} \mathrm{H}_{162} \mathrm{~N}_{30} \mathrm{SO}_{34}\right)$ is a globulin; but it is distinguished from the other globulins of the plasma by its behavior in the presence of certain reagents. It is precipitated by halfsaturation of the plasma with sodium chloride and is coagulated at a relatively low temperature, $133^{\circ} \mathrm{Fahr}$. $\left(56^{\circ} \mathrm{C}\right.$.). Under the influence of the so-called fibrin-ferment, its molecule splits into fibrin, which is insoluble, and a soluble globulin. Its proportion in the blood is about three parts per thousand.

Scrum-globulin. - Serum-globulin $\left(\mathrm{C}_{117} \mathrm{H}_{182} \mathrm{~N}_{30} \mathrm{SO}_{38}+\frac{1}{2} \mathrm{H}_{2} \mathrm{O}\right)$ exists in the blood in the proportion of about thirty-one parts per thousand. It coagulates at a temperature of about $158^{\circ}$ Fahr. $\left(70^{\circ} \mathrm{C}\right.$.). It may be separated from the neutral salts by dialysis. Both fibrinogen and serumglobulin are precipitated by magnesium sulphate. It directly or indirectly nourishes the proteids of the tissues, perhaps undergoing previously a change into serum-albumin. It may be derived from food, but it is thought by some to be a product of disintegration of leucocytes.

Sermm-albumin. - Serum-albumin $\left(\mathrm{C}_{78} \mathrm{H}_{122} \mathrm{~N}_{20} \mathrm{SO}_{24}\right)$ is the most important nutritive proteid of the blood. It is the only proteid that is not precipitated by magnesium sulphate. It coagulates at a temperature of $158^{\circ} \mathrm{Fahr}$. $\left(70^{\circ} \mathrm{C}\right.$.). Its proportion in the blood is about forty-five parts per thousand. It is probable that it is not a simple albumin but is composed of two or three different albumins. It is highly osmotic, a property that distinguishes it from ordinary egg-albumin. 


\section{TABLE OF COMPOSITION OF BLOOD-PLASMA}

Specific gravity, I028.

Water, 779 parts per 1000 in the male; 791 parts per 1000 in the female.

Sodium chloride, 3 to 4 parts per 1000.

Potassium chloride, 0.359 parts per 1000.

Ammonium chloride, proportion not determined.

Potassium sulphate, 0.288 parts per Iooo.

Sodium sulphate, proportion not determined.

Potassium carbonate, proportion not determined.

Sodium carbonate (with sodium bicarbonate), I.200 parts per 1000.

Magnesium carbonate, proportion not determined.

Calcium phosphate of the bones, and neutral phosphate,

Magnesium phosphate,

Potassium phosphate,

Ferric phosphate (probable),

Basic phosphates and neutral sodium phosphate,

( Silica, copper, lead and magnesia, traces occasionally.

Sodium lactate, proportion not determined.

Calcium lactate (probable), proportion not determined.

Sodium oleate,

" palmitate,

" stearate,

" valerate,

“ butyrate, 1.475 parts per 1000.

\section{Olein,}

Palmitin,

Stearin,

Lecithin, containing nitrogen and called phosphorized fatty matter, 0.400 parts per 1000 .

Glucose, 0.002 parts per IOOO.

Glycogen, proportion not determined.

Inosit, proportion not determined.

Carbon dioxide in solution.

Urea, 0.177 parts per 1000, in arterial blood; 0.088 , in the blood of the renal vein.

Sodium urate, proportion not determined.

Potassium urate (probable), proportion not determined.

Calcium urate, " " " " "

Magnesium urate, " " " " " "

Ammonium urate, " " " " " "

Sodium sudorates, etc., "

Inosates, " " " " " "

Oxalates,

Creatin,

Creatinin,

Leucin,

Xanthin,

Hypoxanthin,

Cholesterin, $0.455^{\text {to }} 0.75^{\mathrm{I}}$ parts per $\mathrm{I}, 000$, in the entire blood (Flint).

Fibrinogen, 3 parts per 1000 .

Serum-albumin, 45 parts per Iooo.

Serum-globulin, 31 parts per 1000.

Peptones, 4 parts per I000."

Coloring matters of the plasma, proportion and characters not determined. 


\section{Extractives ANd SAlts}

Grouped under the head of extractives, may be included various nitrogenous and non-nitrogenous matters, such as urea, urates, creatin, creatinin, xanthin, hypoxanthin, the lecithins, fats, waxes, sugar and cholesterin. In addition, inorganic salts exist in the proportion of six to eight parts per thousand. The most abundant of the inorganic salts is sodium chloride, the proportion of which is sixty to ninety per cent of the total saline matters. Potassium chloride is found in much smaller quantity and exists chiefly in the red corpuscles. The other saline matters are in the form of sulphates and phosphates. Calcium salts are present and are important in connection with coagulation. Gases are in solution or in a condition of feeble combination. Of the gases, carbon dioxide is important in retaining certain of the salts in solution.

\section{Coagulation of the Blood}

The blood remains liquid so long as it is contained in the vessels and the circulation is not interrupted; but soon after circulation ceases or the blood is drawn from the body, it coagulates, or "sets" into a jelly-like mass. In a few hours, contraction of the clot will have taken place, and a clear straw-colored serum is then expressed. The serum contains all the constituents of the blood except the corpuscles and the fibrin-factors, which together form the clot. In the human subject, when the blood is received into a moderately deep, smooth vessel, the phenomena of coagulation present themselves in the following order:

First, a gelatinous pellicle forms on the surface, which occurs in one minute and forty-five seconds to six minutes; in two to seven minutes, a gelatinous layer has formed next the sides of the ressel; the whole mass of blood becomes of a jelly-like consistence in seven to sixteen minutes. Contraction then begins, and drops of clear serum make their appearance on the surface of the clot. This liquid increases in quantity, and in ten or twelve hours separation is complete. The clot, which is heavier, sinks to the bottom of the vessel, unless it contains bubbles of gas or the surface is very concave. In most warm-blooded animals the blood coagulates more rapidly than in man. Coagulation is peculiarly rapid in the blood of birds, and sometimes it takes place almost instantaneously. Coagulation is more rapid in arterial than in venous blood. In the former, the proportion of fibrin formed is notably greater and the characters of the fibrin are somewhat different. A solution of sodium chloride dissolves the fibrin of venous blood but does not dissolve the fibrin of an arterial clot. 
The relative proportions of serum and clot are variable, unless that portion of the serum retained between the meshes of the coagulated mass is included in the estimate. As the clot is composed of corpuscles and fibrin, and as these in their moist state represent, in general terms, about one-half of the blood, it may be stated that after coagulation, the actual proportions of clot and serum are about equal. Simply taking the serum that separates spontaneously, there is a large quantity, when the clot is densely contracted, and a smaller quantity, when it is loose and soft. Usually the clot retains about one-fifth of the serum.

On removing the clot, after the separation of the serum is complete, it presents a gelatinous consistence, and is more or less firm according to the degree of contraction. As a general rule, when coagulation has been rapid, the clot is soft and but slightly contracted. When, on the other hand, coagulation has been slow, the clot contracts for a long time and is much denser. When coagulation is slow, the clot frequently presents what is known as the buffed and cupped appearance.

Blood flowing slowly from a small opening in a vessel coagulates more rapidly than when it is discharged in a full stream from a large opening. If received into a shallow vessel, it coagulates much more rapidly than in a deep vessel. If the inner surface of the vessel is rough, coagulation is more rapid than when it is smooth and polished. If the blood as it flows is received on a cloth or a bundle of twigs, it coagulates almost instantaneously. In short, it appears that conditions that favor evaporation from the blood hasten its coagulation.

Coagulation of the blood is prevented by rapid freezing, but it occurs afterward if the blood is carefully thawed. Between $32^{\circ}$ and $140^{\circ} \mathrm{Fahr}$. $\left(0^{\circ}\right.$ and $60^{\circ} \mathrm{C}$.), elevation of temperature increases the rapidity of coagulation.

Various chemical substances retard or prevent coagulation. Among these may be mentioned potassium and sodium hydrate, sodium carbonate, ammonium carbonate, potassium carbonate, ammonia, and sodium sulphate. In the menstrual flow the blood remains liquid on account of mixture with the abundant secretions of the vagina.

The blood coagulates in the vessels after death, though less rapidly than when drawn from the body. It occurs in twelve to twenty-four hours after circulation has ceased, and the blood then is found chiefly in the venous system. The existence of projections into the calibre of vessels or the passage of a fine thread through an artery or vein will determine the formation of a small coagulum on the foreign substance, while the circulation is neither interrupted nor retarded. In the present state of knowledge, explanation of these facts is difficult if not impossible. The process, under these conditions, cannot be subjected to direct 
experiment as in the case of blood coagulating out of the body. Leechdrawn blood remains liquid in the body of the animal; and the blood flowing from a leech-bite presents the same persistent fluidity. This explains the well-known fact that this insignificant wound gives rise to considerable hemorrhage.

With the intention to simplify as much as possible the exposition of the theory of coagulation and to remove sources of confusion, I shall adopt in its description the theory of Schmidt as modified chiefly by the researches of Hammarsten and Lilienfeld. In this theory it is assumed that a substance is developed in coagulating blood that is capable of reacting with fibrinogen to produce fibrin. By the disintegration of certain of the leucocytes and the blood-platelets, a nucleo-albumin is given off, which unites with calcium to form thrombin. This nucleo-albumin is called prothrombin. In the reaction that results in the formation of fibrin, the fibrinogen molecule is split into a body that unites with thrombin, leaving a new substance called fibrin-globulin. Sixty to ninety per cent, however, of the fibrinogen molecule enters into the composition of fibrin. The presence of calcium salts is necessary to the change of prothrombin into thrombin; but Hammarsten has succeeded in producing a fibrin containing only 0.005 per cent of calcium. The agency of the blood-platelets in the production of fibrin can hardly be doubted. Microscopical observations have revealed filaments of fibrin radiating from these bodies partially disintegrated. The formation of fibrin, indeed, may be simply described as follows:--

Prothrombin and Thrombin. - As the result of disintegration of blood-platelets (Lilienfeld) and of certain leucocytes, a nucleo-albumin is formed called prothrombin; prothrombin unites with calcium to form thrombin ; finally, thrombin splits the molecule of fibrinogen, combines with part of this molecule to form fibrin and leaves a part that is called fibrin-globulin.

Uses of Congulation. - The property of the blood under consideration has an important office in the arrest of hemorrhage. The effect of absence of or great diminution in coagulability is exemplified in instances of what is called the hemorrhagic diathesis, or hemophilia, a condition in which slight wounds are followed with alarming and sometimes fatal hemorrhage. This condition may exist for years and is not characterized by any peculiar symptoms except an obstinate flow of blood from slight wounds.

Hemophilia is the most striking example in medicine of transmission by inheritance. It has been traced through successive generations in a single family for more than two hundred years. It affects males much more frequently than females, the ratio being more than ten to 
one. It is a curious fact that the diathesis comes through the females, who are not themselves "bleeders." The daughters transmit it to their sons, but male bleeders seldom transmit the diathesis except to daughters, whose sons usually are bleeders. It has also been observed that the females in bleeder-families are very prolific. Hemophilia may develop spontaneously, without heredity, but it nearly always is congenital.

During coagulation, fibrin assumes a filamentous form, presenting, under the microscope, the appearance of rectilinear fibrillæ. These fibrillæ gradually increase in number, and as contraction of the clot occurs, they become irregularly crossed. They are always straight, however, and never assume the wavy appearance characteristic of true fibrous tissue.

The blood of the renal and hepatic veins, capillary blood, and the blood which passes from the capillary system into the veins after death usually does not coagulate or coagulates very imperfectly; in other words, blood from these parts does not readily form fibrin. The reason of this peculiarity is not known; but the fact affords a partial explanation of the normal fluidity of the blood; for this liquid, passing over the entire course of the circulation in about thirty seconds, seems to be losing its coagulability in its passage through the liver, kidneys, and the general capillary system as fast as its coagulability is increased in the other parts. Taking into consideration the rapidity of the circulation, it is evident that coagulation can not take place while the normal circulation is maintained and while the blood is undergoing the changes incident to general metabolism. 


\section{CHAPTER II}

\section{CIRCULATION OF THE BLOOD THROUGH THE HEART}

Discovery of the circulation - Physiological anatomy of the heart - Tricuspid valve -- Pulmonic valves - Mitral valve - Aortic valves - Iovements of the heart - Cardiac cycle - Sounds of the heart - Frequency of the heart's action - Cause of the rhythmical cuntractions of the heart - Accelerator nerves - Direct inhibition of the heart - Work of the heart.

HARVEY "set forth for the first time his discovery of the circulation," in his public lectures in 1616. In 1628, he published the Excrcitatio Anatomica de Motu Cordis et Sanguinis in Animalibus. This discovery, from the isolated facts bearing upon it observed by anatomists to its culmination in the experiments of Harvey, illustrates so well the gradual development of physiological truth, that it does not seem out of place to begin the study of the circulation with a brief sketch of its history.

The facts bearing on the circulation, developed before the time of Harvey, are chiefly anatomical. The writings of Hippocrates are indefinite on all points connected with the circulatory system; and no clear and positive statements are to be found in ancient works before the time of Aristotle. The book of Aristotle most frequently quoted by physiologists is his History of Animals; and in this occurs a passage which seems to indicate that he thought that air passed from the lungs to the heart; but in his work, De Partibus Animalinm, it is stated that there are two great bloodvessels, the vena cara and aorta, arising from the heart, and that the aorta and its branches carry blood. Galen, however, demonstrated experimentally the presence of blood in the arteries, by including a portion of one of these vessels between two ligatures, in a living animal; but his ideas of the communication between the arteries and veins were erroneous, for he believed in the existence of small orifices in the septum between the ventricles of the heart, a mistake that was corrected by Vesalius about the middle of the sixteenth century.

In 1546 , seven years before the description of the pulmonary circulation by Servetus, Rabelais, probably the most learned man of his time, gave a very fair description of the circulation of the blood through the lungs. An account of this, with a literal translation from 
the book Treating of the Heroic Deeds and Sayings of the Good Pantagmel, was published in 1903 (Flint).

In I 553, Michael Servetus, who is commonly regarded as the discoverer of the passage of the blood through the lungs, or the pulmonary circulation, described the course of the blood through the lungs, from the right to the left side of the heart. This description, complete as it is, was merely incidental to the development of a theory in regard to the formation of the soul and the production of what were called animal and vital spirits (spiritus).

A few years later, Colombo, professor of anatomy at Padua, and Cesalpinus, of Pisa, described the passage of the blood through the lungs, probably without a knowledge of what had been written by Servetus. To Cesalpinus is attributed the first use of the expression "circulation of the blood"; and he also remarked that after ligature or compression of a vein, the swelling is always below the point of obstruction.

The history of the discovery of the valves in the veins is somewhat obscure, although priority of observation is almost always conceded to Fabricius. As regards this point, only the dates of published memoirs are to be considered, notwithstanding the assertion of Fabricius that he had seen the valves in 1574 . In 1545 , Etienne described, in branches of the portal vein, "valves, which he called apophyses, and which he compared to the valves of the heart." In I55I, Amatus Lusitanus published a letter from Cannanus, in which it is stated that he had found valves in certain of the veins. In 1563 , Eustachius published an account of the valves of the coronary vein. In I 586, a clear account, by Piccolhominus, of the valves of the veins was published. Fabricius gave the most accurate descriptions and delineations of the valves, and his first publication is said to have appeared in 1603 . $\mathrm{He}$ demonstrated the valves to Harvey, at Padua; and it is probable that this was the origin of the first speculations by Harvey on the mechanism of the circulation.

In the work of Harvey are described first the movements of the heart, which he exposed and studied in living animals. He described minutely all the phenomena that accompany its action; its diastole, when it is filled with blood, and its systole, when the ventricles contract simultaneously, and "by an admirable adjustment all the internal surfaces are drawn together, as if with cords, and so is the charge of blood expelled with force." From the description of the action of the ventricles, he passed to the auricles, and showed how these, by their contraction, filled the ventricles with blood. By experiments on serpents and fishes, he proved that the blood fills the heart from the 
veins and is sent out into the arteries. Exposing the heart and the great vessels in these animals, he applied a ligature to the veins, which had the effect of cutting off the supply from the heart so that it became pale and flaccid; and by removing the ligature the blood could be seen flowing into the organ. When, on the other hand, a ligature was applied to the artery, the heart became unusually distended, which continued so long as the obstruction remained. When the ligature was removed, the heart soon returned to its normal condition. Harvey completed his description of the circulation by experiments showing the course of the blood in the arteries and veins and the uses of the valves of the veins. By these simple observations the chain of experimental evidence establishing the fact of the circulation of the blood was completed.

Although Harvey accurately described the course of the blood and left no doubt as to the communication between the arteries and veins, it remained for others actually to see the blood in movement and follow it from one system of vessels to the other. In 166I, Malpighi saw the blood circulating in the vessels of the lung of a living frog, examining it with magnifying glasses; and a little later, Leeuwenhoek saw the circulation in the wing of a bat. These observations completed the discovery of the circulation.

In man and in the warm-blooded animals, the organism requires blood that has been oxygenated in the lungs; and to meet this demand fully, the circulatory system is divided into pulmonic and systemic. The heart is double, having a right side and a left side, which are entirely distinct from each other. The right heart receives the blood as it is brought from the general system by the veins and sends it to the lungs; the left heart receives the blood from the lungs and sends it to the general system. It must be borne in mind, howerer, that although the two sides of the heart are distinct from each other, their action is simultaneous; and in studying the motions of the heart, it will be found that the blood is sent at the same time from the right side to the lungs and from the left side to the system. It will not be necessary, therefore, to separate the two circulations in the study of their mechanism; for the simultaneous action of both sides of the heart renders it possible to study its action as a single organ; and the constitution and operations of the two kinds of vessels do not present any material differences.

There are three kinds of bloodvessels: arteries, which carry blood from the heart to the general system; capillaries, which distribute the blood in the parts; and veins, which return the blood from the general system to the heart. 
Physiological Anatomy of the Heart. - The heart of the human subject is a pear-shaped muscular organ, situated in the thoracic cavity, its base lying in the median line and its apex at the fifth intercostal space, three inches ( 7.6 centimeters) to the left of the median line, or one inch (2.5 centimeters) within the line of the left nipple. The weight of the heart is ten to twelve ounces ( 283 to 340 grams) in the male, and eight to ten ounces (227 to 283 grams) in the female. It has four distinct cavities, - a right and a left auricle and a right and a left ventricle, the ventricles being the more capacious. The heart is held in place by the attachment of the great vessels to the posterior wall of the thorax; while the apex is free and is capable of a certain extent of movement. The entire organ is enveloped in a fibrous sac called the pericardium. This sac is lined with a serous membrane, which is attached to the great vessels at the base and is reflected over the surface of the heart. This membrane is lubricated with about a drachm (3.7 cubic centimeters) of liquid, so that the movements of the heart are attended with but slight friction. The se-

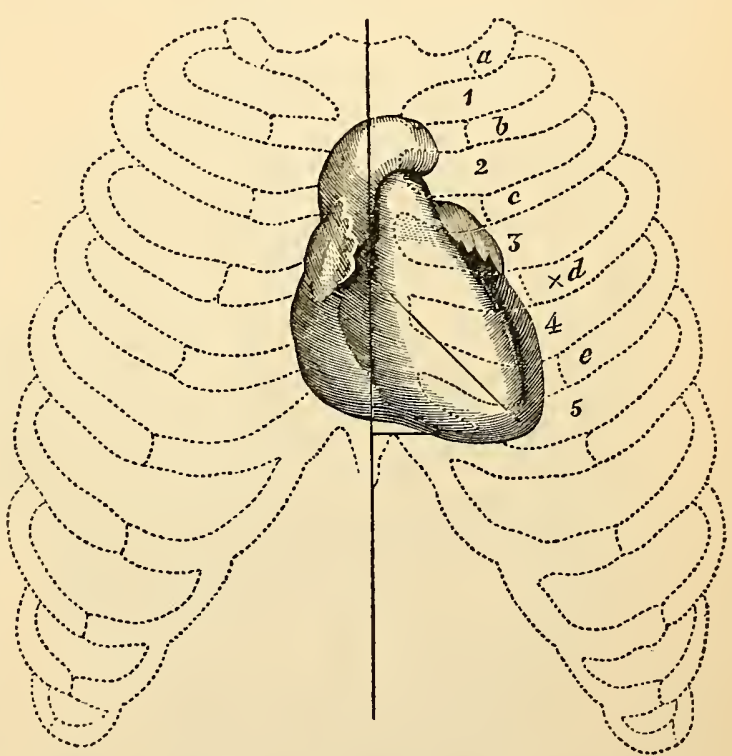

Fig. 16. - Heart in situ (Dalton, in Flint, On the Heart).

$a, b, c, d, e$, ribs; $\mathrm{I}, 2,3,4,5$, intercostal spaces; vertical line, median line; triangle, superficial cardiac region; $x$ on the fourth rib, nipple.

rous pericardium does not present any differences from serous membranes in other situations, which form a part of the great lymphatic system. The cavities of the heart are lined with a smooth membrane, called the endocardium, which is continuous with the lining membrane of the bloodvessels.

The right auricle receives the blood from the venæ cavæ and empties it into the right ventricle. The auricle consists of a principal cavity, or sinus, as it is called, with a little appendix, called, from its resemblance to the ear of a dog, the auricular appendix. It presents two large openings, for the vena cava ascendens and the vena cava descendens respectively, with a small opening for the coronary vein. It also has another 
large opening, called the auriculo-ventricular opening, through which the blood flows into the rentricle. The auricular walls are thinner than the walls of the ventricles, measuring about one line (2.I millimeters). They are composed of muscular fibres arranged in two layers, one of which, the external, is common to both auricles, and the other, the internal, is proper to each. These muscular fibres, although involuntary in their action, belong to the striated variety and are nearly the same in structure as the fibres of the ventricles. Some of the auricular fibres are looped, arising from a cartilaginous ring which separates the auricles and ventricles and passing over the auricles; and others are circular, surround-

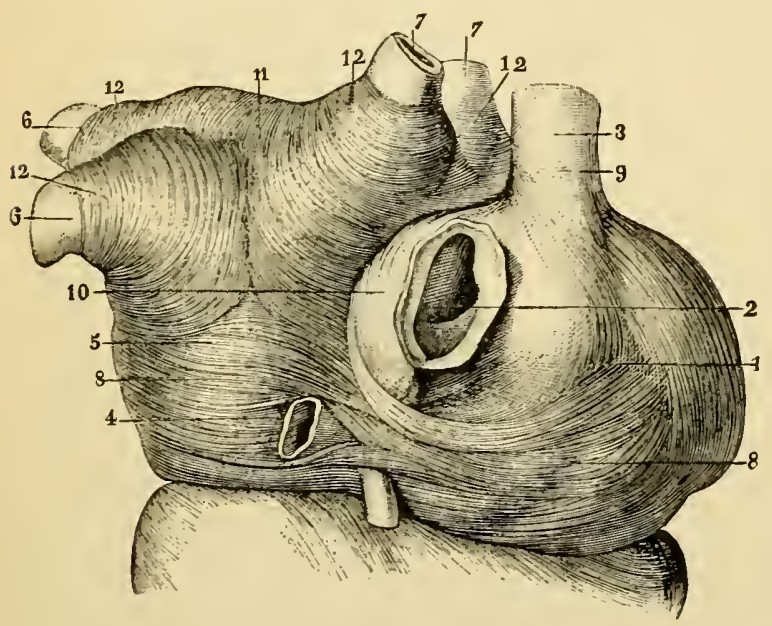

Fig. 17. - Muscular fibres of the auricles (Bonamy and Beau).

I, right auricle; 2 , inferior vena cava; 3 . superior vena cava; 4. coronary vein; 5 , left auricle; 6,6 , left pulmonary veins; 7,7 , right pulmonary veins; 8,8 , muscular fibres surrounding the right and left auriculo-ventricular openings; 9 , muscular fibres surrounding the opening of the superior vena cava; Io, muscular fibres surrounding the opening of the inferior vena cava; II, I2, I2, I2, I2, circular fibres surrounding the openings of the pulmonary veins. ing the auricular appendages and the openings of the veins, extending, also, for a short distance along the course of these vessels. One or two valvular folds are found at the orifice of the coronary vein, preventing a reflux of blood; but there are no valves at the orifices of the venæ cavæ.

The left auricle receives the blood from the lungs by the pulmonary veins. It does not differ materially in its anatomy from the right. It is a little smaller, and its walls are thicker, measuring about a line and a half (3.I 5 millimeters). It has four openings by which it receives blood from the four pulmonary veins. These openings have no valves. Like the right auricle, it has a large opening through which blood flows into the corresponding ventricle. The arrangement of the muscular fibres is essentially the same as in the right auricle. In adult life the cavities of the auricles are distinct from each other. Before birth they communicate by a large opening, the foramen ovale, and the orifice of the inferior vena cava is provided with a membranous fold, the Eustachian valve, which serves to direct the blood from the lower part of the body through 
the opening into the left auricle. After birth the foramen ovale is closed and the Eustachian valve gradually disappears.

The ventricles, in the human subject and in warm-blooded animals, constitute the bulk of the heart. They have a capacity somewhat greater than that of the auricles and are provided with thick muscular walls. The action of this portion of the heart sends the blood, on the one hand, to the lungs and back to the left side of the heart, and on the other, through the entire system of the greater circulation, to the right side.

The capacity of the cavities in the right side of the heart is one-tenth to one-eighth greater than that of the corresponding cavities on the left side. The capacity of the ventricles exceeds that of the auricles by one-fourth to one-third. The absolute capacity of the left ventricle, when distended to its utmost, is 4.8 to 7 ounces ( 433 to 2 I 2 cubic centimeters). This is much greater than most estimates, which place the capacity of each of the various cavities, moderately distended, at about three

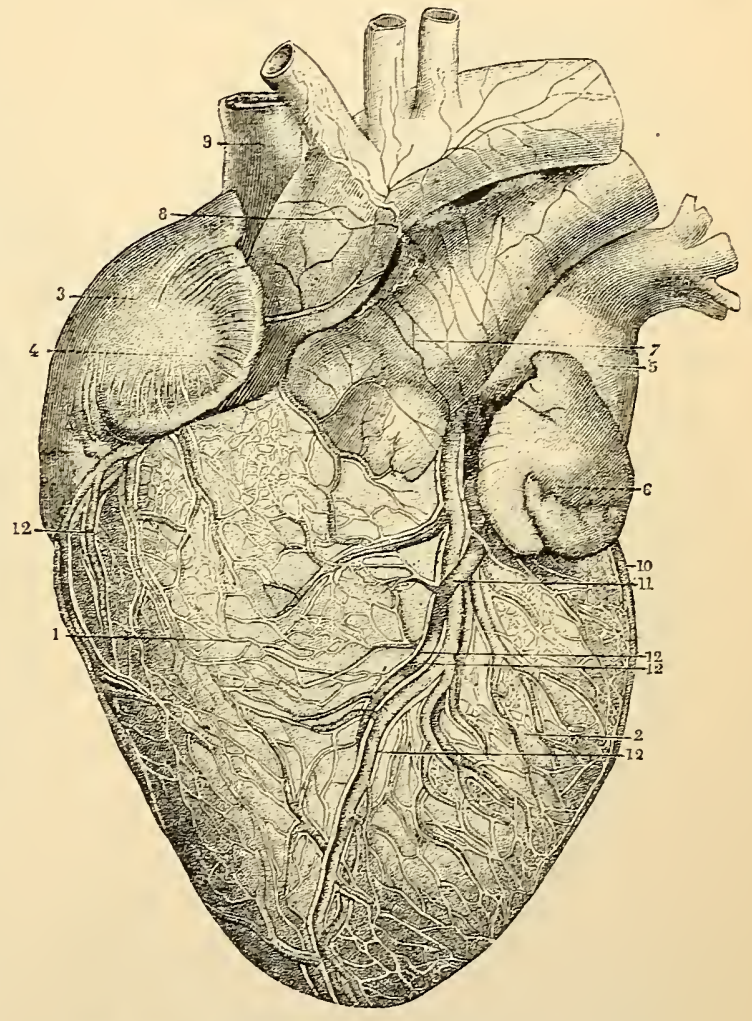

Fig. 18. - Heart, anterior view (Bonamy and Beau).

I, right ventricle ; 2 , left ventricle ; 3, 4, right auricle; 5, 6, left auricle; 7 , pulmonary artery; 8 , aorta; 9 , superior vena cava; IO, anterior coronary artery; II, branch of the coronary vein; 12, I2, I2, lymphatic vessels.

ounces ( 90 cubic centimeters). Notwithstanding the disparity in the extreme capacity of the various cavities, the quantity of blood which enters these cavities necessarily is equal to that which is expelled. This is estimated at about three ounces ( 90 cubic centimeters).

The cavities of the ventricles are conoidal, the right being broader and shorter than the left, which latter extends to the apex. The inner surfaces of both cavities are marked by ridges and papillæ, called columnæ carnex. Some of these are fleshy ridges projecting into the cavity; 
others are columns attached by either extremity and free at the central portion; and others are papillæ, giving origin to the chordæ tendineæ, which are attached to the free borders of the auriculo-ventricular valves. These fleshy columns interlace in every direction and give the inner surfaces of the cavities a reticulated appearance. This arrangement

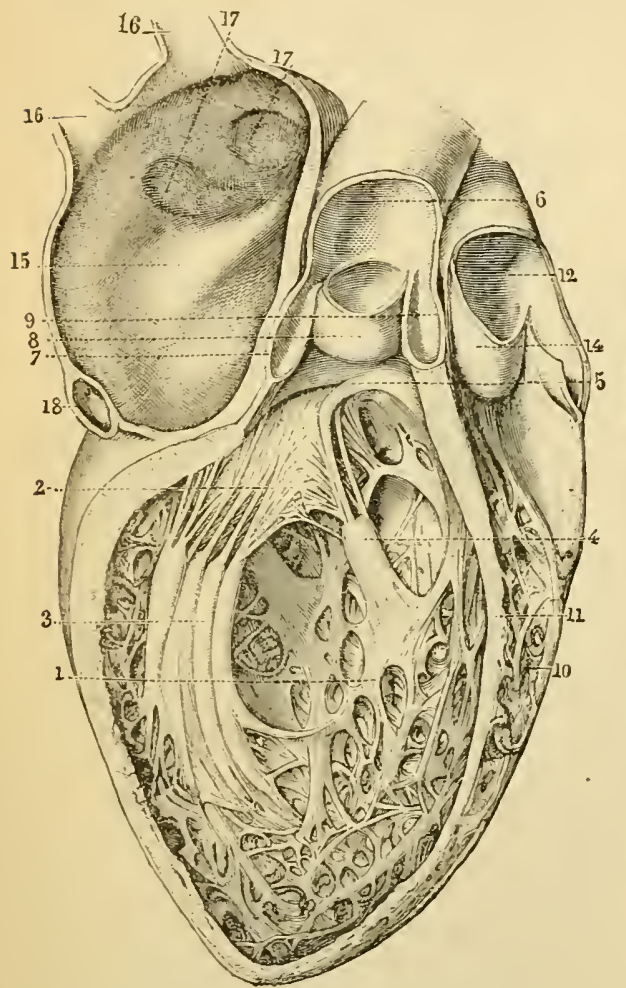

Fig. I9. - Left cavities of the heart (Bonamy and Beau).

1 , left ventricular cavity; 2, mitrul valve; 3,4 , columna carnea ; 5 , aortic cpening; 6 , aorta; $7,8,9$, aortic valves; IO, right ventricular cavity; II, interventricular septum; I2, pulmonary artery; I3, I4, pulmonic valves; I5, left auricular cavity; I6, I6, right pulmonary veins, with 17,17 , openings of the veins; 18 , section of the coronary vein.

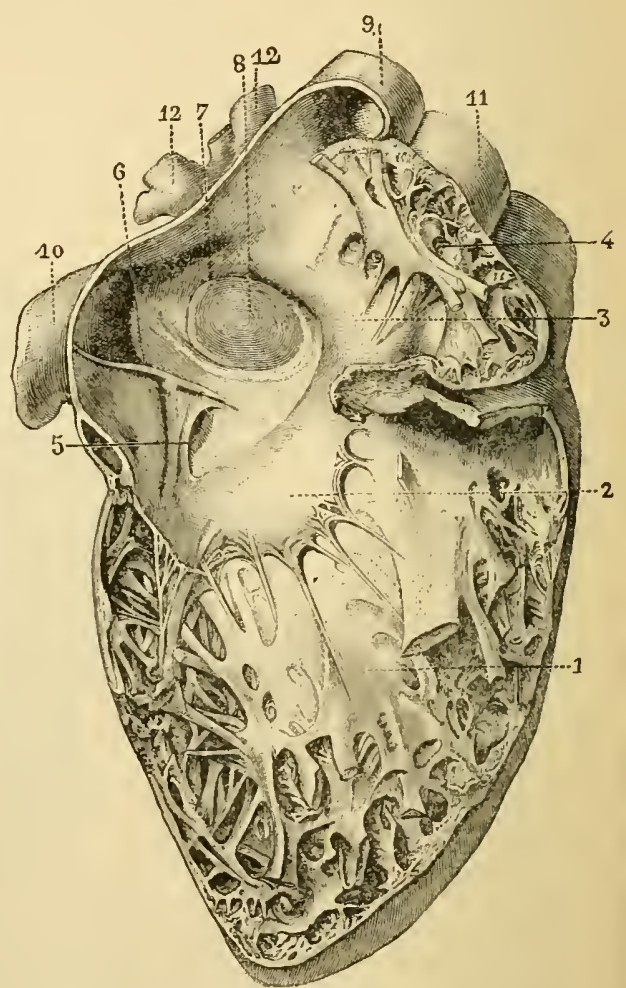

Fig. 20. - Right cavities of the heart (Bonamy and Beau).

I, right ventricular cavity; 2, posterior curtain of the tricuspid valve: 3, right auricular cavity; 4 columnce carnece of the right auricle; 5 , section of the coronary vein; 6, Eustachian valve; 7 , ring of Vieussens; 8 , fossa ovalis; 9. superjor vena cava; Io, inferior vena cava; I1, aorta; I2, I2, right pulmonary veins.

facilitates the complete emptying of the ventricles during their contraction.

The walls of the left ventricle are thicker than those of the right side. The average thickness of the right ventricle at the base is two and a half lines ( 5.25 millimeters), and the thickness of the left ventricle at the corresponding part is seven lines (I4.7 millimeters), or a little more than half an inch. 
The arrangement of the muscular fibres of the ventricles is more regular than in the auricles; but their direction cannot be well made out unless the heart has been boiled for a number of hours, when part of the intermuscular tissue is dissolved. They present two principal layers, - a superficial layer common to both ventricles, and a deep layer proper to each ventricle. The superficial fibres pass obliquely from right to left from the base above to the apex below; here they take a spiral course, become deep and pass into the interior to form the columnæ carneæ. These fibres envelop both ventricles. They may be said to arise from cartilaginous rings that surround the auriculo-ventricular orifices. The external surface of the heart is marked by a groove that indicates the division between the two ventricles. The deep fibres are circular, or transverse, and surround each ventricle separately.

The muscular tissue of the heart is of a deep red color and resembles, in its gross characters, the tissue of ordinary voluntary muscles; but as already intimated, it presents certain peculiarities in its minute anatomy. The fibres are considerably smaller and are more granular than those of ordinary muscles.

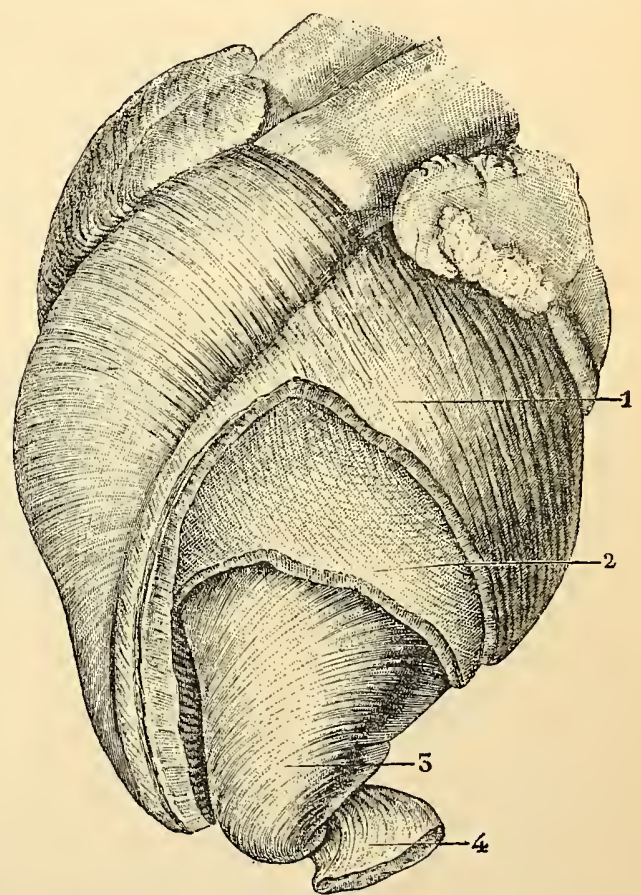

Fig. 21. - Muscular fibres of the ventricles (Bonamy and Beau).

I, superficial fibres common to both ventricles; 2 , fibres of the left ventricle; 3 , deep fibres passing upward toward the base of the heart; 4 , fibres penetrating the left ventricle.

They are, moreover, connected with each other by short inosculating branches and have no sarcolemma. The muscular-fibre cells have each a single oval nucleus which presents a network of chromatin. Sometimes, though rarely, a cell contains two nuclei. The cells are joined together by their ends with what seems to be a cement-substance, with delicate protoplasmic processes extending between two contiguous cells. The branching fibres form a close muscular reticulum in the meshes of which are bloodvessels, nerves and a small quantity of areolar tissue. This arrangement favors the complete expulsion of the 
contents of the cavities, especially the ventricles, with each systole (see Plate II, Fig. 3).

The distribution of the nerves to the heart and the arrangement of the ganglia and nerve-terminations in its substance will be described in connection with the influence of the nervous system on the circulation.

Each ventricle has two orifices, - one by which it receives blood from the auricle, and the other by which the blood passes from the right side to the lungs and from the left side to the general system. All these openings are provided with valves, which are so arranged as to allow the blood to flow in but one direction.

Tricuspid Valie. - This valve is situated at the right auriculo-ventricular opening. It has three curtains, formed of a thin but resisting

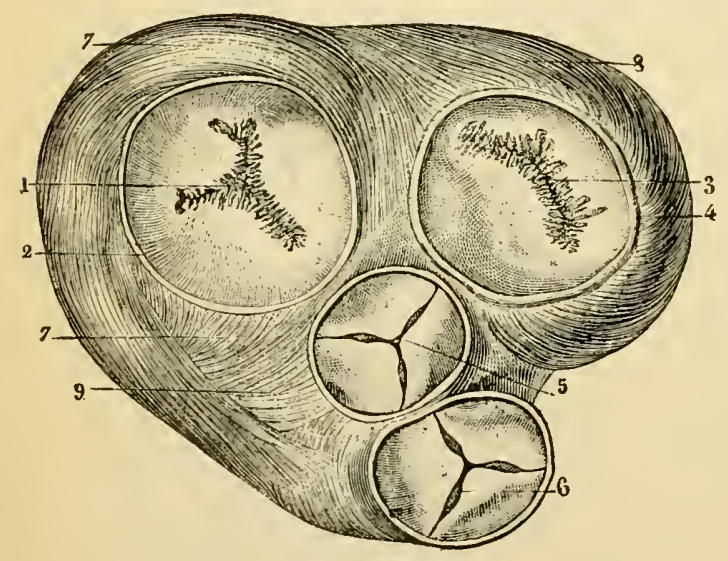

Fig. 22. - Valves of the heart (Bonamy and Beau).

$I$, right auriculo-ventricular opening, closed by the tricuspid valve $; 2$, left auriculo-ventricular opening, closed by the mitral valve; 4 , fibrous ring; 5 , aortic opening and valves; 6 , pulmonic opening and valves; 8,9 , muscular fibres. membrane, which are attached around the opening. Their free borders are attached to the chordæ tendineæ.

The Pulmonic Valves. - The three pulmonic valves, also called the semilunar or the sigmoid valves of the right side, are situated at the orifice of the pulmonary artery. They are strong membranous pouches, with their convexities, when closed, looking toward the ventricle. They are attached to the borders of the orifice of the pulmonary artery, and when closed, their free edges meet and prevent regurgitation of blood. At the centre of the free border of each valve is a little body called the corpuscle of Arantius. Just above the attached margins of the valves, the vessel presents three dilatations, or sinuses, called sinuses of Valsalva. The corpuscles of Arantius aid in the close adaptation of the free borders of the valves to each other to prevent regurgitation of blood.

Mitral Valie. - The mitral valve, sometimes called the bicuspid, is situated at the left auriculo-ventricular orifice. It is attached to the borders of this opening, and its free margins are held in place, when the valve is closed, by the chordæ tendinex, to which they are attached. It 
presents no material difference from the tricuspid valve, except that it is divided into two curtains instead of three.

Aortic Valves. - The three aortic semilunar valves are the same in their arrangement as the pulmonic valves. They prevent regurgitation from the aorta into the left ventricle.

\section{MoveMents of the Heart}

Dilatation of the cavities of the heart is called diastole, and contraction, systole. A complete revolution, or cycle of the heart consists in the filling and emptying of all its cavities, during which they present alternations of rest and contraction. As these occupy less than one second, it is evident that their exact relations to each other require careful study.

Except during the short time occupied in contraction of the auricles, these cavities are constantly receiving blood, on the right side from the venæ cavæ and on the left side from the pulmonary veins. When the auricles have become fully distended, they contract and send the blood on the right side into the right ventricle and on the left side into the left ventricle. During this contraction, the blood ceases to flow from the veins into the auricles, and a small quantity is regurgitated, as the openings are not provided with valves; but the arrangement of the muscular fibres of the auricles around the openings of the veins limits this regurgitation, and the greater part of the blood is forced into the ventricles.

Immediately following contraction of the auricles, there is contraction of the ventricles. This is the chief active operation of the heart, and it usually is spoken of as the systole. Regurgitation of blood, during contraction of the ventricles, is prevented by closure of the auriculo-ventricular valves. This act accomplished, the heart has a period of rest, the blood flowing into the auricles and from them very slowly into the ventricles, until the auricles are again completely filled, when another cycle of the heart begins.

The position of the heart in the thoracic cavity is with its base directed slightly to the right and its apex to the left. The movement of the apex from left to right is a necessary consequence of the direction of the superficial fibres from right to left. The fibres on the anterior surface are longer than the posterior fibres, and therefore the point of the heart is moved upward and to the right during their contraction. In the ventricular systole, the heart itself is propelled forward by the sudden distention of the great vessels at the base, aided by the recoil of the ventricles. By reason of the spiral course of the superficial fibres of 
the ventricles, their contraction produces a twisting of the point upon itself. An untwisting occurs during the ventricular diastole.

If the heart is grasped with the hand during its action, it is observed that the systole is attended with a palpable hardening. Like any other muscle, the heart is sensibly hardened during contraction.

The projection of the point of the heart forward during the systole is not due to elongation of the ventricles. When the ventricles contract, their transverse diameter is slightly diminished and the antero-posterior diameter is correspondingly increased. The ventricles themselves are sensibly shortened during their systole.

Cardiac Cycle. - A cardiac cycle includes the alternate contraction and dilatation of its several cavities. It is to be remembered that the two sides of the heart act together. Dividing the cycle into eighths and beginning with the contraction of the auricles, the following represents a complete revolution: one-eighth, the auricles contract and the ventricles dilate; three-eighths, the ventricles contract and the auricles are passive; four-eights, the auricles dilate and the ventricles are passive. If the condition of the walls of the several cavities is now compared with the condition of the valves, it will be found that the auriculo-ventricular valves are closed during the thrce-eighths of the cycle occupied in contraction of the ventricles, and the semilunar valves are open; and that the semilunar valves are closed during the remaining five-eighths of the cycle, while the auriculo-ventricular valves are open. It is possible, also, to connect the action of the valves with the condition of the walls of the different cavities, and the direction of the blood-currents with what are known as the heart-sounds.

Sounds of the Heart. - The first sound of the heart accompanies contraction of the ventricles. It is heard at its maximum of intensity over the apex in the fifth intercostal space and is conducted upward toward the base. This is a compound sound. Its elements are, vibration of the mitral and tricuspid valvular curtains at the time of their closure, a sound due to muscular contraction and an impression conveyed to the ear from the impulse of the heart against the walls of the chest. The first sound is relatively low in pitch, prolonged and "booming." Its low pitch is due to the length of the free borders of the valvular curtains as compared with the semilunar valves: the prolongation of the sound is attributed to the prolongation of the ventricular contraction during three-eighths of the cardiac cycle; the impulsion element comes from the striking of the apex against the thoracic walls.

The second sound which follows the first - practically without an interval - is relatively high in pitch and is shorter than the first sound. This is a simple sound and is due to the vibration of the valves during their 
closure. It is relatively high in pitch on account of the shorter length of the free borders of the valves. It is heard at its maximum of intensity over the semilunar valves on either side of the upper part of the sternum and is conducted upward along the great vessels.

Placing the stethoscope over the apex of the heart and a little to the left, it is possible to distinguish the sound produced by closure of the mitral valve; and with the stethoscope just below the ensiform cartilage, the sound produced by the tricuspid valve may be distinguished. In the same way, if the stethoscope is placed a little to the right of the sternum, near the aortic valves, the sound produced by these valves may be distinguished, while to the left of the sternum, near the pulmonic valves, the sound produced by these valves becomes more distinct.

A cardiac cycle with reference to the heart-sounds is somewhat different from the cycle described with reference to the contractions of the heart. The cycle, as it relates to the sounds, is as follows: during four-eighths of the cycle, the first sound is heard; this is followed immediately by the second sound, which occupies three-eighths of the cycle. One-eighth of the cycle is silence.

It is of great importance to connect the heart-sounds with the bloodcurrents. During the first sound, the auriculo-ventricular valves are closed, the semilunar valves are open, and, as a consequence, the blood is flowing from the left ventricle into the aorta and from the right ventricle into the pulmonary artery. During the second sound, the blood is flowing into the auricles, a small quantity is passing into the ventricles, the semilunar valves are closed and the auriculo-ventricular valves are open.

Frequency of the Heart's Action. - The number of pulsations of the heart is not far from 70 per minute in an adult male and is between 70 and 80 in the female. There are cases, however, in which the pulse is normally much slower or more frequent than this, a fact that must be remembered when examining the pulse in disease. It is said that the pulse of Napoleon I was only 40 per minute, and that the pulse of Sir William Congreve was never less than I28 per minute in health. It is not unfrequent to find a normal pulse of a hundred or more a minute; but in the cases reported in which the pulse has been found to be 40 or less, it is possible that each alternate beat of the heart was so feeble as to produce no perceptible arterial pulsation. In such instances, the fact may be ascertained by listening to the heart while the finger is placed upon the artery.

In both the male and the female, observers have constantly found a difference in the rapidity of the heart's action at different periods of life. The pulsations of the heart in the fœtus are about I 40 per minute. 
At birth the pulse is 136 . It gradually diminishes during the first year to about I28. The second year, the diminution is quite rapid, I07 being the mean frequency at two years of age. After the second year, the frequency progressively diminishes until adult life, when it is at its minimum, which is about 70 per minute. At the later periods of life the movements of the heart become slightly accelerated, ranging between 75 and 80 .

During early life there is no marked and constant difference in the rapidity of the pulse in the sexes; but near the age of puberty the development of the peculiarities relating to sex is accompanied with an acceleration of the heart's action in the female, which continues into old age.

The condition of the digestive system has a marked influence on the rapidity of the pulse, and there usually is an increase of five or ten beats per minute after each meal. Prolonged fasting diminishes the frequency of the pulse by about twelve beats. Alcohol first diminishes and afterward accelerates the pulse. Coffee is said to accelerate the pulse in a marked degree. It has been ascertained that the pulse is accelerated in a greater degree by animal than by vegetable food.

It has been observed that the position of the body has a decided influence on the rapidity of the pulse. In the male, there is a difference of about Io beats between standing and sitting, and I 5 beats between standing and the recumbent posture. In the female, the variations with position are not so great. The average is, for the male: standing, 8I ; sitting, 7I ; lying, 66;-for the female: standing, 9I; sitting, 84; lying, 80. These are given as averages of a large number of observations. There were a few instances, however, in which there was scarcely any variation with posture, and some in which the variation was much greater than the average. In the inverted posture, the pulse was found to be reduced about i 5 beats (Guy).

The question at once suggests itself whether the acceleration of the pulse in sitting and standing may not be due, in some measure, to the muscular effort required in making the change of posture. This is answered by the experiments of Guy, in which the subjects were placed on a revolving board and the position of the body was changed without any muscular effort. Nearly the same results as those cited above were obtained in these experiments. In a single observation, the pulse, standing, was 89 ; lying, 77 ; difference, I 2 . With the posture changed without muscular effort, the results were as follows: standing, 87; lying, 74 ; difference, I3. Different explanations of these variations have been offered by physiologists; but Guy seems to have settled experimentally the fact that the acceleration is due in part to the 
muscular effort required to maintain the body in the sitting and standing positions. The following are the results of experiments bearing on this point.

"I. Difference between the pulse in the erect posture, without support, and leaning in the same posture, in an average of twelve experiments on the writer, I 2 beats; and on an average of eight experiments on other healthy males, 8 beats.

" 2 . Difference in the frequency of the pulse in the recumbent posture, the body fully supported, and partially supported, 14 beats on an average of five experiments.

" 3 . Sitting posture (mean of ten experiments on the writer), back supported, 80; unsupported, 87; difference, 7 beats.

" 4. Sitting posture with the legs raised at right angles with the body (average of twenty experiments on the writer), back unsupported, 86; supported, 68 ; difference, 18 beats. An average of fifteen experiments of the same kind on other healthy males gave the following numbers : back unsupported, 80 ; supported, 68 ; a difference of I 2 beats."

Muscular exercise increases the frequency of the pulsations of the heart; and the experiments just cited show that the difference in rapidity, which is by some attributed to change in posture, - some positions, it is fancied, offering fewer obstacles to the current of blood than others, - is due mainly to muscular exertion. According to Bryan Robinson ( 734 ), a man in the recumbent posture has 64 pulsations per minute; sitting, 68; after a slow walk, 78 ; after walking four miles in an hour, IOO; and I 40 to I 50 after running as fast as he could.

The influence of sleep on the action of the heart reduces itself almost entirely to the proposition that during this condition there usually is entire absence of muscular effort, and consequently the number of beats is less than when the individual is awake. It has been found that there is no difference in the pulse between sleep and perfect quiet in the recumbent posture. This was noted in the adult male; but there is a marked difference in females and young children, the pulse being always slower during sleep (Quetelet).

The influence of extremes of temperature on the heart is very decided. The pulse may be doubled by remaining a very few minutes exposed to extreme heat. Bence Jones and Dickinson have ascertained that the pulse may be much reduced in frequency, for a short time, by the cold douche. It has also been remarked that the pulse is more rapid in warm than in cold climates.

Influence of Respiration on the Action of the Heart. - The relations between the circulation and respiration are very intimate, and one process can not go on without the other. If circulation is arrested, 
the muscles, being no longer supplied with fresh blood, soon lose their contractility and respiration ceases. Circulation, also, is impossible if respiration is permanently arrested. When respiration is imperfectly performed, the action of the heart is slow and labored. The effects of arrest of respiration are marked in all parts of the circulatory system, arteries, capillaries and veins; but the disturbances thus produced all react on the heart.

If the heart is exposed in a living animal and artificial respiration is kept up, although the pulsations are increased in frequency and diminished in force, after a time they become regular and continue thus so long as air is adequately supplied to the lungs. Under these conditions, respiration is under control and the effects of its arrest on the heart can easily be studied. If respiration is interrupted, the following changes in the action of the heart are observed: For a few seconds pulsations go on as usual; but in about a minute they begin to diminish in frequency. At the same time, the heart becomes engorged with blood and the distention of its cavities rapidly increases. For a time its contractions are competent to discharge the entire contents of the left ventricle into the arterial system, and a cardiometer applied to an artery then indicates an increase in the blood-pressure. A corresponding increase in the movements of the mercury will be noted at each contraction of the heart, indicating that the organ is acting with abnormal vigor. If respiration is still interrupted, the engorgement becomes intense, the heart at each diastole being distended to its utmost capacity. It now becomes incapable of emptying itself, the contractions become unfrequent, perhaps three or four in a minute, and are progressively enfeebled. The organ is dark, almost black, owing to the circulation of venous blood in its substance. If respiration is not resumed, this distention continues, the contractions become less frequent and more feeble and in a few minutes they cease.

The arrest of the action of the heart, under these conditions, is chiefly mechanical. The unaërated blood passes with difficulty through the capillaries of the system, and as the heart is constantly at work, the arteries become greatly distended. This is shown by the great increase in the pressure while the arteries are filled with black blood. If, now, the heart and great vessels are closely examined, the order in which they become distended is readily observed. These phenomena show that in asphyxia the obstruction to the circulation begins, not in the lungs, but in the capillaries of the system and is propagated backward to the heart through the arteries. The distention of the heart in asphyxia is therefore due to the fact that unaërated blood cannot circulate freely in the systemic capillaries. When thus distended, the heart 
becomes paralyzed, like any muscle after a severe strain. It is probable that the impediment to the capillary circulation results from contraction of the arterioles of supply, due to irritation produced by the excess of carbon dioxide in the blood.

Cause of the Rhythmical Contractions of the Heart. - The heart in its structure bears a close resemblance to the voluntary muscles; but it has a constant office to perform and seems to act without external stimulation. Its action resembles, in this regard, the movements of cilia. The movements of the heart are involuntary. Its pulsations can be neither arrested, retarded nor accelerated directly by an effort of the will, except, of course, when retarded by voluntary arrest of respiration or accelerated by violent muscular exercise or other indirect means. Its property of rhythmical contraction, however, seems to depend on the circulation of blood in its substance. If the coronary arteries are tied, the heart ceases to beat in about twenty-three minutes (Erichsen). The regular and efficient contractions of the heart, also, are promoted by the passage of blood through its cavities. Although the heart removed from the body will continue to contract spontaneously and rhythmically for a time, its contractions soon cease, at least in warm-blooded animals; but during intervals of rest, a contraction may be excited by direct stimulation. The nature of the liquid passing through the heart has an influence on the character of its contractions. When blood passes through its cavities, the pulsations are regular and powerful; but if water is substituted, the beats become more frequent and are not so vigorous (Flint, 186I).

It is certain that the muscular tissue of the heart has an inherent property of rhythmical contraction. Under normal conditions, this contraction seems to be propagated from the auricles to the ventricles; but it is also true that the contractions of the heart are regulated through the nervous system. When the heart is divided transversely at about the middle of the ventricles, the upper portion continues to pulsate, while the lower portion does not. The lower portion, however, still possesses contractility and will respond to direct stimulation. This is thought to be due to sympathetic ganglia that exist in the upper part, but not in the lower part of the ventricles. In the frog there are three sympathetic ganglia situated near the auricles: one, the ganglion of Remak, is at the point where the inferior vena cava opens into the right auricle; another is between the left auricle and the right ventricle; another is between the two auricles. In man there is a chain of ganglia between the auricles and the ventricles. It is probable that these ganglia are important in regulating the action of the heart.

In view of the results of experiments on the cold-blooded animals 
especially, it may be stated that the muscular fibres of the auricles and of the upper part of the ventricles have the property of intermittent and regular contraction, which is dependent, to a great extent, on the influence of the so-called motor ganglia of the heart; and that the wave of contraction is transmitted to the lower portion of the ventricles, the fibres of which do not seem to possess the property of independent contraction. The muscular tissue of the heart, however, may be thrown into contraction during diastole by the application of a stimulus, a property observed in all muscular fibres. The excitability manifested in this way is more marked in the interior than on the exterior of the organ. Blood in contact with the lining membrane of the heart acts as a stimulus in a remarkable degree and is even capable of restoring excitability after it has become extinct. The passage of blood through the heart is a natural stimulus and is an important element in the production of regular pulsations, although it does not endow the fibres with their contractile properties. $^{1}$

Accelerator Nerics. - Experiments on the influence of the sympathetic nerves on the heart have not been entirely satisfactory. It has been observed that the action of the heart is arrested by destroying the cardiac plexus; but in regard to this, the difficulty of the operation and the disturbance of the heart consequent on the necessary manipulations must be taken into account. It has been shown, however, that stimulation of the sympathetic in the neck has the effect of accelerating the cardiac movements.

In the bulb is a centre, stimulation of which increases the rapidity of the heart's action ; and from this centre, fibres descend in the substance of the spinal cord, pass out with the communicating branches of the lower cervical and upper dorsal nerves to the sympathetic and go to the cardiac plexus. In the cat, the accelerator fibres pass through the first thoracic sympathetic ganglion. Taking all precautions to eliminate the influence of variations in blood-pressure, it has been shown that after division of the pneumogastrics, stimulation of the accelerator fibres increases the number of beats of the heart. This action is direct and not reflex.

1 The well-known experiments of Stannius, published in 1852, have lately received much attention. In these observations it was found that a ligature applied exactly at the line of junction of the sinus venosus with the right auricle, in the frog, arrests for a time the contractions of other parts of the heart, while the sinus continues to beat regularly; but the walls of the other cavities will contract under direct stimulation. The wave of contraction seems to pass from the sinus to the auricle and ventricle. If, now, another ligature is applied to the line of junction of the auricle with the ventricle, the ventricle resumes its rhythmical contractions, while the auricle continues at rest. Explanations of these phenomena - which are not observed in animals higher in the scale - are so unsatisfactory that it does not seem worth while to discuss them in the body of the text. 
Direct Inhibition of the Heart. - Division of the pneumogastric nerves in the neck increases the frequency and diminishes the force of the contractions of the heart. To anticipate a little of the history of the pneumogastric nerves, it may be stated that while they are exclusively sensory at their origin, they receive, after having emerged from the cranial cavity, a number of communicating filaments from various motor nerves. That they influence certain muscles, is shown by the paralysis of these muscles after division of the nerves in the neck, as, for example, arrest of the movements of the glottis.

A moderate faradic current passed through both pneumogastrics arrests the action of the heart in diastole. This observation has been made on living animals, both with and without exposure of the heart; and this kind of action is known as inhibitory, or restraining. Its nervous mechanism is direct and not reflex; and the inhibitory influence is conveyed to the heart through filaments in the pneumogastrics, derived from the spinal accessory.

It is said that direct stimulation of the bulb has the same effect on the heart as stimulation of the pneumogastrics; but it is difficult to limit the stimulation to a particular point in the bulb and to avoid complicating conditions. A sufficiently powerful stimulus applied to one pneumogastric will arrest the cardiac pulsations, and in some animals the inhibitory action is confined to the nerve of the right side. It is not known that any such difference between the two nerves exists in the human subject, and certainly there is no marked difference in most of the mammalia.

If both pneumogastrics are faradized for two or three minutes, the contractions of the heart return, even though the stimulation is continued, provided the current be not too powerful but of sufficient strength to promptly arrest the pulsations. It is probable that this is due to the fact that the conductivity of the nerve after a time becomes exhausted by prolonged excitation, and its inhibitory influence is for the time destroyed.

Stimulation of the pneumogastrics in any part of their course is followed by the usual inhibitory phenomena, and the same results sometimes follow stimulation of the thoracic cardiac branches. It has also been observed that when the heart's action has been arrested and the organ is quiescent in diastole, direct mechanical stimulation of the heart is followed by a single contraction, showing that the excitability of the fibres has not been entirely suspended.

After section of both pneumogastrics in the neck, digitalis fails. to diminish the number of beats of the heart; showing that separation of the heart from its connections with the cerebro-spinal nerves 
removes the organ from the characteristic and peculiar effects of the poison.

Feeble stimulation of one or both pneumogastrics, when it produces any effect, almost always slows the action of the heart. In some animals, however, the pneumogastrics contain a few accelerator fibres, and feeble excitation sometimes is followed by a slight increase in the rapidity of the cardiac pulsations, but this is unusual.

Reflex Inlizition of the Heart. - Like most of the direct operations of nerves that can be imitated by electric stimulation, the inhibitory action of the pneumogastrics can be produced by reflex action. The action of the heart may be arrested in the frog by sharply tapping the exposed intestines. The same effect has been produced by stimulation of the splanchnic nerves or the cervical sympathetic. In some animals, if one pneumogastric is divided in the neck, the other being intact, stimulation of the central end of the divided nerve will produce inhibition of the heart by an action induced in the undivided nerve. In all these instances the inhibition is reflex. The stimulation is carried by the afferent fibres of the nerves stimulated to the inhibitory centre in the bulb and is reflected to the heart through the efferent fibres of the pneumogastric.

While moderate stimulation of ordinary sensory nerves is sometimes followed by inhibition of the heart, powerful stimulation may arrest the cardio-inhibitory action of the pneumogastrics as well as certain other reflexes.

The inhibitory fibres of the pneumogastrics undoubtedly have an important office in connection with the regulation of the rapidity and force of the cardiac pulsations. It is important, of course, that the heart should act at all times with nearly the same force and frequency. It has been seen that the inherent properties of its fibres and the action, probably, of the cardiac ganglia are competent to make it contract, and the necessary intermittent dilatation of its cavities makes the contractions assume a certain regularity; but the quantity and density of the blood are subject to considerable variations within the limits of health, which, without some regulating influence, would cause variations in the heart's action so considerable as to be injurious. This is shown by the irregular action of the heart when the pneumogastrics have been divided. These nerves convey to the heart a constant influence, which may be compared to the insensible tonicity imparted to voluntary muscles by the general motor system. When a set of muscles on one side is paralyzed, as in facial palsy, their tonicity is lost, they become flaccid, and the muscles on the other side, without any effort of the will, distort the features. An exaggeration of this 
force may be imitated by a feeble faradic current, which renders the pulsations of the heart less frequent and more powerful, or it may be still further exaggerated by a more powerful current, which arrests the action of the heart. Phenomena are not wanting in the human subject to verify these views. Causes operating through the nervous system frequently produce palpitation and irregular action of the heart. Cases are not uncommon in which palpitation habitually occurs after a full meal. There are instances on record of death from arrest of the heart's action as a consequence of fright, anger, grief or other severe mental emotions. Syncope from these causes is by no means uncommon. In the latter instance, when the heart resumes its contractions, the nervous shock carried along the pneumogastrics is sufficient only to arrest its action temporarily. When death takes place, the shock is so great that the heart does not recover from its effects.

Work of the Heart. -- The total work of the heart is easily computed by multiplying the weight of the blood discharged at each contraction of the ventricles by the blood-pressure. Taking the weight of blood discharged by both ventricles as about 4.5 ounces (I 30 grams) and estimating the blood-pressure as equal to about five feet (I.j meter), the result would be about I.25 foot-pounds (O.I75 kilogrammeter) of work for each contraction of the heart. Assuming that the heart beats seventy-two times in a minute, the total work for twentyfour hours would be about I 30,000 foot-pounds (I 8,000 kilogrammeters). These figures are given as representing the probable work and are not to be taken as anything more than approximate estimates. 


\section{CHAPTER III}

\section{CIRCULATION OF BLOOD IN THE VESSELS}

Circulation of blood in the arteries - Physiological anatomy of the arteries - Locomotion of the arteries and production of the pulse - Form of the pulse - Pressure of blood in the arteries - Pressure in different arteries - Influence of respiration - Influence of muscular action, etc. - Influence of hemorrhage, etc. - Rapidity of the current of blood in the arteries - Circulation of blood in the capillaries - Physiological anatomy of the capillaries - Pressure of blood in the capillaries - Rapidity of the capillary circulation - Relations of the capillary circulation to respiration - Causes of the capillary circulation-Influence of temperature on the capillary circulation - Circulation of blood in the veins - Structure and properties of the veins - Valves of the veins - Pressure of blood in the veins - Rapidity of the current of blood in the veins - Causes of the venous circulation - Influence of muscular contraction - Influence of aspiration from the thorax - Uses of the valves of the veins - Conditions that impede the venous circulation - Circulation in the cranial cavity - Circulation in erectile tissues - Derivative circulation - Pulmonary circulation - Circulation in the walls of the heart - Migration and diapedesis - Rapidity of the circulation - Phenomena in the circulatory system after death.

Is man and in all animals with a double heart, each cardiac contraction forces a charge of blood from the right ventricle into the pulmonary artery and from the left ventricle into the aorta; and the valves guarding the orifices of these vessels prevent regurgitation during the intervals of contraction. There is, therefore, but one direction in which the blood can flow in obedience to this rhythmic action; and the fact that even in the smallest arteries there is an acceleration in the current coincident with each contraction of the heart, which disappears when the action of the heart is arrested, shows that the ventricular systole is the cause of the arterial circulation. The supply of blood regulates, to a considerable extent, the processes of nutrition and has an important bearing on the general and special functions; and the various physiological processes necessarily demand considerable modifications in the quantity of arterial blood furnished to parts at different times. The force of the heart, however, varies but little within the limits of health; and the conditions necessary to the proper distribution of blood are regulated almost exclusively by the arterial system. These vessels are endowed with elasticity, by which the circulation is considerably facilitated, and with contractility, by which the supply to any part may be modified independently of the action of the heart. 


\section{Circulation of Blood in the Arteries}

The vessels that carry venous blood to the lungs are branches of a great trunk which takes its origin from the right ventricle. They do not differ in structure from the vessels carrying blood to the general system, except in the fact that their coats are somewhat thinner and more distensible. The aorta, branches and ramifications of which supply all parts of the body, is given off from the left ventricle. Just at its origin, behind the semilunar valves, this vessel has three sacculated pouches called the sinuses of Valsalva. Beyond this point the vessels are cylindrical. The arteries branch, divide and subdivide, until they àre reduced to microscopic size. The branches, with the exception of the intercostal arteries, which make nearly a right angle with the thoracic aorta, are given off at an acute angle. As a rule the arteries are nearly straight, taking the shortest course to the parts which they supply with blood; and while the branches progressively diminish in size, but few are given off between the great trunk and small vessels that empty into the capillary system. So long as a vessel gives off no branches, its calibre does not progressively diminish; as the common carotids, which are as large at their bifurcation as they are at their origin. There are one or two instances in which vessels, although giving off many branches in their course, do not diminish in size for some distance; as the aorta, which is as large at the point of division into the iliacs as it is in the chest, and the vertebral arteries, which do not diminish in calibre until they enter the foramen magnum. It has long been remarked that the combined calibre of the branches of an arterial trunk is greater than that of the main vessel; so that the arterial system, as it branches, increases in capacity. A single exception to this rule is in the instance of the common iliacs, the combined calibre of which is less than the calibre of the abdominal aorta.

Usually the arteries are so situated as not to be exposed to pressure and consequent interruption of the current of blood; but in certain situations, as about some of the joints, there is necessarily some liability to occasional compression. In certain parts, also, as in the vessels going to the brain, particularly in some of the inferior animals, it is necessary to moderate the force of the blood-current on account of the delicate structure of the organs in which they are distributed. Here there is a provision in the shape of anastomoses, by which, on the one hand, compression of a vessel simply diverts and does not arrest the current of blood, and on the other hand, the current is rendered more equable and the force of the heart is moderated.

The arteries are provided with fibrous sheaths, of greater or less strength as the vessels are situated in parts more or less exposed to 
disturbing influences or accidents. They have three well-defined coats. As these vary considerably in arteries of different sizes, it will be convenient, in their description, to divide the vessels into three classes :-

I. The largest arteries; in which are included all that are larger than the carotids and common iliacs.

2. The arteries of medium size; that is, between the carotids and iliacs and the smallest.

3. The smallest arteries; or those less than $\frac{1}{15}$ to $\frac{1}{12}$ of an inch (I.7 to 2. I millimeters) in diameter.

The external coat of the arteries, called the tunica adventitia, is the strongest of the three. It is composed of white fibrous tissue with a network throughout its extent of yellow elastic fibres. A few longitudinal muscular fibres are found in this coat. It is largely the external coat that gives strength to the vessels. The nutrient vessels of the arteries (vasa vasorum) ramify in the external coat.

The middle coat is called the tunica media and is composed of both muscular and elastic fibres. In the larger arteries it is the thickest coat of the three. The muscular fibres are of the non-striated, or involuntary variety and encircle the vessel. The general direction, also, of the elastic fibres is transverse. These fibres interlace with each other in every direction. Arteries of different sizes present differences in the thickness and constitution of the middle coat. In the larger vessels, the elastic elements predominate; and in the smaller arteries, the muscular fibres are more abundant. In the very smallest vessels of supply to the capillary system, the middle coat presents muscular fibres only. The vasomotor nerves are distributed in the middle coat.

The internal coat is called the tunica intima and is composed of three layers. This is sometimes spoken of as the fenestrated membrane of Henle. The innermost of the three layers of the intima is the endothelial lining, made up of oblong endothelial cells with their long diameter in the direction of the vessel. Just beneath the endothelial lining is the subendothelial layer, composed of fine branching connective-tissue corpuscles and fibres. The external layer of the intima is a lamella of fine fibres of elastic tissue. These layers are very thin in the smallest arteries (see Plate III, Figs. 2, 3, 4).

The elasticity of the arteries has an important influence on the general circulation. It provides for what is practically a continuous flow of blood from the smallest arteries into the capillary system. If it is possible to imagine the arterial system as consisting of inert tubes, it is evident that the intermittent force of the heart would be quite as apparent in the small as in the large arteries; but the elasticity of the walls of the vessels, especially of the larger arteries, produces a recoil following the 
contraction of the ventricles, which forces the blood onward in the intervals of the heart's action. This recoil produces what is known as the dicrotic pulse. It is in this way that the physical property of elasticity of the vessels favors the blood-current. The arteries are not only elastic but contractile; and this property, which gives tonicity to the vessels, aids in adapting them to the quantity of blood which they contain and regulates the arterial pressure as well as the supply of blood to different parts of the capillary system.

A study of the elasticity and contractility of the arteries naturally leads to a consideration of the pulse. With each ventricular systole there is a wave of dilatation which extends from the aorta to the smallest arteries. There is, however, a slight delay in the pulse in the smaller vessels. The difference in time between the ventricular systole and the pulse in the feet is about one-seventh of a second.

Locomotion of the Arteries and Production of the Pulse. - With each contraction of the heart, the arteries are increased in length and many of them undergo a considerable locomotion. This may be readily observed in vessels that are tortuous in their course and is frequently quite marked in the temporal artery in old persons. The elongation may also be observed by watching attentively the point where an artery bifurcates, as at the division of the common carotid. It is simply the mechanical effect of sudden distention, which, while it enlarges the calibre of the vessel, causes an elongation that is even more distinct.

The finger placed over an exposed artery or one that lies near the surface experiences a sensation at every beat of the heart as though the vessel were striking against it. Ordinarily this is appreciated when the current of blood is subjected to a certain degree of obstruction, as in the radial, which can readily be compressed against the bone. In an artery imbedded in soft parts which yield to pressure, the actual dilatation of the vessel being very slight, pulsation is felt with difficulty if at all. When obstruction of an artery is complete, as after tying a vessel, the pulsation above the point of ligature is quite marked and can easily be appreciated by the eye. The explanation of this exaggeration of the movement is the following: Normally, the blood passes freely through the arteries and produces, in the smaller vessels, very little movement or dilatation; when, however, the current is obstructed, as by ligation or even compression with the finger, the force of the heart is not sent through the vessel to the periphery, but is arrested and therefore becomes more easily appreciated. In vessels that have become undilatable and incompressible from calcareous deposits, the pulse cannot be felt. The character of the pulse indicates, to a certain extent, the condition of the heart and vessels. 
It is evident from what is known of the modifications that occur in the force of the heart and the quantity of blood in the vessels, and from the changes which may take place in the calibre of the arteries, that the characters of the pulse must be subject to great variations. Many of these may be appreciated simply by the sense of touch. Writers treat of the soft and compressible pulse, the hard pulse, the wiry pulse, the thready pulse, etc., as indicating various conditions of the arterial system. The character of the pulse in disease, aside from its frequency, has always been regarded as of great importance.

Form of the Pulse. - But few of the characters of a pulsation, occupying as it does only one-seventieth part of a minute, can be ascertained by the sense of touch alone. This fact has been appreciated by physiologists; and within the last sixty years, instruments for registering the pulse have been devised, with the view of analyzing the dilatation and movements of the vessels. The instrument now used for this purpose is called the sphygmograph. Vierordt (1855) constructed a complex

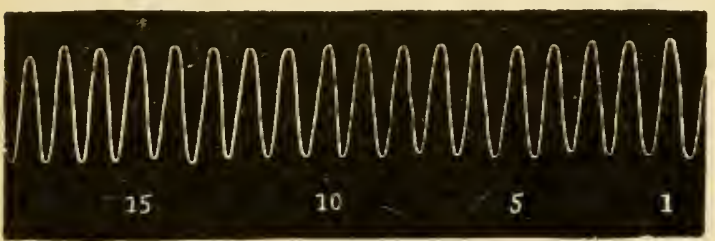

Fig. 23. - Trace of Vierordt. apparatus, so arranged that the impulse from an accessible artery, like the radial, was conveyed to a lever, which marked the movement on a revolving cylinder. These traces, họ̧vever, were perfectly regular and simply marked the extremes of dilatation - exaggerated, of course, by the length of the lever - and the number of pulsations in a given time. The instruments now in use differ from each other mainly in the convenience with which they are applied, the principle in all being substantially that of the sphygmograph of Marey. The modern instruments, applied to the radial artery, give traces quite different from those obtained by Vierordt, which were simply series of regular elevations and depressions. A comparison of these with the traces first obtained gives an idea of the defects that were remedied by Marey; for it is evident that the dilatation and contraction of the arteries can not be so regular and simple as would be inferred merely from the trace made by the instrument of Vierordt.

Analyzing the traces taken by Marey, it is seen that there is a dilatation following the systole of the heart, marked by an elevation of the lever, more or less sudden, as indicated by the angle of the trace, and of greater or less amplitude. The dilatation, having arrived at its maximum, is followed by reaction, which may be slow and regular, or may be, and ordinarily is, interrupted by a second and slighter upward movement of 
the lever. This second impulse varies very much in amplitude. In some rare instances, it is nearly as marked as the first and may be appreciated by the finger, giving the sensation of a double pulse following each contraction of the heart. This is called the dicrotic pulse. As a rule, the first dilatation of the vessel is sudden and is indicated by an almost vertical line. This is followed by a comparatively slow reaction, indicated by a gradual descent of the trace, which is not, however, absolutely regular, but is marked by a slight elevation indicating a second impulse. The amplitude of the trace, or the distance between the highest and the lowest points marked by the lever, depends on the degree of constant tension of the vessels. Marey has found that the amplitude is in an inverse ratio to the tension; which is easily understood, for when the arteries are but little distended, the force of the heart must be more marked in its effects than when the pressure of blood is greater.

In nearly all the traces given by Marey, the descent of the lever indicates more or less oscillation of the mass of blood. The physical

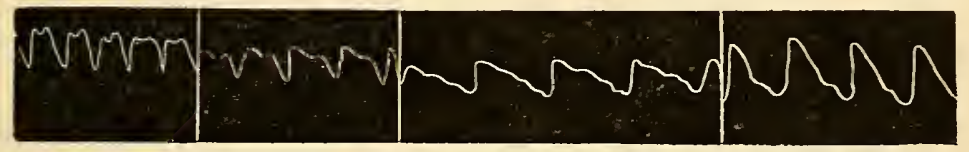

Fig. 24. - Trace of Marey.

Portions of four traces taken in different conditions of the pulse.

properties of the larger arteries render this inevitable. As they yield to the distending force of the heart, reaction occurs after this force is taken off, and if the distention is very great, gives a second impulse to the blood. This is quite marked, unless the tension of the arterial system is so great as to offer too much resistance. One of the most favorable conditions for the manifestation of dicrotism is diminished tension, which is always found coexisting with a marked exhibition of this phenomenon.

Marey accurately determined and registered these various phenomena and demonstrated that an important and essential element in the production of dicrotism is the tendency to oscillation of the fluid in the vessels during the intervals between the contractions of the heart. This can occur only in a fluid that has a certain weight and acquires a velocity from the impulse; and when air is introduced into the apparatus, dicrotism can not be produced under any conditions, as the fluid does not possess weight enough to oscillate between the impulses. Water showed a well-marked dicrotic impulse under favorable conditions; and with mercury, the oscillations made two, three or more distinct impulses. By these experiments, he proved that the blood oscillates in the vessels, if this movement is not suppressed by too great pressure or tension. This 
oscillation gives the successive rebounds that are marked in the descending line of the pulse, and is capable, in some rare instances when the arterial tension is slight, of producing a second rebound of sufficient force to be felt with the finger. The smallest vessels and those of medium size possess to an eminent degree what is called tonicity, or the property of maintaining a certain continued degree of contraction, which is antagonistic to the distending force of the blood. This is shown by opening a portion of an artery included between two ligatures in a living animal, when the contents will be forcibly discharged and the calibre of that portion of the vessel be much diminished. Too great distention of the vessels by pressure of blood seems to be prevented by this constant action of the muscular coat; and thus the conditions are maintained that give to the pulse the characters just described.

By excessive and continued heat, the muscular structure in the arteries may be dilated so as to offer less resistance to the distending force of the heart. Under these conditions, the pulse, as felt with the finger, will be found to be larger and softer than normal. Cold, either general or local, has an opposite effect; the arteries become contracted, and the pulse assumes a harder and more wiry character. As a rule, prolonged contraction of the arteries is followed by relaxation, as is seen in the full pulse and glow of the surface observed in reaction after exposure to cold.

\section{Pressure of Blood in the Arteries}

The reaction of the elastic walls of the arteries during the intervals of the heart's action gives rise to a certain degree of pressure, by which the blood is continually forced toward the capillaries. The discharge of blood into the capillaries has a constant tendency to diminish this pressure; but the contractions of the left ventricle, by forcing repeated charges of blood into the arteries, have a compensating action. By the equilibrium between these two agencies, a certain tension is maintained in the arteries, which is called the arterial pressure.

The first experiments in regard to the extent of the arterial pressure were made by Hales, and were published in I733. This observer, adapting a long glass tube to the artery of a living animal, ascertained the height of the column of blood that could be sustained by the arterial pressure. In experiments on the carotid of the horse, the blood mounted to the height of eight to ten feet (243 to 304 centimeters).

If a large artery, like the carotid, is exposed in a living animal, and a metallic point, connected with a vertical tube of smaller calibre and seven or eight feet (213 or 243 centimeters) long by a bit of elastic tubing, is secured in the vessel, the blood will rise to the height of about 
five feet ( 1.5 meters) and remain at this point almost stationary, indicating, by a slight pulsatile movement, the action of the heart. Carefully watching the level in the tube, in addition to the rapid oscillation coincident with the pulse, another oscillation will be observed, which is less frequent and which corresponds with the movements of respiration. The pressure, as indicated by an elevation of the liquid, is slightly increased during expiration and diminished during inspiration. In such experiments, it is necessary to fill part of the tube, or whatever apparatus is used, with a solution of sodium carbonate, in order to prevent coagulation of the blood as it passes out of the vessels. The experiment with the long tube gives, perhaps, the best general idea of the arterial pressure, which is equal to about five feet of blood ( .5 meters) or a few inches more of water. The oscillations produced by the contractions of the heart are not very extensive, on account of the friction in so long a tube; but this is favorable to the study of the constant pressure.

The experiments of Hales were made with a view of calculating the force of the heart and were not directed particularly to the modifications and variations of the arterial pressure. It is only since the experiments performed by Poiseuille with the hemodynamometer, in 1828 , that physiologists have had reliable data on this latter point. Poiseuille's instrument for measuring the force of the blood is a graduated U-tube, half filled with mercury, with one arm bent at a right angle, so that it can easily be connected with the artery. The pressure of blood is indicated by a depression in the level of the mercury on one side and a corresponding elevation on the other. This instrument possesses certain advantages over the long glass tube; but for estimating simply the arterial pressure, it is much less useful, as it is more sensitive to the impulses of the heart. For the study of the cardiac pressure, it has the disadvantage, in the first place, of considerable friction, and again, the weight of the column of mercury produces an extent of oscillation by mere impetus, greater than that which would actually represent the alternation of systole and diastole of the heart.

An important improvement in the hemodynamometer was made by Magendie. This apparatus, the cardiometer, in which Bernard made some modifications, is the one now commonly used. It consists of a small but thick glass bottle, with a fine graduated tube about twelve inches (30.5 centimeters) in length, communicating with it, either through the stopper or an orifice in the side. The stopper is pierced with a bent tube which is to be connected with the bloodvessel. The bottle is filled with mercury so that it will rise in the tube to a point which is marked zero. It is evident that the pressure on the mercury in the bottle will be indicated by an elevation in the graduated tube; and, moreover, from 
the fineness of the column in the tube, some of the inconveniences due to the weight of mercury in the hemodynamometer are avoided, and there is, also, less friction. This instrument is appropriately called the cardiometer, as it indicates accurately, by the extreme elevation of the mercury, the force of the heart; but it is not so useful in measuring the mean arterial pressure, for in the abrupt descent of the mercury during the diastole of the heart, the impetus causes the level to fall below the real standard of the constant pressure. Marey corrected this difficulty in the " compensating " instrument, which is constructed on the following principle: Instead of a simple glass tube which communicates with the mercury in the bottle, as in Magendie's cardiometer, there are two tubes; one is like the tube already described and represents oscillations produced by the heart, while the other is larger, and has, at the lower part, a constriction of its calibre, which is here reduced to capillary fineness. The latter tube is designed to give the mean arterial pressure; the constricted portion offering such an obstacle to the rise of the mercury that the intermittent action of the heart is not felt, the mercury rising slowly to a certain level, which is constant and varies only with the constant pressure in the vessels.

The instruments in use in physiological laboratories at the present day are modifications of the "kymograph" devised by Ludwig. Ludwig's instrument consists of a U-tube containing mercury, one arm of which is connected with an artery. The mercury in the distal arm carries a float to which is attached a wire terminating in a writingpoint by means of which the oscillations may be recorded in the usual way. The principle, however, is the same as that of the instrument constructed by Poiseuille.

Pressure in Different Arteries. - The experiments of Hales, Poiseuille, Bernard and others seem to show that the constant arterial pressure does not vary much in arteries of different sizes. These physiologists experimented particularly on the carotid and crural, and found the pressure in these two vessels about the same. From their experiments they concluded that the force is nearly equal in all parts of the arterial system. The experiments of Volkmann, however, have shown that this conclusion is not correct. With the registering apparatus of Ludwig, he took the pressure in the carotid and the metatarsal arteries and always found a considerable difference in favor of the former. In an experiment on a dog, he found the pressure equal to about seven inches ( 172 millimeters) in the carotid, and 6.6 inches ( 65 millimeters) in the metatarsal. In an experiment on a calf, the pressure was 4.64 inches (I 6 millimeters) in the carotid, and 3.56 inches ( 89 millimeters) in the metatarsal; and in a rabbit, 3.64 inches 
(9I millimeters) in the carotid, and 3.44 inches ( 86 millimeters) in the crural. These experiments show that the pressure is not the same in all parts of the arterial system, that it is greatest in the arteries nearest the heart and that it gradually diminishes toward the capillaries. The difference is slight, almost inappreciable, except in vessels of small size; but here the pressure is directly influenced by the discharge of blood into the capillaries. The cause of this diminution of pressure in the smallest vessels is the proximity of the great outlet of the arteries, the capillary system; for, as will be seen farther on, the flow into the capillaries has a constant tendency to diminish the pressure in the arteries.

Influence of Respiration. - It is easy to see, in studying the arterial pressure, that there is a marked increase with expiration and a diminution with inspiration. In tranquil respiration the influence on the flow of blood is due simply to the mechanical action of the thorax. With every inspiration the air-cells are enlarged, as well as the bloodvessels of the lungs, the air rushes in through the trachea and the movement of the blood in the veins near the chest is accelerated. At the same time the blood in the arteries is somewhat retarded in its flow from the thorax, or at least does not feel the expulsive influence which follows with the act of expiration. The arterial pressure at that time is at its minimum. ' With the expiratory act the air is expelled by compression of the lungs, the flow of blood into the thorax by the veins is retarded to a certain extent, while the flow into the arteries is favored. This is shown in the increased force, with expiration, in the jet from a divided artery. Under these conditions, the arterial pressure is at its maximum. In perfectly tranquil respiration, the changes due to inspiration and expiration are slight, presenting a difference of not more than half an inch or an inch (I2.7 or 25.4 millimeters) in the cardiometer. When the respiratory movements are exaggerated, the oscillations are more extensive.

Interruption of respiration is followed immediately by a great increase in the arterial pressure. This is due, not to causes within the chest, but to obstruction to the circulation in the capillaries. With an interruption of the respiratory movements, the non-aërated blood passes into the arteries but can not flow readily through the capillaries; and as a consequence the arteries are abnormally distended and the pressure is increased. If respiration is permanently arrested, the arterial pressure becomes, after a time, diminished below the normal standard, and is finally abolished on account of the stoppage of the action of the heart. When respiration is resumed before the action of the heart has become arrested, the pressure soon returns to the normal standard. 
Influence of Mtuscular Action, ctc. - Muscular effort considerably increases the arterial pressure. This is due to two causes. In the first place, the chest is compressed, and this favors the flow of blood into the great vessels. In the second place, muscular exertion produces a certain degree of obstruction to the discharge of blood from the arteries into the capillaries. Experiments on the inferior animals show a great increase in pressure in the struggles that occur during severe operations. It has been shown that stimulation of the sympathetic in the neck and of certain of the cerebro-spinal nerves increases the arterial pressure, probably from an influence on the muscular coats of some of the arteries, causing them to contract and thereby diminishing the total capacity of the arterial system.

Effects of Hemorrhage, etc. - Diminution in the quantity of blood has a remarkable effect on arterial pressure. If, in connecting the instrument with the arteries, even one or two jets of blood are allowed to escape, the pressure will be found diminished perhaps one-half or even more. It is hardly necessary to discuss the mechanism of the effect of the loss of blood on the tension in the vessels, but it is remarkable how soon the pressure in the arteries regains the normal standard after it has been lowered by hemorrhage. As the pressure depends largely on the quantity of blood, so soon as the vessels absorb serosities in sufficient quantity to repair the loss, the pressure is increased. This takes place in a short time, if the loss of blood is not too great.

Experiments on arterial pressure with the cardiometer have verified the fact mentioned in treating of the form of the pulse; namely, that the pressure in the vessels bears an inverse ratio to the distention produced by the contractions of the heart. In the cardiometer, the mean height of the mercury indicates the constant, or arterial pressure; and the oscillations, the distention produced by the heart. It is found that when the pressure is great, the extent of oscillation is small, and vice versa. It will be remembered that the researches of Marey demonstrated that an increase in arterial pressure diminishes the amplitude of the pulsations, as indicated by the sphygmograph, and that the amplitude is great when the pressure is slight. It is also true, as a generai rule, that the force of the heart, as indicated by the cardiometer, bears an inverse ratio to the frequency of its pulsations.

An instrument called the sphygmomanometer has been devised for measuring the arterial pressure in the human subject. This is for use chiefly in clinical work and its description here would be out of place. The principle on which it is constructed is to measure by means of a column of mercury the pressure required to arrest the pulse at the 
wrist, the pressure being applied to the brachial artery just above the elbow.

The following is a summary of the conditions that influence the arterial pressure :-

The pressure is increased by -

I. Increase in the power of the ventricular systole.

2. Increase in the total quantity of blood.

3. Increased contraction of the small vessels that supply the capillary system.

The pressure is decreased by -

I. Decrease in the power of the ventricular systole.

2. Decrease in the total quantity of blood.

3. Decreased contraction, or relaxation of the small vessels that supply the capillary system.

\section{Rapidity of the Current of Blood in the Arteries}

The question of the rapidity of the flow of blood in the arteries has long engaged the attention of physiologists; but the experiments of Volkmann with the hemodrometer, and of Vierordt with a peculiar instrument which he devised for the purpose, did not lead to results that were entirely satisfactory. The apparatus devised by Chauveau, however, is more reliable. This will give, by calculation, the actual rapidity of the current, and it also indicates the variations in velocity that occur at different stages of the heart's action.

The instrument to be applied to the carotid of the horse consists of a thin brass tube, about an inch and a half (38.I millimeters) in length and of the diameter of the artery (about three-eighths of an inch, or 9.5 millimeters), which is provided with an oblong longitudinal opening, or window, near the middle, about two lines ( 4.2 millimeters) long and one line (2.I millimeters) wide. A piece of thin vulcanized rubber is wound around the tube and firmly tied so as to cover this opening. Through a transverse slit in the rubber is introduced a very light metallic needle, an inch and a half (38. I millimeters) in length and flattened at its lower part. This is made to project about halfway into the calibre of the tube. A flat semicircular piece of metal, divided into an arbitrary scale, is attached to the tube, to indicate the deviations of the point of the needle.

The apparatus is introduced into the carotid of a horse, by making a slit in the vessel, introducing first one end of the tube directed toward the heart, then allowing a little blood to enter the instrument, so as to expel the air, and, when full, introducing the other end, securing the whole by ligatures above and below. 
When the circulation is arrested, the needle should be vertical and mark zero on the scale. When the flow is established, a deviation of the needle occurs, which varies in extent with the rapidity of the current. Having removed all pressure from the vessel so as to allow the current to resume its normal character; the deviations of the needle are carefully noted, as they occur with the systole and with the diastole of the heart. After withdrawing the instrument, it is applied to a tube of the same size of the artery, in which a current of water is made to pass with a rapidity that will produce the same deviations as occurred when the instrument was connected with the bloodvessel. The rapidity of the current in this tube may be easily calculated by receiving the water in a graduated vessel and noting the time occupied in discharging a given quantity. By this means the rapidity of the current of blood is ascertained.

It has been found that three currents, with different degrees of rapidity, may be distinguished in the carotid :-

I. With each ventricular systole, as the average of the experiments of Chauveau, the blood moves in the carotids at the rate of about 20.4 inches (5 IO millimeters) per second. After this, the rapidity quickly diminishes and the needle returns quite or nearly to zero, which would indicate complete arrest.

2. Immediately succeeding the ventricular systole, a second impulse is given to the blood, which is synchronous with the closure of the semilunar valves, the blood moving at the rate of about 8.6 inches $(215$ millimeters) per second. This is the dicrotic impulse.

3. After the dicrotic impulse, the rapidity of the current gradually diminishes until just before the systole of the heart, when the needle is nearly at zero. The average rate, after the dicrotic impulse, is about 5.9 inches (I 47.5 millimeters) per second.

The experiments of Chauveau correspond with the experiments of Marey on the form of the pulse. Marey showed that there is a marked oscillation of the blood in the vessels, due to a reaction of their elastic walls following the first distention by the heart; that at the time of closure of the semilunar valves, the arteries present a second, or dicrotic distention, much less than the first; and following this, there is a gradual decline in the distention until the minimum is reached. According to the observations of Chauveau, corresponding with the first dilatation of the vessels, the blood moves with great rapidity; following this, the current suddenly becomes nearly arrested; this is followed by a second acceleration in the current, less than the first ; and following this, there is a gradual decline in the rapidity, to the time of the next pulsation. 
From the fact that the arterial system progressively increases in capacity, there should be found a corresponding diminution in the rapidity of the flow of blood. There are, however, many conditions, aside from simple increase in the capacity of the vessels, which modify the blood-current and render inexact calculations made on purely physical principles. These are the tension of the blood and the conditions of contraction or relaxation of the smallest arteries. It is necessary, therefore, to have recourse to actual experiments to arrive at definite results on this point. Volkmann found a great difference in the rapidity of the current in the carotid and metatarsal arteries, the averages being about Io inches (254 millimeters) per second in the carotid, and about 2.2 inches ( 56 millimeters) in the metatarsal. The same difference, although not quite so marked, was found by Chauveau, between the carotid and the facial. As the vessels are farther and farther removed from the heart, the systolic impulse rapidly diminishes, being reduced in one experiment by about two-thirds; the dicrotic impulse becomes feeble or may even disappear; but the constant flow is much increased in rapidity. The rapidity of the flow in any given artery must be subject to modifications due to the condition of the arterioles which are supplied by it. When these little vessels are dilated, the artery, of course, supplies blood with greater facility and the rapidity of the flow is increased.

\section{Circulation of Blood in the Capillaries}

Before beginning the study of the capillary circulation, it should be understood what is meant by capillary vessels as distinguished from the smallest arteries and veins. From a physiological point of view, the capillaries are to be regarded as beginning where the blood is brought near enough to the tissues to enable them to separate the matters necessary for their regeneration and to give up the products of their physiological wear; but at present it is impossible to assign any limit where the vessels cease to be simple carriers of blood, and it is not known to what part of the vascular system the processes of nutrition are exclusively confined. The divisions of the bloodvessels must be, to a certain extent, arbitrarily defined. The most simple view is to regard as capillaries vessels that have but a single coat; for in these, the blood is brought in closest proximity to the tissues. Vessels that are provided, in addition, with a muscular or with muscular and fibrous coats may be considered as either small arteries or veins.

Physiological Anatomy of the Capillaries. - If the arteries are followed out to their minutest ramifications, they will be found progressively diminishing in size as they branch, and their coats, especially the mus- 
cular coat, becoming thinner and thinner, until at last they present an internal structureless coat lined by endothelium with oval longitudinal nuclei, a middle coat formed of but a single layer of circular muscular fibres, and an external coat composed of a thin layer of longitudinal bundles of fibrous tissue. These vessels are $\frac{1}{400}$ to $\frac{1}{2} \frac{1}{00}$ of an inch $(62.5$ to $125 \mu)$ in diameter. They undoubtedly possess contractility, which is particularly marked in the arterial system. Following the course of the vessels, when they are reduced in size to about $\frac{1}{800}$ of an inch ( $\left.3 \mathrm{I} \mu\right)$, the fibrous coat is lost, and the vessel then presents only the internal coat and a single layer of muscular fibres. The vessels become smaller as they branch, finally lose the muscular fibres and have then but a single coat. These last may be regarded as the true capillary vessels.

The single coat of the capillaries consists of a layer of fusiform or polygonal nucleated endothelium of excessive tenuity. The borders of the endothelial cells may be seen after staining the vessels with silver nitrate. In the smallest capillaries the cells are narrow and elongated or fusiform; and in the larger vessels they are more polygonal, with irregular borders. In staining with silver nitrate, irregular nonnucleated areas frequently are brought into view; and it has been supposed by some that these indicate the presence of stomata, or orifices in the walls of the vessels.

The diameter of the capillaries usually is as small as that of the blood-corpuscles; or it may be smaller, so that these bodies move in a single line and must become deformed in passing through the smallest vessels, recovering their normal shape, however, when they pass into vessels of larger size. The capillaries are smallest in the nervous and muscular tissue, retina, and patches of Peyer, where they have a diameter of $\frac{1}{6000}$ to $\frac{1}{4000}$ of an inch $(4.25$ to $6.25 \mu)$. In the papillary layer of the skin and in the mucous membranes they are $\frac{1}{4000}$ to $\frac{1}{2400}$ of an inch $(6.25$ to $10 \mu)$ in diameter. They are largest in the glands and bones, where they are $\frac{1}{30} \overline{0}$ to $\frac{1}{200}$ of an inch $(8.3$ to $12.5 \mu)$ in diameter. It is only the largest vessels that allow the passage of blood-disks without change in form. The average length of the capillary vessels is about $\frac{1}{50}$ of an inch (0.5 millimeter).

Unlike the arteries, which grow smaller as they branch, and the veins, which become larger in following the course of the blood, by union with each other, the capillaries form a true plexus of vessels of nearly uniform diameter, branching and inosculating in every direction and distributing blood to the parts as their physiological necessities demand. This mode of inosculation is peculiar to these vessels, and the plexus is rich in the tissues, as a general rule, in proportion to the activity of their nutrition. 
Although their arrangement presents certain differences in different organs, the capillary vessels have everywhere the same general characters, the most prominent of which are a nearly uniform diameter and an apparent absence of any definite direction in their branchings. The network thus formed is rich in the parenchyma of the glands and in the organs of absorption. In the walls of the pulmonary alveoli the meshes are particularly close. In other parts the vessels are not so abundant, presenting great variations in different tissues. In the muscles and nerves, in which nutrition is very active, the supply of blood is greater than in other parts, like fibro-serous membranes, tendons, etc. In none of the tissues do the capillaries penetrate the true anatomical elements of the parts, as the ultimate muscular or nervous fibres. Some tissues receive no blood - at least they contain no vessels that are capable of carrying red blood - and are nourished by imbibition of nutrient matters from the bloodplasma.

The capacity of the capillary system is very large. It is necessary only to consider the great vascularity of the skin, mucous membrane or muscles, to appreciate this fact. In injections of

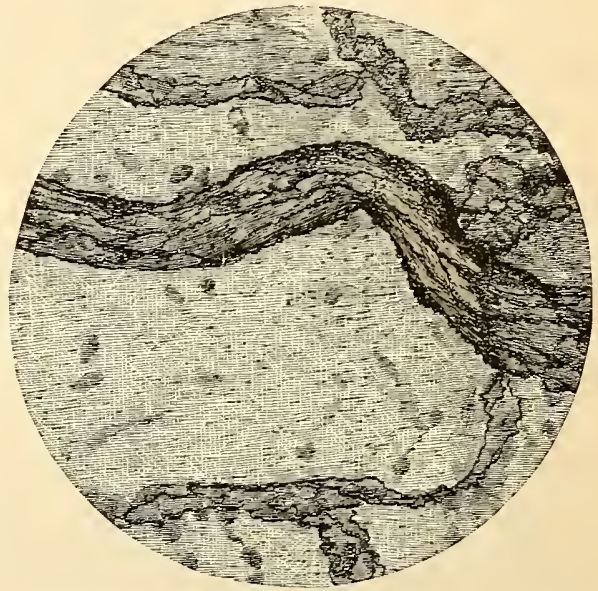

Fig. 25. - Small artery and capillaries from the muscular coat of the urinary bladder of a frog, $\times 400$ (from a photograph taken at the United States Army Medical Museum).

This preparation shows the endothelium of the vessels. It is injected with silver nitrate and mounted in Canada balsam.

these parts, it seems, on microscopical examination, as though they contained nothing but capillaries; but in preparations of this kind, the coats of the capillaries are distended to their utmost limit. Under some conditions in health, they are largely distended with blood, as in the mucous lining of the alimentary canal during digestion, the whole surface presenting a vivid red color, indicating the richness of the capillary plexus. Estimates of the capacity of the capillary system, as compared with the arterial system, have been made, but they are simply approximative. The various estimates given are founded on calculations from microscopical examinations of the rapidity of the capillary circulation as compared with the circulation in the arteries. In this way, it has been calculated that the capacity of the capillary system is between five hundred and eight hundred times that of the arterial system. 
The most convenient part for direct observation of the capillary circulation is the tongue or the web of the frog. Here may be studied, not only the movement of the blood in the capillaries, but the circulation in the smallest arteries and veins, the variations in calibre of these vessels, especially the arterioles, by the action of their muscular coat, and, indeed, the action of vessels of considerable size. This has been a valuable means of studying the circulation in the capillaries as contrasted with the flow in the small arteries and veins.

In studying the circulation under the microscope, the anatomical division of the blood into corpuscles and a clear plasma is observed. This is peculiarly evident in cold-blooded animals, the corpuscles being comparatively large and floating in a plasma that forms a distinct layer next the walls of the vessel. The leucocytes, which are much fewer than the red corpuscles, are found usually in the layer of plasma.

In vessels of considerable size, as well as in some capillaries, the corpuscles, occupying the central portion, move with greater rapidity than the rest of the blood, leaving a comparatively still layer of plasma at the sides. A red corpuscle occasionally becomes involved in the "still layer," when it moves slowly, turning over and over, or even may remain stationary for a time, until it is taken up again and carried along with the central current. A few leucocytes are constantly seen in this layer. They move along slowly and apparently have a tendency to adhere to the walls of the vessel. This is due to the adhesive character of the surface of the white corpuscles as compared with the red, which can easily be observed in examining a drop of blood between glass surfaces, the red corpuscles moving about freely, while the white corpuscles have a tendency to adhere to the glass.

Great differences exist in the character of the flow of blood in the three varieties of vessels under observation. In the arterioles, which may be distinguished from the capillaries by their size and the presence of the muscular and fibrous coats, the movement is distinctly pulsatile, even in their most minute ramifications. The blood moves in them with greater rapidity than in either the capillaries or veins. They become smaller as they branch, and carry the blood always in the direction of the capillaries. The veins, which are relatively larger than the arteries, carry the blood more slowly and in a continuous stream from the capillaries toward the heart. In both the arteries and veins, the current is frequently so rapid that the form of the corpuscles can not be distinguished. Only a few of the leucocytes occupy the still layer, the others being carried on in the central current. 
In the true capillaries, the blood is distributed in every direction, in vessels of nearly uniform diameter. The vessels usually are so small as to admit but a single row of corpuscles. In a single vessel, a line of corpuscles may be seen moving in one direction at one moment, and a few moments after, taking an opposite course. When the .circulation is normal, the movement in the capillaries is always quite slow as compared with the current in the arterioles, and is continuous. Here, at last, the intermittent impulse of the heart is lost. The corpuscles do not necessarily circulate in all the capillaries that are in the field of view. Certain vessels may not receive a corpuscle for some time, but afterward, one or two corpuscles become engaged in them and a current is established. In some of the vessels of smallest size, the corpuscles are slightly deformed as they pass through. The scene is changed with every different part that is examined. In the tongue, in addition to the arterioles and venules and the rich network of capillaries, dark-bordered nerve-fibres, striated muscular fibres, and epithelium can be distinguished. In the lungs large polygonal air-cells are observed, bounded by capillary vessels in which the corpuscles move with great rapidity. It has been observed, also, that the larger vessels in the lungs are crowded to their utmost capacity with red corpuscles.

Pressire of Blood in the Capillaries. - There is, apparently, no way of directly estimating the pressure of blood in the capillaries. If, however, a glass plate is placed on a part in which the capillary circulation is active and is weighted until the subjacent capillaries are emptied, an approximate idea of the blood-pressure in the vessels may be obtained. Experiments made in this way, by Von Kries, show that the pressure in the capillaries of the hand raised above the head is equal to a little less than one inch ( 24 millimeters) of mercury; in the hand hanging down, a little more than two inches ( 54 millimeters); and in the ear, about 0.8 of an inch ( 20 millimeters.)

Rapidity of the Capillary Circulation. - The current in the capillaries of a part is subject to such variations, and the differences in different situations are so considerable, that it is impossible to give a definite rate that will represent the general rapidity of the capillary circulation; and in view of the uncertainty of the methods employed, it seems unnecessary to discuss this question fully. Volkmann calculated the rapidity in the mesentery of the dog and found it to be 0.02 to 0.03 of an inch ( 0.5 to 0.75 millimeters) per second.

Relations of the Capillary Circulation to Respiration. - The immediate effects of asphyxia on the circulation are referable to the general capillary system. In a series of experiments made on frogs, in I 857 (Flint), 
the buib was broken up and the web of the foot was submitted to microscopical examination. The cutaneous surface was then coated with collodion, care only being taken to avoid the web under observation. The effect on the circulation was immediate. It instantly became less rapid, until, at the expiration of twenty minutes, it had entirely ceased. The entire coating of collodion was then instantly peeled off. Quite a rapid. circulation immediately began, but it soon declined and in twenty minutes had nearly ceased. In another observation, the coating of collodion was applied without destroying the bulb. The circulation was affected in the same manner as before and ceased in twenty-five minutes. These experiments, taken in connection with observations on the influence of asphyxia on the arterial pressure, show that non-aërated blood can not circulate freely in the systemic capillaries, probably on account of contraction of the muscular coat of the small arteries, due to irritation from an excess of carbon dioxide in the blood.

Canses of the Capillary Circulation. - The pressure in the arteries, which forces the blood toward the capillaries, is competent, unless opposed by contraction of the arterioles, not only to send the blood through the capillaries but to return it to the heart by the veins. A distinct impulse, following each ventricular systole, is observed in the smallest arteries; the blood flows from them directly and freely into the capillaries; and there is no ground for the supposition that the force is not propagated to this system of vessels. There is, therefore, a force the action of the heart - that is capable of producing the capillary circulation; and there is nothing in the phenomena of the circulation in these vessels inconsistent with its full operation.

Influcnce of Tempcrature on the Capillary Circulation. - Within moderate limits, a low temperature, produced by local applications, has been found to diminish the quantity of blood sent to the capillaries and retard the circulation, while a high temperature increases the supply of blood and accelerates its current. Poiseuille found that when a piece of ice was applied to the web of a frog's foot, the mesentery of a small warmblooded animal or to any part in which the capillary circulation could be observed, the number of corpuscles circulating in the arterioles was much diminished, "those which carried two or three rows of corpuscles giving passage to but a single row." The circulation in the capillaries first became slower and then entirely ceased in parts. On removing the ice, in a very few minutes the circulation regained its former characters. When, on the other hand, the part was covered with water at $104^{\circ} \mathrm{Fahr} .\left(40^{\circ} \mathrm{C}\right.$. $)$, the rapidity of the current in the capillaries was so much increased that the form of the corpuscles could with difficulty be distinguished. 


\section{Circulation of Blood in the Veins}

The blood, distributed to the capillaries of all the tissues and organs by the arteries, is collected from these parts in the veins and carried to the right side of the heart. In studying the anatomy of the capillaries or in observing the passage of the blood from the capillaries to larger vessels in parts of the living organism that can be submitted to microscopical examination, it is seen that the capillaries-vessels of nearly uniform diameter and anastomosing in every direction - empty into a system of vessels, which, by union with others, become larger and larger, and carry the blood away in a continuous current, in which the intermittent impulse derived from the left ventricle is not manifest. These vessels are called venules, or venous radicles. They are the peripheral radicles of the vessels that carry the blood to the heart.

The venous system may be considered as divided into two sets of vessels: one, which is deep-seated and is situated in proximity to the arteries, and the other, which is superficial and receives the greatest part of the blood from the cutaneous surface. The entire capacity of

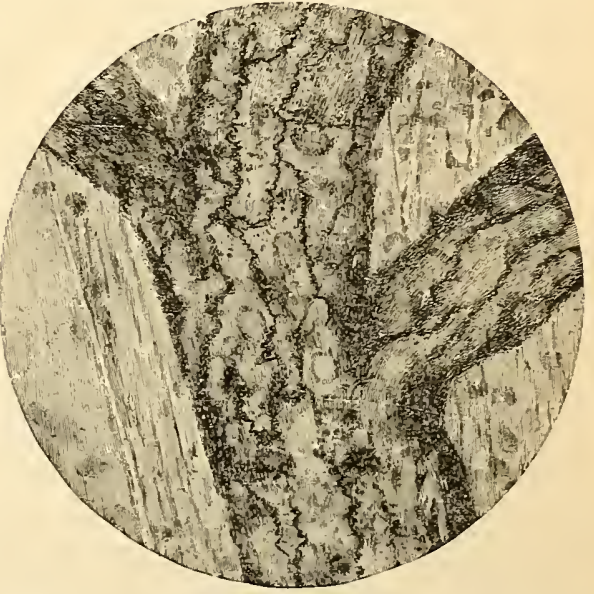

Fig. 26. - Venous radicles uniting to form a small vein, from the muscular coat of the urinary bladder of a frog, $\times 400$ (from a photograph taken at the United States Army Mledical Museum).

This preparation shows the endothelium of the vessels. It is injected with silver nitrate and mounted in Canada balsam.

the venous system, as compared with that of the arteries, is very great. As a rule, each vein, when fully distended, is larger than its adjacent artery. Many arteries are accompanied by two veins, as the arteries of the extremities; while certain of them, like the brachial or spermatic, have more than two. Added to these, are the superficial veins, which have no corresponding arteries. It is true that some arteries have no corresponding veins, but examples of this kind are not sufficient in number to diminish, in any marked degree, the great preponderance of the veins, both in number and volume. It is impossible to give anything like an accurate estimate of the extreme capacity of the veins as compared with the arteries, but it must be much greater. Borelli estimated that the capacity of the veins was to the capacity of the 'arteries, as 4 to I; and Haller, as $2 \frac{1}{4}$ to $\mathrm{I}$. The proportion is variable 
in different parts of the body. In some situations the capacity of the veins and arteries is about equal; while in others, as in the pia mater, the veins contain, when fully distended, six times as much blood as the arteries.

In attempting to compare the quantity of blood normally circulating in the veins with that contained in the arteries, such variations are found at different times and in different parts, both in the quantity of blood, rapidity of circulation, pressure etc., that a definite estimate is impossible. It would be unprofitable to attempt even an approximate comparison, as the variations in the venous circulation constitute one of its most important physiological peculiarities, which must be fully appreciated in order to form a just idea of the uses of the veins. The arteries are always full, and their tension is subject to comparatively slight changes. Following the blood into the capillaries, important modifications in the circulation are observed under varying physiological conditions of the parts. As would naturally be expected, the condition of the veins varies with the changes in the capillaries from which the blood is received.

Following the veins in their course, it is observed that anastomoses with each other form the rule, and not the exception, as in the arteries. There is always a number of channels by which the blood may be returned from a part; and if one vessel is obstructed from any cause, the current is diverted into another. The veins do not present a true anastomosing plexus, such as exists in the capillary system, but simply an arrangement by which the blood may readily find its way back to the heart, and by which the vessels can accommodate themselves to variations in the quantity of their contents.

Structure and Properties of the Veins. - The structure of the veins is more complex than that of the arteries. Their walls, which are always much thinner than the walls of the arteries, may be divided into a number of layers; but for convenience of physiological description, they may be regarded as presenting three coats.

The internal coat of the veins is a continuation of the single coat of the capillaries and of the internal coat of the arteries. It is in the form of a fenestrated membrane, somewhat thinner than in the arteries, lined with a delicate layer of polygonal endothelium, the cells of which are shorter and broader than the endothelial cells lining the arteries.

The middle coat is divided by some anatomists into two layers; an internal layer, composed chiefly of longitudinal fibres, and an external layer, in which the fibres have a circular direction. These two layers are intimately adherent and are quite closely attached to the internal coat. The longitudinal fibres are composed of connective-tissue fibres 
with a large number of the smallest variety of the elastic fibres. This layer contains capillary vessels (vasa vasorum). The circular fibres are composed of elastic tissue, some of the fibres of the same variety as is found in the longitudinal layer, some of medium size, and some in the form of a "fenestrated membrane." In addition, there are inelastic fibres interlacing in every direction and mingled with capillary bloodvessels and non-striated muscular fibres. In the human subject, in the veins of the central portion of the nervous system, the dura mater, the pia mater, the bones, the retina, the vena cava descendens, the thoracic portion of the vena cava ascendens, the external and internal jugulars and the subclavian veins, there are no muscular fibres in the middle coat. In the larger veins, such as the abdominal vena cava, the iliac, crural, popliteal, mesenteric and axillary veins, there are both longitudinal and circular fibres. In the smaller veins the fibres are circular. In the smallest veins the middle coat is composed of fine fibres of connective tissue with a very few muscular fibres.

The external coat of the veins is composed of ordinary fibrous tissue, like that of the corresponding coat of the arteries. In the largest veins, particularly those of the abdominal cavity, this coat contains a layer of longitudinal non-striated muscular fibres. In the veins near the heart are found a few striated fibres, which are continued on to the veins from the auricle (see Plate III, Fig. I).

The venous sinuses and the veins that pass through bony tissue have only the internal coat, to which are added a few longitudinal fibres, the ressels being closely attached to the surrounding parts. As examples may be mentioned the sinuses of the dura mater and the veins of the large bones of the skull. In the first instance, there is little more than the internal coat of the vein firmly attached to the surrounding layers of the dura mater. In the second instance, the same thin membrane is adherent to canals formed by a layer of compact bony tissue. The veins are more closely adherent to the surrounding tissues than the arteries, particularly when they pass between layers of aponeurosis. When a vein is cut across, its walls fall together, if not supported by adhesions to surrounding tissues, so that its calibre is nearly or quite obliterated. The elastic tissue, which gives to the larger arteries their great thickness, is scanty in the veins, and the thin walls collapse when not sustained by liquid in the interior of the vessels.

Although with much thinner walls, the veins, as a rule, will resist a greater pressure than the arteries. Wintringham (I740) showed that the inferior vena cava of a sheep, just above the opening of the renal veins, was ruptured by a pressure of one hundred and seventy-six pounds (79.8 kilos), while the aorta, at a corresponding point, yielded to a 
pressure of one hundred and fifty-eight pounds (7I.7 kilos). The strength of the portal vein was even greater, supporting a pressure of nearly five atmospheres, bearing a relation to the vena cava of six to five; yet these vessels had hardly one-fifth the thickness of the arteries. In the lower extremities in the human subject, the veins are thicker and stronger than in other situations, a provision against the increased pressure to which they are habitually subjected in the upright posture. Wintringham noticed a singular exception to the general rule just given. In the vessels of the glands and of the spleen, the strength of the arteries was greater than that of the veins. The splenic vein gave way under a pressure of little more than one atmosphere, while the artery supported a pressure of more than six atmospheres.

The veins possess a considerable degree of elasticity, although this property is not so marked as it is in the arteries. If a portion of a vein distended with blood is included between two ligatures and a small opening is made in the vessel, the blood will be ejected with some force, and the vessel becomes much reduced in calibre.

It has been shown by direct experiment that the veins are endowed with the peculiar contractility characteristic of the action of the nonstriated muscular fibres. On electric or mechanical stimulation, they contract slowly and gradually, the contraction being followed with a correspondingly gradual relaxation. There is never any rhythmical or peristaltic movement in the veins sufficient to assist the circulation. The only regular movements that occur are seen in vessels in immediate proximity to the right auricle, which are provided with a few fibres similar to those which exist in the walls of the heart.

Nerves from the vasomotor system have been demonstrated in the walls of the larger veins but have not been followed out to the smaller ramifications.

Valies of the Veins. - In all parts of the venous system, except, in general terms, in the abdominal, thoracic and cerebral cavities, there exist little membranous semilunar folds, resembling the aortic and pulmonic valves of the heart. When the valves are closed, their convexities look toward the periphery. In the great majority of instances, the valves exist in pairs; but they are occasionally, although very rarely in the human subject, found in groups of three. They are seldom if ever found in veins of a less diameter than one line (2.I millimeters). The valves are formed in part of the lining membrane of the veins, with fine fibres of connective tissue, elastic fibres and non-striated muscular fibres. There exists, also, a fibrous ring following the line of attachment of the valvular curtains to the vein, which renders the vessel stronger and less dilatable here than in the portions between the valves. The valves are 
most abundant in the veins of the lower extremities. They usually are situated just below the point where a small vein empties into one of larger size, so that the blood as it enters finds an immediate obstacle to passage in the wrong direction. The situation of the valves may be readily observed in any of the superficial veins. If the flow of blood is obstructed, little knots will be formed in the congested vessels, which indicate the position and action of the valves. When the vein is thus congested and knotted, if the finger is pressed along the vessel in the direction of the blood-current, a portion situated between two valves may be emptied of blood; but it is impossible to empty any part of the vessel by pressing the blood in the opposite direction (Harvey). On slitting open a vein, it is easy to observe the shape, attachment and delicacy of structure of the valves. When the vessel is empty or when the blood moves toward the heart, the valves are closely applied to the walls; but if liquid or air is forced in the opposite direction, they project into its calibre, and by the application of their free edges to each other, effectually prevent any backward current. When closed, the application of their free edges forms a line which runs across the vessel. It is found that in successive sets of valves these lines are at right angles to each other, so that if in one set this line has a direction from before backward, in the sets above and below, the lines run from side to side (Fabricius).

There are exceptions to the general proposition that the veins of the great cavities are not provided with valves. Valves are found in the portal system of some of the inferior animals, as in the horse. They do not exist, however, in this situation in the human subject. Usually, in following out the branches of the inferior vena cava, no valves are found until the crural vein is reached; but occasionally there is a double valve at the opening of the external iliac. In some of the inferior animals, there exists constantly a single valvular fold in the vena cava at the openings of the hepatic, and one at the opening of the renal vein. These are not constant in the human subject. Valves are found in the spermatic, but not in the ovarian veins. A single valvular fold has been described at the opening of the right spermatic into the vena cava. There are two valves in the azygos vein near its opening into the superior vena cava. There is a single valve at the opening of the coronary vein. There are no valves at the opening of the brachio-cephalic into the superior vena cava; but there is a strong double valve at the point where the internal jugular opens into the brachio-cephalic. Between this point and the capillaries of the brain, the vessels have no valves, except in rare instances, when one or two are found in the course of the jugular. 
In addition to the double, or more rarely triple valves just described, there is another variety, found in certain parts, at the point where a tributary vein opens into a main trunk. This consists of a single fold, which is attached to the smaller vessel but projects into the larger. The veins are adapted to the return of blood to the heart in a comparatively slow and unequal current. Distention of certain portions is provided for; and the vessels are so protected with valves, that whatever influences the current must favor its flow in the direction of the heart.

The experiments of Hales and Sharpey, showing that defibrinated blood can be made to pass from the arteries into the capillaries and out at the veins by a pressure less than that which exists in the arterial system, and the observations of Magenclie on the circulation in the leg of a living dog, showing that ligation of the artery arrests the flow in the vein, have established the fact that the force exerted by the left ventricle is sufficient to account for the venous circulation. The force of the heart, therefore, must be regarded as the prime cause of the movement of blood in the veins.

As a rule, in the normal circulation, the flow of blood in the veins is continuous and uniform. The intermittent impulse of the heart, which progressively diminishes toward the periphery, but is still felt even in the smallest arteries, is lost in the capillaries. Here, for the first time, the blood moves in a constant current; and as the pressure in the arteries is continually supplying fresh blood, that which has circulated in the capillaries is forced into the venous radicles in a steady stream. As the supply to the capillaries of different parts is regulated by the action of the small arteries, and as this supply is subject to great variations, there must necessarily be corresponding variations in the current in the veins and in the quantity of blood which these vessels receive.

It often occurs that a vein becomes obstructed from some cause that is entirely physiological, such as the action of muscles. The great number of veins, as compared with the arteries, and their free communications with each other, provide that the current, under these conditions, simply is diverted, passing to the heart by another channel. When any part of the venous system is distended, the vessels react on the blood and exert a certain influence on the current, always pressing it toward the heart, for the valves oppose a flow in the opposite direction.

\section{Pressure of Blood in the Veins}

The pressure in the veins is always much less than in the arteries, and is variable in different parts of the venous system and in the same part 
at different times. As a rule, it is in an inverse ratio to the arterial pressure. Whatever favors the passage of blood from the arteries into the capillaries has a tendency to diminish the arterial pressure, and as it increases the quantity of blood passing into the veins, it must increase the venous pressure. The great capacity of the venous system, its frequent anastomoses and the presence of valves which may shut off a portion from the rest, are conditions that involve considerable variations in pressure in different vessels. Muscular effort has a decided influence on the force of the circulation in certain veins and produces an elevation in the pressure. As the reduced pressure in the veins is due in a measure to the great relative capacity of the venous system and the free communications between the vessels, it would seem that if it were possible to reduce the capacity of the veins in a part and force all the blood to pass to the heart by a single vessel corresponding to the artery, the pressure in this vessel would be greatly increased. Poiseuille has shown this to be the fact by the experiment of tying all the veins coming from a part, except one which had the volume of the artery by which the blood was supplied, forcing all the blood to return by this single channel. This being done, he found that the pressure in the vein was much increased, becoming nearly equal to the pressure in the artery.

\section{Rapidity of the CuRRent of Blood in the Veins}

It is impossible to fix on any definite rate as representing the rapidity of the current of blood in the veins. It will be seen that various conditions are capable of increasing very considerably the rapidity of the flow in certain veins, and that the current in some parts of the venous system may be much retarded. Undoubtedly, the general movement of blood in the veins is much slower than in the arteries, from the fact that the quantity of blood is greater. If it is assumed that the quantity of blood in the veins is double that contained in the arteries, the general average of the current would be diminished onehalf. Near the heart, however, the flow becomes more uniform and progressively increases in rapidity, as the quantity of blood received by the heart is equal to the quantity discharged into the arteries.

As the effect of the heart's action on the venous circulation is subject to many modifying influences through the small arteries and capillaries, and as there are other forces influencing the current, that are by no means uniform in their operation, estimates of the general rapidity of the venous circulation or of the variations in different vessels must necessarily be indefinite. 


\section{Causes of the Venous Circulation}

In the veins, the blood is farthest removed from the influence of the contractions of the left ventricle; and although these are felt, there are many other causes that combine to carry on the venous circulation, and many influences by which it is retarded or obstructed. The principal and uniform force operating on the circulation in these vessels is the ris a torgo. Reference has been made to the adequacy of the arterial pressure, extending through the capillaries, to account for the movement of blood in the veins, provided there be no great obstacles to the current. The other forces which concur to produce movement of blood in the veins are the following :-

I. Muscular action, by which many of the veins are at times compressed, thus forcing the blood toward the heart, regurgitation being prevented by the action of the valves.

2. A suction force exerted by the action of the thorax in inspiration, operating, however, only on veins in the immediate neighborhood of the chest.

3. A possible influence from contraction of the coats of the vessels themselves. This is marked in the veins near the heart, in some of the inferior animals.

4. The force of gravity, which operates only on vessels that carry blood from above downward to the heart, and a slight suction force which may be exerted on the blood in a small vein as it passes into a larger vessel in which the current is more rapid.

The obstacles to the venous circulation are: pressure sufficient to obliterate the calibre of a vessel, when, from the free communications with other vessels, the current is diverted into another channel; expiratory efforts; the contractions of the right side of the heart; and the force of gravity, which operates, in the erect posture, on the current in all excepting the veins of the head, neck and parts of the trunk above the heart.

Influence of Muscular Contraction. - That the action of muscles has considerable influence on the current of blood in the veins situated between them and in their substance, has long been recognized; and this action is so marked, that the vessels distributed to muscular tissue have been compared by Chassaignac to a sponge full of liquid, vigorously pressed by the hand. It must be remembered, however, that although the muscles are capable of acting on the blood contained in veins in their substance with great vigor, the heart is fully competent to carry on the venous circulation without their aid; a fact exemplified in the venous circulation in paralyzed parts. 
It has been shown by actual observations with the hemodynamometer, that muscular action is capable of increasing the pressure in certain veins. Bernard found that the pressure in the jugular of a horse, in repose, was I.4 inch (3I.8 millimeters); but the action of the muscles in raising the head increased it to a little more than five inches (I 27 millimeters), or nearly four times. Such observations show at once the great variations in the current and the important influence of muscular contraction on the venous circulation.

In order that contractions of muscles shall assist the venous circulation, two conditions are necessary:-

I. The contractions must be intermittent. This is always the case in the voluntary muscles. It is a view entertained by many physiologists that each muscular fibre relaxes immediately after its contraction, which is instantaneous, and that a certain period of rest is necessary before it can contract again. However this may be, it is well known that all active muscular contraction, as distinguished from the efforts necessary to maintain the body in certain ordinary positions, is intermittent and not very prolonged. Thus the veins, which are partly emptied by the compression, are filled again during the repose of the muscle.

2. There should be no possibility of a retrograde movement of the blood. This condition is fulfilled by the action of the valves. Anatomical researches have shown, also, that these valves are most abundant in veins situated in the substance of or between the muscles; and they do not exist in the veins of the cavities, which are not subject to the same kind of compression.

Force of Aspiration from the Thorax. - During the act of inspiration, the enlargement of the thorax, by depression of the diaphragm and elevation of the ribs, affects the movements of fluids in all the tubes in its vicinity. The air enters by the trachea and expands the lungs so that they follow the movements of the thoracic walls. The flow of blood into the great arteries is somewhat retarded, as is indicated by a diminution in the arterial pressure; and finally, the blood in the great veins passes to the heart with greater facility and in increased quantity. This lastmentioned phenomenon can be readily observed, when the veins are prominent, in profound or violent inspiration. The veins at the lower part of the neck are then seen to empty themselves of blood during inspiration, and they become distended during expiration, producing a sort of pulsation that is synchronous with respiration. This can always be observed after exposure of the jugular in the lower part of the neck. Direct observations on the jugulars, however, show that the influence of inspiration can not be felt much beyond these vessels. They are seen to collapse with each inspiratory act, a condition which limits this influence 
to the veins near the heart. The flaccidity of the wall of the veins will not permit the extended action of any suction force. In the circulation, the veins are moderately distended with blood by the vis a tergo, and, to a certain extent, they are supported by connections with surrounding tissues, so that the force of aspiration is felt farther than in experinents on vessels removed from the body. The blood, as it approaches the thorax, impelled by other forces, is considerably accelerated in its flow ; but it is evident that beyond a certain distance, and that very near the chest, ordinary aspiration has no influence, and violent efforts rather retard than favor the venous current.

In the liver the influence of inspiration becomes an important element in the mechanism of the circulation. This organ presents a vascular arrangement that is exceptional. The blood, distributed by the arteries in a capillary plexus in the mucous membrane of the alimentary canal and in the spleen, instead of being returned directly to the heart by the veins, is collected into the portal vein, carried to the liver and is there distributed in a second set of capillary vessels. It is then collected in the hepatic veins and carried by the vena cava to the heart. The three hepatic veins open into the inferior vena cava near the diaphragm, where the force of aspiration from the thorax would materially assist the current of blood. On following these vessels into the substance of the liver, it is found that their walls are so firmly adherent to the hepatic tissue, that when cut across, they remain patulous; and it is evident that they must remain open under all conditions. The thorax can therefore exert a powerful influence on the hepatic circulation; for it is only the flaccidity of the walls of the vessels which prevents this influence from operating throughout the venous system. Although this must be an important element in the circulation in the liver, the fact that the blood circulates in this organ in the fœetus before any movements of the thorax take place shows that it is not essential.

Aside from the pressure exerted by the contraction of muscles and the force of aspiration from the thorax, the influences that assist the venous circulation are insignificant. There is a slight contraction in the venæ cavæ in the immediate proximity of the heart, which is more extended in many of the lower vetebrate animals and may be mentioned as having an influence - very small it is true - on the flow of blood from the great veins.

In the veins that pass from above downward, the force of gravity favors the flow of blood. This is shown by the turgescence of the veins of the neck and face when the head is kept for a short time below the level of the heart. If the arm is elevated above the head, the veins of the back of the hand are reduced in size, from the greater facility with 
which the blood passes to the heart, while they are distended when the hand is allowed to hang by the side and the blood has to rise against the force of gravity.

Some physiologists are of the opinion that the right ventricle exerts an active suction force during its diastole; but experiments on animals do not sustain this view, and if such a force be exerted, its effect on the circulation, even in the veins near the heart, must be very slight. In the great irregularity in the rapidity of the circulation in different veins, it must frequently happen that a vessel empties its blood into another of larger size in which the current is more rapid. In such an instance, as a physical necessity, the more rapid current in the large vein exerts a certain suction force on the blood in the smaller vessel.

\section{Uses of the Valves of the Veins}

There are two distinct conditions under which the valves of the veins may be closed. One is the arrest of circulation, from any cause, in veins in which the blood has to rise against the force of gravity; and the other, compression of veins, from any cause - usually from muscular contraction - which tends to force the blood from the vessels compressed, into others, when the valves offer an obstruction to a flow toward the capillaries and necessitate a current in the direction of the heart. In the first of these conditions, the valves are antagonistic to the force of gravity; and when a vessel is tenuporarily obstructed, they aid in directing the current into anastomosing vessels. It is but rarely, however, that they act thus in opposition to the force of gravity; and it is only when many of the veins of a part are simultaneously compressed that they aid in diverting the current. When a single vein is obstructed, it is not probable that the valves are necessary to divert the current into other vessels, for this would take place in obedience to the vis a tergo; but when many veins are obstructed in a dependent part and the avenues to the heart become insufficient, the valves divide the columns of blood, so that the pressure is equally distributed throughout the extent of the vessels. This is, however, but an occasional action of the valves; and it is evident that their influence is only to prevent the weight of the entire column of blood from operating on the smallest veins and the capillaries.

It is in connection with the intermittent compression of the veins that the valves have their principal use. Their situation alone leads to this supposition. They are found in greatest numbers throughout the muscular system, having been demonstrated in vessels one line (2. I millimeters) in diameter. They are also found in the upper parts of the body, where they certainly do not operate against the force of gravity, while 
they do not exist in the cavities, where the venous trunks are not subject to compression. In the action of muscles, the skin frequently is stretched over the part, and the cutaneous veins are somewhat compressed. This may be seen in the hand, by letting it hang by the side until the veins become swollen, and then contracting the muscles, when the skin will become tense and the veins much less prominent. Here the valves have an important action. The compression of the veins is greater in the substance of and between the muscles than in the skin; but the blood is forced from the muscles into the skin, and the valves act to prevent it from taking a retrograde course.

Conditions that impede Venous Circulation. - Expiration, in its influence on the circulation in veins near the thorax is directly opposed to inspiration. As inspiration has a tendency to draw the blood from these vessels into the chest, expiration assists in forcing the blood out from the vessels of the thorax and opposes a flow in the opposite direction. The effect of prolonged and violent expiratory efforts is quite marked, being followed by congestion of the veins of the face and neck and a sense of fulness in the head, which may become distressing. The opposition to the venous current usually extends only to vessels in the immediate vicinity of the thorax, or it may be stated in general terms, to those veins in which the flow of blood is assisted by the movements of inspiration; but while the inspiratory influence is confined to a very restricted circuit of vessels, the obstructive influence of violent and prolonged expiration may be extended much farther, as is seen when the vessels of the neck, face and conjunctiva become congested in prolonged vocal efforts, blowing etc. This is not simply a reflux from the large trunks of the thoracic cavity; for in this case, it would be necessary to assume an insufficiency of certain valves, which does not exist.

It is in the internal jugular that the influence of expiration is most important, both on account of its great size in the human subject, as compared with the other vessels, and the importance and delicacy of the parts from which it collects the blood. At the opening of this vessel into the innominate vein, is a pair of strong and perfect valves, which effectually close the orifice when there is a tendency to regurgitation. When the act of expiration arrests the onward flow in the veins near the thorax, these valves are closed and protect the brain from congestion by regurgitation. The blood accumulates behind the valves, but the free communication of the internal jugular with the other veins of the neck relieves the brain from congestion, unless the effort is extraordinarily violent and prolonged.

It is evident that there are other conditions that may impede the 
venous circulation. Accidental compression may temporarily arrest the flow in some one vein. When the volume of blood is materially increased, as after a full meal with copious ingestion of liquids, the additional quantity of blood accumulates mainly in the venous system and proportionally diminishes the rapidity of the venous circulation.

The force of gravity also has an important influence. It is more difficult for the blood to pass from below upward to the heart than to flow downward from the head and neck. The action of this is seen if comparison is made between the circulation in the arm elevated above the head and hanging by the side. In the one case the veins are readily emptied and contain but little blood, and in the other, the circulation is more difficult and the vessels are moderately distended. The walls of the veins are thickest and the valves are most abundant in parts of the body that are habitually dependent.

\section{Circulation in Special Parts}

Circulation in the Cranial Cavity. - In the encephalic cavity there are certain peculiarities in the anatomy of some of the vessels, with exceptional conditions of the blood as regards atmospheric pressure, that have been regarded as capable of considerably modifying the circulation. In the adult the cranium is a closed air-tight box, containing the incompressible cerebral substance, blood, lymph and the cephalorachidian fluid; and the blood is here under conditions quite different from those presented in other parts. The venous passages in the brain, which correspond to the great veins in other situations, are in the form of sinuses between the layers of the dura mater and are but slightly dilatable. In the perfectly consolidated adult head, the blood is not subjected to atmospheric pressure, as in other parts, and the semisolids and liquids which make up the encephalic mass can not increase in size in congestion and diminish in anemia. Nothwithstanding these conditions, the fact remains that examinations of the vessels of the brain after death show differences in the quantity of blood. The question then arises as to what is displaced to make room for the blood in congestion, and what supplies the place of the blood in anemia. An anatomical peculiarity not yet considered offers an explanation of these conditions. Between the pia mater and the arachnoid of the brain and spinal cord there exists a liquid, the cephalo-rachidian fluid, that may pass from the surface of the brain to the spinal canal and communicates with the contents of the ventricles (Magendie). The communication between the cranial cavity and the spinal canal is quite free. It is easy to see one of the physiological uses of this liquid. 
When the pressure of blood in the arteries going to the brain is increased or when there is an obstacle to the return of blood by the veins, more or less congestion takes place, and the blood forces the liquid from the cranial cavity into the spinal canal. The reverse takes place when the supply of blood to the brain is diminished.

The influence of gravity on the cerebral circulation may be considerable, as is shown by the following experiment: If an ordinary "hutch" rabbit is held by the ears with the body dependent, the supply of blood to the brain becomes so far reduced that the animal soon becomes unconscious and will die if kept in this position for half an hour; but if placed in the horizontal position, it is soon restored to consciousness. This is due to large accumulation of blood in the pendulous abdominal cavity and a consequent deficient supply to the encephalon.

Circulation in Erectile Tissues. - In the organs of generation of both sexes, there exists a tissue that is subject to increase in volume and hardness when in a condition of what is called erection. The parts in which the erectile tissue exists are, in the male, the corpora cavernosa of the penis, the corpus spongiosum and the glans penis; and in the female, the corpora cavernosa of the clitoris, the gland of the clitoris and the bulb of the vestibule.

The vascular arrangement in erectile organs is peculiar and is not found in any other part of the circulatory system. Taking the penis as an example, the arteries, which have an unusually thick muscular layer, after they have entered the organ, do not simply branch and subdivide, as in most other parts, but send off large numbers of arborescent branches, which immediately become tortuous and are distributed in the cavernous and spongy bodies in anastomosing vessels, which have but a single thin homogeneous coat. These vessels are larger, even, than the arterioles which supply them with blood, some having a diameter of $\frac{1}{25}$ to $\frac{1}{17}$ of an inch (I to I.5 millimeters). The cavernous bodies have an external investment of strong fibrous tissue of considerable elasticity, which sends bands, or trabeculæ, into the interior, by which they are divided up into cells. The trabeculæ are composed of fibrous tissue mixed with a large number of non-striated muscular fibres. These cells lodge the bloodvessels, which ramify in the tortuous manner already indicated and finally terminate in the veins. The anatomy of the corpora spongiosa is essentially the same, the only difference being that the fibrous envelope and the trabeculæ are more delicate and the cells are smaller.

During sexual excitement, or when erection occurs from any cause, the thick muscular walls of the arteries of supply relax and allow the arterial pressure to distend the capacious vessels lodged in the cells of the cavernous and spongy bodies. This produces the characteristic change in 
the volume and position of the organ. It is evident that erection depends on the peculiar arrangement of the bloodvessels, and is not simply a congestion, such as might occur in any vascular part. During erection there is not a stasis of blood; but if it continues for a certain time, the quantity passing out by the veins must be equal to that which passes in by the arteries.

Derivative Circulation. - In some parts of the circulatory system, there exists a direct communication between the arteries and the veins, so that all the blood does not necessarily pass through the true capillaries. This peculiarity, which had been noted by Todd and Bowman, Paget and others, has been closely studied by Sucquet. By using a black solidifiable injection, he found certain parts of the upper and lower extremities and the head, which became colored by the injection while other parts were not penetrated. Following the vessels by dissection, he showed that in the upper extremity, the skin of the fingers and part of the palm of the hand and the skin over the olecranon were provided with vessels of considerable size, which allowed the fluid injected by the axillary artery to pass directly into some of the veins, while in other parts the veins were entirely empty. Extending his researches to the lower extremity, he found analogous communications between the vessels in the knee, toes and parts of the sole of the foot. He also found communications in the nose, cheeks, lips, forehead and tips of the ears, parts that are peculiarly liable to changes in color from congestion of vessels. These observations have been in the main confirmed by the more recent researches of Hoyer. It is evident that under certain conditions a larger quantity of blood than usual may pass through these parts without necessarily penetrating the capillaries.

Pulmonary Circulation. - The vascular system of the lungs merits the name, which is frequently applied to it, of the lesser circulation. The right side of the heart acts simultaneously with the left, but is anatomically distinct from it, and its muscular walls are less powerful. The pulmonary artery has thinner and more distensible walls than the aorta and distributes its blood to a single system of capillaries, situated but a short distance from the heart. In the lungs, the pulmonary artery is broken up into capillaries, most of them just large enough to allow the passage of blood-corpuscles in a single row. These vessels are provided with a single coat and form a close network surrounding the air-cells. From the capillaries the blood is collected in the pulmonary veins and is carried to the left auricle. There is no great disparity between the arteries and veins of the pulmonary system as regards capacity. The pulmonary veins in the human subject have no valves.

The blood in its passage through the lungs does not meet with the 
resistance that is presented in the systemic circulation; and the anatomy of the pulmonary vessels and of the right side of the heart shows that the blood must circulate in the lungs with comparative facility. The right ventricle has about one-third the force of the left, and the pulmonary artery will sustain a much less pressure than the aorta.

The pressure of blood in the pulmonary artery, measured by connecting a cardiometer with a trocar introduced into the pulmonary artery of a living horse through one of the intercostal spaces, was found to be about one-third as great as the pressure in the aorta, which nearly corresponds with an estimate of the comparative power of the two ventricles, judging from the thickness of their muscular walls (Chauveau and Faivre).

On microscopical examination of the circulation in the lower animals, as in the frog, the movement of blood in the capillaries of the lungs does not present any differences from the capillary circulation in other parts, except that the vessels seem more crowded with corpuscles and there is no "still layer" next their walls.

Circulation in the Walls of the Heart. - The circulation in the walls of the heart does not present important peculiarities. It has been

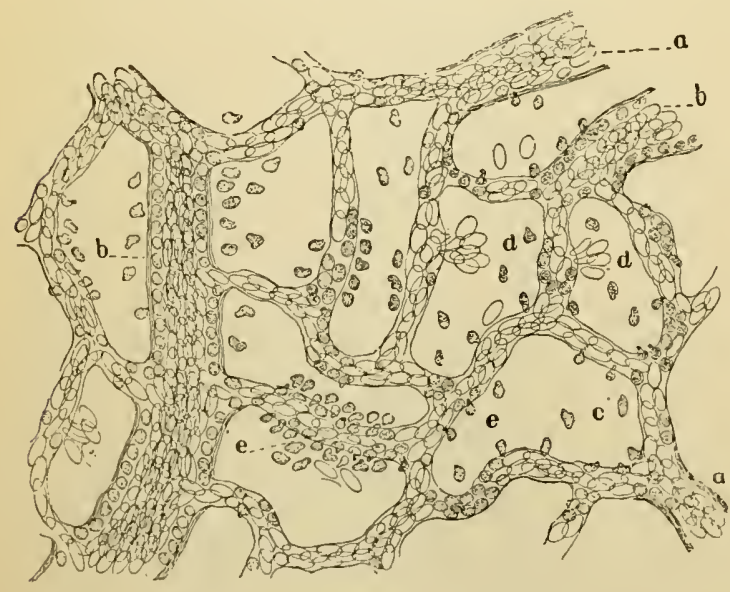

Fig. 27. - Migration of leucocytes and diapedesis of red corpuscles (Councilman).

This figure represents a portion of the mesentery of a frog two hours after exposure. $a, a$, small arteries; $b, b$, small veins; $c$, a few leucocytes in connective tissue; $d, d$, diapedesis of red corpuscles; $e, e$, emigration of leucocytes. shown that the pressure of blood in the coronary arteries in the dog, during the ventricular systole, is sufficient to supply the arterioles in the substance of the heart with blood, precisely as it is supplied to the general arterial system. In a number of experiments in which simultaneous traces of the pulse-beats were obtained, it was found that the coronary and carotid pulses were practically synchronous (Martin).

Passage of Blood-Cor-

puscles through the Walls of the Vessels (Migration and Diapedesis). In the frog it has been observed that leucocytes sometimes pass through the walls of the bloodvessels, either by means of small orifices (stro- 
mata) or by a kind of filtration through the substance which unites the borders of the endothelial cells. This phenomenon was described by Waller. in $\mathrm{I} 84 \mathrm{I}$, but has attracted much attention since the more recent researches of Cohnheim. In this process it is observed that the leucocytes, which first adhere to the vascular walls, send out little projections which penetrate the membrane, so that a point appears outside of the vessel. This point becomes larger and larger, until the corpuscle has passed through. The corpuscles then migrate a certain distance by means of the movements known as ameboid, which have already been described. It was supposed by Cohnheim that this was one of the early pheriomena of inflammation, the migrating corpuscles afterward multiplying by division, constituting the so-called pus-corpuscles. Following stasis of blood in the small vessels, the red corpuscles, it is supposed, may pass out in the same way. It is not certain that these are normal processes or that they take place in the human subject. According to Hering, red corpuscles pass through the walls of the vessels (diapedesis), only when the blood-pressure is sufficient to produce transudation of the plasma.

\section{Rapidity of the Circulation}

Questions of considerable physiological importance arise in connection with the general rapidity of the circulation :-

1. What length of time is occupied in the passage of the blood through both the lesser and the greater circulations?

2. What is the time required for the passage of the entire mass of blood through the heart?

3. What influence has the number of pulsations of the heart on the general rapidity of the circulation?

The first of these questions is the one that has been most satisfactorily answered by experiments on living animals. In 1827 Hering made the experiment of injecting into the jugular vein of a living animal a solution of potassium ferrocyanide, noting the time which elapsed before it could be detected in the blood of the vein of the opposite side. This gave the first correct idea of the rapidity of the circulation. He drew the blood at intervals of five seconds after beginning the injection, and thus, by repeated observations, ascertained pretty nearly the rapidity of a circuit of blood in the animals on which he experimented. Vierordt (I 858 ) collected the blood as it flowed, in little vessels fixed on a disk revolving at a known rate, which gave more exactness to the observations. The results obtained in these two experiments were nearly identical.

The only reasonable objection to these experiments is that a saline 
solution, introduced into the circulation, would have a tendency to diffuse itself throughout the whole mass of blood, it might be, with considerable rapidity. This certainly is an element that should be taken into account; but from the definite data obtained concerning the rapidity of the arterial circulation and the inferences that are unavoidable in regard to the rapidity of the venous circulation, it would seem that the saline solution must be carried on by the mere rapidity of the arterial flow to the capillaries, which are very short, taken up from them, and carried on by the veins, and thus through the entire circuit, before it has had time to diffuse itself to any considerable extent. It is not apparent how this objection can be overcome; for a substance must be used that will mix with the blood, as otherwise it could not pass through the capillaries.

There seems no reason why, with the above restrictions, the results obtained by Hering and Vierordt should not be accepted and their application be made to the human subject.

Hering found that the rapidity of the circulation in different animals had an inverse ratio to their size and a direct ratio to the rapidity of the action of the heart.

The following are the mean results in certain of the domestic animals, taking the course from jugular to jugular, when the blood passes through the lungs and through the capillaries of the head:-

In the Horse, the circulation is accomplished in 27.3 seconds.

In the Dog, the circulation is accomplished in 15.2 seconds.

In the Goat, the circulation is accomplished in 12.8 seconds.

In the Rabbit, the circulation is accomplished in 6.9 seconds.

Applying these results to the human subject and taking into account the size of the body and the rapidity of the heart's action, the duration of the circuit from one jugular to the other may be estimated at $2 \mathrm{I} .4$ seconds, and the general average through the entire system, at 23 seconds. This estimate is simply approximate; but the results in the inferior animals may be received as nearly accurate.

Estimates of the time required for the passage of the whole mass of blood through the heart are even less definite than the estimate of the general rapidity of the circulation. To arrive at a satisfactory result, it is necessary to know the entire quantity of blood in the body and the exact quantity which passes through the heart at each pulsation. If the entire mass of blood is divided by the quantity discharged from the heart with each ventricular systole, the result will be the number of pulsations required for the passage of the blood through the heart; and knowing the number of beats per minute, the length of time thus 
occupied is ascertained. The objection to this kind of estimate is the inaccuracy of the data respecting the quantity of blood in the system as well as the quantity which passes through the heart with each pulsation. Nevertheless, an estimate can be made, which, if not entirely accurate, can not be far from the truth.

The entire quantity of blood, according to estimates based on the most reliable data, is about one-twentieth the weight of the body, or seven pounds ( 3.7 kilograms), in a man weighing one hundred and forty pounds (63.5 kilograms). The quantity discharged at each ventricular systole is estimated at three ounces ( 90 cubic centimeters). It would require, therefore, forty-five pulsations for the passage through the heart of the entire mass of blood. Assuming the pulsations to be seventy-two per minute, this would occupy a little more than thirty-one seconds.

The relation of the rapidity of the circulation to the frequency of the heart's action is a question not neglected in the experiments of Hering. It is evident that if the charge of blood sent into the arteries is the same, or nearly the same, under all conditions, an increase in the number of pulsations of the heart would produce a corresponding acceleration of the general current of blood. This is a proposition, however, which can not be taken for granted; and there are many facts that favor a contrary opinion. It may be stated as a general rule, that when the acts of the heart increase in frequency they diminish in force; and this renders it probable that the ventricle is most completely distended and emptied when its action is moderately slow. When, however, the pulse is much accelerated, the increased number of pulsations of the heart might be sufficient to overbalance the diminished force of each act and would increase the rapidity of the circulation. In regard to the relations between the rapidity of the heart's action and the general rapidity of the circulation, the following conclusions may be accepted as the results of experimental inquiry :-

I. In physiological increase in the number of beats of the heart, as the result of exercise, for example, the general circulation is somewhat increased in rapidity, though not in proportion to the increase in the rapidity of the pulse.

2. In pathological acceleration of the heart's action, as in febrile conditions, the rapidity of the general circulation usually is diminished, it may be to a considerable extent.

3. Whenever the number of beats of the heart is considerably increased from any cause, the quantity of blood discharged at each ventricular systole is much diminished, either from lack of complete distention or from imperfect emptying of the cavities. 
Phenomena in the Circulatory Sy'stem after Death. - Nearly every autopsy shows that after death the blood does not remain equally distributed in the arteries, capillaries and veins. Influenced by gravitation, it accumulates in and discolors the most dependent parts of the body. The arteries are always found empty, and blood accumulates in the venous system and capillaries; a fact which was observed by the ancients and gave rise to the belief that the arteries were air-bearing tubes. This is readily explained by the post-mortem contraction of the muscular coat of the arteries. If the artery and vein of a limb are exposed in a living animal and all the other vessels are tied, compression of the artery does not immediately arrest the current in the vein, but the blood will continue to flow until the artery is emptied (Magendie). The artery, when relieved from the distending force of the heart, reacts on its contents by virtue of its contractile coat and completely empties itself of blood. An action similar to this takes place throughout the arterial system after death. The vessels react on their contents and gradually force the blood into and through the capillaries, which are very short, to the veins, which are capacious, distensible and but slightly contractile. This begins immediately after death, while the contractility of the muscular coat of the arteries remains, and is seconded by subsequent cadaveric rigidity, which affects involuntary as well as voluntary muscular fibres. Once in the venous system, the blood can not return on account of the valves. Thus, after death, the blood is found in the veins and capillaries. 


\section{CHAPTER IV}

\section{RESPIRATORY MOVEMENTS}

Physiological anatomy of the respiratory organs - Movements of respiration - Action of the diaphragm - Action of the muscles which raise the ribs - Scalene muscles - Intercostal muscles - Levatores costarum - Auxiliary muscles of inspiration - Expiration - Influence of the elasticity of the pulmonary structure and walls of the chest - Action of muscles in expiration - Internal intercostals - Infracostales - Triangularis sterni-Obliquus externus - Obliquus internus - Types of respiration - Frequency of the respiratory movements - Respiratory sounds - Coughing, sneezing, sighing, yawning, laughing, sobbing and hiccough - Quantity of air changed in the respiratory acts - Diffusion of air in the lungs.

THE tide of air in the lungs does not strictly constitute respiration, these organs serving merely to facilitate the introduction of oxygen into the blood and the exhalation of carbon dioxide. When the system is drained of blood or if the blood is rendered incapable of interchanging its gases with the air, respiration ceases and all the phenomena of asphyxia are presented, although air may be introduced into the lungs with perfect regularity. As in the nutrition of tissue the nitrogenous constituents of the blood, united with inorganic substances, are transformed into the tissue itself, finally changed into excrementitious products, such as the urinary matters, and discharged from the body, so the oxygen of the blood is appropriated, and carbon dioxide, which is an excrementitious substance, is produced and is discharged in the expired air.

The essential conditions for respiration in animals that have a circulating nutritive liquid are air and blood separated by a membrane that will allow the passage of gases. The effete products of respiration contained in the blood, the most important of which is carbon dioxide, pass out and vitiate the air. The air is deprived of a certain proportion of its oxygen, which passes into the blood, to be conveyed to the tissues. Thus the air must be changed to supply fresh oxygen and get rid of carbon dioxide.

\section{Physiological Anatomy of the Respiratory Organs}

Passing from the mouth to the pharynx, two openings are observed; a posterior opening, which leads to the œsophagus, and anteriorly the opening of the larynx, which is the beginning of the passages concerned exclusively in respiration. 
Beginning with the larynx, it is seen that the cartilages of which it is composed are sufficiently rigid and unyielding to resist the pressure produced by any inspiratory effort. Across its opening are the vocal chords, which are four in number and have a direction from before backward. The two superior are called the false vocal chords, because they are not concerned in the production of the voice. The two inferior

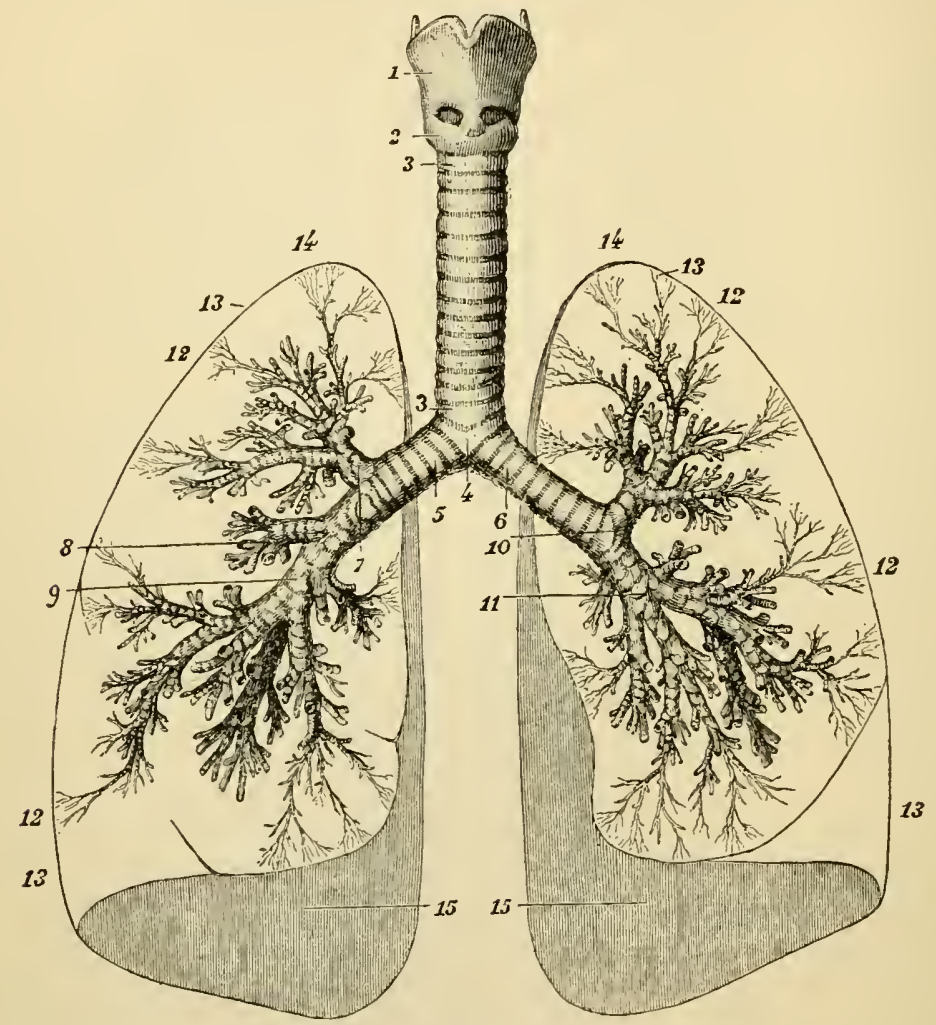

Fig. 28. - Trachea and bronchial tubes (Sappey).

I, 2, larynx; 3, 3, trachea; 4, bifurcation of the trachea ; 5 , right bronchus ; 6 , left bronchus; 7 , bronchial division to the upper lobe of the right lung; 8 , division to the midd?e lobe; 9 , division to the lower lobe; IO, division to the upper lobe of the left lung; II, division to the lower lobe; I2, I2, I2, I2, ultimate ramifications of the bronchia; I3, I3, I3, I3, lungs, represented in contour; I4, I4, summit of the lungs; 15,15 , base of the lungs.

are the true vocal chords. They are ligamentous bands covered with folds of mucous membrane, which is quite thick on the superior chords and very thin and delicate on the true vocal chords. These bands are attached anteriorly to a fixed point between the thyroid cartilages, and posteriorly, to the movable arytenoid cartilages. Air is admitted to the trachea through an opening between the chords, which is called the rima glottidis. Little muscles, arising from the thyroid and cricoid and at- 
tached to the arytenoid cartilages, are capable of separating and approximating the points to which the vocal chords are attached posteriorly, so as to open and close the rima glottidis.

In man, the respiratory movements of the glottis have been studied by means of the laryngoscope. In tranquil respiration, the rima glottidis is opened to a certain extent in inspiration, by the tonic contraction of the posterior crico-arytenoid muscles, and its form is triangular; it is a little narrower in expiration; but in forced, rapid or difficult breathing, the crico-arytenoids act vigorously and the glottis is widely opened with each inspiration, the opening being somewhat elliptical. In the cadaver, the width of the opening of the glottis is reduced about one-half. The muscles concerned in the respiratory movements of the glottis are animated by the recurrent laryngeal nerves, but the filaments going to these muscles are not derived from the spinal accessory. While the recurrents also contain filaments, derived from the accessories, that preside over the vocal movements of the glottis, these movements are quite distinct from those connected with the act of inspiration. The true vocal sounds are expiratory.

Attached to the anterior portion of the larynx, is the epiglottis, a leafshaped lamella of elastic cartilage, which, during ordinary respiration, projects upward and lies against the base of the tongue. During deglutition, respiration is momentarily interrupted, and the air-passages are protected by the muscles that approximate the vocal chords and by the tongue, which presses backward, carrying the epiglottis before it and completely closing the glottis. Physiologists have questioned whether the epiglottis be necessary to the complete protection of the air-passages; and it has frequently been removed from the lower animals, apparently without interfering with the deglutition of solids or liquids. It is a question, however, whether the results of this experiment can be absolutely applied to the human subject. In a case of loss of the entire epiglottis, observed in Bellevue Hospital, the patient experienced slight difficulty in swallowing, from the passage of little particles into the larynx, which produced cough. This case and others of a similar character show that the presence of the epiglottis, in the human subject at least, is necessary to the complete protection of the air-passages in deglutition.

Passing down the neck from the larynx toward the lungs, is the trachea, which is four to four and a half inches (IO.I6 to I I.43 centimeters) in length and about three-quarters of an inch ( I9. I millimeters) in diameter. It is provided with cartilaginous rings, sixteen to twenty in number, which partly surround the tube, leaving one-third of its posterior portion occupied by fibrous tissue mixed with non-striated muscular fibres. Passing into the chest, the trachea divides into the two primitive bronchia, 
the right being shorter, larger and more nearly horizontal than the left. These tubes, provided, like the trachea, with imperfect cartilaginous rings, enter the lungs, divide and subdivide, until the minute ramifications of the bronchial tree open directly into the air-cells. After penetrating the lungs, the cartilages become irregular and are in the form

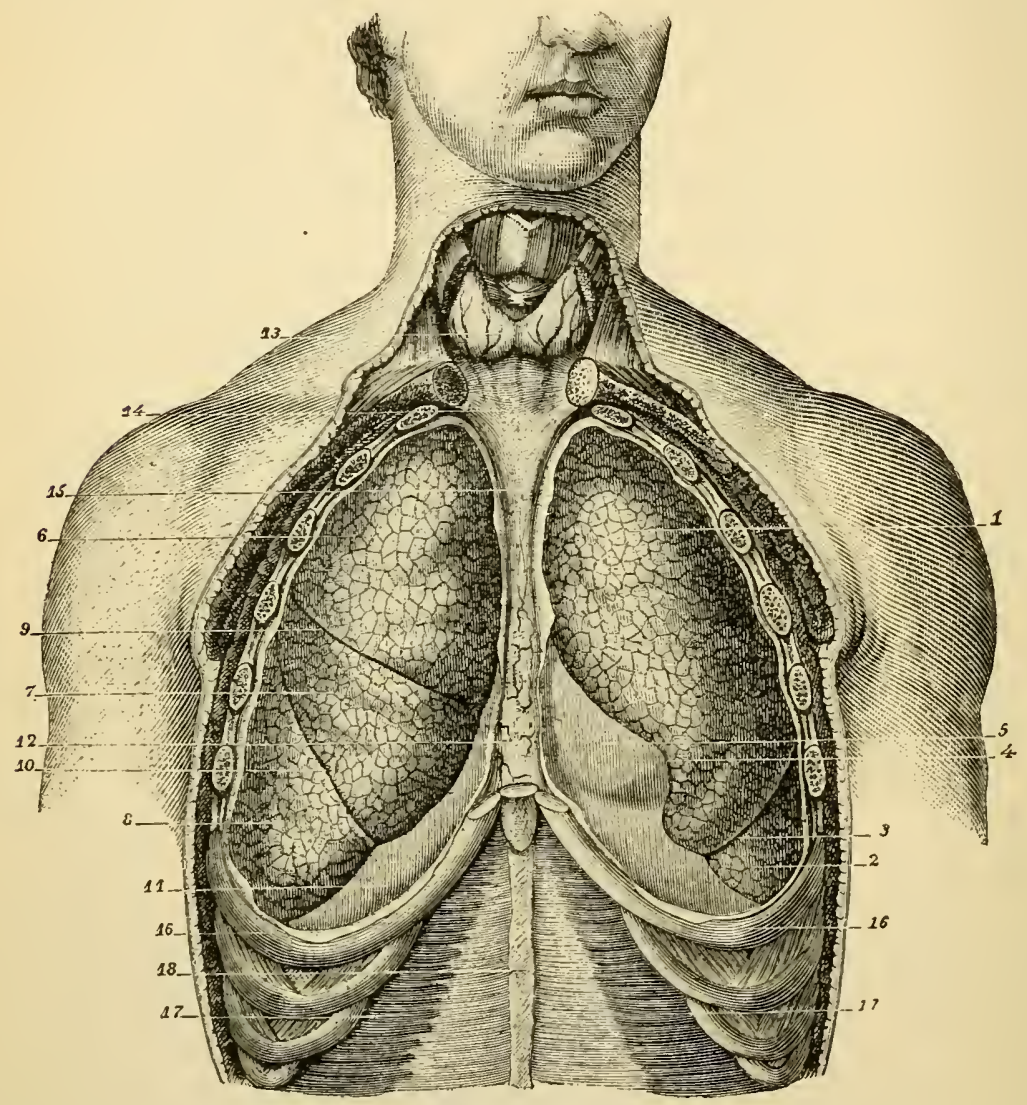

Fig 29. - Lungs, anterior vzew (Sappey).

$\mathrm{I}$, upper lobe of the left lung; 2, lower lobe; 3 , fissure; 4, notch corresponding to the apex of the heart; 5 , pericardium; 6 , upper lobe of the right lung; 7 , middle lobe; 8 ,lover lobe; 9 , fissure; I0, fissure; II, diaphragm; 12 , anterior mediastinum; 13, thyroid gland; 14 , middle cervical aponeurosis; 15. process of attachment of the mediastinum to the pericardium; 16,16 , seventh ribs; 17,17 , transversales muscles; 18, linea alba.

of oblong angular plates, which are so disposed as to completely encircle the tubes. In tubes of very small size, these plates are fewer than in the larger bronchia, until in tubes of a diameter less than $\frac{1}{50}$ of an inch ( 0.5 millimeter), they are lost.

The walls of the trachea and bronchial tubes are composed of two distinct membranes; an external membrane, between the layers of which 
the cartilages are situated, and a mucous membrane. The external membrane is composed of inelastic and elastic fibrous tissue. Posteriorly, in the space not covered with cartilaginous rings, these fibres are mixed with a certain number of non-striated muscular fibres, which exist in two layers; a thick, internal layer, in which the fibres are transverse, and a thinner, longitudinal layer, which is external. The collection of muscular fibres in the posterior part of the trachea is sometimes called the trachealis muscle. Throughout the bronchial tubes, there are circular

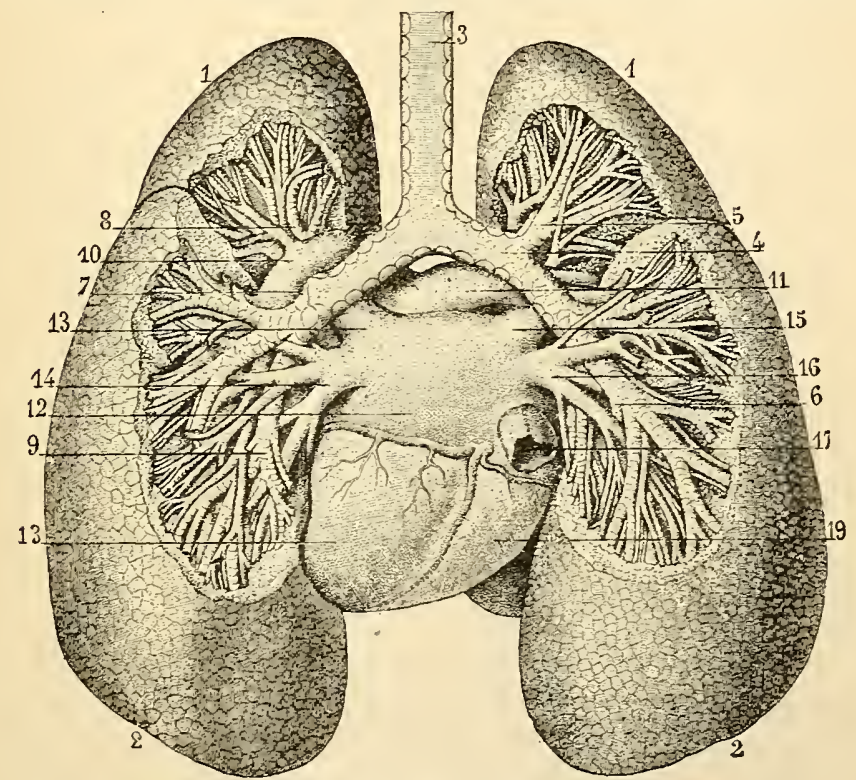

Fig.30.-Bronchia and lungs, posterior view (Sappey).

I, I, summit of the lungs; 2, 2, base of the lungs; 3 , trachea; 4, right bronchus; 5 , division to the upper lobe of the lung; 6 , division to the lower lobe; 7 , left bronchus; 8 , division to the upper lobe; 9 . division to the lowver lobe; Io, left branch of the pulmonary artery; II, right branch; I2, left auricle of the heart; $\mathrm{I}_{3}$, left superior pulmonary vein; $\mathrm{I}_{4}$, left inferior pulmonary vein; $\mathrm{I}_{5}$, right superior pulmonary vein; 16 , right inferior pulmonary vein; 17 , inferior vena cava; 18 , left ventricle of the heart; 19 , right ventricle.

fasciculi of non-striated muscular fibres lying just beneath the mucous membrane, with a number of longitudinal elastic fibres. The character of the bronchia abruptly changes in tubes less than $\frac{1}{50}$ of an inch $(0.5 \mathrm{milli}-$ meter) in diameter. They then lose the cartilaginous rings, and the external and the mucous membranes become so closely united that they can no longer be separated by dissection. The circular muscular fibres continue as far as the air-cells. The mucous membrane is smooth, covered with ciliated epithelium, the movements of the cilia heing from within outward, and is provided with mucous glands. These glands are of the racemose variety, and in the larynx they are of considerable size. 
In the trachea and bronchia, racemose glands exist in the membrane on the posterior portion of the tubes; but anteriorly are small follicles, terminating in a single, and sometimes a double, blind extremity. These follicles are lost in tubes measuring less than $\frac{1}{50}$ of an inch ( 0.5 millimeter) in diameter (see Plate II, Fig. 4).

When moderately inflated, the lungs have the appearance of irregular cones, with rounded apices, and concave bases resting upon the diaphragm. They fill that part of the cavity of the thorax which is not occupied by the heart and great vessels, and are completely separated from each other by the mediastinum. The lungs are in contact with the thoracic walls, each lung being covered with a reflection of the serous membrane that lines the cavity of the corresponding side. Thus they necessarily follow the movements of expansion and contraction of the thorax. Deep fissures divide the right lung into three lobes and the left lung into two. The surface of the lungs is divided into irregularly polygonal spaces, $\frac{1}{4}$ of an inch to an inch (6.4 to 25.4 millimeters) in diameter, which mark what are sometimes called the pulmonary lobules; but this term is incorrect, as each of these divisions includes a number of the true lobules.

Following out the bronchial tubes from the diameter of $\frac{1}{50}$ of an inch (0.5 millimeter), the smallest, which are $-\frac{1}{12} \overline{0}$ to $\frac{1}{75}$ of an inch (0.2 I to 0.33 millimeter) in diameter, open into a collection of oblong vesicles, which are the air-cells. Each collection of vesicles constitutes one of the true pulmonary lobules and is $\frac{1}{50}$ to $\frac{1}{12}$ of an inch (0.5 to 2.1 millimeters) in diameter. After entering the lobule, the tube forms a tortuous central canal, sending off branches which terminate in groups of eight to fifteen pulmonary cells, or alveoli. The cells are a little deeper than they are wide and have each a rounded blind extremity. Some are smooth, but many are marked by little circular constrictions. In the normal lung of the adult, after death, they measure $\frac{1}{200}$ to $\frac{1}{120}$ or $\frac{1}{70}$ of an inch (0. 125 to 0.2 I or 0.36 millimeter) in diameter, but are capable of great distention. The smallest cells are in the deep portions of the lungs, and the largest are near the surface. There are considerable variations in the size of the cells at different periods of life. The smallest cells are found in young children, and they progressively increase in size with age. The walls of the air-cells contain abundant small elastic fibres, which do not form distinct bundles for each air-cell, but anastomose freely with each other, so that the same fibres belong to two or more cells. This structure is peculiar to the lungs and gives to these organs their great distensibility and elasticity, properties which play an important part in expelling the air from the chest, as a consequence simply of cessation of the action of the inspiratory muscles. Interwoven with these elastic fibres, is the richest plexus of capillary bloodvessels found in the economy. The 
vessels are larger than the capillaries in other situations, and the plexus is so close that the spaces between them are narrower than the vessels themselves. When distended, the bloodvessels form the greatest part of the walls of the cells.

Lining the air-cells, are thin cells of flattened epithelium, $\frac{1}{2500}$ to $\frac{1}{2000}$ of an inch (IO to $\mathrm{I} 2.5 \mu$ ) in diameter, which are applied directly to the walls of the bloodvessels. The epithelium here does not seem to be regularly desquamated as in other situations. Examination of injected specimens shows that the bloodvessels are so situated between the cells that the blood in the greater part of their circumference is exposed to the air (see Plate II, Fig. 5).

The entire mass of venous blood is distributed in the lungs by the pulmonary artery. Arterial blood is conveyed to the lungs by the bronchial arteries, which ramify and subdivide on the bronchial tubes and follow their course into the lungs, for the nourishment of these parts. It is possible that the tissue of the lungs may receive some nourishment from the blood of the pulmonary artery; but as this vessel does not send branches to the bronchial tubes, the bronchial arteries supply the matters for their nutrition and for the secretion by the mucous glands (see Plate II, Fig. 4).

\section{Movements of Respiration}

In man and in the warm-blooded animals generally, inspiration takes place as a consequence of enlargement of the thoracic cavity and the entrance of air through the respiratory passages, corresponding with the increased capacity of the lungs. In the mammalia, the chest is enlarged by the action of muscles; and in ordinary respiration, inspiration is an active process, while ordinary expiration is mainly passive.

The walls of the thorax are formed by the dorsal vertebræ and ribs posteriorly, by the upper ten ribs laterally, and by the sternum and costal cartilages anteriorly. The direction of the ribs, their mode of connection with the sternum by the costal cartilages, and their articulation with the vertebral column are such that by their movements, the antero-posterior and transverse diameters of the chest may be considerably modified.

Inspiration. - The ribs are somewhat twisted upon themselves and have a general direction forward and downward. The first rib is nearly horizontal, but the obliquity of the ribs progressively increases from the upper to the lower part of the chest. They are articulated with the bodies of the vertebræ so as to allow of considerable motion. The upper seven ribs are attached by the costal cartilages to the sternum, 
these cartilages running upward and inward. The cartilages of the eighth, ninth and tenth ribs are joined to the cartilage of the seventh. The eleventh and twelfth are floating ribs and are attached only to the vertebræ.

It may be stated in general terms that inspiration is effected by descent of the diaphragm and elevation of the ribs; and expiration, by elevation of the diaphragm and descent of the ribs.

Arising severally from the lower border of each rib and attached to the upper border of the rib below, are the eleven external intercostal muscles, the fibres of which have an oblique direction from above down-

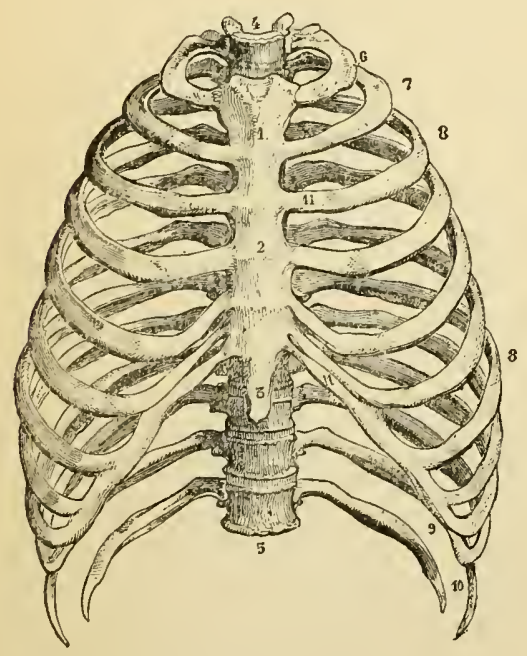

Fig. 31. - Thorax, anterior view' (Sappey).

I, 2,3 , sternum; 4 , circumference of the upper portion of the thorax: 5 , circumference of the base of the thorax; 6 , first rib; 7 , second rib; 8,8 , last five sternal ribs; 9 , upper three false ribs; Io, last two, or floating ribs; II, costal cartilages.

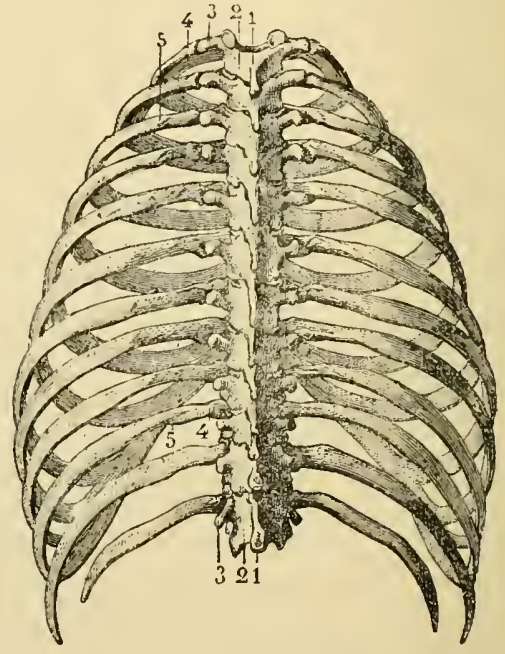

Fig. 32. - Thorax, posterior view (Sappey).

I, I, spinous processes of the dorsal vertebræ; 2,2 , laminæe of the vertebræ; 3,3 , transverse processes; 4,4 , dorsal portions of the ribs: 5,5 , angles of the ribs.

ward and forward. Attached to the inner borders of the ribs, are the internal intercostals, which have a direction from above downward and backward, nearly at right angles to the fibres of the external intercostals. There are also certain muscles attached to the thorax and spine, thorax and head, upper part of humerus etc., that are capable of elevating either the entire chest or the ribs. These act as muscles of inspiration when the attachments to the thorax become the movable points. Some of them are called into action during ordinary respiration; others act as auxiliaries when respiration is moderately exaggerated, as after exercise, and are called ordinary auxiliaries; while others, which ordi- 
narily have different uses, act only when respiration is difficult, and are called extraordinary auxiliaries.

The following are the principal muscles concerned in inspiration :-

\title{
MUSCLES OF INSPIRATION
}

\author{
MUSCLE
}

Diaphragm . . . . . . . . Circumference of lower border of thorax.

Scalenus anticus . . . . . . Transverse processes of third, fourth, fifth and sixth cervical vertebræ - tubercle of first rib.

Scalenus medius . . . . . . . Transverse processes of lower six cervical vertebræ - upper surface of first rib.

Scalenus posticus . . . . . . . Transverse processes of lower two or three cervical vert ebræ - outer surface of second rib.

External intercostals . . . . . . Outer borders of the ribs.

Sternal portion of internal intercostals . . Borders of the costal cartilages.

Twelve levatores costarum . . . . . . Transverse processes of dorsal vertebræ - ribs, between the tubercles and angles.

Ordinary Auxiliaries

Serratus posticus superior . . . . . Ligamentum nuchæ, spinous processes of last cervical and upper two or three dorsal vertebræ upper borders of second, third, fourth and fifth ribs, just beyond the angles.

Sterno-mastoideus . . . . . . Upper part of sternum - mastoid process of temporal bone.

\section{Extraordinary Auxiliaries}

Levator anguli scapulæ . . . . . Transverse processes of upper three or four cervical vertebræ- posterior border of superior angle of scapula.

Trapezius (superior portion) . . . . Ligamentum nuchæ and seventh cervical vertebra - upper border of spine of scapula.

Pectoralis minor

Coracoid process of scapula - anterior surface and upper margins of third, fourth and fifth ribs, near the cartilages.

Pectoralis major (inferior portion) . . . Bicipital groove of humerus - costal cartilages and lower part of sternum.

Serratus magnus . . . . . . . Inner margin of posterior border of scapula - external surface and upper border of upper eight ribs.

Action of the Diaphragm. - The descriptive and general anatomy of the diaphragm gives an idea of its uses in respiration. It arises from the border of the lower circumference of the thorax and mounts into the cavity of the chest, forming a vaulted arch, or dome, with its concavity toward the abdomen and its convexity toward the lungs. In the central portion, there is a tendon of considerable size and shaped something like the club on a playing-card, with middle, right and left leaflets. The rest of the diaphragm is composed of radiating fibres of striated muscular tissue. The œesophagus, aorta and inferior vena cava pass through the diaphragm from the thoracic to the abdominal cavity, by three openings. 
The opening for the œesophagus is surrounded with muscular fibres, by which it is partly closed when the diaphragm contracts in inspiration, as the fibres simply surround the tube and are not attached to its walls.

The opening for the aorta is bounded by the bone and aponeurosis posteriorly, and in front, by a fibrous band to which the muscular fibres are attached, so that their contraction has a tendency rather to increase than to diminish the calibre of the vessel.

The opening for the vena cava is surrounded by tendinous structure, and contraction of the diaphragm, although it may render the form of the opening more nearly circular, can have no effect on its size.

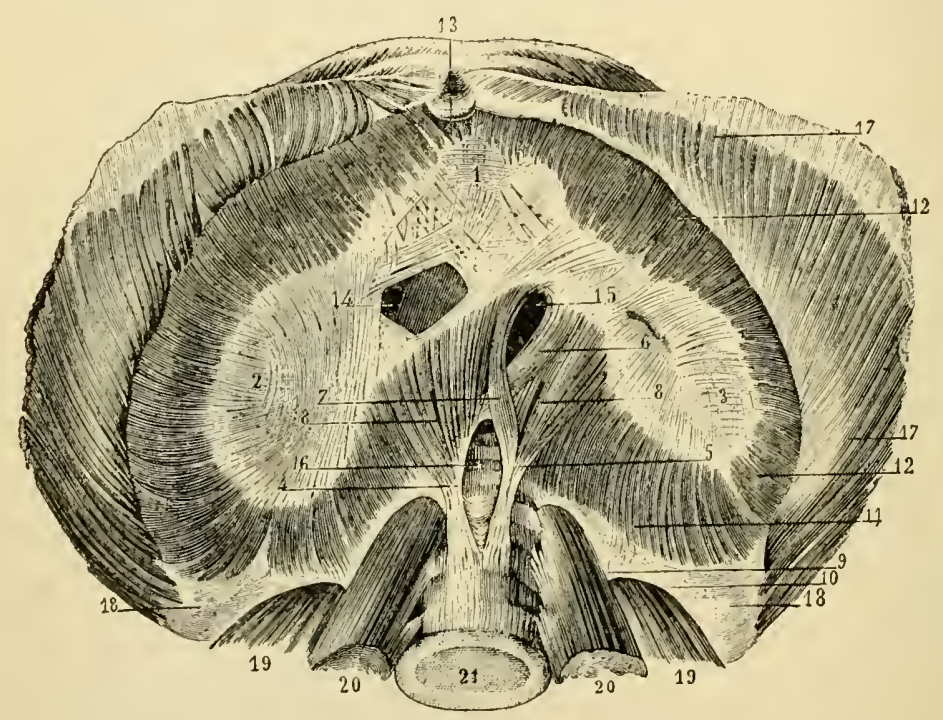

Fig. 33.-Diaphragm (Sappey).

1, 2, 3, central tendon; 4, right pillar; 5 , left pillar; 6,7 , processes between the pillars; 8,8 , openings for the splanchnic nerves; 9 , fibrous arch passing over the psoas magnus; Io, fibrous arch passing over the quadratus lumborum; II, muscular fibres arising from these two arches; I2, I2, muscular fibres arising from the lower six ribs; 13 , fibres from the ensiform cartilage; 14 , opening for the vena cava; 15, opening for the osophagus; 16 , opening for the aorta; 17,17 , part of the transversalis muscle; I8, I8, aponeurosis; I9, I9, quadratus lumborum; 20, 20, psoas magnus; 2I, fourth lumbar vertebra.

In ordinary inspiration, the descent of the diaphragm and its approximation to a plane are the chief phenomena observed; but as there is some resistance to the depression of the central tendon, it is probable that there is also a slight elevation of the inferior ribs.

The phenomena referable to the abdomen which coincide with the descent of the diaphragm can easily be observed in the human subject. As the diaphragm is depressed, it necessarily pushes the viscera before it, and inspiration is therefore accompanied with protrusion of the abdomen. 
The effects of the action of the diaphragm on the size of its openings are limited chiefly to the œsophageal opening. The anatomy of the parts is such that contraction of the muscular fibres has a tendency to constrict this opening. The contraction of the diaphragm is auxiliary to the action of the muscular walls of the œsophagus itself, by which the cardiac opening of the stomach is regularly closed during inspiration. This may become important when the stomach is much distended; for descent of the diaphragm compresses all the abdominal organs and might otherwise cause regurgitation of food.

The contractions of the diaphragm are animated by the phrenic nerve; a nerve which, having the office of supplying the most important respiratory muscle, derives its filaments from a number of sources. It arises from the third and fourth cervical nerves, receiving a branch from the fifth and sometimes from the sixth. It then passes through the chest, penetrates the diaphragm and is distributed on its under surface. Stimulation of this nerve produces convulsive contractions of the diaphragm, and its section paralyzes the muscle almost completely.

From the great increase in the capacity of the chest produced by the action of the diaphragm and its constant and universal action in respiration, it must be regarded as by far the most important and efficient of the muscles of inspiration.

Hiccough, sobbing, laughing and crying are due mainly to the action of the diaphragm, particularly hiccough and sobbing, which are produced by spasmodic contractions of this muscle, usually not under the control of the will.

Action of the Muscles which raise the Ribs. - Scalene Muscles. - In ordinary respiration, the ribs and the entire chest are elevated by the combined action of a number of muscles. The three scalene muscles are attached to the cervical vertebræ and the first and second ribs. These muscles, which act particularly on the first rib, must elevate with it, in inspiration, the rest of the thorax. The articulation of the first rib with the vertebral column is very movable, but it is joined to the sternum by a short cartilage, which allows of very little movement, so that its elevation necessarily carries with it the sternum. This movement increases both the transverse and antero-posterior diameters of the thorax, on account of the mode of articulation and direction of the ribs, which are somewhat rotated as well as rendered more nearly horizontal.

Intercostal Muscles. - Concerning the mechanism of the action of these muscles, there is considerable difference of opinion among physiologists ; so much, indeed, that the question is still left in some uncertainty. The most extended researches on this point are those of Beau and Maissiat (1843), and of Sibson (1846). The latter seem to settle the question of 
the mode of action of the intercostals and explain satisfactorily certain points which even now are not commonly appreciated. Onimus and, more recently, Laborde have shown by experiments on decapitated criminals that the external intercostals raise and the internal intercostals depress the ribs, confirming the views of Sibson.

In the dorsal region, the spinal column forms an arch, with its concavity looking toward the chest, and the ribs increase in length progressively, from above downward, to the deepest portion of the arch, where they are longest, and then progressively become shorter. "During inspiration the ribs approach to or recede from each other according to the part of the arch with which they articulate; the four superior ribs approach each other anteriorly and recede from each other posteriorly; the fourth and fifth ribs, and the intermediate set (sixth, seventh and eighth), move farther apart to a moderate, the diaphragmatic set (four inferior), to a great extent. The upper edge of each of these ribs glides toward the vertebræ in relation to the lower edge of the rib above, with the exception of the lowest rib, which is stationary" (Sibson). These movements increase the antero-posterior and transverse diameters of the thorax. As the ribs are elevated and become more nearly horizontal, they push forward the lower portion of the sternum. Their configuration and mode of articulation with the vertebræ are such that they can not be elevated without undergoing a considerable rotation, by which the concavity looking directly toward the lungs is increased, and with it the bilateral diameter of the chest. All the intercostal spaces posteriorly are widened in inspiration.

The ribs are elevated by the action of the external intercostals, the sternal portion of the internal intercostals and the levatores costarum. The external intercostals are situated between the ribs only and are wanting in the region of the costal cartilages. As the vertebral extremities of the ribs are the pivots on which these levers move, and as the sternal extremities are movable, the direction of the fibres of the intercostals from above downward and forward renders elevation of the ribs a necessary consequence of their contraction, if it can be assumed that the first rib is fixed or at least does not move downward. The scalene muscles elevate the first rib in ordinary inspiration; and in deep inspiration, this takes place to such an extent as palpably to carry with it the sternum and the lower ribs. Theoretically, then, the external intercostals can do nothing but render the ribs more nearly horizontal.

If the external intercostals are exposed in the dog - in which the costal type of respiration is quite prominent-close observation can hardly fail to show that these muscles enter into action with inspiration. If attention is directed to the sternal portion of the internal intercostals, 
situated between the costal cartilages, their fibres having a direction from above downward and backward, it is equally evident that they enter into action with inspiration. By artificially inflating the lungs after death, it is seen that when the lungs are filled with air, the fibres of these muscles are shortened (Sibson). In inspiration the ribs are all separated posteriorly; but laterally and anteriorly, some are separated (all below the fourth), and some are approximated (all above the fourth). Thus all the interspaces, except the anterior portion of the upper three, are widened in inspiration. Sibson has shown by inflation of the chest, that although the ribs are separated from each other, the attachments of the intercostals are approximated. The ribs, from an oblique position, are rendered nearly horizontal; and consequently the inferior attachments of the intercostals are brought nearer the spinal column, while the superior attachments to the upper borders of the ribs are slightly removed from it. Thus these muscles are shortened. If, by separating and elevating the ribs, the muscles are shortened, it follows that shortening of the muscles will necessarily elevate and separate the 'ribs. In the three superior interspaces, the constant direction of the ribs is nearly horizontal, and the course of the intercostal fibres is not so oblique as in those situated between the lower ribs. These spaces are narrowed in inspiration. The muscles between the costal cartilages have a direction opposite to that of the external intercostals and act on the ribs from the sternum, as the others act from the spinal column. The superior interspace is narrowed, and the others are widened in inspiration.

Levatores Costanum. - The action of these muscles can not be mistaken. They have immovable points of origin, the transverse processes of twelve vertebræ from the last cervical to the eleventh dorsal, and spreading out like a fan, are attached to the upper edges of the ribs between the tubercles and the angles. In inspiration they contract and assist in the elevation of the ribs.

Auxiliary Muscles of Inspiration. - The muscles which have just been considered are competent to increase the capacity of the thorax sufficiently in ordinary respiration; but there are certain muscles attached to the chest and the upper part of the spinal column or the upper extremities, which may act in inspiration, although ordinarily the chest is the fixed point and they move the head, neck or arms. These muscles are brought into action when the movements of respiration are exaggerated. When this exaggeration is but slight and is physiological, as after exercise, certain of the ordinary auxiliaries act for a time, until the tranquillity of the movements is restored; but when there is obstruction in the respiratory passages or when respiration is difficult from any 
cause, threatening suffocation, all the muscles that can by any possibility raise the chest are brought into action. These are classed in the table as extraordinary auxiliaries. Most of these muscles can voluntarily be brought into play to raise the chest, and the mechanism of their action may in this way be demonstrated.

Serratus Posticus Superior. - This muscle, by reversing its ordinary action, is capable of increasing the capacity of the thorax.

Sterno-mastoideus. - That portion of the muscle which is attached to the mastoid process of the temporal bone and the sternum, when the head is fixed, is capable of acting as a muscle of inspiration. It does not act in ordinary respiration, but its contractions may be observed whenever respiration is hurried or exaggerated.

The following muscles as a rule act as muscles of inspiration only when respiration is difficult or labored :-

Levator Anguli Scapule and Superior Portion of the Trapesius. Movements of the scapula have often been observed in labored respiration. Its elevation during inspiration is effected chiefly by the levator anguli scapulæ and the upper portion of the trapezius.

Pectoralis Minor and Inferior Portion of the Pectoralis Major. These muscles act together to raise the ribs in difficult respiration. The pectoralis minor is the more efficient. With the coracoid process as the fixed point, this muscle is capable of powerfully assisting in the elevation of the ribs. That portion of the pectoralis major which is attached to the lower part of the sternum and costal cartilages is capable of acting from its insertion into the bicipital groove of the humerus, when the shoulders are fixed, in concert with the pectoralis minor.

Serratus Magnus.-Acting from the scapula as the fixed point, this muscle is capable of assisting the pectorals in raising the ribs and becomes a powerful auxiliary in difficult inspiration.

The division into muscles of ordinary inspiration, ordinary auxiliaries and extraordinary auxiliaries, must not be taken as absolute. In the male, in ordinary respiration, the diaphragm, intercostals and levatores costarum are the principal inspiratory muscles, and the action of the scaleni, with the consequent elevation of the sternum, is commonly very slight or it may be wanting. In the female, the movements of the upper parts of the chest are more prominent, and the scaleni, the serratus posticus superior, and sometimes the sterno-mastoid, are brought into action in ordinary respiration. In the different types of respiration, the action of the muscles necessarily presents considerable variations.

Expiration. - The air is expelled from the lungs, in ordinary expiration, by a simple and comparatively passive process. The lungs contain a large number of elastic fibres surrounding the air-cells and the small- 
est ramifications of the bronchial tubes, which give them great elasticity. The thoracic walls are also very elastic, particularly in young persons. After the muscles which increase the capacity of the thorax cease their action, the elasticity of the costal cartilages and the tonicity of the muscles that have been put on the stretch restore the chest to what may be called its passive dimensions. This elasticity is likewise capable of acting as an inspiratory force when the chest has been compressed in any way. There are also certain muscles, the action of which is to draw the ribs downward and which, in tranquil respiration, are antagonistic to those which elevate the ribs. Aside from this, many operations, such as speaking, blowing, singing etc., require powerful, prolonged or complicated acts of expiration, in which many muscles are brought into play.

Expiration may be considered as depending on two causes :-

I. The passive influence of the elasticity of the lungs and thoracic walls.

2. The action of certain muscles, which either diminish the transverse and antero-posterior diameters of the chest by depressing the ribs and sternum, or the vertical diameter, by pressing the abdominal viscera upward against the diaphragm.

Infuence of the Elasticity of the Pulmonary Structure and Walls of the Chest. - It is easy to understand the influence of the elasticity of the pulmonary structure in expiration. From the collapse of the lungs when openings are made in the chest, it is seen that even after the most complete expiration, these organs have a tendency to expel part of their gaseous contents, which can not be fully satisfied until the chest is opened. They remain partially distended, on account of the impossibility of retraction of the thoracic walls beyond a certain degree; and by virtue of their elasticity, they exert a suction force on the diaphragm, causing it to form a vaulted arch, or dome, above the level of the lower circumference of the chest. When the lungs are collapsed, the diaphragm hangs loosely between the abdominal and thoracic cavities. In inspiration and in expiration, then, the relations between the lungs and diaphragm are reversed. In inspiration, the descending diaphragm exerts a suction force on the lungs, drawing them downward; in expiration, the elastic lungs exert a suction force on the diaphragm, drawing it upward. This antagonism is one of the causes of the great power and importance of the diaphragm as an inspiratory muscle.

The elasticity of the lungs operates chiefly on the diaphragm in reducing the capacity of the chest; for the walls of the thorax, by reason of their own elasticity, have a reaction which succeeds the movements produced by the inspiratory muscles. Although this is the main 
action of the lungs themselves in expiration, their relations to the walls of the thorax are important. By virtue of their elasticity, they assist the passive retraction of the chest. When they lose this property to any considerable extent, as in vesicular emphysema, they offer a notable resistance to the contraction of the thorax; so much, indeed, that in old cases of this disease the thoracic movements are restricted, and the chest presents a characteristic rounded and distended appearance.

Little more need be said concerning the passive movements of the thoracic walls. When the action of the inspiratory muscle ceases, the ribs regain their oblique direction, the intercostal spaces are narrowed, and the sternum, if it has been elevated and drawn forward, falls back to its place, simply by virtue of the elasticity of the parts.

Action of Muscles in Expiration. - The following are the principal muscles concerned in expiration :-

\section{MUSCLES OF EXPIRATION}

MUSCLE

Ordinary K'espiration

ATTACHMENTS

Osseous portion of internal intercostals . . Inner borders of the ribs.

Infracostales . . . . . . . . Inner surfaces of the ribs.

Triangularis sterni . . . . . . . Ensiform cartilage, lower borders of sternum, lower three or four costal cartilages - cartilages of the second, third, fourth and fifth ribs.

\section{Auxiliaries}

Obliquus externus . . . . . . External surface and inferior borders of eight inferior ribs-anterior half of the crest of the ileum, Poupart's ligament, linea alba.

Obliquus internus . . . . . . Outer half of Poupart's ligament, anterior twothirds of the crest of the ileum, lumbar fascia - cartilages of four inferior ribs, linea alba, crest of the pubis, pectineal line.

Transversalis . . . . . . . Outer third of Poupart's ligament, anterior twothirds of the crest of the ileum, lumbar vertebre, inner surface of cartilages of six inferior ribs - crest of the pubis, pectineal line, linea alba.

Sacro-lumbalis . . . . . . . Sacrum - angles of six inferior ribs.

Internal Intercostals. - The internal intercostals have different uses in different parts of the thorax. They are attached to the inner borders of the ribs and costal cartilages. Between the ribs they are covered by the external intercostals, but between the costal cartilages they are covered simply by aponeurosis. Their direction is from above downward and backward, nearly at right angles to the external intercostals. The action of that portion of the internal intercostals situated between the costal cartilages has already been noted. They assist the external 
intercostals in elevating the ribs in inspiration. Between the ribs these muscles are directly antagonistic to the external intercostals. They are more nearly at right angles to the ribs, particularly in that portion of the thorax where the obliquity of the ribs is greatest. They are elongated when the chest is distended, and are shortened when the chest is retracted. This fact, taken in connection with experiments on living animals, shows that they are muscles of expiration. Their contraction tends to depress the ribs and consequently to diminish the capacity of the chest.

Infracostales. - These muscles, situated at the posterior part of the thorax, are variable in size and number. They are most common at the lower part of the chest. Their fibres arise from the inner surface of one rib to be inserted into the inner surface of the first, second or third rib below. The fibres follow the direction of the internal intercostals, and acting from their lower attachments, their contractions assist these muscles in drawing the ribs downward.

Triangularis Sterni. - There has never been any doubt concerning the expiratory action of the triangularis sterni. From its origin, the ensiform cartilage, lower borders of the sternum, and lower three or four costal cartilages, it acts on the cartilages of the second, third, fourth and fifth ribs, to which it is attached, drawing them downward and thus diminishing the capacity of the chest.

The above-mentioned muscles are called into action in ordinary tranquil respiration, and their sole office is to diminish the capacity of the chest. In labored or difficult expiration, and in the acts of blowing, phonation etc., other muscles, called auxiliaries, play a more or less important part. These muscles all enter into the formation of the walls of the abdomen, and their general action in expiration is to press the abdominal viscera and diaphragm into the thorax and diminish its vertical diameter. Their action is voluntary; and by an effort of the will, it may be opposed more or less by the diaphragm, by which means the duration or extent of the expiratory act is regulated. They are also attached to the ribs or costal cartilages, and while they press the diaphragm upward, they depress the ribs and thus diminish the anteroposterior and transverse diameters of the chest. In this action, they may be opposed by voluntary contraction of the muscles that raise the ribs, also for the purpose of regulating the force of the expiratory act.

In labored respiration in disease and in the hurried respiration which follows violent exercise, the auxiliary muscles of expiration, as well as of inspiration, are called into action to a considerable extent.

Obliquus Extemus. - This muscle, in connection with the obliquus internus and transversalis, is efficient in forced or labored expiration, by 
pressing the abdominal viscera against the diaphragm. Acting from its attachments to the linea alba, the crest of the ileum and Poupart's ligament, by its attachment to the eight inferior ribs, it draws the ribs downward.

Obliquns Intcrnus. - This muscle also acts in forced expiration by compressing the abdominal viscera. The direction of its fibres is from below upward and forward. Acting from its attachments to the crest of the ileum, Poupart's ligament and the lumbar fascia, by its attachments to the cartilages of the four inferior ribs, it draws them downward. The direction of the fibres of this muscle is the same as that of the internal intercostals. By its action the ribs are drawn inward as well as downward.

Transversalis. - The expiratory action of this muscle is exerted mainly in compressing the abdominal viscera.

Sacro-lumbalis. - This muscle is situated at the posterior portion of the abdomen and thorax. Its fibres pass from its origin at the sacrum, upward and a little outward, to be inserted into the six inferior ribs at their angles. In expiration it draws the ribs downward, acting as an antagonist to the lower levatores costarum.

There are other muscles that may be used in forced expiration, assisting in the depression of the ribs, such as the serratus posticus inferior, the superior fibres of the serratus magnus and the inferior portion of the trapezius; but their action in respiration is unimportant.

Types of Respiration. - In the movements of expansion of the chest, although all the muscles that have been classed as ordinary inspiratory muscles are brought into action to a greater or less extent, the fact that certain sets may act in a more marked manner than others has led physiologists to recognize different types of respiration. Three types usually are given in works on physiology :-

I. The Abdominal Type. - In this, the action of the diaphragm, and the consequent movements of the abdomen are most prominent.

2. The Inferior Costal Type. - In this, the action of the muscles that expand the lower part of the thorax, from the seventh rib inclusive, is most prominent.

3. The Superior Costal Type. - In this, the action of the muscles that dilate the thorax above the seventh rib and elevate the entire chest is most prominent.

The abdominal type is most marked in children less than three years of age, irrespective of sex, respiration being carried on almost exclusively by the diaphragm.

At a variable period after birth, a difference in the types of respiration in the sexes is observed. In the male the abdominal conjoined 
with the inferior costal type is predominant, and this continues through life. In the female the inferior costal type is insignificant and the superior costal type predominates. The cause of the pronounced movements of the upper part of the chest in the female has been the subject of much discussion. It is probably due, in a great measure, to the mode of dress now so common in civilized countries, which confines the lower part of the chest and renders movements of expansion somewhat difficult. In a series of observations by Dr. Thomas J. Mays (I887), on eighty-two Indian girls at the Lincoln Institution in Philadelphia, between ten and twenty years of age, who had never worn tight clothing, the abdominal type of respiration was found to predominate, the respiratory tracings hardly differing from the tracings in the male. These observations seem to show, in opposition to the views of Hutchinson and others, that the predominance of the superior costal type in the female is confined to civilized races; but it is certain that females accommodate themselves more readily than the male to the superior costal type; and this probably is a provision for the physiological enlargement of the uterus in pregnancy, which nearly arrests respiratory movements except those of the upper part of the chest.

Frequency of the Respiratory Movements. - In counting the respiratory acts, it is desirable that the subject be unconscious of the observation, otherwise their normal rhythm is likely to be disturbed. Of all who have written on this subject, Hutchinson has presented the largest and most reliable collection of facts. This observer ascertained the number of respiratory acts per minute, in the sitting posture, in 1897 males. The results of his observations as to frequency are given in the following table:

\begin{tabular}{|c|c|c|c|c|c|c|c|c|c|c|c|c|c|c|c|c|c|c|c|c|}
\hline $\begin{array}{l}\text { RESPIRATIONS } \\
\text { MINUTE }\end{array}$ & & & & & & & & & $\begin{array}{c}\text { NUMBER OF } \\
\text { CASES }\end{array}$ & $\begin{array}{l}\text { RESPIRATIONS } \\
\text { MINUTE }\end{array}$ & & & & & & & & & & $\begin{array}{l}\text { AUMBER oF } \\
\text { CASES }\end{array}$ \\
\hline 9 to 16. & . & & . & • & . & • & . & . & 79 & $2 I$. & . & • & . & . & . & . & . & - & . & . 129 \\
\hline 16. & . & • & . & • & . & • & . & . & . 239 & 22 . & . & . & . & . & . & . & . & - & . & . 143 \\
\hline I7. & . & . & . & . & . & . & . & - & . 105 & 23 . & . & . & . & . & . & - & . & $\cdot$ & . & 42 \\
\hline IS. & . & . & . & . & . & • & . & - & . $\quad 195$ & 24 . & . & . & . & . & - & . & $\cdot$ & - & - & 243 \\
\hline I9. & - & • & - & • & & • & .. & . & 74 & 24 to 40 & - & · & - & · & - & - & - & $\cdot$ & - & \\
\hline 20 & . & . & . & • & & • & . & - & . $5^{6 \mathrm{I}}$ & & & & & & & & & & & \\
\hline
\end{tabular}

Although this table shows considerable variations in different individuals, the great majority (I73I) breathed sixteen to twenty-four times per minute. Nearly a third breathed twenty times per minute, a number that may be taken as the average.

The relations of the respiratory acts to the pulse are quite constant in health. It has been shown by Hutchinson that the proportion in the great majority of instances is one respiratory act to four pulsations of the 
heart. The same proportion usually obtains when the pulse is accelerated in disease, except when the pulmonary organs are involved.

Age has an influence on the frequency of the respiratory acts, corresponding with what has already been noted in regard to the pulsations of the heart.

The following are the results of observations on three hundred males (Quetelet):-

Forty-four respirations per minute, soon after birth ;

Twenty-six, at the age of five years;

Twenty, between fifteen and twenty years;

Nineteen, between twenty and twenty-five years;

Sixteen, about the thirtieth year;

Eighteen, between thirty and fifty years.

The influence of sex is not marked in very young children. There is no difference between males and females at birth; but in young women, the respirations are a little less frequent than in young men of the same age.

The various physiological conditions which have been noted as affecting the pulse have a corresponding influence on respiration. In sleep the number of respiratory acts is diminished by about twenty per cent (Quetelet). Muscular effort accelerates the respiratory movements pari passu with the movements of the heart.

Relations of Inspiration and Expiration to each other. - Respiratory Sounds. - In ordinary respiration, inspiration is produced by the action of muscles, and expiration, by the passive reaction of the lungs and of the elastic walls of the thorax. The inspiratory and expiratory acts do not follow each other immediately. Beginning with inspiration, it is found that this act maintains about the same intensity throughout. There is then a very brief interval, when expiration follows, which has its maximum of intensity at the beginning of the act and gradually dies away. Between the acts of expiration and inspiration is an interval somewhat longer than the interval between inspiration and expiration.

The duration of expiration usually is a little longer than that of inspiration, although the two acts may be nearly, or in some instances, quite equal. After five to eight ordinary respiratory acts, an effort commonly is made which is rather more profound than usual, by which the air in the lungs is more thoroughly changed. Temporary arrest of the acts of respiration in violent muscular efforts, in straining, in parturition etc., is sufficiently familiar.

Ordinarily respiration is not accompanied with any sound that can be heard without applying the ear directly, or by the intervention of a stethoscope, to the chest, except when the mouth is closed and breathing is 
carried on exclusively through the nasal passages, when a soft breezy sound accompanies both acts. If the mouth is opened sufficiently to admit the free passage of air, no sound is to be heard in health. In sleep the respirations are more profound; and if the mouth is closed the sound is rather more intense.

Snoring, which sometimes accompanies the respiratory acts during sleep, occurs when the air passes through both the mouth and the nose. It is more marked in inspiration, sometimes accompanying both acts, and sometimes it is not heard in expiration. It is not necessary to describe the character of a sound so familiar. Snoring is an idiosyncrasy in many individuals, although those who do not snore habitually may do so when the system is unusually exhausted and relaxed. It occurs when the mouth is open, and the sound is produced by vibration and a sort of flapping of the velum pendulum palati, between the two currents of air from the mouth and nose, together with a vibration in the column of air itself.

Applying the stethoscope over the larynx or trachea, a sound is heard, of a distinctly and purely tubular character, accompanying both acts of respiration. In inspiration, according to the late Dr. Austin Flint, "it attains its maximum of intensity quickly after the development of the sound and maintains the same intensity to the close of the act, when the sound abruptly ends, as if suddenly cut off." After a brief interval, the sound of expiration follows. This also is tubular in quality. It soon attains its maximum of intensity, but unlike the sound of inspiration, it gradually dies away and is lost imperceptibly. It is seen that these phenomena correspond with the nature of the two acts of respiration. Sounds approximating in character the foregoing are heard over the bronchial tubes before they penetrate the lungs.

Over the lungs, a sound may be heard entirely different in its character from that heard over the larynx, trachea or bronchial tubes. In inspiration the sound is much less intense than over the trachea and has a breezy, expansive, or what is called in auscultation, a vesicular character. It is much lower in pitch than the tracheal sound. It is continuous and rather increases in intensity from its beginning to its termination, ending abruptly, like the tracheal inspiratory sound. The sound is produced in part by the movement of air in the small bronchial tubes, but chiefly by the expansion of the air-cells of the lungs. It is followed, without an interval, by the sound of expiration, which is shorter - one-fifth or one-fourth as long - lower in pitch and much less intense. A sound is not always heard in expiration.

Variations in the intensity of the respiratory sounds in different individuals are considerable. As a rule they are more intense in young per- 
sons; which has given rise to the term "puerile respiration," when the sounds are exaggerated in parts of the lung in certain cases of disease. The sounds usually are more intense in females than in males, particularly in the upper regions of the thorax.

It is difficult by any description or comparison to convey an adequate idea of the character of the sounds heard over the lungs and air-passages, and it is unnecessary to make the attempt, when they can be so easily studied in the living subject.

Coughing, Sneczing, Sighing, Yauning, Laughing, Sobbing and Hiccongh. - These peculiar acts demand a few words of explanation. Coughing and sneezing usually are involuntary acts, produced by irritation in the air-tubes or nasal passages, although coughing often is voluntary. In both these acts, there is first a deep inspiration followed with a convulsive action of the expiratory muscles, by which the air is violently expelled with a characteristic sound, in the one case by the mouth, and in the other by the mouth and nares. Foreign bodies lodged.in the airpassages frequently are expelled in violent fits of coughing. In hypersecretion of the bronchial mucous membrane, the accumulated mucus is carried by the act of coughing either to the mouth or well into the larynx, when it may be expelled by the act of exspuition. When either of these acts is the result of irritation from a foreign substance or from secretions, it may be modified or partly smothered by the will, but is not completely under control. The sensibility of the mucous membrane at the summit of the air-passages usually protects them from the entrance of foreign matters, both liquid and solid; for the slightest impression received by the membrane gives rise to a violent and involuntary cough, by which the offending substance may be removed. The glottis, also, is spasmodically contracted.

In sighing, a prolonged and deep inspiration is followed with a rapid and usually an audible expiration. This occurs, as a rule, once in five to eight respiratory acts, for the purpose of changing the air in the lungs more completely, and it is due to an exaggeration of the cause that gives rise to the ordinary acts of respiration. When due to depressing emotions, it has the same cause; for at such times respiration is less efficiently performed. Yawning is an analogous process, but it differs from sighing in the fact that it is involuntary and can not be produced by an effort of the will. It is characterized by a wide opening of the mouth and a profound inspiration. Yawning commonly is assumed to be an evidence of fatigue, but it often occurs from a sort of contagion. When not the result of imitation, it has the same exciting cause as sighing - deficient oxygenation of the blood - and it is followed with a sense of satisfaction, which shows that it meets some decided want on the part of the system. 
Laughing and sobbing, although expressing opposite conditions, are produced by nearly the same action. The characteristic sounds accompanying these acts are the result of short, rapid and convulsive movements of the diaphragm, attended with contractions of the muscles of the face, which produce the expressions characteristic of hilarity or grief. Although to a certain extent under the control of the will, these acts are mainly involuntary. Violent and convulsive laughter may be excited in many individuals by titillation of certain portions of the surface of the body. Laughter and sometimes sobbing, like yawning, may be the result of involuntary imitation.

Hiccough is a peculiar modification of the act of inspiration, to which it is exclusively confined. It is produced by a sudden, convulsive and entirely involuntary contraction of the diaphragm, accompanied with spasmodic constriction of the glottis. The contraction of the diaphragm is more extensive than in laughing and sobbing and occurs only once in every four or five respiratory acts.

Capacity of the Lungs, and the Quantity of Air changed in the Respiratory Acts

The volume of air ordinarily contained in the lungs is about two hundred cubic inches ( 3277 cubic centimeters); but it is evident, from the simple experiment of opening the chest, when the elastic lungs collapse and expel a certain quantity of air which can not be removed while the lungs are in situ, that a part of the gaseous contents of these organs necessarily remains after the most forcible expiration. After an ordinary act there is a certain quantity of air in the lungs, which can be expelled by a forced expiration. In ordinary respiration a comparatively small volume of air enters with inspiration, and a nearly equal quantity is expelled by the succeeding expiration. By the extreme action of all the inspiratory muscles in a forced inspiration, a supplemental quantity of air may be taken into the lungs, which then contain much more than they ever do in ordinary respiration. For convenience of description, physiologists have adopted the following names, which are applied to these various volumes of air :-

I. Residual Air; that which is not and can not be expelled by a forced expiration.

2. Reserve Air; that which remains after an ordinary expiration, deducting the residual air.

3. Tidal, or Ordinary Breathing Air; that which is changed in the ordinary acts of inspiration and expiration. 
4. Complemental Air; the excess over the ordinary breathing air, which may be introduced by a forcible inspiration.

In measuring the air changed in ordinary breathing, it has been found that the acts of respiration are so easily influenced and it is so difficult to experiment on any individual without his knowledge, that the results of many good observers are not entirely reliable. This is one of the most important of the questions under consideration. The difficulties in the way of estimating with accuracy the residual, reserve or complemental volumes, will readily suggest themselves. The observations on these points which may be taken as the most definite and exact are those of Herbst and of Hutchinson. Those of the last-named observer are very elaborate and were made on a large number of subjects of both sexes and of all ages and occupations. They are commonly accepted by physiologists as the most extended and accurate.

Residual Air. - Perhaps there is not one of the questions under consideration more difficult to answer definitely than that of the quantity of air remaining in the lungs after a forced expiration; but it fortunately is not one of great practical importance. The residual air remains in the lungs as a physical necessity. The lungs in health are always in contact with the walls of the thorax; and when the chest is reduced to its smallest dimensions, it is impossible that more air should be expelled. The volume which thus remains has been variously estimated. The residual volume has been put at about one hundred cubic inches ( 639 cubic centimeters), but the quantity varies very considerably in different individuals (Hutchinson). Taking everything into consideration, it may be assumed that this estimate is as nearly correct as any.

Reserve Air. - This name is given to the volume of air that may be expelled and changed by a voluntary effort, but which remains in the lungs, added to the residual air, after an ordinary act of expiration. It may be estimated, without reference to the residual air, by forcibly expelling air from the lungs after an ordinary expiration. The average volume, according to Hutchinson, is one hundred cubic inches (I639 cubic centimeters).

More or less of the reserve air is changed whenever there is a necessity for more complete renovation of the contents of the lungs than ordinary. It is encroached upon in the unusually profound inspiration and expiration which occur once in every five to eight acts. It is used in certain prolonged vocal efforts, in blowing etc. Added to the residual air, it constitutes the minimum capacity of the lungs in ordinary respiration. As it is continually receiving watery vapor and carbon dioxide, it is always more or less vitiated, and when reënforced by the breathing air, which enters with inspiration, is continually in circulation, in obedience 
to the law of the diffusion of gases. Those who are in the habit of arresting respiration for a time, learn to change the reserve air as completely as possible by several forcible acts and then fill the lungs with fresh air. In this way they are enabled to suspend the respiratory acts for two or three minutes without much inconvenience. The introduction of fresh air with each inspiration and the constant diffusion which is going on and by which the proper quantity of oxygen finds its way to the air-cells give, in ordinary breathing, a composition to the air in the deepest portions of the lungs, which insures constant aëration of the blood.

Tidal, or Ordinary Breathing Air. - The volume of air changed in the ordinary acts of respiration is subject to certain physiological variations; and the respiratory movements, as regards their extent, are so easily influenced, that care is necessary to avoid error in estimating the volume of ordinary breathing air. As a mean of the results obtained by Herbst and by Hutchinson, the average volume of breathing air, in a man of ordinary stature, is twenty cubic inches ( 327.7 cubic centimeters). According to Hutchinson, in perfect repose, when the respiratory movements are hardly perceptible, not more than seven to twelve cubic inches ( I 4.7 to I96.6 cubic centimeters) are changed; while, under excitement, the volume may be increased to seventy-seven cubic inches (I 26I.8 cubic centimeters). The breathing volume progressively increases in proportion to the stature of the individual and bears no definite relation to the apparent capacity of the chest (Herbst).

Complemental Air. - The thorax may be so enlarged by an extreme inspiratory effort as to contain a quantity of air much larger than after an ordinary inspiration. The additional volume of air thus taken in may be estimated by measuring all the air that can be expelled from the lungs after the most profound inspiration, and deducting the sum of the reserve air and breathing air. This quantity has been found by Hutchinson to vary in different individuals, bearing a close relation to stature. The mean complemental volume is one hundred and ten cubic inches (I802.9 cubic centimeters).

The complemental air is drawn upon whenever an effort is made which requires a temporary arrest of respiration. Brief and violent muscular exertion usually is preceded by a profound inspiration. In sleep, as the volume of breathing air is somewhat increased, the complemental air is encroached upon. A part or all of the complemental air also is used in certain vocal efforts, in blowing, in yawning, in the deep inspiration which precedes sneezing, in straining etc.

Extreme Breathing Capacity. - By the extreme breathing capacity is meant the volume of air that can be expelled from the lungs by the 
most forcible expiration after the most profound inspiration. This has been called by Hutchinson, the vital capacity, as signifying " the volume of air which can be displaced by living movements." Its volume is equal to the sum of the reserve air, the breathing air and the complemental air, and it represents the extreme capacity of the chest, less the residual air. Its physiological importance is due to the fact that it can be determined by an appropriate apparatus, the spirometer, and comparisons may thus be made between different individuals, both healthy and diseased. The number of observations on this point made by Hutchinson amounts in all to a little less than five thousand.

The extreme breathing capacity in health is subject to variations that bear a close relation to the stature of the individual. Hutchinson begins with the proposition that in a man of medium height (five feet eight inches, or 170.2 centimeters), it is equal to two hundred and thirty cubic inches ( 3768.6 cubic centimeters).

The most striking result of the experiments of Hutchinson, in regard to the modifications of the vital capacity, is that it bears a definite relation to stature, without being much affected by weight or by the circumference of the chest. This is especially remarkable, as it is well known that height does not depend so much on the length of the body as on the length of the lower extremities. He ascertained that for every inch ( 2.5 centimeters) in height, between five and six feet (I 52.4 and 182.9 centimeters), the extreme breathing capacity is increased by eight cubic inches (I 3 I.I cubic centimeters).

Age has an influence, though less marked than stature, on the extreme breathing capacity. As the result of 4800 observations on males, it was shown that the volume increased with age up to the thirtieth year, and progressively decreased, with tolerable regularity, from the thirtieth to the sixtieth year. The figures given above, although subject to certain individual variations, may be taken as a basis for examinations of the extreme breathing capacity in disease.

Relations in Volume of the Expired to the Inspired Air. - A certain proportion of the inspired air is lost in respiration, so that the air expired is always a little less in volume than that taken into the lungs. The loss was put by Davy at one-seventieth, and by Cuvier at one-fiftieth of the volume of air introduced. Observations on this point, to be exact, must include a considerable number of respiratory acts; and from the difficulty in continuing respiration in a regular and normal manner when attention is directed to the respiratory movements, the most accurate results may probably be obtained from experiments on the lower animals. Despretz caused six young rabbits to respire for two hours in a confined space containing 2990 cubic inches $(49,000$ 
cubic centimeters) of air, and ascertained that the volume had diminished by sixty-one cubic inches (I000 cubic centimeters), or a little more than one-fiftieth. Adopting the approximations of Davy and Cuvier, applied to the human subject, as nearly correct, it may be assumed that in the lungs one-seventieth to one-fiftieth of the inspired air is lost.

Diffusion of Air in the Lungs. - When it is remembered that with each inspiration, but about twenty cubic inches ( 327.7 cubic centimeters) of fresh air are introduced, sufficient only to fill the trachea and larger bronchial tubes, it is evident that some forces must act by which this fresh air finds its way into the air-cells, and the vitiated air is brought into the larger tubes, to be expelled with the succeeding expiration.

The interchange between the fresh air in the upper portions of the respiratory apparatus and the air in the deeper parts of the lungs is constantly going on by diffusion aided by the active currents or impulses produced by the alternate movements of the chest. In the respiratory apparatus, at the end of an inspiration, the atmospheric air, composed of a mixture of oxygen and nitrogen, is introduced into the tubes with a considerable impetus and is brought into contact with the gas in the lungs, which is heavier, as it contains a certain quantity of carbon dioxide. Diffusion then takes place, aided by the elastic lungs, which are gradually forcing the gaseous contents out of the cells, until a certain portion of the air loaded with carbon dioxide finds its way to the larger tubes, to be thrown off in expiration, its place being supplied with fresh air.

In accordance with the law that the diffusibility of gases is in inverse ratio to the square root of their densities, the penetration of atmospheric air, which is the lighter, to the deep portions of the lungs would take place with greater rapidity than the ascent of air charged with carbon dioxide; so that eighty-one parts of carbon dioxide should be replaced with ninety-five parts of oxygen. It is found, indeed, that the volume of carbon dioxide exhaled is always less than the volume of oxygen absorbed. This diffusion is constantly going on, so that the air in the pulmonary alveoli, where the interchange of gases with the blood takes place, maintains a nearly uniform composition. The process of aëration of the blood, therefore, has little of that intermittent character which attends the muscular movements of respiration. 


\title{
CHAPTER V
}

\author{
CHANGES WHICH THE AIR AND THE BLOOD UNDERGO IN \\ RESPIRATION
}

Composition of the air - Consumption of oxygen - Exhalation of carbon dioxide - Influence of age-Influence of sex-Influence of digestion - Influence of diet - Influence of muscular activity - Influence of moisture and temperature - Influence of the season of the year - Relations between the oxygen consumed and the carbon dioxide exhaled Sources of carbon dioxide in the expired air - Respiratory quotient-Exhalation of watery vapor - Exhalation of ammonia, organic matter etc. - Exhalation of nitrogen Changes of the blood in respiration - Analysis of the blood for gases - Nitrogen of the blood - Oxygen of the blood - Carbon dioxide of the blood - Respiration by the tissues - Respiratory efforts before birth-Asphyxia.

\section{Changes of the Air in Respiration}

Composition of the Air. - Pure atmospheric air is a mixture of 79.05 parts of nitrogen with 20.95 parts of oxygen. Dewar and others, however, have discovered in the air a small quantity of hydrogen, with new elements called argon, metargon, neon, xenon and helium, all to be included in the proportion assigned to nitrogen. The air usually contains in addition about one part in two thousand of carbon dioxide. The air is never free from moisture, which is variable in quantity, being usually more abundant at a high than at a low temperature. Floating in the atmosphere are large numbers of minute organic bodies, and various odorous and other gaseous matters are accidental constituents.

It is necessary to the regular performance of respiration that the air should contain about four parts of nitrogen to one of oxygen and should have about the density that exists on the surface of the earth. When the density is much increased, as in mines, respiration is more or less disturbed. Under great pressure, such as exists in caissons, a quantity of nitrogen - which is sparingly soluble under ordinary conditions may be forced into the blood. Return to the outer air should be gradual; for if the excessive pressure is suddenly removed, bubbles of nitrogen may be rapidly disengaged, often with a fatal result. Exposure to a rarefied atmosphere, as in the ascent of high mountains or in aërial voyages, may seriously disturb respiration, from the fact that less oxygen than usual is presented to the respiratory surface and the reduced atmospheric pressure diminishes the capacity of the blood for retaining 
gases. Allen and Pepys confined animals for twenty-four hours in an atmosphere of pure oxygen without any notable results; but these experiments do not show that it would be possible to respire unmixed oxygen indefinitely without inconvenience. The only gas beside oxygen, that has the power of maintaining respiration, even for a time, is nitrogen monoxide. This is appropriated by the blood-corpuscles with great avidity; and for a time it produces excitement with delirium etc., which has given it the common name of the laughing gas; but this condition is followed by anesthesia, and finally by asphyxia, probably because the gas has so strong an affinity for the blood-corpuscles as to remain to a certain extent fixed, interfering with the interchange of gases that is essential to life. Notwithstanding this, experimenters have confined rabbits and other animals in an atmosphere of nitrogen monoxide for a number of hours without fatal results. In all cases they became asphyxiated, but in some instances they were restored on being brought again into the ordinary atmosphere.

Other gases that may be introduced into the lungs either produce asphyxia, negatively, from the fact that they are incapable of carrying on respiration, like hydrogen or nitrogen, or positively, by a poisonous effect on the system. The most important of the gases that act as poisons are carbon monoxide, hydrogen monosulphide and arsenious hydride. Carbon monoxide unites with the red corpuscles, forming carbon-monoxide-hemoglobin. This union is so stable that it paralyzes the corpuscles as oxygen-carriers and produces death by asphyxia. It is probable that carbon dioxide is not in itself poisonous. Regnault and Reiset exposed animals (dogs and rabbits) for many hours, to an atmosphere containing twenty-three per cent of carbon dioxide, artificially introduced, with between thirty and forty per cent of oxygen, without any ill effects.

Consumption of Oxygen. - The determination of the quantity of oxygen removed from the air in respiration is important; and on this point, there is an accumulated mass of observations that are comparatively unimportant from the fact that they were made before the methods of analysis of gases were as accurate as now. In the observations of Regnault and Reiset, animals were placed in a receiver filled with air, a measured quantity of oxygen was introduced as fast as it was consumed in respiration, and the carbon dioxide was constantly removed and carefully estimated. In most of the experiments, the confinement did not appear to interfere with the functions of the animal, which ate and drank in the apparatus and was in as good condition at the end as at the beginning of the observation. This method is more accurate than that of simply causing an animal to breathe in a confined space, 
when the consumption of oxygen and accumulation of carbon dioxide and other matters interfere more or less with the respiratory function. As employed by Regnault and Reiset, it is adapted only to experiments on animals of small size. These give an approximate idea, however, of the processes as they take place in the human subject. Pettenkofer constructed a chamber large enough to admit a man and allow freedom of motion, eating, sleeping etc., into which air could be introduced in definite quantity, and from which the products of respiration were removed and estimated. This method had been adapted to the human subject on a small scale in 1843 , by Scharling; but there was no arrangement for estimating the quantity of oxygen consumed.

Estimates of the quantities of oxygen consumed or of carbon dioxide exhaled, based on analyses of the inspired and expired air, calculations from the average quantity of air changed with each respiratory act and the average number of respirations per minute, are not so reliable as analyses showing the actual changes in the air, like those of Regnault and Reiset. Where there is so much multiplication and calculation, a slight inaccuracy in the estimates of the quantities consumed or produced in a single respiration will make a large error in the estimate for a day or even for an hour. Bearing in mind these sources of error, from the experiments of Valentin and Brunner, Dumas, Regnault and Reiset and others, a sufficiently accurate approximation of the proportion of oxygen consumed by the human subject may be made. The air, which contains, when inspired, about twenty-one per cent of oxygen, is found on expiration to contain but about sixteen per cent. In other words, the volume of oxygen absorbed in the lungs is about five per cent of the volume of air inspired.

The quantity of oxygen consumed in respiration is subject to great variations, depending on temperature, the condition of the digestive system, muscular activity etc. The following conclusions, the results of the observations of Lavoisier and Séguin, give at a glance the variations from the above-mentioned causes:-

"I. A man, in repose and fasting, with an external temperature of about $90^{\circ}$ Fahr. $\left(32.5^{\circ}\right.$ C. $)$, consumes 1465 cubic inches (24 liters) of oxygen per hour.

" 2 . The same man, in repose and fasting, with an external temperature of $59^{\circ} \mathrm{Fahr}$. ( $15^{\circ} \mathrm{C}$.), consumes 1627 cubic inches (26.66 liters) of oxygen per hour.

" 3 . The same man, during digestion, consumes 2300 cubic inches (37.69 liters) of oxygen per hour.

"4. The same man, fasting, accomplishing the labor necessary to raise, in fifteen minutes, a weight of about 16 pounds 3 ounces $(7.343$ 
kilograms) to the height of 656 feet (200 meters), consumes 3874 cubic inches (63.48 liters) of oxygen per hour.

" 5. The same man, during digestion, accomplishing the labor necessary to raise, in fifteen minutes, a weight of about 16 pounds 3 ounces (7.343 kilograms) to the height of 692 feet (2 I r.I46 meters), consumes 5568 cubic inches (91.24 liters) of oxygen per hour."

Immediately after birth the consumption of oxygen in warm-blooded animals is relatively very slight. It has been shown that just after birth, dogs and other animals will live for half an hour or longer under water; and cases are on record in which life has been restored in newborn children after seven, and, it has been stated, after twenty-three, hours of asphyxia (Milne-Edwards). During the first periods of extra-uterine life, the condition of the newly born is nearly that of a cold-blooded animal. The lungs are relatively small, and it is some time before they fully assume their office. There is, also, very little power of resistance to a low temperature. Although accurate researches regarding the comparative quantities of oxygen in the venous and arterial blood of the fœtus are wanting, it has frequently been observed that the difference in color is not so marked as it is after pulmonary respiration has become established. The direct researches of W. F. Edwards have shown that the absolute consumption of oxygen by very young animals is quite small; and the observations of Legallois, on rabbits, made every five days during the first month of life, show a rapidly increasing demand for oxygen.

The consumption of oxygen is greater in lean than in fat animals, provided they are in perfect health. The consumption is greater, also, in carnivorous than in herbivorous animals; and in animals of different sizes, it is relatively greater in those which are very small. In small birds, such as the sparrow, the relative quantity of oxygen absorbed was ten times greater than in the fowl.

During sleep the quantity of oxygen consumed is considerably diminished; and in hibernation it is so small, that Spallanzani could not detect any difference in the composition of the air in which a marmot in a state of torpor had remained for three hours. In experiments on a marmot in hibernation, Regnault and Reiset observed a reduction in the oxygen consumed to about one-thirtieth of the ordinary quantity.

It has been shown by experiments that the consumption of oxygen bears a nearly constant ratio to the production of carbon dioxide; and as observations on the influence of sex, the number of respiratory acts etc., on the activity of the respiratory processes have been made chiefly with reference to the carbon dioxide exhaled, these influences will be considered in connection with the products of respiration.

Experiments on the effect of increasing the proportion of oxygen in 
the air have led to various results at the hands of different observers. Regnault and Reiset did not discover any increase in the consumption of oxygen when this gas was largely in excess in the atmosphere.

The results of confining an animal in an atmosphere composed of twenty-one parts of oxygen and seventy-nine parts of hydrogen are remarkable. When hydrogen is substituted for nitrogen, the consumption of oxygen is largely increased. This has been attributed to the greater refrigerating power of the hydrogen; but a more rational explanation is in its greater diffusibility. Hydrogen is the most diffusible of gases; and when introduced into the lungs in place of nitrogen, the vitiated air, charged with carbon dioxide, is undoubtedly more readily removed from the deep portions of the lungs, giving place to the mixture of hydrogen and oxygen. It is probably for this reason that the quantity of oxygen consumed is increased. It is probable that the nitrogen of the air plays an important part in the phenomena of respiration, by virtue of its degree of diffusibility.

In view of the great variations in the consumption of oxygen, dependent on different physiological conditions, such as digestion, exercise, temperature etc., it is impossible to fix on any number that will represent, even approximately, the average quantity consumed per hour. The estimate arrived at by Longet, from a comparison of the results obtained by different reliable observers, is perhaps as near the truth as is possible. This estimate puts the hourly consumption at 1220 to 1525 cubic inches ( 20 to 25 liters), "in an adult male, during repose and under normal conditions of health and temperature."

In passing through the lungs, the air, in addition to losing a certain proportion of its oxygen, undergoes the following changes:-

I. Elevation in temperature.

2. Gain of carbon dioxide.

3. Gain of watery vapor.

4. Gain of ammonia.

5. Gain of a small quantity of organic matter.

6. Gain, and occasionally loss, of nitrogen.

The elevation in temperature of the air that has passed through the lungs has been studied by Gréhant. He found that with an external temperature of $72^{\circ} \mathrm{Fahr} .\left(22.22^{\circ} \mathrm{C}\right.$.), respiring seventeen times per minute, the air taken in by the nares and expired by the mouth through an apparatus containing a thermometer carefully protected from external influences, marked a temperature of $95.4^{\circ} \mathrm{Fahr} .\left(35.22^{\circ} \mathrm{C}\right.$. $)$. Taking in the air by the mouth, the temperature of the expired air was $93^{\circ} \mathrm{Fahr}$. $\left(33.89^{\circ} \mathrm{C}.\right)$. At the beginning of the expiration, Gréhant noted a temperature of $94^{\circ}$ Fahr. $\left(34.44^{\circ} \mathrm{C}\right.$.). After a prolonged expiration, the tem- 
perature was $96^{\circ} \mathrm{Fahr} .\left(35.55^{\circ} \mathrm{C}\right.$.). In these observations the temperature taken beneath the tongue was $98^{\circ} \mathrm{Fahr} .\left(36.67^{\circ} \mathrm{C}\right.$.).

Exhalation of Carbon Dioxide. - On account of variations in the quantities of carbon dioxide exhaled at different times of the day, and particularly the influence of the rapidity of the respiratory movements, it is difficult to fix on any number that will represent the average proportion of this gas contained in the expired air. The same influences have been found affecting the consumption of oxygen, and the same difficulties are experienced in forming an estimate of the proportion of this gas consumed. As it may be assumed, after a comparison of the results obtained by different observers, that the volume of oxygen consumed is about five per cent of the entire volume of air, it may be stated, as an approximation, that in the intervals of digestion, in repose and under normal conditions as regards the frequency of the pulse and respiration, the volume of carbon dioxide exhaled is about four per cent of the volume of the expired air. As the volume of oxygen that enters into the composition of a definite quantity of carbon dioxide is equal to the volume of the carbon dioxide, it is seen that a certain quantity of oxygen disappears in respiration and is not represented in the carbon dioxide exhaled.

There are great differences in the proportion of carbon dioxide in the expired air, depending on the time during which the air has remained in the lungs. This point was studied by Vierordt, in a series of ninety-four experiments made on his own person, with the following results :-

"When the respirations are frequent, the quantity of carbon dioxide expelled at each expiration is much less than in a slow expiration; but the quantity of carbon dioxide produced during a given time by frequent respirations is greater than that which is thrown off by slow expirations."

The air that escapes during the first part of an expiration is less rich in carbon dioxide than that which is last expelled and comes directly from the deeper portions of the lungs. Dividing, as nearly as possible, the expiration into two equal parts, Vierordt found, as the mean of twenty-one experiments, a percentage of 3.72 in the first part of the expiration and 5.44 in the second part.

Temporary arrest of respiratory movements has a marked influence in increasing the proportion of carbon dioxide in the expired air, although the absolute quantity exhaled in a given time is diminished. In a number of experiments on his own person, Vierordt ascertained that the percentage of carbon dioxide becomes uniform in all parts of the respiratory organs after holding the breath for forty seconds. Holding the breath after an ordinary inspiration, for twenty seconds, the percentage of carbon dioxide in the expired air was increased by 1.73 per cent above the normal standard; but the absolute quantity exhaled was diminished by 
2.642 cubic inches ( 43.3 cubic centimeters). After taking the deepest possible inspiration and holding the breath for a hundred seconds, the percentage was increased 3.08 above the normal standard; but the absolute quantity was diminished more than fourteen cubic inches (229.4 cubic centimeters). Allen and Pepys noted that air which had passed nine or ten times through the lungs contained 9.5 per cent of carbon dioxide.

Vierordt has given the following formula representing the influence of the frequency of the respirations on the production of carbon dioxide: Taking 2.5 parts per hundred as the constant value of the gas exhaled by the blood, the increase over this proportion in the expired air is in exact ratio to the duration of the contact of the air and blood.

Among the most reliable observations on the quantity of carbon dioxide exhaled by the human subject in a definite time and the variations to which it is subject, are those of Andral and Gavarret and of Edward Smith. The observations of Lavoisier and Séguin, Prout, Davy, Dumas, Allen and Pepys, Scharling and others, do not seem to have fulfilled the necessary experimental conditions so completely. The observations of Andral and Gavarret were made on sixty-two persons of either sex and different ages and under identical conditions as regards digestion, time of the day, barometric pressure and temperature; and the observations on males, between the ages of sixteen and thirty, between I and 2 P.M., under identical conditions of the digestive and muscular systems, each experiment lasting eight to thirteen minutes, showed an exhalation of about 1220 cubic inches (20 liters) of carbon dioxide per hour.

Edward Smith employed the following method for the estimation of the carbon dioxide exhaled: He used a mask, fitting closely to the face, which covered only the air-passages. The air was admitted after having been measured by an ordinary dry gas-meter. The expired air was passed through a drying apparatus, and the carbon dioxide was absorbed by a solution of potassium hydrate, arranged in a number of layers so as to present a surface of about seven hundred square inches (45 square decimeters), and was carefully weighed. This apparatus was capable of collecting all the carbon dioxide exhaled in an hour. The estimates were made for eighteen waking hours and six hours of sleep. The observations occupied ten minutes each and were made every hour and halfhour for eighteen hours. The average for the eighteen hours gave 20,082 cubic inches ( 329 liters) of carbon dioxide for the whole period. Observations during the six hours of sleep showed a total exhalation of 4126 cubic inches (7.145 liters). This, added to the quantity exhaled during the day, gives as the total exhalation in the twenty-four hours, 
during complete repose, 24,208 cubic inches (about 14.24 cubic feet, or 336.145 liters), containing 7.I44 ounces (202.47 grams) of carbon. In view of the great variations in the exhalation of carbon dioxide, this estimate can be nothing more than an approximation.

One of the important modifying influences is muscular exertion, by which the production of carbon dioxide is largely increased. This would indicate a large quantity during ordinary conditions of exercise, and a much larger quantity in the laboring classes. Dr. Smith has given the following approximate estimates of these differences:-

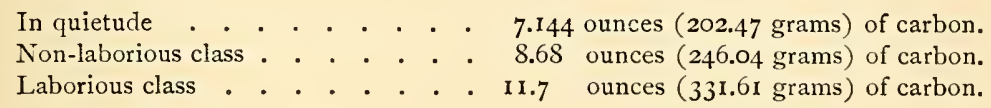

Influence of Age. - During the first few days of life, the infant does little more than sleep and take the small quantity of colostrum furnished by the mammary glands of the mother. While the animal functions are so imperfectly developed and until alimentation becomes more abundant and the infant begins to increase rapidly in weight, the quantity of carbon dioxide exhaled is very small. After the respiratory function has become fully established, it is probable, from the greater number of respiratory movements in early life, that the production of carbon dioxide, in proportion to the weight of the body, is greater in infancy and childhood than in adult life. Direct observations, however, are wanting on this point.

The observations of Andral and Gavarret show the comparative exhalation of carbon dioxide in the male, between the ages of twelve and eighty-two, and give the results of a single observation at the age of one hundred and two years. They show an increase in the absolute quantity exhaled, from the age of twelve to thirty-two; a slight diminution, from thirty-two to sixty; and a considerable diminution, from sixty to eightytwo. Taking into consideration the increase in the weight of the body with age, it is evident that the respiratory activity is much greater in youth than in adult life, and there can be no doubt that there is a rapid diminution in the relative quantity of carbon dioxide produced in old age. Scharling, in a series of observations on a boy nine years of age, an adult of twenty-eight and one of thirty-five years, showed that the respiratory activity in the child was nearly twice as great, in proportion to his weight, as the average in the adults.

Influence of Sex. - All observers have found a notable difference between the sexes, in favor of the male, in the proportion of carbon dioxide exhaled. Andral and Gavarret noted an absolute difference of about forty-five cubic inches (737.4 cubic centimeters) per hour, but did not 
take into consideration differences in the weight of the body. Scharling, taking the proportion exhaled to the weight of the body, noted a marked difference in favor of the male. The difference in muscular activity in the sexes is sufficient to account for the greater elimination of carbon dioxide in the male, for this substance is exhaled in proportion to the muscular development of the individual; but there is an important difference connected with the variations with age, which depends on the condition of the generative system of the female. The absolute increase in the exhalation of carbon dioxide with age in the female is arrested at the time of puberty and remains stationary until the cessation of the menses, provided the menstrual flow occurs with regularity. During this time the average exhalation per hour is 7I4 cubic inches (II.69 liters). After the cessation of the menses the quantity gradually increases, until, at the age of sixty, it amounts to 9I 5 cubic inches ( 5 liters) per hour. From the age of sixty to eighty-two the quantity diminishes to 793 ( 13 liters), and finally to 670 cubic inches (about I I liters). When the menses are suppressed, there is an increase in the exhalation of carbon dioxide, which continues until the flow becomes reëstablished. In a case of pregnancy observed by Scharling the exhalation was increased to about 885 cubic inches (I 4.5 liters).

Influcnce of Digestion. - Lavoisier and Séguin found that in repose and fasting, the quantity of carbon dioxide exhaled per hour was I 2 IO cubic inches (I 9.82 liters), which was raised to I 800 and 1900 (29.5 and 3 I.I 4 liters) during digestion. A series of observations on this point was made by Vierordt upon his own person. Taking his dinner between I 2.30 and I P.M., having noted the frequency of the pulse and respirations and the exhalation of carbon dioxide at I 2 M., he found at 2 P.M., the pulse and respirations increased in frequency, the volume of expired air augmented, and the carbon dioxide exhaled increased from 15.77 to I 8.22 cubic inches ( 258.43 to 298.6 cubic centimeters) per minute. In order to ascertain that this variation did not depend on the time of day, independently of the digestive process, he made a comparison at I 2 м., at $I$ and at 2 P.M. without taking food, which showed no notable variation, either in the pulse, number of respirations, volume of expired air or quantity of carbon dioxide exhaled.

The effect of inanition is to diminish the exhalation of carbon dioxide. Bidder and Schmidt noted the daily production in a cat subjected to eighteen days of inanition, at the end of which time it died. The quantity diminished gradually from day to day, until just before death it was reduced by a little more than one-half. Edward Smith noted in his own person the influence of a fast of twenty-seven hours. There was a marked diminution in the quantity of air respired, in the quantity 
of vapor exhaled, in the number of respirations and in the rapidity of the pulse. The exhalation of carbon dioxide was diminished by onefourth. An important point in this observation was that the quantity was as small four and a half hours after eating as at the end of the twenty-seven hours.

Infuence of Diet. - The most extended series of investigations on the influence of diet upon the absolute quantity of carbon dioxide exhaled are those of Edward Smith. This observer made a large number of experiments on the influence of various kinds of food and extended his inquiries into the influence of certain beverages, such as tea, coffee, cocoa, malt liquors and fermented liquors. He divided food into two classes: one which increased the exhalation of carbon dioxide, which he called respiratory excitants, and the other, which diminished the exhalation, he called non-exciters. The following are the results of a large number of observations upon four persons : -

"The excito-respiratory are nitrogenous food, milk and its components, sugars, rum, beer, stout, the cereals, and potato.

"The non-exciters are starch, fat, certain alcoholic compounds, the volatile elements of wines and spirits, and coffee-leaves.

"Respiratory excitants have a temporary action; but the action of most of them commences very quickly, and attains its maximum within one hour.

"The most powerful respiratory excitants are tea and sugar; then coffee, rum, milk, cocoa, ales, and chiccory; then casein and gluten, and lastly, gelatin and albumen. The amount of action was not in uniform proportion to their quantity. Compound ailments, as the cereals, containing several of these substances, have an action greater than that of any of their elements.

"Most respiratory excitants, as tea, coffee, gluten, and casein, cause an increase in the evolution of carbon greater than the quantity which they supply, while others, as sugar, supply more than they evolve in this excess, that is, above the basis. No substance containing a large amount of carbon evolves more than a small portion of that carbon in the temporary action occurring above the basis-line, and hence a large portion remains unaccounted for by these experiments."

The comparative observations on the four subjects of experiment demonstrated one important fact; namely, that the action of different kinds of food on respiration is modified by idiosyncrasies and the tastes of different individuals.

The following are the results of observations on the effects of different alcoholic beverages taken during the intervals of digestion:-

"Brandy, whiskey, and gin, and particularly the latter, almost always 
lessened the respiratory changes recorded, while rum as commonly increased them. Rum-and-milk had a very pronounced and persistent action, and there was no effect on the sensorium. Ale and porter always increased them, while sherry wine lessened the quantity of air inspired, but slightly increased the carbonic acid evolved.

"The volatile elements of alcohol, gin, rum, sherry, and port-wine, when inhaled, lessened the quantity of carbonic acid exhaled, and usually lessened the quantity of air inhaled. The effect of fine old port-wine was very decided and uniform; and it is known that wines and spirits improve in aroma and become weaker in alcohol by age. The excito-respiratory action of rum is probably not due to its volatile elements."

From these facts it would seem that the most constant effect of alcohol and of alcoholic liquors, such as wines and spirits, is to diminish the exhalation of carbon dioxide. This effect is almost instantaneous, when the articles are taken into the stomach fasting; and when taken with the meals, the increase in carbon dioxide, which habitually accompanies the process of digestion, is materially lessened. Rum, which was found to be a respiratory excitant, is an exception. Malt liquors seem to increase the exhalation of carbon dioxide. "The action of pure alcohol was much more to increase than to lessen the respiratory changes, and sometimes the former effect was well pronounced."

Influence of Slcep. - All who have directed attention to the influence of sleep on the respiratory products have noted a marked diminution in the exhalation of carbon dioxide. According to Edward Smith, the quantity during the night is to the quantity during the day, in complete repose, as ten to eighteen. It has already been stated that there is great diminution in the quantity of oxygen consumed in hibernating animals. Regnault and Reiset found that a marmot in hibernation consumed only one-thirtieth of the oxygen ordinarily appropriated in the active condition. In the same animal, they noted an exhalation of carbon dioxide equal to but little more than half the weight of oxygen absorbed.

Influcnce of Muscular Activity. - The results of the experiments of Dr. Edward Smith on the influence of exercise are as follows:-

In walking at the rate of two miles ( 3.22 kilometers) per hour, the exhalation of carbon dioxide during one hour was equal to the quantity produced during $\mathrm{I} \frac{4}{5}$ hour of repose with food or $2 \frac{1}{2}$ hours of repose without food.

Walking at the rate of three miles ( 4.828 kilometers) per hour, one hour was equal to $2_{4}^{3}$ hours with food or $3 \frac{1}{2}$ hours without food.

One hour's labor at the tread-wheel, while actually working the wheel, was equal to $4 \frac{1}{2}$ hours of rest with food or 6 hours without food. 
It has been observed, however, that when muscular exertion is carried so far as to produce great fatigue and exhaustion, the exhalation of carbon dioxide is notably diminished.

Influence of Moisture and Temperature. - It has been shown that the exhalation of carbon dioxide is greater in a moist than in a dry atmosphere. It has also been ascertained that the exhalation is much greater at low than at high temperatures, within the limits of heat and cold that are easily endured, amounting, according to the experiments of Vierordt on the human object, to an increase of about one-sixth, under the influence of a moderate diminution in temperature. It was found, also, that the quantity of air taken into the lungs was slightly increased at low temperatures.

Influence of the Season of the Year, etc. - It has been shown by the researches of Edward Smith, that spring is the season of the greatest, and fall the season of the least activity of the respiratory function.

The months of maximum are January, February, March and April.

The months of minimum are July, August and a part of September.

The months of decrease are June and July.

The months of increase are October, November and December.

Observations on the influence of barometric pressure have not been sufficiently definite in their results to warrant any exact conclusions.

Some physiologists have attempted to fix certain hours of the day when the exhalation of carbon dioxide is at its maximum and at its minimum; but the respiratory activity is influenced by such a variety of conditions that it is impossible to do this with any degree of accuracy.

\section{Relations between the Oxygen consumed and the Carbon Diox-} IDE EXHALED

Oxygen unites with carbon in a certain proportion to form carbon dioxide, the volume of which is equal to the volume of the oxygen that enters into its composition. It is possible, therefore, to study the relations of the volumes of these gases in respiration, by simply comparing the volumes of the inspired and expired air. It is now commonly recognized that the volume of air expired is less, at a given temperature, than the volume of air inspired. Assuming, then, that the changes in the expired air, as regards nitrogen and all gases except oxygen and carbon dioxide, are insignificant, it must be admitted that a certain quantity of the oxygen consumed is unaccounted for by the oxygen that enters into the composition of the carbon dioxide exhaled. It has already been noted that $\frac{1}{70}$ to $\frac{1}{50}$ ( 1.4 to 2 per cent) of the inspired air is lost in the lungs; or it may be said in general terms that the oxygen 
absorbed is equal to about five per cent of the volume of air inspired, and the carbon dioxide exhaled, only about four per cent. A part of the deficiency in volume of the expired air is to be accounted for, then, by a deficiency in the exhalation of carbon dioxide.

The experiments of Regnault and Reiset have an important bearing on the question under consideration. As these observers were able to measure accurately the quantities of oxygen consumed and carbon dioxide produced in a given time, the relation between the two gases was kept constantly in view. They found great variations in this relation, dependent mainly on the regimen of the animal. The total loss of oxygen was found to be greater in carnivorous than in herbivorous animals; and in animals that could be subjected to a mixed diet, by regulating the food this was made to vary between the two extremes. The mean of seven experiments on dogs showed that for every 1000 parts of oxygen consumed, 745 parts were exhaled in the form of carbon dioxide. In six experiments on rabbits, the mean was 919 for every IOOO parts of oxygen.

In animals fed on grains, the proportion of carbon dioxide exhaled was greatest, sometimes passing a little beyond the volume of oxygen consumed.

"The relation is nearly constant for animals of the same species when subjected to a uniform alimentation, as is easy to realize as regards dogs; but it varies notably in animals of the same species, and in the same animal, submitted to the same regimen, but in which we can not regulate the alimentation, as in fowls."

When herbivorous animals were deprived of food, the relation between the gases was the same as in carnivorous animals.

The final result of the experiments of Regnault and Reiset was that the "relation between the oxygen contained in the carbon dioxide and the total oxygen consumed, varies, in the same animal, between 0.62 and 1.04, according to the regimen to which it is subjected." These observations on animals have been confirmed in the human subject by Doyère, who found great differences in the relations of the two gases in respiration; the volume of carbon dioxide exhaled varying between 0.862 and 1.087 for I part of oxygen consumed.

As regards the destination of the oxygen that is not represented in the carbon dioxide exhaled, it is certain that a part of it, at least, unites with hydrogen to form water, this contributing to the production of animal heat, a question that will be fully discussed in another connection.

The variations in the relative volumes of oxygen consumed and carbon dioxide produced in respiration are not favorable to the hypothe- 
sis that the carbon dioxide is always a result of the direct action of oxygen upon carbohydrates and fats. Such a definite relation between these two gases can not be assumed to exist, in view of the fact that carbon dioxide may be given off by the tissues in the absence of oxygen.

Many of the points that have been considered in relation to the variations in the exhalation of carbon dioxide have been investigated in Pettenkofer's chamber, and the results very nearly correspond with the observations quoted from Scharling, Edward Smith and others.

Sources of Carbon Dioxide in the Expired Air. - All the carbon dioxide in the expired air comes from the venous blood, where it exists in two forms; in a condition either of simple solution or of association with certain of the constituents of the plasma, and in union with bases, forming the carbonates and bicarbonates. The fact that carbon dioxide, as regards the quantity absorbed by the blood, does not obey, in all regards, the laws which regulate the absorption of gases by liquids under different conditions of pressure, has led some physiologists to regard all this gas as existing in the blood in combination; the greater part being loosely united with certain other substances, and a small quantity of that which is thrown off in the expired air being in a condition of union much more stable.

The greater part of the carbon dioxide exhaled comes from the plasma, where it is in a condition of what is known as association. Another and a smaller part probably is set free by the action of the oxyhemoglobin, which is distinctly acid. It has been shown that more carbon dioxide can be extracted by means of a vacuum from the entire blood than from the serum; and it is more readily extracted from arterial than from venous blood. The mechanism by which carbon dioxide is discharged from the venous blood is probably the following :-

Carbon dioxide is carried from the tissues to the lungs, in the venous blood. Here it exists mainly in the plasma, a small quantity, only, existing in the corpuscles. As the venous blood passes through the lungs, the greater part of the carbon dioxide of the plasma either simply diffuses from the blood into the air-cells or passes out by a process known as dissociation. It is certain that the oxyhemoglobin, which is constantly forming in the lungs, assists materially in this process, possibly acting as an acid.

Respiratory Quotient. - The result of the division of the carbon dioxide exhaled by the oxygen taken up by the blood is called the respiratory quotient. Under ordinary conditions of respiration, this is equal to about ninety $(4 \cdot 5 \div 5)$. The greater the proportion of oxygen consumed to the carbon dioxide exhaled, the less is the respiratory q110- 
tient; and the less the proportion of oxygen to carbon dioxide, the greater is the quotient. It is evident from this that the observations of physiologists on the consumption of oxygen and the elimination of carbon dioxide, when applied to the respiratory quotient, must undergo some revision.

The respiratory quotient is lowered by animal food and increased by a vegetable diet. It is much lowered by fasting.

The quotient is lower in children than in adults.

The quotient is higher during the day than during the night.

The quotient is much diminished during sleep.

The quotient is much increased by muscular work.

The quotient is much diminished by low external temperature.

The quotient is much increased by breathing a mixture containing twenty-one parts of oxygen and seventy-11ine parts of hydrogen.

Exhalation of Watery Vapor. - From a large number of observations on his own person and on eight others, collecting the water by sulphuric acid, Valentin made the following estimates of the quantities of water exhaled from the lungs in twenty-four hours:-

In his own person the exhalation in twenty-four hours was 5934 grains (384.48 grams).

In a young man of small size the quantity was 540 I grains $(350$ grams).

In a student rather above the ordinary height the quantity was I I,929 grains (773 grams).

The mean of his observations gave a daily exhalation of 8333 grains (540 grams), or about a pound and a half.

The extent of respiratory surface has a marked influence on the quantity of watery vapor exhaled. This fact is well shown by a comparison of the exhalation in the adult and in old age, as in advanced life the extent of respiratory surface is much diminished. Barral found the exhalation in an old man less than half that of the adult. It is evident that the quantity of vapor exhaled is increased when respiration is accelerated. The quantity of water in the blood also exerts an important influence. Valentin found that the pulmonary transpiration was more than doubled in a man immediately after drinking a large quantity of water.

The vapor in the expired air is derived from the entire surface over which the air passes in respiration, and not exclusively from the air-cells. The air that passes into the lungs derives a certain quantity of moisture from the mouth, nares and trachea. The great vascularity of the mucous membranes in these situations, as well as of the air-cells, and the great number of mucous glands which they contain, serve to keep the respira- 
tory surfaces constantly moist. This is important, for only moist membranes allow the free passage of gases, which is of course essential to the process of respiration.

Exhalation of Ammonia, Organic Matter, etc. - A small quantity of ammonia is exhaled by the lungs in health, and this is increased in certain diseases, particularly in uremia. Its characters in the expired air are frequently so marked that patients, who presumably are unacquainted with the pathology of uremia, sometimes recognize an ammoniacal odor in their own breath.

The pulmonary surface exhales a small quantity of organic matter. This has not been collected in sufficient quantity for analysis, but its presence may be demonstrated by the fact that a sponge saturated with the exhalations from the lungs, or the vapor from the lungs condensed in a glass vessel, will undergo putrefaction, which is a property distinctive of organic substances.

It is well known that certain substances which are but occasionally found in the blood may be eliminated by the lungs. Certain odorous matters in the breath are constant in those who take liquors habitually in considerable quantity. The odor of garlics, onions, turpentine and of many other articles taken into the stomach may be recognized in the expired air.

The lungs eliminate certain gases that are poisonous in small quantities when absorbed in the lungs and carried to the general system in the arterial blood. Hydrogen monosulphide, which produces death in a bird when it exists in the atmosphere in the proportion of one to eight hundred, may be taken in solution into the stomach with impunity and even be injected into the venous system ; in both instances being eliminated by the lungs with great promptness and rapidity. The lungs, while they present an immense and rapidly absorbing surface for volatile poisonous substances, are capable of relieving the system of some of these by exhalation when they find their way into the veins.

Exhalation of Nitrogen. - The most accurate direct experiments, particularly those of Regnault and Reiset, show that the exhalation of a small quantity of nitrogen by the lungs is nearly constant. As the result of a large number of experiments, these observers came to the conclusion that when animals are subjected to their habitual regimen, they exhale a quantity of nitrogen equal in weight to $\frac{1}{100}$ or $\frac{1}{50}$ of the weight of oxygen consumed. In birds, during inanition, they sometimes observed an absorption of nitrogen, but this was rarely seen in mammals. Boussingault, estimating the nitrogen taken into the body and comparing it with the entire quantity discharged, arrived at the same results in experiments upon a cow. Barral, by the same method, con- 
firmed these observations by experiments on the human subject. Notwithstanding the conflicting testimony of physiologists, there can be little doubt that under ordinary physiological conditions there is an exhalation of a small quantity of nitrogen by the lungs.

\section{Changes of the Blood in Respiration (Hematosis)}

There is a marked difference in color, composition and properties, between the blood in the arteries and in the veins, the change from venous to arterial blood being effected almost instantaneously in its passage through the lungs. The blood which goes to the lungs is collected from all parts of the body and presents great differences in its composition in different veins. In some veins it is almost black, and in some it is nearly as red as in the arteries. In the hepatic vein it contains sugar, and its nitrogenous constituents and the corpuscles are diminished; in the portal vein, during digestion, it contains matters absorbed from the alimentary canal; and finally, there is every reason to suppose that parts which require different substances for their nutrition and produce different excrementitious matters exert different influences on the constitution of the blood which passes through them. After this mixture of different kinds of blood has been collected in the right side of the heart and passed through the lungs, it is returned to the left side and sent to the system, and as arterial blood, it has a nearly uniform composition. The change, therefore, which the blood undergoes in its passage through the lungs, is the transformation of the mixture of venous blood from all parts of the organism into a liquid of uniform character, which is capable of nourishing every tissue and organ of the body.

The capital phenomena of respiration, as regards the air in the lungs, are loss of oxygen and gain of carbon dioxide, the other phenomena being comparatively unimportant. As the blood is capable of absorbing gases, the essential changes which it undergoes in respiration are to be looked for in connection with the proportions of oxygen and carbon dioxide before and after it has passed through the lungs.

The elements of the blood which absorb the greatest part of the oxygen are the red corpuscles. While the plasma will absorb, perhaps, twice as much gas as pure water, it has been shown that the volume of oxygen fixed by the corpuscles is about twenty-five times that which is dissolved in the plasma (Fernet, Lothar Meyer).

Analysis of the Blood for Gases. - In disengaging and estimating the gases of the blood, it is necessary to complete the analysis as soon as possible after the blood has been drawn, for the reason that delay in- 
volves a disappearance of a considerable quantity of oxygen, which is replaced by carbon dioxide. This accounts for the indefinite results obtained by the earlier observers. Modern experimenters make use of the mercurial gas-pumps, either of Ludwig or of Pfluger, in which all the gases of the blood are disengaged by removing atmospheric pressure. By means of a "froth-chamber," the gases can be collected and analyzed with but little loss of time; but it is probable that there is always a slight error in estimates, made in this way, of the relative proportions of oxygen and carbon dioxide, the proportion of oxygen being too small, and of carbon dioxide, too large. Nevertheless, the results obtained by this method correspond pretty closely with what is known of the nature of the respiratory process; and analyses of the blood taken at different times show variations in the quantities of oxygen in the arterial blood and of carbon dioxide in the venous blood, corresponding with some of the variations that have been noted in the loss of oxygen and gain of carbon dioxide in the air in respiration. Nearly all the gases contained in the blood may be disengaged by means of the gas-pump, but according to most observers, a small quantity of carbon dioxide remains in the blood in combination. This may be removed by the introduction into the apparatus of a small quantity of tartaric acid. It was justly remarked by Bert, that as the apparatus for the exhaustion of air has been made more and more nearly perfect, the quantity of carbon dioxide in combination has seemed less and less. By far the greatest quantity of the excrementitious carbon dioxide in the blood is extracted by the removal of atmospheric pressure in the most carefully prepared apparatus.

The quantity of carbon dioxide varies considerably in different parts of the venous system. It is well known that the venous blood coming from certain glands is dark during the intervals of secretion and is nearly as red as arterial blood during secretion. In the venous blood from the submaxillary gland of a dog, Bernard found 18.07 per cent of carbon dioxide during repose and I0.14 per cent during secretion. The blood coming from the muscles is the darkest in the body and contains the greatest quantity of carbon dioxide. The quantity of carbon dioxide, also, is increased in the venous blood during digestion.

These facts coincide with the views now held regarding the essential processes of respiration. The blood going to the lungs contains carbon dioxide and but a small proportion of oxygen. In the lungs carbon dioxide is given off, appearing in the expired air, and the oxygen which disappears from the air is carried away by the arterial blood.

Nitrogen of the Blood. - So far as is known, nitrogen has no important office connected with respiration. There is sometimes a slight exhalation of this gas by the lungs, and analyses have demonstrated its 
existence in solution in the blood. There is no evidence that nitrogen enters into combination with the blood-corpuscles. It exists simply in solution in the blood, which is capable of absorbing about ten times as much as water. Nothing is known in regard to the relations of the free nitrogen of the blood to the processes of nutrition.

Using the mercurial gas-pump, it is possible to extract about sixty volumes of gas from one hundred parts of blood. In experiments on the blood of the dog, the average percentage volumes of oxygen, carbon dioxide and nitrogen in arterial and venous blood were as follows (Kirkes):-

Oxygen
Carbon dioxide
Nitrogen

$\begin{array}{cc}\text { ARTERIAL BLOOD } & \text { VENOUS BLOOD } \\ 20 & 8 \text { to I } 2 \\ 40 & 46 \\ \text { I to } 2 & \text { I to } 2\end{array}$

Oxygen of the Blood. - When oxygen exists in a condition of simple solution in any liquid, the quantity dissolved is in direct ratio to the pressure; and when the pressure is increased, the amount held in solution is increased in exact proportion. This law, however, is not operative in the case of the oxygen of the blood. When the condition is one of saturation, the amount does not vary with the pressure. In the case of a mechanical mixture of gases, what is known as the "partial pressure" of each gas is the force which leads to its diffusion, and this may be measured by means of a column of mercury.

The following application of this law may be made to the oxygen of the inspired air: The partial pressure of oxygen in the atmosphere, expressed in percentages of the pressure of one atmosphere $=20.96$; the pressure of oxygen in the air contained in the air-cells $=18$; the tension of oxygen in the arterial blood $=14$; in the tissues, the tension of the oxygen is zero (Fredericq).

This reduction in pressure, from 20.96 to zero, indicates the direction of diffusion of oxygen. The oxygen of the inspired air is diffused into the air-cells because the pressure is 18 instead of 20.96 ; it is diffused into the blood because the tension is here reduced to 14 ; it passes into the tissues because the pressure in the tissues is zero. The tension of gases held in solution in liquids is the force which holds them in solution, or which prevents their escape by diffusion. The oxygen contained in the blood is "associated" with hemoglobin, a condition of union that has already been considered in treating of the composition of the blood.

Carbon Dioxide of the Blood. - The relations of carbon dioxide to the tissues, the blood and the contents of the air-cells are rather more complex than in the case of oxygen, especially as regards the blood. 
The tension of carbon dioxide in the tissues $=5$ to 9 ; in the venous blood it $=3.8$ to 5.4 ; in the air-cells it $=2.8$; in the external air it $=$ 0.03. For the same reason that oxygen diffuses from the external air, where the pressure is 20.96 , to the tissues, where the tension is zero, carbon dioxide passes from the tissues, where the tension is 5 to 9 , to the external air, where it is 0.03 (Fredericq).

The carbon dioxide of the blood is contained chiefly in the plasma, but a small quantity exists in the red corpuscles, in which this gas is slightly more soluble than in pure water. It is somewhat difficult to determine the exact condition of carbon dioxide in the plasma. It is estimated that about five per cent exists in simple solution, seventy-five to eighty-five per cent in a condition of loose combination and ten to twenty per cent in firmer combination. These conditions render it difficult to determine the exact tension of the gas in the plasma; and, indeed, the estimates of the tension of carbon dioxide are much less exact than of oxygen.

Respiration in the Tissues. - Nearly all tissues contain coloring matters known as histohematins. The most important of these is found in the muscles and is called myohematin. It is thought that the oxygen of the blood combines with these histohematins, the process involving true tissue-respiration. At all events, the interchange of gases between the tissues and the blood are exactly opposite to the changes that take place in the lungs. In the lungs, the blood loses carbon dioxide and gains oxygen; in the tissues, the blood loses oxygen and gains carbon dioxide. The real seat of respiration is in the tissues.

\section{Respiratory Processes before Birth}

It is commonly admitted that one of the most important uses of the placenta - the one, indeed, most immediately connected with the life of the fœtus - is a respiratory interchange of gases, analogous to that which takes place in the gills of aquatic animals. The placental villi are bathed in the blood of the uterine sinuses, and this is the only way in which the fœtal blood can receive oxygen. Legallois observed a bright red color in the blood of the umbilical vein; and on alternately compressing and releasing the vessel, he saw the blood change in color successively from red to dark and from dark to red. Zweifel has demonstrated the presence of oxyhemoglobin in the blood of the umbilical vessels by means of the spectroscope, showing that it contains oxygen. As oxygen is thus adequately supplied to the system, the fœtus is in a condition similar to that of animals in which artificial respiration is maintained. The want of oxygen is fully met, and there- 
fore no respiratory efforts take place. Respiratory movements occur, however, even in very young fœtuses, when there is a deficient supply of oxygen.

\section{Cutaneous Riespiration}

Respiration by the skin, although important in many of the lower orders of animals, is inconsiderable in the human subject and is even more insignificant in animals covered with hair or feathers; still, an appreciable quantity of oxygen is absorbed by the skin of the human subject, and a quantity of carbon dioxide, which is relatively larger, is exhaled. Carbon dioxide is given off with the general emanations from the surface, being found, also, in solution in the urine and in most of the secretions.

An estimate of the extent of the cutaneous, as compared with pulmonary respiration, has been made by Scharling, by comparing the relative quantities of carbon dioxide exhaled in the twenty-four hours. According to this observer, the skin performs $\frac{1}{50}$ to $\frac{1}{40}$ of the respiratory office. It is difficult, however, to collect all the carbon dioxide given off by the skin under normal conditions. In the observations by Aubert, the estimate is much lower than that given by Scharling.

\section{AsPhyxia}

A remarkable power of resisting asphyxia exists in newborn animals that have never breathed. Legallois found that young rabbits will live for fifteen minutes deprived of air by submersion, but that this power of resistance diminished rapidly with age. W. F. Edwards has shown that there exists a great difference in this regard in different species. Dogs and cats, which are born with the eyes shut and in which there is at first a very small development of animal heat, will show signs of life after submersion for more than half an hour; while Guinea pigs, which are born with the eyes open, are much more active and produce a greater amount of heat, will not live for more than seven minutes. The explanation of this is that in most warm-blooded animals, during the first periods of extra-uterine life, the demands on the part of the system for oxygen are comparatively slight. At this time there is very little activity in the general processes of nutrition and in the consumption of oxygen and the exhalation of carbon dioxide. The actual difference between the consumption of oxygen immediately after birth and at the age of a few days is sufficient to explain the remarkable power of resisting asphyxia just after birth.

Breathing in a Confined Space. - An important question connected with the physiology of asphyxia is the effect on the system of air vitiated 
by breathing in a confined space. There are here several points that present themselves for consideration. The effect of respiration on the air is to take away a certain proportion of oxygen and to add certain matters regarded as deleterious. The emanation which commonly has been considered as having the most decided influence on the system is carbon dioxide; but this has been much overestimated. In death from charcoal-fumes, carbon monoxide is the poisonous agent. Regnault and Reiset exposed dogs and rabbits for many hours to an atmosphere containing twenty-three per cent of carbon dioxide artificially introduced, and between thirty and forty per cent of oxygen, without ill effects. They took care, however, to keep up a free supply of oxygen.

These experiments are at variance with results obtained by others; but Regnault and Reiset explained this difference by the supposition that the gases in other observations were impure, containing chlorin or carbon monoxide. This view is sustained by the experiments of Bernard with carbon monoxide. In animals killed by this gas, the blood, both venous and arterial, is of a bright red color, which is due to the fixation of the gas by the blood-corpuscles. In this way, the red corpuscles are paralyzed and the animal dies from asphyxia.

In breathing in a confined space, the distress and the fatal results are produced, in all probability, more by animal emanations and a deficiency of oxygen than by carbon dioxide. When the latter gas is removed as fast as it is produced, the effects of diminution in the proportion of oxygen are soon very marked, and they progressively increase until death occurs. The influence of emanations from the lungs and general surface is considerable; and this fact, which almost all have experienced more or less, has been illustrated in instances of large numbers of persons confined without proper change of air.

In crowded assemblages, the slight diminution of oxygen, the elevation of temperature, increase in moisture, and particularly the presence of organic emanations combine to produce unpleasant sensations. The effects of this carried to an extreme degree were exemplified in the confinement of the one hundred and forty-six English prisoners, for eight hours only, in the "Black Hole of Calcutta," a chamber eighteen feet (5.486 meters) square, with only two small windows, and these obstructed by a veranda. Out of this number, ninety-six died in six hours, and one hundred and twenty-three, at the end of the eight hours. Many of those who immediately survived died afterward of putrid fever (Ammal Register, 1758). The incident of the "Black Hole of Calcutta" has frequently been repeated on emigrant and slave ships, by confining great numbers in the hold of the vessel, where they were entirely shut off from the fresh air. 


\title{
CHAPTER VI
}

\author{
ALIMENTATION
}

Hunger and thirst - Nitrogenous alimentary substances - Non-nitrogenous alimentary substances - Carbohydrates - Dextrose - Levulose - Galactose - Saccharose - Lactose Naltose - Starch - Glycogen - Cellulose, inosite and gums - The fats: triolein; tripalmitin; tristearin - Saponification - Emulsification - Inorganic alimentary substances Water - Sodium chloride - Calcium phosphate - Iron - Alcohol - Coffee - Tea Chocolate - Condiments and flavoring articles - The daily ration - Necessity of a varied diet - Meats - Bread - Potatoes - Milk - Eggs.

IN the organism of animals every part is continually undergoing what may be called physiological wear, or katabolism; the nitrogenous constituents of the body are being constantly transformed into effete matter; and as these constituents never exist without inorganic matters, with which they are closely and inseparably associated, it is found that the products of their katabolism are always discharged from the body in connection with inorganic substances. This process of molecular change is a necessary condition of life. Its activity may be increased or retarded by various means, but it can not be arrested. The excrementitious matters thus formed are produced constantly by the tissues and must be as constantly eliminated. It is evident, from the amount of matter that is daily discharged, that the process of katabolism must be very active. Its constant operation necessitates a constant appropriation of new matter by the parts, in order that they may maintain their integrity of composition and be always ready to perform their offices in the economy. The blood contains all the materials necessary for the regeneration of the organism. Its inorganic constituents are found usually in the form in which they exist in the substance of the tissues; but the organic constituents of the parts are formed in the substance of the tissues themselves, by a transformation of matters furnished by the blood. The physiological wear of the organism is, therefore, being constantly repaired by the blood; but in order to keep the great nutritive fluid from becoming impoverished, the matters which it is constantly losing must be supplied from some source out of the body, and this necessitates the ingestion of articles known as food. Food is taken into the body in obedience to a want on the part of the system, which is 
expressed by the sensation of hunger, when it relates to solid or semisolid matters, and of thirst, when it relates to water.

\section{HUNGer AND THIRst}

The term hunger may be applied to all degrees of that peculiar want felt by the system, that leads to the ingestion of nutritive substances. Its first manifestations are, perhaps, best expressed by the term "appetite"; a sensation by no means disagreeable, and one that may be excited by the sight, smell, or even the recollection of savory articles, at times when it does not depend on a want in the system. In the ordinary and moderate development of appetite for food, it is impossible to say that the sensation is referable to any distinct part or organ. It is influenced in some degree by habit; in many persons, the feeling being experienced at or near the hours when food ordinarily is taken. If not gratified, the appetite is rapidly intensified until it becomes actual hunger. Except when the quantity of food taken is unnecessarily large, the appetite simply disappears on the introduction of food into the stomach, and gives place to the sense of satisfaction that accompanies the undisturbed and normal action of the digestive organs.

It has been observed that children and old persons do not endure deprivation of food so well as adults. This was noted in the case of the wreck of the frigate Medusa. After the wreck, one hundred and fifty persons, of all ages, were exposed on a raft for thirteen days, with hardly any food. Out of this number only fifteen survived; and the children, the young persons and the aged were the first to succumb.

In cold climates and during the winter season in temperate climates, the desire for food is notably increased and the tastes are somewhat modified. Animal food, particularly fats, are more agreeable at that time, and the quantity of nutriment demanded by the system is considerably increased. In many persons the difference in the appetite in warm and in cold seasons is very marked.

If food is not taken in obedience to the demands of the system as expressed by the appetite, the sensation of hunger becomes most distressing. It is then manifested by a peculiar and indescribable sensation in the stomach, which soon becomes developed into actual pain. This usually is accompanied with intense pain in the head and a feeling of general distress, which soon render the satisfaction of this imperative demand on the part of the system the absorbing idea of existence. Furious delirium frequently supervenes after a few days of complete abstinence; and this usually is the immediate precursor of death. It is unnecessary to cite the many instances in which murder and cannibalism have been 
resorted to when starvation had become imminent; suffice it to say that the extremity of hunger or of thirst, like the sense of impending suffocation, is a demand on the part of the system so imperative that it must be satisfied if within the range of possibility.

When the system is suffering from defective nutrition, as after prolonged abstinence or during convalescence from diseases attended with defective assimilation, the mere filling of the stomach produces a sensation of repletion of this organ, but the sense of hunger is not relieved; but if, on the other hand, the nutrition is active and sufficient, the stomach frequently is entirely empty for a considerable time without the development of the sense of hunger. The appetite is preserved and hunger is felt by persons who suffer from extensive organic disease of the stomach, and the sensation occasionally has been relieved by nutritious enemata or by injections into the veins. It is certain that appetite and the sense of hunger are expressions of a want on the part of the organism, referred by the sensations to the stomach, but really existing in the general system. This can be completely satisfied only by the absorption of digested alimentary matters by the blood and their assimilation by the tissues.

The sense of hunger undoubtedly is appreciated by the cerebrum, and it has been a question whether there be any special nerves that convey this impression to the encephalon. The nerve which naturally would be supposed to have this office is the pneumogastric; but notwithstanding certain observations to the contrary, it has been shown that section of both these nerves by no means abolishes the desire for food. It has been observed that dogs eat, apparently with satisfaction, after section of the glosso-pharyngeal and lingual nerves. It has been thought, also, that the sensation of hunger is conveyed to the brain through the sympathetic system. Although there are varions considerations that render this somewhat probable, it is not apparent how it can be demonstrated experimentally. It is undoubtedly the sympathetic system of nerves that presides specially over nutrition; and hunger, which depends upon deficiency of nutrition, is certainly not conveyed to the brain by any of the cerebro-spinal nerves.

Thirst is the peculiar sensation that leads to the ingestion of water. In its moderate development, this usually is an indefinite feeling, accompanied by more or less dryness and heat of the throat and fauces, and sometimes, after the ingestion of a quantity of very dry food, by a sensation referred to the stomach. When the sensation of thirst has become intense, the immediate satisfaction following the ingestion of a liquid, particularly water, is very great. Thirst is very much under the influence of habit; some persons experiencing a desire to take liquids 
only two or three times daily, while others do so much more frequently. The sensation is also sensibly influenced by the condition of the atmosphere as regards moisture, by exercise and by other conditions that influence the discharge of water from the body, particularly by the skin. A copious loss of blood is always followed by intense thirst; and in diseases characterized by increased discharge of liquids, thirst usually is excessive.

The demand on the part of the system for water is much more imperative than for solids; and in this respect it is second only to the demand for oxygen. Animals live much longer when deprived of solid food but allowed to drink freely than if deprived of both food and drink. A man, supplied with dry food but deprived of water, will not survive more than a few days. Water is necessary to the processes of nutrition, and acts, moreover, as a solvent in removing from the system the products of katabolism.

After deprivation of water for a considerable time, the intense thirst becomes most distressing. The dryness and heat of the throat and fauces are increased and accompanied with a sense of constriction. A general febrile condition supervenes, the blood is diminished in quantity and becomes thickened, the urine is scanty and scalding, and there seems to be a condition of the principal viscera approaching inflammation. Death takes place in a few days, usually preceded by delirium.

The sensation of thirst is instinctively referred to the mouth, throat and fauces; but it is not appeased necessarily by the passage of water over these parts, and it may be effectually relieved by the introduction of water into the system by other channels, as by injecting it into the veins. Bernard has demonstrated, by the following experiment, that water must be absorbed before the demands of the system can be satisfied: He made a section of the œesophagus in a horse, tied the lower opening and allowed the animal to drink after he had been deprived of water for a number of hours. The animal drank an immense quantity, but the water did not pass into the stomach and the thirst was not relieved. He modified this experiment by causing dogs to drink, with a fistulous opening into the stomach by which the water was immediately discharged. They continued to drink without being satisfied, until the fistula was closed and the water could be absorbed. In a case repported by Gairdner (1820), in the human subject, all the liquids swallowed passed out at a wound in the neck, by which the œsophagus had been cut across. The thirst in this case was insatiable, although buckets of water were taken in the day; but on injecting water into the stomach, the sensation was soon relieved. 
Although the sensation of thirst is referred to special parts, it is an expression of the want of liquids in the system and is to be effectually relieved only by their absorption by the blood. There are no nerves belonging to the cerebro-spinal system that have the office of conveying this sensation to the brain, division of which will abolish the desire for liquids. Experiments show that no effectual relief of the sensation is afforded by simply moistening the parts to which the heat and dryness are referred. As a demand on the part of the system, it is analogous to the sense of want of air and of hunger, differing only in the manner in which it is manifested.

The duration of life after complete deprivation of food and drink is variable. The influence of age has already been referred to. Taking no account of certain remarkable individual instances of starvation in the human subject that have been reported, it may be stated, in general terms, that death occurs within five to eight days after total deprivation of food. In the instance of the one hundred and fifty persons wrecked on the frigate $M e d u s a$, in I 816 , who were exposed on a raft in the open sea for thirteen days, only fifteen were found alive. Dr. Savigny, one of the survivors, gave, in an inaugural thesis, an instructive and accurate account of this occurrence, which has often been quoted in works on physiology. Authentic instances are on record in which life has been prolonged much beyond the period above mentioned; but they usually occurred in persons who were so situated as not to suffer from cold, which the system, under this condition, has but little power to resist. In these cases, also, there was no muscular exertion, and water was taken in abundance.

Bérard quoted the example of a convict who died of starvation after sixty-three days, but in this case water was taken. An instance of eight miners who survived after five days and sixteen hours of almost complete deprivation of food is referred to in works on physiology. Bérard has also quoted from various authors instances of deprivation of food for periods varying between four months and sixteen years; but these accounts have been discredited by physiologists. They occurred usually in hysterical females; and their consideration belongs to psychology rather than to physiology. According to the observations of Chossat, death from starvation occurs after a loss of four-tenths of the weight of the body, the time of death, however, being very variable in different classes of animals.

Thirty to thirty-five days may be taken as the average duration "of life in dogs deprived entirely of food and water. It is important to bear in mind this fact in connection with observations on the nutritive value of different articles of food. 


\section{Alimentation}

Under the name of aliment, in its widest signification, it is proposed to include all articles composed of or containing substances in a form in which they can be used for the nourishment of the body, either by being themselves appropriated by the organism, by influencing favorably the processes of nutrition or by retarding katabolism. The substances that are themselves appropriated may be called direct aliments; and those which simply assist nutrition without contributing reparative material, together with those which retard katabolism, may be termed indirect aliments. In this definition of aliment, nothing is excluded that contributes to nutrition. Oxygen must be considered in this light, as well as water and all articles commonly called drinks.

In the various articles used as food, nutritious substances frequently are combined with each other and with indigestible and innutritious matters. The constituents of the food directly used in nutrition are the true alimentary substances, embracing, thus, only those capable of absorption and assimilation. The ordinary food of warm-blooded animals contains alimentary matters united with innutritious substances from which they are separated in digestion. This necessitates a complicated digestive apparatus. In some of the inferior animals, the quantity of nutritious matter forms so small a part of the ingesta that the digestive apparatus is more complicated than in the human subject. This is specially marked in the herbivora, the flesh of which is an important part of the diet of man. In addition to what are distinctly recognized as alimentary substances, food has many constituents that exert an important influence on nutrition, which have never been isolated and analyzed, but which render it agreeable. Many of these are developed in the process of cooking.

Alimentary substances belong to the inorganic, vegetable and animal kingdoms. They may be divided into the following classes :-

I. Organic nitrogenous substances (albumin, fibrin, casein, myosin etc.), belonging to the animal kingdom, and vegetable nitrogenous substances, such as gluten and legumin.

2. Organic non-nitrogenous substances (sugars, starch and fats).

3. Inorganic substances.

Nitrogenous Alimentary Substances. - In the nutrition of certain classes of animals, these substances are derived exclusively from the animal kingdom, and in others, exclusively from the vegetable kingdom; but in man, both animals and vegetables contribute nitrogenous matters. In both animal and vegetable food, nitrogenous substances are always found associated with inorganic matters (water, sodium chloride, the 
phosphates, sulphates etc.), and frequently with non-nitrogenous matters, especially the carbohydrates.

The most important nitrogenous alimentary constituents of food are contained in muscular tissue, eggs, milk, the juices of vegetables, cereal grains etc. Among the most important are the following: myosin, the chief proteid constituent of muscle, the various albumins found in eggs and in animal liquids, analogous substances existing in vegetables, casein in milk, a substance sometimes called vegetable casein, vitellin in yolk of egg, fibrin, gelatin, and gluten, an important alimentary substance found in the cereal grains. A distinctive character of these substances is that they contain nitrogen, being composed of carbon, oxygen, hydrogen and nitrogen, probably with a small quantity of sulphur. They are either liquid or semisolid in consistence and are coagulable by various reagents. The type of substances of this class is albumin, which has the empirical formula, $\mathrm{C}_{72} \mathrm{H}_{112} \mathrm{O}_{22} \mathrm{~N}_{18} \mathrm{~S}$. Certain of these are called proteids, after a hypothetical substance described by Mulder, under the name of protein.

Nitrogenous substances are found in animal bodies, as has already been stated. They originate in vegetables by a union of nitrogen, derived chiefly from saline matters, with carbohydrates, the carbohydrates in vegetables being produced from carbon dioxide and water.

A distinctive character of nitrogenous matters is that under favorable conditions of heat and moisture they undergo a peculiar form of decomposition called putrefaction. In the processes of digestion, these substances are converted into peptones, and afterward, it is thought, a part may be changed into leucin, tyrosin and other substances not well defined. An analogous decomposition is said to take place under the influence of dilute hydrochloric acid, at a temperature of $104^{\circ}$ Fahr. $\left(40^{\circ} \mathrm{C}\right.$.), and of dilute sulphuric acid, at a temperature of $2 \mathrm{I} 2^{\circ} \mathrm{Fahr} .\left(100^{\circ} \mathrm{C}\right.$.). The chemical history of these substances would require an elaborate description such as properly belongs only to special works on physiological chemistry.

Non-nitrogenous Alimentary Substances. - The important non-nitrogenous alimentary matters are sugars, starch and fats. These are composed of carbon, hydrogen and oxygen. In sugars and starch, the hydrogen and oxygen exist in the proportion to form water, and these matters are therefore called carbohydrates.

Sugars. - Many varieties of sugar occur in food, and this substance may be derived from both the animal and vegetable kingdoms. The most common varieties derived from animals are sugar of milk, and honey, beside a small quantity of liver-sugar, which is taken whenever the liver is used as food. Only a few of the carbohydrates are of 
physiological interest. The formulæ given are merely empirical, and the molecular weights in many instances are indefinite. In the group of amyloses (starch-groups) the molecules are very large - 20,000 to 30,000 (Brown and Morris). The various changes which substances belonging to this group undergo are very complex, and it will be more convenient to discuss these in connection with the physiology of digestion. Here little more than an enumeration of some of the carbohydrates will be given. The following is the usual classification. The sign + indicates that the substance is dextro-rotatory and the sign - , that it is levo-rotatory.

$\begin{array}{ccl}\text { I. Glucoses } & \text { 2. Saccharoses } & \text { 3. Amyloses } \\ \left(\mathrm{C}_{6} \mathrm{H}_{12} \mathrm{O}_{6}\right) & \left(\mathrm{C}_{12} \mathrm{H}_{22} \mathrm{O}_{11}\right) & \left(n \mathrm{C}_{6 \mathrm{i}} \mathrm{H}_{10} \mathrm{O}_{5}\right) \\ + \text { Dextrose } & + \text { Saccharose } & + \text { Starch } \\ - \text { Levulose } & + \text { Lactose } & + \text { Glycogen } \\ + \text { Galactose } & + \text { Maltose } & + \text { Dextrin } \\ & & \text { Cellulose } \\ & & \text { Gums }\end{array}$

Dextrose. - This carbohydrate - often called grape-sugar - is found in fruits, honey, and sometimes in small quantity in the blood and in various tissues. Its empirical formula is $\mathrm{C}_{6} \mathrm{H}_{12} \mathrm{O}_{6}$. It is soluble in hot and cold water and in alcohol. It reduces the copper salts and is fermentible.

Levulose. - This carbohydrate is obtained by treating saccharose with a dilute mineral acid. The saccharose then undergoes what is known as inversion, appropriating water and changing into a mixture of equal parts of levulose and dextrose. This mixture is levo-rotatory, the levo-rotatory power being greater than the dextro-rotatory. It has the same general reactions as dextrose. It crystallizes with difficulty. Its empirical formula is the same as the formula for dextrose $\left(\mathrm{C}_{6} \mathrm{H}_{12} \mathrm{O}_{6}\right)$.

Galactose. - This carbohydrate is formed by the action of dilute mineral acids on lactose. It is dextro-rotatory and has the same general reactions as dextrose. Its empirical formula is $\mathrm{C}_{6} \mathrm{H}_{12} \mathrm{O}_{6}$.

Saccharose. - This carbohydrate is derived from the vegetable kingdom. It crystallizes readily and is dextro-rotatory. It does not reduce the salts of copper. On the addition of yeast, it first undergoes change into invert-sugar and then ferments. It is by far the most important of the sugars used as food. By boiling with a dilute mineral acid, it undergoes inversion into equal parts of dextrose and levulose. It is inverted, also, in the processes of digestion. Its empirical formula is $\mathrm{C}_{12} \mathrm{H}_{22} \mathrm{O}_{11}$.

Lactose. - This is the sugar found in milk. It assumes water under the same conditions as saccharose and splits into dextrose and galac- 
tose by the following process: Lactose $\left(\mathrm{C}_{12} \mathrm{H}_{22} \mathrm{O}_{11}+\mathrm{H}_{2} \mathrm{O}=\right.$ dextrose, $\mathrm{C}_{6} \mathrm{H}_{12} \mathrm{O}_{6}+$ galactose, $\mathrm{C}_{6} \mathrm{H}_{12} \mathrm{O}_{6}$ ). It does not of itself undergo fermentation, but when it has been inverted by the action of yeast, it slowly ferments.

Maltose. - This carbohydrate is the product of the action of maltdiastase on starch. It is strongly dextro-rotatory. Boiled with dilute mineral acids or exposed to the action of inverting ferments, it assumes water and is converted into dextrose. It undergoes this change in digestion. It reduces the salts of copper and promptly undergoes alcoholic fermentation when mixed with yeast. Its empirical formula is $\mathrm{C}_{12} \mathrm{H}_{22} \mathrm{O}_{11}$.

Starch. - This carbohydrate is of vegetable origin. It is composed of hard granules that are insoluble in cold water. Treated with boiling water, the granules swell, press together and gelatinize on cooling. Under the influence of the diastatic ferments, it is first converted into soluble starch, then into dextrin, and finally into dextrose. The empirical formula of soluble starch is $\left(\mathrm{C}_{6} \mathrm{H}_{10} \mathrm{O}_{5}\right)_{20}$ (?). Starch gives an intense blue reaction with iodine, even in very small quantity. This color is discharged by heat but reappears on cooling.

Glycogen. - Glycogen, or animal starch, is found in the liver, muscular tissue, leucocytes and the blood of the hepatic veins. In the liver the products of digestion of the carbohydrates of food are stored up in the form of glycogen. Treated with iodine, glycogen gives a reddish reaction. It is dextro-rotatory.

Cellulose, Inosite and Gums. - The hard covering of starch-granules is composed of cellulose. By boiling starch, these coverings are ruptured, the starch is set free and it may be acted on slightly by the digestive secretions. It exists in vegetable food, but on account of its difficult solubility it is not readily digested. Treated with dilute mineral acids for a long time, like starch, it is converted into glucose.

Inosite, or the sugar of muscular tissue, exists in muscle, the liver, the kidney and in various other parts in small quantity.

The gums exist in food and in the blood in very small quantity. They are unimportant in the processes of nutrition.

The Fats. - Fats exist in the body either in the form of adipose tissue or in granules and in some instances in molecular union with nitrogenous matters. They present three forms, or varieties: triolein, liquid at a temperature of $23^{\circ} \mathrm{Fahr} .\left(-5^{\circ} \mathrm{C}\right.$. $)$; tripalmitin, liquid at I I $5^{\circ}$ Fahr. $\left(45^{\circ}\right.$ C. $)$; tristearin, liquid at $128^{\circ}$ Fahr. $\left(53.66^{\circ}\right.$ C. $)$. Fats are regarded by chemists as compounds of fatty acids with glycerin, and on this account they are sometimes called glycerids. It is incorrect to call them hydrocarbons, a name by which they were formerly known. 
The fatty acids, with the exception of oleic acid, are derived by oxidation from the monatomic alcohols. Oleic acid, however, belongs to the acrylic series. Glycerin is a triatomic alcohol, being formed of three atoms of hydroxyl with glyceril. The type of the neutral fats is triacetin; and the fats should be called triolein, tripalmitin and tristearin, for the reason that in the glycerin which enters into their composition, the three atoms of hydrogen of the hydroxyls are replaced by the acid radicles.

The following are the empirical formulæ for the fats found in the body; triolein, $\mathrm{C}_{3} \mathrm{H}_{5}\left(\mathrm{OC}_{17} \mathrm{H}_{33} \mathrm{CO}\right)_{3}$; tripalmitin, $\mathrm{C}_{3} \mathrm{H}_{5}\left(\mathrm{OC}_{15} \mathrm{H}_{35} \mathrm{CO}\right)_{3}$; tristearin, $\mathrm{C}_{3} \mathrm{H}_{5}\left(\mathrm{OC}_{17} \mathrm{H}_{35} \mathrm{CO}\right)_{3}$. At the temperature of the body, triolein holds the two other fats in solution; and the fats of the body, therefore, are composed of the three varieties and are not solid.

Boiled with an alkali in the presence of water, fats are decomposed into glycerin, which remains free, and the fatty acid, which latter unites with the alkali to form a soap. The following is an example of this decomposition :-

$$
\mathrm{C}_{3} \mathrm{H}_{5}\left(\mathrm{OC}_{15} \mathrm{H}_{31} \mathrm{CO}\right)_{3}+3 \mathrm{KHO}=\mathrm{C}_{3} \mathrm{H}_{5}(\mathrm{OH})_{3}+3 \mathrm{C}_{15} \mathrm{H}_{31} \mathrm{CO} . \mathrm{OK} \text {. }
$$

A similar decomposition takes place under the influence of superheated steam. When a soap is formed, the decomposition is called saponification; when no soap is formed, it is called acidification. The pancreatic juice has the property of acidifying fats.

Shaken up with gummy or mucilaginous mixtures, liquid fats are subdivided into small granules or globules and held permanently in suspension. This is called emulsification; and the form of the fats is changed in this way in digestion. Examples of fatty emulsions in the body are milk and chyle.

The lecithins are very complex fats, the formula of which is $\mathrm{C}_{42} \mathrm{H}_{84} \mathrm{NPO}_{9}$. By decomposition they yield glycerin, phosphoric acid, a fatty acid and cholin. These have been called phosphorized fats.

Cholesterin is a crystallizable monatomic alcohol with the formula $\mathrm{C}_{27} \mathrm{H}_{46} \mathrm{O}$. It is found in large quantity in nervous tissue and also exists in the bile, crystalline lens, spleen, protoplasm of cells and in some other situations. It is taken up from the nervous tissue by the blood, separated from the blood by the liver, discharged into the small intestine with the bile and is eliminated in the form of stercorin $\left(\mathrm{C}_{27} \mathrm{H}_{48} \mathrm{O}\right)$. Stercorin is to be regarded as a product of katabolism, particularly of the nervous tissue. Cholesterin will be treated of more fully in connection with the bile.

Inorganic Alimentary Substances. - It has been shown that all the organs, tissues and liquids of the body contain inorganic matter in 
greater or less quantity. The same is true of vegetable products. All the organic nitrogenous matters contain mineral substances which can not be separated without incineration. When new organic matter is appropriated by the tissues to supply the place of that which has become effete, mineral substances are deposited with them; and the organic matters, as they are transformed into excrementitious substances and discharged from the body, are always thrown off in connection with the mineral substances with which they are associated. This constant discharge of inorganic matters, forming, as they do, an essential part of the organism, necessitates their introduction with the food in order to maintain the normal constitution of the parts. As these matters are necessary to the proper constitution of the body, they must be regarded as alimentary substances.

Water. - This is one of the most important of the constituents of the organism, is found in every tissue and part without exception, is introduced with all kinds of food and is the basis of all drinks. As a rule it is taken in greater or less quantity in a nearly pure state. Although, as a drink, water should be colorless, odorless and tasteless, it always contains more or less saline and other matters in solution, with a certain quantity of air. The air and gases may be driven off by boiling or by reducing the atmospheric pressure. The demand on the part of the system for water is regulated to a certain extent by the quantity discharged from the organism, and this is subject to great variations. The quantity taken as drink also depends on the constitution of the food as regards the water that enters into its composition.

Water is beyond comparison the most important compound in Nature. It is essential to all chemical changes that occur out of the body and to all processes of digestion, anabolism and katabolism. It is the most powerful dissocient known. According to modern views, molecules have no chemical activity and chemical combinations occur only when they are dissociated into ions. Anhydrous hydrochloric acid will not decompose carbonates; pure sulphuric acid has no action on dry litmus; dried ammonia and hydrochloric acid will not combine if brought together; and other illustrations might be given of the action of water in chemical combinations. ${ }^{1}$

Although organic compounds dissociate into ions slowly and feebly, water is the most important agent in their chemical changes. The subject, however, of the possible and probable reactions between proteids and water is so vast, and as yet the results of experiments have

1 According to Dewar, a chemical combination can occur, in the absence of water, at very low temperatures, such as that of liquid air ; but at present, this is the only exception to the general proposition stated above. 
been so indefinite in their applications to physiology, that its discussion would be out of place in this work. It is sufficient to say that these reactions involve hydrolytic cleavages accelerated by various conditions - such as elevation of temperature and the presence of acids and enzymes - and retarded by others. These changes are thought to be due to the dissociation of water and the action of the hydrogen ion. ${ }^{1}$ Applications of these ideas to what may be called general metabolic changes in the living body are evident; and theoretical considerations of this kind might be extended to the digestion of proteids. The hydrolytic changes in carbohydrates have been closely studied and many of them accurately described. It is not too much to say that the most efficient agent in the most important of the digestive, nutritive and katabolic processes probably is dissociated water, its action being favored or retarded by varying conditions.

Sodium Chloride. - Of all saline substances, sodium chloride is the one most widely distributed in the animal and the vegetable kingdoms. It exists in all varieties of food; but the quantity taken in combination with other matters usually is insufficient for the purposes of the economy, and common salt is added to certain articles of food as a condiment, when it improves their flavor, promotes the secretion of certain of the digestive fluids and meets a nutritive demand. Experiments and observations have shown that a deficiency of sodium chloride in food has an unfavorable influence on the general processes of nutrition.

Calcium Phosphate. - This is almost as common a constituent of vegetable and animal food as sodium chloride. It is seldom taken except in combination, particularly with nitrogenous alimentary matters. Its importance in alimentation has been experimentally demonstrated, it having been shown that in animals deprived as completely as possible of this salt, the nutrition of the body, particularly in parts that contain it in considerable quantity, as the bones, is seriously affected.

Iron. - Hemoglobin, the coloring matter of the blood, contains, intimately united with organic matters, a certain proportion of iron. Examples of simple anemia, which are frequently met with in practice and are almost always relieved in a short time by the administration of iron, are evidences of the importance of this substance in alimentation. The quantity of iron discharged from the body is slight, only a trace being discoverable in the urine. A small quantity of iron frequently is introduced in solution in the water taken as drink, and it is a constant constituent of milk and eggs. When its supply in the food is insufficient, it is necessary, in order to restore the normal processes of nutrition, to

1 A very lucid exposition of the action of the hydrogen ion in catalysis is given by Jones, in "The Elements of Physical Chemistry," New York, 1902, p. 456. 
administer it in some form, until its proportion in the organism shall have reached the proper standard.

It is hardly necessary even to enumerate the other inorganic alimentary substances, as nearly all are in such intimate association with nitrogenous matters that they may be regarded as part of their substance. Suffice it to say, that all inorganic matters existing in the organism are found in food. That these are essential to nutrition can not be doubted; but it is evident that by themselves they are incapable of supporting life, as they can not be converted into either nitrogenous or non-nitrogenous organic matters.

Alcohol. - All distilled and fermented liquors and wines contain a greater or less proportion of alcohol. As these are so commonly used as beverages and as the effects of their excessive use are so serious, the influence of alcohol on the organism must be regarded as one of the most important questions connected with alimentation. Some alcoholic beverages influence the functions solely through the alcohol which they contain; while others, as beer and porter, with a comparatively small proportion of alcohol, contain a considerable quantity of solid matter.

Alcohol $\left(\mathrm{C}_{2} \mathrm{H}_{6} \mathrm{O}\right)$, from its composition, is to be classed with the nonnitrogenous substances. It has already been stated that sugar and fat are essential to proper nutrition and that they undergo important changes in the organism. Alcohol is absorbed and taken into the blood; and it becomes a question of importance to determine whether it be consumed in the economy or discharged unchanged by the various emunctories.

Alcohol has long since been recognized in the expired air after it has been taken into the stomach; and late researches have confirmed the earlier observations in regard to its elimination in its original form, and have shown, also, that after it has been taken in quantity, it exists in the blood and all the tissues and organs, particularly the liver and nervous systẹm. Lallemand and Perrin and Duroy have stated, also, that there is a considerable elimination of alcohol by the lungs, skin and kidneys; but the accuracy of the experiments by which these results were arrived at has been questioned. The observations of Anstie and of Dupré have, indeed, thrown doubt on the chromic-acid test for alcohol, which was employed by the French observers above mentioned. Nevertheless, when alcohol has been taken in narcotic doses, there is some alcoholic elimination in the urine, as was shown long ago by Percy. It is certain that most of the alcohol taken in quantities not sufficient to produce alcoholic intoxication is consumed in the organism, and but a trivial quantity is thrown off, either in the urine, the feces, the breath or the cutaneous transpiration. This question is of importance in regard 
to the moderate use of alcohol under normal conditions, and especially in its bearing on the therapeutical action of the various alcoholic drinks administered in cases of disease.

Taken in moderate quantity, alcohol usually produces a certain degree of nervous exaltation which gradually passes off. In some individuals the mental faculties are sharpened by alcohol, while in others they are blunted. There is nothing, indeed, more variable than the immediate effects of alcohol on different persons. In large doses the effects are the well-known phenomena of intoxication, delirium, more or less anesthesia, coma, and sometimes, if the quantity is excessive, death. As a rule, the mental exaltation produced by alcohol is followed by reaction and depression, except in debilitated or exhausted conditions of the system, when alcohol seems to supply a decided want.

The views of physiologists concerning the influence of a moderate quantity of alcohol on the nervous system are somewhat conflicting. That it may temporarily give tone and vigor to the system when the energies are unusually taxed, can not be doubted; but this effect is not produced in all individuals. The constant use of alcohol may create an apparent necessity for it, producing a condition of the system that must be regarded as pathological.

The immediate effects of the ingestion of a moderate quantity of alcohol, continued for a few days, are decided. It notably diminishes the exhalation of carbon dioxide and the discharge of other excrementitious matters, particularly urea. These facts have long since been experimentally demonstrated. Proper mental and physical exercise, tranquillity of the nervous system, and all conditions that favor vigorous nutrition and development of the organism physiologically increase, rather than diminish, the quantity of the excretions, correspondingly increase the demand for food, and if continued, are of permanent benefit. Alcohol, on the other hand, diminishes the activity of nutrition. If its use is long continued, the assimilative powers become so weakened that the proper quantity of food can not be appropriated, and alcohol is craved to supply a self-engendered want. The organism may, in many instances, be restored to its physiological condition by discontinuing the use of alcohol; but it usually is some time before the nutritive powers become active, and alcohol, meanwhile, seems absolutely necessary to existence.

Under ordinary conditions, when the organism can be adequately supplied with food, alcohol is injurious. When the quantity of food is insufficient, alcohol may supply the want for a time and temporarily restore the powers of the body; but the effects of its continued use, conjoined with insufficient nourishment, show that it can not take the 
place of assimilable matters. These effects are too well known to the physician, particularly in hospital-practice, to need further comment. Notwithstanding these undoubted physiological facts, alcohol, in some form, is used by almost every people on the face of the earth, civilized or savage. Whether this be in order to meet some want occasionally felt by and peculiar to the human organism, is a question on which physiologists have found it impossible to agree. That alcohol, at certain times, taken in moderation, soothes and tranquillizes the nervous system and relieves exhaustion dependent on unusually severe mental or physical exertion; can not be doubted. It is by far too material a view to take of existence, to suppose that the highest condition of man is that in which the functions, possessed in common with the lower animals, are perfectly performed. Inasmuch as temporary insufficiency of food, great exhaustion of the nervous system, and various conditions in which alcohol seems to be useful, must of necessity often occur, it is hardly proper that this agent should be absolutely condemned; but it is the article, par excellence, which is liable to abuse; and its effects on the mind and body, when habitually taken in excess, are most serious.

Although alcohol imparts a certain warmth when the system is suffering from excessive cold, it is not proved that it enables men to endure a very low temperature for a great length of time. This end can be effectually attained only by an increased quantity of food. The testimony of Dr. Hayes, the Arctic explorer, is very strong upon this point. He says: "While fresh animal food, and especially fat, is absolutely essential to the inhabitants and travellers in Arctic countries, alcohol is, in almost any shape, not only completely useless but positively injurious. . . Circumstances may occur under which its administration seems necessary; such, for instance, as great prostration from long-continued exposure and exertion, or from getting wet; but then it should be avoided, if possible, for the succeeding reaction is always to be dreaded; and, if a place of safety is not near at hand, the immediate danger is only temporarily guarded against, and becomes, finally, greatly augmented by reason of decreased vitality. If given at all, it should be in very small quantities frequently repeated, and continued until a place of safety is reached. I have known the most unpleasant consequences to result from the injudicious use of whiskey for the purpose of temporary stimulation, and have also known strong able-bodied men to have become utterly incapable of resisting cold in consequence of the longcontinued use of alcoholic drinks." In a paper by General Greely (1887) is the following, which confirms the results of the experience of Hayes: "It seems to me to follow from these Arctic experiences that the regular use of spirits, even in moderation, under conditions of great 
physical hardship, continued and exhausting labor, or exposure to severe cold can not be too strongly deprecated, and that when used as a mental stimulus or as a physical luxury they should be taken in moderation. When habit or inclination induces the use of alcohol in the field, under conditions noted above, it should be taken only after the day's work is done, as a momentary stimulus while waiting for the preferable hot tea and food; or better, after the food, when going to bed, for then it may quickly induce sleep and its reaction pass unfelt."

It is not demonstrated that alcohol increases the capacity to endure severe and protracted bodily exertion. Its influence as a therapeutic agent, in promoting assimilation in certain conditions of defective nutrition, in relieving shock and nervous exhaustion, in sustaining the powers of life in acute diseases attended with rapid emaciation and abnormally active disassimilation, etc., can hardly be doubted; but the consideration of these questions does not belong to physiology. ${ }^{1}$

Coffee. - Coffee is an article consumed daily by many millions of human beings in all quarters of the globe. In armies it has been found almost indispensable, enabling men on moderate rations to perform an amount of labor which would otherwise be impossible. After exhausting efforts of any kind, there is no article that relieves the overpowering sense of fatigue so completely as coffee. Army-surgeons say that at night, after a severe march, the first desire of the soldier is for coffee, hot or cold, with or without sugar, the only essential being a sufficient quantity of the pure article. Almost every one can bear testimony from personal experience to the effects of coffee in relieving the sense of fatigue after mental or bodily exertion and in increasing the capacity for labor, especially mental work, by producing wakefulness and clearness of intellect. From these facts, the importance of coffee, either as an alimentary substance or as taking the place, to a certain extent, of aliment, is apparent.

Except in persons who, from idiosyncrasies, are unpleasantly affected by it, coffee, taken in moderate quantity and at proper times, produces an agreeable sense of tranquillity and comfort, with, however, no disinclination to exertion, either mental or physical. Its immediate influence on the system, which undoubtedly is stimulant, is peculiar

${ }^{1}$ Inasmuch as my views in regard to the physiological effects of alcohol have undergone no material change, I repeat here what has appeared in my "Text-book of Human Physiology" (1888). Recent observations have shown that when alcohol is taken in moderate quantity, about ninety per cent is consumed in the organism (Strassmann). The observations of Rosemann have shown that alcohol may be substituted isodynamically for fats and carbohydrates, when it acts as an alimentary substance. These observations, however, are not opposed to the fact that alcohol in large doses acts as a poison and that its use, in perfect health, is unnecessary and likely to be deleterious. 
and is not followed by reaction or unpleasant after-effects. Habitual use renders coffee almost a necessity, even in those who are otherwise well nourished and subjected to no extraordinary mental or bodily strain. Taken in excessive quantity, or in those unaccustomed to its use, particularly at night, it may produce persistent wakefulness. These effects are so well known that it is often taken for the purpose of preventing sleep.

It has been shown that the use of coffee permits a reduction in the quantity of food, in workingmen especially, much below the standard that would otherwise be necessary to maintain the organism in proper condition. In the observations of De Gasparin on the regimen of the Belgian miners, it was found that the addition of a quantity of coffee to the daily ration enabled them to perform their arduous labors on a diet which was even below that found necessary in prisons where this article was not used. Experiments have shown, also, that coffee diminishes the absolute quantity of urea discharged by the kidneys. In this respect, so far as has been ascertained, the action of coffee is like that of alcohol; and it may reasonably be supposed to retard katabolism, with the important difference that it is followed by no unfavorable after-effects and can be used in moderation for an indefinite time with advantage.

Coffee usually is roasted before an infusion is made. During this process, the grains are considerably swollen, but they lose sixteen or seventeen per cent in weight. A peculiar aromatic substance also is developed by roasting. If torrefaction is pushed too far, much of the agreeable flavor of coffee is lost, and an acrid empyreumatic substance is produced that is disagreeable to the taste.

Tea. - An infusion of the dried and prepared leaves of the tea-plant is perhaps as common a beverage as coffee ; and taking into consideration its large consumption in China and Japan, it actually is used by a greater number of persons. Its effects on the system are similar to those of coffee, but they usually are not so marked. Ordinary tea, taken in moderate quantity, like coffee, relieves fatigue and increases mental activity, but does not usually produce such persistent wakefulness.

Both tea and coffee contain peculiar organic substances. The active principle of tea is called thein, and the active principle of coffee, caffein. As they are supposed to be particularly efficient in producing the peculiar effects on the nervous system characteristic of both tea and coffee, there is good reason to suppose that they are nearly identical in their physiological action. Analyses have shown that thein, or caffein $\left(\mathrm{C}_{8} \mathrm{H}_{10} \mathrm{~N}_{4} \mathrm{O}_{2}+\mathrm{H}_{2} \mathrm{O}\right)$, exists in greater proportion in tea than in coffee; but as a rule, a greater quantity of soluble matter is extracted in the 
preparation of coffee, which may account for its more marked effects on the system.

Chocolate. - Chocolate is made from the seeds of the cocoa-tree, roasted, deprived of their husks and ground with warm rollers into a pasty mass with sugar, flavoring substances being sometimes added. It is then made into cakes, cut into small pieces or scraped to a powder, and boiled with milk or milk and water, when it forms a thick, gruellike drink, which is highly nutritive and has some of the exhilarating properties of coffee or tea. Beside containing a large proportion of nitrogenous matter resembling albumin, the cocoa-seed is rich in fatty matter and contains a peculiar substance, theobromin $\left(\mathrm{C}_{7} \mathrm{H}_{8} \mathrm{~N}_{4} \mathrm{O}_{2}\right)$, analogous to caffein and thein, which is supposed to possess similar physiological properties.

Condiments and Flavoring Articles. - The refinements of cookery involve the use of many articles that can not be classed as alimentary substances. Pepper, capsicum, vinegar, mustard, spices and other articles of this class, so commonly used, have no decided influence on nutrition, except in so far as they promote the secretion of the digestive juices. Common salt, however, is very important, and this has been considered in connection with inorganic alimentary substances. The various flavoring seeds and leaves, truffles, mushrooms etc. have no physiological importance except as they render articles of food more palatable.

Quantity and Variety of Food necessary to Nutrition. - The inferior animals, especially those not subjected to the influence of man, regulate by instinct the quantity and kind of food. The same is true of man during the earliest periods of his existence; but later in life, the diet is variously modified by taste, habit, climate and what may be termed artificial wants. It is usually a safe rule to follow the appetite in regard to quantity, and the tastes, when they are not manifestly vitiated or morbid, in regard to variety. The cravings of nature indicate when to change the form in which nutriment is taken; and that a sufficient quantity has been taken is manifested by a sense, not exactly of satiety, but of evident satisfaction of the demands of the system. During early life, the supply must be a little in excess of the actual loss, in order to furnish materials for growth; during the later periods, the quantity of nitrogenous matter assimilated is somewhat less than the loss; but in adult age, the system is maintained at a tolerably definite standard by the assimilation of matter about equal in quantity to that discharged in the form of excretions.

Although the loss of substance by katabolism creates and regulates the demand for food, it is an important fact, never to be lost sight of, 
that the supply of food has a great influence on the quantity of the excretions. An illustration of this is the influence of food on the exhalation of carbon dioxide; and this is but an example of what takes place in regard to other excretions. The quantity of the excretions is even more strikingly modified by exercise, which, within physiological limits, increases the vigor of the system, provided the increased quantity of food required be supplied.

The Daily Ration. - The daily loss of substance which must be supplied by matters introduced from without is considerable. A large portion of this discharge takes place by the lungs, and a consideration of the mode of introduction of gases to supply part of this waste belongs to the subject of respiration. The most abundant discharge which is compensated by absorption from the alimentary canal is that of water both in a liquid and vaporous condition. The entire quantity of water eliminated daily has been estimated at about four and a half pounds (204I grams), and it is probable that about the same quantity is introduced in the form of drink and as a constituent of the so-called solid articles of food. The quantity taken in the form of drink varies with the character of the food. When the solid articles contain a large proportion of water, the quantity of drink may be diminished; and it is possible, by taking a large quantity of the watery vegetables, to exist entirely without drink. There is no article more frequently taken than water, merely as a matter of habit, any excess being readily removed by the kidneys, skin and lungs. Dalton estimates the daily quantity necessary for a full-grown healthy male, at fifty-four fluidounces ( 530 grams), or 3.38 pounds.

The quantity of solid food necessary to nutrition is shown by estimating the solid matter in the excretions; and the facts thus ascertained correspond closely with the quantity of material which the system has been found to actually demand. The following are the estimated daily losses of the organism:-

Carbon (or its $\left\{\begin{array}{l}\text { Respiration, } 8.825 \text { ounces (250 grams) } \\ \text { Excretions, 2.116 ounces ( } 60 \text { grams) }\end{array} 10941\right.$ ounces (310 grams). equivalent). \{ Excretions, 2.116 ounces ( 60 grams)

Nitrogenous substances (containing 308.64 grains, or 20 grams of nitrogen)

4.586 ounces (130 grams). 15.527 ounces (440 grams).

From this it is estimated that the normal ration, supposing the food to consist of lean meat and bread, is as follows:-

Bread

Meat (without bones)
35.300 ounces (1000 grams). I 0.088 ounces (286 grams). $45 \cdot 388$ ounces (I 286 grams). 
Bread contains Meat contains .

2.469 ounces (70.00 grams) and 10.582 ounces (300.00 grams). 2.125 ounces (60.26 grams) and 1.109 ounces (31.46 grams).

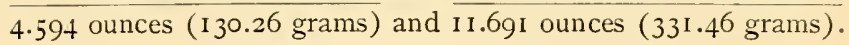

This daily ration, which is purely theoretical, is shown by actual observation to be nearly correct. Dalton says: "According to our own observations, a man in full health, taking active exercise in the open air, and restricted to a diet of bread, fresh meat, and butter, with water and coffee for drink, consumes the following quantities per day:-

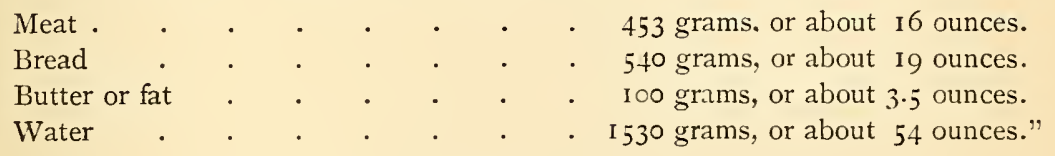

Bearing in mind variations in the nutritive demands of the system in different persons, it may be stated, in general terms, that in an adult male, ten to twelve ounces (282 to 340 grams) of carbon and four to five ounces (II 3 to I 42 grams) of nitrogenous matters, estimated dry, are discharged from the organism and must be replaced by the ingesta; and this demands a daily consumption of between two and three pounds (907 and I36I grams) of solid food, the quantity of food depending, of course, greatly on its proportion of nutritive constituents.

It is true that the daily ration frequently is diminished considerably below the physiological standard in charitable institutions, prisons and elsewhere; but when there is complete inactivity of body and mind, this produces no other effect than that of slightly diminishing the weight and strength. The system then becomes reduced without any actual disease, and there is simply a diminished capacity for labor; but in the alimentation of large bodies of men subjected to exposure and frequently called upon to perform severe labor, the question of food is of great importance, and the men collectively are like a machine in which a certain quantity of material must be furnished in order to produce the required amount of force. This important fact is strikingly exemplified in armies; and the history of the world presents few examples of warlike operations in which the efficiency of the men has not been impaired by insufficient food.

The influence of diet on the capacity for labor was well illustrated by a comparison of the amount of work accomplished by English and French laborers, in I84I, on a railway from Paris to Rouen. The French laborers engaged on this work were able at first to perform only about two-thirds of the labor accomplished by the English. It was suspected that this was due to the more substantial diet of the 
English, which proved to be the fact; for when the French laborers were subjected to a similar regimen, they were able to accomplish an equal amount of work. In all observations of this kind, it has been shown that an animal diet is more favorable to the development of the physical forces than one consisting mainly of vegetables.

It is commonly acknowledged that the consumption of all kinds of food is greater in cold than in warm climates, and almost every one has experienced in his own person a considerable difference in the appetite at different seasons of the year. Travellers' accounts of the quantity of food taken by the inhabitants of the frigid zone seem almost incredible. They speak of men consuming more than a hundred pounds ( 45.36 kilograms) of meat in a day; and a Russian admiral, Saritcheff, gave an instance of a man who, in his presence, ate at a single meal a mess of boiled rice and butter weighing twenty-eight pounds (I 2.7 kilograms). Although it is difficult to regard these statements with entire confidence, the common opinion that the appetite is greater in cold than in warm climates undoubtedly is well founded. Hayes stated, from his personal observation, that the daily ration of the Esquimaux is twelve to fifteen pounds ( 5.443 to 6.804 kilograms) of meat, about one-third of which is fat. On one occasion he saw an Esquimau consume ten pounds (4.536 kilograms) of walrus-flesh and blubber at a single meal, which lasted, however, several hours. The continued low temperature he found had a remarkable effect on the tastes of his own party. With the thermometer ranging from $-60^{\circ}$ to $-70^{\circ} \mathrm{Fahr}$. $\left(-5 \mathrm{I}^{\circ}\right.$ to $-57^{\circ} \mathrm{C}$.), there was a persistent craving for a strong animal diet, particularly fatty substances. Some members of the party were in the habit of drinking the contents of the oil-kettle with evident relish.

Necessity of a Varied Diet. - In considering the nutritive value of the various alimentary substances, the fact that no single article is capable of supplying all the material for the regeneration of the organism has frequently been mentioned. The normal appetite - which is the best guide as regards the quantity and the selection of food-indicates that a varied diet is necessary to proper nutrition. This fact is exemplified in a marked degree in long voyages and in the alimentation of armies, when, from necessity or otherwise, the necessary variety of aliment is not presented. Analytical chemistry fails to show why this change in alimentation is necessary or in what the deficiency in a single kind of diet consists; but it is nevertheless true that after the organic constituents of the organism have appropriated the nutritious elements of particular kinds of food for a certain time, they lose the power of effecting the changes necessary to proper nutrition. This fact is particularly well marked when the diet consists in great part of salted meats, 
although it sometimes occurs when a single kind of fresh meat is constantly used. After long confinement to a diet restricted as regards variety, a supply of other matters, such as fresh vegetables, the organic acids, and articles which are usually called antiscorbutics, becomes indispensable; otherwise, the modifications in nutrition and in the constitution of the blood incident to the scorbutic condition are almost always developed.

It is thus apparent that adequate quantity and proper quality of food are not all that is required in alimentation; and those who have the responsibility of regulating the diet of large numbers of persons must bear in mind the fact that the organism demands considerable variety. Fresh vegetables, fruits etc. should be taken at the proper seasons. It is almost always found, when there is of necessity some sameness in diet, that there is a craving for particular articles, and these, if possible, should be supplied. This was frequently exemplified in the civil war. At times when the diet was necessarily somewhat monotonous, there was an almost universal craving for onions and raw potatoes, which were found by army-surgeons to be excellent antiscorbutics.

With those who supply their own food, the question of variety in diet usually regulates itself; and in institutions, it is a good rule to follow as far as possible the reasonable tastes of the inmates. In individuals, particularly females, it is not uncommon to observe marked disorders in nutrition attributable to want of variety in the diet as well as to an insufficient quantity of food as a matter of education or habit.

A full consideration of the varieties of food and of the different methods employed in its preparation belongs properly to special works on dietetics. Among the ordinary articles of diet, the most important are meats, bread, potatoes, milk, butter and eggs; and it is necessary only to treat of these very briefly.

Meats. - Among the various kinds of muscular tissue, beef has been found to possess the greatest nutritive value. Other varieties of flesh, even that of birds, fishes and animals in a wild state, do not present an appreciable difference, so far as can be ascertained by chemical analysis ; but when taken daily for a long time, they become distasteful, the appetite fails and the system seems to demand a change. The flesh of carnivorous animals is rarely used as food; and animals that eat animal as well as vegetable food, such as pigs or ducks, acquire a disagreeable flavor when they are not fed on vegetables. Soups, broths, and most of the liquid extracts of meat really possess but little nutritive value and they can not replace the ordinary cooked meats. The following is the composition of roasted meat, no dripping being lost, according to the analysis of Ranke, quoted by Pavy :- 
Nitrogenous matters

Fat

Saline matters

Water

27.60

15.45

2.95

54.00

100.00

Bread. - Bread presents a considerable variety of alimentary constituents and is a very important article of diet. The constituents of flour undergo peculiar changes in panification, which give to good bread its characteristic flavor. Bread, especially coarse brown bread, as a single article of food, is capable of sustaining life for a long time. It contains a large proportion of starch, but its important nitrogenous constituent is gluten, which is not a simple substance but contains vegetable fibrin, vegetable albumins, a peculiar substance soluble in alcohol, called glutin, with fatty and inorganic matters. The following is the composition of bread (Letheby):-

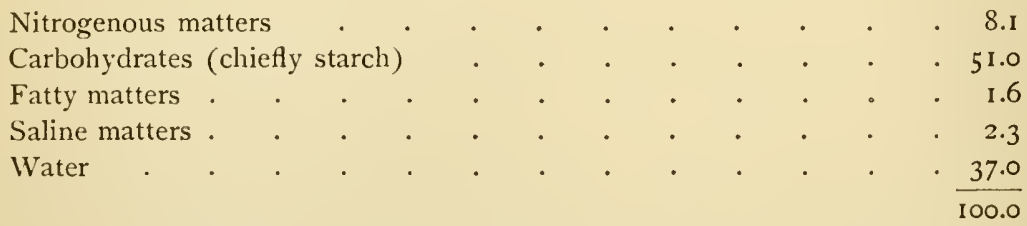

Potatoes. - Potatoes are very useful as an article of diet, especially on account of the agreeable form in which starchy matter is presented; but they contain a small proportion of nitrogenous matter and do not possess so much nutritive value as bread. They are selected from the vegetable foods for description because they are almost universally used in civilized countries throughout the year. They usually are thoroughly cooked, but the raw potato is a valuable antiscorbutic. The following is the composition of potato (Letheby):-

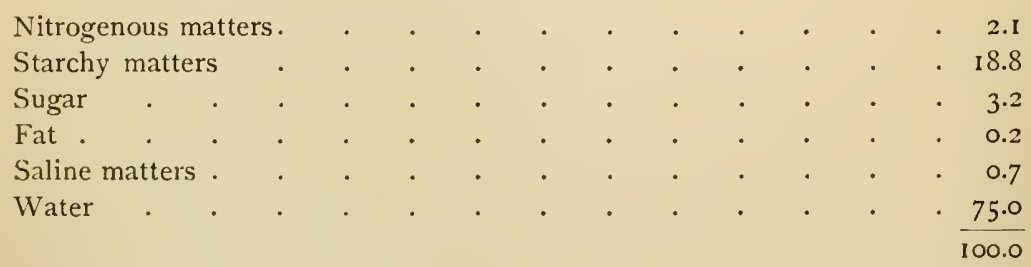

Milk. - Milk, and articles prepared from milk, such as butter and cheese, are important articles of food. In the treatment of disease, milk frequently is used as a single article of diet. On account of the variety of alimentary matters which it contains, including a great number of inorganic salts and even a small quantity of iron, milk will meet all the nutritive demands, probably for an indefinite time. It is largely 
used in the preparation of other articles of food by cooking. Pure butter, which represents the fatty constituents of milk, contains, in IOO parts, 30 parts of olein, 68 parts of palmitin, and 2 parts of other fats peculiar to milk. The following is the composition of cow's milk (Letheby):-

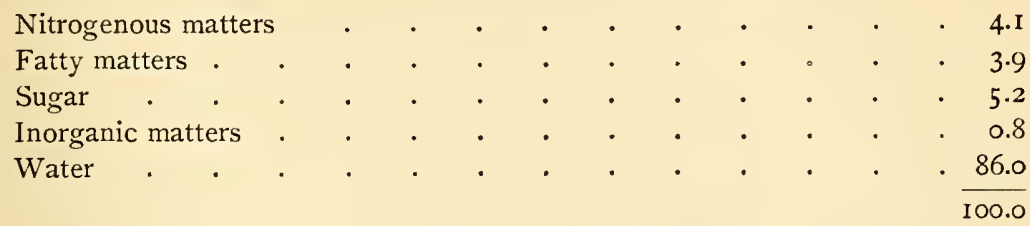

In connection with the composition of human milk, to be given farther on, the great variety of its constituents will be more fully considered.

Eggs. - As regards nutrition, the analogy between eggs and milk is evident when it is remembered that the constituents of eggs furnish materials for the growth of the chick, to which must be added certain saline matters absorbed from the shell during the process of incubation. Among the inorganic constituents of eggs, there is always a small quantity of iron. The following is the composition of the entire contents of the egg (Pavy):-

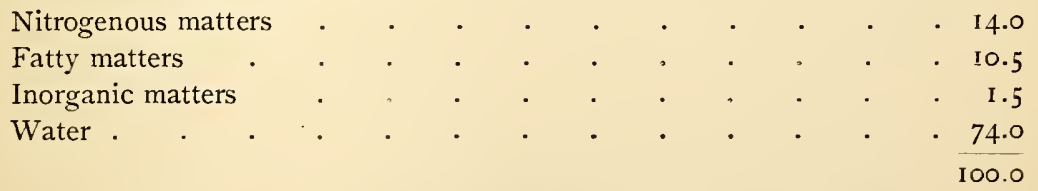

A number of different nitrogenous and fatty matters, a small quantity of saccharine matter, as well as a great variety of inorganic salts, exist in eggs.

The physiological effects of a diet restricted to a single constituent of food or to a few articles have been closely studied both in the human subject and in the inferior animals. Animals subjected to a diet composed exclusively of non-nitrogenous matters die in a short time with symptoms of inanition. The same result follows when dogs are confined to white bread and water; but these animals live very well on the military brown bread, as this contains a greater variety of alimentary matters (Magendie). Facts of this nature were multiplied by the "gelatin commission," and the experiments were extended to nitrogenous substances and articles containing a considerable variety of alimentary matters. In these experiments, it was shown that dogs could not live on a diet of pure myosin, the appetite entirely failing at the forty-third to the fifty-fifth day. They were nourished perfectly well by gluten, 
which is composed of a number of different alimentary substances Among the conclusions arrived at by this commission, which bear particularly on the questions under consideration, were the following:-

"Gelatin, albumin, fibrin, taken separately, do not nourish animals except for a very limited period and in a very incomplete manner. In general, these substances soon excite an insurmountable disgust, to the point that animals prefer to die of hunger rather than touch them.

"The same substances artificially combined and rendered agreeably sapid by seasoning are accepted more readily and longer than if they were isolated, but ultimately they have no better influence on nutrition, for animals that take them, even in considerable quantity, finally die with all the signs of complete inanition.

"Muscular flesh, in which gelatin, albumin and fibrin are united according to the laws of organic nature, and when they are associated with other matters, such as fat, salts etc., suffices, even in very small quantity, for complete and prolonged nutrition" (Paris, I 84I).

In I 769 Dr. Stark, a young English physiologist, fell a victim at an early age to experiments on his own person on the physiological effects of different kinds of food. He lived for forty-four days on bread and water, for twenty-nine days on bread, sugar and water, and for twentyfour days on bread, water and olive-oil. He finally died from the effects of his experiments. 


\section{CHAPTER VII}

\section{MASTICATION, INSALIVATION AND DEGLUTITION}

Physiological anatomy of the organs of mastication - The teeth - Enamel of the teeth Dentin - Cement - Pulp-carity - Superior maxillary bones - Inferior maxilla - Tem. poro-maxillary articulation - Nuscles of mastication - Saliva - Parotid saliva - Submaxillary saliva - Sublingual saliva - Secretions from the smaller glands of the mouth, tongue and pharynx - Mixed saliva - General properties and composition of the saliva Uses of the saliva - Deglutition - Mechanism of deglutition - Protection of the posterior nares during the second period of deglutition - Protection of the opening of the larynx and uses of the epiglottis in deglutition.

INORGANIC alimentary substances, with few exceptions, are introduced in the form in which they exist in the blood and require no preparation or change before they are absorbed; but organic nitrogenous substances are always associated with more or less matter possessing no nutritive properties, from which they must be separated; and even when pure, they undergo certain changes before they are taken up by the blood. The non-nitrogenous matters also undergo changes in constitution or in form preparatory to absorption.

\section{Mastication}

In order that digestion may take place in a perfectly natural manner, it is necessary that the food, as it is received into the stomach, should be so far comminuted and incorporated with the liquids of the mouth as to be readily acted on by the gastric juice; otherwise, gastric digestion is prolonged and difficult.

Physiological Anatomy of the Organs of Mastication. - In the adult, each jaw is provided with sixteen teeth, all being about equally developed. The canines, so largely developed in the carnivora but rudimentary in the herbivora, and the incisors and molars, so fully developed in the herbivora, are, in man, of nearly the same length. Each tooth presents for anatomical description a crown, a neck and a root. The crown is the portion not covered by the gums; the root is the portion embedded in the alveolar cavities of the maxillary bones; and the neck is the portion, sometimes slightly constricted, between the crown and the root and covered by the edge of the gum. Each tooth presents on section several distinct structures. 
Enamel of the Tecth. - The crown is covered with the enamel, which is by far the hardest structure in the economy. This is white and glistening and is thickest on the lower portion of the tooth, especially over the surfaces which, from being opposed to each other on either jaw, are most exposed to wear. It here exists in several concentric layers. The incrustation of enamel gradually becomes thinner

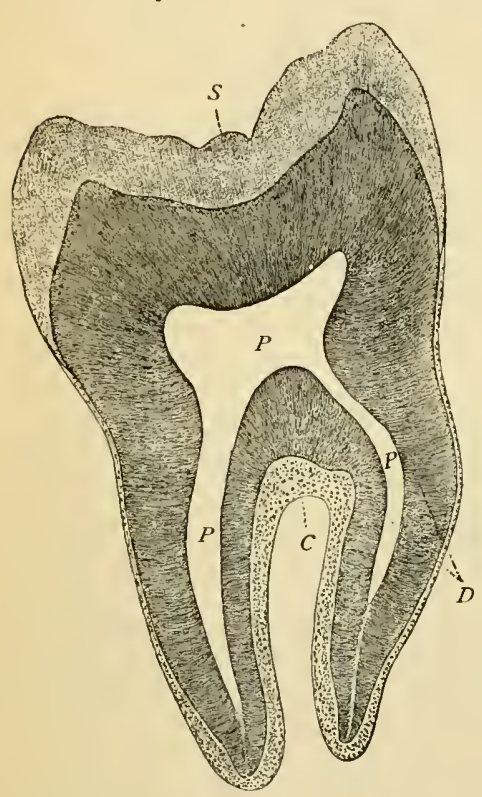

Fig. 34. - Longitudinal section of a molar tooth of man, $\times 4$-reduced onehalf (Sobotta).

This figure gives a general view of the structure of the tooth. The pulpcavity is not cut its whole length in the two roots seen in the section. We recognize the three main elements of the tooth - dentin, enamel and cementum - and their division into crown and root. On account of the low magnification, the interglobular spaces appear only as a dark zone on the surface of the dentin. C, cementum; D, dentin; P, pulpcavity; S, enamel.

toward the neck, where it ceases. The enamel is made up of pentagonal or hexagonal rods, one end resting on the subjacent structure, and the other, when there exists but a single layer of enamel, terminating just beneath the cuticle of the teeth.

The exposed surfaces of the teeth are still further protected by a membrane, $\frac{1}{30000}$ to $\frac{1}{15000}$ of an inch (0.8 to I.7 $\mu$ ) in thickness, closely adherent to the enamel, called the cuticle of the enamel (Nasmyth's membrane). The cuticle presents a strong resistance to reagents and is useful in protecting the teeth from the action of acids that may find their way into the mouth.

Dentin. - The largest portion of the teeth is composed of dentin. In many respects, particularly in its composition, this resembles bone; but it is much harder and does not present the lacunæe and canaliculi, which are characteristic of true osseous structure. The dentin bounds and encloses the central cavity of the tooth, extending in the crown to the enamel, and in the root, to the cement. It is formed of a homogeneous fundamental substance, which is penetrated by a large number of canals radiating from the pulp-cavity toward the exterior. These are called the dentinal tubules or canals. They are $\frac{1}{25000}$ to $\frac{1}{1200 \overline{0}}$ of an inch ( 1 to $2 \mu$ ) in diameter, with walls of a thickness a little less than their calibre. Their course is slightly wavy or spiral. Beginning at the pulp-cavity, into which these canals open, they are found to branch and occasionally anastomose, their communications and branches becoming more frequent as they approach 
the external surface of the tooth. The canals of largest diameter are found next the pulp-cavity, and they become smaller as they branch. The structure forming the walls of these tubules is somewhat denser than the intermediate portion, which is sometimes called the intertubular substance of the dentin; but in some portions of the tooth, the tubules are so abundant that their walls touch each other. Near the origin and near the peripheral ends of the dentinal tubules, are sometimes found solid globular masses of dentin, called dentin-globules, which irregularly bound triangular or stellate cavities of variable size (interglobular spaces). Sometimes these cavities form regular zones near the peripheral ends of the tubules. The dentin is sometimes marked by concentric lines, indicating a lamellated arrangement. In the natural condition, the dentinal tubules are filled with a clear liquid, which penetrates from the vascular structures contained in the pulp-cavity.

Cement. - Covering the dentin of the root, is a thin layer of true bony structure, called cement, or crusta petrosa. This is thickest at the summit and at the deeper portions of the root, where it sometimes is lamellated, and it becomes thinner near the neck. It finally becomes continuous with the enamel of the crown, so that the dentin is everywhere completely covered. The roots of the teeth, covered with the cement, are firmly embedded in the alveolar cavities of the jaws. The alveoli are lined with what is called dental periosteum, which is reflected over the roots as far as the neck, when it becomes continuous with the fibrous tissue of the gums.

Pulp-cavity. - In the interior of each tooth, extending from the apex of the root or roots into the crown, is the pulp-cavity, which contains minute bloodvessels and nervous filaments, held together by longitudinal fibres of connective tissue. This is the only portion of the tooth endowed with sensibility. The bloodvessels and nerves penetrate by a foramen at the extremity of each root.

The dentin and enamel of the teeth must be regarded as perfected structures; for when the permanent teeth are lost, they are not reproduced; and when these parts are invaded by wear or by decay, they are not restored.

The thirty-two permanent teeth are classified as follows:-

I. Eight incisors, four in each jaw, called the central and lateral incisors.

2. Four canines, or cuspidati, two in each jaw, just back of the incisors. The upper canines are sometimes called the eye-teeth, and the lower canines, the stomach-teeth.

3. Eight bicuspids - the small, or false molars - just back of the canines; four in each jaw. 
4. Twelve molars, or multicuspid, situated just back of the bicuspid ; six in each jaw.

The incisors are wedge-shaped, flattened antero-posteriorly, and bevelled at the expense of the posterior face, giving them a sharp cutting edge. Each incisor has a single root. The permanent incisors make their appearance between the seventh and the eighth years.

The canines are more conical and pointed than the incisors, and have longer and larger roots, especially those in the upper jaw. Their roots are single. The permanent canines make their appearance between the eleventh and the twelfth years.

The bicuspid teeth are shorter and thicker than the canines. Their opposed surfaces are rather broad and are marked by two eminences. The upper bicuspids are larger than the lower. The roots are single, but in the upper jaw they are slightly bifurcated at their extremities. The permanent bicuspids make their appearance between the ninth and the tenth years.

The molar teeth, called respectively - counting from before backward - the first, second and third molars, are the largest of all. Their form is that of a cube, rounded laterally and provided with four or five eminences on their opposed surfaces. The first molars are the largest. They usually have three roots in the upper jaw and two in the lower, although they sometimes have four or even five roots. The second molars are but little smaller than the first and resemble them in nearly every particular. The third molars, called the wisdom-teeth, are much smaller than the others and are by no means so useful in mastication. The first molars are the first of the permanent teeth, making their appearance between the sixth and the seventh years. The second molars appear between the twelfth and the thirteenth years; and the third molars, between the seventeenth and the twenty-first years, and sometimes even much later. In some instances the third molars are never developed.

The upper jaw has ordinarily a somewhat longer and broader arch than the lower; so that when the mouth is closed the teeth are not brought into exact apposition, but the upper teeth overlap the lower teeth both in front and laterally. The lower teeth are all somewhat smaller than the corresponding teeth of the upper jaw and usually make their appearance a little earlier.

The superior maxillary bones are immovably articulated with the other bones of the head and do not usually take any active part in mastication. Their inferior borders present fixed surfaces against which the food is pressed by the action of the muscles that elevate the lower jaw. 
The inferior maxilla is a single bone. Its body is horizontal, of a horseshoe shape, and in the alveolar cavities in its superior border, are the lower teeth. Below the teeth, both externally and internally, are surfaces for the attachments of the muscles concerned in the various movements of the jaw and for one of the muscles of the tongue.

Temporo-maxillary Articulation. - In man the articulation of the lower jaw with the temporal bone is such as to allow an antero-posterior sliding movement and a lateral movement, in addition to the movements of elevation and depression. The condyloid process is convex, with an ovoid surface, the general direction of its long diameter being transverse, and slightly oblique from without inward and from before backward. This process is received into a cavity of corresponding shape in the temporal bone - the glenoid fossa - which is bounded anteriorly by a rounded eminence called the eminentia articularis.

Between the condyle of the lower jaw and the glenoid fossa, is an oblong interarticular disk of fibro-cartilage. This is thicker at the edges than in the centre. It is pliable and is so situated that when the lower jaw is projected forward, making the lower teeth project beyond the upper, it is applied to the convex surface of the eminentia articularis and presents a concave surface for articulation with the condyle. One of the uses of this cartilage is to present constantly a proper articulating surface on the articular eminence and thus permit the antero-posterior sliding movement of the lower jaw. It is also important in the lateral movements of the jaw, in which one of the condyles remains in the glenoid cavity and the other is projected, so that the bone undergoes a slight rotation.

Muscles of Mastication. - To the lower jaw are attached certain muscles by which it is depressed, and others by which it is elevated, projected forward, drawn backward and moved from side to side. The following are the principal muscles concerned in the production of these varied movements :-

\section{MUSCLES OF MASTICATION}

\section{Muscles that depress the lower jaw}

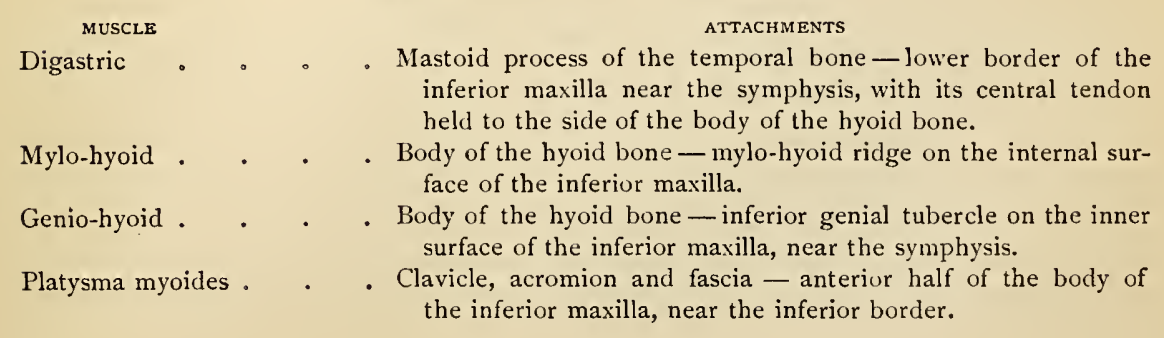


Muscles that elezate the lower jaw and move it laterally and antero-posteriorly

ML'SCLE

Temporal

Masseter

Internal pterygoid .

External pterygoid
ATTACHMENTS

Temporal fossa - coronoid prucess of the inferior maxilla.

- Malar process of the superior maxilla, lower border and internal surface of the zygomatic arch-surface of the ramus of the inferior maxilla.

- Pterygoid fossa - inner side of the ramus, and angle of the inferior maxilla.

Pterygoid ridge of the sphenoid, the surface between it and the pterygoid process, external pterygoid plate, tuberosity of the palate and the superior maxillary bone-inner surface of the neck of the condyle of the inferior maxilla and the interarticular fibro-cartilage.

Action of the Muscles that depress the Lower Jaw. - The most important of these muscles have for their fixed point of action the hyoid bone, which is fixed by the muscles extending from it to the upper part of the thorax. The central tendon of the digastric, as it perforates the stylo-hyoid, is connected with the hyoid bone by a loop of fibrous tissue; and acting from this bone as the fixed point, the anterior belly must of necessity depress the jaw. The attachments of the mylo-hyoid and the genio-hyoid render their action in depressing the jaw sufficiently evident, which is also the case with the platysma myoides, acting from its attachments to the upper part of the thorax. In ordinary mastication the upper jaw undergoes a slight movement of elevation, and this becomes somewhat exaggerated when the mouth is opened to the fullest extent.

Action of the Muscles that elevate the Lower Jaw and move it laterally and antero-posteriorly. - The temporal, masseter and internal pterygoid muscles are chiefly concerned in the simple act of closing the jaws. Their anatomy alone gives a sufficiently clear idea of their mode of action; and their great power is explained by the number of their fibres, by the attachments of many of these fibres to the strong aponeuroses by which they are covered, and by the fact that the distance from their origin to their insertion is very short.

The attachments of the internal and external pterygoids are such that by their alternate action on either side, the jaw may be moved laterally, as their points of origin are situated in front of and internal to the temporo-maxillary articulation. The articulation of the lower jaw is such that in its lateral movements the condyles themselves can not be sufficiently displaced from side to side; but with the condyle on one side fixed or moved slightly backward, the other may be brought forward against the articular eminence, producing a slight movement of rotation.

The above explanation of the lateral movements of the jaw presupposes the possibility of movements in an antero-posterior direction 
Movements in a forward direction, so as to make the lower teeth project beyond the upper, are effected by the pterygoids, the oblique fibres of the masseter and the anterior fibres of the temporal. By the combined action of the posterior fibres of the temporal, the digastric, mylo-hyoid and genio-hyoid, the jaw is brought back to its position. By the same action it may also be drawn back slightly from its normal position while at rest.

Action of the Tongue, Lips and Cheeks, in Mastication. - The varied and complex movements of the tongue during mastication are not easily described. After solid food is taken into the mouth, the tongue prevents its escape from between the teeth, and by its constant movements, rolls the alimentary bolus over and over and passes it at times from one side to the other, so that the food may undergo thorough trituration. Aside from the uses of the tongue as an organ of taste, its surface is endowed with peculiar sensibility as regards the consistence, size and form of different articles; and this is important in determining when mastication is completed, although the thoroughness of mastication is much influenced by habit.

Tonic contraction of the orbicularis oris is necessary to keep the liquids within the mouth during repose; and this muscle is sometimes brought into action when the mouth is full, to assist in keeping the food between the teeth. This latter office, however, is performed mainly by the buccinator; the action of which is to press the food between the teeth and keep it in place during mastication, assisting, from time to time, in turning the alimentary bolus so as to subject new portions to trituration.

Mastication is regulated to a considerable extent by the sensibility of the teeth to impressions of hard and soft substances. It is necessary only to call attention to the ease and certainty with which the presence and the consistence of the smallest substance between the teeth are recognized, to show the importance of this tactile sense in mastication.

\section{Saliva}

The liquid which is mixed with the food in mastication, which moistens the mucous membrane of the mouth and which may be collected at any time in small quantity by the simple act of sputation, is composed of the secretions of a considerable number and variety of glands. The most important of these are the parotid, submaxillary and sublingual, which usually are called the salivary glands. The labial and buccal glands, the glands of the tongue and general mucous surface and certain glandular structures in the mucous membrane of the pharynx also con- 
tribute to the production of the saliva. The liquid that becomes more or less thoroughly incorporated with the food before it reaches the stomach, which must be regarded as the digestive fluid of the mouth, is known as the mixed saliva; but the study of the composition and properties of this as a whole should be prefaced with a consideration of the different secretions of which it is composed. The salivary glands belong to the variety of glands called racemose; and they resemble in their general characters the other glands belonging to this class. One peculiarity, however, in the histology of the secreting alveoli, may be mentioned: Next the basement-membrane, usually near the blind extremity of the tube, are a few crescentic cells called demilunes (Giannuzzi's crescents). In the case of the submaxillary and sublingual glands, at least, these bodies are thought to produce a serous secretion (see Plate IV, Fig. I).

Parotid Saliva. - The parotid is the largest of the three salivary glands. It is situated below and in front of the ear and opens by the duct of Steno into the mouth, at about the middle of the cheek, opposite the second large molar tooth of the upper jaw. The secretion of this gland possesses little or no viscidity.

The organic matter of the parotid saliva is coagulable by heat $\left(212^{\circ}\right.$ Fahr., or $100^{\circ} \mathrm{C}$.), alcohol or the strong mineral acids. The secretion is not so strongly alkaline as the submaxillary and the sublingual saliva and contains a larger proportion of ptyalin. A sulphocyanate is a nearly constant constituent of the parotid saliva. This can not be recognized by the ordinary tests in the fresh saliva taken from the duct of Steno, but in the clear filtered liquid that passes after the precipitation of organic matters, there is a distinct red color on the addition of ferric sulphate. As this reaction is more marked in the mixed saliva, the methods by which the presence of a sulphocyanate is to be recognized will be considered in connection with that liquid. In the human subject, the parotid secretion is more abundant than that of any other of the salivary glands; but the entire quantity in the twentyfour hours has not been directly estimated.

In the horse, ass and ox, it has been found that when mastication is performed on one side of the mouth, the flow from the gland on that side is increased, exceeding by several times the quantity produced on the opposite side. The flow of saliva from the parotid takes place with increased activity during mastication. The opening of the parotid duct is so situated that the liquid is discharged directly upon the food as it is undergoing trituration by the teeth; and as the secretion is more abundant on the side on which mastication is going on and the consistence of the liquid is such as to enable it to mix readily with the food, 
the office of this gland is supposed to be particularly connected with mastication, although its flow continues in small quantity during the intervals. Its quantity is regulated somewhat by the character of the food, being greater when articles taken into the mouth are dry than when they contain considerable moisture. In the human subject, the stimulus produced by sapid substances will sometimes cause a considerable increase in the flow of the parotid saliva. The supposition that the flow from the parotid is dependent on the mechanical pressure of the muscles or of the condyle of the lower jaw during mastication has no foundation in fact.

Submaxillary Saliva. - In the human subject, the submaxillary is the second of the salivary glands in size. Its minute structure is nearly the same as that of the parotid. As its name implies, it is situated beneath the inferior maxillary bone. It is in the anterior part of what is known as the submaxillary triangle of the neck. Its excretory duct - the duct of Wharton - is about two inches ( 5 centimeters) in length and passes from the gland, beneath the tongue, to open by the side of the frenum.

The pure submaxillary saliva presents many important points of difference from the secretion of the parotid. It may be obtained by exposing the duct and introducing a tube, when, on the introduction of any sapid substance into the mouth, the secretion will flow in large pearly drops. This kind of saliva is much more viscid than the parotid secretion. It is clear, and on cooling, it frequently becomes of a gelatinous consistence. Its organic matter is not coagulable by heat. It contains a sulphocyanate, but in small quantity.

The submaxillary gland discharges its secretion in greatest abundance when sapid substances are introduced into the mouth; but unlike the parotid saliva, the secretion does not alternate on the two sides with alternation in mastication. Although sapid articles excite an abundant secretion from the submaxillary glands, they also increase secretion from the parotids and sublinguals; and, on the other hand, movements of mastication increase somewhat the flow from the submaxillaries, and these glands secrete a certain quantity of liquid during the intervals of digestion.

Sublingual Saliva. - The sublinguals, the smallest of the salivary glands, are situated beneath the tongue, on either side of the frenum. In minute structure they resemble the parotid and submaxillary glands. Each gland has a number of excretory ducts, - eight to twenty, which open into the mouth by the side of the frenum; and one of the ducts, larger than the others, joins the duct of the submaxillary gland near its opening in the mouth.

The secretion of the sublingual glands is more viscid, even, than 
the submaxillary saliva, but it differs in the fact that it does not gelatinize on cooling. It is so glutinous that it adheres strongly to any vessel and flows with difficulty from a tube introduced into the duct. Like the secretion from the other salivary glands, its reaction is distinctly alkaline. Its organic matter is not coagulable by heat, acids or the metallic salts.

Secretions from the Smaller Glands of the Mouth, Tongue and Pharynx. - Beneath the mucous membrane of the inner surface of the lips, are small, rounded, glandular bodies, opening into the buccal cavity, called the labial glands; and in the submucous tissue of the cheeks, are similar bodies, called the buccal glands. The latter are somewhat smaller than the labial glands. Two or three of the buccal glands are of considerable size and have ducts opening opposite the last molar tooth. These are sometimes distinguished as the molar glands. There are also a few small glands in the mucous membrane of the posterior half of the hard palate; but the glands on the under surface of the soft palate are larger and form a continuous layer. The glands of the tongue are situated beneath the mucous membrane, mainly on the posterior third of the dorsum ; but a few are found at the edges and the tip, and there is a gland of considerable size on either side of the frenum near the tip. All these are small racemose glands, similar in structure to the true salivary glands. In addition to these structures, the mucous membrane of the tongue is provided with simple and compound follicular glands, which extend over its entire surface, but are most abundant at the posterior portion, behind the circumvallate papillæ.

In the pharynx and the posterior portion of the buccal cavity, are the pharyngeal glands and the tonsils. In the pharynx, particularly the upper portion, racemose glands, like those found in the mouth, exist in large number. The mucous membrane is provided, also, with simple and compound mucous follicles. The tonsils, situated on either side of the fauces between the pillars of the soft palate, consist of an aggregation of compound follicular glands. The number of glands entering into the composition of each tonsil is ten to twenty. The secretion from these glands and follicles can not be obtained, in the human subject, unmixed with the products of the true salivary glands. It has been collected in small quantity, however, from the inferior animals, after ligature of all the salivary ducts. This secretion is simply a grayish viscid mucus, containing a number of leucocytes and desquamated epithelial scales. It is this which gives the turbid and opaline character to the mixed saliva, as the secretions of the salivary glands are all clear. The products of these glands in the mouth are 
mixed with the salivary secretions; and that from the posterior part of the tongue, the tonsils, and the pharyngeal glands passes down to the stomach with the alimentary bolus.

Mixed Saliva. - Although the study of the distinct secretions discharged into the mouth possesses considerable physiological importance, it is only the liquid resulting from their mixture that can properly be considered in connection with the general process of insalivation. On the introduction of food, the quantity of saliva is increased; and the influence of the sight, odor and occasionally even the thought of agreeable articles has already been mentioned.

Quantity of Saliva. - It is not easy to estimate in the human subject the entire quantity of saliva secreted in the twenty-four hours; and considerable variations in this regard exist in different persons and in the same individual at different times. An approximate estimate may be arrived at by noting as nearly as possible the average quantity secreted during the intervals of digestion and adding to it the quantity absorbed by articles of food. The following represents the quantities of saliva secreted during mastication and during the intervals of meals (Dalton):-

Saliva during mastication .

Saliva secreted in intervals of mastication .

Total quantity per day

I7.32 ounces (49I grams).

27.93 ounces (792 grams).

45.25 ounces (I 283 grams).

The total daily quantity of saliva, therefore, is a little more than two and three-fourths pounds.

General Properties and Composition of the Saliva. - The mixed saliva taken from the mouth is colorless, somewhat opaline, frothy and slightly viscid. It has a faint and somewhat disagreeable odor soon after its discharge. If allowed to stand, it deposits a whitish sediment, composed mainly of desquamated epithelial scales with a few leucocytes, leaving the supernatant fluid nearly clear. Its specific gravity is variable, ranging between 1004 or 1006 and 1008 . Its reaction is almost constantly alkaline; although, under certain abnormal conditions, it has been found neutral, and sometimes, though rarely, acid. It becomes slightly opalescent by boiling or on the addition of strong acids; and the addition of absolute alcohol produces an abundant whitish flocculent precipitate. Almost invariably the mixed saliva presents a more or less intense blood-red tint on the addition of a persalt of iron, which is due to the presence of a sulphocyanate either of potassium or of sodium.

A number of analyses of the human mixed saliva have been made by different chemists, presenting, however, few differences, except in 
the relative proportions of water and solid ingredients, which probably are quite variable.

COMPOSITION OF HUMAN SALIVA

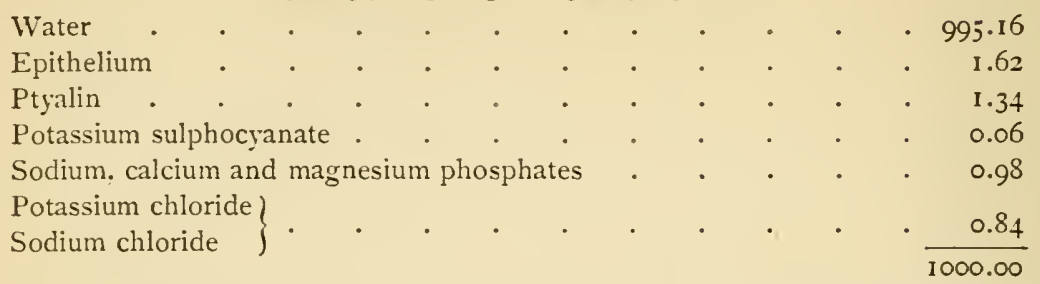

The organic matter of the mixed saliva, called ptyalin, on the addition of an excess of absolute alcohol, is coagulated in the form of whitish flakes, which may readily be separated by filtration. This is the substance described by Mialhe under the name of animal diastase. It has no direct influence on nitrogenous alimentary matters, but when brought in contact with hydrated or soluble starch, readily transforms it, first into dextrin and afterward into maltose. The energy of this action is such that one part is sufficient to effect the transformation of more than two thousand parts of starch.

The presence of a certain quantity of potassium sulphocyanate in the mixed saliva can be demonstrated by the addition of a per-salt of iron. This is a nearly constant and a normal ingredient of the human saliva.

Very little need be said concerning the other inorganic constituents of saliva, except that they are of such a nature as almost invariably to give a distinctly alkaline reaction. They exist in small proportion and do not appear to be connected in any way with the action of the saliva as a digestive secretion.

\section{Uses of the Saliva}

In $183 \mathrm{I}$, Leuchs observed that hydrated starch, mixed with fresh saliva and warmed, was converted into sugar. This fact has since been repeatedly confirmed; and it is now a matter of common observation that boiled starch taken into the mouth almost instantly loses the property of striking a blue color with iodin and responds to the coppertests for sugar. Of the rapidity of this action one can easily convince himself by the simple experiment of taking a little boiled starch into the mouth, mixing it well with the saliva, and testing in the ordinary way. This can hardly be done so rapidly that the reaction does not appear, and the presence of sugar is also indicated by the taste. Although human mixed saliva will finally exert the same action on uncooked starch, the transformation takes place much more slowly. 
Several carbohydrates are formed as intermediate products between starch and sugar by the action of the salivary enzyme. These are not thoroughly understood by physiological chemists for the reason that the size of the different molecules has not been definitely ascertained. The following, however, is perhaps the simplest explanation of these changes. The formula for starch is $\left(\mathrm{C}_{6} \mathrm{H}_{10} \mathrm{O}_{5}\right) n$, the " $n$ " indicating that the molecule is represented by the formula multiplied by an unknown number not less than five. This is changed into soluble starch, which forms a clear solution in water, which filters readily and strikes a blue color with iodin. Its formula is the same as for starch, but the molecule probably is larger. Soluble starch is then converted into dextrin, which has the same formula, but a smaller molecule. At least two kinds of dextrin have been recognized; one, erythrodextrin, strikes a red color with iodin and is readily converted into maltose, while the other, achroödextrin, has no reaction with iodin and is slowly converted into maltose. Leaving out of consideration the size of the molecules, $2\left(\mathrm{C}_{6} \mathrm{H}_{10} \mathrm{O}_{5}\right)+$ $\mathrm{H}_{2} \mathrm{O}=\mathrm{C}_{12} \mathrm{H}_{22} \mathrm{O}_{11}$, which is the formula for maltose. It is probable that some of the starch acted on by the saliva passes into the stomach in the condition of achroödextrin. A certain part, also, may be changed into dextrose $\left(\mathrm{C}_{6} \mathrm{H}_{12} \mathrm{O}_{6}\right)$. A full discussion of these changes, however, belongs to special treatises on physiological chemistry.

The action of the saliva on starch is due entirely to the presence of ptyalin, although its intensity is increased in moderately alkaline solutions or by the addition of certain salts, especially sodium chloride. Feeble acids diminish the activity of this change, and it is arrested by strong mineral acids; although direct experiments have shown that the action of the saliva is slowly and feebly continued in the stomach. The temperature at which the action of the salivary enzyme is most vigorous is about $100^{\circ} \mathrm{Fahr}$. $\left(38^{\circ} \mathrm{C}\right.$.); and any considerable variation from this temperature arrests the process.

In early infancy the action of the saliva on starch is not so vigorous as in the adult; and it is said that immediately after birth the parotid product is the only one of the salivary secretions that contains ptyalin. In a few months, however, ptyalin appears in the submaxillary and sublingual secretions.

It is evident that the saliva, in addition to its mechanical action, transforms a considerable part of starch into sugar; but it is by no means the only secretion engaged in its digestion, similar properties belonging to the pancreatic and the intestinal juices. The last-named secretions are probably more active, even, than the saliva. The saliva acts slowly and imperfectly on raw starch, which becomes hydrated in the stomach and is digested mainly by the liquids of the small intestine. 
In all probability the saliva does not digest all the starch taken as food, the greater part passing unchanged from the stomach into the intestine.

It is undoubtedly the abundant secretion of the parotid glands which becomes most completely incorporated with the food during mastication and which serves to unite the dry particles into a coherent mass. The secretions from the submaxillary and sublingual glands and from the small glands and follicles of the mouth, being more viscid and less in quantity than the parotid secretion, penetrate the alimentary bolus less easily and form a glairy coating on its exterior, agglutinating the particles near the surface.

When the processes of mastication and insalivation have been completed and the food has passed into the pharynx, it meets with the products of the pharyngeal glands, which still further coat the surface with the viscid secretion that covers the mucous membrane in this situation, thus facilitating the first part of deglutition.

It has been observed that the saliva engages bubbles of air in the alimentary mass. In mastication, a considerable quantity of air is mixed with the food, and this facilitates the penetration of the gastric juice. It is well known that moist bread and articles that can not become impregnated in this way with air are not easily acted on in the stomach.

\section{Deglutition}

Deglutition is the act by which solid and liquid articles are passed from the mouth into the stomach. The process involves first, the passage, by an automatic movement, of the alimentary mass through the isthmus of the fauces into the pharynx; then, rapid contraction of the constrictors of the pharynx, by which it is forced into the œesophagus; and finally, a peristaltic action of the muscular walls of the osophagus, extending from its opening at the pharynx to the stomach.

Physiological Anatomy of the Parts concerncd in Deglutition. - The parts concerned in this process are the tongue, the muscular walls of the pharynx and the osophagus. In the passage of food and drink through the pharynx, it is necessary to protect from the entrance of foreign matters a number of openings that are exclusively for the passage of air. These are the posterior nares and the Eustachian tubes above, and the opening of the larynx below.

The tongue is the chief agent in the passage of the alimentary bolus into the pharynx; but a study of all the muscles brought into action would involve anatomical descriptions out of place in this work. The 
movements of the tongue, however, will be described farther on in connection with the mechanism of the first period of deglutition.

The pharynx, in which the most complex of the movements of deglutition take place, is an irregularly funnel-shaped cavity, its longest diameter being transverse and opposite the cornua of the hyoid bone, with its smallest portion at the opening into the œsophagus. Its length

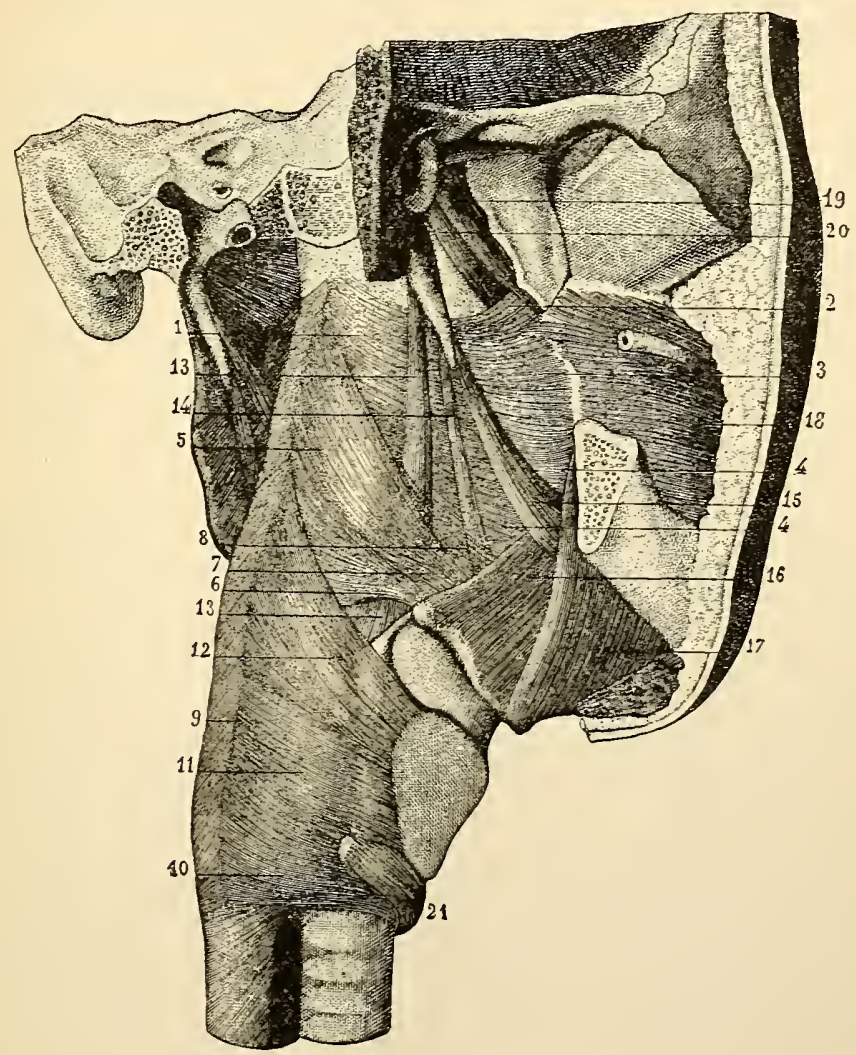

Fig. 35. - Muscles of the pharynx (Sappey).

$\mathrm{I}, 2,3,4,4$, superior constrictor; $5,6,7,8$, middle constrictor; 9 , IO, II, I2, inferior constrictor; I3, I3, stylo-pharyngeus; $x_{4}$, stylo-hyoid muscle; I5, stylo-glossus; I6, hyo-glossus; I7, mylo-hyoid muscle; 18 , buccinator muscle; 19 , tensor palati; 20 , levator palati.

is about four and a half inches (I I.43 centimeters). It is connected superiorly and posteriorly with the basilar process of the occipital bone and with the upper cervical vertebræ. It is incompletely separated from the cavity of the mouth by the velum pendulum palati, a movable musculo-membranous fold continuous with the roof of the mouth and marked by a line in the centre, which indicates its original development by two lateral halves. This, which is called the soft palate, when 
relaxed, presents a concave surface looking toward the mouth, a free arched border, and a conical process hanging from the centre, called the uvula. On either side of the soft palate, are two curved pillars, or arches.

The anterior pillars of the palate are formed by the palato-glossus muscle on either side and run obliquely downward and forward, their membrane becoming continuous with the mucous membrane covering the base of the tongue. The posterior pillars are more closely approximated to each other than the anterior. They run obliquely downward and backward, their mucous membrane becoming continuous with the membrane covering the sides of the pharynx. Between the lower portion of the anterior and posterior pillars, are the tonsils; and in the substance of and beneath the mucous membrane of the palate and pharynx, are small glands, which have already been described.

The isthmus of the fauces, or the strait through which the food passes from the mouth to the pharynx, is bounded above by the soft palate and the uvula; laterally, by the pillars of the palate and the tonsils; and below, by the base of the tongue.

The openings into the pharynx above are the posterior nares and the Eustachian tubes. Below, are the openings of the œsophagus and the larynx.

The muscles of the pharynx are the superior constrictor, the stylopharyngeus, the middle constrictor and the inferior constrictor; and it is easy to see, from the situation of these muscles, how, by their successive action from above downward, the food is passed into the œesophagus.

The muscles forming the fleshy portions of the soft palate are likewise important in deglutition. These are the levator palati, the tensor palati, the palato-glossus and the palato-pharyngeus. The azygos uvulæ, which forms the fleshy portion of the uvula, has no important action in deglutition.

The mucous membrane of the pharynx, aside from the various glands situated beneath it and in its substance, presents certain peculiarities. In the superior portion, which forms a cuboidal cavity just behind the posterior nares, the membrane is darker and much richer in bloodvessels than in other parts. Its surface is smooth and is provided with ciliated epithelium like that which covers the membrane of the posterior nares. Laterally, below the level of the opening of the Eustachian tubes, and posteriorly, at the point where it becomes vertical, the mucous membrane abruptly changes its character. The epithelial covering is here composed of stratified cells similar to those which cover the mucous membrane of the œsophagus; and the membrane is paler and less 
vascular. It is provided with papillæ, some of which are simple conical elevations, while others present two to six conical processes with a single base. These papillæ are rather sparsely distributed over all that portion of the mucous surface which is covered with stratified epithelium.

The contractions of the muscular walls of the pharynx force the alimentary bolus into the œesophagus, a tube with thick muscular walls, extending to the stomach. The œsophagus is about nine inches (23 centimeters) in length. It is cylindrical and slightly constricted at its superior and inferior extremities. Its upper extremity is in the median line behind the lower border of the cricoid cartilage and opposite the fifth cervical vertebra. At first, as it descends, it passes a little to the left of the cervical vertebra. It then passes from left to right from the fourth or fifth to the ninth dorsal vertebra, to give place to the aorta. It finally passes a little to the left again, and from behind forward, to its opening into the stomach. In its passage through the diaphragm, it is surrounded by muscular fibres, so that when this muscle is contracted in inspiration, its action has a tendency to constrict the opening.

The coats of the oesophagus are two in number, unless there be included, as a third coat, the fibrous tissue which attaches the mucous membrane to the subjacent muscular coat.

The external coat is composed of an external longitudinal, and an internal circular, or transverse layer of muscular fibres. In the superior portion, the longitudinal fibres are arranged in three distinct fasciculi; one in front, which passes downward from the posterior surface of the cricoid cartilage, and one on either side, extending from the inferior constrictors of the pharynx. As the fibres descend, the fasciculi become less distinct and finally form a uniform layer. The circular layer is somewhat thinner than the external layer. Its fibres are transverse near the superior and inferior extremities of the tube and are somewhat oblique in the intermediate portion. The muscular coat is $\frac{1}{50}$ to $\frac{1}{12}$ of an inch ( 0.5 to 2.1 millimeters) in thickness.

In the upper third of the œsophagus, the muscular fibres are of the red or striated variety, with some anastomosing bundles; but lower down, there is a mixture of non-striated fibres, which appear first in the circular layer. These latter fibres become gradually more abundant, until, in the lower fourth, they largely predominate. A few striated fibres, however, are found as low down as the diaphragm.

The mucous membrane of the osophagus is attached to the muscular tissue by a dense fibrous layer. It is quite vascular and reddish above, but gradually becomes paler in the inferior portion. The mucous membrane ordinarily is thrown into longitudinal folds, which are obliter 
ated when the tube is distended. Its epithelium is thick, of the squamous variety, and is continuous with and similar to the covering of the lower portion of the pharynx. It is provided with papillæ of the same structure as those found in the pharynx, the conical variety predominating. Racemose mucous glands are found throughout the tube, forming, by their aggregation at the lower extremity just before it opens into the stomach, a glandular ring (see Plate IV, Fig. 2).

Mechanism of Deglutition. - For convenience of description, physiologists usually have divided the process of deglutition into three periods. The first period is occupied by the passage of the alimentary bolus backward to the isthmus of the fauces. This may appropriately be considered as a distinct period, because the movements are effected by the action of muscles under the control of the will. The second period is occupied by the passage of the food from the isthmus of the fauces, through the pharynx, into the upper part of the œsophagus. The third period is occupied by the passage of the food through the œsophagus into the stomach.

In the first period the tongue is the important agent. At the beginning of this period, the mouth is closed and the tongue becomes slightly increased in width, and with the alimentary bolus behind it, is pressed from before backward against the roof of the mouth. The act of swallowing is always performed with difficulty when the mouth is not completely closed; for the tongue, from its attachments, must follow, to a certain extent, the movements of the lower jaw. The first part of the first period of deglutition, therefore, is simple; but when the food has passed beyond the hard palate, it comes in contact with the hanging velum, and muscles are brought into action which render this membrane tense and oppose it in a certain degree to the backward movement of the base of the tongue. This is effected by the action of the tensorpalati and the palato-glossus. The moderate tension of the soft palate admits of its being applied to the smaller morsels, while the opening is dilated somewhat forcibly by masses of greater size.

It is easy to see, in analyzing the first period of deglutition, that liquids and the softer articles of food are assisted in their passage to the isthmus of the fauces by a slight suction force. This is effected by the action of the muscles of the tongue, elevating the sides and depressing the centre of the dorsum, while the soft palate is applied to the base.

The movements in the first period of deglutition are under the control of the will but usually are automatic. When the food has been thoroughly masticated, it requires an effort to prevent the act of swallowing. In this respect, the movements are like the acts of respiration, except that the imperative necessity of air in the system must, in a short 
time, overcome any voluntary effort by which respiration has been arrested.

The second period of deglutition involves more complex and important muscular action than the first. By a rapid succession of movements, the food is made to pass through the pharynx into the œsophagus. The movements are then beyond the control of the will and belong to the kind called reflex. After the alimentary mass has passed beyond the isthmus of the fauces, it is easy to observe a sudden movement of elevation of the larynx, by the action of muscles which usually depress the lower jaw, but are now acting from this bone as the fixed point. The muscles which produce this movement act chiefly on the hyoid bone. They are the digastric (particularly the anterior belly), the mylo-hyoid, the genio-hyoid, the stylo-hyoid and some of the fibres of the genio-glossus. It is probable, also, that the thyro-hyoid acts at this time to draw the larynx toward the hyoid bone. With this elevation of the larynx, there is necessarily an elevation of the anterior and inferior parts of the pharynx, which are, as it were, slipped under the alimentary bolus as it is held by the constrictors of the isthmus of the fauces.

Contraction of the constrictor muscles of the pharynx takes place almost simultaneously with the movement of elevation; and the superior constrictor is so situated as to grasp the morsel of food, and with it the soft palate. The muscles, the constrictors acting from the median raphe, draw up the anterior and inferior walls of the pharynx and pass the food rapidly into the upper part of the osophagus. These complex movements are accomplished with great rapidity, and the larynx and pharynx are afterward returned to their original position.

Protection of the Posterior Nares during the Second Period of Deglutition. - When the act of deglutition is performed with regularity, no part of the liquids and solids swallowed finds its way into the airpassages. The entrance of foreign substances into the posterior nares is prevented in part by the action of the superior constrictors of the pharynx, which embrace, during their contraction, not only the alimentary mass, but the velum pendulum palati itself, and in part, also, by contraction of the muscles forming the posterior pillars of the soft palate. During the first part of the second period of deglutition, the soft palate is slightly raised, being pressed upward by the morsel of food.

While the food is passing through the pharynx, the palato-pharyngeal muscles, which form the posterior pillars of the soft palate, are in a condition of contraction by which the edges of the pillars are nearly approximated, forming, with the uvula between them, almost a complete 
diaphragm between the postero-superior and the antero-inferior parts of the pharynx. This, with the application of the posterior wall of the pharynx to the superior face of the soft palate, completes the protection of the posterior openings of the nasal fossæ.

Protection of the Opening of the Larynx and Uses of the Epiglottis in Deglutition. - The entrance of the smallest quantity of solid or liquid foreign matter into the larynx produces a violent cough. This accident is of not infrequent occurrence, especially when an act of inspiration is inadvertently performed while solids or liquids are in the pharynx. During inspiration, the glottis is opened, and at this time only can a substance of any considerable size find its way into the respiratory passages. Respiration is interrupted, however, during each and every act of deglutition; and there can, therefore, be hardly any tendency at this time to the entrance of foreign substances into the larynx. During a regular act of swallowing, nothing can find its way into the respiratory passages, so complete is the protection of the larynx during the period when the food passes through the pharynx into the œesophagus.

It is evident, from the anatomy of the parts and the necessary results of the contractions of the muscles of deglutition, that while the food is passing through the pharynx, the larynx, by its elevation, passes under the tongue as it moves backward, and the soft base of this organ is, as it were, moulded over the glottis. With the parts removed from the human subject or from one of the inferior animals, the natural movements of the tongue and larynx may be roughly imitated, and it is seen that they must be sufficient to protect the larynx from the entrance of solid or semisolid particles of food. It is impossible for the muscles of the pharynx to contract without drawing together the sides of the larynx, to which they are attached, and assisting to close the glottis. At the same time, as the movements of respiration are arrested during deglutition, the lips of the glottis are more nearly approximated. In addition to this passive and incomplete approximation of the vocal chords, it has been observed that the lips of the glottis are accurately and firmly closed, during each act of deglutition, by contraction of the adductor muscles.

Importance is justly attached to the acute sensibility of the top of the larynx in preventing the entrance of foreign substances. The experiments of dividing all the nervous filaments distributed to the intrinsic muscles show that their action is not essential; but after division of the superior laryngeal - the nerve which gives sensibility to the parts - it has been found that liquids occasionally pass in small quantity into the trachea.

With reference to the action of the epiglottis in contributing to the 
protection of the larynx during the second period of deglutition, observations on the human subject only are to be relied upon. Such observations, in cases of loss of the epiglottis especially, show that this part is necessary to the complete protection of the larynx. While loss of the epiglottis may not interfere always with the perfect deglutition of solids, and even of liquids, particles of food and liquids frequently find their way into the larynx, and deglutition often is effected with difficulty, showing that complete protection of the larynx at all times does not exist unless the epiglottis is intact.

To appreciate the mechanism by which the opening of the larynx is protected during the deglutition of solids and liquids, one has only to carefully follow the articles as they pass over the inclined plane formed by the back of the tongue and the anterior and inferior part of the pharynx. As the food is making this passage in obedience to the contraction of the muscles that carry the tongue backward, draw up the larynx and constrict the pharynx, the soft base of the tongue and the upper part of the larynx are applied to each other, with the epiglottis, which is now inclined backward, between them. At the same time the glottis is closed, in part by the action of the constrictor muscles attached to the sides of the thyroid cartilages and in part by the action of the intrinsic muscles. If the food is tolerably consistent and in the form of a single bolus, it slips easily from the back of the tongue along the membrane covering the anterior and inferior part of the pharynx; but if it is liquid or of soft consistence, a portion takes this course, while another portion passes over the epiglottis, being directed by it into the two grooves by the side of the larynx. It is by these means, together with those by which the posterior nares are protected, that all solids and liquids are passed into the œsophagus and the second period of deglutition is safely accomplished.

The third period of deglutition is the most simple of all. It merely involves contractions of the muscular walls of the œsophagus, by which the food is passed into the stomach. The longitudinal fibres shorten the tube and slip the mucous membrane, lubricated with its glairy secretion, above the bolus; while the circular fibres, by a progressive peristaltic contraction from above downward, propel the food into the stomach. In experiments on the lower animals, it has been observed that while the peristaltic contractions of the upper two-thirds of the tube are immediately followed by a relaxation, which continues until the next act of deglutition, the lower third remains contracted for about thirty seconds after the passage of the food into the stomach. During its contraction, this part of the esophagus is hard, like a cord firmly stretched. This is followed by relaxation; and alternate con- 
traction and relaxation continue, even when the stomach is empty, although, during digestion, the contractions are frequent in proportion to the quantity of food in the stomach. The contraction is always increased by pressing the stomach and attempting to pass some of its contents into the oesophagus. This provision is important in preventing regurgitation of the contents of the stomach, especially when the organ is exposed to pressure, as in urination or defecation.

An approximate estimate of the duration of the acts of deglutition is given in the following quotation from Landois :-

"According to Meltzer and Kronecker, the duration of deglutition in the mouth is 0.3 second; then the constrictors of the pharynx contract 0.9 second; afterward, the upper part of the œesophagus; then after I.8 second, the middle; and after another 3 seconds, the lower constrictor. The closure of the cardia, after the entrance of the bolus into the stomach, is the final act in the total series of movements."

A complete act of deglutition occupies about six seconds. The first act usually is automatic but is under the control of the will. The second act is involuntary when once begun, but it may be excited by the voluntary passage of solids or liquids beyond the velum pendulum palati. It is impossible to perform the second act of deglutition unless there be some article, either solid or liquid, in the pharynx. It is easy to make three or four successful efforts consecutively, in which there is elevation of the larynx, with the other characteristic movements; but a little attention will show that with each act a small quantity of saliva is swallowed. When the efforts have been frequently repeated, the movements become impossible, until time enough has elapsed between them for the saliva to collect.

The position of the body has little to do with the facility with which deglutition is effected. Liquids or solids may be swallowed indifferently in all postures. I have seen a juggler pass a pint of liquid from the mouth to the stomach, while standing on his hands.

It is not very uncommon to find persons who have gradually acquired the habit of swallowing air in order to relieve uncomfortable sensations in the stomach; and when confirmed, this practice occasions persistent disorder in digestion. A number of cases of this kind were reported by Magendie, and in several it was carried to such an extent as to produce great distention of the abdomen. A curious case of habitual airswallowing was observed by the late Dr. Austin Flint and is reported in his work on the Practice of Medicine. 


\title{
CHAPTER VIII
}

\author{
GASTRIC DIGESTION
}

Physiological anatomy of the stomach - Peritoneal coat - Muscular coat - Mucous coat Glands of the stomach - Closed follicles - Gastric juice - Secretion of gastric juice Quantity of gastric juice - Properties and composition of gastric juice - Saline constituents of the gastric juice - Pawlow's experiments on the gastric juice - Action of the gastric juice on meats - Action on albumin, fibrin, casein and gelatin-Action on vegetable nitrogenous substances - Peptones - Action on fats, sugars and amylaceous substances Duration of stomach digestion-Conditions that influence stomach digestion - Movements of the stomach.

\section{Physiological Anatomy of the Stomach}

THE stomach serves the double purpose of a receptacle for food and an organ in which certain important digestive processes take place. It is situated in the upper part of the abdominal cavity and is held in place by folds of the peritoneum and by the osophagus. Its form is not easily described. It has been compared to a bagpipe, which it resembles somewhat, when moderately distended. When empty, it is flattened, and in many parts its opposite walls are in contact. When moderately distended, its length is thirteen to fifteen inches ( 33 to 38 centimeters), its greatest diameter, about five inches ( 12.7 centimeters), and its capacity, one hundred and seventy-five cubic inches (2868 cubic centimeters), or about five pints. The parts usually noted in anatomical descriptions are the following: a greater and a lesser curvature; a greater and a lesser pouch; a cardiac, or œsophageal opening; a pyloric opening, which leads to the intestinal canal. The great pouch is sometimes called the fundus.

The coats of the stomach are three in number; the peritoneal, muscular and mucous. By some anatomists the fibrous tissue which unites the mucous to the muscular coat is regarded as a distinct covering and is called the fibrous coat.

Peritoneal Coat. - This coat is a layer of peritoneum, similar in structure to the membrane covering the other abdominal viscera. It is a reflection of the membrane that lines the general abdominal cavity, which, on the viscera, is somewhat thinner than it is on the walls of the cavity. Over the stomach the peritoneum is $\frac{1}{300}$ to $\frac{1}{200}$ of an inch $(83$ to $125 \mu)$ in thickness. It is a serous membrane and consists of 
ordinary fibrous tissue with a considerable number of elastic fibres. It is closely adherent to the subjacent muscular coat and is not very abundantly supplied with bloodvessels and nerves. Lymphatics have been demonstrated only in the subserous structure. The surface of the peritoneum is everywhere covered with regularly-polygonal cells of endothelium, closely adherent to each other and presenting a smooth surface moistened with a small quantity of liquid.

IIuscular Coat. - Throughout the alimentary canal, from the cardiac opening of the stomach to the anus, the muscular fibres forming the middle coat are of the non-striated variety. These fibres are pale, with

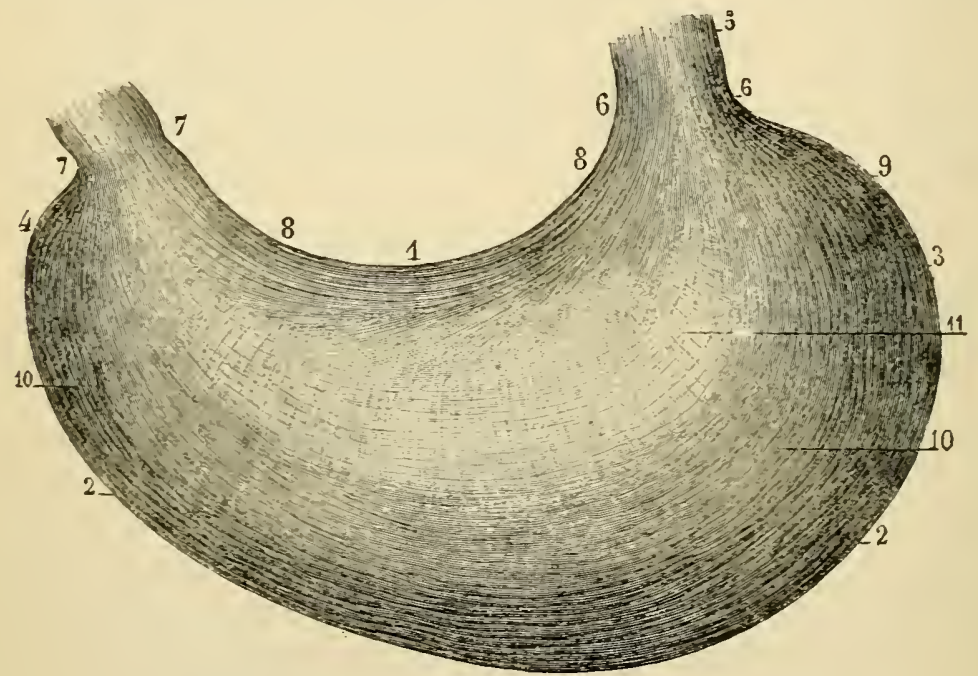

Fig. 36. - Longitudinal fibres of the stomach (Sappey).

I, lesser curvature; 2,2 , greater curvature; 3 , greater pouch; 4 , lesser pouch; 5,6 , 6, lower end of the œsophagus; 7,7 , pylorus; 8,8 , longitudinal fibres at the lesser curvature; 9 , fibres extending over the greater curvature; IO, IO, a very thin layer of longitudinal fibres over the anterior surface of the stomach; II, circular fibres seen through the thin layer of longitudinal fibres.

faint outlines, fusiform or spindle-shaped, containing each an oval longitudinal nucleus. They are closely adherent by their sides and are so arranged as to dovetail into each other, forming sheets of greater or less thickness depending upon the number of their layers. The muscular coat of the stomach varies in thickness in different animals. In the human subject it is thickest in the region of the pylorus and is thinnest at the fundus. Its average thickness is about $\frac{1}{25}$ of an inch ( 1 millimeter). In the pylorus its thickness is $\frac{1}{16}$ to $\frac{1}{12}$ of an inch (I.6 to 2.I millimeters), and in the fundus, $\frac{1}{50}$ to $\frac{1}{36}$ of an inch ( 0.5 to 0.7 millimeter).

The muscular fibres are in two principal layers; an external longitudinal layer and an internal circular layer, with a third layer of oblique 
fibres extending over the great pouch only, which is internal to the circular layer. The longitudinal fibres are continued from the œsophagus and are most marked over the lesser curvature. They are not continued very distinctly over the rest of the stomach. The circular and oblique fibres are best seen with the organ everted and the mucous membrane removed. The circular layer is not very distinct to the left of the cardiac opening, over the great pouch. Toward the pylorus, the layers of fibres are thicker, and at the opening into the duodenum, they form a powerful muscular ring, sometimes called the sphincter of the pylorus, or the pyloric muscle. At this point they project considerably

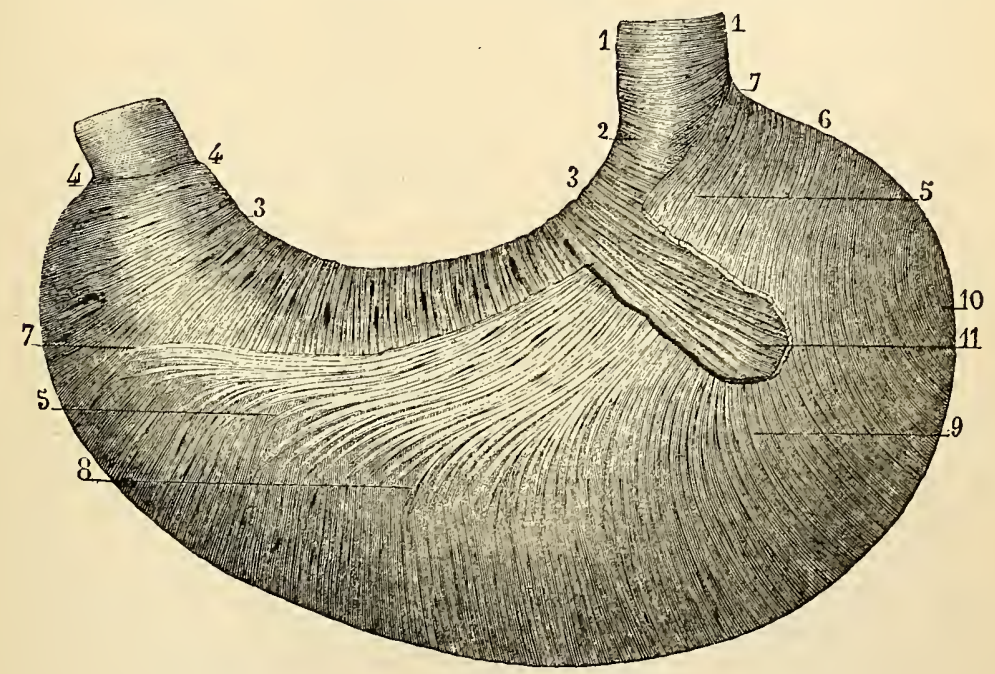

Fig. 37. - Fibres seen with the stomach everted (Sappey).

I, I, œsophagus; 2, circular fibres at the œsophageal opening; 3,3 , circular fibres at the lesser curvature; 4,4 , circular fibres at the pylorus; $5,5,6,7,8$, oblique fibres; 9 , 10, fibres of this layer covering the greater pouch; II, portion of the stomach from which these fibres have been removed to show the subjacent circular fibres.

into the interior of the organ and cease abruptly at the opening into the duodenum, so as to form a sort of valve, presenting, when contracted, a flat surface looking toward the intestine. The oblique layer takes the place, in great part, of the circular fibres over the great pouch. It extends obliquely over the fundus from left to right and ceases at a distinct line extending from the left margin of the œsophagus to about the junction of the middle with the last third of the great curvature. At about the line where the oblique,layer of fibres ceases, the stomach becomes constricted during the movements incident to digestion, dividing the organ into two tolerably distinct compartments. The pyloric division has lately been called the antrum pylori; and the rather 
thickened band of fibres next the line of division is known as the sphincter antri pylori (Hofmeister and Schütz).

The bloodvessels of the muscular coat are quite abundant and are arranged in the peculiar rectangular network which they always present in the non-striated muscular tissue. The nerves come from the pneumogastrics and the sympathetic system and are demonstrated with difficulty.

Mucous Coat. - The mucous membrane of the stomach is soft and velvety in appearance and of a reddish gray color. It is loosely attached to the submucous muscular tissue and is thrown into wide longitudinal folds, which become effaced as the organ is distended. If the mucous membrane is stretched or if the stomach is everted and dis-

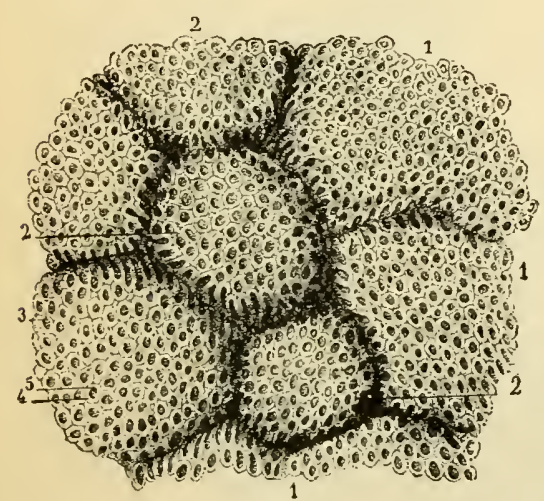

Fig. 38. - Pits in the mucous membrane of the stomach and openings of the glands, $\times 20$ (Sappey).

$I, I, I, 2,2,2,3$, pits of different sizes ; 4,5 , orifices of the gastric glands.

tended and the mucus gently removed under a stream of water, the membrane will be found marked with polygonal pits, or depressions, enclosed by ridges, which, in some parts of the organ, are quite regular. These are best seen with the aid of a simple lens, as many of them are quite small. The diameter of the pits is variable, but the average is about $\frac{1}{2} \frac{1}{0}$ of an inch (O.125 millimeter). This appearance is not distinct toward the pylorus; the membrane here presenting irregular conical projections and well-marked villi resembling those found in. the small intestine. The surface of the mucous membrane is covered with columnar or prismoidal epithelium, the cells being regular in shape, each with a clear nucleus and a distinct nucleolus.

The thickness of the mucous membrane of the stomach varies in different parts. Usually it is thinnest near the œesophagus and thickest near the pylorus. Its thinnest portion measures $\frac{1}{75}$ to $\frac{1}{50}$ of an inch ( 0.34 to 0.5 millimeter); its thickest portion, $\frac{1}{16}$ to $\frac{1}{12}$ of an inch ( 1.6 to 2. I millimeters), and the intermediate portion, about $\frac{1}{25}$ of an inch (I millimeter).

Glands of the Stomach. - Extending from the bottoms of the pits in the mucous membrane of the stomach to the submucous connective tissue, are large numbers of glands. These are arranged often in distinct groups, surrounded with fibrous tissue, each group belonging to one of the polygonal depressions. The tissue which connects the tubes 
is dense but not abundant. There are marked differences in the anatomy of the glands in different parts of the stomach, which are supposed to correspond with differences in the uses of various parts of the mucous membrane. There are, indeed, two distinct varieties of glands; the peptic glands, which secrete propepsin, and the acid-glands, which are supposed to secrete free hydrochloric acid. The peptic glands are most abundant in the pyloric portion of the stomach and around the

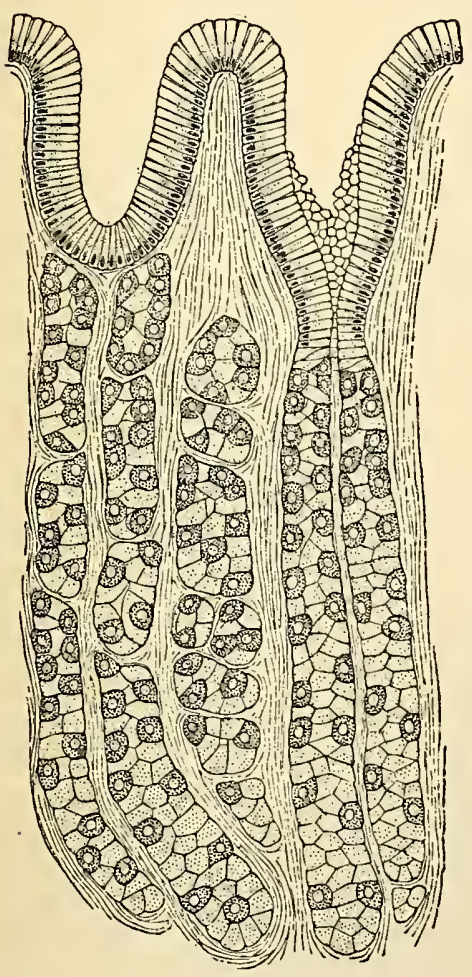

Fig. 39. - Glands of the greater pouch of the stomach (Heidenhain).

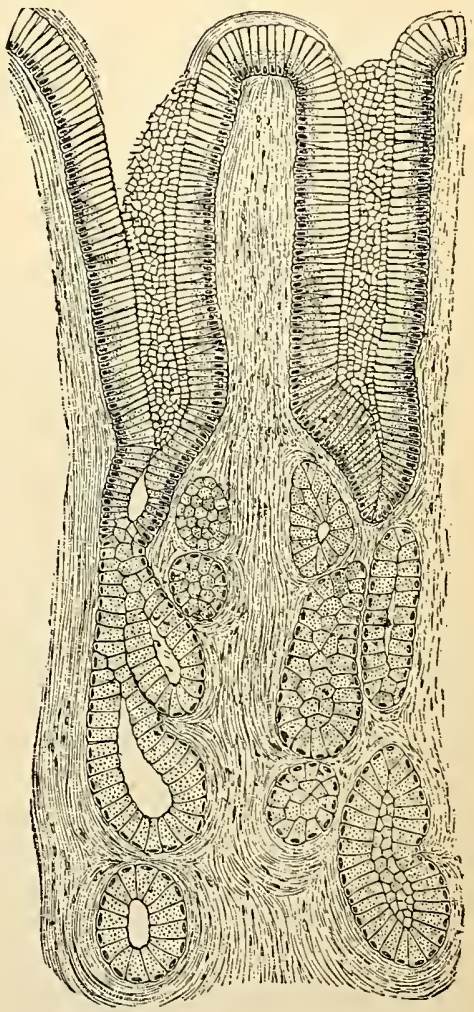

Fig. 40.-Pyloric glands (Ebstein).

cardiac opening. The so-called acid-glands are found throughout the mucous membrane, especially in the greater pouch. The secretion in the pyloric portion of the stomach is not acid at any time, while the secretion in the greater pouch during digestion is always strongly acid.

The pyloric glands are lined with cells that may be called peptic cells (the "chief cells" of some writers). These are conoidal or cuboidal in form, and relatively clear, especially during the intervals of digestion. Similar cells are found, in connection with the so-called acid-cells 
(parietal cells), in the secreting portion of the glands of the greater pouch.

The acid-glands are found throughout the stomach except near the pylorus. The secreting portion of these glands contains peptic cells, but near the tubular membrane are rounded cells, larger than the peptic cells, darker and more granular, which are the acid, or parietal cells. These are strongly stained when treated with osmic acid. It is probable that the so-called acid-glands secrete propepsin as well as an acid, while the pyloric glands secrete propepsin but no acid. In accordance with the views just stated, in the glands of the greater pouch, the acid is secreted by the rounded acid-cells, while the propepsin is secreted by cells (peptic cells) similar to those lining the secreting portion of the pyloric glands. During the intervals of digestion, propepsin is in process of formation by the peptic cells, and no acid is produced; but acid begins to be secreted soon after food is received into the stomach. The peptic cells, therefore, do not produce pepsin directly, but a substance sometimes called zymogen, but more properly propepsin or pepsinogen, which is changed into pepsin, possibly by the action of hydrochloric acid.

The glands of the stomach have an excretory portion and a secreting portion, the latter presenting two or more branches. The excretory portion is lined with cells nearly like those found on the surface of the mucous membrane. The secreting portion is lined with the peptic and the acid-cells already described (see Plate IV, Fig. 3).

Closed Follicles. - In the substance of the mucous membrane, between the tubes and near their cæcal extremities, are occasionally found closed follicles, like the solitary glands and patches of Peyer of the intestines. These are not always present in the adult but frequently are found in children. They usually are most abundant over the greater curvature, though they may be found in other situations. In their anatomy they are identical with the closed follicles of the intestines and do not demand special consideration in this connection.

Gastric Juice. - The observations of Beaumont on Alexis St. Martin, the Canadian who had a large fistulous opening into the stomach, gave the first definite knowledge of the most important of the physiological properties of the gastric juice. The following was the method employed in collecting the secretion: The subject was placed on the right side in the recumbent posture, the valve of mucous membrane partially closing the fistula was pressed into the stomach, and a gum-elastic tube, of the size of a large quill, was passed in to the extent of five to six inches ( 12 to 5 centimeters). On turning him upon the left side until the opening became dependent, the stimulation of the tube caused the secretion to flow, sometimes in drops and sometimes in a small stream. 
Since the publication of Beaumont's experiments, many observations have been made on animals in which permanent gastric fistulæ had been established. In these experiments the dog is most frequently used, as in this animal the operation usually is successful. The animals operated upon by Bassow, who was the first to establish a gastric fistula (1842), were merely objects of curiosity; but Blondlot (1843) and others fixed a tube in the stomach, collected the juice and made important observations in regard to its action in digestion.

Although instances of gastric fistula in the human subject had been reported before the case of St. Martin, and have been observed since that time, the remarkably healthy condition of the subject and the extended experiments of Beaumont have rendered this case memorable in the history of physiology. This was the only instance on record in which normal gastric juice had been obtained from the human subject; and it has served as a standard of comparison for subsequent experiments on the inferior animals.

An artificial gastric juice, prepared by extracting the active prin-

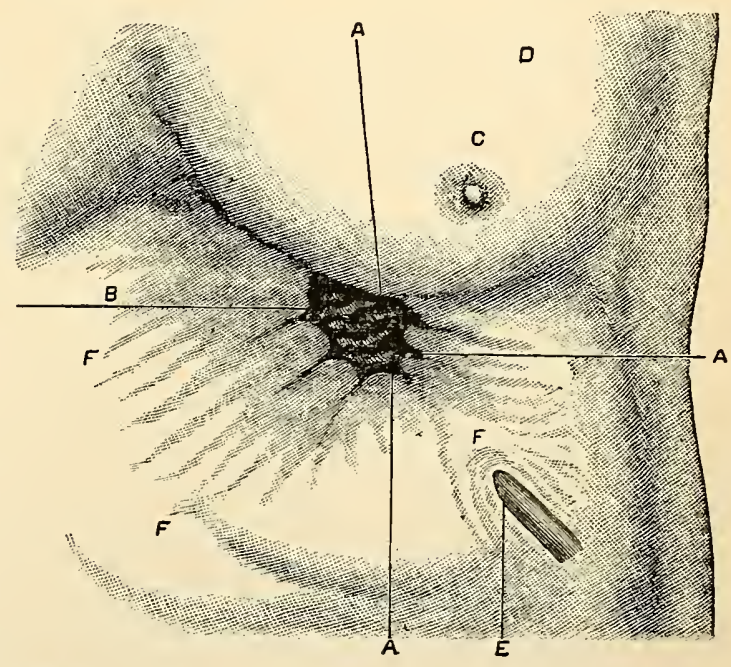

Fig. 4I. - Gastric fistula in the case of St. Martin (Beaumont).

$A, A, A, B$, borders of the opening into the stomach; $C$, left nipple; $D$, chest; $E$, cicatrices from the wound made for the removal of a piece of cartilage; $F, F, F$, cicatrices of the original wound.

ciple from the mucous membrane of the stomach of different animals and adding hydrochloric acid, is useful in observations in regard to the chemistry of the peculiar enzyme, but liquids prepared in this way are not identical with the natural secretion. Extracts of the mucous membrane were made by Eberle (1834), Von Wittich, Brücke and many others.

Secretion of Gastric Juice. - It was observed by Beaumont that during the intervals of digestion the mucous membrane is comparatively pale, "and is constantly covered with a very thin, transparent, viscid mucus, lining the whole interior of the organ." On the introduction of food, the membrane changes its appearance. It then becomes red and turgid with blood; small pellucid points begin to appear in various 
parts, which are drops of gastric juice; and these gradually increase in size until the liquid trickles down the sides in small streams. The membrane is now invariably of a strongly acid reaction, while at other times it is either neutral or faintly alkaline. The thin watery liquid thus produced is the true gastric juice.

While natural food is the proper stimulus for the stomach, and while, in normal digestion, the quantity of gastric juice is perfectly adapted to the work it has to perform, it has been noted that savory and highly seasoned articles ordinarily produce a more abundant secretion than those which are comparatively insipid. An abundant secretion is likewise excited by some of the vegetable bitters and even by the sight or odor of articles of food.

The recent observations of Pawlow, of St. Petersburg, recall the early brilliant experiments of Claude Bernard. Pawlow established an opening in the stomach in the following way: He separated a small portion of the organ from the main cavity completely, so that there remained only an external opening into the portion thus isolated. This is called Pawlow's pouch, or miniature stomach. After feeding, the gastric juice flowed from this pouch clear and unmixed with débris of food. He was thus enabled to obtain pure gastric juice and study its properties, and to avoid sources of error in experiments made with gastric juice artificially prepared. ${ }^{1}$

In the experiments of Pawlow - some of whose conclusions are certainly remarkable, not to say extravagant - it appeared that the juice flowing from the miniature stomach after feeding with different articles presented marked differences in proteolytic activity. The proteolytic action was most vigorous after feeding with bread; and the juice collected at this time he called "bread-juice." The proteolytic power was measured by noting the quantity of food, contained in a graduated tube, that was dissolved in a certain time; and bread-juice was found to have an activity of 6.64 millimeters. The juice obtained after feeding with meat had an activity of 3.39 millimeters. The activity after feeding with milk was still less, being only 3.26 millimeters. Perhaps the most striking observations were made on dogs with "sham-feeding." In these experiments, the œesophagus was divided in the neck, leaving two openings, so that the food swallowed did not pass into the stomach. When food was taken in this way, pure gastric juice flowed in abun-

${ }^{1}$ MM. Cade and Latarjet have recently observed a case of " miniature stomach" of nearly twenty years' standing in a young girl, following a hernia of part of the stomach in the median line, occurring in early infancy. This little pouch had become isolated from the rest of the organ. Nearly all the observations of Pawlow on dogs were confirmed in this case. The fistula was finally closed by operation. - Journal de physiologie et de pathologie générale, Paris, Mars, 1905, tome vii, p. 221. 
dance from the stomach. It was noted, also, that the sight, odor, or even idea of food, particularly in animals having a "passionate longing," excited a flow of juice. The secretion thus obtained, Pawlow called psychic juice. He found also that all the digestive excretions were much increased in quantity when the appetite was good and food was relished. According to these observations, "the properties of the juice correspond with the requirements of the food. The starchholding diet receives a juice rich in amylolytic ferment, the fat-containing, a juice with much fat-splitting ferment. This is manifest from the strength of the juice, but still more so from the absolute quantities of the ferment."

It has long been known that impressions made on the gustatory nerves have a marked influence in exciting the action of the mucous membrane of the stomach; and that in most animals, particularly when they are very hungry, the sight and odor of food often will set up a secretion of both saliva and gastric juice. Febrile conditions, the depression resulting from an excess in eating and drinking, and even purely mental conditions, such as anger or fear, vitiate, diminish and sometimes entirely suppress secretion by the stomach. At some times, under these conditions, the mucous membrane becomes red and dry, and at others it is pale and moist.

After the food has been in part liquefied and absorbed and in part reduced to a pultaceous consistence, the secretion of gastric juice ceases; the movements of the stomach having gradually forced that portion of the food which is but partially acted on in this organ or is digested only in the small intestines out at the pylorus. The stomach is thus entirely emptied, the mucous membrane becomes pale, and its reaction loses its acid character, becoming neutral or faintly alkaline.

Quantity of Gastric Juice. - Data for determining the quantity of gastric juice secreted in the twenty-four hours are so uncertain that it seems impossible to fix on any estimate that can be accepted even as an approximation. Still, the quantity must be considerable, in view of the large amount of alimentary matter acted on in gastric digestion. It probably is not less than six pounds (2.72 kilograms) or more than fourteen pounds ( 6.35 kilograms). After this secretion has performed its office in digestion, it is reabsorbed, and but a small quantity exists in the stomach at any one time.

Properties and Composition of Gastric Juice. - The juice taken from the stomach during the first moments of its secretion and separated from mucus and foreign matters by filtration is a clear liquid, of a faint yellowish or amber tint and possessing little or no viscidity. Its reaction is always strongly acid. The specific gravity of the gastric juice 
in the case of St. Martin, according to the observations of Beaumont and Silliman, was I005; but later, F. G. Smith found it in one instance, I008, and in another, I009. There is every reason to suppose that the secretion, in the case of St. Martin, was normal, and I005 to Ioog may be taken as the range of specific gravity in the human subject.

The gastric juice, if kept in a well-stoppered bottle, will retain its chemical and physiological properties for an indefinite period. The only change that it undergoes is the formation of a pellicle, consisting of a vegetable confervoid growth, some of which breaks up and falls to the bottom of the vessel, forming a whitish flocculent sediment. In addition to this remarkable property of resisting putrefaction, putrefactive changes are arrested in decomposing animal matters, both when taken into the stomach and when exposed to the action of the gastric juice out of the body.

The following analysis by Bidder and Schmidt gives the mean of nine observations on dogs:-

\section{COMPOSITION OF THE GASTRIC JUICE OF THE DOG}

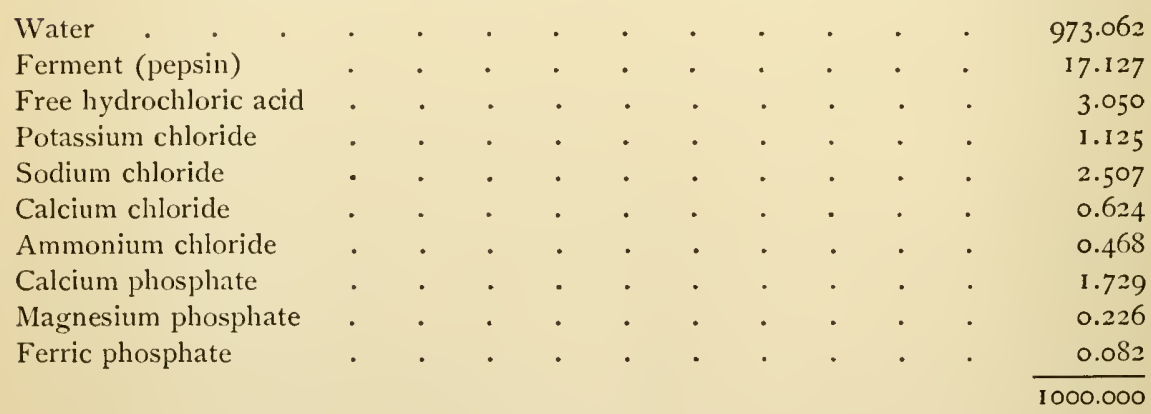

In another series of three observations, in which the saliva was allowed to pass into the stomach, the proportion of free acid was 2.337, and the proportion of organic matter was slightly increased.

Organic Constituent of the Gastric Juice. - Pepsin is a ferment, or enzyme, peculiar to the gastric juice and essential to its digestive properties. When the gastric secretion was first obtained, even by the imperfect methods employed anterior to the observations of Beaurnont and of Blondlot, an organic matter was spoken of as one of its constituents. The gastric juice also contains a milk-curdling enzyme.

Frce Acid of the Gastric Juice. - The character of the free acid was long a question of uncertainty and dispute; but physiologists now adopt the view that the gastric juice contains free hydrochloric acid, with possibly a small quantity of lactic acid. It is admitted, however, that the 
degree of acidity is variable, and that the normal acid may be replaced, without loss of the digestive properties of the secretion, by lactic, oxalic, acetic, formic, succinic, tartaric, citric, phosphoric, nitric or sulphuric acid.

Saline Constituents of the Gastric Juice. - It has been shown that artificial mixtures containing the organic matter of the gastric juice and the proper proportion of free acid are endowed with the digestive properties of the normal secretion from the stomach, and that these properties are rather impaired when an excess of its normal saline constituents is added or when the relation of the salts to the water is disturbed by concentration; but physiologists attach little importance to the saline constituents, except sodium chloride, which is thought to be concerned in the production of hydrochloric acid.

Action of the Gastric Juice in Digestion. - Certain of the substances most readily attacked by the gastric juice are acted on by weak acid solutions containing no organic matter; but it is now well established that the presence of a peculiar organic matter is a condition indispensable to actual digestion. It has also been shown that liquids containing the organic constituent of the gastric juice have no digestive properties unless they also possess the proper degree of acidity; and it is as well settled that liquids containing acids alone have no action on albumins similar to that which takes place in digestion, and that when these substances are dissolved, it is simply accidental.

The presence of any one particular acid does not seem essential to the digestive properties of the gastric juice, so long as the proper degree of acidity is preserved; and it is important that the normal acid can be replaced by other acids, for in case any salt were introduced into the stomach which would be decomposed by the acid of the gastric juice, digestion would be interfered with, unless the liberated acid could take its place.

In studying the physiological action of the gastric juice, it must be borne in mind that the general process of digestion is accomplished by the combined as well as the successive action of the different digestive secretions. The act should be viewed in its ensemble, rather than as a process consisting of several successive and distinct operations, in which different classes of alimentary matters are dissolved by distinct liquids. The food meets with the gastric juice, after having absorbed a large quantity of saliva; and it passes from the stomach to be acted on by the intestinal secretions, having imbibed both saliva and gastric juice.

When the acts which take place in the mouth are properly performed, the following alimentary substances, comminuted by the action 
of the teeth and thoroughly insalivated, are taken into the stomach: muscular tissue with the muscular substance enveloped in its sarcolemma, bloodvessels, nerves, ordinary fibrous tissue holding the muscular fibres together, interstitial fat, and a small quantity of albumins and corpuscles from the blood, all combined with a considerable quantity of inorganic salts; albumin, sometimes unchanged, but usually more or less thoroughly coagulated; fatty matters, sometimes in the form of oil and sometimes enclosed in vesicles, constituting adipose tissue; gelatin and animal matters in a liquid form extracted from meats, as in soups; casein, in its liquid form, united with butter and salts in milk, and coagulated, in connection with various other matters, in cheese ; vegetable nitrogenous matters, of which gluten may be taken as the type; vegetable fats and oils; sugars, from both the animal and vegetable kingdoms, but chiefly from vegetables; different kinds of amylaceous substances; and finally, organic acids and salts, derived chiefly from vegetables. These matters, particularly those from the vegetable kingdom, are united with more or less innutritious matter, such as cellulose. They also are seasoned with aromatic substances, condiments etc., which are not directly used in nutrition.

The various articles described as drinks are taken without any considerable admixture with the saliva. They embrace water and certain nutritious or stimulant infusions (including alcoholic beverages) with a small proportion of inorganic salts in solution.

Action of the Gastric Juice on Meats. - The digestion of meat in the stomach is far from complete. The parts of the muscular structure most easily attacked are the fibrous tissue holding the muscular fibres together, and the sarcolemma, or sheath of the fibres themselves. If the gastric juice of the $\mathrm{dog}$ is placed in a vessel with finely chopped lean meat and kept in contact with it for a number of hours at about $100^{\circ} \mathrm{Fahr} .\left(37.78^{\circ} \mathrm{C}\right.$.), agitating the vessel occasionally so as to subject, so far as possible, every particle of the meat to its action, the filtered liquid will be found increased in density, its acidity diminished, and presenting all the evidences of having dissolved a considerable portion of the tissue. There always, however, will remain a certain portion that has not been dissolved. Its constitution is nevertheless materially changed; for it no longer possesses the ordinary character of muscular tissue and easily breaks down between the fingers into a pultaceous mass. On subjecting this residue to microscopical examination, it is found not to contain any ordinary fibrous tissue; and the fibres of muscular tissue, although presenting the well-marked and characteristic striæ, are broken into short pieces and have very little tenacity. It is evidently only the muscular substance that remains, the connective 
tissue and the sarcolemma having been dissolved. Even on adding fresh juice to the undigested matter, this is not dissolved to any considerable extent, the residue not being sensibly diminished in quantity, and the muscular substance still presenting the characteristic striation.

Whether the gastric juice be incapable of acting on the muscular substance or not, the above-mentioned facts clearly show that muscular tissue usually is not completely digested in the stomach. The action of the gastric juice is to dissolve the intermuscular fibrous tissue and the sarcolemma, or sheath of the muscular fibres, setting the true muscular substance free and breaking it up into small particles. The mass of tissue is thus reduced to a thin pultaceous condition, and it passes into the small intestine, where its digestion is completed. The constituents of the blood, albumins, corpuscles etc., which may be introduced in small quantity in connection with muscular tissue, probably are completely dissolved in the stomach.

Action on Albumin, Fibrin, Casein and Gelatin. - The action of the gastric juice on uncooked white of egg is to disintegrate its structure, separating and finally disșolving the membranous sacs in which the albumins are contained. It also acts on the albumins, forming albumin-peptones, which, unlike albumin, are not coagulated by heat or acids, but are precipitated by alcohol, tannin and many of the metallic salts. The digestion of raw or imperfectly-coagulated albumins takes place with considerable rapidity in the stomach; and the digestion of albumins in this form is more rapid than when they have been coagulated by heat. It is a matter of common as well as of scientific observation, that eggs when hard-boiled are less easily digested than when they are soft-boiled or uncooked. The products of the digestion of raw or of coagulated albumins (albumin-peptones) are essentially the same. It is probable that the entire process of digestion and absorption of albumins takes place in the stomach; and if any albumins pass out of the pylorus, the quantity is small.

Fibrin, as distinguished from myosin, is not a very important article of food. The action of the gastric juice upon it is more rapid and complete than on albumins. The well-known action on fibrin, of water slightly acidulated with hydrochloric acid, has led some physiologists to assume that the acid is the only constituent in the gastric juice necessary to the digestion of this substance; but observations on the comparative action of acidulated water and of artificial or natural gastric juice show that the presence of the organic matter is necessary to the digestion of this as well as of other nitrogenous alimentary substances. The action of water containing a small proportion of acid is to render fibrin soft and transparent, frequently giving to the entire mass a jelly- 
like consistence. The result of the digestion of fibrin, in the gastric juice or in an acidulated fluid to which pepsin has been added, is its complete solution and transformation into a substance that is not affected by heat, acids or by rennet. The substance resulting from the action of gastric juice on fibrin, called fibrin-peptone, resembles the albumin-peptones, but has certain distinctive characters.

Liquid casein is immediately coagulated by the gastric juice, by the action of both the free acid and an organic matter called rennin (chymosin). Once coagulated, casein is acted on in the same way as coagulated albumen. Casein taken as an ingredient of cheese is digested in the same way. The casein of human milk, which coagulates only into a sort of jelly, is more easily digested than casein from cow's milk. The product of the digestion of casein is a soluble substance, not coagulable by heat or the acids, called casein-peptone.

Gelatin is rapidly dissolved in the gastric juice, when it loses the characters by which it is ordinarily recognized and no longer forms a jelly on cooling. This substance is much more rapidly disposed of than the tissues from which it is formed; and the products of its digestion in the gastric juice resemble the substances resulting from the digestion of the albumins.

Action on Vegetable Nitrogenous Substances. - These substances, of which gluten may be taken as the type, are digested to a considerable extent in the stomach. Uncooked gluten is acted on much in the same way as fibrin, and cooked gluten behaves like coagulated albumen. Vegetable articles of food usually contain gluten in greater or less quantity, or substances resembling it, as well as various non-nitrogenous matters and cellulose. The fact that these articles are not easily attacked in any portion of the alimentary canal, unless they have been well comminuted in the mouth, is shown by the passage of grains of corn, beans etc., in the feces. When properly prepared by mastication and insalivation, the action of the gastric juice is to disintegrate them, dissolving out a portion of the nitrogenous matters, freeing starch and other matters so that they may be more easily acted on in the intestines, and leaving the hard indigestible matters, such as cellulose, to pass away in the feces. The nitrogenous constituents of bread are probably acted on in the stomach in the same way and to the same extent as albumins, fibrin and casein.

Peptones. - The peptones in solution form colorless liquids, having a feeble odor resembling that of meat. They are not coagulable by heat or by most acids, a property that distinguishes them from nearly all the nitrogenous constituents of food. They are coagulated, however, by many of the metallic salts, and by chlorin or tannin in slightly 
acidulated solutions. On evaporating peptones to dryness, the residue consists of a yellowish white substance, resembling desiccated white of egg. This is soluble in water, when it regains its characteristic properties, but is insoluble in alcohol.

It is evident that the gastric juice, aside from its action in preparing certain articles for digestion by the intestinal secretions, does not simply liquefy certain of the alimentary matters, but changes them in such a way as to render them osmotic and provides against the coagulation which is so easily induced in other proteids. Peptones readily pass through membranes.

Another, the most important and the essential change exerted by the gastric juice on proteids, is that by which they are rendered capable of assimilation by the system after their absorption. Pure albumin and gelatin, injected into the blood, are not assimilable and are thrown off by the kidneys; but albumin and gelatin that have been digested in gastric juice are assimilated in the same way as though they had penetrated by the natural process of absorption from the alimentary canal. The same is true of casein and fibrin. The action of pepsin is essential to the changes that occur in the albuminous alimentary matters, resulting in the formation of the various peptones; and the change into peptones takes place in all nitrogenous substances that are dissolved in the stomach. This may occur even when the albuminous matters are somewhat advanced in putrefaction; and the gastric juice possesses antiseptic properties, which accounts for the frequent innocuousness of animal substances in various stages of decomposition when taken into the stomach.

The change of proteids into peptones in the stomach is not direct; but the intermediate changes, probably, simply are hydrolytic processes. The proteids are changed by the gastric juice into a class of substances known as proteoses, which includes products from the albumins, globulins, gelatins etc., in their various stages of change. The actual conditions between the proteids of food and peptones, treating them as albumin, probably are the following: Albumin is first converted into parapeptone (acid-albumin, syntonin); this is converted into propeptone, which includes $(a)$ protoalbumose, $(b)$ heteroalbumose and $(c)$ deuteroalbumose. These finally are converted into peptone and their digestion is complete.

Action on Fats, Sugars and Amylaceous Substances. - Most of the fatty constituents of food are liquefied at the temperature of the body; and when taken in the form of adipose tissue, the vesicles in which the fatty matters are contained are dissolved, the fat is set free, is melted and floats in the form of drops of oil on the alimentary mass. The 
action of the stomach, then, seems to be to prepare the fats, chiefly by dissolving the adipose vesicles, for the complete digestion that takes place in the small intestine.

The varieties of sugar of which glucose is the type undergo little if any change in digestion and probably are to some extent directly absorbed by the mucous membrane of the stomach. This is not the case, however, with the varieties classed with cane-sugar. It has been shown that cane-sugar injected into the veins of a living animal is not assimilated but is immediately rejected by the kidneys. When, however, it has been inverted into dextrose and levulose by the action of a dilute acid or by digestion in the gastric juice, it no longer behaves as a foreign substance and does not appear in the urine. Experiments have shown that cane-sugar, after digestion for several hours in the gastric juice, is slowly inverted. This action does not depend on any constituent of the gastric juice except the free acid; and a dilute mixture of hydrochloric acid had an equally marked effect. Experiments in artificial digestion have shown that cane-sugar is inverted by the gastric juice very slowly, the action of this secretion in no way differing from that of very dilute acids. In the natural process of digestion, this action may take place to a certain extent; but it is not shown to be constant or important.

The action of gastric juice, unmixed with saliva, on starch is negative, so far as any transformation into sugar is concerned. When the starch is enclosed in vegetable cells, it is set free by the action of the gastric juice on the nitrogenous parts. Raw starch in the form of granules becomes hydrated in the stomach, on account of the elevated temperature and the acidity of the contents of the organ. This is not the form, however, in which starch usually is taken by the human subject; but when it is so taken, the stomach evidently assists in preparing it for the more complete processes of digestion that are to take place in the small intestine.

Cooked or hydrated starch, the form in which it exists in bread, farinaceous preparations generally and ordinary vegetables, is not affected by the pure gastric juice and passes out at the pylorus unchanged. It must be remembered, however, that the gastric juice does not entirely prevent a continuance of the action of the saliva; and experiments have shown that gastric juice taken from the stomach, when it contains a notable quantity of saliva, has, to a certain extent, the power of transforming starch into sugar.

The changes which vegetable acids and salts, the various inorganic constituents of foods and the liquids that are classed as drinks undergo in the stomach are very slight. Most of these substances can hardly be 
said to be digested; for they are either liquid or in solution in water and are capable of direct absorption and assimilation. In regard to most of the inorganic salts, they either exist in small quantity in the ordinary water taken as drink or are united with organic nitrogenous substances. In the latter case, they become intimately combined with the organic matters resulting from gastric digestion. It has been noted that the various peptones contain the same inorganic salts that existed in the nitrogenous substances from which they were formed.

Duration of Stomach Digestion. - Inasmuch as comparatively few articles, and these belonging exclusively to the class of organic nitrogenous substances, are completely dissolved in the stomach, it is evident that the length of time during which food remains in this organ, or the time occupied in the solution of food by gastric juice out of the body, does not represent the absolute digestibility of different articles.

There has certainly never been presented so favorable an opportunity for determining the duration of gastric digestion as in the case of St. Martin. From a great number of observations made on digestion in the stomach itself, Beaumont came to the conclusion that "the time ordinarily required for the disposal of a moderate meal of the fibrous parts of meat, with bread etc., is three to three and a half hours." The observations of F. G. Smith, made upon St. Martin many years later, gave two hours as the longest time that aliments remained in the stomach. In a case of intestinal fistula reported by Busch, it was noted that food began to pass out of the stomach into the intestines fifteen minutes after its ingestion and continued to pass for three or four hours, until the stomach was emptied. The average time that food remains in the stomach after an ordinary meal may be stated to be between two and four hours.

Milk is one of the articles digested in the stomach with greatest ease. Its highly nutritive properties and the variety of its nutritious constituents render it most valuable as an article of diet, particularly when the digestive powers are impaired and when it is important to supply the system with considerable nutriment. Eggs are likewise highly nutritious and are easily digested. Raw and soft-boiled eggs are more easily digested than hard-boiled eggs. "Whipped" eggs are apparently disposed of with great facility. As a rule the flesh of fish is more easily digested than that of the warm-blooded animals. Oysters, especially when raw, are quite easy of digestion. The flesh of mammals seems to be more easily digested than the flesh of birds. Of the different kinds of meat, venison, lamb, beef and mutton are easily digested, while veal and fat pork are digested with difficulty. Soups usually are very easily digested. The animal substances that are 
digested most rapidly, however, are tripe, pigs' feet and brains. Vegetable articles are digested in about the same time as ordinary animal food; but a great part of the digestion of these substances takes place in the small intestine. Bread is digested in about the time required for the digestion of the ordinary meats.

Conditions that influence Stomach Digestion. - The various conditions that influence gastric digestion, except those relating exclusively to the character or the quantity of food, operate mainly by modifying the quantity and quality of the gastric juice. It is seldom that temperature has any influence, for the temperature of the stomach in health does not present variations sufficient to have any marked effect on digestion.

As a rule, gentle exercise, with repose or agreeable and tranquil occupation of the mind, is more favorable to digestion than absolute rest. Violent exercise or severe mental or physical exertion is always undesirable immediately after the ingestion of a large quantity of food, and as a matter of common experience, has been found to retard digestion.

The effects of sudden and considerable loss of blood on gastric digestion are very marked. After a full meal, the whole alimentary tract is deeply congested, and this condition undoubtedly is necessary to the secretion, in proper quantity, of the various digestive liquids. When the entire quantity of blood in the economy is greatly diminished from any cause, there is difficulty in supplying the amount of gastric juice necessary for a full meal, and disorders of digestion are likely to occur. This is also true in inanition, when the quantity of blood is greatly diminished. In this condition, although the system craves nourishment and the appetite frequently is ravenous, food should be taken in small quantities at a time.

As a rule children and young persons digest food that is adapted to them more easily and in larger relative quantity than those in adult life or in old age; but ordinarily in old age digestion is carried on with more vigor and regularity than the other vegetative processes, such as general assimilation, circulation and respiration.

Influence of the Nervous System on the Stomach. - It is well known that mental emotions frequently have a marked influence on digestion, and this, of course, can take place only through the nervous system. Of the two nerves that are distributed to the stomach, the pneumogastric has been the more carefully studied, experiments on the sympathetic being more difficult. The history of the influence of the pneumogastrics on digestion, however, properly belongs to the physiology of the nervous system. 
Movements of the Stomach. - As food is passed into the stomach, the organ gradually changes its form, size and position. When the stomach is empty, the opposite surfaces of its lining membrane are in contact in many parts and are thrown into longitudinal folds. As the organ is distended, these folds are effaced, the stomach itself becoming more rounded, and as the two ends, with the lesser curvature, are comparatively immovable, the stomach undergoes a movement of rotation, by which the anterior face becomes superior and is applied to the diaphragm. At this time the great pouch has nearly filled the left hypochondriac region; and the great curvature presents anteriorly and comes in contact with the abdominal walls. Aside from these changes, which are due merely to distention, the stomach undergoes important movements, which continue until its contents have been dissolved and absorbed or have passed out at the pylorus; but while these movements are taking place, the two orifices are guarded, so that the food shall remain for the proper time exposed to the action of the gastric juice. By the rhythmical contractions of the lower extremity of the osophagus, regurgitation of food is prevented; and the circular fibres, which form a thick ring at the pylorus, are constantly contracted, so that - at least during the first periods of digestion — only liquids and that portion of food which has been reduced to a pultaceous consistence can pass into the small intestine. It is well known that this resistance at the pylorus does not endure indefinitely, for indigestible articles of considerable size, such as stones, have been passed by the anus after having been introduced into the stomach; but observations have shown that masses of digestible matter are passed by the movements of the stomach to the pylorus, over and over again, and that they do not find their way into the intestine until they have become softened and more or less disintegrated.

The contractions of the walls of the stomach are of the kind characteristic of non-striated muscular fibres. If the finger is introduced into the stomach of a living animal during digestion, it is gently but rather firmly grasped by a contraction, which is slow and gradual, enduring for a few seconds and as slowly and gradually relaxing and extending to another part of the organ. The movements during digestion present certain differences in different animals; but there can be no doubt that the phenomenon is universal. In dogs, when the abdomen is opened soon after the ingestion of food, the stomach appears pretty firmly contracted on its contents. In a case reported by Todd and Bowman, in the human subject, in which the stomach was much hypertrophied and the walls of the abdomen were very thin, the vermicular movements could be distinctly seen. These movements were active, resembling the 
peristaltic movements of the intestines, for which, indeed, they were mistaken, as the nature of the case was not recognized during life.

A peculiarity in the movements of the stomach - which has been repeatedly observed in the lower animals, particularly dogs and cats, and in certain cases has been confirmed in the human subject - is that at about the junction of the cardiac two-thirds with the pyloric third, there is a transverse band of fibres (the sphincter antri pylori) so firmly contracted as to divide the cavity into two almost distinct compartments. It has also been noted that the contractions in the cardiac division are much less vigorous than near the pylorus; the stomach seeming simply to adapt itself to the food by a gentle pressure as it remains in the great pouch, while in the pyloric portion, the movements are more frequent, vigorous and expulsive.

As the result chiefly of the observations of Beaumont, the following may be stated as a summary of the physiological movements of the stomach in digestion:-

The stomach normally undergoes no movements until food is passed into its cavity. When food is received, at the same time that the mucous membrane becomes congested and the secretion of gastric juice begins, contractions of the muscular coat occur, which are at first slow and irregular, but become more vigorous and regular as the process of digestion advances. After digestion has become fully established, the stomach usually is divided, by the firm and almost constant contraction of an oblique band of fibres, into a cardiac and a pyloric portion (the antrum pylori); the former occupying about two-thirds, and the latter, one-third of the length of the organ. The contractions of the cardiac division of the stomach are uniform and rather gentle; while in the pyloric division they are intermittent and more expulsive. The effect of the contractions of the stomach on the food contained in its cavity is to subject it to a nearly uniform pressure in the cardiac portion, the general tendency of the movement being toward the pylorus along the greater curvature and back from the pylorus toward the great pouch along the lesser curvature. At the constricted part which separates the cardiac from the pyloric portion, there is an obstruction to the passage of the food until it has been sufficiently acted on by the secretions in the cardiac division to have become reduced to a pultaceous consistence. The alimentary mass then passes into the pyloric division, and by a more powerful contraction than occurs in other parts of the stomach, it is passed into the small intestine. The classical observations of Beaumont have been in the main confirmed by Hofmeister and Schuitz, Cannon and others.

The revolutions of the alimentary mass, thus accomplished, take 
place slowly, by gentle and persistent contractions of the muscular coat; the food occupying two or three minutes in its passage entirely around the stomach. Every time that a revolution is accomplished, the contents of the stomach are somewhat diminished in quantity; probably, in a slight degree, from absorption of digested matter by the stomach itself, but chiefly by the gradual passage of the softened and disintegrated mass into the small intestine. This process continues until the stomach is emptied, lasting between two and four hours ; after which, the movements of the stomach cease until food is again introduced.

Regurgitation of food by contractions of the muscular coats of the stomach, eructation, or the expulsion of gas, and vomiting are not physiological acts. It has been shown that vomiting is produced by contractions of the abdominal muscles and the diaphragm, compressing the stomach, which is passive, except that the pyloric opening is firmly closed, the cardiac opening being relaxed. Eructation, although usually involuntary, is sometimes under the control of the will. When it occurs, while it is difficult or impossible to prevent the discharge of the gas, the accompanying sound may easily be suppressed. Eructation frequently becomes a habit, which in many persons is so developed by practice that the act may be performed voluntarily at any time. The gaseous contents of the stomach during digestion consist of oxygen, carbon dioxide, hydrogen and nitrogen, in variable proportions. 


\section{CHAPTER IX}

\section{INTESTINAL DIGESTION}

Physiological anatomy of the small intestine - Mucous membrane - Intestinal juice - Action of the intestinal juice in digestion - Pancreatic juice - Internal secretion by the pancreas - Composition and properties of the pancreatic juice-Action of the pancreatic juice on carbohydrates - Action of the pancreatic juice on proteids - Action of the pancreatic juice on fats - Action of bile in digestion-Movements of the small intestine-Physiological anatomy of the large intestine - Ileo-crcal valve - Peritoneal coat - Muscular coat Mucous coat - Processes of fermentation in the intestinal canal - Contents of the large intestine - Stercorin - Indol, scatol, phenol, cresol etc. - Movements of the large intestine - Defecation - Giases found in the alimentary canal.

\section{Physiological Axatomy of the Simall Intestine}

THE small intestine, extending from the pyloric opening of the stomach to the ileo-crecal valve, is loosely held to the spinal column by the mesentery. As the peritoneum lining the cavity of the abdomen passes from either side to the spinal column, it comes together in a double fold in front of the great vessels along the spine, and passing forward, divides again into two layers, which become continuous with each other and enclose the intestine, forming its external coat. The width of the mesentery usually is three to four inches (7.62 to I0.16 centimeters); but at the beginning and at the end of the small intestine, it abruptly becomes shorter, binding the duodenum and that portion of the intestine which opens into the caput coli closely to the subjacent parts. The mesentery thus keeps the intestine in place, but it allows a certain degree of motion, so that the tube may become convoluted, accommodating itself to the size and form of the abdominal cavity. The form of these convolutions is irregular and is constantly changing. The length of the small intestine, according to Gray, is about twenty-five feet ( 7.6 meters); but the canal is very distensible, and its dimensions are subject to frequent variations. Its average diameter is about an inch and a quarter ( 3 . I 8 centimeters).

The small intestine is divided into three portions, which present anatomical and physiological peculiarities more or less marked. These are the duodenum, the jejunum and the ileum.

The duodenum has received its name from the fact that it is about the length of the breadth of twelve fingers, or eight to ten inches 
( 20.32 to 25.4 centimeters). This part of the intestine is wider than the constricted pyloric end of the stomach, with which it is continuous, and is also much wider than the jejunum.

The coats of the duodenum, like those of the other divisions of the intestinal tube, are three in number. The external is the serous, or peritoneal coat, which has already been described. The middle, or muscular coat is composed of nonstriated muscular fibres, such as exist in the stomach, arranged in two layers. The external longitudinal layer is not very thick, and the direction of its fibres can be made out easily only at the outer portions of the tube, opposite the attachment of the mesentery. Near the mesenteric border the outlines of the fibres are faint. This is true throughout the entire small intestine, although the fibres are most abundant in the duodenum. The internal layer of fibres is considerably thicker than the longitudinal layer. These fibres encircle the tube, running usually at right angles to the external layer, but some having an oblique direction. The

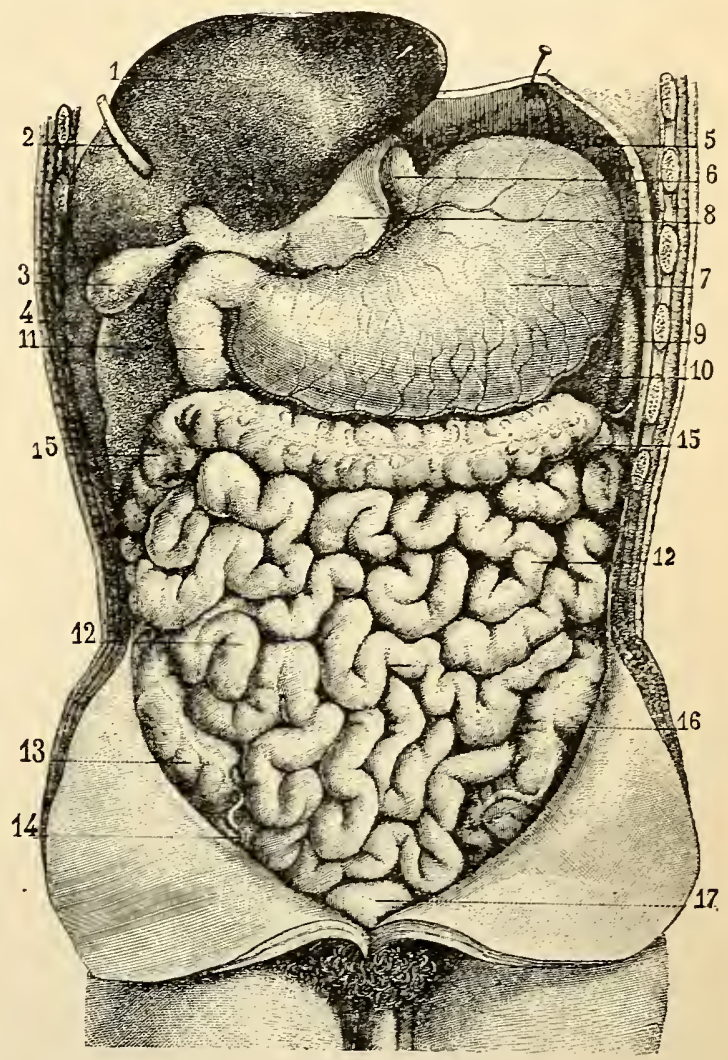

Fig. 42.- Stomach, liver and small intestine (Sappey).

I, inferior surface of the liver; 2, round ligament of the liver; 3. gall-bladder; 4, superior surface of the right lobe of the liver; 5. diaphragm; 6 , lower portion of the cesophagus; 7 , stomach; 8, gastro-hepatic omentum; 9 , spleen; IO, gastro-splenic omentum; II, duodenum; I2, I2, small intestine: I3, cocum; I4,

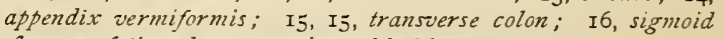
flexure of the colon; $\mathrm{I} 7$, urinary bladder. circular layer is thickest in the duodenum, diminishing gradually in thickness to the middle of the jejunum, but afterward maintaining a nearly uniform thickness throughout the canal to the ileo-cæcal valve.

The jejunum, the second division of the small intestine, is continuous with the duodenum. It presents no well-marked line of separation from the third division, but is considered as including the upper two- 
fifths, the lower three-fifths being called the ileum. It has received the name jejunum from the fact that it is almost always found empty after death.

The ileum is somewhat narrower and thinner than the jejunum, otherwise possessing no marked peculiarities except in its mucous membrane. This division of the intestine opens into the colon.

Mucous Membrane of the Small Intestine. - The mucous coat of the small intestine is somewhat thinner than the lining membrane of the stomach. It is thickest in the duodenum and gradually becomes thinner toward the ileum. It is highly vascular, presenting, like the mucous membrane of the stomach, a great increase in the quantity of blood during digestion. It has a peculiar soft and velvety appearance, and during digestion is of a vivid red color, being pale pink during the intervals. It presents for anatomical description the following parts: I, folds of the membrane, called valvulæ conniventes; 2, duodenal racemose glands, or glands of Brunner; 3 , intestinal tubules, or follicles of Lieberkühn; 4, intestinal villi; 5 , solitary glands or follicles; 6 , agminated glands, or patches of Peyer.

The valvulæ conniventes, simple transverse duplicatures of the mucous membrane, are particularly well marked in man, although they are found in some of the inferior animals belonging to the class of mammals, as the elephant and the camel. They render the extent of the mucous membrane much greater than that of the other coats of the intestine. Beginning at about the middle of the duodenum, they extend, with no diminution in number, throughout the jejunum. In the ileum they progressively diminish in number until they are lost at about its lower third. There are about six hundred of these folds in the first half of the small intestine and two hundred to two hundred and fifty in the lower half. Where they are most abundant, they increase the length of the mucous membrane to about double that of the tube itself; but in the ileum they do not increase the length more than one-sixth. The folds are always transverse and occupy usually one-third to onehalf of the circumference of the tube, although a few may extend entirely around it. The greatest width of each fold is at its centre, where it measures a quarter to half an inch (6.4 to 12.7 millimeters). From this point the width gradually diminishes until the folds are lost in the membrane as it is attached to the muscular coat. Between the folds are found fibres of connective tissue similar to those which attach the membrane throughout the alimentary tract. This, though loose, is constant, and it prevents the folds from being effaced, even when the intestine is distended to its utmost. Between the folds are also found bloodvessels, nerves and lymphatics. 
The position and arrangement of the valvulæ conniventes are such that they move freely in both directions and may be applied to the inner surface of the intestine either above or below their lines of attachment. It is evident that the food, as it passes along in obedience to the peristaltic movements, must, by insinuating itself beneath the folds and passing over them, be exposed to a greater extent of mucous membrane than if these valves did not exist. This is about the only definite use that can be assigned to them.

Thickly set beneath the mucous membrane in the first half of the duodenum, and scattered here and there throughout the rest of its extent, are the duodenal racemose glands, or the glands of Brunner. These are not found in other parts of the intestinal canal. In their structure they closely resemble the racemose glands of the osophagus. On dissecting the muscular coat from the mucous membrane, they may be seen with the naked eye, in the areolar tissue, in the form of small rounded bodies, about one-tenth of an inch ( 2.5 millimeters) in diameter. Examined microscopically, these bodies are found to consist of a large number of rounded follicles held together by a few fibres of connective tissue (see Plate IV, Fig. 4). They have bloodvessels ramifying on their exterior and are lined with glandular epithelium. They communicate with an excretory duct which penetrates the mucous membrane and opens into the intestinal cavity. When these structures are examined in a fresh preparation, the excretory duct frequently is found to contain a clear viscid mucus of an alkaline reaction. This secretion, however, has not been obtained in quantity sufficient to admit of the determination of its chemical or physiological properties.

The intestinal tubules, the follicles or crypts of Lieberkühn, are found throughout the small and the large intestine. In a thin vertical section they are seen closely packed together, extending through nearly the entire thickness of the mucous membrane (see Plate IV, Fig. 4). Between the tubules are bloodvessels embedded in a dense stroma of fibrous tissue, with non-striated muscular fibres. In vertical sections the only situations where the tubules are not seen are in that portion of the duodenum occupied by the ducts of the glands of Brunner and immediately over the centre of the larger solitary glands and some of the closed follicles which are collected to form the patches of Peyer. The tubes are not entirely absent in the patches of Peyer, but are here collected in rings, twenty or thirty tubes deep, which surround each of the closed follicles. Microscopical examination of the surface of the mucous membrane by reflected light shows that the openings of the tubules are between the villi.

The tubules in the human subject usually are simple, though some- 
times bifurcated, and are composed externally of a structureless basement-membrane, lined with a layer of cylindrical epithelium resembling the cells that cover the villi, but shorter. Mixed with these cells are the so-called goblet-cells, which will be described in connection with the intestinal villi. The goblet-cells are thought to secrete mucus. They exist in variable number, often being as abundant as the ordinary epithelial cells. There is much difference of opinion in regard to the
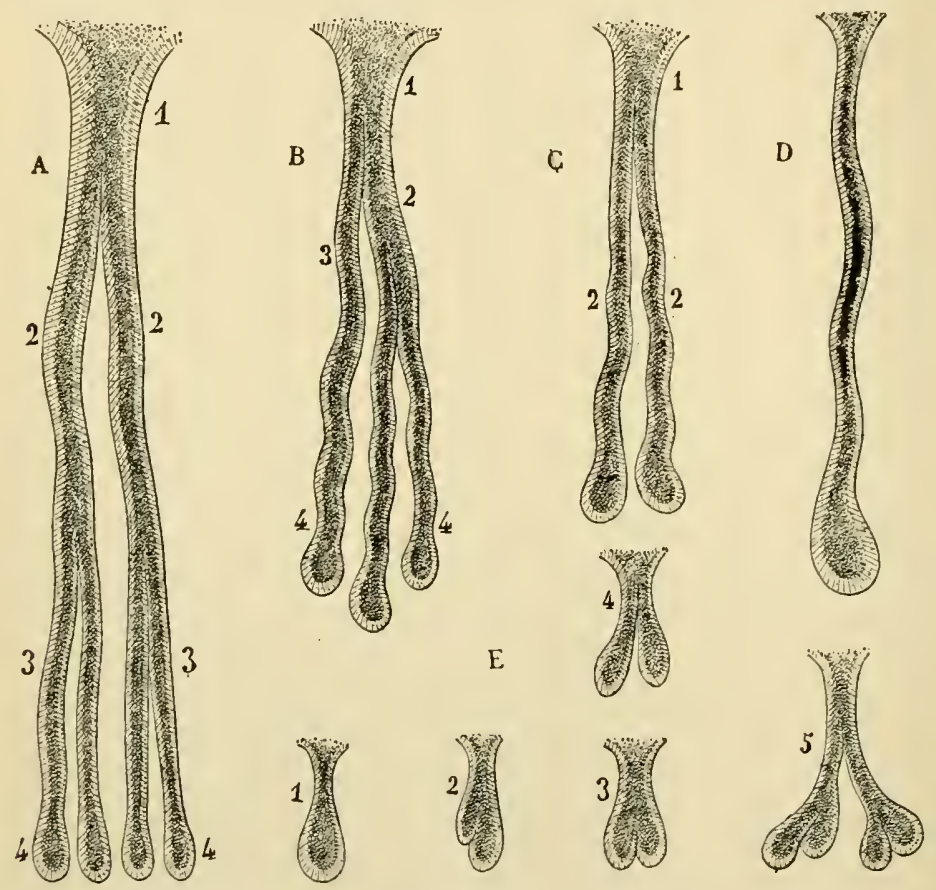

Fig. 43. - Intestinal tubules, $\times$ roo (Sappey).

A. From the $\operatorname{dog}: I$, excretory canal; 2,2 , primary branches; 3,3 , secondary branches; 4,4 , terminal culs-de-sac. $B$. From the ox: I, excretory canal; 2 , principal branch dividing into two; 3 , branch undivided; 4,4 , terminal culs-de-sac. C. From the sheep: I, trunk; 2, 2, branches. $D$. Single tube, from the pig. $E$. From the rabbit and hare: I, simple gland; 2, 3, 4, bifid glands; 5 , compound gland from the duodenum.

office of the epithelium, which many histologists regard as non-secretory. The lumen of the tubules often contains, however, a mucus-like secretion. The length of the tubules is about equal to the thickness of the mucous membrane and is about $\frac{1}{75}$ of an inch (0.33 millimeter). Their diameter is about $\frac{1}{360}$ of an inch ( 0.07 millimeter). In man they are cylindrical, terminating in a single rounded blind extremity, which frequently is a little larger than the rest of the follicle (see Plate IV, Fig. 4). These follicles present considerable differences in the lower animals. In some they are bifid or branched, but in man nearly all are simple. They are 
larger in children than in the adult and are larger in the colon than in the small intestine.

The intestinal villi, although concerned chiefly in absorption, are most conveniently considered in this connection. These exist throughout the small intestine, but they are not found beyond the ileo-cæcal valve, although they cover that portion of the valve which looks toward the ileum. Their number is very large, and they give to the membrane its peculiar and characteristic velvety appearance. They are found on the valvulæ conniventes as well as on the general surface of the mucous membrane. They are most abundant in the duodenum and jejunum. Sappey estimated, as an average, about 6450 to the square inch (IOOO in a square centimeter), and more than ten millions ( I O, I 25,000) throughout the small intestine. In the human subject the villi are in the form of flattened cylinders or cones. In the duodenum, where they resemble somewhat the elevations found in the pyloric portion of the stomach, they are shorter and broader than in other situations and are more like flattened conical folds. In the jejunum and ileum, they are in the form of long, flattened cones and cylinders.

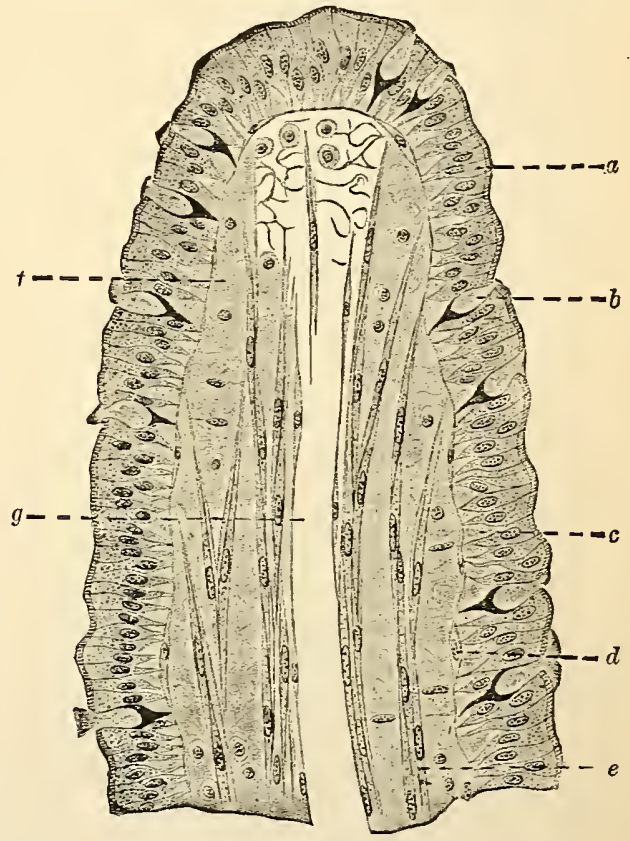

Fig. 44. - Axial section of a villus of a dog (Kultschitzky).

$a$, layer of cuticularized epithelium; $b$, goblet-cells; $c$, space between the attached ends of the epithelium; $d$, nucleated cell of the basement membrane; $e$, nonstriated muscular fibres; $f$, reticulum from the tunica propria; $g$, lumen of the central lymphatic. (Most of lymphoid cells have been removed and the bloodvessels are not shown.)

As a rule the cylindrical form predominates in the lower portion of the intestine. In the jejunum they attain their greatest length, measuring here $\frac{1}{30}$ to $\frac{1}{20}$ of an inch (0.83 to 1.25 millimeter) in length by $\frac{1}{\tau_{0}}$ to $\frac{1}{12} \overline{0}$ of an inch (0.36 to $0.2 \mathrm{I}$ millimeter) in breadth at their base.

The structure of the villi shows them to be simple elevations of the mucous membrane, provided with bloodvessels and with lacteals, or intestinal lymphatics. Externally is found a single layer of cylindrical epithelial cells resting on a nucleated basement-membrane (see Plate 
IV, Fig. 6). They adhere together firmly and are isolated with difficulty. The borders of their free ends are thickened and finely striated, this portion forming a thin membrane or cuticle covering the villi. These cells are called cuticularized cells. Between the cylindrical cells, in variable number, are the so-called goblet-cells. These probably are modified cylindrical cells, the change in form being due to a secretion of mucin near their free extremities which become swollen, this giving to the cells their peculiar appearance. The mucus finally is discharged, leaving a goblet-shaped cavity, the nucleus and granular protoplasm remaining below. Some histologists regard these as a distinct kind of cells (sce Plate IV, Fig. 5).

The substance of the villi is composed of amorphous matter in which are embedded nuclei, a few fine fibres, connective-tissue cells, lymphoid cells and non-striated muscular fibres. The bloodvessels are very abundant; four or five, and sometimes as many as twelve or fifteen arterioles entering at the base, ramifying through the substance of the villus, but not branching or anastomosing or even diminishing in calibre until, by a slightly wavy turn or loop, they communicate with the venous radicles, which are somewhat larger than the arterioles. The veins all converge to two or three branches, finally emptying into a large trunk situated nearly in the long axis of the villus (see Plate V, Fig. I).

The muscular fibres of the villi are longitudinal, forming a thin layer surrounding the villus, about halfway between the periphery and the centre and continuous with the muscular coat of the intestine.

In the central portion of each villus is a small lacteal, one of the vessels of origin of the lacteal system, with an extremely delicate wall composed of endothelial cells with frequent stomata between their borders. This vessel probably is in the form of a single tube, either simple or presenting a few short rounded diverticula.

The stomata of the lacteal vessels are thought to communicate with lymph-spaces or canals in the substance of the villus. Owing to the tenuity of the walls of the lacteals in the villi, it has been found impossible to fill these vessels with an artificial injection, although the lymphatics subjacent to them may easily be distended and studied in this way.

No satisfactory account has been given of nerves in the intestinal villi. If any exist in these structures, they probably are derived from the sympathetic system.

The solitary glands, or follicles (lymph-nodes), and the patches of Peyer, or agminated glands, have one and the same structure, the only difference being that those called solitary are scattered singly in very variable numbers throughout the small and large intestine, while the agminated glands consist of these follicles collected into patches of dif- 
ferent sizes. These patches usually are found in the ileum. The number of the solitary glands is variable and they are sometimes absent. The patches of Peyer are situated in that portion of the intestine opposite the attachment of the mesentery. They are likewise variable in number and are irregular in size. They usually are irregularly-oval in form, and measure half an inch to an inch and a half ( 12.7 to 38.1 millimeters) in length by three-fourths of an inch (I9. I millimeters) in breadth. Sometimes they are three to four inches ( 7.6 to IO.I centimeters) long, but the largest always are found in the lower part of the ileum. Their number is about twenty, and usually they are confined to the ileum; but when they are very abundant, - for they sometimes exist to the number of sixty or eighty, - they may be found in the jejunum or even in the duodenum.

Two varieties of the patches of Peyer have been described; in one, the patch is some-

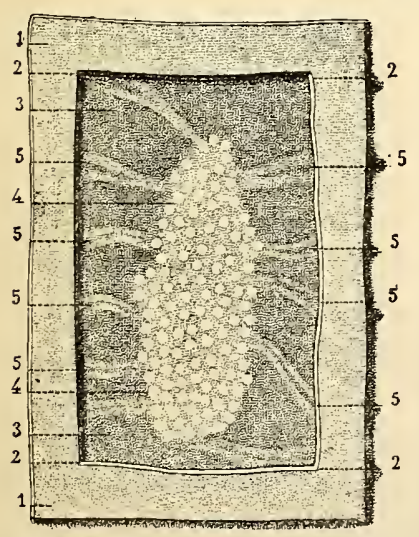

Fig. 46. - Patch of Peyer seen from its attached surface (Sappey).

I, I, serous coat of the intestine; $2,2,2,2$, serous coat removed to show the patch; 3,3 , fibrous coat of the intestine ; 4,4 , patch ; $5,5,5,5,5,5,5$, 5 , valvulæ conniventes. what promi-

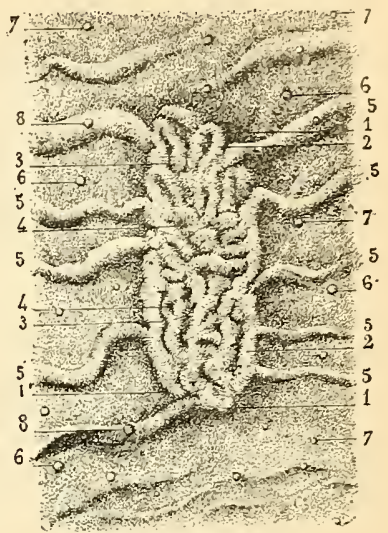

Fig. 45. - Patch of Peyer (Sappey).

I, I, I, patch of Peyer; 2, 2, folds seen on the surface; 3,3 , grooves between the folds; 4,4 , fossettes between some of the folds; $5,5,5,5,5$, $5,5,5$, valvulæ conniventes; $6,6,6$, 6 , solitary glands; $7,7,7,7$, smaller solitary glands; 8,8 , solitary glands on the valvulæ conniventes. nent, its surface being slightly raised above the general mucous surface; in the other, the surface is smooth, and the patch is distinguished at first with some difficulty. The prominent patches are covered with mucous membrane arranged in folds something like the convolutions on the surface of the brain. The valvulæ conniventes cease at or near their borders. These are the only patches that are commonly described as the glands of Peyer, the others, which may be called the smooth patches, frequently being overlooked. The latter are covered with a smooth, thin and closely adherent mucous membrane. Their follicles are small and abundant. The borders of these patches are much less strongly marked than in those of the first variety. As they are seen only on close examination and as they are the only patches present in certain individuals, it is sometimes said that the 
patches of Peyer are wanting. They usually are in less number than the first variety.

The villi are large and prominent on the mucous membrane covering the first variety of Peyer's patches, especially at the summit of the folds. In the second variety the villi are the same as over other parts of the mucous membrane, except that they are placed more irregularly and are not so abundant.

The follicles of the patches of Peyer are closed and are somewhat pear-shaped, with their pointed projections directed toward the lumen of the intestine. Just above the follicle, there usually is a small opening in the mucous membrane, surrounded with a ring of intestinal tubules, and leading to a cavity, the base of which is convex and is formed by the conical projection of the follicle. The diameter of the follicles is $\frac{1}{75}$ to $\frac{1}{25}$ or $\frac{1}{12}$ of an inch (0.34 to 1 or 2 millimeters). The small follicles usually are covered by mucous membrane and have no opening leading to them. Each follicle consists of a rather strong capsule composed of an almost homogeneous or slightly fibrous membrane, enclosing a semifluid, grayish substance, cells, bloodvessels and possibly lymphatics. The semifluid matter is of an albuminous character. The cells are small, rounded and interspersed with small free nuclei. The bloodvessels have rather a peculiar arrangement. In the first place they are clistributed between the follicles, so as to form a rich network surrounding each one. Capillary branches are sent from these vessels into the interior of the follicle, returning in the form of loops. Lymphatic vessels have not been distinctly shown within the investing membrane. They have been demonstrated surrounding the follicles, but it is still doubtful whether they exist in their interior. All that is known is that, during digestion, the number of lacteals coming from the Peyerian patches is greater than in other parts of the mucous membrane; but vessels containing a milky liquid are not seen within the follicles.

The description of the follicles of the patches of Peyer answers, in general terms, for the solitary glands, except that the latter exist in both the small and large intestines. They are sometimes called lymph-nodes - a name given to the lymphatic glands - from their close resemblance to these bodies in their general structure (see Plate V, Fig. 5).

\section{INTESTINAL JUice}

Nearly all observers agree that the intestinal juice which they have been able to collect is yellow, thin and strongly alkaline; but some have found it thin and opalescent, while others state that it is viscid 
and clear. In a case of fistula into the upper third of the intestine in the human subject, produced by a penetrating wound of the abdomen, Busch found a secretion that was white or of a pale rose-color, rather viscid and strongly alkaline. The maximum proportion of solid matter which it contained was 7.4 and the minimum, 3.87 per cent. The secretion apparently could not be obtained in sufficient quantity for ultimate analysis. The nature of this case made it impossible that there should be any admixture of food, pancreatic juice, bile or the secretion of the duodenal glands ; and during the process of digestion, the lower part of the intestine undoubtedly produced a normal fluid.

From what has been ascertained by experiments on the lower animals and observations on the human subject, the intestinal juice has been shown to possess the following characters: Its quantity in any portion of the mucous membrane that can be examined is small; but when the extent of the canal is considered, it is evident that the entire quantity of intestinal juice must be considerable. Vella estimated the quantity secreted by a dog in one hour at about I 1.6 ounces ( 360 grams). The secretion is viscid and adheres to the mucous membrane. It usually is either colorless or of a pale rose-tint. Its reaction is alkaline. In regard to its composition, little of a definite character has been learned. All that can be said is that its solid constituents are in a proportion of about five and a half parts per hundred (Busch) or about two and a half parts per hundred (Thiry). There is reason to believe that in most observations on secretions from the small intestine, the normal intestinal juice was not obtained; and beyond the fact that the mucous membrane produces a secretion or secretions, nothing is known of the mode of action of its glandular structures. Among the enzymes that have been described, is erepsin (Otto Cohnheim). This is supposed to act on proteoses and peptones, splitting them into simpler substances; but the statements of observers in regard to this special action are far from satisfactory. As regards the relative importance of secretions by the glands of Brunner and the follicles of Lieberkühn, nothing definite can be said. Action of the Intestinal Juice in Digestion. - The physiological action of the intestinal juice has lately been studied in dogs by Pawlow, with important results. He discovered, especially in the duodenal secretion, a new ferment to which he gave the name enterokinase. This is a true proteid ferment, its activity being destroyed by boiling. Mixed with the pancreatic juice, the intestinal secretion increases the activity of both the amylolytic and fat-splitting ferments. The duodenal secretion increases especially the activity of the proteolytic ferment.

In 1858 Busch reported a case of fistula in the human subject, in which the opening was supposed to be in the upper third of the small 
intestine. Later, following the observations of Thiry (1864), the intestinal juice was obtained by what is known as the Thiry-Vella method. The experiment of Thiry, modified by Vella, is the following: The abdomen of a dog is opened and a portion of the small intestine, twelve to twenty inches ( 30 to 50 centimeters) in length, is cut away from the rest of the tract. The two cut ends of the intestine are then connected, leaving a portion isolated but attached to the mesentery, each end of which is connected with an opening in the abdominal walls so as to leave two fistulous openings. The parts are then allowed to heal, and what is supposed to be pure intestinal juice may be collected from the isolated portion. While it must be admitted that this is an imperfect way of obtaining a normal secretion, the observations are of considerable value. The result of these experiments has been to show that the intestinal secretion inverts cane-sugar into dextrose and levulose (invertsugar) and may have a like action on milk-sugar and maltose. The majority of observations have failed to show any action on starch or fats. The ferment acting on the sugars has been called invertin (Paschutin).

\section{Pancreatic Juice}

The pancreas is situated transversely in the upper part of the abdominal cavity and is closely applied to its posterior wall. Its form is elongated, presenting an enlarged thick portion, called the head, which is attached to the duodenum, a body and a pointed extremity, which latter is in close relation to the hilum of the spleen. Its average weight is four to five ounces (114.4 to 141.7 grams); its length is about seven inches ( 17.78 centimeters); its greatest breadth, about an inch and a half ( $3.8 \mathrm{I}$ centimeters); and its thickness, three-quarters of an inch ( 1.9 I centimeters). It lies behind the peritoneum, which covers only its anterior surface.

There are nearly always, in the human subject, two pancreatic ducts opening into the duodenum; one which opens in common with the ductus communis choledochus, and one which opens about an inch (25.4 millimeters) above the main duct. The main duct is about an eighth of an inch (3.2 millimeters) in diameter and extends along the body of the gland, becoming larger as it approaches the opening. The second duct is smaller and becomes diminished in calibre as it passes to the duodenum. In general appearance and in minute structure, the pancreas nearly resembles the parotid. It differs, however, from the salivary glands in some important particulars. It is rather softer and looser in texture and has no distinct fibrous covering. Its alveoli contain secreting cells, but here and there are found groups 
of smaller, spindle-shaped bodies called centro-acinar cells, the groups constituting the islands of Langerhans (see Plate V, Fig. 4). It is thought that these cells are not concerned in the production of pancreatic juice, but produce, by what is known as internal secretion, a substance taken up by the blood or lymph, which is instrumental in the normal consumption of sugar. Removal of the entire pancreas is followed by glycosuria, which may be relieved by transplantation of parts of the pancreas in any of the tissues or even under the skin.

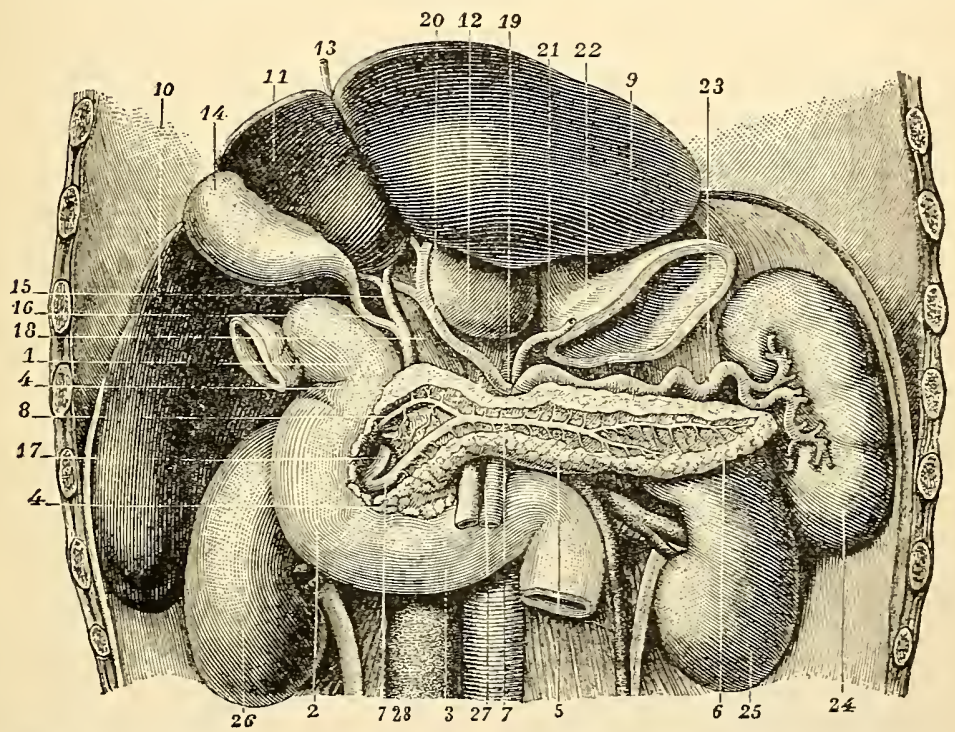

Fig. 47. - The pancreas; its direction, relations and two excretory ducts (Sappey).

I, first portion of the duodenum; 2 , second portion; 3 , third portion; 4 , 4 , head of the pancreas; 5. middle portion; 6 , terminal extremity; 7,7 , principal duct; 8 , accessory duct; 9 , left lobe of the liver; Io, right lobe; II, anterior portal eminence; I2, lobus Spigelii; I3, antero-posterior groove; I4, gall-bladder; I5, hepatic duct; I6, cystic duct; I7, ductus communis choledochus; I8, trunk of the portal vein; I9, cœliac axis; 20 , hepatic artery; $2 \mathrm{I}$, coronary gastric artery; 22 , cardiac portion of the stomach; 23 , splenic artery; 24 , spleen; 25 , left kidney; 26, right kidney; 27 , superior mesenteric artery and vein; 28 , inferior vena cava.

The glycolytic enzyme, however, has not been isolated. A few cases of what is now known as pancreatic diabetes have been observed in the human subject. The pancreas is abundantly supplied with blood. The larger arterial branches ramify in the loose connective tissue between the lobes, into which they send twigs that break up into capillary plexuses surrounding the individual acini, lying in close relation with the epithelium (see Plate V, Fig. 3).

Composition and Properties of the Pancreatic Juice. - One of the most ingenious of the many experiments on digestion made by Pawlow is the establishment of a permanent pancreatic fistula. It is not neces- 
sary to go into the technical details of this operation, and it is sufficient to say that he succeeded in maintaining an open communication with the duodenum at the site of the opening of the principal pancreatic duct and secured normal pancreatic juice in considerable quantity. The liquid thus obtained is viscid, slightly opalescent and has a strongly alkaline reaction. Bernard found the specific gravity of the pancreatic juice of the dog to be I040. The normal secretion from a temporary fistula in a dog has been observed with a specific gravity of rorg (Flint). The quantity of organic matters in the normal juice is very great, so that the liquid is solidified by heat. This coagulability is one of the properties by which the normal secretion may be distinguished from that which has undergone alteration.

COMPOSITION OF THE PANCREATIC JUICE OF THE DOG (BERNARD)

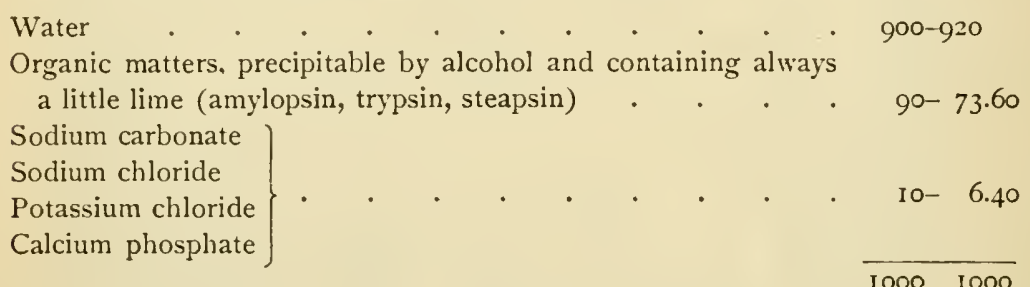

An enzyme, almost if not quite identical with ptyalin, may be extracted from the normal juice by nearly the same processes as those employed in the isolation of the active principle of the saliva. On account of its vigorous action on starch this substance has been called amylopsin.

Trypsin is an enzyme capable of acting on the proteids, changing them into peptones. The secreting cells of the gland produce a substance called trypsinogen, which is changed into trypsin. The action of trypsin on the proteids is increased by the addition of small quantities of sodium chloride, sodium glycocholate or sodium carbonate and is diminished by acids.

A substance called steapsin, capable of decomposing fats into fatty acids and glycerin, has been described as one of the organic constituents of the pancreatic juice. This action upon fats, which was noted by Bernard, though slight, probably assists in their emulsification.

The inorganic constituents of the pancreatic juice, beyond giving the secretion an alkaline reaction, do not possess any special physiological interest, inasmuch as they do not seem to be essential to its peculiar digestive properties.

The entire quantity of pancreatic juice secreted in the twenty-four hours has been variously estimated by different observers. Bernard 
was able to collect from a dog of medium size eighty to one hundred grains ( 5.2 to 6.5 grams) in an hour. There is no accurate basis for an estimate of the quantity secreted in the twenty-four hours in the human subject or of the quantity necessary for the digestion of a given quantity of food.

Unlike the gastric juice, the pancreatic juice, under ordinary conditions of heat and moisture, rapidly undergoes decomposition, and in warm and stormy weather the alteration is marked in a few hours; but at a temperature of $50^{\circ}$ to $70^{\circ} \mathrm{Fahr}$. ( $10^{\circ}$ to $2 \mathrm{I}^{\circ} \mathrm{C}$.), it occupies two or three days. As it decomposes, the liquid acquires an offensive putrefactive odor, and its coagulability diminishes until finally it is not affected by heat. The alkalinity, however, increases in intensity, and when neutralized with an acid, there is a considerable evolution of carbon dioxide.

Pawlow found that an abundant pancreatic secretion was excited by acids; and it is probable that the acid contents of the stomach, passing into the duodenum, act as a powerful stimulus to the gland. $\mathrm{He}$ also showed that dogs with a permanent fistula suffered from erosion of the parts with which the secretion came in contact. This trouble, however, was obviated by having the animals rest on a bed of sand or sawdust, which absorbed the liquid as it was discharged.

The secretion from the pancreas was collected by Pawlow by attaching over the opening in the abdomen the large end of a funnel. The liquid thus obtained had a powerful action on proteids, starch, canesugar and fats. Like the gastric juice, the pancreatic secretion presented marked differences in activity after feeding with different articles. Its proteolytic action was strongest after feeding with milk and less with meat and bread; its amylolytic action was strongest with breadfeeding and less with meat and milk; and its fat-splitting action was strongest after milk-feeding, less with bread, and intermediate with meat.

In addition to the great increase in the activity of the pancreatic secretion produced by the presence of the intestinal juice, it is supposed that the spleen secretes a kinase which is carried to the pancreas, unites with trypsinogen and forms tripsin. It can not be thought, however, that this union is essential to the production of an active trypsin; for this enzyme is formed in animals from which the spleen has been removed, perhaps by the action of intestinal secretions containing enterokinase. The activity of the pancreatic secretion, particularly in the acidification of fats, is much increased by the addition of bile. This well-known fact was confirmed by Pawlow. Added to its other action, the pancreatic juice curdles milk, by virtue of a milkclotting ferment. 
Action of the Pancratic Juice on Carbohydrates. - The action of the pancreatic juice in transforming starch into sugar was first observed in 1844 , by Valentin, who experimented with an artificial liquid made by infusing pieces of the pancreas in water. Bouchardat and Sandras first noted this property in the normal pancreatic secretion. In man, some of the amylaceous matters are acted on by the saliva, but most of the starch taken as food is rapidly digested in the small intestine. It is possible that the bile assists in this process to a slight extent. In the transformation of starch into sugar in the small intestine, the same intermediate processes are observed as occur in the action of the saliva; but the change in the intestine into glucose is more rapid. It is stated that amylopsin is not present in the pancreas of the newborn infant (Korowin), and that in early infancy - before the second or third month - the pancreatic extract will not digest starch.

As cane-sugar passes from the stomach into the duodenum, it is almost instantly inverted; but this is due entirely to the action of the intestinal juice. The pancreatic juice does not act on the sugars.

Action of the Pancreatic Juice on Protcids. - Reference has already been made to the relative importance of intestinal digestion; and it has been apparent that the process of disintegration of food in the stomach is not final, even as regards many of the nitrogenous substances, but is rather preparatory to the complete liquefaction of these matters, which takes place in the small intestine. In experiments in which the pancreas has been partially destroyed in dogs, there was rapid emaciation, with great voracity, and the passage, not only of unchanged fats and starch, but of undigested nitrogenous matters in the dejections. The voracious appetite, progressive emaciation and the passage of all classes of alimentary substances in the feces, after this operation, indicate the great importance of the pancreatic juice in digestion; but the precise mode of action on the proteids is even now a question of some obscurity. If the bile is shut off from the intestine and discharged externally by a fistulous opening, the same voracity and emaciation are observed; and yet there is no single alimentary substance on which the bile, of itself, has been shown to exert a decided digestive action. Furthermore, the pancreatic juice evidently is adapted to act on alimentary matters after they have been subjected to the action of the stomach, a preparation that is essential to proper intestinal digestion; and once passed into the intestine, the food comes in contact with a mixture of pancreatic juice, intestinal juice and bile. Nitrogenous alimentary substances, when exposed to the action of the pancreatic juice out of the body, rapidly become softened and dissolved in some of their parts, but soon undergo putrefaction. Analogous changes take 
place in starchy and fatty matters when they are exposed to the action of the pancreatic juice out of the body, and they pass through the various stages of transformation respectively into lactic acid and the fatty acids. Putrefactive action, however, does not readily take place in proteids that have been precipitated after having been cooked, or in raw gluten or casein. The presence of fat also interferes with putrefaction.

Trypsin, in an alkaline medium, changes proteids into their respective peptones, in much the same way and involving nearly the same intermediate processes as in the digestion of these substances by the gastric juice, except that the first change is into alkali-albumin instead of acid-albumin. In the upper part of the intestine the vigor of this proteolytic action is much increased by admixture with the intestinal secretions; and in the lower part, where the contents are more strongly alkaline, the activity of the process is increased by sodium carbonate. If the action of the pancreatic juice on proteids is prolonged in vitro, the changes continue and substances are formed which yield leucin, tyrosin and other analogous products. The final putrefactive changes resulting in indol, skatol, phenol, etc., some of which have a distinctly fecal odor, probably are due to the action of micro-organisms.

Action of the Pancreatic Juice on Fats. - The pancreatic juice is the only digestive secretion that is capable of forming a complete and permanent emulsion with fats, and this persists when the emulsion is diluted with water. Steapsin, extracted from the fresh pancreas, has the property of decomposing the fats into their fatty acids and glycerin ; but the fatty acids do not appear in the chyle. The emulsification of the fats by the pancreatic juice is to a great extent a mechanical process, dependent on the general physical characters of the liquid; but although the fat contained in the lacteal vessels is always neutral, it is thought that steapsin assists in rendering the emulsion fine and permanent.

\section{Action of the Bile in Digestion}

The physiological anatomy of the liver and the general properties and composition of the bile will be considered again in connection with the physiology of secretion and excretion; and here it will be necessary only to study the action of the bile in digestion.

The question whether the bile is simply excrementitious or is concerned in digestion was formerly the subject of much discussion; but it is now admitted by all physiologists that the action of the bile in digestion and absorption, whatever its office may be as an excretion, is essential to life. The experiments of Swann, Nasse, Bidder and Schmidt, 
Bernard and others, who have discharged all the bile by a fistula into the gall-bladder, communication between the bile-duct and the duodenum having been cut off, show that dogs operated on in this way have a voracious appetite, but die of inanition after having lost four-tenths of the body-weight. The following is an example of experiments of this kind (Flint, I 86I): A fistula was made into the gall-bladder of a dog, after excising a great part of the common bile-duct. The animal suffered no immediate effects from the operation, but died at the end of thirty-eight days, having lost $37 \frac{1}{2}$ per cent in weight. He had a voracious appetite, was fed as much as he would eat, was protected from cold and was carefully prevented from licking the bile. During the progress of the experiment, various observations were made on the flow of bile. During the last five or six days, the animal was ravenous but was not allowed to eat all that he would at one time. At that time he was fed twice a day, but he would not eat fat, even when very hungry. During the last day, when too weak to stand, he attempted to eat while lying down.

Human bile is moderately viscid, of a dark golden-brown color, an alkaline reaction and a specific gravity of about I028. Among other constituents - which will be described in connection with the physiology of secretion - it contains sodium united with two acids peculiar to the bile, called glycocholic and taurocholic acids. Sodium taurocholate is much more abundant than the glycocholate. The viscidity of the bile is due to mucus derived in part from the lining membrane of the gall-bladder and in part, probably, from little racemose glands attached to the larger bile-ducts in the substance of the liver. The so-called biliary salts, sodium taurocholate and sodium glycocholate, are probably the constituents of the bile that are concerned in digestion.

Although the bile is constantly discharged in certain quantity into the duodenum, its flow presents marked variations corresponding with certain stages of the digestive process. In fasting animals, the gallbladder is distended with bile; but in animals opened soon after feeding, it is nearly always found empty. The actual secretion of bile by the liver also is influenced by digestion.

Disregarding slight variations, it may be stated, in general terms, that the bile begins to increase in quantity immediately after eating; that its flow is at its maximum from the second to the eighth hour, during which time the quantity does not vary to any great extent; after the eighth hour it begins to diminish, and from the twelfth hour to the time of feeding it is at its minimum.

One of the uses which has been ascribed to the bile is that of stimulating peristaltic movements of the small intestine and of preventing 
putrefactive changes in the intestinal contents and the abnormal development of gas; but observations on this point have been somewhat conflicting. During the first few days of the experiment just described, the dejections were very rare; but they afterward became regular, and at one time there was even a tendency to diarrhœa. There can be little doubt, however, that the bile restrains putrefaction of the contents of the intestinal canal, particularly when animal food has been taken. The feces in the dog with biliary fistula were always extremely offensive. Bidder and Schmidt found this to be the case in dogs fed entirely on meat; but the feces were nearly odorless when the animals were fed on bread alone.

It has been shown that the bile of itself has little action on the different classes of alimentary substances. In the feces of animals with biliary fistula, the only peculiarity observed - aside from the putrefactive odor and the absence of the coloring matter of the bilehas been the presence of an abnormal proportion of fat. This was noted in the feces of a patient suffering from jaundice apparently due to temporary obstruction of the bile-duct (Flint).

It is difficult to associate the bile directly with the digestion of proteids or of carbohydrates, although imperfectly digested meats as well as fats are found in the feces when bile has been shut off from the small intestine. It was noted, however, by Bidder and Schmidt, in an animal with a biliary fistula and the bile-duct obliterated, that the proportion of fat was 1.90 parts per 1000 of chyle; while in an animal with the biliary passages intact, the proportion was 32.79 parts per I000. In animals operated on in this way, there frequently is a distaste for fatty articles of food. In the observation made in I86I (Flint) the dog refused fat meat even when very hungry and when lean meat was taken with avidity. Later observations have shown that the bile assists the pancreatic secretion in the splitting and emulsification of fats, and it is probable that it also facilitates their absorption, although in what way is not apparent. While it is the digestion and absorption of fats that seem to be most seriously disturbed in cases of biliary fistula, the rapid loss of weight and strength shows great interference with the digestion and absorption of other constituents of food. It is important to note, also, that the secretion of bile, though constant, is greatly increased when food passes into the intestinal canal.

Although it has been shown that the presence of bile in the small intestine is necessary to proper digestion and even essential to life, and although the variations in the flow of bile with digestion are now well established, physiologists have but little definite information concerning the exact mode of action of the bile in intestinal digestion and absorp- 
tion. Nearly all that can be said on this subject is that the action of the bile seems to be auxiliary to that of the other digestive secretions.

\section{Movements of the Small Intestine}

By the contractions of the muscular coat of the small intestine, the alimentary mass is made to pass along the canal, sometimes in one direction and sometimes in another, the general tendency, however, being toward the cæcum; and the partially digested matters that pass out at the pylorus are prevented from returning to the stomach by the peculiar arrangement of the fibres which constitute the pyloric muscle. Once in the intestine, the food is propelled along the canal by peculiar movements that have been called peristaltic, when the direction is toward the large intestine, and antiperistaltic, when the direction is reversed. These movements are of the character peculiar to the non-striated muscular fibres; they are slow and gradual, the contraction enduring for a certain time and being followed by a correspondingly slow and gradual relaxation. Both the circular and the longitudinal muscular layers participate in these movements.

So far as has been ascertained by observations on the human subject and warm-blooded animals, the regular intestinal movements are excited by the passage of alimentary matters from the stomach through the tube during the natural process of digestion. By a slow and gradual action of the muscular coat of the intestine, its contents are passed along, occasionally the action being reversed for a time, until the indigestible residue, mixed with a certain quantity of intestinal secretion, more or less modified, is discharged into the caput coli. These movements apparently are not continuous, and they depend in some degree on the quantity of matter contained in different parts of the intestinal tract. Judging from the movements in the inferior animals after the abdomen has been opened, the intestines are always changing their position, mainly by the action of their longitudinal muscular fibres, so that the force of gravity does not oppose the onward passage of their contents so much as if the relative position of the parts were constant.

The gases found in the intestine have an important mechanical office. They are useful, in the first place, in keeping the canal constantly distended to the proper degree, thus avoiding the liability to disturbances in the circulation and facilitating the passage of the alimentary mass in obedience to the peristaltic contractions. They also support the walls of the intestine and protect these parts against concussions, in walking, leaping etc. The gases are useful, likewise, in 
offering an elastic but resisting mass upon which the compressing action of the abdominal muscles may be exerted in straining and in expiration.

There can be hardly any question that the normal movements of the intestine are due principally to the impression made on the mucous membrane by the alimentary matters, to which may be added, perhaps, the stimulating action of the bile. The vigorous peristaltic movements that occur soon after death have been explained in various ways. It has been shown that these movements are not due to a lowering of the temperature or to exposure of the intestines to the air.

The nerves distributed to the small intestine are derived from the sympathetic and from branches of the pneumogastric, which latter come from the nerve of the right side and are distributed to the entire intestinal tract, from the pylorus to the ileo-cæcal valve. The intestine receives no filaments from the left pneumogastric. Throughout the intestinal tract, is a plexus of non-medullated nerve-fibres with groups of nerve-cells, lying between the longitudinal and circular layers of the muscular coat. This is known as Auerbach's plexus. From this plexus, fine non-medullated filaments are given off, which form a wider plexus, also with ganglion-cells, situated just beneath the mucous membrane. This is called the plexus of Meissner. In experiments on the lower animals it has been shown that stimulation of the pneumogastrics excites peristaltic movements of the intestines; but in some animals these nerves seem to contain inhibitory as well as motor fibres, although it is probable that the principal inhibitory nerves belong to the sympathetic system. Observations on these points, however, are somewhat conflicting.

It is difficult to estimate the time occupied in intestinal digestion and absorption in man. In the dog the small intestine is nearly empty in six to nine hours after full feeding, but in the carnivora the intestine is relatively short and the indigestible residue of food is small (Halliburton). In the human subject, the passage of an ordinary meal through the small intestine occupies probably about twelve hours (Kirkes).

\section{Physiological Anatomy of the Large Intestine}

The length of the large intestine is about five feet ( 1.5 meter). Its diameter is greatest at the cæcum, where it measures, when moderately distended, two and a half to three and a half inches ( 6.35 to 8.89 centimeters). The average diameter of the tube beyond the cæecum is one and two-thirds to two and two-thirds inches ( 4.23 to 6.77 centimeters). Passing from the cæcum, the canal diminishes in calibre, gradually and very 
slightly, to where the sigmoid flexure opens into the rectum. This is the narrowest portion of the canal. Beyond this, the rectum gradually increases in diameter, forming a kind of pouch, which abruptly diminishes in size near the external opening, to form the anus.

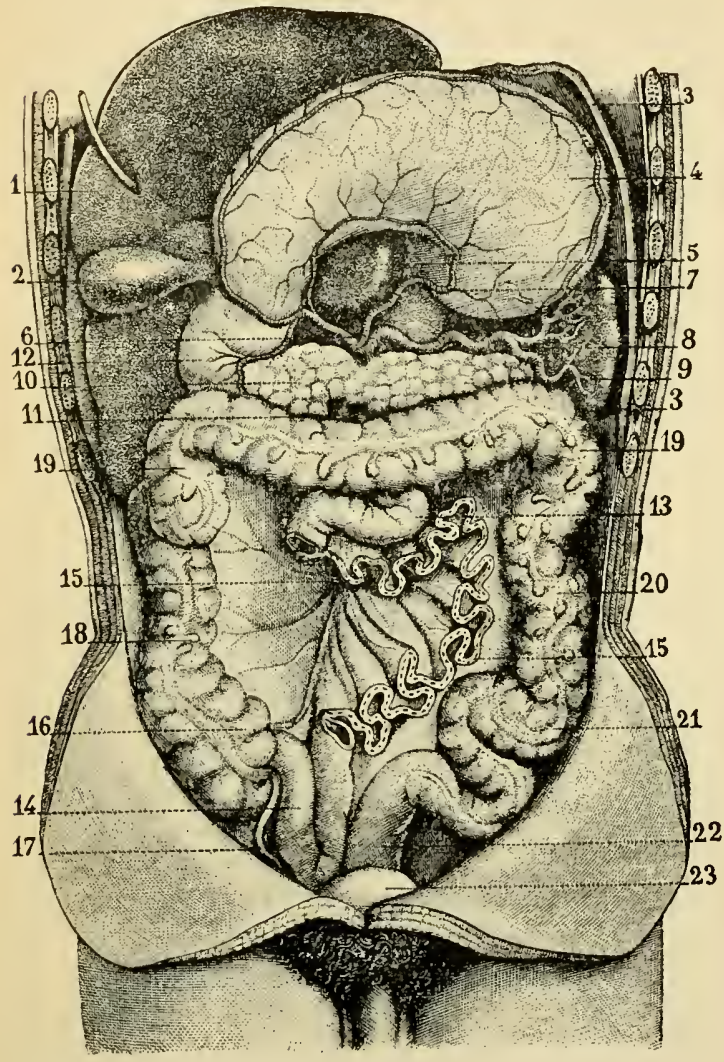

Fig. 48. - Stomach, pancreas and large intestine (Sappey).

I, anterior surface of the liver; 2, gall-bladder; 3,3 , section of the diaphragm: 4 , posterior surface of the stomach; 5 , lobus Spigelii of the liver; 6 , cœliac axis; 7 , coronary artery of the stomach; 8, splenic artery; 9, spleen; Io, pancreas; II, superior mesenteric vessels; I2, duodenum; $\mathbf{I}_{3}$, upper extremity of the small intcstine; 14 , Jower end of the ileum; 15, I5, mesentery; 16. cacum; I7, appendix vermiformis; 18 , ascending colon; 19 , 19, transverse colon; 20, descending colon; $2 \mathrm{I}$, sigmoid flexure of the colon; 22 , rectum; 23 , urinary bladder.

The general direction of the large intestine is from the cæcum, in the right iliac fossa, to the left iliac fossa, thus encircling the convoluted small intestine, in the form of a horseshoe. From the crecum to the rectum, the canal is known as the colon. The first division of the colon, called the ascending colon, passes almost directly upward to the under surface of the liver; the canal here turns at nearly a right angle, passes across the upper part of the abdomen and is called the transverse colon; it then passes downward at nearly a right angle, forming the descending colon. The last division of the colon, called the sigmoid flexure, is situated in the left iliac fossa and is in the form of the italic letter $S$. This terminates in the rectum, which is not straight, as its name would imply, but presents at least three distinct curvatures, as follows: it passes first in an oblique direction from the left sacro-iliac symphysis to the median line opposite the third piece of the sacrum; it then passes downward in the median line, following the concavity of the sacrum and coccyx; and the lower portion, which is about an 
inch (2.54 centimeters) in length, turns backward to terminate in the anus.

The cæcum, or caput coli, presents a rounded dilated cavity continuous with the colon above and communicating by a transverse slit with the ileum. At its lower portion is a small cylindrical tube, opening below and a little posterior to the opening of the ileum, called the vermiform appendix. This is covered with peritoneum and has a muscular and a mucous coat. It is sometimes free and sometimes provided with a short fold of mesentery for a part of its length. The coats of the appendix are very thick. The muscular coat consists of longitudinal fibres only. The mucous membrane is provided with tubules and closed follicles, the latter frequently being very abundant. This little tube usually contains a quantity of clear viscid mucus. The uses of the vermiform appendix are unknown.

Ileo-cacal Valve. - The opening by which the small intestine communicates with the crecum is provided with a valve, known as the ileo-crecal valve, situated at the inner and posterior portion of the cacum. The small intestine, at its termination, presents a shallow concavity, which is provided with a horizontal buttonhole slit, opening into the cæcum. The surface of the valve which looks toward the small intestine is covered with a mucous membrane provided with villi and in all respects resembling the general mucous lining of the small intestine. Viewed from the cxcum, a convexity is observed corresponding to the concavity upon the other side. The cacal surface of the valve is covered with a mucous membrane identical with the general mucous lining of the large intestine. It is evident, from an examination of these parts, that pressure from the ileum opens the slit and allows the easy passage of the semifluid contents of the intestine; but pressure from the cacal side approximates the lips of the valve, and the greater the pressure the more firmly is the opening closed. The valve itself is composed of folds formed of the fibrous tissue of the intestine, and circular muscular fibres from both the small and the large intestine, the whole being covered with mucous membrane. The lips of the valve unite at either extremity of the slit and are prolonged on the inner surface of the cacum, forming two raised bands, or bridles; and these become gradually effaced and are thus continuous with the general lining of the canal. The posterior bridle is a little longer and more prominent than the anterior. These assist somewhat in enabling the valve to resist pressure from the cæcal side. The longitudinal layer of muscular fibres and the peritoneum pass directly over the attached edge of the valve and are not involved in its folds. These give strength to the part, and if they are divided over the valve, gentle traction suffices to draw out and obliterate the 
folds, leaving a simple and unprotected communication between the large and the small intestine. Some modern observers attribute closing of the ileo-cæcal opening to contraction of circular muscular fibres, which constitute a sphincter (ileo-colic sphincter). These, indeed, have been demonstrated in the cat, dog and rabbit. It is possible that such an arrangement and action may exist in man.

Peritoneal Coat. - Like most of the other abdominal viscera, the large intestine is covered by peritoneum. The cæcum is covered by this membrane only anteriorly and laterally. It usually is bound down closely to the subjacent parts, and its posterior surface is without a serous investment ; although sometimes it is completely covered and there may be even a short mesocæcum. The ascending colon is likewise covered with peritoneum only in front and is closely attached to the subjacent parts. The same arrangement is found in the descending colon. The transverse colon is almost completely invested with peritoneum; and the two folds forming the transverse mesocolon separate to pass over the tube above and below, uniting again in front, to form the great omentum. The transverse colon consequently is quite movable. In the course of the colon and the upper part of the rectum, particularly on the transverse colon, are found a number of little sacculated pouches filled with fat, called the appendices epiploïcæ. The sigmoid flexure of the colon is covered by peritoneum, except at the attachment of the iliac mesocolon. This division of the intestine is quite movable. The upper portion of the rectum is almost completely covered by peritoneum and is loosely held in place. The middle portion is closely bound down, and is covered by peritoneum only anteriorly and laterally. The lowest portion of the rectum has no peritoneal covering.

Muscular Coat. - The muscular fibres of the large intestine have an arrangement quite different from that which exists in the small intestine. The external longitudinal layer, instead of extending over the whole tube, is arranged in three distinct bands, which begin in the cæcum at the vermiform appendix. Passing along the ascending colon, one of the bands is situated anteriorly, and the others, latero-posteriorly. In the transverse colon the anterior band becomes inferior and the two lateroposterior bands become respectively postero-superior and postero-inferior. In the descending colon and the sigmoid flexure, the muscular bands resume the relative position which they had in the ascending colon. As these longitudinal fibres pass to the rectum, the anterior and the external bands unite to pass down on the anterior surface of the canal, while the posterior band passes down on its posterior surface. Thus the three bands here become two. These two bands as they pass downward, though remaining distinct, become much wider; and longitudinal mus- 
cular fibres beginning at the rectum are situated between them, so that this part of the canal, especially in its lower portion, is covered with longitudinal fibres in a nearly uniform layer.

Mucous Coat. - The mucous lining of the large intestine presents several points of difference from the corresponding membrane in the small intestine. It is paler, somewhat thicker and firmer, and is more closely adherent to the subjacent parts. In no part of this membrane are there any folds, like those which form the valvula conniventes of the small intestine; and the surface is smooth and free from villi.

Throughout the entire mucous membrane, from the ileo-cæcal valve to the anus, are orifices that lead to simple follicular glands. These structures resemble in all respects the follicles of the small intestine, except that they are a little longer, owing to the greater thickness of the membrane, are wider and rather more abundant. Among these small follicular openings are found, scattered irregularly throughout the membrane, larger openings which lead to utricular glands, resembling the closed follicles in general structure, except that they have an opening into the cavity of the intestine, that is sometimes so large as to be visible to the naked eye (see Plate V, Fig. 5). The number of these glands is variable, and they exist throughout the intestine, together with the closed follicles, except in the rectum. In the cæcum and colon, isolated closed follicles usually are found, which are identical in structure with the solitary glands of the small intestine. These are variable both in number and size.

The mucous membrane of the rectum, in the upper three-fourths of its extent, does not differ materially from that of the colon. In the lower fourth, the fibrous tissue by which the lining membrane is united to the subjacent muscular coat is loose, and the membrane, when the canal is empty, is thrown into a great number of irregular folds. At the site of the internal sphincter, five or six little semilunar valves have been observed, with their concavities directed toward the colon. These form an irregular festooned line which surrounds the canal; their folds, however, are small and have no tendency to obstruct the passage of fecal matters. The simple follicles are particularly abundant in the rectum, and the membrane is constantly covered with a thin coating of mucus. Another peculiarity to be noted in the mucous membrane of the lower portion of the rectum is its great vascularity, the veins, especially, being very abundant.

The rectum terminates in the anus, a buttonhole orifice, situated a little in front of the coccyx, which is kept closed and somewhat retracted, except during the passage of the feces, by the powerful 
external sphincter. This muscle is composed of striated fibres, arranged in the form of an ellipse, its long diameter being antero-posterior.

It is now almost universally admitted that the digestion of all classes of alimentary substances is completed either in the stomach or in the small intestine, and that the mucous membrane of the large intestine does not produce a secretion endowed with well-marked digestive properties. The simple follicles, the closed follicles and the utricular glands produce a glairy mucus, which, so far as is known, serves merely to lubricate the canal. This has never been obtained in sufficient quantity to admit of any accurate investigation into its properties.

In the human subject those portions of the food which resist the successive and combined action of the different digestive secretions are derived chiefly from the regetable kingdom. Hard regetable seeds, the cortex of the cereals, spiral vessels, and, indeed, all parts composed largely of cellulose pass through the intestinal canal without nuch change. These substances form, in the feces, the greatest part of what can be recognized as the residue of matters taken as food. It is well known that an exclusively animal diet, particularly if the nutritious matters are taken in a concentrated and readily assimilable form, leaves very little undigested matter to pass into the large intestine, and gives to the feces a character quite different from that which is observed in herbivorous animals or in man when subjected to an exclusively vegetable diet. The characters of the residue of the digestion of albuminous substances are not very distinct. As a rule the proteids can not be recognized in the healthy feces by the ordinary tests.

Absorption of rarious articles of food in a liquid form may take place with great activity in the large intestine, although it has not been shown that the secretions in this part of the alimentary canal have any distinct digestive properties; still, as is seen in rectal alimentation, eggs, milk and meat-extracts may be taken up by the mucous membrane, and they enter the circulation in such a form that they contribute to nutrition.

Processes of Fermentation in the Intestinal Canal. - The processes of fermentation in the intestines are not properly digestive and are to a great extent due to the action of micro-organisms, which exist here in great numbers and variety. Some of the substances resulting from intestinal fermentations have already been described. Indol $\left(\mathrm{C}_{8} \mathrm{H}_{7} \mathrm{~N}\right)$, skatol $\left(\mathrm{C}_{9} \mathrm{H}_{9} \mathrm{~N}\right)$, phenol $\left(\mathrm{C}_{6} \mathrm{H}_{5} \mathrm{OH}\right)$ and cresol $\left(\mathrm{C}_{6} \mathrm{H}_{4} . \mathrm{OH} . \mathrm{CH}_{3}\right)$ probably result from the action of micro-organisms; but their production may be arrested or retarded by the action of certain drugs, such as calomel, salicylic acid and other so-called antiseptics. The fermentative changes in the intestines involve the formation of certain gases, which will be described at the close of this chapter. 


\section{Contents of the Large Intestine}

When the contents of the small intestine have passed the ileo-cæcal valve, they become changed in their general character, partly from admixture with the secretions of this portion of the canal, and are then known as the feces. The most notable changes relate to consistence, color and odor. The odor, especially, of normal fecal matter is decidedly characteristic.

Fecal matter has a much firmer consistence than the contents of the ileum, which is due to partial absorption of the liquid portions. As a rule the consistence is great in proportion to the length of time that the feces remain in the large intestine; and this is variable in different persons and in the same person, in health, depending somewhat on the character of food. The color changes from the yellow, more or less bright, which is observed in the ileum, to the dark yellowish-brown, characteristic of feces. Although the bile-pigment can not usually be recognized by the ordinary tests, it is a product of bilirubin that gives to the contents of the large intestine their peculiar color, which is lost when the bile is not discharged into the duodenum. In the large intestine, bilirubin $\left(\mathrm{C}_{32} \mathrm{H}_{36} \mathrm{~N}_{4} \mathrm{O}_{6}\right)$ is changed into hydrobilirubin, or stercobilin $\left(\mathrm{C}_{32} \mathrm{H}_{44} \mathrm{~N}_{4} \mathrm{O}_{7}\right)$. In a specimen of normal human feces, which had been dried, extracted with alcohol, the alcoholic extract precipitated with ether and the precipitate dissolved in distilled water, it was impossible to detect the biliary salts by Pettenkofer's test. The color of the feces varies considerably under different forms of diet. With a mixed diet the color is yellowish brown; with an exclusively flesh-diet it is much darker; and with a milk-diet it is more yellow.

The odor of the feces, which is characteristic and quite different from that of the contents of the ileum, is variable and is due in part to the peculiar decomposition of the residue of the food, in part to putrefactive products and in part to matters secreted by the mucous membrane of the colon and of the glands near the anus. Indol has what may be called a fecal odor. The odor of skatol is intensely fetid. Skatol, however, may be prepared artificially from indigo, when it is odorless.

The quantity of feces in the twenty-four hours, according to Wehsarg, is about 4.6 ounces ( 128 grams). This was the mean of seventeen observations; the largest quantity being 10.8 ounces ( 306 grams), and the smallest, 2.4 ounces (68 grams).

The reaction of the feces is variable, depending chiefly on the character of the food. Marcet found the human excrements always alkaline. Wehsarg, on the other hand, usually found the reaction acid, but frequently it was alkaline or neutral. 
The proportions of water and solid matter in the feces are variable. Berzelius found in the healthy human feces, 73.3 parts of water and 26.7 parts of solid residue. The average of seventeen observations by Wehsarg was the same. In the observations of Wehsarg, the mean quantity of solid matter discharged in the feces in the twenty-four hours was 463 grains ( 30 grams), the extremes being 882.8 grains $(57.2$ grams), and 251.6 grains ( 16.28 grams). The proportion of undigested matters in the solid residue was very small, averaging but little more than ten per cent, the mean quantity in the twenty-four hours in ten observations being but 52.5 grains ( 3.4 grams). This was found, however, to be very variable; the largest quantity being 126.5 grains (8.2 grams), and the smallest, I 2.5 grains (O.8 I gram).

Microscopical examination of the feces reveals various vegetable and animal structures that have escaped the action of the digestive fluids. Wehsarg also found a "finely divided fecal matter" of indefinite structure, but containing partly disintegrated intestinal epithelium. Crystals of cholesterin were never observed. Whenever the matter is neutral or alkaline, crystals of ammonio-magnesian phosphate are found. Mucus is also found in variable quantity, with desquamated epithelium and a few leucocytes. In addition, recent microscopical researches have shown the presence of spores of yeast and a great variety of bacteria, which latter exist in the feces in great abundance. These organisms probably excite many of the so-called putrefactive changes in the intestinal contents, which result in the formation of indol, phenol, skatol, cresol etc. According to Senator, these putrefactive products do not exist in the meconium. The quantity of inorganic salts in the feces is not great. In addition to ammonio-magnesian phosphate, magnesium phosphate, calcium phosphate and a small quantity of iron have been found. The chlorides are either absent or are present only in small quantity.

Marcet has pretty generally found in the human feces a substance possessing the characters of margaric acid, and volatile fatty acids; the latter free, however, from butyric acid. He also found a coloring matter, which probably is a modification of bile-pigment. Cystin is mentioned as an occasional constituent.

In addition to the matters just enumerated, the following substances have been extracted from the normal feces:-

Excrtin and Excretolcic Acid.-- Excretin $\left(\mathrm{C}_{20} \mathrm{H}_{36} \mathrm{O}\right)$ was obtained from the normal feces, by Marcet, in 1854 . This substance crystallizes from an ethereal solution in two or three days, in the form of long silky crystals. Examined with the microscope, these are found to consist of acicular, four-sided prisms of variable size. Excretin is insoluble in 
water, slightly soluble in cold alcohol, but very soluble in ether and in hot alcohol. Its alcoholic solutions are faintly though distinctly alkaline. Its fusing-point is between $203^{\circ}$ and $205^{\circ}$ Fahr. $\left(95^{\circ}\right.$ and $96^{\circ} \mathrm{C}$.). It may be boiled with potassium hydrate for hours without undergoing change. The quantity of excretin contained in the feces is not large. Only I 2.6 grains (0.8I6 gram) were obtained by Marcet from nine evacuations. In I857 Marcet assigned to excretin the formula $\mathrm{C}_{78} \mathrm{H}_{78} \mathrm{O}_{6}$; but the formula $\mathrm{C}_{20} \mathrm{H}_{36} \mathrm{O}$ is now given in works on physiological chemistry.

There exists little definite information regarding the production of excretin. Marcet examined on one occasion the contents of the small intestine of a man who had died of disease of the heart, without finding any excretin. It is probable that this substance is formed in the large intestine, although further observations are needed on this point.

The substance called excretoleic acid is indefinite in its composition and properties. It is described as an olive-colored fatty acid, insoluble in water, non-saponifiable and very soluble in ether and in hot alcohol. It fuses between $77^{\circ}$ and $79^{\circ}$ Fahr. $\left(25^{\circ}\right.$ and $26 . I^{\circ} \mathrm{C}$.), but its existence is doubtful.

Stercorin. - This substance was discovered in the feces in 1862 (Flint). As it is one of the most abundant and characteristic constituents of stercoraceous matters, it may properly be called stercorin. Stercorin may be extracted in the following way: The feces are first evaporated to dryness, pulverized and treated with ether. The etherextract is then passed through animal charcoal, fresh ether being added until the original quantity of the ether-extract has passed through. It is impossible to entirely decolorize the solution by this process; but it should pass through perfectly clear and of a pale-amber color. The ether is then evaporated and the residue is extracted with boiling alcohol. The alcoholic solution is evaporated, and the residue is treated with a solution of potassium hydrate for one or two hours at a temperature a little below the boiling-point, by which the fats are dissolved. The mixture is then largely diluted with water, thrown upon a filter and washed until the liquid which passes through is neutral and perfectly clear. The filter is then dried and the residue is washed out with ether. The ether-solution is then evaporated, extracted with boiling alcohol and the alcoholic solution is evaporated. The residue of this last evaporation is stercorin, which may be purified by recrystallization.

When first obtained, stercorin is a clear, slightly amber, oily substance, of about the consistence of Canada balsam used in microscopical preparations. In four or five days it begins to show characteristic crystals. These are few in number at first, but soon the entire mass 
assumes a crystalline form. In one analysis, from seven and a half ounces (202.5 grams) of normal human feces (the entire quantity for the twenty-four hours), 10.417 grains $(0.675 \mathrm{gram})$ of stercorin were obtained, the extract consisting entirely of crystals. This was all the stercorin to be extracted from the regular daily evacuation of a healthy male twenty-six years of age, weighing about one hundred and sixty pounds ( 72.58 kilograms). In the absence of other investigations, the daily quantity of this substance excreted may be assumed to be not far from ten grains (0.648 gram).

In many regards stercorin bears a close resemblance to cholesterin, which is a monatomic alcohol. It is neutral, inodorous and insoluble

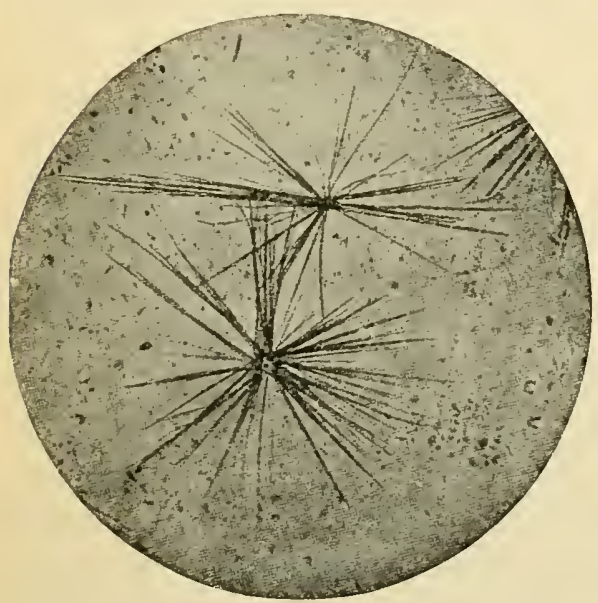

Fig. 49. - Crystals of stercorin, $\times 20$ (Flint, I862; recrystaliized, 1897). in water and in a solution of potassium hydrate. It is soluble in ether and in hot alcohol, but is almost insoluble in cold alcohol. A red color is produced when it is treated with strong sulphuric acid. It may easily be distinguished from cholesterin, however, by the form of its crystals.

Stercorin crystallizes in the form of thin delicate needles, frequently mixed with clear rounded globules, which probably are composed of the same substance in a non-crystalline form. The crystals often are arranged in bundles. They are not to be confounded with excretin, which crystallizes in the form of regular four-sided prisms, or with the thin rhomboidal or rectangular tablets of cholesterin.

There can be no doubt in regard to the origin of the stercorin found in feces. When the bile is not discharged into the duodenum, as probably is the case for a time in icterus accompanied with clay-colored evacuations, stercorin is not to be discovered in the dejections. In one case of this kind, in which the feces were subjected to examination, the matters extracted with hot alcohol were entirely dissolved by boiling for fifteen minutes with a solution of potassium hydrate, showing the absence of cholesterin and stercorin. In another examination of the feces from this patient, made nineteen days after, when the icterus had almost entirely disappeared and the evacuations had become normal, stercorin was discovered. These facts show that the cholesterin of the 
bile, in its passage through the intestine, is changed into stercorin. Both these substances are crystallizable, non-saponifiable, are extracted by the same chemical manipulations and behave in the same way when treated with sulphuric acid. Stercorin must be regarded as a modification of cholesterin, which is the excrementitious constituent of the bile.

The change of cholesterin into stercorin is directly connected with the process of intestinal digestion. If an animal is kept for some days without food, cholesterin will be found in the feces, although, for a few days, stercorin is also present. It is a fact commonly recognized by those who have analyzed the feces, that cholesterin does not exist in the normal evacuations; but whenever digestion is arrested, the bile being constantly discharged into the duodenum, cholesterin is found in large quantity. For example, in hibernating animals, cholesterin is always present in the feces. The same is true of the contents of the intestines during fotal life; the meconium always containing a large quantity of cholesterin, which disappears from the evacuations when the digestive function becomes established. The formula for stercorin is $\mathrm{C}_{2}-\mathrm{H}_{48} \mathrm{O}$. Its physiological relations will be considered in connection with the excretory office of the liver.

Indol, Skatol, Phenol, Cresol etc. - The so-called putrefactive processes, which begin in the small intestine, are more marked in the large intestine and give rise to certain products which have the characteristic fecal odor. Certain of these substances may be produced by the prolonged action, out of the body, of the pancreatic juice on proteids. The pancreatic juice, in an alkaline medium, changes the trypsin-peptones into leucin, tyrosin, hypoxanthin and asparaginic acid. By still further prolonging this action, indol, skatol, phenol and cresol, with some other analogous substances and volatile fatty acids, are formed, and there is an evolution of certain gases. It is probable that these products are formed in abnormal quantities in the small intestine in certain cases of intestinal dyspepsia. Methyl mercaptan $\left(\mathrm{CH}_{3} \mathrm{SH}\right)$ is sometimes found in small quantity in the intestines. This substance has a peculiarly penetrating and disagreeable odor. It probably is produced by bacterial decomposition of proteids.

Mor'ements of the Large Intestine. - Movements of the general character noted in the small intestine occur in the large intestine, although the peculiarities in the arrangement of the muscular fibres and the more solid consistence of the contents render these movements in the large intestine somewhat distinctive. In all instances where the movements have been observed in the human subject or in the lower animals, they have been found to be less vigorous and rapid than the 
contractions of the small intestine. Indeed, when the abdominal organs are exposed, either in a living animal or immediately after death, movements of the large intestine ordinarily are not observed, except on the application of mechanical or electric stimulation; and they are then more circumscribed and much less marked than in any other part of the alimentary canal. That the feces remain for a considerable time in some of the sacculated pouches of the colon, is evident from the appearance which they sometimes present of having been moulded to the shape of the canal. This appearance is frequently observed in the dejections, which are then said to be "figured."

In the cæcum, the pressure of matters received from the ileum forces the mass onward into the ascending colon, and the contractions of its muscular fibres probably are slight and inefficient. Once in the colon, it is easy to see how the contractions of the muscular structure - the longitudinal bands shortening the canal, and the transverse fibres contracting below and relaxing above - are capable of passing the fecal mass slowly onward. Although the transverse fibres are thin and apparently of little power, their contraction is sufficient to empty the sacculi, when assisted by the movements of the longitudinal fibres, especially as the canal is never completely filled and the feces are frequently in the form of small moulded lumps. By these slow and gradual movements, the contents of the large intestine are passed toward the sigmoid flexure of the colon, where they are arrested until the period arrives for their final discharge. The time occupied in the passage of the feces through the ascending, transverse and descending colon undoubtedly is variable in different persons, as great variations are observed in the intervals between the acts of defecation. During their passage along the colon, the contents of the canal assume more and more of the normal fecal consistence and odor and become slightly coated with the mucous secretion of the parts.

The accumulation of feces usually takes place in the sigmoid flexure of the colon; and under normal conditions, the rectum is found empty and contracted. This part of the colon is much more movable than other portions of the large intestine. At certain intervals, the fecal matter is passed into the rectum and is then almost immediately discharged from the body.

Defccation. - In health, expulsion of fecal matters takes place with regularity usually once in the twenty-four hours. This rule, however, is by no means invariable, and dejections may occur habitually twice in the day or every second or third day within the limits of health. At the time when defecation ordinarily takes place, a peculiar sensation is experienced calling for an evacuation of the bowels; and if this is disre- 
garded, the desire may pass away, after a little time the act becoming impossible. It is probable that the feces are then passed out of the rectum by antiperistaltic action. The sensation that leads to an effort to discharge the feces is due to the accumulation of matters in the sigmoid flexure, which finally present at the contracted upper portion of the rectum.

The above is the mechanism of the descent of fecal matter into the rectum in defecation, as the act is usually performed; but under certain conditions, feces must accumulate in the dilated portion of the rectum. Ordinarily, the discharge of feces takes place only after the efforts have been continued for a certain time, and when the evacuation is "figured," the whole length discharged frequently exceeds so much the length of the rectum, that it is evident that a portion of it must have come from the colon; but in cases in which the feces are very liquid, or when the usual call for an alvine evacuation has not been regarded and has become imperative, the discharge of matters at the moment when the sphincter is relaxed shows that the rectum has been more or less distended.

The diaphragm and the abdominal muscles compress the abdominal organs, and consequently those contained in the pelvis, and assist in the expulsion of the contents of the rectum. The diaphragm is the most important of the voluntary muscles concerned in this process; and during the act of straining, the lungs are moderately filled and respiration is for the time interrupted. The vigor of these efforts depends to a considerable extent on the consistence of the fecal mass, violent contractions frequently being required for the expulsion of hardened feces that have accumulated during long constipation. Although more or less straining usually takes place, the contractions of the muscular coats of the rectum frequently are competent of themselves to expel the feces, especially when they are soft.

\section{Gases found in the Alimentary Canal}

The gases in the stomach appear to have no definite office. They usually exist in small quantity and are sometimes absent. The oxygen and nitrogen are derived from bubbles of air incorporated with the alimentary bolus during mastication and insalivation. The other gases probably are evolved from the food during digestion; at least, there is no satisfactory evidence that they are produced in any other way. Magendie and Chevreul collected and analyzed a small quantity of gas from the stomach of an executed criminal a short time after death and ascertained that it had the following composition :- 
GASES CONTAINED IN THE STOMACH

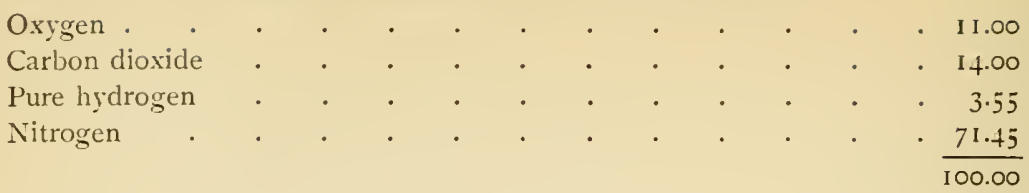

Magendie and Chevreul found three gases in the small intestine. Their examinations were made on three criminals soon after execution. The first was twenty-four years of age, and two hours before execution, he had eaten bread and Gruyère cheese and had drunk red wine and water. The second, who was executed at the same time, was twentythree years of age, and the conditions as regards digestion were the same. The third was twenty-eight years of age, and four hours before death, he ate bread, beef and lentils, and drank red wine and water. The following was the result of the analyses:-

GaSES CONTAINED IN THE SMaLl intestine

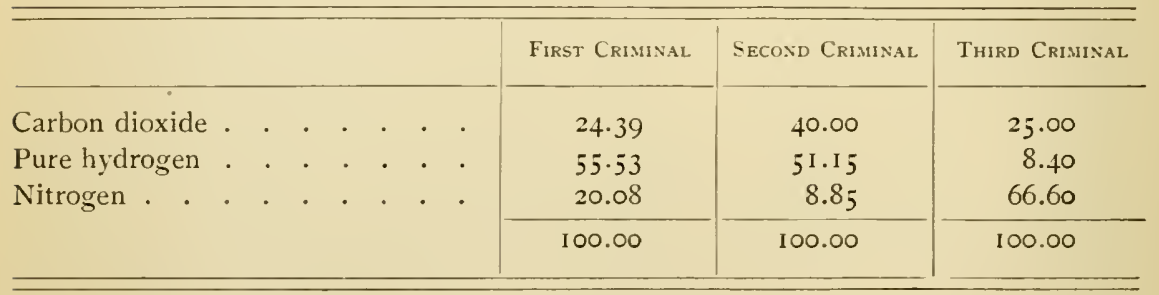

No oxygen was found in either of the examinations, and the quantities of the other gases were so variable as to lead to the supposition that their proportion is not at all definite. Reference has already been made to the mechanical office of these gases in intestinal digestion. The presence of any considerable quantity of gas, however, in the stomach does not seem necessary to normal gastric digestion, except as bubbles of air render the alimentary mass spongy and thus facilitate the penetration of the gastric juice. The importance of this has already been considered in connection with mastication and insalivation.

In the large intestine, the constitution of the gases presented the same variability as in the small intestine. Carburetted hydrogen was found in all the analyses. In the large intestine of the first criminal and in the rectum of the third, were found traces of hydrogen monosulphide. The following is the result of the analyses in the cases just cited. In the third, the gaseous contents of the cæcum and the rectum were analyzed separately:- 
GASES CONTAINED IN THE LARGE INTESTINE

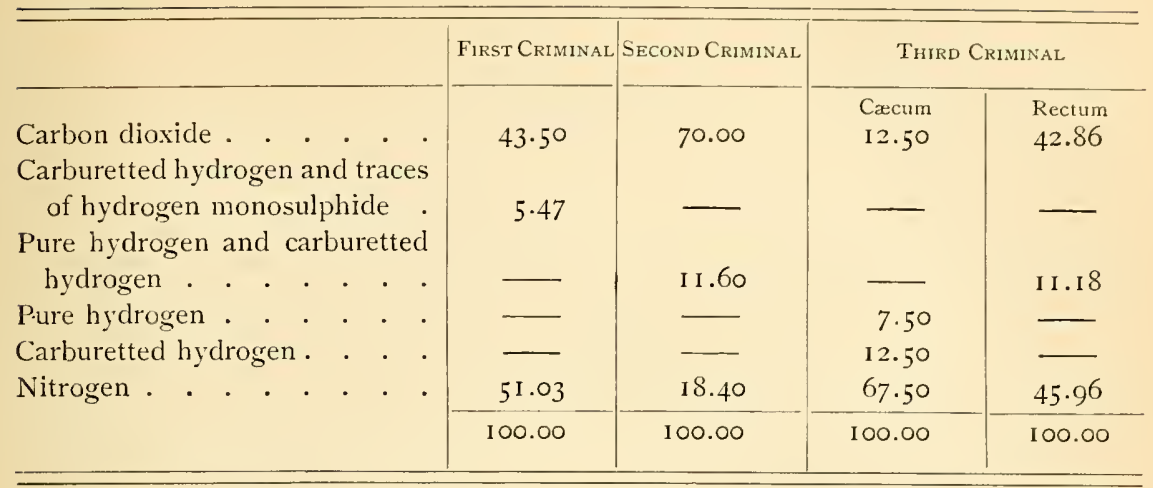

Origin of the Intestinal Gases. - The most reasonable view to take of the origin of the gases normally found in the intestines is that they are given off from the articles of food in their various stages of digestion and decomposition. That this is the principal source of the intestinal gases, there can be no doubt; and it is well known that certain articles of food, particularly vegetables, generate much more gas than others. The principal gases found in the intestinal canal may all be obtained from the food. Some of them, as hydrogen and carburetted hydrogen, do not exist in the blood; and it is difficult to conceive how they can be generated in the intestine except by decomposition of certain of the articles of food. Gases are not found in the alimentary canal of the fotus. 


\section{CHAPTER X}

\section{ABSORPTION - LYMPH AND CHYLE}

Absorption by bloodvessels - Absorption by lymphatic and lacteal vessels - Physiological anatomy of the lymphatic and lacteal vessels - Structure of the lymphatic and lacteal vessels - Lymphatic glands - Absorption of proteids by the lacteals - Absurption of sugar and salts by the lacteals - Absorption of water by the lacteals - Absorption by the skin - Absorption by the respiratory surface - Absorption from closed cavities, reservoirs of glands, etc. - Absorption of fats and insoluble substances - Influence of the condition of the blood and of the vessels on absurption - Influence of the nervous system on absorption-Osmosis - Mechanism of the passage of liquids through membranes - Osmotic pressure - Lymph and chyle - Properties and composition of lymph - Corpuscular elements of the lymph - Origin and uses of the lymph - Properties and composition of chyle - Composition of chyle - Microscopical characters of the chyle - Movements of the lymph and chyle.

\section{Absorption By Bloodvessels}

THAT solutions pass through the walls of the capillaries and of the small veins and that absorption actually takes place in great part by bloodvessels are facts which hardly demand discussion at the present day. Soluble substances that have disappeared from the alimentary canal have been found repeatedly in the blood coming from this part, even when the lymphatics had been divided and communication existed only through the bloodvessels; and it has been shown that during absorption, the blood of the portal vein is rich in proteids, sugar and other matters resulting from digestion.

In the mouth and œesophagus, the sojourn of alimentary matters is so brief and the changes which they undergo are so slight, that no considerable absorption takes place. It is evident, however, that the mucous membrane of the mouth is capable of absorbing certain soluble matters, from the effects that are constantly observed when the smoke or the juice of tobacco is retained in the mouth even for a short time. A certain proportion of the constituents of food that are dissolved by the gastric juice and converted into peptones is taken up directly by the bloodvessels of the stomach. It may, indeed, be assumed, as a general law, that alimentary matters are in great part absorbed so soon as their digestive transformations in the alimentary canal have been accomplished.

In the passage of the food along the intestinal canal, as the digestion of the proteids is completed, these matters are absorbed. The greatest part of the food is absorbed by the intestinal mucous mem- 
brane, and, with the alimentary substances proper, a large quantity of secreted fluid is reabsorbed. This is particularly marked as regards the bile. The biliary salts disappear as the alimentary mass passes down the intestine and undoubtedly are absorbed, although they are so changed that they can not be detected in the blood by the ordinary tests. In this portion of the alimentary canal, it will be remembered, a large absorbing surface is provided by the arrangement of the mucous membrane in folds, forming the valvulæ conniventes, and by the presence of villi, which are found throughout the small intestine. A certain portion of the gaseous contents of the intestines is also taken up, although it is not easily ascertained what particular gases are thus absorbed.

\section{Absorption by Lymphatic and Lacteal Vessels}

Physiological Anatomy of the Lymphatic and Lacteal Vessels. - The lacteals are the intestinal lymphatics; and during the intervals of intes-
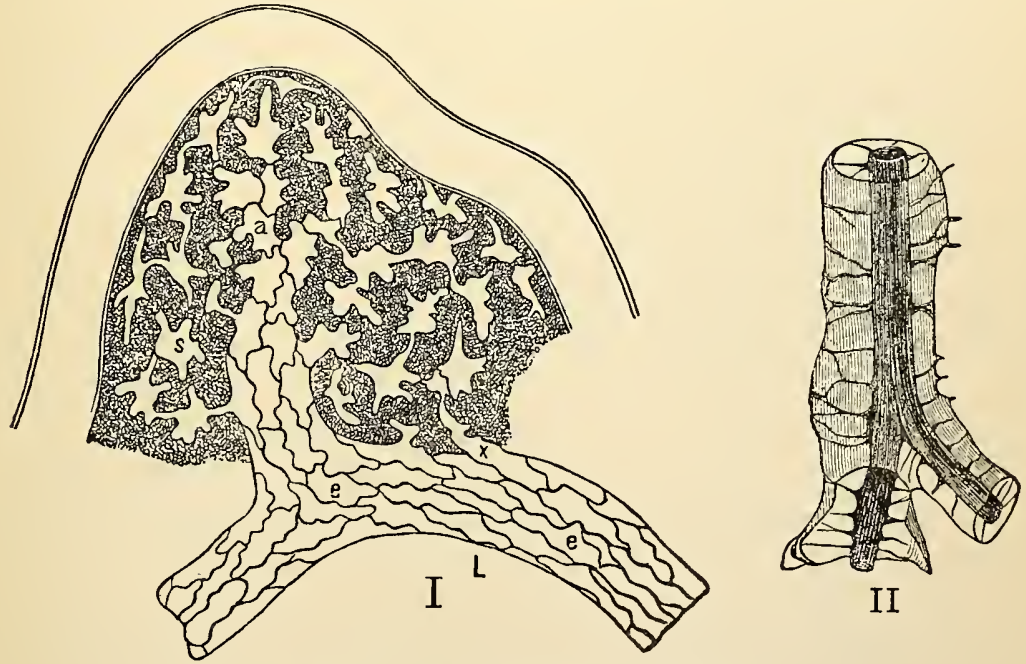

Fig. 50. - Origin of lymphatics (Landois).

I. From the central tendon of the diaphragm of the rabbit (semidiagrammatic); $s$, lymph-canals communicating by $x$ with the lymphatic vessel $L$; $a$, origin of the lymphatic by a union of lymphcanals; $e, e$, endothelium. II. Perivascular canal.

tinal absorption they carry a liquid that is identical with the contents of other lymphatic vessels. In their structure, also, the lacteals are identical with the general lymphatics.

In the connective tissues - which are so widely distributed in the body - there are always found irregularly-shaped stellate spaces, which communicate with each other by branching canals, called lymph-spaces. 
These spaces contain a liquid and large numbers of leucocytes. The leucocytes in these spaces may be called lymph-corpuscles, as they eventually find their way into the true lymphatic vessels; but they are thought to be corpuscles that have passed through the stomata of the capillary bloodvessels. The connective-tissue lymph-spaces, by certain of their branches, finally communicate with the lymph-capillaries, through what have been regarded as the stomata of these vessels. These anatomical data have led to the following view in regard to the relations between the blood, the lymph and the tissues.

Nutrient matters are supplied to the parts by transudation through the walls of the capillary bloodvessels; and effete matters pass from the lymph-spaces into the true lymphatic vessels to be carried to the venous system. In certain tissues and organs, however, such as the cornea and fibrous membranes, the lymph-spaces or canals supply the nutrient liquid; and in the glands, possibly they supply part of the material used in the formation of the secretions.

In the serous membranes and in analogous structures, there are large numbers of openings into the cavities; and the peritoneum, pleura, pericardium, tunica vaginalis testis, chambers of the eye, labyrinth of the internal ear and subarachnoid space are to be regarded as lymphsacs, the contained liquids being lymph, without, however, presenting the so-called lymph-corpuscles.

The relations between the bloodvessels and the smallest lymphatics are very close in certain parts. In the cerebro-spinal centres, the smallest vessels of bone, the retina and the liver, is a system of canals which completely surround the small bloodvessels and are connected with the lymphatic trunks, or reservoirs, described by Fohmann, and found beneath the pia mater. These are called perivascular canals; and the contained liquid is true lymph, containing leucocytes. They exceed the bloodvessels in diameter by $\frac{1}{1250}$ to $\frac{1}{400}$ of an inch (2O to $62 \mu)$.

The capillary lymphatics have been studied in various parts by means of mercurial injections; but the presence of valves in the small trunks renders it necessary to make these injections from the periphery. The vessels have been injected in certain situations with mercury, by simply puncturing with a fine-pointed canula the parts in which the plexus is supposed to exist, and allowing the liquid to diffuse itself gently. Following the course of the vessels, the injection passes into the larger trunks and thence to the lymphatic glands. The regularity of the plexus through which the liquid is first diffused and the passage of the injection through the larger vessels to the glands are proof that the lymphatics have been penetrated and that the appearances observed 
are not the result of infiltration in the tissue. It does not appear that the vessels composing this plexus vary much in size. They are quite elastic, and after distention by injection, they return to a very small diameter when the fluid is allowed to escape.

The lacteal system presents essentially the same anatomical characters as the general lymphatics, and the vessels are filled with colorless lymph during the intervals of digestion. In many situations the lymphatics present in their course little solid structures, called lymphatic glands, although, as regards structure and office, they are not true glandular organs. The smallest capillary lymphatics have a diameter of about $\frac{1}{300}$ of an inch $(83 \mu)$. This may be taken as their average diameter in primitive plexuses. The plexus, when the vessels are abundant, as they are in certain parts of the cutaneous surface, resembles an ordinary plexus of capillary bloodvessels, except that the walls of the lymphatics are thinner and their diameter is greater. The vessels are lined by endothelial cells, the borders of which are brought into view by the action of silver nitrate.

The smallest lymphatic vessels are by far the most abundant. They are arranged in the form

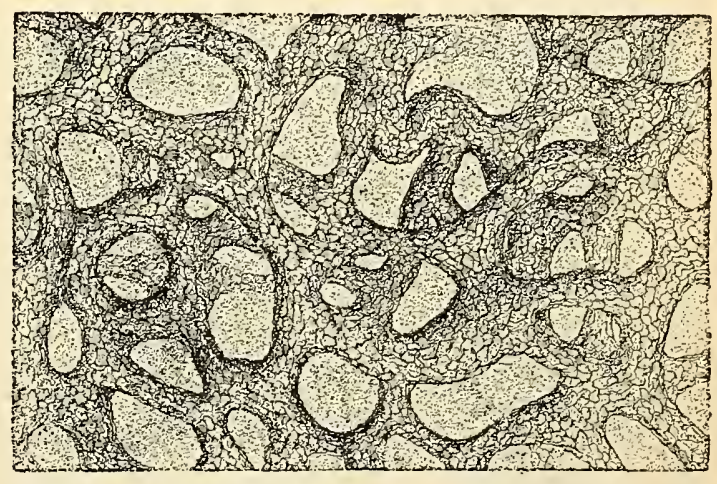

Fig. 51. - Lymphatic plexus, showing the endothelium (Belaieff).

of a fine plexus, superficially situated in the skin. A second plexus exists just beneath the skin, composed of vessels of much greater diameter. The skin is thus enclosed between two plexuses of capillary lymphatics. A plexus analogous to the superficial plexus of the skin is found just beneath the surface of the mucous membranes. These may, indeed, be classed with the superficial lymphatics. The deep lymphatics are much larger and less abundant, and their origin is less easily made out. These accompany the deeper veins in their course. They receive the lymph from the superficial vessels.

No valvular arrangement is found in the smallest lymphatics; but the vessels coming from the primitive plexuses, as well as the large vessels, contain valves in great numbers. These valves, being closely set in the vessels, give to them, when filled with injection, a peculiar and characteristic beaded appearance.

The course of the lymphatics usually is direct. As they pass toward 
the great trunks by which they communicate with the venous system, they present a peculiar anastomosis with the adjacent vessels, called anastomosis by bifurcation; that is, as a vessel passes along with other vessels nearly parallel with it, it bifurcates, and the two branches pass into the nearest vessels on either side. These anastomoses are quite frequent, and they usually occur between vessels of equal size. In

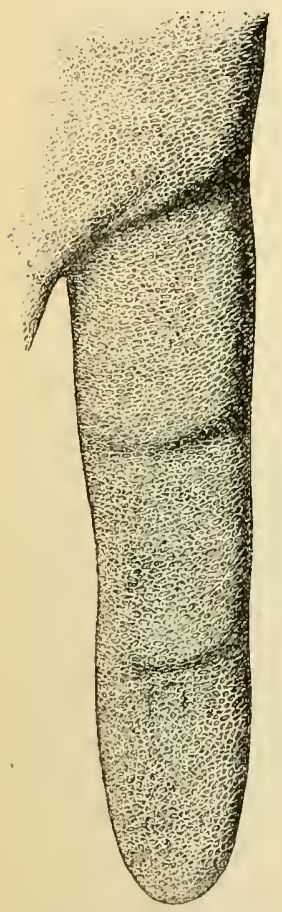

Fig. 52. - Superficial lymphatics of the skin of the palmar surface of the finger (Sappey).

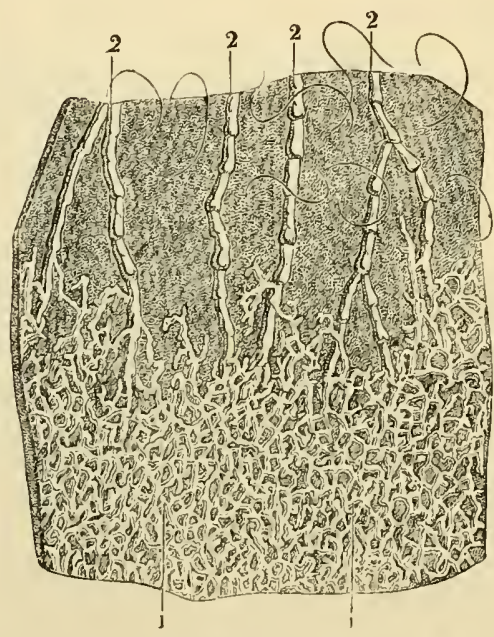

Fig. 53. - Deep lymphatics of the skin of the finger (Sappey).

I, I, deep network of cutaneous lymphatics; $2,2,2,2$, lymphatic trunks connected with this network.

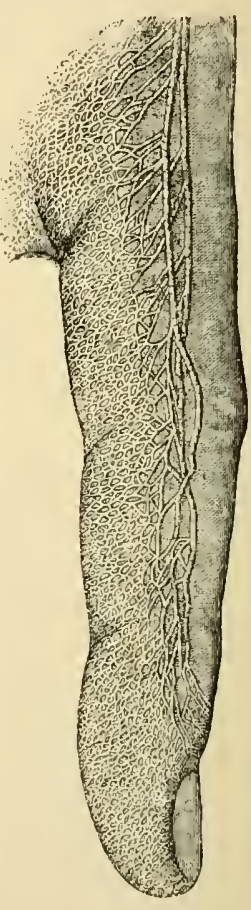

Fig. 54. - The same finger, lateral view, showing lymphatic trunks connected with the superficial network (Sappey).

their course the vessels pass through the so-called lymphatic glands, or nodes.

A notable peculiarity in the lymphatic vessels is that they vary very little in size, being nearly as large at the extremities as they are near the trunk. In their course they are always much smaller than the veins and do not progressively enlarge as they pass on to the great lymphatic trunks. The largest vessels that pass from the skin are $\frac{1}{25}$ to $\frac{1}{12}$ of an inch ( $\mathrm{I}$ to 2 millimeters) in diameter, and the larger vessels, in their course, have a diameter of $\frac{1}{12}$ to $\frac{1}{8}$ of an inch ( 2 to 3 millimeters). As in the case of the smallest lymphatics of the primi- 
tive plexuses, the elasticity of the walls of the vessels renders their diameter greatly dependent on the pressure of liquid in their interior. Many anatomists have noticed that vessels which are hardly perceptible while empty are capable of being dilated to the diameter of half a line (about I millimeter) or more, returning to their original size so soon as the distending liquid is removed.

In the lymphatics of the skin, the only important peculiarity not yet mentioned is that the vessels appear to be unequally distributed in different parts of the surface. They are particularly abundant in the scalp over the biparietal suture, the soles of the feet and the palms of the hand, the fingers at the lateral portion of the last phalanges, and the scrotum. In the median portion of the scrotum they attain their highest degree of development. They are also found, though in less number, originating from around the median line on the anterior and posterior surface of the trunk, the posterior median portion of the extremities, the skin over the mammæ

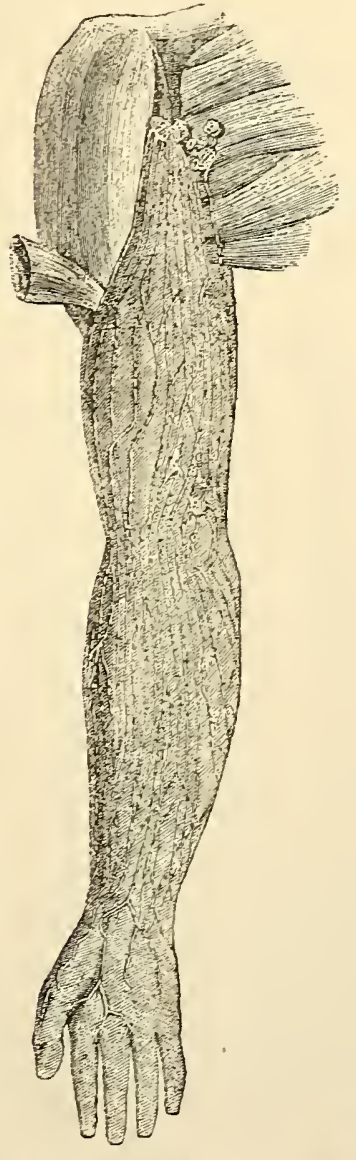

Fig. 55. - Superficial lym-
phatics of the arm (Sappey).

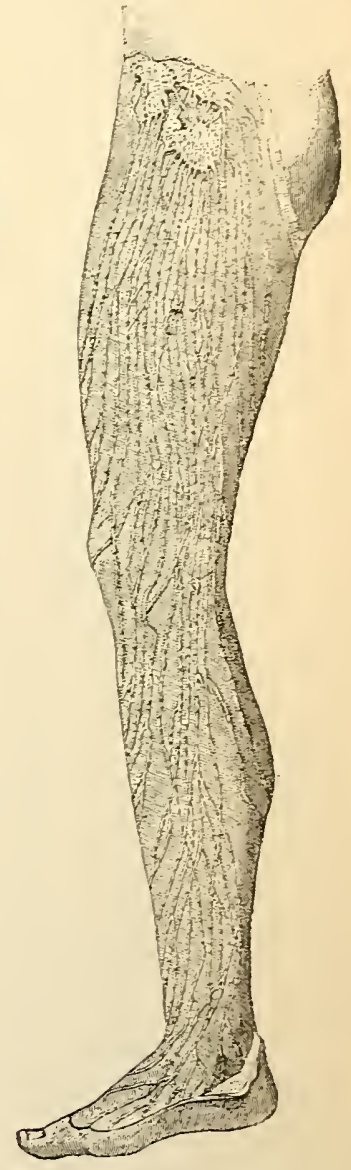

Fig. 56. - Superficial lymphatics of the leg (Sappey). and around the orifices of the mucous passages. Lymphatic vessels have been demonstrated in the anterior portion of the forearm, the thigh and the leg, and in the middle portion of the face, although they are demonstrated with difficulty in these situations. If they exist at all in other portions of the cutaneous surface, they are not abundant.

In the mucous membranes the lymphatics are very abundant. Here are found, as in the skin, two distinct layers which enclose between them the entire thickness of the mucous membrane. The 
more superficial of these layers is composed of a rich plexus of small vessels, and beneath the mucous membrane is a plexus consisting of vessels of larger size. The superficial plexus is very rich in the mixed structure which forms the lips and the glans penis, and around the orifices of the mouth, the nares, the vagina and the anus. There are certain mucous membranes in which the lymphatics have never been injected. In the serous membranes lymphatics have been demonstrated in great abundance. Lymphatics have also been observed taking their origin in the voluntary muscles, the diaphragm, the heart and the non-striated muscular coats of the hollow viscera, although their investigation in these situations is difficult.

Lymphatics are found coming from the lungs in great numbers. These arise in the walls of the air-cells and surround each pulmonary lobule with a close plexus. The deep vessels follow the course of the bronchial tubes, passing through the bronchial glands and the glands at the bifurcation of the trachea, to empty into the thoracic duct and the great lymphatic duct of the right side.

In the glandular system, including the ductless glands, and in the ovaries, the lymphatic vessels are, as a rule, more abundant than in any other parts of the body. They are especially abundant in the testicles, the ovaries, the liver and the kidneys.

The lymphatic vessels from the superficial and deep portions of the head and face on the right side, and those from the superficial and deep portions of the right arm, the right half of the chest, and the mammary gland, with a few vessels from the lungs, pass into the great lymphatic duct (ductus lymphaticus dexter), which empties into the venous system at the junction of the right subclavian with the internal jugular. This vessel is about an inch (25.4 millimeters) in length and $\frac{1}{12}$ to $\frac{1}{8}$ of an inch ( 2 to 3 millimeters) in diameter. It is provided with a pair of semilunar valves at its opening into the veins, which effectually prevents the ingress of blood. The vessels from the inferior extremities and those from the lower portions of the trunk, the pelvic viscera, the abdominal organs generally and the left half of the body above the abdomen empty into the thoracic duct.

In their course, the lymphatics pass through the small, flattened, oval bodies, called the lymphatic glands, or nodes, which are abundant in the groin, the axilla, the pelvis and some other parts. Two to six vessels, called vasa afferentia, penetrate each node, having first broken up into a number of smaller vessels just before they enter. They pass out by a number of small vessels which unite to form one, two or three trunks, usually of larger size than the vasa efferentia. The vessels which thus emerge from the glands are called vasa efferentia. 
The lymphatics of the small intestine, called lacteals, pass from the intestine between the folds of the mesentery to empty, sometimes by one and sometimes by four or five trunks, into the receptaculum chyli. In their course, the lacteals pass through several sets of lymphatic glands, which are here called mesenteric glands.

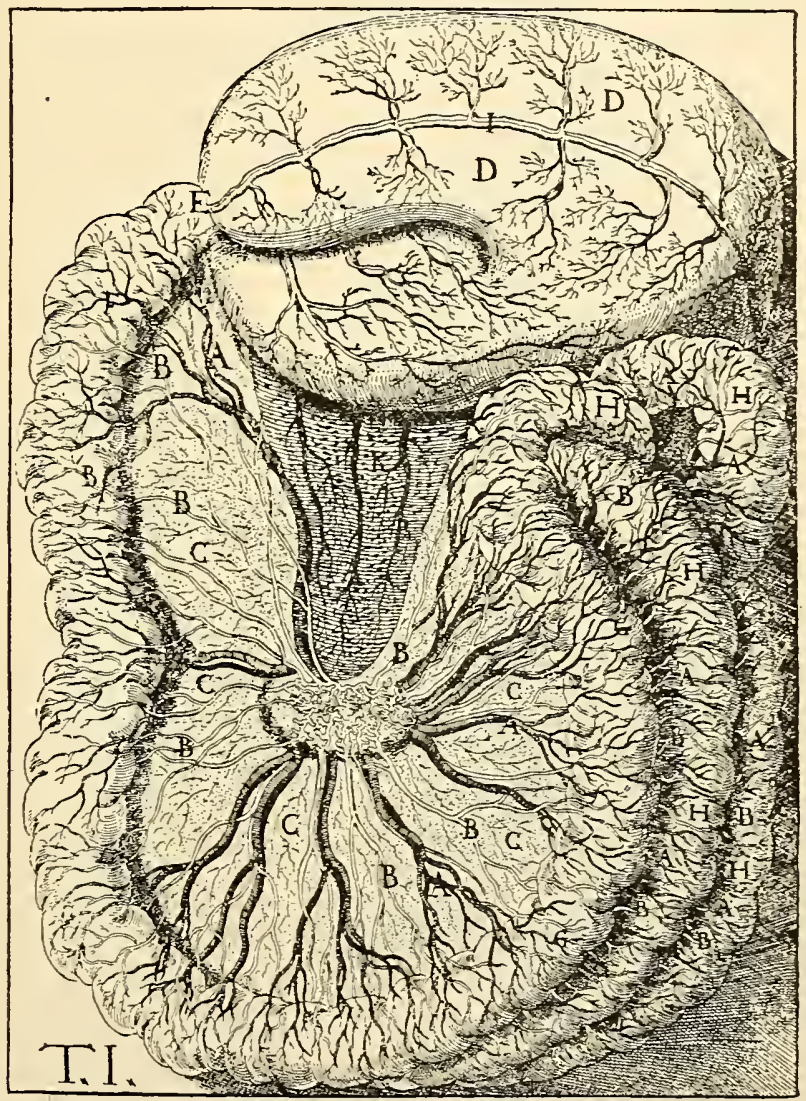

Fig. 57. - Stomaich, intestine and mesentery, with the mesenteric bloodvessels and lucteals (copied and reduced about one-half from a figure in the original work of Asellius, published in 1628 ).

$A, A, A, A, A$, mesenteric arteries and veins; $B, B, B, B, B, B, B, B, B, B$, lacteals; $C, C, C, C$, mesentery; $D, D$, stomach; $E$, pyloric portion of the stomach; $F$, duodenum; $G, G, G$, jejunum; $H, H, H, H, H$, ileum ; $I$, artery and vein on the fundus of the stomach; $K$, portion of the omentum.

The thoracic duct, into which most of the lymphatic vessels empty, is a vessel with delicate walls and about the size of a goose-quill. It begins by a dilatation, more or less marked, called the receptaculum chyli. This is situated on the second lumbar vertebra. The canal passes upward in the median line for the inferior half of its length. It then inclines to the left side, forms a semicircular curve something like the 
arch of the aorta, and empties at the junction of the left subclavian with the internal jugular vein. It diminishes in size from the receptaculum to its middle portion and becomes larger again near its termination. It occasionally bifurcates near the middle of the thorax, but the branches become reunited a short distance above. At its opening into the venous system, there usually is a valvular fold, but this is not constant. There is always, however, a pair of semilunar valves in the duct, three-quarters

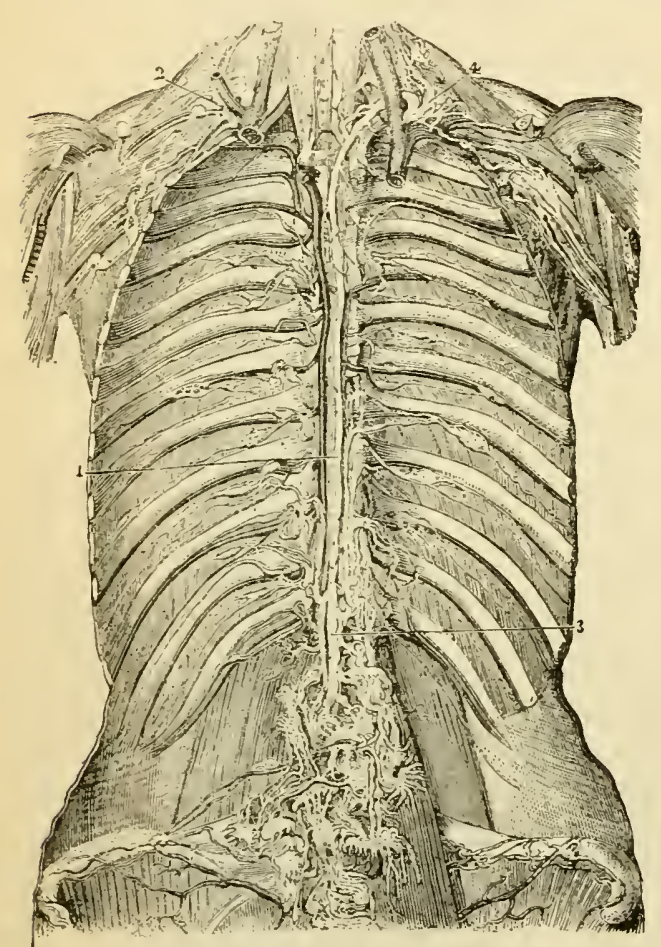

Fig. 58. - Thoracic duct (Mascagni).

I, thoracic duct; 2. great lymphatic duct ; 3 , receptaculum chyli ; 4 , curve of the thoracic duct just before it empties into the venous system. chyle. On account of the difficulty in studying the lymphatics at their origin, except by means of injections or by reagents which stain the vessels, investigations into the structure of the smallest vessels have not been very satisfactory. It is supposed, however, that the vessels here consist of a single coat, resembling, in this regard, the capillary bloodvessels. Belaieff has described in the capillary lymphatics of the penis a lining of endothelial cells arranged in a single layer. These cells are oval, polygonal, fusiform or dentated, with their long diameter in the direction of the axis of the vessels.

of an inch to an inch (19 to 25 millimeters) from its termination, which prevents the entrance of blood from the venous system.

The foregoing sketch of the descriptive anatomy of what has been called the absorbent system of vessels shows that they may collect liquids, not only from the intestinal canal during digestion, but from nearly every tissue and organ in the body, and that these are finally received into the venous circulation.

Structure of the Lymphatic and Lacteal l'essels. - The lymphatic vessels, even those of largest size, are remarkable for the delicacy and transparency of their walls. This is illustrated in the case of the lacteals, which are hardly visible in the transparent mesentery, unless filled with 
In all but the capillary lymphatics, although the walls are very thin, three distinct coats can be distinguished. The internal coat consists of an elastic membrane lined with oblong endothelial cells. This coat readily gives way when the vessels are forcibly distended. The middle coat is composed of longitudinal fibres of connective tissue, with delicate elastic fibres, and non-striated muscular fibres arranged transversely. The external coat is composed of the same structures as the middle coat, but most of the fibres are arranged longitudinally. In this coat the muscular fibres do not form a continuous sheet, but are collected into separate fasciculi, which have a direction either longitudinal or oblique. The fibres of connective tissue are abundant and unite the vessels to the surrounding parts. The internal and the middle coats are closely adherent to each other; but the external coat may readily be separated from the others. Bloodvessels have been found in the walls of the lymphatics, and the existence of vasomotor nerves is probable.

The walls of the lymphatic vessels are closely adherent to the surrounding tissues; so closely, indeed, that even a small portion of a vessel is detached with difficulty, and the vessels, even those of large size, can not be followed out and isolated for any considerable distance.

In all the lymphatic vessels, beginning a short distance from their plexuses of origin, are semilunar valves, usually arranged in pairs with their concavities looking toward the larger trunks. These folds are formed of the middle and inner coats; but the fold formed from the lining membrane is by far the wider, so that the free edges of the valves are thinner than the portion attached to the vessel. The valves are most abundant in the

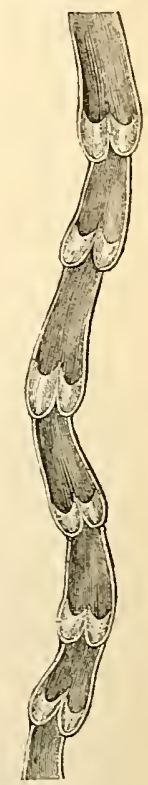

Fig. 59.-Valies of the lymphatics (Sappey). superficial vessels. The distance between the valves is $\frac{1}{12}$ to $\frac{1}{8}$ of an inch ( 2 to 3 millimeters) near the origin of the vessels, and $\frac{1}{4}$ to $\frac{1}{3}$ of an inch ( $\sigma$ to 8 millimeters) in their course. In the lymphatics between the muscles the valves are less abundant. They are relatively few in the vessels of the head and neck and in all that have a direction from above downward. Although there are valves in the thoracic duct, they are not so abundant as in the smaller vessels.

In their anatomy and general properties, the lymphatics bear a close resemblance to the veins. Although much thinner and more transparent, their coats have nearly the same arrangement. The arrangement of valves is the same; and in both systems, the folds prevent reflux of liquids when the vessels are subjected to pressure. 
The lymphatics are very elastic; and it has been shown that the larger vessels and those of medium size are contractile, although the action of their muscular fibres, like that of all fibres of the non-striated variety, is slow and gradual.

An important point in connection with the anatomy of the lymphatic vessels is the question of the existence of openings in their walls, which might allow the passage of solid particles or of emulsions. Anatomical observations have indicated the existence of stomata, of variable size and irregular shape, in the smallest vessels; and an argument in favor of the existence of these openings is the fact of the actual passage, through the walls of the vessels, of fatty particles, the entrance of which can not be explained by the well-known laws of endosmosis. The anatomical evidence of the existence of openings is derived mainly from preparations stained with silver nitrate. It is assumed that silver nitrate stains the solid parts of tissues and the borders of the endothelial cells, and that non-nucleated areas which do not present any staining are necessarily open. In preparations of the lymphatics, the solution of silver is seen staining the tissues and especially the borders of the enclothelial cells lining the vessels; but there are areas between these cells where no staining is observed and in which no nuclei are brought out by staining with carmin.

Lymplatic Glands. - In the course of the lymphatic vessels, are small lenticular bodies, called lymphatic glands, or nodes. The number of these is very great, although it is estimated with difficulty, from the fact that many of them are very small and may escape observation. It may be stated as an approximation that there are six or seven hundred lymphatic glands in the body. Their size and form are also variable within the limits of health. They usually are flattened and lenticular, some as large as a bean and others as small as a small pea or even a pin's-head. They are arranged in two sets; one superficial, corresponding with the superficial lymphatic vessels, and a deep set, corresponding with the deep vessels. The superficial glands are most abundant in the folds at the flexures of the great joints and about the great vessels of the head and neck. The deep-seated glands are most abundant around the vessels coming from the glandular viscera. A distinct set of large glands is found connected with the lymphatic vessels between the folds of the mesentery. These are known as the mesenteric glands. All the lymphatic vessels pass through glands before they empty into the great lymphatic trunks, and most of them pass through several glands in their course.

The normal glands are of a grayish white or reddish color, of about the consistence of the liver, presenting a hilum where the larger blood- 
vessels enter and the efferent vessels emerge, and are covered, except at the hilum, with a delicate membrane composed of inelastic fibres, a few elastic fibres and non-striated muscular fibres. Their exterior is somewhat tuberculated, from the projections of the follicles just beneath the investing membrane. The interior of the glands is soft and pulpy. It presents a coarsely-granular cortical substance, of a reddish white or gray color, which is $\frac{1}{6}$ to $\frac{1}{4}$ of an inch (4 to 6 millimeters) in thickness in the largest glands. The medullary portion, which comes to the surface at the hilum, is lighter colored and is coarser than the cortical substance. Throughout the gland are found delicate fasciculi of fibrous tissue connected with the investing membrane, which serve as a fibrous skeleton for the gland and divide its substance into alveoli. The structure is far more delicate in the cortical than in the medullary portion. Within the alveoli are irregularly-oval closed follicles (lymph-nodules), about $\frac{1}{2} \frac{1}{50}$ of an inch $(100 \mu)$ in diameter, filled with liquid and with cells like those contained in the solitary glands of the intestines and the patches of Peyer. The cells - sometimes called lymph-cells are situated at the periphery

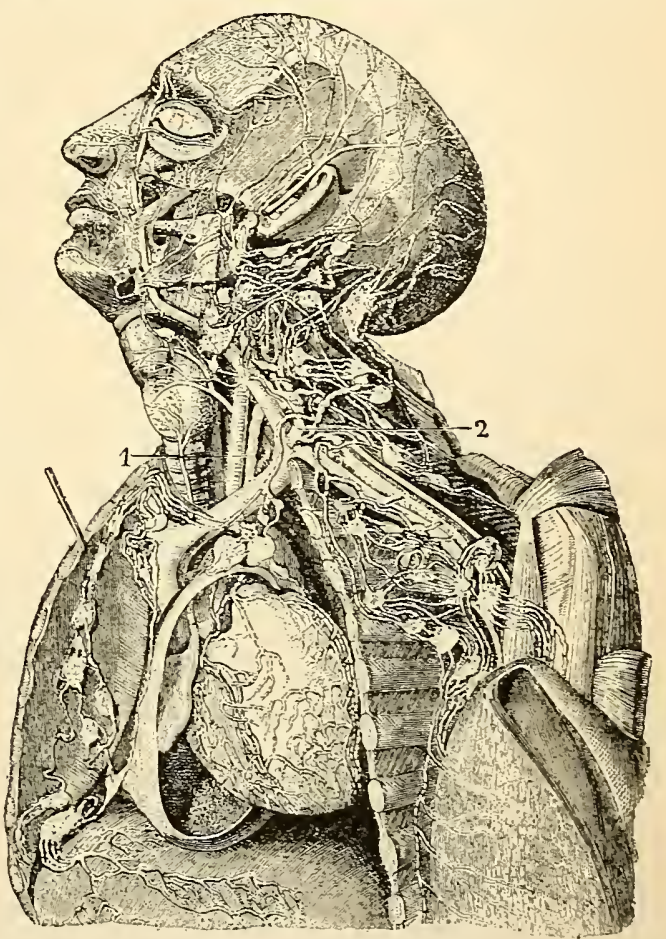

Fig. 60. - Lymphatics and lymphatic glands (Mascagni).

I, upper extremity of the thoracic duct, passing behind the internal jugular vein; 2 , opening of the thoracic duct into the internal jugular and left subclavian veins. The lymphatic glands are seen in the course of the vessels. of the nodules; and in the centre is a relatively clear zone called the germ-centre. The nodules do not occupy the medullary portion of the glands, which is composed chiefly of a network of lymphatic capillaries mixed with rather coarse bands of fibrous tissue. The follicular structures in the lymphatic glands resemble the closed follicles in the mucous membrane of the intestinal canal and the Malpighian bodies of the spleen (see Plate VIII).

In the substance of the lymphatic glands are great numbers of lymphspaces or canals, which probably are lined with endothelium; and these 
spaces communicate with the efferent vessels by stomata. The afferent vessels, two to six in number, penetrate the gland and probably empty their contents into the lymph-spaces. The lymph is then collected from the lymph-spaces by the vasa efferentia, one to three in number, which are always larger than the afferent vessels.

The lymphatic glands are supplied with blood, sometimes by one but usually by several small arteries, which penetrate at the hilum. These vessels pass directly to the medullary portion and there break up into several coarse branches to be distributed to the cortical substance, where they ramify in a delicate capillary network, with rather wide meshes, in the closed follicles of this portion of the gland. This capillary plexus also receives branches from small arterial twigs which penetrate the capsule at different points. Returning on themselves in loops, the vessels unite to form one or more large veins, which emerge usually at the hilum.

Very little is known regarding the distribution of nerves in the lymphatic glands. A few filaments from the sympathetic system enter with the arteries, but they have not been traced to their final distribution. The entrance of filaments from the cerebro-spinal system has not been demonstrated.

The uses of the lymphatic glands are somewhat obscure. They are supposed, however, to have an important office in the elaboration of the corpuscular elements of the lymph and chyle; and it has been observed that the lymph contained in vessels that have not passed through glands is relatively poor in corpuscles, while the large trunks and the efferent vessels contain them in large numbers.

Absorption of Protcids by the Lacteals. - Comparative analyses of the lymph and chyle show in the latter an excess of proteid constituents; and it is natural to infer that the excess of these matters in the chyle is due to absorption of proteids from the intestinal canal. Lane collected the chyle from the lacteals of a donkey, seven and a half hours after a full meal of oats and beans, and compared its composition with that of the lymph. The analyses were made by Rees, who found that the chyle contained about three times as much albumin and fibrin as the lymph. While by far the greatest part of the products of digestion of proteids is absorbed by bloodvessels, there can be no doubt that a small portion is also taken up by the lacteals.

Absorption of Sugar and Salts by the Lacteals. - Small quantities of sugar and sometimes lactic acid have been detected in chyle from the thoracic duct in the herbivora; and the presence of sugar in both lymph and chyle has also been observed. While the products of the digestion of saccharine and amylaceous matters are taken up mainly by blood- 
vessels, a small quantity is also absorbed by the lacteals. In the comparative analyses of the chyle and lymph by Rees, the proportion of inorganic salts was found to be considerably greater in the chyle. The great excess in the quantity of blood coming from the intestine, and the rapidity of its circulation, as compared with the chyle, explain the more rapid penetration by osmosis of the soluble products of digestion.

Absorption of Water by the Lacteals. - There can be no doubt that a small portion of the liquids taken as drink finds its way into the circulation by the lacteals, although the greatest part passes directly into the bloodvessels. When an animal has taken solid food only and is killed during digestion, the thoracic duct contains but a small quantity of chyle ; but when the animal has taken liquids with the food, the thoracic duct and the lacteals are distended.

Aside from the entrance of gases into the blood from the pulmonary surface, physiological absorption is confined almost entirely to the mucous membrane of the alimentary canal. It is true that liquids may find their way into the circulation through the skin, the lining membrane of air-passages, the reservoirs, ducts and parenchyma of glands, the serous and other closed cavities, areolar tissue, the conjunctiva, muscular tissue, and, in fact, all parts that are supplied with bloodvessels; but here the absorption of foreign matters is occasional or accidental and is not connected with general nutrition.

Absorption by the Skin. - It is now admitted that absorption may take place from the general surface, although at one time this was a question much discussed by physiologists. The proofs, however, of the entrance of certain medicinal preparations from the surface of the body are conclusive. The question of most importance in this connection relates to the normal action of the skin as an absorbing surface. Looking at this subject from a purely physiological point of view, absorption from the skin, under ordinary conditions, must be very slight, if, indeed, it takes place at all. There are, nevertheless, facts that render it certain that water may be absorbed by the skin and relieve in some degree the sensation of thirst.

Absorption by the Respiratory Surface. - Animal and vegetable emanations may be taken into the blood by the lungs and produce well-marked pathological conditions. Many contagious diseases are propagated in this way, as well as some fevers and other general diseases that are not contagious. In regard to certain poisonous gases and volatile matters, the effects of their absorption by the lungs are even more striking. Carbon monoxide and arsine produce death almost instantly, even when inhaled in very small quantity. The vapor of pure hydrocyanic acid acts frequently with great promptness 
through the lungs. Turpentine, iodin and many medicinal substances may be introduced with great rapidity by inhalation of their vapors; and the serious effects produced by the emanations from lead or mercury, in persons who work in these articles, are well known. Water and substances in solution, when injected into the respiratory passages, are rapidly absorbed; and poisons administered in this way manifest their peculiar effects with great promptness. Experimenters on this subject have shown the facility with which liquids may be absorbed from the lungs and the air-passages, but it must be remembered that the natural conditions are seldom such as to admit of this action.

Absorption from Closed Cavities, Reservoirs of Glands, etc. - Facts in pathology, showing absorption from closed cavities, areolar tissue, the muscular and nervous tissues, the conjunctiva, and other parts, are sufficiently well known. In cases of effusion of serum into the pleural, peritoneal, pericardial or synovial cavities, in which recovery takes place, the liquid becomes absorbed. It has been shown by experiment that warm water injected into these cavities is disposed of in the same way. Effusions into the areolar tissue usually are removed by absorption. In cases of penetration of air into the pleura or the general areolar tissue, absorption likewise takes place, showing that gases may be taken up in this way as well as liquids. Effusions of blood beneath the skin or the conjunctiva or in the muscular or nervous tissue may become entirely or in part absorbed. As regards absorption from the areolar tissue, the administration of remedies by the hypodermatic method is a familiar evidence of the facility with which soluble substances are taken into the blood when introduced beneath the skin.

Under some conditions, absorption takes place from the reservoirs of glands, the watery portions of the secretions usually being taken up, leaving the solid and the organic matters. It is supposed that the bile becomes inspissated when it has remained for a time in the gall-bladder, even when the natural flow of the secretion is not interrupted. Certainly, when the duct is obstructed, absorption of a portion of the bile takes place, as is shown by coloration of the conjunctiva and even of the general surface. The serum of the blood, under these conditions, is strongly colored with bile. It is probable, also, that some of the watery portions of the urine are reabsorbed by the mucous membrane of the urinary bladder when the urine has been long confined in its cavity, although this reabsorption is ordinarily very slight. Absorption may take place from the ducts and the parenchyma of glands, although this occurs chiefly when foreign substances have been injected into these parts.

Absorption of Fats and Insoluble Substances. - In studying the mechanism of the penetration of fatty particles into the intestinal villi, 
it has been ascertained that the epithelium plays an important part in this process. During the digestion of fat, these cells become filled with fatty granules. It has not been demonstrated precisely how fatty particles penetrate the epithelium, but the fact can not be doubted. From the epithelium, the particles of emulsion pass into the substance of the villi - probably into the lymph-spaces and canals - and from these they readily find their way into the lymphatic capillaries. It has been shown that fatty emulsion will pass more easily through porous septa that have been moistened with bile; and it is probably in this way mainly that the bile aids in the passage of the fine particles of fat into the lacteals.

As a rule, insoluble substances, with the exception of the fats, are never regularly absorbed, no matter how finely they may be divided. The apparent exceptions to this are mercury in a state of minute subdivision like an emulsion, and carbonaceous particles. As regards mercury, it is well known that minute particles in the form of unguents may be introduced into the system by prolonged frictions; but this can not be taken as an instance of physiological absorption. The passage of small carbonaceous particles through the pulmonary membrane seems to be purely mechanical. The same thing possibly may occur when fine sharp particles of carbon are introduced into the alimentary canal.

Influence of the Condition of the Blood and of the Vessels on Absorption. - After loss of blood or its deterioration and concentration from prolonged abstinence, absorption takes place with great activity. This is well known, both as regards the entrance of water and alimentary substances and the absorption of drugs. It was at one time quite a common practice to bleed before administering certain remedies, in order to produce a more speedy action.

The rapidity of the circulation has an important influence in facilitating absorption; and this process usually is active in proportion to the vascularity of different parts. During intestinal absorption, the increase in the activity of the circulation in the mucous membrane is very marked and undoubtedly has an influence on the rapidity with which the products of digestion are taken up.

Influence of the Nervous System on Absorption. - It is certain that absorption, especially in the stomach, is subject to certain variations which can hardly be dependent on anything but nervous action. Liquids that usually are readily absorbed from the stomach are sometimes retained for a time and are afterward rejected in nearly the condition in which they were taken. It is probable, however, that the most important influences thus exerted by the nervous system are effected through the circu- 
lation. When it is remembered that the small arteries may become so contracted under the influence of the vasomotor nerves that their calibre is nearly obliterated, of course retarding in a corresponding degree the capillary and venous circulation in the parts, and again, that the same vessels may be so dilated as to admit to a particular part many times more blood than it ordinarily receives, it becomes apparent that absorption may be profoundly affected through this system of nerves. It has been ascertained that while a section of some of the nerves distributed to the alimentary canal will slightly retard the absorption of the poisonous substances the process is never entirely arrested.

\section{Osmosis}

If liquids pass through the substance of an animal membrane, it is evident that the membrane itself must be capable of taking up a certain portion by imbibition; and this must be considered as the starting-point in absorption. Imbibition is, indeed, a property common to animal tissues; but it is a well-known fact that the tissues do not imbibe all solutions with the same degree of activity. Distilled water is the liquid taken up in greatest quantity, and saline solutions enter the substance of the tissues usually in an inverse ratio to their density. In regard to mixtures of alcohol and water, imbibition is in an inverse proportion to the quantity of alcohol present. Among the other conditions that have a marked influence on imbibition, is temperature; and it is a familiar fact that dried animal membranes may be more rapidly softened in warm than in cold water. While nearly all the structures of the body, after desiccation, will imbibe liquids, the membranes through which the processes of absorption are most active are, as a rule, most easily permeated; and the character of the liquid, the temperature, etc., have a great influence on the activity of this process.

Mechanism of the Passage of Liquids through Membranes. - The passage of liquids through membranes is called osmosis, and this is in obedience to what is known as osmostic pressure. In the case of two liquids passing in opposite directions, the stronger current is called endosmotic, and the weaker, exosmotic. In the passage of liquids into the vessels, in physiological absorption, the process usually is called endosmosis.

It is now definitely ascertained that the following conditions are necessary for the operation of endosmosis and exosmosis:-

I. That both liquids be capable of "wetting" the interposed membrane, or in other words, that the membrane be capable of imbibing both liquids. If but one of the liquids can wet the membrane, the current takes place in only one direction. 
2. That the liquids be miscible with each other and be differently constituted. Although it is found that the currents are most active when liquids are of different densities, this condition is not indispensable; for currents will take place between solutions of different substances, such as salt, sugar or albumin, even when they have the same density.

Diffusion of liquids takes place in accordance with the law of diffusion of gases, formulated by Boyle. The molecules of gases are in constant movement and would pass into space unless restrained or confined by the walls of a containing vessel or by the force of gravity. The pressure of a gas is equal to the pressure required to keep it at a certain degree of concentration. At the surface of the earth, this is measured by the barometer and is equal to about 30 inches (I meter) of mercury. One atmosphere is equal to a pressure of I4.7 pounds to the square inch (about I kilogram to the square centimeter). The pressure of a gas increases with elevation in temperature, by $\frac{1}{2} \frac{1}{3}$ of the pressure at $32^{\circ} \mathrm{Fahr} .\left(0^{\circ} \mathrm{C}\right.$.) for each rise of $\mathrm{I}^{\circ} \mathrm{C}$. From this the hypothetical, or absolute, zero has been calculated to be $-273^{\circ} \mathrm{C}$. Van't Hoff and others have shown that osmotic pressure obeys this law within certain limits.

Osmotic pressure is the force exerted by various solutions - such as a salt or a cane-sugar solution - which draws pure water through a permeable layer, such as an animal membrane or unglazed porcelain. If both liquids are capable of penetrating the membrane, currents pass in both directions until diffusion is complete and both solutions are of equal density.

In order to measure osmotic pressure, it is necessary to use a membrane that will allow a current in one direction only. This is called a semipermeable membrane. If unglazed porcelain is impregnated with copper ferrocyanide, it is rendered impermeable to certain solutions, such as common salt and cane-sugar, while it admits the passage of water. A good example of a semipermeable membrane is the membrane of a hen's egg. This is impermeable to albumin but not to water. Exposing this membrane by removing part of the shell, an egg-endosmometer may be constructed that will measure the osmotic pressure of albumin.

It has been ascertained that a one per cent solution of sodium chloride has an osmotic pressure of five meters (about two hundred inches) of mercury, which is equal to six and a half atmospheres. A two per cent solution has double this pressure. While the pressure is in direct ratio to the concentration of the solution, it has not been possible, thus far, to measure the pressure of solutions higher than six 
per cent of cane-sugar in water, which is only about one-fifth of a normal solution. Physicists are still unacquainted with the cause of osmosis; and while the bearing of the prevailing theories of osmotic pressure, electrolysis and dissociation into anions and cations on absorption, nutrition and secretion are undoubtedly of the highest physiological importance, this is not yet entirely clear.

In no experiments performed out of the body, can the conditions favorable to the passage of liquids through membranes in accordance with purely physical laws be realized as they exist in the living organism. The great extent of the absorbing surfaces; the delicacy and permeability of the membranes; the rapidity with which substances are carried on by the torrent of the circulation, so soon as they pass through these membranes; the uniformity of the pressure, notwithstanding the penetration of liquids, - all these favor the physical phenomena of absorption in a way that can not be imitated in artificially-constructed apparatus. Within the bloodvessels albuminous matters exist in a form that does not permit them to pass through membranes, while the peptones are highly osmotic. The sugars, also, pass through the walls of the vessels with facility, as well as various salts and medicinal substances in solution. The fats, as has been stated, pass mainly into the lacteals, by a process already described, which can not be fully explained by the laws of osmosis.

\section{Lymph and Chyle}

It is estimated that the total quantity of lymph and chyle produced in the twenty-four hours in a man weighing one hundred and forty-three pounds ( 65 kilograms) is about 6.6 pounds ( 3000 grams). Reasoning from experiments made on dogs thirteen hours after feeding, when the liquid which passes up the thoracic duct may be assumed to be pure unmixed lymph, the total quantity of lymph alone, produced in the twenty-four hours by a man of ordinary weight, would be about 4.4 pounds (2000 grams). These estimates can be accepted only as approximate, and they do not indicate the entire quantity of lymph actually contained in the organism.

There are no very satisfactory recent researches in regard to the physiological variations in the quantity of lymph. In comparing the quantity of liquid in the lymphatics of the neck in dogs, during digestion and absorption, with the quantity which they contained soon after digestion was completed, it has been found that while digestion and absorption were going on actively, the vessels of the neck contained scarcely any liquid; but the quantity gradually increased after these processes were completed (Collard de Martigny). 
Properties and Composition of Lymph. - Lymph taken from the vessels in various parts of the system, or the liquid discharged from the thoracic duct during the intervals of digestion, is either transparent and colorless or of a slightly yellowish or greenish tint. When allowed to stand for a short time, it becomes faintly tinged with red, and frequently it has a pale rose-color when first discharged. Microscopical examination shows that this reddish color is dependent on the presence of a few red blood-corpuscles, which are entangled in the clot as the lymph coagulates, thus accounting for the deepening of the color when the liquid has been allowed to stand.

Lymph has no decided or characteristic odor. It is slightly saline in taste, almost insipid. Its specific gravity is much lower than that of the blood. In the dog it is about I022. A few minutes after discharge from the vessels, both lymph and chyle undergo coagulation. This process, as regards the chemical changes involved, is identical with the coagulation of the blood, in which the leucocytes play an important part. Lymph collected from the thoracic duct in the large ruminants coagulates at the end of five, ten or twelve minutes, and sets into a mass having exactly the form of the vessel in which it is contained. The clot is tolerably consistent, but there is never any spontaneous separation of serum.

Although many analyses have been made of lymph from the human subject, the conditions under which it has been obtained render it probable that in the majority of instances it was not entirely normal. It will be necessary, therefore, to compare these analyses with observations on the inferior animals; as in the latter, it has been collected under conditions which leave no doubt as to its normal character. The following is an analysis by Lassaigne of specimens of lymph collected by Colin from the thoracic duct of a cow, under the most favorable conditions :--

COMPOSITION OF LYMPH FROM A COW

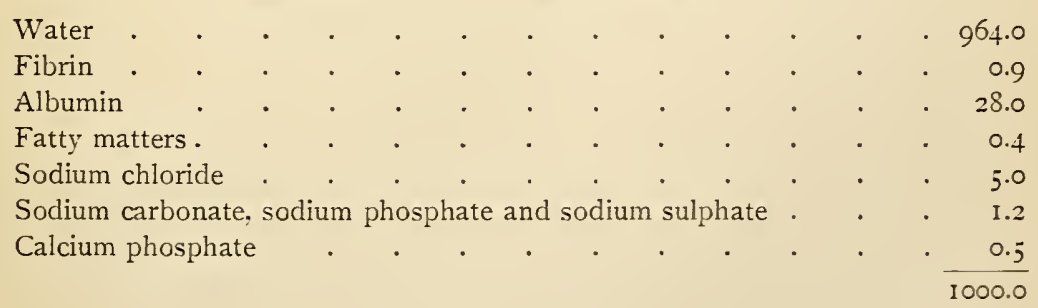

The proportions given in the table are by no means invariable, the differences in coagulability indicating differences in the proportion of fibrin-factors, and the degree of lactescence showing variations in the 
quantity of fatty matters. The table may be taken, however, as an approximation of the average composition of the lymph of these animals during the intervals of digestion.

Analyses of human lymph show a larger proportion of solid constituents than was found by Lassaigne in the lymph of the cow. This excess is pretty uniformly distributed throughout all the constituents, with the exception of the fatty matters and fibrin; the former existing largely in excess in the human lymph, while the latter is smaller in quantity than in the lymph of the cow.

The distinctive characters of the different constituents of the lymph do not demand extended consideration, inasmuch as most of them have already been treated of in connection with the blood. In comparing, however, the composition of the lymph with that of the blood, the great excess of solid constituents in the latter is at once apparent.

In nearly all analyses the proteids have been found to be very much less in quantity in lymph than in the blood. This usually is most marked in regard to the fibrin-factors; but as before stated, the proportion of all these substances is variable. On account of this deficiency, lymph is much inferior to the blood in coagulability; and the coagulum, when formed, is soft and friable. There does not appear, however, to be any actual difference between the coagulating constituents of the lymph and of the blood. Fatty matters usually have been found to be more abundant in the lymph than in the blood; but their proportion is even more variable than that of the proteid constituents. Very little is to be said concerning the ordinary inorganic constituents of the lymph. It has been shown that nearly if not quite all the inorganic matters found in the blood are contained in lymph in varying proportions. Some analyses have given a small quantity of iron. These facts indicate a remarkable correspondence in composition between lymph and blood. All the constituents of the blood, except the red corpuscles, exist in lymph, the only difference being in their relative proportions. In addition to the constituents of the lymph ordinarily given, the presence of glucose, and more lately, the existence of a considerable proportion of urea, have been demonstrated. It has not been ascertained how the sugar contained in the lymph takes its origin.

The lymph of the dog contains about forty parts per hundred in volume of carbon dioxide, of which seventeen parts may be extracted by the gas-pump and twenty-three parts, by acids. In addition, the lymph contains a trace of oxygen and one or two parts of nitrogen.

Corpuscular Elements of the Lymph. - In every part of the lymphatic system, in addition to a few minute fatty granules, there are found certain corpuscular elements known as lymph-corpuscles (leuco- 
cytes). These exist, not only in the clear lymph, but in the opaque liquid contained in the lacteals during absorption. Eighty-two hundred leucocytes have been counted in 0.06I cubic inch (I cubic centimeter) of lymph from a dog.

The leucocytes found in lymph and chyle are rather less uniform in size and general appearance than the leucocytes of the blood. Their average diameter is about $\frac{1}{2500}$ of an inch ( IO $\mu$ ); but some are larger, and others are as small as $\frac{1}{5000}$ of an inch $(5 \mu)$. Some of these corpuscles are quite clear and transparent, presenting but few granules and an indistinct nuclear appearance in their centre; but others are granular and quite opaque. They present the same adhesive character in the lymph as in the blood, and frequently they are found collected in masses in different parts of the lymphatic system. In other regards, these bodies present the same characters as the leucocytes of the blood, and they need not, therefore, be further described, but the variety known as lymphocytes predominates. In addition to leucocytes and a certain number of fatty granules, a few small clear globules or granules, about $\frac{1}{7500}$ of an inch $(3.3 \mu)$ in diameter are almost constantly present in the lymph. They are insoluble in ether and acetic acid but are dissolved by ammonia.

Origin and Uses of the Lymph. - There can hardly be any doubt concerning the source of most of the liquid portions of the lymph, for they can be derived only from the blood. Although the exact relations between the smallest lymphatics and the bloodvessels have not been made out in all parts of the system, there is manifestly no anatomical reason why water, mixed with albuminous matters and holding salts in solution, should not pass from the blood into the lymphatics; and this is rendered nearly certain by the fact that the lymphatics surround many of the bloodvessels. In comparing the composition of the lymph with that of blood-plasma, it is seen that their constituents are nearly identical, the only variations being in their relative proportions.

One of the most important physiological facts in the chemical history of the lymph is the constant existence of a considerable proportion of urea. This can not be derived from the blood, for its proportion is greater in the lymph, notwithstanding the fact that this liquid is being constantly discharged into the bloodvessels. The urea existing in the lymph probably is derived in some way from the liver; it is discharged then into the blood, and is constantly being eliminated by the kidneys.

The positive facts on which to base any precise ideas in regard to the general office of the lymph are not many. From the composition of this liquid, its mode of circulation and the fact that it is being constantly discharged into the blood, it would not seem of itself to have 
important uses in the active processes of nutrition. The quantity and the proportion of solid constituents are rather increased than diminished in animals deprived of food and drink for several days; while starvation always impoverishes the blood from the first. On the other hand, urea, one of the most important of the products of katabolism, undoubtedly is taken up by the lymph and conveyed to the blood.

Properties and Composition of Chyle. - During the intervals of digestion, the intestinal lymphatics and the thoracic duct carry lymph; but so soon as absorption of the products of digestion begins, certain nutritive matters are taken up in quantity by these vessels, and their contents are known under the name of chyle.

In the human subject and in carnivorous animals, the chyle, taken from the lacteals near the intestine, where it is nearly pure, or from the thoracic duct, when it is mixed with lymph, is a white, opaque, milky liquid, of a slightly saline taste and an odor said to resemble that of the semen. The odor is also said to be characteristic of the animal from which chyle is taken; although this is not very marked, except on the addition of a concentrated acid. The reaction of the chyle is either alkaline or neutral. Its specific gravity is less than that of the blood; but it is variable and depends on the quality of food and particularly the quantity of liquids ingested.

The differences in the appearance of the chyle in different animals depend chiefly on the food. The chyle is milky in the carnivora, especially after fats have been taken in quantity; but in dogs nourished with articles containing but little fat, its appearance is hardly lactescent. The - chyle is almost transparent in herbivora fed with hay or straw.

It is impossible to give an accurate estimate of the entire quantity of chyle taken up by the lacteal vessels. When it finds its way into the thoracic duct, it is immediately mixed with all the lymph from the lower extremities; and the large quantities that have been collected from this vessel give no idea of the quantity of chyle absorbed from the intestinal canal. No attempt will be made, therefore, to give even an approximate estimate of the absolute quantity of chyle; but it is evident that this is variable, depending on the nature of the food and the quantity of liquids ingested.

Like the lymph, the chyle, when removed from the vessels, undergoes coagulation. Different specimens vary considerably as regards rapidity of coagulation. Chyle from the thoracic duct usually coagulates in a few minutes. The first portion of the liquid collected from the human subject by Rees - the chyle was collected in this case in two portions - coagulated in an hour. Received into an ordinary glass vessel, the chyle separates more or less completely, after coagulation, into 
clot and serum. The serum is quite variable in quantity and is never clear. Its milkiness does not depend entirely on the presence of particles of emulsified fat, and it is not rendered transparent by ether. It contains, also, leucocytes and granular matter.

Observations have been made with reference to the influence of different kinds of food on the chyle; but these have not been followed by any definite results that can be applied to the human subject. It is usual to find the chyle liquid in the lacteals and in the thoracic duct for many hours after death; but it soon coagulates after exposure to the air. Although the entire lacteal system is sometimes found, in the human subject and in the inferior animals, filled with opaque coagulated chyle, the liquid does not often coagulate in the vessels.

Composition of Chyle. - Analyses of the milky liquid taken from the thoracic duct during full digestion by no means represent the composition of pure chyle; and it is only by collecting the liquid from the mesenteric lacteals, that it can be obtained without a large admixture of lymph. In the human subject, it is rare even to have an opportunity to take the contents of the thoracic duct in cases of sudden death during digestion; and in most of the inferior animals, it is difficult to obtain liquid from the small lacteals in quantity sufficient for accurate analysis.

In the analysis by Rees, the liquid was taken from the thoracic duct of a vigorous man, a little more than an hour after his execution by hanging. The subject was apparently in perfect health up to the moment of death. The evening before, he ate two ounces (56.7 grams) of bread and four ounces (I 3.4 grams) of meat. At seven A.M., one hour before execution, he took two cups of tea and a piece of toast; and he drank a glass of wine just before mounting the scaffold. When the dissection was made, the body was yet warm, although the weather was quite cold. The thoracic duct was rapidly exposed and divided, and about six fluidrachms (22.2 cubic centimeters) of milky chyle were collected. The liquid was neutral and had a specific gravity of I024. The following was its approximate composition:-

\section{COMPOSITION OF HUMAN CHYLE FROM THE THORACIC DUCT}

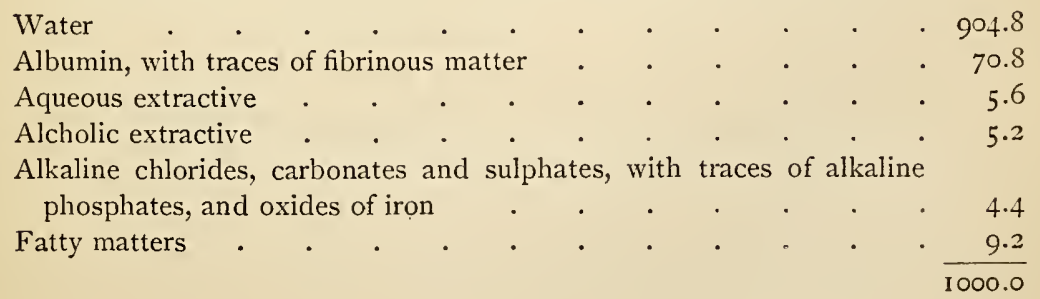


The difference in chemical composition between the unmixed lymph and the chyle is illustrated in a comparative examination of these two liquids taken from a donkey. They were collected by Lane, the chyle being taken from the lacteals before reaching the thoracic duct. The animal was killed seven hours after a full meal of oats and beans. The following analyses were made by Rees:-

COMPOSITION OF CHYLE AND LYMPH BEFORE REACHING THE THORACIC DUCT

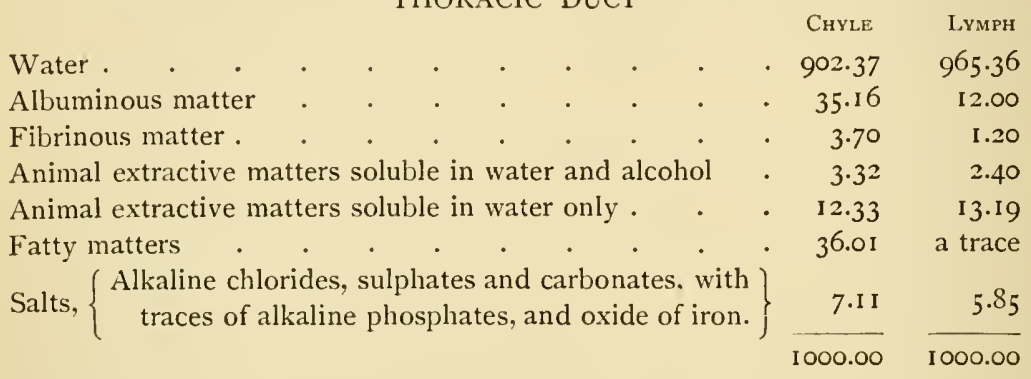

The above analyses show a marked difference in the proportion of solid constituents. The chyle contained about three times as much albumin and fibrin as the lymph, with a larger proportion of salts. The proportion of fatty matters in the chyle was very great, while in the lymph there existed only a trace. The individual constituents of the chyle given in the above tables do not demand further consideration than they have already received under the head of lymph. The albuminous matters are in part derived from the food, and in part from the blood through admixture with lymph. The fatty matters are derived in greatest part from the food. So far as has been ascertained by analyses of the chyle for inorganic salts, it has been found to contain essentially the same inorganic constituents as the blood-plasma.

Microscopical Characters of the Chyle. - The milky appearance of the chyle as contrasted with the lymph is due to the presence of a large number of minute fatty granules. The liquid becomes much less opaque when treated with ether, which dissolves many of the fatty particles. In fact, the chyle of the thoracic duct is nothing more than lymph to which an emulsion of fat in a liquid containing albuminous matters and salts is temporarily added during the process of intestinal absorption. The quantity of fatty granules in the chyle varies considerably with the diet, and it usually diminishes progressively from the smaller to the larger vessels, on account of the constant admixture of lymph. The size of the granules is pretty uniformly $\frac{1}{25000}$ to $\frac{1}{12} \frac{1}{50} \overline{0}$ of an inch ( 1 to $2 \mu$ ). Thcy are much smaller and more uniform in size in the lacteals than 
in the intestine. Their constitution is not constant; and they are composed of the different varieties of fat taken as food, mixed in various proportions. The ordinary corpuscular elements of the lymph are also found in variable quantity in the chyle.

\section{Movements of the Lymph and the Chyle}

Compared with the current of blood, the movements of the lymph and chyle are feeble and irregular; and the character of these movements is such that they are evidently due to a variety of causes. As regards constituents derived directly from the blood, the lymph may be said to undergo a true circulation; inasmuch as there is a constant transudation at the peripheral portion of the vascular system, of liquids that are returned to the circulating blood by the communications of the lymphatic system with the great veins. The constituents of the lymph, however, are not derived entirely from the blood, a considerable portion resulting from interstitial absorption in the general lymphatic system; and the chyle contains certain nutritive matters absorbed by the lacteal vessels. These are, physiologically, the most important constituents of the lymph and chyle; and they are taken up simply to be carried to the blood and do not pass again from the general vascular system into the lymphatics.

So far as the mode of origin of the lymph and chyle has any bearing on the movements of these liquids in the lymphatic vessels, there is no difference between the imbibition of new matters from the tissues or from the intestinal canal and the transudation of the liquid portions of the blood; for the mechanism of the passage of liquids from the bloodvessels is such that the motive power of the blood can not be felt. An illustration of this is in the mechanism of the production of the liquid portions of the secretions. So far as the flow in the vessels of medium size is concerned, the movement probably is continuous, subject only to certain momentary obstructions or accelerations from various causes ; but in the large vessels situated near the thorax and in those within the chest, the movements are in a marked degree remittent, or they may even be intermittent. All experimenters who have observed the flow of lymph or chyle from a fistula in the thoracic duct have noted a constant acceleration with each act of expiration; and an impulse synchronous with the pulsations of the heart has frequently been observed.

The fact that the lymphatic system is never distended, and the existence of valves, by which different portions may become isolated, render it impossible to estimate the general pressure in these vessels. This undoubtedly is subject to great variations in the same vessels at different times as well as in different parts of the lymphatic system. 
The general rapidity of the current in the lymphatic vessels has never been accurately estimated. As a natural consequence of the variations in the distention of these vessels, the rapidity of the circulation must be subject to frequent modifications. It has been calculated that the rapidity of the flow in the thoracic duct is about one inch (25.4 millimeters) per second. This estimate, however, can be only approximate ; and it is evident that the flow must be much less rapid in vessels near the periphery than in the large trunks, as the liquid moves in a space that rapidly becomes contracted as it approaches the openings into the venous system.

Various influences combine to produce the movements of liquids in the lymphatic system, some being constant in their operation, and others, intermittent or occasional. These will be considered, as nearly as possible, in the order of their relative importance.

The forces of osmosis and transudation are undoubtedly the main causes of the lymphatic circulation, more or less modified, however, by influences that may accelerate or retard the current; but this action is capable in itself of producing the regular movement of the lymph and chyle. It is a force that is in constant operation, as is seen in cases of ligation of the thoracic duct, a procedure which must finally abolish all other forces that aid in producing the lymphatic circulation. When the receptaculum chyli is ruptured as a consequence of obstruction of the thoracic duct, the vessel gives way as the result of the constant osmotic action, in the same way that the exposed membranes of an egg may be ruptured when immersed in water.

The situations in which the osmotic force originates are at the periphery, where the single wall of the vessels is thin, and where the extent of absorbing surface is large. If liquids can penetrate with such rapidity and force through the walls of the bloodvessels, where their entrance is opposed by the pressure of liquid already in their interior, they certainly must pass without difficulty through the walls of the lymphatics, where there is no lateral pressure to oppose their entrance, except that produced by the weight of the column of liquid. This pressure is readily overcome; and the valves in the lymphatic system effectually prevent any backward current.

In describing the anatomy of the lymphatic system, it has already been stated that the large vessels and those of medium size are provided with non-striated muscular fibres and are contractile. This has been demonstrated by physiological as well as anatomical investigations; and it is not uncommon to see the lacteals become reduced in size to mere threads, even while under observation. Although experiments usually have failed to demonstrate any rhythmical contractions in the lymphatic 
system, it is probable that the vessels contract on their contents, when they are unusually distended, and thus assist the circulation, the action of the valves opposing a regurgitating flow. This action, however, can not have any considerable and regular influence on the general current.

Contractions of voluntary muscles, especially compression of the abdominal organs by contraction of the abdominal muscles, peristaltic movements of the intestines and pulsations of large arteries situated against the lymphatic trunks, particularly the thoracic aorta, are all capable of increasing the rapidity of the circulation of the lymph and chyle. The contractions of voluntary muscles assist the lymphatic circulation in precisely the way in which they influence the flow of blood in the venous system; and there is nothing to be added to what has been said on this subject in connection with the venous circulation. While a vis a tergo must be regarded as by far the most important agent in the production of the lymphatic circulation, movement of the contents of the thoracic duct receives constant and important aid from the respiratory acts. 


\title{
CHAPTER XI
}

\author{
SECRETION
}

Classification of the secretions - Mechanism of the production of the true secretions - Mechanism of the production of the excretions - Influence of the composition and pressure of the blood on secretion - Influence of the nervous system on secretion - Paralytic secretion by glands - Anatomical classification of glandular organs - Secreting membranes - Follicular glands - Tubular glands - Racemose glands, simple and compound - Ductless, or bloodglands - Secretions and excretions - Synovial membranes and synovia - Mucous membranes and mucus - Mechanism of the secretion of mucus - Composition and varieties of mucus - General uses of mucus - Physiological anatomy of the sebaceous, ceruminous and Meibomian glands - Ordinary sebaceous matter - Smegma of the prepuce and labia minora - Vernix caseosa - Cerumen - Mammary secretion - Mechanism of the secretion of milk-General conditions which modify the lacteal secretion-Properties and composition of milk - Microscopical characters of milk - Composition of the milk Colostrum - Lacteal secretion in the newly born.

In the sense in which the term secretion usually is received, it embraces most of the processes in which there is a separation of matters from the blood or lymph by glandular organs or a formation, by such organs, of new liquids, out of materials furnished by the blood or lymph. It is probable, however, that most of the secretions are derived directly from the blood and not from the lymph contained in the glandular substance. While secretion may be treated of as a distinct process, it is intimately connected with general metabolism. As a rule, secretions are homogeneous liquids without formed anatomical elements, except as accidental constituents, such as desquamated epithelium in mucus or in sebaceous matter. The secretions are either discharged from the body, when they are called excretions, or, after having performed their proper office as secretions, are absorbed in a more or less modified form. Secretions, therefore, are liquids holding certain substances in solution and sometimes containing peculiar ferments - but not necessarily possessing formed anatomical elements - separated from the blood or formed by special organs out of materials furnished by the blood. Secreting parts may be membranes, follicles, collections of follicles, or tubes. In the latter instances they are called glands. This definition includes the excretions. It is not strictly correct to speak of formed anatomical elements as products of secretion, except in the instance of fatty particles in milk. The leucocytes found in pus, the spermatozoids of the seminal fluid, and the ovum, which are sometimes 
spoken of as products of secretion, are anatomical elements developed in the way in which such structures are ordinarily formed.

Classification of the Secretions. - Certain secretions are formed by special organs and have important uses that do not involve their discharge from the body. These may be classed as the true secretions; and the most striking examples are the digestive liquids. Each one of these is formed by a special gland or set of glands, which usually has no other office; and they are never produced by any other part. It is the gland that produces the characteristic constituent or constituents of the true secretions; and the matters thus formed do not preëxist either in the blood or in the lymph. The office which the true secretions have to perform usually is not continuous; and when this is the case, the flow of the secretion is intermittent, taking place only when its action is required. When the parts that produce one of the true secretions are destroyed, the characteristic constituents of this secretion do not accumulate in the blood nor are they formed vicariously by other organs; the effect is absence of the secretion, with the disturbances consequent to the loss of its physiological action.

Certain other of the liquids of the body are composed of water, holding one or more characteristic constituents in solution that result from the physiological wear of the tissues. These matters have no office to perform in the economy and are separated from the blood to be discharged from the body. Such products may be classed as excretions, the urine being the type of liquids of this kind. The characteristic constituents of the excretions have their origin in the tissues and are products of the changes going on in all organized living structures. They preëxist in the blood or lymph and may be eliminated, either constantly or occasionally, by a number of organs. As they are produced continually in the substance of the tissues or organs and are taken up by the blood, they are constantly separated from the blood by the proper eliminating organs. When the glands which thus eliminate these substances are destroyed or when their efficiency is seriously impaired, the excrementitious matters may accumulate in the blood and give rise, directly or indirectly, to toxic phenomena. These effects, however, are often retarded by the vicarious action of other organs.

There are some liquids, such as the bile, that have important uses as secretions and nevertheless contain excrementitious matters. In these instances, it is only the excrementitious matters that are discharged from the organism. In the sheaths of some tendons and of muscles, in the substance of muscles and in some other situations, liquids are found which simply lubricate the parts and which contain very little organic matter, with but a small proportion of inorganic salts. 
It is difficult to draw a line rigorously between transudation and some of the phenomena of secretion; particularly as experiments on dialysis have shown that simple osmotic membranes are capable of separating complex solutions, allowing certain constituents only to pass. This fact explains why the transuded liquids do not contain all the soluble constituents of the blood in the proportions in which they exist in the plasma. All secreted liquids, both the true secretions and the excretions, contain many of the inorganic salts of the blood-plasma.

The secretions proper usually are classified as serous (albuminous) and mucous; the former being entirely liquid and the latter presenting more or less viscidity. It is thought, also, that cells producing these two kinds of secretions differ somewhat from each other in their histological characters.

Mechanism of the Production of the True Secretions. - Although the characteristic constituents of the true secretions are not to be found in the blood or in any other of the animal liquids, they often can be extracted from the glands, particularly during their intervals of so-called repose. This has been repeatedly demonstrated in regard to many of the digestive secretions, as the saliva, the gastric juice and the pancreatic juice; and artificial liquids, possessing certain of the physiological properties of the natural secretions, have been prepared by simply extracting the glandular tissue with an appropriate menstruum. There can be no doubt, therefore, that during the periods when the secretions are not discharged, the glands are taking from the blood matters which are to be transformed into the characteristic constituents of the individual secretions, and that this is constant, bearing a close resemblance to the general process of nutrition. Certain anatomical elements in the glands have the power of selecting proper materials from the blood and causing them to undergo peculiar transformations; in the same way that the muscular tissue takes from the blood albuminous matters and transforms them into its own substance. The exact nature of this process is not understood.

In all secreting organs, epithelium is found which produces the peculiar constituents of the different secretions. The epithelial cells lining the tubes or follicles of the glands constitute the only peculiar structures of these parts, the rest being made up of basement-membrane, connective tissue, bloodvessels, nerves, and other structures that are distributed generally in the economy; and these cells alone contain the constituents of the secretions. It has been found, for example, that the liver-cells contain the glycogen formed by the liver; and it has been further shown that when the cellular structures of the pancreas have been destroyed, the secretion is no longer produced. There can be hardly any doubt in 
regard to the application of this principle to the glands generally, both secretory and excretory. Indeed, it is well known to pathologists, that when the secreting tubes of the kidney have become denuded of their epithelium, they are no longer capable of separating from the blood the peculiar constituents of the urine.

As regards the origin of the characteristic constituents to the true secretions, it is impossible to entertain any other view than that they are produced in the epithelial structures of the glands. While the secretions contain inorganic salts in solution derived from the blood, the organic constituents, such as ptyalin, pepsin and trypsin, are readily distinguished from all other albuminous substances by their peculiar physiological properties.

It may be stated, then, as a general proposition, that the characteristic constituents of the true secretions, as contradistinguished from the excretions, are formed by the epithelial structures of the glands, out of materials furnished mainly by the blood. Their formation is not confined to what is usually termed the period of activity of the glands, or the time when the secretions are poured out, but it takes place more or less constantly when no liquid is discharged. It is more than probable, indeed, that the formation of the peculiar and characteristic constituents of the secretions takes place with as much, or even more activity in the intervals of secretion as during the discharge of liquid; and most of the glands connected with the digestive system seem to require certain intervals of repose and are capable of discharging their secretions for a limited time only. This condition of a gland is called resting.

When a secreting organ is called into activity - like the gastric mucous membrane or the pancreas, following the introduction of food into the alimentary canal - a marked change in its condition occurs. The circulation in the part is then much increased in activity, thus furnishing water and the inorganic constituents of the secretion. This difference in the quantity of blood in the glands during their activity may be observed when the organs are exposed in a living animal and is one of the important conditions bearing on the mechanism of secretion. In all the secretions proper, then, there are intervals, either of complete repose, as is the case with the gastric juice or the pancreatic juice, or periods when the activity of the secretion is greatly diminished, as in the case of the saliva. The resting periods are necessary to the proper action of the secreting glands; forming a marked contrast with the constant action of organs of excretion. It is well known, for example, that digestion is seriously disturbed when the act is too prolonged on account of the habitual ingestion of an excessive quantity of food.

While the mechanism of secretion is not understood in all its details 
as regards all the secretions, in certain glands the processes have been studied with fairly definite results. In some of the salivary glands, in the peptic cells and in the cells of the pancreas, it has been shown that the so-called ferments, or enzymes, are not formed directly. The secreting cells are divided into two portions, or zones; an outer zone, next the tubular membrane, and an inner zone, next the lumen of the tube or follicle. In the inner zone, during the intervals of actual secretion, there appears a substance which is afterward changed into the true ferment; so that there probably is a zymogenic, or ferment-forming substance, first produced by the cells. It is thought that the substance produced by the secreting cells of the salivary glands is not ptyalin but a zymogen that is readily converted into ptyalin, called ptyalinogen; but this substance has not been isolated. The parotid is classed as a serous gland. In the viscid forms of saliva, there appears to be first formed a substance called mucinogen, afterward changed into mucin, on which the viscidity of the secretion depends.

A good example of the changes which secreting cells undergo when a resting gland becomes active may be observed in the pancreas. If the pancreas is removed from a fasting animal and its structure is fixed and stained, the cells lining the secreting alveoli are seen rather sharply divided into the two zones already mentioned. The outer zone, next the alveolar membrane, is narrow and stains deeply. The inner zone is wide and granular and is but slightly stained. When the gland, however, is taken from an animal in full digestion, the outer zone occupies the greater part of the cell, being deeply stained; and the inner zone has become very narrow. It seems, indeed, that the granular matter of the inner zone is used in the production of the active principles of the secretion. A short time after secretion has ceased, the gland will be found to have returned to its resting condition and the granules of the inner zone are restored. These processes take place in all glands and mark their resting and active conditions.

In the salivary glands that produce viscid secretions - the submaxillary and sublingual - the parenchyma presents two kinds of acini, serous and mucous. The so-called serous acini are the more abundant and are thought to produce true saliva, while the mucous acini secrete only the viscid matters that are mixed with the saliva.

In the production of pepsin, the peptic cells first form pepsinogen, which is afterward changed into pepsin. In the pancreas, trypsinogen is formed in the cells, and this is changed into trypsin. The general name zymogen has been given to the substances that are changed into the digestive ferments; although, as is evident, this is not identical in the different glands. 
The theory that the discharge of the secretions is due simply to mechanical causes and is attributable solely to the increase in the pressure of blood can not be sustained. Blood-pressure undoubtedly has considerable influence on the activity of secretion; but the flow will not always take place in obedience to simple pressure, and secretion may be excited for a limited time without any increase in the quantity of blood circulating in the gland.

The glands possess a peculiar excitability, manifested by their action in response to proper stimulation. During secretion they usually receive an increased quantity of blood ; but this is not indispensable, and secretion may be excited without any modification of the circulation. This excitability disappears after the artery supplying the part with blood has been tied for a number of hours; and secretion can not then be excited even when the blood is again allowed to circulate. If the gland is not deprived of blood for too long a period, however, the excitability is soon restored.

Mechanism of the Production of the Excretions. - Certain glands separate from the blood excrementitious matters that are of no use in the economy and are discharged from the body. These matters are different in their mode of production from the characteristic constituents of the secretions. Their formation takes place in the tissues and is connected with the general process of nutrition; and in the excreting glands there is simply a separation of products already formed, probably by cellular action similar to that observed in secreting organs. The action of the excreting organs is constant, and there is not that regular periodic increase in the activity of the circulation observed in secreting organs; but it has been noted that the blood coming from the kidneys is nearly as red as arterial blood, showing that the quantity of blood which these organs receive is greater than the amount required for mere nutrition, the excess, as in the secreting organs, furnishing the water and inorganic salts that are found in the urine. It has also been shown that when the secretion of urine is interrupted, the blood of the renal veins becomes dark like the blood in the general venous system.

Excretion is not, under all conditions, confined to the ordinary excretory organs. When their action is disturbed, certain of the secreting glands, as the follicles of the stomach and intestine, may for a time eliminate excrementitious matters; but this is abnormal and is analogous to the elimination of foreign matters from the blood by the glands.

Influence of the Composition and Pressure of the Blood on Secretion. Under normal conditions, the composition of the blood has little to do with the action of secreting organs, as it simply furnishes the materials out of which the characteristic constituents of the secretions are formed; 
but when certain foreign matters are taken into the system or are injected into the bloodvessels, they are eliminated by the different glandular organs, both secretory and excretory. These organs seem to possess a power of selection in the elimination of different substances. Thus, sugar and potassium ferrocyanide are eliminated in greatest quantity by the kidneys; the salts of iron, by the kidneys and the gastric tubules; and iodin, by the salivary glands.

The discharge of secretions is almost always accompanied with an increase in the pressure of blood in the vessels supplying the glands; and it has been shown, also, that an increase in the pressure, when the nerves of the glands do not exert an opposing influence, increases the activity of secretion; but this does not demonstrate that secretion is due simply to an increase in the pressure of blood in the glands, although this undoubtedly exerts an important influence. It is necessary that other conditions should be favorable to the act of secretion for this influence to be effective. Experiments have shown that pain may completely arrest the secretion of urine, operating undoubtedly through the nervous system. When the flow of urine is arrested by pain, an increase in the pressure of blood in the part fails to excite secretion.

Influence of the Nervous System on Secretion. - The fact that the secretions usually are intermittent in their flow, being discharged in obedience to impressions which are made only when there is a demand for their physiological action, would naturally lead to the supposition that they are regulated, to a great extent, through the nervous system; particularly as it is now well established that the nerves are capable of modifying and regulating local circulations. This applies to a certain extent to the excretions, which are also subject to considerable modifications.

In regard to many of the glands, it has been shown that the influence of the vasomotor nerves is antagonized by certain other nerves, which latter are called the motor nerves of the glands. The motor nerve of the submaxillary is the chorda tympani; and as both this nerve and the sympathetic, which latter contains the vasomotor filaments, together with the excretory duct of the gland, can easily be exposed and operated on in a living animal, many experiments have been performed on this gland. When the parts are exposed and a tube is introduced into the salivary duct, division of the sympathetic induces secretion, with an increase in the circulation in the gland, the blood in the vein becoming red. On the other hand, division of the chorda tympani, the sympathetic being intact, arrests secretion, and the venous blood coming from the gland becomes dark. If the nerves are now stimulated alternately, it will be found that stimulation of the 
sympathetic produces contraction of the vessels of the gland and arrests secretion, while a stimulus applied to the chorda tympani increases the circulation and excites secretion. Enough is known of the nervous influences that modify secretion, to admit of the inference that other glands are supplied with nerves through which certain reflex phenomena affecting their secretions take place. As reflex phenomena involve the action of nerve-centres, it becomes a question to determine whether any particular parts of the central nervous system preside over the various secretions. Experiments showing the existence of such centres are not wanting, but it will be more convenient to treat of these in connection with the physiology of the nervous system.

Mental emotions, pain and various conditions, the influence of which on secretion has long been observed, operate through the nervous system. Many familiar instances of this kind may be mentioned: such as the secretion of tears; arrest or production of the salivary secretions; sudden arrest of the secretion of the mammary glands from violent emotion; increase in the secretion of the kidneys or of the intestinal tract from fear or anxiety; with other examples which it is unnecessary to enumerate.

Paralytic Secretion by Glands. - The effects of destruction of the nerves distributed to the parenchyma of some of the glandular organs are remarkable. If the nerves distributed to the kidney are destroyed, not only is secretion arrested in the great majority of instances, but the renal tissue becomes softened and broken down. After division of the nerves of the salivary glands, these organs become atrophied, but they do not undergo the peculiar putrefactive changes observed in the kidneys. The same effect is produced when the nerves are paralyzed by introducing a few drops of a solution of curare at the origin of the artery distributed to the submaxillary gland. Other glands have socalled motor nerves, stimulation of which excites secretion, but such nerves have been most satisfactorily isolated and studied in connection with the salivary secretions. When the motor nerves of the salivary glands are divided, in the course of a day or two the secretion becomes abundant and watery and loses its normal characters. After about eight days, the secretion begins to diminish and the glands undergo atrophy. The increased secretion first observed is called "paralytic."

Anatomical Classification of Glandular Organs. - The organs which produce the different secretions are susceptible of a classification according to their anatomical peculiarities, which greatly facilitates their study. They may be divided as follows :-

I. Secreting Membranes. - Examples of these are the synovial membranes. 
2. Follicular Glands. - Examples of these are the simple mucous follicles and the simple follicles of Lieberkühn.

3. Tubular Glands. - Examples of these are the ceruminous glands, the sudoriparous glands and the kidneys.

4. Racemose Glands, Simple and Compound.-Examples of the simple racemose glands are the sebaceous and Meibomian glands, the tracheal glands and the glands of Brunner. Examples of the compound racemose glands are the salivary glands, the pancreas, the lachrymal glands and the mammary glands.

5. Ductless, or Blood-Glands. - Examples of these are the thymus, the thyroid, the suprarenal capsules and the spleen.

The liver is a glandular organ that can not be placed in any one of the above divisions. The lymphatic glands and other parts connected with the lymphatic and the lacteal system are not true glandular organs. These are sometimes called conglobate glands.

The general structure of secreting membranes and of the follicular glands is quite simple. The secreting parts consist of a membrane, usually homogeneous, covered on the secreting surface with epithelial cells. Beneath this membrane ramify the bloodvessels which furnish materials for the secretions. The follicular glands are simply digital inversions of this structure, with rounded blind extremities, the epithelium lining the follicles.

The tubular glands have essentially the same structure as the follicles, except that the tubes are long and more or less convoluted. The more complex of these organs contain connective tissue, bloodvessels, nerves and lymphatics.

The compound racemose glands are composed of branching ducts, around the extremities of which are arranged collections of rounded follicles, like bunches of grapes. In addition to the epithelium, basementmembrane and bloodvessels, these organs contain connective tissue, lymphatics, non-striated muscular fibres and nerves. In the simple racemose glands the excretory duct does not branch.

The ductless glands contain bloodvessels, lymphatics, nerves, sometimes non-striated muscular fibres, and a peculiar structure called pulp, which is composed of liquid with cells and occasionally with closed vesicles. These are sometimes called blood-glands, because they are supposed to modify the blood as it passes through their substance.

The testicles and the ovaries are not simple glandular organs; for in addition to the production of mucous or watery secretions, their principal office is to develop certain anatomical elements, the spermatozoids and the ova. The physiology of these organs will be considered in connection with embryology. 
Secretions and Excretions. - The products of the various glands may be divided, according to their uses, into secretions proper and excretions. Some of the true secretions have certain mechanical uses; some, like mucus, are thrown off in small quantity without being actually excrementitious; while others, like the digestive secretions, are produced at certain intervals and are taken up again by the blood.

The serous cavities are now regarded as sacs connected with the lymphatic system; and the liquids of these cavities are not classed with the secretions.

Synovial Membranes and Synovia. - The true synovial membranes are found in the diarthrodial, or movable articulations; but in various parts of the body are found closed sacs, sheaths etc., which resemble synovial membranes both in structure and in their office. Every movable joint is enveloped in a capsule, which is closely adherent to the edges of the articular cartilage and even is reflected over its surface for a short distance; but it is now the common opinion that the cartilage which incrusts the articulating extremities of the bones, though bathed in synovia, is not itself covered with a distinct membrane.

The fibrous portion of the synovial membranes is dense and resisting. It is composed of ordinary fibrous tissue, with a few elastic fibres and bloodvessels. The internal surface is lined with small cells of flattened endothelium with rather large rounded nuclei. These cells exist in one, two, three or sometimes four layers.

In most of the joints, especially those of large size, as the knee and the hip, the synovial membrane is thrown into folds which contain adipose tissue. In nearly all the joints, the membrane presents fringed vascular processes called synovial fringes. These are composed of looped vessels of considerable size; and when injected they bear a certain resemblance to the choroid plexus. The edges of these fringes present a number of leaf-like membranous appendages, in a great variety of curious forms. They usually are situated near the attachment of the membrane to the cartilage.

The arrangement of the synovial bursæ is very simple. Wherever a tendon plays over a bony surface, there is a delicate membrane in the form of an irregularly-shaped closed sac, one layer of which is attached to the tendon, and the other, to the bone. These sacs are lined with an endothelium like that found in the synovial cavities, and they secrete a true synovial fluid. Bursa are also found beneath the skin, especially in parts where the integument moves over bony prominences, as the olecranon, the patella and the tuberosities of the ischium. These sacs, sometimes called bursæ mucosæ, are much more common in man than in the inferior animals, and they have essentially the same uses as 
the deep-seated bursæ. The form of both the superficial and deepseated bursæ is irregular, and their interior frequently is traversed by small bands of fibrous tissue. The synovial sheaths, or vaginal processes, line the canals in which the long tendons play, particularly the tendons of the flexors and extensors of the fingers and toes. They have essentially the same structure as the bursæ, and present two layers, one of which lines the canal, while the other is reflected over the tendon. The vascular folds, described in connection with the articular synovial membranes, are found in many of the bursæ and the synovial sheaths.

The quantity of synovia in the joints is sufficient to lubricate freely the articulating surfaces. When perfectly normal it is either colorless or of a pale yellowish tinge. It is so viscid that it is with difficulty poured from one vessel into another. This peculiar character is due to the presence of an organic substance called synovin, a kind of mucin. When this organic matter has been extracted and mixed with water, it gives to the liquid the peculiar viscidity of the synovial secretion. The reaction of the synovia is faintly alkaline, on account of the presence of a small quantity of sodium carbonate. The secretion, especially when the joints have been much used, usually contains in suspension pale endothelial cells and a few leucocytes. The synovia of the human subject contains about sixty-four parts per thousand of organic matter, with sodium chloride, sodium carbonate, calcium phosphate and ammonio-magnesian phosphate.

The synovial secretion is produced by the general surface of the membrane and not by any special glands. The folds and fringes were at one time supposed to be most active in secreting the organic matter, but there is no evidence that they have any such special office.

Mucous Membranes and Mucus. - A distinct anatomical division of the mucous membranes may be made into two classes; first, those provided with squamous epithelium, and second, those provided with columnar or conoidal epithelium. All the mucous membranes line cavities or tubes communicating with the exterior.

The following are the principal situations in which the first variety of mucous membranes, covered with squamous epithelium, is found: the mouth, the lower part of the pharynx, the œsophagus, the conjunctiva, the female urethra and the vagina. In these situations the membrane is composed of a chorion made up of inelastic and elastic fibrous tissue with capillaries, lymphatics and nerves. The elastic fibres are small and quite abundant. The membrane itself is loosely united to the subjacent parts. The chorion is provided with vascular papillæ, more or less marked; but in all situations except the pharynx, the epithelial 
covering fills the spaces between the papillæ, so that the membrane presents a smooth surface. Between the chorion and the epithelium, is an amorphous basement-membrane. The mucous glands open on the surface of the membrane by their ducts; but the glandular structure is situated in the submucous tissue. Certain of these glands have been described in connection with the anatomy of the mucous membrane of the mouth, pharynx and œsophagus. They usually are simple racemose glands, presenting a collection of follicles arranged around the end of a single excretory duct and lined or filled with rounded nucleated epithelium. The squamous epithelium covering these membranes usually exists in several layers and presents great variety, both in form and size. This is called stratified epithelium. The most superficial layers are of large size, flattened and irregularly polygonal. The deeper layers are smaller and more rounded. The size of the cells is $\frac{1}{2500}$ to $\frac{1}{300}$ of an inch ( IO to $83 \mu$ ). They are pale and slightly granular, each with a small ovoid nucleus and one or two nucleoli.

The second variety of mucous membranes, covered with columnar epithelium, is found lining the alimentary canal below the cardiac opening of the stomach, the biliary passages, the excretory ducts of all the glands, the nasal passages, the upper part of the pharynx, the uterus and Fallopian tubes, the bronchia, the Eustachian tubes and the male urethra. In certain situations this variety of epithelium is provided on its free surface with little hair-like processes called cilia. During life the cilia are in constant motion, producing a current usually in the direction of the mucous orifices. Ciliated epithelium is found throughout the nasal passages, beginning about three-quarters of an inch (I9 millimeters) within the nose; in the upper part of the pharynx; the posterior surface of the soft palate; the Eustachian tube; the tympanic cavity; the larynx, trachea and bronchial tubes, until they become less than $\frac{1}{50}$ of an inch (0.5 millimeter) in diameter; the neck and body of the uterus; the Fallopian tubes; the internal surface of the eyelids; and the ventricles of the brain. Mucous membranes of this variety are formed of a chorion, a basement-membrane and epithelium. The chorion is composed of inelastic and elastic fibres, a few non-striated muscular fibres, amorphous matter, bloodvessels, nerves and lymphatics. It is less dense and less elastic than the chorion of the first variety and is more closely united to the subjacent parts. The surface of these membranes is smooth, the only exception being the mucous membrane of the pyloric portion of the stomach and of the small intestines. These membranes are provided with follicular glands, extending through their entire thickness and terminating in rounded extremities, sometimes single and sometimes double, which rest on 
the submucous structure. Many of them are provided, also, with simple racemose glands, the ducts passing through the membrane, and the glandular structure being situated in the submucous areolar tissue. The columnar epithelium covering these membranes rests on an amorphous structure called basement-membrane. The epithelium usually presents but few layers, and sometimes, as in the intestinal canal, there is only a single layer. The cells are prismoidal, with a large free extremity and a pointed attached end. The cells of the lower strata are shorter and more rounded than those in the superficial layer. The cells are pale and closely adherent to each other by their sides, each with a moderate-sized oval nucleus and one or two nucleoli. The length of the cells is $\frac{1}{800}$ to $\frac{1}{600}$ of an inch $(30$ to $40 \mu$ ), and their diameter, $\frac{1}{3000}$ to $\frac{1}{2500}$ of an inch ( 8 to $\left.10 \mu\right)$. When villosities exist on the surface of the membranes, the cells follow the elevations and do not fill up the spaces between them, as in most of the membranes covered with stratified epithelium.

The mucous membrane of the urinary bladder, of the ureters and of the pelvis of the kidneys can not be classed in either of the above divisions. In these situations the membrane is covered with mixed epithelium, presenting all varieties of form between the squamous and the columnar, some of the cells being caudate and quite irregular in shape.

Mechanism of the Secretion of Mucus. - Nearly every one of the many secretions known under the general name of mucus is made up of the products of several different glandular structures. Certain membranes which do not possess glands, as the mucous lining of the ureters and of a great portion of the urinary bladder, are capable of secreting mucus. The mucous membrane of the stomach produces an alkaline viscid secretion during the intervals of digestion, when the gastric glands do not act; and the gastric glands, during digestion, secrete a liquid of an entirely different character. The secretion produced by the follicles of the small intestine likewise has peculiar digestive properties. These considerations and the fact that the entire extent of the mucous membranes is covered with more or less secretion show that the general epithelial covering of these membranes is capable of secreting a liquid which forms one of the constituents of what is ordinarily recognized as mucus. It is impossible, however, to separate the secretion of the superficial layer of cells from the other secretions found on the mucous membranes; and it will be more convenient to regard as mucus the secretion found upon mucous membranes, except when, as in the case of the gastric or the intestinal juice, a special secretion can be recognized by certain distinctive physiological properties. 
In the membranes covered with columnar epithelium, which usually are provided with simple follicles, the secretion is produced mainly by these follicles but in part by the epithelium covering the general surface. In certain parts, particularly the intestinal mucous membrane, mixed with the ordinary columnar epithelium, are the so-called gobletcells that are supposed to produce and discharge a viscid secretion containing mucin or mucinogen. These cells have already been described. (See page 212.) The membranes covered with squamous epithelium usually contain but few follicles and are provided with simple racemose glands situated in the submucous structure, which are to be regarded as appendages to the membrane. The secretion is here produced by the epithelium on the free surface and is mixed with secretions of the mucous glands.

There is nothing to be said in regard to the mechanism of the secretion of mucus in addition to what has already been stated in connection with the general mechanism of secretion. Mucous membranes are quite vascular; and the cells covering the membrane and lining the follicles and glands attached to it have the property of taking from the blood the materials necessary for the formation of the secretion. These matters pass out of the cells upon the surface of the membrane in connection with water and inorganic salts in varying proportions. Many of the cells themselves are thrown off and are found in the secretion, together with a few leucocytes, which latter are produced on mucous surfaces with great facility.

Composition and Varieties of Mucus. - All the varieties of mucus are more or less viscid; but this character is variable in secretions from different membranes, in some of them the secretion being quite liquid, and in others, almost semisolid. The different kinds of mucus vary considerably in general appearance. Some are clear and colorless; but the secretion usually is grayish and semitransparent. Examined with the microscope, in addition to the mixture of epithelium and occasional leucocytes, which give to the liquid its semiopaque character, the mass of the secretion presents a finely-striated appearance, as if it were composed of thin layers of nearly transparent substance with many folds. These delicate striæ usually do not interlace with each other, and they are rendered more distinct by the action of acetic acid. This appearance, with the peculiar effect of the acid, is characteristic of mucus. Some varieties of mucus present very fine pale granules and a few small globules of oil.

On the addition of water, mucus is somewhat swollen but is not dissolved. An exception to this is the secretion of the conjunctival mucous membrane, which is coagulated by water. As a rule the re- 
action of mucus is alkaline ; the only exception to this being the vaginal mucus, which is very liquid and is distinctly acid.

It is difficult to get an exact idea of the composition of normal mucus, from the fact that the quantity secreted by the membranes in their natural condition is small, being just sufficient to lubricate their surface. All varieties, however, contain an organic matter called mucin, which gives to the liquid its viscidity. They likewise present a considerable variety of inorganic salts, as sodium chloride, potassium chloride, alkaline lactates, sodium carbonate, calcium phosphate, a small proportion of the sulphates, and in some varieties, traces of iron and silica. Of all these constituents, mucin is the most important, as it gives to the secretion its characteristic properties. Like other nitrogenous substances, mucin is coagulable by various reagents. It is imperfectly coagulated by heat; and after desiccation it can be made to assume its peculiar consistence by the addition of a small quantity of water. It is coagulated by acetic acid and by a small quantity of the strong mineral acids, being redissolved in an excess of the latter. It also is coagulated by strong alcohol, forming a fibrinous clot soluble in hot and cold water. Mucin may be readily isolated by adding water to a specimen of normal mucus, filtering, and precipitating with an excess of alcohol. If this precipitate, after having been dried, is exposed to water, it assumes the viscid consistence peculiar to mucin. This property serves to distinguish it from albumin and other nitrogenous matters.

General Uses of Mucus. - The smooth, viscid and adhesive character of mucus, forming, as it does, a coating for the mucous membranes, serves to protect these parts, enables their surfaces to move freely one upon the other, and modifies to a certain extent the process of absorption. Aside from these mechanical uses, it has been shown that mucus, in connection with the epithelial covering of the mucous membranes, is capable of preventing the absorption of certain substances. It is well known, for example, that venoms may be applied with inpunity to certain mucous surfaces, while they produce poisonous effects if introduced into the circulation. These agents are not neutralized by the secretions of the parts, for they will produce their characteristic effects when removed from the mucous surfaces and introduced into the circulation; and it is reasonable to suppose that the mucous membranes are capable of resisting their absorption.

Physiological Anatomy of the Sebaceons, Centminous and Meibomian Glands. - The true sebaceous glands are found in all parts of the skin that are provided with hair; and as nearly every part of the general surface presents either the long, the short or the downy hairs, 
these glands are very widely distributed. They exist, indeed, in greater or less numbers in all parts of the skin except the palms of the hands and the soles of the feet. In the labia minora in the female, and in portions of the prepuce and glans penis of the male, parts not provided with hair, small racemose sebaceous glands are found, which produce secretions differing somewhat from that formed by the ordinary glands.
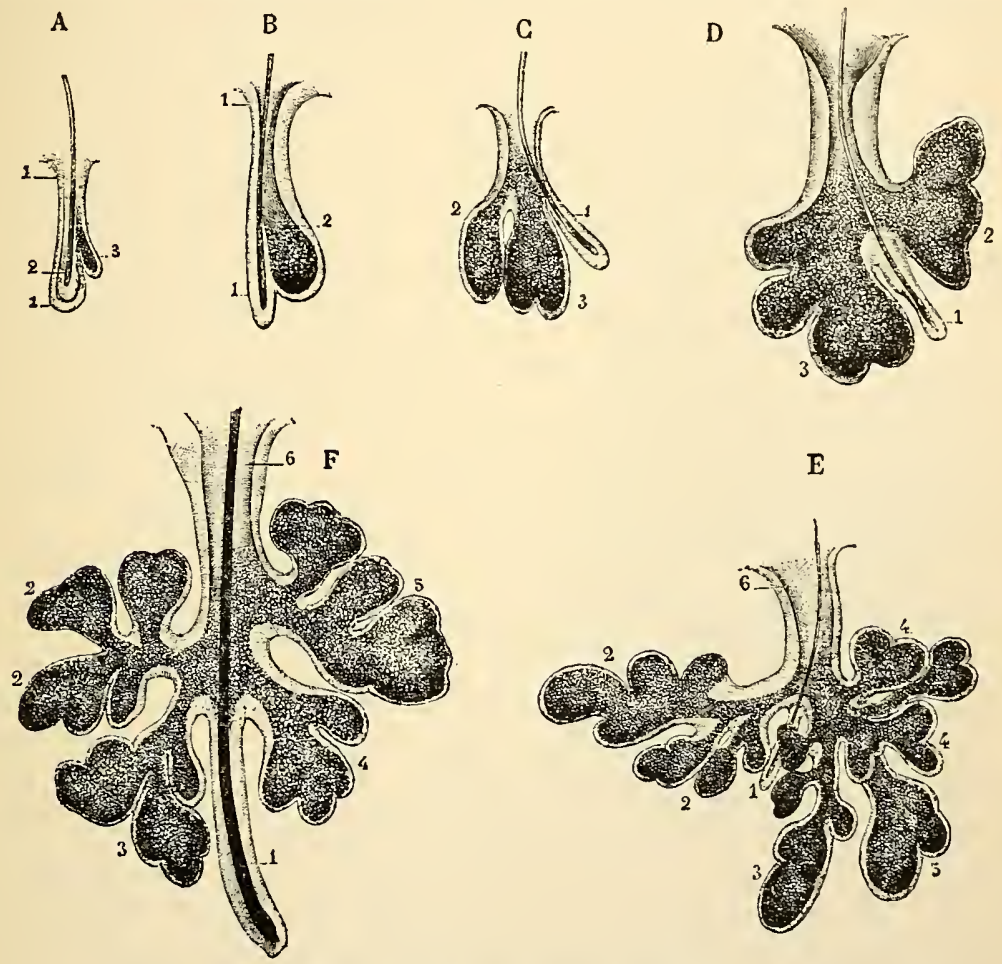

Fig. 6r. - Sebaceous glands (Sappey).

$A$, a gland in its most rudimentary form: I, rudimentary hair-follicle; 2 , downy hair; 3 , simple sebaceous follicle. $B$, a gland more developed: I, hair-follicle; 2 , simple sebaceous follicle. $C$, a gland with two follicles: I, hair-follicle; 2 , simple follicle; 3 , follicle imperfectly divided. $D$, a compound gland: I, hair-follicle; 2 , lobule with three follicles; 3 , lobule with four follicles. $E$, a gland with four lobules: I, hair-follicle; 2,2 , first lobule; 3 , second lobule; 4,4 , third lobule; 5 , fourth lobule; 6 , excretory duct with a hair passing through it. $F$, a gland with four lobules: I, hair-follicle; 2, 2, first lobule; 3 , second lobule; 4 , third lobule; 5 , fourth lobule; 6 , excretory duct.

The glands in the areola of the nipple in the female are large and are connected with small downy hairs.

Nearly all the sebaceous glands are either simple racemose glands, that is, presenting a number of follicles connected with a single excretory duct, or compound racemose glands, presenting several ducts, with their follicles, opening by a common tube. Although there are these variations in the size and arrangement of the glands of the 
general surface, they produce essentially the same secretion, and their anatomical differences consist simply in a multiplication of follicles (see Plate VII, Fig. 7).

The differences in the size of the sebaceous glands bear a certain relation to the size of the hairs with which they are connected; and as a rule, the largest glands are connected with the small downy hairs. These distinctions in size are so marked, that the glands may be divided into two classes; those connected with the long hairs of the head, face, chest, axilla, genital organs and with the coarse short hairs, and those connected with the fine downy hairs.

The glands connected with the larger hair-follicles are of the simple racemose variety and are $\frac{1}{12} \overline{0}$ to $\frac{1}{40}$ of an inch ( 0.21 to 0.64 millimeter) in diameter. Two to five of these glands usually are found arranged around each hair-follicle. They discharge their secretion at about the junction of the upper third with the lower two-thirds of the hair-follicle. The follicles of the long hairs of the scalp usually are provided each with a pair of sebaceous glands, measuring $\frac{1}{120}$ to $\frac{1}{75}$ of an inch (0.2 I to 0.34 millimeter) in diameter. Encircling the hairs of the beard, the chest, axilla and genital organs, are large glands, some of them $\frac{1}{40}$ of an inch (o.64 millimeter) in diameter, arranged in groups of four to eight.

The glands connected with the follicles of the small downy hairs are so large as compared with the hair-follicles that the latter seem rather as appendages to the glandular structures. These glands are of the compound racemose variety and present sometimes as many as fifteen culs-de-sac. The largest are found on the nose, the ear, the caruncula lachrymalis, the penis and the areola of the nipple, where they measure $\frac{1}{50}$ to $\frac{1}{12}$ of an inch (0.5 I to $2 . \mathrm{I}$ millimeters). The glands connected with the downy hairs of other parts usually are smaller. The glands of Tyson, situated on the corona and cervix of the glans penis, are sebaceous glands of the compound racemose variety.

The follicles composing the simple glands and the follicular terminations of the simple and compound racemose glands are formed of a delicate structureless or slightly granular membrane, with an external layer of inelastic and small elastic fibres and are lined with cells. Next the membrane, the cells are polyhedric, pale and granular, most of them presenting a nucleus and a nucleolus; but the follicle itself contains fatty granules and the other constituents of the sebaceous matter, with cells filled with fatty particles. These cells abound in the sebaceous matter as it is discharged from the duct. The great quantity of fatty granules and globules found in the ducts and follicles of the sebaceous glands renders them dark and opaque when examined with the microscope by transmitted light, except when studied in very thin sections, 
and their appearance is quite distinctive. The larger glands are surrounded with capillary bloodvessels.

The ceruminous glands produce a secretion resembling the sebaceous matter in many regards; but in their anatomy they are almost identical with the sudoriparous glands. They belong to the variety of glands called tubular, and they consist of a nearly straight tube which penetrates the skin, and a rounded or ovoid coil situated in the subcutaneous structure. These glands are found only in the cartilaginous portion of the external auditory meatus, where they exist in great number.

The ducts of the ceruminous glands are short and nearly straight, simply penetrating the different layers of the skin, and are $\frac{1}{700}$ to $\frac{1}{500}$ of an inch $(36$ to $50 \mu)$ in diameter. Their openings are rounded and about $\frac{1}{2} \frac{1}{70}$ of an inch $(93 \mu)$ in diameter. They sometimes terminate in the upper part of one of the hair-follicles. They present an external coat of fibrous tissue and are lined with several layers of small, pale, nucleated epithelial cells.

The glandular coil is an ovoid or rounded, brownish mass, $\frac{1}{120}$ to $\frac{1}{50}$ or $\frac{1}{16}$ of an inch (0.2 I to 0.5 I or 1.6 millimeter) in diameter. It is simply a convoluted tube continuous with the excretory duct and terminating in a somewhat dilated rounded extremity. It occasionally presents small lateral protrusions. The diameter of the

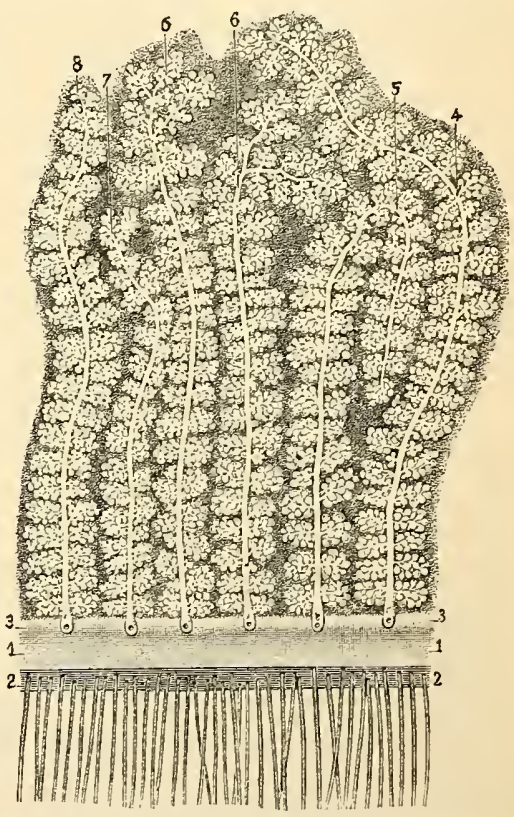

Fig. 62. - Meibomian glands of the upper lid, $\times 7$ (Sappey).

I, I, free border of the lid; 2, 2, anterior lip penetrated by the eyelashes; 3,3 , posterior lip, with the openings of the Meibomian glands; 4 , a gland passing obliquely at the summit; 5 , another gland bent upon itself; 6,6 , two glands in the form of racemose glands at their origin ; $7, a$ very small gland; 8 , a medium-sized gland.

tube is $\frac{1}{300}$ to $\frac{1}{250}$ of an inch $(83$ to IOO $\mu$ ). It has a fibrous coat, with a longitudinal layer of non-striated muscular fibres, and externally a few elastic fibres. It is lined by a single layer of irregularly-polygonal cells, which are $\frac{1}{2000}$ to $\frac{1}{1250}$ of an inch ( 12 to $20 \mu$ ) in diameter. These cells contain a number of brownish or yellowish pigmentary granules. The glandular portion of the tube contains a clear liquid mixed with a granular substance containing cells. In addition to the ceruminous glands, sebaceous follicles are found connected with the hair-follicles.

The Meibomian glands have essentially the same structure as the 
ordinary sebaceous glands. Their ducts, however, are longer, and the terminal follicles are arranged in a peculiar manner by the sides of the tubes along their entire length. These glands are situated partly in the substance of the tarsal cartilages, between their posterior surfaces and the conjunctival mucous membrane. They are placed at right angles to the free border of the eyelids, opening on the inner edge and occupying the entire width of the cartilages. Twenty-five to thirty glands are found in the upper lid, and twenty to twenty-five, in the lower lid.

Each Meibomian gland consists of a nearly straight excretory duct, $\frac{1}{300}$ to $\frac{1}{2} \overline{50}$ of an inch ( 83 to $100 \mu$ ) in diameter, communicating laterally with compound racemose acini, or collections of follicles, measuring $\frac{1}{300}$ to $\frac{1}{12} \overline{5}$ of an inch $(83$ to $200 \mu$ ). Fifteen or twenty of these collections of follicles are found on either side of the duct in glands of medium length. Most of the excretory ducts are nearly straight, but some are turned upon themselves near their upper extremities.

In general structure there is little if any difference between the terminal follicles of the Meibomian glands and the follicles of the ordinary sebaceous glands. They are lined with cells $\frac{1}{2500}$ to $\frac{1}{1250}$ of an inch ( Io to $20 \mu$ ) in diameter. The cells contain fatty globules, but these do not coalesce into large drops, such as are often seen in the ordinary sebaceous cells. The follicles and ducts are filled with the whitish oleaginous matter which constitutes the Meibomian secretion, or the sebum palpebrale.

In addition to the Meibomian secretion, the edges of the palpebral orifice receive a small quantity of secretion from ordinary sebaceous glands of the compound racemose variety (ciliary glands), which are appended in pairs to each of the follicles of the eyelashes, and from sebaceous glands attached to the small hairs of the caruncula lachrymalis.

Ordinary Sebaceous Matter. - Although it may be inferred, from the great number of sebaceous glands opening on the cutaneous surface, that the amount of sebaceous matter must be considerable, it has been impossible to collect the normal secretion in quantity sufficient for ultimate analysis. In some parts, as the skin of the nose, where the glands are particularly abundant, a certain quantity of oily secretion is sometimes observed, giving to the surface a greasy appearance. This may be collected in small quantity on a glass slide and examined microscopically. It then presents a number of strongly refracting fatty globules with a few epithelial cells. The cells, however, are not abundant in the secretion as it is discharged upon the general surface; but if the contents of the ducts and follicles are examined, cells will be 
found in great number. Most of the cells, indeed, remain in the glands, and the oily matter only is discharged. The object of this secretion is to lubricate the general cutaneous surface and give to the hairs the softness characteristic of them in a healthy condition.

The chemical constituents of the sebaceous matter are largely fatty. The solid matters consisted of olein, 270 parts, palmitin, I 35 parts, caseous matter, I 29 parts, gelatin, 87 parts, a little albumin, butyric acid and sodium butyrate, with sodium phosphate, sodium chloride, sodium sulphate and traces of calcium phosphate. Cholesterin, which is present so frequently in the contents of sebaceous cysts, does not exist in the normal secretion.

During the later months of pregnancy and during lactation, the sebaceous glands of the areola of the nipple become distended with a grayish white opaque secretion containing oily globules and granules. Frequently the secretion also contains a large number of epithelial cells. During the periods above indicated, the secretion here is always much more abundant than in the ordinary sebaceous glands.

Smegma of the Prepuce and of the Labia Minora. - In the folds of the prepuce of the male and on the inner surface and folds of the labia minora in the female, a small quantity of a whitish grumous matter, of a cheesy consistence, is sometimes found, particularly when proper attention is not paid to cleanliness. The matter which thus collects in the folds of the prepuce has really little analogy with the ordinary sebaceous secretion. Examination with the miscroscope shows that it is composed almost entirely of irregular scales of epithelium, which do not present the fatty granules and globules usually observed in the cells derived from the sebaceous glands. The production of this substance probably is independent of the secretion of sebaceous matter, as it is formed chiefly in parts of the prepuce in which the sebaceous glands are wanting.

The smegma of the labia minora is of the same character as the smegma preputiale; but it contains drops of oil and the other products of sebaceous glands found in these parts.

Vernix Caseosa. - The surface of the foetus at birth and near the end of utero-gestation usually is covered with a whitish coating, or smegma, called the vernix caseosa. This is most abundant in the folds of the skin; but it nearly always covers the entire surface with a coating of greater or less thickness and of about the consistence of lard. There are great differences in foetuses at term as regards the quantity of vernix caseosa. In some the coating is so slight that it is observed only on close inspection. There are few analyses that give accurately the chemical composition of this substance; and the best idea of its constitution and 
mode of formation can be formed from microscopical examinations. If a small quantity is scraped from the surface and is spread out upon a glass slide with a little glycerin and water, it will be found, on microscopical examination, to consist of a large number of epithelial cells with a few small fatty granules. These cells, after desiccation, constitute about ten per cent of the entire mass. The fatty granules are few and do not seem to be necessary constituents of the vernix, as they are of the sebaceous matter. In fact, the vernix caseosa must be regarded as the residue of the secretion of the sebaceous glands rather than an accumulation of true sebaceous matter.

The office of the vernix caseosa is protective. In making a microscopical preparation of the cells with water, it becomes evident that the coating is penetrated by the liquid with difficulty, even when mixed with it as thoroughly as possible. The protecting coat of vernix caseosa allows the skin to perform its office in utero; and at birth, when this coating is removed, the surface is found in a condition perfectly adapted to extra-uterine existence.

Cerumen. - A peculiar substance of a waxy consistence is secreted by the glands that have been described in the external auditory meatus, under the name of ceruminous glands, mixed with the secretion of sebaceous glands connected with the short hairs in this situation. It is difficult to ascertain what share these two sets of glands have in the formation of the cerumen. The waxy portion of the secretion probably is produced by the sebaceous glands, and the convoluted glands, commonly known as the ceruminous glands, produce a secretion resembling sweat. This view is to a certain extent reasonable; for the sebaceous matter is not removed from the meatus by friction, as in other situations, and would have a natural tendency to accumulate; but the contents of the ducts of the ceruminous glands differ materially from the liquid found in the ducts of the ordinary sudoriparous glands, containing granules and fatty globules such as exist in the cerumen. Although the glands of the ear are analogous in structure, and to a certain extent in the character of their secretion, to the sudoriparous glands, their secretion is peculiar. The perspiratory glands of the axilla and of some other parts also produce secretions differing somewhat from ordinary perspiration. So far as can be ascertained, the cerumen is produced by both sets of glands. The sebaceous glands attached to the hair-follicles probably secrete most of the oleaginous and waxy matter, while the socalled ceruminous glands produce a secretion of much greater fluidity, but containing granular and fatty matters.

The consistence and general appearance of cerumen are variable within the limits of health. When first secreted, it is of a yellowish 
color and about the consistence of honey, becoming darker and more viscid on exposure to the air. It has a decidedly bitter taste. It readily forms a sort of emulsive mixture with water.

Examined microscopically, the cerumen is found to contain semisolid dark granules of an irregularly polyhedric shape, with epithelium from the sebaceous glands, and epidermic scales, both isolated and in layers. Sometimes, also, a few crystals of cholesterin are found.

Chemical examination shows that cerumen is composed of oily matters fusible at a low temperature, a peculiar organic matter resembling mucin, with sodium salts and a certain quantity of calcium phosphate. The yellow coloring matter is soluble in alcohol; and the residue after evaporation of the alcohol is soluble in water and may be precipitated from a watery solution by neutral lead acetate or tin chloride. This extract has a bitter taste.

The cerumen lubricates the external meatus, accumulating about the hairs in the canal. Its bitter taste is supposed to be useful in preventing the entrance of insects.

Meibomian Secretion. - Little is known concerning any special properties of the Meibomian secretion except that it mixes in the form of an emulsion with water more readily than the other sebaceous matters. It is produced in small quantity, mixed with mucus and the secretion from the sebaceous glands attached to the eyelashes and the glands of the caruncula lachrymalis, and smears the edges of the palpebral opening. This oily coating on the edges of the lids, unless the tears are produced in excessive quantity, prevents their overflow upon the cheeks, and the excess is carried away by the nasal duct.

\section{MAMmaRy SECRETION}

The mammary glands are among the most remarkable organs in the economy; not only on account of the peculiar character of their secretion, which is unlike the product of any other of the glands, but from the changes which they undergo at different periods, both in size and structure. Rudimentary in early life and in the male at all periods of life, these organs are fully developed in the adult female only in the later months of pregnancy and during lactation. In the female, after puberty, the mammary glands undergo a rapid increase in size; but even then they are not fully developed.

Physiological Anatomy of the Mammary Glands. - The form, size and situation of the mammæ in the adult female are too well known to demand more than a passing mention. These organs are almost invariably double and are situated on the anterior portion of the thorax, over 
the great pectoral muscles. In women who have never borne children, they usually are firm and nearly hemispherical, with the nipple at the most prominent point. In women who have borne children, the glands during the intervals of lactation are larger, are held more loosely to the subjacent parts and often are flabby and pendulous. The areola of the nipple, also, is darker.

In both sexes the mammary glands are nearly as much developed at birth as at any time before puberty. They make their appearance at about the fourth month, in the form of little elevations of cutaneous structure, which soon begin to send off processes beneath the skin that are destined to be developed into the lobes of the glands. In the fotus at term, the glands measure hardly more than one-third of an inch (8.5 millimeters) in diameter. At this time there are twelve to fifteen lobes in each gland, and each lobe is penetrated by a duct, with but few branches, composed of fibrous tissue and lined with cylindrical epithelium. The ends of these ducts frequently are somewhat dilated; but what have been called the gland-vesicles do not make their appearance before puberty. In the adult male, the glands are half an inch to two inches ( 12.7 to 50.8 millimeters) broad, and $\frac{1}{12}$ to $\frac{1}{4}$ of an inch (2.1 to 6.4 millimeters) in thickness. In their structure, however, they present little if any difference from the rudimentary glands of the infant.

As the time of puberty approaches in the female, the rudimentary ducts of the different lobes become more and more ramified. Instead of each duct having but two or three branches, the lobes, as the gland enlarges, are penetrated by innumerable ramifications which have gradually been developed as processes from the main duct. It is important to remember, however, that these branches are never so abundant or so long during the intervals of lactation as they are when the gland is in full activity.

Between the fourth and fifth months of utero-gestation, the mammary glands of the mother begin to increase in size; and at term they are much larger than during the unimpregnated state. At this time the breasts become quite hard, and the surface near the areola is somewhat uneven, from the great development of the ducts. The nipple also is increased in size, the papillæ on its surface and on the areola are more largely developed and the areola becomes larger, darker and thicker. The glandular structure of the breasts during the latter half of pregnancy becomes so far developed, that if the child is born at the seventh month, the lacteal secretion may be established at the usual time after parturition. Even when parturition takes place at term, a few days elapse before secretion is fully established, and the first product of the glands, called colostrum, is somewhat different from fully-formed milk. 
The only parts of the covering of the breasts that present any peculiarities are the areola and the nipple. The surface of the nipple is covered with papillæ, which are largely developed near the summit. It is covered with epithelium in several layers, the lower strata being filled with pigmentary granules. The true skin covering the nipples is composed of inelastic and elastic fibres, containing a large number of sebaceous glands, but no hair-follicles or sudoriparous glands. These glands are of the racemose variety and not in the form of simple follicles. The nipple contains the lactiferous ducts, fibres of inelastic and elastic tissue, with a large number of non-striated muscular fibres. The muscular fibres have no definite direction, but are so abundant that when contracted the nipple becomes firm and hard.

The areola does not lie, like the general integument covering the gland, on a bed of adipose tissue, but it is closely adherent to the subjacent glandular structure. The skin here is much thinner and more delicate than in other parts, and the pigmentary granules are very abundant in some of the lower strata of epidermic cells, particularly during pregnancy. The true skin of the areola is composed of inelastic and elastic fibres and lies on a distinct layer of non-striated muscular fibres. The arrangement of the muscular fibres - sometimes called the subareolar muscle - is quite regular, forming concentric rings around the nipple. These fibres are supposed to be useful in compressing the ducts during the discharge of milk. The areola presents the following structures : papillæ, considerable smaller than those on the nipple; hairfollicles, containing small rudimentary hairs; sudoriparous glands; and sebaceous glands connected with the hair-follicles. The sebaceous glands are large, and their situation is indicated by little prominences on the surface of the areola, which are especially marked during pregnancy.

The mammary gland itself is of the compound racemose variety. It is covered in front with a subcutaneous layer of fat, and posteriorly it is enveloped in a fibrous membrane loosely attached to the pectoralis major muscle. A considerable quantity of adipose tissue also is found in the substance of the gland between the lobes.

Separated from the adipose and fibrous tissue, the mammary gland is found divided into lobes, fifteen to twenty-four in number. These are subdivided into lobules made up of a greater or less number of acini. The secreting structure is of a reddish yellow color and is distinctly granular, presenting a decided contrast to the pale and uniformly fibrous appearance of the gland during the intervals of lactation. If the ducts are injected from the nipple and followed into the substance of the gland, each one will be found distributing its branches to a dis- 
tinct lobe; so that the organ is really made up of a number of glands identical in structure.

The canals that discharge the milk at the nipple are called lactiferous or galactophorous ducts. They are ten to fourteen in number. The openings of the ducts at the nipple are small, measuring only $\frac{1}{60}$ to $\frac{1}{40}$ of an inch ( 0.42 to 0.64 millimeter). As each duct passes downward, it enlarges in the nipple to $\frac{1}{25}$ or $\frac{1}{12}$ of an inch ( $\mathrm{I}$ or 2 millimeters) in diameter, and beneath the areola it presents an elongated dilatation, $\frac{1}{6}$ to $\frac{1}{3}$ of an inch ( 4.2 to 8.5 millimeters) in diameter, called the sinus of the duct. During lactation a considerable quantity of milk collects in these sinuses, which serve as reservoirs. Beyond the sinuses, the calibre of the ducts measures $\frac{1}{12}$ to $\frac{1}{6}$ of an inch (2.I to 4.2 millimeters). The ducts penetrate the lobes, branching and subdividing, to terminate in the collections of alveoli which form the acini. There is no anastomosis between the different lactiferous ducts, and each one is distributed independently to one or more lobes.

The lactiferous ducts have three distinct coats. The external coat is composed of anastomosing fibres of elastic tissue with some inelastic fibres. The middle coat is composed of non-striated muscular fibres, arranged longitudinally and existing throughout the duct from its opening at the nipple to the secreting alveoli. The internal coat is a thin amorphous membrane, lined with flat polygonal cells during the intervals of lactation and even during pregnancy, the cells being cylindrical in form and frequently presenting multiple nuclei, when milk is secreted.

The acini of the gland, which are very abundant, are visible to the naked eye, in the form of small rounded granules of a reddish yellow color. Between the acini, there exists a certain quantity of ordinary white fibrous tissue with a number of adipose vesicles. The presence of adipose tissue in considerable quantity in the substance of the glandular structure is peculiar to the mammary glands. Each acinus is made up of twenty to forty secreting vesicles, or alveoli. These vesicles are irregular in form, often varicose, and sometimes they are enlarged and imperfectly bifurcated at their extremities. During lactation their diameter is $\frac{1}{400}$ to $\frac{1}{300}$ of an inch $(60$ to $80 \mu$ ).

During the intervals of lactation, as the lactiferous ducts become retracted, the glandular alveoli disappear; and in pregnancy, as the gland takes on its full development, the ducts branch and extend themselves, and the vesicles are gradually developed around their extremities.

Mcchanism of the Sccrction of Milk. - With the exception of water and inorganic matters, all the important and characteristic constituents of the milk are formed in the substance of the mammary glands. The secreting structures separate from the blood a great variety of inorganic 
salts; and milk furnishes all the inorganic matter necessary for the nutrition of the infant, even containing a small quantity of iron.

The lactose, or sugar of milk, the casein and the fatty particles are all produced in the gland. The peculiar kind of sugar here found does not exist anywhere else in the organism. Even when the secretion of milk is most active, different varieties of sugar, such as glucose or canesugar, injected into the bloodvessels of a living animal, are not eliminated by the mammary glands as they are by the kidneys; and their presence in the blood does not influence the quantity of lactose found in the milk.

Casein is produced in the mammary glands, probably by a peculiar transformation of the proteid constituents of the blood. The fatty globules are likewise produced in the substance of the gland, and the peculiar kind of fat that exists in this secretion is not found in the blood. The mechanism of the production of fat in the mammary glands is somewhat obscure. During full activity of the lacteal secretion, however, small fatty globules appear in the cells lining the alveoli, particularly that portion of the cells next the lumen, and these finally are discharged into the ducts.

As regards the mechanism of the formation of the peculiar and characteristic constituents of milk, the mammary glands are to be classed among the organs of secretion and not with those of elimination, or excretion; for none of these matters preëxist in the blood and they all appear first in the substance of the glands.

During the period of secretion, the glands receive a larger supply of blood than at other times. Pregnancy favors the development of the secreting portions of the glands but does not induce secretion. On the contrary, when pregnancy occurs during lactation, it diminishes and modifies, and it may arrest the secretion of milk. The secreting action of the mammary glands is nearly continuous. When the secretion of milk has become fully established, while there may be certain times when it is formed in greater quantity than at others, there is no actual intermission in its production.

General Conditions which modify the Lacteal Secretion. - Very little is known concerning the physiological conditions which modify the secretion of milk. When lactation is fully established, the quantity and quality of the milk secreted become adapted to the requirements of the child at different periods of its existence. In studying the composition of the milk, therefore, it will be found to vary considerably in different stages of lactation. It is evident that as the development of the child advances, a constant increase of nourishment is demanded; and as a rule the mother is capable of supplying all the nutritive requirements of the infant for eight to twenty months. 
During the time when such an amount of nutritive matter is furnished to the child, the quantity of food taken by the mother is sensibly increased; but observations have shown that the secretion of milk is not much influenced by the character of the food. It is necessary that the mother should be supplied with good nutritious articles; but so far as solid food is concerned, there seems to be no great difference between a coarse and a delicate alimentation; and the milk of females in the lower walks of life, when the general condition is normal, is fully as good as in women who are able to live luxuriously. It is, indeed, a fact commonly recognized by physiologists, that the secretion of milk is little influenced by any special diet, provided the alimentation be sufficient and of the quality ordinarily required by the system and that it contain none of the few articles of food known to have a special influence on lactation. It is common, however, for women to become quite fat during lactation; which shows that the fatty constituents of the food do not pass exclusively into the milk, but that there is a tendency, at the same time, to a deposition of adipose tissue in the situations in which it ordinarily is found. It is a matter of common experience, that certain articles, such as acids and fermentable substances, often disturb the digestive organs of the child without producing any change in the milk that can be recognized by chemical analysis. The individual differences in women, in this regard, are very great.

The statements in regard to solid food do not apply to liquids. During lactation there is always an increased demand for water and for liquids generally; and if these are not supplied in sufficient quantity, the secretion of milk is diminished and its quality is almost always impaired. It is a curious fact, which has been fully established by observations on the human subject and the inferior animals, that while the quantity of milk is increased by taking a large amount of simple water, the solid constituents also are increased and the milk retains all its nutritive qualities.

Alcohol, especially when largely diluted, as in malt-liquors and other mild beverages, is well known to influence the secretion of milk. Drinks of this kind almost always temporarily increase the activity of the secretion, and sometimes they produce an effect on the child; but direct and accurate observations on the actual passage of alcohol into the milk are wanting. During lactation the moderate use of drinks containing a small proportion of alcohol frequently is beneficial, particularly in assisting the mother to sustain the unusual drain on the system. There are, however, few instances of normal lactation in which their use is absolutely necessary.

It is well known that the secretion of milk may be profoundly af- 
fected by violent mental emotions. This is the case in respect to many other secretions, as the saliva and the gastric juice. It is hardly necessary, however, to cite many of the instances of modification or arrest of the secretion from this cause that are quoted by authors. Vernois and Becquerel reported a case in which a hospital wetnurse, who lost her only child from pneumonic fever, became violently affected with grief and presented as a consequence an immediate diminution in the quantity of her milk, with a great reduction in the proportion of salts, sugar and butter. In this case the proportion of casein was increased. Astley Cooper reported two cases in which the secretion of milk was instantaneously and permanently arrested by terror. These cases are types of many others, cited by writers, of the influence of mental emotions on secretion.

Direct observations on the influence of spinal nerves on the mammary glands are few and unsatisfactory. The operation of dividing the nerves distributed to these glands, which has occasionally been practised on animals in lactation, has not been observed to produce any sensible diminution in the quantity of the secretion. It is difficult, however, to operate on all the nerves distributed to these organs. There are no observations indicating the situation of a nerve-centre presiding over the secretion of milk, although such a centre may exist.

Quantity of Milk. - It is difficult to form a reliable estimate of the average quantity of milk secreted by the human female in the twentyfour hours. The quantity undoubtedly varies very much in different persons; some women being able to nourish two children, while others, though apparently in perfect health, furnish hardly enough food for one. The quantity that can be drawn from a full breast usually is about two fluidounces ( 60 grams). This may be assumed to be about the quantity contained in the lactiferous ducts when they are moderately distended. Taking into consideration the variations in the quantity of milk secreted by different women, it may be assumed that the daily production is between two and three pints (950 and 1420 grams).

Certain conditions of the female are capable of materially influencing the quantity of milk secreted. It is evident that the secretion usually is somewhat increased within the first few months of lactation, when the progressive development of the child demands an increase in the quantity of nourishment. If the menstrual function becomes reestablished during lactation, the milk usually is diminished in quantity during the periods, but sometimes it is not affected, either in its quantity or composition. Should the female become pregnant, there commonly is a great diminution in the quantity of milk, and that which is secreted is regarded ordinarily as possessing little nutritive value. In obedi- 
ence to a popular prejudice - apparently well founded - the child usually is taken from the breast so soon as pregnancy is recognized. No marked and constant variations have been observed in the quantity of milk in females of different ages.

Propertics and Composition of Milk. - The general appearance and characters of ordinary cow's milk are sufficiently familiar. Human milk is neither so white nor so opaque as cow's milk, having ordinarily a slightly bluish tinge. After secretion has become fully established, milk possesses no viscidity and is nearly opaque. It is almost inodorous, of a peculiar soft and sweetish taste, and when perfectly fresh it has a clecidedly alkaline reaction. The taste of human milk is sweeter than that of cow's milk. A short time after its discharge from the gland, the reaction of milk becomes first amphoteric and afterward faintly acid; but this change takes place more slowly in human milk than in the milk of most of the inferior animals.

The average specific gravity of human milk is I032; although this is subject to considerable variation, the minimum of eighty-nine observations being 1025, and the maximum, 1046 (Vernois and Becquerel). The observations of most physiological chemists have shown that this average is nearly correct.

Milk is not coagulated by heat, even after prolonged boiling; but a thin pellicle then forms on the surface. Although a small quantity of albumin exists in the milk, this does not coagulate on the surface by the action of the heat, for the scum does not form when the liquid is heated in a vacuum or in an atmosphere of carbon dioxide or of hydrogen.

When milk is coagulated by any substance acting on the casein or when it coagulates spontaneously, it separates into a curd, composed of casein with most of the fatty particles, and a nearly clear, greenish yellow serum, called whey. This separation occurs spontaneously at a variable time after the discharge of the milk, taking place much sooner in warm than in cold weather. It is a curious fact that fresh milk frequently is coagulated during a thunder storm, which probably is due to electric action on the ions that enter into the constitution of the casein molecules.

On being allowed to stand for a short time, milk separates, without coagulating, into two tolerably distinct portions. A large proportion of the globules rises to the top, forming a yellowish white and very opaque liquid, called cream, leaving the lower portion poorer in globules and of a decidedly bluish tint. In healthy milk the stratum of cream forms one-fifth to one-third of the entire mass of the milk. In the human subject the skim-milk is not white and opaque, but it is nearly as transparent as whey. The specific gravity of the cream from milk of the 
average specific gravity of 1032 is about I024. The specific gravity of skim-milk is about IO34.

Microscopical Characters of the Milk. - Milk contains a great number of minute spherical globules, of highly refractive power, held in suspension in a clear liquid. These are known as milk-globules and are composed of palmitin, olein, fatty matters peculiar to milk, with a little lecithin and sometimes a very small quantity of cholesterin. The globules also contain a small quantity of a yellow lipochrome, or fatpigment. The specific gravity of pure milk-fat is 949 to 996.

Human milk-globules are $\frac{1}{25000}$ to $\frac{1}{1250}$ of an inch ( $\mathrm{I}$ to $20 \mu$ ) in diameter. They usually are distinct from each other, but they may occasionally become collected into groups without indicating anything abnormal. In a perfectly normal condition of the glands, when the lacteal secretion has become fully established, the milk contains nothing but a clear liquid with these globules in suspension. The proportion of fatty matters in milk is twenty-five to thirty-eight parts per thousand; and this gives an idea of the proportion of globules that are seen on microscopical examination.

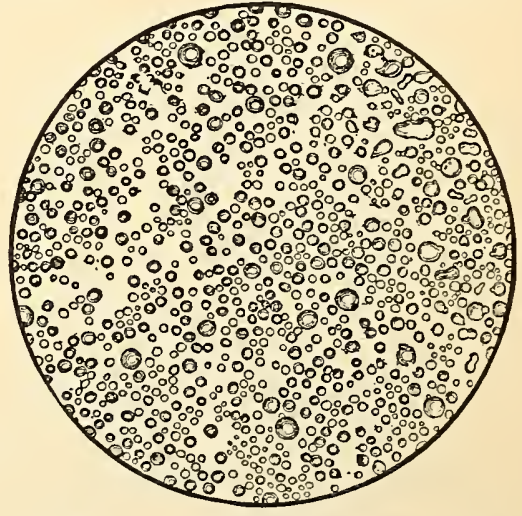

Fig. 63.-Human milk-globules from a

In some regards milk does not healthy lying-in woman eight days after deivery, present the characters of a simple $\times 400$ (Funke).

emulsion. When shaken with ether, the mixture remains opaque; but the fatty matters are dissolved on the addition of potassium hydrate. Dilute acetic acid added to milk causes the globules to run together. These reactions have led to the view that the milk-globules have a membrane that is dissolved by potassium hydrate and by acetic acid. It is probable that the butter in normal milk does not exist precisely in the form of a simple emulsion, but that the globules have a very thin caseous coating.

In view of the action of various reagents on the milk-globules, the only alternative, if the existence of a caseous coating is denied, is the opinion that the addition of potassium hydrate or of acetic acid renders the casein incapable of holding the fat in the condition of an emulsion. There is actually, however, little more than a verbal difference between these two opinions.

Composition of the Milk. - The following table, compiled from analyses by various chemists, gives the constituents of human milk:- 


\section{COMPOSITION OF HUMAN MILK}

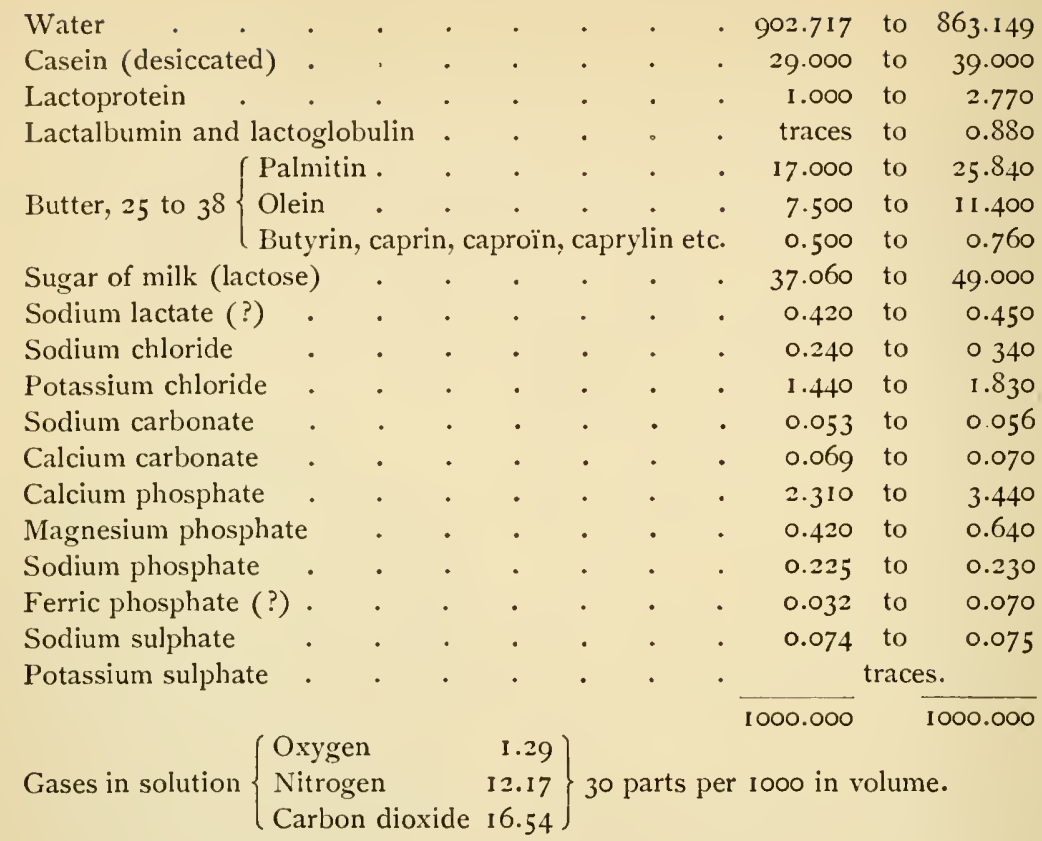

The proportion of water in milk is subject to certain changes, but these are not so considerable as might be expected from the great variations in the entire quantity of the secretion. As regards the quantity of milk in the twenty-four hours, the influence of drinks, even when nothing but pure water is taken, is very marked; and although the activity of the secretion is much increased by fluid ingesta, the quality of the milk usually is not affected, and the proportion of water to the solid matters remains about the same.

Nitrogenous Constituents of Milk. - Little remains to be said concerning the nitrogenous constituents of human milk after what has been stated in connection with alimentation. The different constituents of this class undoubtedly have the same nutritive office and they appear to be identical in all varieties of milk, the only difference being in their relative proportions. It is a matter of common experience, indeed, that the milk of many of the lower animals will take the place of human milk, when prepared so as to make the proportions of its different constituents approximate the composition of the natural food of the child. A comparison of the composition of human milk and of cow's milk shows that the former is poorer in nitrogenous matters and richer in butter and sugar; and consequently, the upper strata of cow's milk, 
properly sweetened and diluted with water, very nearly represent the ordinary breast-milk.

Casein is by far the most important of the nitrogenous constituents of milk, and it supplies nearly all of this kind of nutritive matter demanded by the child. Lactoprotein is not so well defined, and lactalbumin, a proteid resembling serum-albumin, with lactoglobulin, exists in milk in small quantities. These proteids, however, are of little physiological interest.

The coagulation of milk depends on the reduction of casein from a liquid to a semisolid condition. When milk is allowed to coagulate spontaneously, the change is effected by the action of the lactic acid which results from a change in a part of the sugar of milk. Casein, in fact, is coagulated by nearly all acids, even the feeble acids of organic origin. It differs from albumin in this regard and in the fact that it is not coagulated by heat. If fresh milk is slightly raised in temperature and treated with an infusion of the gastric mucous membrane of the calf, coagulation will take place in five or ten minutes, the clear liquid still retaining its alkaline reaction. The action on casein of the rennin of the gastric juice has already been described in connection with gastric digestion.

Non-Nitrogenous Constituents of Milk. - Non-nitrogenous matters exist in abundance in the milk. The liquid casein and the water hold the fats in the condition of a fine and permanent emulsion. This fat may easily be separated from milk and is known under the name of butter. In human milk the butter is much softer than in the milk of many of the inferior animals, particularly the cow; but it is composed of essentially the same constituents, although in different proportions. In different animals, there are developed, even after the discharge of the milk, certain odorous matters that are more or less characteristic of the animal from which the butter is taken.

The greatest part of the butter consists of palmitin. It contains in addition, olein, and a small proportion of peculiar fats - which have not been very well determined - called butyrin, caprin, caproïn, caprylin, with some other analogous substances. Palmitin and olein are found in the fat throughout the body; but the last-named substances are peculiar to the milk. These are especially liable to acidification, and the acids resulting from their decomposition give the peculiar odor and flavor to rancid butter.

Sugar of milk, or lactose, is the most abundant of the solid constituents of the mammary secretion. It is this that gives to the milk its peculiar sweetish taste, although this variety of sugar is much less sweet than cane-sugar. The chief peculiarities of milk-sugar are that 
it readily undergoes change into lactic acid in the presence of nitrogenous ferments, and that it takes on alcoholic fermentation slowly and with difficulty. In the fermentation of milk, the lactose is changed into galactose and dextrose and then into alcohol and carbon dioxide. In some parts of the world alcoholic beverages made from milk are in common use.

Inorganic Constituents of Milk. - It is probable that many inorganic salts exist in the milk, that are not given in the table; and the separation of these from their combinations with organic matters is one of the most difficult problems in physiological chemistry. This must be the case, for during the first months of extra-uterine existence, the child derives all the inorganic as well as the organic matters necessary to nutrition and development, from the breast of the mother. The reaction of milk depends on the presence of alkaline carbonates, and these are important in preserving the fluidity of casein. It is not determined precisely in what form iron exists in the milk, but its presence here is undoubted. A comparison of the composition of the milk with that of the blood shows that most of the important inorganic matters found in the latter exist also in the milk.

Carbon dioxide, nitrogen and oxygen exist in solution in milk. Of these gases, carbon dioxide is the most abundant. It is well known that the presence of gases in solution in liquids renders them more agreeable to the taste, and carbon dioxide increases materially their solvent properties. Aside from these considerations, the uses of the gaseous constituents of the milk are not apparent.

In addition to the constituents given in the table of composition, the milk contains small quantities of nuclein, dextrin, urea, cholesterin, lecithin, hypoxanthin, fluorin and silica.

A study of the composition of the milk fully confirms the fact that this is a typical aliment and presents in itself the proper proportion and variety of material for the nourishment of the body during the period when development is going on with its maximum of activity. The form in which its different nutritive constituents exist is such that they are easily digested and are assimilated with great rapidity. Human breast-milk is the natural food of the infant. It is immune or partially immune and may confer some immunity on the nursing child. It is bacteriologically pure (Welsh). Passing directly from breast to mouth, it is secure from contamination. It can not be adequately replaced by any artificial substitute.

Variations in the Composition of Milk. - If the composition of the milk is compared at different periods of lactation, it will be found to undergo important changes during the first few days. In fact, the first 
liquid secreted, after parturition, is so different from ordinary milk that it has been called by another name. It is then known as colostrum, the peculiar properties of which will be considered under a distinct head. As the secretion of milk becomes established, the liquid, from the first to the fifteenth day, gradually becomes diminished in density and in its proportion of water and of sugar, while there is a progressive increase in the proportion of most of the other constituents - butter, casein and the inorganic salts. The milk, therefore, so far as one can judge from its composition, as it increases in quantity during the first few days of lactation, is constantly increasing in nutritive properties. The differences in the composition of the milk, taken from month to month during the entire period of lactation, are not so distinctly marked. It is difficult, indeed, to indicate any constant variations of sufficient importance to lead to the view that the milk varies much in its nutritive properties at different times during the ordinary period of lactation. The differences between the milk of primiparæ and multiparæ are slight and unimportant. As a rule, however, the milk of primiparæ approaches more nearly the normal standard.

In normal lactation there is no marked and constant difference in composition between milk that has been secreted in.great abundance and milk produced in comparatively small quantity; and the difference between the liquid first drawn from the breast and that taken when the ducts are nearly empty, which is observed in the milk of the cow, has not been noted in human milk.

\section{Colostrum}

Near the end of utero-gestation, during a period which varies considerably in different women and has not been accurately determined, a small quantity of a thickish stringy liquid frequently may be drawn from the mammary glands. This bears little resemblance to perfectlyformed milk. It is small in quantity and usually is more abundant in multiparæ than in primiparæ. This liquid, as well as that secreted for the first few days after delivery, is called colostrum. It is yellowish, semiopaque, of a distinctly alkaline reaction and is somewhat mucilaginous in consistence. Its specific gravity is considerably above that of the ordinary milk, being between I040 and I060. As lactation progresses, the character of the secretion rapidly changes, until the liquid becomes filled with true milk-globules and assumes the characters of ordinary milk.

The opacity of the colostrum is due to the presence of a number of different corpuscular elements. Milk-globules, variable in size and number, are to be found in the secretion from the first. These, how- 
ever, do not exist in sufficient quantity to render the liquid very opaque, and they frequently are aggregated in rounded or irregular masses, held together, apparently, by some glutinous matter. Peculiar corpuscles, supposed to be characteristic of the colostrum, always exist in this secretion. These are known as colustrum-corpuscles. They are spherical, varying in size between $\frac{1}{2500}$ and $\frac{1}{500}$ of an inch (IO and $50 \mu$ ), are sometimes pale, but more frequently quite granular, and they contain very often a large number of fatty particles. They behave in all respects like leucocytes and are described as a variety of these

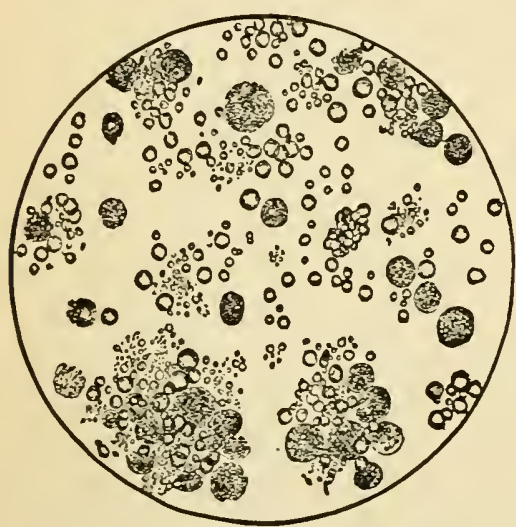

Fig. 64. - Colostrum from a healthy lying-in woman twelve hours after delivery, $\times 400$ (Funke).

The smaller globules are globules of milk. The larger globules, $a, a$, filled with granules, are colostrum-corpuscles. As lactation advances, the colostrum-corpuscles gradually disappear, and the milk-globules become more abundant, smaller and more nearly uniform in size.

sugar and of the inorganic salts. The proportion of fat is at least equal to the proportion in the milk and usually is greater. Instead of casein, colostrum contains a large proportion of serum-albumin; and as the character of the secretion changes, the albumin gradually becomes reduced in quantity and casein takes its place.

The following may be taken as the ordinary composition of colostrum of the human female (Clemm):-

\section{COMPOSITION OF COLOSTRUM}

Water

Albumin, and salts insoluble in alcohol 
Colostrum ordinarily decomposes more readily than milk and rapidly takes on putrefactive changes. If allowed to stand for twelve to twentyfour hours, it separates into a thick, opaque, yellowish cream and a serous liquid. In an observation by Astley Cooper, nine measures of colostrum, taken soon after parturition, after twenty-four hours of repose, gave six parts of cream to three of milk.

The peculiar constitution of colostrum, particularly the presence of an excess of sugar and inorganic salts, renders it somewhat laxative in its effects, and it is supposed to be useful, during the first few days after delivery, in assisting to relieve the infant of the accumulation of meconium.

As the quantity of colostrum that may be pressed from the mammary glands during the later periods of utero-gestation, particularly the last month, is variable, it becomes an important question to determine whether this secretion has any relation to the quantity of milk that may be expected after delivery. This question has been studied by Donné, who arrived at the following conclusions :-

In women in whom the secretion of colostrum is almost absent, the liquid being in exceedingly small quantity, viscid, and containing hardly any corpuscular elements, there is hardly any milk produced after delivery.

In women who, before delivery, present a moderate quantity of colostrum, containing very few milk-globules and a number of colostrum-corpuscles, after delivery the milk will be scanty or it may be abundant, but it is always of poor quality.

When the quantity of colostrum produced is considerable, the secretion being quite fluid and rich in corpuscular elements, particularly milk-globules, the milk after delivery is always abundant and of good quality.

From this, it would seem that the production of colostrum is an indication of the proper development of the mammary glands; and the early production of fatty granules, which are first formed by the cells lining the secreting vesicles, indicates the probable activity in the secretion of milk after lactation shall have become fully established.

The secretion of the mammary glands preserves the characters of colostrum until toward the end of the so-called milk-fever, when the colostrum-corpuscles rapidly diminish in number, and the milk-globules become more abundant, regular and uniform in size. It may be stated in general terms that the secretion of milk becomes fully established and all the characters of the colostrum disappear between the eighth and tenth days after delivery. A few colostrum-corpuscles and masses of agglutinated milk-globules may sometimes be discovered after the 
tenth day, but they are rare. After the fifteenth day, the milk does not sensibly change in microscopical or chemical characters.

\section{Lacteal Secretion in the Newly-Born}

In infants of both sexes there usually is a certain quantity of secretion from the mammary glands, beginning at birth or two or three days after and continuing sometimes for two or three weeks. The quantity of liquid that may be pressed out at the nipples at this time is variable. Sometimes only a few drops can be obtained, but occasionally it amounts to one or two drachms ( 3.7 or 7.4 grams). Although it is impossible to indicate the object of this secretion, which takes place when the glands are in a rudimentary condition, it has been so often observed and described by physiologists, that there can be no doubt in regard to the nature of the liquid and the fact that the secretion is almost always produced in greater or less quantity.

COMPOSITION OF THE MILK OF THE INFANT (GUBLER)

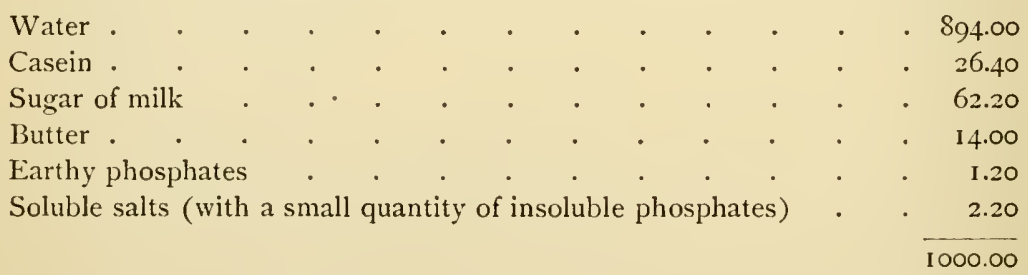

This secretion does not differ much in its composition from ordinary milk. The proportion of butter is less, but the proportion of sugar is greater and the quantity of casein is nearly the same.

Of the other liquids classed as secretions, the saliva, gastric juice, pancreatic juice and the intestinal secretions have already been described in connection with the physiology of digestion. The physiology of the lachrymal secretion will be taken up in connection with the eye, and the bile will be treated of fully under the head of excretion. 


\section{CHAPTER XII}

\section{EXCRETION BY THE SKIN}

Physiological anatomy of the skin - Layers of the skin - The corium, or true akin - The epidermis - Physiological anatomy of the nails - Physiological anatomy of the hairs - Roots of the hairs, and hair-follicles - Growth of the hairs - Sudden blanching of the hair Uses of the hairs - Perspiration - Quantity of cutaneous exhalation - Properties and composition of the sweat.

\section{Physiological Anatomy of the Skin}

THE skin is one of the most complex and important structures in the body and has a variety of uses. In the first place, it forms a protective covering for subjacent parts. It is quite thick over the parts most subject to pressure and friction, is elastic over movable parts and those liable to variations in size, and in many situations is covered with hair. The skin and its appendages are imperfect conductors of caloric, are capable of resisting very considerable variations in temperature, and they thus tend to maintain the normal standard of the animal heat. As an organ of sensibility, the skin has important uses, being abundantly supplied with sensory nerves, some of which present an arrangement peculiarly adapted to the nice appreciation of tactile impressions. The skin assists in preserving the external forms of the muscles. It also relieves the abrupt projections and depressions of the general surface and gives roundness and grace to the contours of the body. In some parts it is closely attached to the subjacent structures, while in others it is less adherent and rests on a layer of adipose tissue.

The skin is important as an organ of excretion; and although the quantity of excrementitious matter exhaled from it is not large, the evaporation of water from the general surface is considerable and is subject to such modifications as may become necessary from the varied conditions of the animal temperature. Thus, while the skin protects the body from external influences, its office is important in regulating the heat that is produced as one of the phenomena attendant on the general process of nutrition.

Extent and Thickness of the Skin. - Without detailing the measurements of different parts, it may be stated in general terms that the cutaneous surface in a good-sized man is equal to a little more than sixteen square feet ( 15,000 square centimeters); and in men of more 
than ordinary size, it may extend to twenty-one or twenty-two square feet ( 2 square meters). In women of medium size, the surface is equal to about twelve and a half square feet (II,500 square centimeters). The true skin is $\frac{1}{12}$ to $\frac{1}{8}$ of an inch (2.I to 3.2 millimeters) in thickness; but in certain parts, particularly in the external auditory meatus, the lips and the glans penis, it frequently measures not more than $\frac{1}{100}$ of an inch (0.254 millimeter).

Layers of the Skin. - The skin is naturally divided into two principal layers, which may be readily separated from each other by maceration. These are the true skin - cutis vera, derma, or corium - and the epidermis, cuticle, or scarf-skin. The true skin is more or less closely attached to subjacent parts by a fibrous structure called the subcutaneous areolar tissue, in the meshes of which there usually is a certain quantity of adipose tissue. This layer is sometimes described under the name of the panniculus adiposus. The thickness of the adipose layer varies in different parts of the general surface and in different persons. There is no fat beneath the skin of the eyelids, the upper and outer part of the ear, the penis and the scrotum. Beneath the skin of the cranium, the nose, the neck, the dorsum of the hand and foot, the knee and the elbow, the fatty layer is about $\frac{1}{12}$ of an inch (2.I millimeters) in thickness. In other parts it usually measures $\frac{1}{6}$ to $\frac{1}{2}$ of an inch (4.2 to 12.7 millimeters). In very fat persons it may measure an inch (25.4 millimeters) or more. On the head and the neck, in the human subject, are muscles attached more or less closely to the skin. These are capable of moving the skin to a slight extent. Muscles of this kind are largely developed and quite extensively distributed in some of the lower animals.

There is no sharply-defined line of demarcation between the cutis and the subcutaneous areolar tissue; and the under surface of the skin is irregular, from the presence of fibres that are divided necessarily in detaching it from the subjacent structures. The fibres which enter into the composition of the skin become looser in their arrangement near its under surface, the change taking place rather abruptly, until they present large alveoli, which usually contain a certain quantity of adipose tissue.

The layer called the true skin is subdivided into a deep, reticulated, or fibrous layer, and a superficial portion, called the papillary layer. The epidermis also is divided into two layers, as follows: an external layer, called the horny layer; and an internal layer, called the Malpighian, or the mucous layer, which is in contact with the papillary layer of the corium.

The Corium, or True Skin. - The reticulated and the papillary layers of the true skin are quite distinct. The lower stratum-the 
reticulated layer - is much thicker than the papillary layer and is dense, resisting, quite elastic and slightly contractile. It is composed of bundles of fibrous tissue, interlacing with each other in every direction, usually at acute angles. Distributed throughout this layer are found anastomosing elastic fibres of the small variety, and with them a number of non-striated muscular fibres. This layer contains in addition a considerable quantity of amorphous matter, which serves to hold the fibres together. The muscular fibres are particularly abundant about the hair-follicles and the sebaceous glands connected with them, and their arrangement is such that when they are excited to contraction by cold or by electricity, the follicles are drawn up, projecting on the general surface and producing the appearance known as "goose-flesh." Contraction of these fibres is especially marked about the nipple, producing the so-called erection of this organ, and about the scrotum and penis, wrinkling the skin of these parts. The peculiar arrangement of the little muscles around the hair-follicles, forming little bands attached to the surface of the true skin and the base of the follicles, explains fully the manner in which the "goose-flesh" is produced (see Fig. 67, page 3I2).

The papillary layer of the skin passes insensibly into the subjacent structure without any marked line of division. It is composed chiefly of amorphous matter like that which exists in the reticulated layer. The papillæ themselves appear to be simple elevations of this amorphous matter, although they contain a few fibres and connective-tissue nuclei.

As regards form, the papillæ may be divided into two varieties: the simple and the compound. The simple papillæ are conical, rounded or club-shaped elevations of the amorphous matter and are irregularly distributed on the general surface. The smallest are $\frac{1}{00}$ to $\frac{1}{400}$ of an inch $(36$ to $62 \mu$ ) in length and are found chiefly on the face. The largest are on the palms of the hands, the soles of the feet and the nipple. These measure $\frac{1}{2} 50$ to $\frac{1}{200}$ of an inch (roo to $125 \mu$ ). Large papillæ, regularly arranged in a longitudinal direction, are found beneath the nails. The regular curved lines observed on the palms of the hands and the soles of the feet, particularly the palmar surfaces of the last phalanges, are formed by double rows of compound papillæ, which present two, three or four elevations attached to a single base. In the centre of each of these double rows of papillæ, is a fine and shallow groove, in which are found the orifices of the sudoriferous ducts.

The papillæ are abundantly supplied with bloodvessels terminating in looped capillary plexuses and with nerves. The termination of the nerves is peculiar and will be fully described in connection with the organs of touch. The arrangement of the lymphatics, which are very 
abundant in the skin, has already been indicated in the general description of the lymphatic system.

The Epidermis and its Appendages. - The epidermis, or external layer of the skin, is composed of cells. It has neither bloodvessels, nerves nor lymphatics. Its external surface is marked with shallow grooves, which correspond to the deep furrows between the papillæ of the derma. Its internal surface is applied directly to the papillary layer of the true skin and follows closely all its inequalities. This portion of the skin is subdivided into two layers. The internal layer is called the rete mucosum, or the Malpighian layer, and the external is called the horny layer. These layers present certain important distinctive characters.

The Malpighian layer is composed of a stratum of prismoidal nucleated cells, containing pigmentary matter, and a number of layers of rounded cells without pigment. The upper layers of cells are semitransparent and nearly colorless; and it is the pigmentary layer chiefly that gives to the skin its characteristic color. All the epidermic cells are somewhat colored in the dark races, but the upper layers contain no pigmentary granules. The thickness of the rete mucosum is $\frac{1}{170} \overline{0}$ to $\frac{1}{75}$ of an inch ( 15 to $333 \mu$ ).

The horny layer is composed of strata of hard flattened cells, irregularly polygonal in shape and usually without nuclei. The deeper cells are thicker and more rounded than those of the superficial layers, are polygonal in form and their borders present a great number of short protoplasmic spines. These are called prickle-cells. There is constantly more or less desquamation of the epidermis, particularly of the horny layer, and the cells are regenerated from the subjacent parts.

Physiological Anatomy of the Nails. - The nails are situated on the dorsal surfaces of the distal phalanges of the fingers and toes. They serve to protect these parts, and in the fingers, they are quite important in prehension. The general appearance of the nails is sufficiently familiar. In their description anatomists have distinguished a root, a body and a free border.

The root of the nail is thin and soft, terminating in rather a jagged edge, which is turned slightly upward and is received into a fold of the skin extending around the nail to its free edge. The length of the root varies with the size of the nail, but it usually is one-fourth to one-third of the length of the body.

The body of the nail extends from the fold of skin covering the root to the free border. This portion of the nail, with the root, is closely adherent by its under surface to the true skin. It is marked by fine but distinct longitudinal striæ and very faint transverse lines. It usually presents a reddish color on account of the great vascularity of the 
subjacent structure. At the posterior part, is a whitish portion, of a semilunar shape, called the lunula, which has this appearance simply from the fact that the corium in this part is less vascular and the papillæ are not so regular as in the rest of the body. The skin beneath the root and body of the nail is called the matrix. It presents highly vascular papillæ arranged in longitudinal rows, and it receives into its grooves corresponding ridges on the under surface of the nail.

On examining the nail in a longitudinal section, the horny layer is found to increase progressively in thickness from the root to near the free border. Examined in transverse section, the nail will also be found much thicker in the central portion than near the edge, and that part which is received into the lateral portions of the fold becomes thin like the rest of the root. The nail becomes thinner at and near the free border.

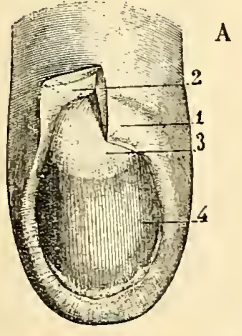

B

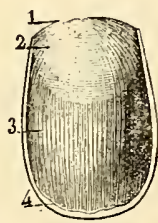

Fig. 65. - Anatomy of the nails (Sappey).
C

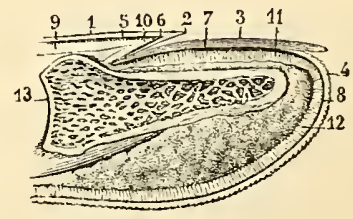

$A$, nail in situ: I, cutaneous fold covering the root of the nail ; 2 , section of this fold, turned back to show the root of the nail ; 3 , lunula; 4 , nail. $B$, concave or adherent surface of the nail: $I$, border of the root; 2 , lunula and root ; 3 , body; 4 , free border. $C$, longitudinal section of the nail: 1,2 , epidermis; 3 , superficial layer of the nail ; 4 , epidermis of the pulp of the finger ; 5,6 , true skin ; 7 , I I, bed of the nail; 8 , Malpighian layer of the pulp of the finger; 9 , Io, true skin on the dorsal surface of the finger; 12, true skin of the pulp of the finger; I3, last phalanx of the finger.

The two layers correspond to the Malpighian and the horny layers of the epidermis, although they are more distinct. The Malpighian layer is applied directly to the ridges of the bed of the nail and presents ridges much less strongly marked than those of the underlying true skin. This layer is rather thinner than the horny layer, is whitish in color and is composed of elongated prismoidal nucleated cells arranged perpendicularly to the matrix.

The horny layer - which constitutes the true nail - is applied by its under surface directly to the ridges of the Malpighian layer. It is composed of strata of flattened nucleated cells which can not be isolated without the use of reagents. After boiling in a dilute solution of sodium or potassium hydrate, it becomes evident that here, as in the horny layer of the epidermis, the lower cells are rounded and those nearer the surface are flattened. The thickness of this layer varies while that of the Malpighian layer is nearly uniform. This layer is constantly growing, and it constitutes the entire substance of the free borders of the nails. 
The connections of the nails with the true skin resemble those of the epidermis; but the relations of these structures to the epidermis itself are somewhat peculiar. Before the fourth month of fœtal life, the epidermis covering the dorsal surfaces of the last phalanges of the fingers and toes does not present any marked peculiarities; but at about the

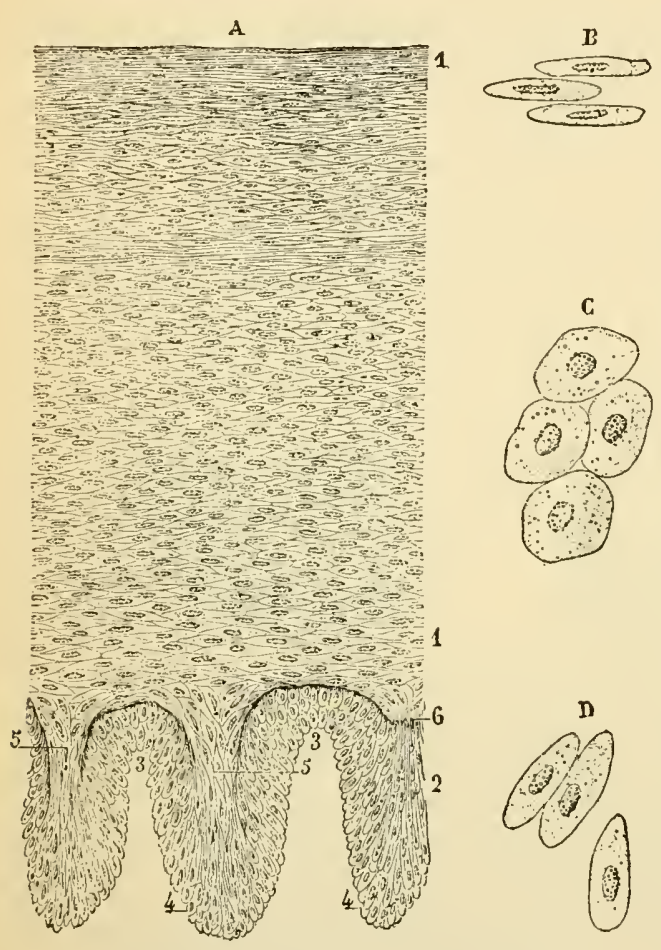

Fig. 66. - Section of the nail (Sappey).

$A$, section of the nail: $\mathrm{I}, \mathrm{I}$, superficial layer; 2 , deep laver; $3,3,4,4$, section of the grooves on the attached surface; 5,5 , union of the superficial with the deep layer; 6 , dark line between the two layers.

$B$, cells of the superficial layer, lateral view.

$C$, cells of the superficial layer, flat view.

$D$, cells of the deep layer.

fourth month, the hard cells of the horny layer of the nails make their appearance between the Malpighian and the horny layers of the epidermis, and at the same time the Malpighian layer beneath this plate, which is destined to become the Malpighian layer of the nails, is thickened and the cells assume a more elongated form. The horny layer of the nails constantly thickens from this time; but until the end of the fifth month, it is covered with the horny layer of the epidermis. After the fifth month, the epidermis breaks away and disappears from the surface; and at the seventh month, the nails begin to increase in length. Thus, at one time the nails are actually included between the two layers of the epidermis; but after they have become developed, they simply are covered at their roots by a narrow border of the horny layer. The nails are therefore to be regarded as modifications of the horny layer of the epidermis, possessing certain anatomical and chemical peculiarities. The Malpighian layer of the nails is continuous with the corresponding layer of the epidermis, but the horny layers are distinct.

Physiological Anatomy of the Hairs. - Hairs, varying greatly in size, cover nearly every portion of the cutaneous surface. The only parts in which they are not found are the palms of the hands and soles of the feet, the palmar surfaces of the fingers and toes, the dorsal surfaces of 
the last phalanges of the fingers and toes, the lips, the upper eyelids, the lining of the prepuce, and the glans penis. Some of the hairs are long, others are short and stiff, and others are fine and downy. These differences have led to a division of the hairs into three varieties:-

The first variety includes the long soft hairs, found on the head, on the face in the male adult, around the genital organs and under the arms in both the male and the female, and sometimes on the breast and over the general surface of the body and extremities, particularly in the male.

The second variety, the short stiff hairs, is found just within the nostrils, on the edges of the eyelids and on the eyebrows.

The third variety, the short, soft, downy hairs, is found on parts of the general surface not provided with the long hairs, and in the caruncula lachrymalis. In early life and ordinarily in the female at all ages, the trunk and extremities are covered with downy hairs; but in the adult male, these frequently become developed into long soft hairs.

The hairs usually are set obliquely in the skin and take a definite direction as they lie on the surface. On the head and face, and, indeed, the entire surface of the body, the general course of the hairs may be followed out; and they present currents or sweeps that have nearly always the same directions.

The diameter and length of the hairs are variable in different persons, especially in the long soft hairs of the head and beard. It may be stated in general terms that the long hairs attain the length of twenty inches to three feet ( 500 to 900 millimeters) in women, and considerably less in men. Like the nails, the hair, when left to itself, attains in three or four years a definite length, but when it is habitually cut it grows constantly. The short stiff hairs are $\frac{1}{4}$ to $\frac{1}{2}$ of an inch (6.4 to I2.7 millimeters) in length. The soft downy hairs measure ordinarily $\frac{1}{12}$ to $\frac{1}{2}$ of an inch (2.I to I 2.7 millimeters) in length.

Of the long hairs, the finest are on the head, where they average about $\frac{1}{400}$ of an inch $(64 \mu)$ in diameter. The hair ordinarily is coarser in women than in men. Dark hair usually is coarser than light hair; and on the same head the extremes of variation are sometimes observed. The hairs of the beard and the long hairs of the body are coarser than the hairs of the head. The average number of hairs on a square inch of the scalp is about 1000 ( 155 in a square centimeter) and the number on the head, about I 20,000 (Wilson).

In a normal condition the hairs are elastic and may be stretched to one-fifth or one-third more than their original length. Their strength varies with their thickness, but an ordinary hair from the head will bear a weight of six to seven ounces (I 70 to 200 grams). A well-known property of the hair is that of becoming strongly electric by friction; and this 
is particularly marked when the weather is cold and dry. The electricity thus excited is negative. Sections of the shaft of the hairs show that

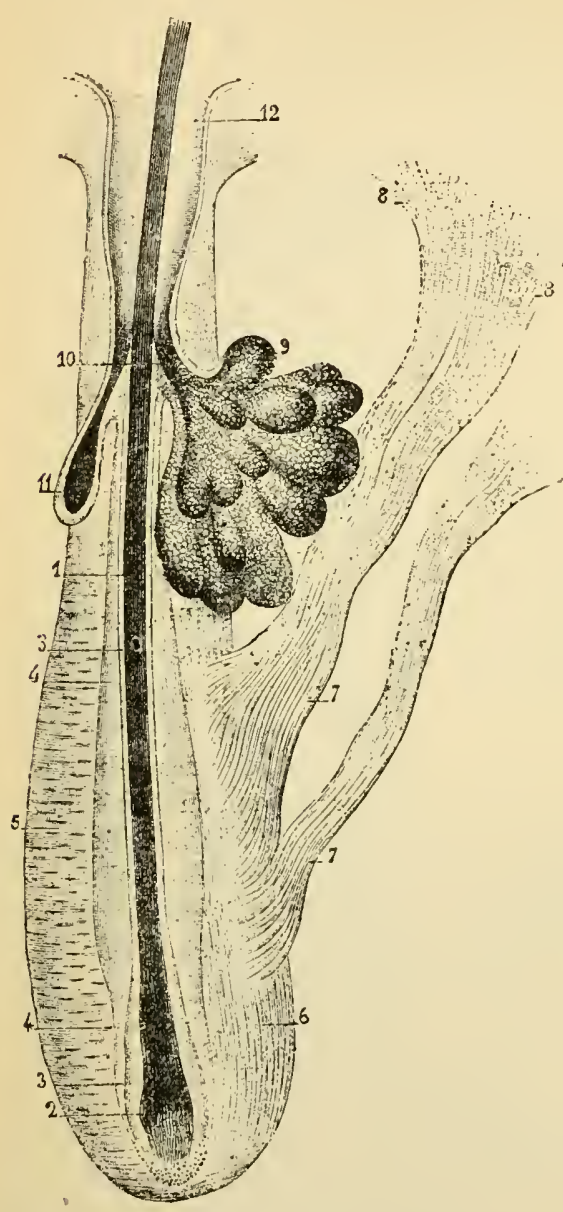

Fig. 67. - Hair and hair-follicle (Sappey).

I, root of the hair; 2 , bulb of the hair; 3 , internal root-sheath ; 4 , external root-sheath ; 5 , membrane of the hair-follicle (the internal, amorphous nembrane of the follicle is very delicate and is not represented in the figure); 6 , external membrane of the follicle; 7,7 , muscular bands attached to the follicle; 8,8 , extremities of these bands passing to the skin; 9 , compound sebaccous gland, with its duct (10) opening into the upper third of the follicle; II, simple sebaceous gland; I2, opening of the hair-follicle. of the follicles is composed of inelastic fibres usually arranged longitudinally. It is provided with bloodvessels, a few nerves and some connective-tissue elements, but no elastic tissue. This is the thickest

they are oval, but their shape is variable, straight hairs being nearly round while curled hairs are quite flat. Another peculiarity of the hairs is that they are hygrometric. They readily absorb moisture and become sensibly elongated, a property that has been made use of by physicists in the construction of hygrometers.

Roots of the Hairs, and Hairfollicles. - The roots of the hairs are embedded in follicular openings in the skin, which differ in the different varieties only in the depth to which they penetrate the cutaneous structure. In the downy hairs, the roots pass into the superficial layers only of the true skin; but in the thicker hairs, the roots pass through the skin and penetrate the subcutaneous cellulo-adipose tissue.

The root of the hair is softer, rounder and a little larger than the shaft. It becomes enlarged into a rounded bulb at the bottom of the follicle, and rests on a fungiform papilla, constricted at its base, to which the hair is closely attached.

The hair-follicles are tubular inversions of the structures that compose the corium, and their walls present three membranes. Their length is $\frac{1}{12}$ to $\frac{1}{4}$ of an inch (2. I to 6.4 millimeters). The membrane that forms the external coat 
of the three membranes and is closely connected with the corium. Next to this, is a fibrous membrane composed of fusiform nucleated fibres arranged transversely. These resemble non-striated muscular fibres. The internal membrane is structureless and corresponds to the amorphous layer of the true skin. The papilla at the bottom of the hair-sac varies in size with the size of the hairs and is connected with the fibrous layers of the walls of the follicle. It is composed of amorphous matter, with a few granules and nuclei, and it probably contains bloodvessels and nerves, although these are not very distinct.

The investment of the root of the hair presents two distinct layers called the external and internal root-sheaths. The external root-sheath is three or four times as thick as the inner membrane, and it corresponds exactly to the Malpighian layer of the epidermis. This sheath is continuous with the bulb of the hair. The internal root-sheath is a transparent membrane composed of flattened cells, usually without nuclei. This extends from the bottom of the hair-follicle and covers the lower two-thirds of the root (see Plate VI).

Structure of the Hairs. - The different varieties of hairs present certain peculiarities in their anatomy, but they are all composed of a fibrous structure. forming the greater part of their substance, covered by a thin layer of imbricated cells. In the short stiff hairs and in the long white hairs, there is a distinct medullary substance; but this is wanting in the downy hairs and is indistinct in many of the long dark hairs.

The fibrous substance of the hairs is composed of hard, elongated, longitudinal fibres, which can not be isolated without the aid of reagents. They may be separated, however, by maceration in warm sulphuric acid, when they

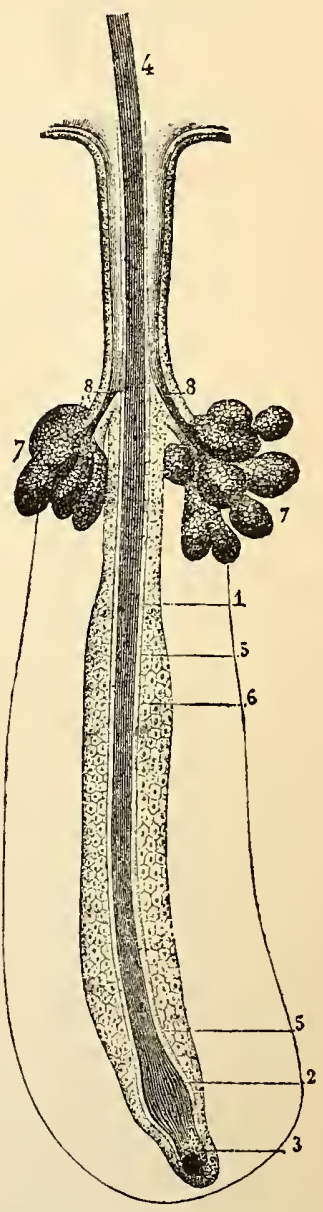

Fig. 68. - Root of the hair (Sappey).

I, root of the hair; 2 , hairbulb; 3 , papilla of the follicle ; 4 , opening of the follicle; 5,5 , internal root-sheath; 6 , external root-sheath ; 7,7 , sebaceous glands; 8,8 , excretory ducts of the sebaceous glands. present themselves in the form of dark irregular spindle-shaped plates. These contain pigmentary matter of various shades of color, occasional cavities filled with air and a few nuclei. The pigment may be of any shade between a light yellow and an intense black; and it is this sub- 
stance that gives to the hair the variety in color observed in different persons. In the lower part of the root the fibres are much shorter, and at the bulb they are transformed into the soft rounded cells found in this situation and covering the papilla.

The epidermis of the hair is thin and is composed of flattened quadrangular plates, overlying each other from below upward. These scales, or plates, are without nuclei, and they exist in a single layer over the shaft of the hair and the upper part of its root; but in the lower part of the root, the cells are thicker, softer, frequently are nucleated, and they exist in two layers.

The medulla is found in the short stiff hairs, and it is often quite distinct in the long white hairs of the head. It occupies one-fourth to one-third of the diameter of the hair. The medulla can be traced from just above the bulb to near the pointed extremity of the hair. It is composed of small, rounded, nucleated cells, which often contain dark granules of pigmentary matter. Mixed with these cells are airglobules; and frequently the cells are interrupted for a short distance and the space is filled with air. The medulla likewise contains a glutinous substance between the cells and surrounding the air-globules (see Plate VII, Fig. I, for transverse sections of hairs).

Growith of the Hairs. - Although not provided with blood and devoid of sensibility, the hairs are connected with vascular parts and are nourished by imbibition from the papillæ. Each hair is first developed in a closed sac, and at about the sixth month of intra-uterine life, its pointed extremity perforates the epidermis. These first-formed hairs are afterward shed, like the milk-teeth, being pushed out by new hairs from below, which latter arise from a second and more deeply seated papilla. The shedding of the hairs usually takes place, between the second and the eighth month after birth.

The differences in the color of the hair depend on differences in the quantity and the tint of the pigmentary matter ; and in old age the hair becomes white or gray from a blanching of the cortex and medulla.

Sudden Blanching of the Hair. - There are a few instances on record in which sudden blanching of the hair has been observed and the causes of this remarkable change fully investigated. One of these cases has been reported by Landois. In this instance the blanching of the hair occurred in a hospital in a single night, while the patient, who had an acute attack of delirium tremens, was under the daily observation of the visiting physician. The microscopical examinations by Landois and others leave no doubt as to the cause of the white color of the hair in cases of sudden blanching; and the fact of the occurrence of this phenomenon can no longer be called in question. All are agreed 
that there is no diminution in the pigment, but that the greater part of the medulla becomes filled with air, small globules being also found in the cortical substance. The hair in these cases presents a marked contrast with hair that has gradually become gray from old age, when there is always a loss of pigment in the cortex and medulla. How the air finds its way into the hair in sudden blanching, it is difficult to understand; and the views that have been expressed on this subject by different authors are entirely theoretical.

The fact that the hair may become white or gray in the course of a few hours renders it probable that many of the cases reported on unscientific authority actually occurred; and these have all been supposed to be connected with intense grief or terror. The terror was very marked in the case reported by Landois. In the great majority of recorded observations, the sudden blanching of the hair has been apparently connected with intense mental emotion; but this is all that can be said on the subject of causation, and the mechanism of the change is not understood.

Uses of the Hair. - The hairs serve an important purpose in the protection of the general surface and in guarding certain of the orifices of the body. The hair on the head and face protects from cold and shields the head from the rays of the sun during exposure in hot climates. Although the quantity of hair on the general surface is small, as it is an imperfect conductor of caloric, it serves in a degree to maintain the heat of the body. It also moderates the friction upon the surface. The eyebrows prevent the perspiration from running from the forehead upon the lids; the eyelashes protect the surface of the conjunctiva from dust and other foreign matters; the mustache protects the lungs from dust, which is very important in persons exposed to dust in long journeys or in their daily work; and the short stiff hairs at the openings of the ears and nose protect these orifices. It is difficult to assign any special office to the hairs in some other situations, but their general uses are sufficiently evident.

\section{Perspiration}

In the fullest acceptation of the term, perspiration embraces the entire action of the skin as an excreting organ and includes the exhalation of carbon dioxide as well as of watery vapor and organic matters. The office of the skin as an eliminator is important; but the quantity of excrementitious matters with the properties of which physiologists are well acquainted, such as carbon dioxide and urea, thrown off from the general surface, is small as compared with what is exhaled by the lungs and discharged by the kidneys. If the surface of the body is covered 
with an impermeable coating, death occurs in a very short time, with great reduction in the body-temperature due to excessive radiation from the varnished surface, the temperature being reduced to about $70^{\circ} \mathrm{Fahr}$. $\left(2 \mathrm{I}^{\circ} \mathrm{C}\right.$. $)$. Warm-blooded animals die usually when more than one-half of the general surface has been varnished. Rabbits die when onefourth of the surface has been covered with an impermeable coating (Laschkewitsch). Valentin and Laschkewitsch found that when the temperature was kept at about the normal standard by artificial means, no morbid symptoms were developed.

Sudoriparous Glands. - With few exceptions, all parts of the skin are provided with sudoriparous glands. They are not found, however, in the skin covering the concave surface of the concha of the ear, the glans penis, the inner lamella of the prepuce and, unless the ceruminous

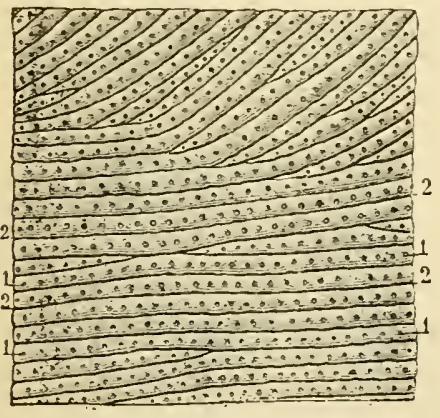

Fig. 69. - Surfuce of the palm of the hand, a portion of the skin about one-half an inch (12.5 millimeters) square, $\times 3^{\frac{2}{4}}$ (Sappey).

I, I, I, I, openings of the sudoriferous ducts; 2, 2, 2, 2, grooves between the papillæe of the skin. glands are regarded as sudoriparous organs, in the external auditory meatus.

On examining the surface of the skin with a low magnifying power, especially on the palms of the hands and the soles of the feet, the openings of the sudoriferous ducts may be seen in the middle of the papillary ridges, forming a regular line in the shallow groove between the two rows of papillæ. The tubes always open on the surface obliquely. In a thin section of the skin, the ducts are seen passing through the different layers and terminating in rounded convoluted coils in the subcutaneous structure. These little rounded or ovoid bodies, which are the sudoriparous, or sweat-producing structures, may be seen attached to the under surface of the skin after it has been removed from the subjacent parts by maceration. A perspiratory gland consists, indeed, of a simple tube, presenting a coiled mass, the sudoriparous portion, beneath the skin, and a tube of greater or less length in proportion to the thickness of the cutaneous layers, which is the excretory duct, or the sudoriferous portion.

The glandular coils are $\frac{1}{12} 5$ to $\frac{1}{25}$ of an inch (0.2 to I millimeter) in diameter ; the smallest coils being found beneath the skin of the penis, the scrotum, the eyelids, the nose and the convex surface of the concha of the ear, and the largest, on the areola of the nipple and on the perineum. Very large glands are found mixed with smaller glands in the axilla, and these produce a peculiar secretion. The coiled portion of the tube is 
about $\frac{1}{370}$ of an inch ( 0.07 millimeter) in diameter, and presents six to twelve turns. It consists of a sharply-defined strong external membrane, which is very transparent, uniformly granular and sometimes indistinctly striated. The tube is of uniform diameter throughout the coil and terminates in a very slightly-dilated, rounded, blind extremity. It is filled with epithelium in the form of finely-granular matter, usually not segmented into cells, and is provided with small oval nuclei. The glandular mass is surrounded with a plexus of capillary bloodvessels, which send a few small branches between the convolutions of the coil. Sometimes the coil is enclosed in a delicate fibrous envelope (see Plate VII, Fig. 2).

The excretory duct is simply a continuation of the glandular coil. Its course through the layers of the true skin is nearly straight. It then passes into the epidermis, between the papillæ of the corium, and presents, in this layer, a number of spiral turns. The spirals vary in number according to the thickness of the epidermis. Six to ten are found in the palms of the hands, and twelve to fifteen in the soles of the feet. As it emerges from the glandular coil, the excretory duct is somewhat narrower than the tube in the secreting portion; but as it passes through the epidermis it again becomes larger. It possesses the same external membrane as the glandular coil and is lined usually by two layers of cells.

In a section of the skin and the subcutaneous tissue, involving several of the sudoriparous glands with their ducts, it is seen that the glandular coils nearly always are situated at different planes beneath the skin.

Sudoriparous glands in the axilla have been described which do not differ so much from the glands in other parts in their anatomy as in the character of their secretion. The coil in these glands is much larger than in other parts, measuring $\frac{1}{25}$ to $\frac{1}{12}$ of an inch (I to 2 millimeters); the walls of the tube are thicker, and they present an investment of fibrous tissue with an internal layer of longitudinal non-striated muscular fibres; and finally, the tubes of the coil itself are lined with cells of epithelium. These glands are abundant in the axilla, forming a continuous layer beneath the skin. Mixed with these are a few glands of the ordinary variety.

Estimates have been made of the number of sudoriparous glands in the body and the probable extent of the exhalant surface of the skin, but they are to be taken as approximate. Krause found great differences in the number of perspiratory openings in different portions of the skin; but taking an average for the entire surface, it was estimated that the entire number of perspiratory glands is $2,38 \mathrm{I}, 248$; and assuming 
that each coil when unravelled measures about $\frac{1}{16}$ of an inch (1.8 millimeter), the entire length of the secreting tubes is about $2 \frac{1}{3}$ miles $\left(3 \frac{3}{4}\right.$ kilometers). It must be remembered, however, that the length of the secreting coil only is given and that the excretory ducts are not included.

Mechanism of the Secretion of Sweat. - The action of the skin as a glandular organ is continuous and not intermittent; but under ordinary conditions the sweat is exhaled from the general surface in the form of vapor. In regard to the mechanism of its separation from the blood, nothing is to be said in addition to the general remarks on the subject of secretion; and it is probable that the epithelium of the secreting coils is the active agent in the selection of the peculiar matters which enter into its composition. There are no examples of the separation by glandular organs of vapor from the blood, and the perspiration is secreted as a liquid, which becomes vaporous as it is discharged upon the surface.

The influence of the nervous system on the secretion of sweat is important. It is well known, for example, that an abundant production of perspiration frequently is the result of mental emotions. Bernard has shown that the nervous influence may be exerted through the sympathetic system. He divided the sympathetic in the neck of a horse, producing as a consequence an elevation in temperature and an increase in the arterial pressure in the part supplied with branches of the nerve. He found, also, that the skin of the part became covered with a copious perspiration. On stimulating the divided extremity of the nerve, the secretion of sweat was arrested. The local secretion of sweat after division of the sympathetic in the neck of the horse was first observed by Dupuy in I 816 .

The stimulation as well as the division of certain nerves induces local secretion of sweat, but this nearly always is associated with dilatation of the bloodvessels of the part; still, sweat frequently is secreted when the surface is pale and bloodless, showing that dilatation of the bloodvessels is not an indispensable condition. The action of so-called vaso-dilator nerves will be treated of in connection with the physiology of the nervous system. In experiments on the cat, excito-secretory fibres have been found to exist in the cerebro-spinal nerves going to the anterior extremities. The fibres for the posterior extremities are in the sheath of the sciatic nerve. In all instances the action of these nerves is direct and not reflex. Experiments on the cat have been quite satisfactory, as this animal sweats only on the soles of the feet and the secretion can readily be observed.

The so-called sweat-centres are in the lower part of the dorsal region of the spinal cord, for the posterior extremities, and in the lower part of 
the cervical region of the cord, for the anterior extremities. According to Adamkiewicz, these centres are subordinate to the principal sweatcentre, which is situated in the bulb. Ott has collected a number of cases of disease of the cord in the human subject which go far to confirm the results of experiments on the inferior animals in regard to the action of excito-secretory nerves and sweat-centres.

When the skin is in a normal condition, after exercise or whenever there is a tendency to elevation of the animal temperature, there is a determination of blood to the surface, accompanied with an increase in the secretion of sweat. This is the case when the body is exposed to a high temperature; and it is by an increase in the transpiration from the surface that the heat of the body is kept down to a normal standard.

Quantity of Cutaneous Exhalation. - The quantity of cutaneous exhalation is subject to variations, depending on conditions of temperature and moisture, exercise, the quantity and character of the ingesta etc. Most of these variations relate to the action of the skin in regulating the temperature of the body; and it is probable that the elimination of excrementitious matters by the skin is not subject, under normal conditions, to the same modifications, although experiments on this point are wanting. When there is such a wide range of variation in different individuals and in the same person under different conditions of season, climate etc., it is possible to give only approximate estimates of the quantity of sweat secreted and exhaled in the twenty-four hours. It is assumed, however, that the average quantity is nearly two pounds, or about 900 grams.

Under violent and prolonged exercise, the loss of weight by exhalation from the skin and lungs may become very considerable. It is stated by Maclaren, the author of a work on training, that in one hour's energetic fencing, the loss by perspiration and respiration, taking the average of six consecutive days, was forty ounces (I 30 grams), with a range of variation of eight ounces (227 grams).

When the body is exposed to a high temperature, the exhalation from the surface is largely increased; and it is by this rapid evaporation that persons have been able to endure for several minutes a dry heat considerably exceeding that of boiling water. Southwood Smith made a series of observations in regard to this point on men employed about the furnaces of gas-works and exposed to intense heat; and he found that in an hour the loss of weight was two to four pounds ( 907 to I8I4 grams), chiefly by exhalation of watery vapor from the skin. In such instances the loss of water by transpiration is compensated by the ingestion of large quantities of liquid. 
Properties and Composition of the Sweat. - An analysis of the sweat was made by Favre in I853. After taking every precaution to obtain the secretion in a perfectly pure state, he collected a very large quantity, nearly thirty pints ( 14 liters), the result of six transpirations from one person, which he assumed to represent about the average in composition. The liquid was perfectly limpid, colorless, and of a feeble but characteristic odor. Almost all observers have found the reaction of the sweat to be acid; but it readily becomes alkaline on being subjected to evaporation, showing that it contains some of the volatile acids. Favre found that the liquid collected during the first half-hour of the observation was acid; during the second half-hour it was neutral or feebly alkaline; and during the third half-hour, it was constantly alkaline. The specific gravity of the sweat is 1003 to 1004 .

\section{COMPOSITION OF THE SWEAT (FAVRE)}

\begin{tabular}{|c|c|c|c|c|c|c|c|c|}
\hline Water & & • & - & . & . & . & 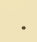 & . 99.5573 \\
\hline Urea . & & . & 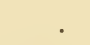 & . & . & . & . & $\quad 0.043$ \\
\hline Fatty matters & & . & . & . & . & & & 0.014 \\
\hline Alkaline lactates. & & . & . & . & . & & & 0.317 \\
\hline Alkaline sudorates & . & - & $\cdot$ & . & • & & & 1.562 \\
\hline Sodium chloride. & & . & 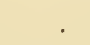 & . & . & & & 2.230 \\
\hline Potassium chloride. & & & & . & . & & & 0.244 \\
\hline Alkaline sulphates, & solub & ble in & water & . & . & & & 0.012 \\
\hline Alkaline phosphates. & . & . & $\cdot$ & . & - & & & a trace \\
\hline Alkaline albuminates, & & . & . & . & . & & & 0.005 \\
\hline Alkaline earthy phospl & nates ( & (solub & & idulated & d water) & & & - a trace \\
\hline Epidermic débris (ins & luble) & ) & . & . & . & . & • & . a trace \\
\hline
\end{tabular}

The sweat is exhaled usually in the form of vapor, when it is known as insensible perspiration. When from any cause it collects on the surface, in the form of a liquid, it is called sensible perspiration.

The peculiar constituents of the sweat are the following: The neutral fats probably are derived in great part from the sebaceous glands, although certain fats, palmitin and stearin, have been found in the secretion of the palms of the hands, which contain no sebaceous glands. The volatile fatty acids are formic, butyric, caproic, capric, acetic etc., some of which exist also in milk. These give to the sweat its peculiar odor. Urea is always present in small quantity, and its proportion may be largely increased when there is a deficiency in elimination by the kidneys. It is a matter, also, of common as well as of scientific observation that the sweat is more abundant when the kidneys are comparatively inactive, and vice versâ. Usually, how- 
ever, when conditions operate to increase the quantity of sweat, the quantity of urine is proportionally diminished. The skin is undoubtedly an important organ of excretion, and it may eliminate excrementitious matters of a character as yet unknown. In regard to the inorganic constituents of the sweat, there is no great interest attached to any but the sodium chloride, which exists in a proportion many times greater than that of all the other inorganic salts combined.

Peculiarities of the Sweat in Certain Parts. - In the axilla, the inguino-scrotal region in the male and the inguino-vulvar region in the female, and between the toes, the sweat always has a peculiar odor, more or less marked, which in some persons is excessively disagreeable. Whenever the secretion has an odor of this kind its reaction is distinctly alkaline; and its peculiar characters are due to a mixture of the secretion of the other follicles found in these situations. Sometimes the sweat about the nose has an alkaline reaction. In the axillary region the secretion frequently has a yellowish color, which may stain the clothing. 


\title{
CHAPTER XIII
}

\author{
EXCRETION BY THE KIDNEYS
}

Physiological anatomy of the kidneys - Pyramidal substance - Cortical substance - Tubes of the cortical substance - Narrow tubes of Henle - Distribution of bloodvessels in the kidney - Mechanism of the production and discharge of urine - Influence of blood-pressure, the nervous system etc., on the secretion of urine - Physiological anatomy of the urinary passages - Mechanism of the discharge of urine - Properties and composition of the urine - Urea - Origin of urea - Influence of the ingesta on the composition of the urine and on the discharge of nitrogen - Influence of muscular exercise on the discharge of nitrogen Uric acid and its compounds - Hippuric acid, hippurates and lactates - Calcium oxalate - Xanthin, hypoxanthin, leucin, tyrosin and taurin - Fatty matters - Inorganic constituents of the urine - Chlorides - Sulphates - Phosphates - Water as a product of excretion - Variations in the composition of the urine - Variations with age and sex - Influence of mental exertion - Internal secretion - Work of the kidneys.

\section{Physiological Anatomy of the Kidneys}

THE kidneys are symmetrical organs, situated in the lumbar region, beneath the peritoneum, invested with a proper fibrous coat, called the capsule, and always surrounded with more or less adipose tissue. They extend usually from the eleventh or twelfth rib downward to near the crest of the ilium, and the right is always a little lower than the left. In shape the kidney is appropriately compared to a bean; and the concavity - the deep, central portion of which is called the hilum - looks inward toward the spinal column. The weight of each kidney is four to six ounces (I 3 to I 70 grams), usually about half an ounce (I 4 grams) less in the female than in the male. The left kidney is nearly always a little heavier than the right.

External to the proper coat of the kidney, is a certain quantity of adipose tissue enclosed in a loose fibrous structure. This is sometimes called the adipose capsule; but the proper coat consists of a close network of ordinary fibrous tissue, interlacing with small elastic fibres. This coat is thin and smooth and may readily be removed from the surface of the organ. At the hilum it is continued inward to line the pelvis of the kidney, covering the calices and bloodvessels.

The kidney in a vertical section presents a cavity at the hilum, bounded internally by the dilated origin of the ureter. This is called the pelvis. It is lined with a smooth membrane, which is simply a continuation of the proper coat of the kidney and which forms little cylinders, called calices, into which the apices of the pyramids are 
received. Some of the calices receive the apex of a single pyramid, while others are larger and receive two or three. The calices unite into three short, funnel-shaped tubes, called infundibula, corresponding respectively to the superior, middle and inferior portions of the kidney. These finally open into the common cavity, the pelvis. The substance of the kidney is composed of two distinct portions, called respectively the cortical substance and the medullary, or pyramidal substance.

The cortical substance is reddish and granular, rather softer than the pyramidal substance, and is about one-sixth of an inch ( 4.2 millimeters) in thickness. This occupies the exterior of the kidney and sends little prolongations, called the columns of Bertin, between the pyramids. The surface of the kidney is marked by little polygonal divisions, giving it a lobulated appearance. This, however, is due mainly to the arrangement of the superficial bloodvessels. The medullary substance is arranged in the form of pyramids, sometimes called the pyramids of Malpighi, twelve, fifteen or eighteen in number, their bases presenting toward the cortical substance and their apices being received into the calices, at the pelvis. Ferrein subdivided the pyramids of Malpighi into smaller pyramids, called the pyramids of Ferrein, each containing about one hundred tubes radiat-

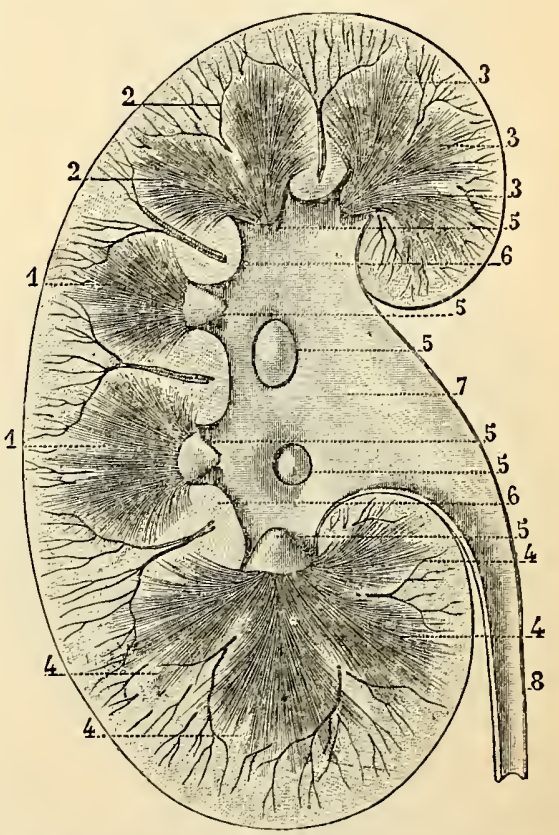

Fig. 70. - Longitudinal section of the kidney (Sappey).

I, I, 2, 2, 3, 3, 3, 4, 4, 4, 4, pyramids of Malpighi $: 5,5,5,5,5,5$, apices of the pyramids, surrounded by the calices; 6,6 , columns of Bertin; 7 , pelvis of the kidney; 8 , upper extremity of the ureter. ing from the openings at the apices of the pyramids toward their bases. The tubes composing these pyramids pass into the cortical substance, forming corresponding pyramids of convoluted tubes, thus dividing this portion of the kidney into lobules more or less distinct.

The medullary substance is firm, of a darker red color than the cortical substance, and is marked by tolerably distinct strix that take a nearly straight course from the bases to the apices of the pyramids. As these strix indicate the direction of the tubes that constitute the greatest part of the medullary substance, this is sometimes called the tubular portion of the kidney. 
From the arrangement of the secreting portion of the kidneys, these organs are classed as tubular glands, presenting a system of tubes, or canals, some of which are supposed simply to carry off the urine, while others separate the excrementitious constituents of the urine from the blood. It is difficult to determine precisely where the secreting tubes

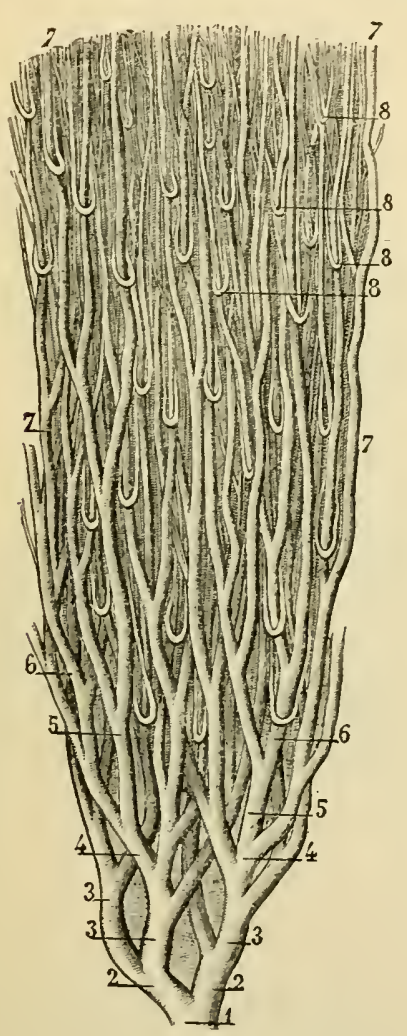

Fig. 7I. - Longitudinal section of the pyramidal substance of the kidney of the foctus (Sappey).

I, trunk of a large uriniferous tube; 2,2 , primary branches of this tube; $3,3,3$, secondary branches; $4,4,5,5,6,6,7,7,7,7$, branches becoming snuller and smaller; $8,8,8,8$, loops of the tubes of Henle.

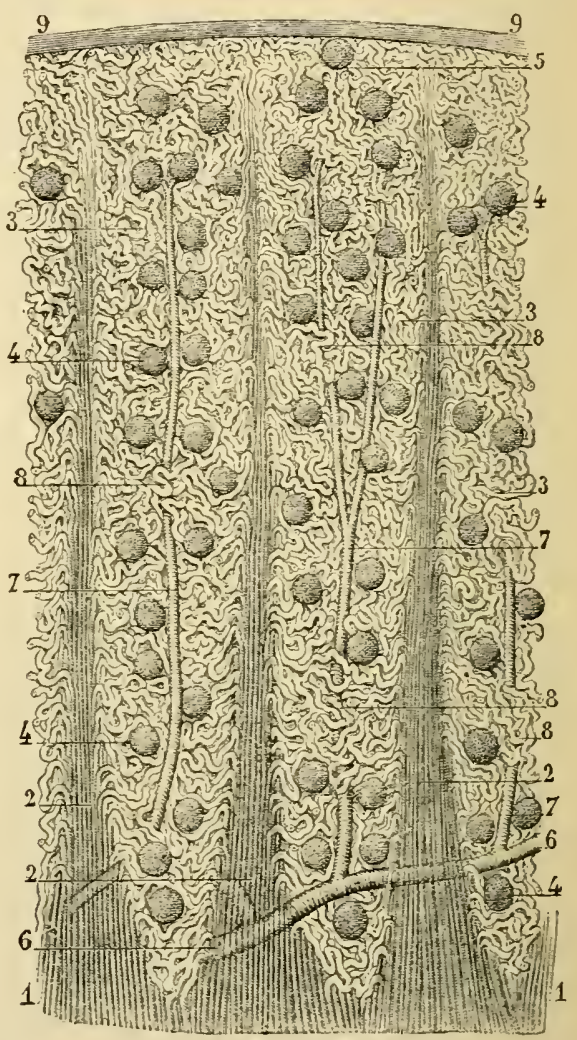

Fig. 72. - Longitudinal section of the cortical substance of the same kidney (Sappey).

I, I, limit of the cortical substance and base of the pyramids; $2,2,2$, tubes passing toward the surface of the kidney; $3,3,3,8,8,8$, convoluted tubes; $4,4,4,4,5$. Malpighian bodies; 6,6 , al tery, with its branches $(7,7,7) ; 9,9$, fibrous covering of the kidney.

merge into the excretory ducts; but it is a common idea, which probably is correct, that the cortical substance is the active portion, while the tubes of the pyramidal portion simply carry off the excretion.

Pyramidal Substance. - Each papilla, as it projects into the pelvis of the kidney, presents ten to twenty-five openings, $\frac{1}{300}$ to $\frac{1}{60}$ of an inch $(85$ to $425 \mu$ ) in diameter. The tubes leading from the pelvis immedi- 
ately divide at very acute angles, usually dichotomously, until a bundle of tubes arises, as it were, from each opening. These bundles constitute the pyramids of Ferrein. In their course the tubes are slightly wavy and are nearly parallel with each other. These are called the straight (collecting) tubes of the kidney, or the tubes of Bellini. They extend from the apices of the pyramids to their bases and pass then into the cortical substance. The pyramids contain, in addition to the straight tubes, a delicate fibrous matrix and bloodvessels, which latter usually pass beyond the pyramids, to be finally distributed in the cortical substance. Small tubes, continuous with the convoluted tubes of the cortical substance, dip down into the pyramids, returning to the cortical substance in the form of loops.

The tubes of the pyramidal substance are composed of a strong structureless basementmembrane lined with granular nucleated cells. They measure $\frac{1}{300}$ to $\frac{1}{200}$ of an inch ( 85 to I $27 \mu$ ) in diameter at the apices, and near the bases of the pyramids their diameter is about $\frac{1}{60} \overline{0}$ of an inch $(42 \mu)$.

The cells lining the straight tubes exist in a single layer applied to the basement-membrane. They are thick and irregularly polygonal in shape, with abundant albuminous granules. They

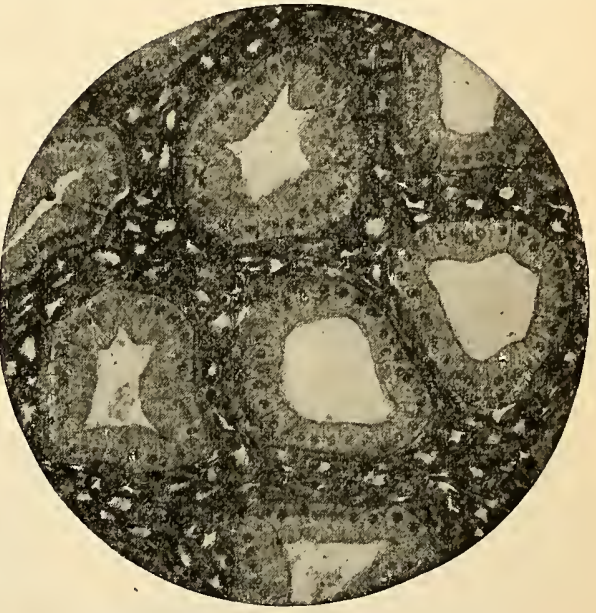

Fig. 73. - Collecting tubes of the kidney, $\times 130$, hematoxylin and eosin (Author's collection).

present one, and occasionally, though rarely, two granular nuclei, with one or two nucleoli. They readily undergo alteration and are seen in their normal condition only in a perfectly fresh healthy kidney. Their diameter is about $\frac{1}{1500}$ of an inch $(17 \mu)$. The calibre of the tubes is reduced by the thickness of their lining epithelium to $\frac{1}{900}$ or $\frac{1}{800}$ of an inch $(28$ or $30 \mu)$.

Cortical Substance. - In the cortical portion of the kidney are found tubes, differing somewhat from the tubes of the pyramidal portion in their size and in the character of their epithelial lining, and presenting a marked difference in their direction. These tubes are rather larger than the tubes of the pyramidal substance, and are convoluted, interlacing with each other in every direction. Scattered pretty uniformly throughout this portion of the kidney, are rounded or ovoid bodies, about four times the diameter of the convoluted tubes, known as the 
Malpighian bodies. These are simply flask-like terminal dilatations of the tubes themselves (see Plate VII, Fig. 4).

The cortical portion of the kidney presents a delicate fibrous matrix, which forms a support for the secreting portion and its bloodvessels. The tubes of the cortical substance present considerable differences in size, and three well-defined varieties can be distinguished:-

I. The first convoluted tubes, directly connected with the Malpighian bodies. 2. Small tubes, continuous with the convoluted tubes, dipping down into the pyramids and returning to the cortical portion in the form of loops. 3. The second convoluted tubes, forming a plexus connecting the different kinds of tubes with each other and finally with the straight tubes of the pyramidal portion.

In tracing out the course of the tubes, it will be found most convenient to begin with a description of the Malpighian bodies and to follow the tubes from these bodies to their connections with the straight tubes of the pyramidal substance.

Malpighian Bodies. - These are ovoid or rounded terminal dilatations of the first convoluted tubes and are $\frac{1}{250}$ to $\frac{1}{100}$ of an inch (IOO to $250 \mu$ ) in diameter. They are composed of a membrane, which is continuous with the external membrane of the convoluted tubes, and is of the same homogeneous character, but somewhat thicker. This envelope, called the capsule of Müller or of Bowman, encloses a mass of convoluted bloodvessels and is lined with a layer of nucleated epithelial cells. In addition to the cells lining the capsule, there are other cells, which are applied to the bloodvessels.

The cells attached to the capsule of Müller are smaller and more transparent than those lining the convoluted tubes. They are ovoid, nucleated and finely granular. The cells covering the vessels, however, are larger and more opaque, and they resemble the epithelium lining the tubes. They measure $\frac{1}{1+00}$ to $\frac{1}{1000}$ of an inch (I6 to $25 \mu$,) in diameter, by about $\frac{1}{2500}$ of an inch ( $10 \mu$ ) in thickness.

Tubes of the Cortical Substance. - Passing from the Malpighian bodies the tubes present first a short constricted portion, called the neck of the capsule, which soon dilates to the diameter of about $\frac{1}{500}$ of an inch $(5 \circ \mu)$, when their course becomes quite intricate and convoluted. These are what are known as the first convoluted tubes. The membrane of these tubes is transparent and homogeneous, but quite firm and resisting. It is lined throughout with a single layer of epithelial cells, $\frac{1}{1+00}$ to $\frac{1}{1000}$ of an inch ( 16 to $25 \mu$ ) in diameter, somewhat larger, therefore, than the cells lining the straight tubes. The cells lining the convoluted tubes present two tolerably distinct portions. The inner portion or zone, which is next the lumen of the tube, is finely granular, 
with sometimes a few small oil-globules. The outer zone presents little fibrils or rods, which are perpendicular to the tubular membrane. These are called "rodded" cells, and a similar appearance is presented by

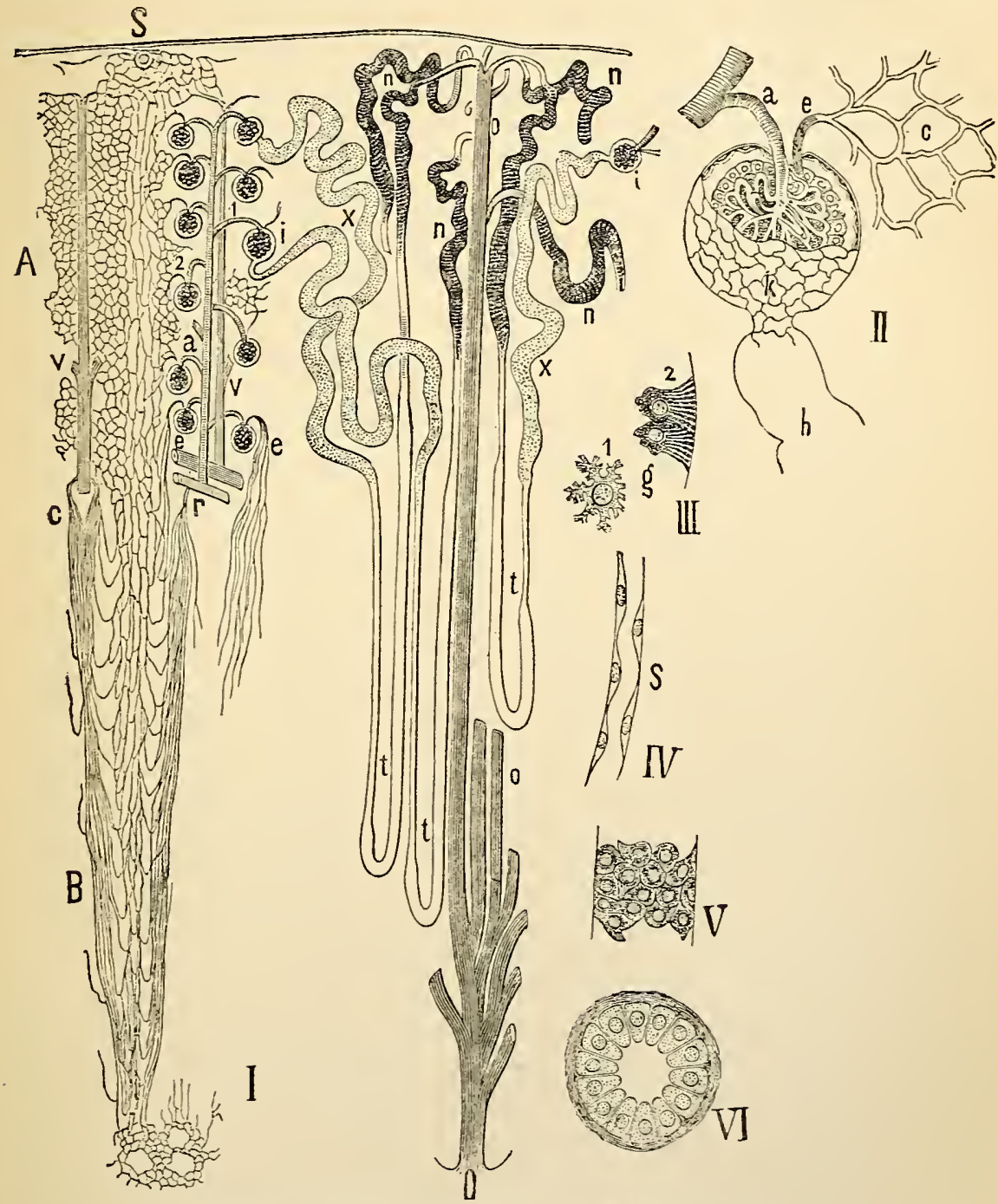

Fig. 74. - Diagram of the structure of the kidney' (Landois).

I. Bloodvessels and tubes (semidiagrammatic) : $A$, capillaries of the cortical substance; $B$, capillaries of the medullary substance; I, artery penetrating a Malpighian body; 2 , vein emerging from a Mlalpighian body; $R$, arteriolæ rectæ; $\dot{C}$, venæ rectæ; $l, l$, interlobular veins; $S$, stellate veins; I, I, capsules of Müller; $X, X$, first convoluted tubes; $T, T, T$, tubes of Henle; $N, \lambda, \Lambda, \Lambda$, second convoluted tubes; $O, O$, straight tubes; $O$, opening into the pelvis of the kidney. II. Malpighian body: $A$, artery; $E$, vein; $C$, capiliaries; $K$, epithelium of the capsule; $H$, beginning of a convoluted tube. III. Rodded cells from a convoluted tube: I, view from the surface; 2 , side view ( $G$, granular zone). IV. Cells lining the tubes of Henle. V. Cells lining the second convoluted tubes. VI. Section of a straight tube. 
some of the cells of the pancreas and of the salivary glands. The nucleus usually is situated between the granular and the rodded zones.

The pyramids of Ferrein extend into the cortical substance to form what are known as the medullary rays. The spaces between the medullary rays constitute the labyrinth. The division between the cortical and the medullary substance is known as the boundary zone.

The greatest part of the solid excrementitious constituents of the urine, such as urea and the urates, is separated from the blood by the cells of the convoluted tubes of the cortical substance and perhaps by the dilated portions of the tubes of Henle, while the water and a certain portion of the inorganic salts of the urine transude through the bloodvessels in the Malpighian bodies.

Narrow Tubes of Hculc. - The convoluted tubes above described, after a tortuous course in the cortical substance, become continuous, near the pyramids, with the tubes of much smaller diameter, which form loops extending to a greater or less depth into the pyramids. The loops formed by these canals (the narrow tubes of Henle) are nearly parallel with the tubes of Bellini and are much greater in number near the bases of the pyramids than toward the apices. The diameter of these tubes is variable, and they present enlargements at irregular intervals in their course. The narrow portions are about $\frac{1}{2000}$ of an inch $(12 \mu)$ in diameter, and the wide portions, about twice this size. The narrow portion is lined with small clear cells with very prominent nuclei. The wider portions are lined with larger, granular cells. Near the bases of the pyramids the wide portion sometimes forms the loop, but near the apices the loop is always narrow. The difference in the size of the epithelium is such that while the diameter of the tube is variable its calibre remains nearly uniform. The membrane of these tubes is quite thick, thicker, even, than the membrane of the tubes of Bellini.

After the narrow tubes of Henle have returned to the cortical substance, they communicate with a system of canals, $\frac{1}{1200}$ to $\frac{1}{100 \overline{0}}$ of an inch $(2 \mathrm{I}$ to $25 \mu)$ in diameter, with very thin walls, lined by rodded epithelium. These are known as the second convoluted tubes. They take an irregular and somewhat angular course between the first convoluted tubes and finally empty into the branches of the straight tubes of Bellini, thus establishing a communication between the tubes coming from the Malpighian bodies and the tubes of the pyramidal substance. They are sometimes called the intermediate tubes, or the canals of communication.

The tubes into which the second convoluted tubes open join with others, usually, two by two, and then pass in a nearly straight direction into the pyramids, where they continue to unite with each other in 
their course, becoming reduced in number until they open at the apices of the pyramids into the infundibula and the pelvis of the kidney.

Distribution of Bloodiessels in the Kidney. - The renal artery, which is quite voluminous in proportion to the size of the kidney, enters at the hilum and divides into four branches. A number of smaller branches penetrate between the pyramids and ramify in the columns of cortical substance that occupy the spaces between the prramids (columns of Bertin). The main vessels, which usually are two in number, occupy the centre of the columns of Bertin, sending off in their course at short intervals regular branches on either side toward the pyramids. When these branches reach the boundary of the cortical substance they turn upward and follow the periphery of the pyramid to its base. Here the ressels form an arched anastomosing plexus, the arterial arcade, situated between the rounded base of the pyramid and the cortical substance. This plexus presents a convexity looking toward the cortical substance, and a concavity, toward the pyranid. It is so arranged that the interstices are just large enough to admit the collections of tubes that form the pyramids of Ferrein.

From the arterial arcade, branches are given off in two opposite directions. From its concavity, small branches, measuring at first $\frac{1}{1200}$ to $\frac{1}{750}$ of an inch ( 2 I to $34 \mu$ ) in diameter, pass downward toward the papillæ, giving off small ramifications at very acute angles and becoming reduced in size to about $\frac{1}{2500}$ of an inch ( $10 \mu$ ). These vessels, called the arteriolæ rectæ, surround the straight tubes and pass into capillaries in the substance of the pyramids and at their apices.

From the convex surface of the arterial arcade, branches are given off at nearly right angles. These pass into the cortical substance, breaking up into a number of little arterial twigs, $\frac{1}{1500}$ to $\frac{1}{600}$ of an inch ( 17 to $40 \mu$ ) in diameter, each one of which penetrates a Malpighian body at a point opposite the neck of the capsule. Once within the capsule, the arteriole breaks up into five to eight branches, which then divide dichotomously into vessels measuring $\frac{1}{30} \frac{1}{0}$ to $\frac{1}{1500}$ of an inch ( 8 to $17 \mu$ ) in diameter, arranged in the form of coils and loops, constituting a dense rounded mass (the Malpighian coil, or glomerulus), filling the capsule. These vessels break up into capillaries without anastomoses (see Plate VII, Fig. 4).

The blood is collected from the vessels of the Malpighian bodies by a vein, which passes out of the capsule by the side of the arteriole and, with veins from other glomeruli, forms a plexus closely surrounding the convoluted tubes. This is a true plexus, the branches anastomosing freely in every direction; and the distribution of ressels in this part resembles essentially the vascular arrangement in most of the glands. 
Bowman called the branches which connect together the vessels of the Malpighian tuft and the capillary plexus surrounding the tubes, the portal system of the kidney. These intermediate vessels form a coarse plexus surrounding the prolongations of the pyramids of Ferrein into the cortical substance.

The renal, or emulgent vein takes its origin in part from the capillary plexus surrounding the convoluted tubes and in part from ressels distributed in the pyramidal substance. A few branches come from vessels in the envelopes of the kidney, but these are comparatively unimportant. The plexus surrounding the convoluted tubes empties into venous radicles which pass to the surface of the kidney, and these present a number of little radiating groups, each converging toward a central vessel. This arrangement gives to the vessels under the fibrous envelope of the kidney a peculiar stellate appearance, forming what are called the stars of Verheyn. The large trunks which form the centres of these stars then pass through the cortical substance to the rounded bases of the pyramids, where they form a vaulted renous plexus corresponding to the arterial plexus already described. The vessels distributed on the straight tubes of the pyramidal substance form a loose plexus around these tubes, except at the papillæ, where the network is much closer. They then pass into the plexus at the bases of the pyramids to join with the veins from the cortical substance. From this plexus a number of larger trunks arise and pass toward the hilum, in the axis of the interpyramidal substance, enveloped in a sheath with the arteries. Passing to the pelvis of the kidney, the veins converge into three or four branches, which unite to form the renal vein. A preparation of all the vessels of the kidneys shows that the veins are much more voluminous than the arteries (see Frontispiece).

The capsule of the kidney has a lymphatic plexus connected with lymph-spaces below; and lymph-spaces, in the form of large slits, exist between and around the convoluted tubes.

The nerves are abundant and are derived from the solar plexus, their filaments following the renal artery in its distribution in the interior of the organ and ramifying on the walls of the vessels.

\section{Mechanism of the Prodectiox axd Discharge of Urine}

The most important constituent of the urine is urea- $\mathrm{CO}\left(\mathrm{NH}_{2}\right)_{2}-$ a crystallizable nitrogenous substance, which is discharged by the skin as well as by the kidneys. This has long been recognized as an excrementitious substance; but the first observations that gave a definite idea of the mechanism of its production were made by Prevost and Dumas 
in $\mathrm{I} 82 \mathrm{I}$. At the time these experiments were made, chemists were not able to detect urea in the normal blood; but Prevost and Dumas extirpated the kidneys from living animals - dogs and cats - and found urea in the blood after certain nervous symptoms had developed. For the first two or three days after the operation there were no marked symptoms; but stupor and other evidences of nervous disturbance finally supervened, when the presence of urea in the blood could easily be determined. These observations were confirmed and extended by Ségalas and Vauquelin in I822. Since that time, as the processes for the determination of urea in the animal liquids have been improved, this substance has been detected in normal blood. Picard ( 1856 ) estimated and compared the proportions of urea in the renal artery and the renal vein and found that the quantity in the blood was diminished by about one-half in its passage through the kidneys. Still later, urea was found in the lymph and chyle, in larger quantity, even, than in the blood (Wurtz). It has been ascertained that urea is an active diuretic when injected in small quantity into the veins of a healthy animal, and is promptly eliminated. When injected into the vascular system of a nephrotomized animal, however, it produces death in a very short time, with characteristic symptoms.

From a review of the important facts bearing on the question under consideration, there seems to be no valid ground for a change in the ideas of physiologists concerning the mode of elimination of urea and other important excrementitious constituents of the urine. There is every reason to suppose that these substances are produced in various tissues and organs of the body during the process of katabolism, are taken up by the lymph and the blood and are separated from the blood by the kidneys. Urea, however, probably is formed almost exclusively in the liver from substances of a like nature, the final process of oxidation taking place in this organ. The mechanism of this will be described farther on.

Extirpation of one kidney from a living animal is not necessarily fatal. When the operation is carefully performed, the wound usually heals without difficulty, and in most instances the remaining kidney seems sufficient for the elimination of urine for an indefinite period. In a large number of experiments, the animals killed long after the wound had healed presented no marked symptoms of retention of excrementitious matters in the blood, except in one or two instances. It is a noticeable fact, however, that in many instances they showed a change in disposition, and the appetite became voracious and unnatural. These animals would sometimes eat feces, the flesh of dogs etc., and, in short, presented certain of the phenomena frequently observed after 
extirpation of the spleen (Flint). After extirpation of one kidney, it has been observed that the remaining kidney increases in weight, although investigations have shown that this is due mainly to an increase in the quantity of blood, lymph and urinary matters, and not to a new development of renal structure.

Influcnee of Blood-pressure, the Nerions System etc., on the Secretion of Urine. - There are many instances in which marked and sudden modifications in the action of the kidneys take place under the influence of fear, anxiety, hysteria etc., which must operate through the nervous system. Although little is known of the final distribution of the nerves in the kidney, it has been ascertained that here, as elsewhere, vasomotor nerves are distributed to the walls of the bloodvessels, and they are capable of modifying the quantity and the pressure of blood in these organs.

It may be stated as a general proposition, that an increase in the pressure of blood in the kidneys increases the flow of urine, and that when the blood-pressure is lowered, the flow of urine is correspondingly diminished. This will in a measure account for the increase in the flow of urine during digestion; but it can not serve to explain all the modifications that may take place in the action of the kidneys.

Inasmuch as the excrementitious matters eliminated by the kidneys are being constantly produced in the tissues by the process of katabolism, the formation of urine is constant, presenting, in this regard, a marked contrast with the intermittent flow of most of the secretions proper as distinguished from the excretions. It was noted by Erichsen, in a case of extroversion of the bladder, and it has been further shown by experiments on dogs, that there is an alternation in the action of the kidneys on the two sides. Bernard exposed the ureters in a living animal and fixed a small silver tube in each, so that the secretion from either kidney could be readily observed; and he noted that a large quantity of liquid was discharged from one side for fifteen to thirty minutes, while the flow from the other side was slight and in some instances was arrested. The flow then began with activity on the other side, while the discharge from the opposite ureter was diminished or arrested.

Physiological Anatomy of the Crinary Passages. - The excretory ducts of the kidneys - the ureters - begin each by a funnel-shaped portion, which is applied to the kidney at the hilum. The ureters themselves are membranous tubes of about the diameter of a goosequill, becoming much reduced in calibre as they penetrate the coats of the bladder. They are sixteen to eighteen inches ( 40 to 46 centimeters) in length, and pass from the kidneys to the bladder, behind the peri- 
toneum. They have three distinct coats: an external coat, composed of ordinary fibrous tissue with small elastic fibres; a middle coat, composed of non-striated muscular fibres; and a mucous coat.

The external coat requires no special description. It is prolonged into the calices and is continuous with the fibrous coat of the kidney.

The fibres of the muscular coat, in the greatest part of the length of the ureters, interlace with each other in every direction and are not arranged in distinct layers; but near the bladder, is an internal layer, in which the direction of the fibres is longitudinal.

The mucous lining is thin, smooth and without follicular glands. It is thrown into narrow longitudinal folds, when the tube is flaccid, which are easily effaced by distention. The epithelium exists in several layers and is remarkable for the irregular shape of the cells. These present, usually, dark granules and one or two clear nuclei with distinct nucleoli. Some of the cells are flattened, some are rounded, and some are caudate with one or two prolongations.

Passing to the base of the bladder, the ureters become constricted, penetrate the coats of this organ obliquely, their course in its walls being a little less than an inch (25 millimeters) in length. This valvular opening allows the free passage of the urine from the ureters, but compression or distention of the bladder closes the orifices and opposes a return of the liquid.

The bladder, which serves as a reservoir for the urine, varies in its relations to the pelvic and abdominal organs as it is empty or more or less distended. When empty, it lies deeply in the pelvic cavity and is then a small sac of an irregularly-triangular form. As it becomes filled, it assumes a globular or ovoid form, rises up in the pelvic cavity, and when excessively distended, it may extend partly into the abdomen. When the urine is voided at normal intervals, the bladder, when filled, contains about a pint (nearly 500 cubic centimeters) of liquid; but under pathological conditions it may become distended so as to contain ten or twelve pints (about 4 or 5 liters), and in some instances of obstruction it has been found to contain even more. The bladder usually is more capacious in the female than in the male.

The coats of the bladder are three in number. The external coat is simply a reflection of the peritoneum, covering the posterior portion completely, from the openings of the ureters to the summit, about onethird of the lateral portion and a small part of the anterior portion.

The middle or muscular coat consists of non-striated fibres, arranged in three tolerably distinct layers: The external muscular layer is composed of longitudinal fibres, which arise from parts adjacent to the neck, and pass anteriorly, posteriorly and laterally over the organ, so 
that when they contract they diminish its capacity chiefly by shortening its vertical diameter. The fibres of the external layer are of a pinkish hue, being much more highly colored than the other layers. The middle layer is formed of circular fibres, arranged, on the anterior surface of the bladder, in distinct bands at right angles to the superficial fibres. They are thinner and less strongly marked on the posterior and lateral surfaces. The internal layer is composed of pale fibres arranged in longitudinal fasciculi, the anterior and lateral bundles anastomosing with each other, as they descend toward the neck of the bladder, by oblique bands of communication, and the posterior bundles interlacing in every direction, forming an irregular plexus. Here they are not to be distinguished from the fibres of the middle layer. This is sometimes called the plexiform layer; and it gives to the interior of the bladder its reticulated appearance. This layer is continuous with the muscular fibres of the urachus, the ureters and the urethra.

The sphincter vesicre is a band of non-striated fibres, about half an inch (12.7 millimeters) in breadth and one-eighth of an inch (3.2 millimeters) in thickness, embracing the neck of the bladder and the posterior half of the prostatic portion of the urethra. The tonic contraction of these fibres prevents the discharge of urine, and during the ejaculation of semen, it offers an obstruction to its passage into the bladder.

The mucous membrane of the bladder is smooth, rather pale, thick, and loosely adherent to the submucous tissue, except over the corpus trigonum. The epithelium is stratified and presents the same diversity in form as that observed in the pelvis of the kidney and the ureters. In the neck and fundus of the bladder, are a few mucous glands, some in the form of simple follicles and others collected to form glands of the simple racemose variety.

The corpus trigonum is a triangular body lying just beneath the mucous membrane at the base of the bladder and extending from the urethra in front to the openings of the ureters. It is composed of ordinary fibrous tissue with a few elastic and muscular fibres. At the opening of the urethra, it presents a small projecting fold of mucous membrane, which is sometimes called the uvula vesica. Over the surface of the trigone, the mucous membrane is closely adherent, and it is not thrown into folds even when the bladder is entirely empty.

The bloodvessels going to the bladder are finally distributed to its mucous membrane. They are not abundant except at the fundus, where the mucous membrane is very vascular. Lymphatics are found in the walls of the bladder. The nerves of the bladder are derived from the hypogastric plexus.

The urethra is provided with muscular fibres, and it is lined by a 
mucous membrane, the anatomy of which will be more fully considered in connection with embryology. In the female the epithelium of the urethra is like that of the bladder. In the male the epithelial cells are small, pale and of the columnar variety.

Mechanism of the Discharge of Urine. - In the human subject, the urine is discharged into the pelves of the kidneys and the ureters by pressure due to the act of separation of liquid from the blood. Once discharged into the ureters, the course of the urine is determined in part by the vis a tergo, and in part, probably, by the action of the muscular coats of these canals.

When the urine has accumulated in certain quantity in the bladder, a peculiar sensation is felt which leads to the act for its expulsion. The intervals at which it is experienced are variable. The urine usually is voided before retiring to rest, on rising in the morning, and two or three times, in addition, during the day. The frequency of micturition, however, depends on habit, on the quantity of liquids ingested and on the degree of activity of the skin.

Evacuation of the bladder is accomplished by the muscular walls of the organ itself, aided by contractions of the diaphragm and the abdominal muscles with certain muscles which operate on the urethra, and it is accompanied by relaxation of the sphincter vesica. This act is at first voluntary, but once begun, it may be continued by the involuntary contraction of the bladder alone. During the first part of the process, the distended bladder is compressed by contraction of the diaphragm and the abdominal muscles; and this after a time excites the action of the bladder itself. A certain time usually elapses then before the urine begins to flow. When the bladder contracts, aided by the muscles of the abdomen and the diaphragm, the resistance of the sphincter is overcome and a jet of urine flows from the urethra. All voluntary action may then cease for a time, and the bladder will nearly empty itself ; but the force of the jet may be increased by voluntary effort.

Toward the end of the expulsive act, when the quantity of liquid remaining in the bladder is small, the diaphragm and the abdominal muscles are again called into action, and there is a convulsive interrupted discharge of the small quantity of urine that remains. At this time the impulse from the bladder, and, indeed, the influence of the abdominal muscles and diaphragm, are very slight, and the flow of urine along the urethra is aided by the contractions of its muscular walls and the action of certain of the perineal muscles, the most efficient being the accelerator urinæ; but with all this muscular action, a few drops of urine remain in the male urethra after the act of urination has been accomplished. The process of evacuation of urine in the female is essen- 
tially the same as in the male, with the exception of the slight modifications due to differences in the direction and length of the urethra.

According to Budge, the influence of the nervous system on the bladder operates through the sympathetic; and he has described a centre in the spinal cord, which presides over the contractions of the lower part of the intestinal canal, the bladder and the vasa deferentia. This is called the genito spinal centre, and it has been located, in experiments on rabbits, in the spinal cord, at a point opposite the fourth lumbar vertebra. From this centre the nervous filaments pass through the sympathetic nerve, communicating with the ganglion which corresponds to the fifth lumbar vertebra.

\section{Properties and Composition of the URine}

The color of the urine in the human subject is quite variable within the limits of health and depends to a considerable extent on the character of food, the quantity of drink and the activity of the skin. As a rule the color is yellowish or amber, with more or less of a reddish tint. The liquid is perfectly transparent, free from viscidity, and exhales, when first passed, a peculiar aromatic odor that is not disagreeable. Soon after the urine cools, it loses this peculiar odor and has an odor known as urinous. This odor remains until the liquid begins to undergo decomposition. The color and odor of the urine usually are modified by the same physiological conditions. When the urine contains a large proportion of solid matters, the color is more intense and the urinous odor is more penetrating; and when its quantity is increased by an excess of water, the specific gravity is low, the color is pale and the odor is faint. The first urine passed in the morning, immediately after rising, usually is more intense in color than that passed during the day and contains a relatively larger proportion of solids in solution.

The temperature of the urine at the moment of its emission, under physiological conditions, varies but a small fraction of a degree from $100^{\circ}$ Fahr. $\left(37.78^{\circ} \mathrm{C}\right.$.).

In estimating the total quantity of urine discharged in the twentyfour hours, it is important to take into consideration the specific gravity as an indication of the amount of solid matter excreted by the kidneys. Variations in quantity constantly occur in health, depending on the proportion of water; but the quantity of solid matters excreted usually is more nearly uniform. It must also be taken into account that differences in climate, habits of life etc., in different countries, have an important influence on the daily quantity of urine. Parkes collected the results of twenty-six series of observations made in America, Eng- 
land, France and Germany, and found the average daily quantity of urine in healthy male adults, between twenty and forty years of age, to be fifty-two and a half fluidounces (I 552.6 cubic centimeters), the average quantity per hour being two and one-tenth fluidounces ( 62 cubic centimeters). The extremes were thirty-five ounces and eighty-one ounces (1035 and 2395 cubic centimeters). The average quantity may be assumed to be about fifty-one fluidounces (I 500 cubic centimeters). The normal range of variation is between thirty and sixty ounces (about 900 and I 775 cubic centimeters). The conditions which lead to a diminution in the quantity of urine usually are more efficient in their operation than those which tend to an increase; and the range below the normal standard is rather wider than it is above. More urine usually is secreted during the day than at night. The quantity of water discharged by the kidneys in the twenty-four hours is a little greater in the female than in the male; but in the female the specific gravity is lower, and the quantity of solid constituents is relatively and absolutely less.

The specific gravity of the urine should be estimated in connection with the absolute quantity in the twenty-four hours. Those who assume that the daily quantity is about fifty-one ounces (I 500 cubic centimeters), give the ordinary specific gravity of the mixed urine of the twenty-four hours as about 1020. The specific gravity is liable to the same variations as the proportion of water, and the density is increased as the water is diminished. The ordinary range of variation in specific gravity is between IOI 5 and IO25; but without positively indicating a pathological condition, it may temporarily be as low as 1005 or as high as 1030 .

The reaction of the urine is acid in the carnivora and alkaline in the herbivora. In the human subject it usually is acid at the moment of its discharge from the bladder; although at certain times of the day it may be neutral or feebly alkaline, the reaction depending on the character of food. The acidity may be measured by neutralizing the urine with an alkali in a solution that has previously been graduated with a solution of oxalic acid of known strength; and the degree of acidity usually is expressed as equivalent to so many grains of crystallized oxalic acid.

The total quantity of acid in the urine of the twenty-four hours in a healthy adult male is equal to between thirty and sixty grains ( 2 and 4 grams) of oxalic acid. The hourly quantity in the observations of Vogel was equal, in round numbers, to between one and a half and three grains (O.I and 0.2 gram) of acid. The proportion of acid was found to be variable in the same person at different times of the day. The urine contains no free acid, but its acidity under an animal or a mixed diet 
depends on the presence of acid salts, the principal salt being acid sodium phosphate, with possibly a little acid calcium phosphate.

\section{COMPOSITION OF THE HUMAN URINE}

Water (in 24 hours, 27 to 50 fluidounces, 800 to 1480 cubic centimeters - Becquerel)

Urea (in 24 hours, 355 to 463 grains, 23 to 30 grams - Robin) . . . . . 15.00 to 23.00 Uric acid . . . . . . . . . . . . . . accidental, or traces Soclium urate, neutral and acid

Ammonium urate, neutral and acid (in

(In 24 hours, 6 to 9 grains, small quantity)

Potassium urate

Calcium urate

Magnesium urate

Sodium hippurate

Potassium hippurate

Calcium hippurate 0.39 to $0.5^{8}$ gram, of uric acid Becquerel-or 9 to 14 grains, $0.5^{8}$ to 0.9 gram, of urates, estimated as neutral urate of soda). . .

1.00 to 1.60

Sodium lactate

Potassium lactate

Calcium lactate

Creatin

Creatinin

(In 24 hours, about 7.5 grains, 0.486 gram, of hippuric acid-Thudichum-equivalent to about 8.7 grains, 0.566 gram, of sodium hippurate) .

Calcium oxalate (daily quantity not estimated) . . . . . . . .

Xanthin . . . . . . . . . . . . . . . . .

Palmitin, olein and other fatty matters . . . . . . . . . . .

Sodium chloride (in 24 hours, about 154 grains, 10 grams - Robin) . . .

Potassium chloride . . . . . . . . . . . . . . . . .

Ammonium chloride

Sodium sulphate

Potassium sulphate

(In 24 hours, 23 to 38 grains, 1.5 to 2.5

Calcium sulphate (traces) grams, of sulphuric acid - Thudichum. About equal parts of sodium sulphate and potassium sulphate - Robin - equivalent to 22.5 to 37.5 grains, 1.45 to 2.43 grams of each) . . .

Potassium indoxyl-sulphate (indican)

Sodium phosphate, neutral ,

Sodium phosphate, acid $\}$

(Daily quantity not estimated)

3.00 to 7.00 not estimated.

Magnesium phosphate (in 24 hours, 7.7 to 11.8 grains, 0.5 to 0.768 gram Neubauer)

$\left.\begin{array}{l}\text { Calcium phosphate, acid } \\ \text { Calcium phosphate, basic }\end{array}\right\} \begin{gathered}\text { (In } 24 \text { hours, } 4.7 \text { to } 5.7 \text { grains, } 0.307 \text { to } \\ 0.372 \text { gram-Neubauer) . . . . . . }\end{gathered}$

Ammonio-magnesian phosphate (daily quantity not estimated) . . . .

(Daily excretion of phosphoric acid, about 56 grains, 3.629 grams Thudichum.)

Silicic acid .

Urochrome

Mucus from the bladder

50 to

$4 \cdot 30$

0.50 to 1.00

0.20 to 1.30

1.50 to 2.40

Proportion of solid constituents, 32.63 to 59.89 parts per 1000 .

Gases of the Urine. (Parts per 1000, in volume)

Oxygen in solution . . . . . . . . . . . . . . . . 0.90 to 1.00

Nitrogen in solution . . . . . . . . . . . . . . 7.00 to 10.00

Carbon dioxide in solution . . . . . . . . . . . 45 to 50.00 
Composition of the Urine. - Regarding the excrementitious constituents of the urine as a measure, to a certain extent, of the general processes of katabolism, it is more important to recognize the quantities of these products discharged in a definite time than to learn simply their proportions in the urine; and in the preceding table of composition of the urine, the absolute quantities of its different constituents excreted in twenty-four hours have been given when practicable.

Urea. - As regards quantity, and probably as a measure of the activity of the process of proteid katabolism, urea- $\mathrm{CO}\left(\mathrm{NH}_{2}\right)_{2}$ - is the most important of the urinary constituents. Regarding the daily excretion of urea as a measure of the physiological wear of certain tissues, its consideration would come properly under the head of nutrition, in connection with other substances known to be products of katabolism; but it is convenient to treat of its general physiological properties and some of its variations in common with other excrementitious matters separated by the kidneys, in connection with the composition of the urine.

The formula for urea, showing the presence of a large proportion of nitrogen, would lead to the supposition that this substance is one of the products of the wear of the nitrogenous constituents of the body. It is found, under normal conditions, in the urine, the lymph and chyle, the blood, the sweat, the vitreous humor and a trace in the saliva. Its presence has been demonstrated, also, in the substance of the healthy liver in both carnivorous and herbivorous animals; and it has been found in minute quantity in the muscular juice.

Urea has been produced synthetically by combining ammonium sulphate with potassium. The products of this combination are potassium sulphate, with cyanic acid and ammonium in a form to constitute urea. Ammonium cyanate is isomeric with urea, and the change is effected by a rearrangement of its elements. It has long been known that urea is readily convertible into ammonium carbonate; and ammonium carbonate, when heated in sealed tubes to the temperature at which urea begins to decompose, is converted into urea.

Urea may readily be extracted from the urine by processes fully described in works on physiological chemistry; and its proportion may now easily be estimated by the various methods of volumetric analysis. It is not so easy, however, to separate it from the blood or from the substance of any of the tissues, on account of the difficulty in getting rid of other organic matters and the readiness with which it undergoes decomposition.

When pure, urea crystallizes in the form of long, four-sided, colorless and transparent prisms, which are without odor, neutral, and in taste 
resemble saltpetre. These crystals are very soluble in water and in alcohol but are insoluble in ether. In its behavior with reagents, urea acts as a base, combining readily with certain acids, particularly nitric and oxalic. It also forms combinations with certain salts, such as mercuric oxide, sodium chloride etc. It exists in the economy in a state of watery solution, with perhaps a small portion modified by the presence of sodium chloride.

Origin of Urea. - It is now admitted by physiologists that urea is not formed in the kidneys but preëxists in the blood. It finds its way into the blood, in part directly from the tissues, and in part from the lymph, which contains a greater proportion of urea than is found in the blood itself. The principal seat of its formation, however, is the liver, although it is produced in other organs in small quantity. The quantity of urea in the blood is kept down by the eliminating action of the kidneys. In certain cases of structural diseases of the liver, the excretion of urea is much diminished, and it may, indeed, disappear from the urine.

Proteids, in the metabolic processes that result in the change of the albuminous constituents of the tissues into urea, are supposed to undergo the following changes. The carbon molecules are oxidized into carbon dioxide, nitrogen is split off as ammonia $\left(\mathrm{NH}_{3}\right)$, and the two combine to form ammonium carbonate. In the liver, ammonium carbonate loses one molecule of water and is converted into ammonium carbamate. This loses a second molecule of water and is converted into carbamid (urea). Carbamic acid in a free state is unknown; but it exists as ammonium carbamate in commercial ammonium carbonate.

Assuming that urea is the most abundant and important of the nitrogenous excrementitious products - which is fully justified by physiological facts - it is difficult to avoid the conclusion that this substance represents, to a great extent, the katabolism of the nitrogenous parts of the tissues and necessarily the physiological wear of the muscular substance. The fact that urea exists in very minute quantity in the muscles - some chemists state that it is absent - probably is due to its constant removal by the blood and lymph.

Uric acid, creatin, creatinin, xanthin, hypoxanthin, leucin, tyrosin and some other analogous substances are to be regarded as formations antecedent to urea, urea being the final and perfect excrementitious product of the liver.

Influence of Ingesta on the Composition of the Urine and on the Elimination of Nitrogen. - Water and other liquid ingesta usually increase the proportion of water in the urine and diminish its specific gravity. This is so marked after the ingestion of large quantities of liquids, that the urine passed under these conditions is sometimes spoken of as the 
urina potus; but when an excess of water has been taken for purposes of experiment, the diet being carefully regulated, the absolute quantity of solid matters excreted is considerably increased. This is particularly marked as regards urea, but it is noticeable in the sulphates and phosphates, though not to any great extent in the chlorides. The results of experiments on this point seem to show that water taken in excess increases the activity of katabolism.

The ordinary meals increase the solid constituents of the urine, the most constant and uniform increase being in the proportion of urea. This, however, depends to a great extent on the kind of food taken. The increase usually is noted during the first hour after a meal and attains its maximum at the third or fourth hour. The inorganic matters are increased as well as the excrementitious substances proper. The urine passed after taking food has been called urina cibi, under the idea that it is to be distinguished from the urine supposed to be derived exclusively from katabolism, which is called the urina sanguinis.

It is an important question, to determine the influence of different kinds of food on the composition of the urine, particularly the comparative effects of a nitrogenous and a non-nitrogenous diet. Lehmann made a number of observations on this point, and his results have been confirmed by many other physiologists. Without discussing fully all these observations, it is sufficient to state that the ingestion of an excess of nitrogenous food always produced a great increase in the proportion of the nitrogenous constituents of the urine, particularly urea. On a non-nitrogenous diet, the proportion of urea was found to be diminished more than one-half. The general results of the experiments of Lehmann are embodied in the following quotation:-

"My experiments show that the amount of urea which is excreted is extremely dependent on the nature of the food which has been previously taken. On a purely animal diet, or on food very rich in nitrogen, there were often two-fifths more urea excreted than on a mixed diet; while, on a mixed diet, there was almost one-third more than on a purely vegetable diet; while, finally, on a non-nitrogenous diet, the amount of urea was less than half the quantity excreted during an ordinary mixed diet."

The influence of food is not confined to the period when any particular kind of food is taken, but is continued for many hours after a return to the ordinary diet.

In regard to the influence of food on the inorganic constituents of the urine, it may be stated in general terms that the ingestion of mineral substances increases their proportion in the excretions.

There are certain articles which, when taken into the system, the 
diet being regular, seem to retard the process of katabolism; or at least they diminish, in a marked manner, the quantity of matters excreted, particularly urea. Alcohol has a decided influence of this kind. Its action may be modified by the presence of salts and other matters in different alcoholic beverages, but in nearly all direct experiments, alcohol either taken under normal conditions of diet, when the diet is deficient or when it is in excess, diminishes the excretion of urea. The same may be stated in general terms of tea and coffee.

Influence of Muscular Exercise on the Elimination of Nitrogen. - In all observations on the influence of muscular exercise on the elimination of nitrogen, account should be taken of the influence of diet; and those observations are most valuable which have given the proportion of nitrogen eliminated to the nitrogen of food. The observations of Fick and Wislicenus (1866) showed a diminution in the elimination of nitrogen during work; but during the time of the muscular work, no nitrogenous food was taken. The same conditions obtained in certain of the observations of Parkes. In a series of observations made in I 880 (Flint), on a man who walked $317 \frac{1}{2}$ miles (about 5 Io kilometers) in five consecutive days, the diet was normal, and the proportionate quantity of nitrogen was calculated for three periods of five days each, with the following results :-

For the five days before the walk, with an average exercise of about eight miles ( 13 kilometers) daily, the nitrogen eliminated was 92.82 parts for 100 parts of nitrogen ingested. For the five days of the walk, for every hundred parts of nitrogen ingested, there were discharged I 53.99 parts. For the five days after the walk, when there was hardly any exercise, for every hundred parts of nitrogen ingested, there were discharged 84.63 parts. During the walk, the nitrogen excreted was in direct ratio to the amount of work; and the excess of nitrogen eliminated over the nitrogen of food almost exactly corresponded to a calculation of the nitrogen of the muscular tissue consumed, as estimated from the loss of weight of the body. In 1876, a similar series of observations was made on the same man by Pavy. In these observations he walked 450 miles (724.2 I kilometers) in six consecutive days. During this period the proportionate elimination of nitrogen was increased, but not to the extent observed in I870. Similar results - although the experiments were made on a less extended scale - were obtained by North in 1878 . These observations are opposed to the views of many physiologists, since the experiments of Fick and Wislicenus, who regard the elimination of nitrogen under ordinary conditions as dependent mainly on the diet and not upon the muscular work performed. The observations of Voit, indeed, are favorable to this view. 
Notwithstanding the results obtained by Fick and Wislicenus, Frankland, Haughton, Voit, and others, the fact remains that severe and prolonged muscular work increases the elimination of nitrogen over and above the quantity to be accounted for by the nitrogenous food taken. Actual observations (Flint, Pavy and others) are conclusive as regards this simple fact; but it is well known that muscular exercise largely increases the elimination of carbon dioxide and the consumption of oxygen. In exercise so violent as to produce dyspnœa, the distress in breathing probably is due to the impossibility of supplying by the lungs sufficient oxygen to meet the increased demand on the part of the muscular system, and the possible amount of muscular work is thereby limited.

The observations and conclusions of Oppenheim (1880) go far to harmonize the results obtained by different experimenters. Oppenheim concludes that muscular work, when not carried to the extent of producing shortness of breath or when moderate and extending over a considerable length of time, does not increase the elimination of urea; but that even less work, when violent and attended with shortness of breath, increases the discharge of urea. According to this view, moderate work draws on the oxygen supplied to the body and at once largely increases the elimination of carbon dioxide; but the less active processes which result in the production of urea are not so promptly affected. Violent muscular work, however, or work which is excessively prolonged, consumes those parts of the tissues the katabolism of which is represented by the discharge of urea. This view, if accepted, harmonizes the apparently contradictory experiments on the influence of muscular work on the elimination of nitrogen.

The daily quantity of urea excreted is subject to considerable variations. It is given in the table as 355 to 463 grains ( 23 to 30 grams). This is less than the estimates frequently given; but when the quantity has been very large, it has depended on an unusual amount of nitrogenous food, or the weight of the body has been above the average. Parkes has given the results of twenty-five different series of observations on this point. The lowest estimate was 286.I grains (I 8.24 grams), and the highest, 688.4 grains (44.6I grams).

Uric Acid and its Compounds. - Uric acid $\left(\mathrm{C}_{5} \mathrm{H}_{4} \mathrm{~N}_{4} \mathrm{O}_{3}\right)$ seldom if ever exists in a free state in normal urine. It is very insoluble, requiring fourteen to fifteen thousand times its volume of cold water or eighteen to nineteen hundred parts of boiling water for its solution. Its presence uncombined in the urine must be regarded as a pathological condition.

In normal urine, uric acid is combined with sodium, ammonium, 
potassium, calcium and magnesium. Of these combinations, the sodium urate and ammonium urate are by far the most important, and they constitute a great proportion of the urates, potassium, calcium and magnesium urates existing only in minute traces. Sodium urate is much more abundant than ammonium urate. The union of uric acid with the bases is very feeble. If from any cause the urine becomes excessively acid after its emission, a deposit of uric acid is likely to occur. The addition of a very small quantity of almost any acid is sufficient to decompose the urates, when the uric acid appears after a few hours, in a crystalline form.

Uric acid, probably in combination with bases, is always found in the substance of the liver in large quantity. The urates also exist in the blood in small quantity and pass ready-formed into the urine. The fact that the urates exist in the liver has led to the opinion that this organ is the principal seat of the formation of uric acid (Meissner). However this may be, uric acid certainly is not formed in the kidneys but is simply separated by these organs from the blood. Meissner did not succeed in finding uric acid in the muscular tissue, although the specimens were taken from animals in which he had found large quantities in the liver. The urates, particularly sodium urate, are products of katabolism of the proteid constituents of the body.

The daily excretion of uric acid, given in the table, is six to nine grains ( 0.39 to 0.58 gram), the equivalent of nine to fourteen grains ( 0.58 to 0.9 gram) of urates estimated as neutral sodium urate. Like urea, the proportion of urates in the urine is subject to certain physiological variations.

Hippuric Acid, Hippurates and Lactates. - The compounds of hippuric acid $\left(\mathrm{C}_{9} \mathrm{H}_{9} \mathrm{NO}_{3}\right)$, which are so abundant in the urine of the herbivora, are now known to be constant constituents of human urine. Hippuric acid is always to be found in the urine of children, but it is sometimes absent temporarily in the adult. The hippurates have been detected in the blood of the ox and have since been found in the blood of the human subject. There can be scarcely any doubt that they pass ready-formed from the blood into the urine. As to the exact mode of origin of the hippurates, there is even less information than in regard to the origin of the other urinary constituents already considered. Experiments have shown that the proportion of hippuric acid in the urine is greatest after taking vegetable food; but it is found after a purely animal diet, and probably it also exists during fasting. The daily excretion of hippuric acid is about 7.5 grains ( 0.486 gram), which is equivalent to about 8.7 grains ( 0.566 gram) of sodium hippurate. 
Hippuric acid itself, unlike uric acid, is soluble in water and in a mixture of hydrochloric acid. It requires six hundred parts of cold water for its solution, but a much smaller proportion of warm water. Under pathological conditions it is sometimes found free in solution in the urine.

Sodium, potassium and calcium lactates exist in considerable quantity in normal urine. They are derived immediately from the blood, passing ready-formed into the urine, where they exist in simple watery solution. The lactates are formed in the muscles, in the substance of which they can readily be detected. Physiologists have little positive information in regard to the precise mode of formation of these salts. It is probable, however, that the lactic acid is the result of transformation of glucose. The lactic acid contained in the lactates extracted from the muscular substance is not identical with the acid resulting from the transformation of the sugars. The former have been called sarcolactates, and they contain one molecule of water less than the ordinary lactates. The compounds of lactic acid in the urine are in the form of sarcolactates.

Creatin and Creatinin. - Creatin $\left(\mathrm{C}_{4} \mathrm{H}_{9} \mathrm{~N}_{3} \mathrm{O}_{2}\right)$ and creatinin $\left(\mathrm{C}_{4} \mathrm{H}_{7} \mathrm{~N}_{3} \mathrm{O}\right)$ probably are identical in their relations to the general process of katabolism, for one is easily converted into the other, out of the body, by very simple chemical means; and there is every reason to suppose that in the organism they are the products of physiological wear of the same tissue or tissues. These substances have been found in the urine, blood, muscular tissue and brain. By certain chemical manipulations, both creatin and creatinin may be converted into urea. These substances are now regarded as excrementitious matters, taken from the tissues by the blood, to be eliminated by the kidneys.

Creatin has a bitter taste, is quite soluble in cold water (one part in seventy-five), and is much more soluble in hot water, from which it separates in a crystalline form on cooling. It is slightly soluble in alcohol and is insoluble in ether. A watery solution of creatin is neutral. It does not readily form combinations as a base; but it has lately been made to form crystalline compounds with some of the strong mineral acids - nitric, hydrochloric or sulphuric. When boiled for a long time with barium hydrate, it is changed into urea and sarcosin. When boiled with the strong acids, creatin loses a molecule of water and is converted into creatinin. This change takes place readily in decomposing urine, which contains neither urea nor creatin but a large quantity of creatinin, when far advanced in putrefaction.

Creatinin is more soluble than creatin, and its watery solution has a strongly alkaline reaction. It is dissolved by eleven parts of cold 
water and is even more soluble in boiling water. It is slightly soluble in ether and is dissolved by one hundred parts of alcohol. This substance is one of the strongest of the organic bases, readily forming crystalline combinations with a number of acids. According to Thudichum, creatin is the original excrementitious substance produced in the muscular substance, and creatinin is formed in the blood by a transformation of a portion of the creatin, somewhere between the muscles and the kidneys; "for, in the muscle, creatin has by far the preponderance over creatinin; in the urine, creatinin over creatin." The fact that creatin has been found in the brain would lead to the supposition that it is also one of the products of katabolism of nervous tissue.

The average daily excretion of creatin and creatinin has been estimated at about II. 5 grains ( 0.745 gram). Of this, Thudichum estimated that 4.5 grains (0.292 gram) consisted of creatin, and 7 grains $(0.453$ gram) of creatinin.

Calcium Oralate. - Calcium oxalate (oxalic acid, $\mathrm{C}_{2} \mathrm{H}_{2} \mathrm{O}_{4}$ ) is not constantly present in normal human urine, although it may exist in certain quantity without indicating any pathological condition. It is exceedingly insoluble, and the appearance of its crystals, which commonly are in the form of small regular octahedra, is quite characteristic. A small quantity may be retained in solution by the acid sodium phosphate in the urine. Calcium oxalate may find its way out of the system by the kidneys after it has been taken with vegetable food or with certain medicinal substances. The ordinary rhubarb, or pie-plant, contains a large quantity of calcium oxalate, which, when this article is taken, passes into the urine. It is probable, however, that a certain quantity is formed in the organism.

Xanthin, Hypoxanthin, Lencin, Tyrosin and Taurin. - Traces of xanthin $\left(\mathrm{C}_{5} \mathrm{H}_{4} \mathrm{~N}_{4} \mathrm{O}_{2}\right)$ have been found in the normal human urine, but its proportion has not been estimated and observers are as yet but imperfectly acquainted with its physiological relations. It has been found in the liver, spleen, thymus, pancreas, muscles and brain. It is insoluble in water but is soluble in both acid and alkaline liquids. Hypoxanthin $\left(\mathrm{C}_{5} \mathrm{H}_{4} \mathrm{~N}_{4} \mathrm{O}\right)$ has not been found in normal urine, although it exists in the muscles, liver, spleen and thymus. Leucin $\left(\mathrm{C}_{6} \mathrm{H}_{13} \mathrm{NO}_{2}\right)$ exists in the pancreas, salivary glands, thyroid, thymus, suprarenal capsules, lymphatic glands, liver, lungs, kidneys and the gray substance of the brain. It has not been detected in the normal urine. The same remarks apply to tyrosin $\left(\mathrm{C}_{9} \mathrm{H}_{11} \mathrm{NO}_{3}\right)$, although it is not so extensively distributed in the economy, to taurin $\left(\mathrm{C}_{2} \mathrm{H}_{7} \mathrm{NO}_{3} \mathrm{~S}\right)$ and to cystin $\left(\mathrm{C}_{3} \mathrm{H}_{7} \mathrm{NSO}_{2}\right)$. The last two, however, contain sulphur, and they may have peculiar physiological and pathological relations that are not at present understood. 
Fatty Matters. - Fat and fatty acids are said to exist in the normal urine in certain quantity. Their proportion, however, is small, and the mere fact of their presence, only, is of physiological interest.

Inorganic Constitucnts of the Urine. - It is by the kidneys that the greatest quantity and variety of inorganic salts are discharged from the organism; and it is probable that even now physiological chemists are not acquainted with the exact proportion and condition of all the constituents of this class found in the urine. In all the processes of nutrition it is found that the inorganic constituents of the blood and tissues accompany the organic matters in their various transformations, although they are themselves unchanged. Indeed, the condition of union of inorganic with organic matters is so intimate that they can not be completely separated without incineration. In view of these facts, it is evident that a certain proportion, at least, of the inorganic salts of the urine is derived from the tissues, of which, in combination with organic matters, they have formed a constituent part. As the kidneys frequently eliminate from the blood foreign matters taken into the system, and are capable sometimes of throwing off an excess of the normal constituents which may be introduced into the circulation, it can readily be understood how a large proportion of some of the inorganic constituents of the urine may be derived from food.

Chlorides. - Almost all the chlorin in the urine is in the form of sodium chloride, the quantity of potassium chloride being insignificant and not of any special physiological importance. By reference to the table of the composition of the urine, it is seen that the proportion of sodium chloride is subject to great variations, the range being between three and eight parts per thousand. This at once suggests the idea that the quantity excreted is dependent to a considerable extent on the quantity taken in with the food; and, indeed, it has been shown by direct observations that this is the fact. The proportion of sodium chloride in the blood seems to be tolerably constant; and any excess that may be introduced is thrown off chiefly by the kidneys. As the chlorides are deposited with the organic matters in all the acts of nutrition, they are found to be eliminated constantly with the products of katabolism of the nitrogenous parts, and their absence from the food does not completely arrest their discharge in the urine. By suppressing salt in the food, its daily excretion may be reduced to between thirty and forty-five grains (I.9 and 2.9 grams). This quantity is less than that ordinarily contained in the ingesta, and under these conditions there is a gradual diminution in the general nutritive activity. In nearly all acute febrile disorders, the chlorin in the urine rapidly diminishes and frequently is reduced to one-hundredth of the normal proportion. The 
quantity rapidly returns to the normal standard during convalescence. Most of the chlorides of the urine are in simple watery solution; but a certain proportion of sodium chloride exists in combination with urea.

The daily elimination of sodium chloride is about one hundred and fifty-four grains (Io grams). The great variations in its proportion in the urine, under different conditions of alimentation, etc., will explain the differences in the estimates given by various authorities.

Sulphates. - In normal urine the sulphates are in the form of salts of potassium and of sodium. It is probable that these in great part pass into the urine with the products of proteid katabolism and are unimportant. A certain proportion of the sulphates, however - about ten per cent - is in a peculiar form of combination with putrefactive products from the large intestine, notably indol, phenol, cresol and skatol. These organic matters are in part absorbed by the blood and in part discharged in the feces. That portion absorbed by the blood is carried to the liver and there combines with sulphates, forming the socalled conjugate, or ethereal sulphates. These sulphates are not toxic, like the organic matters with which they are united, and they are eliminated from the blood by the kidneys. One of the chief products of this kind is potassium indoxyl-sulphate. This may be made to yield indigo by treatment with certain reagents and is called indican. The urinary indican is supposed to represent putrefactive processes in the large intestine.

Although urinary indican is often found in disease, when present in what may be called normal urine it exists in very small quantity. It has been estimated, however, that $\frac{1}{13}$ to $\frac{1}{3}$ of a grain ( 0.005 to 0.02 gram) is discharged in twenty-four hours (Jaffé). It is unfortunate that this product is called indican, as it is likely to be confounded with vegetable indican $\left(\mathrm{C}_{26} \mathrm{H}_{31} \mathrm{NO}_{17}\right)$, which is a glucoside. The formula for indoxylsulphuric acid is $\mathrm{C}_{8} \mathrm{H}_{7} \mathrm{NSO}_{4}$. Urinary indican is $\mathrm{C}_{8} \mathrm{H}_{7} \mathrm{NSO}_{4} \mathrm{~K}$.

Phosphates. - The urine contains phosphates in a variety of forms; but inasmuch as it is not known that any one of the different combinations possesses peculiar relations to the processes of katabolism, as distinguished from the other phosphates, the phosphatic salts may be considered together.

The phosphates exist constantly in the urine and are derived in part from food and in part from the tissues. Like other inorganic matters, they are associated with the nitrogenous constituents of the organism, and when these are changed into excrementitious substances and are separated from the blood by the kidneys, they pass with them and are discharged from the body. 
It is a question of some importance to consider how far the phosphates are derived from the tissues and what proportion comes directly from food. All observers agree that the quantity of phosphates in the urine is in direct relation to the proportion in food, and that an excess of phosphates taken into the stomach is immediately thrown off by the kidneys. It is a familiar fact, indeed, that the phosphates are deficient and the carbonates predominate in the urine of the herbivora, while the reverse obtains in the carnivora, and that variations, in this respect, in the urine may be produced by feeding animals with different kinds of food. Deprivation of food diminishes the quantity of phosphates in the urine, but a certain proportion is discharged that is derived exclusively from the tissues.

In connection with the fact that phosphorus exists in considerable quantity in the nervous matter, it has been assumed that mental exertion is attended with an increase in the elimination of phosphates; and this has been advanced to support the view that these salts are specially derived from katabolism of the brain-substance. Experiments show that it is not alone the phosphates that are increased in quantity by mental work, but urea, the chlorides, sulphates and inorganic matters generally; and in point of fact, physiological conditions that increase the proportion of nitrogenous excrementitious matters increase as well the elimination of inorganic salts. It can not be assumed, therefore, that the discharge of phosphates is specially connected with the activity of the brain. Little has been learned on this point from pathology; for although many observations have been made on the excretion of phosphoric acid in disease - Vogel having made about one thousand different analyses in various affections - no definite results have been obtained. From these facts it is seen that there is no physiological reason why the elimination of the phosphates should be specially connected with the katabolism of any particular tissue or organ, especially as these salts in some form are universally distributed in the organism.

Observations have been made on the hourly variations in the discharge of phosphoric acid at different times of the day; but these do not appear to bear any definite relation to known physiological conditions, not even to the process of digestion.

Of the different phosphatic salts of the urine, the most important are those in which the acid is combined with sodium. These exist in the form of the neutral and acid phosphates. The acid salt is supposed to be the source of the acidity of the urine at the moment of its emission. The so-called neutral salt is slightly alkaline. The proportion of the sodium phosphates in the urine is larger than that of any 
of the other phosphatic salts, but the daily quantity excreted has not been estimated. There exists in the urine a small quantity of the ammonio-magnesian phosphate, but it never in health is in sufficient quantity to form a crystalline deposit. The daily excretion of the phosphates is subject to great variations, but the average quantity of phosphoric acid excreted daily may be estimated at about fifty-six grains (3.629 grams).

The urine contains, in addition to the inorganic salts that have been mentioned, a small quantity of silicic acid; but so far as is known, this has no physiological importance.

Coloring Matter and Mucus. - The peculiar color of the urine is due mainly to the presence of a nitrogenous substance called urochrome. Normal urine, however, contains a number of pigments. A substance called urobilin, although its proportion is very small, is the pigment that has been most carefully studied. This is supposed to result from a transformation of a certain quantity of stercobilin that is taken up by the blood from the intestinal tract and is separated from the blood by the kidneys. After this, however, most of the urobilin is thought to be converted into urochrome. The sum of definite knowledge in regard to the urinary pigments is small; and the physiological relations of these substances are not understood and may be unimportant. It is sufficient to state here that the color of normal urine is due to a mixture of coloring matters, the most important being urochrome. All these matters are originally iron-free derivatives of hemoglobin.

Normal urine always contains a small quantity of mucus, with more or less epithelium from the urinary passages and a few leucocytes. These form a faint cloud in the lower strata of healthy urine after a few hours' repose. The properties of the different kinds of mucus have already been considered. An important peculiarity, however, of the mucus contained in normal urine is that it does not excite decomposition of urea and that the urine may remain for a long time in the bladder without undergoing putrefactive changes.

Gases of the Urine. - In the process of separation of the urine from the blood by the kidneys, certain gases in solution are removed. By using the method employed by Magnus in estimating the gases of the blood, Morin was able to extract about two and a half volumes of gas from a hundred parts of urine. He ascertained, however, that a certain quantity of gas remained in the urine and could not be extracted by the ordinary process. This was about one-fifth of the whole volume of gas. Adding this to the quantity of gas extracted, he obtained the following proportions to one liter of urine, in cubic centimeters (one part per thousand in volume):- 
Oxygen

Nitrogen

Carbon dioxide

The proportion of these gases was found by Morin to be subject to certain variations. For example, after the ingestion of a considerable quantity of water or any other liquid, the proportion of oxygen was considerably increased (from 0.824 to I.024), and the carbon dioxide was diminished more than one-half. The most important variations, however, were in connection with muscular exercise. After walking a long distance, the exercise being taken both before and after eating, the quantity of carbon dioxide was found to be double that contained in the urine after repose. The proportion of oxygen was slightly diminished and the nitrogen was somewhat increased; but the variations of these gases were insignificant.

It is not probable that the kidneys are important as eliminators of carbon dioxide, but the presence of this gas in the urine assists in the solution of some of its saline constituents, notably the phosphates.

Water as a Product of Excretion. - It has been shown by indirect observations that a large proportion of the hydrogen introduced as an ingredient of food, about eighty-five per cent, is not accounted for by the hydrogen of the excreta. Direct observations have shown, also, that under certain conditions an excess of water over that introduced with food and drink is discharged from the body. One of these conditions is abstinence from food (Flint, 1878). The elimination of water is much increased by muscular work (Pettenkofer and Voit, I 868 ; Flint, 1879). These facts point to the actual production of water in the body by a union of oxygen with hydrogen.

While it is not certain that water is constantly produced in the body, there can be no doubt in regard to its formation under some conditions, and the oxidation of hydrogen is important as one of the factors in the production of animal heat. If a certain proportion of the water discharged by the lungs, skin and kidneys is to be regarded as a product of oxidation within the body, the relations which it bears to nutrition probably are the same as those of some of the excretions, especially carbon dioxide, and are subject to nearly the same laws. It has not been shown, however, that water is produced constantly, like the substances commonly regarded as true excretions; and it gives rise to no direct toxic phenomena when retained in the system or when its production is diminished pathologically. Water also has important physiological uses, particularly as a solvent. Still, carbon dioxide, with which water may be compared as regards its mode of production, is not in itself poisonous, its retention in the blood simply interfering with the 
absorption of oxygen; and carbon dioxide probably is useful in increasing the solvent properties of the liquids of the organism.

Variations in the Composition of the Urinc. - The urine not only represents, in its varied constituents, a great part of the physiological disintegration of the organism, but it contains matters evidently derived from food. Its constitution is varying with different conditions of nutrition, with exercise, bodily and mental, with sleep, age, sex, diet, respiratory activity, the quantity of cutaneous exhalation, and, indeed, with every condition that affects any part of the system. There is no liquid in the body that presents such a variety of constituents as a constant condition, but in which the proportion of these constituents is so variable. It is for this reason that in the table of the composition of the urine, the ordinary limits of variation of its different constituents have been given; and it has been found necessary, in treating of the individual excrementitious products, to refer to some of the variations in their proportion in the urine.

Variations with Age and Sex. - There are decided differences in the composition of the urine at different periods of life and in the sexes. These undoubtedly depend in part on the different conditions of nutrition and exercise and in part on differences in the food. Although the quantities of excrementitious matters present great variations, their relations to the organism are not materially modified, except, perhaps, at an early age; and the influence of sex and age operates merely as these conditions affect the diet and the general habits of life.

It has been stated that urea does not exist in the urine of the fœtus; but in a specimen of urine taken from a stillborn child delivered with forceps, examined by Elliot and Isaacs, the presence of urea was determined. Beale found urea in a specimen taken at the seventh month. Observations on children between the ages of three and seven have shown that at this period of life, the urea excreted in proportion to the weight of the body is about double the quantity excreted by the adult.

There are not many definite observations on record in regard to the composition of the urine in the later periods of life. It has been shown, however, that there is a decided diminution at this time in the excretion of urea, and that the absolute quantity of urine is somewhat less.

The absolute quantity of solids excreted is less in women than in men, and the same is true of the quantity in proportion to the weight of the body; still, the differences are not very marked, and the proportion of the urinary constituents being subject to modifications from the same causes as in men, the small deficiency, in the few direct observations on record, may be in part if not entirely explained by the fact 
that women usually perform less mental and physical work than men and that their digestive system is not so active.

Variations at Different Seasons and at Different Periods of the Day. - The changes in the quantity and composition of the urine that may be referred directly to conditions of digestion, temperature, sleep, exercise etc., have long been recognized by physiologists; but it is difficult so to separate these influences that the true modifying value of each can be fully appreciated. For example, there is nothing which produces such marked variations in the composition of the urine as digestion. Under strictly physiological conditions, the modifying influence of digestion must always complicate observations on the effects of exercise, sleep, season, period of the day etc.; and the urine is continually varying in health, with the physiological modifications in the different processes and conditions of life.

At different seasons of the year and in different climates, the urine presents certain variations in its quantity and composition. It seems necessary that a tolerably definite quantity of water should be discharged from the body at all times; and when the temperature or the hygrometric condition of the atmosphere is favorable to the action of the skin, as in a warm, dry climate, the quantity of water in the urine is diminished and its proportion of solid matters is correspondingly increased. On the other hand, the reverse obtains when the action of the skin is diminished from any cause.

At different times of the day the urine presents certain important variations. It is evident that the specific gravity must vary with the relative proportions of water and solid constituents. The urine first discharged in the morning is dense and highly colored; that passed during the forenoon is pale and of a low specific gravity; and in the afternoon and evening it is again deeply colored and its specific gravity is increased. Its acidity is also subject to certain variations, which have already been mentioned.

Influence of Mental Exertion. - Although the influence of mental exertion on the composition of the urine has not been very closely studied, the results of the investigations that have been made are in many regards quite satisfactory. It is a matter of common remark that the secretion of urine often is modified to a considerable extent through the nervous system. Fear, anger and various violent emotions sometimes produce a sudden and copious secretion of urine containing a large proportion of water, and this is often observed in cases of hysteria. Intense mental exertion will occasionally produce the same result.

Internal Secretion. - It is possible that the kidneys are the seat of an internal secretion analogous to the internal secretion of the supra- 
renal capsules. Some observers, indeed, have extracted from the kidneys a substance (renin) that produces an elevation of blood-pressure when injected into the tissues or bloodvessels of living animals, by exciting the vasomotor centres. Renal extract has also been employed in the treatment of uremia, it was thought with favorable results. The entire question, however, demands further investigation.

Work of the Kidncy. - The work of the kidney, as regards the separation of water from the blood-plasma, has been estimated by comparing the osmotic pressure of the urine with that of the bloodplasma, expressed in percentage solutions of sodium chloride. In a typical experiment by Dreser, two hundred cubic centimeters (about $6_{4}^{3}$ fluidounces) of urine were secreted during the night, with a pressure equal to a four per cent solution of sodium chloride. Compared with the blood-plasma, with a pressure equal to a 0.92 per cent solution of sodium chloride, the difference (equal to a 3.08 per cent solution of sodium chloride) is equivalent to 267.88 foot-pounds (thirty-seven kilogrammeters). It must be admitted, however, that this view in regard to the actual work of the kidneys in separating from the blood a given volume of a liquid of a certain osmotic pressure is in some degree speculative. It is not certain that the laws of osmosis can be applied to the processes of secretion absolutely and without reserve, taking no account of a cell-action, with the exact nature of which physiologists are not acquainted. 


\section{CHAPTER XIV}

\section{USES OF THE LIVER - DUCTLESS GLANDS}

Physiological anatomy of the liver - Branches of the portal vein, the hepatic artery and the hepatic duct - Interlobular vessels - Structure of a lobule - Arrangement of the bileducts in the lobules - Anatomy of the excretory passages - Gall-bladder, cystic and common ducts - Chemistry of the liver - Nerves and lymphatics of the liver - Mechanism of the secretion of bile - Quantity of bile - Uses of the bile - Properties and composition of the bile - Biliary salts - Cholesterin and stercorin - Bilirubin - Tests for bile - Excretory action of the liver - Origin of cholesterin - Formation of glycogen in the liver Conditions that influence the quantity of sugar in the blood - Ductless glands and internal secretion - Suprarenal capsules - Cortical substance - Medullary substance - Vessels and nerves - Chemistry of the suprarenal capsules - Addison's disease - The spleen - Fibrous structure - Malpighian bodies - Spleen-pulp - Bloodvessels, nerves and lymphatics Chemical constitution - Variations in volume - Extirpation - Thyroid gland - Structure - Vessels and nerves - Myxœedema - Thymus gland - Pituitary body and pineal gland Acromegaly and giantism - Internal secretion by the testes and ovaries.

\section{Physiological Anatomy of the Liver}

In regard to the descriptive anatomy of the liver, it is sufficient to state that it is situated just below the diaphragm, in the right hypochondriac region and is the largest gland in the body, weighing, when moderately filled with blood, about four and a half pounds ( 2 kilograms). Its weight is somewhat variable, but in a person of ordinary adipose development, its proportion to the weight of the body is about as one to thirtytwo. In early life the liver is relatively larger, its proportion to the weight of the body in the newborn child being as one to eighteen or twenty.

The liver is covered externally with peritoneum, folds or duplicatures of this membrane passing from the surface of the organ to the adjacent parts. These constitute four of the so-called ligaments that hold the liver in place. The proper coat is a thin but dense and resisting fibrous structure, adherent to the substance of the organ, but detached without much difficulty, and very closely united to the peritoneum. This membrane is of variable thickness at different parts of the liver, being especially thin in the groove for the vena cava. At the transverse fissure it surrounds the duct, bloodvessels and nerves, and it penetrates the substance of the organ in the form of a vagina, or sheath, investing the vessels and branching with them. This membrane, as it ramifies in the substance of the liver, is called the capsule of Glisson. It will 
be more fully described in connection with the arrangement and distribution of the hepatic vessels.

The substance of the liver is made up of lobules of an irregularlyovoid or rounded form and about $\frac{1}{25}$ of an inch (I millimeter) in diameter. The space which separates these lobules is about one-quarter of the diameter of the lobule and is occupied by the bloodvessels, nerves and ramifications of the hepatic duct. In certain animals, the pig and the polar bear, the division of the hepatic substance can readily be made out with the naked eye; but in man and in most of the mammalia, the lobules are not so distinct, although their arrangement is essentially the same. The lobules are intimately connected with each other, and branches going to a number of different lobules are given off from the same interlobular vessels; but they are sufficiently distinct to represent, each one, the general anatomy of the secreting portion.

At the transverse fissure, the portal vein, having collected the blood from the abdominal organs, and the hepatic artery, which is a branch of the cœliac axis, penetrate the substance of the liver, with the hepatic duct, nerves and lymphatics, all enveloped in the fibrous vagina, or sheath. The portal vein is by far the larger of the two bloodvessels; and its calibre may be roughly estimated as eight to ten times that of the artery.

The vagina, or capsule of Glisson, is composed of fibrous tissue in the form of a dense membrane, closely adherent to the adjacent structure of the liver, and enveloping the vessels and nerves, to which it is attached by loose areolar tissue. The attachment of the bloodvessels to the sheath is so loose that the branches of the portal vein are collapsed when not filled with blood; presenting a striking contrast to the hepatic veins, which are closely adherent to the substance of the liver and remain open when they are cut across. This sheath is prolonged over the vessels as they branch and follows them in their subdivisions. It varies considerably in thickness in different animals. In man and in the mammalia generally, it is rather thin, becoming more and more delicate as the vessels subdivide. It is lost before the vessels are distributed between the lobules.

The vessels distributed in the liver are the following:-

The portal vein, the hepatic artery and the hepatic duct, passing in at the transverse fissure, to be distributed in the lobules. The bloodvessels are continuous in the lobules with the radicles of the hepatic veins. The duct is to be followed to its branches of origin in the lobules.

The hepatic veins; vessels that originate in the lobules, and collect the blood distributed in their substance by branches of the portal vein and of the hepatic artery. 
Branches of the Portal Vein, the Hepatic Artery and the Hepatic Duct. - These vessels follow out the branches of the capsule of Glisson, become smaller and smaller, and they finally pass directly between the lobules. In their course, however, they send off lateral branches to the sheath, forming the so-called vaginal plexus. The arrangement of the vessels in the sheath is not in the form of a true anastomosing plexus, although branches pass from this so-called vaginal plexus between the lobules. These vessels do not anastomose or communicate with each other in the sheath.

The portal vein does not present any important peculiarity in its course from the transverse fissure to the interlobular spaces. It subdivides, enclosed in its sheath, until its small branches go directly between the lobules; and in its course it sends branches to the sheath (vaginal vessels), which afterward go between the lobules. The hepatic artery has three sets of branches. As soon as it enters the sheath with the other vessels, it sends off minute branches (vasa vasorum) to the walls of the portal vein, to the larger branches of the artery itself, to the walls of the hepatic veins, and a rich network of vessels to the hepatic duct. In its course the hepatic artery also sends branches to the capsule of Glisson (capsular branches), which, with branches of the portal vein, go to form the so-called vaginal plexus. From these vessels a few arterial branches are given off, which pass between the lobules. The hepatic artery can not be followed beyond the interlobular vessels. The terminal branches of the hepatic artery are not directly connected with the radicles of the hepatic veins, but they empty into small branches of the portal vein within the capsule of Glisson.

Interlobular Vessels. - Branches of the portal vein, coming from the terminal ramifications of the vessel within the capsule and from the branches in the walls of the capsule, are distributed between the lobules, constituting the greatest part of the so-called interlobular plexus. These are situated between the lobules and surround them; each vessel, however, giving off branches to two or three lobules, and never to one alone. They do not anastomose and consequently are not in the form of a true plexus. The diameter of the interlobular vessels varies between $\frac{1}{1440}$ and $\frac{1}{20}$ of an inch ( 17 and $34 \mu$ ). In this distribution, the bloodvessels are followed by branches of the duct, which are much fewer and smaller, measuring only $\frac{1}{2500}$ of an inch (10 $\mu$ ), and some, even, have been measured that are not more than $\frac{1}{3000}$ of an inch $(8 \mu)$ in diameter.

Lobular Vessels. - From the interlobular veins eight or ten branches are given off which penetrate the lobule. As the interlobular vessels ramify between different lobules, each one sends branches into two and 
sometimes three of these lobules; so that, so far as vascular supply is concerned, the lobular divisions of the liver are never absolutely distinct.

After passing from the interlobular plexus into the lobules, the vessels immediately break up into an elongated network of capillaries, $\overline{3} \frac{1}{00} \overline{0}$ to $\overline{2} 2 \frac{1}{00}$ of an inch ( 8 to I I $\mu$ ) in diameter, which occupy the lobules with a true plexus. These vessels are very abundant. The blood, having been distributed in the lobules by this lobular plexus, is collected by three or four venous radicles into a single central vessel situated in the long axis of the lobule, called the intralobular vein. A single lobule, surrounded by interlobular vessels, showing the lobular capillary plexus, and the central vein (the intralobular vein) cut across,

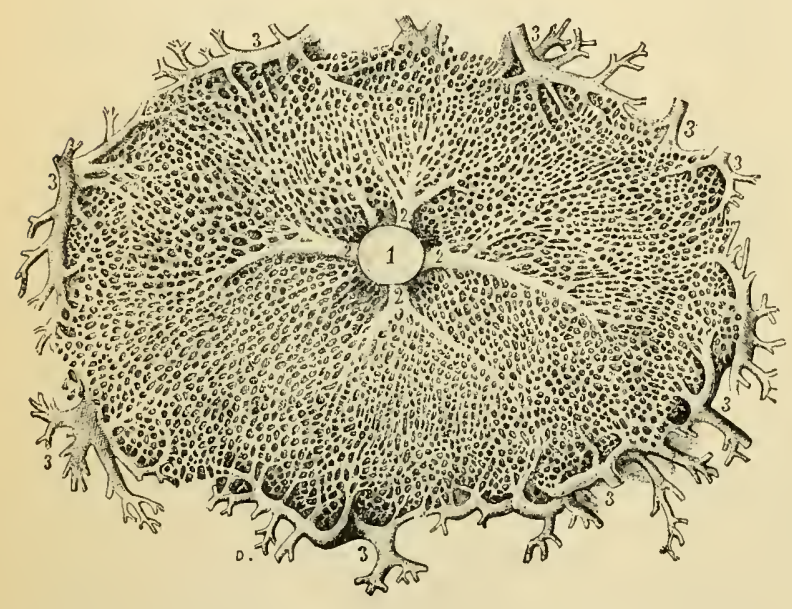

Fig. 75. - Transverse section of a single hepatic lobule (Sappey).

I, intralobular vein, cut across; 2, 2, 2, 2, afferent branches of the intralobular vein; $3,3,3,3,3,3,3,3,3$, interlobular branches of the portal vein, with its capillary branches, forming the lobular plexus, extending to the radicles of the intralobular vein. is represented in Fig. 75 and Plate VII, Fig. 5 .

Intralobular Veins. The capillaries of the lobules converge into three or four venous radicles $(2,2,2,2$, in Fig. 75) which empty into a central vessel. This is the intralobular vein. If a liver is carefully injected from the hepatic veins, and if sections are made in various directions, it will be seen that the intralobular veins follow the long axes of the lobules, receiving vessels in their course, until they empty into a larger vessel situated at what may be called the base of the lobules. These latter are the sublobular veins. They collect the blood, in the manner just described, from all parts of the liver, unite with others, becoming larger and larger, until finally they form the three hepatic veins, which discharge the blood from the liver into the vena cava ascendens.

The hepatic veins differ somewhat in their structure from other veins. Their walls are thinner than those of the portal veins, they are not enclosed in a sheath and they are closely adherent to the hepatic tissue. It has also been noted that the hepatic veins possess a wellmarked muscular tunic, very thin in man but well developed in the pig, 
the ox and the horse, and composed of non-striated muscular fibres interlacing with each other in every direction.

In addition to the bloodvessels just described, the liver receives venous blood from vessels that have been called accessory portal veins, coming from the gastro-hepatic omentum, the surface of the gallbladder, the diaphragm and from the anterior abdominal walls. These vessels penetrate at different points on the surface of the liver, and they may serve as derivatives when the circulation through the portal vein is obstructed.

Structure of a Lobule of the Liver. - Each hepatic lobule, bounded and more or less distinctly separated from the others by the interlobular vessels, contains bloodvessels, radicles of the hepatic ducts and the so-called hepatic cells. The arrangement of the bloodvessels has just been described; but in all preparations made by artificial injection, the space occupied by the bloodvessels is exaggerated by excessive distention, and the difficulties in the study of the relations of the ducts and the liver-cells are thereby much increased.

Liver-cells. - If a scraping from the cut surface of a fresh liver is examined with a moderately high magnifying power, the field of view will be found filled with rounded, ovoid or irregularly-polygonal cells, measuring $\frac{1}{1500}$ to $\frac{1}{100} \overline{0}$ of an inch ( 16 to $25 \mu$ ) in diameter. In their natural condition they are more frequently ovoid than polygonal; and when they have the latter form the corners are always rounded. These cells present one and occasionally two nuclei, sometimes with and sometimes without nucleoli. The presence of small pigmentary granules gives to the cells a peculiar and characteristic appearance; and in addition, nearly all contain a few granules or small globules of fat. Sometimes the fatty and pigmentary granules are so abundant as to obscure the nuclei. The cells also contain more or less glycogen in the form of granules surrounding the nuclei. As regards intimate structure, the liver-cells present a delicate honeycomb network in the meshes of which are contained the granules just described. They have no distinct cellwalls, and they adhere together by portions of their surface so as to form rows, or columns radiating from the centre of each lobule (see Plate VII, Fig. 6).

Arrangement of the Bile-ducts in the Lobules. - In the substance of the lobules is a fine and regular network of vessels of nearly uniform size, about $\frac{1}{10000}$ of an inch ( 2 or $3 \mu$ ) in diameter, which surround the liver-cells, each cell lying in a space bounded by inosculating branches of these canals. This plexus is independent of the bloodvessels, and it seems to enclose in its meshes each individual cell, extending from the periphery of the lobule to the intralobular vein. These canals or inter- 
spaces between the liver-cells in the lobules open into the interlobular hepatic ducts. It is still a question whether these passages are simple spaces between the cells or true vessels lined with a membrane.

Anatomy of the Excretory Biliary Passages. - Between the lobules the ducts are very small, the smallest measuring about $\frac{1}{300}$ of an inch $(8 \mu)$ in diameter. They are composed of a delicate membrane lined with epithelium. The ducts larger than $\frac{1}{1200}$ of an inch (about $20 \mu$ ) have a fibrous coat, formed of inelastic with a few elastic elements, and in the larger ducts, there are in addition a few non-striated muscular fibres. The epithelium lining these ducts is of the columnar variety,

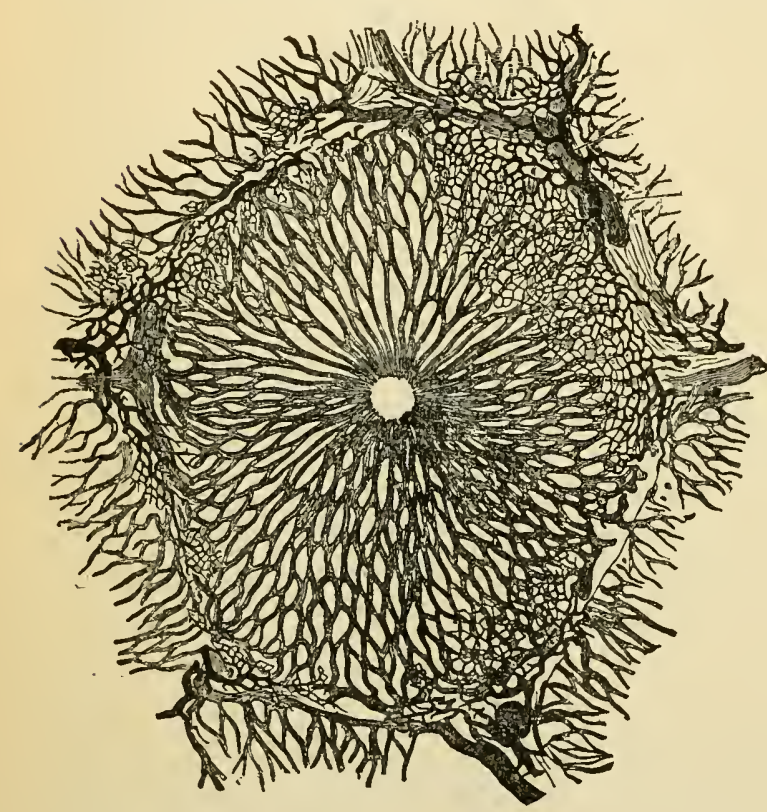

Fig. 76. - Bile-capillaries between the liver-cells-injected liver of the rabbit, $\times 500$ (Pfeiffer). the cells gradually undergoing a transition from the pavement form as the ducts increase in size. In the largest ducts there is a distinct mucous membrane with mucous glands.

Throughout the extent of the biliary passages, from the interlobular canals to the ductus choledochus, are little utricular or racemose glands, varying in size in different portions of the liver. These are situated at short intervals at the sides of the canals. The glands connected with the smallest ducts are simple follicles, $\frac{1}{800}$ to $\frac{1}{400}$ of an inch (3I to $62 \mu$ ) long. The larger glands are formed of groups of these follicles, and they measure $\frac{1}{250}$ or $\frac{1}{100}$ of an inch $(100$ or $250 \mu)$ in diameter. The glands are found connected with the ducts ramifying in the substance of the liver only, and they do not exist in the hepatic, cystic and common ducts. They are composed of a homogeneous membrane lined with small pale cells of epithelium. If the ducts in the substance of the liver are isolated, they are found covered with these little groups of follicles and have the appearance of an ordinary racemose gland, except that the acini are relatively small and scattered. This appearance is represented in Fig. 77.

The excretory biliary ducts, from the interlobular vessels to the point 
of emergence of the hepatic duct, present frequent anastomoses with each other in their course.

Vasa Aberrantia. - In the livers of old persons, and occasionally in the adult, certain vessels are observed ramifying on the surface of the liver but always opening into the biliary ducts, which have been called vasa aberrantia. These are not found in the fœtus or in children. They are appendages of the excretory system of the liver, and are analogous in their structure to the ducts, but are apparently hypertrophied, with thickened fibrous walls, and present in their course irregular constrictions not found in the normal ducts. The racemose glands attached to them are always much atrophied.

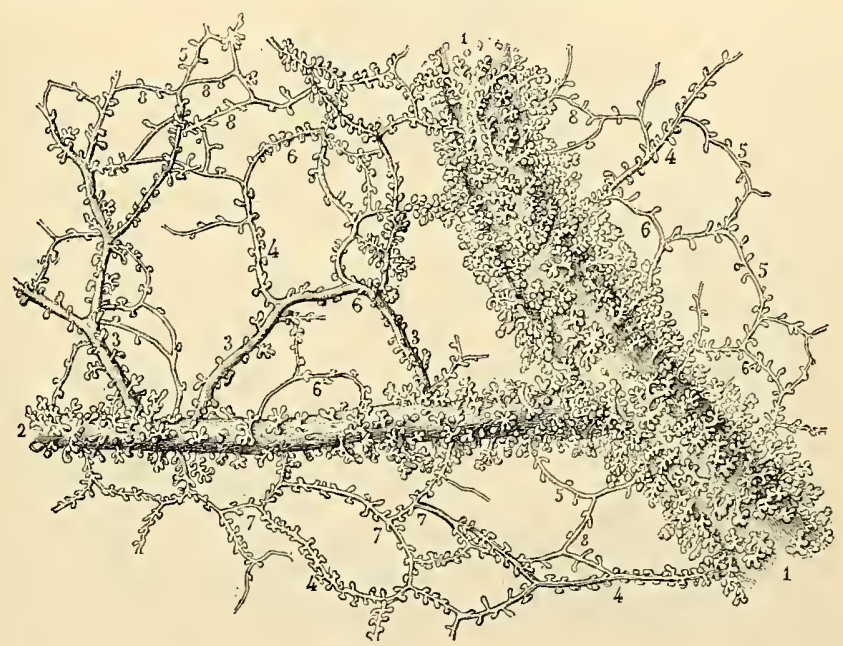

Fig. 77. - Racemose glands attached to the biliary ducts of the pig, $\times$ I8 (Sappey).

I, I, branch of an hepatic duct, with the surface almost entirely covered with racemose glands opening into its cavity; 2 , branch in which the glands are smaller and less abundant; $3,3,3$, branches of the duct with still simpler glands; $4,4,4,4$, biliary ducts with simple follicles attached; $5,5,5,5$, the same, with fewer follicles; $6,6,6,6,6$, anastomoses in arches; $7,7,7$, angular anastomoses; $8,8,8,8$, anastomoses by transverse branches.

Gall-bladder, Hepatic, Cystic and Common Ducts. - The hepatic duct is formed by the union of two ducts, one from the right and the other from the left lobe of the liver. It is about an inch and a half ( 38 millimeters) in length and joins at an acute angle with the cystic duct to form the ductus communis choledochus. The common duct is about three inches ( 76 millimeters) in length, of the diameter of a goose-quill and opens into the descending portion of the duodenum. It passes obliquely through the coats of the intestine and opens into its cavity in connection with the principal pancreatic duct. The cystic duct is about an inch (25 millimeters) in length and is the smallest of the three canals. The structure of these ducts is essentially the same. They 
have a proper coat formed of ordinary fibrous tissue, a few elastic fibres and non-striated muscular fibres. The muscular tissue is not sufficiently distinct to form a separate coat. The mucous membrane is always found tinged yellow with the bile, even in living animals. It presents a large number of minute excavations and is covered with cells of columnar epithelium. This membrane contains a large number of mucous glands.

The gall-bladder is an ovoid or pear-shaped sac, about four inches ( 10 centimeters) in length, one inch ( 25 millimeters) in breadth at its

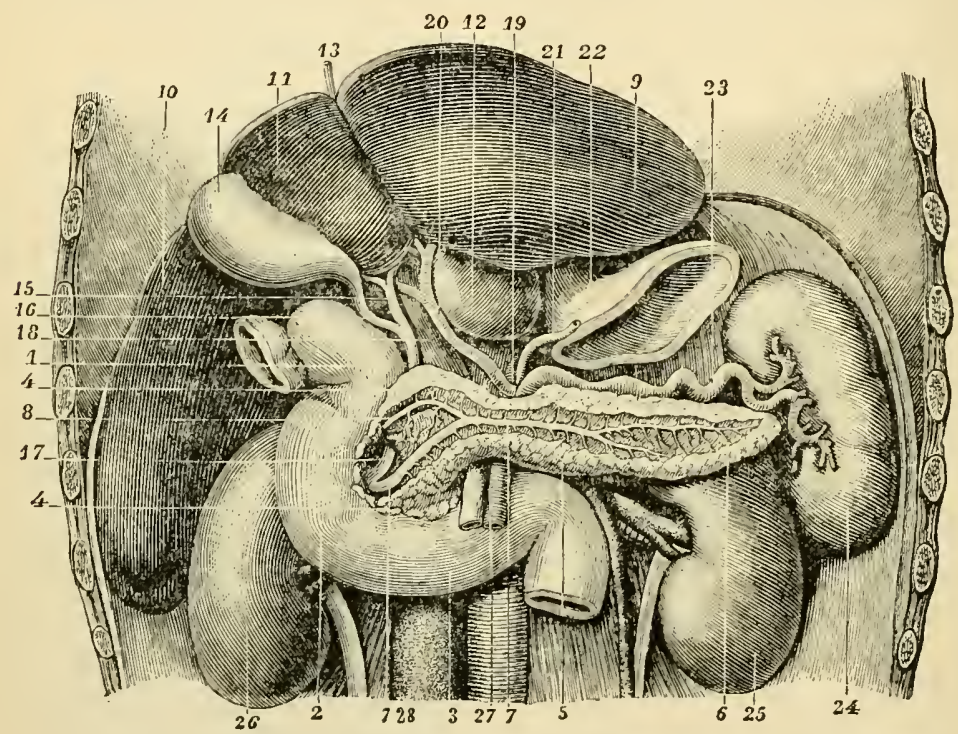

Fig. 78. - Gall-bladder, hepatic, cystic and common ducts (Sappey).

I, 2, 3, duodenum : 4, 4, 5, 6, 7, 7,8, pancreas and pancreatic ducts; 9 , 10, II, 12, 13, liver; 4 , gallbladder; 15, hepatic duct; 16, cystic duct; 17, common duct; 18 , portal vein; 19 , branch from the cœliac axis; 20 , hepatic artery; $2 \mathrm{r}$, coronary artery of the stomach; 22 , cardiac portion of the stomach; 23 , splenic artery; 24, spleen; 25, left kidney; 26, right kidney; 27, superior mesenteric artery and vein; 28 , inferior vena cava.

widest portion, and capable of holding an ounce to an ounce and a half (30 to 45 cubic centimeters) of fluid. Its fundus is covered entirely with peritoneum but this membrane passes only over the lower surface of its body.

The proper coat of the gall-bladder is composed of ordinary fibrous tissue with a few elastic fibres. In some of the lower animals there is a distinct muscular coat, but a few scattered fibres only are found in the human subject. The mucous coat is of a yellowish color, with very small interlacing folds that are very vascular. The mucous membrane of the gall-bladder has a general lining of columnar epithelium with a 
few goblet-cells. In the gall-bladder are found small racemose glands formed of four to eight follicles lodged in the submucous structure. These are essentially the same as the glands opening into the ducts in the substance of the liver and secrete a mucus that is mixed with the bile.

Nerves and Lymphatics of the Liver. - The nerves of the liver are derived from the pneumogastric, the phrenic, and the solar plexus of the sympathetic. The branches of the left pneumogastric penetrate with the portal vein, while the branches from the right pneumogastric, the phrenic and the sympathetic, surround the hepatic artery and the hepatic duct. All these nerves penetrate at the transverse fissure and follow the bloodvessels in their distribution. They have not been traced beyond the final ramifications of the capsule of Glisson and their exact mode of termination is unknown.

The lymphatics of the liver are very abundant. They are divided into two layers; the superficial layer, situated just beneath the serous membrane, and the deep layer. The superficial lymphatics from the under surface of the liver and that portion of the deep lymphatics which follows the hepatic veins out of the liver pass through the diaphragm and are connected with the thoracic glands. Some of the lymphatics from the superior, or convex surface join the deep vessels that emerge at the transverse fissure and pass into glands below the diaphragm, while others pass into the thoracic cavity.

The mode of origin of the lymphatics is peculiar. The superficial lymphatics are subperitoneal and are connected with spaces or canals in the general connective tissue of the liver. The deep lymphatics are supposed to originate by perivascular canals surrounding the bloodvessels of the lobules, which are connected with vessels in the walls of small branches of the hepatic and portal veins, afterward surrounding the larger vessels.

Chemistry of the Liver. - As regards the chemistry of the liver, little has been ascertained that is of much physiological importance. The liver-cells contain globulins, nucleo-proteids, cholesterin, urea, uric acid, xanthin, hypoxanthin and sometimes leucin and tyrosin. In addition, a nitrogenous substance $\left(\mathrm{C}_{105} \mathrm{H}_{186} \mathrm{~N}_{5} \mathrm{SP}_{3} \mathrm{O}_{45}\right)$ containing phosphorus has been found. This has been called jecorin (Drechsel), although it is not confined to the liver but exists in the spleen, brain, muscle and some other tissues. In most of its properties it resembles lecithin.

Mechanism of the Secretion and Discharge of Bile. - In its anatomy the liver differs greatly from other glandular organs, both secretory and excretory. The liver-cells are not enclosed in ducts, but are surrounded with a plexus of small vessels or spaces that receive the bile as it is 
formed. The liver, also, is supplied with both venous and arterial blood, the venous blood largely predominating. In addition it is now recognized that the bile is necessary to intestinal digestion, that it contains excrementitious matters and that the cells constantly produce glycogen. The liver produces urea, which is excreted, however, chiefly by the kidneys. It also effects certain important changes in digested and foreign matters that are absorbed from the alimentary canal.

As regards the bile, the only view that is consistent with actual knowledge is that this secretion is produced by the liver-cells and is taken up by the plexus of canals that surrounds these cells. The little glandular organs attached to the larger branches of the duct secrete mucus that gives the viscidity observed in the bile of some animals. The bile, indeed, is viscid in different animals in proportion to the development of these mucous glands; and in the rabbit, in which the glands do not exist, the bile has no viscidity.

Of course the circulation of blood in the liver is a condition necessary to the secretion of bile. As regards the question of the production of bile from venous or arterial blood, it has been shown that the materials out of which the bile is formed may be supplied by either the hepatic artery or the portal vein. Bile is secreted after the hepatic artery has been tied, and also after the portal vein has been gradually obliterated, the hepatic artery being intact. Bile is produced in the liver from the blood distributed in its substance by the portal vein and the hepatic artery, and not from the blood of either of these vessels exclusively; and bile may continue to be secreted, if either one of these vessels is obliterated, provided the supply of blood be sufficient.

The influence of the nervous system on the secretion of bile has been little studied; and the question is one of some difficulty and obscurity. The liver is supplied abundantly with nerves, both cerebro-spinal and sympathetic, and some observations have been made on the influence of the nerves on its glycogenic action; but in regard to the secretion of bile, there is little to be said beyond what has already been stated concerning the influence of the nervous system on other secretions.

The bile is discharged through the hepatic ducts like the secretion of any other gland. During digestion the liquid accumulated in the gall-bladder passes into the ductus communis, in part by contractions of its walls, and in part, probably, by compression exerted by the distended and congested digestive organs adjacent to it. It seems that this secretion - which necessarily is produced by the liver without intermission, separating from the blood certain excrementitious matters - is retained in the gall-bladder for use during digestion.

Quantity of Bile. - The estimates of the daily quantity of bile in the 
human subject must be merely approximate; and the ideas of physiologists on this point are derived chiefly from experiments on the inferior animals. There are great variations in the daily quantity in different classes of animals, the quantity in the carnivora being the smallest. Applying the results of experiments on the lower animals to the human subject, and assuming that the amount is about equal to the quantity secreted by the carnivora, the daily secretion in a man weighing one hundred and forty pounds ( 63.5 kilograms) would be about two and a half pounds (I 34 grams).

\section{UsEs OF THE BILE}

The uses of the bile in digestion have already been fully described; but before considering its characters as an excretion, it will be necessary to study its general properties and composition.

Properties and Composition of the Bile. - The secretion as it comes directly from the liver is somewhat viscid; but after it has passed into the gall-bladder, its viscidity is much increased by a further admixture of mucus.

The color of the bile is very variable within the limits of health. It may be of any shade between a dark yellowish green and a reddish brown. It is semitransparent except when the color is very dark. In different classes of animals the variations in color are considerable. In the pig it is bright yellow; in the dog it is dark brown; and in the ox it is greenish yellow. As a rule the bile is dark green in the carnivora and greenish yellow in the herbivora.

The specific gravity of human bile from the gall-bladder is 1026 to I032. When perfectly fresh it is almost inodorous but it readily undergoes putrefactive changes. It has a disagreeable and bitter taste. It is not coagulated by heat. When mixed with water and shaken, it becomes frothy, probably on account of the tenacious mucus and its saponaceous constituents.

It usually is stated that the bile is alkaline. This is true of the liquid discharged from the hepatic duct, although the alkalinity is not strongly marked; but the reaction varies after it has passed into the gall-bladder. In the hepatic ducts the reaction always is alkaline; and there are no observations on human bile that show that it is not alkaline in all the biliary passages.

The epithelium of the biliary passages is strongly tinged with yellow even in living animals. This is due to the facility with which the coloring matter of the bile stains the animal tissues.

Perfectly normal and fresh bile, examined with the microscope, presents a certain quantity of mucus, the characters of which have already 
been described. There are no formed anatomical elements characteristic of this secretion. The fatty and coloring matters are in solution and not in the form of globules or granules.

\section{COMPOSITION OF HUMAN BILE (ROBIN)}

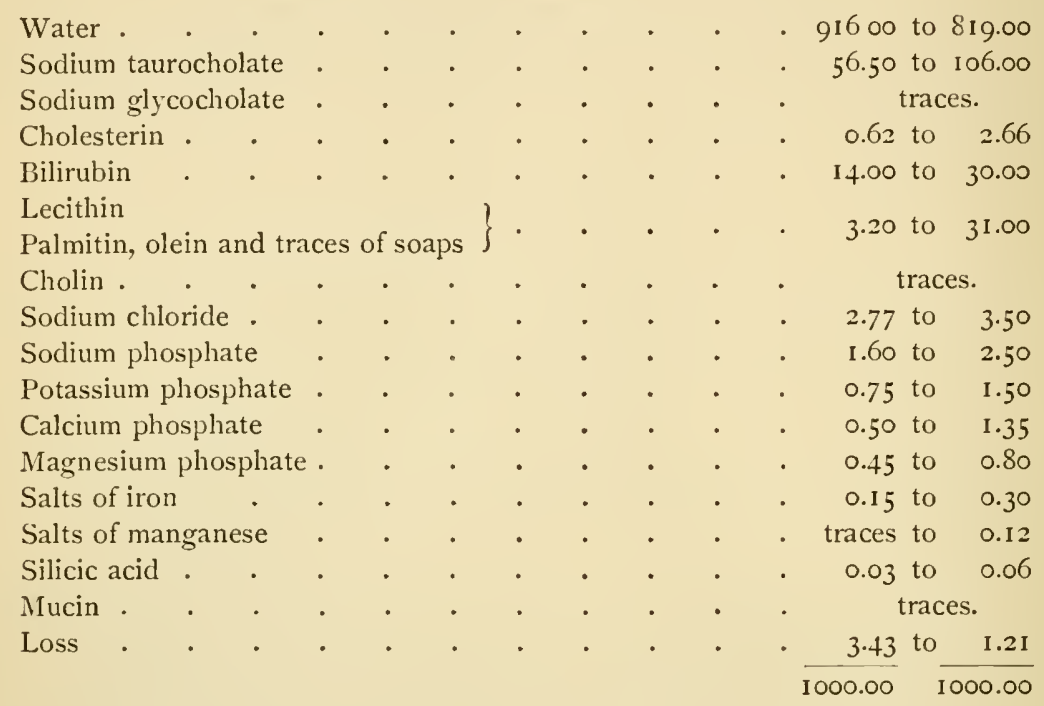

The bile contains no coagulable organic matters except mucin, and all its constituents simply are solids in solution. The quantity of solid matter is large and the proportion of water is relatively small. Among the inorganic salts, sodium chloride exists in considerable quantity, with a large proportion of phosphates. There exist, also, salts of iron and of manganese, with a small quantity of silicic acid.

The fatty and saponaceous constituents of the bile demand hardly any more extended consideration. A small quantity of palmitin and olein are held in solution, partly by the soaps, but chiefly by the sodium taurocholate. The fats sometimes exist in larger quantity, when they may be discovered in the form of globules. The proportion of soaps is small. Lecithin $\left(\mathrm{C}_{44} \mathrm{H}_{90} \mathrm{NPO}_{9}\right)$ is a neutral fatty substance extracted from the bile, and may be decomposed into phosphoric acid and glycerin. Cholin $\left(\mathrm{C}_{5} \mathrm{H}_{15} \mathrm{NO}_{2}\right)$ is found in the bile in minute quantity, and when present, it is supposed to be one of the decomposition-products of lecithin.

Biliary Salts. - In human bile the characteristic biliary salt is a combination of taurocholic acid $\left(\mathrm{C}_{26} \mathrm{H}_{45} \mathrm{NSO}\right)$ with sodium. A small quantity of sodium exists in combination with glycocholic acid $\left(\mathrm{C}_{26} \mathrm{H}_{43} \mathrm{NO}_{6}\right)$. Sodium glycocholate exists in quantity in ox-gall. Both these salts may be precipitated from an alcoholic extract of bile by an 
excess of ether. The taurocholate is precipitated in the form of dark resinous drops which crystallize with difficulty. The glycocholate is readily crystallizable. The biliary salts are very soluble in water and in alcohol. Their reaction is neutral.

There can be no doubt that the biliary salts are products of secretion and are formed in the substance of the liver. In no instance have they been discovered in the blood in health; and although they present certain points of resemblance with some of the constituents of the urine, they are not found in the excreta, except in very rare instances in the urine. There is no reason, therefore, for supposing that these salts are products of katabolism. Once discharged into the intestine, they undergo certain changes and can no longer be recognized by the usual tests; but experiments have shown that, changed or unchanged, they are absorbed with the products of digestion. They probably are concerned in the digestive action of the bile.

Cholesterin. - Cholesterin $\left(\mathrm{C}_{27} \mathrm{H}_{46} \mathrm{O}\right)$ is a normal constituent of various of the tissues and liquids of the body. Most authors state that it is found in the bile, blood, liver, nervous tissue, crystalline lens, meconium and fecal matter. It is to be found in all these situations, with the exception of the feces, where it does not exist normally, being transformed into stercorin in its passage down the intestinal canal.

In the liquids of the body cholesterin exists in solution; but by virtue of what constituents it is held in this condition is a question not entirely settled. It is stated that the biliary salts have the power of holding cholesterin in solution in the bile, and that the small quantity of fatty acids contained in the blood also holds it in solution; but direct experiments on this point are wanting. In the nervous tissue and in the crystalline lens, it is united with the other substances that go to make up these parts. After it is discharged into the intestinal canal, when it is not changed into stercorin it is to be found in a crystalline form, as in the meconium and in the feces of certain animals in hibernation. In pathological fluids and in tumors, it is found in a crystalline form and may be detected by microscopical examination.

Cholesterin is a monatomic alcohol. It is neutral, inodorous, crystallizable, insoluble in water, soluble in ether, and very soluble in hot alcohol though sparingly soluble in cold alcohol. It is inflammable and burns with a bright flame. When treated with strong sulphuric acid it strikes a peculiar red color. It may easily and certainly be recognized under the microscope by the form of its crystals. These are rectangular or rhomboidal, very thin and transparent, of variable size, with distinct and usually regular borders, and frequently arranged in layers with the borders of the lower strata showing through those which are super- 
imposed. The plates of cholesterin often show a cleavage at one corner, the lines running parallel to the borders. Frequently the plates are rectangular and sometimes they are lozenge-shaped.

The proportion of cholesterin in the bile usually is estimated at 0.62 to 2.66 parts per thousand. In a single examination of the human bile, however, the proportion was 0.618 of a part per thousand (Flint).

Bilinubin. - The coloring matter of the bile, bilirubin $\left(\mathrm{C}_{32} \mathrm{H}_{36} \mathrm{~N}_{4} \mathrm{O}_{6}\right)$, bears a certain resemblance to the coloring matter of the blood and is supposed to be formed from it in the liver. It gives to the bile its peculiar tint and has the property of coloring the tissues with which it comes in contact. Whenever the flow of bile is obstructed for any considerable time, the coloring matter is absorbed by the blood and can readily be detected in the serum and in the urine. It also colors the skin and the conjunctiva. It is soluble in chloroform, by which it is distinguished from biliverdin, and forms soluble combinations with alkalies, in which form it is thought to exist in the bile. It probably is formed in the liver from the hemoglobin of the red blood-corpuscles. When exposed to the air or to the influence of certain oxidizing agents, it assumes a greenish color and is changed into biliverdin. It is unnecessary to follow the various other changes produced by spontaneous decomposition or by the action of reagents.

Tests for Bile. - A simple test for bile-pigment is the following: A ten per cent solution of iodin in alcohol is floated on the suspected solution in a test tube. If the coloring matter of bile is present, a green ring will appear between the two liquids.

A delicate test for the biliary salts in a clear solution not containing albumin is what is known as Pettenkofer's test: To the suspected liquid are added a few drops of a strong solution of cane-sugar. Sulphuric acid is then slowly added, to the extent of about two-thirds of the bulk of the liquid. It is recommended to add the acid slowly so that the temperature shall be but little raised. If a large quantity of the biliary salts is present, a red color shows itself almost immediately at the bottom of the test tube, and this soon extends through the entire liquid, rapidly deepening until it becomes dark lake or purple. If the biliary matter exists in small proportion, it may be several minutes before a red color makes its appearance, and the change to a purple is correspondingly slow, the whole process occupying fifteen to twenty minutes.

\section{Excretory Action of the Liver}

Although the liver produces a greater or less quantity of urea, this substance is discharged from the body chiefly in the urine and mere 
traces exist in the bile. The excretory action of the liver will be considered, in this connection, with reference to the bile itself. At the present day it is admitted that the bile is an excretion as well as a secretion; and this question has been fully discussed in connection with the physiology of digestion. The constant and invariable presence of cholesterin in the bile assimilates it in every regard to the excretions, of which the urine may be taken as the type. Cholesterin always exists in the blood and in certain of the tissues of the body. It is not produced in the substance of the liver, but is merely separated from the blood by this organ. It is constantly passed into the intestine, and is discharged, although in a modified form, in the feces. Physiologists know of no office which it has to perform in the economy, any more than urea or any other of the excrementitious constituents of the urine. It accumulates in the blood in certain cases of organic disease of the liver and gives rise to grave nervous symptoms.

Origin of Cholesterin. - Cholesterin exists in largest quantity in the substance of the brain and nerves. It is also found in the substance of the liver - probably in the bile contained in this organ - the crystalline lens and the spleen; but with these exceptions, it is found only in the nervous tissue and blood. It is either deposited in the nervous matter from the blood or it is formed in the brain and taken up by the blood. This is a question, however, that can be settled experimentally.

In a series of experiments made in 1862 , it was invariably found that the proportion of cholesterin in the blood of the internal jugular vein and the femoral vein was greater than in the arterial blood. In experiments made on dogs not etherized, the blood of the jugular vein contained, in one instance 23.3 and in another 59.8 per cent more cholesterin than the arterial blood of the same animals. The blood of the femoral vein contained about 6.3 per cent more cholesterin than arterial blood. In three cases of hemiplegia, cholesterin was found in normal quantity in blood taken from the arm of the sound side, while blood from the paralyzed side contained no cholesterin (Flint).

These observations point to the production of cholesterin in the tissues; and the fact of its existence, under normal conditions, in the nervous tissue renders it probable that the chief seat of its formation is the substance of the nerve-centres and nerves. The question of its production in the spleen is one that has not been investigated.

In another series of experiments, it was shown that the blood lost cholesterin in passing through the liver. In one observation it was found that the arterial blood lost a little more than 23 per cent and the portal blood about $4 \frac{1}{2}$ per cent, in passing through the liver (Flint).

The portal blood, as it goes to the liver, contains but a small per- 
centage of cholesterin over the blood of the hepatic veins, while the percentage in the arterial blood is large. The arterial blood is the mixed blood of the entire system; and as it probably passes through no organ which diminishes its cholesterin before it goes to the liver, it contains a quantity of this substance which must be removed. The portal blood, coming from a limited part of the system, contains less cholesterin, although it gives up a certain quantity. In the circulation in the liver, the portal system largely predominates and is necessary to other important actions of this organ, such as the production of glycogen; but soon after the portal vein enters the liver, its blood becomes mixed with that from the hepatic artery, and from this mixture the cholesterin is separated. It is necessary only that blood containing a certain quantity of cholesterin, should come in contact with the bile-secreting cells in order that this substance shall be separated. The fact that it is eliminated by the liver is proved with much less difficulty than that it is formed in the nervous system. In fact, its presence in the bile, and the necessity of its constant removal from the blood, consequent on its constant formation and absorption, are almost sufficient in themselves to warrant the conclusion that it is eliminated by the liver.

In treating of the composition of the feces, the changes which the cholesterin of the bile undergoes in its passages down the intestinal canal have been fully considered. But one examination only was made of the quantity of stercorin contained in the daily fecal evacuation; and assuming that the quantity of cholesterin excreted by the liver is equal to the stercorin found in the evacuations, the quantity in twenty-four hours is about ten and a half grains (0.68 gram). This corresponds with the estimates of the daily quantity of cholesterin excreted, calculated from its proportion in the bile and the estimated daily quantity of bile produced by the liver.

To complete the chain of the evidence leading to the conclusion that cholesterin is an excrementitious product formed in certain of the tissues and eliminated by the liver, it is necessary only to show that it may accumulate in the blood when the eliminating action of the liver is interrupted.

In a case of simple jaundice from duodenitis, in which there was no great disturbance of the system, a specimen of blood taken from the arm presented evidences of the coloring matter of the bile, but the proportion of cholesterin was not increased, being only 0.508 of a part per thousand. The feces contained a large proportion of saponifiable fat but no cholesterin or stercorin.

In a case of cirrhosis with jaundice, there was ascites, with great general prostration. This patient died a few days after the blood and 
feces had been examined, and the liver was found in a condition of cirrhosis, with the liver-cells shrunken and the gall-bladder contracted. In this case the blood contained $\mathbf{I} .85$ of a part of cholesterin per thousand, more than double the largest quantity found in health. The feces contained a small quantity of stercorin.

Inasmuch as cases frequently present themselves in which there are evidences of cirrhosis of the liver with little if any constitutional disturbance, while others are attended with grave nervous symptoms, it seemed an interesting question to determine whether it were possible for cholesterin to accumulate in the blood without the ordinary evidences of jaundice. An opportunity occurred to examine the blood in two strongly-contrasted cases of cirrhosis, in neither of which was there jaundice. One of these patients had been tapped repeatedly - about thirty times - but the ascites was the only troublesome symptom and the general health was little impaired. In this case the proportion of cholesterin in the blood was only 0.246 of a part per thousand, considerably below the quantity ordinarily found in health. The other patient had cirrhosis, but he was confined to the bed and was very feeble. The proportion of cholesterin in the blood in this case was 0.922 of a part per thousand, a little above the largest proportion found in health. A few other pathological observations of this kind are on record. Picot, in I872, reported a fatal case of "grave jaundice," in which he determined a great increase in the quantity of cholesterin in the blood, the proportion being I.804 per thousand.

It is probable that organic disease of the liver, accompanied with grave symptoms affecting usually the nervous system, does not differ in its pathology from cases of simple jaundice in the fact of retention of the biliary salts in the blood; but these grave symptoms, it is more than probable, are due to a deficiency in the elimination of cholesterin and its consequent accumulation in the system. This condition may be characterized by the name cholesteremia, a term expressing a pathological condition, but at the same time indicating the physiological relations of cholesterin.

Koloman Müller, in I873, succeeded in injecting cholesterin into the bloodvessels without producing any.effects due to mechanical obstruction of the circulation. He made a preparation by rubbing cholesterin with glycerin and mixing the mass with soap and water. He injected into the veins of dogs 2. I6 fluidounces (about 64 cubic centimeters) of this solution, containing about 69 grains ( 4.5 grams) of cholesterin. In five experiments of this kind, he produced a complete representation of the phenomena of "grave jaundice."

In view of all these facts, an excretory action of the liver, involving 
the separation of cholesterin from the blood and its discharge in the feces in the form of stercorin, may be regarded as established, as well as the existence of cholesteremia as a definite pathological condition (Flint, 1862).

\section{Formation of Glycogen in the Liver}

In addition to the uses of the liver already described, this organ constantly contains in health a substance resembling starch, called glycogen, which is converted into glucose and is carried into the circulation by the hepatic veins.

Glycogen belongs to the class of carbohydrates, is isomeric with starch and is readily converted into glucose. In nearly all regards it has the properties of starch, but it gives a deep red color with iodin instead of a blue. In the liver-cells it exists in the form of amorphous granules surrounding the nuclei. It may be extracted from a decoction of the liver-substance, precipitating the albuminous matters by adding alternately dilute hydrochloric acid and potassio-mercuric iodide, filtering and treating the filtrate with an excess of alcohol. The alcoholic precipitate, washed with alcohol and dried rapidly, is in the form of a white powder that will keep indefinitely. In the adult, glycogen is most abundant in the liver; but it has been found in small quantity in the muscular substance, in cartilage and in certain cells in process of development. In the early months of fœtal life it exists in nearly all the tissues and - is found, also, in cells attached to the villi of the placenta.

The most important of the conditions that influence the quantity of glycogen in the liver relate to alimentation and digestion. The liver always contains more glycogen during digestion than fasting. After a few days of starvation, glycogen may almost or quite disappear from the liver. This also occurs in animals fed for a time exclusively with fats, and the quantity is diminished by a purely albuminous diet as contrasted with a mixed diet. Still, glycogen is invariably present in the livers of healthy carnivorous animals that have always been fed with meat alone.

A great increase in the quantity of glycogen in the liver is produced by feeding animals largely with carbohydrates. Not only are the starches apparently stored up for a time in the form of glycogen in the liver, but sugars undergo a change into glycogen, which accumulates in the liver. This is to be expected, as the starches are changed into sugar before they are absorbed and all the carbohydrates behave in the same way as regards general nutrition.

So far as regards the influence of alimentation on the formation of glycogen, it seems probable that in the herbivora and in man, the chief 
source of hepatic glycogen is the class of alimentary substances called carbohydrates; but the fact that glycogen exists in the livers of the carnivora, and probably in man, under a nitrogenous diet, shows that the liver is capable of forming glycogen from albuminous matters.

Change of Glycogen into Sugar. - It is almost certain that the liver does not contain sugar during life. Many years ago (I858) this fact was recognized by Pavy, and it has since been confirmed by other physiologists. Pavy, however, assumed that there was no such thing as sugar-formation by the liver, under absolutely normal conditions. $\mathrm{He}$ regarded the sugar found in the substance of the liver and in the blood of the hepatic veins as due to post-mortem action, and his observations seemed to be directly opposed to those of Bernard. The views of these two observers and their followers seemed to be harmonized by a series of experiments made in I 868. If the abdomen of a dog, perfectly quiet and not under the influence of an anesthetic, is opened, and a portion of the liver excised, rinsed in cold water, and rapidly cut up into boiling water, the extract will show no reaction with Fehling's test for sugar. In one experiment, in which twenty-eight seconds elapsed between the time of opening the abdomen and the action of the boiling water, the reaction with Fehling's test was doubtful. In an experiment in which the time was only ten seconds, there was no trace of sugar in the extract from the liver (Flint). All observers are now agreed that sugar is formed in the liver very rapidly after death.

If the view is correct - that the glycogen of the liver is being constantly transformed into sugar during life, and that this sugar is carried away in the blood-current, as fast as it is formed - sugar would not necessarily be contained in the liver under normal conditions; and there is no actual antagonism between the results obtained by Bernard and the fact that sugar itself is not a normal constituent of the liver, as is asserted by Pavy, McDonnell, Meissner, Ritter and others.

If the liver is washed by means of a stream of water passed through its vessels until it is free from sugar and is then kept at the temperature of the body for a few hours, sugar will reappear in abundance. This is due to a conversion of the glycogen of the liver into sugar by a ferment, which has been extracted and isolated by a process analogous to that by which similar ferments have been extracted from the saliva and the pancreatic juice. This ferment probably exists originally in the liver and does not appear first in the blood.

The question of the transformation of glycogen into sugar during life depends on the comparative quantities of sugar in the blood going to and coming from the liver. Sugar is found in quantity in the blood of the hepatic veins taken immediately after death and exists in blood drawn 
during life by a catheter introduced into the right cavities of the heart; while in the carnivora, under a purely animal diet, no sugar is contained in the blood of the portal system. The normal blood contains, perhaps, a small quantity of sugar - 0.5 to I part per thousand - but the proportion is always greater in the blood of the hepatic veins.

The characters of animal sugar do not materially differ from those of glucose, except that it ferments more readily and is destroyed in the system with great facility. This property of the sugar resulting from the glycogen formed in the liver probably is of great importance. The sugar which results from digestion is all carried to the liver. Here it is changed into glycogen; and it is probable that without this change into glycogen and its subsequent transformation into what is called liversugar, it is not perfectly adapted to the purposes of nutrition.

The sugar discharged into the venous system by the hepatic veins usually is lost in the passage of the blood through the lungs. The question of the final destination of sugar will be taken up again in connection with the physiology of nutrition.

Conditions that influence the Quantity of Sugar in the Blood. - It is probable that disturbances of the circulation in the liver are the most important conditions influencing the discharge of sugar by the hepatic veins, and these operate mainly through the nervous system.

A notable experiment on the influence of the nervous system on the liver is the one in which artificial diabetes is produced by stimulation of the floor of the fourth ventricle (Bernard). This operation is not difficult. The instrument used is a delicate stilet, with a flat cutting extremity and a small projecting point about $\frac{1}{25}$ of an inch (I millimeter) long. In performing the operation on a rabbit, the head of the animal is firmly held in the left hand, and the skull is penetrated in the median line, just behind the superior occipital protuberance. This can easily be done by a few lateral movements of the instrument. Once within the cranium, the instrument is passed obliquely downward and forward, so as to cross an imaginary line drawn between the two auditory canals, until its point reaches the basilar process of the occipital bone. The point then penetrates the medulla oblongata, between the roots of the auditory nerves and the pneumogastrics, and its projection serves to protect the nervous centre from more serious injury from the cutting edge. The instrument is then carefully withdrawn and the operation is completed. This experiment is almost painless, and it is not desirable to administer an anesthetic, as this, in itself, would disturb the glycogenic process. The urine may be obtained before the operation, by pressing the lower part of the abdomen, taking care not to allow the bladder to pass up above the point of pressure, and it will be found turbid, alka- 
line and without sugar. In one or two hours after the operation, the urine will have become clear and acid, and it will react readily with any of the copper tests. When this operation is performed without injuring the adjacent organs, the presence of sugar in the urine is temporary; and the next day the secretion will have returned to its normal condition. The production of glycosuria in this way, in animals, is important in its relations to certain cases of diabetes in the human subject, in which the affection is traumatic and directly attributable to injury near the bulb. The irritation is not conveyed through the pneumogastric nerves, for the experiment succeeds after both of these nerves have been divided; nevertheless, the pneumogastrics have an important influence on glycogenesis. If both nerves are divided in the neck, in a few hours or days, depending on the length of time that the animal survives the operation, no sugar is to be found in the liver, and there is reason to believe that the glycogenic action has been arrested. After division of the nerves in the neck, stimulation of their peripheral ends does not affect the production of sugar; but stimulation of the central ends produces an impression that is conveyed to the nerve-centre, is reflected to the liver and gives rise to an increased production of sugar. It reaches the liver probably through the sympathetic system.

It has been observed that the inhalation of anesthetics and irritating vapors produces temporary glycosuria; and this has been attributed to an irritation conveyed by the pneumogastrics to the nerve-centre, and reflected, in the form of a stimulus, to the liver. It is for this reason that the administration of anesthetics should be avoided in experiments on glycogenic action.

In addition to the varied uses of the liver that have been described, it is thought that this organ either arrests or in some way influences the condition of certain foreign and poisonous substances absorbed from the alimentary canal; but a study of this action does not properly belong to physiology.

\section{Ductless Glands and Interyal Secretion}

Certain organs in the body, with a structure resembling, in some respects, the true glands, but without excretory ducts, have long been the subject of physiological speculation; and the most extravagant notions concerning their uses prevailed in the early history of physiology. The discovery of the action of the liver in modifying the composition of the blood passing through its substance foreshadowed the probable mode of action of the ductless glands; for so far as the production of glycogen is concerned, the liver belongs to this class. Indeed, 
the supposition that the ductless glands effect certain changes in the blood is now regarded by physiologists as the most reasonable of the many theories that have been entertained concerning their uses in the economy. Under this idea, these organs have been called blood-glands or vascular glands, and their action is now known as internal secretion. Under the head of ductless glands, are classed the suprarenal capsules, the spleen, the thyroid gland, the thymus, and sometimes the pituitary body and the pineal gland.

\section{Suprarenal Capsules}

The suprarenal capsules, or adrenals, as their name implies, are situated above the kidneys. They are small triangular flattened bodies, situated behind the peritoneum and capping the kidneys at the anterior portion of their superior ends. The left capsule is a little larger than the right and is rather semilunar in form, the right being more nearly triangular. Their size and weight are variable in different individuals. It may be stated as an average that each capsule weighs about one hundred grains (6.5 grams). The capsules are about an inch and a half (38 millimeters) in length, a little less in width, and about one-fourth of an inch (6.4 millimeters) in thickness.

The weight of the capsules, in proportion to the weight of the kidneys, presents great variations at different periods of life. They are relatively much larger in the fœtus than after birth. They are easily distinguished in the fœtus of two months; at the end of the third month they are a little larger and heavier than the kidneys; they are equal in size to the kidneys - though a little lighter - at four months; and at the beginning of the sixth month they are to the kidneys as two to five. In the fœtus at term the proportion is as one to three, and in the adult, as one to twenty-three (see Plate XVI, Fig. 3).

The color of the capsules is whitish yellow. They are completely covered by a thin fibrous coat which penetrates their interior in the form of trabeculæ. On section they present a cortical and a medullary substance. The cortex is yellowish and $\frac{1}{25}$ to $\frac{1}{12}$ of an inch (I to 2 millimeters) in thickness. It surrounds the capsule completely and constitutes about two-thirds of its substance. The medullary substance is whitish, very vascular, and is remarkably prone to decomposition, so that it is desirable to study the anatomy of these bodies in specimens that are perfectly fresh.

Cortical Substance. - The cortical substance is divided into two layers. The external layer is pale yellow and is composed of closed vesicles, rounded or ovoid in form, containing an albuminous liquid, cells, nuclei and fatty globules. This layer is very thin. The greater part of the 
cortical substance is of a reddish brown color and is composed either of closed tubes containing cells or of columns of cells surrounded with delicate fibrous trabeculæ. On making thin sections through the cortical substance previously hardened in chromic acid and rendered clear by glycerin, rows of cells are seen, arranged with great regularity, and extending, apparently, from the investing membrane to the medullary substance. The cells appear to be enclosed in tubes measuring $\frac{1}{1000}$ to $\frac{1}{300}$ of an inch $(25$ to $80 \mu)$ in diameter. They are granular, with a distinct nucleus and nucleolus and a variable number of oil-globules. They measure $\frac{1}{1750}$ to $\frac{1}{100} \overline{0}$ of an inch (14 to $25 \mu$ ) in diameter. Between the rows of cells of the cortical substance are bands of fibrous tissue connected with the investing membrane of the capsule.

Medullary Substance. - The medullary substance is much paler and more transparent than the cortex. In its centre are openings that mark the passage of its venous sinuses. It is penetrated in every direction by delicate bands of fibrous tissue, which enclose bloodvessels, nerves and elongated closed vesicles containing cells, nuclei and granular matter. These vesicles, which are $\frac{1}{80}$ of an inch ( 0.32 millimeters) long and about $\frac{1}{40} \overline{0}$ of an inch $(64 \mu)$ broad, have been demonstrated in the $\mathrm{ox}$ and in the human subject. The cells in the human subject are $\frac{1}{170} \overline{0}$ to $\frac{1}{1200}$ of an inch ( 15 to $20 \mu$ ) in diameter. They are isolated with difficulty and are irregular in form. The nuclei measure about $\frac{1}{2500}$ of an inch ( $10 \mu)$. The medullary substance is peculiarly rich in vessels and nerves (see Plate IX, Fig. I).

Vessels and Nerves. - The bloodvessels going to the suprarenal capsules are very abundant and are derived from the aorta, the phrenic artery, the cœliac axis and the renal artery. Sometimes as many as twenty distinct vessels penetrate each capsule. In the cortical substance the capillaries are arranged in elongated meshes, anastomosing freely and surrounding but not penetrating the tubes. In the medullary substance the meshes are more rounded, and here the vessels form a rich capillary plexus. Two large veins pass out, to empty on the right side into the vena cava, and on the left, into the renal vein. Other smaller veins empty into the vena cava, the renal and the phrenic veins.

The nerves are abundant and are derived from the semilunar ganglia, the renal plexus, the pneumogastric and the phrenic. The nerves probably pass directly to the medullary substance, but here their mode of distribution is unknown. In the medullary substance, however, there are two ganglia situated close to the central vein.

Nothing is known of lymphatics in the suprarenal capsules, and the existence of such vessels is doubtful. 
Chomistry of the Suprarenal Capsules. - Vulpian has described (1856), in the medullary portion of the suprarenal capsules, a peculiar substance, soluble in water and in alcohol, which gave a greenish reaction with the salts of iron and a peculiar rose tint on the addition of iodin. He could not determine the same reaction with extracts from any other parts. Later, in conjunction with Cloez, he discovered hippuric and taurocholic acid in the capsules of certain of the herbivora. These bodies contain in addition, leucin, hypoxanthin, taurin, fats and inorganic salts, the latter chiefly phosphates and salts of potassium.

It has lately been observed that extracts of the medullary substance of the suprarenal capsules when injected into the bloodvessels produce slowing of the heart with great increase in the blood-pressure. This occurs in animals under normal conditions; but after division of the pneumogastrics, the effect is an acceleration of the heart's action, with a still greater increase in blood-pressure. This peculiar action is difficult to explain; but the fact that the same results follow the use of extracts from the blood of the adrenal veins shows that the gland is the seat of an internal secretion. It is probable, however, that the increase in blood-pressure is due to a direct action on the muscular coats of the bloodvessels, as it occurs after destruction of the vasomotor centres. The active substance has been isolated and is described under the name of epinephrin, with the formula $\mathrm{C}_{17} \mathrm{H}_{15} \mathrm{NO}_{4}$. This probably is the active principle of the adrenalin now often used in therapeutics.

The suprarenal capsules seem to be essential to life. In Addison's disease, a disorder attended with bronzing of the skin and serious and finally fatal disorder of nutrition, there usually is disorganization of the suprarenal capsules, but this is not invariable. It has not been established, however, that disorganization of the capsules stands always in a causative relation to the discoloration of the skin or to the constitutional disturbance. Investigations into diseased conditions have developed little of importance concerning the physiology of these organs.

\section{The Spleen}

The spleen is situated in the left hypochondriac region, next the cardiac extremity of the stomach. Its color is a dark bluish red and its consistence is rather soft and friable. It is shaped somewhat like the tongue of a dog, presenting above, a rather thickened extremity, which is in relation with the diaphragm, and below, a pointed extremity, in relation with the transverse colon. Its external surface is convex. Its internal surface is concave, presenting a vertical fissure, the hilum, which gives passage to the vessels and nerves. It is connected with the 
stomach by the gastro-splenic omentum and is still further fixed by a fold of peritoneum passing to the diaphragm. It is about five inches (127 millimeters) in length, three to four inches ( 75 to 100 millimeters) in breadth and a little more than an inch ( 25.4 millimeters) in thickness. Its weight is six to seven ounces ( 170 to 198 grams). In the adult it attains its maximum of development, and it diminishes slightly in size and weight in old age. In early life it bears about the same relation to the weight of the body as in the adult.

The external coat of the spleen is the peritoneum, which is closely adherent to the subjacent fibrous structure. The proper coat is dense and resisting; but in the human subject it is quite thin and somewhat translucent. It is composed of ordinary fibrous tissue mixed with abundant small fibres of elastic tissue and a few non-striated muscular fibres.

At the hilum the fibrous coat penetrates the substance of the spleen in the form of sheaths for the vessels and nerves. The number of the sheaths in the spleen is equal to the number of arteries that penetrate the organ. This membrane is sometimes called the capsule of Malpighi. The fibrous sheaths are closely adherent to the surrounding substance, but they are- united to the vessels by a loose fibrous network. They follow the vessels in their ramifications to the smallest branches and are lost in the spleen-pulp. Between the sheath and the outer coat are bands, or trabeculæ, presenting the same structure as the fibrous coat. The presence of elastic fibres in the trabeculæ can easily be demonstrated; and this kind of tissue is very abundant in the herbivora. In the carnivora the muscular tissue is particularly abundant and can readily be demonstrated; but in man this is not so easy, and the fibres are less abundant. These peculiarities in the fibrous structure are important in their relations to certain physiological changes in the size of the spleen. Its contractility may be demonstrated in the dog by the application of a faradic current to the nerves as they enter at the hilum. This is followed by a prompt and energetic contraction of the organ. Contractions may be produced, though they are much more feeble, by applying the current directly to the spleen.

The substance of the spleen is soft and friable; and a portion of it, the spleen-pulp, may be pressed out with the fingers or even washed away by a stream of water. Aside from the vessels and nerves, it presents for study: I, an arrangement of fibrous bands, or trabeculæ, by which it is divided into communicating spaces; 2 , closed vesicles, called Malpighian bodies, attached to the walls of the bloodvessels; 3 , a soft, reddish substance, containing large numbers of cells and free nuclei, called spleen-pulp. 
Fibrous Stmucture of the Splecn (Trabecula). - From the internal face of the investing membrane of the spleen and from the fibrous sheaths of the vessels (capsule of Malpighi) are bands, or trabeculæe, which, by their interlacement, divide the substance of the organ into irregularly-shaped communicating cavities. These bands are $2_{25}^{\frac{1}{5}}$ to $\frac{1}{15}$ of an inch (I to 1.7 millimeter) broad, and are composed, like the proper coat, of ordinary fibrous tissue with elastic fibres and probably a few non-striated muscular fibres. They pass off from the capsule of Malpighi and the fibrous coat at right angles, soon branch, interlace and unite with each other, becoming smaller and smaller, until they measure $\frac{1}{250}$ to $\frac{1}{60}$ of an inch (O.I to 0.42 millimeter). This fibrous network serves as a support for the softer parts.

Malpighian Bodics. - These bodies are sometimes called splenic corpuscles or glands. They are rounded or slightly ovoid, about $\frac{1}{50}$ of an inch ( 0.5 millimeter) in diameter, and are filled with what are thought to be lymph-corpuscles, and free nuclei. The Malpighian bodies have no investing membrane. With this difference, they resemble in structure the solitary glands of the intestine. Both the cells and the free nuclei of the splenic corpuscles bear a close resemblance to cells and nuclei found in the spleen-pulp. The corpuscles are surrounded with bloodvessels - which send branches into the interior, to form a delicate capillary plexus - and with what is thought to be a lymphatic space or sinus.

The number of the Malpighian corpuscles in a spleen of ordinary size has been estimated at about ten thousand. They are readily made out in the ox and sheep, but frequently are not to be discovered in the human subject. The occasional absence of these bodies constitutes another point of resemblance to the solitary glands of the small intestine.

The Malpighian bodies are attached to arteries measuring $\frac{1}{80}$ to $\frac{1}{60}$ of an inch ( 0.32 to 0.42 millimeter) or less in diameter. I hey arc often found in the notch formed by the branching of an artery, but they usually lie by the sides of the vessel (see Plate IX, Fig. 3).

Splecn-pulp. - The spleen-pulp is a dark, reddish, semifluid substance, its color varying in intensity in different specimens. It is so soft that it may be washed by a stream of water from a thin section, and it readily decomposes, becoming then nearly liquid. It is contained in the cavities bounded by the fibrous trabecula, and it contains itself microscopic bands of fibres arranged in the same way. It surrounds the Malpighian bodies and contains the terminal branches of the bloodvessels, nerves and lymphatics. On microscopical examination, it presents free nuclei and cells like those described in the Malpighian bodies; but the nuclei are here relatively much more abundant. In 
addition are found red blood-corpuscles, some natural in form and size and others more or less altered, with pigmentary granules, both free and enclosed in cells.

Bloodvessels, Nerves and Lymphatics of the Spleen. - The quantity of blood which the spleen receives is large in proportion to the size of the organ. The splenic artery is the largest branch of the cœliac axis. It is a vessel of considerable length and is remarkable for its tortuous course. It gives off several branches to the adjacent viscera, and as it passes to the hilum, it divides into three or four branches, which again divide so as to form six to ten vessels. These penetrate the substance of the spleen, enveloped in fibrous sheaths with the veins, nerves and lymphatics. In the substance of the spleen the arteries branch rather peculiarly, giving off many small ramifications in their course, usually at right angles to the parent trunk. These are accompanied by the veins until they are reduced to $\frac{1}{80}$ or $\frac{1}{60}$ of an inch $(0.32$ or 0.42 millimeter) in diameter. The two classes of vessels then separate, and the arteries have attached to them the corpuscles of Malpighi. It is also a noticeable fact that the arteries passing in at the hilum have no inosculations with each other in the substance of the spleen, so that the organ is divided up into six to ten vascular compartments.

The veins join the small branches of the arteries in the spleen-pulp and pass out of the spleen in the same sheath. They anastomose quite freely in their larger as well as their smaller branches. Their calibre is estimated as about twice that of the arteries. The estimates which have put the calibre of the veins at four or five times that of the arteries probably are exaggerated. The number of veins emerging from the spleen is equal to the number of arteries of supply.

By most anatomists two sets of lymphatic vessels have been recognized, the superficial and the deep. The superficial lymphatics are in the investing membrane of the spleen and probably are connected with the deep lymphatics. The origin of the deep vessels is somewhat obscure. Lymphatic spaces, with anastomosing venous spaces or sinuses, however, surround the Malpighian bodies and permeate the spleen-pulp. It is probable that lymph and blood are mixed in these sinuses (see Plate IX, Fig. 2). At the hilum the deep lymphatics are joined by vessels from the surface. The vessels, numbering five or six, then pass into small lymphatic glands and empty into the thoracic duct opposite the eleventh or twelfth dorsal vertebra. No lymphatic vessels have been observed going to the spleen.

The nerves of the spleen are derived from the solar plexus. They follow the vessels in their distribution and are enclosed with them in the capsule of Malpighi. They are distributed ultimately in the spleen- 
pulp, but nothing definite is known of their mode of termination. When these nerves are stimulated, the non-striated muscles in the substance of the spleen are thrown into contraction.

Some Points in the Chemical Constitution of the Spleen. - Little has been learned in regard to the probable uses of the spleen from analyses of its substance; and it would therefore be out of place to discuss its chemical constitution very fully. Cholesterin has been found constantly and in considerable quantity, and the same may be said of uric acid. In addition, chemists have extracted from the substance of the spleen various proteids, hypoxanthin, leucin, tyrosin, lecithin, jecorin, a peculiar crystallizable substance called, by Scherer, lienin, crystals of hematoidin, lactic acid, acetic acid, butyric acid, inosite, amyloid matter and some indefinite fatty matters.

Variations in the Volume of the Spleen.-One of the theories in regard to the uses of the spleen, which merits some consideration, is that it serves as a diverticulum for the blood when there is a tendency to congestion of the other abdominal viscera.

It has been shown that the spleen becomes enlarged in dogs four or five hours after feeding, that its enlargement is at its maximum at about the fifth hour and that it gradually diminishes to its original size during the succeeding twelve hours; but it is not apparent how far these changes are important or essential to normal digestion and absorption. Experiments have shown that animals may live, digest, and absorb alimentary matters after the spleen has been removed, and this has been observed even in the human subject. In view of these facts, it can not be assumed that the office of the spleen as a diverticulum for the blood is essential to the proper action of the other abdominal organs.

Changes in the volume of the spleen may be produced by operating on the nervous system, chiefly through the vasomotor nerves. Section of the nerves at the hilum increases the size of the spleen by increasing the quantity of blood; and stimulation of these nerves produces contraction of the spleen. It is said that stimulation of the bulb diminishes the size of the spleen, and that the same result can be produced by reflex action, stimulating the central ends of the pneumogastrics or of various sensory nerves, provided the splanchnic nerves be intact. Starting from the bulb, the nerve-fibres that influence the size of the spleen pass down the spinal cord to the lower dorsal region, enter the semilunar ganglion by the left splanchnic and are distributed to the spleen through the splenic plexus.

Rhythmical contraction of the spleen, occurring about once a minute, has been observed in dogs and cats (Roy) and was supposed to assist the circulation in this organ; but there is no evidence that 
this takes place in the human subject, and the movements are so slow and inefficient that it does not appear that they can either promote or retard the flow of blood.

Extirpation of the Spleen. - Removal of the spleen is an old and a very common experiment. In the works of Malpighi (I687) is an account of an experiment on a dog, in which the spleen was destroyed and the operation was followed by no serious results. Since then it has been removed so often and the experiments have been so often negative in their results that it is hardly necessary to cite authorities on the subject. There are many instances, also, in which it has been in part or entirely removed from the human subject, which it is unnecessary to refer to in detail. One of the phenomena following extirpation of the spleen is a modification of the appetite. Great voracity in animals after removal of the spleen was noted by the earlier observers. Later experimenters have observed this change in the appetite and have noted that digestion and assimilation do not appear to be disturbed, the animals becoming unusually fat.

In the following observation these phenomena were well marked:-

The spleen was removed from a young dog weighing twenty-two pounds (about io kilograms). Before the operation the dog presented nothing unusual, either in his appetite or disposition. The wound healed rapidly, and after recovery had taken place, the animal was fed moderately once a day. It was noticed, however, that the appetite was voracious. The dog became so irritable and ferocious that it was dangerous to approach him, and it became necessary to separate him from the other aninals in the laboratory. He would eat refuse from the dissecting-room, the flesh of dogs, feces etc. About six weeks after the operation, having been well fed twenty-four hours before, the dog ate at one time a little more than four pounds (1814 grams) of beefheart, nearly one-fifth of his weight. This he digested well, and the appetite was undiminished on the following day. The dog had a remarkably sleek and well-nourished appearance (Flint, I86I).

The above is a striking example of the change in the appetite and disposition of animals after extirpation of the spleen; but these results are by no means invariable. In many instances of removal of the spleen from dogs, the animals were kept for several months and nothing unusual was observed. On the other hand, the change in disposition and the development of an unnatural appetite were observed in animals after removal of one kidney. These effects were also marked in an animal with biliary fistula that lived for thirty-eight days. In the latter instance, the voracity could be accounted for by the disturbance in digestion and assimilation produced by shutting off the bile from the 
intestine; but these phenomena occurring after removal of one kidney are not so readily explained.

Cases are on record of congenital absence of the spleen in the human subject, in which no special phenomena had been observed during life.

Aside from certain uses connected with changes in its volume, it is certain that the spleen has an important relation to the development of blood-corpuscles, both white and red. In certain cases of leucocythemia, the spleen is in a condition of hyperplastic enlargement. The blood coming from the spleen is peculiarly rich in leucocytes, but the proportion of its red corpuscles is diminished. It may be that the spleen destroys a certain number of red corpuscles, the coloring matter being changed into other pigmentary matters, and that it also produces new red corpuscles. After removal of the spleen, the red blood-corpuscles are diminished in number and the proportion of leucocytes is increased. This condition continues for about six months; but after that time, in dogs, the marrow of the long bones, which normally is yellow, becomes red, assuming the character of the marrow concerned in the formation of blood-corpuscles. Temporary diminution of red corpuscles and increase of leucocytes have been observed in the blood in cases of extirpation of the spleen in the human subject. Whatever uses the spleen has in connection with the development of red and of white blood-corpuscles it shares with the red marrow of the bones and the so-called lymphatic glands.

A theory has been proposed that the cells of the Malpighian bodies of the spleen act as phagocytes and destroy certain pathogenic bacteria (Metschnikoff). This notion was based on experiments on monkeys with the spirillum of relapsing fever; but the original observations were not fully confirmed by other investigators. As the result of careful experiments on rabbits, Melkinow-Raswedenkow drew the conclusion that while removal of the spleen diminishes the power of resistance to certain infections, the structures in this organ do not act as phagocytes and destroy micro-organisms. This question, however, belongs to pathology rather than to physiology.

The production, in the spleen, of a kinase, which converts trypsinogen into trypsin, has already been mentioned in connection with the pancreatic secretion.

\section{Thyroid GLand}

The thyroid gland is attached to the lower part of the larynx and follows it in its movements. Its color is brownish red. The anterior face is convex and is covered by certain of the muscles of the neck. 
The posterior surface is concave and is applied to the larynx and trachea. It presents two lateral lobes, each with a rounded thickened base below and a long pointed extremity extending upward, the lobes being connected by an isthmus (see Fig. 79, page 388). Each of these lobes is about two inches ( 50 millimeters) in length, three-quarters of an inch (I9 millimeters) in breadth, and about the same in thickness at its thickest portion. The isthmus connects the lower portion of the lateral lobes, covers the second and third tracheal rings and is about half an inch (I2 millimeters) wide and one-third of an inch ( 8.5 millimeters) thick. From the left side of the isthmus, and sometimes from the left lobe, is a portion projecting upward, called the pyramid. The weight of the thyroid gland is 350 to 380 grains ( 22 to 24 grams). Its weight in proportion to the weight of the adjacent organs does not vary with age. It is a little larger and more prominent in the female than in the male.

Structure of the Thyroid Gland. - The thyroid gland is covered with a thin but resisting coat of ordinary fibrous tissue which is loosely connected with the surrounding parts. From the internal surface of this membrane are fibrous bands, or trabeculæ, giving off, as they pass through the gland, secondary trabeculæ and then subdividing until they become of microscopic size. By this arrangement the gland is divided up into small communicating cavities. The trabeculæ contain many small elastic fibres. Throughout the substance of the gland, lodged in the meshes of the trabeculæ, are rounded or ovoid closed vesicles, measuring $\frac{1}{600}$ to $\frac{1}{250}$ of an inch (40 to $100 \mu$ ). These are formed of a structureless membrane and are lined with a single layer of pale granular nucleated cells, $\frac{1}{3000}$ to $\frac{1}{2000}$ of an inch ( 8 to I $2 \mu$ ) in diameter. The layer of cells sometimes lines the vesicle completely, sometimes it is incomplete and sometimes it is wanting. The contents of the vesicles are a clear, yellowish, slightly viscid, colloid substance, with a few granules, pale cells, and nuclei. The vesicles are arranged in the form of lobules, and between them are the great veins (see Plate IX, Fig. 4).

Four small bodies, two on either side, lie posteriorly to the thyroid. These are called parathyroids. Sometimes two are enclosed in the thyroid itself. They are composed mainly of cells resembling epithelium but present no acinous vesicles. Accessory thyroids in the neck and sometimes extending into the thorax have also been described. These structures, however, have little more than an anatomical interest, except that extirpation of the parathyroids may be followed by nearly all the phenomena resulting from removal of the thyroid, and when death does not follow thyroidectomy, this result is supposed to be due to the existence of accessory thyroids. 
Vessels and Ncries. - The bloodvessels of the thyroid gland are very abundant, this organ being supplied by the superior and inferior thyroid arteries with sometimes a branch from the innominata. The arteries break up into a close capillary plexus, surrounding the vesicles with a rich network, but never penetrating their interior. The veins are large, and like the hepatic veins, they are so closely adherent to the surrounding tissue that they do not collapse when cut across. The veins emerging from the gland form a plexus over its surface and the surface of the trachea, and they then go to form the superior, middle and inferior thyroid veins. The nerves are derived from the pneumogastrics and from the cervical sympathetic ganglia. The lymphatics are abundant but difficult to inject. The exact distribution of the nerves and the origin of the lymphatics are not well understood.

Nearly all that is known in regard to the chemical constitution of the thyroid may be embodied in the statement that it contains leucin, xanthin, lactic acid, succinic acid and some volatile fatty acids. Recently, however, a substance called iodothyrin has been extracted, which contains nearly ten per cent of iodin in combination with proteids. It is probable that this is the active principle of thyroid extract, now often used in therapeutics. The blood of the thyroid veins has been analyzed, but the changes in its composition in passing through the gland are slight and indefinite. "It has been said that one of the uses of the thyroid is to regulate the blood-circulation in the brain, but the observations in support of this view are not satisfactory.

Myxadcma. - Important clinical facts have been developed showing a connection between the thyroid gland and a disease characterized by infiltration of the connective tissues with a gelatinous substance containing mucin. This disease has been described by Ord under the name of myxœdema. It is attended with marked impairment of the mental faculties and a condition like cretinism. This usually is associated with disease of the thyroid gland.

Complete excision of the thyroid gland in the human subject has been followed by the peculiar mental condition characteristic of cretinism. In the lower animals the operation of complete extirpation is fatal. The experiments of Horsley on dogs and monkeys show great differences in the results, depending on age. In young animals death usually occurs in a few days, while old animals survive the operation four, five or six months. So far as could be ascertained from these experiments on the lower animals - dogs and monkeys - the conditions, including the mental phenomena, resembled those observed in cases of myxœedema in the human.subject. The animals operated on were found to be exceedingly sensitive to cold; but when put in a hot-air bath at a tem- 
perature of $105^{\circ} \mathrm{Fahr} .\left(40.5^{\circ} \mathrm{C}\right.$.) after the general symptoms made their appearance, they could be kept alive for several months. Recent experiments on animals (Lanz) and observations on the human subject (Halsted) seem to show that the thyroid has some relation to the generative function, its absence or disease greatly impairing fecundity and sexual activity.

It is difficult to draw from these various observations absolutely definite conclusions in regard to the physiological relations of the thyroid. This organ seems essential to life and its removal profoundly affects the general processes of nutrition. It influences the quantity of mucin in the body, but precisely in what way it is difficult to determine.

\section{Thymus Glaxd}

In its anatomy the thymus resembles the ductless glands, but its office, whatever this may be, is confined to early life. In the adult the organ is wanting, traces, only, of fibrous tissue, with a little fat, existing after puberty, in the situation previously occupied by the gland. As there never has been a plausible theory, even, of the uses of this organ, the existence of which is confined to the first two or three years of life, it seems necessary to give only a brief sketch of its structure.

The thymus appears at about the third month of fœtal life and gradually increases in size until near the end of the second year. It then undergoes atrophy and disappears almost entirely at the age of puberty. It is situated partly in the thorax and partly in the neck. The thoracic portion is in the anterior mediastinum, resting on the pericardium and extending as low as the fourth costal cartilage. The cervical portion extends upward as far as the lower border of the thyroid. The whole gland is about two inches (50.8 millimeters) in length, an inch and a half (38 millimeters) broad at its lower portion, and about one-quarter of an inch (6.4 millimeters) thick. Its color is grayish with a slightly rosy tint. It usually is in the form of two lateral lobes lying in apposition in the median line, although sometimes there exists but a single lobe. It is composed of a number of lobules held together by connective tissue.

The proper coat of the thymus is a delicate fibrous membrane sending processes into the interior of the organ. Its fibrous structure, however, is loose, so that the lobules can be separated with little difficulty. Portions of the gland may be, as it were, unravelled, by loosening the interstitial fibrous tissue; and in this way it is found to be composed of little lobular masses attached to a continuous cord. This arrangement is more distinct in the inferior animals of large size than in man. 
The lobules are composed of rounded vesicles, ten to fifteen in number and $\frac{1}{12} 5$ to $\frac{1}{40}$ of an inch $(200$ to $600 \mu)$ in diameter. The walls of these vesicles are thin, finely granular and fragile. The vesicles contain a small quantity of an albuminous liquid, with cells and free nuclei. The cells are small and transparent, and the nuclei are spherical, relatively large, containing one to three nucleoli. The free nuclei are rounded

A

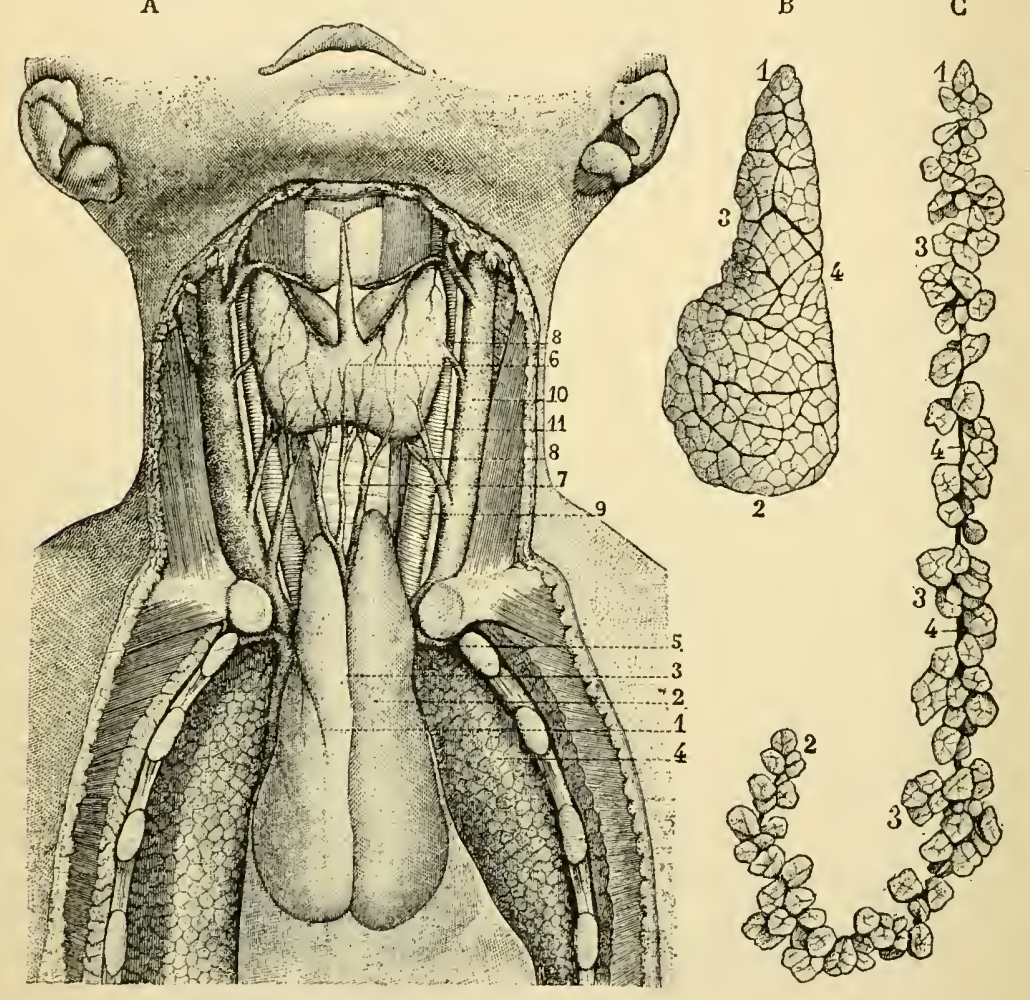

Fig. 79. - Thyroud and thymus glands (Sappey).

A. I, right lobe of the thymus; 2 , left lobe; 3 , groove between the two lobes; 4 , lungs, the anterior borders raised to show the thymus; 5 , terminal branch of the internal mammary vein; 6 , thyroid gland; 7. median inferior thyroid veins; 8 , lateral inferior thyroid veins; 9 , common carotid artery; IO, internal jugular vein; I I, pneumogastric nerve. $B$, right lobe of the thymus with the investing membrane removed: I, upper extremity of the lobe; 2 , lower extremity; 3 , external border; 4 , internal border. $C_{7}$ arrangement of the lobules of the same lobe, around the central cord: I, upper extremity of the lobe; 2 , lower extremity; $3,3,3$, lobules; 4,4 , central cord.

and contain several distinct nucleoli. Histologists describe a cortex and a medullary portion. In the latter are small masses of imbricated epithelium, known as the concentric corpuscles of Hassall (see Plate IX, Fig. 5).

The bloodvessels of the thymus are abundant, but their calibre is small and the gland is not very vascular. They are derived chiefly 
from the internal mammary artery, a few coming from the inferior thyroid, with occasional branches from the superior diaphragmatic or the pericardial. They pass between the lobules, surround and penetrate the vesicles and form a capillary plexus in their interior. The vesicles in this respect bear a certain resemblance to the closed follicles of the intestine. The veins are abundant, but they do not follow the course of the arteries. The principal vein emerges at about the centre of the gland posteriorly and empties into the left brachio-cephalic. Other small veins empty into the internal mammary, the superior diaphragmatic and the pericardial. A few nervous filaments from the sympathetic surround the principal thymic artery and penetrate the gland. Their ultimate distribution is uncertain. The lymphatics are very abundant.

As regards chemical constitution, it may be stated in general terms that the thymus contains matters of about the same character as those found in the other ductless glands.

Inasmuch as the thymus is peculiar to early life, the most important points in its anatomical history relate to its mode of development. This, however, does not present any great physiological interest and is fully treated of in works on anatomy.

Little that is definite can be said in regard to the physiology of the thymus. In some recent experiments on Guinea pigs (Paton and Goodall, 1904) it was found that removal of the thymus soon after birth was not followed by any marked effects on the growth of the animals; but it seemed to diminish the resistance to the toxins of staphylococci and streptococci. The operation had no effect on the resistance to diphtheria. Later observations by Henderson showed that "castration in cattle causes a persistent growth and a retarded atrophy of the thymus gland"; and a similar effect was noted in Guinea pigs and rabbits. "In bulls and unspayed heifers the normal atrophy of the thymus which begins after the period of puberty is greatly accelerated when the bulls have been used for breeding and when the heifers have been pregnant for several months." The exact significance of these experimental results is not apparent.

\section{Pituitary Body and Pineal Gland}

These little bodies, situated at the base of the brain, are quite vascular, contain closed vesicles and but few nervous elements and are sometimes classed with the ductless glands. Physiologists have no definite idea of their uses.

The pituitary body, sometimes called the hypophysis, is of an ovoid form, a reddish gray color, weighs five to ten grains ( 0.324 to 0.648 
gram), and is situated on the sella Turcica of the sphenoid bone. It is said to be larger in the fœtus than in the adult, and in fœtal life it has a cavity communicating with the third ventricle. This little body has been studied by Grandry, in connection with the suprarenal capsules. He regarded it as composed essentially of closed vesicles, with fibres of connective tissue and bloodvessels. The vesicles are formed of a transparent membrane, containing irregularly-polygonal nucleated cells and free nuclei. The nuclei are distinct, with a well-marked nucleolus. Capillary vessels surround these vesicles without penetrating them. Grandry did not observe either nerve-cells or fibres between the vesicles.

The pineal gland is situated just behind the posterior commissure of the brain, between the nates, and is enclosed in the velum interpositum. It is of a conical shape, one-third of an inch (8.5 millimeters) in length and of nearly the color of the pituitary body. It is connected with the base of the brain by several delicate commissural peduncles. It presents a small cavity at its base, and frequently it contains in its substance little calcareous masses composed of calcium phosphate, calcium carbonate, ammonio-magnesian phosphate and a small quantity of organic matter. It is covered with a fibrous envelope which sends processes into its interior. As the result of the researches of Grandry, it has been found to present a cortical substance, analogous in structure to the pituitary body, and a central portion, composed of the ordinary nervous elements found in the gray matter of the brain. Its structure is very like that of the medullary portion of the suprarenal capsules.

Acromegaly and Giantism. - Disease of the pituitary body, especially in adult life, is attended with great enlargement of the bones of the extremities and the features of the face, or what is known as acromegaly. When the disease occurs in early life, as development progresses there is a condition known as giantism, the individual sometimes reaching an enormous stature. That there is some relation between the pituitary body and acromegaly and giantism, there can be no doubt; but the nature of this relation is obscure. In some cases of acromegaly, improvement has been noted following the exhibition of extract of the normal pituitary body.

Internal Secretion by the Testes and Ovaries. - As early as I889, Brown-Séquard described an internal secretion by the testes, and others have included in their investigations the ovaries. While it is by no means certain or even reasonably probable, notwithstanding the redundant literature on the subject, that testicular extracts increase mental and physical vigor in the male or that ovarian extracts have similar effects on the female, these early experiments have the merit of having practically inaugurated important observations on the internal secretions of 
other organs, although they led at first to certain therapeutic extravagancies. It is not to be understood, however, from this comment, that future researches may not show important functions belonging to the sexual organs, in addition to their well-known action in reproduction.

As regards the ovaries, it has been conjectured that they have an internal secretion which may have a general influence on the organism; but although ovariectomy has lately become frequent, sufficient time has not elapsed to warrant definite conclusions concerning the remote effects of this operation. The testicles present a vascular areolar tissue which passes from the fibrous structure between the seminiferous tubes. In this tissue are cytoplasmic cells with some fatty and pigmentary granules; and this structure is now called the interstitial gland of the testis. Some late researches seem to show that these cells have a function resembling internal secretion; but their action is obscure, and it has not been shown that they furnish an extract producing any well-defined results when administered by subcutaneous injection or by the mouth.

Internal secretion by the pancreas and kidneys has already been considered in connection with the other and more familiar functions of these organs. 


\section{CHAPTER XV}

\section{METABOLISM - NUTRITION - ANIMAL HEAT AND FORCE}

Action of glandular cells-Metabolism, anabolism and katabolism-General nutritionLuxus-consumption - Isodynamic values of foods - Animal heat and force - Limits of variations in the normal temperature in man - Variations in different parts of the body - Variations at different periods of life - Variations at different times of the day - Influence of exercise etc., on the heat of the body - Influence of the nervous system on the production of animal heat (heat-centres) - Mechanism of the production of animal heat - Equalization of the animal temperature-Relations of heat to force.

\section{Metabolism, Anabolism and Katabolism}

THE constant change going on in the constituents of the body is called metabolism. A part of this process consists in a repair of the tissues and is known as anabolism. This repair is made necessary by a constant change of the constituents of the organism into effete matters. This change is called katabolism.

In the changes that are involved in metabolism, inorganic as well as organic matters participate. Organic matters, indeed, are never free from inorganic substances; and the latter accompany organic matters in the changes incident to nutrition. The organic constituents of the body are almost all included in the proteids.

The inorganic matters number about twenty-one. The most important of these is water. This is found in all tissues without exception. It is discharged from the body as water and is useful in carrying off effete matters in solution. It is introduced in all forms of food and drink. In addition, it is formed in the body by the union of hydrogen with oxygen, this process contributing to the production of animal heat and force. The water thus formed and discharged from the body may properly be regarded as an excretion.

Many saline compounds exist in the body in solution in water. These are essential to nutrition. They are introduced from without, exist in the body united with organic matters and are discharged in the form in which they entered.

Proteids, as proteids, are not discharged from the body in health. They are converted into excrementitious matters and as such form part 
of the excretions. The twenty-one inorganic matters belong to a class of substances that pass through the body unchanged.

The carbohydrates undergo important changes in the body. They are oxidized and converted into carbon dioxide and water, contributing to the production of animal heat and force. Oxidation of the carbohydrates is one of the sources of water produced in the economy.

The probable mechanism of the transformation of the carbohydrates by oxidation into carbon dioxide and water was foreshadowed by Ford, an American physician, in I872. This observer obtained a small quantity of alcohol from the blood of the ox, which he assumed was derived from the carbohydrates. The results of these experiments, however, were not confirmed by certain German physiologists and were discredited; but recently (I904), Stolaska has extracted from the blood of the ox, the heart of the dog and the pancreas of the pig, a glycolytic enzyme capable of producing alcohol. This writer regards "alcoholic fermentation as the first stage of the respiratory process."

The modern trend of physiological opinion is toward the idea that alcohol, in doses so small that it may be promptly oxidized, is not harmful, although, when alimentation is sufficient, it is unnecessary. Its oxidation produces energy, like the oxidation of so-called carbonaceous foods, notably the carbohydrates. In this connection a study of diabetes mellitus is most instructive. It is thought by some pathologists that this disorder is due, in many instances, to absence of an enzyme, normally produced in the pancreas, that acts on carbohydrates. It is thought, also, that all the energy due to oxidation of carbohydrates comes from the final oxidation of alcohol which results from the action of enzymes in the blood on sugar, and that carbohydrates can not be utilized as producers of heat and force in any other way. All the carbohydrates oxidized in the body pass into the general circulation by the hepatic veins. As early as I888, I wrote - "It is reasonable to suppose that the small quantity of sugar constantly discharged into the blood by the liver is converted into alcohol, which is promptly oxidized, being converted into carbon dioxide and water."

The fats undergo oxidation in the body; and this oxidation contributes to the production of animal heat and force. As a rule the fats exist in the organism in combination with each other but are not combined with proteids.

Oxygen exists in the red blood-corpuscles combined with hemoglobin. Carbon dioxide exists in solution in the blood, lymph, chyle and secreted liquids. Nitrogen, carburetted hydrogen and hydrogen monosulphide exist in a gaseous state in the alimentary canal. A small quantity of nitrogen exists in solution in the blood. 


\section{General Nutrition}

A comparison of the outgo and income of the organism and estimates of the quantity of food necessary for the proper nutrition of a man of ordinary weight and under ordinary conditions have already been given in the chapter treating of alimentation. It may be stated here in general terms that ten to twelve ounces ( 283.5 to 340.2 grams) of carbon, and four to five ounces (II3.4 to I4I.75 grams) of proteids are discharged from the organism daily. To meet these expenditures exactly and maintain the body in a condition of physiological equilibrium would require the introduction of four to five ounces (II3.4 to I4I.75 grams) of proteids, one and one-half ounces ( 28.35 to 42.52 grams) of fat and sixteen to nineteen ounces ( 453.6 to 483.6 grams) of carbohydrates. Practically, however, provision should be made for unusual requirements, and the diet should be in excess of what would exactly meet the outgo of material.

Luxus-Consumption. - An illustration of the necessity of a diet more than sufficient exactly to meet the outgo under ordinary conditions is to be found in what is called luxus-consumption. By this term it is intended to indicate change in a certain quantity of nutritive matter, especially proteids, which does not involve repair of tissue. Voit, who first described this process, divided the proteids of the blood into tissueproteids and circulating proteids, the latter representing the excess of proteids over and above actual nutritive requirements. Voit's theory, however, failed to meet with general acceptance, and the expression luxus-consumption is regarded as misleading and unscientific; but the fact remains that food taken in excess of actual nutritive requirements is readily disposed of when it does not remain in the body in the form of fat.

Isodynamic Values of Foods. - The theory of the isodynamic values of foods is that proteids, fats and carbohydrates are theoretically interchangeable on the basis of equal heat-values in oxidation. This has been formulated by Rubner into a law, of which the following is an illustration : It has been found that one gram of fat is equal in heat-value to about two and one-quarter grams of proteid or carbohydrate. As regards the production of heat and power, therefore, fats, proteids and carbohydrates are interchangeable in the proportion of one part of fat to two and one-quarter parts of either proteid or carbohydrate. In this theory it is assumed that the proteids are oxidized into urea and carbon dioxide, and that their heat-value is the same as when they are burned out of the body. It is probable, also, that isodynamic substitution may include alcohol (Rosemann). 


\section{Aximal Heat and Force}

The processes of nutrition in animals are attended with the development and maintenance of a body-temperature that is more or less independent of external conditions. This is true in the lowest as well as the highest animal organisms; and analogous phenomena have been observed in plants. In cold-blooded animals nutrition may be suspended by a diminished external temperature, and certain of the functions become temporarily arrested, to be resumed when the animal is exposed to a greater heat. This is true, to some extent, in certain warmblooded animals that periodically pass into a condition of torpor, called hibernation; but in man and most of the warm-blooded animals, the general temperature of the body undergoes but slight variations. Certain animals pass into a condition analogous to hibernation under the influence of the intense heat of summer in tropical countries. This condition is called estivation. It has been observed in the tenrec of Madagascar - a kind of hedgehog - to continue for three months during the hot season. The animal heat is nearly the same in cold and in hot climates; and if from any cause the body becomes incapable of keeping up its temperature when exposed to cold, or of moderating it when exposed to heat, death is the result.

Estimated Quantity of Heat produced by the Body. - In order to express quantities of heat, it is necessary to fix on some definite quantity to be taken as a heat-unit. In what is to follow, a heat-unit is to be understood as the heat required to raise the temperature of one pound of water one degree from $32^{\circ} \mathrm{Fahr}$. (pound-degree Fahr.).

It has been calculated that one heat-unit is equal to the force expended in raising one pound 772 feet or 772 pounds one foot (Joule). The equivalent of heat in force has been calculated by estimating the heat produced by a certain weight falling through a certain distance, assuming the falling force to be precisely equal to the force that has been used in raising the weight; but physicists have not actually succeeded in so completely converting heat into force as to raise one pound 772 feet or 772 pounds one foot, by the expenditure of one heat-unit.

The heat-unit and its equivalent in force are, of course, differently expressed according to the metric system. When heat-units or footpounds are given in the text, the equivalents, according to the metric system, are given in parentheses. These equivalents are as follows:-

A heat-unit, according to the metric system, or the heat required to raise the temperature of one kilo of water one degree from zero C., will be designated as a kilo-degree C. A kilo-degree is called a large calorie. A gram-degree - the heat required to raise the temperature of one 
gram of water one degree C. - is called a small calorie. One kilodegree $=1000$ gram-degrees .

One pound degree $=0.252$ kilo-degree C. One kilo-degree C. $=$ 3.96 (nearly 4) pound-degrees. A kilogrammeter represents the force required to raise a weight of one kilogram one meter. One foot-pound $=0.138$ kilogrammeter. One kilogrammeter $=7.24$ foot-pounds. One pound-degree $=772$ foot-pounds. One pound-degree $=106.6$ kilogrammeters. One kilo-degree C. $=422.25$ kilogrammeters. One kilo-degree C. $=3057$ foot-pounds.

Two methods have been employed in arriving at estimates of the actual quantity of heat produced by the body in a definite time:-

I. The direct method consists in placing an animal in a calorimeter and measuring the heat produced, making the necessary corrections. This has repeatedly been done, but the results obtained have been variable and not entirely satisfactory. An important element of inaccuracy in direct observations - one, indeed, which it seems impossible to correct absolutely - is due to the great variations in heat-production with digestion, conditions of muscular repose or exercise, external temperature etc. Another source of error is the difficulty in estimating the heat lost by the body and not actually produced during the time of the observation.

2. The indirect method consists in estimating the heat represented by oxidation, calculated from the quantity of oxygen consumed in the processes that result in the production and discharge of carbon dioxide, water, urea etc. These estimates have been compared with the calculated heat-value of the food consumed, and the results very nearly correspond.

According to the estimates of Helmholtz, Ranke and others, by the indirect method, the heat-production is equal to about 2.5 heat-units per hour per pound-weight of the body (1.39 kilo-degree C. per kilogram). In a man weighing 180.4 pounds ( 82 kilograms) the heat-production in twenty-four hours (Helmholtz) was 10,8 I 8 heat-units (2732 kilo-degrees C.). According to this estimate, a man weighing 140 pounds $(63.5$ kilograms) would produce 8400 heat-units (2II8 kilo-degrees C.) in twenty-four hours. This would be equal to $6,484,800$ foot-pounds, or about 894,500 kilogrammeters.

A study of this subject and of the details of observations both direct and indirect has made it evident that the experimental difficulties and the unavoidable elements of inaccuracy are greater in the direct than in the indirect method. In comparing the estimates of heat actually produced with the heat-value of food - which, of course, is the ultimate source of heat and force in the body - the correspondence is 
much closer if the indirect estimates are adopted. It therefore 'seems more in accordance with ascertained facts to adopt the indirect estimates, although this can not be done without reserve. The heat produced, then, is probably equal to about 2.5 heat-units (pound-degrees) per hour per pound-weight of the body (nearly I.4 kilo-degree C. per kilogram). This is equal to about 8400 heat-units, or about 2 I 20 kilodegrees C., in twenty-four hours; which is equal to about 6,500,000 foot-pounds, or about 900,000 kilogrammeters.

The normal variations in the production of heat are not absolutely and definitely represented by variations in the actual temperature of the body and by the consumption of oxygen. Muscular work may increase the production of heat sixty per cent (Hirn) while it increases the consumption of oxygen about $4 \frac{1}{2}$ times, a large part of the oxidation being expended in the form of work. The production of heat is diminished in fasting animals (dogs) by nearly forty-five per cent (Senator), after deprivation of food for two days. In old age and in infancy there is less heat produced than in adult life. The production of heat is less in females than in males and is less during the night than during the day. These points will be touched upon again, in connection with the normal variations in the temperature of the body.

Limits of Variation in the Normal Temperature in Nan. - One of the most common methods of taking the general temperature has been to introduce a registering thermometer in to the axilla, reading off the degrees after the mercury has become stationary. Nearly all observations made in this way agree with the results obtained by Gavarret, who estimated that the temperature in the axilla, in a perfectly healthy adult man, in a temperate climate, ranges between $97.7^{\circ}$ and $99.5^{\circ} \mathrm{Fahr}$. $\left(36.5^{\circ}\right.$ and $37.5^{\circ}$ C. $)$. Davy, from a large number of observations on the temperature under the tongue, fixed the standard, in a temperate climate, at $98^{\circ} \mathrm{Fahr}$. $\left(36.67^{\circ} \mathrm{C}\right.$.). The axilla and the tongue, however, being more or less exposed to external influences, do not exactly represent the general heat of the organism; but these are the situations, particularly the axilla, in which the temperature is most frequently: taken. As a standard for comparison, it may be assumed that the most common temperature in these situations is $98^{\circ} \mathrm{Fahr} .\left(36.67^{\circ} \mathrm{C}\right.$.) subject to variations, within the limits of health, of about $0.5^{\circ} \mathrm{Fahr} .\left(0.27^{\circ} \mathrm{C}\right.$.) below and $1.5^{\circ}$ Fahr. $\left(0.82^{\circ} \mathrm{C}\right.$.) above.

Variations with Extemal Temperature. - The general temperature of the body varies, though within restricted limits, with extreme changes in climate. The results obtained by Davy, in a large number of observations in temperate and hot climates, show an elevation in the tropics of $0.5^{\circ}$ to $3^{\circ}$ Fahr. $\left(0.27^{\circ}\right.$ to $1.65^{\circ}$ C. $)$. It is well known, also, that the 
human body, the surface being properly protected, is capable of enduring for some minutes a heat greater than that of boiling water. Under these conditions the body-temperature is raised but slightly as compared with the intense heat of the surrounding atmosphere. In the observations of Dobson, the temperature was raised to $99.5^{\circ} \mathrm{Fahr}$. $\left(37.5^{\circ} \mathrm{C}\right.$. $)$ in one instance, 101. $5^{\circ} \mathrm{Fahr} .\left(39.6^{\circ} \mathrm{C}\right.$. $)$ in another, and $102^{\circ}$ Fahr. $\left(38.9^{\circ} \mathrm{C}\right.$.) in a third, when the body was exposed to a heat of more than $212^{\circ}$ Fahr. $\left(100^{\circ} \mathrm{C}\right.$.). Delaroche and Berger, however, found that the temperature in the mouth could be increased by $3^{\circ}$ to $9^{\circ} \mathrm{Fahr}$. ( $1.65^{\circ}$ to $5.05^{\circ}$ C.) after sixteen minutes of exposure to intense heat. This was for the external parts only; and it is not probable that the temperature of the internal organs ever presents such wide variations.

It is difficult to estimate the temperature in persons exposed to intense cold, as in Arctic explorations, because care is always taken to protect the surface of the body as completely as possible; but experiments have shown that the body-heat may be considerably reduced, as a temporary condition, without producing death. In the latter part of the last century, Currie caused the temperature in a man to fall $15^{\circ}$ Fahr. $\left(8.25^{\circ} \mathrm{C}\right.$.) by immersion in a cold bath ; but he could not bring it below $83^{\circ}$ Fahr. $\left(28.33^{\circ} \mathrm{C}\right.$.). This extreme depression, however, lasted only two or three minutes, and the temperature afterward returned to within a few degrees of the normal standard. The results of experiments show that while the normal variations in the temperature in the luman subject, even when exposed to great climatic changes, are

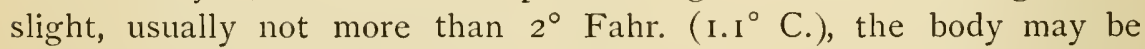
exposed for a time to excessive heat or cold, and the extreme limits consistent with the preservation of life may be reached. So far as has been ascertained by direct experiment, these limits are about $83^{\circ}$ and $107^{\circ}$ Fahr. $\left(28.33^{\circ}\right.$ and $41.67^{\circ} \mathrm{C}$.).

Variations in Differcnt Parts of the Body. - The blood becomes slightly lowered in temperature in passing through the general capillary circulation, but the difference is ordinarily not more than a fraction of a degree. This fact is not opposed to the proposition that heat is produced in greatest part in the general capillary system as one of the results of nutritive action; for the blood circulates with such rapidity that the heat acquired in the capillaries of the internal organs, where little or none is lost, is but slightly diminished before it passes into the arteries, even in circulating through the lungs; and cutaneous evaporation simply moderates the heat acquired in the tissues and keeps it at the proper standard.

The blood usually is $0.36^{\circ}$ to $\mathrm{I} .8^{\circ} \mathrm{Fahr}$. ( $0.2^{\circ}$ to $\mathrm{I}^{\circ} \mathrm{C}$.) warmer in the hepatic veins than in the aorta. The temperature in the hepatic veins 
is $0.18^{\circ}$ to $\mathrm{I} .44^{\circ} \mathrm{Fahr}$. $\left(0 . \mathrm{I}^{\circ}\right.$ to $0.8^{\circ} \mathrm{C}$.) higher than in the portal veins. This shows that the blood coming from the liver is warmer than in any other part of the body. As regards the temperature of the blood in the two sides of the heart, experiments on the lower animals have been somewhat contradictory; but there is no positive evidence of any considerable change in the temperature of the blood in passing through the lungs in the human subject. In the lower animals there probably exist no constant differences in temperature in the two sides of the heart. When the loss of heat by the general surface is active, as in animals with a slight covering of hair, the blood usually is cooler in the right cavities; but in animals with a thick covering, that probably lose considerable heat by the pulmonary surface, the blood is cooler in the left side of the heart.

Variations at Different Periods of Life. - The most important variations in the temperature of the body at different periods of life are observed in infants just after birth. The body of the infant and of young mammalia removed from the mother presents a diminution in temperature of $1^{\circ}$ to $4^{\circ}$ Fahr. $\left(0.55^{\circ}\right.$ to $2.2^{\circ} \mathrm{C}$.). In infancy the ability to resist cold is less than in later years; but after a few days the temperature of the child nearly reaches the standard in the adult, and the variations produced by external conditions are not so great.

In certain animals, particularly dogs and cats, that are born with the eyes closed and in which the foramen ovale remains open for a few days, the temperature rapidly diminishes when they are removed from the body of the mother, and they then become reduced to a condition approximating that of cold-blooded animals; but after about fifteen days this change in temperature can not be effected. In dogs just born, the temperature fell, after three or four hours' separation from the mother, to a point but a few degrees above that of the surrounding atmosphere (W. F. Edwards).

In adult life there does not appear to be any marked and constant variation in the normal temperature; but in old age, while the actual temperature of the body is not notably reduced, the power of resisting refrigerating influences is considerably diminished. There are no observations showing any constant differences in the temperature of the body in the sexes; and it may be assumed that in the female the body-temperature is modified by the same influences and in the same way as in the male.

Variations at Different Times of the Day, etc. - Although the limits of variation in the body-temperature are not very wide, certain fluctuations are observed, depending on muscular repose or activity, digestion, sleep etc. It has been ascertained that there are two well-marked 
periods in the day when the heat is at its maximum: at eleven A.M. and four P.M. The fall in temperature during the night takes place sleeping or waking; and when sleep is taken during the day, it does not disturb the period of the maximum, which occurs at about four P.M. At eleven in the morning, the body-heat is at one of its periods of maximum; it gradually diminishes for two or three hours and is raised again to the maximum at about four in the afternoon, when it again undergoes diminution until the next morning. The variations amount to between $1^{\circ}$ and $2.16^{\circ}$ Fahr. $\left(0.55^{\circ}\right.$ and $1.19^{\circ} \mathrm{C}$.). The minimum is always during the night.

The influence of defective nutrition or of inanition on the heat of the body is very marked. In pigeons the extreme variation in temperature during the day, under normal conditions, was found by Chossat to be I. $3^{\circ}$ Fahr. $\left(0.7^{\circ} \mathrm{C}\right.$ ). During the progress of inanition this variation was increased to $5.9^{\circ} \mathrm{Fahr} .\left(3.25^{\circ} \mathrm{C}\right.$.) with a slight diminution in the absolute temperature, and the periods of minimum temperature were unusually prolonged. Immediately preceding death from starvation, the diminution in temperature became very rapid, the rate being $7^{\circ}$ to II ${ }^{\circ} \mathrm{Fahr}$. $\left(3.85^{\circ}\right.$ to $6^{\circ} \mathrm{C}$.) per hour. Death usually occurred when the diminution had amounted to about $30^{\circ} \mathrm{Fahr}$. (I6.5 ${ }^{\circ} \mathrm{C}$.).

When the surrounding conditions call for the derelopment of an unusual quantity of heat, the diet is modified, both as regards quantity and kind of food; but when food is taken in sufficient quantity and is of a kind capable of maintaining proper nutrition, its composition does not affect the general temperature. The temperature of the body, indeed, seems to be uniform in the same climate, even in persons living on entirely different kinds of food. Nevertheless, the conditions of external temperature have a remarkable influence on the diet. It is well known that in the heat of summer, the quantity of meats and fat taken is relatively small, and of the succulent fresh vegetables and fruits, large, as compared with the diet in the winter; but although the proportion of carbohydrates in many fresh vegetables used during a short season of the year is not great, these articles are also deficient in nitrogenous matters. During the winter the ordinary diet, composed of meat, fat, bread, potatoes etc., contains a large proportion of nitrogenous matters as well as a considerable proportion of carbohydrates; and in the summer the proportion of both these varieties of food is reduced, the more succulent articles taking their place. This is further illustrated by a comparison of the diet in the torrid or temperate and in the frigid zones. It is stated that the daily ration of the Esquimaux is twelve to fifteen pounds ( 5.433 to 6.894 kilograms) of meat, about one-third of which is fat. Hayes noted that with a temperature of $-60^{\circ}$ to $-70^{\circ}$ 
Fahr. (about $-51^{\circ}$ to $-57^{\circ} \mathrm{C}$.), there was a continual craving for a strong, animal diet, particularly fatty substances.

The influence of alcoholic beverages on the animal temperature has been studied chiefly with reference to the question of their use in enabling the system to resist excessive cold. The testimony of scientific Arctic explorers is that the use of alcohol does not enable men to endure a very low temperature for any considerable length of time.

As a rule, when the respiratory activity is physiologically increased - as it is by exercise, bodily or mental, ingestion of food or diminished external temperature - the production of heat in the body is increased correspondingly; and on the other hand, it is diminished by conditions that physiologically decrease the absorption of oxygen and the exhalation of carbon dioxide. The relations of animal heat to the general process of nutrition are most intimate. Any condition that increases the activity of nutrition and of katabolism, or even anything that increases katabolism alone, increases the production of heat. The reverse of this proposition is also true.

Notwithstanding the fact that there is a certain correspondence between the activity of the respiratory processes and the production of heat, this is far from absolute. It has been shown by Senator that digestion increases heat-production rather more than it increases the exhalation of carbon dioxide. Muscular work has been found to increase the quantity of oxygen consumed in much greater proportion than it increased the heat-production (Hirn). Even adding to the heat produced the work, reduced to heat-units, the heat-production was about doubled, while the quantity of oxygen consumed was increased about four and a half times.

Influence of Exercise etc., on the Heat of the Body. - The most nearly complete repose of the muscular system is observed during sleep, when hardly any of the muscles are brought into action except those concerned in tranquil respiration. There is always a notable diminution in the general temperature at this time. In the variations in the heat of the body, the minimum is always during the night; and this is not entirely dependent on sleep, for a depression in temperature is always observed at that time, even when sleep is avoided. It is a matter of common observation that one of the most efficient means of resisting the depressing influence of cold is to exercise the muscles constantly; and it is well known that after long exposure to intense cold, the tendency to sleep, which becomes almost irresistible, if yielded to, is followed by a rapid loss of heat and almost certain death. Muscular work increases the production of heat; but the variations in the actual temperature of the body in man, although distinct, are seldom considerable, for the reason 
that muscular exercise usually is attended with increased action of the skin, which keeps the heat of the body within restricted limits. In violent muscular exertion, as in fast running, the increased production of heat may be so rapid that it can not be entirely compensated by evaporation from the skin, and the temperature may rise to $104^{\circ} \mathrm{Fahr}$. $\left(40^{\circ} \mathrm{C}\right.$.). In about an hour and a half of repose the temperature falls to the normal standard.

The elevation in temperature that attends muscular work is produced directly in the substance of the muscle (Becquerel and Breschet). Introducing a thermo-electric needle into the biceps of a man who used the arm in sawing wood for five minutes, these physiologists noted an elevation of temperature of nearly $2^{\circ} \mathrm{Fahr}$. ( ${ }^{\circ} \mathrm{C}$.).

Observations on the influence of mental exertion on the temperature of the body have not been so many, but they are, apparently, no less exact in their results. Davy observed a slight but constant elevation during "excited and sustained attention." Lombard noted an elevation of temperature in the head during mental exertion of various kinds, but

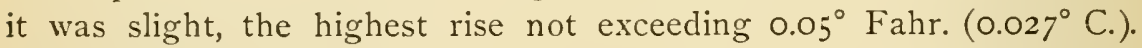
According to Burdach, the temperature of the body is increased by the emotions of hope, joy, anger, and all exciting passions, while it is diminished by fear, fright and mental distress.

It is evident that if animal heat be one of the necessary attendant phenomena of nutrition, it must be greatly influenced by conditions of the circulation. It has been a question, indeed, whether the modifications in temperature produced by operating on the vasomotor nerves are not due entirely to changes in the supply of blood. It is certain that whatever determines an increased supply of blood to any part raises the temperature; and whenever the quantity of blood in any organ or part is considerably diminished, the temperature is reduced. This fact is illustrated in operations for the deligation of large arteries. It is well known that after tying a large vessel, the utmost care is necessary to keep up the temperature of the part to which its branches are distributed, until the anastomosing vessels become enlarged sufficiently to supply the quantity of blood necessary for normal nutrition.

Infuncuce of the Nerrous System on the Production of Animal Heat (Hcat-Ccntres). - The local influences of the vasomotor nerves on calorification operate mainly if not entirely through changes in the nutrition of parts, produced by variations in blood-supply. These influences will be fully considered in connection with the physiology of the nervous system.

The temperature of the body may be modified through the nervous system by reflex action, and this implies the existence of nerve-centres, 
or of a nerve-centre, capable of influencing the general process of calorification. Experiments have been made, chiefly on parts of the encephalon, with the view of determining the existence and location of heat-centres. Ott describes four heat-centres, irritation of which by puncture increases the temperature of the body in rabbits by several degrees $\left(4^{\circ}\right.$ to $6^{\circ} \mathrm{Fahr}$., or $2.2^{\circ}$ to $3.3^{\circ} \mathrm{C}$.). These four centres are the following: $\mathrm{I}$, in front of and beneath the corpus striatum $(\mathrm{Ott}) ; 2$, the median portion of the corpora striata and the subjacent parts (Aronsohn and Sachs); 3, between the corpus striatum and the optic thalamus $(\mathrm{Ott}) ; 4$, the anterior inner end of the optic thalamus (Ott). Puncture of these parts is followed by rise in temperature, which continues for a variable time - two to four days. A similar centre has been described as existing in the dog in the cortex of the anterior portion of the upper surface of the brain near the median line (Eulenberg and Landois). The conductors connected with these centres decussate and pass through the bulb and the spinal cord. The effects of puncture or stimulation of these parts probably is inhibitory.

\section{Mechanism of the Production of Animal Heat}

In man and in the warm-blooded animals generally, the maintenance of the temperature of the organism at a nearly fixed standard is a necessity of life; and while heat is generated with an activity that is constantly varying, it is counterbalanced by physiological loss of heat from the cutaneous and respiratory surfaces. Variations in the activity of calorification are not to be measured by corresponding changes in the body-temperature, but are to be estimated by calculating the quantity of heat lost. The ability of the human race to live in all climates is explained by the adaptability of man to different conditions of diet and exercise and by the power of regulating loss of heat from the surface by appropriate clothing.

So far as it is possible to determine by experiment, not only is there no particular part or organ in the body endowed with the special office of calorification, but every part in which the nutritive forces are in operation produces a certain quantity of heat; and this probably is true of the blood-corpuscles and other anatomical elements of this class. The production of heat in the body is general and is one of the necessary results of the process of nutrition; but, with nutrition, it is subject to local variations, as is illustrated in the effects of operations on the vasomotor nerves and in the phenomena of inflammation.

In 1866 Frankland made a number of calculations of the heat-units and the estimated force-value of various articles of food, which are now 
accepted and used by most writers on subjects connected with the theories of animal heat and the source of muscular power. As regards the heat produced by the oxidation of these substances in the body, if it is assumed that the same quantity of heat is produced by the oxidation, under all circumstances, of a definite quantity of oxidizable matter, it is necessary simply to deduct from the heat-value of articles of food the heat-value remaining in certain parts of the food which pass out of the body in an unoxidized state. It was in this way that Frankland arrived at a determination of the heat-value of articles of food oxidized in the body.

The following selections from Frankland's table give an idea of the heat-value of different articles of food oxidized in the body. In this table the heat-units are calculated as pound-degrees.

HEAT-VALUE OF TEN GRAINS OF THE MATERIAL OXIDIZED INTO CARBON DIOXIDE, WATER AND UREA IN THE ANIMAL BODY (FRANKLAND)

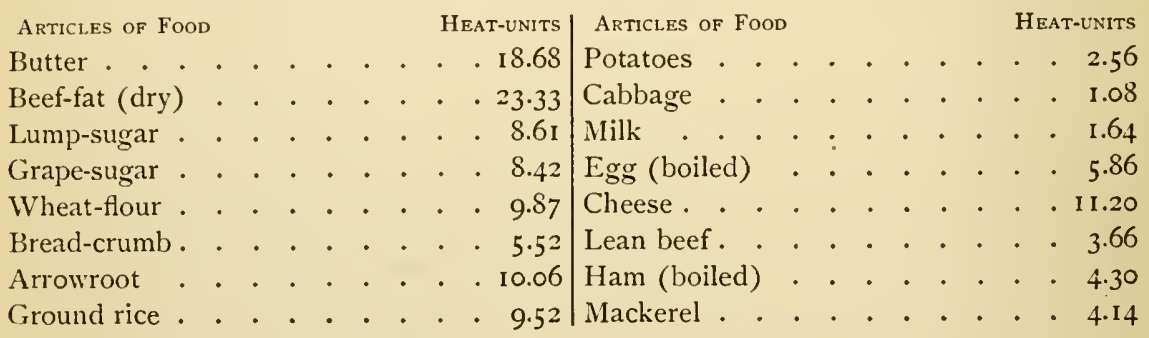

In the following, selected from the table quoted by Chapman, the heat-units are calculated as kilo-degrees C.

HEAT-VALUE OF ONE GRAM OF THE MATERIAL OXIDIZED INTO CARBON DIOXIDE, WATER AND UREA IN THE ANIMAL BODY (FRANKLAND)

\begin{tabular}{|c|c|c|c|c|c|c|c|c|c|c|c|c|c|}
\hline of Foor & & & & & & HEAT-UNITS & Articles of Food & & & & & & HEAT-UNITS \\
\hline utt & & & & & & $\cdot 7.264$ & Potatoes & & - & - & & & . 0.990 \\
\hline (dry) & & . & & & & 9.069 & Cabbage & & & & & & . \\
\hline$r$. & & . & & & & . $3 \cdot 348$ & Milk & & & & & & .028 \\
\hline ar & & . & & & . & .227 & Egg (boiled) & & & . & & & .28 \\
\hline r & & & & & & 3.840 & $\mathrm{Ch}$ & & & & & & . \\
\hline & . & • & & & . & . 1.450 & eef. . & & & & & & +26 \\
\hline & & . & & & . & . 3.912 & oiled) & & & & & & I.680 \\
\hline round & & & & & & 3.760 & | Mackerel . & & & & & & 1.610 \\
\hline
\end{tabular}

The heat-value of one gram of alcohol is equal to 8.958 heat-units (kilo-degrees C.), or the heat-value of 10 grains of alcohol is equal to 23 heat-units (pound-degrees Fahr.). 
As regards the processes of combustion that take place in the living organism, the oxidation of the constituents of food produces carbon dioxide and water ; but it is probable that the quantity of heat produced bears a definite relation to the total consumption of oxygen, the heat, so far as this is concerned, being the same whether the oxygen unite with carbon or with hydrogen. This relation between the quantity of oxygen consumed and the production of heat seems to be disturbed by muscular work; but it has thus far been found impossible to estimate accurately the quantity of heat represented by the force expended in muscular work, circulation, respiration etc.

The heat-producing processes undoubtedly are represented mainly by the exhalation of carbon dioxide and water and to a less degree by the discharge of urea, the quantity of heat produced by other chemical changes being comparatively small. It is also true that the fats are much more important factors in calorification than the proteids; but it seems beyond question that there must be heat evolved in the body by oxidation of nitrogenous matters. When the daily quantity of food is largely increased for the purpose of generating the heat required in excessively cold climates, nitrogenous matters are taken in greater quantity, as well as fats, although their increase is not in the same proportion. From these facts, and from other considerations that have already been fully discussed, it is evident that the physiological metamorphoses of proteids have a certain share in the production of animal heat. The carbohydrates and fats are not concerned in the building up of tissues and organs; except as fats are deposited in the form of adipose tissue. Their addition to the food saves the nitrogenous tissues, which latter must be used in heat-production in starvation and in a restricted diet deficient in non-nitrogenous matters. If the nonnitrogenous constituents of food do not form tissue, are not discharged from the body and are consumed in some of the processes of nutrition, it would seem that their change must involve the production of carbon dioxide and water and the evolution of heat.

Although it may be assumed that the non-nitrogenous constituents of food are specially important in the production of animal heat and that they are not concerned in the repair of tissue, it must be remembered that the body-temperature may be kept at the proper standard on a nitrogenous diet; and it is not possible to connect calorification exclusively with the consumption of any single class of alimentary matters.

The exact mechanism of the oxidation-processes in the body is not understood. All physiologists, however, are agreed that the quantity of heat produced by oxidation is the same, whether the combustion be rapid or slow. The fact that fats are never discharged, but are either 
consumed entirely or are deposited in the body as fat, leaves their oxidation and discharge as oxidation-products the only alternative. The oxidation of proteids has already been considered. As regards the carbohydrates, if it can be shown that alcohol normally exists in the blood, even in very small quantity, the idea that these matters are slowly passed from the liver as sugar into the general circulation and are then converted into alcohol, which is promptly oxidized, is worthy of serious consideration (see page 393). Such a theory explains the destination of the carbohydrates and their relations to calorification. There can be no doubt that in certain cases of fever, alcohol administered in large quantity may be oxidized and "feed" the fever, thereby saving consumption of tissue.

In a series of observations made in 1879 (Flint), it seemed impossible to account for the heat actually produced in the body and expended as force in muscular work etc., by the heat-value of food and of tissue consumed. The estimates of heat-production, made by the direct method, were then adopted; but even the indirect estimates - which were much less - presented difficulty, though in a less degree. In these observations it was shown that water was actually produced in the body, in quantity over and above that contained in food and drink, during severe and prolonged muscular work. It was also shown that water was produced in considerable quantity during twenty-four hours of abstinence from food. It has been shown by Pettenkofer and Voit that "the elimination of water is very much increased by work, and the increase continues during the ensuing hours of sleep." As regards the oxidation of hydrogen in this formation of water, it is probable that the hydrogen of the tissues is used and that the matter thus consumed is supplied again to the tissues in order to maintain the physiological status of the organism. Adding the heat-value of the water thus formed to the heat-value of food, there is little difficulty in accounting for the heat and force actually produced and expended.

The demonstration that water is actually formed within the organism under certain conditions not only completes the oxidation-theory of the production of animal heat, but it affords an explanation of certain physiological phenomena that have heretofore been obscure. It it well known, for example, that a proper system of physical training will reduce the fat of the body to a minimum consistent with health and strength. This involves a diet containing a relatively small proportion of fat and liquids, and regular muscular exercise attended with profuse sweating. Muscular work increases the elimination of water, while it also exaggerates for the time the calorific processes. Muscular exercise undoubtedly favors the consumption of the non-nitrogenous parts of the body, and 
a diminution of the supply of fats, carbohydrates and water in the food prevents, to a certain extent, the new formation of fat. In excessive muscular work, the production of water is increased and the circulation becomes more active. The volume of blood then circulating in the skin and passing through the lungs in a given time is relatively increased, and there is an increased discharge of water from these surfaces. The same condition that produces an increased quantity of water in the body and has a tendency to exaggerate the process of calorification seems to produce also an increased evaporation from the surface, which serves to equalize the animal temperature.

Equalization of the Animal Temperature. - There is always more or less loss of heat by evaporation from the general surface; and when the surrounding atmosphere is very cold it becomes desirable to reduce this loss to the minimum. This is done by appropriate clothing, which certainly must be regarded as a physiological necessity. Clothing protects from excessive heat as well as from cold. Thin porous coverings moderate the heat of the sun, equalize evaporation and afford great protection in hot climates. In excessive cold, clothing moderates the loss of heat from the surface. When the body is not exposed to currents of air, garments are useful chiefly as non-conductors, imprisoning many layers of air, which are warmed by contact with the person. It is also important to protect the body from the wind, which greatly increases the loss of heat by evaporation.

When from any cause there is a tendency to undue elevation of the body-heat, cutaneous transpiration is increased and the temperature is kept at the proper standard. This has already been considered in treating of the action of the skin, and facts were noted showing that men can work when exposed to a heat much higher than that of the body itself. The quantity of vapor that is lost under these conditions is sometimes very large. Tillet recorded an instance of a young girl who remained in an oven for ten minutes without inconvenience, at a temperature of $324.5^{\circ}$ Fahr. $\left(162.5^{\circ} \mathrm{C}\right.$.). Blagden, in his noted experiments in a heated room, made in connection with Banks, Solander, Fordyce and others, found in one series of observations that a temperature of $21 \mathrm{I}^{\circ} \mathrm{Fahr}$. $\left(99.5^{\circ} \mathrm{C}\right.$.) could be easily borne; and at another time the heat was raised to $260^{\circ} \mathrm{Fahr}$. $\left(126.5^{\circ} \mathrm{C}\right.$.). Under these external conditions, the body is protected from the radiated heat by clothing, the air is perfectly dry, and the animal temperature is kept down by increased evaporation from the surface.

It is a curious fact that after exposure of the body to an intense dry heat or to a heated vapor, as in Turkish or Russian baths, when the general temperature is somewhat raised and the surface is bathed in 
perspiration, a cold plunge, which checks the action of the skin almost immediately, is not injurious and is decidedly agreeable. This presents a striking contrast to the effects of sudden cold on a system heated and exhausted by long-continued exertion. In the latter instance, when the perspiration is suddenly checked, serious disorders of nutrition, with inflammation etc., may supervene. When the skin acts to keep down the temperature of the body in simple exposure to external heat, there is no modification in nutrition, and the tendency to an elevation of the body-temperature comes from causes entirely external. It is a practical observation that no ill effects are produced, under these circumstances, by suddenly changing the external conditions; but when the body-heat is raised by a modification of the internal nutritive processes, as in prolonged muscular work, these changes should not be suddenly arrested; and a suppression of the compensative action of the skin is likely to produce disturbances in nutrition, often resulting in inflammations.

\section{Relations of Heat to Force}

Since the development of the theory of the conservation of forces, which had its origin in an essay published by J. R. Mayer, in I842, physiologists have applied the laws of correlation and conservation of forces to operations involving the production of heat and the development and expenditure of force in animals. This theory, if applicable to what were formerly called vital operations, certainly affords, in its definite quantities of heat and force as expressed in heat-units and footpounds, a basis for calculating the value of material changes in the body. Without discussing the purely physical questions involved, the laws of correlation and conservation of forces, as they are applicable to human physiology, may be briefly stated as follows:-

Potential energy is something either residing in or imparted to matter, which is capable of being converted directly or indirectly into heat. The animal body, for example, is a storehouse of potential energy. Its tissues may be made to unite with oxygen and heat is produced. Any body may have potential energy imparted to it. If a weight is raised to a certain height, when the force which has accomplished this work is exhausted, the potential energy imparted to the weight causes it to fall; and in this fall heat is produced. The weight may be supported at the height to which it has been raised for an indefinite time; but it still possesses the potential energy that has been imparted to it, and when the support is removed, this potential energy is converted into force, which may be converted into heat. Potential energy may be converted directly into heat, as when a body is oxidized. 
It is converted indirectly into heat, when movement, falling or other force is produced; for all force may be converted into heat. The conversion into heat, directly or indirectly, affords a convenient measure of potential energy. Using the example of the change of potential energy into heat by oxidation, the energy stored up in matter is measured by estimating the heat produced by oxidation as so many heat-units. Using the example of falling force imparted to a weight, the potential energy imparted to the body is estimated by calculating the heat produced by the body falling.

If the entire body of an animal were to be burned in a calorimeter, the heat produced would be an exact measure of the potential energy of the tissues converted into heat by oxidation. If one could imagine an animal perfectly quiescent, neither losing nor gaining weight, nourished by food, expending no force in circulation and respiration, but supplied with oxygen, the potential energy of the food could be measured by the heat produced. In animal organisms, heat is produced mainly by oxidation, although other chemical processes contribute to the production of heat to some extent. The body contains the potential energy stored up in its tissues. The oxygen taken in by respiration changes a certain part of this potential energy into heat. If food is not supplied in adequate quantity, the body loses weight by this change of tissue into certain matters, such as carbon dioxide, water and urea, which are discharged. Food supplies the waste of tissue and is the ultimate source of the potential energy of the body. If food is supplied in excess, that which is not in some form discharged from the body remains and adds to the total potential energy stored up in the organism.

Kinetic energy is mechanical force. It is the force of a falling body, or as regards animal mechanics, it is muscular force used in respiration, circulation or any kind of muscular work. In physics, kinetic energy, or force, and heat are regarded as mutually convertible. The reasoning by which this law was formulated is the following:-

The force used in raising a weight to a certain height, which is imparted to the weight as potential energy, is precisely equal to the force developed by this body as it falls. If this force could be transmitted to another body of equal weight, without any loss of energy by friction, it would raise the second weight to an equal height. The arbitrary unit of this force is a foot-pound or a kilogrammeter, terms that have already been defined. The falling of a body of a certain weight through a definite distance produces a definite quantity of heat that itself is capable of producing force; and it is assumed that the heat produced by a falling body, if absolutely and entirely converted into force, would raise that body to the height from which it had fallen, or 
would exactly equal the falling force. A heat-unit is therefore said to be equal to a definite number of foot-pounds or kilogrammeters. Calculations have been made showing the conversion of foot-pounds or kilogrammeters into heat-units, but mechanical difficulties have thus far prevented the actual conversion of heat-units into their equivalents in foot-pounds or kilogrammeters. As a matter of reasoning, however, it is assumed that if a certain number of foot-pounds or kilogrammeters is equal to a certain number of heat-units, the reverse of the equation is true; but the application of this law to animal physiology is always by a conversion of heat-units into foot-pounds or kilogrammeters. The experiments on which the law rests have been made by converting footpounds or kilogrammeters into heat-units.

In work by machinery, a large proportion of the force-value of fuel is dissipated in the form of heat. This is well illustrated by Landois: If a steam engine burning a certain quantity of coal, but doing no work, is placed in a calorimeter, the heat produced can be measured. If, now, the engine is made to do a certain work, as in raising a weight, the heat, as measured by the calorimeter, will be less, and the work done is found to be very nearly proportional to the decrease in the measured heat (Hirn). It is estimated by Landois, that of the heat produced by the body, one-fifth may be used as work. In the best steam engine, it is possible to use only one-eighth as work, seven-eighths being dissipated as heat.

Many elaborate and careful estimates have been made of the mechanical work produced by the human body. The basis of such calculations is more or less indefinite, and the reduction of the work to foot-pounds or kilogrammeters is difficult and inexact. Even the general statement, that of the heat-units produced by the body, fourfifths remain as heat and one-fifth is converted into work, must be regarded as approximate.

In the application of the law of correlation and conservation of forces to animal mechanics, the matters consumed in the production of heat and force are food-stuffs. These are oxidized, either directly or indirectly. If oxidized indirectly, it is the substance of the tissues that is thus consumed, the loss being repaired by food. A certain proportion of the heat produced is used in maintaining the heat of the body; another part is dissipated by radiation from the general surface; another part is converted into force and is used by the heart and the respiratory muscles ; and still another part is used in the general work of the muscular system. All this involves a considerable oxidation of matter.

In this connection it is interesting to note again the heat-value of different alimentary matters :- 
One gram of proteid = about 4500 calories (gram-degrees).

One gram of fat = about 9000 calories (gram-degrees).

One gram of carbohydrate $=$ about 4000 calories (gram-degrees).

In the animal organism, a part of the potential energy of the tissues may be converted into force by voluntary effort. In fevers, an abnormally large proportion of the potential energy of the organism is converted into heat, and it is not possible to use much of this energy as force. These and other peculiarities of living bodies, as regards the production of heat and force, are difficult of explanation. In the essential fevers, the conditions which involve the abnormal production of heat finally consume the substance of the tissues. They involve especially an increased production of carbon dioxide and urea and not to any great extent the formation of water. If heat-producing alimentary substances and alcohol can be introduced and consumed, the tissues are thereby proportionally saved from degeneration and destruction. 



\section{PART II}

MUSCULAR MOVEMENTS - VOICE AND SPEECH - NERVOUS SYSTEM - SPECIAL SENSES EMBRYOLOGY 


\title{
CHAPTER XVI
}

\author{
MUSCULAR MOVEMENTS
}

Amorphous contractile substance and ameboid movements - Ciliary movements - Movements due to elasticity - Elastic tissue - Muscular movements - Contraction of involuntary muscular tissue - Physiological anatomy of the voluntary muscular tissue - Connective tissue - Bloodvessels and lymphatics - Connection of the muscles with the tendons - Chemical composition of the muscles - Physiological properties of the muscles - Elasticity of muscles - Muscular tonicity - Sensibility of the muscles - Muscular contractility and excitability - Muscular contraction - Changes in the form of fibres during contraction - Rigor mortis - Passive organs of locomotion - Physiological anatomy of the bones - Lacunæ Canaliculi - Bone-cells or corpuscles - Marrow of the bones - Periosteum - Physiological anatomy of cartilage - Cartilage-cavities - Cartilage-cells - Elastic cartilage and fibrocartilage.

THE processes connected with the nutrition of animals involve certain movements; and almost all animals possess in addition the power of locomotion. Many of these movements have of necessity been considered in connection with the different functions; as the action of the heart and vessels in the circulation, the uses of the muscles in respiration, the muscular acts in deglutition, the peristaltic movements and the mechanism of defecation and urination. There remain, however, certain general facts in regard to various kinds of movement and the mode of action of the different kinds of muscular tissue, that demand more or less extended consideration.

Amorphous Contractile Substance and Ameboid Movements. - In some of the lowest forms of beings, in which hardly anything but amorphous matter and a few granules can be recognized with the microscope, certain movements of elongation and retraction of amorphous substance have been observed. In the higher animals, similar movements have been noted in certain of their structures, such as the leucocytes, the contents of the ovum, epithelial cells and connectivetissue cells. These movements usually are simple changes in the form of the cell; and they depend on an organic substance formerly called sarcode and now known as protoplasm, which has already been described. In the anatomical elements of adult animals of the higher classes, these movements usually appear slow and gradual, even when viewed with high magnifying powers; but in some of the very lowest forms of life, the movements serve as a means of progression and are more rapid. 
Such movements are called ameboid; and they probably are analogous to ciliary movements, the cause of which, however, is obscure.

Ciliary Movements. - The epithelium covering certain of the mucous membranes is provided with little hair-like processes on the borders of the cells, called cilia. These are in constant motion from the beginning to the end of life; and they produce currents on the surfaces of the

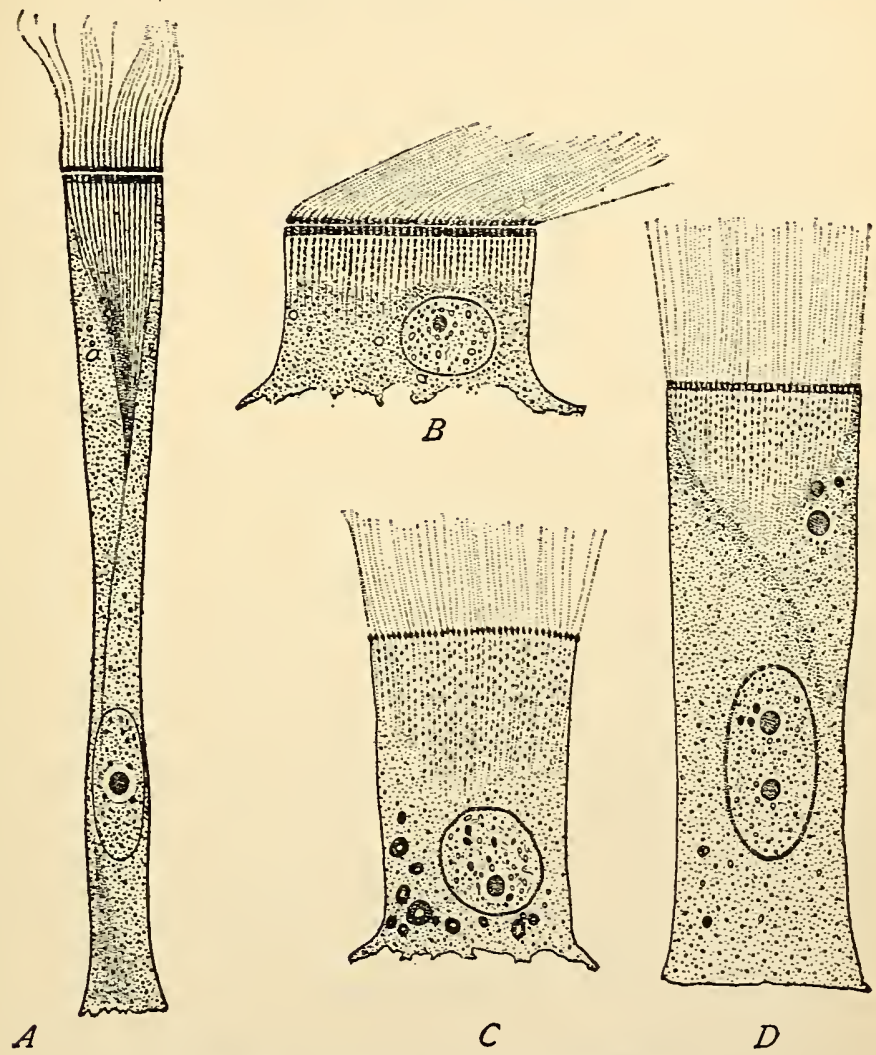

Fig. 80. - Ciliated epithelium (Engelmann).

$A$, from intestinal epithelium of anodonta; $B$, from gill of anodonta; $C, D$, intestinal epithelium of cyclas.

membranes to which they are attached, the direction being usually from within outward. In man and in the warm-blooded animals generally, the ciliated or vibratile epithelium is of the variety called columnar, conoidal or prismoidal. The cilia are attached to the thick ends of the cells, and they form on the surface of the membrane a continuous sheet of moving processes. In general structure the ciliary processes are homogeneous, and they gradually taper from their attachment to the cell to an extremity of excessive tenuity. 
The presence of cilia has been demonstrated on the following surfaces : The respiratory passages, including the nasal fossæ, the pituitary membrane, the summit of the larynx, the bronchial tubes, the superior surface of the velum palati and the Eustachian tubes; the sinuses about the head; the lachrymal sac and the internal surface of the eyelids; the genital passages of the female, from the middle of the neck of the uterus to the fimbriated extremities of the Fallopian tubes; some of the seminal passages; the ventricles of the brain and some unimportant situations. In these parts, on each cell of conoidal epithelium are six to twelve processes, about $\frac{1}{25000}$ of an inch ( $\mathrm{I} \mu$ ) in thickness at their base, and $\frac{1}{5000}$ to $\frac{1}{4000}$ of an inch $(5$ to $6 \mu)$ in length. Between the cilia and the substance of the cell, there usually is a thin transparent disk. The appearance of the cilia is represented in Fig. 8o. When seen in situ, they appear regularly disposed over the surface, are of nearly equal length and are slightly inclined in the direction of the opening of the cavity lined by the membrane.

When the ciliary movements are seen in a large number of cells, the appearance is well illustrated by the comparison by Henle to the undulations of a field of wheat agitated by the wind. In watching this movement, it usually is seen to gradually diminish in rapidity, until what at first appeared simply as currents, produced by movements too rapid to be studied in detail, are revealed as distinct undulations, in which the action of individual cilia may be studied. Several kinds of movement have been described, but the most common is a bending of the cilia, simultaneously or in regular succession, in one direction, followed by an undulating return to the perpendicular. The other movements, such as the infundibuliform, in which the point describes a circle around the base, the pendulum-movement etc., are not common and are unimportant.

The combined action of the cilia on the surface of a mucous membrane, moving as they do in one direction, is to produce currents of considerable power. This may be illustrated under the microscope by covering the surface with a liquid holding little solid particles in suspension, when the granules are tossed from one portion of the field to another with considerable force. It is not difficult, indeed, to measure in this way the rapidity of the ciliary currents. In the frog it has been estimated at $\frac{1}{2} \frac{1}{00}$ to $\frac{1}{175}$ of an inch (IOO to I $40 \mu$ ) per second, the number of vibratile movements being seventy-five to one hundred and fifty per minute. In the fresh-water polyp the movements are more rapid, being two hundred and fifty or three hundred per minute. There is no reliable estimate of the rapidity of the ciliary currents in man, but they probably are more active than in animals low in the scale. 
The movements of cilia, like those observed in fully-developed spermatozoids, seem to be independent of nervous influence, and they are affected only by local conditions. They may continue, under favorable circumstances, for more than twenty-four hours after death, and they can be seen in cells detached from the body, when moistened with proper solutions. When the cells are moistened with water, the activity of their movement is at first increased; but it soon disappears as the cells become swollen. Acids arrest the movements, but they may be excited by feebly alkaline solutions. As regards the uses of these movements, it is sufficient to refer to the physiology of the parts in which cilia are found, where the peculiarities of their action are considered more in detail. In the lungs and the air-passages generally and in the genital passages of the female, the currents are of considerable importance; but it is difficult to imagine the use of these movements in certain other situations, as the ventricles of the brain.

Movements due to Elasticity. - There are certain important movements in the body due simply to the action of elastic ligaments or membranes. These are distinct from muscular movements and are not to be classed even with the movements produced by the resiliency of muscular tissue, in which muscular tonicity is more or less involved. Movements of this kind consist simply in the return of movable parts to a certain position after they have been displaced by muscular action, and in the reaction of tubes after forcible distention, as in the walls of the large arteries.

Elastic Tissue. - Most anatomists adopt the division of the fibres of elastic tissue into three varieties. This division relates to the size of the fibres; and all

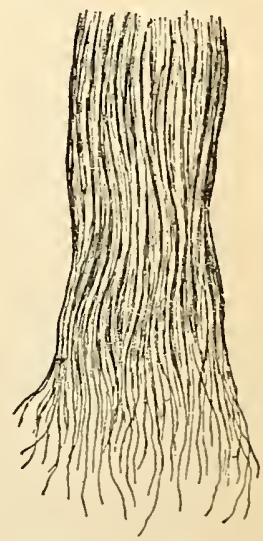

Fig. 81. - White fibrous tissue; one end of the bundle has been teased out so as to display the component fibrille (Piersol). varieties are found to possess essentially the same chemical composition and general properties. On account of the yellow color of this tissue, presenting, as it does, a decided contrast to the white glistening appearance of the inelastic fibres, it frequently is called the yellow elastic tissue.

The first variety of elastic tissue is composed of small fibres, usually intermingled with fibres of the ordinary inelastic tissue. They possess all the chemical and physical characters of the larger fibres but are very fine, measuring $\frac{1}{25000}$ to $\frac{1}{6000}$ or $\frac{1}{5000}$ of an inch ( $\mathrm{I}$ to 4 or $5 \mu$ ) in diameter. If acetic acid is added to a preparation of ordinary connective tissue, the inelastic fibres are rendered semitransparent, but the elastic fibres are unaffected and become quite distinct. They are then 
seen isolated - that is, never arranged in bundles - usually with a dark double contour, branching, brittle, and when broken, their extremities curled and presenting a sharp fracture like a piece of India rubber.

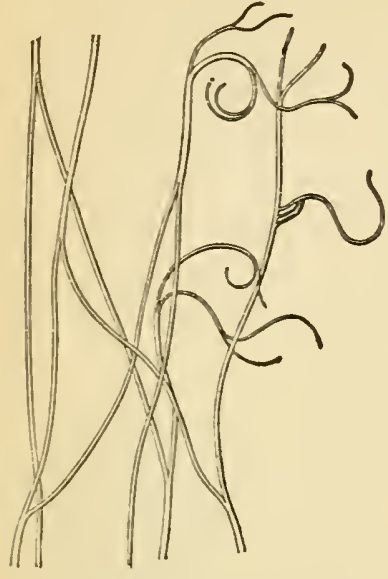

Fig. 82. - Elastic fibres isolated: from the adientitia of the aorta (Piersol, after Schiefferdecker).

These fibres take a wavy course between the bundles of inelastic fibres in the areolar tissue and in most of the ordinary fibrous membranes. They are found in greater or less abundance in the situations just mentioned; in the ligaments, but not the tendons; in the layers of non-striated muscular tissue; the true skin; the true rocal chords; the trachea, bronchial tubes, and largely in the parenchyma of the lungs; the external layer of the large arteries; and, in brief, in nearly all situations in which ordinary connective tissue exists.

The second variety of elastic tissue is composed of fibres, larger than the first, ribbon-shaped, with well-defined outlines, anastomosing, undulating or curved in the form of the letter $\mathrm{S}$, presenting curled ends and sharp fracture, like the smaller fibres. These measure $\frac{1}{5000}$ to $\frac{1}{3000}$ of an inch $(5$ to $8 \mu$ ) in diameter. Their type is found in the ligamenta subflava and the ligamentum nuchæ. They are also found in some of the ligaments of the larynx, the stylo-hyoid ligament and the suspensory ligament of the penis.

The third variety of elastic tissue is found forming the middle coat of the large arteries and has already been described in connection with the vascular system. The fibres are large and flat, inosculating freely with each other by short communicating branches. These anastomosing fibres, forming the so-called fenestrated membranes, are arranged in layers, and the structure is sometimes called the lamellar elastic tissue.

The physical property of elasticity plays an important part in many of the animal functions. Examples of this are in the action of the large arteries in the circulation and in the resiliency of the parenchyma of

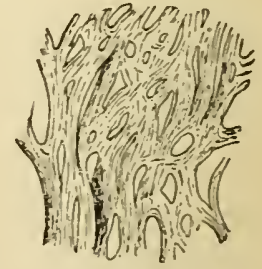

Fig. 83.-Portion of the elastic tissue of the intima of the human aerta; the fibres are so broad and so closely grouped that they constitute an elastic sheet - the so-called fenestrated membrane of Henle (Piersol). the lungs. The ligamenta subflava and the ligamentum nuchæ are important in aiding to maintain the erect posture of the body and head and to restore this position when flexion has been produced by muscular action. Still, the contraction of muscles also is necessary to keep the body in a vertical position. 


\section{Muscular Movenents}

Muscular movements are divided into voluntary and involuntary; and usually there is a corresponding division of the muscles as regards their minute anatomy. The latter, however, is not absolute; for there are certain involuntary actions, like the contractions of the heart or the movements of deglutition, that require the rapid and vigorous contraction characteristic of the voluntary muscular tissue; and here the structure resembles that of the voluntary muscles.

Physiological Anatomy of the Involuntary Muscular Tissue. - The involuntary muscular system presents a striking contrast to the volun-

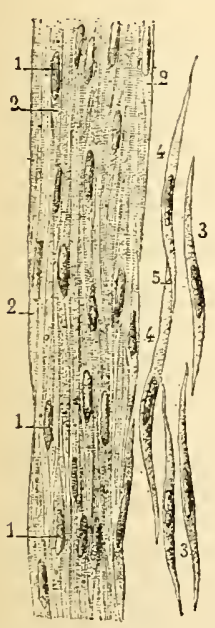

Fig. 84. - Muscular fibres from the urinary bladder of the human subject, $\times 200$ (Sappey).

I, I, I, nuclei ; 2, 2, 2, borders of some of the fibres; 3,3 , isolated fibres; 4,4 , two fibres joined together at 5 .

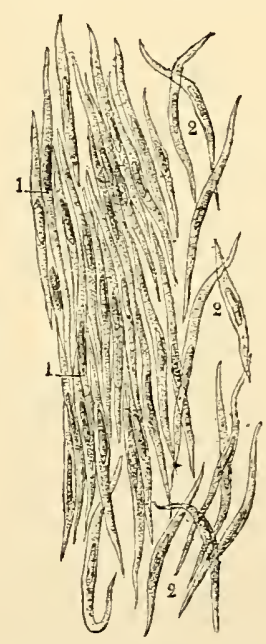

Fig. 85. - .Muscular fibres from the aorta of the calf, $\times 200$ (Sappey).

I, I, fibres joined with each other; 2, 2, 2, isolated fibres.

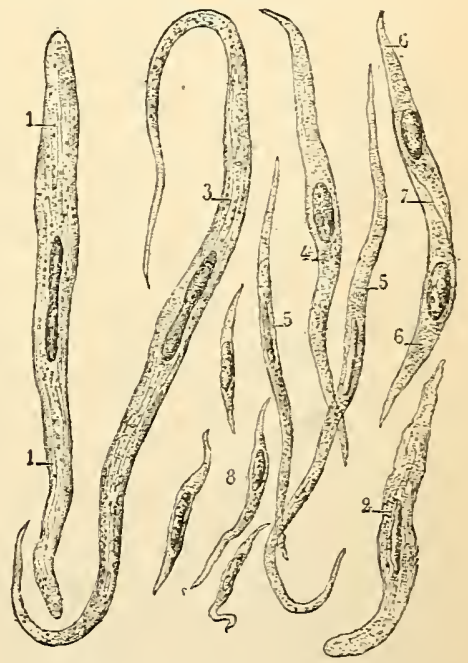

Fig. 86. - Muscular fibres from the uterns of a woman who died at the ninth month of utero-gestation, $\times 35^{\circ}$ (Sappey).

I, I, 2, short wide fibres; $3,4,5,5$, longer and narrower fibres; 6,6 , two fibres united at $7 ; 8$, small fibres in process of development.

tary muscles, not only in its minute anatomy and mode of action, but in the arrangement of its fibres. While the voluntary muscles are almost invariably attached by their extremities to movable parts, the involuntary muscles form sheets or membranes in the walls of hollow organs, and by their contraction they simply modify the capacity of the cavities which they surround. On account of the peculiar structure of the fibres, they have been called muscular fibre-cells, smooth muscular fibres, pale fibres, non-striated fibres, fusiform fibres or contractile cells. The distribution of these fibres to parts concerned in the organic functions, as 
the alimentary canal, has given them the name of organic muscular fibres, or fibres of organic life. In their natural condition, the involuntary muscular fibres are pale, finely granular, flattened, and of an elongated spindle-shape, with a very long, narrow, almost linear nucleus. The nucleus usually has no distinct nucleolus and sometimes it is curved or shaped like the letter $\mathrm{S}$. The ordinary length of these fibres is about $\frac{1}{500}$ of an inch $(50 \mu)$ and their breadth about $\frac{1}{4000}$ of an inch $(6 \mu)$. In the gravid uterus they undergo remarkable hypertrophy, measuring here $\frac{1}{80}$ to $\frac{1}{50}$ of an inch $(300$ to $500 \mu)$ in length, and $\frac{1}{2000}$ of an inch $(12 \mu)$ in breadth.

In the contractile sheets of involuntary muscular tissue, the fibres are arranged side by side, are closely adherent, and their extremities are, as it were, dove-tailed into each other. Usually the borders of the fibres are regular and their extremities are simple; but sometimes the ends are forked and the borders present one or more little projections. The fibres seldom exist in a single layer except in the smallest arterioles. Usually the layers are multiple, being superimposed in regular order.

Contraction of the Inioluntary IIuscular Tissue. - The mode of contraction of the involuntary muscles is peculiar. It does not take place immediately on the reception of a stimulus, applied either directly or through the nerves, but it is gradual, enduring for a time and then followed by slow and gradual relaxation. A description of the peristaltic movements of the intestines gives an idea of the mode of contraction of these fibres, with the gradual propagation of the stimulus along the alimentary canal as the food makes its impression on the mucous membrane. Another illustration is afforded by labor-pains. These are due to the muscular contractions of the uterus, and they last for a few seconds or one or two minutes. Their gradual access, continuation for a certain period, and gradual disappearance coincide with the description of contractions of the involuntary muscular fibres.

The contraction of the involuntary muscular tissue is relatively slow, and the fibres return slowly to a condition of repose. The movements are always involuntary. Peristaltic action is the rule, and the contraction takes place progressively and without oscillations. Contractility persists for a long time after death. Excitation of the nerves has less influence on contraction of these fibres than direct excitation of the muscles. The involuntary muscular tissue is regenerated rapidly, while the structure of the voluntary muscles is restored with difficulty after destruction or division.

Phy'siological Anatomy of the Voluntary Muscular Tissuc. - A voluntary muscle contains, in addition to its contractile substance, fibres of inelastic and elastic tissue, adipose tissue, abundant bloodvessels, nerves 
and lymphatics, with certain nuclear and cellular anatomical elements. The muscular system in a well-proportioned man is equal to about twofifths of the weight of the body. Among the characteristic properties of the muscles are elasticity, a constant and insensible tendency to contraction, called tonicity, the power of contracting forcibly on the reception of a proper stimulus, and a peculiar kind of sensibility. The muscular tissue is made up of a great number of microscopic fibres, known as primitive muscular fasciculi. These are called red, striated or voluntary fibres. Their structure is complex, and they may be subdivided longitudinally into fibrillæ and transversely into disks. In very short muscles, some of the primitive fasciculi may run the entire length of the muscle; but the fasciculi usually are I.2 to I.6 inch (30 to 40 millimeters) in length. The fasciculi, however, do not inosculate with each other, but the end of one fasciculus is united longitudinally with the end of another by a strongly adhesive substance, the line of union being oblique; so that the fibres practically run the entire length of the muscle. Exceptions to this arrangement are the branching muscular fibres of the heart and of the face and tongue. Each fasciculus in ordinary muscular tissue is enclosed in its own sheath, without branching or inosculation. This sheath contains the true muscular substance only, and it is not penetrated by bloodvessels, nerves or lymphatics. The color of the muscular fibres by transmitted light is a delicate amber, resembling the color of the blood-corpuscles.

The primitive fasciculi vary in size in different individuals, in the same individual under different conditions and in different muscles. As a rule they are smaller in young persons and in females than in adult males. They are comparatively small in persons of slight muscular development. In persons of great muscular vigor, or when the general muscular system or particular muscles have been increased in size and power by exercise, the fasciculi are relatively larger. It is probable that physiological increase in the size of a muscle from exercise is due to an increase in the size of the preëxisting fasciculi and not to the formation of new elements. In young persons the fasciculi are $\frac{1}{1700}$ to $\frac{1}{1200}$ of an inch ( 15 to $20 \mu$ ) in diameter. In the adult they measure $\frac{1}{450}$ to $\frac{1}{25} \overline{0}$ of an inch $(55$ to $100 \mu)$.

The appearance of the primitive muscular fasciculi under the microscope is characteristic. They present regular transverse strix, formed of alternating dark and clear bands about $\frac{1}{250} \overline{0}$ of an inch ( $1 \mu$ ) wide. With a high magnifying power, a fine transverse line is observed running through the middle of each one of the clear bands. In addition they present longitudinal striæ, not so distinct, and difficult to follow to any extent in the length of the fasciculus, but tolerably well marked, 
particularly in muscles that are habitually exercised (see Plate IX, Fig. 6). The muscular substance, presenting this peculiar striated appearance, is enclosed in a thin but elastic and resisting tubular membrane, called the sarcolemma or myolemma. This envelope can not be seen in ordinary preparations of the muscular tissue; but it frequently happens that the contractile muscular substance is broken, leaving the sarcolemma intact, which gives a good view of the membrane and conveys an idea of its strength and elasticity. Attached to the inner surface of the sarcolemma are small elongated nuclei with their long diameter in the direction of the fasciculi.

Connective Tissue. - In the muscles there is a membrane surrounding a number of the primitive fasciculi. This is called the perimysium. The fibrous membranes that connect together the secondary bundles, with their contents, are enclosed in a sheath enveloping the entire muscle, sometimes called the external perimysium. The peculiarity of these membranes as distinguished from the sarcolemma is that they have a fibrous structure and are connected together throughout the muscle, while the tubes forming the sarcolemma are structureless and each one is distinct.

The name usually given to ordinary fibrous tissue is connective tissue. It has been called cellular, areolar or fibrous, but most of these names were given without a clear idea of its structure. Its principal anatomical element is a fibre of excessive tenuity, wavy and with a single contour. These fibres are collected into bundles of variable size and are held together by an adhesive amorphous substance. The wavy lines that mark the bundles of fibres give them a characteristic appearance (see Fig. 8I).

The direction and arrangement of the fibres in the various tissues present marked differences. In the loose tissue beneath the skin and between the muscles and in the loose structure surrounding some of the glands and connecting the sheaths of bloodvessels and nerves to the adjacent parts, the bundles of fibres form a large network and are wavy in their course. In the strong dense membranes, as the aponeuroses, the proper coats of many glands, the periosteum and perichondrium and the serous membranes, the waves of the fibres are shorter and the fibres themselves interlace much more closely. In the ligaments and tendons the fibres are more nearly straight and are arranged longitudinally.

The proportion of elastic fibres in different situations is variable; but they are all of the smallest variety and present a striking contrast to the inelastic fibres in form and size. Although they are very small, the elastic fibres always present a double contour.

Certain cellular and nuclear elements are always found in the con- 
nective tissue. The cells are known as connective-tissue cells. They are irregular in size and form, some being spindle-shaped or caudate, and others, star-shaped. They possess one, and sometimes two or three, clear ovoid nuclei with distinct nucleoli. On the addition of acetic acid the cells disappear, but the nuclei are unaffected. It is impossible to give accurate measurements of the cells on account of their great variations in size. Between the muscles, and in the substance of the muscles between the bundles of fibres, there always exists a greater or less quantity of adipose tissue in the meshes of the fibrous structure.

Bloodvessels and Lymphatics. - The muscles are abundantly supplied with bloodvessels, usually by a number of small arteries with two satellite veins. The capillary arrangement in this tissue is peculiar. From the smallest arterioles, capillary vessels are given off, arranged in a network with tolerably regular, oblong, rectangular meshes, their long diameter following the direction of the fibres. These envelop each primitive fasciculus, enclosing it completely, the artery and vein being on the same side. The capillaries are smaller than in any other part of the vascular system (see Plate X, Fig. I).

The arrangement of the lymphatics in the muscles has not been definitely ascertained. There are lymphatics surrounding the large vascular trunks of the extremities and of the abdominal and thoracic walls, which, it would appear, must come from the substance of the muscles; but they have not been traced to their origin.

Connection of the Muscles with the Tendons. - The primitive muscular fasciculi terminate in little conical extremities that are received into corresponding depressions in the bundles of fibres composing the tendons; but this union is so close that the muscle or the tendon may be ruptured without a separation at the point of union. In the penniform muscles this arrangement is quite uniform. In other muscles it is essentially the same, but the perimysium seems to be continuous with the loose fibrous tissue enveloping the corresponding tendinous bundles.

Chemical Composition of the Muscles. - The most important proteid constituent of the muscles is myosin. This resembles fibrin, but it presents certain points of difference in its behavior to reagents, by which it may be readily distinguished. One of its peculiar properties is that it is dissolved at an ordinary temperature by a mixture of one part of hydrochloric acid and ten of water. Myosin is now regarded as a compound substance containing at least four different proteids that may be separated by fractional heat-coagulation: (I) A globulin that coagulates at about II $7^{\circ} \mathrm{Fahr}$. $\left(47^{\circ} \mathrm{C}\right.$. $)$. This is called paramyosinogen. (2) A globulin that coagulates at $133^{\circ} \mathrm{Fahr}$. $\left(56^{\circ} \mathrm{C}\right.$. $)$. (3) A globulin that 
coagulates at $146^{\circ} \mathrm{Fahr} .\left(63^{\circ} \mathrm{C}\right.$.). This is called myoglobulin. (4) An albumin, analogous to serum-albumin, called myo-albumin.

Combined with the organic constituents of the muscular substance are mineral salts in great variety, which cannot be separated without incineration. Certain excrementitious matters have also been found in the muscles; and probably nearly all those eliminated by the kidneys exist here, although they are taken up by the blood as fast as they are produced and consequently are detected with difficulty. The muscles also contain inosite, inosic acid, lactic acid, and certain volatile acids of fatty origin. During life the muscular liquid is slightly alkaline, but it becomes acid soon after death. The muscle itself, during contraction, has an acid reaction. The muscular juice is alkaline or neutral after moderate exercise as well as during repose; but when a muscle is made to undergo excessive exercise, the lactic and other acids exist in greater quantity and the reaction becomes acid.

\section{Physiological Properties of the Muscles}

The important general properties of the striated muscles are the following: I. elasticity; 2. tonicity; 3. sensibility of a peculiar kind; 4. contractility and excitability. These are all necessary to the physiological action of the muscles. Their elasticity is brought into play in opposing muscles or sets of muscles; one set acting to move a part and to extend the antagonistic muscles, which, by virtue of their elasticity, retract when the extending force is removed. Their tonicity is an insensible and a more or less constant contraction, by which the action of opposing muscles is balanced when both are in the condition of what is called repose. Their sensibility is peculiar and is expressed chiefly in the sense of fatigue and in the appreciation of weight and of resistance to contraction. Their contractility and excitability are properties that enable them to contract under stimulation. All these general properties belong strictly to physiology, as do some special acts that are not necessarily involved in the study of ordinary descriptive anatomy.

Elasticity of Muscles. - The true muscular substance contained in the sarcolemma is eminently contractile; and although it may possess a certain degree of elasticity, this property is most strongly marked in the accessory anatomical elements. The interstitial fibrous tissue is loose and presents a certain number of elastic fibres; and the sarcolemma is very elastic. It is probably the sarcolemma that gives to the muscles their retractile power after simple extension.

Muscular Tonicity. - The muscles, under normal conditions, have an insensible and a constant tendency to contract, which is more or less 
dependent on the action of motor nerves. If, for example, a muscle is cut across in a surgical operation, the divided extremities become permanently retracted; or if the muscles of one side of the face are paralyzed, the muscles on the opposite side insensibly distort the features. It is difficult to explain these phenomena by assuming that tonicity is due to reflex action, for there is no evidence that the contraction takes place as the consequence of a stimulus. All that can be said is that a muscle, not excessively fatigued and with its nervous connections intact, is constantly in a state of insensible contraction more or less marked.

Sensibility of the Muscles. - The muscles possess that kind of sensibility which gives an appreciation of resistance, immobility, and elasticity of substances that are grasped, or which, by their weight, are opposed to muscular effort. It is by the appreciation of weight and resistance that the force required to accomplish muscular acts is regulated. These properties refer chiefly to simple muscular efforts. After long-continued exertion, there is a sense of fatigue that is peculiar to the muscles. It is difficult to separate this entirely from the sense of nervous exhaustion, but it is to a certain extent distinct; for when suffering from the fatigue that follows overexertion, it seems as though a nervous stimulus could be sent to the muscles, to which they are for the time unable to respond. When muscles are thrown into tetanic contraction, a peculiar sensation is produced, which is entirely different from painful impressions made on ordinary sensory nerves. In the cramps of cholera, tetanus, or the convulsions from strychnin, these distressing sensations are very marked. The general sensibility of muscles is very slight. The rather indefinite property, called muscular sense, is supposed by some physiologists to be connected with peculiar structures - neuro-muscular spindles - that will be described in connection with the nervous system.

Muscular Contractility and Excitability. - During life and under normal conditions, muscles contract in obedience to a proper stimulus applied either directly or through the nerves. In the natural action of the organism, this contraction is induced by nervous influence either through volition or reflex action. Still, a muscle may be living and yet have lost its contractility. For example, after a muscle has been for a long time paralyzed and disused, the application of the most powerful stimulus will fail to induce contraction; but when examined with the microscope, it is found that the nutrition of the muscle has become profoundly affected and that the contractile substance has disappeared. Muscular contractility persists for a certain time after death and in muscles separated from the body; and this fact has been taken advantage of by physiologists in the study of the properties of the muscular tissue. 
When the motor nerves are divided, the nutrition of the muscles to which they are distributed is disturbed; and although muscular contractility may persist for some time after nervous excitability has disappeared, it is much diminished at the end of six weeks. Some varieties of curare paralyze the end-plates of the motor nerves, leaving the sensory nerves intact. If a frog is poisoned by introducing a little of this agent under the skin, stimulation, electric or mechanical, applied to an exposed nerve, fails to produce muscular contraction; but if the stimulus is applied directly to the muscles, they will contract vigorously. ${ }^{1}$ If a frog is poisoned with potassium sulphocyanate, however, the contrary effect is observed; that is, the muscles become insensible to excitation, while the nervous system is unaffected. This may be demonstrated by applying a tight ligature around the body in the lumbar region, involving all the parts except the lumbar nerves. If the poison is now introduced beneath the skin above the ligature, only the anterior parts are affected, because the vascular communication with the posterior extremities is cut off. If the exposed nerves are now stimulated, the muscles of the legs are thrown into contraction, showing that the nervous conductivity and excitability remain. Reflex movements in the posterior extremities also may be produced by irritation of the parts above the ligature. These experiments leave no doubt as to the existence of an independent excitability in the muscular tissue. Contractions of muscles, it is true, are excited normally through the nervous system, and artificial stimulation of a motor nerve is the most efficient method of producing the simultaneous action of all the fibres of a muscle or of a set of muscles; but direct electric, mechanical or chemical irritation of the muscles themselves will produce contraction after the nervous connections have been destroyed.

\section{Muscular Contraction}

The stimulus of the will, conveyed through the conductors of motor impulses from the brain to a muscle or set of muscles, excites the muscular fibres and causes them to contract. In muscles that have been exercised and educated, this action is regulated with great nicety, so that the most delicate and rapid as well as powerful contractions may be produced. Certain movements not under the control of the will are produced as the result of unconscious reflex action of nervous centres.

${ }^{1}$ It has been shown, by a very simple experiment, that curare paralyzes the end-plates only of the motor nerves. If a frog is poisoned with curare and one leg is ligated above the knee, leaving the nerve out of the ligature, reflex movements may be excited in the ligated leg, but not in other parts. This shows that the motor nerves still retain their conductivity. 
During contraction certain important changes are observed in the muscles themselves: They change in form, consistence, and to a certain extent in their constitution; the different periods of their stimulation, contraction and relaxation are positive and well marked; their nutrition is for the time modified; they develop electric currents; and in short, they present a number of general phenomena, distinct from the results of their action, that are more or less important.

The most prominent of the phenomena accompanying muscular action are shortening and hardening of the fibres. It is necessary only to observe the action of any well-developed muscle to appreciate these changes. The active shortening is shown by the approximation of the points of attachment, and the hardening is sufficiently palpable. The latter phenomenon is marked in proportion to the development of the true muscular tissue and its freedom from inert matter, such as fat. Notwithstanding the marked and constant changes in the form and consistence of the muscles during contraction, their actual volume undergoes modifications so slight that they may be disregarded.

Changes in the Form of the Muscular Fibres during Contraction.-An essential experimental condition in the study of the mechanism of muscular action is to imitate, in a muscle or a part of a muscle that can be subjected to direct observation, the force that naturally excites it to contraction. The application of electricity to the nerve is the most convenient method that can be employed for this purpose. In this way a single contraction may be produced, or by employing a rapid succession of impulses, so-called tetanic action may be excited. While the electric current is not identical with nerve-impulses, it is the best substitute that can be used in experiments on muscular contractility, and it has the advantage of affecting but little the physical and chemical integrity of the nervous and muscular tissues.

A description of the electric current and its generation, with the different forms of electric apparatus, belongs properly to physics and would be out of place in a text-book on physiology. It is sufficient to say that apparatus has been devised for the study of muscular contraction that enables experimenters to stimulate muscles either directly, or indirectly through excitation of motor nerves. The contractions produced by exciting a muscle through stimulation of its motor nerve most nearly resemble normal muscular action.

In the study of the phenomena of muscular contraction, a so-called nerve-muscle preparation is most useful. This is simply the leg of a. frog detached from an animal just killed, the skin removed, and the sciatic nerve remaining attached to the muscles. With this preparation, a stimulus may be applied either to the nerve or directly to the muscle. 
When the nerve is stimulated by means of a single feeble inductive shock, the muscle contracts almost immediately after the electric discharge. There is, however, a short interval between the stimulation and the contraction. This is called the latent period, and it occupies about $\frac{1}{100}$ of a second. Then follows the stage of muscular contraction, which rapidly progresses to its maximum. The period of contraction occupies about $\frac{1}{40}$ of a second. The muscle then rather more slowly returns to its relaxed condition. This period occupies a little less than $\frac{1}{12}$ of a second. Not counting the latent period, the duration of a muscular contraction and relaxation is about $\frac{1}{10}$ of a second. A part of the latent period is occupied in the conduction of the stimulus along the nerve, and the part belonging to the muscle is much shorter. Following the relaxation, there is often a vibratory wave of very slight contractions, due to the elastic reaction of the muscular fibres.

A stimulus may be applied to a nerve, that is of just sufficient strength to produce a muscular contraction. This is called the minimal strength of stimulus. By gradually increasing the strength of the electric current, a maximal stimulus may be reached. The vigor of the muscular contraction is proportional to the strength of the stimulus, between these two extremes. The latent period is shorter with a strong than with a weak stimulation. The vigor of contraction is not increased by increasing the strength of the stimulus beyond the maximal point.

The foot in a muscle-nerve preparation may be loaded with a weight. When this is done, provided the weight be not too great to be lifted by the muscle, the extent of the contraction increases with the weight up to what may be called the maximum of contraction. Beyond this point, however, it diminishes until the weight is increased beyond the power of the muscle. Increasing the weight increases the length of the latent period.

Repeated stimulation, especially when the foot is weighted, is followed by fatigue of the muscle. At first the work seems gradually to increase the power of the contractions, and they progress to a maximum; but then fatigue begins and the power gradually diminishes until the muscle becomes exhausted.

Within certain limits, diminished temperature at first increases the vigor of muscular contractions, which afterward are gradually diminished. Moderate increase of temperature increases the vigor of the contractions and shortens all the periods, including the latent period.

In an entire muscle, the contraction is in the form of a wave. With delicate chronometric apparatus, the rapidity of this wave between two points in a muscle may be measured. In the frog's muscle, the wave moves at the rate of about $\mathrm{I} 2 \mathrm{O}$ inches (3 meters) in a second. 
In a muscle-nerve preparation, the effect of a second stimulus applied to the nerve before the first contraction is concluded is to increase the extent of the first contraction. Indicating these contractions by curves, the curve of the second contraction is superimposed on the first. When a number of successive stimuli are employed, the curves are superimposed until a maximum is reached. Muscular contraction produced in this way is called incomplete tetanus. The rate of the stimuli required to produce this kind of tetanus is fifteen to twenty per second. Incomplete tetanus may be produced by voluntary effort as well as by repeated electric shocks; and this explains ordinary muscular action. In voluntary effort, the normal stimulus that excites muscular contraction is sent from the nerve-centres along the nerves in the form of what are called impulses. The rate at which these impulses are sent out by the nerve-cells is ten to twelve in a second. By the superimposition of the contractions, the force exerted by a muscle may be regulated by the will. Were it not thus, prolonged muscular effort would be impossible. During voluntary contraction a muscle is in a condition of incomplete tetanus; but this must not be confounded with the conditions in the disease known as tetanus or in poisoning with strychnin.

A muscle possesses extensibility as well as elasticity. After it has been stretched to its limit, when the force is removed it returns to its original length. When any elastic body that is stretched afterward returns to exactly its original dimensions, it is said to be endowed with perfect elasticity. Elastic tissue is an example of this. Muscles, also, are perfectly elastic structures.

In a state of rest there is a feeble electric current flowing from the general surface to the cut surface. This is called the current of rest (Du Bois Reymond). When, however, the muscle contracts, the galvanometer, which has been deviated by the current of rest, shows a diminution in this current, and it may return to zero under the influence of a current in the opposite direction. This second current is called the current of action. This diminution or extinction of the currents of rest is known as negative variation of muscle-current.

Delicate apparatus for measuring changes in temperature show that muscular action is attended with elevation of temperature in the muscle itself. In large animals this has been found to amount to several degrees; but a rise in temperature may be noted even in a muscle-nerve preparation from a frog. By the thermopile, variations of $\frac{1}{400} \overline{0}$ of a degree C. may be noted (Helmholtz).

Muscles in contraction undergo certain important chemical changes. The reaction of a resting muscle is alkaline. Muscular action results in 
the production of sarcolactic acid, and consequently the reaction of the muscles becomes acid. During contraction a muscle consumes oxygen and gives off carbon dioxide. It is probable, also, that other katabolic products are formed in the same way.

Rigor Mortis. - At a certain time after death, the entire voluntary muscular system becomes rigid, and the muscles will not contract under stimulation. This is due to a gradual coagulation of the muscle-plasma. During this process the muscles contract and give off heat and carbon dioxide in small quantities. The muscles first affected usually are those of the neck and lower jaw and the rigidity gradually extends to the feet. Cadaveric rigidity begins in fifteen minutes to seven or eight hours after death and lasts until putrefaction sets in. It disappears in the same order, as regards different muscles, in which it began and extended.

\section{Passive Organs of Locomotion}

The study of locomotion involves a knowledge of the physiological anatomy of certain passive organs, such as the bones, cartilages and ligaments. Although a complete history of the structure of these parts trenches somewhat on the domain of anatomy, a brief description of their histology will practically complete the account of the tissues of the body, with the exception of the nervous system and the organs of reproduction, which will be taken up hereafter.

Locomotion is effected by the action of muscles on certain passive movable parts. These are the bones, cartilages, ligaments, aponeuroses and tendons. The fibrous structures have already been described, and it remains only to study the structure of bones and cartilages.

Physiological Anatomy of the Bones. - The bones are composed of what is called the fundamental substance, with cavities and canals of peculiar form. The cavities contain corpuscular bodies called bonecorpuscles. The canals of larger size serve for the passage of bloodvessels, while the smaller canals (canaliculi) connect the cavities with each other and finally with the vascular tube (see Plate X, Fig. 2). Many of the bones present a medullary cavity filled with a peculiar structure called marrow. In almost all bones there are two distinct portions; one, that is exceedingly compact, and the other, more or less spongy, or cancellated. The bones are invested with a membrane, containing vessels and nerves, called periosteum.

The fundamental substance is composed of an organic matter, called ossein, combined with various inorganic salts, in which calcium phosphate largely predominates. In addition to calcium phosphate, the bones contain calcium carbonate, calcium fluoride, magnesium phos- 
phate, sodium phosphate and sodium chloride. The relative proportions of the organic and inorganic constituents are somewhat variable; but the average is about one-third of the former to two-thirds of salts. This proportion is necessary to the proper consistence and toughness of the bones.

Anatomically, the fundamental substance of the bones is arranged in the form of regular concentric lamellæ, about $\frac{1}{3000}$ of an inch $(8 \mu)$ in thickness. This matter is of an indefinitely and faintly striated appearance, but it can not be reduced to distinct fibres. In the long bones the arrangement of the lamellæ is quite regular, surrounding the Haversian canals and forming what are sometimes called the Haversian rods, following in their direction the length of the bone. In the short thick bones the lamellæ are more irregular, frequently radiating from the central portion toward the periphery.

The Haversian canals are found in the compact bony structure. They are either absent or very few in the spongy and reticulated portions. Their form is rounded or ovoid, the larger canals being sometimes quite irregular. In the long bones their direction usually is longitudinal, although they often anastomose by lateral branches. Each one of these

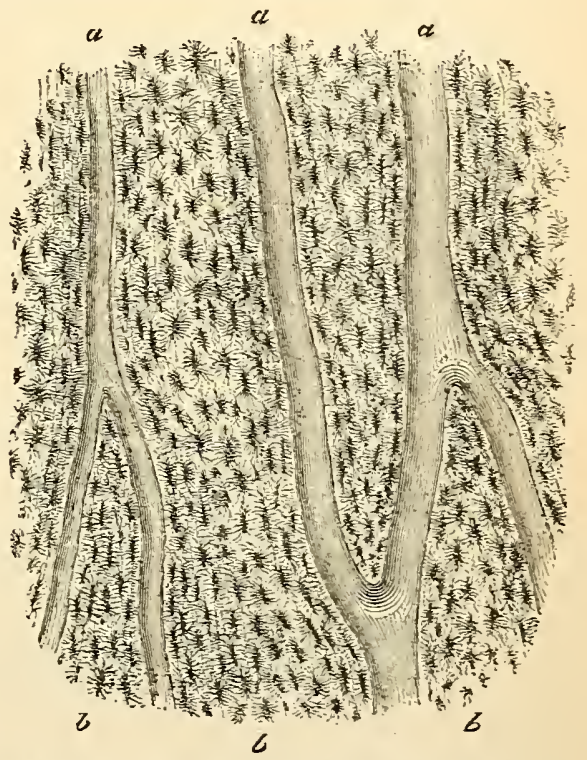

Fig. 87. - Vascular canals and lacunce seen in a longitudinal section of the humerus, $\times 200$ (Sappey).

$a, a, a$, vascular canais; $b, b, b$, lacunæ and canaliculi in the fundamental substance.

canals contains a bloodvessel, and their disposition constitutes the vascular arrangement of the bones. They are all connected with openings on the surfaces of the bones, by which the arteries penetrate and the veins emerge. Their size, of course, is variable. The largest are about $\frac{1}{60}$ of an inch $(400 \mu)$ and the smallest $\frac{1}{500}$ of an inch $(30 \mu)$ in diameter. Their average size is $\frac{1}{250}$ to $\frac{1}{200}$ of an inch (I00 to I $25 \mu$ ). In a transiverse section of a long bone the Haversian canals may be seen cut across and surrounded by twelve to fifteen lamellæ.

Lacunce. - The fundamental substance is everywhere marked by irregular microscopic excavations, of a peculiar form, called lacunæ. They are connected with little canals, giving them a stellate appearance. These canals are most abundant at the sides of the lacunæ. The 
lacunæ measure $\frac{1}{1250}$ to $\frac{1}{800}$ of an inch $(20$ to $30 \mu)$ in their long diameter, by about $\frac{1}{2} \frac{1}{000}$ of an inch $(10 \mu)$ in width.

Canaliculi. - These are little wavy canals, connecting the lacunæ with each other and presenting communications between the first series of lacunæ and the Haversian canals. Each lacuna presents eighteen to twenty canaliculi radiating from its borders. The length of the canaliculi is $\frac{1}{800}$ to $\frac{1}{600}$ of an inch ( 30 to $40 \mu$ ), and their diameter is about $\frac{1}{25000}$ of an inch $(1 \mu)$. The arrangement and relations of the Haversian canals, lacunæ and canaliculi are shown in Figure 88.

Bone-cells, or Corpuscles. - These structures are stellate, granular, with a large nucleus and several nucleoli, and of exactly the size and

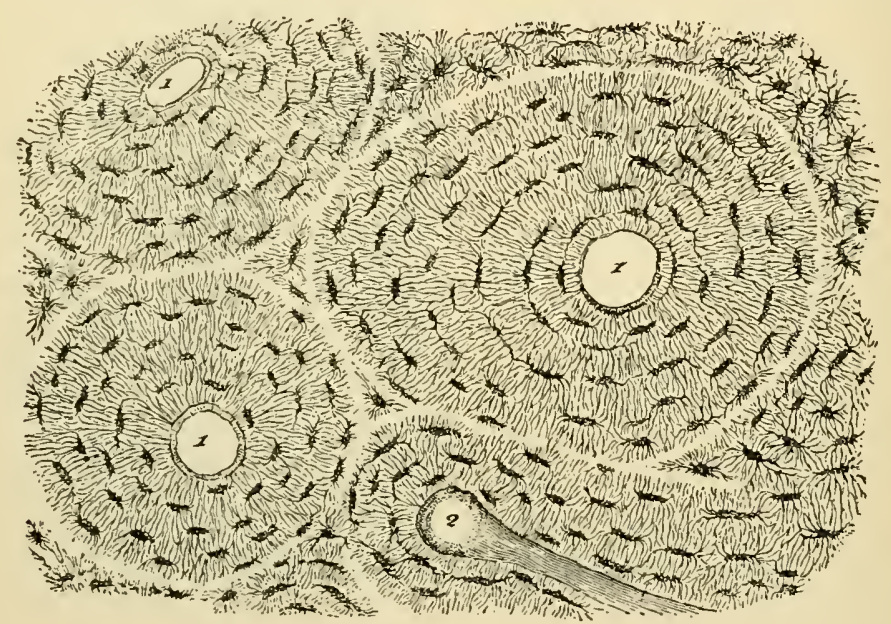

Fig. 88. - Vascular canals and lacunce seen in a transverse section of the humerus, $\times 200$ (Sappey).

I, I, I, section of the Haversian canals; 2, section of a longitudinal canal divided at the point of its anastomosis with a transverse canal. Around the canals, cut across perpendicularly, are seen the lacunæ (with their canaliculi), forming concentric rings.

form of the lacunæ. They send out prolongations into the canaliculi, but it has been impossible to ascertain positively whether or not they form membranes lining the canaliculi throughout their entire length.

Marrow of the Bones. - The marrow is found in the medullary cavities of the long bones, filling them completely and moulded to the irregularities of their walls. It is also found filling the cells of the spongy portion. In other words, with the exception of the vascular canals, lacunæ and canaliculi, the marrow fills all the spaces in the fundamental substance. The cavities of the bones are not lined with a membrane corresponding to the periosteum, and the marrow is applied directly to the bony substance. In the fœtus and in young children the marrow is red and very vascular. In the adult it is yellow in some 
bones and gray or gelatiniform in others. It contains certain peculiar cells and nuclei, with amorphous matter, adipose vesicles, connective tissue, bloodvessels and nerves, with little bodies, called myelocytes, and free nuclei. These are found in greater or less number in the bones at all ages, but they are more abundant in proportion as the amorphous matter and fat-cells are deficient. The myelocytes resemble the leucocytes of the blood but are a little larger and each has a vesicular nucleus. They are capable of ameboid movements. In addition, the marrow contains erythroblasts, acidophile cells, large basophile cells, leucocytes, and erythrocytes. The erythroblasts multiply by karyokinesis, lose their nuclei and become red blood-corpuscles. The uses and destination of the other varieties of cells have not been definitely ascertained (see Plate I). Irregular nucleated patches, described under the name of myeloplaxes, or giant-cells, more abundant in the spongy portions than in the medullary canals, are found applied to the internal surfaces of the bones. They vary in size and form (measuring $\frac{1}{1200}$ to $\frac{1}{2} \frac{1}{50}$ of an inch, or 20 to $100 \mu$ in diameter), are finely granular and present two to twenty or thirty nuclei. The nuclei are clear and ovoid and usually contain a distinct nucleolus. The myeloplaxes are rendered pale by acetic acid and the nuclei are then brought distinctly into view. They are especially abundant in the red marrow.

In addition to the anatomical elements just described, the marrow contains a few delicate bundles of connective tissue, most of which accompany the bloodvessels. In the fotus the adipose vesicles are few or may be absent; but in the adult they are quite abundant, and in some bones they seem to constitute the whole mass of the marrow. They do not differ materially from the fat-cells in other situations. Holding these different structures together, is a variable quantity of semitransparent, amorphous or slightly granular matter.

The nutrient arteries of the bones send branches to the marrow, usually two in number for the long bones, which are distributed between the various anatomical elements and finally surround the fatty lobules and the fat vesicles with a delicate capillary plexus. The veins correspond to the arteries in their distribution. The nerves follow the arteries and are lost when these vessels no longer present a muscular coat. Nothing is known of the presence of lymphatics in any part of the bones or in the periosteum.

The chief physiological interest connected with the marrow of the bones is in its relations to the formation of blood-corpuscles. This question has already been discussed in connection with the development of the corpuscular elements of the blood.

Periosteum. - In most of the bones the periosteum presents a single 
layer of fibrous tissue, but in some of the long bones two or three layers may be demonstrated. This membrane adheres to the bone but can be separated without much difficulty. It covers the bones completely, except at the articular surfaces, where its place is supplied by cartilaginous incrustation. It is composed mainly of ordinary fibrous tissue with small elastic fibres, bloodvessels, nerves and a few adipose vesicles.

The arterial branches ramifying in the periosteum are quite abundant, forming a close anastomosing plexus which sends small branches into the bony substance. There is nothing peculiar in the arrangement of the veins. The distribution of the veins in the bony substance itself has been very little studied.

The nerves of the periosteum are abundant and form in its substance quite a close plexus.

The adipose tissue is variable in quantity. In some parts it forms a continuous sheet, and in others the vesicles are scattered here and there in the substance of the membrane.

The importance of the periosteum to the nutrition and regeneration of the bones is very great. Instances are on record where bones have been removed, leaving the periosteum, and in which the entire bone has been -reproduced. The importance of the periosteum has been still further illustrated by the experiments of Ollier and others, on transplantation of this membrane in the different tissues of living animals, which has been followed by the formation of bone in these situations.

Plyysiological Anatomy of Cartilage. - In this connection the structure of the articular cartilages presents the chief physiological interest. The articular surfaces of all the bones are incrusted with a layer of cartilage, varying in thickness between $\frac{1}{50}$ and $\frac{1}{25}$ of an inch (0.5 and I millimeter). The cartilaginous substance is white, opaline and semitransparent when examined in thin sections. It is not covered with a membrane, but in the non-articular cartilages it has an investment analogous to the periosteum, called perichondrium.

Examined in thin sections cartilage is found to consist of a homogeneous fundamental substance, marked with excavations called cartilage-cavities. The intervening substance has a peculiar organic constituent called chondrin. The organic matter is united with a certain proportion of inorganic salts. The fundamental substance is elastic and resisting. The cartilages are closely united to the subjacent bony tissue. The free articular surface has already been described in connection with the synovial membranes.

Cartilage-cavitics. - These cavities are rounded or ovoid, measuring $\frac{1}{1250}$ to $\frac{1}{300}$ of an inch $(20$ to $80 \mu)$ in diameter. They are smaller in 
the articular cartilages than in other situations, as in the costal cart1lages. They are simple excavations in the fundamental substance, having no lining membrane, and they contain a small quantity of a viscid liquid with one or more cells. They are analogous to the lacunæ of the bones.

Cartilage-cells. - Near the surface of the articular cartilages the cavities contain each a single cell; but in the deeper portions the cavities are long and contain two to twenty cells arranged longitudinally. The cells are about the size of the smallest cavities. They are ovoid, with a large granular nucleus. They often contain a few small globules of oil. In the costal cartilages the cavities are not abundant but are rounded and quite large. The cells contain usually a certain quantity of fatty matter. The appearance of the ordinary articular cartilage is represented in Figure 89.

The ordinary cartilages have neither bloodvessels, lymphatics nor nerves, and are nourished by imbibition from the surrounding parts. In the development of the body, the anatomy of the cartilaginous tissue possesses peculiar importance, from the fact that the deposition of cartilage, with a few exceptions, precedes the formation of bone (see Plate X, Fig. 3).

Elastic Cartilage.-This variety of cartilage presents certain important peculiarities in the structure

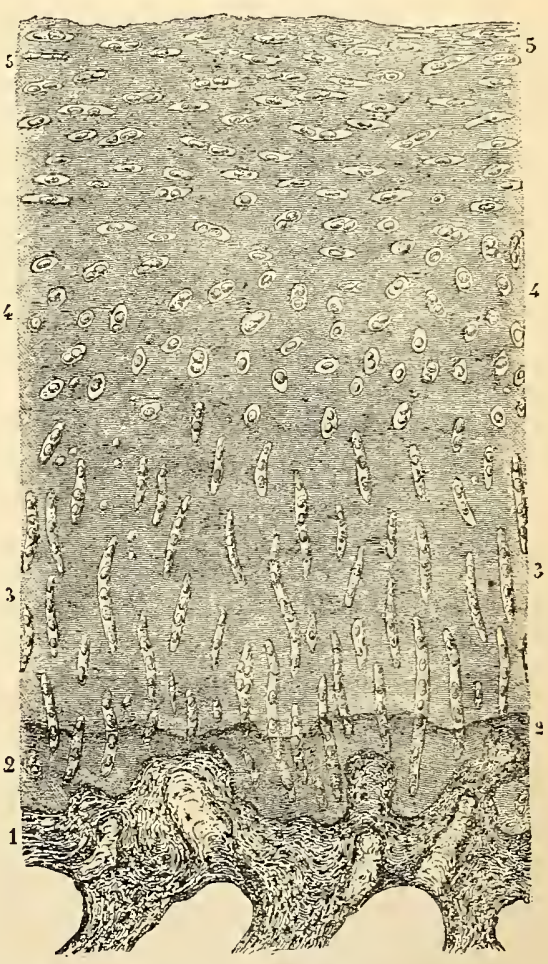

Fig. 89.- Vertical section of diarthrodial cartilage (Sappey).

I, I, osseous tissue; 2,2 , superficial layer of osseous tissue treated with hydrochloric acid; 3,3 , cavities and cells of the deep layer of cartilage ; 4,4 , cavities and cells of the middle layer; 5,5 , cavities and cells of the superficial layer. of its fundamental substance. It exists principally in the cartilages of the ear and of the Eustachian tubes, the cartilages of Santorini and of Wrisberg and the epiglottis. Elastic cartilage is composed of fibrous tissue with a great predominance of elastic fibres, fusiform nucleated fibres, a certain number of adipose vesicles, cartilage-cells, bloodvessels and nerves. The fibrous elements above mentioned take the place of the homogeneous fundamental substance of ordinary cartilage. The most 
important peculiarity in the structure of this tissue is that it is abundantly supplied with bloodvessels and nerves (see Plate X, Fig. 4).

Fibro-cartilage, found in the synchondroses, the interarticular disks and the intervertebral cartilages, is composed of interlacing bundles of fibrous tissue surrounding individual cartilage-cells or small groups of cells. This structure more nearly resembles tendon than it does true cartilage.

The reader is referred to works on anatomy for a history of the action of the muscles. In some works on physiology will be found descriptions of the acts of walking, running, leaping, swimming etc.; but it has been thought better to omit these subjects, rather than to enter so minutely as would be necessary into purely anatomical details and to give descriptions of movements that are simple and familiar. 


\section{CHAPTER XVII}

\section{VOICE AND SPEECH}

Physiological anatomy of the vocal organs-Muscles of the larynx-Crico-thyroid muscles - Arytenoid muscle - Lateral crico-arytenoid muscles - Thyro-arytenoid muscles Mechanism of the production of the voice - Movements of the glottis during phonation - Action of the intrinsic muscles of the larynx in phonation - Action of accessory vocal organs - Laryngeal mechanism of the vocal registers - Vocal registers in the male Vocal registers in the female - Mechanism of speech - Vowels-Consonants - The phonograph and telephone.

THE principal organ concerned in the production of the voice is the larynx. The accessory organs are the lungs, trachea, expiratory muscles, the mouth and the resonant cavities about the face. The lungs furnish the air by which the vocal chords are thrown into vibration, and the mechanism of this action involves merely modifications of expiration. By the action of the expiratory muscles the intensity of vocal sounds is regulated. The trachea not only conducts the air to the larynx, but it may assist, by resonance, in modifying the quality of the voice. Most of the variations in tone and quality, however, are effected by the action of the larynx itself and of the resonant cavities situated above.

Physiological Anatomy of the Vocal Organs. - The vocal chords are stretched across the superior opening of the larynx from before backward. They consist of two pairs. The superior, called the false vocal chords, or the ventricular bands, are not concerned in the production of the voice. They are less prominent than the inferior chords, although they have nearly the same direction. They are covered with a thin mucous membrane that is closely adherent to the subjacent tissue. The chords are composed of ordinary fibrous tissue with a few elastic fibres.

The true vocal chords, or vocal bands, are situated just below the superior chords. Their anterior attachments are near together, at the middle of the thyroid cartilage, and are immovable. Posteriorly they are attached to the movable arytenoid cartilages; and by the action of certain muscles their tension may be modified and the chink of the glottis may be opened or closed. These are much larger than the false vocal chords and they contain a great number of elastic fibres. Like 
the superior vocal chords they are covered with a very thin and closely adherent mucous membrane. The mucous membrane over the borders of the chords is covered with flattened epithelium without cilia. There are no mucous glands in the membrane covering either the superior or

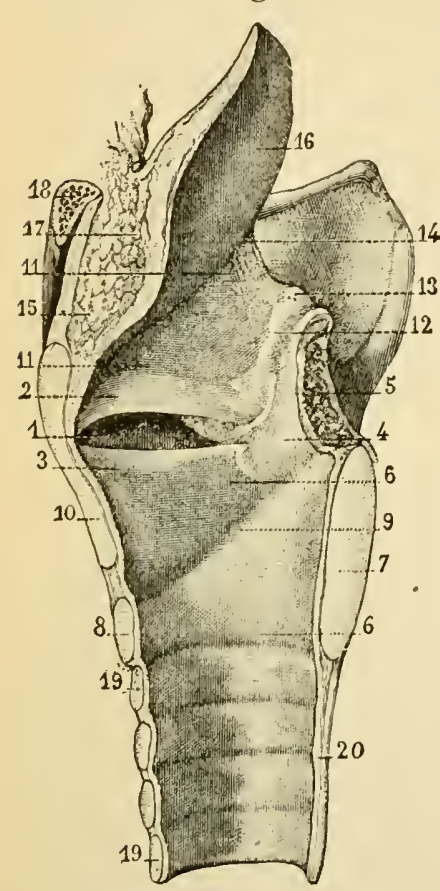

Fig. 90. - Vertical section of the human lary'n, showing the vocal chords (Sappey).

$I$, ventricle of the larynx; 2 , superior vocal chord; 3 , inferior vocal chord; 4 , arytenoid cartilage; 5 , section of the arytenoid muscle; 6,6 , inferior portion of the cavity of the larynx; 7 , section of the posterior portion of the cricoid cartilage; 8 , section of the anterior portion of the cricoid cartilage; 9. superior border of the cricoid cartilage; Io, section of the thyroid cartilage; II, II, superior portion of the cavity of the larynx; I2, 13, arytenoid gland; I4, 16, epiglottis; 15,17 , adipose tissue; 18 , section of the hyoid bone; $19,19,20$, trachea. consequence, the posterior portion, which carries the arytenoid cartilages and the posterior attachments of the vocal chords, is depressed. This action would, of course, increase the distance between the arytenoid cartilages and the anterior portion of the thyroid, elongate the vocal chords, and subject them to a certain degree of tension. Experiments the inferior chords. The inferior vocal chords alone are directly concerned in the production of the voice.

Muscles of the Larynx. - The muscles of the larynx are classified as extrinsic and intrinsic. The extrinsic muscles are attached to the outer surface of the larynx and to adjacent organs, such as the hyoid bone and the sternum. They are concerned chiefly in the movements of elevation and depression of the larynx. The intrinsic muscles are attached to the different parts of the larynx, and by their action on the articulating cartilages are capable of modifying the tension of the vocal chords.

The vocal chords can be rendered tense or lax by muscular action. Their fixed point is in front, where their extremities, attached to the thyroid cartilage, are nearly or quite in contact with each other. The arytenoid cartilages, to which they are attached posteriorly, present a movable articulation with the cricoid cartilage; and the cricoid, which is narrow in front, and is wide behind, where the arytenoid cartilages are attached, presents a movable articulation with the thyroid cartilage. It is evident, therefore, that muscles acting on the cricoid can cause it to swing on its two points of articulation with the inferior cornua of the thyroid, raising the anterior portion and approximating it to the lower edge of the thyroid; and as a 
have shown that such an effect is produced by the contraction of the crico-thyroid muscles.

The articulations of the different parts of the larynx are such that the arytenoid cartilages can be approximated to each other posteriorly, thus diminishing the interval between the posterior attachments of the vocal chords. This action can be effected by contraction of the single muscle of the larynx (the arytenoid) and also by the lateral crico-arytenoids. The thyro-arytenoid muscles, the most complex of all the intrinsic muscles in their attachments and the direction of their fibres, are important in regulating the tension and capacity of vibration of the vocal chords.

The posterior crico-arytenoid muscles, arising from each lateral half of the posterior surface of the cricoid cartilage and passing upward and outward to be inserted into the outer angle of the inferior portion of the arytenoid cartilages, rotate these cartilages outward, separate them and act as dilators of the chink of the glottis. These muscles are concerned chiefly in the respiratory movements of the glottis during inspiration.

The muscles mainly concerned in the modifications of the voice by their action on the vocal chords are the cricothyroids, the arytenoid, the lateral cricoarytenoids and the thyro-arytenoids. The following is a sketch of their attachments and mode of action :-

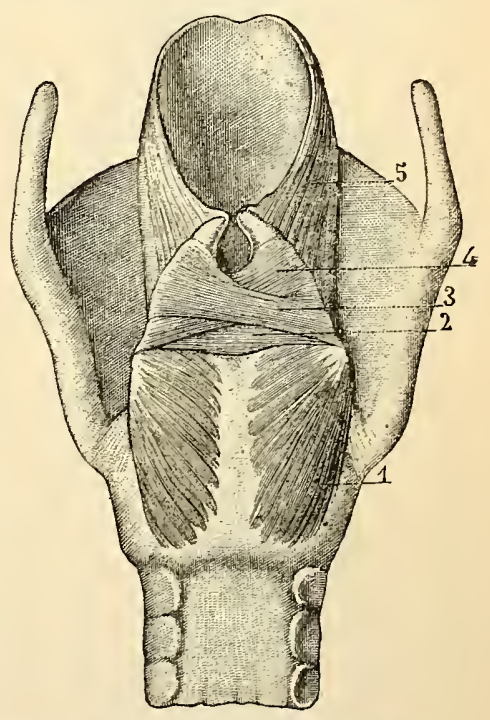

Fig. 9r. - Posterior view of the muscles of the larynx (Sappey).

I, posterior crico-arytenoid muscle; 2,3 , 4 , different fasciculi of the arytenoid muscle; 5, aryteno-epiglottidean muscle.

Crico-thyroid Muscles. - These muscles are situated outside of the larynx, at the anterior and lateral portions of the cricoid cartilage. Each muscle is of a triangular form, the base of the triangle presenting posteriorly. It arises from the anterior and lateral portions of the cricoid cartilage, and its fibres diverge to be inserted into the inferior border of the thyroid cartilage, extending from the middle of this border posteriorly as far back as the inferior cornua. After dividing the nervous filaments distributed to these muscles, a certain degree of hoarseness of the voice is observed, due to relaxation of the vocal chords; and by imitating their action mechanically the cricoid and thyroid cartilages may be approximated in front, carrying back the arytenoid cartilages and rendering the chords tense. 
Arytenoid Muscle. - This single muscle fills the space between the arytenoid cartilages and is attached to their posterior surfaces and borders. Its action is to approximate the posterior extremities of the chords and to constrict the glottis, so far as the articulations of the arytenoid cartilage with the cricoid will permit. This muscle is important in phonation, as it serves to fix the posterior attachments of the vocal

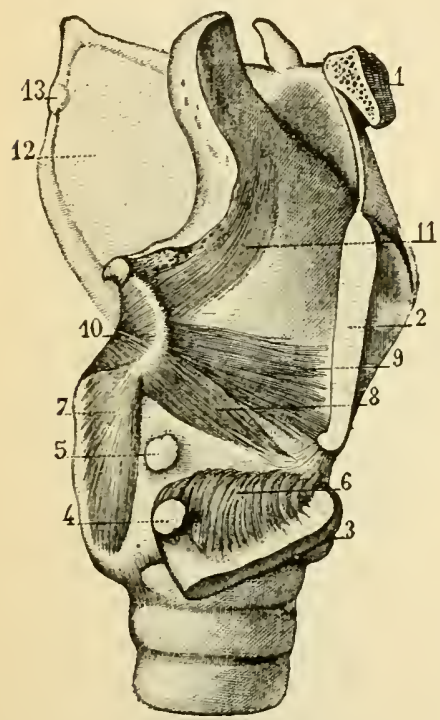

Fig. 92. - Lateral view of the muscles of the larynx (Sappey).

$I$, body of the hyoid bone; 2 , vertical section of the thyroid cartilage; 3 , horizontal section of the thyroid cartilage, turned downward to show the deep attachment of the crico-1hyroid muscle; 4 , facet of articulation of the small cornu of the thyroid cartilage with the cricoid cartilage; 5, facet on the cricoid cartilage; 6 , superior attachment of the crico-thyroid muscle; 7 , posterior cricoarytenoid muscle; 8 , lateral crico-arytenoid muscle; 9 , thyro-arytenoid muscle ; Io, arytenoid muscle; II, aryteno-epiglottidean muscle; 12 , middle thyrohyoid ligament; 13, lateral thyro-hyoid ligament.

of muscles operating directly or indirectly on the vocal chords render the problem of the precise mode of action of the intrinsic muscles of the larynx complicated and difficult. It is certain, however, that in these muscular acts, the thyro-arytenoids play an important part. Their contraction regulates the thickness of the chords, while at the same time it modifies their tension. The swelling of the chords, which certain other of the intrinsic muscles.

Lateral Crico-arytenoid Muscles.-These muscles are in the interior of the larynx. They arise from the sides and superior borders of the cricoid cartilage, pass upward and backward and are attached to the base of the arytenoid cartilages. By dividing all the filaments of the recurrent laryngeal nerves except those distributed to these muscles and then stimulating the nerves, it is shown that they act to approximate the vocal chords and constrict the glottis, particularly in its interligamentous portion. These muscles, with the arytenoid, act as constrictors of the larynx.

Thyro-arytenoid Muscles. - These muscles are situated within the larynx. They are broad and flat and arise in front from the upper part of the crico-thyroid membrane and the lower half of the thyroid cartilage. From this line of origin, each muscle passes backward in two fasciculi, both being attached to the anterior surface and the outer borders of the arytenoid cartilages. Stimulation of the nervous filaments distributed to these muscles renders the vocal chords tense. The variations that may be produced in the pitch and quality of the voice by the action

chords and to increase the efficiency of 
may be rendered regular and progressive by voluntary action, is an important element in determining the timbre of the voice.

Mechanism of the Production of the Voice. - If the glottis is examined with the laryngoscope during ordinary respiration, the wide opening of the chink during forced inspiration, due to the action of the posterior crico-arytenoid muscles, can be observed without difficulty. This action is effected by a separation of the posterior points of attachment of the vocal chords to the arytenoid cartilages. During ordinary expiration, none of the intrinsic muscles seem to act and the larynx is passive, while the air is gently forced out by the elasticity of the lungs and of the thoracic walls; but so soon as a vocal effort is made, the appearance of the glottis undergoes a change and it becomes modified in the most varied manner with the different modifications in pitch and intensity of which the voice is capable. Although sounds may be produced, and even words may be articulated, with the act of inspiration, true and normal phonation takes place during expiration only. It is evident, also, that the inferior vocal chords alone are concerned in this act.

Movements of the Glottis during Phonation. - It is somewhat difficult to observe with the laryngoscope all the vocal phenomena, on account of the epiglottis, which hides a considerable portion of the vocal chords anteriorly, especially during the production of certain notes; but the patience and skill of Garcia enabled him to overcome most of these difficulties, and to settle, by autolaryngoscopy, certain important questions in regard to the action of the larynx in singing. It is fortunate that these observations were made by one versed theoretically and practically in music and possessed of unusual control over the vocal organs. ${ }^{1}$

Garcia, after having observed the respiratory movements of the larynx as they have just been briefly described, noted that so soon as any vocal effort was made, the arytenoid cartilages were approximated, so that the glottis appeared as a narrow slit bounded by two chords of equal length, firmly attached posteriorly as well as anteriorly. The glottis thus undergoes a marked change. A nearly passive organ, opening for the passage of air into the lungs but inactive in expiration, has now become a musical instrument, presenting a slit with borders capable of accurate vibrations.

1 Manoel Garcia, a celebrated teacher of singing in London, is regarded as the inventor of the modern laryngoscope. He used this instrument for the study of the vaice in his own person and presented a memoir - "Observations on the Human Voice" - to the Royal Society, in 1855. It is only since that date that the laryngoscope has been used for medical and surgical purposes. Garcia is now living and celebrated his one-hundredth birthday in London, March 17, 1905. 
The approximation of the posterior extremities of the vocal chords and their tension by the action of certain of the intrinsic muscles are accomplished just before the vocal effort is actually made. The glottis being thus prepared for the emission of a particular sound, the expiratory muscles force air through the larynx with the required power. The power of the voice is due to the force of the expiratory act, which is regulated chiefly by the antagonistic relations of the diaphragm and the abdominal muscles. From the fact that the diaphragm, as an inspiratory muscle, is directly opposed to the muscles that have a tendency to push the abdominal organs, with the diaphragm over them, into the thoracic cavity and thus diminish the pulmonary capacity, the expiratory and inspiratory acts may be balanced so nicely that the most deli-

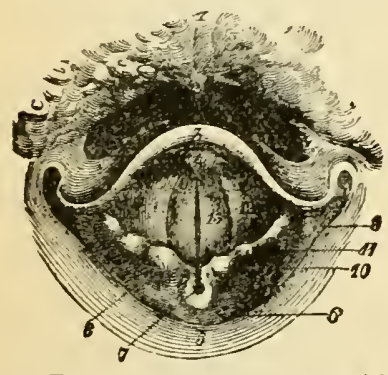

Fig. 93.-Glottis seen with the laryngoscope during the emission of high-patched sounds (Le Bon).

I, 2, base of the tongue; 3,4 , epiglottis; 5,6 , pharynx; 7 , arytenoid cartilages; 8 , opening between the true vocal chords; 9 , aryteno-epiglottidean folds; 10, cartilage of Santorini; II, cuneiform cartilage; 12, superior vocal chords; 13 , inferior vocal chords. cate vocal vibrations can be produced. The glottis, thus closed as a preparation for a vocal act, presents a certain resistance to the egress of air. This is overcome by the action of the expiratory muscles, and with the passage of air through the chink, the edges of the true vocal chords, which bound the opening, are thrown into vibration. Many of the different qualities that are recognized in the human voice are due to differences in the length, breadth and thickness of the vibrating bands; but aside from what is technically known as quality, pitch is dependent on the length of the opening through which the air is made to pass and the degree of tension of the chords. The mechanism of these changes in the pitch of vocal sounds is illustrated by Garcia in the following, which relates to what is known as the chest-voice:-

"If we emit veiled and feeble sounds, the larynx opens at the notes

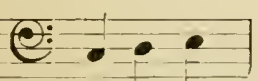
and we see the glottis agitated by large and loose vibrations throughout its entire extent. Its lips comprehended in their length the anterior apophyses of the arytenoid cartilages and the vocal chords; but, I repeat it, there remains no triangular space.

"As the sounds ascend, the apophyses, which are slightly rounded on their internal side, by a gradual apposition commencing at the back, encroach on the length of the glottis; and as soon as we reach the sounds throughout their only solidly fixed

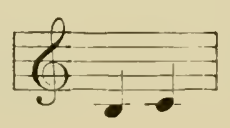
they finish by touching each other whole extent; but their summits are one against the other at the notes 


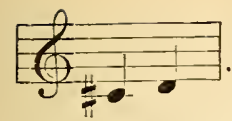

In some organs these summits are a little vacillating when they form the posterior end of the glottis, and two or three half-tones which are formed show a certain want of purity and strength, which is very well known to singers. From $Q_{m-1}$ the vibrations, having become rounder and purer, are accomplished by the vocal ligaments alone, up to the end of the register.

"The glottis at this moment presents the aspect of a line swelled toward its middle, the length of which diminishes still more as the voice ascends. We shall also see that the cavity of the larynx has become very small, and that the superior ligaments have contracted the extent of the ellipse to less than one-half."

These observations have been in the main confirmed by Battaille, Emma Seiler and others who have applied the laryngoscope to the study of the voice in singing.

In childhood the general characters of the voice are essentially the same in both sexes. The larynx is smaller than in the adult and the vocal muscles are more feeble; but the quality of the vocal sounds at this period of life is peculiarly penetrating. While there are certain characters that distinguish the voices of boys before the age of puberty, they present, as in the female, the different qualities of soprano and contralto. After the age of puberty, the female voice does not undergo any very marked change, except in the development of additional strength and extended compass, the quality remaining the same; but in the male there is a rapid change at this time in the development of the larynx, and the voice assumes a different quality. This change usually is arrested if castration is performed in early life; and this operation was frequently resorted to in the seventeenth century and earlier, for the purpose of preserving the qualities of the male soprano and contralto, particularly for church-music. It is only of late years, indeed, that the practice of castration has fallen into disuse in Italy.

The extreme range of all kinds of the human voice taken together is equal to nearly four octaves; but it is rare that any single voice has a compass of more than two and a half octaves. There are examples, however, in which singers have acquired a compass of three octaves. In music the notes are written the same for the male as for the female voice, but the value of the female notes, as reckoned by the number of vibrations in a second, is an octave higher than in the male.

In both sexes there are differences, both in the range and the quality of the voice, which it is impossible for a cultivated musical ear to mistake. The different voices in the male are the bass, the tenor, and an intermediate voice called the barytone. The female voices are the con- 
tralto, the soprano, and the intermediate, or mezzo-soprano. In the bass and barytone, the lower and middle notes are the most natural; and while the higher notes may be acquired by cultivation, they do not possess the same quality as the corresponding notes of the tenor. The same remarks apply to the contralto and soprano.

The following scale gives the ordinary ranges of the different kinds of voice; but it must be remembered that there are individual instances in which these limits are exceeded:-

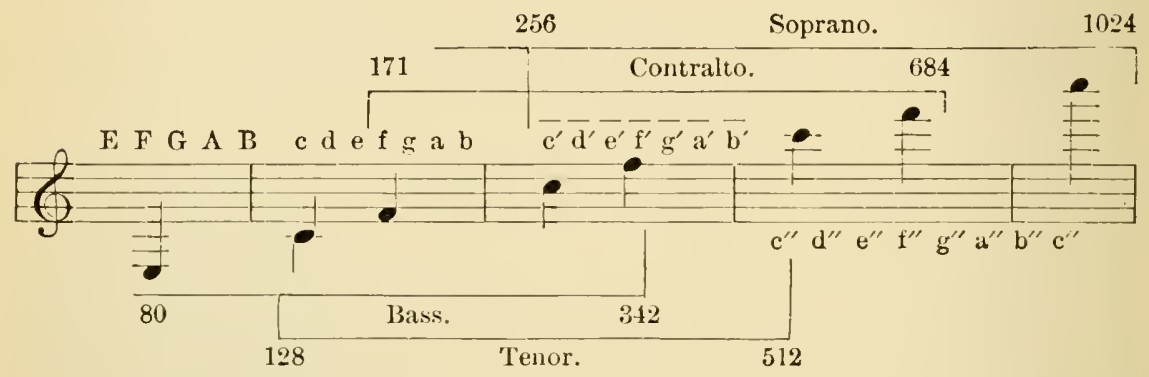

The accompanying figures indicate the number of vibrations per second in the corresponding tone. It is evident that from $c^{\prime}$ to $f^{\prime}$ is common to all voices; nevertheless, they have a different timbre. The lowest note or tone, which, however, is only occasionally sung by bass singers, is the contra-F, with 42 vibrations; the highest note of the soprano voice is $a^{\prime \prime \prime}$, with 1708 vibrations (Landois and Stirling).

There is really no great difference in the mechanism of the different kinds of voice, and the variations in pitch are due chiefly to the greater length of the vocal chords in the low-pitched voices and to their shortness in the higher voices. The differences in quality are due to peculiarities in the conformation of the larynx, to differences in its size and to variations in the size and form of the auxiliary resonant cavities. Great changes in the quality of the voice may be effected by practice. A cultivated note, for example, has a different sound from a harsh, irregular vibration; and by practice, a tenor may imitate the quality of the base, and vice vers $\hat{a}$, although the effort is unnatural. It is not unusual to hear male singers imitate closely the notes of the female; and the contralto sometimes can imitate the voice of the tenor in a surprisingly natural manner.

The wave-length of the ordinary speaking voice in the male is eight to twelve feet. In the speaking voice of the female and in children, it is two to four feet.

Action of the Intrinsic Muscles of the Larynx in Phonation. - In the production of low chest-notes, in which the vocal chords are elongated and are at the minimum of tension that will allow of regular vibrations, 
the crico-thyroid muscles are brought into action, and these are assisted by the arytenoid and the lateral crico-arytenoids, which combine to fix the posterior attachments of the vibrating ligaments. It will be remembered that the crico-thyroids, by approximating the cricoid and thyroid cartilages in front, increase the distance between the arytenoid cartilages and the anterior attachment of the vocal chords.

As the notes produced by the larynx become higher in pitch, the posterior attachments of the chords are approximated, and at this time the lateral crico-arytenoids probably are brought into vigorous action.

The uses of the thyro-arytenoids are more complex; and it is probably in great part by the action of these muscles that the varied and delicate modifications in the rigidity of the vocal chords are produced.

The differences in singers as regards the purity of their notes and intonation are due in part to the accuracy with which some put the vocal chords on the stretch; while in those in whom the voice is of inferior quality, the action of the muscles is more or less vacillating and the tension frequently is incorrect. The fact that some singers can make the voice heard above the combined sounds from a large chorus and orchestra is not due entirely to the intensity of the sound, but in great measure to the mathematical equality of the sonorous vibrations and the comparative absence of discordant waves. ${ }^{1}$

Action of Accessory Vocal Organs. - A correct use of the accessory organs of the voice is of great importance in singing; but the action of these parts is simple and does not require an extended description. The human vocal organs, indeed, consist of a vibrating instrument, the larynx, and of certain tubes and cavities by which the sound is reënforced and modified.

The trachea serves, not only to conduct air to the larynx, but to reënforce the sound to a certain extent by the vibrations of the column of air in its interior. When a powerful vocal effort is made, it is easy to feel, with the finger on the trachea, that the contained air is thrown into vibration.

The capacity of the cavity of the larynx is capable of certain variations. In fact, both the vertical and the bilateral diameters are diminished in high notes and are increased in low notes. The vertical diameter may be modified slightly by ascent and descent of the true vocal chords, and the lateral diameter may be reduced by the action of the inferior constrictors of the pharynx on the sides of the thyroid cartilage.

1 The characters of musical sounds, both vocal and instrumental, will be considered in connection with the physiology of audition. 
The epiglottis, the superior vocal chords and the ventricles are by no means indispensable to, the production of vocal sounds. In the emission of high notes the epiglottis is somewhat depressed, and the superior chords are brought nearer together; but this affects only the form of the resonant cavity above the glottis. In low notes the superior chords are separated. It was before the use of the laryngoscope in the study of vocal phenomena that the epiglottis and the ventricles were thought to be so important in phonation. Undoubtedly the epiglottis has something to do with the character of the voice; but its action is not absolutely necessary or even important, as has been shown in experiments of excising the part in living animals.

The most important modifications of the laryngeal sounds are produced by the resonance of air in the pharynx, mouth and nasal fossæ. This resonance is indispensable to the production of the natural voice. Under ordinary conditions, in the production of low notes the velum palati is fixed by the action of its muscular fibres, so that there is a reverberation of the bucco-pharyngeal and naso-pharyngeal cavities; that is, the velum is in such a position that neither the opening into the nose nor the opening into the mouth is closed, and all the cavities resound. As the notes are raised in pitch, the isthmus contracts, the part immediately above the glottis also is constricted, the resonant cavity of the pharynx and mouth is reduced in size, until finally, in the highest notes of the chest-register, the communication between the pharynx and the nasal fossæ is closed and the sound is reënforced entirely by the pharynx and mouth. At the same time the tongue - a very important organ to singers, particularly in the production of high notes - is drawn backward. The point being curved downward, its base projects upward posteriorly and assists in diminishing the capacity of the bucco-pharyngeal cavity. In the changes which the pharynx thus undergoes in the production of different notes, the uvula acts with the velum and assists in the closure of the different openings. In singing up the scale, this is the mechanism, as far as the chest-notes extend. When, however, a singer changes into what is sometimes called the head-voice (falsetto), the velum palati is drawn forward instead of backward, and the resonance takes place chiefly in the naso-pharyngeal cavity.

Laryngeal Mechanism of the Vocal Registers. - One difficulty, at the very beginning of a discussion of this subject, is in fixing on clear definitions of what are to be recognized as vocal registers. In the first place it must be understood that the singing voice is different from the speaking voice. Without being actually so far discordant as to offend a musical ear, the ordinary voice in speaking seldom has what may 
be called strictly a musical quality, while the perfect singing voice produces true musical notes. This probably is due to the fact that the inflections of the voice in speaking are not in the form of distinct musical intervals, that the vibrations follow each other and are superimposed in an irregular manner, and that no special effort is made to put the vocal chords on any definite tension, unless to meet a more powerful expiratory effort when the voice is increased in force. A shout or a scream is not a singing note. This difference is at once apparent in contrasting recitative with ordinary dialogue in operatic performances.

The divisions of the voice into registers, made by physiologists, are sometimes based on theories in regard to the manner of their production; and if these theories are not correct, the division into registers must be equally faulty. Again, there are such marked differences between male and female voices, that it does not seem possible to apply the same divisions to both sexes. There is no difficulty, however, in recognizing the qualities of voices called bass, barytone and tenor, in the male, or contralto, mezzo and soprano, in the female. A division of the voice into registers should be one easily recognizable by singers and singing teachers; and this must be different for male and for female voices. If a division could be made such as would be readily recognized by the ear, irrespective of theories, it would remain only to ascertain as nearly as possible the exact vocal mechanism of each register. It must be remembered that the voice of a perfect singer shows no recognizable break, or line of division between the vocal registers, except when a difference is made apparent in order to produce certain legitimate musical effects. One great end sought to be attained in training the voice in singing is to make the voice as nearly as possible uniform throughout the extent of its range; and this has been measurably accomplished in certain singers.

Judging of different registers entirely by the effect produced on the ear, both by cultivated and uncultivated singers, the following seem to be the natural divisions of the male voice:-

I. The chest-register. This is the register commonly used in speaking. Though usually called the chest-voice, it has, of course, no connection with any special action of the chest, except, perhaps, with reverberation of air in the trachea and the larger bronchial tubes. This register is sensibly the same in the male and in the female.

2. The head-register. In cultivated male voices, a quality is often produced, probably by diminished power of the voice, with some modification in the form and capacity of the resonant cavities, that is recognized as a "head-voice" by those who do not regard the head-register as equivalent to the falsetto. 
3. The falsetto-register. By the use of this register the male may imitate the voice of the female. Its quality is different from that of the chest-voice, and the transition from the chest to falsetto usually is abrupt and quite marked. It may be called an unnatural voice in the male; still, by careful cultivation, the transition may be made almost imperceptibly. The falsetto never has the power and resonance of the full chest-voice. It resembles the head-voice, but every good singer can recognize the fact that he employs a different mechanism in its production.

Applying an analogous method of analysis to the female voice, the natural registers seem to be the following:-

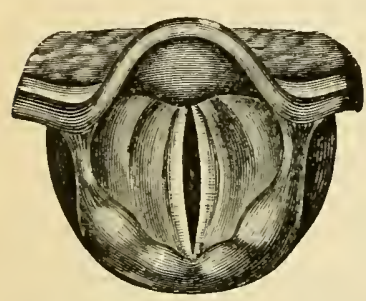

Fig. 94. - Appearance of the vocal chords in the production of the chest-voice (MlandI).

I. The chest-register. This register is the same in the female as in the male.

2. The lower medium register, usually called the medium. This is the register commonly used by females in speaking.

3. The upper medium register. This is sometimes called the head-register and is thought by some to be produced by precisely the same mechanism as the falsetto-register in the male. It has, however, a vibrant quality, is full and powerful and is not an unnatural voice like the male falsetto.

4. The true head-register. This is the pure tone, without much vibrant quality, that seems analogous to the male falsetto.

Vocal Registers in the Male. - According to the division and definitions just given of the vocal registers, in the male voice there is but one natural register, extending from the lowest note of the bass to the falsetto, and this is the chest-register. In the low notes, the vocal chords vibrate and the arytenoid cartilages participate in this vibration to a greater or less extent. In the low notes, also, the larynx is open; that is, the arytenoid cartilages do not touch each other. As the notes are raised in pitch, the arytenoid cartilages are approximated more and more closely, and they touch each other in the highest notes, the vocal chords alone vibrating. It is probable that the degree of approximation of the arytenoid cartilages is different in different singers and that the part of the musical scale at which they actually touch is not invariable.

What has been called, in this classification, the head-register of the male, is not a full round voice, but the notes are more or less sotto voce. This peculiar quality of voice does not seem to have been made the subject of laryngoscopic investigation. It has a vibrant character, that is modified by peculiar action of the resonant cavities, which latter has not been described. It is not probable that its mechanism differs essen- 
tially, as regards the action of the glottis, from that of the full chestregister, shown in Fig. 94.

The falsetto-register in the male undoubtedly involves such a division of the length of the vocal chords that only a portion is thrown into vibration. There is always an approximation of the chords in their posterior portion, and sometimes also in their anterior portion. This is illustrated in Fig. 95.

The mechanism by which the vocal chords are approximated in portions of their length has not been satisfactorily explained; but laryngoscopic examinations leave no doubt of the fact of such action. The extent of this shortening of the chords must vary in different persons and in the same person, probably, in the production of falsetto-notes of different pitch. According to Mrs. Seiler, the shortening is due to the

I

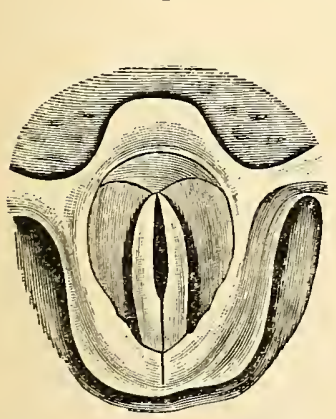

II

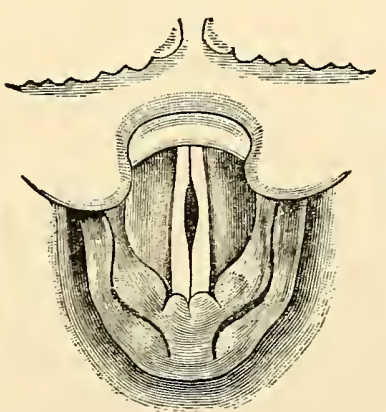

III

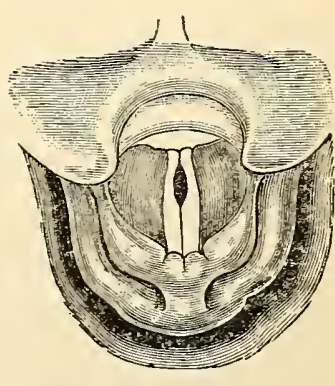

Fig. 95. - Appearances of the vocal chords in the production of the falsetto-voice (Mills).

I, the larynx during falsetto-production; after Mandl. II, the larynx during the emission of falsettotones; middle range; after Holmes. III, the larynx of the female during the production of head-tones, as seen by the author (Mills).

action of a muscular bundle, called the internal thyro-arytenoid, on little cartilages extending forward from the arytenoid cartilage in the substance of the vocal chords as far as the middle of the glottis; but careful dissections have failed to confirm this view.

Some singers, especially tenors, have been able by long practice to pass from the chest to the falsetto so skilfully that the transition is scarcely apparent; but the falsetto is wanting in what is called vibrant quality.

Vocal Registers in the Female. - There is no difference between the vocal mechanism of the chest-voice in the sexes. In the best methods of teaching singing, one important object is to smooth the transition from the chest-voice to the lower medium. The full chest-notes, especially in contraltos, closely resemble the corresponding notes of the tenor. 
The mechanism of the lower medium and upper medium in females does not radically differ from the mechanism of the chest-voice. In these registers the arytenoid cartilages become more and more closely approximated to each other as the voice ascends in the scale until, in the higher notes, they probably are firmly in apposition. It is probable that the vocal chords alone vibrate in the lower and upper medium, while the apophyses of the arytenoid cartilages participate in the vibrations in the female chest-voice.

The vocal chords are much shorter in the female than in the male. The average length in the male is about $\frac{7}{8}$ of an inch (22 millimeters) and in the female, about $\frac{6}{8}$ of an inch ( 17 millimeters). If the chords alone vibrate, without the apophyses of the arytenoid cartilages, the difference in length would account for the differences in pitch of the voice in the sexes. The tenor can not sing above the chest-range of the female voice without passing into the falsetto, to produce which he must actually shorten his vocal chords so that they are as short or shorter than the vocal chords of the female. This is shown by the scale of range of the different voices compared with the length of the vocal chords; and this idea is sustained still further by a comparison of "the larynx during falsetto production" (Fig. 95). In the male falsetto, produced by this shortening of the vocal chords, the more nearly the resonant cavities are made to resemble, in form and capacity, the corresponding cavities in the female, the more closely will the quality of the female voice be imitated. It is probable that the vocal bands in the female present a thinner and narrower vibrating edge than the chords in the male, although there are no exact anatomical observations on this point. This, however, would account for the clear quality of the upper registers of the female voice as compared with the male voice or with the female chest-register. Analogous differences exist in reedinstruments, such as the clarinet and the bassoon. This comparison of the female upper registers with the male falsetto does not necessarily imply a similarity in the mechanism of their production, as is assumed by some writers. In the female lower and upper medium registers, the vocal chords vibrate in their entire length; in the male falsetto, the chords are shortened so that they are approximated in length to the length of the chords in the female.

To reduce to brief statements the views just expressed, based partly on laryngoscopic examinations - that are far from complete - by a number of competent observers, the following may be given as the mechanism of the vocal registers in the female, taking no account of the changes in form and capacity of the resonant cavities:-

I. The chest-voice is produced by "large and loose vibrations" 
(Garcia) of the entire length of the vocal chords, in which the apophyses of the arytenoid cartilages participate to a greater or less extent, these cartilages not being in close apposition.

2. In passing to the lower medium, the arytenoid cartilages probably are not closely approximated, but they do not vibrate, the vocal chords alone acting.

3. In passing to the upper medium, the arytenoid cartilages probably are closely approximated, and the vocal chords alone vibrate, but they vibrate in their entire length.

4. The head-register - which may be called the female falsettobears the same relation to the lower registers in both sexes. The notes are clear but deficient in vibrant quality. They are higher in the female than in the male because the vocal chords are shorter. Laryngoscopic observations demonstrating this fact in the female are as accurate and definite as in the male (see Fig. 95).

The reasons why the range of the different vocal registers is limited are the following: Within the limits of each register, the tension of the vocal chords has an exact relation to the pitch of the sound produced. This tension is of course restricted by the limits of power of the muscles acting on the vocal chords, for high notes, and by the limit of possible regular vibration of chords of a certain length, for low notes. The higher the tension and the greater the rigidity of the chords, the greater is the expiratory force required to throw them into vibration; and this, also, has certain limits. It is not desirable to push the lower registers in female voices to their utmost limits. All competent singing teachers recognize this fact. The female chest-register may be made to meet the upper medium, especially in contraltos; but the singer then has practically two voices, a condition often existing but musically intolerable. In blending the different registers so as to make a perfectly uniform single voice, the arytenoid vibrations should be rendered progressively and evenly less and less prominent, until they imperceptibly cease when the lower medium is fully reached; the arytenoid cartilages should then be progressively and evenly approximated to each other, until they are firmly in contact and the upper medium is fully reached. The female vocal apparatus is then perfect. While single notes of the chest-voice, lower medium and upper medium, contrasted with each other, have different qualities, the voice is even throughout its entire range; and the proper shading called for in musical compositions can be made in any part of the scale. The blending of the male chest-register into the falsetto and of the upper medium into the female falsetto, or true head-voice, is more difficult, but it is not impossible. Theoretically this must be done by shortening the vocal chords gradually and progressively and not 
abruptly, unless the latter method be required to produce a legitimate effect of contrast.

Even in singing identical notes, there are distinctly-recognizable differences in quality between the bass, barytone and tenor, and between the contralto, mezzo and soprano. For the female these may be compared to the differences in identical notes played on different strings of the violin. For the male, they may be compared to the qualities of the different strings of the cello. Falsetto-notes may be compared to harmonics produced on these instruments.

These ideas in regard to the mechanism of the different vocal registers have resulted from a study of these registers, first from an æsthetic point of view; and endeavoring then to find explanations of different qualities of sound appreciated by the ear, in laryngoscopic and other scientific observations; not by reasoning from scientific observations as to what effects on the ear should be produced by certain acts performed by the vocal organs. It may be stated, in this connection, that the works of Bach, Beethoven and other old masters were composed exactly in accordance with purely physical laws, long before these laws were ascertained and defined, as has been done by Helmholtz and others.

\section{Mechanism of Speech}

Articulate language consists of a conventional series of sounds made for the purpose of conveying certain ideas. There being no universal language, it will be necessary to confine the description of speech to the language in which this work is written. Language, as it is naturally acquired, is purely imitative and does not involve of necessity the construction of an alphabet with its combinations into syllables, words and sentences; but as civilization has advanced, certain differences in the accuracy and elegance with which ideas are expressed have become associated with the degree of development and cultivation of intellectual faculties. Philologists have long since established a certain standard varying, to some extent, with usage and the advance of knowledge, but still sufficiently definite - by which the correctness of modes of expression is measured. It is not proposed to discuss the science of language, or to consider, in this connection at least, the peculiar mental operations concerned in the expression of ideas, but to take the language as it exists and to describe briefly the mechanism of the production of the most important articulate sounds.

Almost every language is imperfect, so far as an exact correspondence between its sounds and written characters is concerned. The English language is full of incongruities in spelling, such as silent letters and 
arbitrary and unmeaning variations in pronunciation; but these do not belong to the subject of physiology. There are, however, certain natural divisions of sounds as expressed by the letters of the alphabet.

Vowels. - Certain articulate sounds are called vowel, or vocal, from the fact that they are produced by the vocal chords and are but slightly modified as they pass out of the mouth. The true vowels, $a, e, i, o, u$, can all be sounded alone and may be prolonged in expiration. These are the sounds chiefly employed in singing. The differences in their characters are produced by changes in the position of the tongue, mouth and lips. The vowel-sounds are necessary to the formation of a syllable; and although they usually are modified in speech by consonants, each one may of itself form a syllable or a word. In the construction of syllables and words, the vowels have many different qualities, the chief differences being as they are made long or short. In addition to the modifications in vowel-sounds by consonants, two or three may be combined so as to be pronounced in a single vocal effort, when they are called respectively, diphthongs and triphthongs. In the proper diphthongs, as $o i$, in voice, the two vowels are sounded. In the improper diphthongs, as $e a$, in heat, and in the Latin diphthongs, as $a$, in Cæsar, one of the vowels is silent. In triphthongs, as eau, in beauty, only one vowel is sounded. $Y$, at the beginning of words, usually is pronounced as a consonant; but in other positions it is pronounced as $e$ or $i$.

An important question relates to the differences in the quality of the different vowel-sounds when pronounced with equal pitch and intensity. The cause of these differences was studied very closely in the latter part of the last century, but it has lately been rendered clear by the researches of Helmholtz and of Koenig. In this connection it will be sufficient to indicate the results of the modern investigations very briefly. It will be seen in studying the physics of sound in connection with the sense of hearing, that nearly all sounds, even when produced by a single vibrating body, are compound. Helmholtz, by means of his resonators, has succeeded in analyzing the apparently simple sounds into different component parts and has shown that the quality of such sounds may be modified by reënforcing certain of the overtones, as they are called, such as the third, fifth or octave. For those who are familiar with the physics of sound, the explanation of the mechanism of the production of vowelsounds is readily comprehensible. The reader is referred, however, to the remarks on overtones in another part of this work, under the head of audition, for a more thorough exposition of this subject. The different vowel-sounds may be emitted with the same pitch and intensity, but the sound in each is different on account of variations in the resonant cavities of the accessory vocal organs, especially the mouth. It has 
been ascertained experimentally that the overtones in each instance are different as they are reënforced by the vibrations of air in the accessory vocal organs, in some instances the third, in others, the fifth etc., being increased in intensity. This can hardly be better illustrated than by the following quotation from Tyndall, in which modern researches have been applied to the vowel-sounds of the English language:-

"For the production of the sound $U$ (oo in hoop), I must push my lips forward so as to make the cavity of the mouth as deep as possible, at the same time making the orifice of the mouth small. This arrangement corresponds to the deepest resonance of which the mouth is capable. The fundamental tone of the vocal chords is here reënforced, while the higher tones are thrown into the shade. The $U$ is rendered a little more perfect when a feeble third tone is added to the fundamental.

"The vowel $O$ is pronounced when the mouth is so far opened that the fundamental tone is accompanied by its strong higher octave. A very feeble accompaniment of the third and fourth is advantageous, but not necessary.

"The vowel $A$ derives its character from the third tone, to strengthen which by resonance the orifice of the mouth must be wider, and the volume of air within it smaller than in the last instance. The second tone ought to be added in moderate strength, whilst weak fourth and fifth tones may also be included with advantage.

"To produce $E$ the fundamental tone must be weak, the second tone comparatively strong, the third very feeble, but the fourth, which is characteristic of this vowel, must be intense. A moderate fifth tone may be added. No essential change, however, occurs in the character of the sound when the third and fifth tones are omitted. In order to exalt the higher tones which characterize the vowel-sound $E$, the resonant cavity of the mouth must be small.

"In the production of the sound all! the higher overtones come principally into play; the second tone may be entirely neglected; the third rendered very feebly; the higher tones, particularly the fifth and seventh, being added strongly.

"These examples sufficiently illustrate the subject of vowel-sounds. We may blend in various ways the elementary tints of the solar spectrum, producing innumerable composite colors by their admixture. Out of violet and red we produce purple, and out of yellow and blue we produce white. Thus also may elementary sounds be blended so as to produce all possible varieties of clang-tint. After having resolved the human voice into its constituent tones, Helmholtz was able to imitate these tones by tuning-forks, and, by combining them appropriately together, to produce the clang-tints of all the vowels." 
Consonants. - Some of the consonants have no sound in themselves and serve merely to modify vowel-sounds. These are called mutes. They are $b, d, k, p, t$, and $c$ and $g$ hard. Their office in the formation of syllables is sufficiently apparent.

The consonants known as semivowels are $f, l, m, n, r, s$, and $c$ and $g$ soft. These have an imperfect sound of themselves, approaching in character the true vowel-sounds. Some of these, $l, m, n$ and $r$, from the facility with which they flow into other sounds, are called liquids. Orthoepists have further divided the consonants with reference to the mechanism of their pronunciation : $d, j, s, t, z$, and $g$ soft, being pronounced with the tongue against the teeth, are called dentals; $d, g, j, k$, $l, n$ and $q$ are called palatals; $b, p, f, v$ and $m$ are called labials; $m, n$ and $n g$ are called nasals ; and $k, q$, and $c$ and $g$ hard are called gutturals. After the description already given of the voice, it is not necessary to discuss further the mechanism of these simple acts of articulation.

For the easy and proper production of articulate sounds, integrity of the mouth, teeth, lips, tongue and palate is essential. All are acquainted with the modifications in articulation in persons in whom the nasal cavities resound unnaturally from imperfection of the palate; and the slight peculiarities observed after loss of the teeth and in harelip are sufficiently familiar. The tongue usually is regarded, also, as an important organ of speech, and this is the fact in the great majority of cases ; but instances are on record in which distinct articulation has been preserved after complete destruction of this organ. These cases, however, are unusual and they do not invalidate the importance of the tongue in ordinary speech.

It is thus seen that speech consists essentially in a modification of the vocal sounds by the accessory organs, or by parts situated above the larynx; the latter being the true vocal instrument. While the peculiarities of pronunciation in different persons and the difficulty of acquiring foreign languages after the habits of speech have been formed show that the organs of articulation must perform their office with great accuracy, their movements are simple and vary with the peculiarities of different languages.

Whispering. - Articulate sounds may be produced by the action of the resonant cavities, the lips, teeth and tongue, in which the larynx takes no part. This action occurs in whispering and it can not properly be called vocal. It is difficult to make any considerable variations in the pitch of a whisper, and articulation in this way may be produced in inspiration as well as in expiration, although the act in expiration is more natural and easy. The character of a whisper may readily be distinguished from that of the faintest audible sound involving vibration 
of the vocal chords. In aphonia from paralysis of the vocal muscles of the larynx, patients can articulate distinctly in whispering; but in cases of chronic bulbar paralysis (glosso-labio-laryngeal paralysis) speech is entirely lost.

The Phonograph and Telephone. - In I877 a remarkable invention was made in this country, by Mr. Thomas A. Edison, which possesses considerable physiological importance. Mr. Edison constructed a simple instrument, called the phonograph, which will repeat, with a certain degree of accuracy, the peculiar characters of the human voice both in speaking and singing as well as the pitch and quality of musical instruments. This demonstrates conclusively the fact that the qualities of rocal sounds depend upon the form of the sonorous vibrations. The following are the main features in the construction of this instrument: It consists of a cylinder of iron provided with very fine shallow grooves in the form of a close spiral. Upon the cylinder a sheet of tin-foil is accurately fitted. Bearing on the tin-foil is a steel-point connected with a vibrating plate of mica or of thin iron. The vibrating plate is connected with a mouth-piece which receives the vibrations of the voice or of a musical instrument. The cylinder is turned with a crank, and at the same time the plate is thrown into vibration by speaking into the mouth-piece. As the disk vibrates in consonance with the voice, the vibrations are marked by little indentations upon the tin-foil. When this has been done, the cylinder is moved back to the starting point and is turned again at the same rate as before. As the steel-point passes over the indentations in the tin-foil, the plate is thrown into vibration, and the sound of the voice is actually repeated, although diminished in intensity. Many improvements have lately been made in the phonograph, which have added much to its character illustrating the various qualities of the human voice. The telephone illustrates conduction of the form of sound-wares. As is well known, it is easy to recognize peculiarities of voice in using this instrument. 


\title{
CHAPTER XVIII
}

\author{
STRUCTURE AND PROPERTIES OF THE NERVOUS SYSTEM
}

Divisions and structure of the nervous tissue - Medullated nerve-fibres - Non-medullated nerve-fibres - Gelatinous nerve-fibres (fibres of Remak) - Accessory anatomical elements of the nerves - Branching and course of the nerves - Termination of nerves in voluntary muscles - Termination of nerves in glands - Modes of termination of sensory nerves Corpuscles of Vater, or of Pacini - Tactile corpuscles - End-bulbs - General mode of termination of the sensory nerves - Structure of the nerve-centres - Nerve-cells - Nissl's granules - The neuron - Accessory anatomical elements of the nerve-centres - Degeneration and regeneration of nerves - Motor and sensory nerves - Mode of action of the motor nerves - Associated movements - Mode of action of the sensory nerves - Physiological differences between motor and sensory nerves - Nervous excitability and conductivity - Rapidity of nervous conduction - Personal equation - Action of electricity on the nerves - Law of contraction - Electric current from the exterior to the cut surface of a nerve-Electrotonus, anelectrotonus and catelectrotonus.

THE nervous system is anatomically and physiologically distinct from all other systems and organs. It receives impressions made on the terminal branches of its sensory portion, and it conveys stimulus to parts, determining and regulating their actions; but it gives to no tissue or organ its special excitability or the power of performing its particular office in the economy. The nervous system connects into a coördinated organism all parts of the body. It is the medium through which all impressions are received. It animates or regulates all movements, roluntary and involuntary. It regulates, also, secretion, nutrition, calorification and all the processes of organic life.

In addition to its action as a medium of conduction and communication, the nervous system, in certain of its parts, is capable of receiving impressions and of generating a stimulating influence, or force, peculiar to itself. As there can be no physiological connection or coördination of different parts of the organisms without nerves, there can be no unconscious reception of impressions giving rise to involuntary movements, no appreciation of impressions, general, as in ordinary sensation, or special, as in sight, smell, taste or hearing, no instinct, volition, thought or even knowledge of existence, without nerve-centres.

\section{Divisions and Structure of the Nervous Tissue}

The nervous tissue presents two great divisions, each with distinct anatomical as well as physiological differences. One of these divisions is 
composed of fibres. This kind of nervous matter is incapable of generating a force or impulse, and it serves only as a conductor. The other division is composed of cells, and this kind of nervous matter, while it may serve as a conductor, is capable of generating the so-called nerve-force.

The nerve-fibres and cells are also divided into two great systems, as follows :-

I. The cerebro-spinal system, composed of the brain and spinal cord with the nerves directly connected with these centres. This system is specially connected with the functions of relation, or of animal life. The centres preside over general sensation, the special senses, voluntary and some involuntary movements, intellection, and, in short, all functions that characterize the animal. The nerves serve as the conductors of impressions known as general or special sensations and of the stimulus that gives rise to voluntary and certain involuntary movements, the latter being the automatic movements connected with animal life.

2. The sympathetic, or organic system. This system is specially connected with functions relating to nutrition, operations that have their analogue in the vegetable kingdom and are sometimes called the functions of vegetative life. Although this system presides over functions distinct from those characteristic of and peculiar to animals, the centres of this system all have an anatomical and physiological connection with the cerebro-spinal nerves.

The cerebro-spinal system is subdivided into centres presiding over movements and ordinary sensation and centres capable of receiving impressions connected with the special senses, such as vision, audition, olfaction and gustation. The nerves receiving these special impressions and conveying them to the appropriate centres are more or less insensible to ordinary impressions. The organs to which these special nerves are distributed usually are of a complex and peculiar structure; and they present accessory parts that are important and essential in the transmission of the special impressions to the terminal branches of the nerves.

The physiological division of the nervous system into nerves and nerve-centres is carried out as regards the anatomical structure of these parts, although they are connected together in the so-called neuron. The two great divisions of the system, anatomically considered, are nerve-cells and nerve-fibres.

The cells of the nerve-centres, while they may transmit impressions and impulses, are the only parts capable of generating impulses; and as a rule they do not receive impressions in any other way than through 
nerve-fibres. There are, however, many exceptions to this rule, as in the case of movements following direct stimulation of the sympathetic ganglia and certain centres in the brain and spinal cord; but the cells of many of the ganglia belonging to the cerebro-spinal axis are insensible to direct stimulation and can receive only impressions conducted to them by the nerves.

The nerve-fibres act as conductors and are incapable of generating impulses. There is no exception to this rule, but there are differences in the properties of certain fibres. The nerves generally, for example, receive direct impressions, the motor filaments conducting these to the muscles and the sensory filaments conveying the impressions to the centres. These fibres also conduct impulses generated by the nerve-centres; but there are many fibres, such as those composing the white matter of the encephalon and the spinal cord, that are insensible to direct stimulation, while they convey to the centres impressions conveyed to them by sensory nerves and conduct to the motor nerves impulses generated by nerve-cells.

In the natural classification of nerve-fibres, they are divided into two groups; one embracing fibres that have the conducting element alone, and the other presenting this anatomical element surrounded with certain accessory structures. In the course of the nerves, the simple fibres are the exception and the other variety is the rule; but as the nerves are followed to their terminations in muscles or sensitive parts or are traced to their origin in the nerve-centres, they lose one or another of their coverings. These two varieties are designated as medullated and nonmedullated fibres.

Medullated Nerve-fibres. - These fibres are so called because, in addition to the axis-cylinder, or conducting element, they contain, enclosed in a tubular sheath, a soft substance called medulla. This substance is strongly refractive and gives to the nerves a peculiar appearance under the microscope, from which they are sometimes called dark-bordered nerve-fibres. As the whole substance of the fibre is enclosed in a tubular membrane, these are frequently called nerve-tubes.

If the nerves are examined while fresh and unchanged, their anatomical elements appear in the form of simple fibres with strongly accentuated borders. The diameter of these fibres is $\frac{1}{250} \overline{0}$ to $\frac{1}{170} \overline{0}$ of an inch ( Io to I $5 \mu$ ). In a short time the borders become darker and the fibres assume a different appearance. By the use of certain reagents, it can be demonstrated that a medullated nerve-fibre is composed of three distinct portions: a homogeneous sheath, a semifluid matter contained in the sheath and a delicate central band.

The tubular sheath of the nerve-fibres, the neurilemma, is a some- 
what elastic, homogeneous membrane, presenting oval nuclei with their long diameter in the direction of the tube. This is sometimes called the sheath of Schwann. It exists in all the medullated nerve-fibres, large and small, except those in the white portions of the encephalon and spinal cord and the trunk of the auditory nerve. It possibly exists in non-medullated fibres, although its presence here has not been satisfactorily demonstrated.

The medullary substance fills the tube and surrounds the central band. This is called by various names, as myelin, white substance of Schwann, medullary sheath, nervous medulla etc. It does not exist

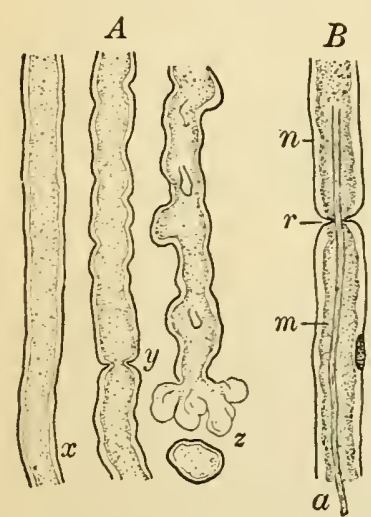

Fig. 96.-Medullated nerve-fibres (Piersol).

$A$, teased in salt solution; $x$, shorily after death; $y$, node of Ranvier; $z$, post-mortem distortions of medullary substance. $B$, an isolated stained fibre; $a$, axiscylinder; $r$, node of Ranvier; $m$, medullary substance; $n$, neurilemma. either at the origin of the nerves in the gray substance of the nerve-centres or at the peripheral termination of the nerves, and it is not a conducting element. When the nerves are perfectly fresh, this substance is transparent, homogeneous, and strongly refracting, like oil ; but as the nerves become altered by desiccation, the action of water, acetic acid and various other reagents, it coagulates into an opaque granular mass. In the white substance of the encephalon and spinal cord, the neurilemma is wanting and the fibres present only the axiscylinder surrounded with the white substance of Schwann. As a post-mortem condition, these fibres present under the microscope varicosities at irregular intervals, which give them a peculiar and characteristic appearance.

The medullated nerve-fibres do not have regular outlines, but present constrictions at various points in their length, called the constrictions or nodes of Ranvier. At these nodes the medullary substance is wanting and the neurilemma is in contact with the axis-cylinder.

When a medullated nerve-fibre is slightly stretched, a number of oblique cuts are observed running across the fibre and extending to the axis-cylinder, called incisures. These involve the medullary substance only and are best observed when this substance has been stained with osmic acid. It is not known that they possess any physiological importance.

The axis-cylinder, occupying one-fifth to one-fourth of the diameter of the nerve-tube, is the conducting portion of the nerve. In the ordinary medullated fibres, the axis-cylinder can not be seen in the natural condition, because it refracts in the same manner as the medullary sub- 
stance; and it can not easily be demonstrated afterward, on account of the opacity of the coagulated matter. If a fresh nerve, however, is treated with strong acetic acid, the divided ends of the fibres retract, leaving the axis-cylinder, which latter is but slightly affected by reagents. It then presents itself in the form of a pale, slightly-flattened band, with outlines tolerably regular, though slightly varicose at intervals. It is somewhat granular and finely striated in a longitudinal direction. This band is elastic but not very resisting. What serves to distinguish it from other portions of the nerve-fibre is its insolubility in most of the reagents employed in anatomical investigations. It is slightly swollen by acetic acid but is dissolved after prolonged boiling. If nerve-tissue is treated with a solution of carmin, the axis-cylinder only is colored. It also stains with gold chloride. It has been observed that the nervefibres treated with silver nitrate present in the axiscylinder well-marked transverse striations (Fromann); and some anatomists regard both the nerve-cells and the axes of the fibres as composed of two substances, the limits of which are marked by the regular striæ thus developed. This, however, is a point of purely anatomical interest. The presence of regular and well-marked striæe in the axis-cylinder after the addition of a solution of silver nitrate and the action of light can not be doubted; but it has not yet been determined whether these markings are artificial or whether the axis-cylinder is really composed of two kinds of substance.

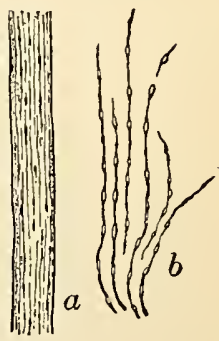

Fig. 97.- Gold-stained axis-cylinder (Piersol).

$a$, shows component fibrillæ; $b$, shows varicose nerve-fibrillæ near their termination.

For some time it has been known that the axiscylinders in the organs of special sense, in the final distribution of sensory nerves and in some other situations, break up into fibrillæ. A fibrillated appearance, indeed, is often observed in nerves in their course, and it is now the common opinion that the axis-cylinders are composed of fibrillæe held closely together by connective substance. This fibrillated structure of the nerves is quite prominent in some of the lower orders of animals.

Non-medullated Nerve-fibres. - These fibres, which are largely distributed in the nervous system, appear to be simple prolongations, without alteration, of the axis-cylinders of the medullated fibres. They are found chiefly in the peripheral terminations of the nerves and in the filaments of origin of the fibres from the nerve-cells. Some anatomists think that they have a delicate investing membrane, but this has not been satisfactorily demonstrated.

Gelatinous Nerve-fibres (Fibres of Remak). - There has been some 
difference of opinion in regard to the physiology of the so-called gelatinous nerve-fibres. Some anatomists have regarded them simply

A

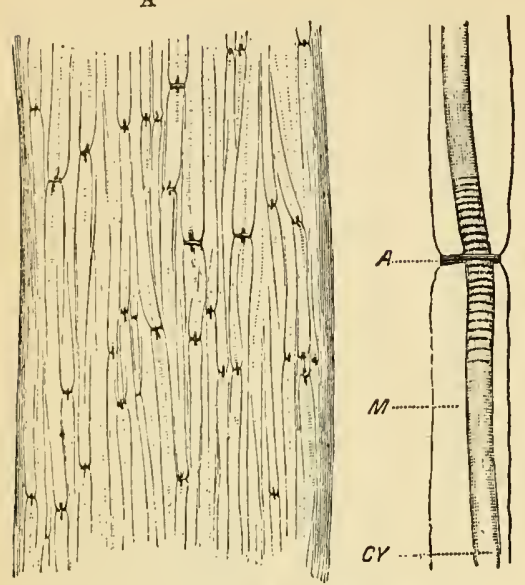

Fig. 98. - Nodes of Ranvier and lines of Fromann (Ranvier).

$A$, intercostal nerve of the mouse, treated with silver nitrate, $\times 200$. B, nerve-fibre from the sciatic nerve of a full-grown rabbit, $\times 600$; $A$, node of Ranvier; $M$, medullary substance rendered transparent by the action of glycerin ; $C Y$, axis-cylinder presenting the lines of Fromann, which are very distinct near the node. The lines are less marked at a distance from the node.

as elements of connective tissue, and others have described them as axiscylinders surrounded with a nucleated sheath; but the fibres do not present the lines of Fromann when treated with silver nitrate. While elements of connective tissue may have been mistaken for true nerve-fibres, there are in the nerves, particularly in those belonging to the sympathetic system, fibres resembling the nerve-fibres of the embryo. These are the gelatinous nerve-fibres, or fibres of Remak. All the nerves have this structure until about the fifth month of intra-uterine life, and in the regeneration of nerves after division or injury, the new elements ordinarily assume this form before they arrive at their full development.

The gelatinous nerve-fibres present the following characters: They are invested with a delicate neurilemma, are flattened, with regular and sharp borders, grayish, pale and fibrillated, with very fine granules and a number of oval longitudinal nuclei, a characteristic which has given them the name of nucleated nerve-fibres. They branch frequently. The diameter of the fibres is about $\frac{1}{8000}$ of an inch $(3 \mu)$. The nuclei have nearly the same diameter as the fibres and are about $\frac{1}{1250}$ of an inch $(20 \mu)$ in length. They are finely granular and present no nucleoli. The fibres are rendered pale by the action of acetic acid, but they are slightly swollen only, and present,

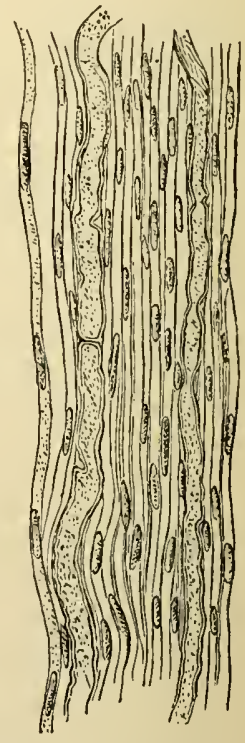

Fig. 99. - Fibres of Re$m a k, \times 300$ (Robin).

With the gelatinous fibres of Remak, are seen two of the ordinary darkbordered nerve-fibres. in this regard, a marked contrast with the elements of connective tissue. They are found chiefly in the sympathetic system and in that particular portion of this system connected with involuntary movements. They usually are not found in the white filaments of the sympathetic. 
Accessory Anatomical Elements of the Nerves. - The nerres present, in addition to the different varieties of true nerve-fibres just described, certain accessory anatomical elements common to nearly all the tissues of the organism, such as connective tissue, bloodvessels and lymphatics.

Like the muscular tissue, the nerves are made up of their true anatomical elements - the nerve-fibres - held together into primitive, secondary and tertiary bundles, and so on, in proportion to the size of the nerve. The primitive fasciculi are surrounded with a delicate membrane called the sheath of Henle. This membrane is homogeneous or very finely granular, sometimes marked with longitudinal striæ, and possessing elongated granular nuclei. There are three kinds of nuclei either attached to or situated near the sheath. These are (I) nuclei attached to the inner surface of the sheath; (2) nuclei belonging to the nervefibres within the sheath; and (3) nuclei of connective-tissue elements near the sheath. Silver nitrate discloses the borders of a lining endothelium. The sheath of Henle begins at the point where the nervefibres emerge from the white portion of the nervous centres and it extends to their terminal extremities, being interrupted by the ganglia in the course of the nerves. This membrane usually envelops a primitive fasciculus of fibres, branching as the bundles divide and pass from one trunk to another. It is sometimes found surrounding single fibres. It usually is not penetrated by bloodvessels, the smallest capillaries of the nerves ramifying in its substance but seldom passing through to the individual nerve-fibres. Within the sheath of Henle are sometimes found elements of connective tissue, with very rarely a few capillary bloodvessels in the largest fasciculi.

The quantity of fibrous tissue in the different nerves is variable and depends on the conditions to which they are subjected. In the nerves within the bony cavities, where they are entirely protected, the fibrous tissue is very scanty; but in the nerves between muscles there is a tolerably strong investing membrane or sheath surrounding the whole nerve and sending into its interior processes which envelop smaller bundles of fibres. This sheath is formed of ordinary fibrous tissue, with small elastic fibres and nucleated connective-tissue cells.

The greatest part of the fibrous sheath of the nerves is composed of bundles of white inelastic tissue, interlacing in every direction; but it contains also many elastic fibres, adipose tissue, a network of arteries and veins, and "nervi nervorum," which are to these structures what the vasa vasorum are to bloodvessels. The adipose tissue is constant, being found even in extremely emaciated persons.

The vascular supply to most of the nerves is rather scanty. The arteries break up into a plexus of fine capillaries, arranged in oblong 
longitudinal meshes surrounding the fasciculi of fibres; but they rarely penetrate the sheath of Henle and usually do not come in contact with the ultimate nervous elements. The veins are rather more voluminous and follow the arrangement of the arteries. Lymph-spaces, lined with delicate endothelium, are found in the connective-tissue sheaths of the bundles of fibres.

Branching and Course of the Neries. - The nerve-fibres in the course of the nerves have no connection with each other by branching

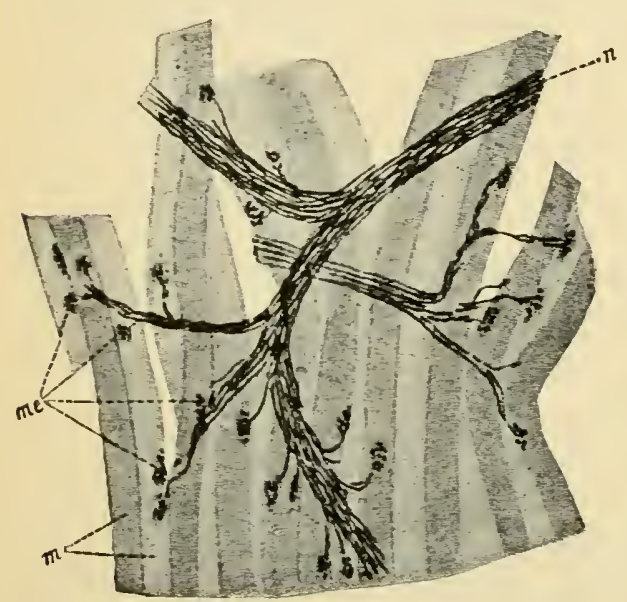

Fig. roo. - Branching of a nerve in an abdominal muscle of a mouse, $\times 120$ (Sobotta).

$m$, muscular fibres; me, motor end-plates; $n$, nerve. or inosculation. A bundle of fibres frequently sends branches to other nerves and receives branches in the same way; but this is simply the passage of fibres from one sheath to another, the fibres themselves maintaining throughout their course their individual physiological properties. The nervefibres do not branch or inosculate except near their terminations. When there is branching of medullated fibres, it is always at the site of one of the nodes of Ranvier.

Termination of Neries in Voluntary. Muscles. - In man and in the warm-blooded animals generally, the medullated nerve-fibres divide dichotomously near their endings in the muscular fibres, the divisions taking place at the nodes of Ranvier. The fibres finally resulting from these divisions pass to the sarcolemma and terminate in a rather prominent mass called an end-plate, with six to twelve or sometimes sixteen nuclei that are distinct from the nuclei of the muscular fibre. The tubular membrane of the nerve-fibre here fuses with the sarcolemma and the medullary substance is lost. By the action of gold chloride, it has been shown that fibrils arise from the under surface of the end-plates, which pass into the substance of the muscular fibres, between the muscular fibrillæ. These fibrils probably are connected with the axis-cylinders, but their exact mode of termination in the muscular substance has not been satisfactorily demonstrated.

Although the sensibility of the muscles is slight as compared with that of the skin and mucous membranes, they are not insensible and they possess nerve-fibres other than those exclusively motor. Through- 
out the substance of most of the voluntary muscles are so-called neuromuscular spindles, made up of small bundles of fibres surrounded with a rather thick covering of connective tissue. These structures are oneeighth to one-third of an inch ( 3 to 8 millimeters) long and about $\frac{1}{12} 5$ inch (0.2 millimeter) in diameter. A medullated nerve-fibre passes to each spindle, loses its medullary sheath, subdivides and the non-medullated fibres form a network surrounding the sarcolemma. It is not certain that they then penetrate the sarcolemma and terminate in the muscular substance, although this view has been advanced. These spindles are abundant near the connections of the muscles with the tendons. They do not exist in the muscles of the eye and of the tongue. The neuro-muscular spindles are regarded by some physiologists as sensory and connected with the so-called muscular sense.

Termination of Neries in the Involuntary Muscular Tissue. Nerve-fibres form a plexus in the connective tissue surrounding the involuntary muscles and then send small fibres into the sheets or layers of muscularfibre cells, which branch and probably go finally to the nuclei of these structures. In many instances, the fine terminal nerve-fibres branch, go into the
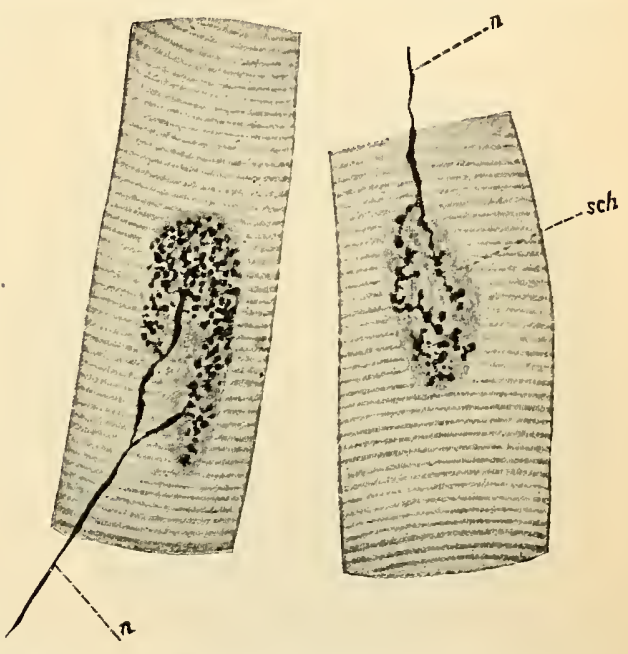
nuclei of the muscular fibres and afterward pass out to join with other fibres and form a plexus.

Termination of Nerves in Glands. - Having formed a more or less branching plexus, non-medullated fibres pass directly into the glandular cells and terminate in the nucleoli. Anatomists have also described and figured multipolar cells, interspersed with the glandular cells, in which some of the nerve-fibres terminate. These, however, are not found in the parotid. These nerve-fibres are regarded as glandular nerves and are distinct from the vasomotor nerves.

Modes of Termination of Sensory Nerves. - There undoubtedly are several modes of termination of the sensory nerves in integument and in mucous membranes, some of which have been quite accurately described. In the first place, anatomists now recognize three varieties of corpuscular terminations, differing in their structure, probably, accord- 
ing to the different properties connected with sensation with which the parts are endowed. In addition it is probable that sensory nerves are connected with the hair-follicles, so largely distributed throughout the cutaneous surface. There are, also, terminal filaments not connected with special organs, some of them, perhaps, ending simply in free extremities and some connected with epithelium.

Corpuscles of Vater or of Pacini.- These bodies were called corpuscles of Pacini until it was shown that they had been seen about a century and a half ago by Vater. In man they are oval or egg-shaped and measure $\frac{1}{25}$ to $\frac{1}{6}$ of an inch (I to 4 millimeters) in length. They are found in the subcutaneous layer on the palms of the hands and the

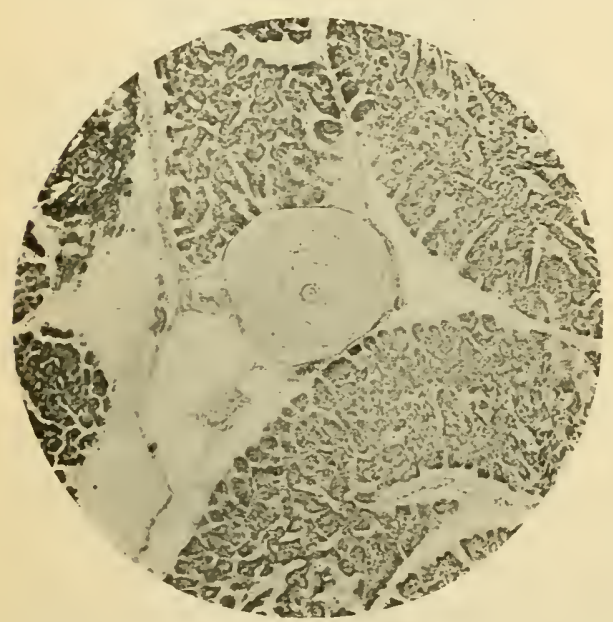

Fig. I02. - Transverse section of a corpuscle of Vater, $\times 30$ (Author's collection).

The corpuscle lies in the pancreas, portions of which are shown in the section, which is deeply stained with alum-carmin.

muscles of the hands and feet. They are always found in the great sympathetic plexuses, in front of and by the sides of the abdominal aorta, and behind the peritoneum, in the vicinity of the pancreas. They have been observed in the mesentery and near the coccygeal gland.

The corpuscles consist of several layers of connective tissue enclosing one, two or three central bulbs in which are found the ends of the nerve. These bulbs are finely granular and nucleated and are regarded by most anatomists as composed of connective tissue. At the base of the corpuscle is a pedicle formed of connective tissue surrounding a medullated nerve-fibre which penetrates the corpuscle. Within the corpuscle the medullary substance of the nerve-fibre is lost and only the axis-cylinder remains. soles of the feet, and are most abundant on the palmar surfaces of the fingers and toes, particularly the third phalanges. In the entire hand there are about six hundred and about the same number in the feet. They are sometimes, but not constantly, found in the following situations: the dorsal surfaces of the hands and feet, on the cutaneous nerves of the arm, the forearm and the neck, the internal pudic nerve, the intercostal nerves, the articular nerves of the extremities, the nerves beneath the mammary glands, the nerves of the nipples and in the substance of the 
The situation of these corpuscles, beneath the true skin instead of in its substance, shows that they can not properly be considered as tactile corpuscles, a name which is applied to other structures found in the papillæ of the corium; and it is impossible to assign to them any

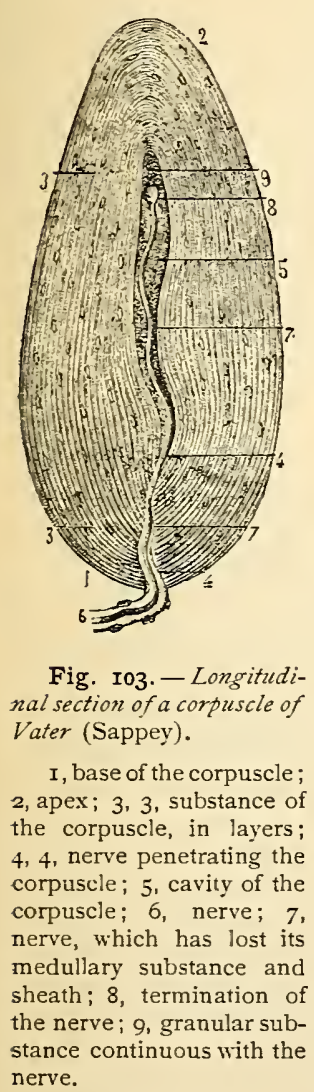

Fig. 103. - Longitudinal section of a corpuscle of Vater (Sappey).

$I$, base of the corpuscle ; 2 , apex; 3,3 , substance of the corpuscle, in layers; 4,4 , nerve penetrating the corpuscle; 5 , cavity of the corpuscle; 6 , nerve; 7 , nerve, which has lost its medullary substance and sheath; 8 , termination of the nerve; 9 , granular substance continuous with the nerve.

Corpuscles do not exist in all papillæ, and they are found chiefly in those called compound. In an area a little more than $\frac{1}{12}$ of an inch square (2.2 millimeters square), on the third phalanx of the index-finger, Meissner counted four hundred papillæ, in one hundred and eight of which he found tactile corpuscles, or about one in four. In an equal area on the second phalanx, he found forty corpuscles; on the first phalanx, fifteen; eight on the skin of the hypothenar eminence; thirty-four on the plantar surface of the ungual phalanx of the great toe; and seven or eight in the skin on the middle of the sole of the foot. In the skin of the forearm the corpuscles are very rare. The tactile corpuscles usually occupy special papillæ that are not

special use connected with sensation, such as the appreciation of temperature, pressure or weight. All that can be said in regard to them is that they constitute one of the several modes of termination

Tactile Corpuscles. - The name tactile corpuscles implies that these bodies are connected with the sense of touch ; and this view is sustained by the fact that they are found almost exclusively in parts endowed in a marked degree with tactile sensibility. They are sometimes called the corpuscles of Meissner and Wagner, after the anatomists by whom they were first described. The true tactile corpuscles are found in greatest number on the palmar surfaces of the hands and fingers and the plantar surfaces of the feet and toes. They exist, also, in the skin on the backs of the hands and feet, the nipples and a few on the anterior surface of the forearm. The largest papillæ of the skin are found on the hands, feet and nipples, where the tactile corpuscle from the human corium (Böhm and Davidoff).

a, upper portion, in which only the epithelial cells are represented ; $b$, shows a dendrite coiled around the epithelial cells; $c$, nerve-fibre.

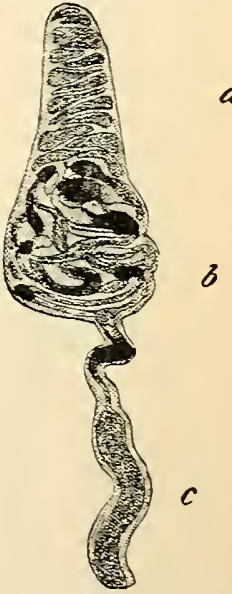

Fig. I04.- Tactile corpuscles are most abundant. 
provided with bloodvessels; so that the papillæ of the hand may be properly divided into vascular and nervous.

The form of the tactile corpuscles is oblong, with the long diameter in the direction of the papillæ. Their length is $\frac{1}{380}$ to $\frac{1}{2} \frac{1}{50}$ of an inch $(66$ to $100 \mu)$. In the palm of the hand they are $\frac{1}{250}$ to $\frac{1}{1} \pm 0$ of an inch ( 100 to $165 \mu$ ) long, and $\frac{1}{550}$ to $\frac{1}{500}$ of an inch $(45$ to $50 \mu$ ) in thickness. They usually are situated at the summits of the secondary eminences of the compound papilla. They consist of a central bulb of homogeneous or slightly granular connective-tissue substance, harder than the central bulb of the corpuscles of Vater, and a covering. The covering is com-

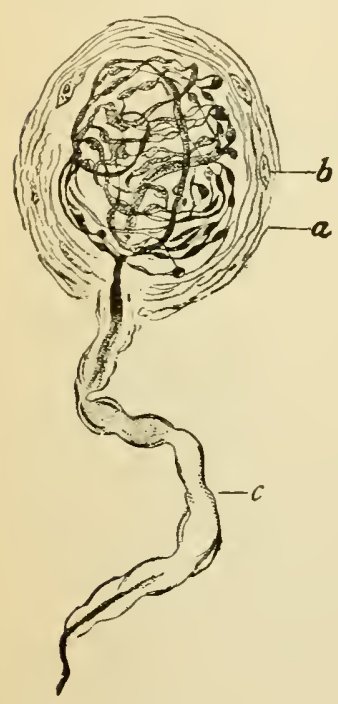

Fig. 105. - Corpuscle of Krause from the human conjunctiva (Dogiel).

$a$, endothelial envelope; $b$, nucleus of a connectivetissue cell; $c$, nerve-fibre. posed of connective tissue with a few fine elastic fibres. One, two, and sometimes three or four dark-bordered nerve-fibres pass from the subcutaneous nervous plexus to the base of each corpuscle. These surround the corpuscle with two or three spiral turns, and they terminate in pale extremities on the surface of the central bulb.

End-bulbs. - Under this name, a variety of corpuscles has been described by Krause as existing in the conjunctiva covering the eye and in the semilunar fold, in the floor of the buccal cavity, the tongue, the glans penis and the clitoris. They bear some analogy to the tactile corpuscles, but they are smaller and more simple in their structure. They form rounded or oblong enlargements at the ends of the nerves, which are composed of homogeneous matter with a delicate investment of connective tissue, lined with endothelial cells. They measure $\frac{1}{100} \overline{0}$ to $\frac{1}{250}$ of an inch $(25$ to $100 \mu)$ in diameter. In the parts provided with papilla, they are situated at the summits of the secondary elevations. The arrangement of the nervefibres in these corpuscles is very simple. One, two, or three medullated fibres pass from the submucous plexus to the corpuscles. The investing sheath of the fibres is here continuous with the connective tissue covering of the corpuscle, and the nerve-fibres pass into the corpuscle, break up into two or three divisions, and terminate in convoluted or knotted coils. The nerve-fibres are medullated for a certain distance, but their terminations are pale. The above is one form of these corpuscles. Sometimes, however, the terminal bulbs are oblong, and sometimes but a single nerve-fibre penetrates the bulb and terminates in a simple pale filament. 
General Mode of Termination of the Sensory Nerves. - The actual termination of the sensory nerves on the general surface and in mucous membranes is still a question of some obscurity. Although anatomists have arrived at a pretty definite knowledge of the sensory corpuscles, it must be remembered that there is an immense cutaneous and mucous surface in which no corpuscles have as yet been demonstrated; and it is in these parts, endowed with what may be called general sensibility, as distinguished from the sense of touch, that the mode of termination of the nerves demands further study.

According to Kölliker, in the great majority of instances the sensory nerves terminate in some way in the hair-follicles; and this would account for the termination of the nerves in by far the greatest portion of the skin, as there are few parts in which hair-follicles do not exist;

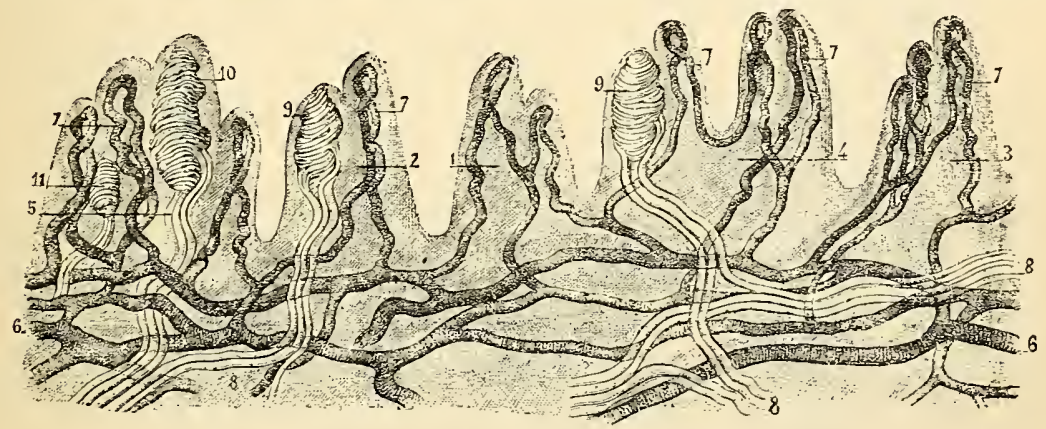

Fig. 106. - Papille of the skin of the palm of the hand (Sappey).

I, papilla with two vascular loops; 2, papilla with a tactile corpuscle; 3 , papilla with three vascular loops; 4, 5, large compound papillæ; 6,6 , vascular network beneath the papillæ; $7,7,7,7$, vascular loops in the papillæ; $8,8,8,8$, nerves beneath the papillæ; 9 , 9 , IO, II tactile corpuscles.

but unfortunately the exact mode of connection of the nerves with these follicles is not apparent. The following seems to be all that is positively known of the terminations of the nerves on the general surface:

Medullated nerve-fibres form a plexus in the corium, which gives off filaments, usually non-medullated, that terminate in the structures just beneath the epidermis. That some fibres go to the hair-follicles, there can be no doubt. It is thought by some histologists that pale fibres form a network around and between the cells of the Malpighian layer of the epidermis. A certain number of fibres pass to the nonstriated muscular fibres of the skin. A certain number pass to papillæ and terminate in tactile corpuscles, and others pass to papillæ that have no tactile corpuscles.

In the mucous membranes the mode of termination is, in general terms, by a delicate plexus just beneath the epithelium, coming from a submucous plexus analogous to the deep cutaneous plexus. In certain 
membranes the nerves terminate in end-bulbs, or corpuscles of Krause (see Fig. I05, p. 466). In the cornea, branching nerve-fibres pass to the nucleoli of the corneal corpuscles and to the nucleoli of the cells of the posterior layer of epithelium.

Structure of the Nerie-centres. - A peculiar pigmentary matter in the nerve-cells and in the surrounding granular substance gives to the nerve-

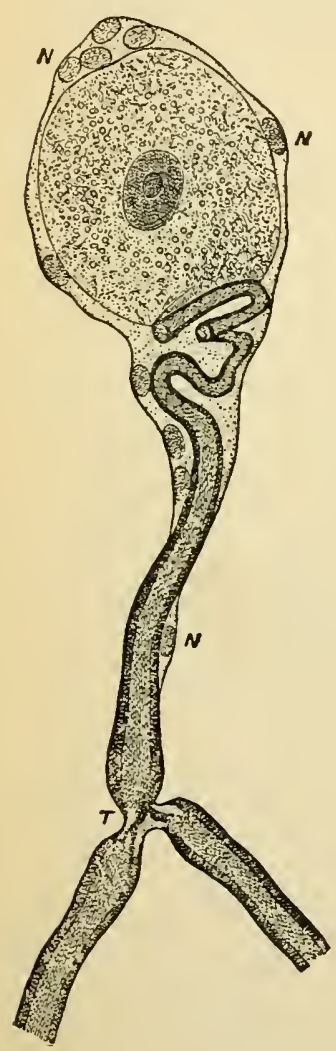

Fig. 107. - Unipolar cell from the Gasserian ganglion (Schwalbe).

$N, N, N$, nuclei of the sheath; $T$, fibre branching at a node of Ranvier. centres a grayish color, by which they are readily distinguished from the white, or fibrous division of the nervous system. Wherever this gray matter is found, the anatomical elements of the tissue are cellular, except in the nerves formed of gray, or gelatinous fibres. Under the general division of nerve-centres are included, anatomically at least, the gray matter of the cerebrospinal centres, the ganglia of the roots of the spinal and certain of the cranial nerves, and the ganglia of the sympathetic system. In these parts are found cells, which constitute the essential anatomical element of the tissue, granular matter resembling the contents of the cells, pale fibres originating in prolongations of the cells, elements of connective tissue, delicate membranes enveloping some of the cells, with bloodvessels and lymphatics.

Nerve-cells. - The following varieties of cells exist in the nerve-centres and constitute their essential anatomical elements: unipolar, bipolar and multipolar cells. These cells present great differences in their size and general appearance, and some distinct varieties are found in particular portions of the nervous system. Unipolar and bipolar cells are found in the ganglia of the cranial nerves and in the ganglia of the posterior roots of the spinal nerves. Small unipolar cells are found in the sympathetic ganglia. Multipolar cells present three or more prolongations. Small cells, with three and rarely four prolongations, are found in the posterior cornua of the gray matter of the spinal cord. From their situation they have been called sensory cells; and they are found in greatest number in parts known to be endowed exclusively with sensory properties. Large, irregularly-shaped multipolar cells, with a number of poles, or prolongations, are found chiefly in the anterior cornua of the gray matter of the spinal cord, 
and these have been called motor cells. They sometimes present as many as ten or twelve poles.

Unipolar cells, such as exist in the ganglia of the nerves as distinguished from the ganglia of the cerebro-spinal axis, have but a single prolongation, which is continuous with a nerve-fibre. These cells frequently have a connective-tissue envelope, or sheath, which is prolonged as a sheath for the nerve. Unipolar cells, with a connectivetissue sheath, the pole surrounded with a spiral fibre, have been observed in the sympathetic ganglia of the frog. These do not exist in the human subject or in the mammalia, and nothing is known of the uses of the spiral fibres.

Bipolar cells seem to be nucleated enlargements in the course of medullated nerve-fibres. Usually the medullary substance does not extend over the cell, although this sometimes occurs.

Multipolar cells have a number of poles, but there is always one pole which does not branch and which becomes continuous with the axis-cylinder of a nervefibre. This is now known as the neurite, or axis-cylinder prolongation. The other poles, called dendrites, or protoplasmic prolongations, branch freely and are lost in the intercellular substance (see Plate XI, Fig. I).

With all the differences in the size and form of the nerve-cells, they present tolerably uniform general characters as regards their structure and contents. With

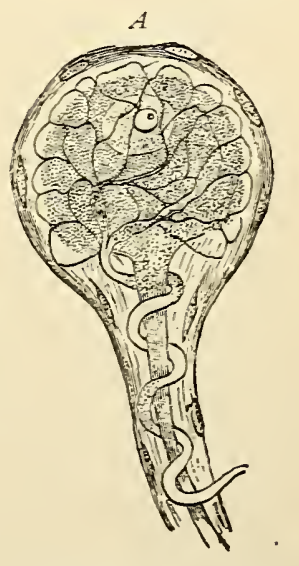

Fig. I08. $-A$, unipolar nerve-cell wit'? a spiral fibre; $B$, bipolar nerve-cell (Landois). the exception of the unipolar and bipolar cells, they are irregular in shape, with strongly-refracting granular contents, frequently a considerable number of pigmentary granules, and always a distinct nucleus and nucleolus. Many cells also contain little angular bodies called Nissl's granules (see Plate XI, Fig. 2). The nucleus in the adult is almost invariably single, although in rare instances two have been noted. Cells with multiple nuclei are often observed in young animals. The nucleoli usually are single, but there may be as many as four or five. The diameter of the cells is variable. They usually measure $\frac{1}{1250}$ to $\frac{1}{500}$ of an inch ( 20 to $50 \mu$ ); but there are many of larger size and some are smaller. The nuclei measure $\frac{1}{2000}$ to $\frac{1}{1250}$ of an inch ( 12 to $20 \mu$ ). The nerve-cells are soft, have no true cell-membrane and are fibrillated the fibrillation extending into the poles. 
Nerve-cells present many peculiarities as they are found in different situations. Some of these will be described here, and others will be considered in connection with the study of special nerve-centres and the structure of what is called the neuron.

In the sympathetic ganglia, the nerve-cells usually are multipolar, and, with their processes, are enclosed in a sheath lined with endothelium. Figure 109 represents an isolated cell from a sympathetic ganglion of man. It is to be noted that this cell, like most of the cells of the central nervous system, is multipolar. Figure I IO represents a cell from the electric organ of the torpedo, isolated and prepared without the use of staining solutions. This cell shows the characteristics of a multipolar nerve-cell: the axis-cylinder prolongation, or neurite, the so-called protoplasmic

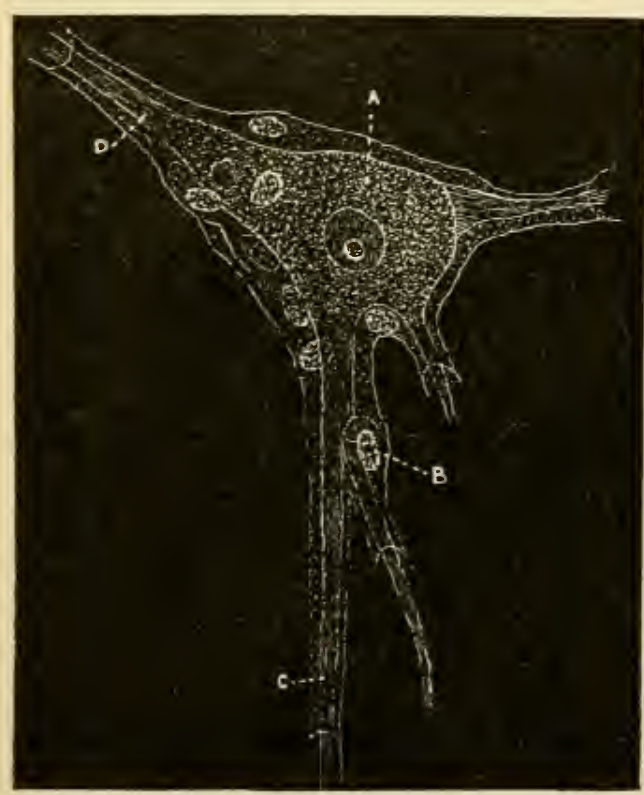

Fig. I09. - Sympathetic ganglion-cell from man, $\times 750$ (Key and Retzius).

$A$, cell with nucleus and nucleolus; $B$, sheath with nucleated-cell lining; $C$, branched process; $D$, axis-cylinder process. prolongations, or dendrites, the nucleus and nucleolus and the structure of the cellbody.

Nissl's Granules. - In the cells of the encephalon and spinal cord, staining deeply with methylene blue, are found small angular granules, first described by Nissl. These extend a short distance into the protoplasmic prolongations, and the stain also is taken up by the nucleus and nucleolus (see Fig. I I 2, page 472, and Plate XI, Fig. 2). The Nissl granules - sometimes called tigroid granules - are composed of chromoplasm, which is a nucleo-proteid. The name kinetoplasm has been given to this substance to indicate its probable
activity of the cell is stored function. It is thought that the potential activity of the cell is stored up in the chromoplasm, which is used in the discharge of nerveimpulses during the activity of the cell; and that during periods of rest the granules are formed again. It has been noted, indeed, that the discharge of nerve-impulses brings about a disintegration of Nissl's granules, which break down into a fine granular substance, part of which temporarily disappears. This result of cell-activity is called 
chromatolysis. When cells undergo degeneration and atrophy, the chromatoplasm disappears. This process is called disuse atrophy.

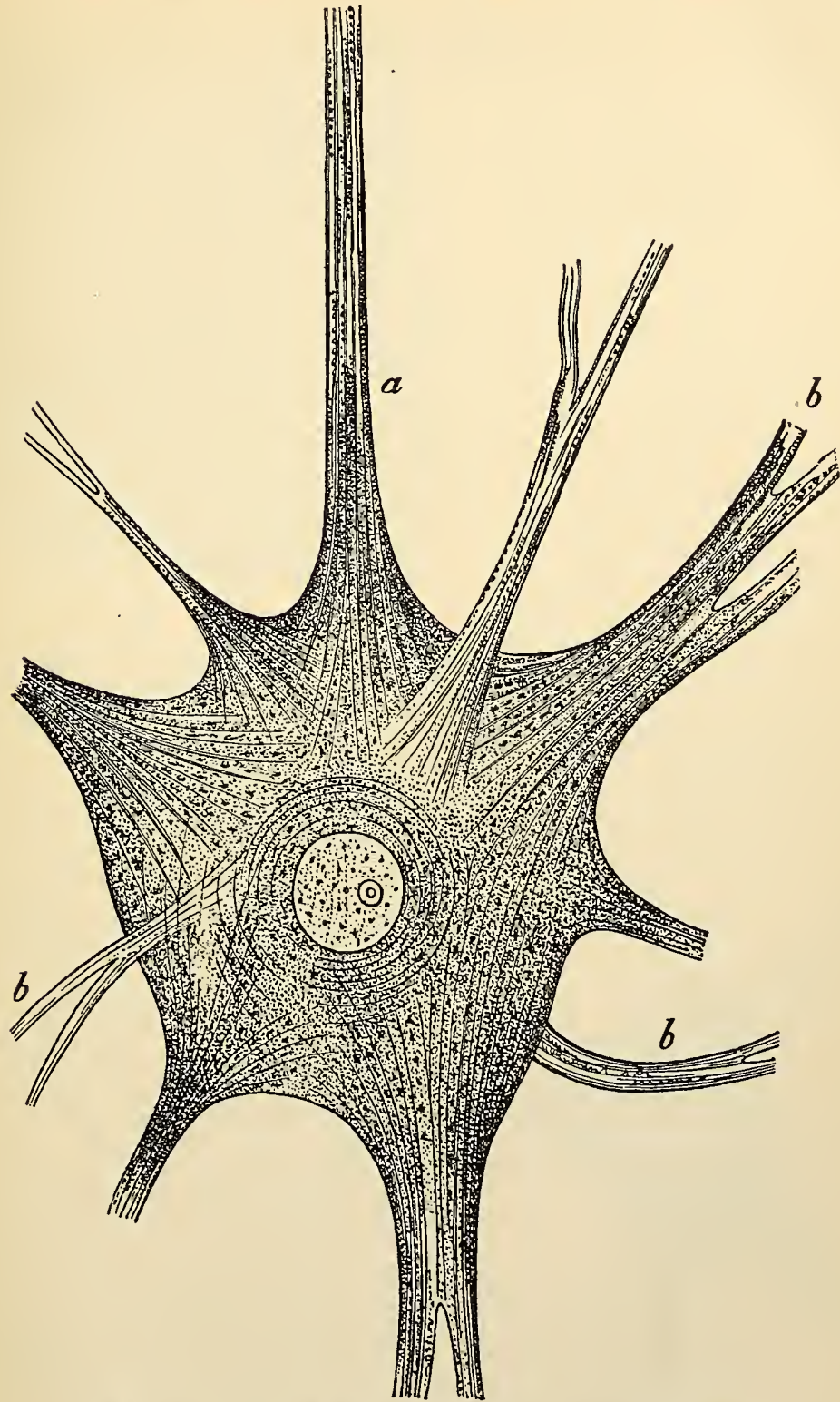

Fig. I10. - Cell from the electric lobe of the torpedo (Schultze). $a$, axis-cylinder; $b, b, b$, dendrites.

The Neuron. - The prevailing idea now is that the nervous system is made up of anatomically and physiologically distinct entities called 
neurons. The neuron is composed of (I) a cell-body, (2) a neurite, formerly known as the axis-cylinder prolongation, (3) dendrites, formerly called protoplasmic prolongations and $(4)$ collaterals, given off by the neurites. The neurites are fibrillated, and their terminal fibrils are called teloneurites. In their course they frequently give off large numbers of collaterals. The terminal fibrils of the dendrites are called telodendrites. The cell-body is identical with what has already been described as the nerve-cell. The unstained Fig. I IO shows the cell-body, the neurite and the protoplasmic prolongations, but not the collaterals, teloneurites and telodendrites. It is supposed that nerve-impulses are sent

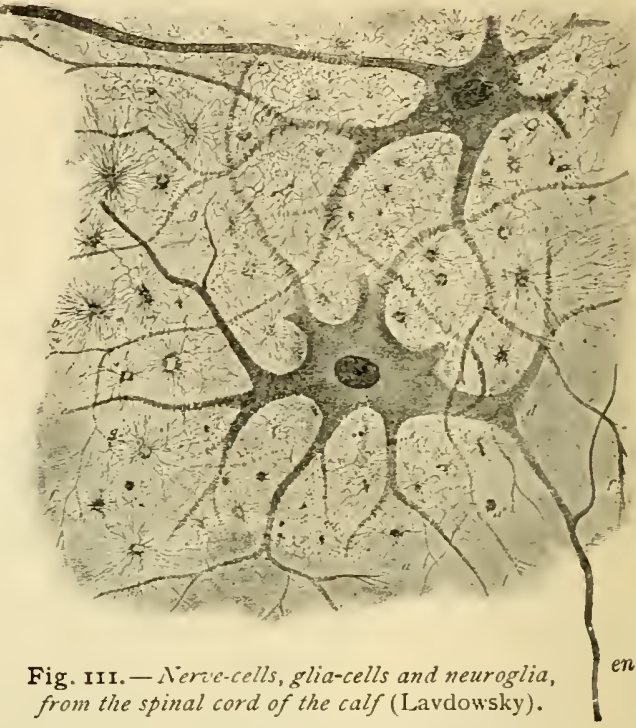

$e n$, neurites; the neurite from the lower cell gives off a process. The arborescent processes are dendrites branching among the fibres of neuroglia.

out by the cell-body through the neurites and that impressions are received

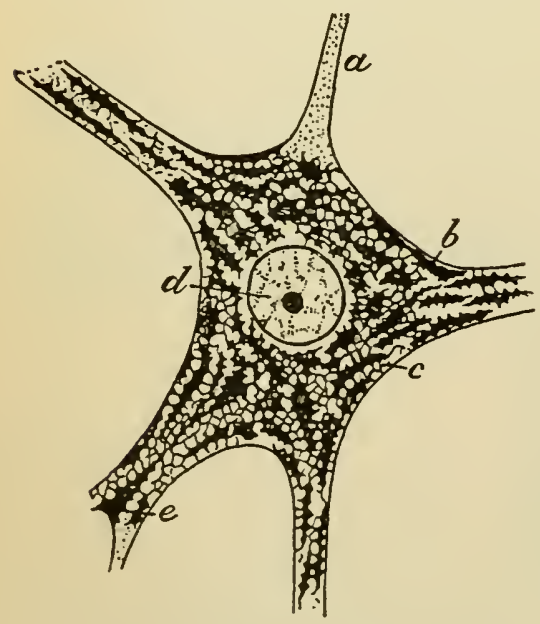

Fig. II2. - Diagram of a nerve-cell to show the Nissl granules (R. y Cajal).

$a$, axis-cylinder process; $b$, Nissl granules; $c$, achromatic substance between them; $d$, nucleus; $e$, dendrite with Nissl granule at division. by the dendrites.

Accessory Anatomical Elements of the Nerve-centres. - In addition to the neurons, which probably are the only structures directly concerned in innervation, are the following accessory anatomical elements: I, outer coverings surrounding some of the cells; 2, intercellular granular matter; 3 , peculiar corpuscles called myelocytes (Robin); 4, connective-tissue elements ; 5 , neuroglia ; 6 , bloodvessels and lymphatics.

Certain of the cells in the spinal ganglia and in the ganglia of the sympathetic system are surrounded with a covering, removed a certain distance from the cell itself so as to be nearly twice the diameter of the 
cell, which is continuous with the sheath of the medullated fibres. This membrane is nucleated and is composed mainly of a layer of very delicate endothelium.

In the gray matter of the nerve-centres, there is a finely granular substance between the cells, which closely resembles the granular contents of the cells themselves. In addition to this granular matter are peculiar elements called by Robin myelocytes. These are found in the cerebro-spinal centres, forming a layer near the boundary of the white substance, particularly in the cerebellum. They exist in the form of free nuclei and nucleated cells, the free nuclei being by far the more abundant. The nuclei are rounded or ovoid, with strongly accentuated borders, are unaffected by acetic acid, are finely granular and usually are without nucleoli. The cells are rounded or slightly polyhedric, pale, clear or very slightly granular, and contain bodies similar to the free nuclei. The free nuclei are $\frac{1}{5000}$ to $\frac{1}{4000}$ of an inch $(5$ to $6 \mu)$ in diameter, and the cells measure $\frac{1}{2500}$ to $\frac{1}{2000}$, and sometimes $\frac{1}{1500}$ of an inch (IO, I2, and I5 $\mu$ ). These elements are not to be confounded with the so-called myelocytes found in bone-marrow.

In the cerebro-spinal centres there is a delicate stroma of connective tissue, chiefly in the form of stellate branching cells, which serves in a measure to support the nervous elements. Neuroglia closely resembles connective tissue, but the cells and their prolongations are much finer. The cells are sometimes called glia-cells, or spider-cells. Neuroglia, however, is derived from the epiblast and is composed of neurokeratin, while the true connective tissues are derived from the mesoblast. With the connective tissue, the neuroglia serves to support the true nervous structures.

The bloodvessels of the nerve-centres form a capillary network with large meshes. The gray substance is richer in capillaries than the white. A peculiarity of the vascular arrangement in the cerebro-spinal centres has already been described in connection with the anatomy of the lymphatic system. The bloodvessels here are surrounded by what have been called perivascular canals. These are radicles of the lymphatic system.

Composition of the Nervous Substance. - The chemistry of the nervous substance, so far as it is understood, throws little light on its physiology. Certain proteids have been extracted which possess no more than a purely chemical interest. The substance called cerebrin is composed of carbon, hydrogen, oxygen and nitrogen, without either sulphur or phosphorus. Protagon is a nitrogenous substance containing phosphorus (Liebreich, I865), and probably is a mixture of cerebrin and lecithin. Lecithin is regarded as a nitrogenous fat. Other 
substances that have been extracted - xanthin, hypoxanthin, inosite, creatin and various volatile fatty acids - have no special physiological interest connected with the nervous system and are found in many other situations. Cholesterin, which always exists in considerable quantity in the nervous tissue, has been considered in connection with the physiology of excretion. The ordinary fats are in combination with other fats or with peculiar acid substances. The reaction of nervetissue is either neutral or faintly alkaline under normal conditions, becoming acid soon after death.

Degeneration and Regeneration of Nerves. - The degenerations observed in nerves separated from the centres to which they normally are attached, first studied by Waller, in I 850 , are now used in following out certain nervous connections too intricate to be revealed by ordinary dissection. This is known as the Wallerian method. If an ordinary mixed nerve is divided in its course, both the motor and sensory fibres of the peripheral portion undergo degeneration and lose their conductivity. As regards the spinal nerves, degeneration occurs in the motor fibres only, when the anterior spinal root has been divided, and the nerve has degenerated fibres (motor) mixed with the sensory fibres, which latter retain their anatomical and physiological integrity. The motor fibres of the spinal nerves are degenerated when separated from their connections with the anterior cornua of gray matter of the cord. If the posterior roots of the spinal nerves are divided beyond the ganglia, the peripheral sensory fibres degenerate; but if the ganglia are exsected, the central as well as the peripheral portions degenerate. These experiments show the existence of centres that preside over the nutrition of the nerves. The centres for the motor filaments of the spinal nerves are in the anterior cornua of gray matter of the cord. The centres for the sensory fibres are the ganglia of the posterior roots. The centres for the sensory cranial nerves are the ganglia on their roots; and the centres for the motor cranial nerves are probably the gray nuclei of origin of these nerves. The Wallerian method has been found useful in studying the paths of conduction in the encephalon and spinal cord, as will be seen in connection with the physiology of these parts.

The excitability of the motor nerves disappears in about four days after their section. Of course, in experiments on this point, it is necessary to excise a portion of the nerve to prevent reunion of the divided extremities; but when this is done, after about the fourth day, stimulation of the nerve will produce no contraction of muscles, although the latter retain their contractility. This loss of excitability is gradual, and it continues, whether the nerve is exposed and stimulated from time to time or is left to itself, progressing from the centres to the periphery. 
The sensibility of the sensory nerves disappears from the periphery to the centres, as is shown in dying animals and in experiments with anesthetics. The sensibility is lost, first in the terminal branches of the nerves, next in the trunks and in the posterior roots of the spinal nerves, and so on to the centres.

Nerves that have been divided may be regenerated if anatomical union of the divided ends can be secured; and this sometimes takes place several months after injury to the nerves, the regeneration occurring by the formation of new fibres. Mixed nerves are regenerated in this way, and conduction in both directions finally is restored. The sensory conduction appears first, and next, the conduction of motor impulses. The restoration of the physiological properties of the nerves occupies several weeks. The central end of a mixed nerve has been made to unite with the peripheral end of another mixed nerve, but it is doubtful whether a divided end of a motor nerve can be united to the divided end of a sensory nerve.

\section{Motor and Sensory Nerves}

Aside from nerves possessing special properties, such as nerves of sight, hearing, smell, taste and, according to some physiologists, nerves of touch, temperature, sense of weight and muscular sense, the cerebrospinal nerves present two kinds of fibres. These are ( I) centrifugal, or motor fibres, and (2) centripetal, or sensory fibres. The motor fibres conduct impulses from the centres to the muscles and excite muscular contraction. The sensory fibres conduct impressions from the periphery to the centres, which are appreciated either as ordinary sensation or as pain. As regards the nerves arising by two roots from the spinal cord, the exact anatomical and physiological divisions into motor and sensory were first made by Magendie, in I 822 . As will be seen farther on, this division is distinct for the cranial nerves, so that it is universal in the cerebro-spinal system. The importance of the discovery of the distinct properties of the two roots of the spinal nerves is such that it merits at least a brief historical account, particularly as this discovery has been quite generally attributed to Charles Bell.

The first definite statement in regard to distinct properties of the two roots of the spinal nerves was made by Alexander Walker, in I809, who said that the posterior roots were for motion and the anterior roots for sensation, - the exact reverse of the truth.

In a pamphlet privately printed by Charles Bell, probably in I $8 \mathrm{I}$, and "submitted for the observations of his friends," the view was advanced that the anterior roots are both motor and sensory and that 
the posterior preside over "the secret operations of the bodily frame, or the connections which unite the parts of the body into a system."

In I 822, Magendie, as the result of experiments on the exposed roots in living dogs, stated that "he was able at that time to advance as positive, that the anterior and the posterior roots of the nerves which arise from the spinal cord have different functions, that the posterior seem more particularly destined to sensibility, while the anterior seem more specially connected with motion."

All observations on the roots of the spinal nerves show that the mixed nerves arising from the cord derive their motor properties from the anterior roots and their sensory properties from the posterior roots : and since the publication of an historical review of the question of priority between Bell and Magendie (Flint, 1868), it has been admitted that the credit of this discovery belongs to Magendie.

The anterior roots possess a certain degree of sensibility in addition to their motor properties (Magendie). This sensibility, which is slight, is derived from fibres from the posterior roots, which turn back to go to the anterior roots. This has been positively demonstrated by the Wallerian method. When a posterior root is divided beyond the ganglion, the sensibility of the corresponding anterior root is lost, and degenerated fibres appear, after a few days, in the anterior roots. This sensibility of the anterior roots is called recurrent sensibility. Similar relations exist between certain of the motor and sensory cranial nerves.

Mode of Action of the Motor Nerves. - As regards the normal action of the motor nerves, impulses, the nature of which is unknown, generated in the centres, are conducted from the centres to the.peripheral distribution of the nerves in the muscles, and are here manifested by contraction. Their mode of action, therefore, is centrifugal. When these motor filaments are divided, the connection between the parts animated by them and the centre is interrupted, and motion in these parts, in obedience to the natural stimulus, becomes impossible. While, however, it is not always possible to induce generation of nerve-force in the centres by the direct application of any agent to them, this force may be imitated by stimulation applied to the nerve itself. A nerve that will thus respond to direct stimulation is said to be excitable.

If a motor nerve is divided, electric, mechanical or other stimulus applied to the extremity connected with the centres produces no effect; but the same stimulus applied to the extremity connected with the muscles is followed by contraction. The phenomena indicating that a nerve retains its physiological properties are always manifested at its peripheral distribution, and these do not vary essentially when the nerve is stimulated at different points in its course. For example, stimulation 
of the anterior roots near the cord produces contraction in the muscles to which the fibres of these roots are distributed; but the same effect follows stimulation of the nerve going to these muscles, in any parts of its course.

So far as their physiological action is concerned, the individual nerve-fibres are independent; and the relations which they bear to each other in nervous fasciculi and in the so-called anastomoses of nerves involve simple contiguity. Comparing the nerve-force to electricity, each individual fibre seems completely insulated; and a stimulus conducted by it to muscles does not extend to the adjacent fibres. That it is the axis-cylinder which conducts and the medullary tube which insulates, it is impossible to say with positiveness; but it is more than probable that the axis-cylinder is the only conducting element.

Generation of motor impulses may be induced by an impression made on sensory nerves and conveyed by them to the centres. If, for example, a certain portion of the central nervous system, as the spinal cord, is isolated, leaving its connections with the motor and sensory nerves intact, these phenomena may readily be observed. An impression made on the sensory nerves will be conveyed to the gray matter of the cord and will induce the generation of a motor impulse by the cells of this part, which will be conducted to the muscles and give rise to contraction. As the impulse, in such observations, seems to be reflected from the cord through the motor nerves to the muscles, this action has been called reflex. These phenomena constitute an important division of the physiology of the nervous system and will be fully considered by themselves.

Associated Movements. - It is well known that the action of certain muscles is with difficulty isolated by an effort of the will. This applies to sets of muscles on one side of the body and to corresponding muscles on the two sides. For example, it is almost impossible, without great practice, to move some of the fingers, at the same time restraining the movements of the others; and the action of certain sets of muscles of the extremities is simultaneous. The toes, which are but little used as the foot is confined in the ordinary dress, are capable of little independent action. It is difficult to move one eye without the other, or to make rapid rotary movements of one hand while a different order of movements is executed with the other; and instances of this kind might be multiplied.

Many associated movements may be influenced in a remarkable degree by education, of which no better examples can be found than in skilful performers on certain musical instruments, such as the piano, harp, violin and other stringed instruments. In the technical study of 
such instruments, not only does one hand become almost independent of the other, but very complex associated movements may be acquired. An accomplished pianist or violinist executes the different scales automatically by a single effort of the will, and pianists frequently execute at the same time scales with both hands, the action being opposed to the natural association of movements.

Looking at the associated movements in their relations to the mode of action of the motor nerves, it seems probable that, as a rule, the anatomical relations of the nerves are such that a motor impulse or an effort of the will can not be conducted to a portion only of a muscle, but must act on the whole muscle, and the same is true, probably, of certain restricted sets of muscles; but the association of movements of corresponding muscles on the two sides of the body, with the exception, perhaps, of the muscles of the eyes, is due mainly to habit and may be greatly modified by education.

Mode of Action of the Sensory Nerves. - The sensory nerve-fibres, like the fibres of the motor nerves, are independent of each other in their action; and in the so-called anastomoses that take place between sensory nerves, the fibres assume no new relations, except as regards contiguity.

As motor fibres convey to their peripheral distribution impulses produced by a stimulus applied in any portion of their course, so an impression made on a sensory nerve is always referred to the periphery. A familiar example of this is afforded by the very common accident of contusion of the ulnar nerve as it passes between the olecranon and the condyle of the humerus. This is attended with painful tingling of the ring and little finger and other parts to which the filaments of this nerve are distributed, without, necessarily, any pain at the point of injury. More striking examples are afforded in neuralgic affections dependent on disease of or pressure on the trunk of a sensory nerve. In such cases, excision of the nerve is often practised, but no permanent relief follows unless the section be made between the affected portion of the nerve and the nerve-centres; and the pain is always referred to the termination of the nerve, even after it has been divided between the seat of the disease and the periphery, leaving the parts supplied by the nerve insensible to direct irritation. In cases of disease it is not unusual to note great pain in parts of the skin that are insensible to direct impressions. The explanation of this is that the nerves are paralyzed near their terminal distribution, so that an impression made on the skin can not be conveyed to the sensorium; but the trunks of the nerves still retain their conductivity and are the seat of diseased action, producing pain referred by the patient to the periphery. In the very 
common operation of restoring the nose by transplanting skin from the forehead, after the operation has been completed, the skin having been entirely separated, and united in its new relations, the patient feels that the forehead is touched when the finger is applied to the artificial nose. After a time, however, the sensorium becomes accustomed to the new arrangement of the parts and this deceptive feeling disappears.

There are certain curious nervous phenomena, that are not without physiological interest, presented in persons who have suffered amputations. It has long been observed that after loss of a limb, the sensation of the part remains; and pain is frequently experienced, which is referred to the amputated member. Thus a patient will feel distinctly the fingers or toes after an arm or a leg has been removed, and irritation of the ends of the nerves at the stump produces sensations referred to the missing member. After a time the sense of presence of the lost limb becomes blunted, and it may in some cases entirely disappear. This may take place a few months after the amputation, or the sensations may remain for years. Examples have been reported by Müller, in which the sense was undiminished thirteen, and in one case twenty, years after amputation. In a certain number of cases, however, the sense of the intermediate part is lost, the feeling in the hand or foot, as the case may be, remaining as distinct as ever, the impression being that the limb is gradually becoming shorter. It was noted by Gueniot, that the sense of the limb becoming shorter exists in about half the cases of amputation in which cioatrization goes on regularly; and in these cases, the patient finally experiences a feeling as though the hand or foot were in direct contact with the stump.

Pliysiological Differences between Motor and Sensory Nerve-fibres. It has not been shown that there is any essential anatomical difference between the conducting elements of motor and sensory nerve-fibres; but the physiological differences are sufficiently distinct, as has already been seen. Under normal conditions, motor fibres conduct motor impulses in but one direction, and these fibres are insensible. Sensory fibres conduct impressions always in the opposite direction, and they do not conduct motor impulses.

Nervous Excitability and Conductivity. - Immediately or soon after death, when the excitability of the nerves is at its maximum, they may be stimulated by mechanical, chemical or electric irritation, all these agents producing contraction of the muscles to which the motor filaments are distributed. Mechanical irritation - simply pinching a portion of the nerve, for example - produces a single muscular contraction; but if the injury to the nerve is such as to disorganize its fibres, that portion of the nerve will no longer conduct an impulse. Among the irritants of 
this kind, are extremes of heat and cold. If an exposed nerve is cauterized, a vigorous muscular contraction follows. The same effect, though less marked, may be produced by the sudden application of intense cold. Among chemical reagents, there are some which excite the nerves and others which produce no effect; but these are not important from a physiological point of view, except common salt, which is sometimes used when it is desired to produce tetanic action. Mechanical stimulation and the action of certain chemicals are capable of exciting the nerves; but when their action goes so far as to disorganize the fibres, the conducting power of these fibres is lost. While, however, stimulation of the nerve above the point of such injury has no effect, stimulation between this point and the muscles is still followed by contraction.

The excitability of a nerve is not to be confounded with its conductivity. A nerve is excitable when its stimulation excites muscular action, the impulse being conducted to the muscle. Acting thus as a conductor, the nerve is said to possess conductivity. It is possible to destroy for a time the excitability of a restricted portion of a nerve without affecting its conductivity; for when a stimulus is applied to the nerve above this portion, it is followed by muscular contraction, the impulse being conducted through the portion that has been rendered inexcitable. Some parts of the nervous system, indeed, normally are inexcitable under direct stimulation, but nevertheless act as conductors.

The most convenient method of exciting the nerves in physiological experiments is by means of electricity. This may be employed without disorganizing the nerve-tissue, and it consequently admits of extended and repeated application. The action of electricity, however, with the methods of preparing the nerves and muscles for experimentation, will be considered under a separate head.

Rapidity of Norvous Conduction. - The first accurate estimates of the rapidity of nervous conduction were made by Helmholtz, in 1850 , and were applied to the motor nerves of the frog. These estimates were arrived at by an application of the graphic method, which was afterward considerably extended and improved by Marey. The process employed by Marey, which is essentially the same as that used in more recent investigations, is the following:-

To mark small fractions of a second, a tuning-fork vibrating at a known rate (five hundred times in a second) is so arranged that a point connected with one of its arms is made to play against a strip of blackened paper. As the paper remains stationary, the point makes but a single mark; but when the paper moves, as the point vibrates, a line is produced with regular curves, each curve representing $\frac{1}{500}$ of a second. If a lever is now attached to a muscle and so arranged as to indicate 
on the paper, moving at the same rate, the instant when contraction takes place, it is evident that the interval between two contractions produced by stimulating the nerve at different points in its course may be accurately measured; and if the length of the nerve between the two points of stimulation is known, the difference in time will represent the rate of nervous conduction. In experiments on frogs the leg is prepared by cutting away the muscles and bone of the thigh, leaving the nerve attached. The lever is then applied to the muscles of the leg, and the nerve is stimulated successively at two points, the distance between them being measured.

Employing the myograph of Marey, Baxt, in the laboratory of Helmholtz, succeeded in measuring the rate of nervous conduction in the human subject. In these experiments, the swelling of the muscle during contraction was limited by enclosing the arm in a plaster-mould, and the contraction was observed through a small opening. By then exciting the contraction by stimulating the radial nerve successively at different distances from the muscle, the estimate was made. The rate in the human subject was thus estimated at one hundred and eleven feet (33.9 meters) per second.

The method used in determining the rate of conduction in motor nerves - an estimation of the difference in time of the passage of a stimulus applied to a nerve at two points situated at a known distance from each other - has been applied to the conduction of sensations. Hirsch made the first attempt to solve this question, in r86r. He employed the delicate chronometric instruments used in astronomy and noted the difference in time between the appreciation of an impression made on a part of the body far removed from the brain, as the toe, and an impression made on the cheek. This process admitted of a rough estimate of about one hundred and eleven feet (33.9 meters) per second as the rate of sensory conduction.

It is not necessary to describe fully the apparatus by means of which the most recent estimates of the rate of nervous conduction have been made. The general results of the observations of Helmholtz, Marey, Baxt, Schleske and of many others nearly correspond with the estimates just given, and they show that the rate is about the same for motor and sensory nerves. This rate is modified by various conditions. It is diminished in the anelectrotonic and increased in the catelectrotonic condition of nerves. In the frog Helmholtz observed that the rate was much reduced by cold; at $32^{\circ} \mathrm{Fahr}$. ( $0^{\circ} \mathrm{C}$.) being not more than onetenth as rapid as at $60^{\circ}$ or $70^{\circ} \mathrm{Fahr}$. ( $15.5^{\circ}$ or 21 . I I ${ }^{\circ} \mathrm{C}$.).

The rate of transmission of impulses and impressions through the spinal cord has been investigated by calculating the distances between 
nerves as they are given off at different points and measuring the time required for the appreciation of certain impressions and the beginning of certain movements. While these observations are not absolutely exact, their general results are of considerable physiological interest. According to Burkhardt, the rate of motor conduction in the cord is about one-third of the normal rate in the motor nerves As compared with the sensory nerves, the cord conducts tactile impressions a little faster and painful impressions less than one-half as fast.

Attempts have been made to estimate the duration of acts involving the central nervous system, such as the reflex phenomena of the spinal cord or the operations of the cerebral hemispheres These have been partially successful, or, at least, they have shown that the reflex and the cerebral acts require a distinctly appreciable period of time. This in itself is an important fact; although the duration of these acts has not been measured with absolute accuracy. As the general result of experiments on these points, it has been found that the reflex action of the spinal cord occupies more than twelve times the period required for the transmission of stimulus or impressions through the nerves. Donders found, in experiments on his own person, that an act of volition required $\frac{1}{2} \bar{s}$ of a second, and one of simple distinction or recognition of an impression, $\frac{1}{25}$ of a second. These estimates, however, are merely approximate; and, until they attain greater accuracy, it is unnecessary to describe in detail the apparatus employed.

Aside from what have been described as reflex acts, it is found that when impulses or impressions pass through cells, there is a distinct retardation or delay in conduction, amounting to about 0.006 of a second for afferent conduction in the spinal cord and about 0.05 for efferent conduction. These figures, however, are approximations and are interesting chiefly as indicating an action of nerve-cells different from ordinary conduction by nerve-fibres, that has not been satisfactorily explained. It is probable that the delay is due to a redistribution of the impulses by the cells to the synapses through which the conduction is continued in one direction or the other.

Personal Equation. - In recording astronomical observations, it has been found that a certain time elapsed between the actual observation of a phenomenon and the moment of its record. This error, which is equal to the interval of time between the impression made on the retina and the muscular act by which a record is made, is not the same in different persons or even in the same person at all times. It may amount to $\frac{1}{8}$ of a second or even more, and it may be as low as $\frac{1}{50}$ of a second. If this difference is due to different rates of nervous conduction, and not entirely to variations in the rapidity of mental operations, 
it is evident that the velocity of the nerve-current must vary very considerably in different individuals.

Action of Electricity on the Nerves. - So long as the nerves retain their excitability and anatomical integrity, they will respond to properly applied electric stimulus. Experiments may be made on the exposed nerves in living animals or in animals just killed; and of all classes, the cold-blooded animals present the most favorable conditions, on account of the persistence of nervous and muscular excitability for a considerable time after death. Experimenters commonly use frogs, on account of the long persistence of the physiological properties of their tissues and the facility with which certain parts of the nervous system can be exposed. For ordinary experiments on nervous conduction, the parts are prepared by detaching the posterior extremities, removing the skin, and cutting away the bone and muscles of the thigh so as to leave the leg with the sciatic nerve attached. A frog's leg thus isolated presents a nervous trunk one or two inches ( 25 or 50 millimeters) in length, attached to the muscles, which will respond to a feeble electric stimulus. It is by experiments made on frogs prepared in this way, that most of the important facts in regard to the action of electricity on the nervous system have been developed.

In physiological experiments it is sometimes necessary to use different forms of electrical apparatus in order to study different properties and phenomena of nerve and muscle. A description of the apparatus thus used would be out of place in this work, and it will be necessary only to enumerate and describe the different currents used and the manner of their application. Many of the phenomena, also, described by electro-physiologists, although curious and interesting, have little apparent application to human physiology or to the practice of medicine. A description of such phenomena may well be brief in a work for the use of students and practitioners of medicine.

In studying the action of nerve and muscle, experimenters often use what is called a single faradic, or induction shock, with a duration of about $\frac{1}{1000}(0.0008)$ of a second. The excitation, therefore, is practically instantaneous. These single shocks are produced by Du Bois-Reymond's apparatus, which is a modification of the faradic, or induction battery. It will be seen farther on that somewhat different effects are produced by the stimulus, due to closing and opening the circuit, and that with a feeble current, no contractions occur at any other time. The contractions thus produced are known respectively as opening and closing. By the use of Du Bois-Reymond's keys, either the closing or the opening excitation may be diverted from the nerve, and a single closing or opening shock may be applied at will. 
What is commonly known as an interrupted current is a faradic or induced current, in which the closing and opening excitations follow each other with greater or less rapidity, and the intervals may be regulated so that they occur at a regular rate. A rapid succession of induction-shocks produces a more or less prolonged muscular action, called tetanic contraction. The number of successive shocks in a second required to produce a tetanic condition of a muscle varies in different animals and in different muscles in the same animal. The minimum seems to be about sixteen per second, with a considerable range of variation. Very rapid stimuli, even more than 3000 per second, will produce tetanic contraction.

The faradic, or induced current is different in its effects, under certain conditions of the nerves and muscles, from an interrupted galvanic, or primary current. This question is important in practical medicine in determining the so-called "reaction of degeneration" of nerve and muscle.

The constant current, under certain conditions, has no effect that is indicated by muscular phenomena, contraction occurring only on closing or opening the circuit. This is known as the galvanic, or primary current. It produces, however, a peculiar condition of nerves and muscles, which will be described under the head of electrotonus. The primary current is derived directly from the cells of a galvanic battery, and this is to be distinguished from the faradic, or induced current. The faradic current is induced in a coil of small insulated wire, brought near and parallel to and partly or entirely surrounding a coil of larger wire carrying the primary current. When the circuit of the primary current is closed, the direction of the induced current is the reverse of that of the primary current. When the primary circuit is opened, the induced current has the same direction as the primary current. The direction of the primary current is uniform, but the direction of the induced current alternates with each interruption of the primary current. These induced currents are of momentary duration, being produced only when the primary current is closed and opened. A rapid interruption of the primary current is produced by what is called a rheotome, or current-interrupter, which is attached to all inductionbatteries.

The points or surfaces used in closing a circuit within which a portion of nerve or muscle is included are called electrodes. They usually are designated as the copper, or positive electrode or pole, and the zinc, or negative electrode or pole. The positive pole is also called the anode, and the negative pole, the cathode. The direction of the current, when the circuit is closed, is from the anode to the cathode. 
When a galvanic current is passed through a liquid or a moist animal tissue, decomposition occurs, by what is known as electrolysis, or internal polarization. The products of this decomposition, called ions, are of course different in different liquids or moist tissues. These accumulate at the poles and after a time disturb the currents and the phenomena produced, the electrolytic process acting in the same way as an exciting electric current. In animal tissues, acids accumulate at the anode, and alkalies, at the cathode. The ions which pass to the anode are called anions, and those which accumulate at the cathode are called cations. In physiological experiments, it often is desirable to eliminate electrolysis, or internal polarization, and this is done by using the non-polarizable electrodes devised by Du Bois-Reymond. These may be described as follows: "The researches of Regnault, Matteucci and Du Bois-Reymond have proved that such electrodes can be made by taking two pieces of carefully amalgamated pure zinc wire, and dipping these in a saturated solution of zinc sulphate contained in tubes, their lower ends being closed by means of modeller's clay moistened with a 0.6 per cent normal saline solution. ${ }^{1}$ The contact of these electrodes with the tissues does not give rise to polarity" (Landois and Stirling).

It is evident that the galvanic current may be applied to a nerve so that the direction may in the one case follow the course of the nerve, that is, from the centre to the periphery, and in the other, be opposite to the course of the nerve. These are called respectively descending and ascending currents. When the positive pole (copper) is placed nearer the origin of the nerve, and the negative pole (zinc) below this point in the course of the nerve, the galvanic current follows the normal direction of the motor conduction, and this is called the descending current. When the poles are reversed and the direction is from the periphery toward the centre, it is called the ascending current. It will be convenient to speak of these two currents respectively as descending and ascending, in detailing experiments on the action of electricity on the nerves.

The main points to be noted in regard to the effects of the application of electricity to an exposed nerve are the action of constant currents, the phenomena observed on closing and on opening the circuit and the effects of an interrupted current.

During the passage of a feeble constant current through a nerve, whatever be its direction, there are no convulsive movements and no evidences of pain. This fact has long been recognized by physiologists,

1 Using ordinary electrodes, when brought in contact with an animal tissue, they become surrounded with positive and negative ions, which form miniature batteries giving off an electric stimulus when the circuit is closed or opened. 
who at first limited the effects of electricity on the nerves to two periods, one at the closing of the circuit and the other at its opening. It will be seen, however, that the passage of electricity through a portion of a nervous trunk produces a peculiar condition in the nerve, described under the name of electrotonus; but the fact remains that neither motion nor sensation is excited in a mixed nerve during the actual passage of a feeble constant current.

Law of Contraction. - All who have experimented on the action of galvanism on the nerves have noted the fact alluded to above, that contraction occurs only on closing or on opening the circuit. Take, for example, a frog's leg prepared with the nerve attached: Place one pole of a galvanic apparatus on the nerve and then make the connection, including a portion of the nerve in the circuit. With the feeblest current, contraction occurs only on closing the circuit. With what is called the "weak" current (Pflüger), contraction occurs only on closing the circuit, for currents in either direction. With the "moderate" current, contraction occurs both on closing and on opening the circuit, for currents in either direction. With the "strong" current, contraction occurs only on closing the circuit, with the descending current, and only on opening the circuit, with the ascending current. The above phenomena constitute what is called Pflüger's "law of contraction." The explanations of this law are the following:-

The stimulus that gives rise to a closing contraction occurs at the cathode, when the electrotonus produced by the passage of the current begins. The stimulus which produces an opening contraction occurs at the anode, when the electrotonus ceases. The impulse is always stronger when the electrotonus begins than when it ceases. Therefore, when the current is so feeble that but one contraction is produced, this contraction occurs only on closing the circuit, for both ascending and descending currents.

With the "moderate" current, the strength of the opening as well as of the closing impulse is sufficient to produce a contraction; and contractions therefore occur both on opening and closing the circuit, for both ascending and descending currents.

"Strong" currents produce closing contraction with the descending current, for the reason that the current destroys the conductivity of that portion of the nerve included between the poles of the battery, and, the stimulus occurring only at the cathode (see above), and the cathode being applied to that portion of the nerve nearer the muscle, the closing impulse only is conveyed to the muscle. The opening impulse (at the anode) is cut off from the muscle by the loss of conductivity in the intrapolar portion of the nerve. With the ascending current, the opening 
impulse, occurring at the anode, which is nearer the muscle, produces an opening contraction, and the closing impulse, which is at the cathode, is not conducted to the muscle.

While the constant current usually does not excite contractions during the actual time of its passage through a nerve, with a certain strength of current the muscle is thrown into a tetanic condition. This is called "closing tetanus." When a constant current, not of sufficient strength to produce closing tetanus, is passed for several minutes through a long extent of nerve, a vigorous contraction occurs on opening the circuit, which is followed by tetanus lasting for several seconds. This is called "opening tetanus." After a time - this varying with the excitability of the nerve and the strength of the current - the descending current will destroy the nervous excitability; but it may be restored by repose, or more quickly, by the passage of an ascending current. If the ascending current is passed first for a few seconds, a contraction follows the opening of the circuit; and this contraction, within certain limits, is more vigorous the longer the current is passed. At the same time, the prolonged passage of the ascending current increases the excitability of the nerve for any kind of stimulus.

After a certain time - which varies in different animals - the nervous excitability becomes somewhat enfeebled by exposure of the parts. The phenomena then observed belong to the conditions involved in the process of "dying" of the nerve. In the later stages of this condition, the phenomena may be formulated as follows :-

If the sciatic nerve attached to the leg of a frog, prepared in the usual way for such experiments, is subjected to a feeble galvanic current, there is a time when muscular contraction takes place only at the instant when the circuit is closed, no contraction occurring when the circuit is opened; and this occurs only with the descending current. With the ascending current, contraction of the muscles occurs only when the circuit is opened, and none takes place when the circuit is closed. These phenomena are distinct after the excitability of the parts has become somewhat diminished by exposure or by electric stimulation of the nerve.

If a sufficiently powerful constant current is passed through a nerve, disorganization of its tissue takes place, and the nerve finally loses its excitability, as it does when bruised, ligatured, or when its structure is destroyed in any other way. It was thought by Galvani that a current directed exactly across a nerve, so as to pass at right angles to its fibres, does not give rise to muscular contraction. This riew is commonly accepted by physiologists.

The muscular contraction produced by electric stimulation of a nerve 
is more vigorous the greater the extent of the nerve included between the poles of the battery. This fact has long been observed, and its accuracy may easily be verified. It would naturally be expected that the greater the amount of stimulation, the more marked would be the muscular action; and the stimulation seems to be increased in proportion to the extent of nerve through which the current is made to pass.

The excitability of a nerve, it is well known, may be exhausted by repeated applications of electricity, whatever be the direction of the current; and it is more or less completely restored by repose. When it has been exhausted for the descending current, it will respond to the ascending current, and vice vers $\hat{a}$; and after it has been exhausted by the descending current, it is restored more promptly by stimulation with the ascending current than by repose, and vice versâ. This phenomenon, observed by Volta, is known as "voltaic alternation."

Many of the phenomena illustrating the law of contraction may be observed without the use of complicated apparatus. A simple form of battery, very convenient for some of these experiments, consists of alternate copper and zinc wires wound around a piece of wood bent in the form of a horseshoe and terminating in two platinum points representing the positive and negative poles. This forms a sort of electric forceps, about eight inches ( 20 centimeters) long, which, when moistened with water slightly acidulated, will give a current of about the proper strength for many simple experiments.

The law of contraction is applicable to inhibitory nerves, as the inhibitory nerve of the heart, the difference being that stimulation produces inhibition instead of contraction. It also holds good for sensory nerves, the effects being observed by noting the reflex contractions produced (Pflüger).

A peculiar phenomenon, described by Matteucci, has been called "induced muscular contraction." If the nerve of a galvanoscopic frog's leg is placed in contact with the muscles of another leg prepared in the same way, stimulation of the nerve, giving rise to contraction of the muscles with which the nerve of the first leg is in contact, will induce contraction in the muscles of both. This experiment may be extended, and contractions may thus be induced in a series of legs, the nerve of one being in contact with the muscles of another. It is shown that "induced contraction" is not due to an actual propagation of the electric current, but to a stimulus attending the muscular contraction itself, by the fact that the same phenomena occur when the first contraction is produced by mechanical or chemical excitation of the nerve. It probably is due to the negative variation of the muscle-current during contraction (see page 427 ). 
Electric Current from the Exterior to the Cut Surface of a Nerve. Before studying certain phenomena presented in nerves of which a portion is subjected to the action of a constant galvanic current, it is important to note the fact that there exists in the nerves, as in the muscles, a current with a direction from the exterior to the cut surface. It has been roughly estimated that the nerve-current has one-eighth to onetenth the intensity of the muscle-current. The existence of the nervecurrent has, so far as known, no more physiological significance than the analogous fact observed in the muscular tissue. Currents exist also in the skin and in mucous membranes, the direction being from the outer surface, which is positive, to the inner surface, which is negative. ${ }^{1}$

Electrotonus, Anelectrotonus and Catelectrotonus. - When a constant galvanic current is passed through a portion of a freshly-prepared nerve, a large part of the entire nerve is brought into a peculiar electric condition. While in this state, the nerve will deflect the needle of a galvanometer and its excitability is modified. This is due to an electric tension of the entire nerve, induced by the passage of a current through a portion of its extent. This condition is called electrotonus. There is also a peculiar condition of that portion of the nerve near the anode, differing from the condition of the nerve near the cathode. Near the anode the excitability of the nerve is diminished, and this condition is called anelectrotonus. Near the cathode the excitability is increased, and this condition is called catelectrotonus. These phenomena have been the subject of extended investigation by electro-physiologists; and although the conditions are not to be included in the physiological properties of the nerves, they have considerable pathological and therapeutic importance. It is well known, for example, that electricity often is one of the most efficient agents at command for the restoration of the properties of nerves affected with disease; and the constant current has been extensively and successfully used as a therapeutic agent. The constant current, in restoring the normal condition of nerves, must influence, not only that portion included between the poles of the battery, but the entire nerve; and the electrotonic condition, with its modifications, in a measure explains how this result may be obtained.

The electrotonic condition is marked in proportion to the excitability of the nerve, and it is either absent or very feeble in nerves that are dead or have lost their excitability. If a strong ligature is applied to the extrapolar portion of a nerve, or if the nerve is divided and the cut ends are brought in contact with each other, the electrotonic condition is

1 The current described above was called by Du Bois-Reymond the "current of rest." Some writers now adopt the view of Hermann that this current does not exist in normal muscle or nerve but is due to injury of the tissue. 
either not observed or is feeble. These facts show that the phenomena of electrotonus depend on the physiological integrity of nerves. A dead nerve or one that has been divided or ligated may present these phenomena under the stimulation of a powerful current - and then only to a slight degree - when the condition depends on the purely physical properties of the nerve as a conductor; but these phenomena are not to be compared with those observed in nerves that retain their physiological properties.

As stated above, the electrotonic condition is not restricted to that portion of the nerve included between the poles of the battery. The condition of the portion between the poles is called intrapolar electroto-

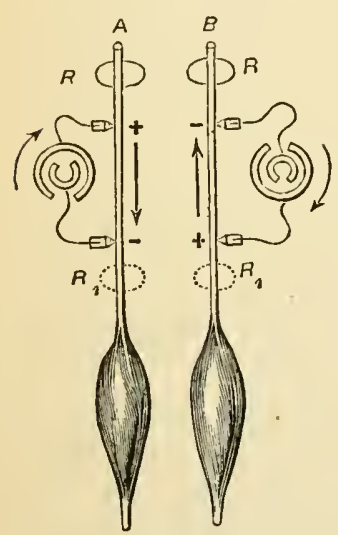

Fig. II3. - Method of testing excitability in electrotonus (Landois).

The positive poles are $t$ and the negative poles are - ; $R, R_{1}, R, R_{1}$, points excited by the saline solution. nus, and the condition of the nerve outside of the poles is called extrapolar electrotonus.

When a portion of a nerve is subjected to a moderately strong constant current, the conditions of the extrapolar portions corresponding to the two poles of the battery are different. Near the anode the excitability of the nerve and the rate of nervous conduction are diminished. If, however, a galvanometer is applied to this portion of the nerve, its electromotive power, measured by the deflection of the galvanometric needle, is increased. On the other hand, near the cathode the excitability of the nerve is increased, as well as the rate of nervous conduction, but the electromotive power is diminished.

The anelectrotonic condition, on the one hand, and the catelectrotonic condition, at the other pole of the battery, are marked in extrapolar portions of the nerve and are to be recognized, as well, in that portion through which the current is passing; but between the poles, there is a point where these conditions meet, as it were, and where the excitability is unchanged. This is called the neutral point. When the galvanic current is of moderate strength, the neutral point is about midway between the poles. "When a weak current is used, the neutral point approaches the positive pole, while in a strong current, it approaches the negative pole. In other words, in a weak current the negative pole rules over a wider territory than the positive pole, whereas in a strong current the positive pole prevails" (Rutherford).

The conditions of extrapolar excitability vary with the direction of the current applied to the nerve. A convenient stimulus with which to measure this excitability is a solution of common salt, which excites 
more or less powerful tetanic contractions of the muscles. These variations are illustrated in Fig. I I 3.

In Fig. I I $3, A$, a descending constant current is applied to the nerve. When the circuit is open, the salt applied to the nerve at $R$ produces contractions of the muscle. If the circuit is ${ }^{2}$ closed, the contractions either become much less vigorous or cease, on account of the diminished excitability near the anode. This is called descending extrapolar anelectrotonus. If the salt is applied at $R_{1}$, the contractions are increased in vigor by closing the circuit, on account of the increased excitability of the nerve near the cathode. This is called descending extrapolar catelectrotonus.

In Fig. I I $3, B$, the conditions are reversed. The polarizing current here must be very weak, as a strong current may destroy the conductivity of the intrapolar portion of the nerve and thus prevent the conduction of the stimulus to the muscle when the salt is applied at $R$. On closing the circuit, there is ascending extrapolar catelectrotonus at $R$, and ascending extrapolar anelectrotonus at $R_{1}$.

Within certain limits, the greater the strength of the constant current applied to the nerve and the greater the length of nerve included between the poles of the battery, the greater is the deflection of the galvanoscopic needle, by which the electrotonic condition is measured.

Electrotonic conditions in sensory nerves are measured by reflex movements produced by the action of a stimulus applied to these nerves. The variations in excitability of inhibitory nerves are indicated by increase or diminution in the inhibitory action. These phenomena are analogous to those observed in motor nerves. The influence of a constant current on the muscle-current is distinct though feeble, producing a kind of electrotonic condition of muscle.

Vegative Variation. - When a rapidly-interrupted current is applied to a nerve so as to produce a tetanic condition of the muscles to which it is distributed, the normal or tranquil nerve-current (current of rest) is overcome, and a galvanoscopic needle applied to the nerve, which was first deviated by the nerve-current, will be observed to retrograde and may return to zero (Du Bois-Reymond). This may also be observed to a slight degree under the influence of mechanical or chemical stimulation of the nerve, the natural nerve-current being diminished, but usually not abolished. The variation of the needle under the influence of these conditions has been called negative variation. It is observed in greater or less degree when impulses of any kind are passing through a nerve, this conduction constituting what is known as the current of action. These phenomena are analogous to the negative variation of muscle-currents, which has already been described. 


\title{
CHAPTER XIX
}

\author{
SPINAL NERVES - MOTOR CRANIAL NERVES
}

Spinal nerves - Cranial nerves - Motor oculi communis (third nerve) - Physiological anatomy - Properties and uses of the motor oculi communis - Patheticus, or trochlearis (fourth nerve) - Physiological anatomy - Properties and uses of the patheticus - Motor oculi externus, or abducens (sixth nerve) - Physiological anatomy - Properties and uses of the motor oculi communis - Nerve of mastication (the small, or motor root of the fifth nerve) - Physiological anatomy - Properties and uses of the nerve of mastication Facial, or nerve of expression (seventh nerve) - Physiological anatomy - General properties of the facial - Uses of branches of the facial given off within the aqueduct of Fallopius Uses of the chorda tympani-Influence of certain branches of the facial on the movements of the palate and uvula - Uses of the external branches of the facial - Spinal accessory (eleventh nerve) - Physiological anatomy - Properties and uses of the spinal accessory Uses of the internal branch from the spinal accessory to the pneumogastric - Influence of the internal branch of the spinal accessory on deglutition - Influence of the spinal accessory on the heart - Uses of the external, or muscular branches of the spinal accessory Sublingual (twelfth nerve) - Physiological anatomy -- Properties and uses of the sublingual.

WiтH a knowledge of the general properties of the nerves belonging to the cerebro-spinal system, it is easy to understand the uses of most of the special nerves, simply from their anatomical relations. This is especially true of the spinal nerves. These, in general terms, are distributed to the muscles of the trunk and extremities, to the sphincters and the integument covering these parts, the posterior segment of the head, and to certain mucous membranes. It is evident, therefore, that an account of the exact office of each nervous branch would necessitate a full description, not only of the nerves, but of the muscles of the body, which is manifestly within the scope only of treatises on descriptive anatomy.

\section{Spinal Nerves}

There are thirty-one pairs of spinal nerves; eight cervical, twelve dorsal, five lumbar, five sacral and one coccygeal. Each nerve arises from the spinal cord by an anterior (motor) and a posterior (sensory) root, the posterior root being the larger and having a ganglion. Immediately beyond the ganglion, the two roots unite into a single mixed nerve, which passes out of the spinal canal by an intervertebral foramen. The nerve thus constituted is possessed of motor and sensory properties. It divides outside of the spinal canal into two branches, 
anterior and posterior, both containing motor and sensory filaments, which are distributed respectively to the anterior and the posterior parts of the body. The anterior branches are the larger, and they supply the limbs and all parts in front of the spinal column.

The anterior branches of the upper four cervical nerves form the cervical plexus; and the four inferior cervical nerves, with the first

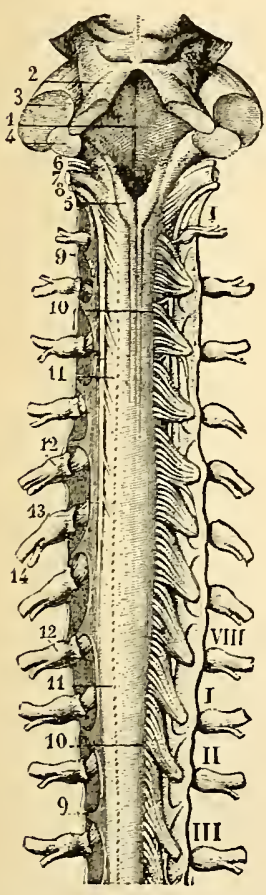

Fig. II4. - Cervical portion of the spinal cord (Hirschfeld).

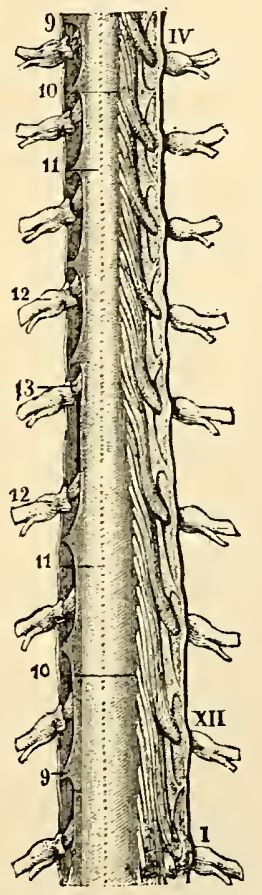

Fig. II5.-Dorsal portion of the spinal cord (Hirschfeld).

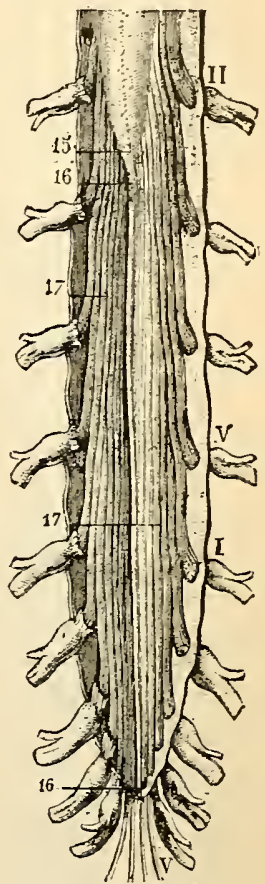

Fig. I16. - Inferior portion of the spinal cord, and cauda equina (Hirschfeld).

I, antero-inferior wall of the fourth ventricle; 2 , superior peduncle of the cerebellum; 3 , middle peduncle of the cerebellum; 4 , inferior peduncle of the cerebellum; 5 , inferior portion of the posterior median columns of the cord; 6 , glosso-pharyngeal nerve; 7 , pneumogastric; 8 , spinal accessory nerve; 9, 9. 9, 9, dentated ligament; IO, IO, IO, IO, posterior roots of the spinal nerves; II, II, I I, I I, posterior lateral grome: I2, I2, I2, I2, ganglia of the posterior roots of the nerves; $\mathrm{I}_{3}, \mathrm{I}_{3}$, anterior roots of the nerves; I4, division of the Herves into two branches; 15 , lower extremity of the cord ; 16, 16, coccygeal ligament; I7, I7, cauda equina; I-VIII, cervical nerves; I, II, III, IV-XII, dorsal nerves; I, II-V, lumbar nerves; I-V, sacral nerves.

dorsal, form the brachial plexus. The anterior branches of the dorsal nerves, with the exception of the first, supply the walls of the chest and abdomen. These nerves go directly to their distribution without forming a plexus. The anterior branches of the upper four lumbar nerves form the lumbar plexus. The anterior branch of the fifth lumbar nerve and a branch from the fourth unite with the anterior branch of the first 
sacral, forming the lumbo-sacral nerve, and enter into the sacral plexus. The upper three anterior sacral nerves, with a branch from the fourth, form the sacral plexus. The greatest portion of the fourth anterior sacral is distributed to the pelvic viscera and the muscles of the anus. The fifth anterior sacral and the coccygeal are distributed to parts about the coccyx.

The posterior branches of the spinal nerves are quite simple in their distribution. With one or two exceptions, of no great physiological importance, these nerves pass backward from the main trunk, divide into two branches, external and internal, and their filaments of distribution go to the muscles and to integument behind the spinal column.

It is further important to note that all the cerebro-spinal nerves anastomose with the sympathetic.

\section{Cranial Nerves}

Many of the cranial nerves are peculiar, either as regards their general properties or in their distribution to parts concerned in special functions. In some of these nerves, the most important facts concerning their distribution have been ascertained only by physiological experimentation, and their anatomy is inseparably connected with their physiology. It would be desirable, if it were possible, to classify these nerves with reference strictly to their properties and uses; but this can be done only to a certain extent. The common classification of the cranial nerves is the arrangement of Sömmerring, in which the nerves are numbered from before backward in the order in which they pass out of the skull, making twelve pairs.

\section{Classification of the Cranial Nerves}

Nerves of Special Sense

Olfactory. (First pair.)

Optic. (Second pair.)

Auditory. (Eighth pair.)

Gustatory, comprising a part of the glosso-pharyngeal (ninth pair) and a small filament from the facial (seventh pair) to the lingual branch of the fifth pair.

\section{Nerves of Motion}

Nerves of motion of the eyeball, comprising the motor oculi communis (third pair), the patheticus (fourth pair), and the motor oculi externus (sixth pair). 
Nerve of mastication, or motor root of the fifth pair.

Facial, sometimes called the nerve of expression. (Seventh pair.)

Spinal accessory. (Eleventh pair.)

Sublingual. (Twelfth pair.)

\section{Nerves of General Sensibility}

Trifacial, or large root of the fifth pair.

A portion of the glosso-pharyngeal. (Ninth pair.)

Pneumogastric. (Tenth pair.)

In the above arrangement the nerves are classified according to their properties at their roots. In their course, some of these nerves become mixed and their branches are both motor and sensory, such as the pneumogastric and the inferior maxillary division of the trifacial.

The nerves of special sense have little or no general sensibility; and with the exception of the gustatory nerves, they do not present ganglia on their roots, in this, also, differing from the ordinary sensory nerves. They are capable of conveying to the nerve-centres only certain peculiar impressions, such as odors, for the olfactory nerves, light, for the optic nerves, and sound, for the auditory nerves. The proper transmission of these impressions, however, involves the action of accessory parts, more or less complex; and the properties of these

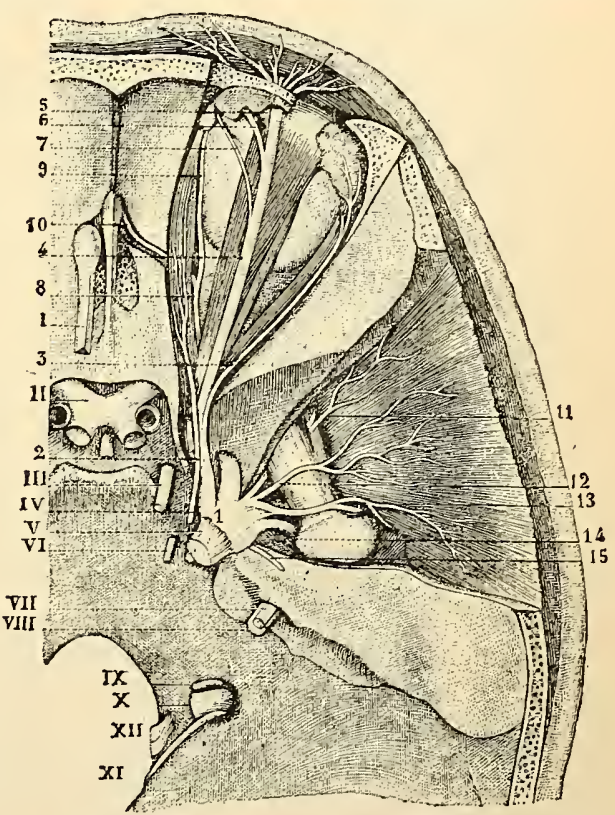

Fig. 117. - Roots of the cranial nerves (Hirschfeld).

I, olfactory; II, optic; III, motor oculi communis; IV, patheticus; $V$, nerve of mastication and trifacial; VI, motor oculi externus; VII, facial; VIII, auditory; IX, glosso-pharyngeal; X, pneumogastric; XI, spinal accessory; XII, sublingual. The numbers I to I5 refer to branches that will be described hereafter. nerves will be considered in connection with the physiology of the special senses.

\section{Motor Oculi Communis (Third Nerve)}

The third cranial nerve is the most important of the motor nerves distributed to the muscles of the eyeball. Its physiology is readily understood in connection with its distribution, the only point at all obscure 
being its relations to the movements of the iris, concerning which the results of experiments are somewhat contradictory.

Physiological Anatomy. - The apparent origin of the third nerve is from the inner edge of the crus cerebri, directly in front of the pons Varolii and midway between the pons and the corpora albicantia. It presents here eight or ten filaments, of nearly equal size, which soon unite into a single rounded trunk.

The deep origin of the nerve has been studied by means of dissections of the encephalon fresh and hardened by different liquids. From

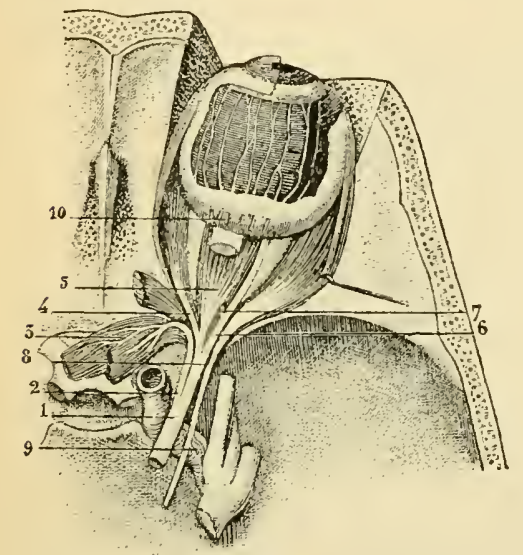

Fig. 118. - Distribution of the motor oculi communis (Hirschfeld).

I, trunk of the motor oculi communis ; 2 , superior branch; 3, filaments which this branch sends to the superior rectus and the levator palpebri superioris; 4 , branch to the internal rectus; 5, branch to the inferior rectus; 6, branch to the inferior oblique muscle; 7 , branch to the lenticular ganglion; 8 , motor oculi externus; 9 , filaments of the motor oculi externus anastomosing with the sympathetic; I0, ciliary nerves.

divides into two branches. The superior, which is the smaller, passes to the superior rectus muscle of the eye and certain of its filaments are continued to the levator palpebræ superioris. The inferior division breaks up into three branches. The internal branch passes to the internal rectus muscle; the inferior branch, to the inferior rectus; the external branch, the largest of the three, is distributed to the inferior oblique muscle, and in its course it sends a short and thick filament to the lenticular, or ophthalmic ganglion of the sympathetic. It is this branch which is supposed, through the short ciliary nerves passing from the lenticular ganglion, to furnish motor filaments to the ciliary muscle and 
the iris. In its course this nerve receives a few delicate filaments from the cavernous plexus of the sympathetic and a branch from the ophthalmic division of the trifacial.

Properties and Uses of the Motor Oculi Communis. - Stimulation of the root of the third nerve in a living animal produces contraction of the muscles to which it is distributed, but no pain. If the stimulus, however, is applied a little farther on in the course of the nerve, there are evidences of sensibility; and this is readily explained by its communications with the ophthalmic branch of the trifacial. At its root, therefore, this nerve is exclusively motor and its office is connected with the action of muscles.

The phenomena observed after section of the motor oculi communis in living animals are the following:-

I. Falling of the upper eyelid, or blepharoptosis.

2. External strabismus, immobility of the eye except in an outward direction, inability to rotate the eye on its antero-posterior axis in certain directions and slight protrusion of the eyeball.

3. Dilatation of the pupil, with a certain degree of interference with the movements of the iris.

The falling of the upper eyelid is constantly observed after division of the third nerve in living animals and always follows its complete paralysis in the human subject. An animal in which the nerve has been divided can not raise the lid but can press the lids together by a voluntary effort. In the human subject the falling of the lid gives to the face a peculiar and characteristic expression. The complete loss of power shows that the levator palpebræ superioris muscle depends on the third nerve entirely for its motor filaments.

The external strabismus and the immobility of the eyeball except in an outward direction are due to paralysis of the internal, superior and inferior recti muscles, the external rectus acting without its antagonist. This condition requires no further explanation. These points are illustrated by the experiment of dividing the nerve in rabbits. If the head of the animal is turned inward, on exposing the eye to a bright light, the globe will turn outward by the action of the external rectus; but if the head is turned outward, the globe remains motionless.

It is somewhat difficult to note the effects of paralysis of the inferior oblique muscle, which also is supplied by the third nerve. This muscle, acting from its origin at the inferior and internal part of the circumference of the base of the orbit to its attachment at the inferior and external part of the posterior hemisphere of the eyeball, gives to the globe a movement of rotation on an oblique horizontal axis, downward and backward, directing the pupil upward and outward. When this 
muscle is paralyzed, the superior oblique, having no antagonist, rotates the globe upward and inward, directing the pupil downward and outward. The action of the oblique muscles is observed when the head is moved alternately toward one shoulder and the other. In the human subject, when the inferior oblique muscle on one side is paralyzed, the eye can not move in a direction opposite to the movements of the head, as it does upon the sound side, so as to keep the pupil fixed, and there is double vision.

When all the muscles of the eyeball except the external rectus and superior oblique are paralyzed, as they are by section of the third nerve, the globe is slightly protruded, simply by the relaxation of most of its muscles. An opposite action is easily observed in a cat with the facial nerve divided so that it can not close the lids. When the cornea is touched, all the muscles, particularly the four recti, act to draw the globe into the orbit, which allows the lid to fall slightly and projects the little membrane which serves as a third eyelid in these animals.

The third nerve sends a filament to the ophthalmic ganglion of the sympathetic, and from this ganglion the short ciliary nerves take their origin and pass to the iris. While it is true that division of the third nerve affects the movements of the iris, it becomes a question whether this be a direct influence or an influence exerted primarily on the ganglion, not, perhaps, differing from the general effects on the sympathetic ganglia that follow destruction of their branches of communication with the motor nerves.

The third nerve animates the muscular fibres that contract the pupil, the contraction produced by stimulation of the optic nerves being reflex in its character. The reflex action by which the iris is contracted is not instantaneous, like most of the analogous phenomena observed in the cerebro-spinal system, and its operations are rather characteristic of the action of the sympathetic system and non-striated muscular tissue. It has been found, also, that the pupil is not immediately dilated after division of the third nerve. Several hours after the operation, however, the pupil usually is found dilated, and it may slowly contract when the eye is exposed to the light.

Experiments have shown, also, that although the pupil contracts feebly and slowly under the stimulus of light after division of the motor oculi, it will dilate under the influence of belladonna and can be made to contract by operating on other nerves. It is well known, for example, that division or stimulation of the fifth nerve produces contraction of the pupil. This takes place after as well as before division of the third nerve. Section of the sympathetic in the cervical region also contracts the pupil, and this occurs after paralysis of the motor oculi. These 
facts show that the third is not the only nerve capable of acting on the iris and that it is not the sole avenue for the transmission of reflex influences.

When the eye is turned inward by a voluntary effort, the pupil is contracted; and when the axes of the two eyes are made to converge strongly, as in looking at near objects, the contraction is very considerable.

The third nerve contains filaments that preside over voluntary movements of the ciliary muscle in the accommodation of the eye to vision at different distances.

The following case illustrates, in the human subject, nearly all the phenomena following paralysis of the motor oculi communis in experiments on the lower animals :-

The patient was a girl, nineteen years of age, with complete paralysis of the nerve on the left side. There was slight protrusion of the eyeball, complete ptosis, with the pupil moderately dilated and insensible to ordinary impressions of light. The sight was not affected, but there was double vision, except when objects were placed before the eyes so that the axes were parallel, or when an object was seen with but one eye. The axis of the left eye was turned outward, but it was not possible to detect deviation upward or downward. On causing the patient to incline the head alternately to one shoulder and the other, it was evident that the affected eye did not rotate in the orbit but moved with the head. This seemed to be a case of complete and uncomplicated paralysis of the third nerve.

\section{Patheticus, or Trochlearis (Fourth Nerve)}

The physiology of the patheticus resolves itself into the action of a single muscle, the superior oblique.

Physiological Anatomy. - The apparent origin of the patheticus is from the superior peduncles of the cerebellum; but it may easily be followed to the valve of Vieussens. The deep roots can be traced, passing from without inward, to the following parts: One filament is lost in the substance of the peduncles; other filaments pass from before backward into the valve of Vieussens and are lost, and a few pass into the frenulum; a few filaments pass backward and are lost in the corpora quadrigemina; but the greatest number pass to the median line and decussate with corresponding filaments from the opposite side. The fibres can be traced to a nucleus in the floor of the aqueduct of Sylvius, beneath the nucleus of the third nerve. The decussation of the fibres of origin of the fourth nerve has the same physiological significance as 
the decussation of the roots of the third. From this origin, the patheticus passes into the orbit by the sphenoidal fissure and is distributed to the superior oblique muscle of the eyeball. In the cavernous sinus it receives branches of communication from the ophthalmic branch of the fifth, but these are not closely united with the nerve. A small branch passes into the tentorium, and one joins the lachrymal nerve, these, however, being exclusively sensory and coming from the ophthalmic

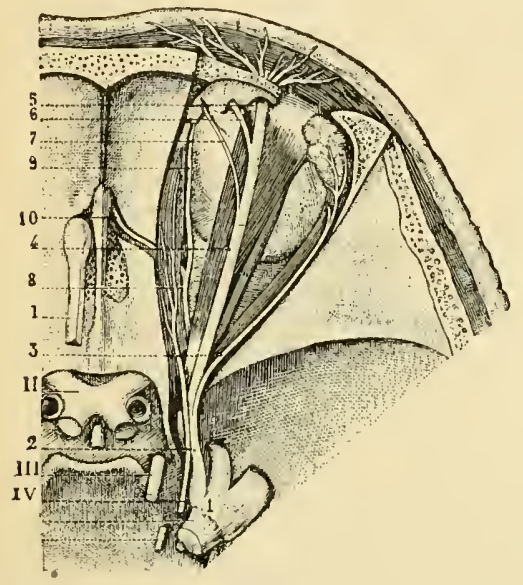

Fig. II9. - Distribution of the patheticus (Hirschfeld).

I, olfactory nerve; II, optic nerves; III, motor oculi communis; IV, patheticus, by the side of the ophthalmic branch of the fifth, and passing to the superior oblique muscle; IV, motor oculi externus; I, ganglion of Gasser; $2,3,4,5,6,7,8,9$, Io, ophthalmic division of the fifth nerve, with its branches.

acts on the eychall from the pulleyc branch of the fifth. It also receives a few filaments from the sympathetic.

Proforties and Uses of the Patheticus. - Direct observations on the patheticus in living animals have shown that it is motor and its stimulation excites contraction of the superior oblique muscle only. This muscle arises just above the inner margin of the optic foramen, passes forward along the upper wall of the orbit at its inner angle to a little cartilaginous ring which serves as a pulley. From its origin to this point it is muscular. Its tendon becomes rounded just before it passes over the pulley, where it makes a sharp curve, passes outward and slightly backward and becomes spread out to be attached to the globe at the superior and external part of its posterior hemisphere. It acts on the eyeball from the pulley at the upper and inner portion of the orbit as the fixed point and rotates the eye on an oblique horizontal axis, from below upward, from without inward and from behind forward. By its action the pupil is directed downward and outward. It is the antagonist of the inferior oblique, the action of which has been described in connection with the motor oculi communis. When the patheticus is paralyzed, the eyeball is immovable so far as rotation is concerned. When the head is moved toward the shoulder, the eye does not rotate to maintain the globe in the same relative position and there is double vision.

Paralysis confined to the fourth nerve in the human subject is rather unusual. "In seventy-seven cases of paralysis of a single oculomotor nerve I found the third nerve affected in thirty-one cases, the fourth in . nine, and the sixth in thirty-seven." - (Nettleship.) 


\section{Motor Oculi Externus, or Abducens (Sixth Nerve)}

Like the patheticus, the motor oculi externus is distributed to but a single muscle. Its uses, therefore, are apparent from a study of its distribution and properties.

Physiological Anatomy. - The apparent origin of the sixth nerve is from the groove separating the anterior corpus pyramidale of the medulla oblongata from the pons Varolii, from the upper portion of the medulla and from the lower portion of the pons next the groove. Its origin at this point is by two roots : an inferior root, which is the larger and comes from the corpus pyramidale; and a superior root, sometimes wanting, which seems to come from the lower portion of the pons. All anatomists are agreed that the deep fibres of origin of this nerve pass to the gray matter in the floor of the fourth ventricle. It is not known that the fibres of the two sides decussate. From this origin the nerve passes into the orbit by the sphenoidal fissure and is distributed exclusively to the external rectus muscle of the eyeball. In the cavernous sinus it anastomoses with the sympathetic through the carotid plexus and receives a filament from Meckel's ganglion. It also receives sensory filaments from the

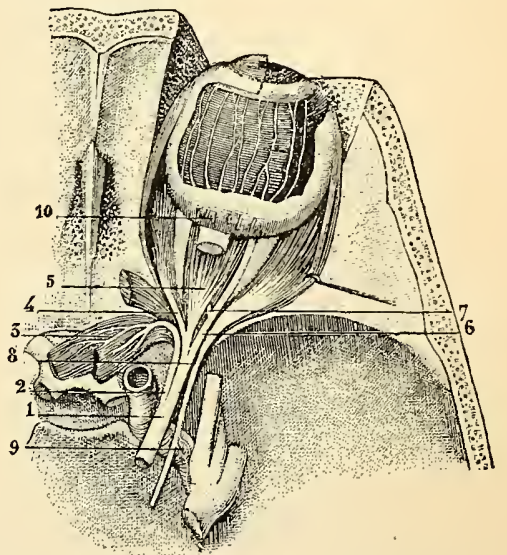

Fig. 120.- Distribution of the motor oculi externus (Hirschfeld).

I, trunk of the motor oculi communis, with its branches $(2,3,4,5,6,7) ; 8$, motor oculi externus, passing to the external rectus muscle; 9, filaments of the motor oculi externus, anastomosing with the sympathetic; Io, ciliary nerves. ophthalmic branch of the fifth. It is thought by some anatomists that this nerve occasionally sends a small filament to the ophthalmic ganglion; and this branch, which is exceptional, exists in those cases in which paralysis of the motor oculi communis, which usually furnishes all the motor filaments to this ganglion, is not attended with immobility of the iris.

Properties and Uses of the Motor Oculi Externus. - Direct experiments have shown that the motor oculi externus is insensible at its origin, its stimulation producing contraction of the external rectus muscle and no pain. The same experiments illustrate the action of the nerve, inasmuch as its stimulation is followed by contraction of the muscle and deviation of the eye outward. Division of the nerve in the lower animals or its paralysis in the human subject is attended with 
internal, or converging strabismus, due to the unopposed action of the internal rectus nuuscle.

In regard to the associated movements of the eyeball, it is important to note that all the muscles of the eye that have a tendency to direct the pupil inward or produce the simple movements upward and downward (the internal, inferior, and superior recti) are animated by a single nerve, the motor oculi communis, this nerve also supplying, however, the inferior oblique; and that each of two muscles that move the globe so as to direct the pupil outward (the superior oblique and the external rectus) is supplied by a special nerve. The movements of the eyeball will be described more in detail in connection with the physiology of vision.

\section{Nerve of Mastication (the Small, or Motor Root of the Fifth Nerve)}

The motor root of the fifth nerve is distinct from its sensory portion until it emerges from the cranial cavity by the foramen ovale. It is then closely united with the inferior maxillary division of the large root; but at its origin it has been shown to be motor, and its section in the cranial cavity has demonstrated its distribution to a particular set of muscles.

Physiological Anatomy. - The apparent origin of the fifth nerve is from the lateral portion of the pons Varolii. The small, or motor root arises from a point a little higher and nearer the median line than the large root, from which it is separated by a few fibres of the white substance of the pons. At the point of apparent origin, the small root presents six to eight rounded filaments. If a thin layer of the pons covering these filaments is removed, the roots will be found penetrating its substance, becoming flattened, passing under the superior peduncles of the cerebellum and going to a gray nucleus, with large multipolar cells, in the anterior wall of the fourth ventricle near the median line. At this point the fibres change their direction, passing from without inward and from behind forward toward the median line, the fibres diverging widely. The posterior fibres pass to the median line and certain of them decussate with fibres from the opposite side. The anterior fibres pass toward the aqueduct of Sylvius and are lost. The fibres become changed in their character when they are followed inward beyond the anterior wall of the fourth ventricle. Here they lose their white color, become gray and present a number of globules of gray substance between their filaments.

From the origin above described, the small root passes beneath the ganglion of Gasser, - from which it sometimes, though not constantly, 
receives a filament of communication, - lies behind the inferior maxillary division of the large root and passes out of the cranial cavity by the foramen ovale. Within the cranium the two roots are distinct; but after the small root passes through the foramen, it is united by a mutual interlacement of fibres with the sensory branch.

The inferior maxillary nerve, made up of the motor root and the inferior maxillary division of the sensory root, just after it passes out

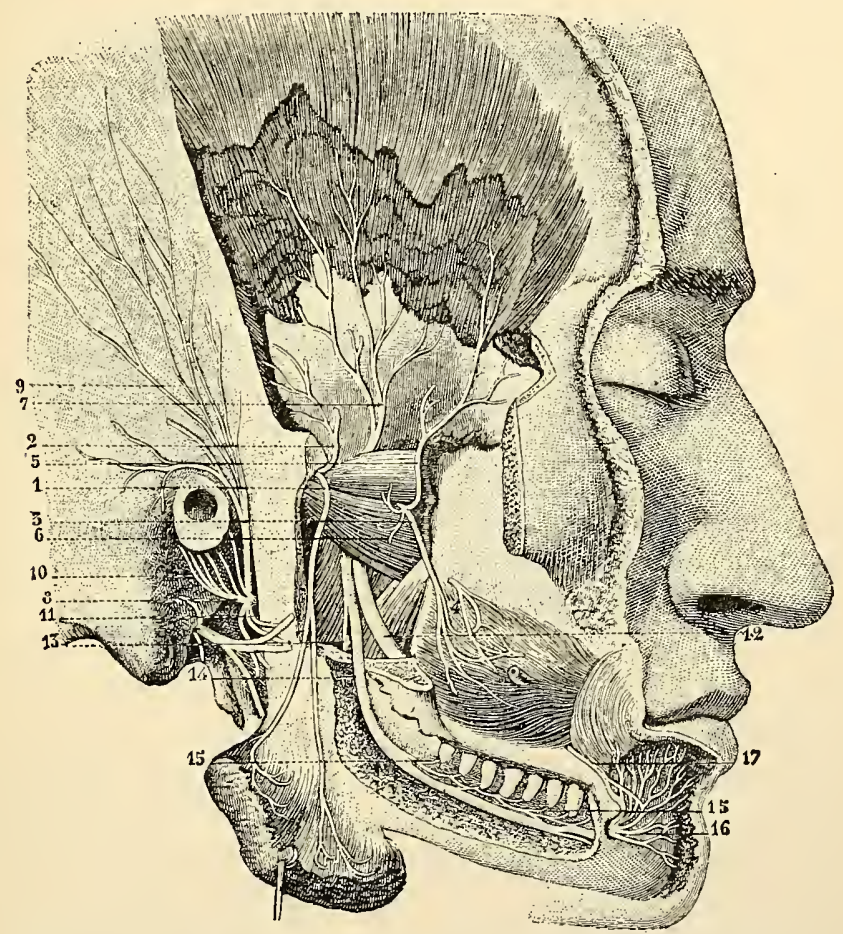

Fig. r2x. - Distribution of the small root of the fifth nerve (Hirschfeld).

I, branch to the masseter muscle; 2 , filament of this branch to the temporal muscle; 3 , buccal branch; 4 , branches anastomosing with the facial nerve; 5 , filament from the buccal branch to the temporal muscle; 6, branches to the external pterygoid muscle; 7 , middle deep temporal branch; 8 , auriculo-temporal nerve; 9 , temporal branches; IO, auricular branches; II, anastomosis with the facial nerve; I2, lingual branch; $1_{3}$, branch of the small root to the mylo-hyoid muscle; $\mathrm{I}_{4}$, inferior dental nerve, with its branches $\left(I_{5}, I_{5}\right)$; I6, mental branch; $I_{7}$, anastomosis of this branch with the facial nerve.

by the foramen ovale, divides into two branches, anterior and posterior. The anterior branch, which is the smaller, is composed almost entirely of motor filaments and is distributed to the muscles of mastication. It gives off five branches. The first passes to be distributed to the masseter muscle, in its course occasionally giving off a small branch to the temporal muscle and a filament to the articulation of the inferior maxilla with the temporal bone. The two deep temporal branches are 
distributed to the temporal muscle. The buccal branch sends filaments to the external pterygoid and the temporal muscles, and a small branch is distributed to the internal pterygoid muscle. From the posterior branch, which is chiefly sensory but contains some motor filaments, branches are sent to the mylo-hyoid muscle and to the anterior belly of the digastric. In addition the motor branch of the fifth sends filaments to the tensor muscles of the velum palati.

Experiments have shown that the buccinator muscle receives no motor filaments from the fifth but is supplied entirely by the facial. The buccal branch of the fifth sends motor filaments to the external pterygoid and the temporal, its final branches of distribution being sensory and going to integument and mucous membrane.

In treating of the physiology of digestion, a table has been given of the muscles of mastication with a description of their action. It will be seen by reference to this table that the following muscles depress the lower jaw : the anterior belly of the digastric, the mylo-hyoid, the geniohyoid and the platysma myoides. Of these the digastric and the mylohyoid are animated by the motor root of the fifth; the genio-hyoid is supplied by filaments from the sublingual; and the platysma myoides, by branches from the facial and from the cervical plexus. All the muscles that elevate the lower jaw and move it laterally and anteroposteriorly, namely, the temporal, masseter, and the internal and external pyterygoids, - the muscles most actively concerned in mastication, - are animated by the motor root of the fifth.

Propertics and Uses of the Nerve of Mastication. - The anatomical distribution of the small root of the fifth nerve points at once to its uses. Charles Bell called it the nerve of mastication, in I $82 \mathrm{I}$, although he did not relate any experiments in regard to its action. Anatomical and physiological writers since that time have adopted this view. It would be difficult if not impossible to stimulate the root in the cranial cavity in a living animal; but its faradization in animals just killed determines movements of the lower jaw. Experiments have demonstrated the physiological properties of the small root, which is without doubt a nerve of motion only.

There is little left to say in regard to the uses of the motor root of the fifth nerve, in addition to the description of the action of the muscles of mastication, contained in the chapters on digestion, except as regards the action of the filaments sent to the muscles of the velum palati. In deglutition the muscles of mastication are indirectly involved. This act can not be well performed unless the mouth is closed by these muscles. When the food comes in contact with the velum palati, muscles are brought into action which render this membrane tense, so that the 
opening is adapted to the size of the alimentary bolus. These muscles are animated by the motor root of the fifth. This nerve, then, is not only the nerve of mastication, animating all the muscles concerned in this act except two of the most unimportant depressors of the lower jaw (the genio-hyoid and the platysma myoides), but it is concerned indirectly in deglutition.

\section{Facial, or Nerve of Expression (Seventh Nerve)}

The anatomical relations of the facial nerve are quite intricate and it communicates freely with other nerves. So far as can be determined by experiments on living animals, this nerve is exclusively motor at its origin; but in its course it presents anastomoses with the sympathetic, and with branches of the fifth and the cervical nerves, receiving sensory filaments.

Physiological Anatomy. - The facial nerve has its apparent origin from the lateral portion of the medulla oblongata, or bulb, in the groove between the olivary and restiform bodies just below the border of the pons Varolii, its trunk being internal to the trunk of the auditory nerve. It is separated from the auditory by two filaments constituting what is known as the intermediary nerve of Wrisberg, or the portio inter duram et mollem. As this little nerve joins the facial it usually is included in its root.

Anatomists have endeavored to trace the fibres of the facial from their point of emergence from the encephalon to their true origin, but with results not entirely satisfactory. Its fibres pass inward, with one or two deviations from a straight course, to the floor of the fourth ventricle, where they spread out and become fan-shaped. In the floor of the fourth ventricle certain of the fibres have been thought to terminate in the cells of the gray substance, and others have been traced to the median line, where they decussate; but the course of many of the fibres has not been satisfactorily established. The fibres of origin of the intermediary nerve of Wrisberg have been traced to the nucleus of the glosso-pharyngeal.

It is evident from physiological experiments that the decussation of the fibres in the floor of the fourth ventricle itself is not very important. Vulpian made, in dogs and rabbits, a longitudinal section in the middle line of the ventricle, which would necessarily have divided the fibres passing from one side to the other, without producing notable paralysis of the facial nerves on either side. This observation shows that the main decussation of the fibres animating the muscles of the face takes place in some other situation. 
Certain pathological facts bearing on the question of decussation of the filaments of origin of the facial have long been recognized. They are in brief as follows: When there is lesion of the brain-substance anterior to the pons Varolii, the phenomena due to paralysis of the facial are observed on the same side as the hemiplegia, opposite the side of injury to the brain. When the lesion is either in or below the pons,

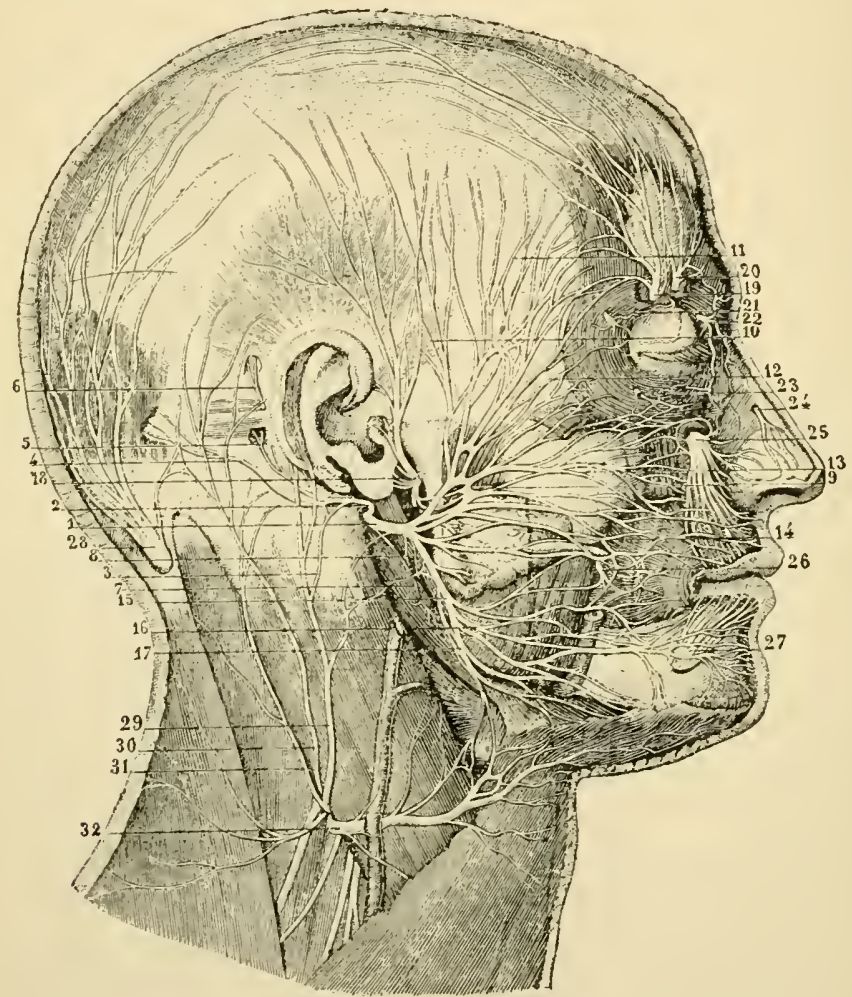

Fig. 122. - Superficial branches of the facial and the fifth (Hirschfeld).

I, trunk of the facial; 2 , posterior auricular nerve; 3, branch which it receives from the cervical plexus; 4, occipital branch; 5, 6, branches to the muscles of the ear; 7 , digastric branches; 8, branch to the stylo-hyoid muscle; 9, superiur terminal branch; 10, temporal branches; II, frontal branches; 12, branches to the orbicularis palpebrarum; 13, nasal, or suborbital branches; 14, buccal branches; 15, inferior terminal branch; 16, mental branches; 17, cervical branches; 18, superficial temporal nerve (branch of the fifth); 19,20 , frontal nerves (branches of the fifth); 21, 22, 23, 24, 25, 26, 27, (branches of the fifth) ; 28,29,30,31, 32 (branches of the cervical nerves).

the face is affected on the same side, and not on the side of the hemiplegia. This is called alternate paralysis. In view of these facts, the phenomenon of hemiplegia of one side and facial paralysis on the other is regarded as indicating, with tolerable certainty, that injury to the brain has occurred on the same side as the facial paralysis, either within or posterior to the pons Varolii. 
As already stated, the fibres of origin of the facial have been traced to the floor of the fourth ventricle, where a few decussate but most of them are lost. The question now is, whether or not the fibres pass up through the pons and decussate above, as the pathological facts just noted would seem to indicate. Anatomical researches on this point are not satisfactory; and the existence of such a decussation has not been clearly demonstrated. The pathological observations nevertheless remain; and however indefinite anatomical researches may have been, there can be no doubt that lesions in one lateral half of the pons affect the facial on the same side, while lesions above the pons have a crossed action. The most that can be said on this point is that it is a reasonable inference from pathological facts that the nerves decussate anterior to the pons.

The main root of the facial, the auditory nerve and the intermediary nerve of Wrisberg pass together into the internal auditory meatus. At the bottom of the meatus, the facial and the nerve of Wrisberg enter the aquæductus Fallopii, following its course through the petrous portion of the temporal bone. In the aqueduct the nerve of Wrisberg presents a little ganglioform enlargement (geniculate ganglion) of a reddish color, which has been shown to contain nerve-cells. The main root and the intermediary nerve then unite and form the common trunk of the facial, which emerges from the cranial cavity by the stylo-mastoid foramen. It is thought by some anatomists that the intermediary nerve of Wrisberg arises from the glosso-pharyngeal, which communicates with the facial in the tympanum. A branch has been described going from the glossopharyngeal to the geniculate ganglion. It has been seen that the fibres of origin of this nerve come from the nucleus of the glossopharyngeal.

In the aquæductus Fallopii the facial gives off the following branches :-

I. The large petrosal branch is given off at the ganglioform enlargement and goes to Meckel's ganglion.

2. The small petrosal branch is given off at the ganglioform enlargement or a very short distance beyond it and passes to the otic ganglion. muscle.

3. A small branch, the tympanic, is distributed to the stapedius

4. The chorda tympani passes through the cavity of the tympanum and joins the lingual branch of the inferior maxillary division of the fifth as it passes between the two pterygoid muscles, with which nerve it becomes closely united.

5. Opposite the point of origin of the chorda tympani, a communi- 
cating branch passes between the facial and the pneumogastric, connecting these nerves by a double inosculation.

The five branches above described are given off in the aquæductus Fallopii. The following branches are given off after the nerve has emerged from the cranial cavity:-

I. Just after the facial has passed out at the stylo-mastoid foramen, it sends a small communicating branch to the glosso-pharyngeal nerve. This branch is sometimes wanting.

2. The posterior auricular nerve is given off by the facial a little below the stylo-mastoid foramen. Its superior branch is distributed to the retrahens aurem and the attollens aurem. In its course this nerve receives a communicating branch of considerable size from the cervical plexus by the auricularis magnus. It sends some filaments to the integument. The inferior, or occipital branch, the larger of the two, is distributed to the occipital portion of the occipito-frontalis muscle and to integument.

3. The digastric branch is given off near the root of the posterior auricular. It is distributed to the posterior belly of the digastric muscle. In its course it anastomoses with filaments from the glosso-pharyngeal. From the plexus formed by this anastomosis, filaments are given off to the digastric and to the stylo-hyoid muscles.

4. Near the stylo-mastoid foramen a small branch is given off which is distributed exclusively to the stylo-hyoid muscle.

5. Near the stylo-mastoid foramen, or sometimes a little above it, a long delicate branch is given off, which is not noticed in many works on anatomy. It is described, however, by Hirschfeld, under the name of the lingual branch. It passes behind the stylo-pharyngeal muscle and then by the sides of the pharynx to the base of the tongue. In its course it receives one or two branches from the glosso-pharyngeal nerve, which are nearly as large as the original branch from the facial. As it passes to the base of the tongue it anastomoses again by a number of filaments with the glosso-pharyngeal. It then sends filaments of distribution to the mucous membrane and finally passes to the stylo-glossus and palatoglossus muscles.

Having given off these branches, the trunk of the facial passes through the parotid gland, dividing into its two great terminal branches :-

I. The temporo-facial branch, the larger, passes upward and forward to be distributed to the superficial muscles of the upper part of the face; namely, the attrahens aurem, the frontal portion of the occipitofrontalis, the orbicularis palpebrarum, corrugator supercilii, pyramidalis nasi, levator labii superioris, levator labii superioris alæque nasi, the 
dilators and compressors of the nose, part of the buccinator, the levator anguli oris and the zygomatic muscles. In its course it receives branches of communication from the auriculo-temporal branch of the inferior maxillary nerve. It joins also with the temporal branch of the superior maxillary and with branches of the ophthalmic. It thus becomes a mixed nerve and is distributed in part to integument.

2. The cervico-facial nerve passes downward and forward to supply the buccinator, orbicularis oris, risorius, levator labii inferioris, depressor labii inferioris, depressor anguli oris and platysma.

General Properties of the Facial Nerve. - It has long been recognized that the facial is the motor nerve of the superficial muscles of the face and that its division produces paralysis of motion and no marked effects on sensation. It is evident, also, from the communications between the facial with the fifth, that it contains in its course sensory fibres. Indeed, all who have operated on this nerve have found that it is slightly sensory after it has emerged from the cranial cavity. It is a question, however, of great importance to determine whether or not the facial be endowed with sensibility by virtue of its own fibres of origin. The main root evidently is from the motor tract, resembles the anterior roots of the spinal nerves and is distributed to muscles; but this root is joined by the intermediary nerve of Wrisberg, which presents a small ganglionic enlargement that is analogous to the ganglia on the posterior roots of the spinal nerves. The testimony of direct experimentation is in favor of the insensibility of the facial at its origin. It is true that the intermediary nerve of Wrisberg has a certain anatomical resemblance to the sensory nerves, chiefly by reason of its ganglioform enlargement; but direct experiments are wanting, to show that it is a nerve of general sensibility.

Uses of the Branches of the Facial given off within the Aqueduct of Fallopius. - The first branch, the large petrosal, is the motor root of Meckel's ganglion. This will be referred to again, in connection with the sympathetic system. The second branch, the small petrosal, is one of the motor roots of the otic ganglion. The third branch, the tympanic, is distributed exclusively to the stapedius muscle. The second and third branches will be considered again, in connection with the physiology of the internal ear. The fourth branch, the chorda tympani, is so important that it demands special consideration. The fifth branch is given off opposite the origin of the chorda tympani and passes to the pneumogastric, to which nerve it probably supplies motor filaments. In this branch, sensory filaments pass from the pneumogastric and constitute a part of the sensory connections of the facial.

Uses of the Chorda Tympani. - This nerve passes between the 
bones of the ear and through the tympanic cavity to the lingual branch of the inferior maxillary division of the fifth, which it joins at an acute angle, between the pterygoid muscles. As regards the portion of the facial that furnishes the filaments of the chorda tympani, it is nearly certain that these come from the intermediary nerve of Wrisberg.

There can be no doubt in regard to the influence of the chorda tympani on the sense of taste in the anterior two-thirds of the tongue. In cases of disease or injury in which the root of the facial is involved so that the chorda tympani is paralyzed, in addition to the ordinary phenomena of paralysis of the superficial muscles of the face, there is

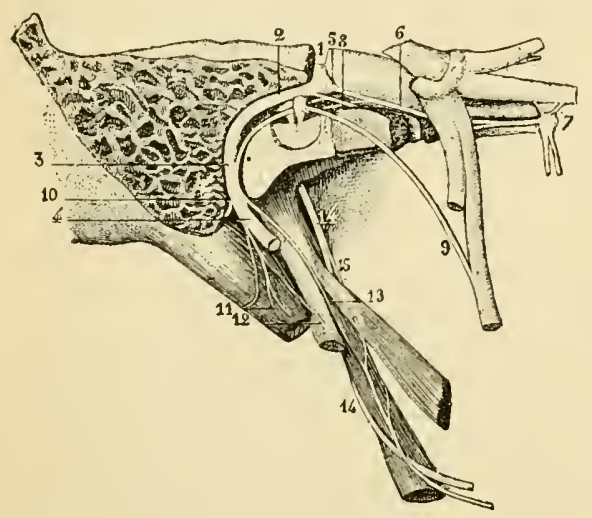

Fig. 123. - Chorda-tympani nerve (Hirschfeld).

I, 2, 3, 4, facial nerve passing through the aquæeductus Fallopii; 5, ganglioform enlargement (geniculate ganglion); 6, great petrosal nerve; 7, spheno-palatine ganglion; 8 , small petrosal nerve; 9. chorda tympani; IO, II, I2, I3, various branches of the facial; 14, I4, 15, glosso-pharyngeal nerve. loss of taste in the anterior twothirds of the tongue on the side corresponding to the lesion. The action of the chorda tympani will be considered again, in connection with the physiology of gustation.

Influence of Certain Branches of the Facial on the Movements of the Palate and Unula.There can be little doubt that filaments from the facial animate certain of the movements of the velum palati and the uvula. It has been observed that in certain cases of facial paralysis the palate on one side is flaccid and the uvula is drawn to the opposite side; but these phenomena do not occur unless the nerve is affected at its root or within the aquæductus Fallopii. It is true that the uvula frequently is drawn to one side or the other in persons unaffected with facial paralysis, but it is none the less certain that it is deviated as a consequence of paralysis of the facial in some instances. The filaments of the facial which influence the levator palati and azygos uvulæ muscles are derived from the large petrosal branch of the nerve, passing to the muscles through Meckel's ganglion, the filaments to the palato-glossus and the palato-pharyngeus being given off from the glosso-pharyngeal, but originally coming from an anastomosing branch of the facial.

In view of examples of paralysis of the palate and uvula in certain cases of facial palsy, the frequent occurrence of contractions of the muscles of these parts following stimulation of the facial and the reflex 
action through the glosso-pharyngeal and the facial, there can be little doubt that the muscles of the palate and uvula receive filaments of distribution from the seventh nerve. The effects of paralysis of these muscles are manifested by more or less trouble in deglutition and in the pronunciation of certain words, with difficulty in the expulsion of mucus collected in the back part of the mouth and the pharynx.

Uses of the External Branches of the Facial. - The general action of the branches of the facial going to the superficial muscles of the face is sufficiently evident, in view of what is known of the distribution of these branches and the general properties of the nerve. It is now recognized as the nerve that presides over the movements of the superficial muscles of the face, not including those directly concerned in the act of mastication. This being its general action, it is easy to assign to each of the external branches its particular office.

Just after the facial has passed out at the stylo-mastoid foramen, it sends to the glosso-pharyngeal the communicating branch, the action of which has just been mentioned in connection with the movements of the palate.

The posterior auricular branch, becoming partly sensory by the accession of filaments from the cervical plexus, gives sensibility to the integument on the back part of the ear and over the occipital portion of the occipito-frontalis muscle. It animates the retrahens and the attollens aurem, muscles that are little developed in man but are important in certain of the inferior animals. It also animates the posterior . portion of the occipito-frontalis.

The branches distributed to the posterior belly of the digastric and to the stylo-hyoid muscle simply animate these muscles, one of the uses of which is to assist in deglutition. The same may be said of filaments that go the stylo-glossus.

The two great branches distributed upon the face, after the trunk of the nerve has passed through the parotid gland, have the most prominent action. Both these branches are slightly sensory, from their connections with other nerves, and are distributed in small part to integument.

The temporo-facial branch animates all the muscles of the upper part of the face. In complete paralysis of this branch, the eye is constantly open, even during sleep, on account of paralysis of the orbicularis muscle. In cases of long standing, the globe of the eye may become inflamed from constant exposure, from abolition of the movements of winking by which the tears are distributed over its surface and little foreign particles are removed, and, in short, from absence of the protective action of the lids. In these cases the lower lid may become slightly everted. The frontal portion of the occipito-frontalis, 
the attrahens aurem and the corrugator supercilii muscles, are also paralyzed. The most prominent symptom of paralysis of these muscles is inability to corrugate the brow on one side.

Paralysis of the muscles that dilate the nostrils has been shown to have an important influence on respiration through the nose. In instances of complete paralysis of the nostril of one side, there frequently is some difficulty in inspiration, even in the human subject.

Physiologists have noted an interference with olfaction, due to the inability to inhale with one nostril, in cases of facial paralysis. The influence of the nerve in the act of conveying odorous emanations to the olfactory membrane is sufficiently evident after what has been said concerning the action of the facial in respiration.

The effects of paralysis of the other superficial muscles of the face are manifested in the distortion of the features, on account of the unopposed action of the muscles of the sound side, a phenomenon that is sufficiently familiar. When facial palsy affects one side and is complete, the angle of the mouth is drawn to the opposite side, the eye on the affected side is widely and permanently opened, even during sleep, and the face has on that side a peculiarly expressionless appearance. When a patient affected in this way smiles or attempts to grimace, the distortion is much increased. The lips are paralyzed on one side, which sometimes causes a flow of saliva from the corner of the mouth. In the lower animals that use the lips in prehension, paralysis of these parts interferes considerably with the taking of food. The flaccidity of the paralyzed lips and cheek in the human subject sometimes causes a puffing movement with each act of expiration, as if the patient were smoking a pipe.

The buccinator is not supplied by filaments from the nerve of mastication but is animated solely by the facial. Paralysis of this muscle interferes materially with mastication, from a tendency to accumulation of the food between the teeth and the cheek. Patients complain of this difficulty, and they sometimes keep the food between the teeth by pressure with the hand. In the rare instances in which both facial nerves are paralyzed, there is very great difficulty in mastication, from the cause just mentioned.

The action of the external branches of the facial is thus sufficiently simple; and it is only as its deep branches affect the sense of taste, the movements of deglutition, etc., that it is difficult to ascertain their exact office. As this is the nerve of expression of the face, it is in the human subject that the phenomena attending its paralysis are most prominent. When both sides are affected, the aspect is remarkable, the face being absolutely expressionless and looking as if it were covered with a mask. 


\section{Spinal Accessory (Eleventh Nerve)}

The spinal accessory nerve, from the great extent of its origin, its important anastomoses with other nerves and its peculiar course and distribution, has long engaged the attention of anatomists and physiologists, who have advanced many theories in regard to its office. Its physiological history, however, may properly be said to begin with the experiments of Claude Bernard.

Physiological Anatomy. - The origin of this nerve is very extensive. A certain portion arises from the lower half of the bulb, and the rest takes its origin below, from the upper two-thirds of the cervical portion of the spinal cord. That portion of the root which arises from the medulla oblongata is called the bulbar portion, the roots from the cord constituting the spinal portion. Inasmuch as there is a marked difference between the uses of these two portions, the anatomical distinction just mentioned is important.

The superior roots arise by four or five filaments from the lower half of the medulla oblongata, below the origin of the pneumogastrics. These filaments of origin pass to a gray nucleus in the medulla.

The spinal portion of the nerve arises from the upper part of the spinal cord, between the anterior and posterior roots of the upper four or five cervical nerves. The filaments of origin are six to eight in number. The most inferior of these usually is

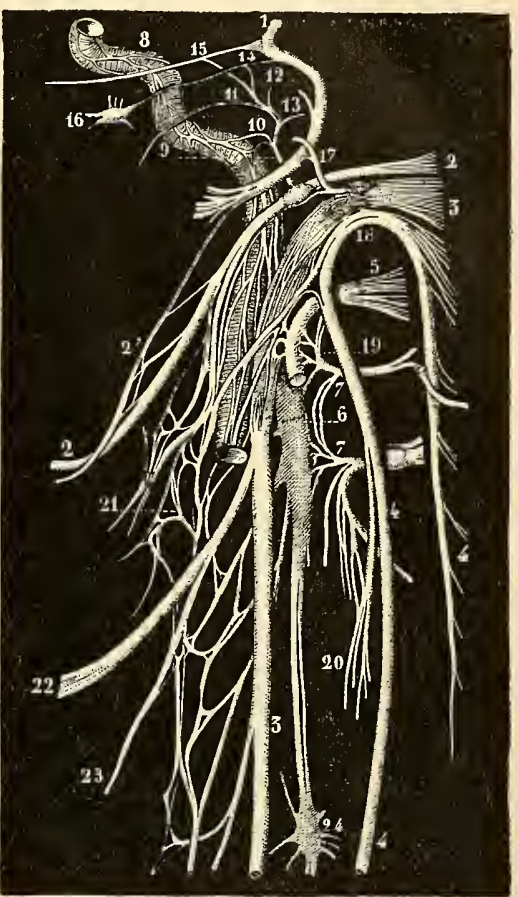

Fig. 124. - Spinal accessory nerve (Hirschfeld).

I, trunk of the facial nerve; 2,2 , glossopharyngeal nerve; 3,3 , pneumogastric; 4.4 , 4 , trunk of the spinal accessory; 5 , sublingual nerve; 6 , superior cervical ganglion; 7,7 , anastomosis of the first two cervical nerves; 8 , carotid branch of the sympathetic; 9,10 , II, I2, I3, branches of the glosso-pharyngeal; I4, I5, branches of the facial; I6, otic ganglion; 17, auricular branch of the pneumogastric; 18, anastomosing branch from the spinal accessory to the pneumogastric; 19. anastomosis of the first pair of cervical nerves with the sublingual; 20, anastomosis of the spinal accessory with the second pair of cervical nerves; 21 , pharyngeal plexus; 22, superior laryngeal nerve; 23, external laryngeal nerve; 24 , middle cervical ganglion. single, the other filaments frequently being arranged in pairs. These take their origin from the lateral portion of the cord and are connected with the anterior cornua of gray matter. 
Following the nerve from its most inferior filament of origin upward, it gradually increases in size by union with its other roots, enters the cranial cavity by the foramen magnum, and passes to the jugular foramen, by which it emerges, with the glosso-pharyngeal, the pneumogastric and the internal jugular vein.

In its course the spinal accessory anastomoses with several nerves. Just as it enters the cranial cavity, it receives filaments of communication from the posterior roots of the upper two cervical nerves. These filaments, however, are not constant. It frequently, though not constantly, sends a few filaments to the superior ganglion, or the ganglion of the root of the pneumogastric. After it has emerged by the jugular foramen it sends a branch of considerable size to the pneumogastric, from which nerve it receives a few filaments of communication. In its course it also receives filaments of communication from the anterior branches of the second, third and fourth cervical nerves.

In its distribution the spinal accessory presents two branches. The internal, or anastomotic branch passes to the pneumogastric just below the plexiform enlargement, which is sometimes called the ganglion of the trunk of the pneumogastric. This branch is composed principally if not entirely of the filaments that take their origin from the bulb. As it joins the pneumogastric it subdivides into two smaller branches. The first of these forms a portion of the pharyngeal branch of the pneumogastric. The second becomes intimately united with the pneumogastric, lying at its posterior portion, and furnishes filaments to the inferior, or recurrent laryngeal branch, which is distributed to all the muscles of the larynx except the crico-thyroid. The passage of the filaments from the spinal accessory to the pharyngeal branch of the pneumogastric is readily observed; but the fact that filaments from this nerve pass to the larynx by the recurrent laryngeal has been ascertained only by physiological experiments. In the chimpanzee, however, the internal branch does not go to the pneumogastric but passes directly to the muscles of the larynx (Vrolik).

The external, or large branch of the spinal accessory, called the muscular branch, penetrates and passes through the posterior portion of the upper third of the sterno-cleido-mastoid muscle. It then goes to the anterior surface of the trapezius, which muscle receives its ultimate branches of distribution. In its passage through the sterno-cleido-mastoid, it joins with branches from the second and third cervical nerves and sends filaments of distribution to the muscle. Although the two muscles just mentioned receive motor filaments from the spinal accessory, they are also supplied from the cervical nerves; and consequently they are not entirely paralyzed when the spinal accessory is divided. 
Properties and Uses of the Spinal Accessory. - Notwithstanding the difficulty in exposing and operating on the roots of the spinal accessory, it has been demonstrated that their stimulation produces convulsive movements in certain muscles. By stimulating the filaments that arise from the bulb, contractions of the muscles of the pharynx and larynx are produced, but no movements of the sterno-mastoid and trapezius. Stimulation of the roots arising from the spinal cord produces movements of the two muscles just mentioned and no movements in the larynx. In view of these experiments, it is evident that the true filaments of origin of the spinal accessory are motor; and it is further evident that the filaments from the bulb are distributed to the muscles of the pharynx and larynx, while the filaments from the spinal cord go to the sterno-cleido-mastoid and trapezius.

The trunk of the spinal accessory, after the nerve has passed out of the cranial cavity, has a certain degree of sensibility. If the nerve is divided, the peripheral extremity manifests recurrent sensibility, but the central end also is sensible, probably from direct filaments of communication from the cervical nerves and the pneumogastric.

Uses of the Internal Branch from the Spinal Accessory to the Pneumogastric. - Bischoff ( 1832 ) attempted to ascertain the uses of this branch by dividing the roots of the spinal accessory on both sides in a living animal. The results of his experiments may be stated in a very few words: $\mathrm{He}$ attempted to divide all the roots of the nerves on both sides by dissecting down to the occipito-atloid space and penetrating into the cavity of the spinal canal. In the first three experiments on dogs, the animals died so soon after section of the nerves that no satisfactory results were obtained. In two succeeding experiments on dogs, the animals recovered. After division of the nerves the voice became hoarse, but a few weeks later it became normal. On killing the animals, an examination of the parts showed that some of the filaments of origin had not been divided. An experiment was then made on a goat, but this was unsatisfactory, as the roots were not completely divided. Finally another experiment was made on a goat. In this the results were more satisfactory. After division of the nerve on one side, the voice became hoarse. As the filaments were divided on the opposite side, the voice was enfeebled, until finally it became extinct. The sound emitted afterward was one that could in no wise be called voice ("qui neutiquam vox appellari potuit").

Bernard, who determined exactly the influence of the spinal accessory over the vocal movements of the larynx, first repeated the experiments of Bischoff; but the animals operated on died so soon from hemorrhage or other causes that his observations were not satisfactory. 
After many unsuccessful trials, he succeeded in overcoming all difficulties by following the trunk of the nerve back to the jugular foramen, seizing it here with a strong forceps and drawing it out by the roots. The operation usually is most successful in cats, although Bernard frequently succeeded in other animals.

When one spinal accessory is extirpated, the vocal sounds are hoarse and unnatural. When both nerves are torn out, in addition to the disturbance of deglutition and the partial paralysis of the sterno-mastoid and trapezius muscles, the voice becomes extinct. Animals operated on in this way move the jaws and make evident efforts to cry, but no vocal sound is emitted. Bernard kept animals, with both nerves extirpated, for several months and did not observe any return of the voice. His observations, which have been fully confirmed (Flint), show that the internal branch of the spinal accessory is the nerve of phonation. The filaments that preside over the vocal movements of the larynx pass in greatest part through the recurrent laryngeal branches of the pneumogastrics; but the recurrent laryngeals also contain filaments from other motor nerves, which latter are concerned in the respiratory movements of the glottis.

Influence of the Internal Branch of the Spinal Accessory on Deglutition. - There are two ways in which deglutition is affected through this nerve: I. When the larynx is paralyzed as a consequence of extirpation of both nerves, the glottis can not be completely closed to prevent the entrance of foreign bodies into the air-passages. In rabbits particularly, it has been noted that particles of food penetrate the trachea and find their way into the lungs. 2. The spinal accessory furnishes filaments to the pharyngeal branch of the pneumogastric, and through this nerve it directly affects the muscles of deglutition; but the muscles animated in this way by the spinal accessory have a tendency to draw the lips of the glottis together, while they assist in passing the alimentary bolus into the osophagus. When these important acts are wanting, there is some difficulty in the process of deglutition itself, as well as danger of passage of foreign particles into the larynx.

Influence of the Spinal Accessory on the Heart. - The spinal accessory furnishes to the pneumogastric the inhibitory fibres that influence the action of the heart. A sufficiently powerful faradic current passed through one pneumogastric will in some animals arrest the cardiac movements; and it has been noted that the influence of the right nerve on the heart is greater than that of the left. Waller found that if he extirpated the spinal accessory on one side, after four or five days the action of the heart could not be arrested by stimulating the pneumogastric of the same side; but inhibition followed stimulation of the pneumo- 
gastric of the opposite side, where the connections with the spinal accessory were intact. In these observations it seemed necessary that a sufficient time should elapse after extirpation of the spinal accessory for the excitability of the filaments that join the pneumogastric to become extinct; but the experiments are sufficient to show the direct inhibitory influence of the spinal accessory on the heart. After extirpation of the spinal accessory, degenerated fibres are found in the trunk of the pneumogastric. The mechanism of inhibition of the heart has already been considered in connection with the physiology of the circulation.

Uses of the External, or Muscular Branch of the Spinal Accessory. Observations have shown that the internal branch of the spinal accessory, and the internal branch only, is directly concerned in the vocal movements of the larynx, and to a great extent in the closure of the glottis during deglutition. It has been noted in addition that animals in which both branches have been extirpated present irregularity of the movements of the anterior extremities and suffer from shortness of breath after violent muscular exertion. The use of the corresponding extremities in the human subject is so different that it is not easy to make a direct application of these experiments; still, certain inferences may be drawn from them in regard to the action of the external branch in man.

In prolonged vocal efforts the vocal chords are put on the stretch, and the act of expiration is different from that in tranquil breathing. In singing, for example, the shoulders frequently are fixed; and this is done to some extent by the action of the sterno-cleido-mastoid and the trapezius. It is probable, then, that the action of the branch of the spinal accessory which goes to these muscles has a certain synchronism with the action of the branch going to the larynx and the pharynx; the one fixing the upper part of the chest so that the expulsion of the air through the glottis may be more nicely regulated by the expiratory muscles, and the other acting on the vocal chords.

In violent muscular effort, the glottis is closed, the thorax is fixed after a full inspiration and respiration is arrested so long as the effort, if it be not too prolonged, is continued. The same synchronism, therefore, obtains in this as in prolonged vocal efforts. In experiments in which the muscular branch only has been divided, shortness of breath, after violent muscular effort, is observed; and this probably is due to lack of synchronous action of the sterno-cleido-mastoid and trapezius. The irregularity in the movements of progression in animals in which either both branches or the muscular branches alone have been divided is due to anatomical peculiarities. 


\section{Sublingual (Twelfth Nerve)}

The last of the motor cranial nerves is the sublingual; and its action is intimately connected with the physiology of the tongue in deglutition and articulation, although the sublingual is also distributed to certain of the muscles of the neck.

Physiological Anatomy. - The apparent origin of the sublingual is from the bulb, in the groove between the olivary body and the anterior pyramid, on the line of the anterior roots of the spinal nerves. At this point its root is formed of ten to twelve filaments, which extend from the inferior portion of the olivary body to about the junction of the upper with the middle third of the bulb. These filaments of origin are separated into two groups, superior and inferior. From this apparent origin, the filaments have been traced into the gray matter of the floor of the fourth ventricle, between the deep origin of the pneumogastric and the glosso-pharyngeal. It is probable that some of the filaments of origin of these nerves decussate in the floor of the fourth ventricle. The superior and inferior filaments of origin of the nerve unite to form two bundles, which pass through distinct perforations in the dura mater. These two bundles then pass into the anterior condyloid foramen and unite into a single trunk as they emerge from the cranial cavity.

After the sublingual has passed out of the cranial cavity it anastomoses with several nerves. It sends a filament of communication to the sympathetic as it branches from the superior cervical ganglion. Soon after it has passed through the foramen it sends a branch to the pneumogastric. It anastomoses by two or three branches with the upper two cervical nerves, the filaments passing in both directions between the nerves. It anastomoses with the lingual branch of the fifth by two or three filaments passing in both directions.

In its distribution the sublingual presents several peculiarities :-

Its first branch, the decendens noni, passes down the neck to the sterno-hyoid, sterno-thyroid and omo-hyoid muscles.

The thyro-hyoid branch is distributed to the thyro-hyoid muscle.

The other branches are distributed to the stylo-glossus, hyo-glossus, genio-hyoid and genio-hyo-glossus muscles, their terminal filaments going to the intrinsic muscles of the tongue.

It is thus seen that the sublingual nerve is distributed to all the muscles in the infra-hyoid region, the action of which is to depress the larynx and the hyoid bone after the passage of the alimentary bolus through the pharynx; to one of the muscles in the supra-hyoid region, the genio-hyoid; to most of the muscles that move the tongue; and to 
the muscular fibres of the tongue itself. The action of these muscles and of the tongue itself in deglutition has already been fully discussed.

Properties and $U$ 'ses of the Sublingual. - The fact that the sublingual nerve arises from a continuation of the motor tract of the spinal cord and has no ganglion on its main root would lead to the supposition that it is an exclusively motor nerve. Experiments on the inferior

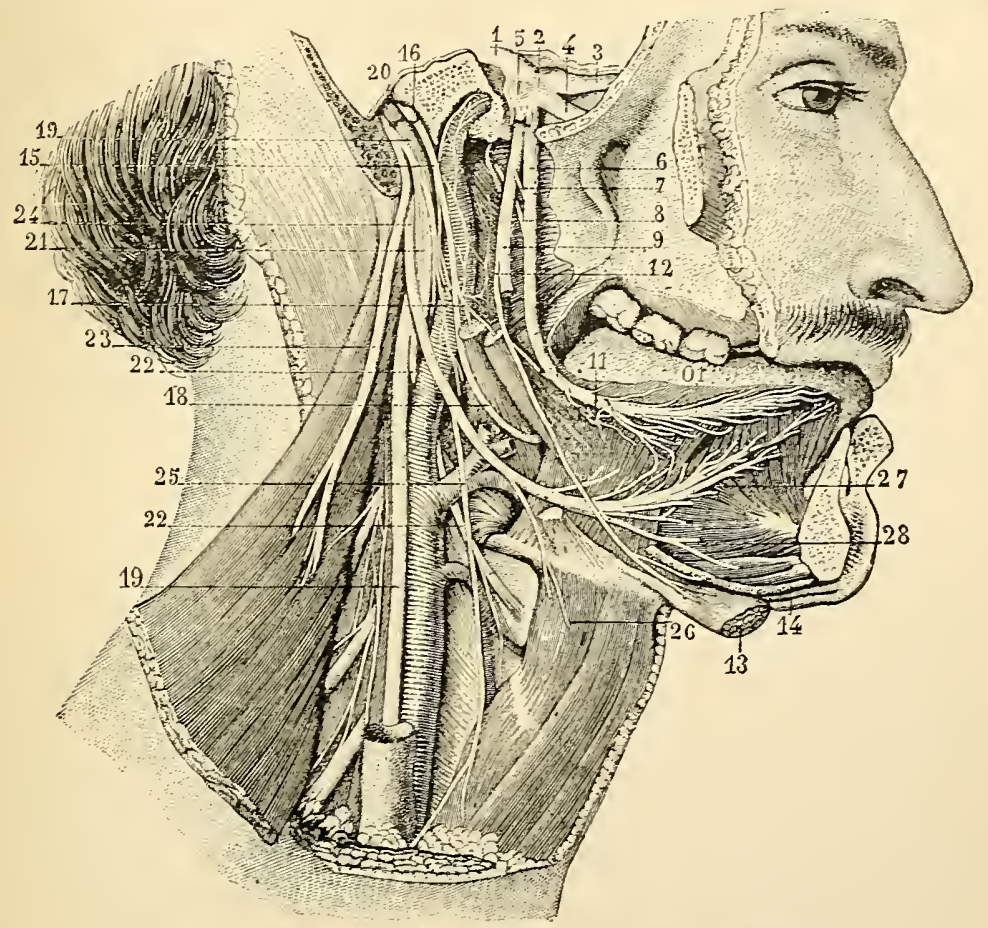

Fig. 125. - Distribution of the sublingual nerve (Sappey).

I, root of the fifth nerve; 2, ganglion of Gasser; $3,4,5,6,7,9$, I0, I2, branches and anastomoses of the filth nerve; II, submaxillary ganglion; I3, anterior belly of the digastric muscle; I4, section of the mylo-hyoid muscle; I5, glosso-pharvngeal nerve; 16, ganglion of Andersch; 17, I8, branches of the glosso-pharyngeal nerve; I9, I9, pneumogastric; 20, 2I, ganglia of the pneumogastric; 22, 22, superior laryngeal branch of the pneumogastric; 23 , spinal accessory nerve; 24 , sublingual nerve; 25, descendens noni; 26 , thyro-hyoid branch; 27 , terminal branches; 28 , two branches, one to the geniohyo-glossus and the other to the genio-hyoid muscle; 8, chorda tympani.

animals, taken in connection with the anatomical characters of the nerve, render it almost certain that its root is devoid of sensibility at its origin. All modern experimenters have confirmed the observations of Mayo and of Magendie, in regard to the sensibility of the sublingual after it has passed out of the cranial cavity. The anastomoses of this nerve with the upper two cervical nerves, with the pneumogastric, and with the lingual branch of the fifth, afford a ready explanation of this. 
The sublingual may easily be exposed in the dog by making an incision just below the border of the lower jaw, dissecting down to the carotid artery and following the vessel upward until the nerve is seen as it crosses its course. On applying a feeble faradic current at this point, there are evidences of sensibility and the tongue is moved at each stimulation.

The phenomena following section of both sublingual nerves point directly to their uses. The most notable fact observed after this operation is that the movements of the tongue are lost, while general sensibility and the sense of taste are not affected. The phenomena following division of these nerves consist simply in loss of power over the tongue, with considerable difficulty in deglutition.

In the human subject the sublingual usually is more or less affected in hemiplegia. In these cases, as the patient protrudes the tongue the point is deviated. This is due to the unopposed action of the geniohyo-glossus on the sound side, which, as it protrudes the tongue, directs the point toward the side affected with paralysis. 


\section{CHAPTER XX}

\section{TRIFACIAL NERVE-PNEUMOGASTRIC NERVE}

Trifacial (large root of the fifth nerve) - Physiological anatomy - Properties and uses of the trifacial - Immediate effects of division of the trifacial - Remote effects of division of the trifacial - Pneumogastric (tenth nerve) - Physiological anatomy - Properties and uses of the auricular nerves - Properties and uses of the pharyngeal nerves - Properties and uses of the superior laryngeal nerves - Properties and uses of the inferior, or recurrent laryngeal nerves - Properties and uses of the cardiac nerves - Depressor nerve - Properties and uses of the pulmonary nerves - Effects of division of the pneumogastrics on respiration - Effects of faradization of the pneumogastrics on respiration - Properties and uses of the cesophageal nerves - Properties and uses of the abdominal nerves - Influence of the pneumogastrics on the liver - Influence of the pneumogastrics on the stomach and intestines - Effects of faradization - Influence of section of the pneumogastrics on the movements of the stomach - Influence of the pneumogastrics on the small intestine.

\section{Trifacial (Large Root of the Fifth Nerve)}

A SINGLE nerve, the large root of the fifth pair, called the trifacial or the trigeminal, gives general sensibility to the face and to the head as far back as the vertex. This nerve is important, not only as the great sensitive nerve of the face, but on account of its connections with other nerves and its relations to the organs of special sense.

Physiological Anatomy. - The apparent origin of the large root of the fifth is from the lateral portion of the pons Varolii, at a point posterior and inferior to the origin of the small root, from which it is separated by a few transverse fibres of white substance. The deep origin is far removed from its point of emergence from the encephalon. The roots pass entirely through the substance of the pons, from without inward and from before backward, without any connection with the fibres of the pons itself. By this course the fibres reach the bulb, where the roots divide into three bundles. The anterior bundle passes from behind forward, between the anterior fibres of the pons and the cerebellar portion of the restiform bodies, to anastomose with the fibres of the auditory nerve. The other bundles, which are posterior, pass, one in the anterior wall of the fourth ventricle to the lateral tract of the bulb and the other, becoming grayish in color, to the restiform bodies, from which they may be followed as far as the point of the calamus scriptorius. A few fibres from the two sides decussate at the median 
line in the anterior wall of the fourth ventricle. From this origin the large root of the fifth passes obliquely upward and forward to the ganglion of Gasser, which is situated in a depression in the petrous portion of the temporal bone, on the internal portion of its anterior face.

The Gasserian ganglion is semilunar in form, with its concavity looking upward and inward. At the ganglion the nerve receives filaments of

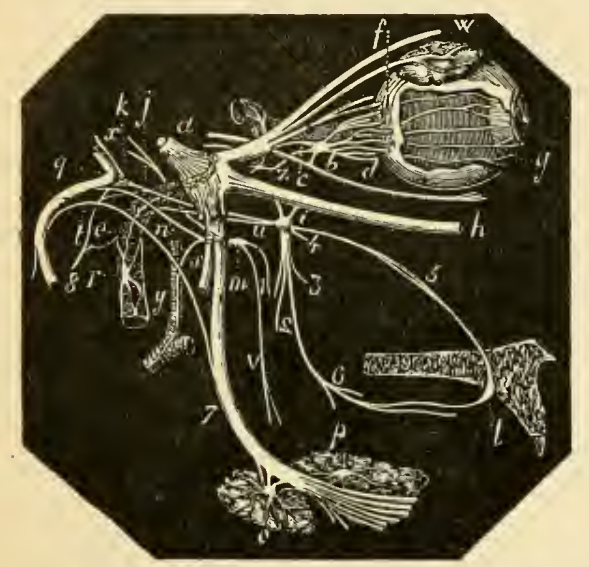

Fig. 126. - Principal branches of the large root of the fifth nerve (Robin).

$a$, ganglion of Gasser; $a-w$, ophthalmic division of the fifth; $b$, ophthalmic ganglion; $c$, branch from the ophthalmic division of the fifth to the ophthalmic ganolion; $d$, motor oculi communis; $e$, carotid; $f$. ciliary nerves; $g$, cornea and iris; $a-h$, superior maxillary division of the fifth; $i$, two branches from the superior maxillary division of the fifth to the spheno-palatine ganglion; $j$, deep petrosal nerve; $k$, filaments from the motor root of the fifth to the internal muscle of the malleus; $l$, naso-palatine ganglion; $m$, otic ganglion: $n$, small superficial petrosal nerve; $o$, branches of the fifth to the submaxillary ganglion; $p$, branches to the sublingual gland; $q$, facial nerve; $r$, sympathetic ganglion; $s$, nerve of mastication; $t$, chorda tympani, joining the lingual branch of the fifth; " Vidian nerve; $v$, branch from the motor root to the internal pterygoid muscle; w, branch of the fifth to the lachrymal gland; $x$, bend of the facial nerve; $y$, middle meningeal artery; $z$, filament from the carotid plexus to the ophthalmic ganglion; ( $I$ and 2 are not in the figure) 3 , external spheno-palatine filaments; 4, spheno-palatine ganglion; 5, naso-palatine nerve; 6, anterior palatine nerve; 7 , inferior maxillary division of the fifth; 8 , nerve of Jacobson.

rent branch which passes between the layers of the tentorium.

Just before the ophthalmic enters the orbit by the sphenoidal fissure it divides in to three branches, the lachrymal, frontal and nasal.

The lachrymal, the smallest of the three, sends a branch to the orbiplexus of the sympathetic. This is important in view of certain remote effects that follow division of the fifth nerve through the ganglion in living animals.

At the ganglion of Gasser, from its anterior and external portion, are given off a few small and unimportant branches to the dura mater and the tentorium.

From the convex border of the ganglion the three great divisions arise, which have given to the nerve the name of trifacial or trigeminal. These are: I, the ophthalmic ; 2 , the superior maxillary; 3 , the inferior maxillary. The ophthalmic and superior maxillary divisions are derived entirely from the sensory root. The inferior maxillary division joins the motor root and with it forms a mixed nerve.

The ophthalmic, the first division of the fifth, is the smallest of the three. Before it enters the orbit it receives filaments of communication from the sympathetic, sends small branches to all the motor nerves of the eyeball and gives off a small recurcommunication from the carotid 
tal branch of the superior maxillary nerve, passes through the lachrymal gland, to which certain of its filaments are distributed, and its terminal filaments go to the conjunctiva and to the integument of the upper eyelid.

The frontal branch, the largest of the three, divides into the supratrochlear and supraörbital nerves. The supratrochlear passes out of the orbit between the supraörbital foramen and the pulley of the superior oblique muscle. It sends in its course a long filament to the nasal branch and is finally lost in the integument of the forehead. The supraörbital passes through the supraörbital foramen, sends a few filaments to the upper eyelid and supplies the forehead, the anterior and the median portions of the scalp, the mucous membrane of the frontal sinus and the pericranium covering the frontal and parietal bones.

The nasal branch, before it penetrates the orbit, gives off a long filament to the ophthalmic ganglion. It then gives off the long ciliary nerves, which pass to the ciliary muscle and iris. Its trunk finally divides into the external nasal, or infratrochlearis, and the internal nasal, or ethmoidal. The infratrochlearis is distributed to the integument

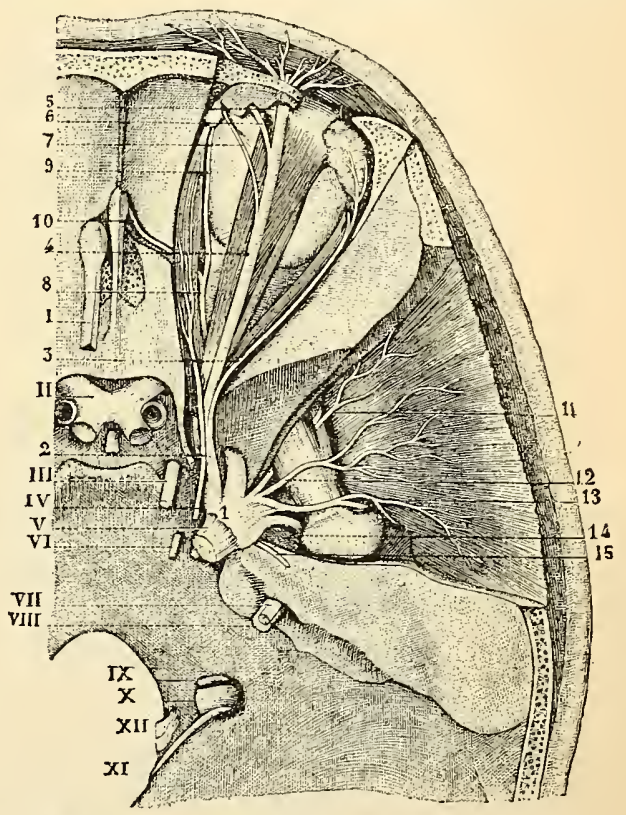

Fig. I27. - Ophthalmic division of the fifth (Hirschfeld).

I, ganglion of Gasser; 2, ophthalmic division of the fifth; 3 , lachrymal branch; 4, frontal branch; 5 , external frontal; 6 , internal frontal; 7 , supratrochlear; 8, nasal branch; 9, external nasal; I0, internal nasal; II, anterior deep temporal nerve; I2, middle deep temporal nerve; I3, posterior deep temporal nerve; $I_{4}$, origin of the superficial temporal nerve; $I_{5}$, great superficial petrous nerve. I to XII, roots of the cranial nerves. of the forehead and nose, to the internal surface of the lower eyelid, the lachrymal sac and the caruncula. The internal nasal is distributed to the mucous membrane and also in part to the integument of the nose.

The superior maxillary division of the fifth passes out of the cranial cavity by the foramen rotundum, traverses the infraörbital canal and emerges on the face by the infraörbital foramen. Branches from this nerve are given off in the spheno-maxillary fossa and the infraörbital canal before it emerges upon the face. In the spheno-maxillary fossa, 
the first branch is the orbital, which passes into the orbit, giving off one branch, the temporal, which passes through the temporal fossa by a foramen in the malar bone and is distributed to the integument on the temple and the side of the forehead. Another branch, the malar, which likewise emerges by a foramen in the malar bone, is distributed to the integument over this bone. In the spheno-maxillary fossa, are also given off two branches, which pass to the spheno-palatine, or Meckel's ganglion. From this portion of the nerve, branches are given off, the two posterior dental nerves, which are distributed to the molar and bicuspid teeth, the mucous membrane of the corresponding

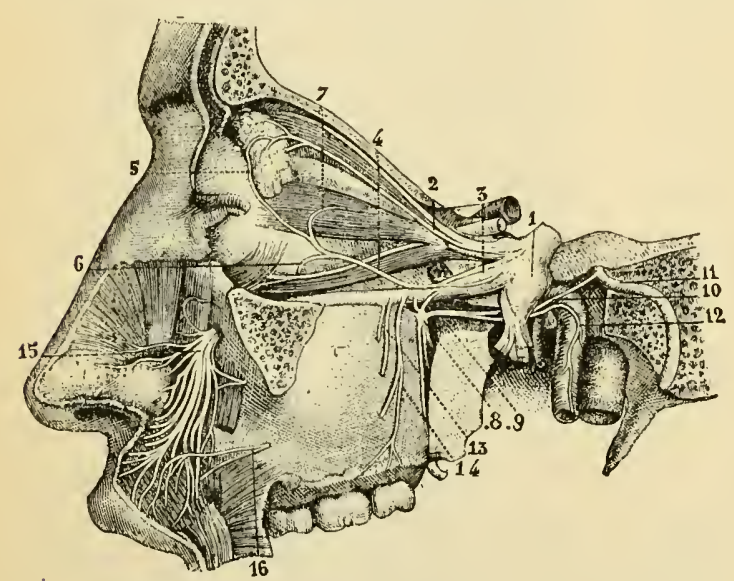

Fig. 128. - Superior maxillary division of the ffth (Hirschfeld).

I, ganglion of Gasser ; 2, lachrymal branch of the ophthalmic division; 3 , superior maxillary division of the fifth; 4 , orbital branch; 5 , lachrymo-palpebral filament; 6 , malar branch; 7 , temporal branch; 8, spheno-palatine ganglion; 9, Vidian nerve; Io, great superficial petrosal nerve; II, facial nerve; I2, branch of the Vidian nerve; 13 , anterior and two posterior dental branches; 14, branch to the mucous membrane of the alveolar processes; 15 , terminal branches of the superior maxillary division; I6, branch of the facial.

the ophthalmic, and to the integument and mucous membrane of the upper lip (the labial branches).

The inferior maxillary is a mixed nerve, composed of the inferior division of the large root and the entire small root. The distribution of the motor filaments has already been described. This nerve passes out of the cranial cavity by the foramen ovale, and then separates into the anterior division, containing nearly all the motor filaments, and the posterior division, which is chiefly sensory. The sensory portion breaks up into the following branches:-

I. The auriculo-temporal nerve supplies the integument in the temporal region, the external auditory meatus, the integument of the ear, the 
temporo-maxillary articulation and the parotid gland. It also sends branches of communication to the facial.

2. The lingual branch is distributed to the mucous membrane of the tongue as far as the point, the mucous membrane of the mouth, the gums and the sublingual gland. This nerve receives a branch from the facial (the chorda tympani) which has already been described. From

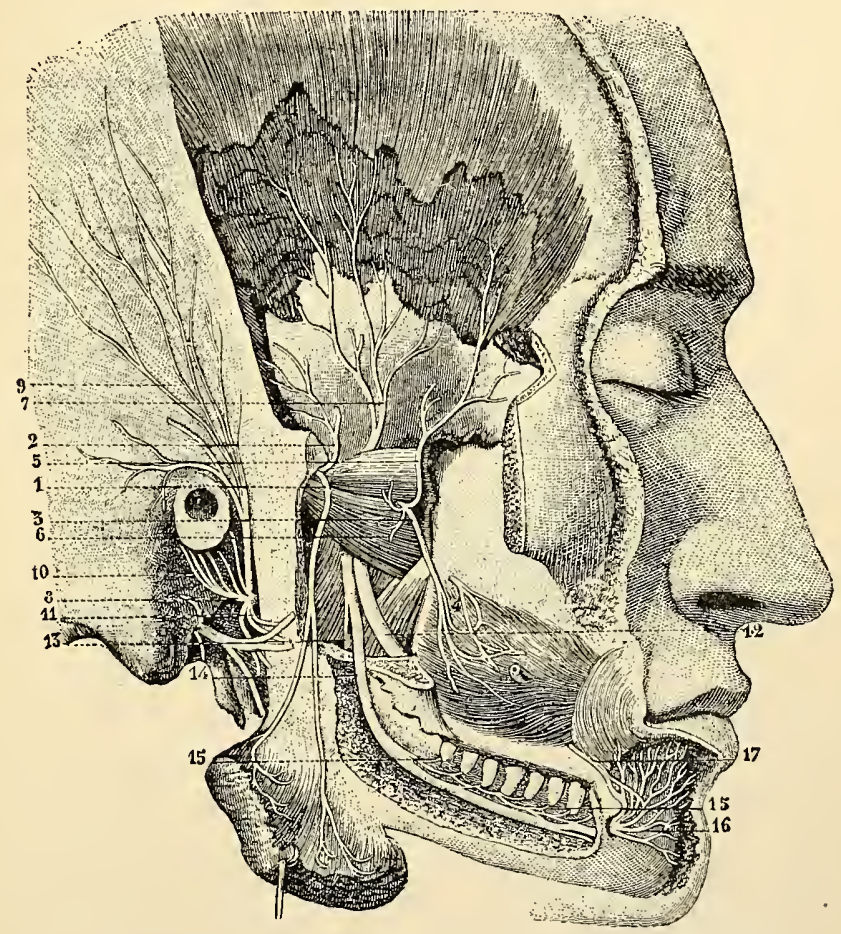

Fig. I29. - Inferior maxillary division of the fifth (Hirschfeld).

$I$, branch from the motor root to the masseter muscle; 2 , filaments from this branch to the temporal muscle ; 3 , buccal branch ; $5,6,7$, branches to the muscles; 8 , auriculo-temporal nerve; 9, temporal branches; I0, auricular branches; II, anastomosis with the facial nerve; 12, lingual branch; I3, branch of the motor root to the mylo-hyoid muscle; $\mathrm{I}_{4}, \mathrm{I}_{5}, \mathrm{I}_{5}$, inferior dental nerve, with its branches; 16, mental branch; I7, anastomosis of this branch with the facial nerve.

this nerve, also, are given off two or three branches that pass to the submaxillary ganglion.

3. The inferior dental nerve, the largest of the three, passes in the substance of the inferior maxillary bone, beneath the teeth, to the mental foramen, where it emerges upon the face. The most important sensory branches are those which supply the pulps of the teeth and the branches upon the face. The nerve emerging on the face by the mental foramen, called the mental nerve, supplies the integument of the chin, 
the lower part of the face and the lower lip. It also sends certain filaments to the mucous membrane of the mouth.

Properties and Lses of the Trifacial. - The trifacial is the great sensory nerve of the face and of the mucous membranes lining the cavities about the head. It is impossible to stimulate this nerve at its origin without seriously involving other parts; but observations in regard to the properties of the large root go to show that it is an exclusively sensory nerve, and that its sensibility is very acute as compared with other nerves. It was divided in the cranial cavity by Mayo (I822-1823), Fodéra (I 823) and Magendie (I 824). Magendie divided the nerve at its root by introducing a small cutting stylet through the skull. He succeeded in keeping the animals alive for several days or weeks and noted in his experiments immediate loss of sensibility in the face on the side on which the nerve had been divided. When this operation is performed without accident, the cornea and the integument and mucous membrane on that side of the head are deprived of sensibility and may be pricked, lacerated or burned without any evidence of pain. Almost always the small root of the fifth is divided as well as the large root, and the muscles of mastication are paralyzed on one side; but with this exception, there is no paralysis of motion, sensation alone being destroyed.

Immediate Effects of Division of the Trifacial. - This nerve has not been exposed in the cranial cavity in living animals; but its branches on the face and the lingual branch of the inferior maxillary division have been operated on and found to be exquisitely sensitive. Physiologists have exposed the roots in animals immediately after death and have found that stimulation of the large root carefully insulated produces no muscular contraction. All who have divided this root in living animals have recognized, not only that it is sensitive, but that its sensibility is far more acute than that of any other nervous trunk in the body.

So far as audition and olfaction are concerned, there are no special effects immediately following section of the trifacial; but there are certain important phenomena observed in connection with the eye and the organs of taste.

At the instant of division of the fifth, the eyeball is protruded and the pupil becomes strongly contracted. The pupil, however, usually is restored to the normal condition in a few hours. After division of the nerve, the lachrymal secretion is diminished, but this is not the cause of the subsequent inflammation, for the eyes are not inflamed, even after extirpation of both lachrymal glands.

Another of the immediate effects of complete division of the fifth nerve is loss of general sensibility in the tongue. Most experiments 
on the influence of this nerve on the general sensibility and the sense of taste in the tongue have been made by dividing the lingual branch of the inferior maxillary division. When this branch is irritated, there are evidences of intense pain. When it is divided, the general sensibility and the sense of taste are destroyed in the anterior portion of the tongue. It will be remembered, however, that the chorda tympani joins the lingual branch of the fifth as it passes between the pterygoid muscles, and that section of this branch of the facial abolishes the sense of taste in the anterior two-thirds of the tongue. If the gustatory properties of the lingual branch of the fifth be derived from the chorda tympani, lesions of the fifth not involving this nerve would be followed by loss of general sensibility, but the taste would be unaffected. This has been shown to be the fact, by cases of paralysis of general sensibility of the tongue without loss of taste in the human subject, which will be discussed more fully in connection with the physiology of gustation.

Among the immediate effects of section of the fifth, is interference with the reflex acts of deglutition. After section of the superior laryngeal branches of the pneumogastrics, no movements of deglutition follow stimulation of the mucous membrane of the top of the larynx. When the fifth is divided on one side, stimulation of the velum on the corresponding side has no effect, while movements of deglutition are produced by irritating the velum on the sound side. These experiments show that the fifth nerve is important in the reflex phenomena of deglutition as a sensory nerve, conveying the impression from the velum palati to the nerve-centres. This action probably takes place through filaments that pass from the fifth to the mucous membrane through Meckel's ganglion.

Remote Effects of Division of the Trifacial. - After section of the fifth nerve in the cranial cavity, the immediate loss of sensibility of the integument and mucous membranes of the face and head usually is supplemented with serious disturbances in the nutrition of the eye, the ear and the mucous membranes of the nose and mouth. After a period varying between a few hours and one or two days following the operation, the eye on the affected side becomes the seat of purulent inflammation, the cornea becomes opaque and ulcerates, the humors are discharged and the organ is destroyed. Congestion of the parts usually is very prominent a few hours after division of the nerve. At the same time there is an increased discharge from the mucous membranes of the nose and mouth on the affected side, and ulcers appear on the tongue and lips. It is probable, also, that disorders in the nutrition of the auditory apparatus follow the operation, although these are not so prominent. Animals affected in this way usually die in fifteen to twenty days. 
In the early experiments of Magendie, it was noted that "the alterations in nutrition are much less marked" when the division is effected behind the ganglion of Gasser than when it is done in the ordinary way through the ganglion. It is difficult enough to divide the nerve completely, within the cranium, and is almost impossible to make the operation at will through or behind the ganglion; and the phenomena of inflammation are absent only in exceptional and accidental instances. Magendie offered no satisfactory explanation of these differences in the consecutive phenomena, but the facts have been repeatedly verified. In a number of experiments in which the nerve was divided in the cranial cavity (Flint), the consecutive inflammatory effects were nearly always observed; but in an experiment made in 1868 , the nerve was completely divided on the left side, as was shown by total loss of sensibility of the parts to which it is distributed, and the animal (a rabbit) lived nearly four months. Four days after the operation the loss of sensibility was still complete. There was very little redness of the conjunctiva of the left eye, and a slight streak of opacity, so slight that it was distinguished with some difficulty. Twelve days after the operation, the sensibility of the left eye was distinct but slight. There was no redness of the conjunctiva, and the opacity of the cornea had disappeared. The animal was in good condition, and the line of contact of the upper with the lower incisors, when the jaws were closed, was very oblique. The animal was kept alive by careful feeding with bread and milk for one hundred and seven days after the operation, and there was no inflammation of the organs of special sense. It died at that time of inanition, having become extremely emaciated. The animal never recovered power over the muscles of the left side, and the incisors grew to a great length, seriously interfering with mastication.

The explanation of the phenomena of disordered nutrition in the organs of special sense, particularly the eye, following division of the fifth, is not afforded by the section of this nerve alone; for when the loss of sensibility is complete after division of the nerve behind the Gasserian ganglion, these results may not follow. They are not explained by deficiency in the lachrymal secretion; for they are not observed when both lachrymal glands have been extirpated. They are not due simply to an enfeebled general condition; for in the experiment just detailed, the animal died of inanition, after section of the nerve, without any evidences of inflammation. In view of the fact that section of the sympathetic filaments is well known to modify the nutrition of parts to which they are distributed, producing congestion and increase in temperature, it is rational to infer that the modifications in nutrition which follow section of the fifth after it receives filaments from the 
sympathetic system, not occurring when these sympathetic filaments escape division, are to be attributed to lesion of the sympathetic and not to the division of the sensory nerve itself.

The following explains, in a measure at least, the consecutive inflammatory effects of. section of the fifth with its communicating sympathetic filaments: By dividing the sympathetic, the eye and the mucous membranes of the nose, mouth and ear are rendered hyperemic, the temperature is raised and the processes of nutrition are exaggerated. This condition of the parts would seem to require a full supply of nutritive material from the blood in order to maintain the condition of exaggerated nutrition; but when the blood is impoverished - probably as the result of deficiency in the introduction of nutritive matter, from paralysis of the muscles of mastication on one side - the nutritive processes in these delicate parts are seriously modified, so as to constitute inflammation. It has been observed, however, that inflammation of the eye does not follow section of the fifth when the part is protected from external irritation, as by serving together the eyelids. It must be, as it seems, that the delicate structures of the organs of special sense, especially vision, are rendered vulnerable by the loss of sensibility and the hyperemia, the congestion following this operation readily passing into inflammation. Under these conditions, when the eye is completely protected from irritation, inflammation may not occur.

Pathological facts in confirmation of experiments on the fifth pair in the lower animals are not wanting; but it must be remembered that in cases of paralysis of the nerve in the human subject, it is not always possible to locate exactly the seat of the lesion and to appreciate fully its extent, as can be done when the nerve is divided by an operation. In these cases it sometimes occurs that the phenomena, particularly those of modified nutrition, are more or less contradictory.

Cases of paralysis of the fifth in the human subject in the main confirm the results of experiments on the inferior animals. In cases in which the fifth nerve alone is involved in the disease, without the facial, there is simply loss of sensibility on one side, the movements of the superficial muscles of the face being unaffected. When the small root is involved, the muscles of mastication on one side are paralyzed; but in certain reported cases in which this root escaped, there was no muscular paralysis. The senses of sight, hearing and smell, except as they are affected by consecutive inflammation, are little if at all disturbed in uncomplicated cases. The sense of taste in the anterior portion of the tongue is perfect, except in those cases in which the facial, the chorda tympani or the lingual branch of the fifth after it had been joined by the chorda tympani is involved in the disease. In some cases 
there is no alteration in the nutrition of the organs of special sense ; but in this respect the facts in regard to the seat of the lesion are not so satisfactory as in experiments on the lower animals, it being difficult to limit exactly the boundaries of the lesion.

\section{Pneumogastric (Tenth Nerve)}

Of all the cranial nerves, the pneumogastric presents the greatest number of anastomoses, the most remarkable course and the most varied uses. Arising from the bulb by a purely sensory root, it communicates with at least five motor nerves and is distributed largely to muscular tissue, both of the voluntary and the involuntary variety. On account of its wide distribution and wandering course, it is often called the vagus or par vagum.

Physiological Anatomy. - The apparent origin of the pneumogastric is from the lateral portion of the bulb, just behind the olivary body, between the roots of the glosso-pharyngeal and the spinal accessory. The deep origin is mainly from what is called the nucleus of the pneumogastric, at the inferior portion of the gray substance in the floor of the fourth ventricle. The course of the fibres, traced from without inward, is somewhat intricate.

The deep origins of the pneumogastric and glosso-pharyngeal nerves appear to be in the main identical. Tracing the filaments from without inward, they may be followed in four directions: (I) The anterior filaments pass from without inward, first very superficially, in the direction of the olivary body; but they then turn and pass deeply into the substance of the restiform body, in which they are lost. (2) The posterior filaments are superficial, and they pass, with the fibres of the restiform body, toward the cerebellum. (3) Of the intermediate filaments, the anterior pass through the restiform body, the greatest number extending to the median line, in the floor of the fourth ventricle. A few fibres are lost in the middle fasciculi of the bulb and a few pass toward the brain. (4) The posterior intermediate filaments traverse the restiform body to the floor of the fourth ventricle, where some pass to the median line and others descend in the substance of the bulb. It is difficult to follow the fibres of origin of the pneumogastrics beyond the median line; but recent observations leave no doubt of the fact that many of these fibres decussate in the floor of the fourth ventricle.

There are two ganglionic enlargements belonging to the pneumogastric. In the jugular foramen, is a well-marked, grayish, ovoid enlargement, one-sixth to one-fourth of an inch (4.2 to 6.4 millimeters) in length, called the jugular ganglion, or the ganglion of the root. This 
is united by two or three filaments with the ganglion of the glossopharyngeal. It is a true ganglion, containing nerve-cells. After the nerve has emerged from the cranial cavity, it presents on its trunk another grayish enlargement, half an inch to an inch ( 12 to 25 millimeters) in length, called the ganglion of the trunk. This has a plexiform structure, the white fibres being mixed with grayish fibres and nerve-cells. The exit of the nerve from the cranial cavity is by the jugular foramen, or posterior foramen lacerum, in company with the spinal accessory, the glosso-pharyngeal nerve and the internal jugular vein.

Anastomoses. - There are occasional filaments of communication that pass from the spinal accessory to the ganglion of the root of the pneumogastric, but these are not constant. After both nerves have emerged from the cranial cavity, an important branch of considerable size passes from the spinal accessory to the pneumogastric, with which it becomes closely united. Experiments have shown that these filaments from the spinal accessory pass in great part to the larynx by the inferior laryngeal nerves.

In the aquaductus Fallopii, the facial nerve gives off a filament of communication to the pneumogastric, at the ganglion of the root. This filament, joined at the ganglion by sensory filaments from the pneumogastric and some filaments from the glosso-pharyngeal, is called the auricular branch of Arnold. By some

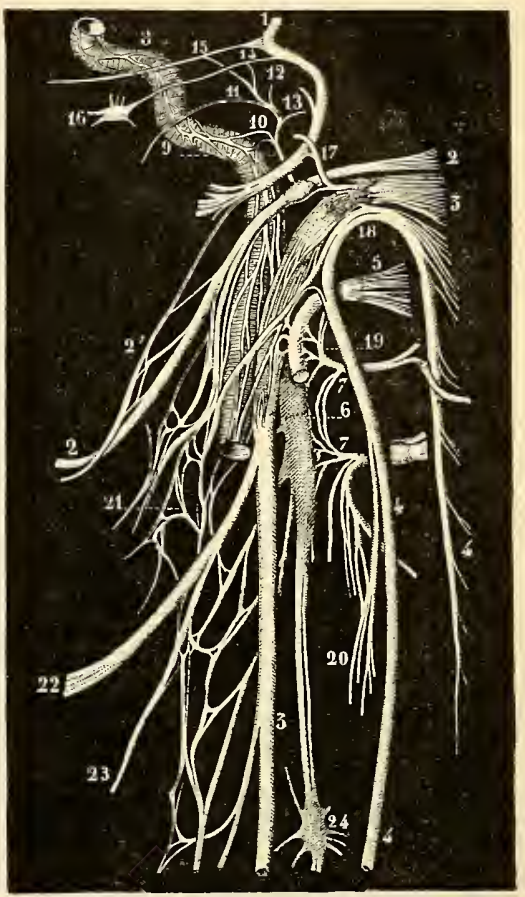

Fig. I30. - Anastomoses of the pneumogastric (Hirschfeld).

I, facial nerve ; 2 , glosso-pharyngeal nerve; 2 ', anastomoses of the glosso-pharyngeal with the facial; 3,3 , pneumngastric, with its two ganglia; 4, 4, spinal accessory; 5, sublingual nerve; 6 , superior cervical ganglion of the sympathetic; 7 , anastomotic arcade of the first two cervical nerves; 8 , carotid branch of the superior cervical ganglion of the sympathetic ; 9. nerve of Jacobson; Io, branches of this nerve to the sympathetic; II, branch to the Eustachian tube; 12, branch to the fenestra ovalis; 13, branch to the fenestra rotunda; $I_{4}$, external deep petrous nerve; I $_{5}$, internal deep petrous nerve; 16 , otic ganglion; 17 , auricular branch of the pneumogastric ; 18 , anastomosis of the pnenmogastric with the spinal accessory; I9, anastomosis of the pneumogastric with the sublingual; 20 , anastomosis of the spinal accessory with the second pair of cervical nerves; 2I, pharyngeal plexus; 22, superior laryngeal nerve; 23 , external laryngeal nerve; 24 , middle cervical ganglion. anatomists it is regarded as a branch of the facial and by others it is described with the pneumogastric. 
Two or three small filaments of communication pass from the sublingual to the ganglion of the trunk of the pneumogastric.

At the ganglion of the trunk, the pneumogastric usually receives filaments of communication from the arcade formed by the anterior branches of the first two cervical nerves. These, however, are not constant.

The pneumogastric is connected with the sympathetic system by a number of filaments of communication from the superior cervical ganglion, passing in part upward toward the ganglion of the root of the pneumogastric, and in part transversely and downward. These filaments frequently are short, and they bind the sympathetic ganglion to the trunk of the nerve. The main trunk of the pneumogastric and its branches receive a few filaments of communication from the middle and inferior cervical and the upper dorsal ganglia of the sympathetic.

The pneumogastric frequently sends a slender filament to the glosso-pharyngeal nerve, at or near the ganglion of Andersch. Branches from the pneumogastric join branches from the glossopharyngeal, the spinal accessory and the sympathetic, to form the pharyngeal plexus.

Distribution. - Although the pneumogastric nerves on the two sides do not present any important differences in the destination of their filaments as far down as the diaphragm, the distribution of the abdominal branches is not the same. The most important branches are the following:-

1. Auricular.

2. Pharyngeal.

3. Superior laryngeal.

4. Inferior, or recurrent laryngeal.
5. Cardiac (cervical and thoracic).

6. Pulmonary (anterior and posterior).

7. Esophageal.

8. Abdominal.

The auricular nerves are sometimes described in connection with the facial. They are given off from the ganglion of the trunk of the pneumogastric and are composed of filaments of communication from the facial and from the glosso-pharyngeal as well as of filaments from the pneumogastric itself. The nerves thus constituted are distributed to the integument of the upper portion of the external auditory meatus and a small filament is sent to the membrana tympani.

The pharyngeal nerves are given off from the superior portion of the ganglion of the trunk, and they contain a large number of the filaments of communication which the pneumogastric receives from the spinal accessory. In their course by the sides of the superior constrictor muscles of the pharynx, these nerves anastomose with filaments from 
the glosso-pharyngeal and the superior cervical ganglion of the sympathetic, to form what is known as the pharyngeal plexus. The ultimate filaments of distribution pass to the muscles and the mucous membrane

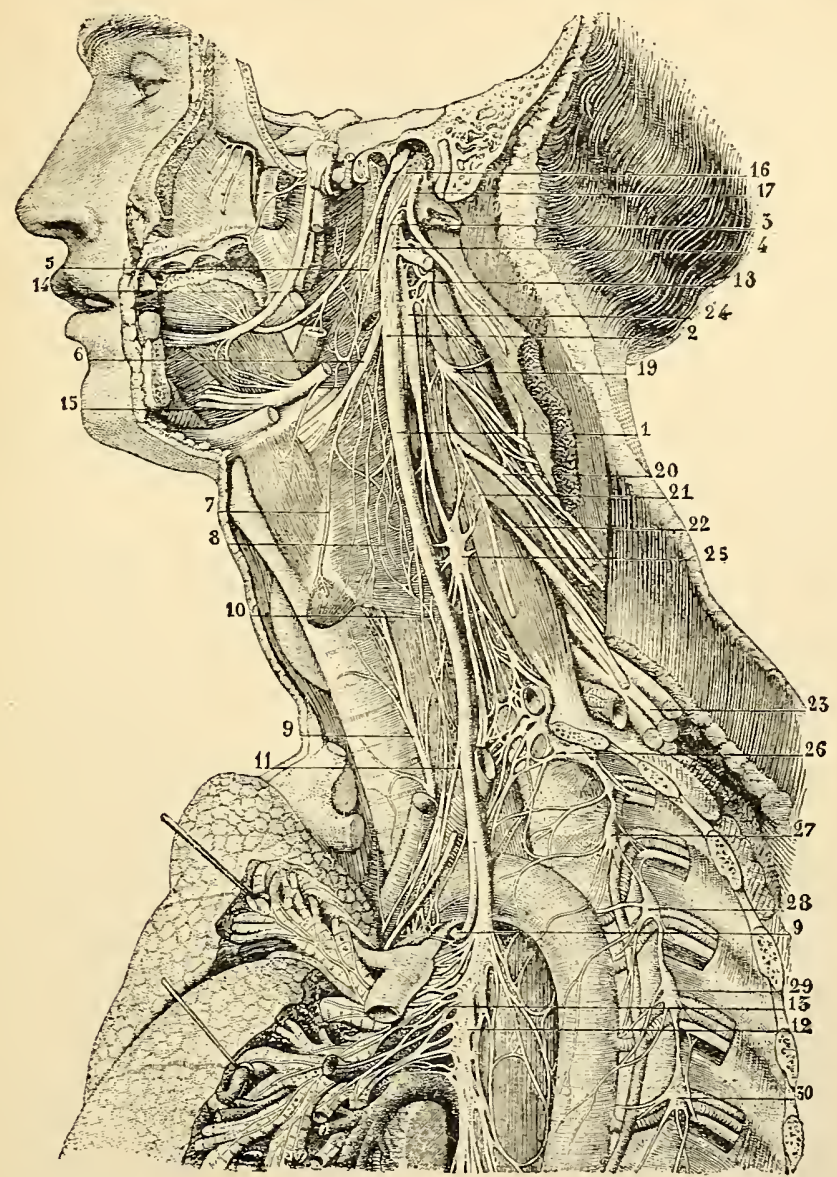

Fig. 131. - Distribution of the pneumogastric (Hirschfeld).

I, trunk of the left pneumogastric; 2, ganglion of the trunk; 3, anastomosis with the spinal accessory; 4 , anastomosis with the sublingual; 5, pharvngeal branch (the auricular branch is not shown in the figure); 6, superior laryngeal branch; 7, external laryngeal nerve; 8 , laryngeal plexus; 9,9 , inferior laryngeal branch; IO, cervical cardiac branch; II, thoracic cardiac branch; I2, I3, pulmonany branckes; I4, lingual branch of the fifth; $\mathrm{I}_{5}$, lower portion of the sublingual; I6, glosso-pharyngeal; I7, spinal accessory; I8, I9, 20, spinal nerves; 2I, phrenic nerve; 22, 23, spinal nerves; $24,25,26,27$, $28,29,30$, sympathetic ganglia.

of the pharynx. Physiological experiments have shown that the motor influence transmitted to the pharyngeal muscles through the pharyngeal branches of the pneumogastric is derived from the spinal accessory.

The superior laryngeal nerves are given off from the lower part of 
the ganglion of the trunk. Their filaments come from the side opposite the point of junction of the pneumogastric with the communicating branch from the spinal accessory, so that probably the superior laryngeals contain few if any motor fibres from the eleventh nerve. The superior laryngeal gives off the external laryngeal, a long, delicate branch which sends a few filaments to the inferior constrictor of the pharynx and is distributed to the crico-thyroid muscle and the mucous membrane of the ventricle of the larynx. The external laryngeal anastomoses with the inferior laryngeal nerve and with the sympathetic. The internal branch is distributed to the mucous membrane of the epiglottis, the base of the tongue, the aryteno-epiglottidean fold and the mucous membrane of the larynx as far down as the true vocal chords. A branch from this nerve, in its course to the larynx, penetrates the arytenoid muscle, to which it sends a few filaments, but these are sensory. This branch also supplies the crico-thyroid muscle. It anastomoses with the inferior laryngeal nerve. An important branch, described by Cyon and Ludwig, in the rabbit, under the name of the depressor nerve, arises by two roots, one from the superior laryngeal and the other from the trunk of the pneumogastric. It passes down the neck by the side of the sympathetic, and in the chest it joins filaments from the thoracic sympathetic, to pass to the heart, between the aorta and the pulmonary artery. This nerve is not isolated in the human subject, but it is probable that analogous fibres exist in man in the trunk of the pneumogastric.

It is important from a physiological point of view to note that the superior laryngeal nerve is the nerve of sensibility of the upper part of the larynx as well as of the supralaryngeal mucous membranes, and that it animates a single muscle of the larynx (the crico-thyroid) and the inferior constrictor of the pharynx.

The inferior, or recurrent laryngeal nerves present some slight differences in their anatomy on the two sides. On the left side the nerve is the larger and is given off at the arch of the aorta. Passing beneath this vessel, it ascends in the groove between the trachea and the osophagus. In its upward course it gives off filaments that join the cardiac branches, filaments to the muscular tissue and mucous membrane of the upper part of the œesophagus, filaments to the mucous membrane and the intercartilaginous muscular tissue of the trachea, one or two filaments to the inferior constrictor of the pharynx and a branch that joins the superior laryngeal. Its terminal branches penetrate the larynx behind the posterior articulation of the thyroid with the cricoid cartilage and are clistributed to all the intrinsic muscles of the larynx, except the crico-thyroids, which latter are supplied by the superior laryngeal. On the right side the nerve winds from before backward around the sub- 
clavian artery and has essentially the same course and distribution as on the left side, except that it is smaller and has fewer filaments of distribution.

The important physiological point connected with the anatomy of the recurrent laryngeals is that they animate all the intrinsic muscles of the larynx except the crico-thyroid. Experiments have shown that these nerves contain a large number of motor filaments derived from the spinal accessory.

The cervical cardiac branches, two or three in number, arise from the pneumogastrics at different points in the cervical portion and pass to the cardiac plexus, which is formed in great part of filaments from the sympathetic. The thoracic cardiac branches are given off from the pneumogastrics below the origin of the inferior laryngeals and join the cardiac plexus.

The anterior pulmonary branches are few and delicate as compared with the posterior branches. They are given off below the origin of the thoracic cardiac branches, send a few filaments to the trachea and then form a plexus that surrounds the bronchial tubes and follows the bronchial tree to its terminations in the air-cells. The posterior pulmonary branches are larger and greater in number than the anterior. They communicate freely with sympathetic filaments from the upper three or four thoracic ganglia and then form the great posterior pulmonary plexus. From this plexus a few filaments go to the inferior and posterior portion of the trachea, a few pass to the muscular tissue and mucous membrane of the middle portion of the œsophagus and a few are sent to the posterior and superior portion of the pericardium. The plexus then surrounds the bronchial tree and passes with its ramifications to the pulmonary tissue, like the corresponding filaments of the anterior branches. The pulmonary branches are distributed to the mucous membrane and not to the walls of the bloodvessels.

The œsophageal branches take their origin from the pneumogastrics above and below the pulmonary branches. These branches from the two sides join to form the osophageal plexus, their filaments of distribution going to the muscular tissue and the mucous membrane of the lower third of the œsophagus.

The abdominal branches are quite different in their distribution on the two sides.

On the left side the nerve, which is here anterior to the cardiac opening of the stomach, immediately after its passage by the side of the œsophagus into the abdomen, divides into a number of branches, which are distributed to the muscular walls and the mucous membrane of the stomach. As the branches pass from the lesser curvature, they take 
a downward direction and go to the liver, and with another branch running between the folds of the gastro-hepatic omentum, they follow the course of the portal vein in the hepatic substance. The branches of this nerve anastomose with the nerve of the right side and with the sympathetic.

The right pneumogastric, situated posteriorly, at the œsophageal opening of the diaphragm sends a few filaments to the muscular coat and the mucous membrane of the stomach, passes backward and is distributed to the liver, spleen, kidneys, suprarenal capsules and finally to the entire small intestine. From the splenic plexus, filaments derived from the pneumogastric go to the pancreas. Before the nerves pass to the intestines, there is a free anastomosis and interchange of filaments between the right and the left abdominal branches.

General Properties of the Roots of Origin of the Pneumogastrics. The sensibility of the pneumogastrics in the neck, while it is dull as compared with the properties of other sensory nerves, is nevertheless distinct. It is impossible, however, to expose the roots of the nerves in living animals before they have received communicating motor filaments, without such mutilation as would interfere with accurate observations; but in animals just killed, if the roots are exposed and divided so as to avoid reflex movements, and if care is taken to avoid stimulation of motor filaments from adjacent nerves, it is found that the application of electricity to the peripheral end of the root from its origin to the ganglion gives rise to no movements. It may therefore be assumed that the true filaments of origin of the pneumogastrics are exclusively sensory, or at least that they have no motor properties.

Properties and Uses of the Auricular Nerves. - The auricular nerves are sometimes described with the facial and sometimes with the pneumogastric. They contain filaments from the facial, the pneumogastric and the glosso-pharyngeal. The sensory filaments of these nerves give sensibility to the upper part of the external auditory meatus and to the membrana tympani. The meatus also receives filaments from the trifacial.

Properties and Uses of the Pharyngeal Nerves. - The pharyngeal branches of the pneumogastric are mixed nerves, their motor filaments being derived from the spinal accessory; and their direct action on the muscles of deglutition belongs to the physiological history of the lastnamed nerve. As already stated in treating of the spinal accessory, the filaments of communication that go to the pharyngeal branches of the pneumogastric are distributed to the pharyngeal muscles.

It is impossible to divide all the pharyngeal filaments in living animals and observe directly how far the general sensibility of the pharynx 
and the reflex phenomena of deglutition are influenced by this section. So far as one can judge from the distribution of the filaments to the mucous membrane, it would seem that they combine with the pharyngeal filaments of the fifth, and possibly with sensory filaments from the glosso-pharyngeal, in giving general sensibility to these parts.

In experiments on the reflex phenomena of deglutition, it has been shown that the action of the pharyngeal muscles can not be excited by stimulation of the mucous membrane of the supralaryngeal region and the pharynx after section of the fifth and of the superior laryngeal branches of the pneumogastrics. This would seem to indicate that the pharyngeal branches of the pneumogastrics are of little importance in these reflex phenomena.

Properties and Uses of the Superior Laryngeal Nerves. - The stimulation of these nerves produces intense pain and contraction of the crico-thyroids; but it has been shown by experiment that the arytenoid muscles, through which the nerves pass, receive no motor filaments. The influence. of the nerves on the muscles resolves itself into the action of the crico-thyroids, which has been treated of fully under the head of phonation. When these muscles are paralyzed the voice becomes hoarse. The filaments to the inferior muscles of the pharynx are few and comparatively unimportant. The superior laryngeals do not receive their motor supply from the spinal accessory.

The sensory filaments of the superior laryngeals have important uses connected with the protection of the air-passages from the entrance of foreign matters, particularly in deglutition; and they also are concerned in the reflex action of the constrictors of the pharynx. When both superior laryngeals have been divided in living animals, liquids often pass in small quantity into the larynx, owing to the absence of the reflex closure of the glottis when foreign matters are brought in contact with its superior surface, and the occasional occurrence of inspiration during deglutition.

Aside from the protection of the air-passages, the superior laryngeal is one of the sensory nerves through which the reflex acts in deglutition operate. There are certain parts that depend for their sensibility entirely on this nerve; namely, the mucous membrane of the epiglottis, of the aryteno-epiglottidean fold and of the larynx as far down as the true vocal chords. When an impression is made on these parts, as when they are touched with a piece of meat, regular and natural movements of deglutition ensue.

If the superior laryngeal nerves are divided and a stimulus is applied to their central ends, movements of deglutition are observed, and there also is arrest of the action of the diaphragm. From these experiments, 
it would seem that the impression which gives rise to the movements of deglutition aids in protecting the air-passages from the entrance of foreign matters by temporarily arresting the inspiratory act.

Properties and Uses of the Inferior, or Recurrent Laryngeal Nerves. The anatomical distribution of these nerves shows that their most important action is connected with the muscles of the larynx. The few filaments given off in the neck to join the cardiac branches are probably not very important. It is proper to note, however, that the inferior laryngeal nerves supply the muscular tissue and mucous membrane of the upper part of the œesophagus and trachea, and one or two branches are sent to the inferior constrictor of the pharynx. The action of these filaments is sufficiently evident.

The inferior laryngeals contain chiefly motor filaments, as is evident from their distribution as well as from the effects of direct stimulation. All who have experimented on these nerves have noted little or no evidence of pain when they are irritated or divided.

One of the most important uses of the recurrents relates to the production of vocal sounds. In connection with the physiology of the internal, or communicating branch from the spinal accessory to the pneumogastric, it has been shown that this branch is the true nerve of phonation. Before the uses of the spinal accessory were fully understood, experiments on the inferior laryngeals led to the opinion that these were the nerves of phonation, as loss of voice follows their division in living animals. It is true that these nerves contain the filaments which preside over the vocal movements of the larynx; but it is also the fact that these vocal filaments are derived from the spinal accessory, and that the recurrents contain as well motor filaments which preside over movements of the larynx not concerned in the production of vocal sounds.

The muscles of the larynx concerned in phonation are the crico-thyroids, animated by the superior laryngeals, and the arytenoid, the lateral crico-arytenoids and the thyro-arytenoids, animated by the inferior laryngeals. The posterior crico-arytenoids are respiratory muscles, and these are not affected by extirpation of the spinal accessories, but the glottis is still capable of dilatation, so that inspiration is not impeded. If, however, the spinal accessories are extirpated and the larynx is then exposed in a living animal, the glottis remains dilated, but will not close when irritated. If the inferior laryngeals are then divided, the glottis is mechanically closed with the inspiratory act, and the animals often die of suffocation. In view of the varied sources from which the pneumogastrics receive their motor filaments, it is easy to understand how certain of these may preside over the vocal movements, and others, from a different source, may animate the respiratory movements of the glottis. 
The obstruction to the entrance of air into the lungs is a sufficient explanation of the increase in the number of the respiratory acts after division of both recurrents. The acceleration of respiration is much greater in young than in adult animals. This does not apply to very young animals, in which section of the recurrents produces almost instant death.

Feeble stimulation of the central ends of the inferior laryngeals, after their division, produces rhythmical movements of deglutition, coincident usually with arrest of the action of the diaphragm. These phenomena are commonly observed in rabbits, but they are not constant. The reflex action of these nerves in deglutition probably is dependent on the communicating filaments which they send to the superior laryngeal nerves.

Properties and Uses of the Cardiac Nerves. - The chief uses of the cardiac branches relate to the influence of the pneumogastrics on the action of the heart. This has already been considered in connection with the physiology of the circulation. The effect of dividing the pneumogastrics in the neck is to remove the heart from the influence of its inhibitory nerves; but at the same time the operation profoundly affects the respiratory movements, and this latter effect must be eliminated so far as possible in studying the influence of the pneumogastrics on the circulation. The same remark applies to the experiment of faradization of the pneumogastrics in the neck. The cardiac branches are operated on with difficulty, and most experiments have been made on the cervical portion of the pneumogastric itself.

Faradization of the pneumogastrics in the neck arrests the action of the heart in diastole. This is a direct action and is due to the excitation of the inhibitory fibres, which are derived from the spinal accessory nerves. The phenomena following stimulation of these nerves have already been described in connection with the physiology of the circulation and the properties and uses of the spinal accessories.

Depressor Nerve. - While this nerve is not isolated in the human subject, it is probable that fibres, the action of which is analogous to that observed in animals in which the nerve is anatomically distinct, exist in the trunk of the pneumogastric. The action of the depressor nerves, which is reflex, has already been described in connection with the physiology of the circulation.

Properties and Uses of the Pulmonary Nerves. - The trachea, bronchia and the pulmonary structure are supplied with motor and sensory filaments by branches of the pneumogastrics. The recurrent laryngeals supply the upper part, and the pulmonary branches, the lower part of the trachea, the lungs themselves being supplied by the pulmo- 
nary branches alone. The sensibility of the mucous membrane of the trachea and bronchia is due to the pneumogastrics; and these parts are insensible to irritation when the nerves have been divided in the neck.

Effects of Division of the Pnenmogastrics on Respiration. - Section of both pneumogastrics in the neck usually is followed by death in two to five days. In very young animals, death may occur almost instantly from paralysis of the respiratory movements of the glottis. It has been found by all experimenters that animals survived and presented no very distinct abnormal phenomena after section of one nerve. Animals operated on in this way present hoarseness of the voice and a slight increase in the number of respiratory acts. Some observers have found the corresponding lung partly emphysematous and partly engorged with blood, and others have not noted any change in the pulmonary structure.

When both nerves are divided in full-grown dogs, the effect on the respiratory movements is very marked. For a few seconds the number of respiratory acts may be increased; but so soon as the animal becomes tranquil, the number is very much diminished and the movements change their character. The inspiratory acts become unusually profound and are attended with excessive dilatation of the thorax. The animal usually is quiet and indisposed to move. Under these conditions the number of respirations may fall from sixteen or eighteen to four per minute.

In most animals that die from section of both pneumogastrics, the lungs are found engorged with blood, and, as it were, carnified, so that they sink in water. This condition probably is not the result of inflammation of the pulmonary parenchyma, although this was the view formerly entertained, and it is now held by some physiologists. Bernard found that the pulmonary lesion did not exist in birds, although section of both nerves was fatal. It had previously been ascertained that in some animals death takes place with no alteration of the lungs. When the entrance of the secretions into the air-passages is prevented by the introduction of a canula into the trachea, solidification of the lungs is nevertheless observed. Those who regard the condition as inflammatory call it a "vagus pneumonia," due to division of "trophic" fibres of the pneumogastrics; but the mechanism and immediate causes of the pulmonary changes are obscure and have not been satisfactorily explained.

The pneumogastrics sometimes reunite after division. The following observation (Flint, I 874) illustrates this fact, which has frequently been noted: Both pneumogastrics were divided in the neck in a mediumsized dog. The pulse was immediately increased from one hundred and 
twenty to two hundred and forty in the minute, and the number of respirations fell from twenty-four to four or six. In ten days the pulse and respirations had become normal. The dog was then killed by section of the medulla oblongata and reunion of the divided ends of the nerves was found to be nearly complete.

Effects of Faradization of the Pnennogastrics on Respiration. - Faradization of the pneumogastrics in the neck, if the current is sufficiently powerful, arrests respiration. This arrest may be produced at any time with reference to the respiratory act, either in expiration or inspiration, although it is more readily effected in expiration. During the passage of the current, the general movements of the animal also are arrested. Although respiration may always be arrested in this way, quite a powerful current is required. During the passage of a very feeble current, the respirations are accelerated. They are then retarded as the current is made stronger, until they finally cease.

The following are the phenomena, observed by Bert, during the passage of a powerful faradic current:-

"If an excitation is employed sufficiently powerful to arrest respiration in inspiration, all respiratory movements may be made to cease at the very moment when the excitation is applied (inspiration, halfinspiration, expiration), either by operating on the pneumogastric, or operating on the laryngeal. . .

"Any feeble excitation of centripetal nerves increases the number of the respiratory movements; any powerful excitation diminishes them. A powerful excitation of the pneumogastrics, of the superior laryngeal, of the nasal branch of the infraörbital, may arrest them completely; if the excitation is sufficiently energetic, the arrest takes place at the very moment it is applied. Finally, sudden death of the animal may follow a too powerful impression thus transmitted to the respiratory centre: all this being true for certain mammalia, birds and reptiles."

The above expresses the most important experimental facts at present known in regard to the influence of stimulation of the pneumogastrics on respiration. The pulmonary branches themselves are so deeply situated that they have not as yet been made the subject of direct experiment, with any positive and satisfactory results. The pneumogastrics undoubtedly are the nerves concerned in the reflex acts of normal respiration. Their relations to these movements, however, will be considered in connection with the action of the respiratory nerve-centres.

Properties and Uses of the Esophageal Nerves. - The muscular walls and the mucous membrane of the œsophagus are supplied entirely by branches from the pneumogastrics. The upper portion is supplied 
by filaments from the inferior laryngeal branches, the middle portion, by filaments from the posterior pulmonary branches, and the inferior portion receives the œesophageal branches. These branches are both sensory and motor; but probably the motor filaments largely predominate, for the mucous membrane, although it is sensible to the extremes of heat and cold, the feeling of distention, and a burning sensation on the application of strong irritants, is not acutely sensitive.

That the movements of the esophagus are animated by branches from the pneumogastrics, has been clearly shown by experiments. In the first place, except in animals in which the anatomical distribution of the nerves is different from the arrangement in the human subject, the entire œesophagus is paralyzed by division of the nerves in the neck. When the pneumogastrics are divided in the cervical region in dogs, if the animals attempt to swallow a considerable quantity of food, the upper part of the œesophagus is found enormously distended. Bernard noted in a dog in which a gastric fistula had been established, that articles of food given to the animal did not pass into the stomach, although he made efforts to swallow. An instant after the attempt, the matters were regurgitated, mixed with mucus, but of course did not come from the stomach.

Direct experiments on the roots of the pneumogastrics have shown that these nerves influence the movements of the oesophagus, and that the motor filaments involved do not come from the spinal accessories; but it is not known from what nerves these motor filaments are derived.

Properties and Uses of the Abdominal Nerves. - In view of the extensive distribution of the terminal branches of the pneumogastrics to the abdominal organs, it is evident that the action of these nerves must be important, particularly since it has been shown that the right nerve is distributed to the entire small intestine.

Influence of the Pneumogastrics on the Liver. - There is very little known in regard to the influence of the pneumogastrics on the secretion of bile; and the most important experiments on the innervation of the liver relate to the production of glycogen. If both pneumogastrics are divided in the neck, and if the aninal is killed at a time varying between a few hours and one or two days after, the liver contains no sugar, under the conditions in which it usually is found. From experiments of this kind, Bernard concluded that the glycogenic processes are suspended when the nerves are divided. The experiments, however, made by irritating the pneumogastrics, were more satisfactory, as in these he looked for sugar in the blood and in the urine and did not confine his examinations for sugar to the substance of the liver.

After division of pneumogastrics in the neck, if the peripheral ends 
are stimulated there is no effect on the liver; but when the stimulus is applied to the central ends, the glycogenic processes become exaggerated and sugar makes its appearance in the blood and in the urine. Bernard made a number of experiments illustrating this point, on dogs and rabbits. The current employed usually was feeble, and it was continued for five or ten minutes two or three times in an hour. In some instances the stimulation was continued for thirty minutes. From these experiments, it is assumed that the physiological production of glycogen by the liver is reflex and is due to an impression conveyed to the nerve-centres through the pneumogastrics. The inhalation of irritating vapors and of anesthetics produces an increased glycogenic action in the liver. The effects of irritating the floor of the fourth ventricle, by which temporary diabetes is produced, have been considered in connection with the glycogenic action of the liver.

Influence of the Pnenmogastrics on the Stomach and Intestines. Little or nothing is known in regard to the action of the pneumogastrics on the spleen, kidneys and suprarenal capsules. The influence of these nerves on the stomach and intestine will be considered under the following heads:-

I. The effects of faradization of the nerves.

2. The effects of section of the nerves on the movements of the stomach in digestion.

3. The influence of the nerves on the small intestine.

Effects of Faradization. - The stomach contracts under stimulation of the pneumogastrics in the neck, not instantly, but after five or six seconds. Stimulation of the splanchnic nerves, however, while it produces movements of the intestines, does not affect the stomach. Judging from the tardy contraction of the stomach and the analogy between the action of the pneumogastrics on this organ and the action of the sympathetic nerves on the non-striated muscular tissue, it has been assumed that the motor action of the pneumogastrics is due, not to the proper filaments of these nerves, but to filaments derived from the sympathetic. This, however, is not exactly in accord with experimental facts; for it has lately been known that the pneumogastrics are the excito-secretory nerves of the stomach and pancreas (Pawlow).

Effects of Section of the Pneumogastrics on the Movements of the Stomach. - If the pneumogastrics are divided in the neck in a dog in full digestion, in which a gastric fistula has been established so that the interior of the organ can be explored, the following phenomena are observed :-

In the first place, before division of the nerves, the mucous membrane of the stomach is turgid, its reaction is intensely acid and the 
finger introduced through the fistula is firmly grasped by the contractions of the muscular walls. When the pneumogastrics are divided, the contractions of the muscular walls cease, the mucous membrane becomes pale, the secretion of gastric juice apparently is arrested and the sensibility of the organ is abolished.

Notwithstanding the apparent arrest of the movements of the stomach in digestion by section of the pneumogastrics, it has been shown that substances may be slowly passed to the pylorus, and that the movements, although they are greatly diminished in activity, are not entirely abolished. The movements occurring after section of the nerves have been attributed to local irritation of the intramuscular terminal nervous filaments.

The influence of the pneumogastrics on the general processes of digestion, the sensations of hunger and thirst and on absorption from the alimentary canal have already been considered in connection with the physiology of digestion and absorption.

Influence of the Pnenmogastrics on the Small Intestine. - The pneumogastrics influence intestinal as well as gastric secretion. After section of the nerves in the cervical region, powerful cathartics (crotonoil, calomel, podophyllin, jalap, arsenic etc.) fail to produce purgation, even in doses sufficient to cause death. The articles used may be given by the mouth just before dividing the nerves or may be injected under the skin.

Stimulation of the pneumogastrics excites peristaltic movements of the small intestine. Experiments on the lower animals have shown, however, that if the nerve is stimulated during peristalsis, the movements are inhibited for a few moments, but afterward are increased in activity.

The cranial nerves that have been considered are the third, fourth, fifth, sixth, seventh, tenth, eleventh and twelfth. The anatomical and physiological history of the olfactory (first), optic (second), auditory (eighth), gustatory (branch of the seventh and a part of the ninth) and of the general sensory nerves so far as they are concerned in the sense of touch, belongs properly to the chapters on the special senses. 


\section{CHAPTER XXI}

THE SPINAL CORD

Membranes of the encephalon and spinal cord-Cephalo-rachidian liquid-Physiological anatomy of the spinal cord - Columns of Türck - Crossed pyramidal tracts - Anterior ground columns - Lateral bundles-Ascending and descending cerebellar fasciculiDirect cerebellar fasciculi - Columns of Burdach - Columns of Goll - Directions of nervefibres in the cord - General properties of the spinal cord - Relations of the posterior white columns of the cord to muscular coördination - Nerve-centres in the spinal cord - Reflex action of the spinal cord-Reflexes in man.

THE nervous structures contained in the cavity of the cranium and in the spinal canal, exclusive of the roots of the cranial and spinal nerves, constitute the cerebro-spinal axis. This portion of the nervous system is composed of white and gray matter. The fibres of the white matter act solely as conductors. The gray matter constitutes a chain of ganglia, which act as nerve-centres, receiving impressions and generating impulses. Certain parts of the gray matter also serve as conductors.

The cerebro-spinal axis is enveloped in membranes for its protection and for the support of its nutrient vessels. It is surrounded to a certain extent with liquid, and it presents cavities, as the ventricles of the brain and the central canal of the cord, that contain liquid. The gray matter is distinct from the white, even to the naked eye. In the spinal cord the white substance is external and the gray is internal. The surface of the brain presents an external layer of gray matter, the white substance being internal. In the white substance of the brain, also, are collections of gray matter. The white matter of the cerebrospinal axis is composed largely of fibres. The gray substance is composed largely of cells.

The encephalon is contained in the cranial cavity and consists of the cerebrum, cerebellum, pons Varolii and the bulb, or medulla oblongata. In the human subject and in many of the higher animals, its surface is marked with convolutions by which the extent of its gray substance is much increased. The cerebrum, the cerebellum and most of the encephalic ganglia are connected with the white substance of the encephalon and with the spinal cord. All the cerebro-spinal nerves are connected with the encephalon and the spinal cord. 
Membranes of the Encephalon and Spinal Cord. - The membranes of the brain and spinal cord are the dura mater, the arachnoid and the pia mater.

The dura mater of the encephalon is a dense membrane in a single layer and is composed chiefly of ordinary fibrous tissue. That portion which lines the cranial cavity is adherent to the bones. In certain situations it is separated into two layers and bounds what are known as the venous sinuses. The dura mater also sends off certain folds or processes. One of these passes into the longitudinal fissure and is called the falx cerebri; another lies between the cerebrum and the cerebellum and is called the tentorium; another is situated between the lateral halves of the cerebellum and is called the falx cerebelli. The dura mater is closely attached to the bone at the border of the foramen magnum. From this point it passes into the spinal canal and forms a loose covering for the cord. In the spinal canal, the membrane is not adherent to the bones, which have, like most other bones in the body, a special periosteum. At the foramina of exit of the cranial and the spinal nerves, the dura mater sends out processes which envelop the nerves, with the fibrous sheaths of which they soon become continuous. The subdural space contains a small quantity of liquid.

The arachnoid is a delicate membrane, resembling the serous membranes, except that it presents but one layer. Its inner surface is covered with a layer of endothelium. There is a considerable quantity of liquid between the arachnoid and the pia mater surrounding the cerebrospinal axis, in what is called the subarachnoid space. This is called the cerebro-spinal, or cephalo-rachidian liquid. The arachnoid does not follow the convolutions and fissures of the encephalon or the fissures of the cord, but it simply covers their surfaces. There is a longitudinal, incomplete, cribriform, fibrous septum in the cord, passing from the inner layer of the arachnoid to the pia mater. A similar arrangement is found in certain situations at the base of the skull.

The pia mater of the encephalon is a delicate fibrous structure, very vascular, seeming to present, indeed, only a skeleton network of fibres for the support of vessels going finally to the nervous substance. This membrane covers the surface of the encephalon immediately, follows the sulci and fissures, and is prolonged into the ventricles, where it forms the choroid plexus and the velum interpositum. From its internal surface small vessels are given off which pass into the nervous substance.

The pia mater of the encephalon is continuous with the corresponding membrane of the cord; but in the spinal canal the membrane is thicker, stronger, more closely adherent to the subjacent parts, and its bloodvessels are not so abundant. In this situation many of the fibres 
are arranged in longitudinal bands. This membrane lines the anterior fissure and a portion of the posterior fissure of the cord. At the foramina of exit of the cranial and the spinal nerves, the fibrous structure of the pia mater becomes continuous with the nerve-sheaths.

Between the anterior and posterior roots of the spinal nerves, on either side of the cord, is a narrow ligamentous band, the ligamentum denticulatum, which assists in holding the cord in place. This extends from the foramen magnum to the terminal filament of the cord, and is attached internally to the pia mater, and externally, to the dura mater.

Cephalo-rachidian Liquid. - The greatest part of the liquid in the cranium and in the spinal canal is contained in the subarachnoid space. The ventricles of the encephalon are in communication with the central canal of the cord, and also are connected with the general subarachnoid space by a narrow triangular orifice at the inferior angle of the fourth ventricle. By this arrangement the liquid in the ventricles of the encephalon and in the central canal of the cord communicates with the liquid surrounding the cerebro-spinal axis, and the pressure on these parts is equalized.

So far as is known, the office of the cephalo-rachidian fluid is simply mechanical, and its properties and composition have no very definite physiological significance. Its quantity has been estimated in the human subject at about two fluidounces (6o cubic centimeters); but this was the smallest quantity obtained by placing the subject upright, making an opening in the lumbar region and a counter-opening in the head to admit the pressure of the atmosphere (Magendie). The exact quantity in the living subject could hardly be estimated in this way; and it is difficult, indeed, to see how anything more than a roughly approximate idea could be obtained. The quantity indicated probably does not represent all the liquid contained in the ventricles and in the subarachnoid space; but as it is the most definite estimate that has been made, it is given as an approximation. It is probable that the quantity is subject to considerable variations.

The general properties and composition of the cephalo-rachidian liquid are in brief the following: It is transparent and colorless, free from viscidity, of a distinctly saline taste, an alkaline reaction and it resists putrefaction for a long. time. It is not affected by heat or acids. It contains a large proportion of water ( 98 I to 985 parts per thousand), a considerable quantity of sodium chloride, a trace of potassium chloride, sulphates, carbonates and alkaline and earthy phosphates. In addition it contains traces of urea, glucose, sodium lactate, fatty matter, cholesterin and albumin. 


\section{Physiological Anatomy of the Spinal Cord}

The spinal cord, with its membranes, the roots of the spinal nerves and the surrounding liquid, occupies the spinal canal and is continuous with the encephalon. Its length is fifteen to eighteen inches ( 38.1 to 45.7 centimeters) and its weight is about an ounce and a half (42.5 grams). Its general form is cylindrical, but it is slightly

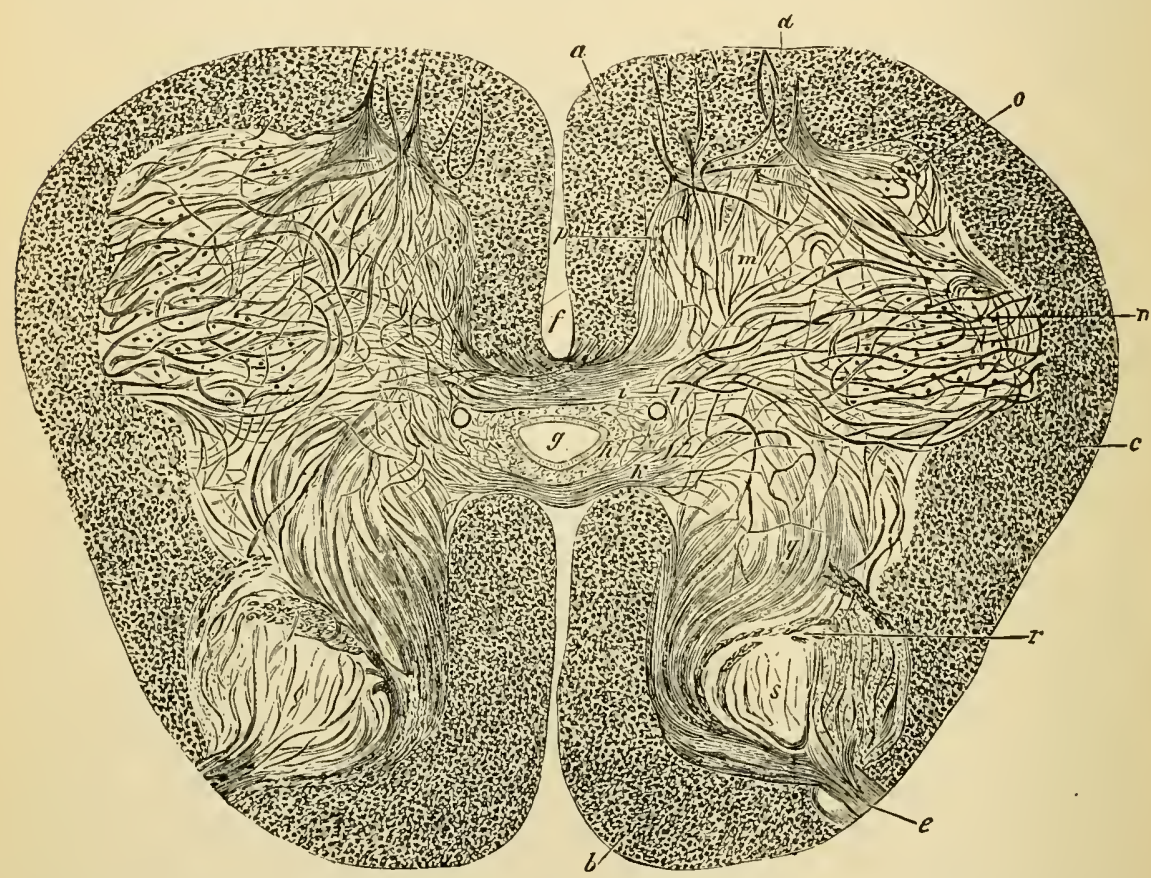

Fig. 132. - Transverse section of the spinal cord of a child six months old, at the middle of the lumbar enlargement, treated with potassium auric chloride and uranium nitrate; $\times 20$. By means of these reagents, the direction of the fibres in the gray substance is rendered unusually distinct (Gerlach).

$a$, anterior columns; $b$, posterior columns ; $c$, lateral columns; $d$, anterior roots; $e$, posterior roots ; $f$ anterior white commissure, in communication with the fasciculi of the anterior cornua and the anterior columns; $g$, central canal, with its epithelium; $h$, surrounding connective substance of the central canal; $i$, transverse fasciculi of the gray commissure in front of the central canal; $k$, transverse fasciculi of the gray commissure behind the central canal; $l$, transverse section of the two central veins; $m$, anterior cornua; $n$, great, lateral cellular layer of the anterior cornua; $o$, lesser, anterior cellular layer; $\not$, smallest, median cellular layer; $q$, posterior cornua; $r$, ascending fasciculi in the posterior cornua; s, substantia gelatinosa.

flattened in certain portions. It extends from the foramen magnum to the lower border of the body of the first lumbar vertebra. It presents, at the origin of the brachial nerves, an elongated ovoid enlargement flattened antero-posteriorly, and a corresponding enlargement at the origin of the nerves that supply the lower extremities. It terminates below in a slender gray filament, called the filum terminale, at the lower 
border of the first lumbar vertebra. The sacral and cocygeal nerves, after their origin from the lower portion of the cord, pass downward to emerge by the sacral foramina and form what is known as the cauda equina. The substance of the cord is composed of white and gray matter, the white matter being external. The pointed inferior extremity of the cord consists entirely of gray matter.

The cord presents an anterior and a posterior median fissure, and imperfect and somewhat indistinct anterior and posterior lateral grooves, from which latter arise the anterior and the posterior roots of the spinal nerves. The posterior lateral groove is fairly well marked, but there is no distinct line at the origin of the anterior roots. The anterior median fissure is more distinct. It penetrates the anterior portion of the cord in the median line for about one-third of its thickness and receives a highly vascular fold of the pia mater. It extends to the anterior white commissure. The posterior fissure is not so distinct as the anterior. It is not lined throughout by a fold of the pia mater but is filled with connective tissue and bloodvessels, which form a septum posteriorly between the lateral halves of the cord. The posterior median fissure extends nearly to the centre of the cord, as far as the posterior gray commissure.

The arrangement of the white and the gray matter in the cord is seen in a transverse section. The gray substance is in the form of a letter $\mathrm{H}$, presenting two anterior and two posterior cornua connected by what is called the gray commissure. The anterior cornua are short and broad and do not extend to the surface of the cord. The posterior cornua are longer and narrower, and they extend nearly to the surface at the point of origin of the posterior roots of the spinal nerves. In the centre of the gray commissure is a narrow canal, lined with cells of ciliated epithelium, called the central canal. This is in communication above with the fourth ventricle and extends below to the filum terminale. That portion of the gray commissure situated in front of this canal is sometimes called the anterior gray commissure, the posterior portion being known as the posterior gray commissure. The central canal is immediately surrounded by connective tissue. In front of the gray commissure is the anterior white commissure.

The proportion of the white to the gray substance is variable in different portions of the cord. In the cervical region, the white substance is most abundant, and, in fact, it progressively increases in quantity from below upward throughout the cord. In the dorsal region, the gray matter is least abundant and it exists in greatest quantity in the lumbar enlargement.

The. white substance of the cord is composed of nerve-fibres, con- 
nective-tissue elements, neuroglia, and bloodvessels, the latter arranged in a wide and delicate plexus. The nerve-fibres are variable in size and are composed of the axis-cylinder and the medullary substance, without the tubular membrane.

The anterior cornua of gray matter contain bloodvessels, connectivetissue elements, neuroglia, very fine nerve-fibres, and large multipolar nerve-cells, which are sometimes called motor cells. The posterior cornua are composed of the same elements, the cells being much smaller, and the fibres exceedingly small, presenting very fine plexuses. The cells in this situation are sometimes called sensory cells. Near the posterior portion of each posterior cornu, is an enlargement, of a gelatiniform character, containing small cells and fibres, called the substantia gelatinosa. The relations between the nerve-cells and the nerve-fibres have already been described in connection with the general structure of the nervous system. The multipolar nerve-cells present certain prolongations which do not branch and are directly connected with the medullated nerve-fibres. These are the neurites, or axis-cylinder prolongations. In addition, fine branching poles are described under the name of dendrites, or protoplasmic prolongations. The neuroglia is particularly abundant in that part of the posteria cornua of gray matter called the substantia gelatinosa (see Plate XII).

The division of the spinal cord into columns has a physiological as well as an anatomical basis. Anatomists usually recognize, on either side of the cord, an anterior column, bounded by the anterior median fissure and the line of origin of the anterior roots of the spinal nerves, a lateral column, bounded by the lines of origin of the anterior and of the posterior roots of the nerves, and a posterior column, bounded by the line of the posterior roots of the spinal nerves and the posterior median fissure. As the anterior or posterior columns include either white or gray matter, they are called respectively anterior or posterior white or gray columns. Physiological and pathological researches, however, have shown that the cord may properly be further divided as follows :- -

I. Columns of Tïrck. - By the sides of the anterior median fissure, are two narrow columns of white matter, one on either side, extending to the white commissure, called the columns of Türck, the direct, or the uncrossed pyramidal tracts. The fibres of these columns descend, probably decussate in the cervical region of the cord, and the columns are lost in the lower dorsal region. Destruction of certain motor parts in the brain is followed by descending secondary degenerations in these columns.

2. Crossed Pyramidal Tracts. - These are situated, one on either 
side, in the posterior portion of the lateral columns and are bounded internally by the posterior cornua of gray matter and externally by a narrow band called the direct cerebellar tract. In following the columns upward, it is found that they pass forward in the upper part of the cervical region and decussate in the lower portion of the anterior pyramids of the bulb. These are descending tracts, and their fibres undergo descending secondary degenerations as the result of destruction of certain motor areas in the brain.

3. Anterior Ground Columns. - These fasciculi are bounded internally by the columns of Türck and externally by the anterior cornua of gray matter and the anterior roots of the spinal nerves. Their fibres are supposed to connect the gray matter of the anterior cornua of the cord with the gray matter of the bulb.

4. Lateral Bundles. - These columns lie in the lateral portion of the cord externally to the anterior cornua of gray matter and the gray commissure. Their fibres are supposed to connect the gray matter of the cord with the gray matter of the bulb.

5. Ascending and Descending Cerebellar Fasciculi. -- These are situated externally to the lateral bundles. They are supposed to connect the gray matter of the cord with the cerebellum.

The fibres of the anterior ground columns and the lateral bundles do not degenerate in either direction as the result of section of the cord. Their fibres seem to connect nerve-cells with each other, and their trophic cells exist at both extremities, which accounts for the absence of degeneration just mentioned.

6. Direct Cerebellar Fasciculi. - These fasciculi are situated at the outer and posterior portion of the lateral columns. Their fibres pass to the funiculi graciles, or posterior pyramids of the bulb, and thence to the cerebellum, by the inferior peduncles. They connect the cells of the posterior cornua of gray matter with the cerebellum. These columns make their appearance first in the lumbar region of the cord, and they increase in size from below upward. After section of the spinal cord, the fibres of the direct cerebellar fasciculi show ascending secondary degenerations. Their trophic centres probably are the cells of the posterior cornua of gray matter.

7. Columns of Burdach (Posterior Lateral Columns). - These columns are in the posterior portion of the cord, between the columns of Goll and the posterior cornua of gray matter. Their fibres connect certain of the cells of the gray matter of the posterior cornua with the cerebellum; or at least the fibres pass upward and are connected with the restiform bodies, going to the cerebellum through the inferior peduncles. The fibres also connect nerve-cells in different planes of the 
cord with each other. A certain number of ascending secondary degenerations has been noted in these columns.

8. Columns of Goll (Posterior Median Columns). - These delicate columns are situated on either side of the posterior median fissure. They are lost in the lower dorsal region. Their fibres pass upward and pass into the funiculi graciles of the bulb. After section of the cord, ascending secondary degenerations are observed in these columns.

In addition to these, which are the principal columns of the cord, anatomists have described a tract called Gower's tract, lying between the ascending and descending cerebellar, the lateral bundles and the crossed pyramidal tracts, Clarke's columns, lying next the posterior

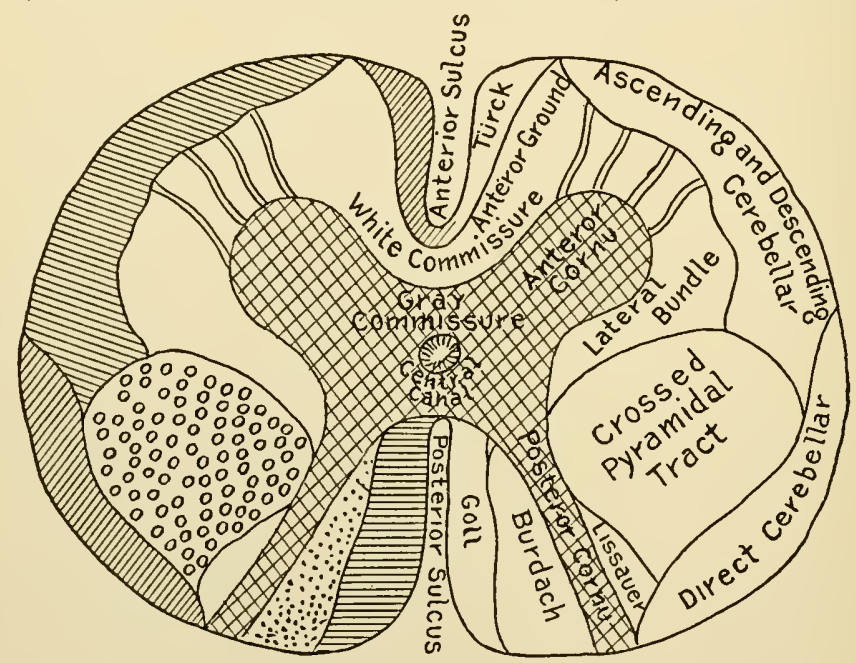

Fig. 133. - Diagram of the columns and conducting paths of the spinal cord in the upper dorsal region.

median fissure and the gray commissure and limited mainly to the dorsal region, and the tract of Lissauer, lying externally to the posterior extremity of the posterior cornu of gray matter. Gower's tract passes to the bulb by the periphery of the anterior pyramids and is thought by some physiologists to contain nerves of pain and nerves of temperature, but the properties of these columns are not very distinctly defined. It is stated that the cells which form the nucleus of the pneumogastric in the bulb are similar to those found in Clarke's columns. It is not known that the fibres of the tract of Lissauer pass to the bulb. It would seem that a description of eight principal columns renders the anatomy of the cord sufficiently complex without subdividing them into fasciculi that are not as yet known to have any special physiological significance. Still, degenerations attacking these smaller tracts have 
been observed and studied, but without developing distinct and positive physiological applications.

Directions of Nerve-fibres in the Cord. - Many of the points in the description of the course and connections of the fibres in the cord are given as probable. Anatomical observations have been somewhat contradictory, but many of these have been corrected or verified by following the paths of degeneration. What are called secondary degenerations are anatomical changes in the nerve-fibres which follow separation of the fibres from the cells which act as their trophic centres, or the centres presiding over their nutrition, these changes being secondary to destruction or degeneration of the centres.

The fibres of the anterior roots of the spinal nerves, following these fibres inward and upward, pass to the large multipolar motor cells of the anterior cornua of gray matter and have no direct connection with the white columns. Their direction through the white columns of the cord is oblique and slightly upward. Surrounding the nerve-cells are arborescent fibrils (synapses) connected with the fibres that pass up the cord in two bundles, median and lateral. So far as conduction is concerned, these fibres may be regarded as continuous with the fibres of the anterior roots. The fibres of the median bundle pass to the anterior white commissure, in which they decussate. The bundles then go each one to the column of Türck on the opposite side and pass upward in the so-called direct pyramidal tracts. The fibres of the lateral bundle go to the crossed pyramidal tract in the lateral column of the same side and pass upward to decussate at the bulb.

The fibres of the columns of Türck and the crossed pyramidal tracts are the only fibres of the cord that are known to convey motor impulses from the brain. Destruction of certain parts of the brain produces descending secondary degenerations in these fibres.

It is probable that fibres arise from the cells of the gray matter of the cord, which connect these cells with each other by means of synapses and are concerned in certain reflex phenomena involving the action of the cord alone. These fibres are in the anterior fundamental fasciculi, the anterior ground columns and the lateral bundle. They present no secondary degenerations.

The fibres of the posterior roots of the spinal nerves pass into the white substance of the cord, where they immediately bifurcate, one limb passing downward and the other upward for a certain distance, before they enter the gray matter. The upper limb of the bifurcation is the longer. In their course they send off collateral branches which penetrate the gray matter and break up into arborescent fibrils (synapses). These mingle and interlace with similar aborescent fibrils from 
the cell-bodies, the connection between them, however, being indirect and not directly continuous. Some neurites from the posterior roots possibly are directly continuous with neurites from the cell-bodies.

It is probable that fibres pass from the cell-bodies and go to the cerebellum in the direct cerebellar fasciculi, the columns of Goll and the ascending cerebellar fasciculi, which show ascending secondary degenerations.

Certain fibres from the posterior roots of the spinal nerves pass to the cells of the posterior cornua of gray matter of the cord and are connected by arborizing processes with arborizing prolongations of these cells (synapses). Processes from these cells pass to the gray commissure and decussate around the central canal, conducting sensory impressions to the brain in the gray matter of the opposite side of the cord. The sensory conductors, therefore, decussate all along the cord. Fibres originating in the nerve-cells of the posterior cornua pass in and out, along the cord, and their synapses connect the cells with each other above and below. These may properly be called longitudinal commissural fibres. They probably constitute the greatest part of the columns of Burdach.

\section{General Properties of the Spinal Cord}

In describing the general properties of the cord, as shown by the effects of stimulus applied to its exterior or to its cut surface, the term "excitability" will be used to express a property indicated by direct muscular contraction following stimulation of the cord, and sensibility, a property which enables it to receive impressions which produce pain. In exciting different parts of the cord with electricity, it is necessary to guard carefully against an extension of the current beyond the points which it is intended to stimulate. Some physiologists regard the cord as inexcitable and insensible, both on its surface and in its deeper portions. With this view, it is supposed that parts of the cord will conduct motor impulses received from the centres situated above but are not excited by a stimulus applied directly. In the same way, it is thought, parts of the cord will convey sensory impressions received through the nerves but are insensible to direct irritation. Certain of the columns, however, react under direct stimulation.

In experiments made in 1863 (Flint) on a living dog, the cord having been exposed in the lumbar region and stimulated mechanically and with an electric current two hours after the operation, certain positive results were obtained which led to the following conclusions:-

The gray substance probably is inexcitable and insensible to clirect stimulation. 
The antero-lateral columns are insensible, but are excitable both on the surface and in their substance; and direct stimulation of these columns produces convulsive movements in certain muscles, which movements are not reflex and are not attended with pain. The lateral columns are less excitable than the anterior columns.

The surface, at least, of the posterior columns is very sensitive especially near the posterior roots of the nerves. The deep portion of the posterior columns are probably insensible except very near the origin of the nerves.

The above conclusions refer only to the general properties of different portions of the cord, as shown by direct stimulation, in the same way that the general properties of the nerves in their course are demonstrated.

Motor Paths in the Cord. - What has been said regarding the direction of the fibres in the cord and the situation and course of the degenerations following destruction of motor centres conveys a definite idea of the motor paths in the cord. This idea is sustained by experiments in which different columns of the cord have been divided in living animals.

The motor paths are in the direct pyramidal tracts (columns of Türck) and in the crossed pyramidal tracts of the lateral columns. The motor impulses are conveyed by the fibres of these tracts to the multipolar cells in the anterior cornua of gray matter and are thence transmitted to the anterior roots of certain spinal nerves. In the lower dorsal region the conduction is confined to the crossed pyramidal tracts in the lateral columns, while above, the direct pyramidal tracts participate in this action.

The motor fibres decussate in the anterior pyramids of the bulb (crossed pyramidal tracts), and in the cervical region to a comparatively slight extent, before the direct pyramidal tracts (columns of Türck) pass to the encephalon. In the cervical region the decussation takes place probably in the anterior white commissure. The fact of this decussation of motor conductors is sustained by pathology paralysis of motion following brain-lesions, occurring on the opposite side of the body - and by experiments in which the fibres as they cross are divided by a longitudinal median section in the bulb and in the cervical region of the cord.

Vasomotor nerve-fibres exist in the lateral columns of the cord and probably are connected with the cells of the gray matter. They pass out in the anterior roots of the spinal nerves and go to the bloodvessels either from the branches of the spinal nerves directly or through filaments sent to the sympathetic. 
Sensory Paths in the Cord. - The gray matter of the cord is the part concerned in the conduction of sensory impressions. This fact has been verified by recent experiments; but it is thought that some of the sensory conductors run in the columns of Goll. The columns of Goll, however, exist only in the cervical and upper dorsal regions.

The sensory conductors do not decussate at any particular point as do the motor conductors in the crossed pyramidal tracts. The fibres from the posterior roots of the spinal nerves pass to the sensory cells of the posterior cornua and decussate throughout the length of the cord. If the cord is divided longitudinally in the median line, there is complete paralysis of sensation on both sides in all parts below the section. In this section, the only fibres that are divided are those passing from one side of the cord to the other. This decussation is by fibres prolonged from the cells of the posterior cornua, which cross in the gray commissure, around the central canal.

When one lateral half of the cord is divided in a living animal, sensibility is impaired or lost on the opposite side of the body below the section, but there is hyperesthesia on the side corresponding to the section. This exaggeration of sensibility has not been satisfactorily explained.

Conduction through Synapses. - Repeated reference has already been made to the so-called synapses that connect nerve-fibres with nervecells. The cell-bodies, as has been seen, present each an axis-cylinder prolongation, the neurite, and branching, or arborizing processes, called dendrites. In the theory of the transmission of nerve-impulses or of centripetal currents through synapses, it is assumed that the conducting neurite, when it reaches a cell-body, branches and arborizes around the cell, its branchings interlacing with branching dendrites, but without direct connection of filaments. This is called a synapse - from $\sigma v \nu a ́ \pi \tau \omega$, to join together. Following out an impulse from a motor area in the cerebral cortex to a muscle, it evidently must pass through several collections of cells, notably in the corpus striatum, the pons and the multipolar cells on the opposite side of the cord. Each synapse has been compared to a relay-station; and the passage of the impulse, which is taken up by the cells and conveyed onward by its neurite, involves a delay which, in the frog, is about 0.002 of a second. In afferent conduction the mechanism of transmission of impressions is nearly the same. In reflex action involving the cord only, arborizing filaments from the afferent nerve-roots form synapses with the dendrites of the motor cells and impulses are sent from these cells to muscles. It is thought, also, that synapses exist in the ganglia of the sympathetic system in the motor paths of conduction to non-striated muscles. The same mechan- 
ism is supposed to exist when different areas in the central nervous system are connected by commissural, or association fibres. The bipolar cells of the ganglia on the posterior roots of the spinal nerves and of the sensory cranial nerves, however, seem to be merely enlargements in the course of the nerve-fibres.

According to this theory of nerve-conduction, impulses are taken up by the dendrites of the nerve-cells and transmitted onward through their neurites; and this may involve the passage of the current through a number of so-called cell-stations. The cells send out impulses through their neurites and receive them through the dendrites, the same mechanism existing for both centripetal and centrifugal conduction, and "delays" always occurring at the cell-stations. The current, therefore, can move only in one direction, this depending on the arrangement of the arborizations of the neurites and of the dendrites. Finally, therefore, the sensory cells of the cerebral cortex receive impressions through their dendrites. If it is assumed that neurites in their course may conduct in both directions, - and some experiments favor this view, - as a logical consequence the synapses must act as valves; so that a centrifugal current can not pass in the opposite direction beyond a synapse, the same being true of a centripetal current.

While the theory of the action of synapses explains many heretofore obscure phenomena of nerve-conduction and is extremely probable, it should be received as such and not as a matter of positive and absolute demonstration. There are certain properties and functions of nervecells, connected with trophic action, degenerations and associations of nerve-centres, that still are imperfectly understood.

Relations of the Posterior White Columns of the Cord to Muscular Coördination. - It was noticed by Todd, many years ago (I839-1847), in cases of that peculiar form of muscular incoördination now known as locomotor ataxia, that the posterior white columns of the cord were diseased. Reasoning from this fact, Todd made the following statement in regard to the office of these columns:-

"I have long been impressed with the opinion, that the office of the posterior columns of the spinal cord is very different from any yet assigned to them. They may be in part commissural between the several segments of the cord, serving to unite them and harmonize them in their various actions, and in part subservient to the function of the cerebellum in regulating and coördinating the movements necessary for perfect locomotion."

The view thus early advanced by Todd has been sustained by experiments on living animals. If the posterior columns are completely divided by two or three sections made at intervals of about three-fourths of an 
inch to an inch and a quarter (20 to 30 millimeters), the most prominent effect is a remarkable trouble in locomotion, consisting in a want of proper coördination of movements. Experiments on the different columns of the cord in living animals, however, are so difficult that physiologists have preferred to take the observations in cases of disease in the human subject as the basis of their ideas in regard to the office of the posterior white columns.

The characteristic phenomenon of locomotor ataxia is inability to coördinate muscular movements, particularly those of the extremities. There is not of necessity any impairment of actual muscular power; and although pain and more or less disturbance of sensibility are usual, these conditions are not invariable and they are always coincident with clisease of sensory conductors. The characteristic pathological condition is disease of the posterior white columns (columns of Burdach). This usually is followed by or is coexistent with disease of the posterior roots of the spinal nerves and disease of the cells of the posterior gray matter of the cord. As the cells are affected, there follow ascending secondary degenerations in the columns of Goll. It is fair to assume that the disease of the cells of the gray matter of the cord and of the posterior roots of the spinal nerves is connected with the disorders of general sensibility. The disease of the columns of Burdach produces the disorder in movements.

Reasoning from the characteristic phenomena and the essential pathological conditions of the cord in typical cases of locomotor ataxia, the posterior white columns of the cord, connecting cells of the gray matter in different planes with each other, assist in regulating and coördinating the voluntary movements. The fibres of these columns also connect the cord with the cerebellum, which has an important office in muscular coördination. It is probable that the appreciation of the muscular sense and the sense of pressure, if these can be separated from what is known as general sensibility, are connected with the action of the fibres of the posterior white columns.

\section{Nerve-centres in the Spinal Cord}

It has long been known that decapitation of animals does not arrest muscular action; and the movements observed after this mutilation present a certain degree of regularity and have been shown to be in accordance with well-defined laws. Under these conditions, the regulation of such movements is effected through the spinal cord and the spinal nerves. If an animal is decapitated, leaving only the cord and its nerves, there is no sensation, for the parts capable of appreciating sensation are 
absent; nor are there any true voluntary movements, as the organ of the will is destroyed. Still, in decapitated animals, the sensory nerves are for a time capable of conducting impressions, and the motor nerves can transmit impulses to the muscles; but the only part capable of receiving impressions or of generating motor impulses is the gray matter of the cord. If, in addition to the removal of all the encephalic ganglia, the cord itself is destroyed, all muscular movements are abolished, except as they may be produced by direct stimulation of the muscular tissue or of individual motor nerves.

The gray matter of the brain and spinal cord is a connected chain of ganglia, capable of receiving impressions through the sensory nerves and of generating motor impulses. The cerebro-spinal axis, taken as a whole, has this general office; but some parts have separate and distinct properties and can act independently of the others. The cord, acting as a conductor, connects the brain with the parts to which the spinal nerves are distributed. If the cord is separated from the brain in a living animal, it may act as a centre independently of the brain ; but the encephalon has no communication with the parts supplied with nerves from the cord, and it can act only on the parts which receive nerves from the brain itself.

When the cord is separated from the encephalon, an impression made on the general sensory nerves is conveyed to its gray substance, and this gives rise to a stimulus, which is transmitted to the voluntary muscles, producing certain movements that are independent of sensation and volition. This impression is said to be reflected back from the cord through the motor nerves; and the movements occurring under these conditions are called reflex. As they are movements excited by stimulation of sensory nerves, they are sometimes called excitomotor.

The term "reflex," as it is now generally understood, may properly be applied to any generation of impulses that occurs as a consequence of an impression received by a nerve-centre ; and it is evident that reflex phenomena are by no means confined to the action of the spinal cord. The movements of the iris are reflex, and yet they take place in many instances without the intervention of the cord. Movements of the intestines and of the involuntary muscles usually are reflex, and they involve the action of the sympathetic system of nerves. Impressions made on the nerves of special sense, as those of smell, sight, hearing, etc., give rise to certain trains of thought. These involve the action of the brain, but still they are reflex. In this last example of reflex action, it sometimes is difficult to connect the operations of the mind with external impressions as an exciting cause; but it is evident, on a little reflection, that this often occurs. 
Reflex Action of the Spinal Cord. - Simple reflex action involves the existence of an afferent (sensory) nerve, a collection of nerve-cells, and an efferent (motor) nerve, the nerves being connected with the nervecells. In a decapitated animal, not only are the movements independent of sensation and volition, but no movements occur if the sensory nerves are protected from any kind of impression or stimulation (Marshall Hall, I832 and I833). If the cord is destroyed, however, no movements follow stimulation of the surface; and if either the afferent or the efferent nerves are divided, no reflex movements can take place. Experiments on decapitated animals are in accord with the results of observations on acephalous fœtuses and cases of complete paraplegia from injury to the cord.

In the simplest form of a reflex movement, the muscular contraction is confined to the muscle or muscles which correspond, in their nervous supply, to the afferent nerve stimulated; but when the stimulus is sufficiently powerful or when the cord is in a condition of exaggerated excitability, the impression is disseminated throughout the gray matter, and the entire muscular system may be thrown into action. With feebler stimulation, one side only of the muscular system may respond. When the reaction extends to the opposite side, it is called crossed reflex. The extension of a stimulus conveyed by a single afferent nerve throughout the cord is called irradiation.

When a feeble stimulus applied to an afferent nerve is repeated frequently and at short intervals, general muscular movements are produced. This follows stimuli applied three times in a second, and the effect is increased up to sixteen shocks in a second, but not beyond this number (Rosenthal).

In studying the paths of conduction in the cord, it has been seen that sensory conduction takes place through the gray matter and possibly through the columns of Goll, that motor impulses are conducted by the direct and the crossed pyramidal tracts, and that the columns of Burdach probably are connected with muscular coördination. The fibres of the cord that are specially concerned in reflex action probably are in the anterior ground columns and the lateral bundles.

It is well known that the reflex excitability of the cord is exaggerated by removal of the encephalon. According to Setschenow, certain parts in the encephalon, particularly the optic lobes in frogs, exert an inhibitory influence over the reflex acts of the cord, and as a consequence, reflex phenomena are more marked when this influence has been removed.

Certain poisons, especially strychnin, have a remarkable influence over reflex excitability. In a frog decapitated and poisoned with 
strychnin, no reflex movements occur unless an impression is made on the sensory nerves; but a slight irritation, such as a current of air, throws the entire muscular system into a condition of violent tetanic spasm. The same phenomena are observed in cases of poisoning with strychnin or of tetanus in the human subject.

The inhalation of anesthetic agents may abolish all the ordinary reflex phenomena. Whether this be due to an action on the cord itself or to a paralysis of the sensory nerves, it is difficult to determine. Ordinarily, in animals rendered insensible by anesthetics, the movements of respiration continue; but these also may be arrested, as has been observed by all who have experimented with anesthetics, especially with chloroform. A common way of determining that an animal is completely under the influence of an anesthetic is by noting an absence of the reflex act of closing the eyelids when the cornea is touched.

It is necessary, after what has gone before, only to indicate in a general way certain phenomena observed in the human subject, which illustrate the reflex action of the cord. It is a common observation, in cases of paraplegia in which the lower portion of the cord is intact, that movements of the limbs follow titillation of the soles of the feet, these movements taking place independently of the consciousness or the will of the subject experimented on. Acephalous monsters will present general reflex movements and movements of respiration and will even suck when the finger is introduced into the mouth. Observations of this kind are so familiar that they need not be cited in detail. Experiments have also been made on criminals after decapitation; and although the reflex phenomena are not so well marked and can not be excited so long after death as in cold-blooded animals, they are sufficiently distinct.

General muscular spasms following stimulation of sensory nerves are pathological and take place only when the reflex excitability of the cord is exaggerated. Examples of this action are the spasms observed in tetanus or in poisoning by strychnin. In experiments on the lower animals, particularly frogs, coördinate reflex movements are often observed, such as the movements of jumping or swimming. This is sometimes called purposive reflex action, as the movements seem to have a definite purpose, or object. The following well-known experiment illustrates a coördinate, or purposive reflex.

Pflüger removed the entire encephalon from a frog, leaving only the spinal cord. He then touched the surface of the thigh over the inner condyle with acetic acid. The animal thereupon rubbed the irritated surface with the foot of the same side, apparently appreciating the seat of the irritation, and endeavoring, by a voluntary effort, to remove it. The foot of this side was then amputated, and the irritation was 
renewed in the same place. The animal made an ineffectual effort to reach the spot with the amputated member, and failing in this, after some general movements of the limbs, rubbed the spot with the foot of the opposite side.

It has been thought that this experiment shows a persistence of sensation and the power of voluntary movements after removal of the entire encephalon; but it must be remembered that the cord contains cells collected probably into groups that correspond to sets of muscles concerned in coördinate movements, and that many movements set in action by an effort of the will continue in an automatic manner, as the ordinary movements of progression. It is more reasonable to suppose that a persistent stimulation of the surface, such as is produced by the action of acetic acid on the skin of a frog, can give rise to coördinate movements of a purely reflex character than to assume that the movements in Pfluger's experiment were voluntary efforts to remove a painful impression. It is certain that in the higher classes of animals after removal of the encephalon, - in experiments on decapitated criminals and in patients suffering from paraplegia, - there is no evidence of centres of true sensation or volition in the spinal cord. In man and the higher animals, all muscular movements that depend solely on the reflex action of the cord must be regarded as automatic and independent of consciousness and volition.

Some of the confusion in regard to the precise nervous mechanism of many movements executed by the adult man may be removed by an exact comprehension of the terms used in their description. There are certain movements that are entirely independent of sensation and volition and of practice and education. Such are the movements of the lower extremities in paraplegia, movements of the stomach and intestines and the movements of tranquil respiration. These may be called purely reflex, depending entirely on the action of the cord or the bulb. So-called purposive movements may be included in this category, at least when they occur in such instances as that of the brainless frog that swims or jumps. The nerve-impulses there may be assumed to be conducted along constant and habitual paths in the cord. Another class of movements, that has its highest illustration in man, embraces those that are habitual or highly skilled. These may be called automatic. They may be initiated by an effort of the will but continue without further thought or attention. Such movements are those of walking, skating, swimming, in certain instances playing on musical instruments and doing mechanical work. It seems more reasonable to regard such movements as due to the action of the spinal cord than to invoke what has been called "unconscious cerebration"; for while these 
movements are executed automatically, the brain may be actively engaged in other directions. It must be remembered, however, that the brain itself often works automatically, intelligent results occurring without an appreciation of the mental processes by means of which they are accomplished. Examples of this kind of action are often afforded by remarkable players at chess or "lightning calculators."

Certain purely reflex movements may be restrained by an effort of the will, as is well known; provided, always, that these be movements that can be executed by voluntary effort. Nevertheless, if the sensory impression is sufficiently powerful or is frequently repeated, it often is impossible to control such movements by the will. Movements that are never in themselves voluntary, such as the ejaculation of semen, when excited by reflex action, can not be restrained by a voluntary effort; while the reflex act of coughing, for example, may be measurably controlled. It is hardly proper to speak of inhibition of the reflexes, in the sense in which the term "inhibition" is commonly used in physiology, for the reason that probably there are no special inhibitory nerves for these movements.

Various reflexes are made use of in pathology as means of diagnosis. The superficial reflexes are those produced by tickling the soles of the feet or by exciting other parts of the skin. The most prominent of the deep reflexes is the patellar reflex, or the knee-jerk, produced by percussion of the ligamentum patellæ.

Reflexes in Man. - The most important of the superficial reflexes are the following:-

Tickling of the soles of the feet produces movements of the lower extremities. In cases of paraplegia in which the properties of the lower portion of the cord are retained, the reflex movements produced by tickling the soles of the feet are often violent.

When the skin is scratched over the gluteus, the muscle is thrown into contraction.

Scratching of the skin on the inner surface of the thigh produces retraction of the testicle on that side. This is called the cremasteric reflex.

The muscles of the abdomen contract when the skin is scratched, and the same occurs with the muscles of the back on stimulation of the skin of that part.

The deep reflexes are sometimes called tendon-reflexes. The "knee-jerk" is produced by contraction of the quadriceps following a smart blow on the tendon of the patella.

The "ankle-clonus" is a contraction of the gastrocnemius when that muscle is forcibly put on the stretch and maintained in this condition for a short time. 
Both the superficial and the deep reflexes often afford important information in regard to diseases of the brain and spinal cord.

The gray matter of the cord is not a single centre, but consists of a number of centres connected with each other and with the brain. Some of these have already been described in connection with the history of various physiological processes, and others will be considered hereafter under appropriate heads. In addition to those already described, are centres for defecation, at the fifth lumbar vertebra in dogs (Budge), the erection-centre, in the lumbar region (Eckhard), and the parturition-centre (Körner), at the first and second lumbar vertebræ. Throughout the gray matter are a number of subsidiary vasomotor centres that are subordinate to similar dominant centres in the bulb. The cord also contains subsidiary sweat-centres. 


\title{
CHAPTER XXII
}

\author{
THE CEREBRUM AND THE BASAL GANGLIA
}

Weights of the encephalon and of certain of its parts - The cerebral hemispheres-Cerebral convolutions - Basal ganglia - Corpora striata, optic thalami and internal capsule Tubercula quadrigemina - Crura cerebri - Pons Varolii - Directions of fibres in the cerebrum - Fibres connecting the cerebrum with the cerebellum - Fibres connecting the two sides of the brain - Fibres connecting different cerebral convolutions on the same side (association fibres) - Fibres connecting the brain with the spinal cord - Cerebral localization-Motor cortical zone (Rolandic area) - General uses of the cerebrum - Extirpation of the cerebrum - Comparative development of the cerebrum in the lower animals Development of the cerebrum in different races of men and in different individuals - Facial angle - Pathological observations - Reaction-time - Centre for the expressions of ideas in language.

THE encephalic ganglia are collections of gray matter found in the encephalon, or what is commonly known as the brain. This part of the cerebro-spinal axis is contained in the cranial cavity. It is provided with membranes, which are similar to the membranes of the spinal cord and have been described in connection with the cord and the general arrangement of the cerebro-spinal axis. The gross anatomical divisions of the encephalon are the cerebrum, cerebellum, pons Varolii and bulb. As regards their physiological uses, the cerebellum, pons and bulb are to a certain extent subordinate to the cerebrum. In treating of the physiology of these parts, it will be convenient to take up first the cerebrum, or the cerebral hemispheres, with their anatomical and physiological connections and their relations to the other parts of the encephalon.

All parts of the encephalon that act as nerve-centres are more or less intimately connected with each other anatomically, and are finally connected, through the bulb, with the spinal cord. The exceptions to this are the centres of olfaction, vision, audition and gustation, which will be considered fully in connection with the physiology of the special senses. The spinal cord, as has been seen, is capable of independent action as a nerve-centre or collection of nerve-centres, also serving as a means of connection between the brain and the parts through the spinal nerves. The motor and sensory cranial nerves are directly connected with the encephalon.

A detailed anatomical description of the brain would be out of place in this work, as there are many anatomical parts, the exact physiological 
relations of which are not understood; still, there are certain parts, which will be referred to by name, a general knowledge of the arrangement of which is necessary. The general relations of these parts are shown in Fig. I34, slightly reduced and modified, from Harrison Allen, which represents a vertical longitudinal section of the brain in the median line.

As bearing on certain points in the physiology of the brain, it is important to note the weight of the entire encephalon and of its great divisions.

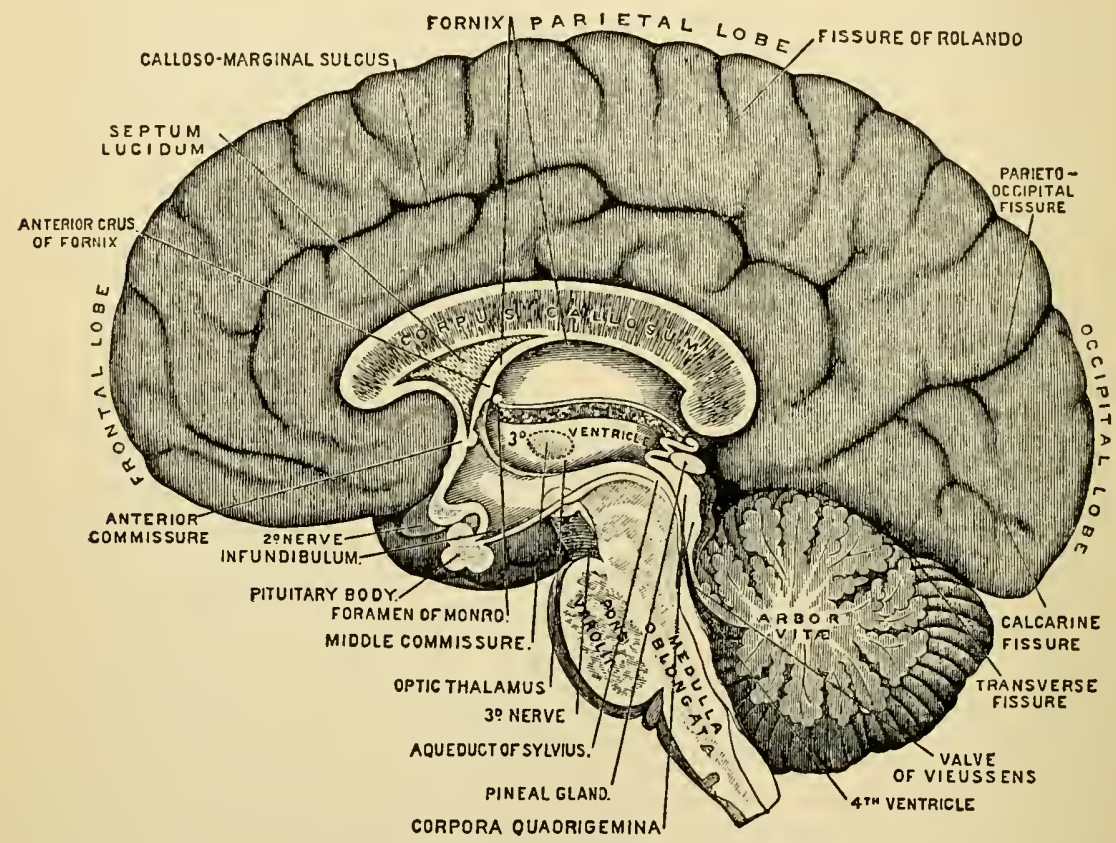

Fig. 134. - View of the structures displayed on the right side of a median longitudinal section of the brain-semidiagrammatic.

Weights of the Encopltalon and of Certain of its Parts. - Most of the tables of weights of the healthy adult brain of the Caucasian, compiled by different writers, give essentially the same figures, the differences amounting to only one or two ounces ( 28.3 or 56.7 grams) for the entire encephalon. The average weight given by Quain, combining the tables of Sims, Clendinning and Reid, is $49 \frac{1}{2}$ ounces (I 408.3 grams) for the male, and 44 ounces (I 247.4 grams) for the female. The number of male brains weighed was 278 , and of female brains, I9r. In males the minimum weight was 34 ounces (963.9 grams), and the maximum, 65 ounces ( 1842.7 grams). In I 70 cases out of the 278 , the weights ranged between 46 and $53^{\circ}$ ounces ( 1304.1 and 1502.5 grams), which may be 
taken as the average limits. In females the minimum was $3 \mathrm{I}$ ounces ( 878.8 grams), and the maximum, 56 ounces ( 1587.6 grams). In 125 cases out of the $19 \mathrm{I}$, the weights ranged between $4 \mathrm{I}$ and 47 ounces (I 62.3 and 1332.4 grams).

Quain assumed, from various researches, that in new-born infants the brain weighs Ir.65 ounces ( 327.8 grams), for the male, and Io ounces (283.5 grams), for the female. In both sexes, "the weight of the brain generally increases rapidly up to the seventh year, then more slowly to between sixteen and twenty, and again more slowly to between thirty-one and forty, at which time it reaches its maximum point. Beyond that period, there appears a slow but progressive diminution in weight of about one ounce (28.3 grams) during each subsequent decennial period ; thus confirming the opinion, that the brain diminishes in advanced life."

The comparative weights of the several parts of the encephalon, calculated by Reid from observations on the brains of fifty-three males and thirty-four females, between the ages of twenty-five and fifty-five, are as follows :-

\begin{tabular}{|c|c|c|}
\hline Divisions OF THE ENCEPHALON & Males & Females \\
\hline Average weight of the cerebrum & $43.9^{8} \mathrm{oz}$. (I $247 \cdot 3$ grams $)$ & $3^{8.75} \mathrm{oz} .($ Iog8.6 grams) \\
\hline $\begin{array}{l}\text { A verage weight of the cerebellum } \\
\text { Average weight of the pons and }\end{array}$ & $5.25 \mathrm{oz} . \quad($ I 48.8 grams $)$ & $4.76 \mathrm{oz} . \quad$ (I34.9 grams) \\
\hline medulla oblongata . . . . & $0.98 \mathrm{oz} . \quad$ (28.2 grams) & I.o I oz. (28.6 grams) \\
\hline $\begin{array}{l}\text { Average weight of the entire en- } \\
\text { cephalon } . . \\
\end{array}$ & $50.2 \mathrm{I} \mathrm{oz} .(\mathrm{I} 423.5 \mathrm{grams})$ & $44.52 \mathrm{oz}$. (I 262.1 grams) \\
\hline
\end{tabular}

The proportionate weight of the cerebellum to that of the cerebrum, in the male, is as I to $8 \frac{4}{7}$, and in the female, as I to $8 \frac{1}{4}$ (Quain).

The specific gravity of the whole encephalon is about 1036, that of the gray matter being 1034, and of the white, I040 (Quain).

\section{The Cerebral Hemispheres}

Cortical Substance. - The surface of the cerebral hemispheres is marked with fissures, sulci and convolutions, which serve to increase the extent of the gray substance. The sulci between the convolutions vary in depth in different parts, the average being about an inch (25.4 millimeters). The gray matter, which is external and follows the convolutions, is $\frac{1}{2}$ to $\frac{1}{8}$ of an inch (2.I to 3.2 millimeters) in thickness. Anatomists have described this substance as existing in several layers, but these divisions are largely artificial. In certain parts, however, particularly in the posterior portion of the cerebrum, the gray substance 
is quite distinctly divided into two layers, by a very delicate intermediate layer of a whitish color.

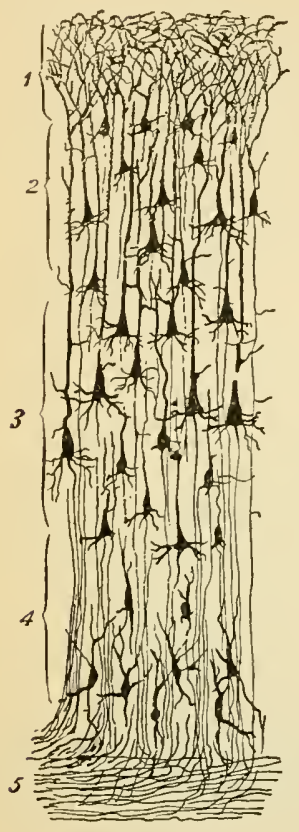

Fig. 135. - Vertical section of the cerebral cortex (R. y Cajal).

I, molecular layer; 2 , the layer of small pyramidal cells : 3. layer of large pyramidal cells; 4, layer of large stellate cells; 5 , white substance.

thickest of the three. These cells present one bifurcating neurite and many dendrites and collaterals. This layer also contains a few large pyramidal cells, with neurites, dendrites, teloneurites and telodendrites.

3. The layer of large pyramidal cells has the same arrangement as that of the cells of the second layer. This layer also contains a few small pyramidal cells. The second and third layers are not very easily separable from each other.

4. This layer presents rather large stellate cells, with neurites, dendrites and collaterals. ${ }^{1}$

${ }^{1}$ Some histologists describe five layers in the cerebral cortex, adding to the four layers described ahove, a layer external to the molecular layer; but this layer is composed largely of neuroglia and does not contain nerve-cells. 
It may be said in general terms that the more superficial cells of the cerebral cortex are small and the deeper cells are larger. The neurites of the molecular layer do not pass to the white matter. The neurites of the three deeper layers extend downward into the white matter. The dendrites and telodendrites pass, some upward to the molecular layer, and some to interlace with each other.

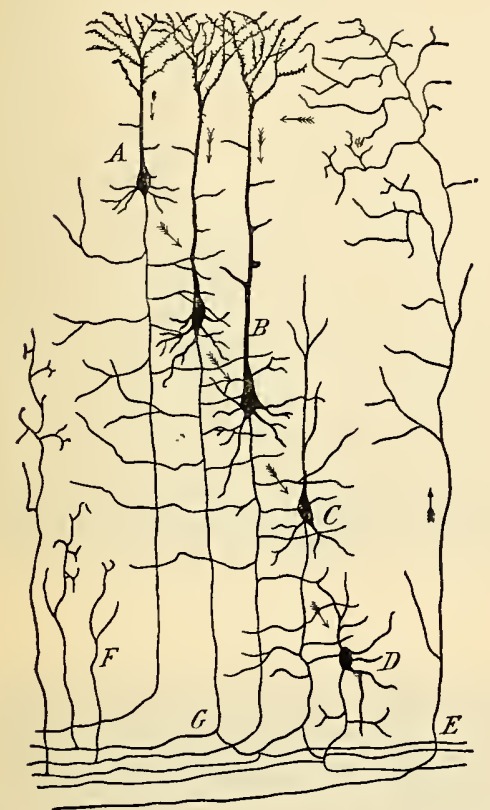

Fig. 137. - Diagrammatic section through the cerebral cortex (R. y Cajal).

$A$, small pyramidal cell of the second layer; $B$, two large pyramidal cells of the third layer; $C, D$, stellate cells of the fourth layer; $E$, centripetal neurite from distant centres; $F$, collaterals from the white substance; $G$, bifurcated neurite from the white substance.

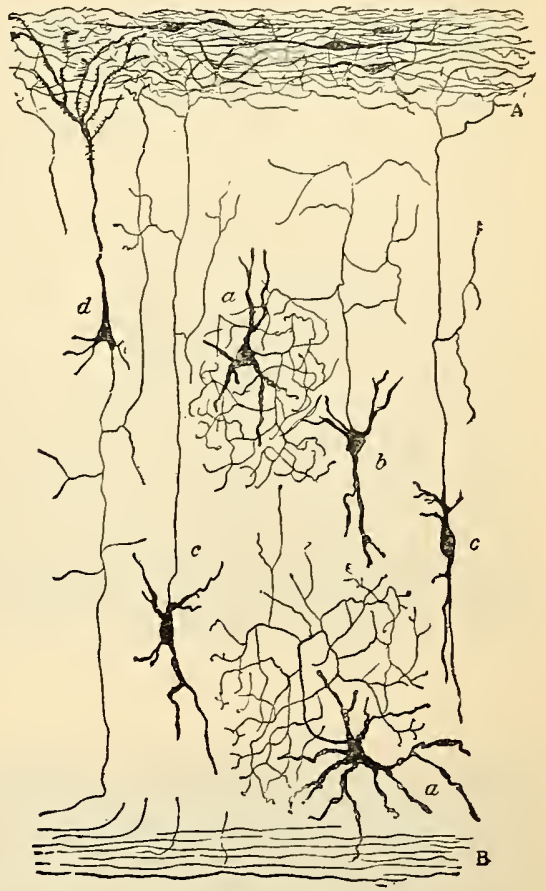

Fig. 138. - Cells with short neurites in the cerebral cortex (R. y Cajal).

$A$, molecular layer; $B$, white substance; $a$, cells with neurites and teloneurites; $b$, cell with a neurite extending downward; $c, c$, cells with neurites extending upward; $d$, small pyramidal cell.

The cerebral cortex receives centripetal fibres from the white matter, which give off abundant collaterals and terminate in the molecular layer by arborizing filaments (synapses) surrounding the nerve-cells.

The white matter of the cerebrum presents four groups of fibres:-

I. Centrifugal, or "projection" fibres pass downward from the three deeper layers of the cortex and finally, with their collaterals, extend into the pyramidal tracts of the cord.

2. Commissural fibres from the smaller pyramidal cells of the cortex connect the two sides of the cerebrum. 


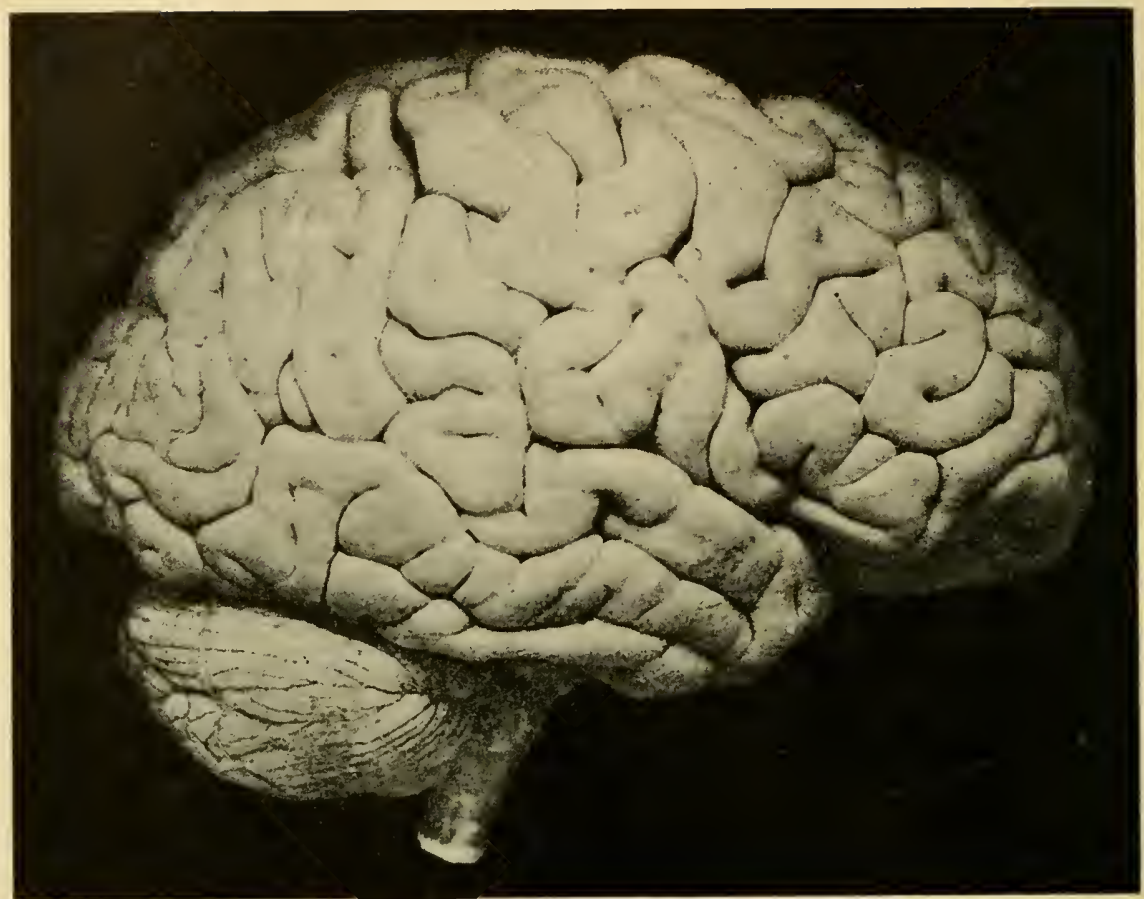

Fig. 139. - Lateral surface of the brain, reduced about one-third (Daiton).

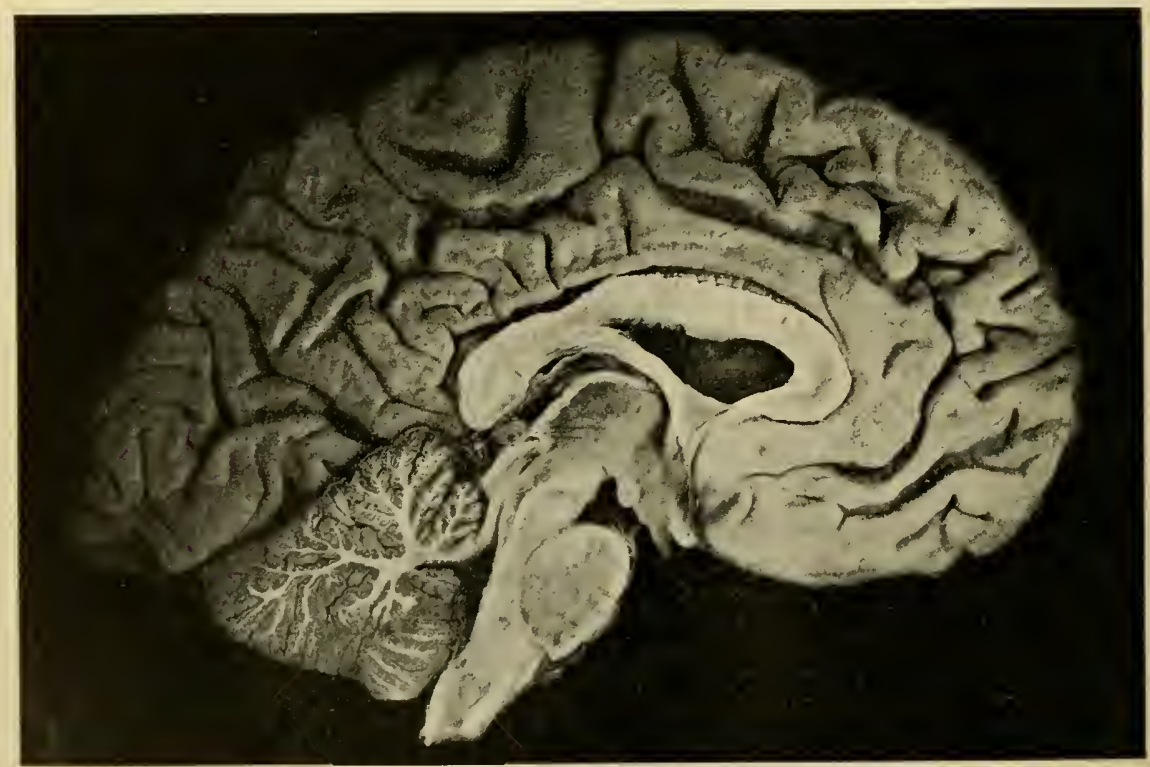

Fig. 140. - Median section of the brain, reduced about one-third (Dalion). 


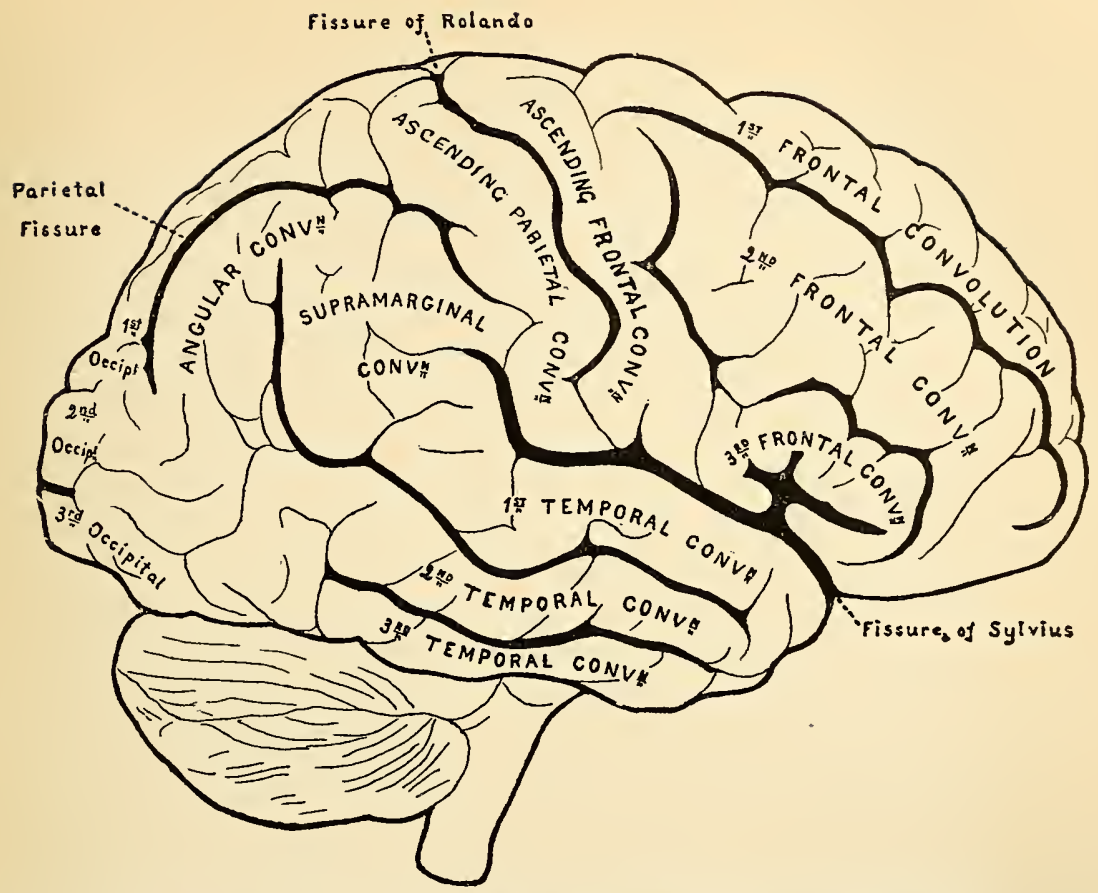

Fig. 139'. - Lateral surface of the brain, reduced about one-third (Dalton).

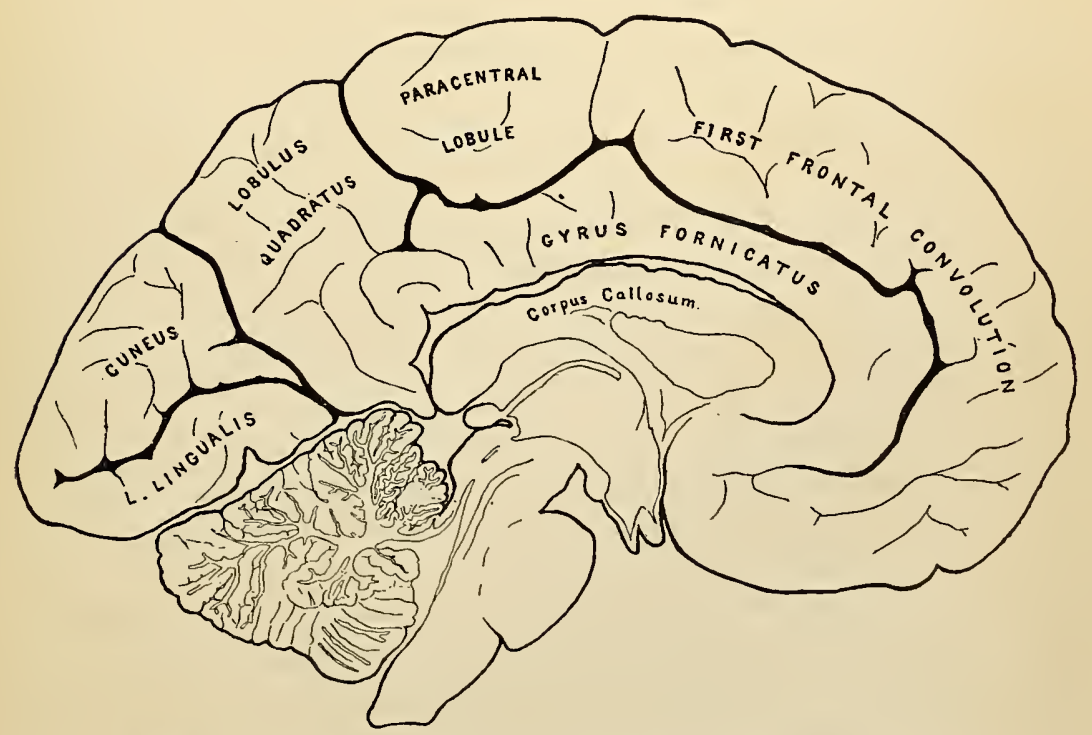

Fig. I40'. - Median section of the brain, reduced about one-third (Dalton). 
3. Association fibres connect different parts of the cortex on the same side. These constitute the greatest part of the white substance.

4. Centripetal fibres, coming from the centripetal tracts of the cord, go to the molecular layer of the cortex.

In addition to the above, there are fibres that connect the cerebrum with the cerebellum, which will be described later.

Cercbral Conolutions. - The cerebrum presents a great longitudinal median fissure by which it is partially divided into two lateral halves, and three great fissures - the fissure of Sylvius, the fissure of Rolando and the parieto-occipital fissure. The lobes of the cerebrum are (I) the frontal lobe, lying in front of and above the fissure of Sylvius and in front of the fissure of Rolando, (2) the parietal lobe, behind the frontal lobe and in front of and above the occipital lobe, (3) the occipital lobe, and (4) the temporo-sphenoidal lobe. The parietal lobe is bounded in front by the fissure of Rolando and below by the fissure of Sylvius and the parieto-occipital fissure (shown in Fig. I34). The occipital lobe lies below the parieto-occipital fissure. The temporo-sphenoidal lobe is situated below the fissure of Sylvius and in front of the occipital lobe.

While the convolutions are not exactly the same in all human brains, or even in both sides of the brain, their arrangement and relations may be described in a general way with sufficient accuracy to enable one to recognize easily the most important physiological points in the descriptive anatomy of the cerebral surface.

The first frontal convolution is bounded internally by the great longitudinal fissure and externally by a shallow fissure nearly parallel to the longitudinal fissure. The second frontal convolution lies next the first frontal convolution, and is bounded externally by two shallow fissures lying in front of the fissure of Sylvius. The third frontal convolution curves around the short branch of the fissure of Sylvius. On either side of the fissure of Rolando are the ascending frontal convolution and the ascending parietal convolution. Curving around the posterior extremity of the fissure of Sylvius, is the supramarginal convolution, which is continuous with the first temporal convolution, the latter lying behind and parallel to the fissure of Sylvius. Internal to the posterior portion of the intraparietal sulcus is the angular convolution, which is continuous with the second temporal convolution. At the inferior border of the temporo-sphenoidal lobe, below the first and second temporal convolutions, is the third temporal convolution. The superior parietal convolution lies by the side of the median fissure and is the posterior continuation of the first frontal convolution. The situation of the occipital convolutions is indicated in Fig. I39. In addition to these convolutions on the general surface of the cerebrum, there 
are convolutions on the surface of the base of the brain and in the gray matter by the sides of the great longitudinal fissure. In the fissure of Sylvius, near its ascending branch, between the anterior and the posterior lobes of the brain and beneath the third frontal convolution, is a group of convolutions constituting the island of Reil, or insula.

Fig. I 40 shows the most important parts observed on the inner surface of the left hemisphere. These parts do not demand any explanation beyond that given in the diagram in outline.

Basal Ganglia. - The ganglia at the base of the brain are the olfactory ganglia, the corpora striata, optic thalami, tubercula quadrigemina and the gray matter of the pons Varolii. The olfactory ganglia will be described in connection with the physiology of the sense of smell. The corpora striata and the optic thalami are important in their relations to the internal capsule and the paths of motor and sensory conduction.

Corpora Striata, Optic Thalami and Internal Capsule. - The corpora striata are pear-shaped bodies, situated at the base of the brain, with their rounded bases directed forward, and the narrower ends, backward and outward. Their external surface is gray, and they present, on section, alternate strix of white and gray matter. They present what is called an intraventricular portion, projecting into the anterior part of the lateral ventricles, and an extraventricular portion, which is embedded in the white substance at the base of the brain. The corpora striata are to be regarded as subsidiary centres connected with motion.

The optic thalami are oblong bodies situated between the posterior extremities of the corpora striata and resting upon the crura cerebri on the two sides. These are white externally, and in their interior they present a mixture of white and gray matter. They are subsidiary nervecentres connected with sensation.

In a horizontal section made through the brain, involving the corpora striata and the optic thalami, the corpora striata present a division into two nuclei. These are the caudate nucleus, which is internal, and the lenticular nucleus, which is external to and behind the caudate nucleus. External to the lenticular nucleus, is a layer of white substance, the external capsule, in which there is a band of gray matter, called the claustrum. External to the external capsule, at its anterior portion, is the insula, or island of Reil.

Between the caudate nucleus and the lenticular nucleus in front, is a broad band of white fibres, continuous with a band of white fibres lying posteriorly, between the lenticular nucleus and the optic thalamus on either side. This band is the internal capsule. The portion of the internal capsule that lies between the caudate nucleus and the lenticu- 
lar nucleus is called its anterior division; and the portion situated between the lenticular nucleus and the optic thalamus is its posterior

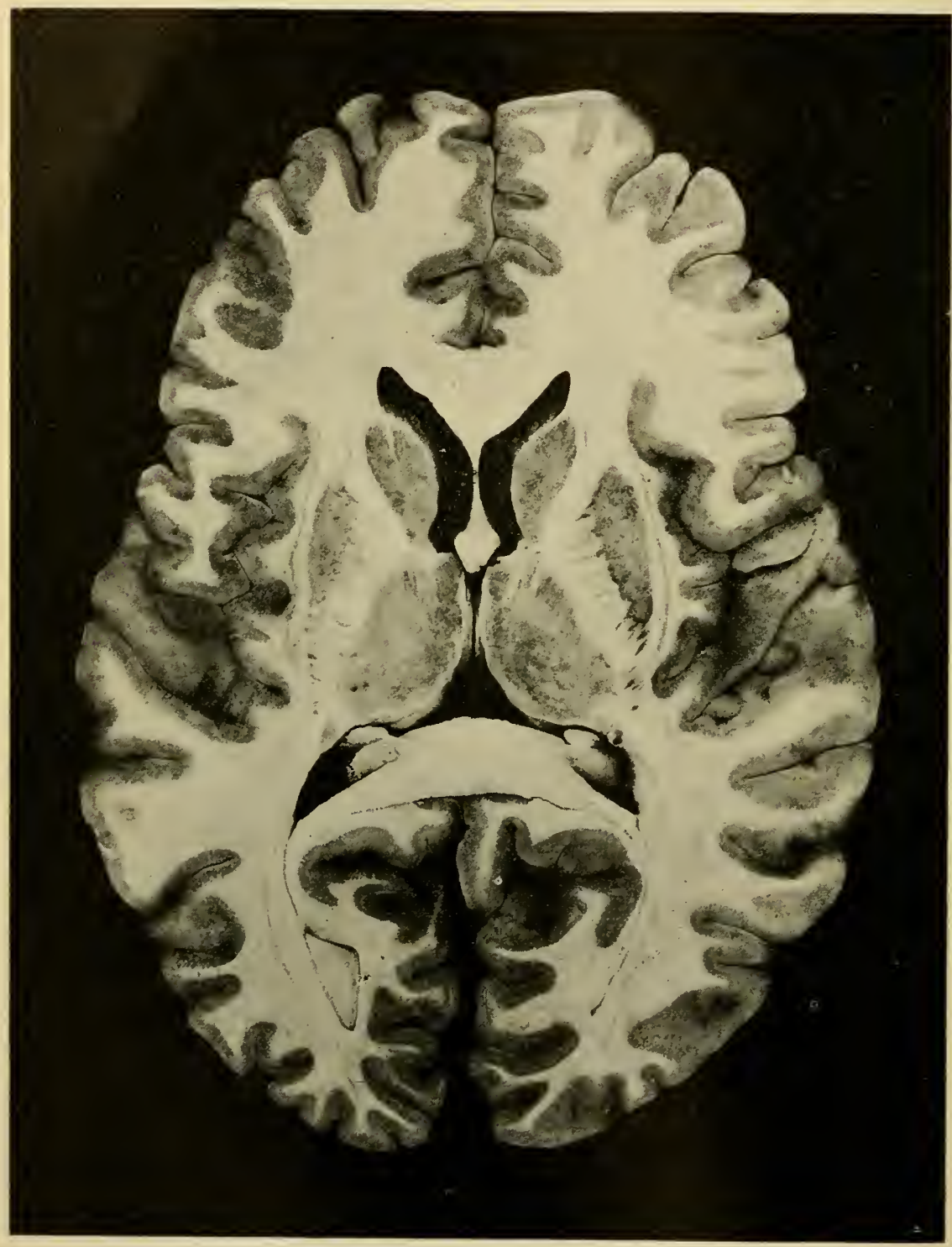

Fig. 141. - Horizontal section of the brain, reduced about one-quarter (Dalton).

division. The bend where the posterior division joins the anterior division is called the knee of the capsule.

The directions of the fibres of the internal capsule are in general 
terms the following: Fibres from the crura cerebri go directly into the corpora striata in front and into the optic thalami behind. This is the course of the greatest part of the fibres, but some fibres go directly

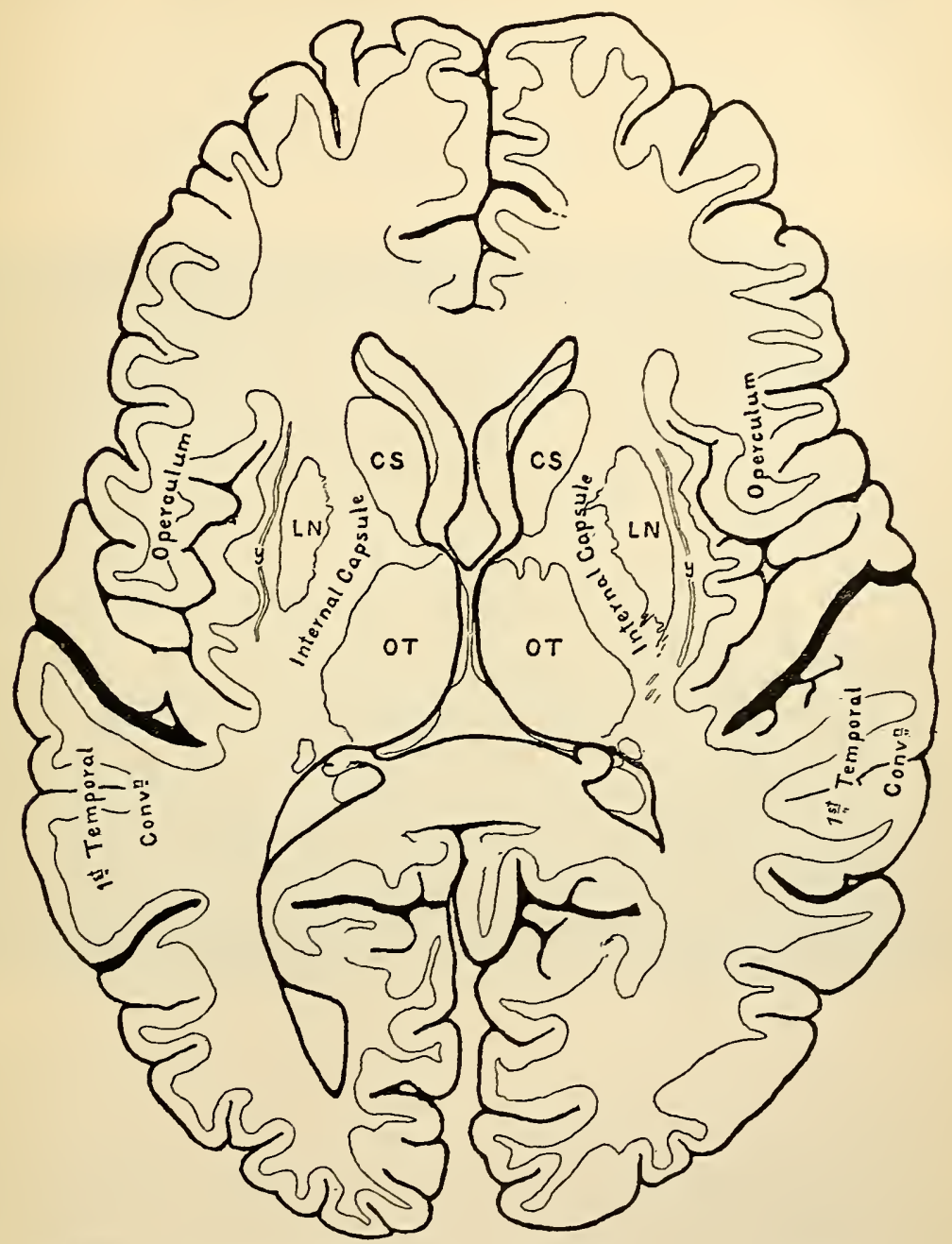

Fig. I4I'. - Horizontal section of the brain, reduced about one-quarter (Dalton).

CS, corpus striatum (caudate nucleus); LN, lenticular nucleus; OT, optic thalamus.

through the internal capsule, and thence to the gray matter of the cerebral convolutions. Most of the fibres, however, which form the internal capsule come from the gray matter of the corpora striata and optic thalami and curve outward and upward to go to the gray matter of the hemispheres. As they pass from the internal capsule 
to the internal surface of the cerebral convolutions, they form the corona radiata.

In the human subject, lesions affecting the anterior two-thirds of the posterior division of the internal capsule produce paralysis of motion only and are followed by descending degenerations. The fibres in this part are connected with the corpora striata. Lesions affecting both the anterior two-thirds and the posterior third of the posterior division of the internal capsule produce paralysis of motion and sensation. The fibres in the posterior third are connected with

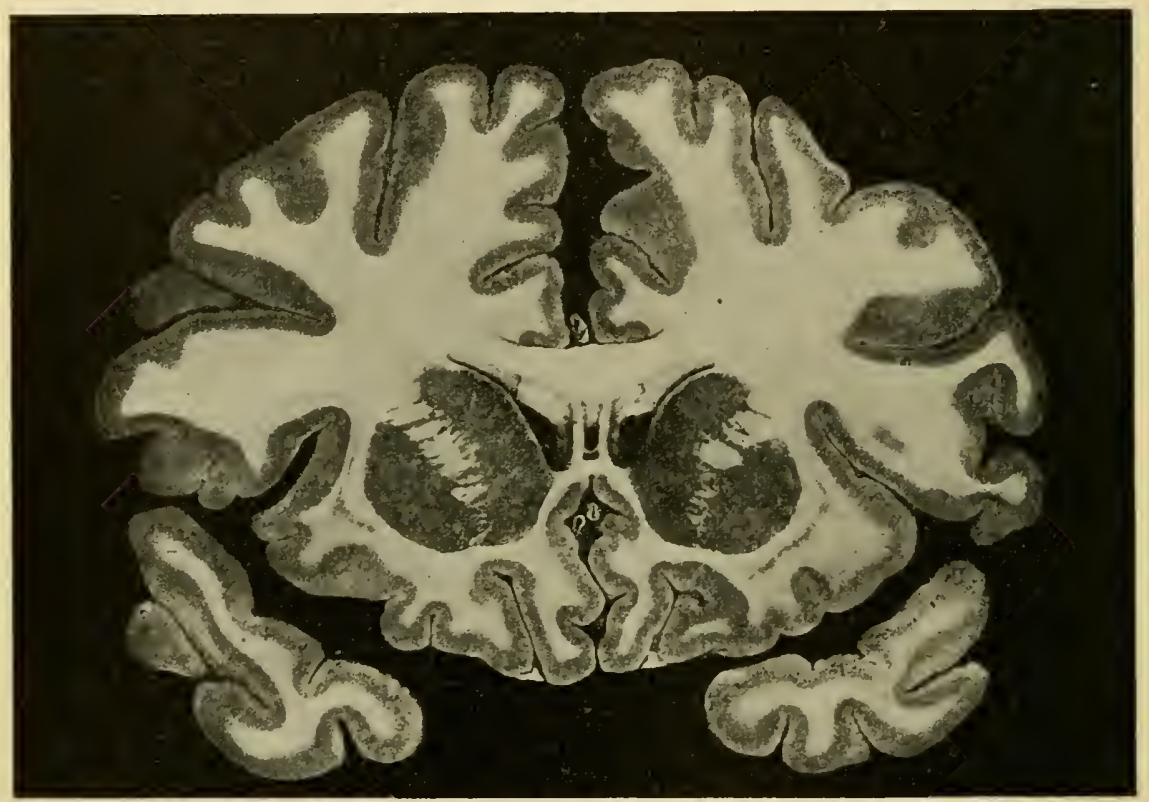

Fig. 142. - lertical section of the brain, reduced about one-quarter (Dalton).

the optic thalami. Ascending degenerations have not been observed in the fibres of the cerebrum.

Tubercula Quadrigemina. - These little bodies, sometimes called the optic lobes, are rounded eminences, two on either side, situated just below the third ventricle. The anterior, called the nates, are the larger. These are oblong and of a grayish color externally. The posterior. called the testes, are situated just behind the anterior. They are rounded and are rather lighter in color than the anterior. Both contain gray matter in their interior. They are the main points of apparent origin of the optic nerves and are connected by commissural fibres with the optic thalami. In birds the tubercles are two in number, instead of four, and are called tubercula bigemina. 
Crura Cerebri. - The crura are short, thick, rounded bands which pass from the cerebral hemispheres to the upper border of the pons Varolii. They are rather broader above than below and are about threequarters of an inch (19 millimeters) in length. They are composed of longitudinal white fibres connecting various parts with the cerebrum. Each crus is divided into a superficial and a deep band, by a layer of gray substance called the locus niger. The locus niger contains small multipolar nerve-cells and abundant pigmentary granules. The lower, or superficial band of the crus is called the crusta. The deep band is

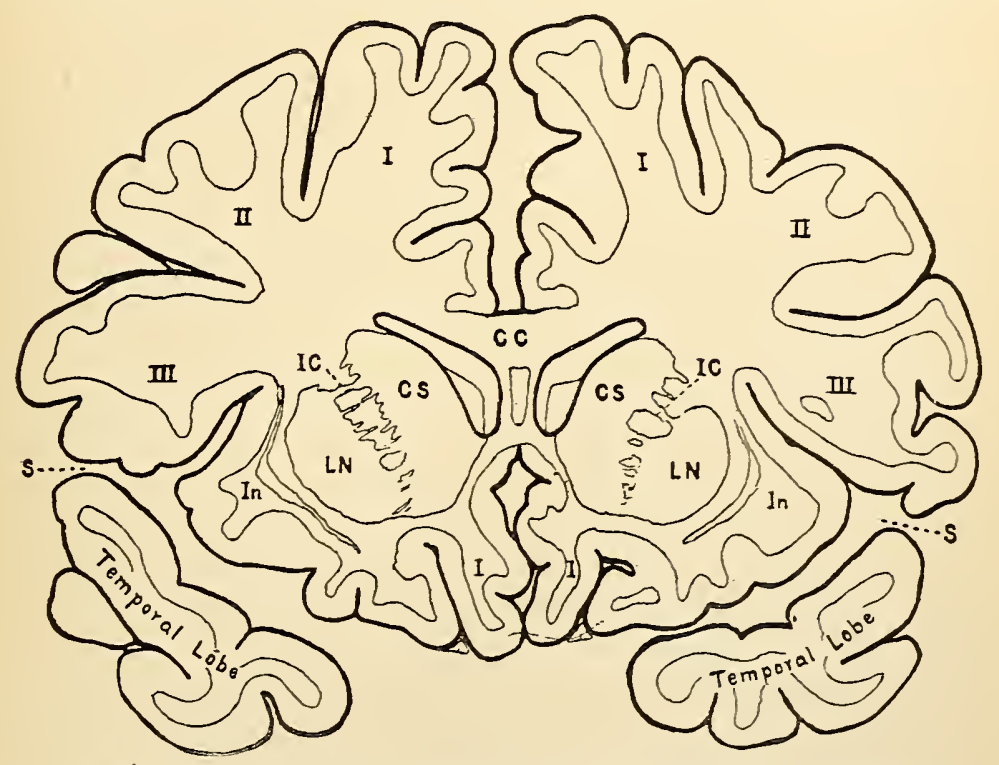

Fig. I42'. - Vertical section of the brain, reduced about one-quarter (Dalton).

I, I, I, I, first frontal convolution; II, II, second frontal convolution; III, III, third frontal convolution; $C C$, corpus callosum; $C S$, corpus striatum; $I C$, internal capsule; $L N$, lenticular nucleus; $I n$, insula; $S$, fissure of Sylvius.

called the tegmentum. The crusta consists of white fibres only. In the tegmentum the fibres are mixed with masses of gray matter.

Pons Varolii. - The pons Varolii, called the tuber annulare, cr the mesocephalon, is situated at the base of the brain just above the bulb. It is white externally and contains in its interior a large admixture of gray matter. It presents both transverse and longitudinal white fibres. Its transverse fibres connect the two halves of the cerebellum. Its longitudinal fibres are connected below with the anterior pyramidal bodies and the olivary bodies of the bulb, the lateral columns of the cord and a certain portion of the posterior columns. The fibres are connected above with the crura cerebri and pass to the brain. 
If the cerebral hemispheres, the olfactory ganglia, the optic lobes, the corpora striata and the optic thalami are removed, the animal loses the special senses of smell and sight and the intellectual faculties, there is a certain degree of enfeeblement of the muscular system, but voluntary motion and general sensibility are retained. So far as voluntary motion is concerned, an animal operated on in this way is nearly in the condition of one simply deprived of the cerebral hemispheres. There are no voluntary movements that show any degree of intelligence, but the animal can stand, and various consecutive movements are executed, which are different from the simple reflex acts depending exclusively on the spinal cord. The coördination of movements is perfect unless the cerebellum has been removed. As regards general sensibility, an animal deprived of all the encephalic ganglia except the pons Varolii and the bulb undoubtedly feels pain. In rabbits, rats and other animals, after removal of the cerebrum, corpora striata and optic thalami, pinching of the ear or foot is immediately followed by prolonged and plaintive cries. Physiologists have insisted on the character of these cries as indicating actual perception of painful impressions and as different from cries that are purely reflex. Voluntary movements and cries are observed in persons subjected to painful surgical operations, when incompletely under the influence of an anesthetic, concerning the character of which there can be no doubt. The movements are voluntary, and the cries are evidence of the acute perception of pain ; but such patients have no recollection of any painful sensation. So far as can be judged from what is known of the action of the encephalic centres, the pain under these conditions is perceived by some nerve-centre, probably in the pons Varolii, but the impression is not conveyed to the cerebrum and is not recorded by the memory.

Taking all the experimental facts into consideration, the following seems to be the most reasonable view in regard to the action of the pons Varolii as a nerve-centre:-

It is an organ capable of originating impulses that give rise to voluntary movements, when the cerebrum, corpora striata and the optic thalami have been removed; and probably it regulates the automatic voluntary action in station and progression. Many voluntary movements, the result of intellectual effort, are made in obedience to impulses transmitted from the cerebrum, through conducting fibres in the pons Varolii, to the cord and the general motor nerves.

The gray matter of the pons Varolii is also capable of receiving painful impressions, which, when all the encephalic ganglia are intact, are conducted to and are perceived by the cerebrum and are 
remembered; but there are distinct evidences of the perception of pain, even when the cerebrum has been removed.

Directions of the Fibres in the Cerebrum. - Fibres pass from the cerebral hemispheres to the cerebellum. Commissural fibres connect the cerebrum and certain of the basal ganglia on the two sides. Fibres connect the gray matter of cerebral convolutions on the same side. Fibres pass from the inner surface of the gray matter of the cerebrum to the internal capsule, corpora striata, optic thalami and pons Varolii, the bulb and thence to the spinal cord. The directions of these four sets of fibres have been quite accurately described.

I. Fibres connecting the Cerebrum with the Cerebellum.-(A) Fibres from the gray matter of the frontal lobe, in front of the anterior central convolution, pass through the anterior division of the internal capsule and thence through the inner portion of the outer layer of the crus cerebri (crusta) to the pons, where they seem to go to the cells of the gray matter, forming synapses. From the pons, fibres go to the lateral and posterior regions of the cerebellum on the opposite side. (B) Fibres from the occipital and temporo-sphenoidal lobes pass in the outer portion of the crusta and go to the upper portion of the cerebellum, near the middle lobe. This connection probably is crossed. (C) Above the pyramidal tract of the crusta, is a small tract of fibres which connects the caudate nucleus of the corpus striatum, through synapses in the pons, with the cerebellum on the opposite side (Gowers).

2. Fibres connecting the Two Sides of the Brain.-(A) Fibres from the inner surface of the gray matter of the convolutions pass from one side to the other, through the corpus callosum, and connect the two hemispheres. These are the transverse fibres of the corpus callosum. (B) Fibres from the gray matter of the temporo-sphenoidal lobes pass through the corpora striata to the anterior commissure. These connect the temporo-sphenoidal lobes, and probably the corpora striata, on the two sides. (C) Fibres from the deeper portion of the crus cerebri (tegmentum) pass to the optic thalamus on either side and thence to the temporo-sphenoidal lobes. They form the posterior commissure and connect the temporo-sphenoidal lobes and the optic thalami of the two sides.

3. Fibres connecting Different Cerebral Convolutions on the Same Side (Association Fibres). - (A) Arcuate fibres, passing from one convolution to another, connect adjacent convolutions. (B) Longitudinal fibres connect distant convolutions. The fibres of the fornix connect the optic thalamus with the hippocampus major and the uncinate gyrus. Fibres in the corpus callosum connect the anterior and posterior extremities of the gyrus fornicatus. These are the longitudinal fibres of the corpus callosum. Other longitudinal fibres, connecting parts more or less dis- 
tant, are found in the tenia semicircularis, the uncinate fasciculus, the fillet of the gyrus fornicatus and the inferior longitudinal fasciculus. The last-mentioned fasciculus connects the gray matter of the temporo-sphenoidal and occipital lobes. ${ }^{1}$

4. Fibres connecting the Brain with the Spinal Cord. - Arising from the internal concave surface of the cortical substance of the cerebrum, converging fibres, at first running side by side with the curved commissural fibres, separate as the latter curve backward to pass again to the cortical substance, and are directed toward the corpora striata and the optic thalami. The limits of the irregular planes of separation of the commissural and the converging fibres contribute to form the boundaries of the ventricular cavities of the brain. In studying the course of the converging fibres arising from all points in the concave surface of the cerebral gray matter, it is found that they take various directions. The fibres from the anterior region of the cerebrum pass backward and form distinct fasciculi which converge to the gray substance of the corpora striata. The fibres from the middle portion converge regularly to the middle region of the external portions of the optic thalami. The fibres from the posterior portion pass from behind forward and are distributed in the posterior portion of the optic thalami. The fibres from the convolutions of the hippocampi and the fascia dentata are lost in the gray substance lining the internal borders of the optic thalami. In the course of most of these fibres toward the corpora striata and the optic thalami, they pass through the internal capsule.

The fibres from the middle and anterior portions of the cerebrum, especially the middle portion, contribute largely to the formation of the anterior two-thirds of the posterior division of the internal capsule. The fibres from the posterior portion of the cerebrum are found in the posterior third of the posterior division of the internal capsule. The middle and anterior fibres undergo descending degenerations following lesions of motor areas. A few of the converging fibres from the hemispheres pass directly through the internal capsule and have no connection with the corpora striata and optic thalami.

1 Recent researches by Flechsig, made by a new process called the embryological method, though incomplete, probably will lead to important results. It has been ascertained that various tracts of fibres receive medullary sheaths at different stages of intra-uterine development, the axis-cylinders appearing first without coverings. As the medullary substance appears, the fibres are said to become "myelinated." Association fibres are myelinated later than projection fibres, the latter receiving the sheath at about the fifth month. In studying embryos by this method, Flechsig assumes to have demonstrated the exact course of various association tracts. and their connections with "association centres." The theoretical deduction from these observations are interesting, but can not as yet be accepted as conclusive. They relate largely to the voluntary action of groups of muscles following sensory impressions of various kinds, cutaneous, auditory, visual, etc. 
From the internal capsule, the fibres pass in the crus cerebri to the upper border of the pons Varolii. The motor.fibres pass through the pons as longitudinal fibres, go to the anterior pyramids of the bulb, where most of them decussate, and thence to the pyramidal tracts of the spinal cord. The sensory fibres go to the posterior part of the cord. The converging cerebral fibres are reënforced, in their downward course, by fibres from the tubercular quadrigemina and the gray matter of the pons Varolii. Certain fibres go to the olivary bodies in the bulb.

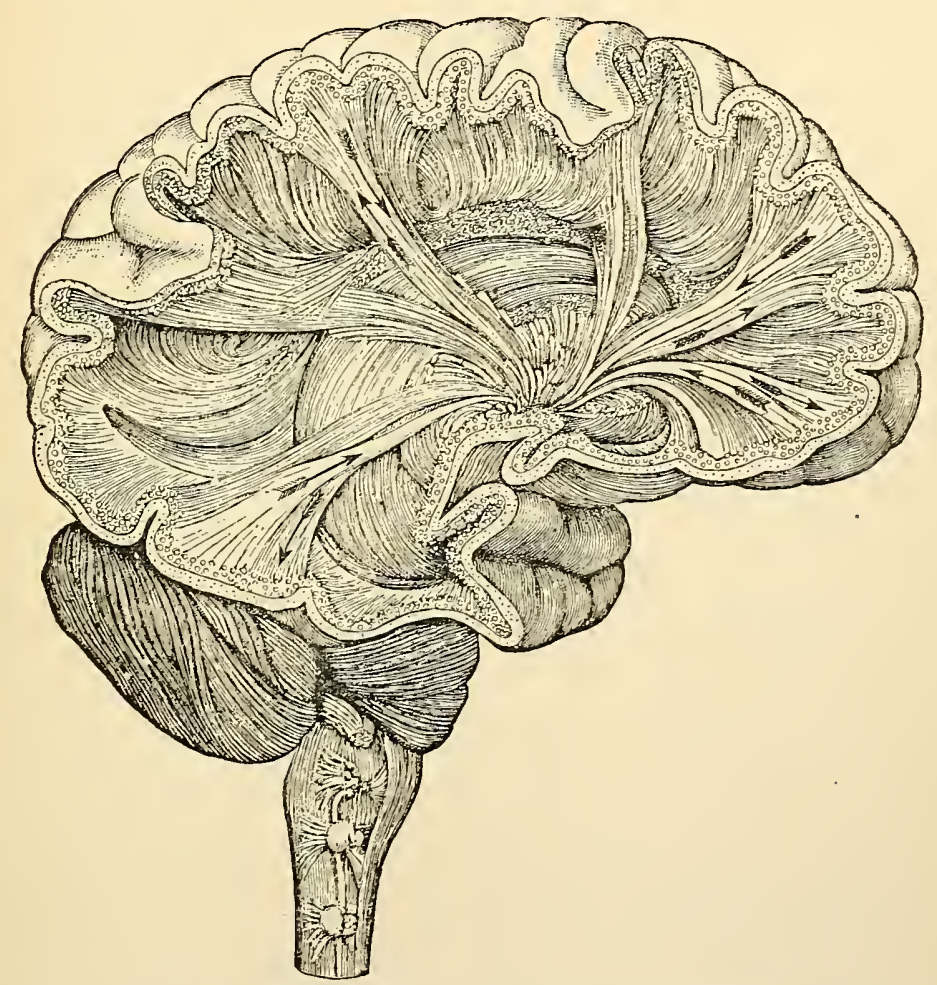

Fig. 143. - Diagrammatic representation of the direction of some of the fibres in the cerebrum (Le Bon).

A more extended description of these fibres will be given in connection with the physiological anatomy of the bulb. As fibres pass through parts containing nerve-cells, they often are "reënforced" in a way that is somewhat obscure. It is probable, however, that this is by fibres originating in arborescent filaments (synapses) surrounding the cells.

Cerebral Localization. - The observations of Flourens (I822 and I823) and his immediate followers, which seemed to show that the cerebrum was neither excitable nor sensible to direct stimulation, have been so completely contradicted by the experiments of Fritsch and 
Hitzig (I870), Ferrier, Munk, Horsley and many others, that the question of the existence of motor and sensory centres - especially motor centres - hardly admits of discussion. The negative results obtained by Flourens were probably due to severe hemorrhage, which, according to Ferrier, rapidly destroys the excitability of the motor cortical areas. Some of the experiments of Goltz, by which it was attempted to prove that circumscribed and invariable motor areas do not exist, are answered by observations showing descending secondary degenerations following injury of certain parts of the cerebral cortex. The earlier observations on cerebral localization were made on dogs. Later, experiments have been made on monkeys, and the results of these have been to a certain extent confirmed by pathological observations on the human subject. Beginning with the observations in which descending degenerations have been noted as a consequence of destruction of parts of the cerebral cortex, it may be assumed that distinct areas exist which preside over certain localized muscular movements.

Motor Cortical Zone (Rolandic Arca). - The motor cortical zone is on either side of the fissure of Rolando. It usually is described as including the ascending frontal and ascending parietal convolutions (see Fig. I 39), and the paracentral lobule (see Fig. I40). Faradization of parts in and adjacent to this zone is followed by localized muscular movements. In fact, the motor areas seem to be subject to nearly the same laws, as regards their reactions to faradic stimulation, as are the motor nerves. Forty faradic shocks per second produce a corresponding number of single muscular contractions. Forty-six shocks per second produce a tetanic contraction (Franck and Pitres). Destruction of motor areas is followed by partial loss of power in certain sets of muscles and by secondary descending degeneration of nerve-fibres extending through the corona radiata, the internal capsule, the crura cerebri, the anterior pyramids of the bulb and finally the pyramidal tracts of the spinal cord.

While it can not be doubted that the Rolandic area is made up of a number of motor centres, it is now thought by many physiologists that it receives sensory fibres from the skin and the muscular system; and therefore it is sometimes called the sensori-motor area. The physiological basis of this view is the close association of sensations and voluntary movements; and it has been proved that extirpation of the Rolandic area in monkeys is followed by impaired sensibility. Recent anatomical researches, made by means of the staining methods of Golgi and Ramon y Cajal, seem to favor this idea, although the tracing of the fibres presents difficulties which render the results somewhat unsatisfactory. 
It remains now to locate the distinct motor areas. This has been done on the brain of the monkey by Ferrier, Horsley and others, who have applied their observations as nearly as possible to the human brain. While the divisions now recognized can not be taken as absolute, experiments on monkeys have been followed by results so nearly constant, that the most important localizations may be accepted as correct.

r. The centre for movements of the upper extremities is on the outer surface of the brain and includes the superior portion of the ascending frontal convolution and nearly all the ascending parietal con-

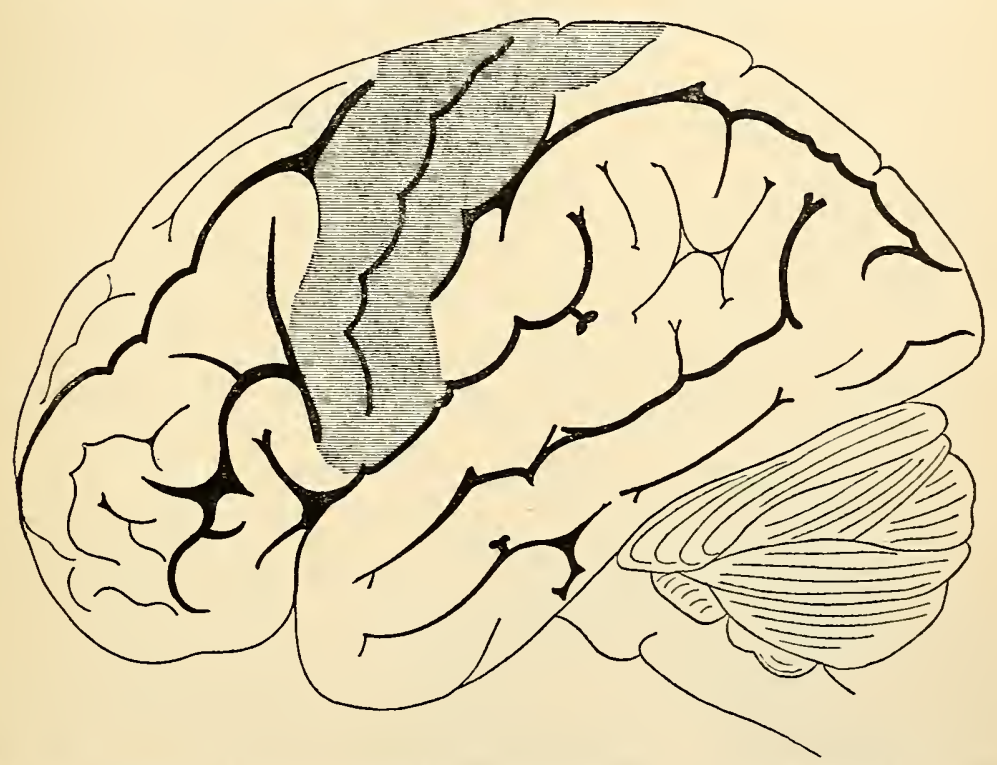

Fig. I44.-Motor cortical zone (Rolandic area) on the outer surface of the cerebrum (Exner).

volution, except the very uppermost portion of these convolutions, next the great longitudinal fissure.

2. The centre for movements of the leg and foot is on the mesial surface of the brain, including the paracentral lobule.

3. The centre for movements of the trunk and shoulders is mainly on the mesial surface of the brain, in front of the upper part of the centre for the leg and foot.

4. The centre for movements of the head, eyes and lids is on the outer surface of the brain, including the posterior portion of the first and second frontal convolutions and extending to the mesial surface.

5. The centre for the muscles of the mouth includes the lower portion of the ascending frontal convolution.

The action of all these motor centres is crossed. 
Attempts have been made to subdivide the centres just indicated, but most of these are hardly justified by experimental results, even in monkeys. Still less can it be assumed that more restricted centres can be accurately mapped out in the human subject. It has been thought proper, therefore, to describe in this work those centres only, the localization of which admits of little doubt. It is probable, however, judging from the very recent experiments of Sherrington and Grünbaum

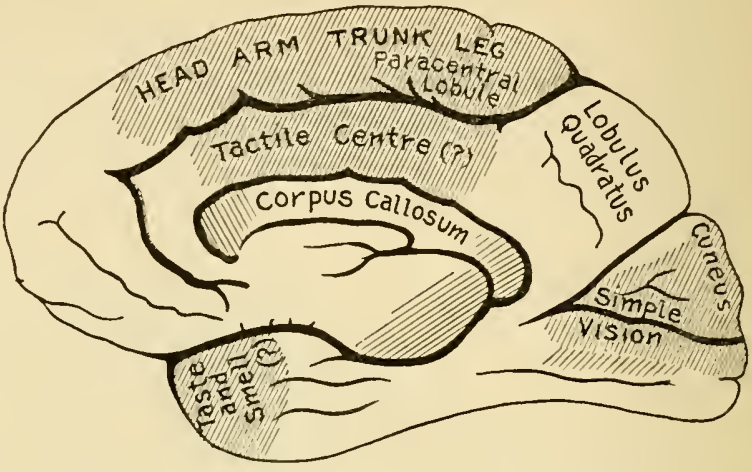

Fig. 145. - Diagram of a median section of the brain. on certain anthropoid apes, that the cortical motor areas are more extensive than has been supposed. The method employed by these observers was to place one electrode of a feeble faradic current on

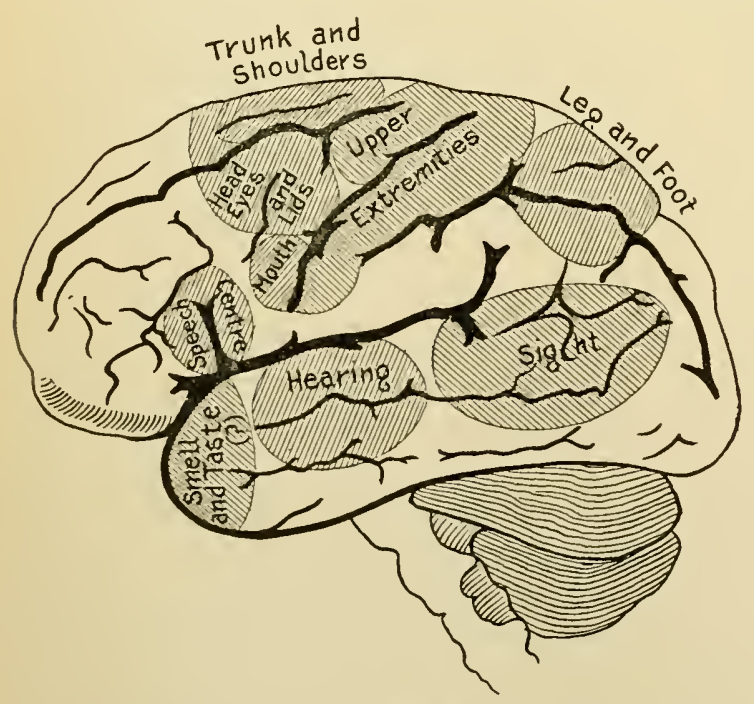

Fig. 146. - Diagram of certain motor cortical areas. certain parts of the exposed cortex and the other on an indifferent point. This was thought to limit the motor areas much more accurately than the older method with the two electrodes applied to the brain.

In man, lesions of parts of the motor-cortical zone produce localized paralysis, or what is called monoplegia, the action being crossed. The following are some of the more common forms of monoplegia that have been observed to attend localized cortical lesions: I, oculomotor monoplegia (isolated ptosis); 2, facial monoplegia, sometimes associated with paralysis of the hypoglossal nerve; 3, brachial monoplegia, or paralysis of the opposite arm; 4, crural monoplegia, or 
paralysis of the opposite leg; 5, brachio-facial monoplegia, or paralysis of the arm and face.

In addition to the motor areas just described, there probably is a group of sensory areas on the mesial surface of the brain that has been called the tactile centre. This is in the gyrus fornicatus, just above the corpus callosum. The centres for the special senses, which will be more fully considered, however, farther on, are the following: olfactory centre and taste centre, at the anterior extremity of the temporal lobe; the simple visual centre, on the mesial surface of the cerebrum above and below the calcarine fissure; the psychical visual centre, on the convex surface of the occipital lobe, behind the auditory centre and on the left side only; the simple auditory centre, in the superior and middle temporal convolutions; the psychical auditory centre, in the superior temporal convolution, on the left side only.

One of the most important of the cerebral centres is the centre for speech, which will be described after the consideration of the general uses of thé cerebral hemispheres.

\section{General Uses of the Cerebrui}

The cortical substance of the cerebral hemispheres not only is capable of generating motor impulses of the kind known as voluntary and of receiving sensory impressions, including those connected with the special senses, but its anatomical and physiological integrity, and its connections, especially with sensory conductors, are essential to what are known as mental operations. The existence of the mind and the possibility of normal operations of the intelligence depend on the existence of the gray matter of the cerebral cortex and its normal physiological condition and relations. Mental operations involve a slight elevation of temperature and slightly increase some of the excretions. It is probable, therefore, that they involve changes of matter; and these changes, if they occur, can be effected only by the cells of the brain. Without defining or analyzing the intellectual faculties or attempting to locate different faculties in special parts, it is sufficient to state that certain of them reside probably in that portion of the brain which is anterior to the motor-cortical zone; that is, in the frontal lobes. These lobes, so far as is known, do not present motor or sensory areas; and recent observations have shown that lesions of the frontal lobes especially are attended with marked impairment of the intellectual faculties and little or no motor disturbance.

The brain and the intellectual power of man are so far superior in their development to this organ and its properties in the lower animals. 
that some psychologists have regarded the human intelligence as distinct in nature as well as in degree. Although physiologists do not commonly accept this proposition, regarding the intelligence of man as simply superior in degree to that of the lower animals, it is evident that this difference in the degree of development is so great as to render the human mind hardly comparable with the intellectual attributes of animals low in the scale. Still, there can be no doubt in regard to the identity of the nature of the faculties of the brain in man and in some of the lower animals, however much these faculties may differ in their development. If this proposition be true, it is reasonable to apply experiments on the brain in the lower animals to the physiology of corresponding parts in the human subject.

Extirpation of the Cerebrum. - Experiments on different classes of animals show clearly that the brain is less important, as regards the ordinary manifestations of animal life, in proportion as its relative development is smaller. For example, if the cerebral hemispheres are removed from fishes or reptiles, the movements that are called voluntary may be but little affected; while if the same mutilation is performed in birds or certain of the mammalia, the diminished power of voluntary motion is much more marked. It would be plainly unphilosophical to assume, because a fish or a frog will swim in water and execute movements after removal of the hemispheres very like those of the uninjured animal, that their feeble intelligence is not destroyed by the operation. It is not only possible but probable that in the very lowest of the vertebrates, the operations of the nervous centres are not the same as in higher animals. There is, for example, a fish (the lancet-fish, amphioxus lanceolatus) that has no brain, all the functions of animal life being regulated by the gray substance of the spinal cord. It is essential, therefore, in endeavoring to apply the results of experiments on the brain in the lower animals to human physiology, to separate, so far as possible, distinct manifestations of intelligence from automatic acts.

Flourens (I822 and 1823) made a series of observations on the different parts of the encephalon. As regards the cerebral hemispheres, he found that complete removal of these parts in living animals (frogs, pigeons, fowls, mice, moles, cats and $\operatorname{dog} s$ ) was invariably followed by stupor, apparent loss of intelligence and absence of even the ordinary instinctive acts. Animals thus mutilated retained general sensibility and the power of voluntary movements, but were thought to be deprived of the special senses of sight, hearing, smell and taste. As regards general sensibility and voluntary movements, Flourens was of the opinion that animals deprived of their cerebral lobes possessed sensation 
but had lost the power of perception; and that they could execute voluntary movements when an irritation was applied to any part, but had lost the power of making such movements in obedience to a spontaneous effort of the will. One of the most remarkable phenomena observed was entire loss of memory and of the power of connecting ideas. The voluntary muscular system was enfeebled but not paralyzed. Removal of one hemisphere produced, in the higher classes of animals experimented on, enfeeblement of the muscles of the opposite side, but the intellectual faculties were in part or entirely retained.

The observations of Flourens have been repeated by many physiologists, and in the main confirmed, except as regards the special senses. Bouillaud (I 826) made a large number of observations on pigeons, fowls, rabbits and other animals, in which, after removal of the hemispheres, he noted the persistence of the senses of sight and hearing. Longet finally demonstrated the fact that both sight and hearing are retained after extirpation of the hemispheres, even more clearly than Bouillaud, by the following experiments: he removed the hemispheres from a pigeon, the animal surviving the operation eighteen days. When this animal was placed in a dark room and a light was suddenly brought near the eyes, the iris contracted and the animal winked; "but it was remarkable, that when a lighted candle was moved in a circle, and at a sufficient distance, so that there should be no sensation of heat, the pigeon executed an analogous movement of the head." An examination after death showed that the removal of the cerebrum had been complete. An animal deprived of the hemispheres also opened the eyes at the report of a pistol and gave other evidence that the sense of hearing was retained.

In regard to the senses of smell and taste, it is more difficult to determine their presence than to ascertain that the senses of sight and hearing are retained. It is probable, however, that the sense of smell is not abolished, if the hemispheres are carefully removed, leaving the olfactory ganglia intact; and there is no direct evidence that extirpation of the cerebrum affects the sense of taste; indeed, in young cats and dogs, Longet noted evidences of a disagreeable impression following the introduction of a concentrated solution of colocynth into the mouth, as distinctly as in the same animals under normal conditions.

Comparative Development of the Cerebrum in the Lower Animals. - It is necessary only to refer briefly to the development of the cerebrum in the lower animals as compared with the human subject, to show the connection of the hemispheres with intelligence. In man the cerebrum presents a large preponderance in weight over other portions of the encephalon; but in some of the lower animals the cerebrum is even less 
in weight than the cerebellum. In man, also, not only the relative but the absolute weight of the brain is greater than in the lower animals, with but two exceptions. Todd has cited a number of observations made on the brains of elephants, in which the weights ranged between nine and ten pounds (about 4000 and 4500 grams). Rudolphi gave the weight of the encephalon of a whale, seventy-five feet long (about 23 meters), as considerably over five pounds (about 2300 grams). With the exception of these animals, man possesses the largest brain in the zoölogical scale.

Another interesting point in this connection is the development of cerebral convolutions in certain animals, by which the relative quantity of gray matter is increased. In fishes, reptiles and birds, the surface of the hemispheres is smooth; but in many mammals, especially in those remarkable for intelligence, the cerebrum presents a greater or less number of convolutions, as it does in the human subject.

Development of the Cerebrum in Different Races of Men and in Different Individuals. - It may be stated as a general proposition, that in the different races of men, the cerebrum is developed in proportion to their intellectual power; and in different individuals of the same race, the same general rule obtains. Still, this presents marked exceptions. Certain brains in an inferior race may be larger than the average in the superior race; and it is frequently observed that unusual intellectual vigor is coexistent with a small brain, and the reverse. These exceptions, however, do not take away from the force of the original proposition. As regards races, the rule is found to be invariable, when a sufficient number of observations are analyzed; and the same holds true in comparing a large number of individuals of the same race. Average men have an advantage over average women of about six ounces ( 70 grams) of cerebral substance; and while many women are far superior in intellect to many men, such instances are not sufficiently frequent to invalidate the general proposition, that the greatest intellectual capacity and mental vigor is coincident with the greatest quantity of cerebral substance. If the view - which is in every way reasonable - be accepted, that the gray substance alone of the cerebral hemispheres is directly connected with the mind, it would be necessary, in comparing different individuals with the view of establishing a definite relation between brain-substance and intelligence, to estimate the quantity of gray matter; but it is not easy to see how this can be done with any degree of accuracy.

It is undoubtedly true that proper training and exercise develop and increase the vigor of the intellectual faculties, and that thereby the brain is increased in power, as are the muscles under analogous conditions. 
This will perhaps explain some of the exceptions above indicated; but an additional explanation may be found in differences in the quality of brain-substance in different individuals, irrespective of the size of the cerebral hemispheres. One evidence that these differences in the quality of intellectual working matter exist is that some small brains actually accomplish more and better work than some large brains. This may be due to differences in training, to the extraordinary development, in some individuals, of certain qualities, to intensity and pertinacity of purpose, capacity for persistent labor in certain directions, a fortunate direction of the mental efforts, opportunity and circumstances, etc. ; but aside from these considerations, it is probable that there are important individual differences in the quality of nervous matter.

Facial Angle. - It is not necessary to enter into an extended discussion of the relations of the facial angle to intelligence. It was proposed by Camper to take the angle made at the junction of two lines, one drawn from the most projecting part of the forehead to the alveolæ of the teeth of the upper jaw, and another passing horizontally backward from the lower extremity of the first line, as the facial angle. This angle is to a certain extent a measure of the projection of the anterior lobes of the brain. A number of observations on the facial angle in different races has been made by Camper and by other physiologists and ethnologists. These show, in general terms, that the angle is larger in man than in any of the inferior animals and is largest in those races that possess the greatest intellectual development.

Pathological Observations. - It is a fact now generally admitted in pathology, that loss of cerebral substance from repeated hemorrhage is sooner or later followed by impairment of the intellectual faculties. This point frequently is difficult to determine in an individual instance; but an analysis of a sufficient number of cases shows impaired memory, tardy, inaccurate and feeble connection of ideas, abnormal irritability of temper with childish susceptibility to petty or imaginary annoyances, easily-excited emotional manifestations and a variety of phenomena denoting abnormally feeble intellectual power following any considerable disorganization of cerebral substance. In short, pathological conditions of the brain all go to show that the intellectual faculties are directly connected with the cerebral hemispheres.

In idiots the brain usually is of small size, although there are exceptions to this rule. In two cases of adult idiots, reported by Tiedemann, the brain was about one-half of the normal weight. The brain of an idiotic woman, forty-two years of age, reported by Gore, weighed ten ounces and five grains (about 284 grams). It has been observed, also, that the cerebellum is not proportionally diminished in size in idiots 
(Bradley). In one instance reported, the proportion of the cerebellum to the cerebrum was as I to 5.5 . In the healthy adult male of ordinary weight, the proportion is as $\mathrm{I}$ to $8 \frac{4}{7}$. The statements just made in regard to the brains of idiots refer to cases characterized by complete absence of intelligence, and furthermore, probably, by very small development of the body. On the other hand, there are instances of idiocy, the body being of ordinary size, in which the weight of the encephalon is little if any below the average. Lélut has reported several cases of this kind. In one of these, a deaf-mute idiot, fortythree years of age, a little above the ordinary stature, presenting "idiocy of the lowest degree ; almost no sign of intelligence ; no care of cleanliness," the encephalon weighed 48.32 ounces ( 1369.8 grams). Other cases of idiots of medium stature are given, in which the brain weighed but little less than the normal average. In the West Riding Lunatic Asylum Reports, London, 1876 , is a report of the case of a congenital imbecile, aged thirty years, height five feet and eight inches ( 172.7 centimeters), died of phthisis, whose brain weighed $70_{2}^{1}$ ounces (2000 grams). This is heavier than the heaviest normal brain on record. The normal brain-weight is $49 \frac{1}{2}$ ounces (1408.3 grams).

Reaction-time. - The time that elapses between the application of sensory stimulus and its appreciation by the sensorium is known as reaction-time. In experiments with reference to this point, the person observed makes an electric signal when the sensation is perceived. The reaction-time is 0.12 of a second for a shock on the hand, O. I 3 for the forehead, 0.17 for the toe and 0.13 for a sudden noise (Exner). The duration is about 0.16 of a second for impressions made on the nerves of special sense. This is the time of conduction of the impression to the brain, its appreciation by the individual, the generation of the voluntary impulse and the conduction of this impulse to the muscles concerned in making the signal. It probably is subject to variations analogous to those observed in the "personal equation."

Centre for the Expression of Ideas in Language. - The location of this centre depends entirely on the study of cases of disease in the human subject. It is evident that there must be a comprehension of the significance of words, the formation of an idea more or less complex and a coördinate action of the muscles concerned in speech as conditions essential to expression in spoken language. One or more of these conditions may be absent in cases of disease ; and the general absence of the power of verbal expression, when this depends on cerebral lesion, is known as aphasia. This is quite different from aphonia, which is simply loss of voice. If the comprehension of the meaning of words is absent, the individual is incapable of receiving ideas expressed in 
language. In cases of aphasia it often is difficult to determine this point. In certain cases it is possible that the individual may understand what is said and may form ideas to which he is unable to give verbal expression. In such instances he can neither speak nor write. There are certain cases in which written or printed words convey no idea, while spoken words are understood, but there is no loss of intelligence and words are spoken without difficulty. This condition is called word-blindness. If there is simple want of coördination of the muscles concerned in speech, words are spoken that may have no connection with the idea to be conveyed, but the individual may be able to express himself in writing. This condition is known as ataxic aphasia. The inability to express ideas in writing is called agraphia, and this usually is an indication of the condition known as amnesic aphasia, in which it is impossible to put ideas into words in any way. In this condition there is loss of memory for words, as its name implies. Persons affected with purely ataxic aphasia may understand and write perfectly, but they can not read aloud or repeat words or sentences spoken to them. In cases of simple amnesic aphasia, patients can sometimes repeat dictated words. In cases in which hemiplegia is marked, the aphasia usually is ataxic. In cases in which there is no hemiplegia, the aphasia usually is amnesic. The ataxic and amnesic forms of aphasia may be combined. A full description, however, of the many and varied forms of aphasia would be out of place in this work.

In 1766 Pourfour du Petit reported a case of aphasia, with lesion of the left frontal lobe of the cerebrum, in which the patient could pronounce nothing but " non."

Marc Dax ( 1836 ) indicated loss or impairment of speech in one hundred and forty cases of right hemiplegia. These observations attracted little attention, until I86r, when the subject was studied by Broca. Since then, many cases of aphasia with lesion of the left frontal lobe have been reported by various writers. In I 863 M. G. Dax, a son of Marc Dax, limited the lesion to the middle portion of the left frontal lobe. It was further stated by Broca and Hughlings Jackson to be that portion of the brain nourished by the left middle cerebral artery (the inferior frontal branch). According to recent observers, the most frequent lesion is in the parts supplied by the left middle cerebral artery, particularly the lobe of the insula, or the island of Reil ; and it is a curious fact that this part is found only in man and monkeys, being in the latter but slightly developed.

While the cerebral lesion in aphasia involves the left frontal lobe in the great majority of cases, there are instances in which the right lobe alone is affected, and these occur in left-handed persons. Aside from 
the anatomical arrangement of the arteries, which seem to furnish a greater quantity of blood to the left hemisphere, it is evident that so far as voluntary movements are concerned, the right hand, foot, eye etc., are used in preference to the left, and that the motor operations of the left hemisphere are superior in activity to those of the right. Bateman has quoted two cases of aphasia dependent on lesion of the right side of the brain, and consequent left hemiplegia, in which the persons were left-handed; and these, few as they are, are important, as showing that a person may use the right side of the brain in speech, as in the other motor acts.

Broadbent has reported an examination of the encephalon of a deaf and dumb woman. In this case the brain was found to be of about the usual weight, but the left third frontal convolution was of " comparatively small size and simple character."

Taking into consideration all the pathological facts bearing on the question, it seems certain that in the great majority of persons the organ or part presiding over the faculty of language is situated on the left side, at or near the third frontal convolution and the island of Reil, mainly in the parts supplied by the middle cerebral artery. In some few instances the organ seems to be in the corresponding part on the right side. It is possible that originally both sides preside over speech, and the superiority of the left side of the brain over the right and its more constant use by preference in right-handed persons may lead to a gradual abolition of the action of the right side of the brain, in connection with speech, simply from disuse. This view, however, is purely hypothetical. In some cases of aphasia from lesion of the speechcentre in the left hemisphere, recovery takes place, and occasionally "speech has been again lost when a fresh lesion occurred in this part of the right hemisphere" (Gowers). In the ataxic form of aphasia, the idea and memory of words remain, and there is loss of speech simply from inability to coördinate the muscles concerned in articulate language. Patients affected in this way can not speak but can write with ease and correctness. In the amnesic form of the disease, the idea and memory of language are lost; patients can not speak, and are affected with agraphia, or inability to write. The motor tracts from the centre for speech pass to the anterior portion of the posterior division of the internal capsule and thence through the left crus, into the pons Varolii, where they decussate and go to the right side of the bulb.

Recently the curious fact has been noted that in the deaf and dumb, who use a sign-language made with the fingers, lesion of the speechcentre is attended with aphasia, in which the power of expressing ideas by means of the sign-language is lost. 


\title{
CHAPTER XXIII
}

\author{
THE CEREBELLUM AND THE BULB
}

The cerebellum - Physiological anatomy - Extirpation of the cerebellum - Pathological ob. servations - The bulb - Physiological anatomy - Uses of the bulb - Nerve-centres in the bulb - Respiratory centre - Vital point (so called) - Rolling and turning movements following injury of certain parts of the encephalon (forced movements).

\section{The Cerebellum}

A FULL description of the anatomy of the cerebellum is not necessary to a comprehension of its uses, so far as these are known. The points, in this connection, that are most important are the following: the division of the substance of the cerebellum into gray and white matter; the connections between the cells and the fibres; the connections of the fibres with the cerebrum and with certain prolongations of the columns of the spinal cord through the bulb; and the passage of

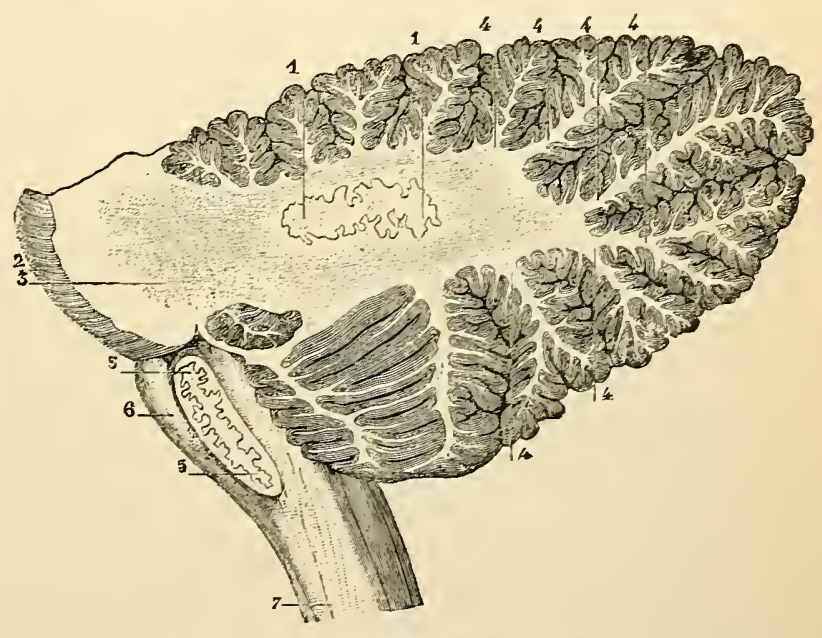

Fig. 147. - Cerebellum and bulb (Hirschfeld).

I, I, corpus dentatum; 2 , pons Varolii; 3 , section of the middle peduncle; $4,4,4,4,4,4$, laminæ forming the arbor vitæ; 5, 5, olivary body of the bulb; 6 , anterior pyramid of the bulb; 7 , upper extremity of the spinal cord.

fibres between the two lateral lobes. These are the only anatomical points that will be considered.

Physiological Anatomy. - The cerebellum, situated beneath the occipital lobes of the cerebrum, weighs about 5.25 ounces (I 48.8 grams) in the male, and 4.7 ounces ( 135 grams) in the female. The proportionate weight to that of the cerebrum is as $I$ to $8 \frac{4}{7}$ in the male, and as I to $8 \frac{1}{4}$ in the female. It is separated from the cerebrum by a strong 
process of the dura mater, called the tentorium. Like the cerebrum, the cerebellum presents an external layer of gray matter, the interior being formed of white, or fibrous nerve-tissue. The extent of the gray substance is much increased by convolutions and by the penetration from the surface of arborescent processes of gray matter. Near the centre of each lateral lobe, embedded in the white substance, is an

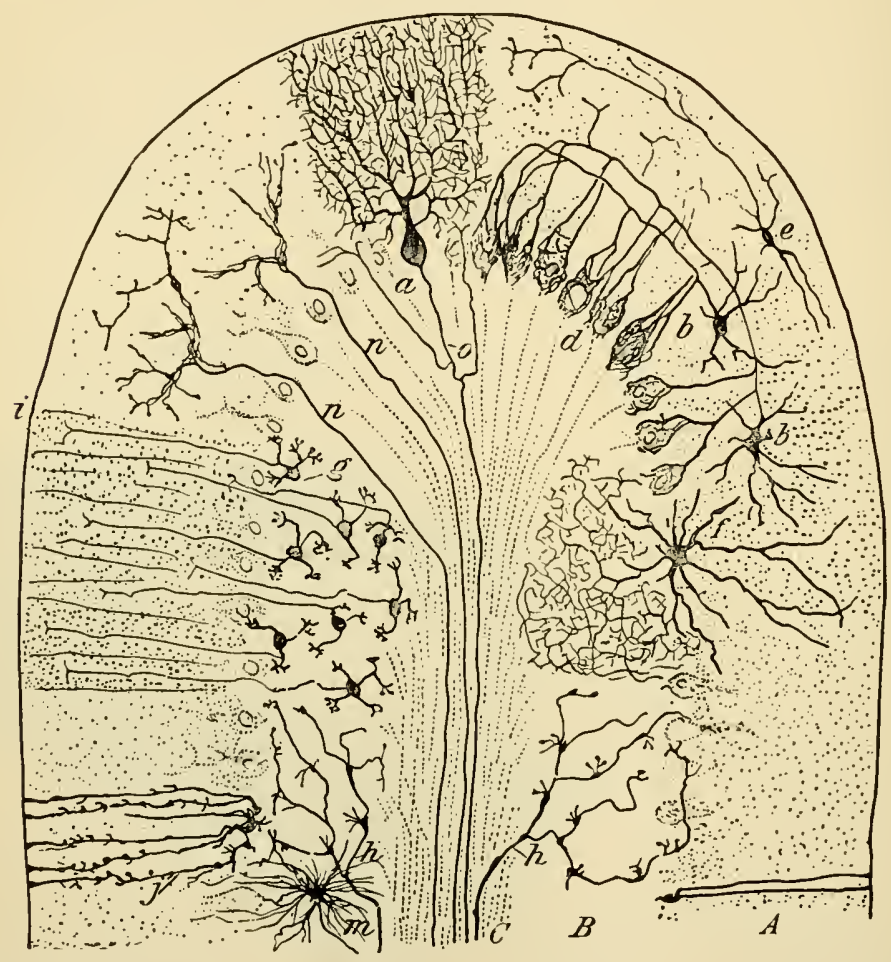

Fig. 148. - Section of a cerebellar lamina perpendicular to its axis (R. y Cajal).

$A$, molecular layer; $B$, granular layer; $C$, white substance; $a$, cell of Purkinje; $o$, its neurite with two collaterals; $b, b$, stellate cells of the molecular layer; $d$, basket-like arrangement of teloneurites around a cell of Purkinje ; $e$, superficial stellate cell; $f$, large stellate cell of the granular layer; $g$, small stellate cell of the granular layer; $h$, centripetal neurite; $n$, centripetal neurite distributed in the molecular layer; $j, m$, neuroglia. The arborescent dendrites of one only of the cells of Purkinje are represented in the figure.

irregularly-dentated mass of gray matter, called the corpus dentatum. The convolutions are finer and more abundant and the gray substance is deeper in the cerebellum than in the cerebrum. These convolutions, also, are present in many of the inferior animals in which the surface of the cerebrum is smooth.

The cerebellum consists of two lateral hemispheres, more largely developed in man than in the inferior animals, and a median lobe. The 
hemispheres are subdivided into smaller lobes, which it is unnecessary to describe. Beneath the cerebellum, bounded in front and below by the bulb and pons Varolii, laterally, by the superior peduncles, and above, by the cerebellum itself, is a lozenge-shaped cavity, called the fourth ventricle.

The gray substance of the cerebellum is divided quite distinctly into two layers. The external layer is called the molecular layer, and the internal, the granular layer.

The molecular layer contains the large cells of Purkinje. These send off arborescent dendrites that extend toward the surface, giving off small telodendrites in their course. The neurites pass downward

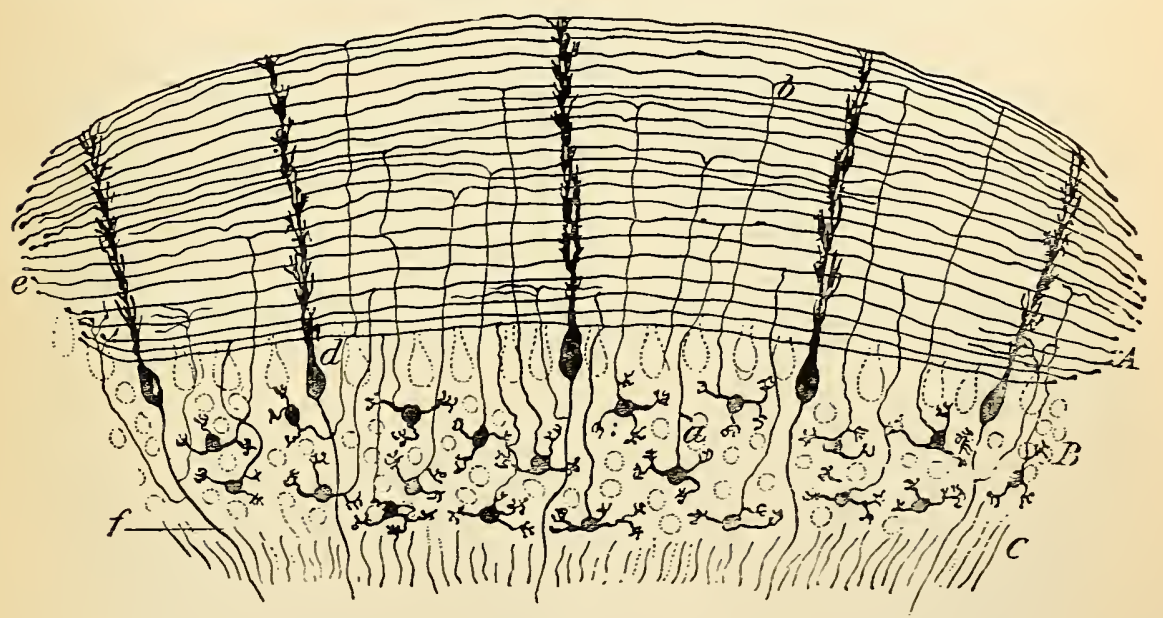

Fig. 149. - Section of a cerebellar lamina parallel to its axis (R. y Cajal).

$A$, molecular layer; $B$, granular layer; $C$, white substance; $a$, small stellate cell of the granular layer, with its bifurcating neurite; $b$, bifurcation; $e$, bulbous end of a neurite; $f$, neurite of a cell of Purkinje.

into the white substance. In passing through the white matter, they give off collaterals, which reascend to the molecular layer. The molecular layer also contains small stellate cells, which give off neurites that send collaterals to form a basket-like arrangement around the cells.

The granular layer presents small stellate cells which have a few short dendrites and long bifurcating neurites, the latter extending into the molecular layer. This layer also has large stellate cells that send their dendrites into the molecular layer and distribute their neurites in the granular layer.

The histology of the structures found in the cerebellum is complex; and its physiological significance is as yet so obscure that it does not seem necessary here to describe it more in detail. 
In the white substance are found arcuate fibres that connect different adjacent areas of gray matter.

The superior peduncles of the cerebellum pass to the cerebrum through the longitudinal tract of the pons Varolii. The middle peduncles connect the two lateral lobes through the transverse tract of

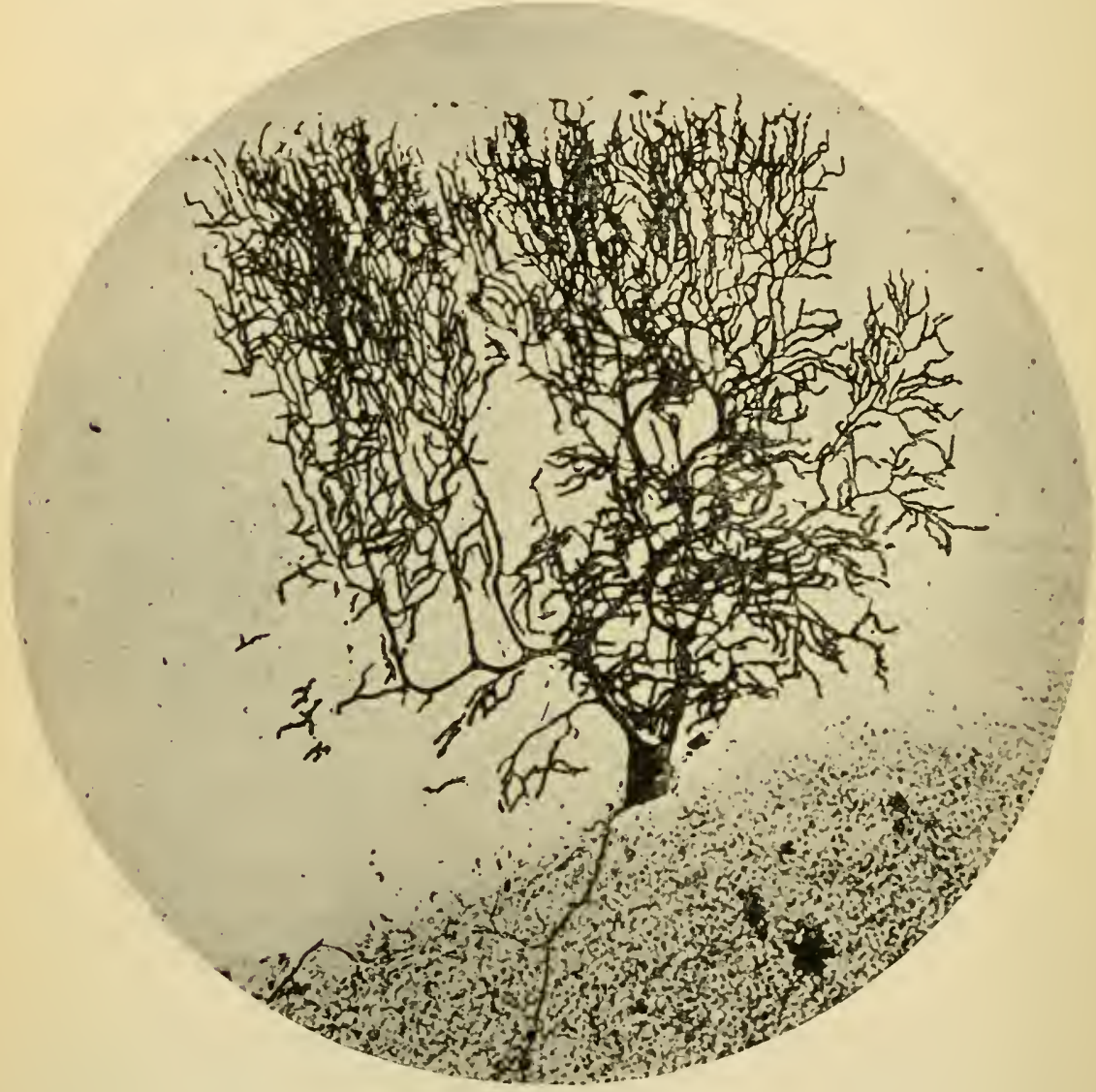

Fig. 150. - Cell of Purkinje, $\times$ i7o (Leaming) ${ }^{1}$

This figure shows the arborizing dendrites extending upward from the cell-body and a neurite, with one collateral passing to the left in the granular layer.

1 This figure is an exact reproduction of an actual object stained by the method of Golgi. The dendrites extend upward toward the border of the gray matter. The cell-body lies near the granular layer. From the cell-body, a neurite passes into the granular layer toward the white matter. The dendrites belong to a cell-body that has, superimposed, another cell-body with a neurite. The two cell-bodies are fused as well as the granular substance below. The dendrites of the superimposed cell-body were wiped away. Aside from the fusing of the cell-bodies and of the granular layers, the picture has not been touched and is an exact reproduction of the actual appearances. It is unusual to see a neurite extending for any considerable distance from a Purkinje cell. 
the pons. The inferior peduncles are connected with the restiform bodies of the bulb and through the bulb with the posterior columns of the cord.

Extirpation of the Cerebellum. - When its greatest part or the entire cerebellum is removed from a bird or a mammal, the animal being before the operation in a normal condition and no other parts being injured, there are no phenomena constantly and invariably observed except certain modifications of the voluntary movements (Flourens). The intelligence, general and special sensibility, the involuntary movements and the simple faculty of voluntary motion remain. The movements, however, are irregular and incoördinate; the animal can not maintain its equilibrium; and on account of the impossibility of making regular movements, it can not feed. This want of equilibrium and of the power of coördinating the muscles of the general voluntary system causes the animal to assume remarkable postures, which, to one accustomed to these experiments, are characteristic. Later experiments on the inferior animals, as well as pathological observations on the human subject, have shown that muscular coördination is disturbed when the median lobe is extirpated, while removal of the lateral lobes has no such effect. It is well known that many muscular acts are more or less automatic, as in standing, and, to a certain extent, in walking. These acts, as well as nearly all voluntary movements, require a certain coördination of the muscles, and this, and this alone, is affected by destruction of the middle lobe of the cerebellum.

Pathological Observations. - Records of cases of lesion of the cerebellum in the human subject have accumulated until the number is quite large. A study of cases in which the phenomena referable to cerebellar injury were not complicated by paralysis, coma or convulsions, shows that serious lesion of the middle lobe is almost always attended with marked muscular incoördination; but cases in which only a portion of one or of both hemispheres is involved may not present any disorder in the muscular movements. These facts are in accord with the results of experiments on the lower animals.

The phenomena observed in cases of cerebellar incoördination are notably different from those presented in simple locomotor ataxia. In cerebellar disease, the gait is staggering, much as it is in alcoholic intoxication. The chief difficulty seems to be in maintaining the equilibrium in progression, even with great care and close attention on the part of the patient. With the idea in mind that there is a coördinating centre for the muscles of progression, and that this centre acts imperfectly, it seems as though an efficient effort at coördination were impossible. In locomotor ataxia, patients seem to make coördinating efforts, but the 
paths by which these efforts find their way to the muscles are disturbed, and the coördinating process, which is more or less automatic in health, requires peculiar care and attention; but with the aid of the sense of sight and artificial supports, progression may be safely though irregularly accomplished. The movements are jerky, and each step seems to require a distinct act of volition. It is possible to imagine that in disorganization of the paths of coördination in the spinal cord, the coördinating centre may act in some degree through the motor paths in the direct and crossed pyramidal tracts. It is certain that the want of normal coördinating power is supplemented by ordinary voluntary acts and by the sense of sight.

Vertigo is not a necessary accompaniment of cerebellar ataxia. Disease of the semicircular canals of the internal ear (Ménière's disease) is attended with vertigo, and this is the main cause of the disturbances of equilibrium.

\section{The BulB}

The medulla oblongata, or bulb, connects the spinal cord with the encephalic ganglia. It is composed of white and gray matter and presents in its substance a number of important nerve-centres. It is not necessary to give anything like a complete anatomical description of the bulb. Its most important conducting parts are those which are continuous with the columns of the cord and pass to the cerebrum and cerebellum. The nuclei of origin of certain of the cranial nerves in the floor of the fourth ventricle have already been mentioned.

Physiological Anatomy. - The bulb is pyramidal in form, with its broad extremity above, and rests in the basilar groove of the occipital. bone, extending from the lower border of the pons Varolii to the atlas. It is about an inch and a quarter (3 I.8 millimeters) in length, threequarters of an inch (19. I millimeters) broad at its widest portion and half an inch (I 2.7 millimeters) thick. It is flattened antero-posteriorly. Like the cord, it has an anterior and a posterior median fissure.

Apparently continuous with the anterior columns of the cord, are the two anterior pyramids, one on either side. Viewed superficially, the innermost fibres of these pyramids are seen to decussate in the median line; but if the fibres are traced from the cord, it is found that they come from the crossed pyramidal tracts of the lateral columns and that none are derived from the anterior columns. The fibres of the external portion of the anterior pyramids come from the direct pyramidal tracts of the cord. At the site of the decussation, the pyramids are composed entirely of white matter; but as the fibres spread out to pass to the 
encephalon above, they present nodules of gray matter between the fasciculi of fibres.

External to the anterior pyramids, are the corpora olivaria. These are oval and are surrounded by a distinct groove. They are white externally and contain a gray nucleus called the corpus dentatum.

External to the corpora olivaria, are the restiform bodies, formed chiefly of white matter and constituting the posterolateral portion of the bulb. They are continuous with the posterior white columns of the cord. The restiform bodies spread out as they ascend, and pass to the cerebellum, forming a large portion of the inferior peduncles. Some fibres from the restiform bodies pass to the cerebrum.

Beneath the olivary bodies and between the anterior pyramids and the restiform bodies, are the lateral tracts of the bulb, sometimes called the intermediary or lateral fasciculi, or the funiculi of Rolando. These are composed of an intimate mixture of white and gray matter and have a yellowish gray color. They receive all that portion of the anterolateral columns of the cord which does not enter into the composition of the anterior pyramids. They usually are described as parts of the restiform bodies; but they are peculiarly important, from the fact that they contain the gray centres presiding over respiration; and for that reason they are here described as distinct fasciculi.

The posterior pyramids (funiculi graciles) are the smallest of all. They pass upward to the cerebellum, without decussating, joining the restiform bodies above. They are composed chiefly of white matter.

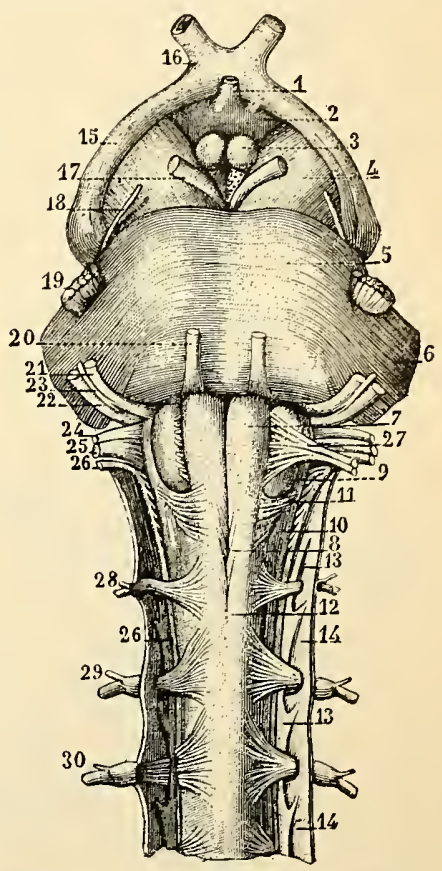

Fig. 151. - Anterior view of the bulb (Sappey).

I, infundibulum ; 2, tuber cinereum ; 3. corpora albicantia; 4, cerebral peduncle; 5, pons Varolii; 6, origin of the middle peduncle of the cerebellum; 7 , anterior pyramids of the bulb; 8 , decussation of the anterior pyramids; 9. olivary bodies; 10 , restiform bodies; II, arciform fibres; I2, upper extremity of the spinal cord; $1_{3}$, ligamentum denticulatum; I4, I4, dura mater of the cord; 15, optic tracts; 16 , chiasm of the optic nerves; 17 , motor oculi communis; I8, patheticus; I9, fifth nerve; 20, motor oculi externus; 2I, facial nerve; 22 , auditory nerve; 23 , nerve of Wrisberg; 24, glosso-pharyngeal nerve; 25. pneumogastric; 26,26 , spinal accessory; 27 , sublingual nerve; $28,29,30$, cervical nerves. As they pass upward in the bulb, they diverge, leaving a space called the fourth ventricle.

The fourth ventricle is the cavity between the pons Varolii, the 
bulb and the cerebellum. It is lozenge-shaped, the acute angles being above and below. The upper angle extends to the upper border of the pons, and the lower angle, to the lower border of the olivary bodies. The triangles which form this lozenge are of nearly equal size. The superior triangle is bounded laterally by the superior peduncles of the cerebellum, as they converge to meet at the corpora quadrigemina. The inferior triangle is bounded laterally by the funiculi graciles and the restiform bodies of the medulla, which diverge at its lower angle. The arched roof of the ventricle is formed by the valve of Vieussens, which is stretched between the superior peduncles of the cerebellum and covers the anterior triangle, and the cerebellum, which covers the

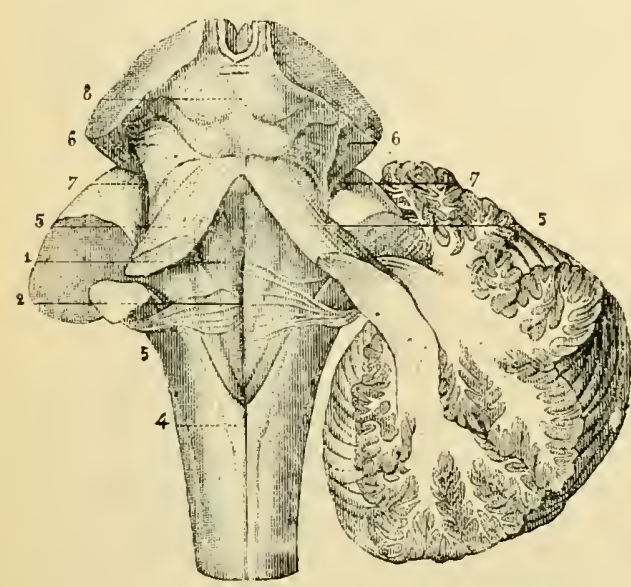

Fig. 152. - Floor of the fourth ventricle (Hirschfeld).

I, median fissure, between the fasciculi teretes: 2 , transverse white stria; 3 , inferior peduncle of the cerebellum; 4, posterior pyramid (funiculus gracilis); 5. 5 , superior peduncles (divided) of the cerebellum; 6 , 6 , bands to the side of the crura cerebri; 7,7 , lateral grooves of the crura cerebri; 8 , corpora quadrigemina. posterior triangle. Beneath the cerebellum is a reflection of the pia mater. The fourth ventricle communicates above with the third ventricle by the aqueduct of Sylvius; below with the subarachnoid space, by the foramen of Magendie; and by a small opening below, with the central canal of the cord. The floor of the ventricle is formed by the posterior surface of the pons above and the bulb below. It presents a fissure in the median line, which terminates below in the calamus scriptorius. By the sides of the median fissure are the fasciculi teretes, which correspond to the intermediary fasciculi of the bulb. Little eminences in the floor indicate the situation of nuclei of origin of cranial nerves. The floor is composed mainly of a layer of gray matter, continuous with the gray commissure of the cord. The lower portion of the floor is marked by transverse lines of white matter emerging from the median fissure.

The two lateral halves of the posterior portion of the bulb are connected together by fibres arising from the gray matter of the lateral tracts, or intermediary fasciculi, passing obliquely, in a curved direction from behind forward, to the raphe in the median line. There also are fibres passing from before backward to form a posterior commissure, and fibres arising from the cells of the olivary bodies, which connect 
the gray substance of the lateral halves. Commissural fibres also connect the gray matter of the lateral tracts with the corpora dentata of the olivary bodies, and the olivary bodies with the cerebellum, their fibres forming part of the inferior peduncles of the cerebellum. In addition, it is probable that fibres, taking their origin from all the gray nodules of the bulb, pass to the parts of the encephalon situated above.

So far as the fibres of origin of the cranial nerves are concerned, it may be stated in general terms that a number of the motor roots arise from the gray matter of the floor of the fourth ventricle, the roots of sensory nerves arising from gray nuclei in the posterior portions.

It is hardly necessary to discuss the action of the bulb as a conductor of sensory impressions and of motor stimulus to and from the brain. It is evident that there is conduction of this kind from the spinal cord to the ganglia of the encephalon, and this must take place through the medulla: a fact which is inevitable, from its anatomical relations, and which is demonstrated by its section in living animals.

The action of the bulb as a reflex nerve-centre depends on its gray matter. When the gray substance is destroyed, certain important reflex phenomena are instantly abolished. From its connection with the cranial nerves, one would expect it to play an important part in the movements of the face, in deglutition, in the action of the heart and of various glands, important points that are fully considered under appropriate heads.

The connections of the bulb with the different columns of the cord are as follows :-

The columns of Türck pass directly from the cord to the cerebrum by fibres at the outer borders of the anterior pyramids.

The crossed pyramidal tracts pass to the anterior pyramids and there decussate.

The anterior ground columns and the lateral bundles connect the gray matter of the cord with the gray matter of the bulb.

The direct cerebellar fasciculi and the columns of Goll pass to the cerebellum through the funiculi graciles, next the posterior fissure.

The columns of Burdach and the descending and ascending cerebellar tracts pass to the restiform bodies.

The fibres of the funiculi graciles and some of the fibres of the columns of Burdach go to the cerebellum without decussating. A few fibres of the restiform bodies go directly to the cerebrum.

Nerve-centres in the Bulb. - The following centres have been located in the bulb:-

I. The centre for closure of the eyelids is related to the nuclei of origin of the facial and the trifacial. 
2. The sneezing centre is related to the trifacial and certain expiratory muscles.

3. The centre for coughing is related to the pneumogastric and certain expiratory muscles.

4. The centre for swallowing is related to the glosso-pharyngeal, the trifacial, the pneumogastric and the muscles of deglutition.

The bulb also contains (5) the vomiting centre, (6) the glycogenic centre, (7) the dominant sweat-centre, (8) the salivary centre, and (9) the dominant vasomotor centres.

IO. The bulb probably contains inhibitory and accelerator cardiac centres. The fibres of the inhibitory nerves, in the human subject, are contained in the sheath of the pneumogastric. Arising from the bulb, passing into the cord and out of the cord with the communicating branches of the lower cervical and upper dorsal nerves to the sympathetic and thence to the cardiac plexus, are the so-called accelerator nerves of the heart. The inhibitory and accelerator nerves are connected with the cardiac centre in the bulb. The inhibitory fibres restrain, or inhibit the heart's action, and the action of the accelerator nerves is to increase the number of pulsations.

II. Respiratory Centres. - There are two respiratory centres in the bulb, one on either side of the median line and connected together by commissural fibres. These centres preside over the respiratory movements. They do not, however, occupy all that portion of the bulb included between the two planes first indicated by Flourens, but are confined to the gray matter of the lateral tracts, or the intermediary fasciculi. This is shown by the fact that respiration persists in animals after division of the anterior pyramids and the restiform bodies. Nearly all experimenters have found that the spinal cord may be divided below the bulb, and that all the encephalic ganglia above may be removed, respiratory movements still persisting. It is a common thing in vivisection to kill an animal by breaking up the bulb. When this is done there are no struggles and no manifestations of the distress of asphyxia. The respiratory muscles simply cease their action, and the animal loses instantly the sense of want of air. A striking contrast to this is presented when the trachea is tied or when all the respiratory muscles are paralyzed without touching the medulla.

Under normal conditions the centres on the two sides probably operate through the pneumogastric nerves, and the respiratory movements on the two sides are synchronous. That there is a respiratory centre on either side is shown by the experiment of dividing the bulb longitudinally in the median line, the respiratory movements afterward continuing with regularity. If, now, the pneumogastric is divided on 
one side, the respiratory movements on that side become slower and are no longer synchronous with the movements on the opposite side. This shows that while the respiratory centres on the two sides normally act together, being connected with each other by commissural fibres, each one has independent connections with the pneumogastric on the corresponding side of the body.

In ordinary tranquil respiration the act of inspiration is reflex and is due to an impression conveyed to the bulb through the pulmonary branches of the pneumogastrics. It has lately been asserted that the impression which gives rise to inspiratory movements is due to partial collapse of the air-vesicles, and that when the vesicles are distended, an impression is received by the bulb that gives rise to movements of expiration. It is difficult, however, to reconcile this theory with the facts. It does not account for the absence of respiratory movements in the fœtus, when the air-cells are entirely collapsed, or for certain phenomena that follow inflation of the lungs with irrespirable gases. It is more reasonable to assume that the impression, in tranquil respiration, is due to the carbon dioxide contained in the air-cells, and that the morements of expiration are almost entirely passive.

In difficult respiration attended with a sense of suffocation and violent inspiratory efforts, the sense is due to the circulation of nonoxygenated blood in the respiratory centres. This was shown by a number of experiments (Flint) begun in I86I and carried on up to I 880 .

Vital Point (so called). - Since it has been definitely ascertained that destruction of a restricted portion of the gray substance of the bulb produces instantaneous and permanent arrest of the respiratory movements, Flourens and others have called this centre the vital knot, destruction of which is immediately followed by death. With the existing knowledge of the properties and uses of the different tissues and organs of which the body is composed, it is almost unnecessary to present any arguments to show the unphilosophical character of such a proposition. One can hardly imagine such a thing as instantaneous death of the entire organism; and still less can it be assumed that any restricted portion of the nervous system is the one essential vital point. Probably, a very powerful electric discharge passed through the entire cerebrospinal axis produces the nearest approach to instantaneous death: but even then it is by no means certain that some parts do not for a time retain their physiological properties. In apparent death the nerves and the heart may be shown to retain their characteristic properties; the muscles will contract under stimulus, and will appropriate oxygen and give off carbon dioxide, or respire; the glands may be made to secrete; and no one can assume that under these conditions the entire organism 
is dead. There seems to be no such thing as death, except as the various tissues and organs which go to make up the entire body become so altered as to lose their physiological properties beyond the possibility of restoration; and this does not occur for all parts of the organism in an instant. A person drowned may be to all appearances dead, and certainly would die without measures for restoration; yet in such instances, restoration may be accomplished, the period of apparent death being simply a blank, so far as the recollection of the individual is concerned. It is as impossible to determine the exact instant when the vital principle, or whatever it may be called, leaves the body in death, as to inclicate the time when the organism becomes a living being. Death is nothing more than a permanent destruction of so-called vital physiological properties; and this occurs successively and at different times for different tissues and organs.

When it is seen that frogs will live for weeks, and sometimes for months, after destruction of the bulb, and that in mammals, by keeping up artificial respiration, many of the most important physiological acts, such as the movements of the heart, may be prolonged for hours after decapitation, one can understand the physiological absurdity of the proposition that there is any such thing as a vital point, either in the bulb or in any other part of the nervous system.

\section{Rolling and Turning Movements following Injury of Certain Parts of the Excephalon (Forced Movements)}

The remarkable movements of rolling and turning, produced by section or injury of certain of the commissural fibres of the encephalon, are not very important in their bearing on the uses of the brain, and they are rather to be classed among the curiosities of experimental physiology. The movements follow unilateral lesions and are dependent, to a certain extent, on a consequent inequality in the power of the muscles on one side, without actual paralysis. These have been called forced movements. Injury to the following parts usually determines movements of rotation:-

"I. Cerebral hemispheres;

"2. Corpora striata;

"3. Optic thalami (Flourens, Longet, Schiff);

"4. Cerebral peduncles (Longet);

"5. Pons Varolii;

"6. Tubercula quadrigemina, or bigemina (Flourens);

"7. Peduncles of the cerebellum, especially the middle, and the lateral portions of the cerebellum (Magendie); 
“8. Olivary bodies, restiform bodies (Magendie);

"9. External part of the anterior pyramids (Magendie);

"Io. Portion of the bulb from which the facial nerve arises (BrownSéquard);

"I I. Optic nerves;

"I2. Semicircular canals (Flourens); auditory nerve (BrownSéquard)."

The movements that follow unilateral injury of the parts mentioned above are of two kinds; namely, rolling of the entire body on its longitudinal axis, and turning, always in one direction, in a small circle, called by the French the movement of manège. A capital point to determine in these phenomena is whether the movements are due to paralysis or enfeeblement of certain muscles upon one side of the body, to a direct or reflex irritation of the parts of the nervous system involved or to both these causes combined. The experiments of Brown-Séquard and others show that the movements may be due to irritation alone, for they occur when parts of the encephalon and the upper portions of the cord are simply pricked, without section of fibres. When there is extensire division of fibres, it is probable that the effects of the enfeeblement of certain muscles are added to the phenomena produced by simple irritation. The most satisfactory explanation of these movements is the one proposed by Brown-Séquard, who attributed them to a more or less convulsive action of muscles on one side of the body, produced by irritation of the nerve-centres. He regarded the rolling as simply an exaggeration of the turning movements, and places both in the same category.

It is not necessary to enter into an extended discussion of these experiments: In some of them, the movements have been observed toward the side operated on, and in others, toward the sound side. These differences probably depend on the fact that in certain experiments the fibres are involved before their decussation, and in others, after they have crossed in the median line. In some instances the movements may be due to a reflex action, from stimulation of afferent fibres, and in others the action of the irritation may be direct. 


\title{
CHAPTER XXIV
}

\author{
SYMPATHETIC SYSTEM - SLEEP
}

Cranial ganglia - Cervical ganglia - Thoracic ganglia - Ganglia in the abdominal and the pelvic cavities - General properties of the sympathetic ganglia and nerves - Direct experiments on the sympathetic - Vasomotor centres and nerves - Reflex vasomotor phenomena - Vaso-inhibitory nerves - Trophic centres and nerves - Sleep - Dreams Condition of the brain and nervous system during sleep.

LIKE the cerebro-spinal system, the sympathetic is composed of centres, or ganglia, and nerves. The ganglia contain nerve-cells, most of which differ but little from the cells of the encephalon and spinal cord. The nerves are composed of fibres, some of which are nearly identical in structure with the ordinary motor and sensory fibres, while many are the so-called gelatinous fibres. The nerve-fibres are connected with the cells in the ganglia, and the ganglia are connected with each other by commissural fibres.

The sympathetic ganglia constitute a continuous chain on either side of the body, beginning above with the ophthalmic ganglia and terminating below in the ganglion impar. It is important to note, however, that the chain of ganglia is not independent, but that each ganglion receives motor and sensory filaments from the cerebro-spinal nerves, and that filaments pass from the sympathetic to the cerebro-spinal system. The general distribution of the sympathetic filaments is to mucous membranes - and possibly to integument, - to the heart, to non-striated muscular fibres, and particularly to the muscular coat of the arteries. So far as has been shown by anatomical investigations, there are no fibres derived exclusively from the sympathetic that are distributed to striated muscles except those which pass to the muscular tissue of the heart. Near the terminal filaments of the sympathetic, in most of the parts to which these fibres are distributed, there exist large numbers of ganglionic cells.

The general arrangement of the sympathetic ganglia and the distribution of the nerves may be stated very briefly; but a knowledge of certain anatomical points is indispensable as an introduction to an intelligent study of the physiology of this system.

In the cranium, are the four cranial ganglia - the ophthalmic, the spheno-palatine, the otic and the submaxillary. In the neck, are the 
three cervical ganglia - the superior, middle, and inferior. In the chest, are the twelve thoracic ganglia, corresponding to the twelve ribs. The great semilunar ganglia, the largest of all and sometimes called the abdominal brain, are in the abdomen, by the side of the cœliac axis. In the lumbar region, in front of the spinal column, are the four lumbar ganglia. In front of the sacrum are the four or five sacral, or pelvic ganglia; and finally, in front of the coccyx, is a small single ganglion, the last of the sympathetic chain, called the ganglion impar. Thus, the sympathetic cord, as it is sometimes called, consists of twenty-eight to thirty ganglia on either side, terminating below in a single ganglion.

Cranial Ganglia. - The ophthalmic, lenticular, or ciliary ganglion is situated deeply in the orbit, is of a reddish color and about the size of a pin's-head. It receives a motor branch from the third pair and sensory filaments from the nasal branch of the ophthalmic division of the fifth. It also is connected with the cavernous plexus and with Meckel's ganglion. Its so-called motor and sensory roots from the third and the fifth pair have already been described in connection with these nerves. Its filaments of distribution are the ten or twelve short ciliary nerves, which pass to the ciliary muscle and the iris. A delicate filament from this ganglion passes to the eye, with the central artery of the retina, in the canal in the centre of the optic nerve.

The uses of the ophthalmic ganglion relate mainly to the action of the ciliary muscle and iris; and it is necessary here only to indicate its anatomical relations, leaving its function to be taken up in connection with the physiology of vision.

The spheno-palatine, or Meckel's ganglion, is the largest of the cranial ganglia. It is triangular in shape, reddish in color, and situated in the spheno-maxillary fossa, near the spheno-palatine foramen. It receives a motor root from the facial, by the Vidian nerve. Its sensory roots are the two spheno-palatine branches from the superior maxillary division of the fifth. It has a large number of branches of distribution. Two or three small filaments enter the orbit and go to its periosteum. Its other branches, which it is unnecessary to describe fully in detail, are distributed to the gums, the membrane covering the hard palate, the soft palate, the uvula, the roof of the mouth, the tonsils, the mucous membrane of the nose, the middle auditory meatus, a portion of the pharyngeal mucous membrane and the levator palati and azygos uvulæ muscles. It is probable that the filaments sent to these two striated muscles are derived from the facial nerve and do not properly belong to the sympathetic system. The ganglion sends also a short branch of a reddish gray color to the carotid plexus.

The otic ganglion, sometimes called Arnold's ganglion, is a small, 


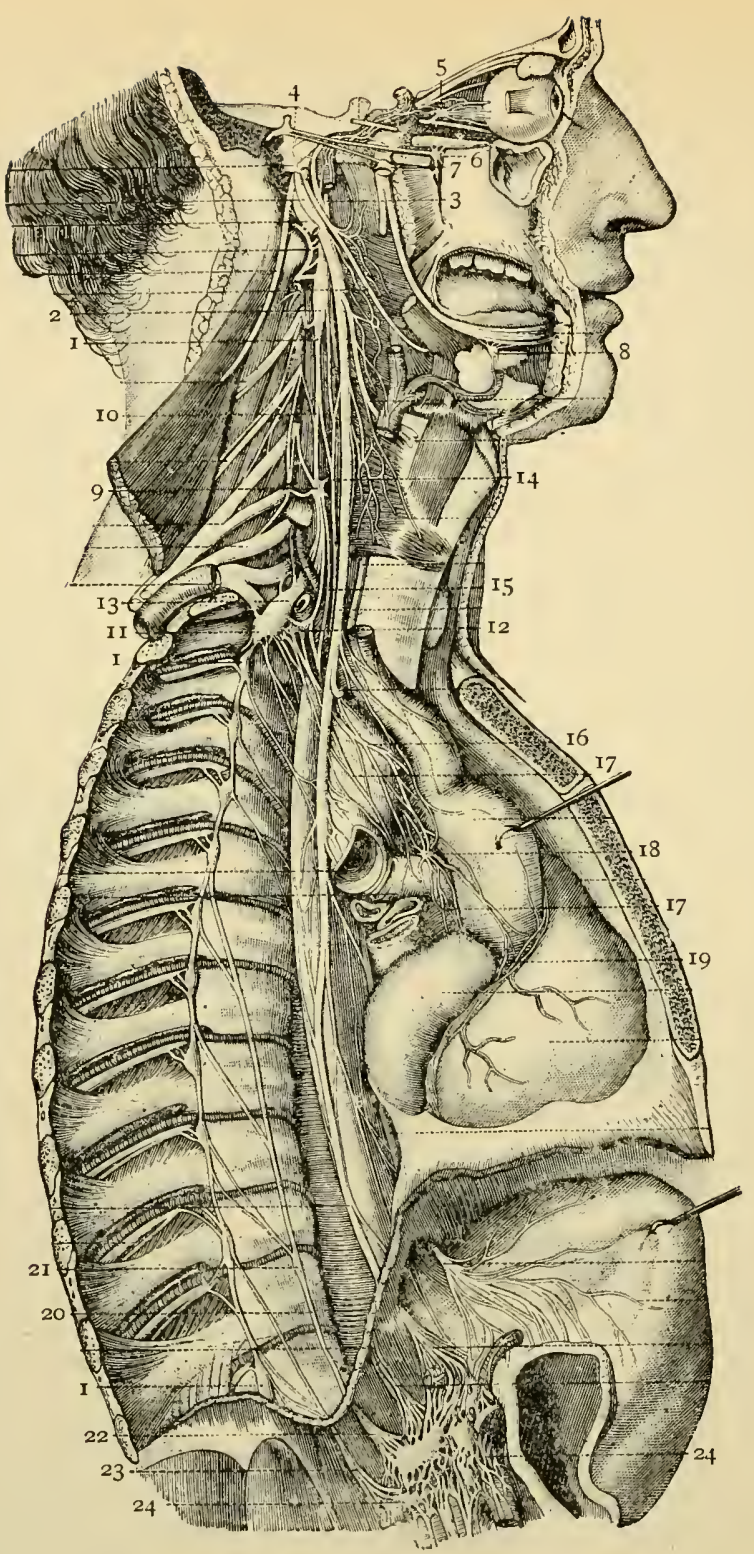

Fig. 153. - Cervical and thoracic portions of the sympathetic (Sappey).

I, I, I, chain of ganglia of the sympathetic ; 2 , superior cervical ganglion ; 3 , branches to the carotid; 4 , filaments from the facial to the spheno-palatine and to the otic ganglion; 5 , ophthalmic ganglion; 6 , spheno-palatine ganglion; 7 , otic ganglion; 8, submaxillary ganglion; 9, middle cervical ganglion: Io, cord connecting the two ganglia; II, inferior cervical ganglion; I2, I3, filaments connecting this with the middle ganglion; 14 , superior cardiac nerve; 15 , middle cardiac nerve; 16 , inferior cardiac nerve; I7, I7, cardiac plexus; 18, ganglion of the cardiac plexus; I9, nerve following the right coronary artery; $20,21,22$, great splanchnic nerve; 23 , lesser splanchnic nerve; 24,24 , solar plexus. 
oval, reddish gray body, situated just below the foramen orale. It receives a motor filament from the facial and sensory filaments from branches of the fifth and of the glosso-pharyngeal. Its filaments of distribution go to the mucous membrane of the tympanic cavity and Eustachian tube and to the tensor tympani and tensor palati muscles. Reasoning from the general mode of distribution of the sympathetic filaments, those going to the striated muscles are derived from the facial. It also sends branches to the carotid plexus.

The submaxillary ganglion, which is situated on the submaxillary gland, is small, rounded, and reddish gray in color. It receives motor filaments from the chorda tympani nerve and sensory filaments from the lingual branch of the fifth. Its filaments of distribution go to Warton's dict, to the mucous membrane of the mouth and to the submaxillary gland.

Cervical Ganglia.The three cervical ganglia are situated opposite the third, fifth and seventh cervical vertebræ respectively. The middle

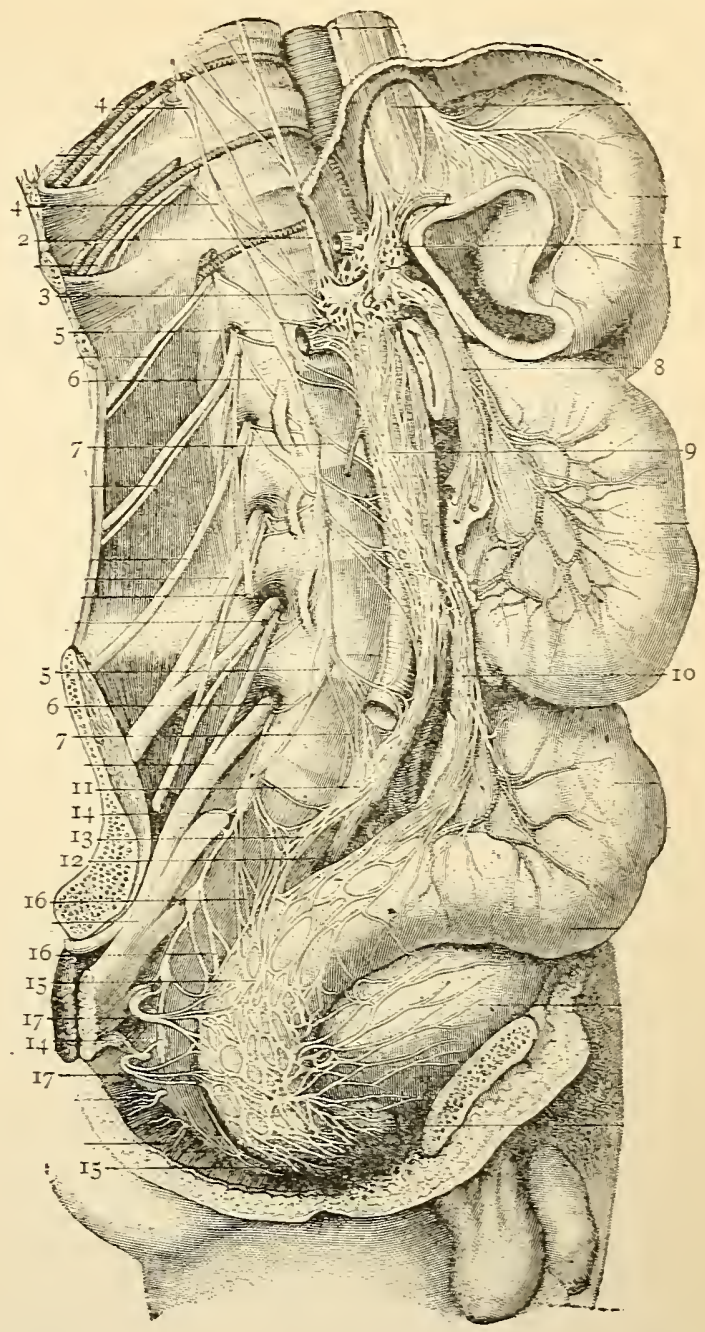

Fig. 154. - Lumbar and sacral fortions of the sympathetic (Sappey).

I, solar plexus; 2 , lower end of the great splanchnic nerve: 3 , lower end of the lesser splanchnic nerve; 4,4 , last two thoracic ganglia: 5,5 , the four lumbar ganglia $; 6,6,7,7$, branches from the lumbar ganglia; 8, superior mesenteric plexus; 9, II, I2, I3, aortic lumbar plexus; Io, inferior mesenteric plexus; I4, I4, sacral portion of the sympathetic; $I_{5}, I_{5}, I 6, I 6, I 7, I 7$, hypogastric plexus. ganglion sometimes is wanting, and the inferior ganglion occasionally is fused with the first thoracic ganglion. These ganglia are connected 
together by the so-called sympathetic cord. They have a number of filaments of communication above with the cranial and the cervical nerves of the cerebro-spinal system. Branches from the superior ganglion go to the internal carotid to form the carotid and the cavernous plexuses, following the vessels as they branch to their distribution. Branches from this ganglion pass to the cranial ganglia. There also are branches that unite with filaments from the pneumogastric and the glosso-pharyngeal to form the pharyngeal plexus, and branches that form a plexus on the external carotid, the vertebral and the thyroid arteries, following the ramifications of these vessels.

From the cervical portion of the sympathetic, the three cardiac nerves arise and pass to the heart, entering into the formation of the cardiac plexus. The superior cardiac nerve arises from the superior ganglion; the middle nerve, the largest of the three, arises from the middle ganglion, or from the sympathetic cord when this ganglion is wanting; and the inferior nerve arises from the inferior cervical ganglion or the first thoracic. These nerves present frequent communications with various of the adjacent cerebro-spinal nerves, penetrate the thorax and form the deep and superficial cardiac plexuses and the posterior and the anterior coronary plexuses. In these plexuses there are found ganglioform enlargements; and on the surface and in the substance of the heart, are collections of nerve-cells connected with the fibres.

Thoracic Ganglia. - The thoracic ganglia are situated in the chest, beneath the pleura, and rest on the heads of the ribs. They usually are twelve in number, but occasionally two are fused into one. They are connected together by the sympathetic cord. They each communicate by two filaments with the cerebro-spinal nerves. One of these is white, like the spinal nerves, and probably passes to the sympathetic, and the other, of a grayish color, is thought to contain the true sympathetic filaments. From the upper six ganglia filaments pass to the aorta and its branches. The branches that form the posterior pulmonary plexus arise from the third and fourth ganglia. The great splanchnic nerve arises mainly from the seventh, eighth and ninth ganglia, receiving a few filaments from the upper six ganglia. This is a large, white, rounded cord, which penetrates the diaphragm and passes to the semilunar ganglion, sending a few filaments to the renal plexus and the suprarenal capsules. The lesser splanchnic nerve arises from the tenth and eleventh ganglia, passes into the abdomen and joins the cœliac plexus. The renal splanchnic nerve arises from the last thoracic ganglion and passes to the renal plexus. The three splanchnic nerves present frequent anastomoses with each other. 
Ganglia in the Abdominal and the Pelvic Cavities. - The semilunar ganglia on the two sides send off radiating branches to form the solar plexus. They are situated by the side of the coeliac axis and near the suprarenal capsules. These are the largest of the sympathetic ganglia. From these arise plexuses distributed to various parts in the abdomen, as follows: The phrenic plexus follows the phrenic artery and its branches to the diaphragm. The cœliac plexus subdivides into the gastric, hepatic and splenic plexuses, which are distributed to organs as their names indicate. From the solar plexus different plexuses are given off, which pass to the kidneys, the suprarenal capsules, the testes in the male and the ovaries in the female, the intestines (by the superior and inferior mesenteric plexuses), the upper part of the rectum, the abdominal aorta and the vena cava. The filaments follow the distribution of the bloodvessels in the solid viscera.

The lumbar ganglia, four in number, are situated in the lumbar region, on the bodies of the vertebræ. They are connected with the ganglia above and below and with each other by the sympathetic cord, receiving, like the other ganglia, filaments from the spinal nerves. Their branches of distribution form the aortic lumbar plexus and the hypogastric plexus and follow the course of the bloodvessels.

The four or five sacral ganglia and the ganglion impar are situated by the inner side of the sacral foramina and in front of the coccyx. These are connected with the ganglia above and with each other and receive filaments from the sacral nerves, there being usually two branches of communication for each ganglion. The filaments of distribution go to all the pelvic viscera and bloodvessels. The inferior hypogastric, or pelvic plexus is a continuation of the hypogastric plexus above and receives a few filaments from the sacral ganglia. The uterine nerves go to the uterus and the Fallopian tubes. In the substance of the uterus the nerves are connected with small collections of ganglionic cells. The sympathetic filaments are prolonged into the upper and lower extremities, following the course of the bloodvessels and terminating in their muscular coat.

The filaments of the sympathetic, at or near their terminations, are connected with ganglionic cells, not only in the heart and the uterus, but in the bloodvessels, lymphatics, the coccygeal gland, the submucous and the muscular layer of the entire alimentary canal, the salivary glands, pancreas, excretory ducts of the liver and pancreas, the larynx, trachea, pulmonary tissue, bladder, ureters, the entire generative apparatus, suprarenal capsules, thymus, lachrymal canals, ciliary muscle and the iris. In these situations nerve-cells have been demonstrated, and it is 
probable that they exist everywhere in connection with the terminal filaments of this system of nerves.

Gencral Propertics of the Sympathetic Ganglia and Nerves. - The sympathetic ganglia and nerves possess a dull sensibility, which is particularly marked in the ganglia. That the nerves contain afferent fibres is shown by certain reflex phenomena.

Stimulation of the sympathetic produces muscular movements, but these are confined usually to non-striated muscular fibres, to which these nerves are largely distributed. The muscular movements do not immediately follow stimulation of the nerves, but there is a long latent period. The muscular contraction, also, persists for a time and the subsequent relaxation is slow. The properties of the vasomotor nerves will be considered separately.

The sympathetic ganglia are connected with the motor and sensory divisions of the cerebro-spinal system. Some of the ganglia and nerveplexuses are directly dependent for their action on the cerebro-spinal system, while others are capable, at least for a time, of independent action. Among the latter, are the ganglia of the heart, the intestinal plexuses, the plexuses of the uterus and Fallopian tubes, of the ureters and of the bloodvessels.

Dircet Experiments on the Sympathetic. - The experiments of Pourfour du Petit (I7I2-I 725.) were the first to give any positive information regarding the action of the sympathetic system; and these observations may be taken as the starting-point of a definite knowledge of the physiology of the sympathetic, although they showed only the influence of the cervical portion on the eye. In I 816 Dupuy removed the superior cervical ganglia in horses, with the effect of producing injection of the conjunctiva, elevation of temperature in the ear and an abundant secretion of sweat on one side of the head and neck. These experiments showed that the sympathetic has an important influence on nutrition, calorification and secretion. In 185 I Bernard divided the sympathetic in the neck on one sicle in rabbits and noted on the corresponding side of the head and the ear increased vascularity and an elevation in temperature of $7^{\circ}$ to $\mathrm{II}^{\circ} \mathrm{Fahr}$. $\left(4^{\circ}\right.$ to $6^{\circ} \mathrm{C}$.). This condition of increased heat and vascularity continues for several months after division of the nerve. In 1852 Brown-Séquard repeated these experiments and attributed the elevation of temperature directly to an increase in the supply of blood to the parts affected. He made an important advance in the history of the sympathetic by demonstrating that its section paralyzed the muscular coat of the arteries, and further, that faradization of the nerve in the neck caused the vessels to contract. This was the discovery of the vasomotor nerves, and it belongs without question to Brown- 
Séquard, who published his observations in August, I852. A few months later in the same year, Bernard made similar experiments and presented the same explanation of the phenomena observed.

The important points developed by the first experiments of Bernard and of Brown-Séquard were that the sympathetic system influences the general process of nutrition, and that many of its filaments are distributed to the muscular coat of the bloodvessels. Before these experiments, it had been shown that filaments from this system influenced the contractions of the muscular coat of the alimentary canal.

When the sympathetic is divided in the neck, the local increase in temperature is attended with a considerable increase in the supply of blood to the side of the head corresponding to the section. The increased temperature is due to a local exaggeration of the nutritive processes, apparently dependent on the hyperemia. There are many instances in pathology of local increase in temperature attending increased supply of blood to restricted parts. In an experiment by Bidder, after excising about half an inch (12.7 millimeters) of the cervical sympathetic in a half-grown rabbit, the ear on that side, in the course of about two weeks, became distinctly longer and broader than the other.

It is easy to observe the effects of dividing the sympathetic in the neck, but similar phenomena have been noted in other parts. Among the most striking of these experiments are those reported by Samuel, who described an intense hyperemia of the mucous membrane of the stomach and intestines following extirpation of the cœliac plexus. By comparative experiments it was shown that this did not result from peritonitis produced by the operation.

As regards secretion, the influence of the sympathetic is very marked. When the sympathetic filaments distributed to a gland are divided, the supply of blood is much increased and an abundant flow of the secretion follows (Bernard). Peyrani has shown that the sympathetic has an influence on the secretion of urine. When the nerves in the neck are stimulated, the quantity of urine and of urea is increased, and this increase is greater with the induced than with the constant current. When the sympathetic is divided, the quantity of urine and of urea sinks to the minimum.

Moreau published in 1870 a series of observations on the influence of the sympathetic nerves on the secretion of liquid by the intestinal canal, which are important as affording a possible explanation of the sudden occurrence of watery diarrhœa. In these experiments the abdomen was opened in a fasting animal, and three loops of intestine, each loop four to eight inches ( 100 to 200 millimeters) long, were isolated by ligatures. All the nerves passing to the middle loop were divided, 
taking care to avoid the bloodvessels. The intestine was then replaced, and the wound in the abdomen was closed with sutures. The next day the animal was killed. The two loops with the nerves intact were found empty, as is normal in fasting animals, and the mucous membrane was dry; but the loop with the nerves divided was found filled with a clear, alkaline liquid, colorless or slightly opaline, which precipitated a few flocculi of organic matter on boiling.

Vasomotor Centres and Neries. - The principal or dominating vasomotor centres are situated in the bulb, one on either side, about onetenth of an inch (2.5 millimeters) from the median line. Each centre, in the rabbit, is about one-eighth of an inch ( 3 millimeters) long and about one-sixteenth of an inch ( 1.5 millimeters) wide. Its lower border is about one-fifth of an inch ( 5 millimeters) above the calamus scriptorius. Each side of the body has its special vasomotor centre, and very few if any of the vasomotor fibres decussate. The situation of the vasomotor centres in the bulb has been determined by successive removal of the nerve-centres above. If the central end of a large cerebro-spinal nerve is stimulated in an animal poisoned with curare, the vasomotor nerves produce contraction of the bloodvessels by reflex action and there is a rise in the blood-pressure. The action is not interfered with by removal of the encephalic ganglia from above downward, until the part of the medulla containing the vasomotor centres is reached. When these centres are destroyed, the reflex vasomotor action is permanently arrested.

Subordinate vasomotor centres exist in the spinal cord. When the vasomotor centre in the bulb is destroyed, there is a fall in the bloodpressure; but if the circulation is continued, after a time the bloodvessels regain their "tone" and the pressure may then be affected by reflex action. It is probable that these spinal centres exist throughout the dorsal and lumbar regions of the cord.

All the vasomotor nerves are derived from the bulb and the spinal cord. Some of the vasomotor fibres to the head pass in the trunks of the motor cranial nerves, but most of them come from the anterior roots of some of the spinal nerves and pass to the head by the filaments of distribution of the cervical sympathetic. The vasomotor fibres pass in the lateral columns of the cord, and from the cord, in the anterior roots of the spinal nerves, in the dog, as far down as the second pair of lumbar nerves. These fibres are medullated but are of small size. They pass to the bloodvessels either through branches from the sympathetic ganglia or through the ordinary cerebro-spinal nerves. They therefore are not confined to branches of the sympathetic.

The vasomotor nerves are capable of influencing local circulations, 
probably through distinct centres for different parts. Direct stimulation of the principal vasomotor centre ( IO to I 2 or more single induction shocks per second for strong currents or 20 to 25 for moderate currents) increases the blood-pressure to the maximum.

The contractile coats of the veins and lymphatics probably are influenced by vasomotor nerves, but little is known of the mechanism of this action.

Reflex Vasomotor Plienomena. - The most important physiological processes connected with the vasomotor nerves are reflex. It is evident from experiments on the inferior animals and observations on the human subject that there are afferent as well as efferent nerves. The reflex acts connected with secretion have already been considered; but there are other phenomena that demand a brief description.

As regards animal heat, the production of which is intimately connected with the supply of blood to the parts, it is important to note the observations of Brown-Séquard and of Lombard, who found that pinching of the skin on one side was attended with a diminution in the temperature in the corresponding member of the opposite side, and that sometimes, when the irritation was applied to the upper extremities, changes were produced in the temperature of the lower limbs. Tholozan and Brown-Séquard found, also, that lowering the temperature of one hand produced a considerable depression in the heat of the other hand, without any notable diminution in the general heat of the body. Brown-Séquard showed that by immersing one foot in water at $4 \mathrm{I}^{\circ}$ Fahr. $\left(5^{\circ} \mathrm{C}\right.$.) the temperature of the other foot was diminished by about $7^{\circ}$ Fahr. $\left(4^{\circ} \mathrm{C}\right.$.) in the course of eight minutes. These experiments show that certain impressions made on the sensory nerves affect the animal heat by reflex action. As section of the sympathetic filaments increases the heat in particular parts with an increase in the supply of blood, and their faradization reduces the quantity of blood and diminishes the temperature, it is reasonable to infer that the reflex action takes place through the vasomotor nerves. If it is assumed that the impression is conveyed to the centres by the nerves of general sensibility, and that the vessels are modified in their calibre and the heat is affected through the sympathetic fibres, it remains only to determine the situation of the centres that receive the impression and generate the stimulus. These centres are situated in the cerebro-spinal axis.

The existence of vasomotor nerves and their connection with centres in the cerebro-spinal axis are now sufficiently well established. It is certain, also, that centres presiding over particular acts may be distinctly located, as the genito-spinal centre, in the spinal cord opposite the fourth lumbar vertebra, and the cilio-spinal centre, in the cervical 
region of the cord. Impulses generated in these centres, sometimes as the result of impressions received through the nerves of general sensibility, produce contraction of the non-striated muscular fibres of the iris, vasa deferentia etc., including the muscular coats of the bloodvessels. The contraction of the muscular walls of the vessels is tonic; and when their nerves are divided, relaxation takes place and the vessels are dilated by the pressure of blood. By this action the local circulations are regulated in accordance with impressions made on sensory nerves, the physiological requirements of certain parts, mental emotions etc. Secretion, the peristaltic movements of the alimentary canal, the movements of the iris etc., are influenced in this way. This action also is illustrated in cases of reflex paralysis, in inflammations as the result of " taking cold," and in many other pathological conditions.

It remains only to show that the phenomena following section of the sympathetic in animals are illustrated in certain cases of disease or injury in the human subject. It is rare to observe traumatic injury confined to the sympathetic in the neck. A single case, however, apparently of this kind, has been reported by Mitchell. A man received a gunshot-wound in the neck. Among the phenomena observed a few weeks after, were contraction of the pupil on the side of the injury, and after exercise, flushing of the face on that side. There was no difference in the temperature of the two sides during repose, but no thermometric observations were made when half of the face was flushed by exercise. Bartholow has reported several cases of unilateral sweating of the head (two observed by himself), in some of which there probably was compression of the sympathetic by an aneurism. In the cases in which the condition of the eye was observed, the pupil was found contracted in some and dilated in others. In none of these cases were there any accurate thermometric observations. In a series of observations by Wagner, on the head of a woman, eighteen minutes after decapitation, strong stimulation of the sympathetic produced enlargement of the pupil. In such a case as this, it would not be possible to make observations on the influence of the sympathetic on the temperature.

Viso-inhibitory Norves. - There are certain nerves the direct action of which under faradic stimulation is to dilate certain bloodvessels. These nerves may also be excited by reflex action through the sensory nerves. In many nerves, as the chorda tympani, the nervi erigentes etc., the existence of inhibitory fibres has been demonstrated. For example, division of the nervi erigentes has no immediate effect on the penis, but faradization of the peripheral ends of the nerves dilates the bloodvessels and produces erection. Fibres possessing this property. 
undoubtedly exist throughout the body, in the sympathetic and in the motor and mixed nerves; and it is possible that there are vasomotor inhibitory centres, although such centres have not been located. The mode of action of these nerves is analogous to that of the inhibitory nerve of the heart, restraining and regulating the action of vasomotor nerves and allowing the pressure of blood to dilate the vessels. It does not, however, seem proper to call them "vaso-dilator" nerves, any more than it would be correct to call the inhibitory nerve of the heart the cardiac dilator nerve.

Trophic Centres and Nerves. - Collections of nerve-cells act as centres, presiding over the nutrition of the nerve-fibres with which they are connected; but it has been found that the nutrition of other parts may be profoundly affected through the nervous system. Many pathologists, relying on the presence of lesions of cells in the cord, in connection with cases of progressive muscular atrophy, admit the existence of trophic cells and nerves. These views, however, rest almost entirely on pathological observations. Experiments on the sympathetic do not positively show any influence on nutrition, except as this system of nerves affects the supply of blood to the parts. When a sympathetic nerve is divided, there is an exaggeration of the nutritive processes in particular parts, and there may be inflammatory phenomena, but atrophy of muscles is not observed. Atrophy of muscles, indeed, follows division of cerebro-spinal nerves only, or, as cases of disease have shown, disorganization of cells belonging to what are recognized as motor'centres. As regards this condition, there can be no doubt of the fact that progressive muscular atrophy is attended with disorganization of certain of the motor cells of the spinal cord.

Without fully discussing this subject - which properly belongs to pathology - the facts may be briefly stated as follows: There may be progressive atrophy of certain muscles, uncomplicated with paralysis except in so far as there is weakness of these muscles due to partial and progressive destruction of their contractile elements. The only constant pathological condition in these cases, aside from the changes in the muscular tissue, is destruction of certain cells in the antero-lateral portions of the cord, with more or less atrophy of the corresponding anterior roots of the nerves. It has not been assumed that there are cells in the cord, presenting anatomical peculiarities by which they may be distinguished from the ordinary motor or sensory elements; but the fact of the degeneration of certain cells (a pigmentary and sclerotic atrophy), others remaining normal, has led to the distinction by writers, of trophic cells, and of course these must be connected with the parts by trophic nerves. 


\section{Sleep}

When it is remembered that about one-third of each day is passed in sleep, and that at this time, voluntary motion, sensation, the special senses and various of the functions of the organism are greatly modified, the importance of a physiological study of this condition is sufficiently apparent. The subject of sleep is most appropriately considered in connection with the nervous system, for the reason that the most important modifications in function are observed in the cerebro-spinal axis and nerves. Repose is as necessary to the nutrition of the muscular system as proper exercise; but repose of the muscles relieves the fatigue due to exercise, without sleep. It is true that after violent and prolonged exertion there frequently is a desire for sleep, but simple repose often will restore the muscular power. After the most violent exertions, a renewal of muscular vigor is most easily and completely effected by rest without sleep, a fact familiar to all who are accustomed to athletic exercises. After prolonged and severe mental exertion, however, or after long-continued muscular effort involving excessive expenditure of the so-called nerve-force, sleep becomes an imperative necessity. If the nervous system is not abnormally excited by effort, sleep follows moderate exertion as a natural consequence, and it is the only physiological means of complete restoration; but the two most important muscular acts, namely, those concerned in circulation and respiration, are not completely arrested, sleeping or walking, although they undergo certain modifications.

In infancy and youth, when the organism is in process of development, sleep is more important than in adult life or in old age. The infant does little but sleep, eat and digest. In adult life, under physiological conditions, a person requires about eight hours of sleep; some need less, but few require more. In old age, unless after extraordinary exertion, less sleep is required than in adult life. Each individual learus by experience how much sleep is necessary in perfect health; and there is nothing which more completely incapacitates one for mental or muscular effort, especially the former, than loss of natural rest.

Sleeplessness is one of the most important of the predisposing calises of certain forms of brain-disease, a fact that is well recognized by practical physicians. One of the most severe methods of torture is long-continued deprivation of sleep; and persons have been known to sleep when subjected to acutely painful impressions. Severe muscular effort, even, may be continued during sleep. In forced marches, regiments have been known to sleep while walking; men 
have slept soundly in the saddle; persons will sometimes sleep during the din of battle; and other instances illustrating the imperative demand for sleep after prolonged vigilance might be cited. It is remarkable, also, how noises to which one has become accustomed may fail to disturb natural rest. Those who have been long habituated to the noise of a crowded city frequently find difficulty in sleeping in the stillness of the country. Prolonged exposure to intense cold induces excessive somnolence; and if this is not resisted, the sleep passes into stupor, the power of resistance to cold rapidly diminishes and death is the result. Intense heat often produces drowsiness, but, as is well known, is not favorable to natural sleep.

Sleep is preceded by a feeling of drowsiness, an indisposition to mental or physical exertion, and a general relaxation of the muscular system. It then requires a decided effort to keep awake. In sleep the voluntary muscles are inactive, the lids are closed, the ordinary impressions of sound are not appreciated, and sometimes there is a dreamless condition, in which the consciousness of existence is lost.

Dreams. - There may be, during sleep, mental operations of which there is no consciousness or recollection, unconscious cerebration, as it has been called. It is well known that dreams are vividly remembered immediately on awakening, but that the recollection of them rapidly fades away, unless they are brought to mind by an effort to recall and relate them. Whatever may be the condition of the mind in sleep, if the sleep is normal, there is repose of the cerebro-spinal system and an absence of voluntary effort, which restore the capacity for mental and physical exertion.

The impressionability and the activity of the human mind are so great, most of the animal functions are so far subordinate to its influence and the organism is so subject to unusual mental conditions, that it is difficult to determine with exactness the phenomena of sleep that are physiological and to separate those that are slightly abnormal. It can not be assumed, for example, that a dreamless sleep, in which existence is, as it were, a blank, is the only normal condition of repose of the system; nor is it possible to determine what dreams are due to previous trains of thought, to impressions from the external world received during sleep, and are purely physiological, and what are due to abnormal nervous influences, disordered digestion etc. It may be assumed, however, that an entirely refreshing sleep is normal.

That reflex ideas originate during sleep, as the result of external impressions, there can be no doubt ; and many remarkable experiments on the production of dreams of a definite character, by subjecting a person during sleep to peculiar influences, have been recorded. The halluci- 
nations produced in this way are called hypnagogic, and they occur usually when the subject is not in a condition favorable to sound sleep.

As regards dreams due to external impressions, it is a curious fact, which has been noted by many observers and is one that accords with the personal experience of all who have reflected on the subject, that trains of thought and imaginary events, which seem to pass over a long period of time in dreams, actually occur in the brain within a few seconds. A person is awakened by a certain impression, which undoubtedly has given rise to a dream that seemed to occupy hours or days, and yet the period of time between the impression and the awakening was hardly more than a few seconds; and persons will drop asleep for a few minutes, and yet have dreams with elaborate details and apparently of great length.

Condition of the Brain and Nerious System during Slecp. - During sleep the brain may be in a condition of absolute repose - at least, so far as there is any subjective knowledge of mental operations - or there may be more or less connected trains of thought. There is, also, as a rule, absence of voluntary effort, although movements may be made to relieve discomfort from position or external irritation, without awakening. The sensory nerves retain their properties, although the general sensibility is somewhat blunted; and the same may be said of the special senses of hearing, smell and probably of taste. There is every reason to believe that the action of the sympathetic system is not disturbed or affected by sleep, if the influence of the vasomotor nerves on the circulation in the brain be excepted.

Two opposite theories were formerly held in regard to the immediate cause of sleep. In one, this condition was attributed to venous congestion and increased pressure of blood in the brain, and this view probably had its origin in the fact that cerebral congestion induces stupor or coma. Stupor and coma, however, are distinct from natural sleep; for in the former the action of the brain is suspended, there usually is no consciousness, no dreaming, and the condition is manifestly abnormal. In animals. rendered comatose by opium, the brain when exposed is found deeply congested with venous blood. The same condition often obtains in profound anesthesia by chloroform, but a state of the brain nearly resembling normal sleep is observed in anesthesia by ether. These facts have been demonstrated by experiments on living animals and have been observed in the human subject in cases of injury of the head. When opium is administered in large doses, the brain is congested during the condition of stupor or coma; but this congestion is relieved when the animal passes, as sometimes happens, from the effects of the agent into a natural sleep. In view of these facts and others that will be stated 
hereafter, it is unnecessary to discuss further the theory that sleep is attended with or is produced by congestion of the cerebral vessels.

The idea that the circulation in the brain is diminished during sleep has been entertained by some physiologists, but it has rested chiefly on theoretical considerations. The experiments of Durham (I860) seem to demonstrate that the supply of blood to the brain is always greatly diminished during sleep. These experiments were made on dogs. A piece of the skull was removed with a trephine, and a watch glass was accurately fitted to the opening and cemented at the edges with Canada balsam. When the animals operated on were awake, the vessels of the pia mater were seen moderately distended and the circulation was active; but during natural sleep, the brain retracted and became pale. "The contrast between the appearance of the brain during its period of functional activity and during its state of repose or sleep was most remarkable." There can hardly be a doubt, after these experiments, that during sleep the cerebral circulation is considerably diminished in activity.

The influence of diminished supply of blood to the brain has been illustrated by compression of both carotid arteries. In an experiment performed on his own person, Fleming produced immediate and profound sleep in this way, and this result invariably followed in subsequent trials on himself and others. Waller produced anesthesia in patients by pressure on both pneumogastric nerves; but the nerves are so near the carotid arteries that they could hardly be compressed, in the human subject, without interfering with the current of blood, and such experiments do not positively show whether the loss of sensibility be due to pressure on the nerves or on the vessels. In some rare instances in which both carotid arteries have been tied in the human subject, it has been stated that there is an unusual drowsiness following the consequent diminution in the activity of the cerebral circulation; but this result is by no means constant, and the morbid conditions involved in so serious an operation usually are such as to interfere with their value as facts bearing on the question under consideration. So far as the human subject is concerned, the most important facts are the results of compression of both carotids in healthy persons. These, as well as experiments on animals, all go to show that the supply of blood to the brain is diminished during natural sleep, and that sleep may be induced by retarding the cerebral circulation by compressing the vessels of supply. When the circulation is interfered with by compressing the veins, congestion is the result, and there is stupor or coma.

If diminished flow of blood through the cerebral vessels is the cause of natural sleep, it becomes important to inquire how this condition of physiological anemia is brought about. It must be that when the system 
requires sleep, the vessels of the brain contract in obedience to a stimulus received through the sympathetic system of nerves, diminishing the supply of blood here as in other parts under varied physiological conditions. The vessels of the brain are provided with vasomotor nerves, and it is sufficient to have noted that the arteries are contracted during sleep, the mechanism of this action being well established by observations on other parts of the circulatory system.

Little is known of the intimate nature of the processes of nutrition of the brain during its activity and in repose; but there can be no doubt of the fact that there is more or less cerebral action at all times when one is awake. Although the mental processes are much less active during sleep, even at this time the operations of the brain are not always suspended. It is equally well established that exercise of the brain is attended with physiological wear of nervous tissue, and like other parts of the organism, its tissue requires periodic repose for regeneration of the substance consumed. Analogies to this are to be found in parts that are more easily subjected to direct observation. The muscles require repose after exertion, and the glands, when not actively engaged in discharging their secretions, present intervals of so-called rest. As regards the glands, during the intervals of rest the supply of blood to their tissue is much diminished. It is probable, also, that muscles in action receive more blood than during rest; but it is mainly when these parts are not active, and when the supply of blood is smallest, that the processes of regeneration of tissue seem to be most efficient. As a rule the activity of parts, while it is attended with an increased supply of blood, is a condition more or less opposed to the processes of repair, the hyperemia being, apparently, a necessity for the marked and powerful manifestations of their peculiar action. When the parts are active, the blood seems to be required to keep at the proper standard the so-called irritability of the tissues and to increase their power of action under proper stimulus. Exercise increases the power of regeneration and favors full development in the repose that follows; but during rest, the tissues have time to appropriate new matter, and this does not seem to involve a large supply of blood. A muscle is exhausted by prolonged exertion; and the large quantity of blood passing through the tissue carries away carbon dioxicle and other products of katabolism, which are increased in quantity, until it gradually uses up its capacity for work. Then follows repose; the supply of blood is reduced, but under normal conditions, the tissue repairs the waste which has been excited by action, the blood furnishing nutritive matter and carrying away a comparatively small quantity of effete products. 
It may safely be assumed that processes analogous to those just described take place in the brain. By absence of voluntary effort, the muscles have time for rest and for the repair of physiological waste, and their action is for the time suspended. As the activity of the brain involves consciousness, volition, the generation of thought, and, in short, the mental condition observed while awake, complete repose of the brain is characterized by the opposite conditions. It is true that the brain may be rested without sleep, by abstaining from mental effort, by the gratification of certain of the senses and by mental distraction of various kinds, and that the mind may work to some extent during sleep; but during the period of complete repose - a condition necessary to perfect health and full mental vigor - consciousness and volition are lost, there is no thought, and the brain, which does not receive blood enough to stimulate it to action, is simply occupied in the insensible repair of its substance and is preparing itself for renewed work. Exhaustion of the muscles produces a sense of fatigue of the muscular system, indisposition to muscular exertion, and a desire for rest, not necessarily involving drowsiness. Fatigue of the brain is manifested by indisposition to mental exertion, dulness of the special senses and a desire for sleep. Simple repose will relieve physiological fatigue of muscles; and when a particular set of muscles has been used, the fatigue often disappears when these muscles alone are at rest, although others be brought into action. Sleep, and sleep alone, relieves fatigue of the brain.

During sleep nearly all the physiological processes, except those directly under the control of the sympathetic nervous system, are diminished in activity. The circulation is slower, and the pulsations of the heart are less frequent, as well as the respiratory movements. These points have already been considered in connection with the physiology of circulation and respiration. Physiologists have little positive information in regard to the relative activity of the processes of digestion, absorption and secretion during sleep. The drowsiness which many persons experience after a full meal may be due in part to a determination of blood to the alimentary canal and a consequent diminution in the supply to the brain.

It must be that fatigue, as well as sleep, has a physical basis. While fatigue may be in part explained by the assumption that during exercise the reparative processes are for the time unequal to the wear or waste, this does not fully account for the nervous phenomena observed. It has been shown, indeed, by Ranke, and later by Mosso, that the blood of a fatigued animal injected into an animal at rest will produce promptly all the phenomena of fatigue. This is explained by the assumption that exercise involves the production of what may be called 
"fatigue-products"; but the characters of these products have not been ascertained. Nevertheless, they probably exist in the blood and affect the nerve-cells.

When fatigue is followed by sleep, it is certain that the supply of blood to the brain is considerably diminished. The only explanation of this is that in some way the somniferous agent in the blood operates through the vasomotor system. The fatigue-product or products induce a desire for sleep ; but the necessity for sleep is represented by certain structural changes that have taken place, probably in the nerve-cells of the brain. These changes are degenerative and their repair requires a more or less extended period of functional rest. Studies of these changes by Hodge, Mann, Vas, Lambert and others have afforded a reasonable physical basis of sleep.

Following nervous cell-activity or repeated stimulation of nerve-cells through the nerves, the cells undergo marked changes in size and configuration. The cells become shrunken, vacuolated and crenated; and the nuclei are even more shrunken and irregular in form, the diminution in size sometimes amounting to fifty per cent. These changes have been observed in cats, dogs, birds and other animals lower in the scale. That they may occur in the human subject is inferred. After a number of hours of repose - about equal to the daily period of sleep in the human subject - the cells and nuclei are found to have returned to their original condition.

In addition to the changes just indicated, it is more than probable that alterations occur in the Nissl bodies. If the view can be accepted that these bodies contain stored-up nervous energy that is consumed in the production of nerve-impulses and may be restored by rest, it may be assumed that the necessity for sleep is due - in part, at least - to destruction of chromatoplasm by active chromatolysis. There is, however, what may be called a passive chromatolysis in "disuse atrophy" of nerve-cells. This has been shown in the cell-degeneration that occurs after nerve-fibres attached to cells have been divided.

While the views of physiologists in regard to the office of Nissl bodies are to some extent speculative, it is certain that nerve-cells undergo degenerative changes during wakefulness and activity and that during sleep they are gradually restored to their normal condition and resume their normal appearance, calling normal the condition following repose. By these restorative processes the nervous system becomes capable of renewed activity. It is to be remembered, however, that this restoration may be in a measure produced by rest without sleep, and that sleep may follow a period of nervous and muscular inactivity as nearly complete as is possible in a waking condition. 


\section{CHAPTER XXV}

\section{SENSE OF TOUCH - OLFACTION - GUSTATION}

Muscular sense-Sense of touch - Appreciation of temperature - Olfaction - Olfactory (first nerve) - Properties and uses of the olfactory nerves - Mechanism of olfaction Relations of olfaction to the sense of taste - Gustation - Nerves of taste - Chorda tympani - Glosso-pharyngeal (ninth nerve) - General properties of the glosso-pharyngeal - Relations of the glosso-pharyngeal to gustation - Mechanism of gustation - Physiological anatomy of the organs of taste - Taste-beakers.

THE senses of smell, taste, sight and hearing involve peculiar organs, provided with nerves having special properties that usually are not endowed with what is described as general sensibility. These nerves have been omitted in the general description of the nervous system, as well as the organs in which they are distributed.

Sensations of pain, touch, contact (localization), temperature, weight, pressure and the so-called muscular sense, are all conveyed to the sensorium by what have been described as centripetal nerves, the sense of touch being perfected in certain parts by peculiar structures at the endings of the nerves. The muscular sense, by which weight and resistance are appreciated, undoubtedly depends largely on the muscular nerves. The above subdivisions are sufficiently distinct so far as the character of the sensations are concerned; and it remains to see whether they pass to distinct centres by special paths of conduction or are conveyed by what are known as nerves of general sensibility. As regards this question, physiologists have to rely mainly on experiments on the human subject, and pathological observations.

The general sensory nerves are sufficiently distinct in their properties from the true nerves of special sense. The latter convey peculiar impressions only, such as those of sight, hearing, smell and taste. The former, when strongly stimulated or irritated, always convey impressions of pain. Separating, then, all other senses, except the venereal sense, from the true special senses, it is proper to inquire whether it be reasonable to assume that any of the varieties of general sensation require special nerves for their conduction.

It is well known that a relatively strong stimulation of a sensory nerve or of sensitive parts is necessary for the production of a painful impression; and it is also well known that very painful impressions 
overpower impressions of touch, weight, pressure, temperature and the so-called muscular sense. In cases of disease, it is sometimes observed that tactile sensibility is retained in parts that are insensible as regards pain. It is possible that sensory nerve-fibres may become so altered in their properties as to be incapable of conducting painful impressions, while they still conduct sensations that are appreciated only as impressions of contact. This is observed in certain cases of artificial anesthesia. In hyperesthesia, or exaggerated sensibility to painful impressions, the tactile sense is necessarily overpowered in a greater or less degree. Impressions made on a sensory nerve in its course are alway's appreciated as painful, and the pain is referred to the terminal distribution of the nerve, this being a law of sensory perception. There is no sense of contact at the ends of the nerve, and there is no contact. The impression, in order to be perceived at all, must be painful. These facts may be in a measure applied to local impressions produced by extremes of heat and cold or by chemical or electric stimulation of sensitive parts.

The internal organs have as a rule no tactile sensibility, although they may be sensitive; and feeble impressions may not be appreciated, while stronger impressions are painful.

Titillation is produced by unusual feeble impressions or slight impressions frequently repeated on the peripheral ends of certain sensory nerves. These impressions are not precisely tactile nor are they painful. They produce peculiar sensations, and they frequently give rise to violent reflex movements, by what is known as a summation of sensory stimulations.

Muscular Sense (so called). - It is difficult to define exactly what is meant by the term "muscular sense," as it is used by some physiologists. In all probability the sense which enables one to appreciate the resistance, immobility or elasticity of substances that are grasped or stood upon, or which are in any way opposed to the exertion of muscular effort, may be greatly modified by education and habit. It is undoubtedly true, however, that general sensibility regulates the action of muscles to a considerable extent. If, for example, the lower extremities are paralyzed as regards sensation, the muscular power remaining intact, frequently the person so affected can not walk unless able to see the ground.

Those who regard the muscular sense as distinct from the sense of touch, weight or pressure, connect it with the neuro-muscular spindles, that have already been described in treating of the terminations of nerves in voluntary muscles.

In general the parts that are most sensitive to the impressions of 
touch, as the fingers, enable one to appreciate differences in pressure and weight with greatest accuracy. The sense of simple pressure, unaided by the estimation of weight by muscular effort, usually is more acute on the left side. Differences in weight can be accurately distinguished when they amount to only one-sixteenth, by estimating the muscular effort in lifting, in addition to the sense of pressure ; but the sense of pressure alone enables most persons to appreciate a difference of not less than one-eighth. When weights are tested by lifting with the hand, the appreciation of slight differences is more delicate if the weights are successively tested with the same hand than when two weights are placed, one in either hand. When the interval between the two trials is more than forty seconds, slight differences in weight - the difference between fourteen and a half and fifteen ounces (4I I and 425 grams), for example-can not be accurately appreciated. In such trials, it is necessary to have the metals used of the same temperature, for cold metals seem heavier than warm.

\section{Sense of TOUCH}

The different modes of termination of the sensory nerves have already been described; and in many instances it is possible to explain, by the anatomical characters of the nerves, the differences that have been observed in the delicacy of the tactile sensibility and sense of contact in different parts - differences which are very important pathologically as well as physiologically.

Variations in the Sense of Contact in Different Parts (Localization of Impressions). - In certain parts of the cutaneous surface the general sensibility is much more acute than in others. For example, a sharp blow on the face is more painful than a similar injury to other parts; and the eye, as is well known, is peculiarly sensitive. The appreciation of temperature varies in different parts, this probably depending to a great extent on habitual exposure. Some parts, as the soles of the feet or the axilla, are peculiarly sensitive to titillation. The sense of touch, also, by which the size, form, character of the surface, consistence etc., of objects are appreciated, is developed in a greater degree in some parts than in others. The tips of the fingers commonly are used to ascertain those properties of objects revealed by the sense of touch. This sense is capable of education and is almost always extraordinarily developed in persons who are deprived of some other special sense, as sight or hearing. The blind learn to recognize individuals by feeling of the face. A remarkable instance of this is quoted in works on physiology, of the blind sculptor, Giovanni Gonelli, who was said to model excellent likenesses, being guided by the sense of touch alone. Other 
instances of this kind are on record. The blind have been known to become proficients in conchology and botany, depending entirely on the touch. It is well known that the blind learn to read with facility by passing the fingers over raised letters but little larger than the letters in an ordinary folio Bible.

An easy method of determining the relative delicacy of the sense of contact in different parts of the cutaneous surface was devised a number of years ago (I 829) by E. H. Weber. This method consists in the application to the skin, of two fine points, separated from each other by a known distance. The individual experimented on should be blindfolded, and the points applied to the skin simultaneously. By carefully adjusting the distance between the points, a limit will be reached where the two impressions upon the surface are appreciated as one; and by gradually approximating them, the subject will suddenly feel both points as one, when an instant before, with the points a little farther removed from each other, he distinctly felt two impressions. This gives a measure of the delicacy of the sense of contact in different parts. An instrument, consisting of a pair of dividers with a graduated bar giving a measure of the separation of the points, combines simplicity, convenience of use and portability. This instrument is called an esthesiometer. The experiments of Weber were made on his own person. They showed some slight variations with the direction of the line of the two points, but these are not important. The following table is made of selections from the observations of Weber, taking those that are most likely to be useful as a guide in pathological investigations. The experiments of Valentin and others on different persons do not vary much in their results from the figures given in the table on opposite page.

By comparing the distribution of the tactile corpuscles with the results given in the table, it will be seen that the sense of contact is most delicate in those situations in which the tactile corpuscles are most abundant. In the space of a little more than $\frac{1}{12}$ of an inch (2.2 millimeters) square, on the palmar surface of the third phalanx of the index finger, Meissner counted the greatest number of corpuscles; namely, one hundred and eight. In this situation the tactile sensibility is more acute than in any other part of the skin, the mean distance indicated by the esthesiometer being 0.603 of a line, or 1.27 millimeter (Valentin). In the same space on the second phalanx, forty corpuscles were counted, the esthesiometer marking 1.558 line, or 3.27 millimeters (Valentin), this part ranking next in tactile sensibility after the red surface of the lips. One can readily understand how the tactile corpuscles, embedded in the amorphous substance of the cutaneous papillæ, might increase 
the delicacy of appreciation of slight impressions, by presenting hard surfaces against which the nerve-filaments can be pressed.

\section{TABLE OF VARIATIONS IN THE SENSE OF CONTACT IN DIFFERENT PORTIONS OF THE SKIN (WEBER)}

The sensibility is measured by the greatest distance between two points at which they convey a single impression when applied simultaneously. The measurements are given in lines ( $\frac{1}{12}$ of an inch, or a little more than 2 millimeters).

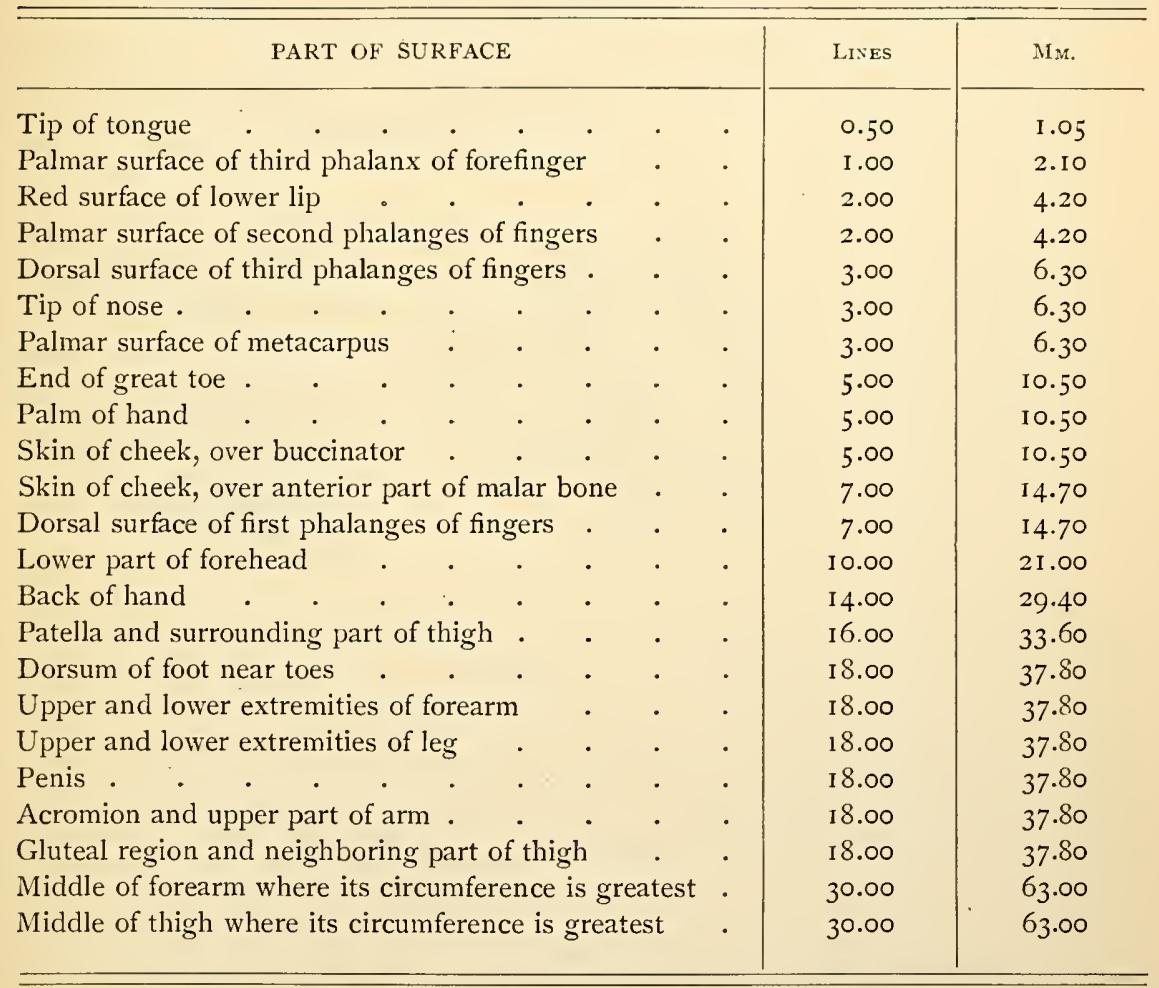

As regards those portions of the general cutaneous surface in which no tactile corpuscles have been demonstrated, it is not easy to connect the variations in the sense of contact with the nervous distribution, as little is known of the comparative richness of the terminal nervous filaments in these situations.

There is much uncertainty in regard to the location of a cerebral tactile centre. Ferrier described a diffused centre in the "hippocampal region," the action of which is crossed; but the observations to determine loss of the sense of contact after destruction of this part, made on monkeys, are by no means satisfactory. It may be stated provisionally 
that the centre for this sense probably is in the gyrus fornicatus, which is on the mesial surface of the brain, above the corpus callosum. However, Munk and many others locate this centre in the Rolandic area, a part containing several motor centres. These differences of opinion illustrate the evident difficulty of studying tactile sensibility in the inferior animals.

Appreciation of Temperature. - As regards the general temperature, the sense is relative and is much modified by habit. This statement needs no explanation. As is well known, what is cold for an inhabitant of the torrid zone would be warm for one accustomed to an excessively cold climate. Habitual exposure also modifies the sense of temperature. Many persons not in the habit of dressing warmly suffer but little in

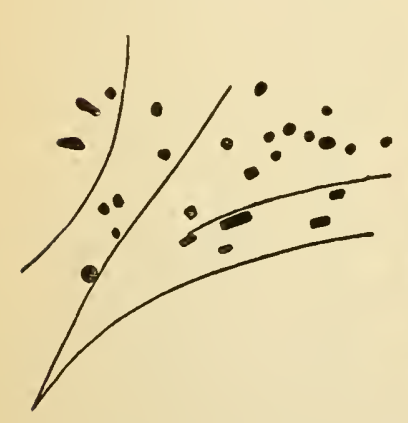

$A$

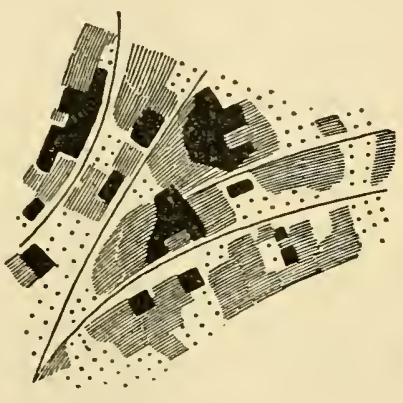

$B$

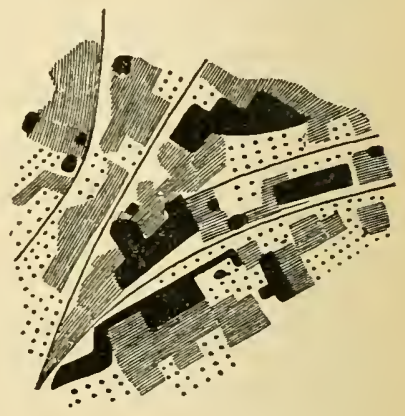

c

Fig. I55. - Map showing the relative distribution of the sensitivity to touch, warmth and cold in the pulm of the left hand (Goldscheider).

"In $A$, the whole surface is, when tested by a small cork applied by a spring, found of approximately equal sensitivity except in the areas marked black; these are relatively insensible for touch.

"In $B$, the areas of sensitivity to warmth stimuli are represented by degrees of shading, the most sensitive, by the black shading; the next, by the lined areas; the next, by the dotted areas; and those of least sensitiveness, by the blank spaces.

"In $C$, the topography of sensitivity to cold stimuli is indicated iu the same way as that to warm stimuli in $B . "$

extremely cold weather. Those who habitually expose the hands or even the feet to cold, render these parts comparatively insensible to temperature; and the same is true of those who often expose the hands, face or other parts to heat. The variations in the sensibility of different parts of the surface to temperature depend, also, on special properties of the parts themselves.

The experiments of Weber and others show that the skin is the chief organ for the appreciation of temperature, if the mouth, palate, vagina and rectum, by which the differences between warm and cold substances are readily distinguished, are excepted. In several instances in which large portions of the skin were destroyed by burns and other injuries, experiments have been made by applying spatulas of different 
temperatures. In one of these, a spatula, plunged in water at $48^{\circ}$ to $55^{\circ} \mathrm{Fahr}$. ( $9^{\circ}$ to $12^{\circ} \mathrm{C}$.), was applied to a denuded surface, and again, a spatula at $\operatorname{II} 3^{\circ}$ to $122^{\circ} \mathrm{Fahr}$. $\left(45^{\circ}\right.$ to $50^{\circ} \mathrm{C}$.). When the patient was requested to tell which was the warmer, the answers were as frequently incorrect as they were correct; but the discrimination was easy and certain when the applications were made to the surrounding healthy skin. When applications at a higher temperature were made to the denuded part, the patient suffered only pain.

Recent experiments have shown that there are distinct areas of the skin which are sensible to heat and others sensible to cold. These are called heat and cold spots. They are irregularly intercommingled with other areas, some of which are sensitive to painful and some to tactile impressions. Figure 155 shows the arrangement of the heat and cold areas.

The venereal sense is unlike any other sensation and is general as well as referable to the organs of generation. In this connection, however, it is interesting to note that the tactile sensibility of the palmar surface of the third phalanx of the fingers, measured by the esthesiometer, compared with the sensibility of the penis, is as 0.802 to 0.034 , or between twenty-three and twenty-four times greater.

\section{Olfaction}

The nerves directly connected with the senses of olfaction, vision and audition have little or no general sensibility. As regards the olfactory nerves, the parts to which they are distributed are so largely supplied with branches from the fifth, that it is somewhat difficult to determine the fact of their sensibility or insensibility to ordinary impressions. These nerves, however, are distributed to the mucous membrane of that portion only of the nasal cavity that is endowed with the special sense of smell.

Nasal Fossa. - The two irregularly-shaped cavities in the middle of the face, opening in front by the anterior. nares and connected with the pharynx by the posterior nares, are called the nasal fossæ. The membrane lining these cavities usually is called the Schneiderian mucous membrane, and sometimes, the pituitary membrane. This membrane is closely adherent to the fibrous coverings of the bones and cartilages by which the nasal fossæ are bounded, and it is thickest over the turbinated bones. It is continuous with the membrane lining the pharynx, the nasal duct and lachrymal canals, the Eustachian tube, the frontal, ethmoidal and sphenoidal sinuses and the antrum. There are openings leading from the nasal fossæ to all these cavities. 
The essential organ of olfaction is the mucous membrane lining the upper half of the nasal fossæ. Not only has it been shown anatomically that this part alone receives the terminal filaments of the olfactory nerves, but experiments have demonstrated that it is the only part capable of appreciating odorous impressions. If a tube is introduced into the nostril, placed horizontally over an odorous substance so that the emanations can not penetrate its calibre, no odor is perceived, though the membrane below the end of the tube might receive the emanations; but if the tube is directed toward the odorous substance, so that the

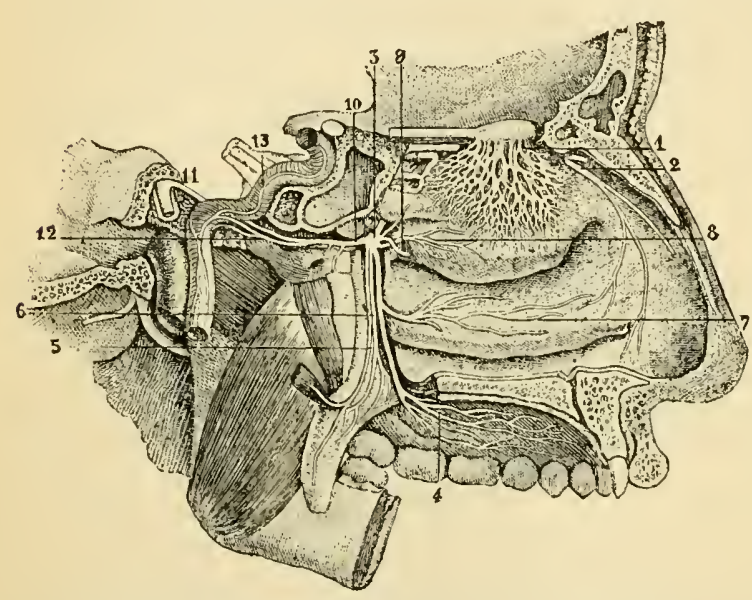

Fig. I56. - Olfactory ganglion and nerves (Hirschfeld).

I, olfactory ganglion and nerves; 2 , branch of the nasal nerve: 3. spheno-palatıne ganglion; 4,7 , branches of the great palatine nerve; 5 , posterior palatine nerve; 6 , middle palatine nerve; 8,9 , branches from the spheno-palatine ganglion: IO, II, I2, Vidian nerve and its branches; $1_{3}$, external carotid branch from the superior cervical ganglion. ish color. It is covered with long, delicate, columnar cells, nucleated, and each one provided with three to eight ciliary processes, the movements of which are from before backward. The olfactory membrane is provided with a large number of long racemose mucous glands, which produce a secretion that keeps the surface moist, a condition essential to the accurate perception of odorous impressions. The olfactory organ in some of the lower animals, in which the sense of smell is very delicate, is relatively larger than in man. In dogs it is much larger.

\section{Olfactory (First Nerve)}

The apparent origin of the olfactory nerve is by three roots from the inferior and internal portion of the frontal lobe of the cerebrum, in 
front of the anterior perforated space. The three roots are an external and an internal white root, and a middle root composed of gray matter. The external white root is long and delicate, passing outward, across the fissure of Sylvius, to the temporo-sphenoidal lobe. The internal white root is thicker and shorter than the external root and arises from the most posterior portion of the frontal lobe. The middle, or gray root arises from a little eminence of gray matter situated on the posterior and inner portion of the inferior surface of the frontal lobe.

The deep origin of the roots of the olfactory nerves is still a matter of discussion. The external root passes through the gray substance of the island of Reil, to a gray nucleus in the temporo-sphenoidal lobe, in front of the pes hippocampi. The fibres of the middle root have not been traced farther than the gray eminence from which they arise. The fibres of the internal root probably are connected with the fibres of the gyrus fornicatus. The three roots converge to form a single cord at the inner boundary of the fissure of Sylvius. This cord passes forward and slightly inward, in a deep groove between two convolutions on the under surface of the frontal lobe covered by the arachnoid membrane, to the ethmoid bone. This portion of the nerve is soft and friable. It is composed of both white and gray matter, the proportions being about two-thirds of the former to one-third of the

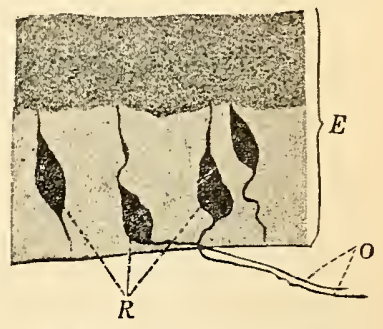

Fig. 157. - Vertical section of the olfactory membrane of an executed criminal, $\times 250$ (Zimmermann and Sobotta).

$E$, epithelium; $O$, olfactory fibres; $R$, olfactory cells. latter. The gray substance, derived from the gray root, is at the upper portion of the nerve, the white substance occupying the inferior and the lateral portions.

By the side of the crista galli of the ethmoid bone, the nerve-trunk expands into an oblong ganglion called the olfactory bulb. This is grayish in color, very soft, and contains the ordinary ganglionic elements. From the olfactory bulb, fifteen to eighteen nervous filaments are given off, which pass through foramina in the cribriform plate of the ethmoid bone. These filaments are composed entirely of nervefibres, and are quite resisting, owing to fibrous elements prolonged from the dura mater. It is strictly correct, perhaps, to regard these as the true olfactory nerves, the cord leading from the olfactory bulb to the cerebrum being properly a commissure. Having passed through the cribriform plate, the olfactory nerves are distributed to the olfactory membrane, in three groups : an inner group, distributed to the mucous membrane of the upper third of the septum; a middle group, to the upper portion of the nasal fossæ; and an outer group, to the mucous 
membrane covering the superior and middle turbinated bones and a portion of the ethmoid.

The mode of termination of the olfactory nerves differs from that of the ordinary sensory nerves, and is peculiar and characteristic, as it is in the other organs of special sense. The olfactory mucous membrane contains terminal nerve-cells, called olfactory cells, which are situated between the cells of epithelium. These are long, delicate, spindle-shaped, varicose structures, each one containing a clear round nucleus. In the frog there is a fine hair-like process projecting from each cell beyond the mucous membrane, which has not been observed in man or in the mammalia.

Propertics and Uses of the Olfactory Nerves. - It is almost certain that the olfactory nerves possess none of the general properties of ordinary nerves belonging to the cerebro-spinal system and are endowed with the special sense of smell alone. The filaments coming from the olfactory bulbs and distributed to the pituitary membrane have not been exposed and stimulated in living animals; but experiments on the nerves behind the olfactory bulbs show that they are insensible to ordinary impressions. Attempts have been made to demonstrate, in the human subject, the special properties of these nerves, by passing an electric current through the nostrils; but their situation is such that these observations are of necessity indefinite and unsatisfactory.

Among the experiments on the higher orders of animals, in which the olfactory nerves have been divided, may be cited, as open to no objections, those of Vulpian and Philipaux, on dogs. It is well known that the sense of smell usually is very acute in these animals. On dividing or extirpating the olfactory bulbs, "after the animal had completely recovered, it was deprived of food for thirty-six or fortyeight hours; then, in its absence, a piece of cooked meat was concealed in a corner of the laboratory. Animals, successfully operated on, then taken into the laboratory, never found the bait; and, nevertheless, care had been taken to select hunting-dogs." This experiment is conclusive; more so than those in which animals deprived of the olfactory bulbs were shown to eat feces without disgust, for this sometimes occurs in dogs that have not been mutilated.

Comparative anatomy shows that the olfactory bulbs usually are developed in proportion to the acuteness of the sense of smell. Pathological facts show, in the human subject, that impairment or loss of the olfactory sense is coincident with injury or destruction of these ganglia. Cases have been reported in which the sense of smell was lost or impaired from injury to the olfactory nerves. In nearly all the cases on record, the general sensibility of the nostrils was not affected. 
The delicacy of the sense of smell, measured by the possible tenuity of odorous particles that can be recognized through the olfactory organs, is very great, even in man, in whom olfaction is much inferior to this sense in some of the lower animals. Fischer and Penzoldt, experimenting with ethyl mercaptan $\left(\mathrm{C}_{2} \mathrm{H}_{5} \mathrm{SH}\right)$ in $\mathrm{I} 885$, were able to recognize the peculiar and disgusting odor of this substance in a dilution of one part in fifty billions of air, the weight of mercaptan distributed in three cubic inches (about 50 cubic centimeters) being estimated at

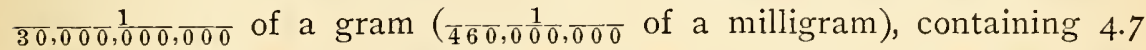
trillion molecules. It is said that the odor of iodoform can be recognized in quantities as small as $\overline{6}, \overline{50} \overline{0}, \frac{1}{000}, \overline{000}$ of a grain, or the one hundred billionth of a gram (Berthelot).

Mechanism of Olfaction. - In experimenting on the sense of smell it has been found difficult to draw an exact line of distinction between impressions of general sensibility and those which attack the special sense, or, in other words, between irritating and odorous emanations; and the vapors of ammonia, acetic acid, nitric acid etc., undoubtedly possess irritating properties that overpower their odorous qualities. It is unnecessary in this connection to discuss the different varieties of odors recognized by some of the earlier writers, as the fragrant, aromatic, fetid, nauseous etc., distinctions sufficiently evident from their mere enumeration; and it is plain enough that there are emanations, like those from delicately-scented flowers, that are easily recognizable by the sense of smell, while they make no impression on the ordinary sensory nerves. The marked individual differences in the delicacy of the olfactory organs in the human subject and in different animals are evidence of this fact. Hunting-dogs recognize odors to which most persons are insensible; and certain races of men are said to possess a remarkable delicacy of the sense of smell. Like the other special senses, olfaction may be cultivated by attention and practice, as is exemplified in the delicate discrimination of wines, qualities of drugs etc., by experts.

After what has been said concerning the situation of the olfactory organ in the upper part of the nasal fossæ and the necessity of particles impinging upon this membrane in order that their odorous properties may be appreciated, it is almost unnecessary to state that the passage of odorous emanations to this membrane by inspiring through the nostrils is essential to olfaction, so that animals or men, after division of the trachea, being unable to pass the air through the nostrils, are deprived of the sense of smell. The act of inhalation through the nose is an illustration of the mechanism by which the odorous particles may be brought at will in contact with the olfactory membrane. 
It is a curious point to determine whether the sense of smell is affected by odors passing from within outward through the nasal fossæ. Persons who have offensive emanations from the respiratory organs usually are not aware, from their own sensations, of any disagreeable odor. This fact has been explained by the assumption that the olfactory membrane gradually becomes accustomed to the odorous impression. This is an apparently satisfactory explanation; for it can hardly be supposed that the direction of the emanations, provided they come in contact with the membrane, could modify their effects. In a case of cancer of the stomach, with vomiting of fetid matters, the patient at first perceived an offensive odor when the gases from the stomach were expired through the nostrils; but this gradually disappeared (Longet).

Relations of Olfaction to the Sense of Taste. - The relations of the sense of smell to the sense of taste are very intimate. In the appreciation of delicate shades of flavor, it is well known that olfaction plays so important a part that it can hardly be separated from gustation. The common practice of holding the nose when disagreeable remedies are swallowed is an illustration of the connection between the two senses. In most cases of anosmia, there is inability to distinguish delicate flavors, and patients can distinguish by the taste, only sweet, saline, acid and bitter impressions.

It undoubtedly is true that the delicacy of the sense of taste is impaired when the sense of smell is lost. The experiment of tasting wines blindfolded and with the nostrils plugged, and the partial loss of taste during a severe coryza, are sufficiently familiar illustrations of this. In the great majority of cases, when there is complete anosmia, the taste is sensibly blunted; and in cases in which this does not occur, it is probable that the savory emanations pass from the mouth to the posterior portion of the nasal fossæ, and that here the mucous membrane is not entirely insensible to special impressions.

It is unnecessary, in this connection, to describe fully the reflex phenomena that follow impressions made on the olfactory membrane. The odor of certain sapid substances, under favorable conditions, will produce an abundant secretion of saliva and even of gastric juice, as has been shown by experiments on animals. Other examples of the effects of odorous impressions of various kinds are sufficiently familiar.

According to Ferrier and others, the olfactory centre is on the mesial surface of the brain, near the anterior extremity of the uncinate gyrus; but this location of the centre can not be regarded as definitely determined. Stimulation of this part in monkeys produces peculiar movements of the nostril and lip of the same side. 


\section{Gustation}

The special sense of taste gives the appreciation of what is known as the savor of certain substances introduced into the mouth; and this sense exists, in general terms, in parts supplied by filaments from the lingual branch of the fifth and the glosso-pharyngeal nerves.

It is assumed by some physiologists, that the true tastes are quite simple, presenting the qualities that are recognized as sweet, acid, bitter and saline; while the more delicate shades of what are called flavors nearly always involve olfactory impressions which it is difficult to separate entirely from gustation. Flavors, indeed, are regarded simply as odors. Applying the term "savor" exclusively to the quality that makes an impression on the nerves of taste, it is evident that the sensation is special in its character and different from the tactile sensibility of the parts involved and from the sensation of temperature. The terminal filaments of the gustatory nerves are impressed by the actual contact of savory substances, which must of necessity be soluble. To a certain extent there is a natural classification of savors, some of which are agreeable, and others, disagreeable; but even this distinction is modified by habit, education, and various other circumstances. Articles that are unpleasant in early life often become agreeable in later years. Inasmuch as the taste is to some extent an expression of the nutritive demands of the system, it is found to vary under different conditions. Chlorotic females, for example, frequently crave the most unnatural articles, and their morbid taste may disappear under appropriate treatment. Inhabitants of the frigid zone crave fatty articles of food and often drink rancid oils with avidity. Patients often become accustomed to the most disagreeable remedies and take them without repugnance. Again, the most savory dishes may even excite disgust, when the appetite has become cloyed; while abstinence sometimes lends a delicious flavor to the simplest articles of food. The taste for certain articles certainly is acquired; and this is almost always true of tobacco, now so largely used in civilized countries.

Anything more than the simplest classification of savors is difficult if not impossible. It is easy to recognize that certain articles are bitter or sweet, empyreumatic or insipid, acid or alkaline etc., but beyond these simple distinctions, the shades of difference are closely connected with olfaction and are too delicate and too many for detailed description. Some persons are comparatively insensible to nice distinctions of taste, while others recognize with facility the most delicate differences. Strong impressions may remove for a time the appreciation of less powerful and decided flavors. The tempting of the appetite by a 
proper gradation of gustatory and odorous impressions is illustrated in the modern cuisine, which aims at an artistic combination and succession of dishes and wines, so that the agreeable sensations are prolonged to the utmost limit. This may often be regarded as a violation of strictly hygienic principles, but it none the less exemplifies the cultivation of the sense of taste.

Nerves of Taste. - Two nerves, the chorda tympani and the glossopharyngeal, are endowed with the sense of taste. These nerves are distributed to distinct portions of the gustatory organ. The chorda tympani has already been referred to as one of the branches of the facial; the glosso-pharyngeal has not yet been described.

Chorda Tympani. - In the description already given of the facial, the chorda tympani is spoken of as the fourth branch. It passes through the tympanum, between the ossicles of the ear, and joins the inferior maxillary division of the fifth at an acute angle, between the two pterygoid muscles, becoming so closely united with it that it can not be followed farther by dissection. The filaments of this branch probably originate from the intermediary nerve of Wrisberg, which some anatomists describe as arising from the glosso-pharyngeal. The course of the filaments of the chorda tympani, after this nerve has joined the fifth, is shown by the effect on the sense of taste and the alteration of the nerve-fibres following its division; and observations on the sense of taste show that this nerve is distributed to the anterior two-thirds of the tongue. The general properties of the chorda tympani have been ascertained only by observations made after its paralysis or division. All experiments in which a stimulus has been applied directly to the nerve in living animals have been negative in their results.

In paralysis of the facial in which the lesion affects the root so deeply as to involve the chorda tympani, there is loss of taste in the anterior two-thirds of the tongue, general sensibility being unaffected; and many cases illustrating this fact have been recorded. Aside from cases of paralysis of the facial with impairment of taste, in which the general sensibility of the tongue is intact, instances are on record of affections of the fifth pair, in which the tongue was insensible to ordinary impressions, the sense of taste remaining.

Passing from the consideration of pathological facts to experiments on living animals, the results are equally satisfactory. Although it is somewhat difficult to observe impairment of taste in animals, experimenters have succeeded in training dogs and cats so as to observe the effects of colocynth and various sapid substances applied to the tongue. In a number of experiments of this kind, it has been observed that after 
section of the chorda tympani - or of the facial so as to involve the chorda tympani - the sense of taste is abolished in the anterior twothirds of the tongue on the side of the section. In a number of cases the introduction of an artificial membrana tympani in the human subject has been followed by loss of taste on the corresponding side of the tongue, and on both sides, when membranes were introduced into both ears. This disappears when the membranes are removed; and the phenomena may properly be referred to pressure on the chorda tympani.

As regards the gustatory properties of the anterior two-thirds of the tongue, certainly in the human subject, it may be stated without reserve that these properties depend on the chorda tympani, its gustatory filaments taking their course to the tongue with the lingual branch of the inferior maxillary division of the fifth. In addition, the lingual branch of the fifth contains filaments derived from the large root of this nerve, which give general sensibility to the mucous membrane.

\section{Glosso-Pharyngeal (Ninth Nerve)}

The glosso-pharyngeal is distributed to those portions of the gustatory mucous membrane not supplied by filaments from the chorda tympani. It undoubtedly is a nerve of taste; and the question of its other uses will be considered in connection with its general properties, as well as the differences between this nerve and the chorda tympani.

Physiological Anatomy. - The apparent origin of the glosso-pharyngeal is from the groove between the olivary and restiform bodies of the medulla oblongata, between the roots of the auditory nerve above and the pneumogastric below. The deep origin is in a gray nucleus in the lower part of the floor of the fourth ventricle, between the nucleus of the auditory nerve and the nucleus of the pneumogastric. From this origin, the nerve passes forward and outward, to the posterior foramen lacerum, by which it emerges with the pneumogastric, the spinal accessory and the internal jugular vein. At the upper part of the foramen, is a small ganglion, the jugular ganglion, including only a portion of the root. Within the foramen, is the main ganglion, including all the filaments of the trunk, called the petrous ganglion, or the ganglion of Andersch.

At or near the ganglion of Andersch the glosso-pharyngeal usually receives a small filament from the pneumogastric. This communication is sometimes wanting. The same may be said of a filament passing to the glosso-pharyngeal from the facial, which is not constant. Branches 
from the glosso-pharyngeal go to the otic ganglion and to the carotid plexus of the sympathetic.

The distribution of the glosso-pharyngeal is quite extensive. The tympanic branch (the nerve of Jacobson) arises from the anterior and external parts of the ganglion of Andersch, and enters the cavity of the tympanum, where it divides into six branches. Of these six branches,

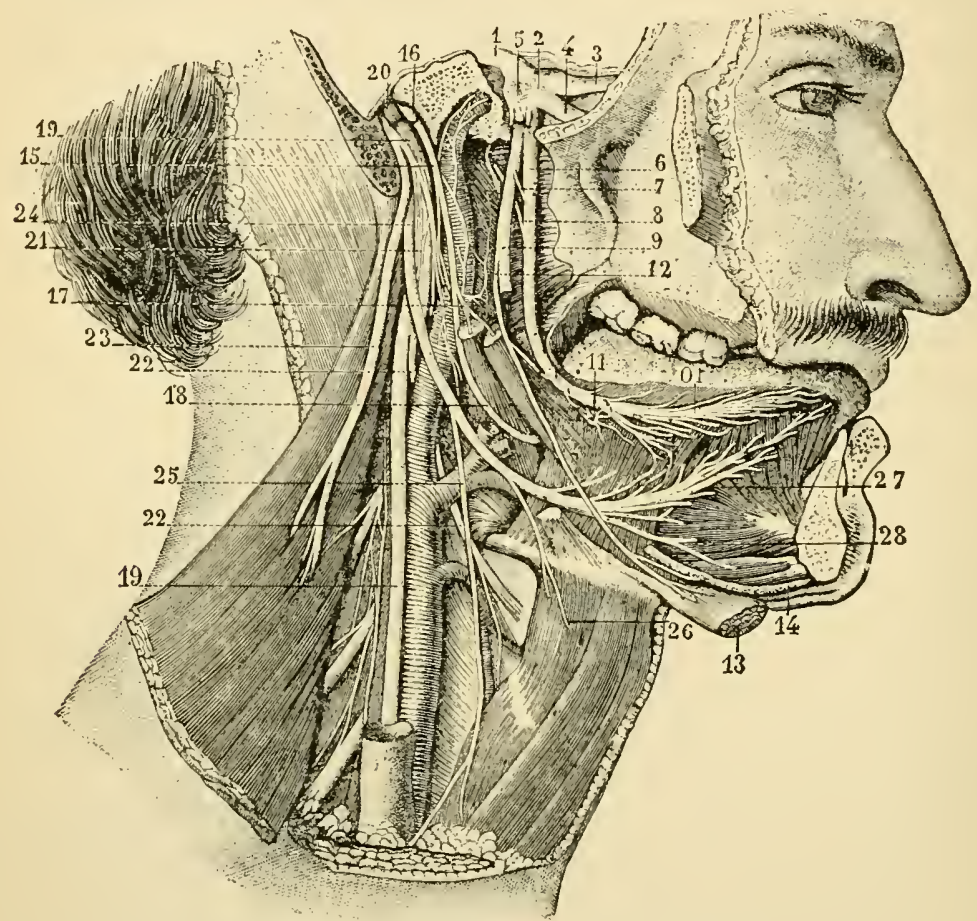

Fig. 158. - Glosso-pharyngeal nerve (Sappey).

I, large root of the fifth nerve; 2 , ganglion of Gasser; 3 , ophthalmic division of the fifth; 4 , superior maxillary division: 5 , inferior maxillary division; 6, 10, lingual branch of the fifth, containing the filaments of the chorda tympani; 7 , branch from the sublingual to the lingual branch of the fifth; 8, chorda tympani; 9, inferior dental nerve; II, submaxillary ganglion; I2, mylo-hyoid branch of the inferior dental nerve; 13 , anterior belly of the digastric muscle; I4, section of the mylo-hyoid muscle; 15, 18, glosso-pharyngeal nerve; I6, ganglion of Andersch; I7, branches from the glossu-pharyngeal to the stylo-glossus and the stylo-pharyingeus muscles; 19, 19, pneumogastric; 20, 2I, ganglia of the pneumogastric; 22,22 , superior laryngeal nerve; 23 , spinal accessory; $24,25,26,27,28$, sublingual nerve and branches.

two posterior are distributed to the mucous membrane of the fenestra rotunda and the membrane surrounding the fenestra ovalis; two anterior are distributed, one to the carotid canal, where it anastomoses with a branch from the superior cervical ganglion, and the other to the mucous membrane of the Eustachian tube; two superior branches are distributed to the otic ganglion and, as is stated by some anatomists, to 
the spheno-palatine ganglion. In addition to these, a branch has been described as going to the geniculate ganglion of the intermediary nerve of Wrisberg.

A little below the posterior foramen lacerum, the glosso-pharyngeal sends branches to the posterior belly of the digastric and to the stylohyoid muscle. There is also a branch which joins a filament from the facial to the stylo-glossus.

Opposite the middle constrictor of the pharynx, three or four branches join branches from the pneumogastric and the sympathetic, to form together the pharyngeal plexus. This plexus contains a number of ganglionic points, and filaments of distribution from the three nerves go to the mucous membrane and to the constrictors of the pharynx. The mucous membrane probably is supplied by the glosso-pharyngeal. It is probable, also, that the muscles of the pharynx are supplied by filaments from the pneumogastric, which are derived originally from the spinal accessory.

Near the base of the tongue, branches are sent to the mucous membrane covering the tonsils and the soft palate.

The lingual branches penetrate the tongue about midway between its border and centre, are distributed to the mucous membrane at its base and are connected with certain of the papillæ.

General Properties of the Glosso-Pharyngeal. - To ascertain the general properties of this nerve, it must be stimulated at its root before it has contracted anastomoses with other nerves; and the nerve must be divided in order to avoid reflex phenomena. Taking these precautions it has been found that stimulation of the peripheral end of the nerve does not give rise to muscular movements. There can be no doubt of the fact that the nerve is sensory, although its sensibility is dull. In experiments in which the nerve has seemed to be insensible to ordinary impressions, it is probable that the animals operated on had been exhausted more or less by pain and loss of blood in the operation of exposing the nerve, which, it is well known, abolish the sensibility of some of the nerves.

Experiments on the glosso-pharyngeal are not very definite and satisfactory in their results as regards the general sensibility of the base of the tongue, the palate and the pharynx. The sensibility of these parts seems to depend chiefly on branches of the fifth, passing to the mucous membrane through Meckel's ganglion. Experiments show, also, that the reflex phenomena of deglutition take place mainly through these branches of the fifth, and that the glosso-pharyngeal has little or nothing to do with the process. In fact, after division of both glosso-pharyngeal nerves, deglutition does not seem to be affected. Stimulation of the 
glosso-pharyngeal by electricity inhibits respiration for a short time. This action is reflex.

Relations of the Glosso-Pharyngcal Neres to Gustation. - Relying on experiments on the inferior animals, particularly dogs, it seems

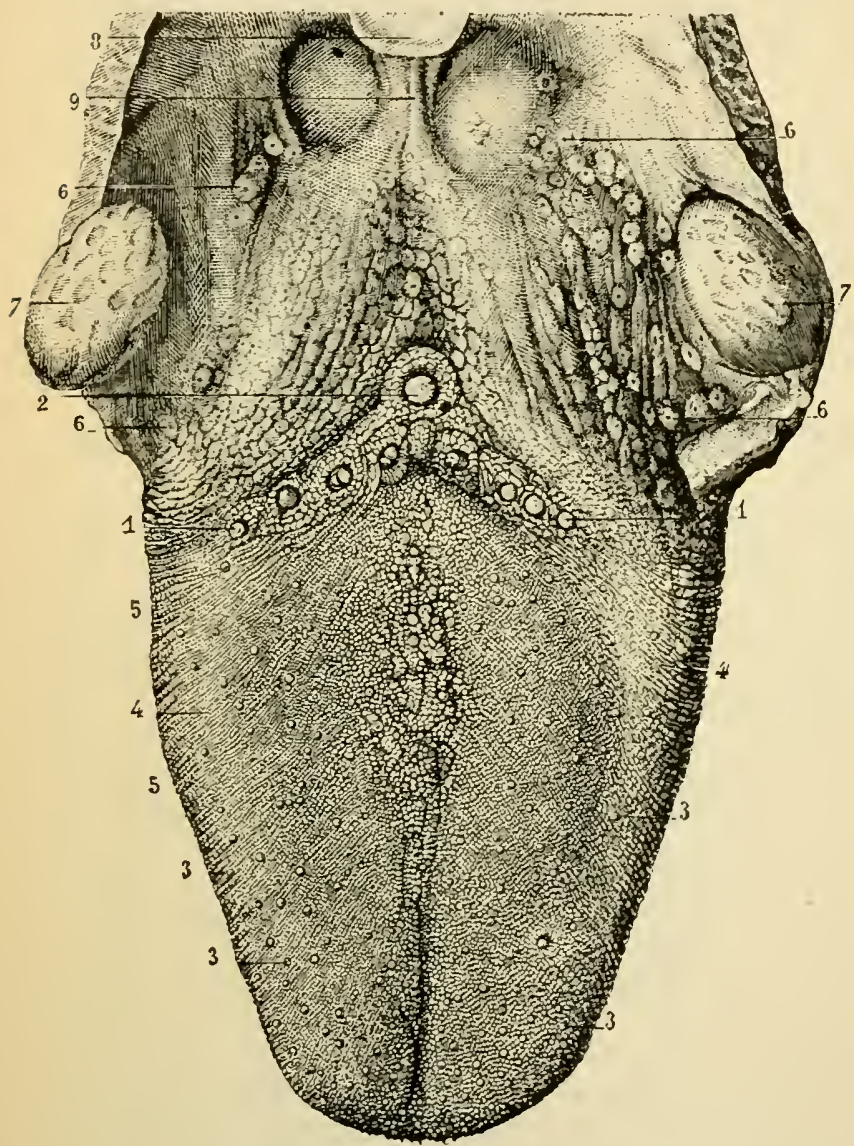

Fig. 159. - Papilla of the tongue (Sappey).

I, I, circumvallate papillæ; 2 , median circumvallate papilla, which entirely fills the foramen crecum; $3,3,3,3$, fungiform papillæ; 4,4 , filiform papillx; 5,5 , vertical folds and furrows of the border of the tongue; $6,6,6,6$, glands at the base of the tongue; 7,7 , tonsils; 8 , epiglottis; 9 , median glosso-epiglottidean fold. certain that there are two nerves presiding over the sense of taste: The chordatympani nerve gives this sense to the anterior two-thirds of the tongue exclusive$\mathrm{ly}$; and the glossopharyngeal supplies this sense to the posterior portion of the tongue. The chorda tympani seems to have nothing to do with general sensibility ; while the glossopharyngeal is an ordinary sensory nerve, as well as a nerve of special sense.

Mcchanism of Gustation. - Articles that make the special impression on the gustatory organ are in solution ; introduced into the mouth, they increase the flow of saliva, the reflex action involving chiefly the submaxillary and sublingual glands; there usually is more or less mastication, which increases the flow of the parotid saliva; and during the acts of mastication and the first stages of deglutition, the sapid substances are distributed over the gustatory membrane, so extensively, indeed, that it is difficult to locate exactly the seat of the special impression. In this way, by the movements of the tongue, aided by an increased flow 
of saliva, the actual contact of the savory articles is rapidly effected. The thorough distribution of these substances over the tongue and the

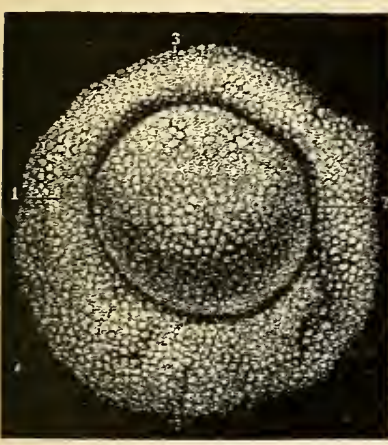

Fig. 160.-Medium-sized circumvallate papilla, $\times 20$ (Sappey).

1, papilla, the base only being apparent (it is seen that the base is covered with secondary papillæ) ; 2 , groove between the papilla and the surrounding wall; 3,3 , wall of

the papilla. $\quad$ it appears that sweets attack especially the tip of the tongue; acids and salines, the sides of the tongue; bitters, the back of the tongue. It is said that an acid taste may be recognized in a solution equivalent to one part of sulphuric acid in one thousand of water. Chewing the leaves of the gymnema sylvestre abolishes for a time the sense of bitters and sweets, but sensibility to acids and salines remains. This plant is used by the natives in India as a remedy for snake-bite.

\section{Physiological Anatomy of the Organs of} Taste. - Anatomical and physiological researches have shown that, at least in the human subject, the organ of taste probably is confined to the dorsal surface of the tongue and the lateral portion of the soft palate. The upper surface of the tongue presents a large number of special papillæ, called in contradistinction to the filiform papillæ, fungiform and circumvallate. These are not found on its under surface or anywhere except on the superior portion; and it is now well established that the circumvallate and

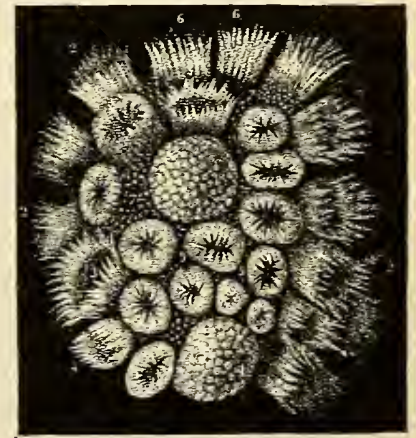

Fig. 161. - Fungiform, fliform, and hemispherical papulle, $\times 20$ (Sappey).

I, I, two fungiform papillæ covered with secondary papillæ; 2,2 , 2, filiform papillæ; 3 , a filiform papilla, the prolongations of which are turned outward; 4 , a filiform papilla with vertical prolongations; 5,5 , small filiform papillæ with the prolongations turned inward ; 6,6 , filiform papillæe with striations at their bases ; 7,7 , hemispherical papillæ, slightly apparent, situated between the fungiform and the filiform papillæ. 
fungiform papillæ alone contain the organs of taste. Experiments on the gustatory organs, by the application of solutions to different parts through fine glass tubes, have shown that the mucous membrane around a papilla has no gustatory sensibility, but that different savors can be distinguished when a single papilla is touched (Camerer).

In Fig. I 59, which represents the dorsal surface of the tongue, the large circumvallate papillæ, usually seven to twelve in number, are seen in the form of an inverted $\mathrm{V}$, occupying the base of the tongue. The fungiform papillæ are scattered over the surface but are most abundant at the point and near the borders. Both varieties of papillæ are distinguishable by the naked eye.

The circumvallate papillæ simply are enlarged fungiform papillæ, each one surrounded with a circular ridge, or wall, and covered with small secondary papillæ. The fungiform papillæ have each a short thick pedicle and an enlarged rounded extremity. One hundred and fifty to two hundred of these can easily be counted. These, also, present small secondary papillæ on their surfaces. When the mucous membrane of the tongue is examined with a low magnifying power, particularly after maceration in acetic or in dilute hydrochloric acid, their structure is readily observed. They are abundantly supplied with bloodvessels and nerves.

Several glandular structures are found beneath the mucous membrane of the tongue. On either side of the frenum, near the point, is a gland about three-quarters of an inch (20 millimeters) long and onethird of an inch ( 8.5 millimeters) broad, which has five or six little openings on the under surface of the tongue. Near the taste-buds, are small racemose glands, which discharge a watery secretion by minute ducts that open into the grooves within the walls of the circumvallate papillæ (Ebner).

Taste-Buds. - Lovén and Schwalbe (1867) described under this name peculiar structures that are supposed to be the true organs of taste. They are found on the lateral slopes of the circumvallate papillæ and occasionally on the fungiform papillæ. They consist of flask-like collections of spindle-shaped cells, which are received into little excavations in the epithelial covering of the mucous membrane, the bottom resting on the connective-tissue layer. Their form is ovoid, and at the neck of each flask is a rounded opening called the taste-pore. Their length is $\frac{1}{350}$ to $\frac{1}{300}$ of an inch ( $7 \mathrm{I}$ to $83 \mu$ ), and their transverse diameter, about $\frac{1}{6} \frac{1}{0}$ of an inch $(4 \mathrm{I} \mu)$. The cavity of the taste-buds is filled with cells, of which two kinds are described. The first variety, the outer cells, or the cover-cells, are spindle-shaped and curved to correspond to the wall of the beaker. These come to a point at the taste- 
pore. In the interior are elongated cells, with large clear nuclei, which are called taste-cells. Delicate hair-like processes are connected with the taste-cells and extend through the taste-pores, in the form of fine filaments. Bodies similar to the taste-buds have been found on the papillæ of the soft palate and uvula, the mucous membrane of the epiglottis and some parts of the top of the larynx. As regards these structures in the tongue, it has been found that four or five months after section of the glosso-pharyngeal on one side in rabbits, the taste-buds

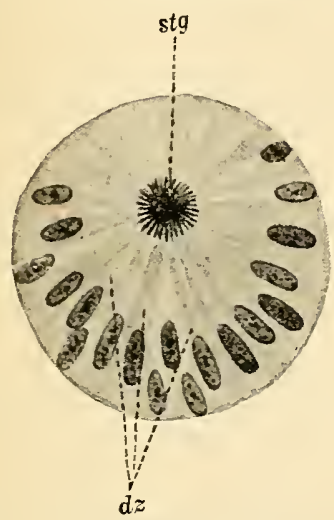

Fig. I62. - Transverse section of a tastebud from a rabbit, $\times 500$ (Sobotta).

$s t g$, taste-pore ; $d z$, lining epithelium.

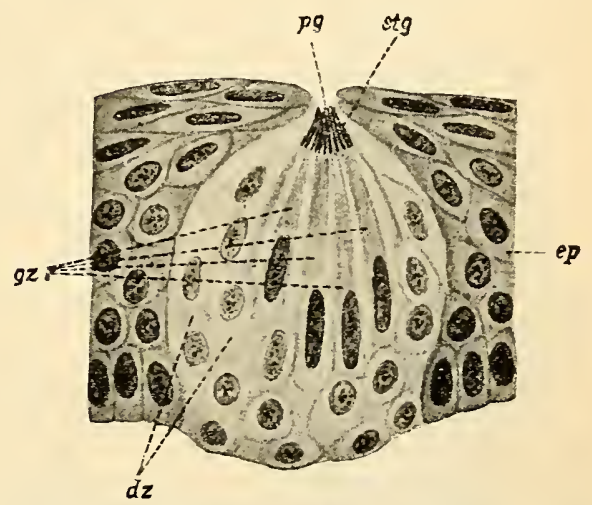

Fig. I63. - Vertical section of a taste-bud, $\times 500$ (Sobotta).

ep, stratified epithelium; $d z$, lining epithelium; $g z$, gustatory cells; $p g$, taste-pore; stg, taste-filaments (gustatory rods).

on the corresponding side of the posterior portion of the tongue disappear, while they remain perfect on the sound side.

According to the views of those who have described the so-called taste-buds, sapid solutions find their way into the interior of these structures through the taste-pores and come in contact with the taste-cells, these cells being directly connected with the terminal filaments of the gustatory nerves.

Ferrier and others have described a taste-centre near the so-called olfactory centre in the uncinate gyrus; but their observations are not very definite, and the location of a centre for gustation must still be regarded as uncertain. 


\title{
CHAPTER XXVI
}

\author{
THE ORGAN OF VISION
}

Optic (second nerve) - General properties of the optic nerves - Physiological anatomy of the eyeball - Sclerotic coat - Cornea - Choroid cuat - Ciliary processes - Iris - Pupillary membrane - Retina - Layer of rods and cones (Jacob's membrane, or bacillar membrane) - Crystalline lens - Suspensory ligament of the lens (zone of Zinn) - Aqueous humor - Vitreous humor - Summary of the anatomy of the globe of the eye.

THE chief points to be considered in the physiology of vision are the following :-

I. The physiological anatomy and the general properties and uses of the optic nerves.

2. The physiological anatomy of the parts essential to normal vision.

3. The laws of refraction, diffusion etc., bearing on the physiology of vision.

4. The action of the different parts of the eye in the production and appreciation of correct images.

5. Binocular vision.

6. The physiological anatomy and uses of accessory parts, as the muscles that move the eyeball.

7. The physiological anatomy and uses of the parts that protect the eye, as the lachrymal glands, eyelids etc.

\section{Optic (Second Nerve)}

The bands that pass from the tubercula quadrigemina to the eyes are divided into the optic tracts, which extend from the tubercula on either side to the chiasm, or commissure; the chiasm, or the decussating portion; and the optic nerves, which pass from the chiasm to the eyes.

The optic tracts arise each by two roots, internal and external. The internal roots, which are the smaller, arise from the anterior tubercula quadrigemina and pass through the internal corpora geniculata to the optic chiasm. The external roots, which are the larger, arise from the posterior part of the optic thalami, pass to the external corpora geniculata, from which they receive fibres, and thence to the chiasm.

Partly by anatomical researches and partly by experiments on the 
cerebral cortex in the lower animals and pathological observations on the human subject, it has been shown that fibres from the apparent origin of the optic tracts pass backward to the gray matter of the occipital lobes of the cerebrum. They then pass to the bulb, extend down as far as the decussation of the pyramids and probably are concerned in the reflex movements of the iris.

The two roots of each optic tract unite above the external corpus geniculatum, forming a flattened band, which takes an oblique course around the under surface of the crus cerebri to the optic commissure.

The optic commissure, or chiasm, is situated just in front of the corpus cinereum, resting upon the olivary process of the sphenoid bone. As its name implies, this is the point of union between the nerves of the two sides. At the commissure the fibres from the optic tracts take three directions ; and in addition, the commissure contains filaments passing from one eye to the other, which have no connection with the optic tracts. The four sets of fibres in the optic commissure are the following:-

I. Decussating fibres, passing from the optic tract on either side to the eye of the opposite side. The greatest part of the fibres take this direction. Their relative situation is internal.

2. External fibres, fewer than the preceding, which pass from the optic tract to the eye on the same side.

3. Fibres situated on the posterior border of the commissure, which pass from one optic tract to the other and do not

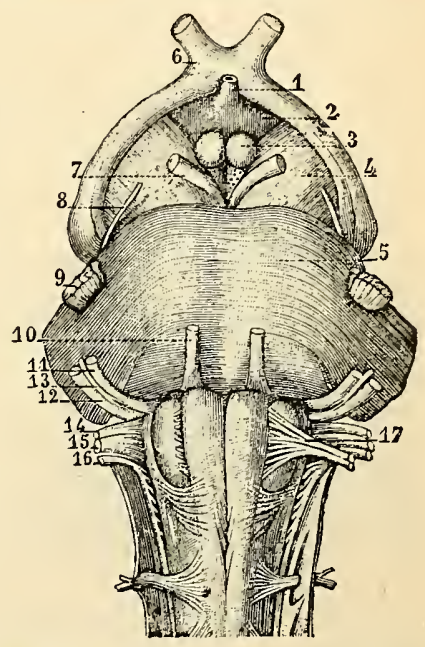

Fig. 164.-Optic tracts, commissure and nerves (Hirschfeld).

I, infundibulum; 2, corpus cinereum; 3 , corpora albicantia; 4 , cerebral peduncle; 5 , pons Varolii; 6, optic tracts and nerves, decussating at the commissure, or chiasm; 7 , motor oculi communis; 8, patheticus; 9, fifth nerve; Io, motor oculi externus; II, facial nerve; I2, auditory nerve; 13, nerve of Wrisberg; 14, glossopharyngeal nerve; i5, pneumogastric; 16, spinal accessory; 17, sublingual nerve. go to the eyes. These fibres are scanty and sometimes wanting.

4. Fibres situated on the anterior border of the commissure, greater in number than the preceding, which pass from one eye to the other and which have no connection with the optic tracts.

The fibres of the optic tracts on the two sides are connected with distinct portions of the retina. This fact is illustrated in cases of hemianopsia, which show that the decussating fibres have the following directions and distribution :-

From the left side of the encephalon, fibres pass to the right eye, 
supplying the inner, or nasal mathematical half of the retina, from a vertical line passing through the macula lutea. Fibres also pass to the left eye, supplying the outer, or temporal half of the retina. The macula lutea, then, and not the point of entrance of the optic nerve, is in the true vertical line of division of the retina.

With the exception of a few grayish filaments, the fibres of the optic tracts and the optic nerves are of the ordinary medullated variety and present no differences in structure from the general cerebro-spinal nerves.

The optic commissure is covered with a fibrous membrane and is more resisting than the optic tracts. The optic nerves are rounded and are enclosed in a double sheath derived from the dura mater and the arachnoid. They pass into the orbit on either side and penetrate the

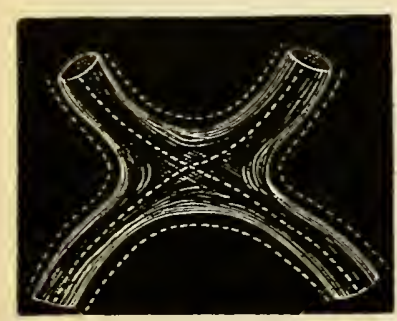

Fig. 165. - Diagram of the decussation of fibres at the optic commissure.

The dotted lines show the four directions of the fibres. sclerotic at the posterior, inferior and internal portion of the globe. As the nerves enter the globe they lose their coverings from the dura mater and arachnoid. The sheath derived from the dura mater is adherent to the periosteum of the orbit at the sphenoidal fissure, and when it reaches the globe, it fuses with the sclerotic coat. Just before the nerves penetrate the globe, they each present a well-marked constriction. At the point of penetration there is a thin but strong membrane, presenting a number of perforations for the passage of the nervous filaments. This membrane, the lamina cribrosa, is in part derived from the sclerotic, and in part, from the coverings of the individual nerve-fibres, which lose their investing membranes at this point. In the interior of each eye there is a little mammillated eminence, formed by the united fibres of the nerve. The retina, with which the optic nerve is connected, will be described as one of the coats of the eye.

In the centre of the optic nerve is a minute canal, lined with fibrous tissue, in which are lodged the central artery of the retina and its corresponding vein, with a delicate nervous filament from the ophthalmic ganglion. The vessels penetrate the optic nerve $\frac{1}{3}$ to $\frac{3}{4}$ of an inch (8.5 to 19. I millimeters) behind the globe. The central canal does not exist behind these vessels.

General Propertics of the Optic Nerves. - There is little to be said regarding the general properties of the optic nerves, except that they are the only nerves capable of conveying to the cerebrum the special impressions of sight and are not endowed with general sensibility. 
Their mechanical or electric stimulation produces luminous impressions.

\section{Physiological Anatomy of the Eyeball}

The eyeball is a spheroidal body, partly embedded in a cushion of fat in the orbit, protected by the surrounding bony structures and the eyelids, its surface bathed with the secretion of the lachrymal gland and movable in various directions by the action of certain muscles. It is surrounded with a thin serous sac, the capsule of Tenon, which exists in two layers. The outer layer lies next the fat in which the globe is embedded and the inner layer invests the sclerotic coat. When the axis of the eye is directed forward, the globe has the form of a sphere in its posterior five-sixths, with the segment of a smaller sphere occupying its anterior sixth. The segment of the smaller sphere, bounded externally by the cornea, is more prominent than the rest of the globe.

The eyeball is made up of several coats enclosing certain refracting media. The external coat is the sclerotic, covering the posterior fivesixths of the globe, which is continuous with the cornea, covering the anterior sixth. This is a dense, opaque, fibrous membrane, for the protection of the inner coats and the contents of the globe. The cornea is dense, resisting and transparent. The muscles that move the globe are attached to the sclerotic coat.

Were it not for the prominence of the cornea, the eyeball would present very nearly the form of a perfect sphere, as will be seen by the following measurements of its various diameters ; but the prominence of its anterior sixth gives the greatest diameter in the antero-posterior direction.

In measurements made by Sappey, one to four hours after death, of the eyes of twelve adult females and fourteen adult males, of different ages, the following mean results were obtained:-

\begin{tabular}{|c|c|c|c|c|}
\hline \multirow{2}{*}{ SUBJECtS EXAMINED } & \multicolumn{4}{|c|}{ DiAMETERS (INCH, AND MILLIMETERS IN PARENTHESES) } \\
\hline & Antero-posterior & Transverse & Vertical & Oblique \\
\hline $\begin{array}{l}\text { Mean of } 12 \text { females, } 18 \text { to } 81 \text { years of age } \\
\text { Mean of } 14 \text { males, } 20 \text { to } 79 \text { years of age }\end{array}$ & $\begin{array}{l}0.941 \text { (23.9 mm.) } \\
0.968(24.6 \mathrm{~mm} .)\end{array}$ & $\begin{array}{l}0.91 \mathrm{I}(23.4 \mathrm{~mm}) \\
0.94 \mathrm{I}(23.9 \mathrm{~mm} .)\end{array}$ & $\begin{array}{l}0.905 \\
0.925(23.0 \mathrm{~mm} .)\end{array}$ & $\begin{array}{l}0.937(23.8 \mathrm{~mm} .) \\
0.949(24.1 \mathrm{~mm} .)\end{array}$ \\
\hline
\end{tabular}

From these results it is seen that all the diameters are less in the female than in the male. The antero-posterior diameter is the greatest of all, and the vertical diameter is the shortest. The measurements at different ages, not cited in the table just given, show that the excess of the antero-posterior diameter over the others diminishes with age. 
Sclerotic Coat. - The sclerotic is the dense, opaque, fibrous covering of the posterior five-sixths of the eyeball. Its thickness is different in different portions. At the point of penetration of the optic nerve, it measures $\frac{1}{35}$ of an inch (I millimeter). It is thinnest at the middle portion of the eye, measuring about $\frac{1}{50}$ of an inch ( 0.5 millimeter), and is a little thicker again near the cornea. This membrane is composed chiefly of bundles of ordinary connective tissue. The fibres are slightly wavy, and are arranged in flattened bands, which are alternately longitudinal and transverse, giving the membrane a lamellated appearance, although it can not be separated into distinct layers. Mixed with these bands of connective-tissue fibres are small fibres of elastic tissue. The vessels of the sclerotic are scanty. They are derived from the ciliary vessels and the vessels of the muscles of the eyeball. The tissue of the sclerotic yields gelatin on boiling.

Cornea. - The cornea is the transparent membrane that covers about the anterior sixth of the globe of the eye. As before remarked, this is the most prominent portion of the eyeball. It is in the form of a segment of a sphere, attached by its borders to the segment of the larger sphere formed by the sclerotic. The thickness of the cornea is about $\frac{1}{30}$ of an inch (0.8 millimeter), in its central portion, and about $\frac{1}{25}$ of an inch (I millimeter) near its periphery. Its substance is composed of transparent fibres, arranged in complete layers something like the layers of the sclerotic. It yields chondrin instead of gelatin on boiling.

On the external, or convex surface of the cornea, are several layers of delicate, transparent, nucleated epithelium. The most superficial cells are flattened, the middle cells are rounded, and the deepest cells are elongated and arranged perpendicularly. These cells become slightly opaque and whitish after death. Just beneath the epithelial covering of the cornea, is a very thin, transparent membrane, described by Bowman under the name of the "anterior elastic lamella." This membrane, with its cells, is a continuation of the conjunctiva. It is sometimes called Bowman's membrane.

The proper corneal structure is composed of flattened bundles of very pale fibres interlacing with each other in every direction. Their arrangement is lamellated, although they can not be separated into complete and distinct layers. Between the bundles of fibres, lie a great number of stellate, anastomosing, connective-tissue corpuscles. In these cells and in the intervals between the fibres, there is a considerable quantity of transparent liquid. The fibres constituting the substance of the cornea are continuous with the fibrous structure of the sclerotic, from which they can not be separated by maceration. At the margin of the cornea the opaque fibres of the sclerotic abruptly become trans- 
parent. The corneal substance is very tough and will resist a pressure sufficient to rupture the sclerotic.

On the posterior, or concave surface of the cornea, is the membrane of Descemet or of Demours. This is elastic, transparent, structureless, rather loosely attached, and covered with a single layer of regularly polygonal, nucleated epithelium. At the circumference of the cornea a portion of this membrane passes to the anterior surface of the iris, in the form of a number of processes which constitute the ligamentum iridis pectinatum, a portion passes into the substance of the ciliary muscle and a portion is continuous with the fibrous structure of the sclerotic.

In the adult the cornea is almost without bloodvessels, but in fotal life it presents a rich plexus extending nearly to the centre. These disappear, however, before birth, leaving a very few delicate looped vessels at the extreme edge.

In the cornea, fine nerve-fibres terminate in the nuclei of the posterior layer of the epithelium of its convex surface. The cornea also contains lymph-spaces and the socalled "wandering cells." The surface of the cornea is exquisitely sensitive.

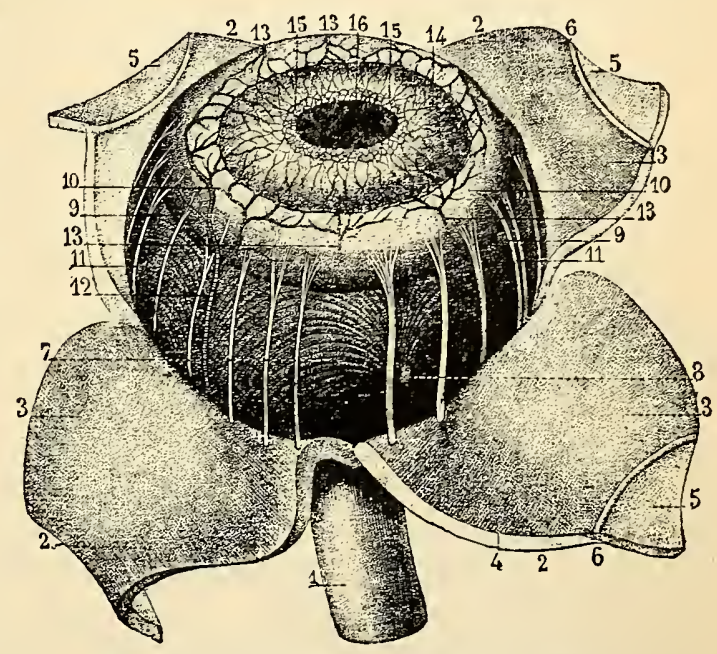

Fig. 166. - Choroid coat of the eye (Sappey).

I, optic nerve; $2,2,2,2,3,3,3,4$, sclerotic coat, divided and turned back to show the choroid; $5,5,5,5$, the cornea, divided into four portions and turned back; 6,6 , canal of Sichlemm; 7 , external surface of the choroid, traversed by the ciliary nerves and one of the long ciliary arteries; 8 , central vessel, into which open the vasa vorticosa; 9,9 , Io, ro, choroid zone; II, II, ciliary nerves; I2, long ciliary artery; I3, I3, I3, I3, anterior ciliary arteries; $I_{4}$, iris; I5 $_{5}$ I5 $_{5}$, vascular circle of the iris; I6, pupil.

Choroid Coat. - Calling the sclerotic and the cornea the first coat of the eyeball, the second is the choroid, with the ciliary processes, the ciliary muscle and the iris. This was called by the older anatomists the uvea, a name which was later applied, sometimes to the entire iris and sometimes to its posterior, or pigmentary layer. The choroid and ciliary processes will be described together as the second coat. The ciliary muscle and the iris will be described separately.

The choroid is distinguished from the other coats by its dark color and its great vascularity. It occupies that portion of the eyeball corresponding to the sclerotic. It is perforated posteriorly by the optic nerve and is 
connected in front with the iris. It is delicate in structure and composed of two or three distinct layers. Its thickness is $\frac{1}{85}$ to $\frac{1}{25}$ of an inch ( 0.3 to I millimeter). Its thinnest portion is at about the middle of the eye. Posteriorly it is a little thicker. Its thickest portion is at its anterior border.

The external surface of the choroid is connected with the sclerotic by vessels and nerves (the long ciliary arteries and the ciliary nerves) and very loose connective tissue. This is sometimes called the membrana fusca, although it can hardly be regarded as a distinct layer. It contains, in addition to bloodvessels, nerves and fibrous tissue, a few irregularly-shaped pigment-cells.

The vascular layer of the choroid consists of arteries, veins and capillaries, arranged in a peculiar manner. The layer of capillary vessels, which is internal, is sometimes called the tunica Ruyschiana. The arteries, which are derived from the posterior short ciliary arteries and are connected with the capillary plexus, lie just beneath the pigmentary layer of the retina. The plexus of capillaries is closest at the posterior portion of the membrane. The veins are external to the other vessels. They are very abundant and are disposed in curves converging to four trunks. This arrangement gives the veins a peculiar appearance, and they have been called vasa vorticosa. The pigmentary portion is composed, over the greatest part of the choroid, of a single layer of regularly-polygonal cells, somewhat flattened, measuring $\frac{1}{200 \overline{0}}$ to $\frac{1}{150} \overline{0}$ of an inch ( 12 to $16 \mu$ ) in diameter. These cells are filled with pigmentary granules of uniform size and give to the membrane its characteristic dark-brown or chocolate color. The pigmentary granules in the cells are less abundant near their centre, where a clear nucleus can readily be observed. In the anterior portion of the membrane, in front of the anterior limit of the retina, the cells are smaller, more rounded, more completely filled with pigment and present several layers. Beneath the layer of hexagonal pigment-cells, the intervascular spaces of the choroid are occupied by stellate pigment-cells. The cells next the layer of rods and cones are regarded as constituting the outer, or pigmentary layer of the retina. These cells send little hairlike processes inward between the rods and cones.

Ciliary Processes. - The anterior portion of the choroid is arranged in the form of folds or plaits projecting internally, called the ciliary processes. The largest of these folds are about $\frac{1}{10}$ of an inch $(2.5$ millimeters) in length. They are sixty to eighty in number. The larger folds are of nearly uniform size and are arranged regularly around the margin of the crystalline lens. Between these folds, which constitute about two-thirds of the entire number, are smaller folds, lying, without 
regular alternation, between the larger. Within the folds, are received corresponding folds of the thick membrane, continuous anteriorly with the hyaloid membrane of the vitreous humor, called the zone of Zinn.

The ciliary processes present bloodvessels, which are somewhat larger than those of the rest of the choroid. The pigmentary cells are smaller and are arranged in several layers. The anterior border of the processes is free and contains little or no pigment.

Ciliary Muscle. - This muscle, formerly known as the ciliary ligament and now sometimes called the tensor of the choroid, is the agent for the accommodation of the eye to vision at different distances. Under this view, the ciliary muscle is an organ of great importance; and it is essential, in the study of accommodation, to have an exact idea of its relations to the coats of the eye and to the crystalline lens.

The form and situation of the ciliary muscle are as follows: It surrounds the anterior margin of the choroid, in the form of a ring about $\frac{1}{8}$ of an inch (3.2 millimeters) wide and $\frac{1}{50}$ of an inch (0.5 millimeter) in thickness at its thickest portion, which is its anterior border. It becomes thinner from before backward, until its posterior border apparently fuses with the fibrous structure of the choroid. It is semitransparent and of a grayish color. Its situation is just outside of the ciliary processes, these processes projecting in front of its anterior border, about $\frac{1}{25}$ of an inch (I mil-

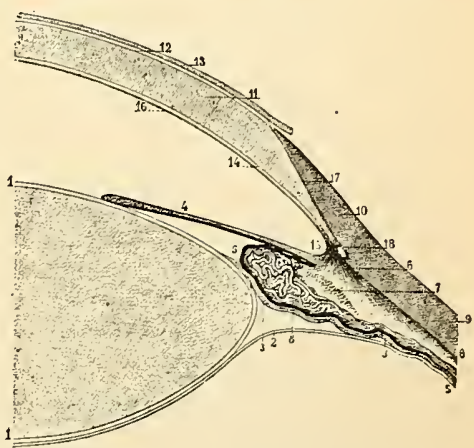

Fig. I67. - Ciliary muscle, $\times 5$ (Sappey). I, I, crystalline lens; 2, hyaloid membrane; 3, zone of Zinn; 4, iris; 5, 5, one of the ciliary processes; 6,6 , radiating fibres of the ciliary muscle; 7 , section of the circular portion of the ciliary muscle; 8 , venous plexus of the ciliary process; 9 , 10, sclerotic coat; II, I2, cornea; I3, epithelial layer of the cornea; 14 , membrane of Descemet; 15, ligamentum iridis pectinatum; r6, epithelium of the membrane of Descemet; I7, union of the sclerotic coat with the cornea; 18 , section of the canal of Schlemm. limeter). Regarding the anterior border of this muscle as its origin and the posterior border as its insertion, it arises in front from the circular line of junction of the cornea and sclerotic, the border of the membrane of Descemet, and the ligamentum iridis pectinatum. Its fibres, which are chiefly longitudinal, pass backward and are lost in the choroid, extending somewhat farther back than the anterior limit of the retina. In addition, a network of circular muscular fibres has been described, lying over the anterior portion of the ciliary body, at the periphery of the iris, beneath the longitudinal fibres. Some of these fibres have an oblique direction.

The ciliary muscle is composed mainly of muscular fibres. These fibres, anatomically considered, belong to the non-striated variety. 
They are pale, present a number of oval longitudinal nuclei and have no strixe.

It is evident, from the arrangement of the fibres of the ciliary muscle, that its action must be to approximate the border of connection of the sclerotic and cornea and the circumference of the choroid, compressing the vitreous humor and relaxing the suspensory ligament of the lens. This action enables the lens to change its form; and it adapts the curvature of the lens to vision at different distances. The nerves of the ciliary muscle are derived from the long and the short ciliary.

Iris. - The iris corresponds to the diaphragm of optical instruments. It is a circular nembrane, situated just in front of the crystalline lens, with a round perforation (the pupil) near its centre.

The attachment of the greater circumference of the iris is to the line of junction of the cornea and sclerotic, near the origin of the ciliary muscle, the latter passing backward to be inserted into the choroid, and the former passing directly over the crystalline lens. The diameter of the iris is about half an inch ( 12.5 millimeters). The pupil is subject to considerable variations in size. When at its medium of dilatation, the diameter of the pupil is $\frac{1}{8}$ to $\frac{1}{6}$ of an inch ( 3.2 to 4.2 millimeters). The pupillary orifice is not in the mathematical centre of the iris but is situated a little toward the nasal side. The thickness of the iris is a little greater than that of the choroid, but it is unequal in different parts, the mernbrane being thinnest at its great circumference and its pupillary border, and thickest at about the junction of its inner third with the outer two-thirds. It slightly projects anteriorly and divides the space between the lens and the cornea into two chambers, anterior and posterior, the anterior chamber being much the larger. Taking advantage of a property of the crystalline lens called fluorescence, which enables an observer, by concentrating on it a blue light, to see the boundaries in the living eye, Helmholtz has demonstrated that the posterior surface of the iris and the anterior surface of the lens are actually in contact, except, perhaps, for a certain distance near the periphery of the iris. This being the case, the posterior chamber is very small and exists only near the margins of the lens and the iris.

The color of the iris is different in different individuals. Its anterior surface usually is very dark near the pupil and presents colored radiations toward its periphery. Its posterior surface is of a darkpurple color and is covered with pigment-cells.

The iris presents three layers. The anterior layer is continuous with the membrane of the aqueous humor. At the greater circumference, it presents little fibrous prolongations, forming a delicate dentated membrane continuous with the ligamentum iridis pectinatum. The 
membrane covering the general anterior surface of the iris is extremely thin and is covered with cells of tessellated epithelium. Just beneath this membrane are a number of irregularly-shaped pigment-cells.

The posterior layer of the iris is thin, easily detached from the middle layer, and contains a number of small cells rich in pigmentary granules. Some anatomists recognize this membrane only as the uvea.

The middle layer constitutes by far the greatest part of the substance of the iris. It is composed of connective tissue, muscular fibres of the non-striated variety, many bloodvessels and probably nerve-terminations. Directly surrounding the pupil, forming a band about $\frac{1}{50}$ of an inch ( 0.5 millimeter) in width, is a layer of non-striated muscular fibres, called the sphincter of the iris. In addition to the sphincter, are radiating fibres, which can be traced from near the circumference of the iris almost to its pupillary border, lying both in front of and behind the circular fibres.

The bloodvessels of the iris are derived from the arteries of the choroid, from the long posterior ciliary and from the anterior ciliary arteries. The long ciliary arteries are two branches, running along the sides of the eyeball, between the sclerotic and choroid, to form finally a circle surrounding the iris. The anterior ciliary arteries are derived from the muscular branches of the ophthalmic. They penetrate the sclerotic a little behind the iris and join the long ciliary arteries in the vascular circle. From this circle, the vessels branch and pass into the iris, to form a smaller arterial circle around the pupil. The veins of the iris empty into a circular sinus situated at the junction of the cornea with the sclerotic. This is called the circular venous sinus, or the canal of Schlemm.

The nerves of the iris are the long ciliary, from the fifth, which are sensory, and the short ciliary, from the ophthalmic ganglion. The circular fibres are animated by filaments from the third nerve, which pass through the ophthalmic ganglion. The radiating fibres receive filaments derived from the cervical sympathetic, probably through the carotid plexus and the ganglion of Gasser.

Pupillary Membrane. - At a certain period of fœtal life the pupil is closed with a membrane connected with the lesser circumference of the iris, called the pupillary membrane. This is not distinct during the first months; but between the third and the fourth months, it is readily seen. It is most distinct at the sixth month. The membrane is thin and transparent and completely separates the anterior from the posterior chamber of the eye. It is provided with vessels derived from the arteries of the iris, anastomosing with each other and turning back in the form of loops near the centre. At about the seventh month it begins to give 
way at the centre, gradually atrophies and scarcely a trace of it can be seen at birth. The presence and condition of the pupillary membrane often are important as an aid in determining the age of a fœetus.

Retina. - The retina is described by anatomists as the third tunic of the eye. It is closely connected with the optic nerve, and the most important structures entering into its composition are continuous with prolongations from the nerve-cells. This is the membrane endowed with the special sense of sight, the other structures in the eye being accessory.

If the sclerotic and choroid are removed from the eye under water, the retina is seen, in perfectly fresh specimens, in the form of a delicate transparent membrane covering the posterior portion of the vitreous humor. A short time after death it becomes slightly opaline. It extends over the posterior portion of the eyeball to a distance of about $\frac{1}{15}$ of an inch ( $\mathrm{I} .7$ millimeter) behind the ciliary processes. When torn from its anterior attachment, it presents a finely-serrated edge, called the ora serrata. This edge adheres very closely, by mutual interlacement of fibres, to the zone of Zinn. In the middle of the membrane, its thickness is about $\frac{1}{120}$ of an inch $(200 \mu)$. It becomes thinner nearer the anterior margin, where it measures only about $\frac{1}{300}$ of an inch $(80 \mu)$. Its external surface is in contact with the choroid, and its internal, with the hyaloid membrane of the vitreous humor.

The optic nerve penetrates the retina about $\frac{1}{8}$ of an inch (3.2 millimeters) within, and $\frac{1}{1^{2}}$ of an inch (2.I millimeters) below the anteroposterior axis of the globe, presenting at this point a small rounded elevation on the internal surface of the membrane, perforated in its centre for the passage of the central artery of the retina. At a point $\frac{1}{12}$ to $\frac{1}{8}$ of an inch (2.I to 3.2 millimeters) external to the point of penetration of the nerve, is an elliptic spot, its long diameter being horizontal, about $\frac{1}{8}$ of an inch (2. I millimeters) long and $\frac{1}{36}$ of an inch ( 0.7 millimeter) broad, called the yellow spot of Sömmerring, or the macula lutea. In the centre of this spot is a depression called the fovea centralis. This depression is in the axis of distinct vision. The yellow spot exists only in man and the quadrumana.

The structures in the retina that present the greatest physiological importance are the layer of pigment-cells, the layer of rods and cones, the layer of nerve-cells, and the filaments that connect the rods and cones with the cells. These are the only anatomical elements of the retina, so far as is known, that are directly concerned in the reception of optical impressions; and they will be described rather minutely, while the intermediate layers will be considered more briefly. 
Most anatomists recognize ten layers in the retina :-

I. Inner limitary membrane.

2. Expansion of fibres of the optic nerve.

3. Layer of ganglion-cells.

4. Inner molecular layer.

5. Inner nuclear layer.
6. Outer molecular layer.

7. Outer nuclear layer.

8. Outer limitary membrane.

9. Layer of rods and cones.

Io. Layer of pigment-cells.

I. The inner limitary membrane is a delicate structure, with fine striæ and nuclei, composed of connective-tissue elements. It is about $\frac{1}{2500 \overline{0}}$ of an inch ( $1 \mu$ ) in thickness. From this membrane, connective-tissue elements (fibres of Müller) are sent into the various layers of the retina, where they form a framework for the support of the other structures.

2. The layer formed by the expansion of the optic nerve is composed of pale transparent nerve-fibres, $\frac{1}{50000}$ to $\frac{1}{2000}$ of an inch (0.5 to I $\mu$ ) in diameter. These do not require special description.

3. The layer of ganglion-cells is composed of multipolar nerve-cells, measuring $\frac{1}{3000}$ to $\frac{1}{50}$ of an inch $(8$ to $32 \mu$ ) in diameter. In the centre of the retina, at the macula lutea, the cells present eight layers, and they diminish to a single layer near the periphery. The smaller cells are situated near the centre, and the larger, near the periphery. Each cell sends off several filaments (two to twenty-five), probably going to the layer of rods and cones, and a single filament which becomes continuous with one of the filaments of the optic nerve.

4. The inner molecular layer consists of a plexus of minute fibrils and fine granules.

5. The inner nuclear layer is composed of nuclear bodies, rather large and some of them stellate in form.

6. The outer molecular layer consists of a plexus of fine fibrils and. nuclei.

7. The outer nuclear layer presents clear, oval, nuclear bodies, connected with the rods and cones by one process to each rod or cone, and sending fibrils to the adjacent molecular layer.

8. The outer limitary membrane is composed of delicate connectivetissue elements.

9. The layer of rods and cones is the most important of all and will receive special description.

IO. The layer of pigment-cells has already been described in connection with the choroid coat.

Layer of Rods and Cones (Jacob's .Membrane - the Bacillar. Membrane). - The layer of rods and cones is composed of rods, or cylinders, extending through its entire thickness, closely packed, and giving to the 
external surface a regular mosaic appearance; and between these, is a greater or less number of flask-shaped bodies, the cones. This layer is about $\frac{1}{33}$ of an inch $(76 \mu)$ in thickness at the middle of the retina; $\frac{1}{400}$ of an inch $(62 \mu)$, about midway between the centre and the periphery; and near the periphery, about $\frac{1}{450}$ of an inch $(55 \mu)$. At the macula lutea the rods are wanting. Over the rest of the membrane, the rods predominate and the cones become less and less frequent toward the periphery.

The rods are regular cylinders, their length corresponding to the thickness of the layer, terminating above in truncated extremities, and

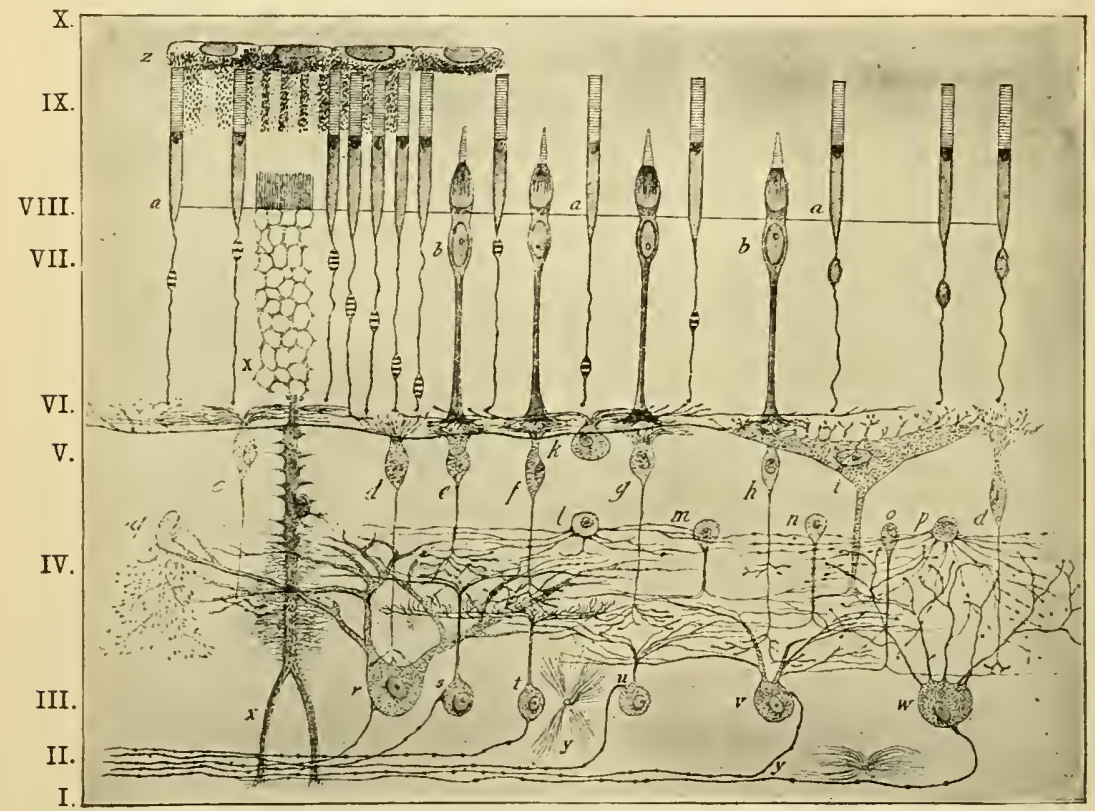

Fig. 168. - Diagram of the retina (Kallius).

I, inner limitary membrane; II. expansion of fibres of the optic nerve; III, layer of ganglioncells; IV, inner molecular layer; $V$, inner nuclear layer ; VI, outer molecular laver; VII, outer nuclear layer ; VIII, outer limitary membrane ; IX, layer of rods and cones; X, layer of piginentcells.

NotF. - The small letters in this figure refer to minute details of structure that have not been considered in the text. The lower border of the cut may be taken as representing the inner limitary membrane. The numbering of the layers is from below upward, reversing the order in the original diagram, and a number for the inner limitary membrane has been added.

below in points which probably are continuous with the filaments of connection with the nerve-cells. Their diameter is about $\frac{1}{130 \overline{0}}$ of an inch $(2 \mu)$. They are clear, of rather a fatty lustre, soft and pliable, but somewhat brittle, and so alterable that they are with difficulty seen in a natural state. They should be examined in perfectly fresh preparations, moistened with liquid from the vitreous humor or with serum. When 
perfectly fresh it is difficult to make out anything but an entirely homogeneous substance; but shortly after death each rod seems to be divided by a delicate line into an outer and an inner segment, the outer being a little the longer. At the upper extremity of the inner segment, is a hemispherical body, with its convexity presenting inward, called the lentiform body. The entire inner segment is somewhat granular and often presents a granular nucleus at its inner extremity. The outer segment apparently differs in its constitution from the inner segment and is not similarly affected by reagents. Treated with dilute acetic acid the outer segment becomes broken up transversely into thin disks.

The cones probably are of the same constitution as the rods, but the portion called the inner segment is pyriform. The straight portion above (the outer segment) is sometimes called the cone-rod. The entire cones are about half the length of the rods and occupy the inner portion of the layer. The outer segment is in its constitution precisely like the outer segment of the rods. The inner segment is slightly granular and contains a nucleus. The cones are connected below with filaments passing into the deeper layers of the retina.

At the fovea centralis, Jacob's membrane is composed entirely of elongated cones with no rods. These are slightly increased in thickness at the macula lutea, but are diminished again in thickness, by about one-half, at the fovea centralis. At the forea the optic nervefibres are wanting; and the ganglion-cells, which exist in a single layer over other portions of the retina, here present six to eight layers, except at the very centre, where there are but three layers. Of the layers between the cones and the ganglion-cells, the outer nuclear layer and the outer molecular layer remain in the fovea, while the inner nuclear layer and the inner molecular layer are wanting. At the forea, indeed, those elements of the retina which may be regarded as purely accessory disappear, leaving only the structures that are concerned directly in the reception of visual impressions.

The retina becomes progressively thinner from the centre to the periphery. The molecular and granular layers and the nervous layers are lost in the anterior half of the membrane.

The following is the probable mode of connection between the rods and cones and the ganglion-cells: The filaments from the bases of the rods and cones pass inward, presenting in their course the corpuscles which have been described in the molecular and granular layers, and finally become, as is thought, directly continuous with the poles of the ganglion-cells. The cells send filaments to the layer formed by the expansion of the optic nerve, which are continuous with the nervefibres. 
The following description, with Fig. I69, was furnished by Loring:-

"The arteries and veins of the retina are subdivisions of the arteria and vena centralis. The larger branches run in the nerve-fibre layer and are immediately beneath the inner limitary membrane. The vessels lie so superficially that in a cross-section examined with the microscope, they are seen to project above the general level of the retina, toward the vitreous humor. While the large vessels are in the plane of the inner surface of the retina, the smaller branches penetrate the substance of the retina, to the inner nuclear layer. They do not extend, however, as far as the outer nuclear and the layer of rods and cones. These two layers, therefore, have no bloodvessels.

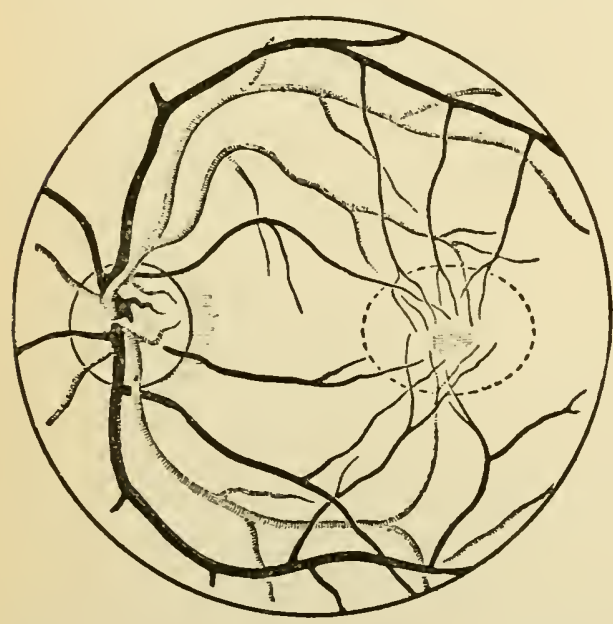

Fig. I6g. - Bloodvessels of the retina, $\times 7^{\frac{1}{3}}$ (Loring).

"The ramifications of the vessels present an arborescent appearance when seen with the ophthalmoscope. The manner in which the vessels are distributed and the way in which the circulation is carried on can be better understood by a study of Fig. I 69 than by any detailed description. The figure represents the ophthalmoscopic appearance of a normal eye in young adult life. The darker vessels are the veins, and the lighter vessels, the arteries. The dotted oval line is diagrammatic and indicates the position and extent of the macula lutea. It is seen that this oval space contains a number of fine vascular twigs which, coming from above and below, extend toward the spot in the centre of the oval, which marks the position of the fovea centralis. In opposition, then, to the general opinion, which is that the macula lutea has no bloodvessels, it is the spot of all others in the retina which is most abundantly supplied with minute vascular branches. These vessels can be distinctly seen even with the ophthalmoscope; and microscopical examination shows that the capillary plexus in the macula lutea is closer and richer than in any other part of the retina." (The figure shows an inverted image of the right fundus.)

The arteries of the retina send branches to the periphery, where they supply a wide plexus of small capillaries in the ora serrata. These capillaries empty into an incomplete venous circle, branches from which pass back by the sides of the arteries, to the vena centralis. 
Crystalline Lens. - The crystalline is a double-convex lens, which is transparent and very elastic. Its action in the refraction of the rays of light is analogous to that of convex lenses in optical instruments. It is situated behind the pupil in what is called the hyaloid fossa of the vitreous humor, which is exactly moulded to its posterior convexity. In the fotus the capsule of the lens receives a branch from the arteria centralis, but it is non-vascular in the adult. The anterior convexity of the lens is just behind the iris, and its borders are in relation with what is known as the suspensory ligament. The convexities do not present regular curvatures, and they are so subject to variations after death that measurements, post mortem, are of little value. During life,

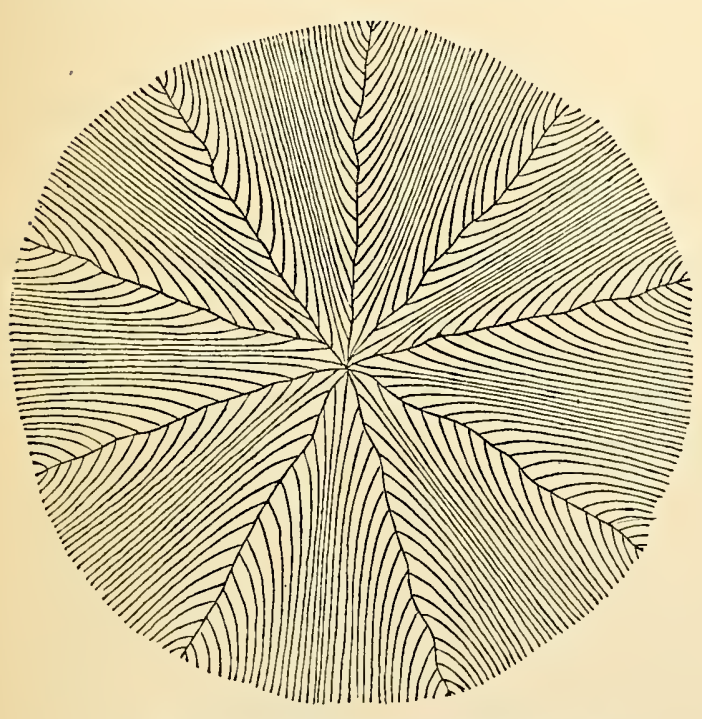

Fig. I70. - Crystalline lens, anterior viezo (Babuchin).

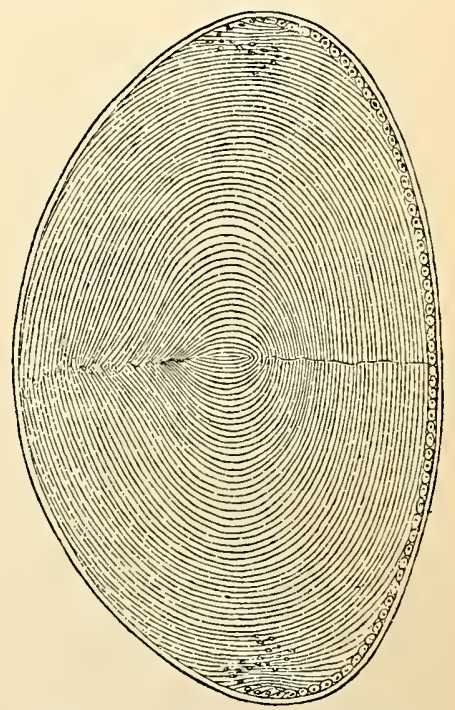

Fig. $\mathbf{7}$ x. - Section of the crystalline lens (Babuchin).

however, they have been exactly measured in different conditions of accommodation. The diameters of the lens in the adult are about $\frac{1}{3}$ of an inch (8.5 millimeters) transversely and $\frac{1}{4}$ of an inch (6.4 millimeters) antero-posteriorly. The convexity is greater on its posterior than on its anterior surface. In fœtal life the convexities of the lens are greater than in the adult and its structure is much softer. In old age the convexities are diminished and the lens becomes harder and less elastic. The substance of the lens is made up of layers of fibres of different degrees of density, and the whole is enveloped in a delicate membrane called the capsule.

The capsule of the lens is a thin transparent membrane and is very elastic. It usually is from $\frac{1}{2500}$ to $\frac{1}{1500}$ of an inch (IO to $17 \mu$ ) thick, 
but is much thinner at the periphery, measuring here only $\frac{1}{600}$ of an inch $(4 \mu)$. Its thickness is increased in old age. The anterior portion of the capsule is lined on its inner surface with a layer of delicate nucleated epithelium. The posterior half of the capsule has no epithelial lining. The cells are regularly polygonal, measuring $\frac{1}{2000}$ to $\frac{1}{250}$ of an inch $(\mathrm{I} 2$ to $20 \mu$ ) in diameter, with large round nuclei. After death they are said to break down into a liquid, known as the liquid of Morgagni, though by some this liquid is supposed to be exuded from the substance of the lens. At all events, the cells disappear soon after death.

If the lens is viewed entire with a low magnifying power, it presents upon either of its surfaces, a star with nine to sixteen radiations extending from the centre to about one-half or two-thirds of the distance to the periphery. The stars seen on the two surfaces are not coincident, the rays of one being situated between the rays of the other. In the fœtus the stars are more simple, presenting only three radiations on either surface. These stars are not fibrous, like the rest of the lens, but are composed of a homogeneous substance, which extends, also, between the fibres.

The greatest part of the substance of the lens is composed of delicate, soft and pliable fibres, which are transparent but perfectly distinct. These fibres are flattened six-sided prisms, closely packed together, so that their transverse section presents a regularly-tessellated appearance. They are $\frac{1}{500 \overline{0}}$ to $2 \frac{1}{250}$ of an inch $(5$ to $10 \mu)$ broad, and $\frac{1}{13000}$ to $\overline{90} \frac{1}{00}$ of an inch $(2$ to $3 \mu)$ in thickness. Their flat surfaces are parallel with the surface of the lens. The direction of the fibres is from the centre and from the rays of the stellate figures to the periphery, where they turn and pass to the star of the opposite side. The outer layers of fibres near the equator, or circumference of the lens, contain distinct oval nuclei with one or two nucleoli. These become smaller in passing more deeply into the substance of the lens and gradually disappear.

The regular arrangement of the fibres of the lens makes it possible to separate its substance into laminæ, which have been compared by anatomists to the layers of an onion; but this separation is artificial, and the number of apparent layers depends largely on the dexterity of the manipulator. It is to be noted, however, that the exernal portions of the lens are soft, even gelatinous, and that the central layers are much harder, forming a sort of central kernel, or nucleus.

The lens is composed of a globulin, called crystallin, combined with various inorganic salts. One of the constant constituents of this body is cholesterin. In an examination of four fresh crystalline lenses of the 
ox, cholesterin was found in the proportion of 0.907 of a part per 1000 (Flint). In some cases of cataract, cholesterin exists in the lens in a crystalline form; but under normal conditions it is united with the other constituents.

Suspensory Ligament of the Lens (Zone of Zinn). - The vitreous humor occupies about the posterior two-thirds of the globe and is enveloped in a delicate capsule called the hyaloid membrane. In the region of the ora serrata of the retina this membrane divides into two layers. The posterior layer lines the depression in the vitreous humor into which the lens is received. The anterior layer passes forward toward the lens and divides into two secondary layers, one of which passes forward to become continuous with the anterior portion of the capsule of the lens, while the other passes to the posterior surface of the lens to become continuous with this portion of its capsule. The anterior of these layers is corrugated or thrown into folds that correspond with the ciliary processes, with which it is in contact. This corrugated portion is called the zone of Zinn. The two layers thus surround the lens and are properly called its suspensory ligament. As the two layers of the suspensory ligament separate a certain distance from the lens, one passing to the anterior and the other to the posterior portion of the capsule, there remains a triangular canal, about $\frac{1}{10}$ of an inch (2.5 millimeters) wide,

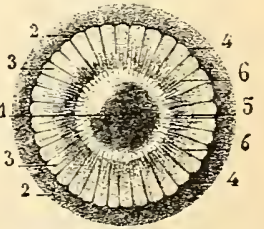

Fig. I72. - Zone of Zinn (Sappey).

I, crystalline lens; 2 , 2 , vitreous humor; 3,3 , zone of Zinn; 4,4 , posterior portion of the zone of Zinn, thrown into folds; $5,6,6$, anterior and middle portions of the zone of Zinn. surrounding the border of the lens, called the canal of Petit. Under natural conditions the walls of this canal are nearly in apposition, and it contains a small quantity of clear liquid.

The membrane forming the suspensory ligament is composed of pale longitudinal and transverse fibres of rather a peculiar appearance, which are much less affected by acetic acid than fibres of ordinary connective tissue.

Aqueous Humor. - The space bounded in front by the cornea, posteriorly, by the crystalline lens and the anterior face of its suspensory ligament, and at its circumference, by the tips of the ciliary processes, is known as the aqueous chamber. This contains a clear liquid called the aqueous humor. The iris separates this space into two divisions, which communicate with each other through the pupil; namely, the anterior chamber, situated between the anterior face of the iris and the cornea, and the posterior chamber, between the posterior face of the iris and the crystalline. It is evident, from the position of the iris, that the anterior chamber is much the larger; and, indeed, the 
posterior surface of the iris and the anterior surface of the lens are in contact, except, perhaps, near their periphery or when the iris is much dilated. The liquid filling the chambers of the eye is rapidly reproduced after it has been evacuated, as occurs in many surgical operations.

The aqueous humor is colorless and transparent, faintly alkaline, of a specific gravity of about 1005 , and with nearly the same index of refraction as that of the cornea and the vitreous humor. It contains a small quantity of an albuminous matter, but it is not rendered turbid by heat or other agents that coagulate albumin. Various inorganic salts (the chlorides, sulphates, phosphates and carbonates) exist in small proportions in this liquid. It contains also traces of urea and glucose.

The anterior and the posterior chambers of the eye are regarded as lymph-spaces communicating with the lymphatics of the conjunctiva, cornea, iris and ciliary processes. In addition a lymph-space is described as existing between the choroid and the sclerotic. This space is supposed to communicate with a perivascular-canal system around the vasa vorticosa, and through these vessels, with the space between the capsule of Tenon and the sclerotic (Schwalbe). The latter is connected with lymph-channels that surround the optic nerve (Key and Retzius).

Vitrcous Humor. - The vitreous humor is a clear glassy substance, occupying about the posterior two-thirds of the globe. It is enveloped in a delicate structureless capsule, called the hyaloid membrane, which is about $\frac{1}{600}$ of an inch $(4 \mu)$ in thickness. This membrane adheres rather strongly to the limitary membrane of the retina. In front, at the ora serrata, the hyaloid membrane is thickened and becomes continuous with the suspensory ligament of the lens.

The vitreous humor itself is gelatinous, of feeble consistence and slightly alkaline in its reaction, with a specific gravity of about 1005 . On section there oozes from it a watery and slightly mucilaginous liquid. This humor is not affected by heat or alcohol but is coagulated by certain mineral salts, especially lead acetate. When thus solidified it presents regular layers, like the white of an egg boiled in its shell; but these are artificial. In the embryo the vitreous humor is divided into a number of little cavities and contains cells and leucocytes. It is also penetrated by a branch from the central artery of the retina, which passes through its centre to ramify on the posterior surface of the lens. This structure, however, is not found in the adult, the vitreous humor being then without bloodvessels. The vitreous humor is divided into compartments formed by delicate membranes radiating from the point of penetration of the optic nerve to the anterior boundary where the hyaloid membrane is in contact with the capsule of the lens. In this way the humor is divided up, something like the half of an orange, 
into about one hundred and eighty membranous processes of extreme delicacy, which do not interfere with its transparency.

Summary of the Axatomy of the Globe of the Eye

This summary is intended simply to indicate the relations and the physiological importance of the various parts of the eye, in connection with Fig. I73.

The eyeball is nearly spherical in its posterior five-sixths, its anterior sixth being formed of the segment of a smaller sphere, which is slightly projecting. It presents the following parts, indicated in the figure:-

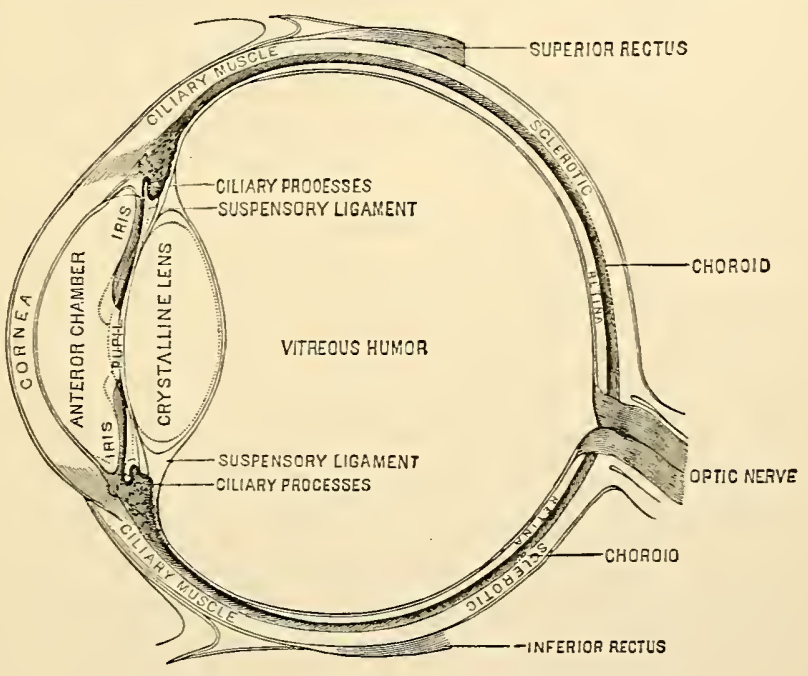

Fig. I73. - Diagrammatic section of the eye.

The sclerotic; a dense fibrous membrane, chiefly for the protection of the more delicate structures of the globe, and giving attachment to the muscles that move the eyeball. Attached to the sclerotic are the tendons of the recti and the oblique muscles.

The cornea; a transparent structure, forming the anterior projecting sixth of the globe; dense and resisting, allowing, however, the passage of light; covered, on its convex surface, with several layers of transparent epithelial cells.

The choroid coat; lining the sclerotic and extending only as far forward as the cornea; connected with the sclerotic by loose connective tissue, in which ramify bloodvessels and nerves, and presenting an external vascular layer and an internal pigmentary layer (described with retina), which latter gives its characteristic dark-brown color. 
The ciliary processes; folds of the choroid, which form its anterior border and which embrace the folds of the suspensory ligament of the lens.

The ciliary muscle; situated just outside of the ciliary processes, arising from the circular line of junction of the sclerotic with the cornea, passing over the ciliary processes, and becoming continuous with the fibrous tissue of the choroid. The action of this muscle is to tighten the choroid over the vitreous humor and to relax the ciliary processes and the suspensory ligament of the lens, when the lens, by virtue of its elasticity, becomes more convex. This action is indicated by the dotted lines in the figure.

The iris; dividing the space in front of the lens into two chambers occupied by the aqueous humor. The anterior chamber is much the larger. The iris, in its central portion surrounding the pupil, is in contact with the lens. Its circumference is just in front of the line of origin of the ciliary muscle.

The retina; a delicate transparent membrane, lining the choroid and extending to about $\frac{1}{15}$ of an inch ( 1.7 millimeters) behind the ciliary processes, the anterior margin forming the ora serrata. The optic nerve penetrates the retina a little internal to and below the antero-posterior axis of the globe. The layer of rods and cones is situated next the pigmentary layer, which is external. Internal to the layer of rods and cones and the outer limitary membrane, are the molecular and nuclear layers; next, the layer of nerve-cells; next, the expansion of the fibres of the optic nerve; and next, in apposition with the hyaloid membrane of the vitreous humor, is the inner limitary membrane.

The crystalline lens; elastic, transparent, enveloped in its capsule and surrounded by the suspensory ligament.

The suspensory ligament; the anterior layer connected with the anterior portion of the capsule of the lens, and the posterior, with the posterior portion of the capsule. The folded portion of this ligament, which is received between the folds of the ciliary processes, is the zone of Zinn. The triangular canal between the anterior and the posterior layers of the suspensory ligament and surrounding the equator of the lens is the canal of Petit.

The vitreous humor; enveloped in the hyaloid membrane, which membrane is continuous in front with the suspensory ligament of the lens. 


\title{
CHAPTER XYVII
}

\author{
REFRACTION IN THE EYE-ACCOMMODATION
}

Refraction in the eye - Certain laws of refraction, dispersion etc, bearing on the physiology of vision - Refraction by lenses - Spherical monochromatic aberration-Chromatic aberra. tion - Formation of images in the eye - Visual purple and visual yellow and accommodation of the eye for different degrees of illumination - Mechanism of refraction in the eye Astigmatism - Movements of the iris - Direct action of light on the iris - Accommodation of the eye for vision at different distances - Changes in the crystalline lens in accommodation - Changes in the iris in accommodation - Erect impressions produced by.images inverted upon the retina - Field of indirect vision - Binocular vision - Corresponding points - The horopter - Duration of luminous impressions (after-images) - Irradiation.

In applying some of the laws of refraction of light to the action of the transparent media of the eye, it is necessary to have in mind certain general facts in regard to vision, that have as yet been considered very briefly or have been omitted. The eye is not a perfect optical instrument, looking at it from a purely physical point of view. This statement, however, should not be understood as implying that the arrangement of the parts is not such as to adapt them perfectly to their uses in connection with the appreciation of visual impressions. By physical tests it can be demonstrated that the eye is not entirely achromatic; but in ordinary vision the dispersion of colors is not appreciated. There is but a single point in the retina, the fovea centralis, where vision is absolutely distinct; and it is upon this point that images are made to fall when the eye is directed toward any particular object.

The refracting apparatus is not exactly centred, - a condition essential to the satisfactory performance of perfect optical instruments. For example, in a compound microscope or a telescope, the centres of the different lenses entering into the construction of the instrument are situated in a straight line. Were the eye a perfect optical instrument, the line of vision would coincide exactly with the optical axis; but this is not the case. The visual line - a line drawn from an object to its image on the fovea centralis - deviates from the optical axis, in normal eyes, to the nasal side. The visual line, therefore, forms an angle with the optical axis, which is known as the angle alpha. This deviation of the visual line is observed both in the horizontal and in the vertical planes. The horizontal deviation varies by two to eight degrees, and the vertical, by one to three degrees. Of course this want of exact centring 
of the dioptric apparatus in normal eyes does not practically affect distinct vision; for when the eyes are directed toward any object, this object is brought in the line of the visual axis; but the angle alpha is an important element to be taken into account in certain mathematical calculations connected with the physics of the eye.

The area of distinct vision is quite restricted; but were it larger, it is probable that the mind would become confused by the extent and variety of the impressions, and that it would not be so easy to observe minute details and fix the attention on small objects.

Although certain objects are seen with distinctness only in a restricted field, the angle of vision is very wide, and rays of light are admitted from an area equal to nearly the half of a sphere. Such a provision is eminently adapted to visual requirements. The eyes are directed to a particular point and a certain object is seen distinctly, with the advantage of an image in the two eyes, exactly at the points of distinct vision; the rays coming from without the area of distinct vision are received upon different portions of the surface of the retina and produce an impression more or less indistinct, not interfering with the observation of the particular object to which the attention is for the moment directed; but even while looking intently at any object, the attention may be attracted by another object of an unusual character, which might, for example, convey an idea of danger, and the point of distinct vision can be turned in its direction. Thus, while but few objects are seen distinctly at one time, the area of indistinct vision is large ; and the attention may readily be directed to unexpected or unusual objects that come within any part of the field of view. The small extent of the area of distinct vision, especially for near objects, may readily be appreciated in watching a person who is reading, when the eyes will be seen to follow the lines from one side of the page to the other.

Certain Laws of Refraction, Dispersion etc., bearing on the Physiology of Vision. - Physiologists have little to do with the theory of light, except as regards the modifications of luminous rays in passing through the refracting media of the eye. It will be sufficient to state that nearly all physicists of the present day agree in accepting what is known as the theory of undulation, rejecting the emission-theory proposed by Newton. It is necessary to the theory of undulation to assume that all space and all transparent bodies are permeated with what has been called a luminiferous ether ; and that light is propagated by vibration or undulation of this hypothetical substance. This theory assimilates light to sound in the mechanism of its propagation; but in sound the waves are longitudinal, following the line of propagation, while in light the particles vibrate transversely, or at right angles to the line of propagation. 
The different calculations of physicists in regard to the velocity of light have been remarkably uniform in their results. The lowest calculations put it at about 185,000 miles (297,725 kilometers) in a second, and the highest, at about 195,000 miles (3 13,8 I 8 kilometers). The rate of propagation usually is assumed to be about 192,000 miles (309,000 kilometers).

The intensity of light is in proportion to the amplitude of the vibrations. The intensity diminishes in inverse ratio to the square of the distance.

In the theory of the colors into which white light may be decomposed by prisms, it is a matter of demonstration that the waves of the different colors are not of the same length. The decomposition of light is due to differences in the refrangibility of the different colored rays as they pass at an angle through a medium denser than the air.

The analysis of white light into the colors of the spectrum shows that it is compound; and by synthesis, the colored rays may be brought together, producing white light. Colors may be obtained by decomposition of light by transparent bodies, the different colored rays being refracted, or bent, by a prism, at different angles. It is not in this way, however, that the colors of different objects are produced. Certain objects have the property of reflecting the rays of light. A smooth polished surface, like a mirror, may reflect all the rays; and the object then has no color, only the reflected light being appreciated by the eye. Certain other objects do not reflect all the rays of light, some of them being lost to view, or absorbed. When an object absorbs all the rays it has no color and is called black. When an object absorbs the rays equally and reflects a portion of these rays without decomposition, it is gray or white. There are many objects, however, that decompose white light, absorbing certain rays of the spectrum and reflecting others. The rays not absorbed, but returned to the eye by reflection, give color to the object. Thus, if an object absorbs all the rays of the spectrum except the red, the red rays strike the eye, and the color of the object is red. So it is with objects of different shades, the colors of which are given by the unabsorbed rays.

A mixture of different colors in certain proportions will result in white. Two colors, which, when mixed, result in white, are called complementary. The following colors of the spectrum bear such a relation to each other: Red and greenish blue; orange and cyanogen-blue; yellow and indigo-blue; greenish yellow and violet.

The fact that impressions made on the retina persist for an appreciable length of time affords an illustration of the law of complementary colors. If a disk, presenting divisions with two complementary colors, is 
made to revolve so rapidly that the impressions made by the two colors are blended, the resulting impression is of white.

Refraction by Lenses. - A ray of light is an imaginary pencil so small as to present but a single line; and the light admitted to the interior of the eye by the pupil is supposed to consist of an infinite number of such rays. In studying the physiology of vision, it is important to recognize the laws of refraction of rays by transparent bodies bounded by curved surfaces, with particular reference to the action of the crystalline lens.

The action of a double-convex lens, like the crystalline, in the refraction of light, may be readily understood by an application of the wellknown laws of refraction by prisms. A ray of light falling on the side of a prism at an angle is deviated toward a line perpendicular to the surface of the prism. As the ray passes from the prism to the air, it is again refracted, but the deviation is then from the perpendicular of the second surface of the prism. In passing through a prism, therefore, the pencil of light is bent, or refracted, toward the base.

A circle is equivalent to a polygon with an infinite number of sides. A regular double-convex lens is a transparent body bounded by segments of a sphere. Theoretically, a double-convex lens may be assumed to be composed of an infinite number of sections of prisms (Fig. I 74, I), or to make the comparison with prisms more striking, though less accurate, the lens may be assumed to be composed of prisms, Fig. I74, II (Weinhold).

If these prisms or sections of prisms are infinitely small, so that the surface of each receives but a single infinitely small pencil of light, these pencils will be refracted toward the bases of the prisms, and different rays of light from all points of an object may be brought to an infinite number of foci, all these foci, for a plane object, being in the same plane. If the number of sections is equal on every side of the centre of the lens, the bases looking toward the axis of the !ens, the rays of light will cross at a certain point and the image will be inverted. This is illustrated in Fig. 174, which represents a section of a lens theoretically dissected into six sections of prisms.

If the lens A B (Fig. I74) is assumed to be free from what is known as spherical aberration, the rays from the point $C$ will be refracted and brought to a focus at the point $D$. In the same way the rays from $E$ will be brought to a focus at $\mathrm{F}$, the two sets of rays crossing before they reach their focal points. The same is true for all the rays from every point in the image $\mathrm{C} \mathrm{E}$, which strike the lens at an angle, but the ray $\mathrm{G}$ $\mathrm{H}$, which is perpendicular to the lens, is not deviated. The rays of light are refracted in this way by the cornea and by the crystalline lens. The 
retina is normally at such a distance from the lens that the 1 ays are brought to a focus exactly at its surface. Inasmuch as the rays cross before they reach the retina, the image is inverted.

Supposing the crystalline lens to be free from spherical and chromatic aberration, the formation of a perfect image depends on the following conditions :-

The object must be at a certain distance from the lens. If the object is too near, the rays as they strike the lens are too divergent and are brought to a focus beyond the plane F H D, or behind the retina; and as a consequence the image is confused. In optical instruments the adjustment is made for objects at different distances by moving the lens itself. In the eye, however, the adjustment is effected by increasing or diminishing the curvatures of the lens, so that the rays are
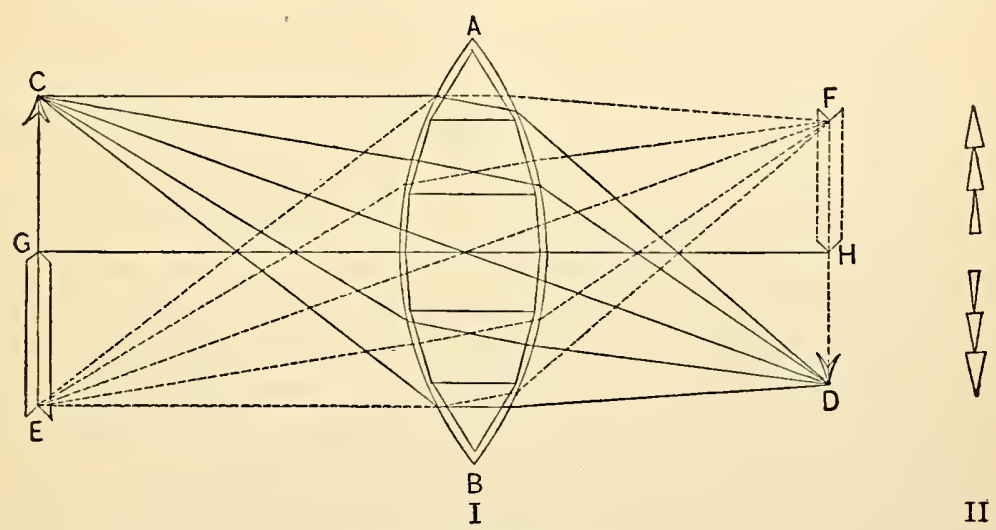

Fig. 174. - Refraction by convex lenses.

brought to a focus on the visual surface of the retina. The faculty of thus changing the curvatures of the crystalline lens is called accommodation. This power, however, is restricted within certain well-defined limits.

In some individuals the antero-posterior diameter of the eye is too long, and the rays, for most objects, come to a focus before they reach the retina. This defect may be remedied by placing the object very near the eye so as to increase the divergence of the rays as they strike the lens. Such persons are said to be near-sighted (myopic), and objects are seen distinctly only when very near the eye. This defect may be remedied for distant objects, by placing concave lenses before the eyes, by which the rays falling upon the lens are diverged. The opposite condition, in which the antero-posterior diameter is too short (hypermetropia), is such that the rays are brought to a focus behind the retina. This is corrected by converging the rays of incidence by placing convex 
lenses before the eyes. In old age the crystalline lens becomes flattened, its elasticity is diminished and the power of accommodation is lessened; conditions which also tend to bring the rays to a focus behind the retina. This condition is called presbyopia. To render near vision, as in reading, distinct, objects are placed farther from the eye than under normal conditions. The defect may be remedied, as in hypermetropia, by placing convex lenses before the eyes, by which the rays are converged before they fall on the lens.

Sphcrical Monochromatic Abcration. - In a convex lens in which the surfaces are segments of a sphere, the rays of light from an object are not converged to a uniform focus, and the production of a distinct image is impossible. For example, if the lens had regular curvatures, the rays refracted by its peripheral portion would be brought to a focus in front of the retina; the focus of the rays converged by the lens near its centre would be behind the retina; a few, only, of the rays would have their focus at the retina itself; and as a consequence, the image would appear confused. This is illustrated in imperfectly-corrected lenses, and is called spherical aberration. It is also called monochromatic aberration, because it is to be distinguished from an aberration that involves decomposition of light into the colors of the spectrum. If an object is examined under the microscope with an imperfectly-corrected objective, it is evident that the field of view is not uniform and that there is a different focal adjustment for the central and the peripheral portions of the lens. In the construction of optical instruments, this error may be in part corrected if the rays of light are cut off from the periphery of the lens by a diaphragm, which is an opaque screen with a circular perforation allowing the rays to pass to a restricted portion of the lens, near its centre. The iris corresponds to the diaphragm of optical instruments, and it corrects the spherical aberration of the lens in part, by eliminating a portion of the rays that would otherwise fall upon its peripheral portion. This correction, however, is not sufficient for high magnifying powers; and it is only by the more or less perfect correction of this kind of aberration by other means, that powerful lenses have been rendered available in optics.

The spherical aberration of lenses that diverge the rays of light is precisely opposite to the aberration of converging lenses. In a compound lens, therefore, it is possible to fulfil the conditions necessary to the convergence of all the incident rays to a focus on a uniform plane, so that the image produced behind the lens is not distorted. Given, for example, a double-convex lens, by which the rays are brought to innumerable focal points situated in different planes. The fact that but few of these focal points are in the plane of the retina renders the image 
indistinct. If a concave or a plano-concave lens is placed in front of this convex lens, which will diverge the rays more or less, the inequality of the divergence by different portions of the second lens will have the following effect: As the angle of divergence gradually increases from the centre toward the periphery, the rays near the periphery - which are most powerfully converged by the convex lens - will be most widely diverged by the peripheral portion of the concave lens; so that if the opposite curvatures are accurately adjusted, the aberrant rays may be blended. It is evident that if all the rays were equally converged by the convex lens and equally diverged by the concave lens, the action of the latter would be simply to elongate the focal distance; and it is equally evident that if the aberration of the one is exactly opposite to the aberration of the other, there will be perfect correction. Mechanical art has not effected correction of every portion of powerful convex lenses in this way; but by a combination of lenses and diaphragms together, highly-magnified images, nearly perfect, have been produced. Lenses in which spherical aberration has been corrected are called aplanatic.

It is evident that for distinct vision at different distances, the lens must be nearly free from spherical aberration. This is not effected by a combination of lenses, as in ordinary optical instruments, but by the curvatures of the lens itself and by certain differences in the consistence of different portions of the lens, which will be fully considered hereafter.

Chromatic Aberration. - A refracting medium does not act equally on the different colored rays into which white light may be decomposed; in other words, as the white ray falling on the inclined surface of a glass prism is bent, it is decomposed into the colors of the spectrum. As a convex lens is practically composed of an infinite number of prisms, the same effect would be expected. Indeed, a simple convex lens, even if the spherical aberration is corrected, always produces more or less decomposition of light. The image formed by such a lens consequently is colored at its borders; and this defect in simple lenses is called chromatic aberration.

In prisms the chromatic dispersion may be corrected by allowing the colored rays from one prism to fall on a second prism, which latter is inverted, so that the colors are brought together and produce white light. Two prisms thus applied to each other constitute, in fact, a flat plate of glass, and the rays of light pass without deviation. If this principle is applied to lenses, it is evident that the dispersive power of a convex lens may be exactly opposite to that of a concave lens. By the convex lens the colored rays are separated by convergence and cross each other; and in the concave lens the colored rays are diverged in the opposite direction. If, then, a convex is combined with a concave lens, the white 
light decomposed by the one will be recomposed by the other, and the chromatic aberration will thus be corrected; but in using a convex and a concave lens composed of the same material, the convergence by the one will be neutralized by the divergence of the other, and there will be no amplification of the object. Newton supposed that dispersion, or decomposition of light, by lenses was always in exact proportion to refraction, so that it would be impossible to correct chromatic aberration and retain magnifying power; but it has been ascertained that there are great differences in the dispersive power of different kinds of glass, without corresponding differences in refraction. This discovery rendered it possible to construct achromatic lenses (Dollond, I757). According to Ganot, Hall was the first to make achromatic lenses, I753, but his discovery was not published.

In the construction of modern optical instruments, chromatic aberration is corrected, with a certain diminution in amplification, by cementing together lenses made of different materials, as of flint-glass and crown-glass. Flint-glass has a much greater dispersive power than crown-glass. If, therefore, a convex lens of crown-glass is combined

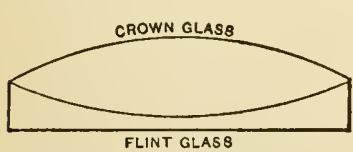

Fig. 175. - Achromatic lens. with a concave or plano-concave lens of flintglass, the chromatic aberration of the convex lens may be corrected by a concave lens with a curvature that will reduce the magnifying power about one-half. A compound lens, with the spherical aberration of the convex element corrected by the curvature of a concave lens, and the chromatic aberration corrected in part by the curvature and in part by the greater refractive power of flint-glass, will produce a perfect image.

Although the eye is not absolutely achromatic, the dispersion of light is not sufficient to interfere with distinct vision ; but the chromatic aberration is practically corrected in the crystalline lens, probably by differences in consistence and in the refractive power of its different layers.

\section{Formation of Images in the Eye}

It is necessary only to call to mind the general arrangement of the structures in the eye and to apply the simple laws of refraction, in order to comprehend how images are formed on the retina.

The eye corresponds to a camera obscura. Its interior is lined with a layer of dark pigment-cells, the immediate action of which is to prevent the confusion of images by internal reflection. The rays of light are admitted through a circular opening (the pupil), the size of which is regulated by the movements of the iris. The pupil is con- 
tracted when the light striking the eye is intense and is dilated as the quantity of light is diminished. In accommodation of the eye, the pupil is dilated for distant objects and contracted for near objects; for in looking at near objects, the aberrations of sphericity and achromatism in the lens are more marked, and the peripheral portion is cut off by the action of this movable diaphragm, thus aiding correction. The rays of light from an object pass through the cornea, the aqueous humor, the crystalline lens and the vitreous humor; and they are refracted with so little spherical and chromatic aberration, that the image formed upon the retina is practically perfect. The layer of rods and cones of the retina is the only part in the eye endowed with special sensibility, the impressions of light being conveyed to the brain by the optic nerves. This layer is situated next the pigmentary layer; but the other layers of the retina, through which the light passes to reach the rods and cones, are transparent.

It has been shown that the rods and cones are the only structures capable of directly receiving visual impressions, by the following experiment, first made by Purkinje: With a convex lens of short focus, an intense light is concentrated on the sclerotic, at a point as far as possible removed from the cornea. This passes through the translucent coverings of the eye at this point, and the image of the light reaches the retina. In then looking at a dark surface, the field of vision presents a reddish yellow illumination, with a dark arborescent appearance produced by the shadows of the large retinal vessels; and as the lens is moved slightly, the shadows of the vessels move with it. Without going elaborately into the mechanism of this phenomenon, it is sufficient to state that Heinrich Müller has arrived at a mathematical demonstration that the shadows of the vessels are formed on the layer of rods and cones, and that this layer alone is capable of receiving impressions of light. His explanation is generally accepted and is regarded as proof of the peculiar sensibility of this portion of the retina.

Theoretically, an illuminated object placed in the angle of vision would form upon the retina an image, diminished in size and inverted. This is capable of demonstration by means of the ophthalmoscope; as with this instrument the retina and the images formed upon it may be seen during life.

All parts of the retina, except the point of entrance of the optic nerve, are sensitive to light; and the arrangement of the cornea and pupil is such that the field of vision is, at the least estimate, equal to the half of a sphere. If a ray of light falls on the border of the cornea at a right angle to the axis of the eye, it is refracted by its surface and will pass through the pupil to the opposite border of the 
retina. Above and below, the circle of vision is cut off by the overhanging arch of the orbit and the malar prominence; but externally the field is free. With the two eyes, therefore, the lateral field of vision must be equal to at least one hundred and eighty degrees. It is easy to demonstrate, however, with the ophthalmoscope, as well as by taking cognizance of the impressions made by objects far removed from the axis of distinct vision, that images formed upon the lateral and peripheral portions of the retina are confused and imperfect. One has a knowledge of the presence and an indefinite idea of the general form of large objects situated outside the area of distinct vision; but when it is desired to note such objects exactly, the eyeball is turned by muscular effort so as to bring them at or near the visual axis. This fact, with what is known of the mechanism of refraction by the cornea and lens, makes it evident that the area of the retina on which images are formed with perfect distinctness is quite restricted. A moment's reflection is sufficient to convince one that in order to see an object distinctly, it is necessary to bring the visual axis to bear upon it directly.

In examining the fundus of the eye with the ophthalmoscope, the yellow spot, with the fovea centralis, can be seen, free from large bloodvessels and composed chiefly of those elements of the retina which are sensitive to light. If at the same time an image for which the eye is perfectly adjusted is observed, it will be seen that this image is perfect only at the fovea centralis; and if the object is removed from the visual axis, there is a confused image upon the retina, removed from the fovea, at the same time that the subject is conscious of indistinct vision.

At the point of penetration of the optic nerve, the retina is insensible to luminous impressions; or at least, its sensibility is here so obtuse as to be inadequate for the purposes of vision. This point is called the punctum cæcum; and its want of sensibility was demonstrated many years ago ( 1668 ) by Mariotte.

The existence of this spot - which is not in the area of distinct vision - gives no inconvenience for the reason that in binocular vision the rays from an object do not fall at the same time on both blind spots. It is not noticed subjectively, even in monocular vision.

The relative sensibility of different portions of the retina has been found to be, in an inverse ratio, equal to about the square of the distance from the axis of most perfect vision (Volkmann). This observer calculated the distance between the sensitive elements of the retina at which he supposed that two parallel lines would appear as one. In the axis of vision, the distance was 0.00029 inch $(7.366 \mu)$, and at a deviation inward of $8^{\circ}$, it was 0.03 I 86 inch $(809.244 \mu)$, a diminution in acuteness, of more than a hundred times. 
Visual Purple and Visual Yellow and Accommodation of the Eye for Different Degrees of Illumination. - The outer segments of the rods of the retina sometimes present a peculiar red or purple color which disappears after ten or twelve seconds of exposure to light. This was first observed by Boll ( 1876 ) in the retinæ of frogs that had been kept for a certain time in the dark. From his preliminary researches Boll concluded that this coloration of the retina exists only during life and persists for but a few moments after death; that it is constantly destroyed during life by the action of light and reappears in the dark; and finally that it plays an important part in the act of vision. Kühne and others have since confirmed and extended the original observations of Boll; and the visual purple (rhodopsin) has been noted in the mammalia and in man. It has been extracted from the retinæ of frogs and dissolved in a five-per-cent solution of crystallized ox-gall, still presenting in solution its remarkable sensitiveness to light. Finally it has been found possible to fix images of simple objects, such as strips of black paper pasted on a plate of ground glass, upon the retina, by a process very like photography.

The visual purple is produced by the cells of the pigmentary layer of the retina and from them is absorbed by the outer segments of the rods. It is not present in any part of the cones and does not exist, therefore, in the area of distinct vision, at the fovea centralis. The rapid disappearance of the color under the influence of actinic rays of light renders it necessary to examine the retina under a non-actinic (monochromatic) sodium-flame. When thus examined and gradually exposed to actinic rays, the color quickly fades into a yellow and finally disappears, being restored, however, in the dark. If the choroid and the pigmentary layer of the retina are removed, the rods are bleached, and the color is restored in the dark when the choroid is replaced. In the eye of the frog, kept in the dark, the hair-like processes that extend from the pigmentary layer of the retina downward between the rods and cones are retracted, and the pigment is then contained chiefly in the cells themselves. After prolonged exposure of the retina to light, these processes, loaded with pigment, extend between the cones as far as the limitary membrane.

The fact that visual purple is not found in the fovea centralis is opposed to the theory that its existence is directly essential to distinct vision; nevertheless, certain phenomena observed in passing from a bright light to comparative obscurity, and the reverse, show that the purple has, at least, an important indirect action. In passing from the dark to a bright light, the eye is dazzled and distinct vision is difficult. It may be assumed that this is due to unusual general sensitiveness of 
the retina to light, on account of the excessive quantity of visual purple which has accumulated in the dark; and that distinct vision is restored when the retina is bleached to a yellow, which seems to be the most favorable condition for the exact appreciation of visual impressions under full illumination. On the other hand, it requires time for the eye to become accustomed to a dim light; and during this time the yellow is changing to purple. Investigations of the absorption-spectra of the purple and yellow have shown that the purple allows the actinic rays to pass perfectly, while the yellow completely absorbs these rays. The existence of visual purple seems to be most favorable to the imperfect and shadowy vision which occurs under dim illumination, when the exact appreciation of minute details is impossible. In the condition known as night-blindness, it is probable that the visual purple has become exhausted beyond the possibility of prompt restoration such as is normal; and persons so affected can not see at night, although minute vision under a bright light may not be affected. In certain cases of this kind, the normal conditions may be restored by a few days' seclusion in the dark. What is called functional night-blindness frequently occurs in sailors during long tropical voyages, and is due to the excessive action of diffused light on the retina.

Change of visual purple to yellow is readily effected, but the further change to white is slower and more difficult. Conversely, change from white to yellow is slow and change from yellow to purple is comparatively prompt. One use of the colors, purple and yellow, seems to be to accommodate the retina for vision under different degrees of illumination. The purple adapts the eye to a feeble illumination, and the yellow, to a full illumination. This being the case, it is manifestly proper to speak of a visual yellow as well as of visual purple.

That the accommodation of the eye to different degrees of illumination is due to changes in the colors produced by the pigmentary layer of the retina and not to different degrees of dilatation of the pupil, is shown by the fact that a person does not see better in the dark when the pupil has been dilated by atropin. In a very dim light there is no possibility of exact accommodation for near objects, which, when small, can not be seen distinctly; and the contraction of the pupil that attends accommodation for near vision does not occur. It is possible that under dim illumination, parts outside of the fovea, that are insensible to vision under a bright light, receive visual impressions. Under these conditions the pupil is dilated and rays impinge on portions of the retina not used in direct vision. A natural extension of this idea would confine distinct vision and the appreciation of minute details to the action of the fovea centralis, in which there is no visual purple, other parts of the retina, 
under full illumination, not being used. To express this in a few words, the fovea centralis is used by day, and the adjacent parts of the retina, by night.

\section{Mechanism of Refraction in the Eye}

An object that is seen reflects rays from all points of its surface to the cornea. If the object is near, the rays are divergent as they strike the eye. Rays from distant objects are practically parallel. It is evident that refraction for diverging rays must be greater than for parallel rays, as a necessity for distinct vision; in other words, the eye must be accommodated for vision at different distances. Leaving, however, the mechanism of accommodation for later consideration, it may be stated simply that the important agents in refraction in the eye are the surfaces of the cornea and the crystalline lens. Calculations have shown that the index of refraction of the aqueous humor is sensibly the same as that of the substance of the cornea, so that practically the refraction is the same as if the cornea and the aqueous humor were one and the same substance. The index of refraction of the vitreous humor is practically the same as that of the aqueous humor, both being about equal to the index of refraction of pure water. ${ }^{1}$ Refraction by the crystalline lens, however, is more complex in its mechanism; depending first, on the curvatures of its two surfaces, and again, on differences in consistence of different portions of its substance. In view of these facts, the conditions of refraction in the eye in distinct vision may be simplified by assuming the following arrangement:-

The cornea presents a convex surface on which the rays of light are received. At a certain distance behind its anterior border, is the crystalline, a double-convex lens, corrected sufficiently for practical purposes, both for spherical and chromatic aberration. This lens is suspended in a liquid with an index of refraction equal to that of pure water, as both the aqueous humor in front and the vitreous humor behind have the same refractive index. Behind the lens, in its axis and exactly in the plane upon which the rays of light are brought to a focus by the action of the cornea and the lens, is the fovea centralis, in which is the centre for distinct vision. The anatomical elements of the fovea are capable of receiving visual impressions, which are conveyed to the brain by the optic nerves. All impressions made on other portions of the retina are comparatively indistinct; and the point of entrance of the optic nerve is insensible to light. Inasmuch as the punctum crecum is situated in

1 The index of refraction is the ratio between the sine of the angle of incidence and the sine of the angle of refraction. This is practically the same for the cornea, the crystalline lens, the aqueous humor and the vitreous humor. For the aqueous and vitreous humors, it is 1.34 to I.36 ; for the crystalline lens, it is 1.40 to 1.45 , a little higher. 
either eye upon the nasal side of the retina, in normal vision, rays from the same object can not fall on both blind points at the same time. Thus, in binocular vision, the insensibility of the punctum cæcum does not interfere with sight; and the movements of the globe prevent any notable interference in vision, even with one eye. The sclerotic coat is for the protection of its contents and for the insertion of muscles. The iris has an action similar to that of the diaphragm in optical instruments. The suspensory ligament of the lens, the ciliary body, and the ciliary muscle are for the fixation of the lens and its accommodation for vision at different distances. The pigment-cells are for the absorption of light, preventing confusion of vision from reflection within the eye. They also produce the visual purple.

Refraction by the cornea is effected simply by its external surface. The rays of light from a distant point are deviated by its convexity so that, if they were not again refracted by the crystalline lens, they would be brought to a focus at a point situated about $\frac{4}{10}$ of an inch (Io millimeters) behind the retina. Without the crystalline lens, therefore, clistinct unaided vision usually is impossible, although the sensation of light is appreciated. In cases of extraction of the lens for cataract (aphakia), the place of the crystalline may be supplied by a convex lens placed before the eye.

The rays of light, refracted by the anterior surface of the cornea, are received upon the anterior surface of the crystalline lens, by which they are still further refracted. Passing through the substance of the lens, they undergo certain modifications in refraction, dependent on the differences in the various strata of the lens. These modifications have not been accurately calculated; but it is sufficient to state that they contribute to the accuracy of the formation of a retinal image practically free from chromatic dispersion. As the rays pass out of the lens, they are again refracted by its posterior curvature and are brought to a focus at the area of distinct vision.

The rays from all points of an object distinctly seen are brought to a focus, if the accommodation of the lens is correct, on a restricted surface in the macula lutea; but the rays from different points cross before they reach the retina, and the image is inverted.

Calculating the curvatures of the refracting surfaces in the eye and the indices of refraction of its transparent media, it has been pretty clearly shown, by mathematical formula, that the eye - viewed simply as an optical instrument, and not practically, as the organ of vision presents a certain degree of spherical and chromatic aberration; but these calculations are not important in a purely physiological consideration of the sense of sight. 
In most calculations of the size of images, the positions of conjugate foci, etc., in normal and abnormal eyes, a schematic eye reduced by Donders, after the example of Listing, is regarded as sufficiently exact for all practical purposes. This simple scheme represents the eye as reduced to a single refracting surface, the cornea, and a single liquid assumed to have an index of refraction equal to that of pure water. The distance between what are called the two nodal points and between the two principal points of the dioptric system of the eye is so small, amounting to hardly $\frac{1}{100}$ of an inch ( 0.254 millimeter), that it can be neglected. In this simple eye, there is assumed to be a radius of curvature of the cornea of about $\frac{1}{5}$ of an inch ( 5 millimeters) and a single optical centre situated $\frac{1}{5}$ of an inch ( 5 millimeters) back of the cornea, the "principal point " being in the cornea, in the visual axis. The posterior focal distance, that is, the focus, at the bottom of the eye, for rays parallel in the air, is about $\frac{4}{5}$ of an inch (20 millimeters). The anterior focal distance, that is, for rays parallel in the vitreous humor, is about $\frac{3}{5}$ of an inch (I 5 millimeters). The measurements in this simple schematic eye can easily be remembered and used in calculations.

\section{Astigmatism *}

In the normal human eye the visual line does not coincide exactly with the mathematical axis; but there is still another normal deviation from mathematical exactness in the refraction of rays by the cornea and the lens, which is of considerable importance. If two threads, crossing each other at right angles in the same plane, are placed before the eyes, one of these threads being vertical, and the other, horizontal, when the optical apparatus is adjusted so that one line is seen with distinctness, the other is not well defined. In other words, when the eye is accommodated for the vertical thread, the horizontal thread is indistinct, and vice vers $\hat{a}$. When the horizontal line is seen distinctly, in order to see the vertical line without modifying the accommodation, it must be removed to a greater distance. This depends chiefly on a difference in the vertical and the horizontal curvatures of the cornea, so that the horizontal meridian has a focus slightly different from the focus of the vertical meridian. A condition opposite to that observed in the cornea usually exists in the lens; that is, the difference which exists between the curvatures of the lens in the vertical and the horizontal meridians is such that the deeper curvature in the lens is situated in the meridian of the shallower curvature of the cornea. In this way, in normal eyes, the aberration of the lens has a tendency to correct the aberration in the cornea; but this correction is incomplete, and there still remains, 
in all degrees of accommodation, a certain difference in vision as regards vertical and horizontal lines.

The condition just described is known under the name of normal regular astigmatism; but the aberration is not sufficient to interfere with distinct vision. The degree of regular astigmatism presents normal variations in different eyes. In some eyes there is no astigmatism; but this is rare. When the astigmatism amounts to $\frac{1}{40}$ or more, it is to be considered abnormal; which simply means that beyond this point the aberration interferes with distinct vision.

From the simple definition of regular astigmatism, it is evident that this condition and the degree in which it exists may easily be determined by noting the differences in the foci for vertical and horizontal lines, and it may be exactly corrected by the application of cylindrical glasses of proper curvature. Indeed, the curvature of a cylindrical glass that will enable a person to distinguish vertical and horizontal lines with perfect distinctness at the same time, is an exact indication of the degree of aberration. Regular astigmatism, such as just described, may be so exaggerated as to interfere seriously with vision, when it becomes abnormal. This kind of aberration, however, which usually is dependent on an abnormal condition of the cornea, is remediable by the use of properly-adjusted cylindrical glasses.

Irregular astigmatism, excluding cases of pathological deformation, opaque spots etc., in the cornea, depends usually on irregularity in the different sectors of the crystalline lens. Instead of a simple and regular aberration, consisting in a difference between the depth of the vertical and the horizontal curvatures of the cornea and lens, there are irregular variations in the curvatures of different sectors of the lens. As a consequence of this, when the irregularities are very great, there is impairment of the sharpness of vision. The circles of diffusion, which are regular in normal vision, become irregularly radiated, and single points appear multiple, an irregularity described under the name of polyopia monocularis. Accurate observations have shown that this condition exists in a moderate degree in normal eyes; but it is so slight as not to interfere with ordinary vision. In what is called normal irregular astigmatism, the irregularity depends entirely on the crystalline lens. If a card with a very small opening is placed before the eye and is moved in front of the lens, so that the pencil of light falls successively on different sectors, it can be shown that the focal distance is different for different portions. The radiating lines of light observed in looking at remote luminous points, as the fixed stars, are produced. by this irregularity in the curvatures of the different sectors of the lens.

While regular astigmatism, both normal and abnormal, may be per- 
fectly corrected by placing cylindrical glasses before the eyes, it is impossible, in the majority of cases, to construct glasses that will correct entirely what is called irregular astigmatism.

\section{MOVEMENTS OF THE IRIS}

There are two physiological conditions under which the size of the pupil is modified: The first of these depends on the degree of illumination to which the eye is exposed. When the illumination is dim, the pupil is widely dilated. When the eye is exposed to a bright light, the retina is protected by contraction of the iris. The muscular action by which the iris is contracted is characteristic of the smooth muscular fibres, as can be readily seen by exposing an eye, in which the pupil is dilated, to a bright light. Contraction does not take place instantly, but an appreciable interval elapses after the exposure, and a more or less gradual diminution in the size of the pupil is observed. This is seen both in solar and in artificial light. The second of these conditions depends indirectly on the voluntary action of muscles. The effort of converging the axes of the eyes, in looking at a very near object, contracts the pupils; and accommodation of the eye for near objects produces the same effect, even when the eyes are not converged. This action will be fully considered under the head of accommodation.

Direct Action of Light on the Iris. - The variations in the size of the pupil under different physiological conditions are effected almost exclusively through the nerrous system, either by reflex action from variations in the intensity of light, or by a direct influence, as in accommodation for distances; but it is nevertheless true that the muscular tissue of the iris will respond directly to the stimulus of light. Harless noted, in subjects dead of various diseases, five to thirty hours after death, that the iris contracted under the stimulus of light; and he regarded this as probably due to direct action on its muscular tissue. The experiments of Harless were made on the two eyes, one being exposed to the light, while the other was closed. The contraction, however, took place slowly, requiring an exposure of several hours. This mode of contraction is different from the action of the iris during life; but it is precisely like the contraction observed after division of the motor oculi communis, which is slow and gradual and depends on the direct action of light on the muscular fibres.

Action of the Nervous System on the Iris. - This subject, so far as it relates to the third pair, has been considered in connection with the physiology of these nerves; and it is unnecessary to refer again in 
detail to the experiments that have already been cited. The reflex phenomena observed are sufficiently distinct. When light is admitted to the retina, the pupil contracts, and the same result follows mechanical irritation of the optic nerves. When the third pair of nerves has been divided, no such reflex phenomena are observed. It is well known, also, that division of the third nerves in the lower animals or their paralysis in the human subject produces permanent dilatation of the pupil, the iris responding, only in the slow and gradual manner already indicated, to the direct action of light.

Taking all the experimental data into consideration, it is certain that the third nerve has an important influence on the iris. Filaments from the ophthalmic ganglion animate the circular fibres, or sphincter, and these filaments are derived from the third cranial nerve. If this nerve is divided, the iris becomes permanently dilated and is immovable, except that it responds slowly to the direct action of light. The reflex action by which the pupil is contracted under the stimulus of light operates through the third nerve, and no such action can take place after this nerve has been divided. In view of these facts, there can be no doubt in regard to the nervous action on the sphincter of the pupil, this muscle being animated exclusively by filaments from the motor oculi communis, coming through the ophthalmic ganglion.

Most anatomists admit the existence of radiating muscular fibres in the iris, the action of which is antagonistic to the circular fibres, and which dilate the pupil. That these fibres are subjected to nervous influence, is rendered certain by experiments on the sympathetic system. There can be no doubt that the action of the sympathetic on the pupil is directly antagonistic to that of the third pair, the former presiding over the radiating muscular fibres; and the only question to determine is the course taken by the sympathetic filaments to the iris. Experiments on the influence of the fifth pair on the pupil have been somewhat contradictory in different animals. In rabbits, section of this nerve in the cranial cavity produces contraction of the pupil; but in dogs and cats, the same operation produces dilatation. In the human subject, of course, it is impossible to determine this point by direct experiment; and the varying results obtained in observations on different animals probably depend upon differences in the anatomical relations of the nerves. It is probable, however, that the filaments of the sympathetic that animate the radiating fibres join the fifth nerve near the ganglion of Gasser and from this nerve pass to the iris. In observations on the human subject it has been noted that division of the sympathetic in the neck was attended with contraction of the pupil on the side of the injury (Mitchell). Stimulation of the cervical sympathetic, in the head of 
a woman eighteen minutes after decapitation, produced dilatation of the pupil (Wagner).

There seem to be two distinct nerve-centres corresponding to the two sets of nerves that regulate the movements of the iris. One of these centres presides over the reflex contractions of the iris, and the other is the centre of origin of the nervous influence through which the pupil is dilated.

The mechanism of reflex contraction of the iris under the stimulus of light is sufficiently simple. An impression is made on the retina, which is conveyed by the optic nerves to the centre, and in obedience to this impression, the sphincter of the iris contracts. If the optic nerves are divided, so that the impression can not be conveyed to the centre, or if the third nerve is divided, no movements of the iris can take place. The centres which preside over the reflex phenomena of contraction of the pupil are situated in the bulb. The action of these centres is crossed in animals in which the decussation of the optic nerves is complete. In man the axes of both eyes are habitually brought to bear upon objects, and it is well known that there is a physiological unity in the action of the two eyes in ordinary vision. It has been observed that when one eye only is exposed to light, the pupil becoming contracted under this stimulus, the pupil of the other 'eye also contracts. There is, indeed, a direct contraction and dilatation of the pupil of the eye which is exposed to the light, and an indirect, or "consensual" movement of the iris on the opposite side. The consensual contraction occurs about $\frac{2}{5}$ of a second later than the direct action, and the consensual dilatation, about $\frac{1}{2}$ of a second later. The condition of the pupillary reflexes often gives important information in cases of cerebral disease. They are particularly important in the early stages of paresis.

The filaments of the sympathetic that produce dilatation of the pupil take their origin from the spinal cord. In the spinal cord, between the sixth cervical and the second thoracic nerves, is the inferior cilio-spinal centre. When the cord is stimulated in this situation, both pupils are dilated. If the cord is divided longitudinally and the two halves are separated from each other by a glass plate, stimulation of the right half produces dilatation of the right pupil, and vice versô. This does not occur when the sympathetic in the neck has been divided. In addition to the inferior cilio-spinal centre, there is a superior centre, which is in communication with the superior cervical ganglion and is situated near the sublingual nerve. The influence of this centre over the pupil can not be demonstrated by direct stimulation, because it is too near the origin of the fifth, irritation of which affects the iris; but it is shown by division of its filaments of communication with the iris. 


\section{Accommodation of the Eye for Vision at Different Distances}

Supposing the eye to be adapted to vision at an infinite distance, in which the rays from an object as they strike the cornea are practically parallel, it is evident that the foci of the rays, as they form a distinct image upon the retina, are all situated in the proper plane. Under these conditions, in a normal eye, the image, appreciated by the individual or seen by means of the ophthalmoscope, is clear and distinct. If the foci are situated in front of the retina, the rays, instead of coming to a focus on a point in the retina, will cross, and, from their diffusion or dispersion, will produce indistinct vision. Under these conditions a distinct point is not perceived, but every point in the image is surrounded with an indistinct circle. These are called "circles of diffusion." If, now, the eye, adjusted for vision at an infinite distance, is brought to bear on a near object, the rays from which are divergent as they strike the cornea, the image will no longer be distinct but will be obscured by circles of diffusion. It is the adjustment by which these circles of diffusion are removed that constitutes accommodation. This fact has been demonstrated by Helmholtz by means of the ophthalmoscope. "If the eye is adjusted to the observation of an object placed at a certain distance, it is found that the image of a flame, placed at the same distance, is produced with perfect distinctness upon the retina, and, at the same time, upon the illuminated plane of the image, the vessels and the other anatomical details of the retina are seen with equal distinctness. But, when the flame is brought considerably nearer, its image becomes confused while the details of the structure of the retina remain perfectly distinct."

It is evident that there is a certain condition of the eyes adapted to vision at an infinite distance; and that for the distinct perception of near objects the transparent media must be so altered in their arrangement or in the curvatures of their surfaces that the refraction will be greater; for without this, the rays would be brought to a focus beyond the retina.

The changes in the eye by which accommodation is effected are now known to consist mainly in an increased convexity of the lens for near objects; and the only points in dispute are a few unimportant details in the mechanism of this action. The simple facts to be borne in mind in studying this question are the following:-

When the eye is accommodated to vision at an infinite distance the parts are passive.

In the adjustment of the eye for near objects the convexities of the lens are increased by muscular action. 
In accommodation for near objects the pupil is contracted; but this action is accessory and is not essential.

The ordinary range of accommodation varies between a distance of about five inches ( 12.7 centimeters) and infinity.

Changes in the Crystalline Lens in Accommodation. - It is important to determine the extent and nature of the changes of the lens in accommodation; and these changes have been accurately measured in the living subject. It has been ascertained that the lens becomes increased in thickness in accommodation for near objects, chiefly by an increase in its anterior curvature, by which this surface of the lens is made to project toward the cornea. As the iris is in contact with the anterior surface of the lens, this membrane, is made to project in the act of accommodation. The posterior curvature of the lens is also increased, but this is slight as compared with the increase of the curvature of its anterior surface. The distance between the posterior surface of the lens and the cornea is not sensibly altered. It is unnecessary to describe minutely the methods employed in making these calculations, and it is sufficient to state that it is done by accurately measuring the comparative size of images formed by reflection from the anterior surface of the lens. The results obtained by Helmholtz, in observations on two persons, are the following:-

\begin{tabular}{|c|c|c|c|}
\hline \multirow{2}{*}{$\begin{array}{l}\text { Persons } \\
\text { Examined }\end{array}$} & \multicolumn{2}{|c|}{$\begin{array}{c}\text { Radius of Curvature of the ANterior Surface of } \\
\text { THE Lexs }\end{array}$} & \multirow{2}{*}{$\begin{array}{l}\text { DisplacemeNt of the Pupit iN AC- } \\
\text { COMMOdation For Near Objects }\end{array}$} \\
\hline & Distant Vision & Near Visioy & \\
\hline O. H. & 0.464 I in. (I I.9 mm.) & 0.3354 in. $(8.6 \mathrm{~mm})$. & $0.0140 \mathrm{in} .(0.36 \mathrm{~mm})$. \\
\hline B. P. & 0.3432 in. ( $8.8 \mathrm{~mm})$. & $0.270 \mathrm{I}$ in. $(5.9 \mathrm{~mm})$. & 0.0172 in. (0.44 mm.) \\
\hline
\end{tabular}

The mechanism of the changes in the thickness and in the curvatures of the lens in accommodation can be understood only by keeping clearly in mind the physical properties of the lens itself and its anatomical relations. In situ, in what has been called the indolent state of the eye, the lens is adjusted for vision at an infinite distance and is flattened by the tension of its suspensory ligament. After death, indeed, it is easy to produce changes in its form by applying traction to the zone of Zinn. Remembering the exact relations of the suspensory ligament, the ciliary muscle and the lens, and keeping in mind the tension within the globe, it is evident that when the ciliary muscle is at rest, the capsule will compress the lens, increasing its diameter and diminishing its convexity. It is in this condition that the eye is adapted to vision at 
an infinite distance. It is evident, also, that slight changes in the convexity of the lens are sufficient for the range of accommodation required. If any near object is fixed with the eye there is a conscious effort, and prolonged examination of near objects produces a sense of fatigue. This may be illustrated by the very familiar experiment of looking at a distant object through a gauze. When the object is seen distinctly, the gauze is scarcely perceived; but by an effort the eye can be brought to see the meshes of the gauze distinctly, when the impression of the distant object is either lost or becomes indistinct.

The ciliary muscle arises from the circular line of junction of the cornea and sclerotic, passes backward, and is lost in the tissue of the choroid, extending as far as the anterior border of the retina. Most of the fibres pass directly backward but some become circular or spiral. When this muscle contracts, the choroid is drawn forward, with probably a slightly spiral motion of the lens, the contents of the globe, situ-

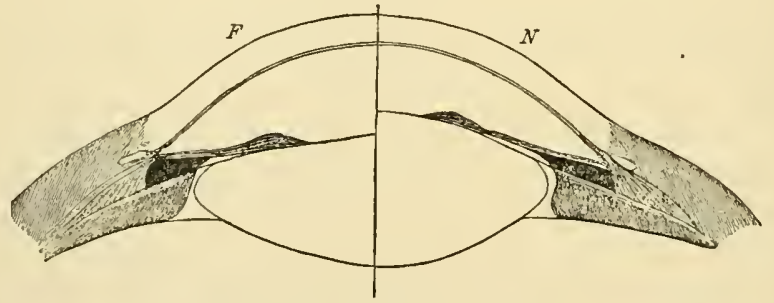

Fig. 176. - Section of the lens, etc., showing the mechanism of accommodation (Fick).

The left side of the figure $(F)$ shows the lens adapted to vision at infinite distances. The right side of the figure $(N)$ shows the lens adapted to the vision of near objects, the ciliary muscle being contracted and the suspensory ligament of the lens consequently relaxed.

ated behind the lens, are compressed, and the suspensory ligament is relaxed. The lens itself, the compressing and flattening action of the suspensory ligament being diminished, becomes thicker and more convex, by virtue of its own elasticity, in the same way that it becomes thicker after death, when the tension of the ligament is artificially diminished. The theory of Tscherning that the ciliary muscle tightens the suspensory ligament, compresses the lens near its borders and produces bulging of its central portion is not tenable in view of demonstrations that the ligament actually is relaxed during accommodation for near objects (Hess).

This is in brief the mechanism of accommodation. Near objects are seen distinctly by a voluntary contraction of the ciliary muscle, the action of which is perfectly adapted to the requirements of vision. In early life the lens is soft and elastic, and the accommodating power is at its maximum; but in old age the lens becomes flattened, harder and less elastic, and the power of accommodation necessarily is diminished. 
Changes in the Iris in Accommodation. - The size of the pupil is sensibly diminished in accommodation of the eye for near objects. Although the movements of the iris are directly associated with the muscular effort by which the form of the lens is modified, the contraction of the pupil is not one of the essential conditions of accommodation. Helmholtz reported a case in which the iris was completely paralyzed, the power of accommodation remaining perfect; and he described another case, reported by Von Graefe, in which accommodation was not disturbed after loss of the entire iris.

It has already been noted that the pupil contracts when the eyes are made to converge by the action of the muscles animated by the third pair of nerves; and it is evident that convergence of the eyes occurs in looking at very near objects. Increased convergence of the visual lines without change of accommodation makes the pupil contract, as is easily proved by simple experiments with prismatic glasses. When accommodation is effected without converging the visual axes, "each stronger tension is combined with contraction of the pupil" (Donders). Contraction of the pupil, therefore, occurs both in convergence of the visual axes without accommodation and in accommodation for near objects without convergence of the eyes.

The action of the iris, as is evident from the facts just stated, is to a certain extent under the control of the will; but it can not be disassociated, first, from the voluntary action of the muscles that converge the visual axes, and second, from the action of the ciliary muscle. Donders, by alternating the accommodation for a remote and a near object, was able to contract and dilate the pupil voluntarily more than thirty times in a minute. Brown-Séquard, in discussing the voluntary movements of the iris, has mentioned a case in which "the pupil could be contracted or dilated without changing the position of the eye or making an effort of adaption for a long or a short distance." As further evidence of the connection of accommodation with muscular action, cases are cited in works on ophthalmology in which there is paralysis of the ciliary muscle, as well as cases in which the act of accommodation is painful.

A curious phenomenon connected with accommodation may be observed in looking at a near object through a very small opening, like a pinhole. The shortest distance at which one can see a small object distinctly is about five inches (12.7 centimeters); but in looking at the same object through a pinhole in a card, it can be seen distinctly at the distance of about one inch (25.4 millimetres), and it then appears considerably magnified. In this experiment, the card serves as a diaphragm with a very small opening, so that only the centre of the lens is used; 
and the apparent increase in the size of the object probably is due to the fact that its distance from the eye is many times less than the distance at which distinct vision is possible under ordinary conditions. It is well known that myopic persons, by being able to bring the eye nearer to objects than is possible in ordinary vision, can see minute details with peculiar distinctness.

Erect Impressions produced by Images inverted upon the Retina. The images that make visual impressions are necessarily inverted on the retina; but the cerebral visual centre takes no cognizance of this, and

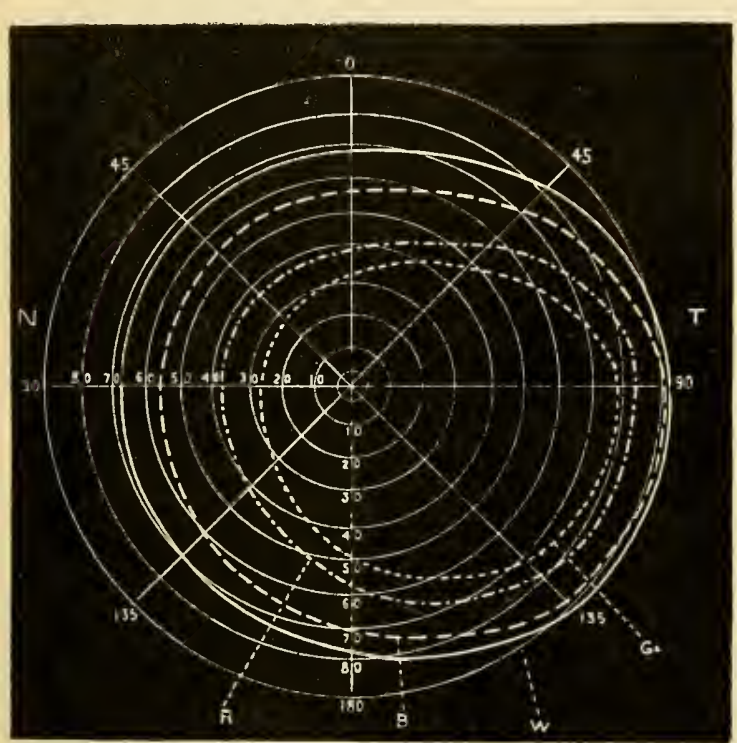

Fig. 177. - Field of vision of the right eye, as projected by the patient on the inner surface of a hemasphere, the pole of which forms the object of regard-semidiagrammatic (Landolt).

$T$, temporal side; $N$, nasal side; $W$, boundary for white; $B$, boundary for biue ; $R$, boundary for red; $G$, boundary for green.

objects are seen in their actual position. It seems almost absurd to enter into a serious discussion of this fact. Quoting the words of von Helmholtz, "our natural consciousness is completely ignorant even of the existence of the retina and of the formation of images: how should it know anything of the position of images formed upon it?"

Ficld of Indirect Vision. - If the eye is kept fixed on a certain point, and an object is moved from this point as a centre in lines radiating in different directions until it passes from the field of view, the limits of indirect vision are indicated. Eight or ten such points of limit, connected by a curved line, give a map of the visual field. This may be done roughly on a flat surface, such as a blackboard, placed at a distance of twelve to eighteen inches ( 3 to 4.5 centimeters) from the eye, or a chart may be made with an instrument called the perimeter, by which the field is marked on the inner surface of a hemisphere. The field of vision thus delineated is an irregular oval, extending from the fixed point farther to the temporal side than to either the nasal side or above and below. The extent from the fixed point is about $90^{\circ}$ on the temporal side, and about $70^{\circ}$ to the nasal side and above and below. The field for white light is larger than 
for colors, especially on the nasal side, as is seen in Fig. I77. The field is smallest for green, a little larger for red, and is still larger for blue. Investigation of the field of indirect vision with the perimeter is useful in ophthalmology; but the chief physiological interest, as regards the sensibility of the retina, is connected with direct vision.

\section{BiNocular Vision}

Thus far the mechanism of the eye and its action as an optical instrument have been described for monocular vision only; but it is evident that both eyes are habitually used, and that their axes are practically parallel in looking at distant objects and are converged when objects are approached to the nearest point at which there is distinct vision. In fact an image is formed simultaneously on the retina of either eye, but it is nevertheless appreciated as a unit. If the axis of one eye is slightly deviated by pressure on the globe, so that the images are not formed upon corresponding points in the retina of either eye, vision is more or less indistinct and is double. In strabismus, when this condition is recent, temporary or periodical, as in recent cases of paralysis of the external rectus muscle, when both eyes are normal, there is double vision. When the strabismus is permanent and has existed for a long time, double vision may not be observed, unless the subject directs the attention strongly to this point. As but one eye is capable of fixing objects accurately, images are formed on the fovea of this eye only. Images formed upon the retina of the other eye are indistinct, and in many instances are habitually disregarded; so that practically the subject uses but one eye, and presents the errors of appreciation that attend monocular vision, such as a want of exact estimation of the solidity and distance of objects. It is stated as the rule that when strabismus of long standing is remedied, so far as the axes of the eyes are concerned, by an operation, binocular vision is not restored. This is explained on the supposition that the perceptive power of the retina of the affected eye has been gradually lost from disuse. In normal binocular vision the images are formed on the fovea centralis of either eye; that is, on corresponding points, which are, for each eye, the centres of distinct vision. The concurrence of both eyes is necessary to the exact appreciation of distance and form; and when the two images are formed on corresponding points, the visual centre receives a correct impression of a single object. When vision is perfect, the sensation of the situation of any single object is referred to one and the same point; and the impression of a double image can not be received unless the conditions of vision are abnormal. 
Corresponding Points. - While it is evident, after the statements just made, that images must be formed on the fovea of each eye in order to produce the effect of a single object, it becomes important to ascertain how far it is necessary that the correspondence of points should be carried out in the retina. It is almost certain that for absolutely perfect single vision with the two eyes, the impressions must be made on exactly corresponding points, even to the ultimate sensitive elements of the retina. It may be assumed, indeed, that each rod and each cone of one eye has its corresponding rod and cone in the other, situated at exactly the same distance and in corresponding directions from the visual axis. When the two images of an object are formed on these corresponding points, they appear as one; but when the images do not correspond, the impression is as though the images were formed on different points in one retina, and of necessity they appear double.

The effect of a slight deviation from the corresponding points may be illustrated by the following experiment: If a small object, like a lead-pencil, held at a distance of a few inches, is fixed with the eyes, it is seen distinctly as a single object. Holding another small object in the same line, a few inches farther removed, when the first is seen distinctly, the second appears double. If the second object is fixed with the eyes, the first appears double. . It is evident here, that when the axes of the eyes bear on one of these objects, the images of the other must be formed at a certain distance from the corresponding retinal points.

The Horopter. - The above-mentioned experiment affords an explanation of the horopter. If both eyes are fixed on a point directly in front and are kept in this position, an object moved to one side or the other, within a certain area, may be seen without any change in the direction of the axis of vision; but the distance from the eye at which there is single vision of this object is fixed, and at any other distance the object appears double. The explanation of this is that at a certain distance from the eye, the images are formed on corresponding points in the retina; but at a shorter or longer distance, this can not occur. This illustrates the fact that there are corresponding points in a large part of the sensitive layer of the retina as well as in the forea centralis. By these experiments the following facts have been ascertained: With both eyes fixed on an object, another object moved to one side or the other can be seen distinctly only when it is carried in a certain curved line. On either side of this line, the object appears double. This line, or area - for the line may have any direction - is called the horopter. It was supposed at one time to be a regular curve, or a portion of a circle drawn through the fixed point and the points of intersection of the rays of light in each eye. Although it has been ascertained that 
the line varies somewhat from a regular curve, and also varies in different meridians, this is due to differences in refraction, etc., and the principle is not altered.

If the visual areas of the two retinæe are superimposed, the fixationpoints coinciding, it becomes evident that a portion only of the two fields can have corresponding points. This is the light portion shown in Fig. $\mathrm{x} 78$, which may be called the binocular field of vision. Binocular vision must be impossible in the temporal portion of each visual area.

It is undoubtedly true that education and habit have much to do with the correction of visual impressions and the just appreciation of the size, form and distance of objects. In the remarkable case of Kaspar Hauser, who is said to have been kept in total darkness and seclusion from the age of five months until he was nearly seventeen years old, the appreciation of size, form and distance were acquired by correcting and supplementing the sense of sight by experience. This boy at first had no idea of the form of objects or of distance, until he had learned by touch, by walking etc., that certain objects were round and others were square, and had actually traversed the distance from one object to another. At first all objects appeared as if painted upon a screen. Such points as

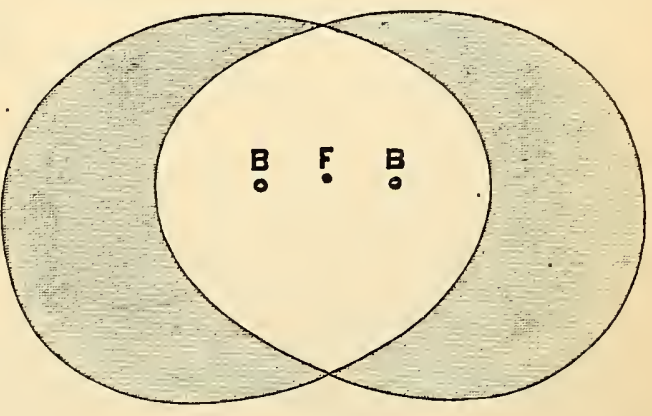

Fig. I78. - Binocular fleld of vision (Förster). $\mathrm{F}$, fixation-point; $\mathrm{B}, \mathrm{B}$, blind spots. these it would be impossible to observe accurately in infants; but young children often grasp at distant objects, apparently under the impression that they are within reach. It must be admitted, however, that the account of the case of Kaspar Hauser is rather indefinite; but it is certain that even in the adult, education and habit greatly improve the faculty of estimating distances.

Careful observations leave no doubt of the fact that monocular vision is incomplete and inaccurate, and that it is only when two images are formed, one upon either retina, that vision is perfect. The sum of actual knowledge on this important point is expressed in the following quotation from Giraud-Teulon :-

"Monocular vision only indicates to us immediately, visual direction, and not precise locality. At whatever distance a luminous point may be situated in the line of direction, it forms its image upon the same point in the retina. 
"In the physiological action of a single eye, in order to arrive at an idea of the clistance of a point in a definite direction, we have only the following elements :-

"I. The consciousness of an effort of accommodation.

"2. Our own movement in its relations to the point observed.

" 3 . Facts brought to bear from recollection, education, our acquired knowledge with regard to the form and size of objects: in a word, experience.

" 4 . The geometric perspective of form and position.

"5. Aërial perspective.

"All these are elements wanting in precision and leaving the problem without a decisive solution.

"And, indeed :-

"We place before one of our eyes, the other being closed, the excavated mould of a medallion: we do not hesitate, after a few seconds, to mistake it for the relief of the medallion. This illusion ceases at the instant that both eyes are opened.

"Or again :-

"A miniature, a photograph, a picture, produces for a single eye a perfect illusion; but if both eyes are open, the picture becomes flat, the prominences and the depressions are effaced.

"We may repeat the following experiment described by Malebranche: 'Suspend by a thread a ring, the opening of which is not directed toward us; step back two or three paces; take in the hand a stick curved at the end; then, closing one eye with the hand, endeavor to insert the curved end of the stick within the ring, and we shall be surprised at being unable to do in a hundred trials what we should believe to be very easy. If, indeed, we abandon the stick and endeavor to pass one of the fingers through the ring, we shall experience a certain degree of difficulty, although it is very near. This difficulty ceases at the instant that both eyes are opened.

"As regards precision, exactitude of information concerning the relative distance of objects, that is to say, the idea of the third dimension, or of depth, there is then a notable difference between binocular vision and that which is obtained by means of one eye alone."

It is evident that an accurate idea of the distance of near objects can not be obtained except by the use of both eyes, and this fact will partly explain the errors of monocular vision in looking with one eye at objects in relief; for under these conditions it is impossible to determine with accuracy whether the points in relief are nearer or farther from the eye than the plane surface. This will not fully explain, however, the idea of solidity of objects, which is obtained by the use of both 
eyes; for the estimation of distance is obtained by bringing the axes of both eyes to bear on a single object, be it near or remote. The fact is as was distinctly stated by Galen in the second century - that in looking at any solid object not so far removed as to render the visual axes practically parallel, a portion of the surface seen with the right eye is not seen with the left eye, and vice versa $\hat{a}$. The two impressions, therefore, are not identical for each retina; the image on the left retina including a portion of the left side of the object, not seen with the right eye, the right image in the same way including a portion of the right surface, not seen with the left eye. These slightly dissimilar impressions are fused and produce the impression of a single image, when vision is normal; and this gives the idea of relief or solidity and an exact appreciation of the form of objects, when they are not too remote.

Although an opposite opinion is held by some experimenters, Helmholtz, with many others, has stated that when one color is seen with one eye and another color, with the other eye, in the stereoscope, the impression is not of a single color resulting from the combination of the two. It is true that there is an imperfect mingling of the two colors, but this is different from the resulting color produced by the actual fusion of the two. There is, in other words, a sort of confusion of colors, without the complete combination observed in ordinary experiments. One additional point of importance, however, is that the binocular fusion of two pictures, unequally illuminated or of different colors, produces a single image of a peculiar lustre, even when both surfaces are dull. This may be shown by making a stereoscopic combination of images of crystals, one with black lines on a white ground, and the other with white lines on a black ground. The resulting image has then the appearance of dark brilliant crystals, like graphite.

Duration of Luminous Impressions (After-Images). - The time required for a single visual stimulation of the retina is short. The letters on a printed page are distinctly seen when illuminated by an electric spark, the duration of which is not more than forty-billionths of a second (Rood). An impression made on the retina, however, endures for a length of time that bears a certain relation to the intensity of the luminous excitation. If the eyes are closed after looking steadily at a bright object, the object is more or less distinctly seen after the rays have ceased to pass to the eye and the image fades away gradually. When there is a rapid succession of images, they may be fused into one, as the spokes of a rapidly revolving wheel are indistinct and produce a single impression. This is due to the persistence of the successive retinal impressions; for if a revolving wheel or even a falling body is illuminated for the brief duration of an electric spark, it appears to be 
stationary, as the period of time necessary for distinct vision and the duration of the illumination are so short that there is no time for any appreciable movement of the object. The familiar experiments made with revolving disks illustrate these points. In a disk marked with alternate radiating lines of black and white, the rays become indistinguishable during rapid revolution, and the disk appears of a uniform color, such as would be produced by a combination of the black and white. The effects of an artificial combination of colors may be produced in this way, the resultant color appearing as if the individual colors had been ground together. The duration of retinal impressions varies considerably for the different colors. According to Emsmann, the duration for yellow is 0.25 of a second; for white, 0.25 of a second; for red, 0.22 of a second; and for blue, $0.2 \mathrm{I}$ of a second.

The impressions that remain on the retina after an object has been looked at steadily are called after-images. When these are bright and of the same character as the object, they are called positive after-imáges. When the stimulation of the retina has been powerful and prolonged, the after-image frequently is dark. Such images are called negative after-images.

It is unnecessary to describe further in detail the well-known phenomena which illustrate the point under consideration. The circle of light produced by rapidly revolving a burning coal, the track of a meteor and other illustrations, are sufficiently familiar, as well as many scientific toys producing optical illusions of various kinds.

Irradiation. - It has been observed that luminous impressions are not always confined to the elements of the retina directly involved, but are sometimes propagated to those immediately adjacent. This gives to objects a certain degree of amplification, which usually is in proportion to their brightness. An illustration of this is afforded by the simple experiment of looking at two circles, one black on a white ground, and the other white on a black ground. Although the dimensions of the two circles are identical, the irradiation of rays from the white circle makes this appear the larger. In a circle with one half black and the other white, the white portion will appear larger, for the same reason. These phenomena are due to what has been called irradiation; and their explanation is very simple. It is probable that luminous impressions are never confined absolutely to those parts of the retina on which the rays of light directly impinge, but that the sensitive elements immediately contiguous are always more or less involved. In looking at powerfully-illuminated objects, the irradiation is considerable, as compared with objects which send fewer luminous rays to the eye.

In experiments analogous to those just described, made with strongly 
colored objects, it has been observed that the border of irradiation takes a color complementary to that of the object itself. This is particularly well marked when the objects are steadily looked at for some time. Illustrations of this point also are very simple. In looking steadily at a red spot or figure on a white ground, a faint areola of a pale green soon appears surrounding the red object; or if the image is yellow, the areola will appear pale blue. These appearances are called accidental areolæ. 


\section{CHAPTER XXVIII}

MOVEMENTS OF THE EYEBALL-PARTS FOR PROTECTION OF THE EYE

Action of the recti muscles - Action of the oblique muscles - Associated action of the muscles of the eyeball - Centres for vision - Perception of colors - Parts for the protection of the eyeball - Muscles that open and close the eyelids - Conjunctival mucous membrane The lachrymal apparatus - The tears.

THE eyeball nearly fills the carity of the orbit, resting by its posterior portion upon a bed of adipose tissue, which is never absent, even in extreme emaciation. External to the sclerotic, is a fibrous membrane, the tunica vaginalis oculi, or capsule of Tenon, which is useful in maintaining the equilibrium of the globe. This membrane surrounds the posterior two-thirds of the globe and is loosely attached to the sclerotic. It is perforated by the optic nerve posteriorly, and by the tendons of the recti and oblique muscles of the eyeball in front, being reflected over these muscles. It also is continuous with the palpebral ligaments and is attached, by two tendinous bands, to the border of the orbit at. the internal and the external angles of the lids.

The muscles that move the globe are six in number for either eye. These are the external and internal recti, the superior and inferior recti and the two oblique muscles. The four recti and the superior oblique arise posteriorly from the apex of the orbit. The recti pass directly forward by the sides of the globe and are inserted by short tendinous bands into the sclerotic, at a distance of one-fourth to one-third of an inch (6.4 to 8.5 millimeters) from the margin of the cornea. The superior oblique, or trochlearis muscle passes along the upper and inner wall of the orbit to a point near the inner angle. It here presents a rounded tendon, which passes through a ring, or pulley, of fibro-cartilage; and it is from this point that its action is exerted on the globe. From the pulley, or trochlea, the tendon becomes flattened, passes outward and backward beneath the superior rectus, and is inserted into the sclerotic about midway between the superior and the external rectus and just behind the equator of the globe. The inferior oblique muscle arises just within the anterior margin of the orbit near the inner angle of the eye and passes around the anterior portion of the globe beneath the inferior rectus and between the external rectus and the eyeball, taking a direction outward and slightly backward. Its tendon is inserted into 
the sclerotic a little below the insertion of the superior oblique. The general arrangement of these muscles is shown in Fig. I 79.

The movements of the eyeball are easily understood from a study of the associated movements of the muscles just enumerated, at least so far as is necessary to the comprehension of the mechanism by which the eyes are directed toward any particular object. The centre of distinct vision is in the fovea ; and it is evident that in order to see any object distinctly, it is necessary to bring it within the axes of vision of both eyes. As the globe is so balanced in the orbit as to be capable of rotation, within certain limits, in every direction, it is necessary only to

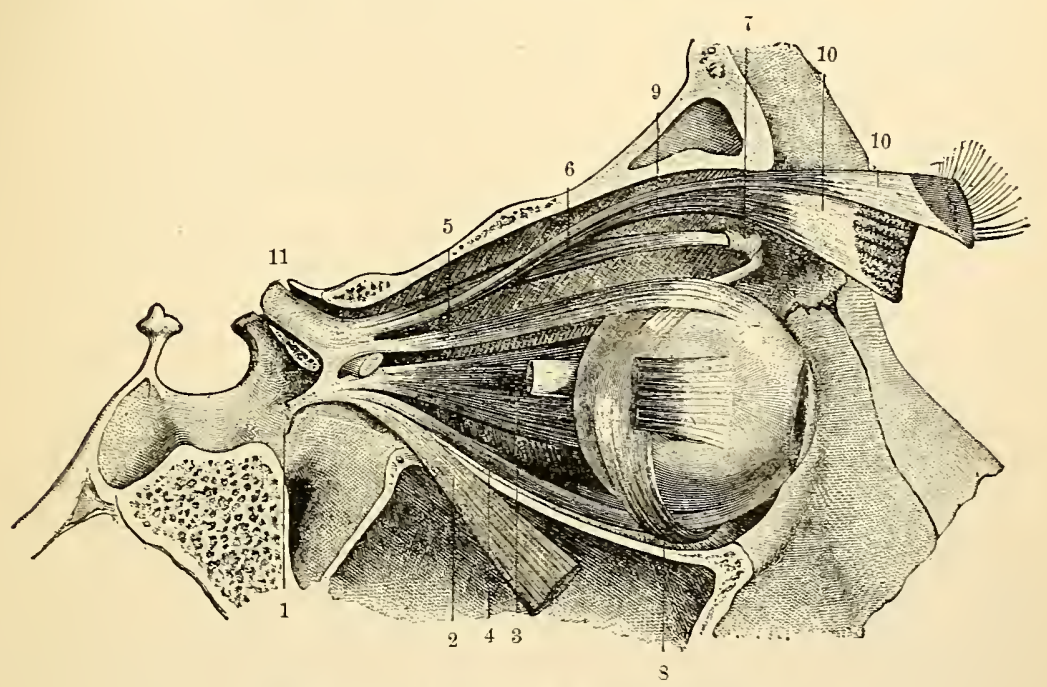

Fig. 179. - Muscles of the eyeball (Sappey).

I, attachment of the tendon connected with the inferior rectus, internal rectus and external rectus; 2 , external rectus, divided and turned downward, to expose the inferior rectus; 3 , internal rectus; 4 , inferior rectus ; 5 , superior rectus; 6 , superior oblique; 7 , pulley and reflected portion of the superior oblique; 8 , inferior oblique; 9 , levator palpebri superioris; I0, Io, middle portion of the levator palpebri superioris; II, optic nerve.

note the exact mode of action of each of the muscles, in order to comprehend how the different movements are accomplished; and it is sufficient for practical purposes to admit that approximately there is a common axis of rotation for each pair of muscles.

Under ordinary conditions in the human subject, the action of the six ocular muscles is confined to movements of rotation and torsion of the globe. It is said that in the human subject there is no such thing as protrusion of the eye from general relaxation of these muscles, and that it is impossible, by a combined action of the four recti muscles, to retract the globe in the orbit; but those who have operated on the eyes assert that this statement is erroneous, and that the globe is almost 
always suddenly and powerfully drawn within the orbit when a painful impression is made on the cornea. This is a matter of common observation by ophthalmic surgeons.

The extent to which the line of vision may be turned by a voluntary effort varies in different individuals, even when the eyes are normal. In myopic eyes, the centre of rotation is deeper in the orbit than normal and the extent of the possible deviation of the visual line is correspondingly diminished. Helmholtz stated that in his own person, with the greatest effort that he was capable of making, he could move the line of vision in the horizontal plane to the extent of about fifty degrees, and in the vertical plane, about forty-five degrees; but he added that these extreme rotations were very forced, and that they could not be sustained for any considerable length of time. It is probable that the eyeball is seldom moved in an angle of forty-five degrees, the direction of the visual line being more easily accomplished by movements of the head.

Action of the Recti Muscles. - The internal and external recti rotate the globe on a vertical axis that is perpendicular to the axis of the eye. The isolated action of these muscles, particularly of the external rectus, is often illustrated in certain forms of paralysis, which have been alluded to in connection with the history of the cranial nerves.

The superior and inferior recti rotate the globe on a horizontal axis, which is not at right angles with the axis of the eye but is inclined from the nasal side slightly backward. The line that serves as the axis of rotation for these muscles forms an angle of about seventy degrees with the axis of the globe; and as a consequence, their action is not so simple as that of the internal and external recti. The insertion of the superior rectus is such, that when it contracts, the pupil is directed upward and inward, the inferior rectus directing the pupil downward and inward.

The above represents the isolated action of each pair of recti muscles; but it is easy to see how, without necessarily involving the action of the oblique muscles, the globe may be made to perform a great variety of rotations and the line of vision may be turned in nearly every direction by the action of the recti muscles alone.

Action of the Oblique Muscles. - It is sufficient for all practical purposes to assume that the superior and the inferior oblique muscles act as direct antagonists to each other. The most exact measurements show that the axis of rotation for these muscles is horizontal and has an oblique direction from before backward and from without inward. The angle formed by the axis of rotation of the oblique muscles with the axis of the globe is thirty-five degrees; and the angle between the axis of the oblique muscles and the axis of the superior and inferior recti muscles is seventy-five degrees. 
Given the direction of the axis of rotation and the direction of the superior oblique muscle, it is easy to understand the effects of its contraction. As this muscle, passing obliquely backward and forward over the globe, acts from the pulley near the inner angle of the eye to its insertion just behind the anterior half of the globe on its external and superior surface ( 7 , Fig. I79), it must rotate the globe so as to direct the pupil downward and outward.

The inferior oblique, passing outward and slightly backward under the globe, acts from its origin at the margin of the orbit near the inner angle of the eye to its insertion, which is just below the insertion of the superior oblique. This muscle rotates the globe so as to direct the pupil upward and outward.

The action of the oblique muscles is specially connected with the movements of torsion of the globe. It is necessary to distinct single vision with both eyes, that the images should be formed on corresponding points on the retina, and that they should bear, for the two eyes, corresponding relations to the perpendicular. Thus it is that when the head is inclined to one side, the eyes are twisted on an oblique anteroposterior axis; as can be readily seen by observing little spots on the iris during these movements.

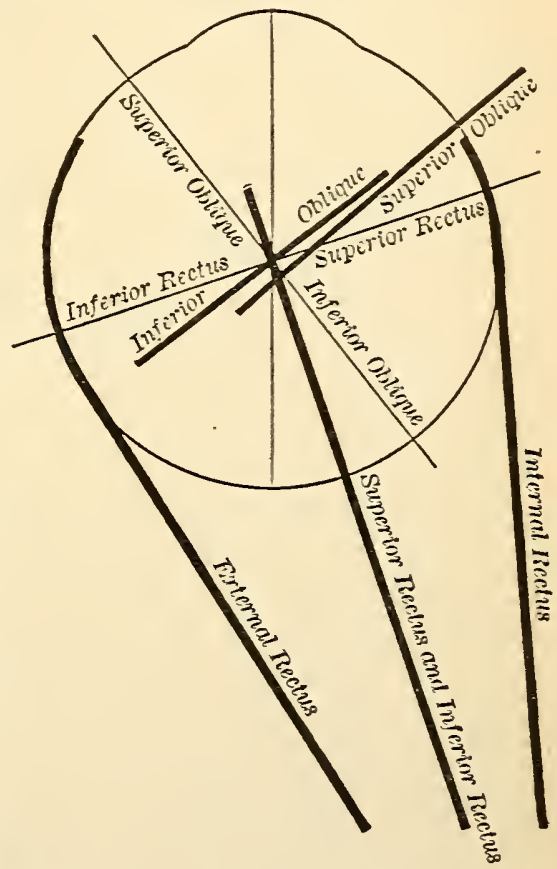

Fig. I80. - Diagram illustrating the action of the muscles of the eyeball (Fick).

The heary lines represem the muscles of the eyeball, and the fine lines, the axes of the superior and the inferior recti and the axes of the oblique muscles.

The superior oblique muscle is supplied by a single nerve, the patheticus. When this muscle is paralyzed, the inferior oblique acts without its antagonist, and the eyeball is immorable so far as the twisting of the globe is concerned. When the head is moved toward the shoulder, the globe can not rotate to maintain a position corresponding to that of the other eye and there is double vision.

Associated Action of the Muscles of the Eyeball. - It is almost unnecessary to add, after the description just given of the actions of the individual muscles of the globe, that their contractions may be associated so as to produce a great variety of morements. There is no 
consciousness, under ordinary conditions, of the muscular action by which the globe is rotated and twisted in various directions, except that by an effort of the will the line of vision is directed toward different objects. By a strong effort the axes of the eyes may be converged by contracting both internal recti, and some persons can produce extreme divergence by using both external recti; but this is abnormal.

In looking at distant objects the axes of vision are practically parallel. In looking at near objects the effort of accommodation is attended with the degree of convergence necessary to bring the visual axes to bear on identical points. In looking around at different objects the head is moved more or less and the globes are rotated in various directions. In the movements of the globes vertically the axes are kept parallel, or at the proper angle, by the internal and external recti, and the superior and inferior recti on the two sides act together. In rotating the globe from one side to the other on a vertical axis, the external rectus on one side acts with the internal rectus on the other. In the movements of torsion on an antero-posterior axis, there must be an associated action of the oblique muscles and the recti.

An important point, not to be lost sight of in the study of the associated action of the muscles of the globe, relates to the associated movements of the two eyes. Perfect binocular vision is possible only when impressions are made on corresponding points in the retina of each eye. If one eye is deviated in the horizontal plane, the points no longer correspond and there is double vision, the same as if two impressions were made on one retina; for when the impressions exactly correspond, the two retinæ act practically as a single organ. The same is true in deviation of the globe in the vertical plane. If it be supposed, for the sake of argument, that the retina is square, it is evident that a torsion or twisting of one globe on an antero-posterior axis must be attended with an analogous movement of the other globe in order to bring the visual rays to bear on the corresponding points; in other words, the obliquity of the assumed square of the retina must be exactly the same for the two eyes or the coincidence of the corresponding points would be disturbed and there would be double vision. Deviation of one eye in the horizontal or the vertical plane disturbs the relation of the corresponding points, and a deviation from exact coincidence of action in torsion of the globes, twists, as it were, the corresponding points, so that their relation is also disturbed. It is evident, therefore, that the varied movements of the globes, by the combined action of the recti and oblique muscles, must correspond for either eye, in the movements of torsion on an antero-posterior axis as well as in movements of rotation on the horizontal or the vertical axis. 


\section{Centres for Vision}

Experiments have been made on the lower animals by Ferrier, Munk, Exner and many others, with the object of locating in the cerebrum a centre for vision. It is important, however, to compare the results of such experiments with cases of cerebral lesions in the human subject. As the general result of experiments, both on dogs and monkeys, and of pathological observations, the present opinion is that the centres for vision are in the occipital lobes. The convolutions immediately above and below the calcarine fissure, on the mesial surface of the cerebrum (Figs. I 34 and 140, pages 566 and 570), seem to be the cerebral terminations of fibres that are continuous with the optic tracts. These fibres are not crossed in the cerebrum, but the conductors decussate at the optic chiasm as they pass to the eyes. Cases have been observed in the human subject in which lesion of these parts on one side has been followed by loss of vision in one lateral half of the retina in either eye. This condition is called hemianopsia. In these instances the blindness is confined to the temporal side of the retina corresponding to the lesion and the nasal side of the retina of the opposite eye. This is called lateral homonymous hemianopsia, and this is the form that occurs in unilateral cerebral lesion. In dogs and in monkeys, destruction of both occipital lobes and both angular convolutions produces total and permanent blindness of both eyes.

The complete and perfect perception of visual impressions involves intellectual action connected with the simple visual sense. An individual may see objects and yet not be able to appreciate their significance. In the condition known as word-blindness, words are seen, but they convey no idea. A dog with part of the occipital lobes removed may see objects such as food, but does not recognize their character. There are, indeed, psychical centres, which elaborate the impressions received by the simple visual centres.

What seems at present to be the most rational view to take in regard to the location and action of the visual centres is the following:-

I. The centre for simple visual impressions is on the inner surface of the cerebrum, on either side of the calcarine fissure, between the cuneus and the lobulus lingualis. This part is connected with homonymous halves of the retina of either eye - the temporal half of the retina of the same side and the nasal half of the retina of the opposite side. The part above the calcarine fissure is connected with the upper portion of the retina, and the part below, with the lower portion of the retina.

2. The action of the cortex of the convex surface of the temporal lobe - perhaps only on the left side - is necessary for full visual per- 
ception and recognition and for the production of visual memories. This may be called the psychical visual centre. Psychical blindness may exist, indeed, without loss of visual sensation.

3. The angular convolution is not a visual centre, as was claimed by Ferrier. It is related to visual perception only in so far as it affects "the memories of the appearance of written or printed words" (Hun). In cases of word-blindness lesions have been found in this situation.

Perception of Colors. - By far the most obscure question connected with vision relates to the perception of colors. In treatises on physiology, the difficulty of this subject is well illustrated in the indefinite manner in which it is discussed; and I am tempted to dismiss the question with the remark - which appeared in earlier writings on vision - that "nothing is known of the mechanism of color-perception." It is well known, however, that white light may be decomposed into the colors of the spectrum and that different colors have different —often widely different - wave-lengths. If a beam of white light, with a certain wave-length, impinges on the retina, it produces a certain impression that is appreciated by the visual centres. If, now, beams of light, different in color and with different wave-lengths, reach the retina, they must produce different impressions and a different impression for each color and combination of colors. When these impressions have been frequently repeated and the visual centres have, so to speak, become educated, different colors are recognized. It seems a question, indeed, if there be anything more than this in color-perception. Certain it is, that if the waves that make the impression on the retina which gives the sensation of light and color are perceived in a certain way, differences in wave-lengths must involve differences in the nature of the impressions, corresponding with necessary differences in the nature of the stimuli. It almost seems better to take this view, however unsatisfactory it may appear, than to enter into a discussion that is not likely to lead to positive conclusions. In olfaction, of course different odorous impressions are made by different substances composed of different numbers of molecules. It has been found that the odor of ethyl mercaptan is recognizable in a dilution of one part in fifty billions of air. If such infinitesimally small particles can make a decided and peculiar impression on the nerves of smell, it is not unreasonable to suppose that the parts concerned in vision may appreciate the difference between red and violet, with $392,000,000,000,000$ and $757,000,000,000,000$ vibrations in a second respectively. There are, indeed, quite as wide differences, as regards number of molecules, between red and violet as there is between ethyl mercaptan $\left(\mathrm{C}_{6} \mathrm{H}_{5} \mathrm{SH}\right)$ and benzene sulphide $\left(\mathrm{C}_{6} \mathrm{H}_{5}\right)_{2}$ and between many other distinctively odorous substances. 
Color-blindness is an abnormal condition in which the power of discrimination between different colors is impaired or lost. Some persons are insensible to all colors and others to certain colors only. Red-green blindness is the most common form. Cases of disease of the brain, in which ordinary visual perception remains while the sense of color is absent, seem to show that parts of the visual centres are specially concerned in the appreciation of colors. That this defect, however, may depend on the retina, is shown in cases in which one eye is color-blind while the other is normal. There are cases, also, in which these defects seem to be due exclusively to atrophy of the disk and contraction of the visual field, the acuteness of vision not being much impaired; but unfortunately even these striking pathological conditions throw little light on the physiology of normal color-perception.

\section{Parts for the Protectiox of the Erteball}

The orbit, formed by the union of certain of the bones of the face, receives the eyeball, the ocular muscles, the muscle of the upper lid, bloodressels, nerves and a part of the lachrymal apparatus. It contains, also, a certain quantity of adipose tissue, which latter never disappears, even in extreme marasmus. The bony walls of this cavity protect the globe and lodge the parts enumerated above. The internal, or nasal wall of the orbit projects considerably beyond the external wall, so that the extent of vision is greater in an outward than in an inward direction. As the globe is more exposed to accidental injury from an outward direction, the external wall of the orbit is strong, while the bones that form its internal wall are comparatively fragile. The upper border of the orbit (the superciliary ridge) is provided with short stiff hairs (the eyebrows) which serve to shade the eye from excessive light and to protect the eyelids from perspiration from the forehead.

The eyelids are covered with a thin integument and are lined with the conjunctival mucous membrane. The subcutaneous connective tissue is thin and loose and is free from fat. The skin presents a large number of short papille and small sudoriparous glands. At the borders of the lids, are short, stiff, curved hairs arranged in two or more rows, called the eyelashes, or cilia. Those of the upper lid are in greater number and longer than the lower cilia. The curve of the lashes is from the eyeball. They serve to protect the globe from dust, and to a certain extent to shade the eye.

The tarsal cartilages are small, elongated, semilunar plates, extending from the edges of the lids toward the margin of the orbit, between the skin and the mucous membrane. Their length is about an inch 
(25.4 millimeters). The central portion of the upper cartilage is about one-third of an inch ( 8.5 millimeters) broad, and the corresponding part of the lower cartilage measures about one-sixth of an inch (4.2 millimeters). At the inner canthus, or angle of the eye, is a small delicate ligament, or tendon, the tendo palpebrarum, which is attached to the lachrymal groove internally, passes outward and divides into two lamellæ that are attached to the two tarsal cartilages. At the outer canthus the cartilages are attached to the malar bone by the external tarsal ligament. The tarsal cartilages receive additional support from the palpebral ligament, a fibrous membrane attached to the margin of the orbit and the convex border of the cartilages and lying beneath the orbicularis muscle. This membrane is strongest near the outer angle of the eye.

On the posterior surface of the tarsal cartilages, partly embedded in them and lying just beneath the conjunctiva, are the Meibomian glands. The structure and uses of these glands have already been described in connection with the physiology of secretion. They produce an oily liquid, which smears the edges of the eyelids and prevents the overflow of tears.

Muscles that open and close the Eyclids. - The corrugator supercilii draws the skin of the forehead downward and inward; the orbicularis palpebrarum closes the lids; and the levator palpebræ superioris raises the upper lid. The tensor tarsi, called the muscle of Horner, is a very thin delicate muscle, which is regarded by some anatomists as a deep portion of the orbicularis. Considering this as a distinct muscle, it consists of two delicate slips, which pass from either eyelid behind the lachrymal sac, uniting here to go to its attachment at the posterior portion of the lachrymal bone. When this acts with the orbicularis, it compresses the lachrymal sac.

The orbicularis palpebrarum is a broad thin muscle, closely attached to the skin, surrounding the free margin of the lids and extending a short distance over the bones beyond the margin of the orbit. This muscle may be described as arising from the tendo palpebrarum, the surface of the nasal process of the superior maxillary bone and the internal angular process of the os frontis. From this origin at the inner angle of the eye, its fibres pass elliptically around the fissure of the lids, as indicated above. Its action is to close the lids. In the ordinary moderate contraction of this muscle, only the upper lid is moved; but in forcible contraction, the lower lid moves slightly and the lids are drawn toward the nose.

The levator palpebræ superioris is situated within the orbit. It arises from a point a little above and in front of the optic foramen at the apex 
of the orbit, passes forward above the eyeball and spreads into a thin tendon, which is inserted into the anterior surface of the superior tarsal cartilage. Its action is to raise the upper lid. This muscle and its relations are shown in Fig. 179 (9, I0, I0), page 699.

In the act of opening the eyes the levator muscles alone are brought into play. Closing the lids is accomplished by the orbicular muscles. Both these sets of muscles act to a great extent without the intervention of the will. The eyes are kept open almost involuntarily, except in extreme fatigue; although when the will ceases to act the lids are closed. Nevertheless there is hardly a conscious effort usually in keeping the eyes open, and an effort is required to close the eyes. During sleep the eyes are closed and the globes are turned upward. The contractions of the orbicular muscles which take place in winking usually are involuntary. This act occurs at short intervals, and it is useful in spreading the lachrymal secretion over the exposed portions of the globes. The action of both sets of muscles ordinarily is simultaneous, although they may be educated so as to close one eye while the other is open. The action of the orbicularis is so far removed from the control of the will, that when the surface of the globe is touched or irritated or when the impression of light produces intense pain, it is almost impossible to keep the eye open.

Conjunctival Mucous Membrane. - The entire inner surface of the upper and lower eyelids is lined with a mucous membrane which is reflected forward from the inner periphery of the lids over the eyeball. The membrane lining the lids is called the palpebral conjunctiva, and that covering the eyeball, the ocular conjunctiva. The latter presents a sclerotic and a corneal portion. The conjunctiva presents a superior and an inferior fold, where it is reflected over the globe. In the superior conjunctival fold, are glandular follicles, or accessory lachrymal glands, which secrete a certain portion of the liquid that moistens the surface of the eyeball. These usually are described as forming a part of the lachrymal gland. At the inner canthus there is a vertical fold, the plica semilunaris, with a reddish spongy elevation at its inner portion, called the caruncula lacrymalis. The caruncula presents a collection of follicular glands, with a few delicate hairs on its surface. The conjunctiva is continuous with the membrane of the lachrymal ducts, of the puncta lacrymalia and of the Meibomian glands. Beneath the conjunctiva, except in the corneal portion, is a loose connective tissue.

The palpebral conjunctiva is reddish, thicker than the ocular portion, furrowed, and presents small isolated papillæ near the borders of the lids, which increase in number and size toward the folds. This portion of the membrane presents large capillary bloodvessels and lymphatics 
and is covered with a layer of cells of flattened epithelium. The sclerotic portion is thinner, less vascular and has no papillæ. It is covered with conical and rounded epithelial cells in two to four layers. Over the cornea the epithelium of the sclerotic portion is continued in delicate transparent layers, without a distinct basement-membrane.

The Lachrymal Apparatus. - The eyeball is constantly bathed in a watery liquid, which is secreted by the lachrymal gland, is spread over the globe by the movements of the lids and of the eyeball and is prevented, under ordinary conditions, from overflowing upon the cheek by the Meibomian secretion. The excess of this secretion is collected into the lachrymal sac, and is carried into the nose by the nasal duct. The

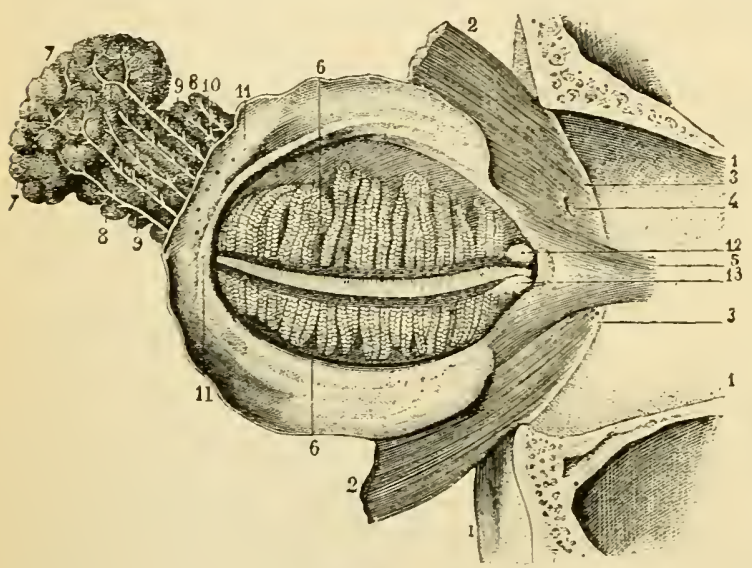

Fig. 181. - Lachrymal and Meibomian glands (Sappey).

I, I, internal wall of the orbit ; 2, 2, internal portion of the orbicularis palpebrarum; 3,3 , attachment of this muscle to the orbit; 4 , orifice for the passage of the nasal artery; 5 , muscle of Horner ; 6,6 , posterior surface of the eyelids, with the Meibomian glands; $7,7,8,8,9,9$, IO, lachrymal gland and ducts; II, openings of the lachrymal ducts. lachrymal gland, the lachrymal canals, duct and sac, and the nasal duct constitute the lachrymal apparatus.

The lachrymal gland is an ovoid flattened gland of the racemose variety, resembling the salivary glands in its general structure. It is about the size of a small almond and is lodged in a shallow depression in the bones of the orbit at its upper and outer portion. It is closely attached to the periosteum by its upper surface and is moulded below to the convexity of the globe. Its anterior portion is separated from the rest by a well-marked groove, is comparatively thin and adheres to the upper lid. It presents six to eight (usually seven) ducts, which form a row of openings into the conjunctival fold. Five or six of these openings are situated above the outer canthus, and two or three open below. In its minute structure this gland presents no points of special physiological importance as distinguished from the ordinary racemose glands. It receives nervous filaments from the fifth cranial and the sympathetic.

The channels by which the excess of tears is conducted into the nose begin by two little points situated on the margin of the upper and the lower lid near the inner canthus, called the puncta lacry- 
malia, which present each a minute opening. These open respectively into the upper and the lower lachrymal canals, which together surround the caruncula lacrymalis. At the inner angle just beyond the caruncula, the two canals join to empty into the lachrymal sac, which is the dilated upper extremity of the nasal duct. The duct is about half an inch (12.7 millimeters) in length and empties into the inferior meatus of the nose, taking a direction nearly vertical and inclined slightly outward and backward. This portion of the lachrymal apparatus is fibrous and is lined by a reddish mucous membrane that presents several well-marked folds. Near the puncta are two folds, one for each lachrymal canal. Another pair of folds exists near the horizontal portions of the canals. At the opening of the duct into the nose, is an overhanging fold of the nasal mucous membrane. These folds are supposed to prevent the reflux of liquid from the lachrymal canals and the entrance of air from the nose. The mucous membrane of the lachrymal canals is covered with flattened epithelium like that of the conjunctiva. The lachrymal sac and duct are lined with a continuation of the ciliated epithelium of the nose. The disposition of the apparatus just described is shown in Fig. 182.

The Tears. - The secretion of the lachrymal gland is constant, although its flow may be increased under various conditions. The actual quantity of the secretion has never been estimated. During sleep it is much diminished; and when the eyes are open, the quantity is sufficient to moisten the eyeball, the excess being carried into the nose

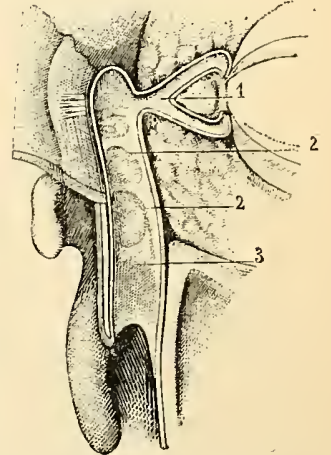

Fig. I82. - Lachrymal canals, lachrymal sac and nasal canal, opened by their anterior portion (Sappey).

I, walls of the lachrymal passages, smooth and adherent; 2,2 , walls of the lachrymal sac, presenting delicate folds of the mucous membrane; 3 , a similar fold belonging to the nasal mucous membrane. so gradually that this process is not appreciated. That this drainage of the excess of tears takes place, is shown by cases of obstruction of the nasal duct, when the liquid constantly overflows upon the cheeks, producing considerable inconvenience.

It is probable that the openings at the puncta lacrymalia take up the lachrymal secretion, like little pipettes; this action being aided by the movements in winking, by which, when the lids are closed, the points are compressed and turned backward, opening and drawing in the tears when the lids are opened. It is possible that the lachrymal sac is compressed in the act of winking, by the contractions of the muscle of Horner; and that this, while it empties the sac, may in the subsequent relaxation assist in the introduction of liquid from the orbit. 
Little is known in regard to the chemical composition of tears beyond an analysis made many years ago by Frerichs. According to this observer, the following is the composition of the lachrymal secretion :-

\section{COMPOSITION OF THE TEARS}

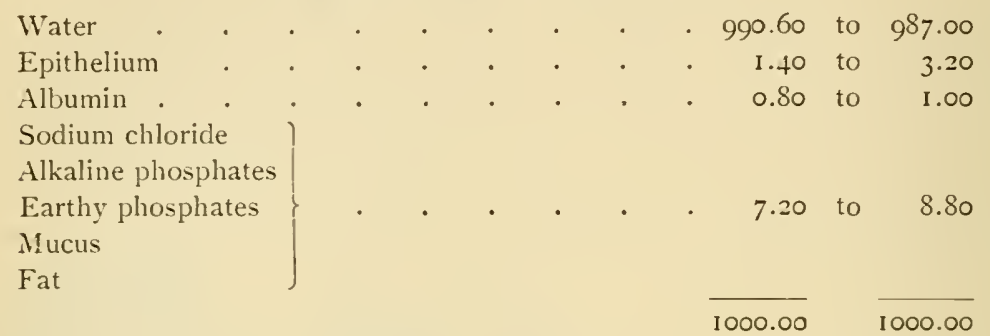

The specific gravity of the tears has not been ascertained. The liquid is clear, colorless, of a saltish taste and a feebly alkaline reaction. The albumin given in the table is called by some authors, lachrymin, thrænin or dacryolin. This substance, whatever it may be called, resembles mucus in many regards and probably is secreted by the conjunctiva and not by the lachrymal glands. Unlike ordinary mucus it is coagulated by water. It is supposed to be a globulin (Halliburton).

The secretion of tears is readily influenced through the nervous system. Aside from the increased flow of this secretion from emotional causes, which probably operate through the sympathetic, a hypersecretion almost immediately follows irritation of the mucous membrane of the conjunctiva or of the nose. The same result follows violent muscular effort, laughing, coughing, sneezing etc. The secretion of tears following stimulation of the mucous membrane is reflex. 


\title{
CHAPTER XXIX
}

\author{
AUDITION
}

Auditory (eighth nerve) - General properties of the auditory nerves - Topographical anatomy of the parts essential to the appreciation of sound - The external ear - General arrangement of parts composing the middle ear - General arrangement of the bony labyrinth - Physics of sound - Pitch of musical sounds - Musical scale - Quality of musical sounds - Harmonics, or overtones - Harmony - Discords and dissonance-Tones by influence Uses of different parts of the middle ear - Structure of the membrana tympani - Lses of the membrana tympani - Mechanism of the ossicles of the ear - Physiological anatomy of the internal ear - General arrangement of the membranous labyrinth - Liquids of the labyrinth - Distribution of the nerves in the labyrinth - Urgan of Corti — Uses of different parts of the internal ear - Uses of the semicircular canals - Uses of the parts contained in the cochlea - Centres for audition.

IMPRESSIONS of sound are conveyed to the brain by special nerves; but in order that these impressions shall reach these nerves so as to be properly appreciated, a complex accessory apparatus is required, the integrity of which is essential to normal audition. The study of the arrangement and action of these accessory parts is even more important and is far more intricate than the physiology of the auditory nerves. The auditory nerves conduct impressions of sound, as the optic nerves conduct impressions of light; but there is an elaborate arrangement of parts by which the waves are collected, conveyed to a membrane capable of vibration, and finally carried to the nerves by which the intensity and the varied qualities of sound are appreciated.

\section{Auditory (Eighth Nerve)}

The origin of the auditory nerve can easily be traced to the floor of the fourth ventricle, where it presents two roots. The external, or superficial root, sometimes called the posterior root, can be seen usually without preparation. It consists of five to seven grayish filaments, which decussate in the median line and pass outward, winding from the fourth ventricle around the restiform body. The deep root consists of a number of distinct filaments arising from the gray matter of the fourth ventricle, two or three of which pass to the median line to decussate with corresponding filaments from the opposite side. Filaments from this root have been traced to a gray nucleus in the inferior peduncle of the cerebellum and thence to the white substance of the cerebellum 
itself. The deep root passes around the restiform body inward, so that this portion of the bulb is encircled by the two roots. Passing from the superior and lateral portion of the bulb, the trunk of the nerve is applied to the superior and anterior surface of the facial. It then passes around the middle peduncle of the cerebellum and receives a process from the arachnoid membrane, which envelops it in a common sheath with the facial. It finally penetrates the internal auditory meatus. In its course it receives filaments from the restiform body and probably from the pons Varolii. Within the meatus the nerve divides into an anterior and a posterior branch, the anterior being distributed to the cochlea, and the posterior, to the vestibule and semicircular canals. The distribution of these branches will be clescribed in connection with the anatomy of the internal ear.

The auditory nerves are grayish in color and their consistence is soft, thus differing from the ordinary cerebro-spinal nerves and resembling in a certain degree the other nerves of special sense. On the external, or superficial root, is a small ganglioform enlargement, containing fusiform nerve-cells. The filaments of the trunk of the nerve consist of very large axis-cylinders, surrounded with a medullary sheath but having no tubular membrane. In the course of these fibres, are found small, nucleated, ganglionic enlargements.

General Properties of the Auditory Norves. - There can be no doubt, as regards the eighth, that it is the only nerve capable of receiving and conveying to the brain the special impressions produced by waves of sound; but it is an important question to determine whether this nerve be endowed also with general sensibility. Analogy with most of the other nerves of special sense would indicate that the auditory nerves are insensible to ordinary impressions; and this view has been sustained by direct experiments. In experiments made by passing electric currents through the ears, some physiologists have thought that auditory sensations were produced; but it is probable that the sensations observed were due to clonic spasm of the stapedius muscle and not to impressions of sound produced by the action of the stimulus on the auditory nerves. In cases of complete facial paralysis from otitis, in which paralysis of the auditory nerve could be excluded, it has not been possible to produce subjective auditory sensations, even by powerful faradization by means of a catheter passed through the Eustachian tube into the tympanic cavity or by the external meatus. In addition there are well-established clinical observations which sustain the theory of muscular contraction and are opposed to the idea of impressions of sound produced by direct stimulation of the auditory nerves. The results, then, as regards stimulation of the auditory nerves, have been negative. Were it practicable to 
subject the nerves to mechanical or electric stimulation in the human subject without involving other parts, it might be possible to arrive at a definite conclusion; but the difficulties in the way of such an experiment have thus far proved insurmountable.

Topographical Anatomy of the Parts essential to the AppreCiATION OF SOUND

Perfect audition involves the anatomical integrity of a complex apparatus, which, for convenience of anatomical description, may be divided into the external, middle and internal ear.

I. The external ear includes the pinna and the external auditory meatus and is bounded internally by the membrana tympani.

2. The middle ear includes the cavity of the tympanum, or drum, with its boundaries. The parts here to be described are the membrana tympani, the form of the tympanic cavity, its openings, its lining membrane, and the small bones of the ear, or ossicles, with their ligaments, muscles and nerves. The cavity of the tympanum communicates by the Eustachian tube with the pharynx and it also presents openings into the mastoid cells.

3. The internal ear contains the terminal filaments of the auditory nerve. It includes the vestibule, the three semicircular canals and the cochlea, which together form the labyrinth.

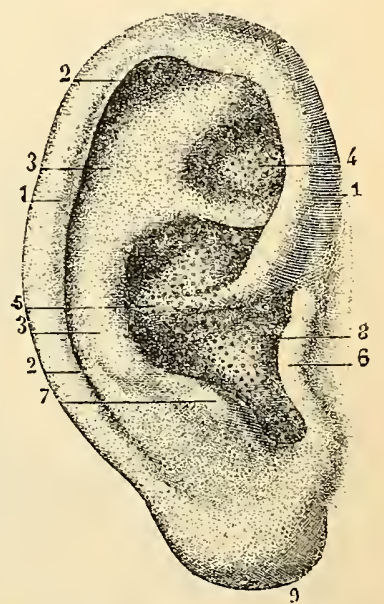

Fig. 183. - The pinna (Sappey).

I, I, helix ; 2, 2, fossa of the helix; 3,3 , antihelix; 4 , fossa of the antihelix; 5 , concha; 6 , tragus; 7 , antitragus; 8 , external auditory meatus; 9 , lobule.

The pinna and the external meatus simply conduct the waves of sound to the tympanum. The parts entering into the structure of the middle ear are accessory and are analogous in their uses to the refracting media of the eye. Structures contained in the labyrinth constitute the true sensory organ.

The External Ear. - The pinna, auricle, or pavilion is that portion projecting from the head, which first receives the waves of sound. The outer ridge of the pinna is called the helix. Just within this is a groove called the fossa of the helix. This fossa is bounded anteriorly by a prominent but shorter ridge, bifurcating above and anteriorly, called the antihelix; and above the concha, between the bifurcating arms of the antihelix and the anterior portion of the helix, is a shallow fossa, called the fossa of the antihelix. The deep fossa immediately sur- 
rounding the opening of the meatus is called the concha. A small lobe projects posteriorly, covering the anterior portion of the concha, which is called the tragus; and the projection at the lower extremity of the antihelix is called the antitragus. The fleshy dependent portion of the pinna is called the lobule.

The form of the pinna and its consistence depend on the presence of elastic cartilage, which occupies the entire external ear except the lobule. The structure of this kind of cartilage has already been described (see Plate X, Fig. 4).

The integunent covering the ear does not vary much from the integument of the general surface. It is thin, closely attached to the subjacent parts, and possesses small rudimentary hairs, with sudoripa-

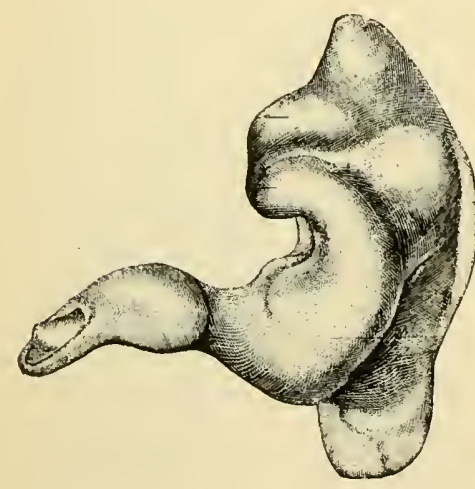

Fig. 184. - Posterior view of a mould in wax of the cavity of the concha and the external auditory meatus (Sappey). rous and sebaceous glands.

The muscles of the external ear are not important in the human subject; and excluding a few exceptional cases, they are not uncler the control of the will. The extrinsic muscles are the superior, or attollens, the anterior, or attrahens, and the posterior, or retrahens aurem. In addition there are the six small intrinsic muscles, situated between the ridges upon the cartilaginous surface. The pinna is attached to the sides of the head by two distinct ligaments and a few delicate ligamentous fibres.

The external auditory meatus is about an inch and a quarter (3r.8 millimeters) in length and extends from the concha to the membrana tympani. Its course is somewhat tortuous. Passing from without inward, its direction is at first somewhat upward, turning abruptly over a bony prominence near the middle, from which it has a slightly downward direction to the membrana tympani. Its general course is from without inward and slightly forward. The inner termination of the canal is the membrana tympani, which is quite oblique, the upper portion being inclined outward, so that the inferior wall of the meatus is considerably longer than the superior.

The walls of the external meatus are partly cartilaginous and fibrous, and partly bony. The cartilaginous and fibrous portion occupies a little less than one-half of the entire length and consists of a continuation of the cartilage of the pinna, with fibrous tissue. The lower twothirds of this portion of the canal is cartilaginous and the upper third 
is fibrous. The rest of the tube is osseous and is a little longer and narrower than the cartilaginous portion. Around the inner extremity of the canal, except at its superior portion, is a narrow groove which receives the greater portion of the margin of the membrana tympani.

The skin of the external meatus is continuous with the integument covering the pinna. It is very delicate, becoming thinner from without inward. In the osseous portion it adheres closely to the periosteum, and at the bottom of the canal it is reflected over the membrana tympani, forming its outer layer. In the cartilaginous and fibrous portion are short stiff hairs, with sebaceous glands attached to their follicles, and the coiled tubes known as the ceruminous glands. The structure of these glands and the properties and composition of the cerumen have already been described in connection with the physiology of the glands of the skin.

General Arrangement of the Parts composing the Middle Ear.A minute and purely anatomical description of the middle ear would be out of place in this work, where it is desired to give only such an account of the anatomy as will enable the student to comprehend the physiology of the ear, reserving for special description certain of the most important structures. It will be useful, however, to give a general outline of the different parts, with their names.

The middle ear presents a 11arrow cavity of irregular shape, situated between the external ear and the labyrinth, in the petrous portion of the temporal bone. The general arrangement of its parts is shown in Fig. I85. The outer wall of the tympanic cavity is formed by the membrana tympani (6, Fig. I 85$)$. This membrane is concave, its concavity looking outward, and oblique, inclining usually at an angle of forty-five degrees with the perpendicular. This angle, however, varies considerably in different individuals. The roof is formed by a thin plate of bone. The floor is bony and is much narrower than the roof. The inner wall, separating the tympanic cavity from the labyrinth, is irregular, presenting several small elevations and foramina. The fenestra ovalis, an ovoid opening near its upper portion, leads to the cavity of the vestibule. This is closed in the natural state by the base of the stapes and its annular ligament. Below is a smaller opening, the fenestra rotunda, which leads to the cochlea. This is closed in the natural state by a membrane called the secondary membrana tympani. The principal portion of the tympanic cavity is sometimes called the atrium, although this name is applied to other parts not connected with the ear. From the general tympanic cavity is a prolongation, extending upward and backward, in which are lodged the head of the malleus and a great part of the incus. This is called the epitympanic recess, or attic. It 
communicates with the mastoid antrum, into which open little canals leading to the mastoid cells. The tympanum also presents an opening leading to the Eustachian tube and a small foramen that gives passage to the tendon of the stapedius muscle. The Eustachian tube extends. from the upper part of the pharynx to the tympanum.

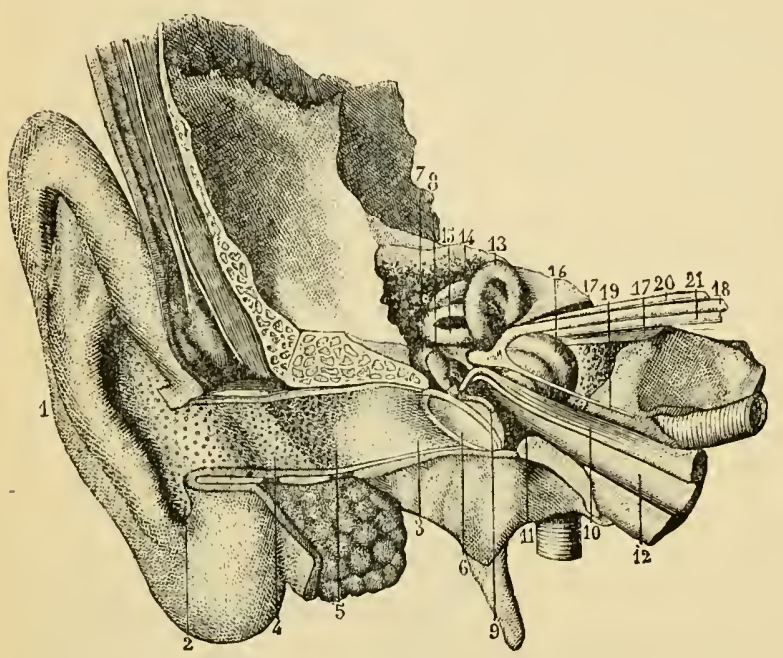

Fig. 185. - General view of the organ of hearing (Sappey).

I, pinna; 2 , cavity of the concha, on the walls of which are seen the orifices of a great number of sebaceous glands; 3 , external auditory meatus; 4 , angular projection formed by the union of the anterior portion of the concha with the posterior wall of the auditory canal; 5, openings of the ceruminous glands, the most internal of which form a curved line which corresponds with the beginning of the osseous portion of the external meatus; 6 , membrana tympani and the elastic fibrous membrane which forms its border; 7 , anterior portion of the incus; 8 , malleus; 9 , handle of the malleus, applied to the internal surface of the membrana tympani, which it draws inward toward the projection of the promontory; ro, tensor-tympani muscle, the tendon of which is reflected at a right angle, to become attached to the superior portion of the handle of the malleus; $\mathbf{I I}$, tympanic cavity; 12, Eustachian tube, the internal or pharyngeal extremity of which has been removed by a section perpendicular to its curve; I3, superior semicircular canal; I4 $_{4}$ posterior semicircular canal; 15, external semicircular canal; 16, cochlea; 17 , internal auditory canal; 18 , facial nerve; 19 , large petrosal branch, given off from the ganglioform enlargement of the facial and passing below the cochlea, to go to its distribution; 20 , vestilsular branch of the auditory nerve; 21 , cochlear branch of the auditory nerve.
The small bones of the ear are three in number - the malleus, the incus and the stapes, - forming a chain and connected together by ligaments (Fig. I 86). These bones are situated in the upper part of the tympanum. The handle of the malleus (II, 3, Fig. I86) is closely attached to the membrana tympani, and the long process (II, 2, Fig. I86) is attached to the Glasserian fissure of the temporal bone. The malleus is articulated with the incus. The incus ( $I, 2$, Fig. I 86) is connected with the posterior wall of the tympanum, near the openings of the mastoid cells. It is articulated with the malleus, and by the extremity of its long process, with the stapes. The stapes (I, 3, Fig. I86) is the most internal bone of the middle ear. It is articulated by its smaller extremity with the long process of the incus. Its base is oval and, with its annular ligament, is applied to the fenestra ovalis. The direction of the stapes is nearly at a right angle with the long process of the incus, in the natural state. Some anatomists describe a fourth bone as existing be- 
tween the long process of the incus and the stapes; but this is seldom distinct, usually being united either with the incus or with the stapes.

There are two well-defined muscles connected with the ossicles of the middle ear. One of these is attached to the malleus, and the other, to the stapes. The so-called laxator tympani probably is not composed of muscular fibres and should not be enumerated with the muscles of the tympanum.

The larger of the two muscles is the tensor tympani. Its fibres arise from the cartilaginous portion of the Eustachian tube, the spinous process of the sphenoid bone and the adjacent portion of the temporal. From this origin it passes backward, almost horizontally, to the tympanic cavity. In front of the fenestra ovalis it turns at nearly a right

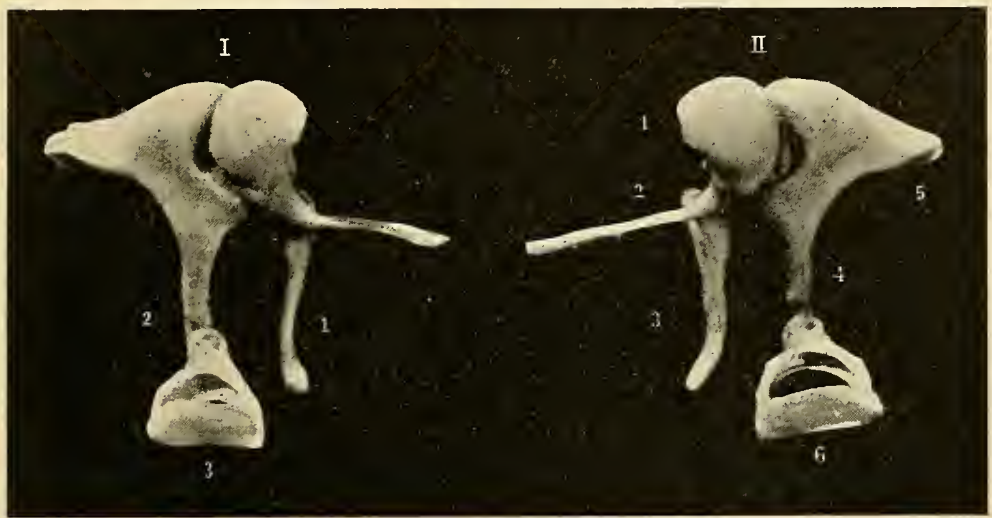

Fig. 186. - Ossicles of the tympanum, $\times 4$ (modified from Rüdinger).

I, ossicles of the left ear; I, malleus; 2 , incus; 3 , stapes. II, ossicles of the right ear; I, malleus; 2 , long process; 3 , handle; 4 , long process of the incus; 5 , short process of the incus; 6 , stapes.

angle over a bony process, and its tendon is inserted into the handle of the malleus at its inner surface near the root. The tendon is very delicate, and the muscular portion is about half an inch (I 2.7 millimeters) in length (IO, Fig. I 85). The muscle and its tendon are enclosed in a distinct fibrous sheath. The action of this muscle is to draw the handle of the malleus inward, pressing the base of the stapes against the membrane of the fenestra ovalis and producing tension of the membrana tympani. The fibres of this, and of all the muscles of the middle ear, are of the striated variety. The tensor tympani is supplied with motor filaments from the otic ganglion, which probably are derived from the facial nerve.

The stapedius muscle is situated in the descending portion of the acquæductus Fallopii and in the cavity of the pyramid on the posterior wall of the tympanic cavity. Its tendon emerges from a foramen at 
the summit of the pyramid. In the canal in which this muscle is lodged, its direction is vertical. At the summit of the pyramid it turns at nearly a right angle, its tendon passing horizontally forward to be attached to the head of the stapes. Like the other muscles of the ear, this is enveloped in a fibrous sheath. Its action is to draw the head of the stapes backward, relaxing the membrana tympani. This muscle receives filaments from the facial nerve, by a distinct branch, the tympanic.

The posterior wall of the tympanic cavity presents foramina that open directly into a number of irregularly-shaped cavities (mastoid

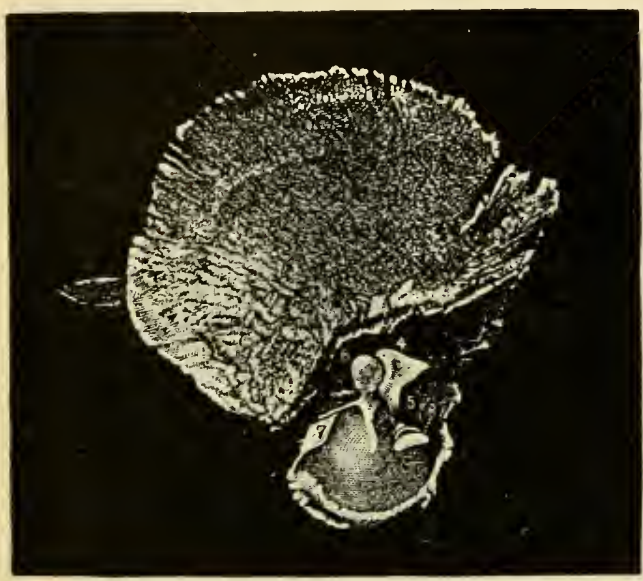

Fig. 187. - The right temporal bone, the fetrous portion removed, showing the ossicles seen from within - reduced about one-half. From a photograph (Rüdinger).

4 , the incus, the short process of which is directed nearly in a horizontal direction backward; 5 , the long process of the incus, free in the tympanic cavity, articulated with the stapes; 6 , the malleus, articulated with the incus: 7 , the long process of the malleus, in the Glasserian fissure; 8 , the stapes, articulated with the incus. This is drawn somewhat outward; otherwise the base of the stapes alone would be visible. This figure shows the handle of the malleus attached to the membrana tympani.

and from this constricted portion it gradually dilates to its opening into the pharynx, the entire canal presenting the appearance of two cones. The osseous portion extends from the tympanum to the spine of the sphenoid bone. The cartilaginous portion is an irregularly-triangular cartilage, bent on itself above, forming a furrow, with its concavity presenting downward and outward. The fibrous portion occupies about one-half of the tube, beyond the osseous portion, and completes the canal, forming its inferior and external portion. In its structure the 
cartilage of the Eustachian tube is intermediate between hyaline and elastic cartilage.

The circumflexus, or tensor palati muscle, which has already been described in connection with deglutition, is attached to the anterior margin, or the hook of the cartilage. The attachments of this muscle have been accurately described by Rüdinger, who called it the dilator of the tube.

The action of certain of the muscles of deglutition dilates the pharyngeal opening of the Eustachian tube. If the mouth and nostrils are closed and several repeated acts of deglutition are made, air is drawn from the tympanic cavity, and the atmospheric pressure renders the membrane of the tympanum tense, increasing its concavity. By one or two lateral movements of the jaws, the tube is opened, the pressure of air is equalized and the ear returns to its former condition. The nerves animating the dilator tubæcome from the pneumogastric and are derived originally from the spinal accessory.

A smooth mucous membrane forms a continuous lining for the Eustachian tube, the cavity of the tympanum and the mastoid cells. In all parts it is closely adherent to the subjacent tissues, and in the cavity of the tympanum it is very thin. In the cartilaginous portion of the Eustachian tube there are mucous glands, which are most abundant near the pharyngeal orifice, and gradually diminish in number toward the osseous portion, in which there are no glands. Throughout the tube the surface of the mucous membrane is covered with conoidal cells of ciliated epithelium. The mucous membrane of the tympanic cavity is very thin, consisting of little more than epithelium and a layer of connective tissue. It lines the walls of the cavity and the inner surface of the membrana tympani, is prolonged into the mastoid cells and covers the ossicles and those portions of the muscles and tendons which pass through the tympanum. On the floor of the tympanic cavity and on its anterior, inner and posterior walls, the epithelium is of the conoidal ciliated variety. On the promontory, roof, ossicles and muscles, the cells are of the pavement-variety and not ciliated, the transition from one form to the other being gradual. The entire mucous membrane contains lymphatics, a plexus of nerve-fibres and nerve-cells, with some peculiar cells, the physiology of which is not understood.

The above is merely a general sketch of the physiological anatomy of the middle ear, and it will not be necessary to treat more fully of the cavity of the tympanum, the mastoid cells or the Eustachian tube, except as regards certain points in their physiology. The minute anatomy of the membrana tympani and the articulations of the ossicles can be more conveniently considered in connection with the physiology of these parts. 
General Arrangement of the Bony Labyrinth. - The internal portion of the auditory apparatus is contained in the petrous portion of the temporal bone. It consists of an irregular cavity, called the vestibule, the three semicircular canals (I3, I4, I5, Fig. I85) and the cochlea (16, Fig. I 85). The general arrangement of these parts in situ and their relations to the adjacent structures are shown in Fig. 185. Figure 188 , showing the bony labyrinth isolated, is from a photograph in

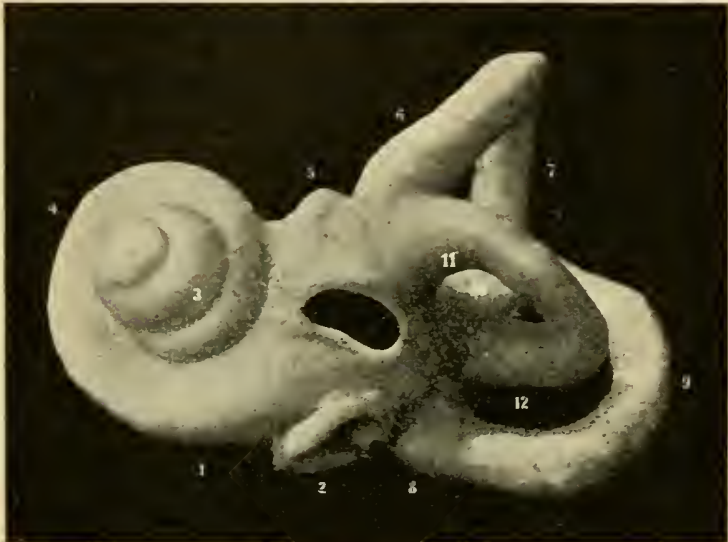

Fig. I88. - The left bony labyrinth of a newborn child, forward and outward view, $x+$. From a photograph, and slightly reduced (Rüdinger).

$I$, the wide canal, the beginning of the spiral canal of the cochlea; 2 , the fenestra rotunda; 3 , the second turn of the cochlea; 4 , the final half-turn of the cochlea; 5 , the border of the bony wall of the vestibule, situated between the cochlea and the semicircular canals; 6 , the superior, or sagital semicircular canal; 7 , the portion of the semicircular canal bent outward; 8 , the posterior, or transverse semicircular canal; 9 , the portion of the posterior connected with the superior semicircular canal; ro, point of junction of the superior and the posterior semicircular canals ; II, the ampulla ossea externa; I2, the horizontal, or external semicircular canal. The explanation of this figure has been modified and condensed from Rüdinger.
Rüdinger's atlas.

The vestibule is the central chamber of the labyrinth, communicating with the tympanic cavity by the fenestra ovalis, which is closed in the natural state by the base of the stapes. This is the central ovoid opening shown in Fig. 188. The inner wall of the vestibule presents a round depression, called the fovea hemispherica, perforated by a number of small foramina through which pass nervous filaments from the internal auditory meatus. Behind this depression is the opening of the aqueduct of the vestibule. In the posterior wall of the vestibule are five small round openings leading to the semicircular canals, with a larger opening below leading to the cochlea.

The general arrangement of the semicircular canals is shown in Fig. I $88(6,7,8,9$, I0, I I, I 2$)$.

The arrangement of the cochlea - the anterior division of the labyrinth - is shown in Fig. $188(1,3,4)$. This is a spiral canal, about an inch and a half (38.1 millimeters) long, and one-tenth of an inch (2.5 millimeters) wide at its beginning, gradually tapering to the apex, and making, in its course, two and a half turns. Its interior presents a central pillar around which winds a spiral lamina of bone. The fenestra 
rotunda (2, Fig. 188 ), closed in the natural state by a membrane, - the secondary membrana tympani, - lies between the lower portion of the cochlea and the cavity of the tympanum.

What is called the membranous labyrinth is contained within the bony parts just described. Some of the anatomical points connected with its structure and the distribution and connections of the auditory nerve have direct and important relations to the physiology of hearing, while many are of purely anatomical interest. Such facts as bear directly on physiology will be considered in connection with the uses of the internal ear.

\section{Physics of Sound}

Most of the points in acoustics that are essential to the comprehension of the physiology of audition are definitely settled. The theories of the propagation of sound involve wave-action, concerning which there is no question. For the conduction of sound a ponderable medium is essential; and it is not necessary, as in the case of the undulatory theory of light, to assume the existence of an imponderable ether. The human ear, though perhaps not so acute as the auditory apparatus of some of the inferior animals, not only appreciates irregular waves, such as produce noise as distinguished from sounds called musical, but is capable of distinguishing regular waves, as in simple musical sounds and harmonious combinations.

In music certain successions of regular sounds are agreeable to the ear and constitute what is called melody. Again, there is appreciation, not only of the intensity of sounds, both noisy and musical, but of pitch and different qualities, particularly in music. Still further, musical notes may be resolved into certain invariable component parts, such as the octave, the third, fifth etc. These components of what were formerly supposed to be simple sounds - which may be isolated by artificial means, to be described farther on - are called tones; while the sounds themselves, produced by the union of the different tones, are called notes, which may themselves be combined to form chords.

The quality of musical sounds may be modified by the simultaneous production of others which correspond to certain of the components of the predominating note. For example, if there is added to a single note, the third, fifth and octave, the result is a major chord, the sound of which is different from that of a single note or of a note with its octave. If the third is diminished by a semitone, there is a different quality, which is peculiar to minor chords. In this way a great variety of musical sounds may be made on a single instrument, as the piano; and by the harmonious combinations of the notes of different instru- 
ments and of different registers of the human voice, as in choral and orchestral compositions, shades of effect, almost innumerable, may be produced. The modification of sounds in this way constitutes harmony; and an educated ear not only experiences pleasure from these musical combinations, but can distinguish their different component parts.

A chord may convey to the ear the sensation of completeness in itself or it may lead to a succession of notes before this sense of completeness is attained. Different chords in the same key may be made to follow each other, or by transition-notes, may pass to the chords of other keys. Each key has its fundamental note; and the transition from one key to another, in order to be agreeable to the ear, must be made in certain ways. These regular transitions constitute modulation. The ear becomes fatigued by long successions of notes or chords always in one key, and modulation is essential to the enjoyment of elaborate musical compositions; otherwise the notes would not only become monotonous, but their correct appreciation would be impaired, as the appreciation of colors becomes less distinct after looking for a long time at an object presenting a single vivid tint.

Law's of Sonorous Vibrations. - Sound is produced by vibrations in a ponderable medium; and the sounds ordinarily heard are transmitted to the ear by means of vibrations of the atmosphere. A simple and very common illustration of this fact is afforded by the experiment of striking a bell carefully arranged in iacuo. Although the stroke and the vibration can readily be seen, there is no sound; and if air is gradually introduced, the sound will become appreciable and progressively more intense as the surrounding medium is increased in density. The oscillations of sound are to and fro in the direction of the line of conduction and are said to be longitudinal. In the undulatory theory of light, the vibrations are supposed to be at right angles to the line of propagation, or transverse. A complete oscillation to and fro is called a sound-wave.

It is evident that vibrating bodies may be made to perform and impart to the atmosphere oscillations of greater or less amplitude. The intensity of sound is in proportion to the amplitude of the vibrations. In a vibrating body capable of producing a definite number of waves of sound in a second, it is evident that the greater the amplitude of the wave, the greater is the velocity of the particles thrown into vibration. It has been ascertained that there is an invariable mathematical relation between the intensity of sound, the velocity of the conducting particles and the amplitude of the waves; and this is expressed by the formula, that the intensity is proportional to the square of the amplitude. It is evident, also, that the intensity of sound is diminished by distance. 
The sound, as the waves recede from the sonorous body, becomes distributed over an increased area. The propagation of sound has been reduced also to the formula, that the intensity diminishes in proportion to the square of the distance.

Sonorous vibrations are subject to many of the laws of reflection of light. Sound may be absorbed by soft and non-vibrating surfaces in the same way that certain surfaces absorb the rays of light. By carefully arranged convex surfaces, the waves of sound may readily be collected to a focus. These laws of the reflection of sonorous waves explain echoes and the conduction of sound by confined strata of air, as in tubes. To extend the parallel between sonorous and luminous transmission, it has been ascertained that the waves of sound may be refracted to a focus by being made to pass through an acoustic lens, as a balloon filled with carbon dioxide. The waves of sound also may be deflected around solid bodies, when they produce what have been called shadows of sound.

Observing the sound produced by the blow of an axe, it is seen that sound is transmitted with much less rapidity than light. At a short distance the view of the blow is practically instantaneous; but there is a considerable interval between this and the sound. This interval represents the velocity of sonorous conduction. This fact is also illustrated by the interval between a flash of lightning and the sound of thunder. The velocity of sound depends on the density and elasticity of the conducting medium. The rate of conduction of sound by atmospheric air at the freezing-point of water is about I I 8 feet ( 340 meters) per second. This rate presents comparatively slight variations for the different gases, but it is much more rapid in liquids and in solids.

Noise and Musical Sounds. - There is a well-defined physical as well as an æsthetic distinction between noise and music. Taking as examples, single sounds, a sound becomes noise when the air is thrown into confused and irregular vibrations. A noise may be composed of musical sounds when these are not in accord with each other, and sounds called musical are not always free from discordant vibrations. A noise possesses intensity, varying with the amplitude of the vibrations, and it may have different qualities depending on the form of its vibrations. A noise may be dull, sharp, ringing, metallic, hollow etc., these terms expressing qualities that are readily understood. A noise also may be called sharp or low in pitch, as the rapid or slow vibrations predominate, without answering the requirements of musical sounds.

A musical sound consists of vibrations following each other at regular intervals, provided that the succession of waves be not too slow or too rapid. When the vibrations are too slow, there is an appreciable succession of impulses and the sound is not musical. When they are too 
rapid, the sound is excessively sharp, but it is painfully acute and has no pitch that can be accurately determined by the auditory apparatus. Such sounds may be occasionally employed in musical compositions, but in themselves they are not strictly musical.

Musical sounds have the characters of duration, intensity, pitch and quality. Duration depends on the length of time during which the vibrating body continues in action. Intensity depends on the amplitude of the vibrations and has no relation to pitch. Pitch depends on the rapidity of the regular vibrations. Quality depends on combinations of different notes in harmony, the character of the harmonics of fundamental tones and the form of the vibrations.

Pitch of Husical Sonnds. - Pitch depends on the number of vibrations. A musical sound may be of greater or less intensity; it may at first be quite loud and gradually die away; but the number of vibrations in a definite note is invariable, be it weak or powerful. The rapidity of the conduction of sound does not vary with its intensity or pitch; and in the harmonious combination of the sounds of different instruments, be they high or low in pitch, intense or feeble, it is always the same in the same conducting medium. Distinct musical notes may present a great variety of qualities, but all notes of the same pitch have equal rates of vibration. Notes equal in pitch are said to be in unison. An educated ear can distinguish slight differences in pitch in ordinary musical notes; but this power of appreciation of pitch is restricted within well-defined limits that vary slightly in different individuals. According to Helmholtz, the range of sounds that may be legitimately employed in music is between 40 and 4000 vibrations in a second, embracing about seven octaves. In an orchestra the double bass gives the lowest note, which has 40.25 vibrations in a second, and the highest note, given by the small flute, has 4752 vibrations. In grand organs there is a pipe that gives a note of 16.5 vibrations, and the deepest note of modern pianos has 27.5 vibrations; but delicate shades of pitch in these low notes are not appreciable to most persons. Sounds above the limits just indicated are audible but are painfully sharp, and their pitch can not be exactly appreciated by the ear.

The limits of appreciation of musical sounds do not apply to ordinary audition; and the extreme range of hearing is much greater, being about eleven octaves. Shrill sounds, that can not be recognized as musical, still are audible and the number of their vibrations may be measured mechanically. "Galton's whistle" produces sounds that have between 30,000 and 40,000 vibrations in a second; but beyond these limits the vibrations are inaudible. It is possible, however, that vibrations inaudible to the human subject may be heard by some of the lower animals. 
Musical Scale. - A knowledge of the relations of different notes to each other lies at the foundation of the science of music; and without a clear idea of certain of the fundamental laws of music, it is impossible to comprehend thoroughly the mechanism of audition.

It requires but little cultivation of the ear to enable one to comprehend the fact that the successions and combinations of notes must obey certain laws; and long before these laws were subjects of mathematical demonstration, the relations of the different notes of the scale were established, merely because certain successions and combinations were agreeable to the ear, while others were discordant and apparently unnatural.

The most convenient sounds for study are those produced by vibrating strings, and the phenomena here observed are essentially the same for all musical sounds; for it is by means of vibrations communicated to the air that the waves of sound find their way to the auditory apparatus. Take, to begin with, a string vibrating 48 times in a second. If this string is divided into two equal parts, each part will vibrate 96 times in a second. The note thus produced is the octave, or the 8 th of the primary note, called the 8 th, because the natural scale contains eight notes, of which the first is the lowest, and the last, the highest. The half may be divided again, producing a second octave, and so on, within the limits of appreciation of musical sounds. If the string is divided so that $\frac{2}{3}$ of its length will vibrate, there are 72 vibrations in a second, and this note is the 5 th in the scale. If the string is divided again, so as to leave $\frac{4}{5}$ of its length, there are 60 vibrations, which give the $3 \mathrm{~d}$ note in the scale. These are the most prominent subdivisions of the note; and the Ist, $3 \mathrm{~d}$, 5 th and 8th, when sounded together, make what is known as the common major chord. Three-fourths of the length of the original string make 64 vibrations, and give the $4^{\text {th }}$ note in the scale. With $\frac{8}{9}$ of the string, there are 54 vibrations, and the note is the $2 \mathrm{~d}$ in the scale. With $\frac{3}{5}$ of the string, there are 80 vibrations, or the 6 th note in the scale. With $\frac{8}{15}$ of the string, there are 90 vibrations, or the 7 th note in the scale. The original note, which may be called $\mathrm{C}$, is the key-note, or the tonic. In this scale, which is called the natural, or diatonic, there is a regular mathematical progression from the ist to the 8 th. This is called the major key. Melody consists in an agreeable succession of notes, which may be assumed, for the sake of simplicity, to be pure. In a simple melody every note must be one of those in the scale. When a different note is sounded, the melody passes into a key that has a different fundamental note, or tonic, with a different succession of $3 \mathrm{ds}$, 5 ths etc. Every key, therefore, has its Ist, $3 \mathrm{~d}$, 5 th and 8 th, as well as the intermediate notes. If a note formed by a string $\frac{5}{6}$ the length of the tonic instead of $\frac{4}{5}$, is substituted for the major $3 \mathrm{~d}$, the key is converted into the minor. The minor 
chord, consisting of the Ist, the minor $3 \mathrm{~d}$, the 5 th and the 8 th, is harmonious, but it has a quality quite different from that of the major chord. The notes of a melody may progress in the minor key as well as in the major. Taking the small numbers of vibrations merely for convenience, the following is the mode of progression in the natural scale, which may be assumed to be the scale of $\mathrm{C}$ major:-

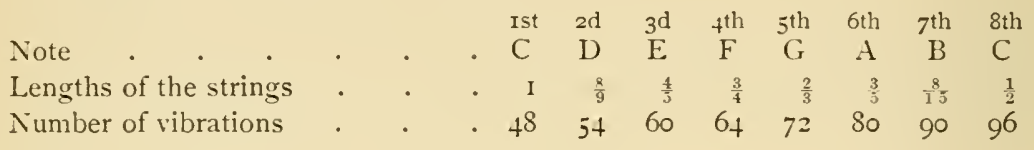

The intervals between the notes of the scale, it is seen, are not equal. The smallest, between the $3 \mathrm{~d}$ and 4 th and the 7 th and 8 th, are called semitones. The other intervals are either full perfect tones or small perfect tones. Although there are semitones, not belonging to the key of $C$, between $C$ and $D, D$ and $E, F$ and $G, G$ and $A$, and $A$ and $B$, these intervals are not all composed of exactly the same number of vibrations; so that, taking the notes on a piano, with $\mathrm{D}$ as the tonic, the 5 th would be $A$. It is assumed that $D$ has 54 vibrations, and $A$, 80 , giving a difference of 26 . With $\mathrm{C}$ as the tonic and $\mathrm{G}$ as the fifth, there is a difference of 24 . It is on account of these differences in the intervals, that each key in music has a more or less peculiar and distinctive character.

Even in melody, and still more in harmony, in long compositions, the ear becomes fatigued by a single key, and it is necessary in order to produce the most pleasing effects to change the tonic, by what is called modulation, returning afterward to the original key.

Quality of Musical Sounds. - Nearly all musical sounds, which seem at first to be simple, can be resolved into certain well-defined constituents; but with the exception of the notes of great stopped pipes in the organ, there are few absolutely simple sounds used in music. These simple sounds are pure, but are of unsatisfactory quality and wanting in richness. Almost all other musical sounds have a fundamental tone which is at once recognized; but this tone is accompanied by harmonics caused by secondary vibrations of subdivisions of the sonorous body. The number, pitch and intensity of these harmonic, or aliquot vibrations affect what is called the quality, or timbre of musical notes, by modifying the form of the sonorous waves. A string vibrating a certain number of times in a second, if the vibrations were absolutely simple, would produce, according to the laws of vibrating bodies, a simple musical tone; but as the string subdivides itself into different portions, one of which gives the $3 \mathrm{~d}$, another, the 5 th, and so on, of the fundamental, it is evident that the form of the vibrations must be considerably modi- 
fied, and with these modifications in form, the quality, or timbre of the note is changed.

From what has just been stated, it follows that nearly all musical notes consist, not only of a fundamental sound, but of harmonic vibrations subordinate to the fundamental and qualifying it in a particular way. These harmonics may be feeble or intense; certain of them may predominate over others; some that usually are present may be eliminated; and, in short, there may be a great diversity in their arrangement, and thus the timbre may present an infinite variety. This is one of the elements entering into the composition of notes, and it affords a partial explanation of quality.

Another element in the quality of notes depends on their reënforcement by resonance. The vibrations of a stretched string not connected with a resonant body are almost inaudible. In musical instruments the sound is taken up by some mechanical arrangement, as the soundboard of the organ, piano, violin, harp or guitar. In the violin, for example, the sweetness of the notes depends mainly on the construction of the resonant part of the instrument and but little on the strings themselves, which latter are frequently changed; and the same is in a measure true of the human voice.

In addition to the harmonic tones of sonorous bodies, various discordant sounds usually are present, which modify the timbre, producing a certain roughness, such as the grating of a violin-bow, the friction of the columns of air against the angles in wind-instruments, etc. All these conditions have their effect on the quality of tones; and these discordant sounds may exist in infinite number and variety. These sounds are composed of irregular vibrations and consequently are inharmonious. Nearly all notes that are spoken of in general terms as musical are composed of musical, or harmonic aliquot tones, with the discordant elements to which allusion has just been made.

Aside from the relations of the various component parts of musicai notes, the quality depends largely on the form of the vibrations. To quote the words of Helmholtz, "the more uniformly rounded the form of the wave, the softer and milder is the quality of the sound. The more jerking and angular the wave-form, the more piercing the quality. Tuning-forks, with their rounded form of waves, have a remarkably soft quality; and the qualities of sound produced by the zither and violin resemble in harshness the angularity of their wave-forms."

Hamonics, or Overtones. - As before stated, nearly all sounds are composite; but some contain many more aliquot, or secondary vibrations than others. The notes of vibrating strings are peculiarly rich in harmonics; and these may be used for illustration, remembering that the 
phenomena here observed have their analogies in nearly all varieties of musical sounds. If a stretched string is made to vibrate, the secondary tones, which qualify the fundamental, are called harmonics, or overtones.

While it is difficult at all times to distinguish by the ear the individual overtones of vibrating strings, their existence can be demonstrated by certain simple experiments. Take, for example, a string, the fundamental tone of which is C. If this string is damped with a feather at one-fourth of its length and a violin-bow is drawn across the shorter section, not only the fourth part of the string across which the bow is drawn is made to vibrate, but the remaining three-fourths; and if little riders of paper are placed on the longer section at distances equal to one-fourth of the entire string, they will remain undisturbed, while riders placed at any other points on the string will be thrown off. This experiment shows that the three-fourths of the string have been divided. This may be illustrated by connecting one end of the string with a tuning-fork. When this is done and the string is brought to the proper degree of tension, it will first vibrate as a whole, then, when a little tighter, will spontaneously divide into two equal parts, and under increased tension, into three, four and so on. By damping a string with the light touch of a feather, it is possible to suppress the fundamental tone and bring out the overtones, which exist in all vibrating strings but usually are overpowered by the fundamental. The points that mark the subdivisions of the string into segments of secondary vibrations are called nodes. When the string is damped at its centre, the fundamental tone is quenched and there are overtones an octave above; damping it at a distance of one-fourth, there is the second octave above, and so on. When the string is damped at a distance of one-fifth from the end, the four-fifths sound the $3 \mathrm{~d}$ of the fundamental, with the second octave of the $3 \mathrm{~d}$. If it is damped at a distance of two-thirds, there is the 5 th of the fundamental, with the octave of the 5th. Every vibrating string thus possesses a fundamental tone and overtones. Qualifying the fundamental, there is first, as the most simple, a series of octaves; next, a series of 5 ths of the fundamental and their octaves; and next, a series of 3 ds. These are the most powerful overtones, and they form the common chord of the fundamental; but they are so far concealed by the greater intensity of the fundamental that they can not easily be distinguished by the unaided ear, unless the fundamental has been quenched in some way. In the same way the harmonic 5 ths and $3 \mathrm{ds}$ overpower other overtones; for the string is subdivided again and again into overtones, which are not harmonious like the notes of the common chord of the fundamental.

The presence of overtones, resultant tones and additional tones, 
which latter will be described hereafter, can be demonstrated, without damping the strings, by resonators. It is well known that if a glass tube, closed at one end, which contains a column of air of a certain length, is brought near a resounding body emitting a note identical with that produced by the vibrations of the column of air, the air in the tube will resound in consonance with the note, while no other note will have this effect. The resonators of Helmholtz are constructed on this principle. A glass globe or tube (Fig. I 89) is constructed so as to produce a certain note. This has a larger opening (a) and a smaller opening (b), which latter is fitted in the ear with warm sealing-wax, the other ear being closed. When the proper note is sounded, it is reënforced by the resonator and is greatly increased in intensity, while all other notes are heard very faintly. By using resonators graduated to the musical scale, it is easy to analyze a note and distinguish its overtones. The resonators of Helmholtz that are open at the larger extremity are more delicate than those in which this is closed with a membrane.

A striking and instructive point in the present discussion is the following: All the overtones are produced by vibrations of divisions of the string in-
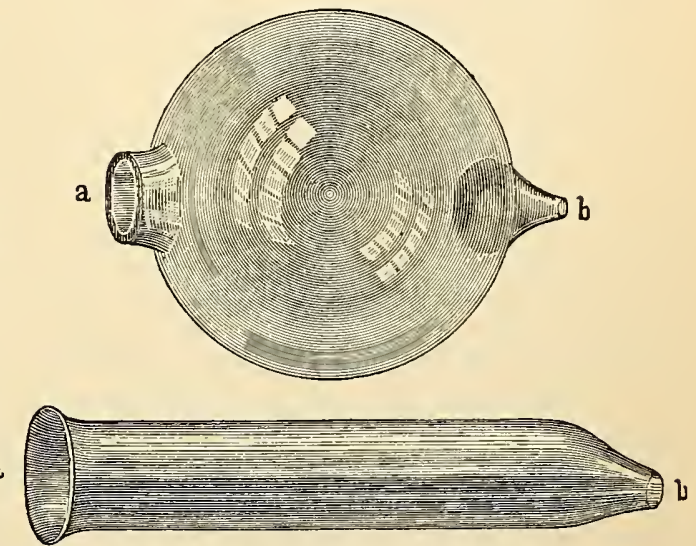

Fig. 189. - Resonators of Helmholtz. cluded between the comparatively still points, called nodes; and if a string is thrown into vibration by plucking or striking it at one of these nodal points, the overtones which vibrate from this node at a fixed point are abolished. It is readily understood that when a string is plucked at any point, it will vibrate so vigorously at this point that no node can be formed. This fact has long been recognized by practical musicians, although many probably are unacquainted with its explanation. Performers on stringed instruments habitually attack the strings near one of their extremities. In the piano, where the strings may be struck at almost any point, the hammers are placed at a distance of $\frac{1}{9}$ to $\frac{1}{7}$ of the length of the strings from their extremities; and it has been ascertained by experience that this gives the richest notes. The nodes formed at these points would produce the 7 ths and 9 ths as overtones, which do not belong to the perfect major chord, while the nodes for the harmonious overtones are undisturbed. The reason, then, why the 
notes are richer when the strings are attacked at this point, is that the harmonious overtones are full and perfect, and certain of the discordant overtones are suppressed.

When two harmonious notes are produced under favorable conditions, one can hear, in addition to the two sounds, a sound differing from both and much lower than the lower of the two. This sound is too low for an harmonic, and it has been called a resultant tone. The formation of a new sound by combining two sounds of different pitch is analogous to the blending of colors in optics, except that the primary sounds are not lost. The laws of the production of these resultant sounds are very simple. When two notes in harmony are sounded, the resultant tone is equal to the difference between the two primaries. For example, C, with 48 vibrations, and its 5 th, with 72 vibrations in a second, give a resultant tone equal to the difference, which is 24 vibrations, and it is consequently the octave below C. These resultant tones are feeble as compared with the primary tones, and they can be heard under only the most favorable experimental conditions. In addition to these sounds, Helmholtz has discovered sounds, even more feeble, which he calls additional, or summation-tones. The value of these is equal to the sum of vibrations of the primary tones. For example, C (48) and its 5 th $(72)$ would give a summation-tone of 120 vibrations, or the octave of the $3 \mathrm{~d}$; and $C(48)$ with its $3 \mathrm{~d}(60)$ would give 108 vibrations, the octave of the $2 \mathrm{~d}$. These tones can be distinguished by means of resonators.

It is thus seen that musical sounds are complex. With single notes there are many different harmonics, or overtones, and in chords there are series of resultants, that are lower than the primary notes, and series of additional, or summation-tones, that are higher; but both the resultant and the summation-tones bear exact mathematical relations to the primary notes.

Harmony. - Overtones, resultant tones and summation-tones of strings have been discussed rather fully, for the reason that in studying the physiology of audition, it will be seen that the ear is capable of recognizing single sounds or successions of single sounds; but at the same time certain combinations of sounds are appreciated and are even more agreeable than those which apparently are produced by simple vibrations. Combinations of notes which thus produce an agreeable impression are called harmonious. They seem to become blended with each other into a complete sound of peculiar quality, all the different vibrations entering into their composition being simultaneously appreciated by the ear. The blending of notes which bear to each other certain mathematical relations is called harmony; but two or more 
notes, though each one be musical, are not necessarily harmonious. The most prominent overtone, except the octave, is the 5 th, with its octaves, and this is called the dominant. The next is the $3 \mathrm{~d}$, with its octaves. The other overtones are comparatively feeble. Reasoning, now, from a knowledge of the relations of overtones, it might be inferred that the reënforcement of the 5 th and $3 \mathrm{~d}$ by other notes bearing similar relations to the tonic would be agreeable. This is the fact; and it was ascertained empirically long before the pleasing impression produced by such combinations was explained mathematically.

It is a law in music that the simpler the ratio between the number of vibrations in two sounds, the better the harmony. The simplest relation, of course, is $\mathrm{I}: \mathrm{I}$, when the two sounds are said to be in unison. The next in order is $I: 2$. In sounding $C$ and its 8 th, for example, there are 48 vibrations of one to 96 of the other. These sounds can produce no discord, because the waves never interfere with each other, and the two sounds can be prolonged indefinitely, always maintaining the same relations. The combined impression, therefore, is continuous. The next in order are the Ist and 5 th, their relations being $2: 3$. In other words, with the Ist and 5 th, for two waves of the Ist there are three waves of the 5 th. The two sounds may thus progress indefinitely, for the waves coincide for every second wave of the ist and every third wave of the 5 th. The next in order is the $3 \mathrm{~d}$. The $3 \mathrm{~d}$ of $\mathrm{C}$ has the 8 th of $\mathrm{C}$ for its 5 th, and the 5 th of $\mathrm{C}$ for its minor $3 \mathrm{~d}$. The Ist, $3 \mathrm{~d}, 5$ th and 8 th form the common major chord; and the waves of each note blend with each other at such short intervals of time that the ear experiences a continuous impression, and no discord is heard. This explanation of the common major chord illustrates the law that the smaller the ratio of vibration between different tones, the better is their harmony. Sounded with the Ist, the 4 th is more harmonious than the $3 \mathrm{~d}$; but its want of harmony with the 5 th excludes it from the common chord. The Ist, $4^{\text {th }}$ and 8 th are harmonious, but to make a complete chord the 6th must be added.

Discords and Dissonance. - A knowledge of the mechanism of simple accords leads naturally to a comprehension of the rationale of discords and dissonance. Two inharmonious notes that can not be resolved into harmony by the addition of another note or other notes produce discord. When the inharmonious sound is resolved into a harmony, the notes first sounded produce what is called dissonance. The fact that certain combinations of musical notes produce a disagreeable impression was first ascertained empirically, with no knowledge of the exact cause; but the mechanism of discord is now regarded by most physicists as settled. 
The sounds produced by two tuning-forks giving precisely the same number of vibrations in a second are in unison. If one of the forks is loaded with a bit of wax, so that its vibrations are slightly reduced, and if both are put in vibration at the same instant, there is discord. Taking the illustration given by Tyndall, it may be assumed that one fork has 256 , and the other, 255 vibrations in a second. While these two forks are vibrating, one is gradually gaining on the other; but at the end of half a second, one will have made 128 vibrations, while the other will have made $127 \frac{1}{2}$. At this point the two waves are moving in exactly opposite directions; and as a consequence, the sounds neutralize each other and there is an instant of silence. The perfect sounds, as the two forks continue to vibrate, are thus alternately reënforced and diminished, and this produces what is known in music as beats. As the difference in the number of vibrations in a second is one, the instants of silence occur once in a second; and in this illustration the beats occur once a second. Unison takes place when two sounds can follow each other indefinitely, their waves blending perfectly; and discord or dissonance is marked by successive beats, or pulses. If the forks are loaded so that one will vibrate 240 times in a second, and the other 234 , there will be six instants in a second when the interference will be manifest; or in other words in $\frac{1}{6}$ of a second, one fork will make 40 vibrations, while the other is making 39. This will give 6 beats in a second. From these experiments the law may be deduced that the number of beats produced by two tones not in harmony is equal to the difference between the two rates of vibration. An analogous interference of undulations is observed in optics, when waves of light are made to interfere and produce darkness.

It is evident that the number of beats will increase as two discordant notes are produced higher and higher in the scale. According to Helmholtz, beats can be recognized up to 132 in a second. Beyond that point they become confused and there is only a general sensation of discord. Beats, then, are due to interference of sound-waves. There is no interference of the waves of notes in unison, provided waves start at the same instant; the intensity of the sound being increased by reënforcement. The differences between the Ist and 8 th, the Ist and $5^{\text {th, the }}$ s st and $3 \mathrm{~d}$, and other harmonious combinations, is so great that there are no beats and no discord, the more rapid waves reënforcing the harmonics of the primary sound. It is important to remember, in this connection, that resultant tones are equal to the difference in the rates of vibration of two harmonious notes. Taking a note of 240 vibrations, and its 5 th, with 360 vibrations, these two have a difference of $\mathrm{r} 20$, which is the lower octave of the Ist and is an harmonious tone. 
The laws just stated are applicable to overtones, resultant tones and additional tones, which, like the primary notes, have their beats and discords.

Tones by Influence. - After what has been stated in regard to the laws of musical vibrations, it will be easy to comprehend the production of sounds by influence. If a key of the piano is lightly touched so as to raise the damper but not to sound the string, and then a note is sung in unison, the string will return the sound, by the "influence" of the sound-waves of the voice. The sound thus produced by the string will have its fundamental tone and overtones; but the series of overtones will be complete; for none of the nodes are abolished, as in striking or plucking a string at any particular point. If, instead of a note in unison, any of the octaves are sounded, the string will return the exact note sung; and the same is true of the $3 \mathrm{~d}$, 5 th etc. If a chord in harmony with the undamped string is struck, this chord will be exactly returned by influence. In other words, a string may be made to sound by influence, its fundamental tone, its harmonics and harmonious combinations. To carry the observation still farther, the string will return, not only a note of its exact pitch and its harmonics, but notes of the peculiar quality of the primary note. This is an important point in its applications to the physiology of hearing and can be readily illustrated. Taking identical notes in succession, produced by the voice, trumpet, violin, clarinet or any other musical instrument, it can easily be noted that the quality of the sound, as well as the pitch, is rendered by a resounding string; and the same is true of combinations of notes. The laws of tones by influence have been illustrated by strings merely for the sake of simplicity; but they are applicable more or less to all bodies capable of producing musical sounds, except that some are thrown into vibration with more difficulty than others.

A thin membrane, like a piece of bladder or thin rubber, stretched over a circular orifice, such as the mouth of a wide bottle, may readily be tuned to a certain note. When arranged in this way, the membrane can be made to sound its fundamental tone by influence. In addition, the membrane, like a string, will divide itself so as to sound the harmonics of the fundamental, and it will likewise be thrown into vibration by the 5 th, $3 \mathrm{~d}$ etc., of its fundamental, thus obeying the laws of vibrations of strings, although the harmonic sounds are produced with greater difficulty. ${ }^{1}$

1 The account just given of some of the laws of sonorous vibrations and their relations to musical effects and combinations, although by no means complete, may seem rather extended for a work on physiology; but it should be borne in mind that the mechanism of the appreciation of musical sounds includes the entire physiology of audition. This subject can not be 


\section{Uses of Different Parts of the Middle Ear}

The uses of the pavilion and of the external auditory meatus are sufficiently plain. The pavilion serves to collect the waves of sound, and probably it inclines them toward the external meatus as they come from various directions. Although this action is simple, it has some importance, and the various curves of the concavity of the pavilion tend more or less to concentrate sonorous vibrations. Such has long been the opinion of physiologists, and this seems to be carried out by experiments in which the concavities of the external ear have been obliterated by wax. There probably is no resonance or vibration of much importance until the waves of sound strike the membrana tympani. The same remarks may be made in regard to the external auditory meatus. It is not known precisely how the obliquity and the curves of this canal affect the waves of sound, but it is probable that the deviation from a straight course protects, to a certain extent, the tympanic membrane from impressions that might otherwise be too violent.

Structure of the Mcmbrana Tympani. - The general arrangement of the membrana tympani has already been described in connection with the topographical anatomy of the auditory apparatus. The membrane is elastic, about the thickness of ordinary gold-beater's skin, and is subject to various degrees of tension by the action of the muscles of the middle ear and under different conditions of atmospheric pressure within and without the tympanic cavity. Its form is nearly circular; and it has a diameter in the adult, according to Sappey, of a little more than $\frac{2}{5}$ of an inch ( Io to I I millimeters) vertically and about $\frac{2}{5}$ of an inch (IO millimeters) antero-posteriorly. The excess of the vertical over the horizontal diameter is about $\frac{1}{50}$ of an inch (0.5 millimeter).

The periphery of the tympanic membrane is received into a little ring of bone, which may be separated by maceration in early life but is consolidated with the adjacent bony structures in the adult. This bony ring is incomplete at its superior portion, but aside from this, it resembles the groove which receives the crystal of a watch. At the periphery of the membrane, is a ring of condensed fibrous tissue which is received into the bony ring. This ring also presents a break at its superior portion.

The concavity of the membrana tympani presents outward, and it comprehended without a general knowledge of the physics of sound and of some of the laws of harmony ; for not only is there a perception of single notes by the auditory apparatus, but the most elaborate combinations of sounds in harmony are appreciated together and at one and the same instant, as will be seen in sturlying the action and uses of clifferent parts concerned in audition. Many of the laws of musical combinations are directly applicable to the physiology of hearing. 
may be increased or diminished by the action of the muscles of the middle ear. The point of greatest concavity, where the extremity of the handle of the malleus is attached, is called the umbo. On the inner surface of the membrane are two pouches, or pockets. One is formed by a small, irregular, triangular fold, situated at the upper part of its posterior half and consisting of a process of the fibrous layer. This, which is called the posterior pocket, is open below and extends from the posterior upper border of the membrane to the handle of the malleus, which it assists in holding in position. "After it has been divided, the bone is much more movable than before" (Tröltsch). The anterior pocket is lower and shorter than the posterior. It is formed by a small bony process turned toward the neck of the malleus, by the mucous membrane, by the bony process of the malleus, by its anterior ligament, the chorda tympani and the anterior tympanic artery. The handle of the malleus is inserted between the two layers of the fibrous structure of the membrana tympani and occupies the upper half of its vertical diameter, extending from the periphery to the umbo.

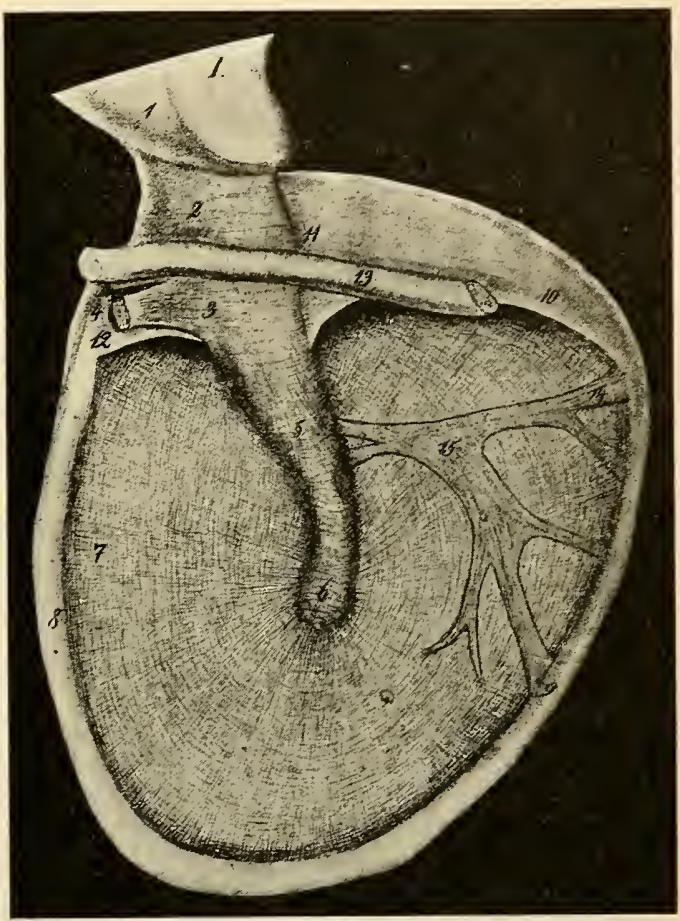

Fig. r9o. - Right membrana tympani, seen from within, $\times 6$. Reduced about one-fifth from a photograph (Rüdinger).

I, head of the malleus, divided; 2 , neck of the malleus; 3 , handle of the malleus, with the tendon of the tensor-tympani muscle; 4 , divided tendon of the tensor tympani; 5,6 , portion of the malleus between the layers of the membrana tympani; 7 , outer (radiating) and inner (circular) fibres of the membrana tympani; 8 , fibrous ring of the membrana tympani ; 9, I4, I5, dentated fibres, discovered by Gruber; Io, posterior pocket; II, connection of the posterior pocket with the malleus; I2, anterior pocket; I3, chorda-tympani nerve.

The membrana tympani, though thin and translucent, presents three distinct layers. Its outer layer is a very thin extension of the integument lining the external meatus, presenting, however, neither papillæ nor glands. The inner layer is a delicate continuation of the mucous membrane lining the tympanic cavity and is covered with tessellated epithelial cells. The fibrous portion, or lamina propria, is formed of 
two layers. The outer layer consists of fibres radiating from the handle of the malleus to the periphery. These are best seen near the centre. The inner layer is composed of circular fibres, which are most abundant near the periphery and diminish in number toward the centre.

The color of the membrana tympani, when it is examined with an aural speculum by daylight, is peculiar, and it is rather difficult to describe, as it varies in the normal ear in different individuals. Politzer described the membrane, examined in this way, as translucent, and of a color which "most nearly approaches a neutral gray, mingled with a weaker tint of violet and light yellowish brown." This color is modified, in certain portions of the membrane, by the chorda tympani and the bones of the ear, which produce some opacity. The entire membrane in health has a soft lustre. In addition there is seen, with proper illumination, a well-marked triangular cone of light, with its apex at the end of the handle of the malleus, spreading out in a downward and forward direction, and $\frac{1}{16}$ to $\frac{1}{12}$ of an inch ( 1.6 to 2 . I millimeters) broad at its base. This appearance is regarded by physiologists as indicating a normal condition of the membrane. It is undoubtedly due to reflection of light and not to a peculiar structure of that portion of the membrane on which it is seen.

Uses of the Membrana Tympani. - It is unquestionable that the membrana tympani is very important in audition. In cases of disease in which the membrane is thickened, perforated or destroyed, the acuteness of hearing is more or less affected. That this is in great part due to the absence of a vibrating surface for the reception of waves of sound, is shown by the relief experienced by patients who can tolerate the presence of an artificial membrane of rubber. As regards the mere acuteness of hearing, aside from the pitch of sounds, the explanation of the action of the membrane is very simple. Sonorous vibrations are not readily transmitted through the atmosphere to solid bodies, like the bones of the ear; and when they are thus transmitted they lose considerably in intensity. When, however, the aërial vibrations are received by a membrane, under the conditions of the membrana tympani, they are transmitted with little loss of intensity; and if this membrane is connected with solid bodies, like the bones of the middle ear, the vibrations are readily conveyed to the sensory portions of the auditory apparatus. The parts composing the middle ear are well adapted to the transmission of sonorous waves to the auditory nerves. The membrane of the tympanum is delicate in structure, stretched to the proper degree of tension, and vibrates under the influence of the waves of sound. Attached to this membrane, is the angular chain of bones, which conducts its vibrations, like the bridge of a violin, to the liquid of the 
labyrinth. The membrane is fixed at its periphery and has air on both sides, so that it is under conditions favorable for vibration.

A study of the mechanism of the ossicles and muscles of the middle ear shows that the membrana tympani is subject to certain physiological variations in tension, due to the contraction of the tensor tympani. It is also evident that this membrane may be drawn in and rendered tense by exhausting or rarefying the air in the drum. If the mouth and nose are closed and an attempt is made to breathe forcibly by expanding the chest, the external pressure tightens the membrane. In this condition the ear is rendered insensible to grave sounds, but high-pitched sounds appear to be more intense. If the tension is removed, as may be done by an act of swallowing, the grave sounds are heard with normal distinctness. This experiment, tried at a concert, produces the curious effect of abolishing a great number of the lowest tones, while the shrill sounds are heard very acutely. The same phenomena are observed when the external pressure is increased by descent in a diving bell.

Undoubted cases of voluntary contraction of the tensor tympani have been observed by otologists; and in these, by bringing this muscle into action, the limit of perception of high tones may be greatly increased. In two instances of this kind, recorded by Blake, the ordinary limit of perception was found to be three thousand single vibrations, and by contraction of the muscle, this was increased to five thousand.

The concave form of the membrana tympani and the presence of a bony process between its layers, which is part of the chain of bones of the middle ear, are conditions under which it is impossible that it should have a single fundamental tone. This has been shown by experiments with stretched membranes depressed in their central portion by means of a solid rod. No membrane can have a single fundamental tone unless it be in a condition of uniform tension, like a string, and this is impossible in the membrana tympani. Nevertheless, the membrana tympani repeats sounds by influence and is capable of repeating in this way a much greater variety of sounds than if it had itself a fundamental tone and were capable of a uniform degree of tension. This has been shown by experiments with stretched elastic membranes made to assume a concave form. If the membrana tympani had a single fundamental tone, it would vibrate by influence only with certain tones in unison with it and the overtones would be eliminated. It would then act like a resonator closed with a membrane; and the tone with which it happened to be in unison would overpower all others. The fact is that all tones, the vibrations of which reach the membrane, are appreciated at their proper value as regards intensity. Again, if the membrana tympani had its own fundamental tone, it would have overtones of the 
fundamental, which would produce errors and confusion in auditory appreciation. The chain of bones, also, attached to the membrane acts as a damper and prevents the persistence of vibrations after the waves of sound cease in the air. This provision enables rapid successions of sounds to be distinctly and accurately repeated.

The arrangement of the muscles and bones of the middle ear is such that the tension of the membrana tympani may be regulated and graduated with great nicety. It does not seem to be necessary to perfect audition that this should be done for every single note or combination of notes, but the membrane probably is brought by voluntary effort to a definite degree of tension for notes within a certain range as regards pitch or for successions and progressions of sounds in a particular key. So far as the consciousness of this muscular action is concerned, it may be revealed only by the fact of the correct appreciation of certain musical sounds. Some persons can educate the ear so as to acquire what is called the faculty of absolute pitch ; that is, without the aid of a tuningfork or any musical instrument, they are able to recognize the exact musical value of any given note. A possible explanation of this is that such persons may have educated the muscles of the ear so as to put the tympanic membrane in such a condition of tension as to respond to a given note and to recognize the position of this note in the musical scale. Finally, an accomplished musician, in conducting an orchestra, can by a voluntary effort direct his attention to certain instruments and hear their notes distinctly, separating them from the general volume of sound, can distinguish discords and designate a single instrument making a false note.

Destruction of both tympanic membranes does not necessarily produce total deafness, although this condition involves considerable impairment of hearing. So long as there is simple destruction of these membranes, the bones of the middle ear and the other parts of the auditory apparatus being intact, the waves of sound are conducted to the auditory nerves, although this is done imperfectly. In a case reported by Astley Cooper, one membrana tympani was entirely destroyed, and the other was nearly gone, there being some parts of its periphery remaining. In this person the hearing was somewhat impaired, although he could distinguish ordinary conversation without much difficulty. Fortunately, he had considerable musical taste, and it was ascertained that his musical ear was not seriously impaired; "for he played well on the flute and had frequently borne a part in a concert. I speak this, not from his authority only, but also from that of his father, who is an excellent judge of music, and plays well on the violin: he told me that his son, besides playing on the flute, sang with much taste, and perfectly in tune." 
There is an important consideration that must be kept in view in studying the uses of any distinct portion of the auditory apparatus, like the membrana tympani. This membrane, like all other parts of the apparatus, except the auditory nerves themselves, has simply an accessory action. If the regular waves of a musical sound are conveyed to the terminal filaments of the auditory nerves, these waves make their impression and the sound is correctly appreciated. It makes no difference, except as regards intensity, how these waves are conducted; the sound is appreciated by the impression made on the nerves, and on the nerves only. The waves of sound are not like the waves of light, refracted, decomposed, perhaps, and necessarily brought to a focus as they impinge on the retina; but so far as the action of the accessory parts of the ear are concerned, the waves of sound are unaltered; that is, the rate of their succession remains the same, though they are reflected by the concavities of the concha and repeated by the tympanic membrane. Even if it be assumed that the membrane under normal conditions repeats musical sounds by vibrations produced by influence, and that sounds are exactly repeated, the position of these sounds in the musical scale is not and can not be altered by the action of any of the accessory organs of hearing. The fact that a person may retain his musical ear with both membranes destroyed is not an argument against the view that the membrane repeats sounds by influence; for if musical sounds or irregular vibrations are conducted to the auditory nerves, the impression produced must of necessity be dependent exclusively on the character, regularity and number of the sonorous vibrations. And again, the physical laws of sound teach that a membrane like the membrana tympani must reproduce sounds with which it is more or less closely in unison much better than discordant or irregular vibrations. In a loud confusion of sounds, one can readily distinguish melody or harmony, even when the vibrations of the latter are comparatively feeble.

It has been shown that the appreciation of the pitch of sounds bears a certain relation to the degree of tension of the tympanic membrane. When the membrane is rendered tense, there is insensibility to low notes. When the membrane is brought to the highest degree of tension by voluntary contraction of the tensor tympani, the limit of appreciation of high notes may be raised from three thousand to five thousand vibrations. It is a fact in the physics of the membrana tympani that the vibrations are more intense the nearer the membrane approaches to a vertical position; and it has been observed that the membrane has a position more nearly vertical in musicians than in persons with an imperfect musical ear (Tröltsch).

Experiments have shown that the tympanic membrane vibrates more 
forcibly when relaxed than when it is tense. In certain cases of facial palsy, in which it is probable that the branch of the facial going to the tensor tympani was affected, the ear has been found painfully sensitive to powerful impressions of sound. This probably has no relation to pitch, and most sounds that are painfully loud are comparatively grave. Artillerists are in danger of rupture of the membrana tympani from sudden concussions. To guard against this injury, it is recommended to stop the ear, draw the shoulder up against the ear most in danger, and particularly to inflate the middle ear after Valsalva's method. "This method consists in making a powerful expiration, with the mouth and nostrils closed" (Tröltsch).

Mechanism of the Ossicles of the Ear. - The ossicles of the middle ear, in connection with the muscles, have a twofold office: First, by the action of the muscles the membrana tympani may be brought to different degrees of tension. Second, the angular chain of bones serves to conduct sonorous vibrations to the labyrinth. It must be remembered that the handle of the malleus is closely attached to the membrana tympani, especially near its lower end. Near the short processes which is a conical projection at the root of the handle - the attachment is looser and there is even an incomplete joint-space. The long process is attached closely to the Glasserian fissure of the temporal bone.

The malleus is articulated with the incus by a peculiar joint. This is so arranged, presenting a sort of cog, that the handle can rotate only outward; and when a force is applied that would have a tendency to produce a rotation inward, the malleus must carry the incus with it. This mechanism has been compared to that of a watch-key with cogs that are fitted together and allow the key to turn in one direction, but are separated so that only the upper portion turns when the force is applied in the opposite direction (Helmholtz). In the articulation between the malleus and the incus, the only difference is that there is but one $\operatorname{cog}$; but this is sufficient to prevent an independent rotation of the malleus inward.

The body of the incus is attached to the posterior bony wall of the tympanum. Its articulation with the malleus has just been indicated. By the extremity of its long process, it also is articulated with the stapes, which completes the chain. In situ, the stapes forms nearly a right angle with the long process of the incus.

The stapes is articulated with the incus, as indicated above, and its oval base is applied to the fenestra ovalis. Surrounding the base of the stapes is a ring of fibro-cartilage that is closely united to the bony wall of the labyrinth by an extension of the periosteum.

The articulations between the malleus and the incus and between 
the incus and the stapes are so arranged that when the membrana tympani is forced outward - as it may be by inflation of the tympanic cavity - there is no danger of tearing the stapes from its attachment to the fenestra ovalis; for when the handle of the malleus is drawn outward, the cog-joint between the malleus and the incus is loosened and no considerable traction can be exerted on the stapes.

The tensor tympani is by far the more important of the two muscles of the middle ear. Its action is to tighten the cog-like joint between the malleus and the incus, to tighten, also, all the ligaments of the incus, to draw the long process of the malleus inward, thereby increasing the tension of the membrana tympani, and to press the base of the stapes against the fenestra ovalis. By the action of this muscle the chain of ossicles becomes practically a solid and continuous angular rod.

Although experiments have demonstrated the mechanism of the ossicles and the action of the tensor tympani, both as regards the chain of bones and the membrana tympani, direct observations are wanting to show the exact relations of these different conditions of the ossicles and of the membrane to the physiology of audition. One very important physical point, however, which has been the subject of much discussion, is settled. The chain of bones acts as a single solid body in conducting vibrations to the labyrinth. It is a matter of physical demonstration that vibrations of the bones themselves would be inconceivably rapid as compared with the highest tones that can be appreciated by the ear, if it were possible to induce in these bones regular vibrations. Practically, then, the ossicles have no independent vibrations that can be appreciated. This being the fact, the ossicles simply conduct to the labyrinth vibrations induced in the membrana tympani by sound-waves; and their arrangement is such that these vibrations lose but little in intensity. While it has been shown experimentally that the amplitude of vibration in the membrana tympani and the ossicles diminishes as the tension of the membrane is increased, it would seem that when the tensor tympani contracts, it must render the conduction of sound-waves to the labyrinth more delicate than when the auditory apparatus is in a relaxed condition, which may be compared with the "indolent" condition of accommodation of the eye. When the membrana tympani is relaxed and the cog-like articulation between the malleus and the incus is loosened, the vibrations of the membrane and of the malleus may have a greater amplitude; but when the malleoincudal joint is tightened and the stapes is pressed against the fenestra ovalis, the loss of intensity of vibration, in conduction through the bones to the labyrinth, must be reduced to the minimum. With this view, 
the tensor-tympani muscle, while it contracts to secure for the membrana tympani the degree of tension most favorable for vibration under the influence of certain sounds, puts the chain of bones in the condition best adapted to the conduction of the vibrations of the membrane to the labyrinth, with the smallest loss of intensity.

\section{Physiological Anatomy of the Internal Ear}

The internal ear consists of the labyrinth, which is divided into the vestibule, semicircular canals and cochlea. The general arrangement of these parts has already been described; and it remains only to study the structures contained within the bony labyrinth, in so far as their anatomy bears directly on the physiology of audition. Passing inward from the tympanum, the first division of the internal ear is the vestibule. This cavity communicates with the tympanum by the fenestra ovalis, which is closed in the natural state by the base of the stapes. It communicates, also, with the semicircular canals and with the cochlea.

General Arrangement of the Membranous Labyrinth. - The bony labyrinth is lined with a moderately-thick periosteum, consisting of connective tissue, a few delicate elastic fibres, nuclei and bloodvessels and spots of calcareous concretions. This membrane adheres closely to the bone and extends over the fenestra ovalis and the fenestra rotunda. Its inner surface is smooth and is covered with a single layer of cells of endothelium, which in some parts is segmented and in others forms a continuous nucleated sheet. In certain portions of the vestibule and semicircular canals, the periosteum is united to the membranous labyrinth more or less closely by fibrous bands, which have been called ligaments of the labyrinth. The fenestra rotunda, which lies between the cavity of the tympanum and the cochlea, is closed with a membrane formed by an extension of the periosteum lining the cochlea, on one side, and the mucous membrane lining the tympanic cavity, on the other.

In the bony vestibule, occupying about two-thirds of its cavity, are two distinct sacs: a large ovoid sac, the utricle, situated in the upper and posterior portion of the cavity, and a smaller rounded sac, the saccule, situated in its lower and anterior portion. These two sacs communicate with each other through a small canal in the form of the letter $Y$, which is represented in the upper diagram in Fig. 19I. The utricle communicates with the three semicircular canals, and the saccule is connected with the true membranous cochlea by the canalis reuniens. At a point in the utricle corresponding to the entrance of a branch of the auditory nerve, is a round whitish spot, called the acoustic spot (macula acustica), containing otoliths, or otoconia, which are attached to the inner surface 
of the membrane. A similar spot containing otoliths exists in the saccule at the point of entrance of its nerve. Otoliths are also found in the ampullæ of the semicircular canals. These calcareous masses are composed of crystals of calcium carbonate, which are hexagonal and pointed at their extremities. Nothing definite is known of the uses of these calcareous bodies, which exist in man, mammals, birds and reptiles.

The membranous semicircular canals occupy about one-third of the cavity of the bony canals. They present small ovoid dilatations, called ampullæ, corresponding to the ampullate enlargements of the bony canals. They are held in place by a large number of little fibrous bands extending to the bony labyrinth.

The membranes of the cochlea include the periosteum lining the bony canal, and the true membranous cochlea. Viewed externally, the true membranous cochlea appears as a single tube following the

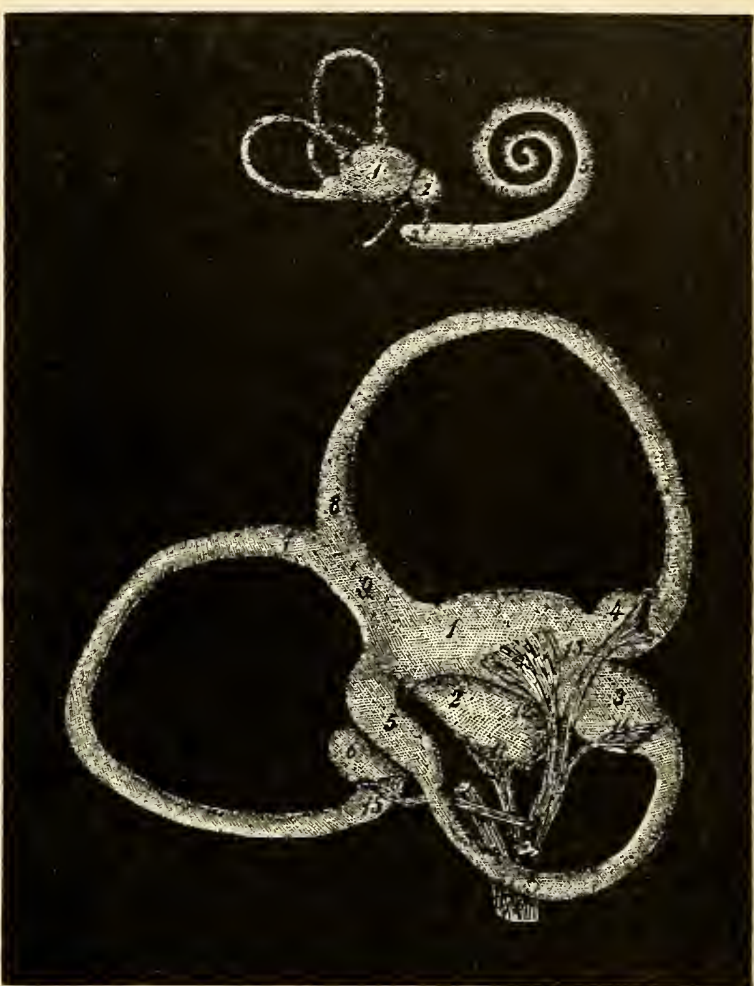

Fig. I9I. - Diagram of the labyrinth. From a photograph, and slightly reduced (Rüdinger).

Upper figure: $I$, utricle; 2 , saccule; 3,5 , membranous cochiea ; 4 , canalis reuniens; 6 , semicircular canals.

Lower figure: 1 , utricle ; 2 , saccule ; $3,4,6$, ampullæ; $5,7,8,9$, semicircular canals; Io, auditory nerve (partly diagrammatic); II, I2, I3, I4, I5, distribution of the branches of the nerve to the vestibule and the semicircular canals.

turns of the bony cochlea, beginning below by a blind extremity and terminating in a blind extremity at the summit of the cochlea. If a section of the cochlea is made in a direction vertical to the spiral, it will be seen that the bony canal is divided, partly by bone and partly by membrane, into an inferior portion, a superior portion, and a triangular canal, lying between the two, which is external. The bony septum is in the form of a spiral plate extending from the central column (the modiolus) into the 
cochlea, about halfway to its external wall, and terminating above in a hook-shaped extremity called the hamulus. The free edge of this bony lamina is thin and dense. Near the modiolus it divides into two plates, with an intermediate spongy structure, in which are lodged vessels and nerves. The surface of the bony lamina looking toward the base of the cochlea is marked by a number of regular transverse ridges.

Attached to the free margin of the bony lamina is a membrane, the membrana basilaris, which extends to the outer wall of the cochlea. In

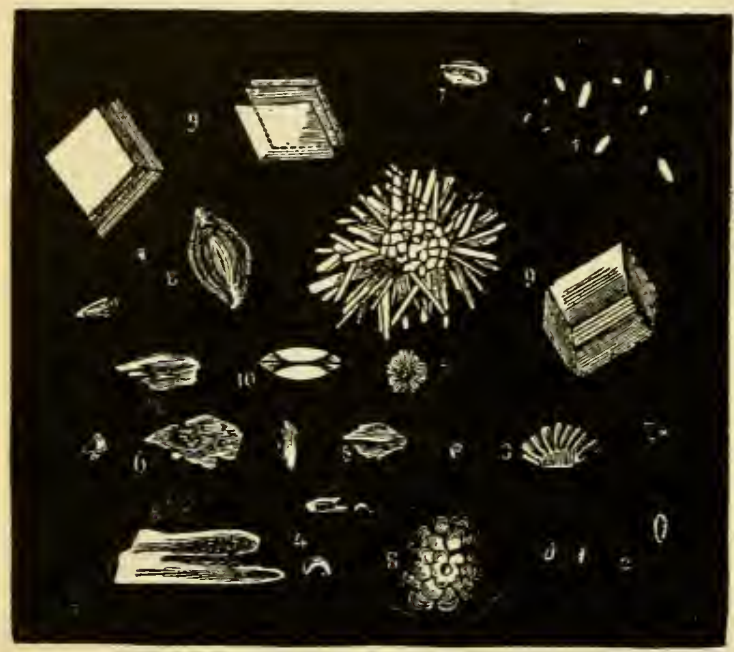

Fig. 192. - Otoliths from various animals (Rüdinger).

I, from the goat; 2 , from the herring; 3 , from the devil-fish; 4, from the mackerel; 5 , from the flying-fish; 6 , from the pike; 7 , from the carp; 8 , from the ray; 9 , from the shark; Io, from the grouse. brane of Corti. Between the membrana tectoria and the membrana basilaris is the quadrilateral canal, which contains the organ of Corti. The membrane of Reissner extends from the inner portion of the limbus upward and outward to the outer wall of the cochlea. This divides the portion of the cochlea situated above the scala tympani into two portions: an internal portion, the scala vestibuli, and an external triangular canal, called the canalis cochlea, or the true membranous cochlea.

In the anatomical description of the contents of the bony cochlea, the scalæ and membranous parts may be designated as follows: The canalis cochleæ is divided, as just indicated, by the membrana tectoria. The portion between the membrana tectoria and the membrane of Reissner is called the scala media. This communicates with the saccule by the canalis reuniens. 
I. The portion below the bony and membranous septum, called the scala tympani. This is bounded by the periosteum lining the corresponding portion of the bony cochlea and the under surface of the bony lamina and by the membrana basilaris.

2. The scala vestibuli. This is bounded by the periosteum lining the corresponding portion of the bony cochlea and the upper surface of the bony septum and by the membrane of Reissner.

3. The true membranous cochlea. This is the spiral, triangular canal, bounded externally by the periosteum of the corresponding portion of the wall of the cochlea, internally, by the membrane of Reissner, and on the other side, by the membrana basilaris. What is thus called the membranous cochlea is divided by the limbus laminæ spiralis and the membrana tectoria into two portions; a triangular canal above (scala media), which is the larger, and the quadrilateral canal below, between the lim-

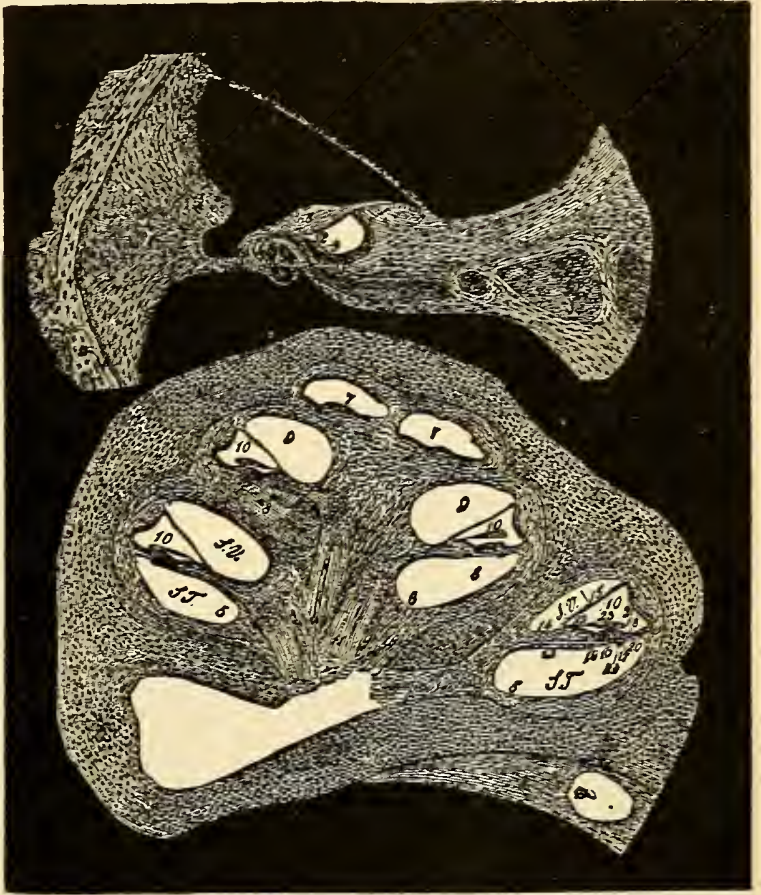

Fig. 193. - Section of the first turn of the spiral canal of a cat newly born. - Section of the cochlea of a human fotus at the fourth month. From a photograph, and slightly reduced (Rüdinger).

Upper figure: I, 2, 6, lamina spiralis; 2 , lower plate; $3,4,5,5$, nervus cochlearis; 7 , membrane of Reissner; 8, membrana tectoria; 9, epithelium; Io, II, pillars of Corti; I2, inner hair-celis; I3, outer hair-cells; I4, I6, membrana basilaris; I5, epithelium in the sulcus spiralis; I7, I8, I9, ligamentum spirale; 20 , spiral canal, below the membrana basilaris.

Lower figure, $S T, S T, 5,5,7,7,8,8$, scala tympani; $S V, S V$, 9,9 , scala vestibuli; $I$, base of the cochlea; 2 , apex; 3,4 , central column; IO, IO, IO, IO, ductus cochlearis; II, branches of the nervus cochlearis; I2, I2, I2, spiral ganglion; I3, I4, limbus laminæe

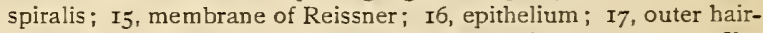
cells; I8, epithelium of the membrana basilaris; I9, nervous filaments; 20, union of the membrana basilaris with the ligamentum spirale; 2 I, epithelium of the peripheral wall of the ductus cochlearis; 22,23 , membrana tectoria; 24 , spiral canal, below the membrana basilaris.

bus and membrana tectoria and the membrana basilaris. The quadrilateral canal contains the organ of Corti and various complex anatomical structures. The relations of these parts are shown in Fig. 193, but the numbers in the figure are made out with some difficulty. 
The membranous cochlea, as just described, includes the scala media and the quadrilateral canal, follows the spiral course of the cochlea, terminates superiorly in a pointed blind extremity at the cupola, beyond the hamulus, and is connected below with the saccule of the vestibule, by the canalis reuniens. The relations of the different portions of the membranous cochlea to each other and to the scalæ of the cochlea also are shown in Fig. 193.

Liquids of the Labyrinth. - The labyrinth contains a certain quantity of a clear watery liquid, called the humor of Cotugno or of Valsalva. A portion of this liquid surrounds the utricle and saccule, the semicircular canals and the true membranous cochlea; and this is known as the perilymph of Breschet. Another portion of the liquid fills the true membranous labyrinth and is sometimes called the humor of Scarpa; but it is known more commonly as the endolymph of Breschet. The perilymph occupies about one-third of the cavity of the bony vestibule and semicircular canals and both scalæ of the cochlea. Both this liquid and the endolymph are clear and watery, becoming somewhat opalescent on the addition of alcohol. The spaces in the labyrinth are directly connected with the lymphatic system. The space occupied by the perilymph communicates with lymphatics chiefly through the aqueduct of the cochlea, but there also is a communication through the internal auditory meatus with the space beneath the dura mater. The endolymph passes to the subarachnoid space beneath the arachnoid covering of the auditory nerve. So far as is known, the uses of the liquid of the internal ear are to sustain the delicate structures contained in this portion of the auditory apparatus and to conduct sonorous vibrations to the terminal filaments of the auditory nerves and the parts with which they are connected.

Distribution of the Nerves in the Labyrinth. - As the auditory nerves enter the internal auditory meatus, they divide into an anterior, or cochlear, and a posterior, or vestibular branch. The vestibular branch divides into three smaller branches, a superior and anterior, a middle, and a posterior branch. The superior and anterior branch, the largest of the three, is distributed to the utricle, the superior semicircular canal and the external semicircular canal. The middle branch is distributed to the saccule. The posterior branch passes to the posterior semicircular canal. The nerves distributed to the utricle and saccule penetrate at the points occupied by the otoliths, and the nerves going to the semicircular canals pass to the ampullæ, which also contain otoliths (see Fig. I9I). In each ampulla, at the point where the nerve enters, is a transverse fold projecting into the canal and occupying about one-third of its circumference, called the septum transversum. 
The nerves terminate in essentially the same way in the sacs of the vestibule and the ampullæ of the semicircular canals. At the points where the nerves enter, in addition to the otoliths, are cylindrical cells of various forms, which pass gradually into the general endothelium of the cavities. In addition to these cells, are fusiform nucleated bodies, the free ends of which are provided with hair-like processes, called fila acustica. These are about $\frac{1}{800}$ of an inch $(31 \mu)$ in length and are distributed in quite a regular manner around the otoliths. The nerves form an anastomosing plexus beneath the endothelium and terminate probably in the fusiform bodies just described as presenting the fila acustica at their free extremities. In the sacs of the vestibule and in the semicircular canals, nerves exist only in the maculæ acusticæ and the ampullæ.

The cochlear division of the auditory nerve breaks up into a number of small branches which pass through the foramina at the base of the cochlea in what is called the tractus spiralis foraminulentus. These follow the axis of the cochlea and pass in their course toward the apex, between the plates of the bony spiral lamina. Between these plates of bone, the dark-bordered nerve-fibres pass each one

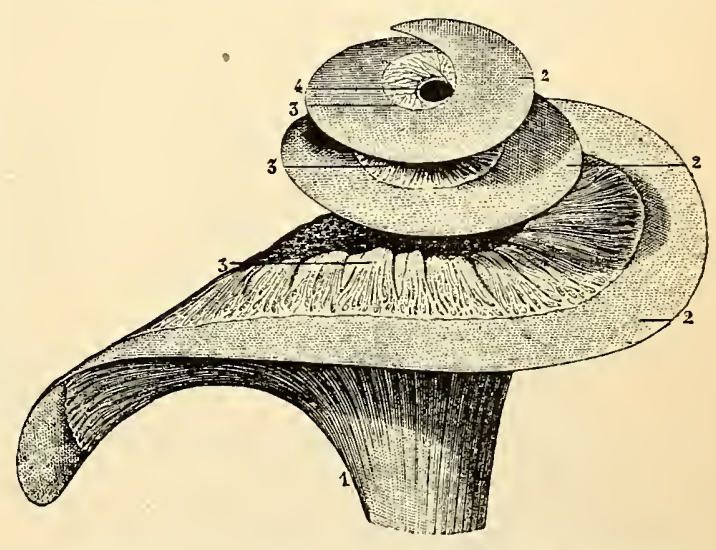

Fig. 194.-Distribution of the cochlear nerve in the spiral lamina. The cochled is from the right side and is seen from its antero-inferior part (Sappey).

I, trunk of the cochlear nerve; $2,2,2$, membranous zone of the spiral lamina; $3,3,3$, terminal expansion of the cochlear nerve, exposed in its whole extent by the removal of the superior plate of the lamina spiralis; 4 , orifice of communication of the scala tympani with the scala vestibuli.

through a bipolar cell, these cells together forming a spiral ganglion known as the ganglion of Corti. Beyond this ganglion the nerves form an anastomosing plexus and finally enter the quadrilateral canal, or the canal of Corti. As they pass into this canal they suddenly become pale and exceedingly fine. They probably are connected finally with the organ of Corti, although their exact mode of termination has not yet been determined. The course of the nerve-fibres to their distribution in the cochlea is shown in Fig. 194.

Organ of Corti. - In the quadrilateral canal, bathed in the endolymph throughout its spiral course, is an arrangement of pillars, or rods, which are regular, like the strings of a harp in miniature. These are the pillars of Corti. These pillars are external and internal, with their 
bases attached to the basilar membrane and their summits articulated above, so as to form a regular spiral arcade enclosing a triangular space that is bounded below by the basilar membrane. The number of the elements of the organ of Corti is estimated at about 4500 , for the outer, and 6500 , for the inner rods (Pritchard). The relations of these structures to the membranous labyrinth are seen in Fig. 193. The external pillars are longer, more delicate, and more rounded than the internal pillars. The form of the pillars is more exactly shown in Figs. 195 and 196 , the latter figure, however, exhibiting other structures which enter into the constitution of the organ of Corti. It will be remarked that a small nucleated body is attached to the base of either pillar. At the summit, where the internal and the external pillars are joined
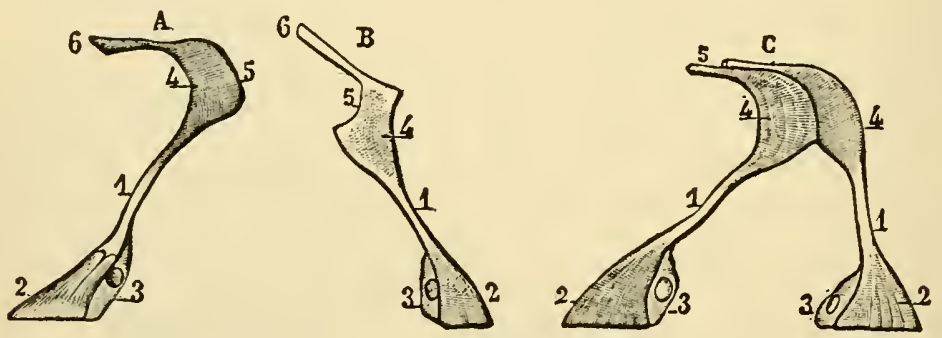

Fig. 195. - The two pillars of the organ of Corti (Sappey).

A, external pillar of the organ of Corti : 1 , body, or middle portion ; 2, posterior extremity, or base ; 3 , cell on its internal side; 4 , anterior extremity; 5 , convex surface, by which it is joined to the internal pillar; 6 , prolongation of this extremity.

$\mathrm{B}$, internal pillar of the organ of Corti: I, body, or middle portion; 2, posterior extremity; 3 , cell on its external side; 4 , anterior extremity; 5 , concave surface, by which it is joined to the external pillar; 6 , prolongation, lying above the corresponding prolongation of the external pillar.

$\mathrm{C}$, the two pillars of the organ of Corti, united by their anterior extremity, and forming an arcade, the concavity of which presents outward; I, I, body, or middle portion of the pillars; 2,2, posterior extremities; 3,3 , cells attached to the posterior extremities; 4,4 , anterior extremities joined together; 5, terminal prolongation of this extremity.

together, is a delicate prolongation, directed outward, which is attached to the covering of the quadrilateral canal.

The above description comprises about all that is definitely known of the arrangement of the pillars or rods of Corti. They are nearly homogeneous, except when treated with reagents, and are said to be of about the consistence of cartilage. They are closely set together, with narrow spaces between them, and it is difficult to see how they can be stretched to any considerable degree of tension. The arch is longer at the summit than at the base of the cochlea, the longest rods, at the summit, measuring, according to Pritchard, about $\frac{1}{200}$ of an inch (125 $\mu$ ), and the shortest, at the base, about $\frac{1}{500}$ of an inch $(50 \mu)$. At the base of the cochlea the two sets of rods are about equal in length. From the base to the apex, both sets, outer and inner, progressively increase in 
length, and the outer rods become the longer, so that near the apex they are nearly twice as long as the inner. The anatomical relations between the pillars and the terminal filaments of the auditory nerves are not definitely settled.

In addition to the pillars just described, various cellular elements enter into the structure of the organ of Corti. The most important of these are the inner and the outer hair-cells. These are 16,400 to 20,000 in number (Hensen, Waldeyer). The inner hair-cells are arranged in a single row, and the outer hair-cells, in four rows. Nothing definite is known of the uses of these cells. The relations of these parts are shown in Fig. 196. It is supposed by some anatomists that the fila-

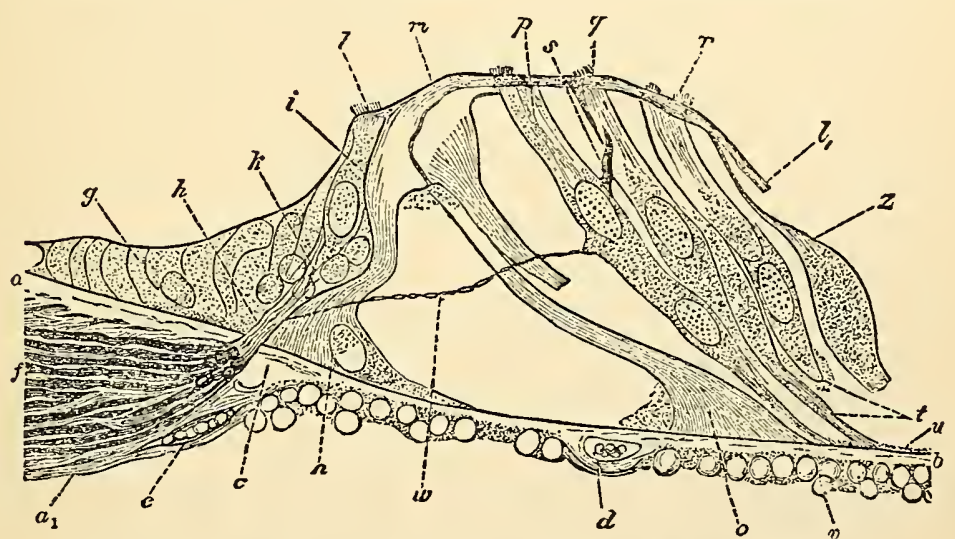

Fig. 196. - Vertical section of the organ of Corti of the dog (Waldeyer).

$a-b$, homogeneous layer of the basilar membrane; $v$, tympanic layer, with nuclei, granular cellprotoplasm and connective tissue; $a_{1}$, tympanic lip of the crista spiralis; $c$, thickened portion of the basilar membrane; $d$, spiral vessel; $e$, bloodvessel $f, h$, bundle of nerves; $g$, epithelium; $i$, inner hair-cell, with its basilar process, $k ; l$, head-plate of the inner pillar; $m$, union of the two pillars; $n$, base of the inner pillar; 0 , base of the outer pillar; $p, q, r$, outer hair-cells, with traces of the cilia; $t$, bases of two other hair-cells; $z$, Hensen's prop-cell; $l-l_{1}$, lamina reticularis; $w$, nerve-fibre passing to the first hair-cell, $p$.

ments of the auditory nerves terminate in the cells above described; but this point is not definitely settled.

\section{Uses of Different Parts of the Internal Ear}

The precise uses of the different parts found in the internal ear are somewhat obscure, notwithstanding the careful researches that have been made into the anatomy and the physiology of the labyrinth. There are several points, however, bearing on the physiology of this portion of the auditory apparatus, concerning which there can be no doubt:-

First, it is certain that impressions of sound are received by the 
terminal filaments of the auditory nerves and by these nerves are conveyed to the brain.

Second, the uses of the parts composing the external and the middle ear are chiefly accessory. The sonorous waves are collected by the pinna and are conveyed by the external meatus to the middle ear; the membrana tympani vibrates under their influence; and they are thus collected, repeated and transmitted to the internal ear.

Uses of the Semicircular Canals. - In the experiments of Flourens, on pigeons and rabbits (I824), it was shown that destruction of the semicircular canals had apparently no effect on the sense of hearing, while destruction of the cochlea on both sides produced total deafness. In addition it was observed that destruction of the semicircular canals on both sides was followed by remarkable disturbances in equilibration. The animals could maintain the standing position, but so soon as they made any movements, "the head began to be agitated; and this agitation increasing with the movements of the body, walking and all regular movements finally became impossible, in nearly the same way as when equilibrium and stability of movements are lost after turning several times or violently shaking the head." These observations of Flourens, at least so far as regards the influence of the semicircular canals on equilibration, have been confirmed by Goltz and are sustained by observations on the human subject, in the condition known as Ménière's disease. So far as can be determined from experimental data, it does not seem probable that the nerves directly concerned in audition are distributed to any considerable extent in the semicircular canals. Indeed, the uses of these parts is obscure; for it can hardly be admitted, on purely anatomical grounds, that they are concerned in the discrimination of the direction of sonorous vibrations, an idea that has been advanced by some physiologists. ${ }^{1}$

Uses of the Parts contained in the Cochlea. - There can be no doubt in regard to the capital point in the physiology of the cochlea; namely, that those branches of the auditory nerve which are essential to the sense of hearing and which receive the impressions of sound are distributed in the cochlea. An analysis of sonorous impressions shows that they possess various attributes, such as intensity, quality and pitch. So far as the terminal filaments of the auditory nerve are concerned, it is evident that the intensity of sound is appreciated in proportion to the force of the impression made on these nerves. In regard to quality of

${ }^{1}$ Although the physiological literature of the semicircular canals is of immense volume, little of a convincing and definite character has been learned concerning their function since the observations of Flourens; and their further discussion would be out of place in this work. The directions of the canals may be studied in Fig. 18S, page 720 . 
sound, it has been seen that this is due to the form of sonorous vibrations ; and that musical sounds usually are compound, their quality depending largely on the relative loudness of the harmonics, partial tones etc. It has also been seen that resonant bodies may repeat by influence, not only the actual pitch of tones, but their quality. If there is in the cochlea an anatomical arrangement of rods or fibres by which the sonorous vibrations conveyed to the ear are repeated, there is reason to believe that the quality as well as the pitch is reproduced.

The arrangement of the rods entering into the structure of the organ of Corti and of the fibres of the membrana basilaris has afforded a theoretical explanation of the final mechanism of the appreciation of pitch. With the exception of the internal ear, the action of different portions of the auditory apparatus is simply to conduct and repeat sonorous vibrations; and the sole use of these accessory parts, aside from the protection of the organs, is to convey the vibrations to the terminal nervous filaments. Whatever may be the uses of the membrana tympani in repeating sounds by influence, it is certain that this membrane possesses no true auditory nerves, and that the auditory nerves only are capable of receiving impressions of sound. Thus, hearing, and even the appreciation of pitch, is not necessarily lost after destruction of the membrana tympani ; and if sonorous vibrations reach the auditory nerves, they will be appreciated and appreciated correctly.

In view of the arrangement of the organ of Corti, with its eleven thousand or more rods of different lengths arranged with a certain degree of regularity, a number more than sufficient to represent all the notes of the musical scale, it is not surprising that they should be regarded as capable of repeating all the notes heard in music. Helmholtz formulated this idea in the theory that sounds conveyed to the cochlea throw into vibration only those elements of the organ of Corti which are tuned, so to speak, in unison with them. According to this hypothesis, the rods of Corti constitute a harp of several thousand strings, played on, as it were, by the sonorous vibrations. Theories analogous to that proposed by Helmholtz, but of course lacking the basis of exact anatomical and physical details developed by modern researches and experiments, were advanced by Du Verney (1683) and by Le Cat (I 767 ).

Viewing the question anatomically, it is by no means certain that the rods of Corti are so attached and stretched that they are capable of separate and individual vibrations. It has not been demonstrated that certain of these rods vibrate under the influence of certain notes or that they are tuned in accord with certain notes. Hensen and others have rejected the theory of Helmholtz, basing their opinions mainly on the anatomical arrangement of the organ of Corti. Hensen assumed it to be 
a physical impossibility for the different rods to vibrate individually, and he regarded it as improbable that the rods are tuned in accord with different musical notes. The present idea is that strands of the membrana basilaris, stretched between the lower extremities of the pillars of Corti, being of different lengths, vibrate in obedience to the waves of sound conveyed to them through the auditory nerves; but even this does not afford an entirely satisfactory explanation of the mechanism of the final appreciation of pitch. It is the fact, however, that disease of the lower part of cochlea is attended with deafness to high tones, and disease affecting the summit produces deafness to low tones.

It is not necessary that sonorous vibrations should pass to the cochlea through the external ear and parts in the middle ear. Sounds may be conducted to the auditory nerves through the bones of the head or through the Eustachian tubes, as is shown by the simple and familiar experiment of placing a tuning-folk in contact with the head or between the teeth, the ears being closed.

The action of the two ears does not seem to be essential to the correct appreciation of auditory impressions; but variations in the force of such impressions made on either ear aid in determining the direction of sounds, although errors in regard to this of ten occur.

The estimate of the distance of sounds is made by judging of their intensity, in connection with information obtained through other senses, especially the sense of sight. The power of estimating distance is largely influenced by experience and education.

Centres for Audition. - The centres for audition in dogs and monkeys are in the superior temporo-sphenoidal convolution (Ferrier, Munk). In man these centres are in the first and second temporal convolutions of the temporo-sphenoidal lobe, parts supplied by the fourth branch of the middle cerebral artery. This has been ascertained by pathological observations as well as by experiments on the lower animals. In man the action of these centres is not completely crossed, and destruction of the centre upon one side does not cause total deafness in either ear. Complete destruction of the centres on both sides, however, produces deafness in both ears. Injury of the first temporal convolution often is followed by the condition known as word-deafness, in which the subject hears the sound of words, but these sounds convey no idea. This is the psychical auditory centre and is confined to the first temporal convolution on the left side (Wernicke). Word-deafness is analogous to the condition already described under the name of word-blindness, and the centre usually is confined to the left side of the cerebrum. It has been suggested by Westphal that this centre may be on the right side of the cerebrum in left-handed persons. 


\section{CHAPTER XXX}

\section{EMBRYOLOGY}

Female organs of generation - The ovaries - Graafian follicles - The parovarium - The uterus - The Fallopian tubes - Structure of the ovum - Discharge of the ovum - Passage of ova into the Fallopian tubes - Puberty and menstruation - Changes in the Graafian follicles after their rupture (corpus luteum) - Corpus luteum of pregnancy - Male organs of generation - Interstitial gland of the testis - Vas deferens - Vesiculæ seminales - Prostate - Glands of the urethra - Male element of generation - Spermatozoids.

Generation is one of the most important of the animal functions and as such usually is treated of quite fully in works on physiology; but a more or less extended account is also to be found. in every complete treatise on anatomy and in most works on obstetrics. The physiological history of the human organism, however, would not be complete without an account of generation and development; although this will involve, to some extent, a repetition of what usually is found in works on other subjects.

In what is known as sexual generation, the two anatomical elements - spermatozoid and ovum - are developed in separate beings, male and female, and are brought together, in man and in the higher animals, by sexual connection, or copulation. In this way, the life of animals is prolonged and individual forms are preserved in future beings. This can not be secured without the acquisition by the ovum of a new element. The animal cell may and does multiply by the process already described under the name of karyokinesis; but this can not continue indefinitely. After a cell has undergone this process about one hundred and fifty times, the reproductive power of the protoplasm, having gradually become enfeebled, is finally lost. It has been estimated that the cell increases in mass by the appropriation of nutritive matters, as the cube of its diameter, while the absorbing surface increases as the square of the diameter (Herbert Spencer). If this view is accepted, it is evident that a time will inevitably come in its life-history when the cell can no longer absorb enough material for growth ; but with every division, there is a relative increase in surface ; and thus it becomes possible for growth to continue. The process of growth, indeed, might continue indefinitely were it not that the cytoplasm gradually loses its power of appropriating new matter. By the introduction of a new element, however, in the 
union of male with female cells, the power of the resulting cell to perpetuate itself is secured.

\section{Female Organs of Generation}

A knowledge of certain points in the anatomy of the female organs of generation is essential to a comprehension of the most important of the processes of reproduction. Following a fruitful intercourse, the function of reproduction, as regards the male, ceases with the comparatively simple process of penetration of the male element through the protective covering of the ovum and its fusion with the female element. The fertilized ovum then passes through certain changes, forms attachments to the body of the mother, continues its development and is nourished and grows, until the fotus at term is brought into the world.

The female organs of generation are divided anatomically into internal and external. The external organs are the vulva, the adjacent parts and the vagina. The internal organs are the uterus, Fallopian tubes and the ovaries. The ovaries are the true female organs, in which alone the female element can be produced. The Fallopian tubes and the uterus are accessory in their uses, the female element, the ovim, passing through the tubes to the uterus, where it forms the attachments to the body of the mother that are essential to its nourishment and full development.

The vagina has a direction, slightly curved anteriorly, that is nearly coincident with the axis of the outlet, or the inferior strait of the pelvis. Projecting into the vagina, at its upper extremity, is the lower part of the neck of the uterus. The uterus extends from the vagina nearly to the brim of the pelvis. It is situated between the bladder and the rectum, and has an antero-posterior inclination when the bladder is moderately distended, which brings its axis nearly coincident with that of the superior strait of the pelvis. With the body erect, the angle of the uterus with the perpendicular is about forty-five degrees.

The uterus is held in place by ligaments, certain of which are formed of folds of the peritoneum. The anterior ligament is reflected from the anterior surface to the bladder; the posterior ligament extends from the posterior surface to the rectum; the round ligaments extend from the upper angle of the uterus, on either side, between the folds of the broad ligament and through the inguinal canal, to the symphysis pubis; the broad ligaments extend from the sides of the uterus to the walls of the pelvis.

The uterus and the broad ligaments partially divide the pelvis into two portions; and these ligaments, which consist of a double fold of 
peritoneum, present a superior, or posterior surface, and an inferior, or anterior surface. The superior, or anterior border of this fold is occupied by the Fallopian tubes, the peritoneum constituting their outer coat. Laterally, at the free extremities of the tubes, the peritoneum ceases, and there is an actual opening of each Fallopian tube into the peritoneal cavity. Attached to the broad ligament and projecting on its posterior surface, is the ovary, which is connected with the fibrous tissue between the two layers of the ligament. At the hilum of the ovary, as will be seen farther on, the structure of the peritoneum undergoes a marked change.

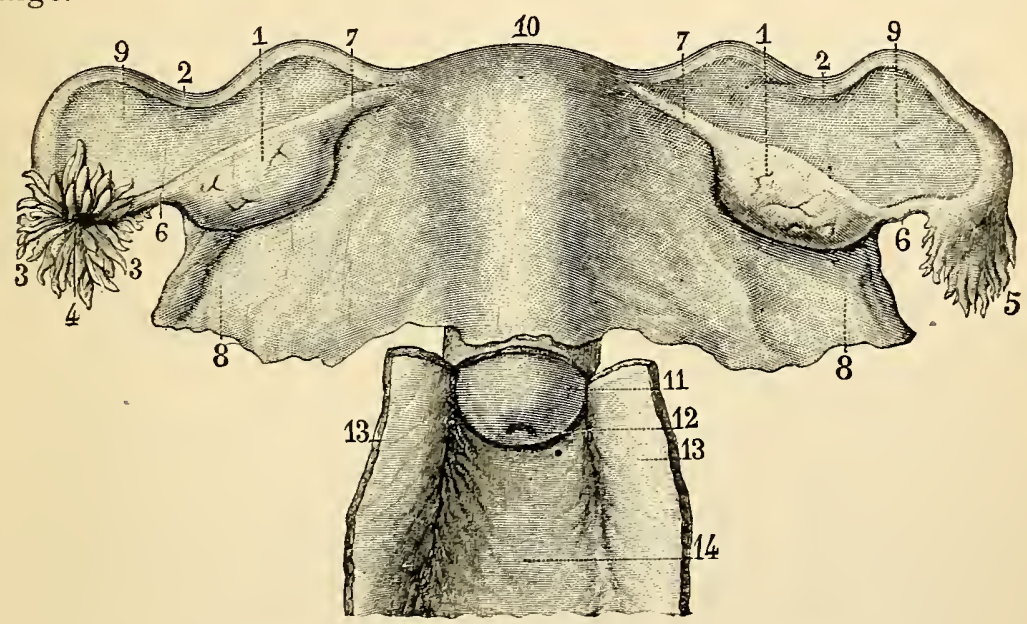

Fig. 197. - Uterus, Fallopian tubes and ovaries - posterior view (Sappey).

I, ovaries; 2, 2, Fallopian tubes; 3, 3, finbriated extremity of the left Fallopian tube, seen from its concavity; 4 , opening of the left tube; 5 , fimbriated extremity of the right tube, posterior view; 6,6 , fimbriæ which attach the extremity of each tube to the ovary; 7,7 . ligaments of the ovary; $8,8,9,9$, broad ligaments; Io, uterus; II, cervix uteri; I2, os uteri; I3, I3, It, vagina.

The Ovaries. - The ovaries, attached to the broad ligament and projecting on its posterior surface, lie nearly horizontally in the pelvic cavity, on either side of the uterus. They are of a whitish color, and their form is ovoid and flattened, with the anterior border, sometimes called the base, attached to the broad ligament. By closely examining their mode of connection with the broad ligament, it is seen that at the margin of the attached surface of the ovary, the posterior layer of the ligament ceases, and that the fibrous stroma of the medullary portion of the ovary is continuous with the fibrous tissue lying between the two layers.

The ovary is about an inch and a half (38. I millimeters) in length, half an inch (I2.7 millimeters) in thickness, and three-quarters of an inch (I9.I millimeters) in width at its widest portion. The outer 
extremity is somewhat rounded and is attached to one of the fimbrix of the Fallopian tube. The inner extremity is more pointed and is attached to the side of the uterus by means of the ligament of the ovary. This ligament is shown in Fig. I97 $(7,7)$. It is a rounded cord, composed of non-striated muscular fibres spread out on the attached extremity of the ovary and the posterior surface of the uterus, and is covered by peritoneum. The weight of the ovary is sixty to one hundred grains ( 3.9 to 6.5 grams), and it is largest in the adult virgin. Its attached border is called the hilum; and at this portion the vessels and nerves penetrate. The surface presents rounded translucent elevations, produced by distended Graafian follicles, with little cicatrices indicating the situation of ruptured follicles. There may also be seen, between the distended follicles, corpora lutea in different stages of atrophy.

After the peritoneum has reached the ovary, its fibrous layer becomes indistinct and fuses with the fibrous stroma of the ovary itself. The peritoneal endothelium here undergoes a change, and the cells on the surface of the ovary are cuboidal and in a single layer. This change in the structure of the peritoneum is abrupt and is indicated by a distinct line surrounding the hilum of the ovary.

On making a section of the ovary, it is readily seen with the naked eye that the organ is composed of two distinct structures; a cortical substance, sometimes called the tunica albuginea, which is about $\frac{1}{25}$ of an inch (I millimeter) in thickness, and a medullary substance containing a large number of bloodvessels. The cortical substance alone contains the Graafian follicles. The external layer of this is denser than the deeper portion, but there is no distinct fibrous membrane such as is sometimes described under the name of tunica albuginea.

The cortical substance of the ovary consists of connective tissue in several layers, the fibres of which are continuous with the looser fibres of the medullary portion. In the substance of this layer, are embedded the ova, enclosed in the sacs called Graafian follicles. This layer contains a few bloodvessels coming from the medullary portion, which surround the follicles.

The medullary portion of the ovary is very vascular and is composed of small bands, or trabeculæ of connective tissue, with non-striated muscular fibres. The bloodvessels, which penetrate at the hilum, are large and convoluted, especially at the hilum itself, where there is a mass of convoluted veins, forming a sort of vascular bulb. In the medullary portion, which is sometimes called the vascular zone, the muscular fibres follow the vessels, in the form of muscular sheaths.

In addition to the bloodvessels, the ovary receives nerves from the spermatic plexus of the sympathetic, the exact mode of termination of 
which has not been ascertained. Lymphatics have also been demonstrated at the hilum.

Graafian Follicles. - These vesicles, or follicles, were described and figured by DeGraaf in 1672, and are known by his name. They contain the ova, undergo a series of peculiar changes, enlarge, approach the surface of the ovary, and finally are ruptured, discharging their contents into the fimbriated extremity of the Fallopian tube. The Graafian follicles are developed exclusively in the cortical substance. If the ovary is examined at any period of life, no follicles are found in the medullary substance; but a few of the larger may project downward, so as to encroach somewhat upon it, being actually of a diameter greater than the thickness of the cortex. The entire number of follicles of all sizes in either ovary is about 36,000 (Henle). According to the table of measurements given by Waldeyer, the primordial follicles in the human embryo, at the seventh month, measure $\frac{1}{800}$ to $\frac{1}{250}$ of an inch (30 to IOO $\mu$ ) in diameter, and the primordial ova, $\frac{1}{1650}$ to $\frac{1}{1000}$ of an inch ( 5 to $25 \mu$ ).

The ovary appears early in embryonic life, in the form of a cellular outgrowth from the Wolffian body. Most of its cells are small, but as early as the fourth or fifth day, in the chick, some of them are to be distinguished by their large size, their rounded form and the presence of a large nucleus. These cells are supposed to be primordial ova. In the process of development, some of the peripheral cells penetrate in the form of tubes (the so-called ovarian tubes); and at the same time, delicate processes, formed of connective tissue and bloodvessels, extend from the fibrous stroma underlying the epithelium and enclose collections of cells. It is probable that there are two modes of formation of follicles; one, by the penetration of epithelial tubes from the surface, which become constricted and divided off into closed cavities, and the other, by the extension of fibrous processes from below, which enclose little collections of cells. By both these processes, little cavities are formed, which contain a number of cells. In each of these cavities, there is a single, large, rounded cell, with a large nucleus, this cell being a primordial ovum; and in addition, in the same cavity, there are other cells, which are the cells of the Graafian follicle. The exact nature of the processes just described has been studied in the chick; but it is probable that the same kind of development occurs in mammalia and in the human subject.

From birth until just before puberty, the cortical substance of the ovary contains several thousands of what are termed primordial follicles enclosing the primordial ova; and it is probable that after the ovaries are fully developed at birth, no additional ova or Graafian follicles make 
their appearance. The prevailing idea is, indeed, that the great majority of these never arrive at maturity and that they undergo atrophy at various stages of their development. In the adult, according to Waldeyer, the smallest Graafian follicles measure $\frac{1}{800}$ to $\frac{1}{600}$ of an inch (30 to $40 \mu$ ), and the smallest ova, a little more than $\frac{1}{1000}$ of an inch $(26 \mu)$. The primordial ova have the form of rounded cells, each with a large clear nucleus and a nucleolus. Other structures are developed in and surrounding these cells as the ova arrive at their full development.

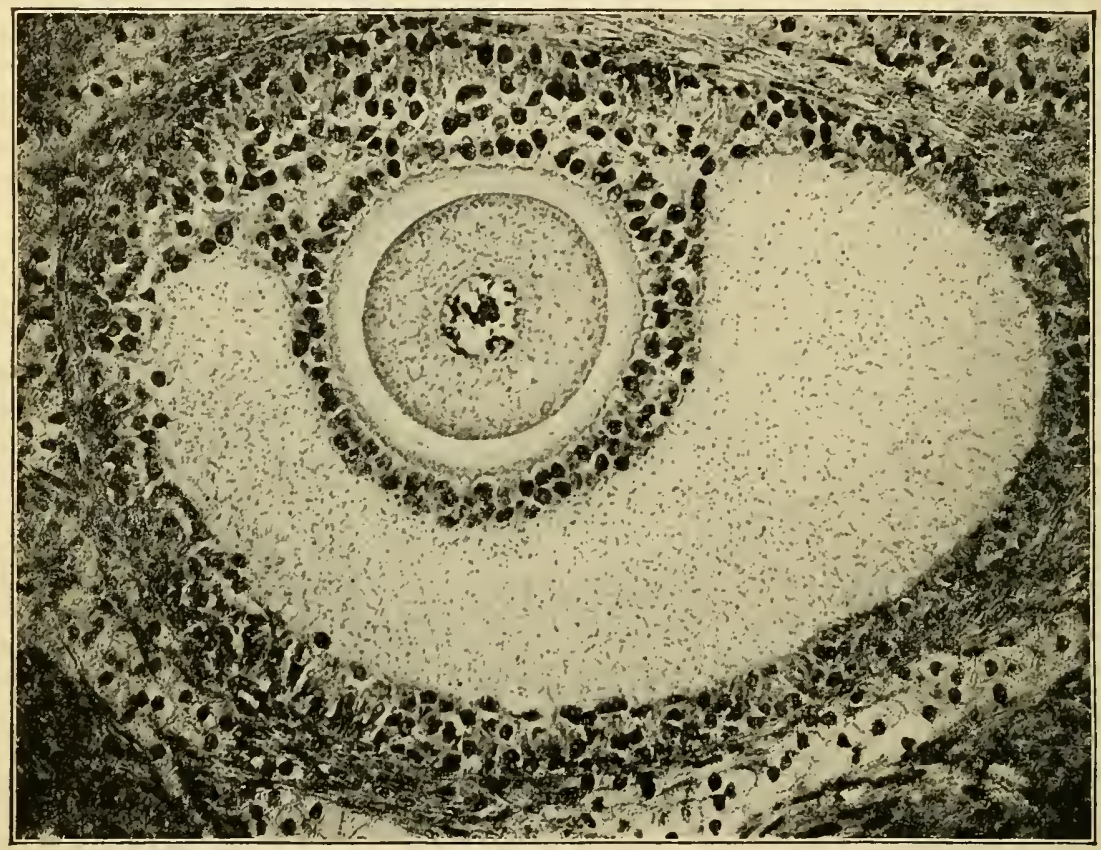

Fig. 198. - Ovum of the cat, within the ovary, directly reproduced from a photograph of a preparation by Dahlgren, $\times 235$ (Wilson).

The ovum lies in the discus proligerus within the Graafian follicle.

The most important stage in the development of the ova and Graafian follicles is observed at about the beginning of puberty. At this time a number of follicles (twelve, twenty, thirty or even more) enlarge, so that all sizes are observed, between the smallest primordial follicles, $\frac{1}{80} \overline{0}$ of an inch $(30 \mu)$, and the largest, nearly $\frac{1}{2}$ an inch ( 2 millimeters) in diameter. In follicles that have attained any considerable size, there are the fully-developed ova, one in each follicleexcept in rare instances, when there are two; and these ova have a diameter of about $\frac{1}{12}$ of an inch $(200 \mu)$. In the process which culminates in the discharge of the ovum into the fimbriated extremity of 
the Fallupian tube, the Graafian follicle gradually enlarges, becomes distended with liquid which finally breaks through, and the follicle ruptures on the surface of the ovary.

At or near the period of their maturity the Graafian follicles present several coats and are filled with an albuminous liquid. The mature follicles project just beneath the surface and form little rounded translucent elevations. The smallest follicles are near the surface, and as they enlarge, at first they become deeper, becoming superficial only as they approach the condition of fullest distention.

Taking one of the largest follicles as an example, two fibrous layers can be distinguished; an outer layer, of ordinary connective tissue, and an inner layer, the tunica propria, formed of the same kind of tissue, with the difference that as the follicle enlarges the inner layer becomes vascular. The vascular tunica propria is lined with cells of epithelium, forming the so-called membrana granulosa. At a certain point in this membrane, is a mass of cells, called the discus or cumulus proligerus, in which the ovum is embedded. The situation of the discus proligerus is not invariable; sometimes it is at the most superficial, and sometimes it is at the deepest part of the Graafian follicle.

The liquid of the Graafian follicle is alkaline, slightly yellowish and not viscid. It contains a small quantity of albuminous matter coagulable by heat, alcohol and acids. This liquid is supposed to be secreted by the cells lining the inner membrane of the follicle.

The Parovarium. - The parovarium, or organ of Rosenmuiller, is simply the remains of the Wolffian body, lying in the folds of the broad ligament, between the ovary and the Fallopian tube. It consists of twelve to fifteen tubes of fibrous tissue, lined with ciliated epithelium. It has no physiological importance.

The Uterns. - The form, situation and relations of the uterus and Fallopian tubes have already been indicated and are shown in Fig. 197. It is a pear-shaped body, somewhat flattened antero-posteriorly, presenting a fundus, a body and a neck. At its lower extremity, is an opening into the vagina, called the os externum. At the upper portion of the neck, is a constriction, which indicates the situation of the os internum. The form of the uterus is shown in Fig. 199 (A). It usually is about three inches ( 76.2 millimeters) in length, two inches ( 50.8 millimeters) in breadth at its widest portion, and one inch (25.4 millimeters) in thickness. Its weight is one and a half to two and a half ounces ( 42.5 to 7 I grams). It is somewhat loosely held in place by the broad and round ligaments and by the folds of the peritoneum in front and behind. The delicate layer of peritoneum that forms its external covering extends behind as far down as the vagina, where it is reflected back 
upon the rectum, and anteriorly, a little below the upper extremity of the neck (os internum), where it is reflected upon the urinary bladder. At the sides of the uterus, the peritoneal covering, a little below the entrance of the Fallopian tubes, becomes loosely attached and leaves a line for the penetration of the vessels and nerves. Fig. I99 (C), giving a view of the interior of the uterus, shows a triangular cavity, with two cornua corresponding to the openings of the Fallopian tubes, and very thick walls, the greatest part of which is composed of layers and bands of non-striated muscular fibres.

The muscular walls of the uterus are composed of non-striated fibres arranged in several layers. These fibres are spindle-shaped and always

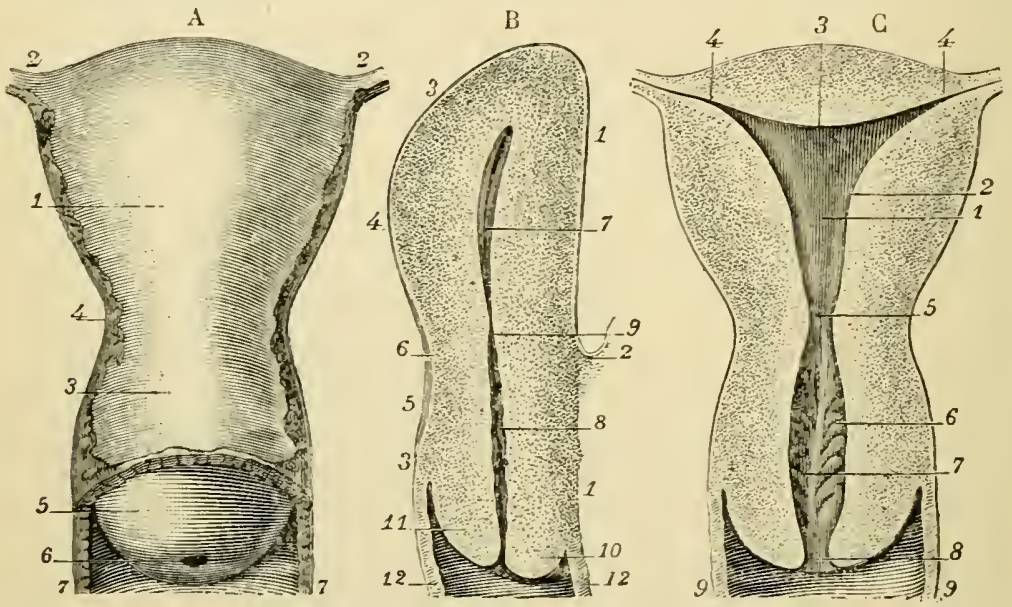

Fig. 199. - Virgin uterus (from a zooman twenty-two years old). A.- anterior view. B. - median longitudinal section. C. - transverse longitudinal section (Sappey).

A. I, body; 2, 2, angles; 3 , cervix; 4 , site of the os internum; 5 , vaginal portion of the cervix; 6 , external os: 7,7 , vagina.

B. I, I, profile of the anterior surface; 2, vesico-uterine cul-de-sat; 3, 3, profile of the posterior surface; 4 , body; 5 , neck; 6 , isthmus; 7 , cavity of the body; 8 , cavity of the cervix; 9 , os internum; IO, anterior lip of the os externum; II, posterior lip; I2, I2, vagina.

C. I, cavity of the body; 2, lateral wall; 3 , superior wall; 4, 4, cornua; 5 , os internum; 6, cavity of the cervix; 7 , arbor vita of the cervix; 8 , os externum; 9,9 , vagina.

nucleated, the nucleus presenting one or two large granules that have been taken for nucleoli. They are closely bound together, so that they are isolated with great difficulty. In addition to an amorphous adhesive substance between the muscular fibres, there are many rounded and spindle-shaped cells of connective tissue, and a few elastic fibres. The muscular fibres of the uterus enlarge immensely during gestation, becoming at that time ten or fifteen times as long and five or six times as broad as they are in the unimpregnated state. They are united into bundles, or fasciculi, which in certain of the layers interlace with each other in every direction. The fibres are divided into external, middle and internal layers. 
The external muscular layer, which is very thin but distinct, is closely attached to the peritoneum. When the uterus is somewhat enlarged after impregnation, there are observed oblique and transverse superficial fibres passing over the fundus and the anterior and posterior surfaces to the sides. Here they are prolonged upon the Fallopian tubes, the round ligament and the ligament of the ovary, and they also extend between the layers of the broad ligament. This external layer is so thin that it can not be very efficient in the expulsive contractions of the uterus; but from its connections with the Fallopian tubes and the ligaments, it is useful in holding the uterus in place. It does not extend entirely over the sides of the uterus.

A

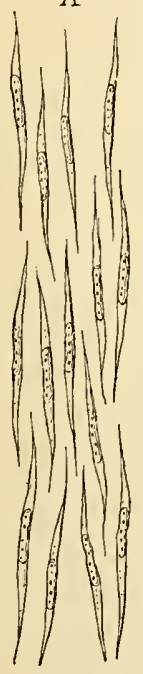

B

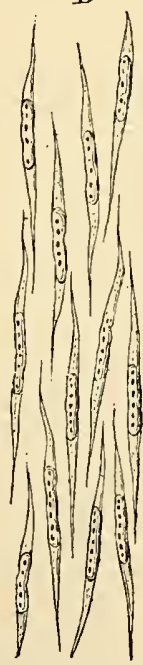

C

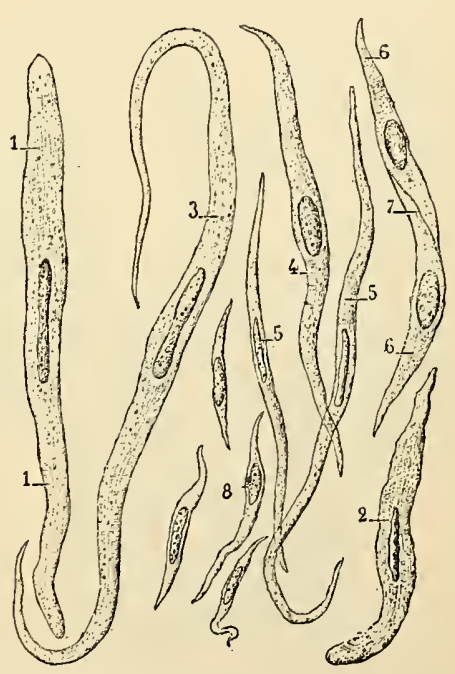

Fig. 200. - Muscular fibres of the uterus (Sappey).

$A$, fibres of the uterus of the fœetus at term; $B$, of a woman twenty years of age; $C$, of a woman just delivered.

The middle muscular layer is the one most efficient in the parturient contractions of the uterus. It is composed of a thick and intricate network of fasciculi interlacing with each other in every direction.

The inner muscular layer is arranged in the form of broad rings, which surround the Fallopian tubes, become larger as they extend over the body of the uterus and meet at the centre of the organ, near the neck.

The mucous membrane of the uterus is of a pale reddish color; and that portion lining the body is smooth and is so closely attached to the subjacent structures that it can not be separated to any great extent by dissection. There is, indeed, no proper submucous areolar tissue, 
the membrane being applied directly to the uterine walls. It is covered by a single layer of cylindrical epithelial cells, with delicate cilia, the movements of which are from without inward, toward the openings of the Fallopian tubes. Examination of the surface of the membrane with a low magnifying power shows the openings of a great number of tubular glands. These glands usually are simple, sometimes branched, dividing, about midway between the opening and the lower extremity, into two and very rarely into three secondary tubules. Their course usually is tortuous, so that their length frequently exceeds the thickness of the mucous membrane. The openings of these tubes are about

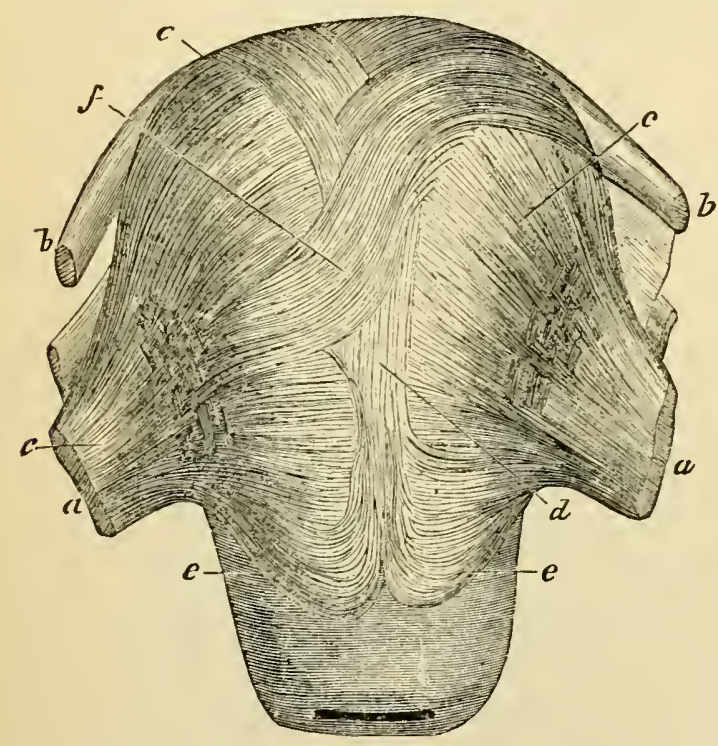

Fig. 201. - Superficial muscular fibres of the anterior surface of the uterus (Liégeois).

$a, a$, round ligaments; $b, b$, Fallopian tubes; $c, c, c, e, e$, transverse fibres; $d, f$, longitudinal fibres. $\frac{1}{3} \overline{0}$ of an inch $(72 \mu)$ in diameter. Their secretion, which forms a thin layer of mucus on the surface of the membrane in health, is grayish, viscid and feebly alkaline. The tubes themselves have very thin structureless walls and are lined with cylindrical ciliated epithelial cells.

The changes which the mucous membrane of the body of the uterus undergoes during menstruation are remarkable. Under ordinary conditions its thickness is $\frac{1}{25}$ to $\frac{1}{14}$ of an inch ( 1 to I. 8 millimeters); but it measures during the menstrual period $\frac{1}{6}$ to $\frac{1}{4}$ of an inch (4.2 to 6.4 millimeters).

In the cervix the membrane is paler, firmer and thicker than the membrane of the body of the uterus, and between these two mucous surfaces there is a distinct line of demarcation. It is more loosely attached to the subjacent tissue in the cervix, and the anterior and posterior surfaces of the neck present an appearance of folds radiating from the median line, forming what has been called the arbor vitæ uteri, or plicæ palmatæ. Throughout the cervical membrane, are mucous glands, and in addition, in the lower portion, are a few rounded, semitransparent, closed follicles, called the ovules of Naboth, which are 
cystic enlargements of obstructed follicles. The upper half of the cervical membrane is smooth but the lower half presents a large number of villi. The epithelium of the cervix presents great variations in its character in different individuals. Before puberty the entire membrane of the cervix is covered with ciliated epithelium. After puberty, however, the epithelium of the lower portion changes its character, and there are cylindrical cells above, with squamous cells in the inferior portion. The latter extend upward in the neck for a variable distance.

The bloodvessels of the uterus are very large and present certain important peculiarities in their arrangement. The uterine arteries pass between the layers of the broad ligament, to the neck, and then ascend by the sides of the uterus, presenting a rich plexus of vessels, anastomosing above with branches from the ovarian arteries, sending branches over the body of the uterus, and finally penetrating the organ, to be distributed mainly in the middle layer of muscular fibres. In their course these vessels present a convoluted arrangement and form a sort of mould of the body of the uterus. Rouget has called this the erectile tissue of the internal generative organs. It lacks, however, certain of the characters of true erectile tissue.

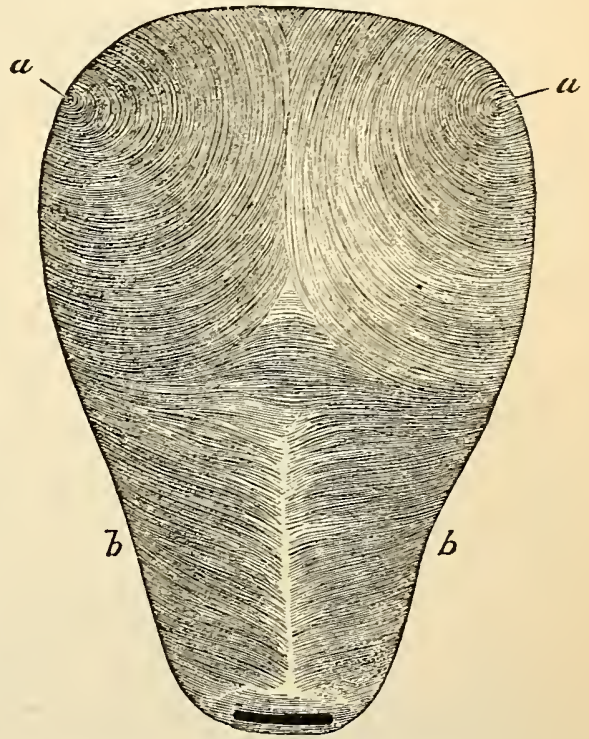

Fig. 202. - Inner layer of muscular fibres of the uterus (Liégeois).

By placing the pelvis in a bath $a$, $a$, rings around the openings of the Fallopian tubes; of warm water and injecting

what he called the spongy bodies of the ovaries and uterus by the ovarian veins, he produced a distention of the vessels and a sort of erection, the uterus executing a movement upward.

In the muscular walls of the uterus, are large veins, the walls of which are closely adherent to the uterine tissue. During gestation these vessels become enlarged, forming the so-called uterine sinuses.

Lymphatics are not very abundant in the unimpregnated uterus, but they become largely developed during utero-gestation. They exist in a superficial and a deep layer, the deeper vessels being connected with lymph-spaces in the muscular walls and in the mucous membrane.

The uterine nerves are derived from the inferior hypogastric and 
the spermatic plexuses and the third and fourth sacral. In the substance of the uterus they present in their course small collections of ganglionic cells, and it is said that the nerves pass finally to the nucleoli of the muscular fibres (Frankenhaeuser).

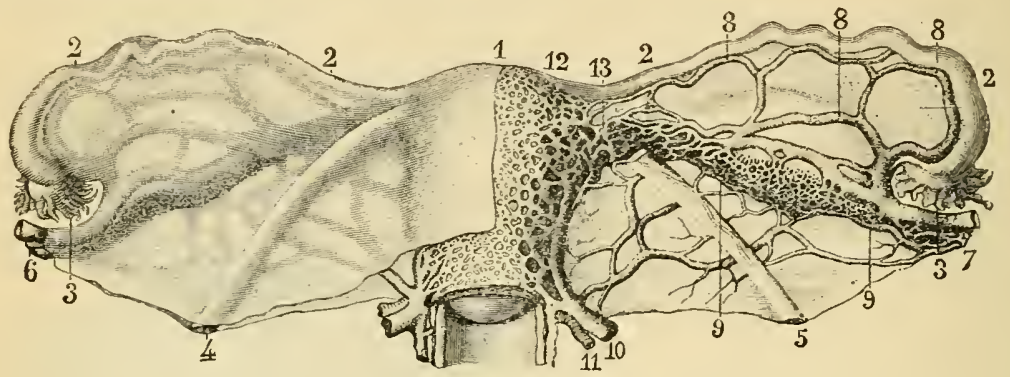

Fig 203. - Uterine and utero-ovarian veins (Sappey).

I, Anterior face of the uterus, the right side covered with peritoneum - on the left side the uteroovarian plexus is exposed; 2, 2, 3, 3, Fallopian tubes; 5,5 , round ligaments; 6 , utero-ovarian veins covered with peritoneum; 7 , same vessels exposed; $8,8,8$, veins in the broad ligament; 9 , plexus at the hilum of the ovary; Io, uterine vein; II, uterine artery; I2, venous plexus covering the uterus; 13, anastomoses of the uterine with the utero-ovarian veins.

The Fallopian Tubes. - The Fallopian tubes, or oviducts, lead from the ovaries to the uterus. They are three to four inches ( 7.6 to IO.I centimeters) long, but their length is not always equal on the two sides. They lie between the folds of the broad ligament at its upper border. Opening into the uterus on either side at the cornua, they present each a

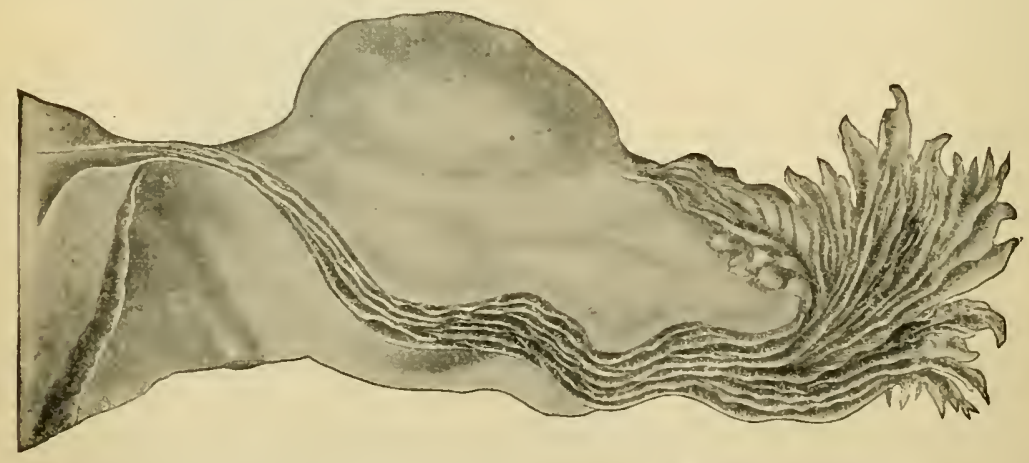

Fig. 204. - Section through the left Fallopian tube (Williams, after Sappey).

small orifice, about $\frac{1}{25}$ of an inch (I millimeter) in diameter. From the cornua they take a somewhat undulatory course outward, gradually increasing in size, so that they are rather trumpet-shaped. Near the ovary they turn downward and backward. The extremity next the ovary is marked by ten to fifteen fimbriæ, or fringes, which have given this the name of the fimbriated extremity, or morsus diaboli. All 
these fringe-like processes are free except one; and this one, which is longer than the others, is attached to the outer angle of the ovary and presents a little gutter, or furrow, extending from the ovary to the opening of the tube. At this extremity, is the abdominal opening of the tube, which is two or three times larger than the uterine opening. Passing from the uterus, the calibre of the tube gradually increases as the tube itself enlarges, and there is an abrupt constriction at the abdominal opening.

Beneath the peritoneal coat, which is formed by the layers of the broad ligament, is a layer of connective tissue, containing a rich plexus

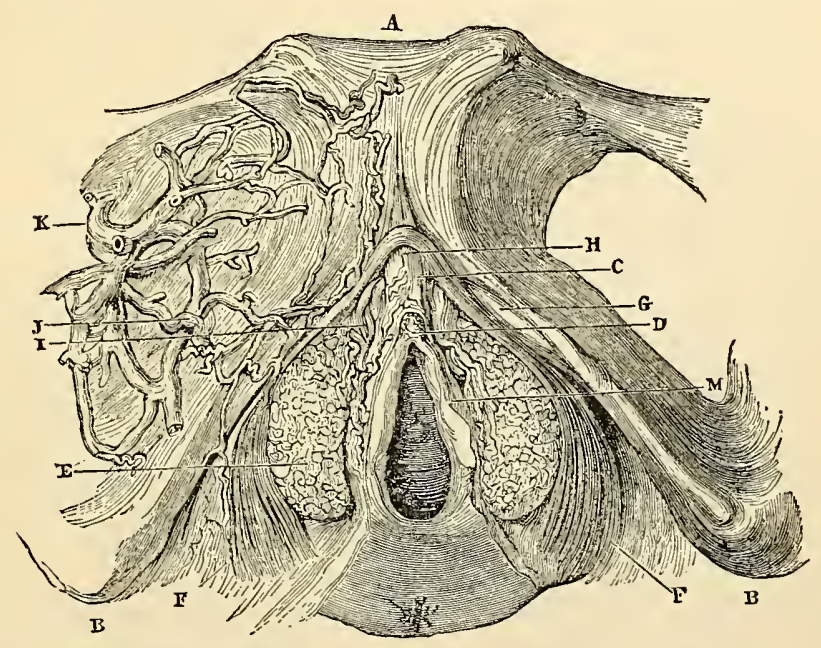

Fig. 205. - External erectile organs of the female (Liégeois).

$A$, pubis; $B, B$, ischium; $C$, clitoris; $D$, gland of the clitoris; $E$, bulb; $F$, constrictor muscle of the vulva; $G$, left pillar of the clitoris; $H$, dorsal vein of the clitoris; $I$, intermediary plexus; $J$, vein of communication with the obturator vein; $K$, obturator vein ; $M$, labia minora.

of bloodvessels. This constitutes the proper fibrous coat of the Fallopian tubes.

The muscular layer is composed mainly of circular fibres of the nonstriated variety, with a few longitudinal fibres prolonged over the tube from the external muscular layer of the uterus. This coat is quite thick and sends bands between the layers of the broad ligament to the ovary.

The mucous membrane of the tube is thrown into folds, which are longitudinal and transverse near the uterus and are more complicated at the dilated portion. In this portion, next the ovary, embracing about the outer two-thirds, the folds project far into the calibre of the tube. These are sometimes simple, but more frequently they present secondary folds, often meeting as they project from opposite sides. This arrangement gives an arborescent appearance to the membrane on transverse 
section of the tube. The mucous membrane is covered with cylindrical ciliated epithelium, the movement of the cilia being from the ovary toward the uterus. The membrane of the tubes has no mucous glands (see Plate X, Fig. 5).

It is not necessary to give a minute description of the external organs of the female. Opening by the vulva externally, and terminating at the neck of the uterus, is a membranous tube, the vagina. This lies between the bladder and the rectum. It has a curved direction, being $3 \frac{1}{6}$ to $3 \frac{1}{2}$ inches ( 8 to 9 centimeters) long in front, and $3 \frac{1}{3}$ to 4 inches ( 9 to io centimeters) long posteriorly. At the constricted portion of the outer opening, there is a muscle, called the sphincter vagina, and the tube is somewhat narrowed at its upper end, where it embraces the cervix uteri. The inner surface presents a mucous membrane, marked by transverse rugæ, with papillæe and mucous glands. Its surface is covered with stratified epithelium in several layers. The vagina is quite extensible, as it must be during parturition to allow the passage of the child. It presents a proper coat of dense fibrous tissue, with longitudinal and circular muscular fibres of the non-striated variety. Surrounding it, is a rather loose so-called erectile tissue, which is most prominent at its lower portion.

The parts composing the external organs are abundantly supplied with vessels and nerves. In the clitoris, which corresponds to the penis of the male, and on either side of the vestibule, there is a true erectile tissue.

Structure of the Ovum. - The ovum lies in the Graafian follicle, embedded in the mass of granular cells which forms the discus proligerus. Surrounding the ovum are cells similar to those found in other parts of the membrana granulosa, and two or three layers of columnar cells, the latter lying next the zona pellucida. These columnar cells constitute the corona radiata. The ovum itself presents the following structures: ( I) Zona pellucida; (2) perivitelline space; (3) a clear outer zone of the vitellus; (4) protoplasmic zone (formative yolk); (5) deutoplasmic zone (nutritive yolk); (6) germinal vesicle (Purkinje); germinal spot (Wagner). The thin membrane within the zona pellucida and immediately surrounding the vitellus, described under the name of vitelline membrane by some anatomists, was not observed by Nagel in the human ovum.

The ovum is globular, with a diameter of about $\frac{1}{150}$ of an inch ( 165 to $\mathrm{I} 7 \mathrm{O} \mu$ ) measured from the outer border of the zona pellucida.

The zona pellucida (zona radiata, or vitelline membrane) is $\frac{1}{1250}$ to $\frac{1}{1000}$ of an inch ( 20 to $24 \mu$ ) in thickness. It is a strong membrane, appearing in the form of a clear zone in the mass of surrounding cells. 
It is marked by striæ, which are thought by some anatomists to indicate the presence of small pores; but the large single opening called a micropyle, which is found in many of the osseous fishes and in mollusks, has not been demonstrated in the human ovum.

Between the zona pellucida and the vitellus, is a narrow space, about $\frac{1}{20000}$ of an inch $(1.3 \mu)$ in diameter. This is called the perivitelline space.

The vitellus is contained within the zona pellucida. It presents a clear outer zone $\frac{1}{6000}$ to $\frac{1}{4000}$ of an inch $(4$ to $6 \mu)$ in diameter. This can not be distinguished from the protoplasmic zone, except in perfectly fresh

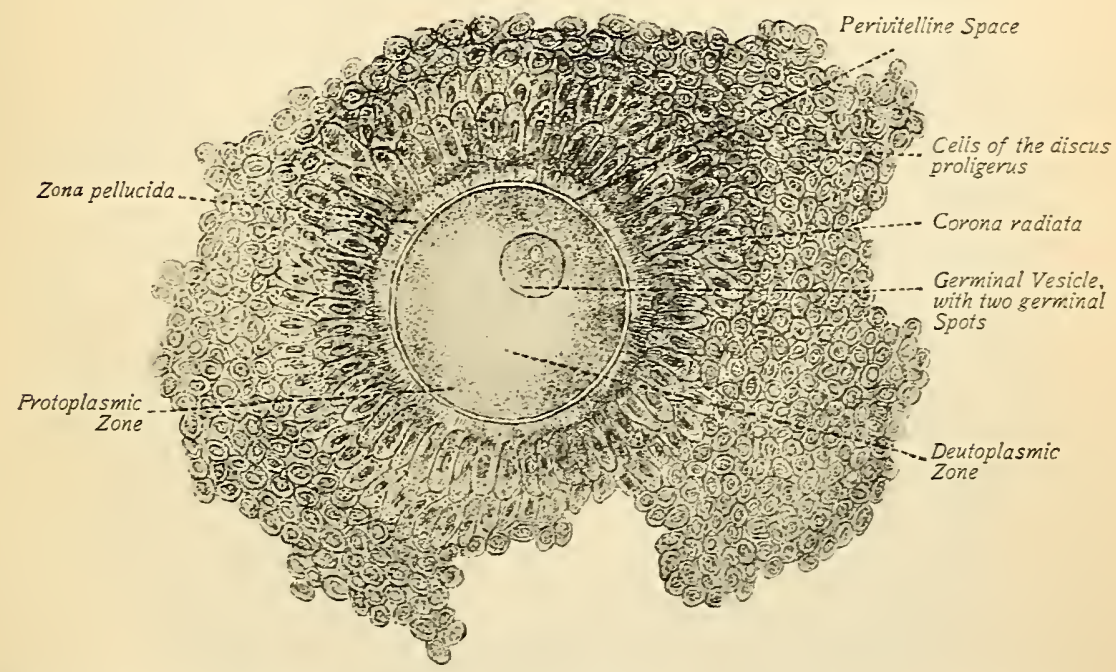

Fig. 206.-Deutoplasm-forming ovum from a Graafian follicle of a woman twenty-seven years old, X I6o (Nagel).

This ovum was taken from a fresh ovary removed from the living subject.

ova. It is composed of clear protoplasm without granules and represents that portion of the protoplasm of the vitellus which is not at any time converted into deutoplasm. Within the clear zone, is the protoplasmic zone (formative yolk). This presents very fine granules, and the zone is $\frac{1}{2} \overline{0} \overline{0}$ to $\frac{1}{12} \overline{0}$ of an inch (IO to 2 I $\mu$ ) in thickness. Occupying the central portion of the vitellus, is the deutoplasm (nutritive yolk), forming a mass about $\frac{1}{30} \overline{0}$ of an inch $(82$ to $87 \mu)$ in diameter. The deutoplasm presents granules of different sizes and different refractive power. Treated with eosin, the protoplasm becomes rose-colored, but the deutoplasm is unaffected. As the ovum reaches its final stage of development, the protoplasmic zone, as far as that portion which forms the so-called outer zone, is gradually changed into deutoplasm. In 
Fig. 206, a human ovum is represented in which this change of protoplasm into deutoplasm is in progress.

The germinal vesicle lies always in the protoplasmic zone, just outside of the deutoplasm. As the mass of deutoplasm extends, the

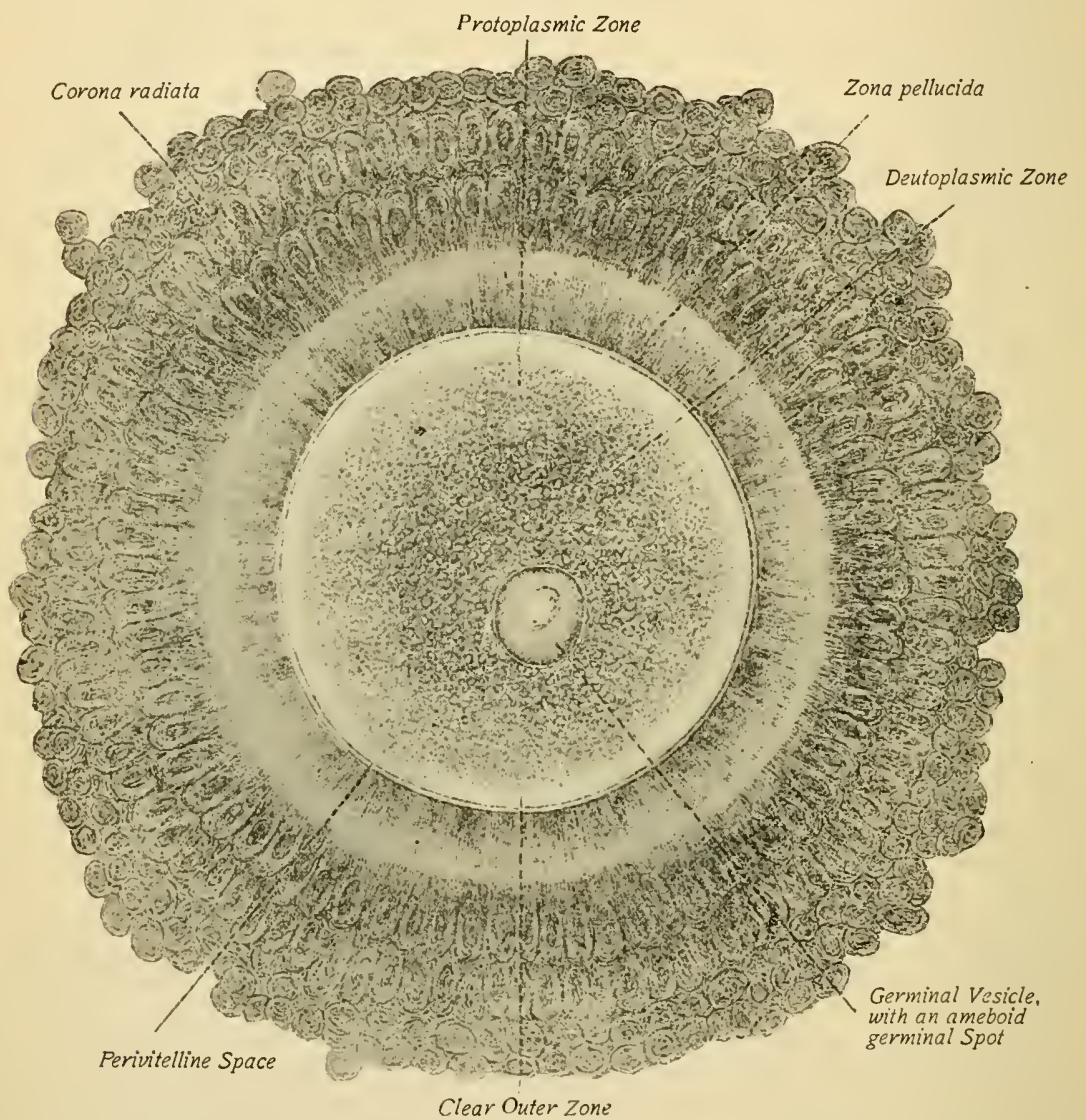

Fig. 207. - Ovum from a Graafian follicle of a woman thirty years old, $\times 375$ (Nagel).

This ovum was taken from a fresh ovary removed from the living subject.

germinal vesicle is pushed toward the periphery of the vitellus. The vesicle measures about $\frac{1}{1000}$ of an inch $(25$ to $27 \mu)$ in diameter. It is globular, with a double contour. In hardened preparations it presents a frame-work of fine anastomosing fibres. In the fully-cleveloped human ovum, no ameboid movements have as yet been observed in the germinal vesicle. In Fig. 207, the germinal vesicle is seen lying upon and not within the deutoplasmic zone. The mature ovum presents but one germinal vesicle. Two germinal vesicles, however, are sometimes found in primordial ova (see Fig. 208). 
The germinal spot (Wagner) is contained in the germinal vesicle. Some vesicles present two germinal spots. In perfectly fresh ova the germinal spots have been observed to undergo ameboid movements.

Discharge of the Ovum. - A ripe Graafian follicle measures $\frac{2}{5}$ to $\frac{1}{2}$ of an inch (IO to 12 millimeters) in diameter, and presents a rounded elevation, containing a plexus of bloodvessels, on the surface of the ovary. At its most prominent portion, is an ovoid spot in which the membranes are entirely free from bloodvessels. At this spot, which is called the macula folliculi, or stigma, the coverings finally give way and the contents of the follicle are discharged. For a short time anterior to the rupture of the follicle important changes have been going on in its structure. In the first place, the portion situated at the very surface of the ovary undergoes degeneration, by which this part of the wall gradually becomes weakened. At the same time, at the other portions of the follicle, there is a proliferation of cells which project into the interior, and an extension into the interior of bloodvessels in the form of loops. These changes, with an increase in the pressure of liquid and the degeneration at the macula, cause the follicle to burst; and with the liquid, the discus proligerus and the ovum are expelled. The formation of a cell-growth in the interior of the follicle is the beginning of the corpus luteum; and this occurs some time before the discharge of the ovum takes place.

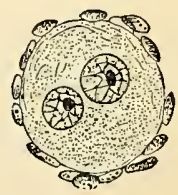

Fig. 208. - Primordial ovum with two germinal vesicles and follicular epithe. lium - from the ovary of a newborn child (Nagel).

The time at which the follicle ruptures, particularly with reference to the menstrual period, is not definite; but it is certain that while sexual excitement probably hastens the discharge of an ovum by producing a greater or less tendency to congestion of the internal organs, ovulation takes place independently of coition. The opportunities for determining this fact in the human female are not frequent; but it has been fully demonstrated by observations on the inferior animals, and there is now no doubt in regard to the identity of the phenomena of rut and of menstruation. At stated periods marked by the phenomena of menstruation, one Graafian follicle - and sometimes more than onebecomes distended and usually ruptures and discharges its contents into the Fallopian tubes. This discharge of an ovum or ova may occur at the beginning, at the end, or at any time during the continuance of the menstrual flow. On this point the observations of Coste seem entirely conclusive. In a woman who died on the first day of menstruation, he found a recently-ruptured follicle; in other instances, at a more advanced period and toward the decline of the menstrual flow, he found evidences that the rupture had occurred later; in the case of a female 
who drowned herself four or five days after the cessation of the menses, a follicle was found in the right ovary, so distended that it was ruptured by very slight pressure; and other instances were observed in which follicles were not ruptured during the menstrual period.

\section{Passage of Ova into the Fallopian Tubes}

The fact that the ova in the great majority of instances pass into the Fallopian tubes is sufficiently evident. The fact, also, that ova may fall into the cavity of the peritoneum is illustrated by the occasional occurrence of extra-uterine pregnancy, a rare accident, which shows that in all probability the failure of unimpregnated ova to enter the tubes is exceptional. As regards the mechanism of the passage of the ova into the tubes, however, the explanation is difficult. At the present time there are two theories in regard to this process; one, in which it is supposed that the fimbriated extremities of the Fallopian tubes, at the time of rupture of the Graafian follicles, become adapted to the surface of the ovaries, and the other, that the ova are carried to the openings of the tubes, by ciliary currents. Neither of these theories, however, is susceptible of actual demonstration; and their value is to be judged from anatomical facts. It is not difficult to understand, taking into account the situation of the ovaries and the relations of the Fallopian tubes, how an ovum may pass into the tube, without invoking the aid of muscular action. It may be supposed, for example, that a Graafian follicle is ruptured when the fimbriated extremity of the tube is not applied to the surface of the ovary. One of the fimbriæ, longer than the others, is attached to the outer angle of the ovary and presents a little furrow, or gutter, leading to the opening of the tube. This furrow is lined with ciliated epithelium, as, indeed, is the mucous membrane of all the fimbriæ, the movements of which produce a current in the direction of the opening, which would apparently be sufficient to carry the ovum into the tube. At the same time there probably is a constant flow of liquid over the ovarian surface, directed by the ciliary current toward the tube; and when the liquid of the ruptured follicle is discharged this, with the ovum, takes the same course. This probably is the mechanism of the passage of ova into the Fallopian tubes; and it is possible that the fimbriated extremity may be drawn toward the ovarian surface, although it is difficult to understand how it can be closely applied to the ovary and exert any considerable pressure on the distended follicle. It is proper to note, also, that the conditions dependent on the currents of liquid directed by the movements of cilia are constant and could influence the passage of an ovum at whatever time it might be discharged, while a 
muscular action would be more or less intermittent. The time occupied in the passage of an ovum from the ovary to the uterus has been estimated at four to eight days.

Puberty and Menstruation. - At a certain period of life, usually between the ages of thirteen and fifteen, the human female undergoes a remarkable change and arrives at what is termed the age of puberty. At this time there is a marked increase in the general development of the body; the limbs become fuller and more rounded; a growth of hair makes its appearance upon the mons Veneris; the mammary glands increase in size and take on a new stage of development; Graafian follicles enlarge, and one or more approach the condition favorable to rupture and the discharge of ova. The female becomes capable of impregnation, and continues so, in the absence of pathological conditions, until the cessation of the menses.

The age of puberty is earlier in warm than in cold climates; and many instances are on record in which the menses have appeared exceptionally much before the usual period. Usually, at the age of forty or forty-five, the menstrual flow becomes irregular, occasionally losing its sanguineous character, and it ceases at about the age of fifty years. The time of cessation of the menses is called the menopause, climacteric, or change of life. Ova then are no longer developed and discharged, and impregnation does not follow intercourse. It is said, however, that sometimes the menses return, with a second period of fecundity, although this is rare. According to most writers, while climate has a certain influence over the time of cessation as well as the first appearance of the menses, this is not very marked. When the menses appear early in life, they usually cease at a correspondingly early period; but this is by no means constant. There are, also, many exceptions to the ordinary limits to the period of fecundity. An instance of childbirth at the age of sixty-three years, with menstruation following at the usual time, has been reported by Kennedy.

In the human female, near the time of puberty, there sometimes is a periodical sero-mucous discharge from the genital organs, preceding, for a few months, the regular establishment of the menstrual flow. Sometimes, also, after the first discharge of blood, the female passes several months without another period, when the second flow takes place and the menses become regular. In a condition of health the periods recur every month until they cease at the climacteric. In the majority of cases the flow recurs on the twenty-seventh or the twenty-eighth day; but sometimes the interval is thirty days. As a rule, also, utero-gestation, lactation, and severe diseases, acute and chronic, suspend the periods; but this has exceptions, as some females menstruate regularly during 
pregnancy, and it is not very uncommon for the menses to appear during lactation.

When a cow gives birth to twins, one a male and the other apparently a female, the latter is called a free-martin and has no ovaries. John Hunter, in his paper on the free-martin, gave a full description of this anomalous animal and stated that it does not breed or show any inclination for the bull. In an examination of a free-martin, raised and killed by the late Professor James R. Wood, in $\mathrm{I} 868$, the uterus was found rudimentary and there were no ovaries (Flint).

A menstrual period presents three stages: first, invasion; second, a sanguineous discharge; third, cessation.

The stage of invasion is variable in different females. There is usually, anterior to the establishment of the flow, more or less of a feeling of general malaise and a sense of fulness and weight in the pelvic organs, accompanied with an increase in the quantity of vaginal mucus, which becomes brownish or rusty in color and has a peculiar odor. At this time, also, the breasts become slightly enlarged. This stage may continue for one or two days, although in many instances the first evidence of the access of a period is a discharge of blood.

When the symptoms above indicated occur, the general sense of uneasiness usually is relieved by the discharge of blood. During this, the second stage, blood flows from the vagina in variable quantity, and the discharge continues for three to five days. In regard to the duration of the flow, there are great variations in different individuals. Some women have a flow of blood for one or two days only; while in others the flow continues for five to eight days, within the limits of health. A fair average, perhaps, is four days. It is difficult to arrive at even an approximation of the total quantity of the menstrual flow; but it has been estimated at five to six ounces (I 50 to I 75 grams).

Supposing the menstrual discharge to continue for four days, on the first day the quantity is comparatively small; on the second and third the flow is at its height; and the quantity is diminished on the fourth day. During this, the second stage, the flow has the appearance of pure arterial blood, not coagulated, and mixed with epithelium from the vagina, cylindrical cells from the uterus, leucocytes and a certain quantity of sero-mucous secretion. Chemical examinations of the liquid have not shown any marked peculiarities, except that the quantity of fibrin is either not estimated or is given as much less than in ordinary blood.

The mechanism of the hemorrhage probably is the same as in epistaxis. There is a rupture of small bloodvessels, probably capillaries, and blood is thus exuded from the entire surface of the membrane lining the uterus, and sometimes, but rarely, from the membrane of the Fallopian 
tubes. The blood is then discharged into the vagina and is kept liquid by the vaginal mucus. The mucus of the body of the uterus is viscid and alkaline; the mucus secreted at the neck is gelatinous, viscid and tenacious, and also is alkaline; the vaginal mucus is decidedly acid, creamy and not viscid, containing epithelium and leucocytes.

The third stage is that of cessation of the menses. During the latter part of the second stage, the flow of blood gradually diminishes. The discharge becomes rusty, then lighter in color, and in the course of about twenty-four hours, it assumes the characters observed in the intermenstrual period.

When the menstrual flow has become fully established, there is no marked general disturbance, except a sense of lassitude, which may become exaggerated if the discharge be unusually abundant.

If the mucous membrane of the uterus is examined during the menstrual flow, it is found smeared with blood, which sometimes extends into the Fallopian tubes. It is then much thicker and softer than during the intermenstrual period. Instead of measuring about $\frac{1}{14}$ of an inch (I.8 millimeters) in thickness, as it does under ordinary conditions, its thickness is $\frac{1}{6}$ to $\frac{1}{4}$ of an inch ( 4.2 to 6.4 millimeters). It becomes more loosely attached to the subjacent parts, is somewhat rugous and the glands are enlarged. At the same time there are developed, in the substance of the membrane, large numbers of spherical and fusiform cells. This condition probably precedes the discharge of blood by several days, during which time the membrane is gradually preparing for the reception of the ovum. There also is a degeneration of the different elements entering into the structure of the mucous membrane, including the bloodvessels, this change being most marked at the surface; and it is on account of the weakened condition of the vascular walls that the hemorrhage takes place. A short time after the flow has ceased, the mucous membrane returns to its ordinary condition. There is a considerable desquamation of epithelium from the uterus, with the flow of blood, during the menstrual period. Sometimes, in normal menstruation, the epithelium thrown off is in the form of patches.

Changes in the Graafian Follicles after their Rupture (Corpus Luteum). - After the discharge of an ovum, its Graafian follicle undergoes certain retrograde changes, involving the formation of what is called the corpus luteum. Even when the discharged ovum has not been fertilized, the corpus luteum persists for several weeks, so that, ovulation occurring every month, several of these bodies, in different stages of retrogression, may sometimes be seen in the ovaries.

For a certain time anterior to the discharge of the ovum, there is a cell-proliferation from the proper coat of the Graafian follicle, and prob- 
ably from the membrana granulosa, with a projection of looped bloodvessels into the interior of the follicle. This is the first formation of the corpus luteum. At the time of rupture of the follicle, the ovum, with a great part of the membrana granulosa, is discharged. Usually, at the time of rupture of the follicle, there is a discharge of blood into its interior; but this is not invariable, although there is always a gelatinous exudation more or less colored with blood. At the same time the follicular wall undergoes hypertrophy, and it becomes convoluted or folded, and highly vascular. This convoluted wall, formed by the proper coat of the follicle, is surrounded with the fibrous tunic, and its thickening is most marked at the deepest portion of the follicle. At the end of about three weeks, the body - which is now called the corpus luteum, on account of its yellowish or reddish yellow color - has arrived at its maximum of development and measures about half an inch (I 2.7 millimeters) in depth, by about three-quarters of an inch (I9. I millimeters) in length, its form being ovoid. The convoluted wall then contains a layer of large, pale, finely-granular cells, which are internal and are supposed to be the remains of the epithelium of the follicle. The great mass of this wall, however, is composed of large nucleated cells (leutein cells), containing fatty globules and granules of reddish or yellowish pigmentary matter. The thickness of the wall is about one-eighth of an inch ( 3.2 millimeters) at its deepest portion.

After about the third week the corpus luteum begins to contract; its central portion and the convoluted wall become paler; and at the end of seven or eight weeks, a small cicatrix marks the point of rupture of the follicle.

The above are the changes which occur in the Graafian follicles after their rupture and the discharge of ova, when the ova have not been fertilized; and the bodies thus produced are called false corpora lutea, as distinguished from corpora lutea formed after conception, which latter are called true corpora lutea.

Corpus Luteum of Pregnancy. - When a discharged ovum has been fertilized, the corpus luteum passes through its various stages of development and retrogression more slowly than the ordinary corpus luteum of menstruation. The retrogression begins toward the end of the third month. "During the fourth month, the corpus luteum diminishes by nearly a third, and toward the end of the fifth, it ordinarily is reduced one-half. It still forms, however, during the first days after parturition, and in the greatest number of cases, a tubercle which has a diameter of not less than $\frac{2}{7}$ to $\frac{1}{3}$ of an inch $(7.3$ to 8.5 millimeters $)$. The tubercle afterward diminishes quite rapidly ; but it is nearly a month before it is reduced to the condition of a little, hardened nucleus, which persists 
more or less as the last vestige of a process so slow in arriving at its final term. Nevertheless, there is nothing absolute in the retrograde progress of this phenomenon. I have seen women, dead at the sixth and even the eighth month of pregnancy, present corpora lutea as voluminous as others at the fourth month" (Coste, 1849).

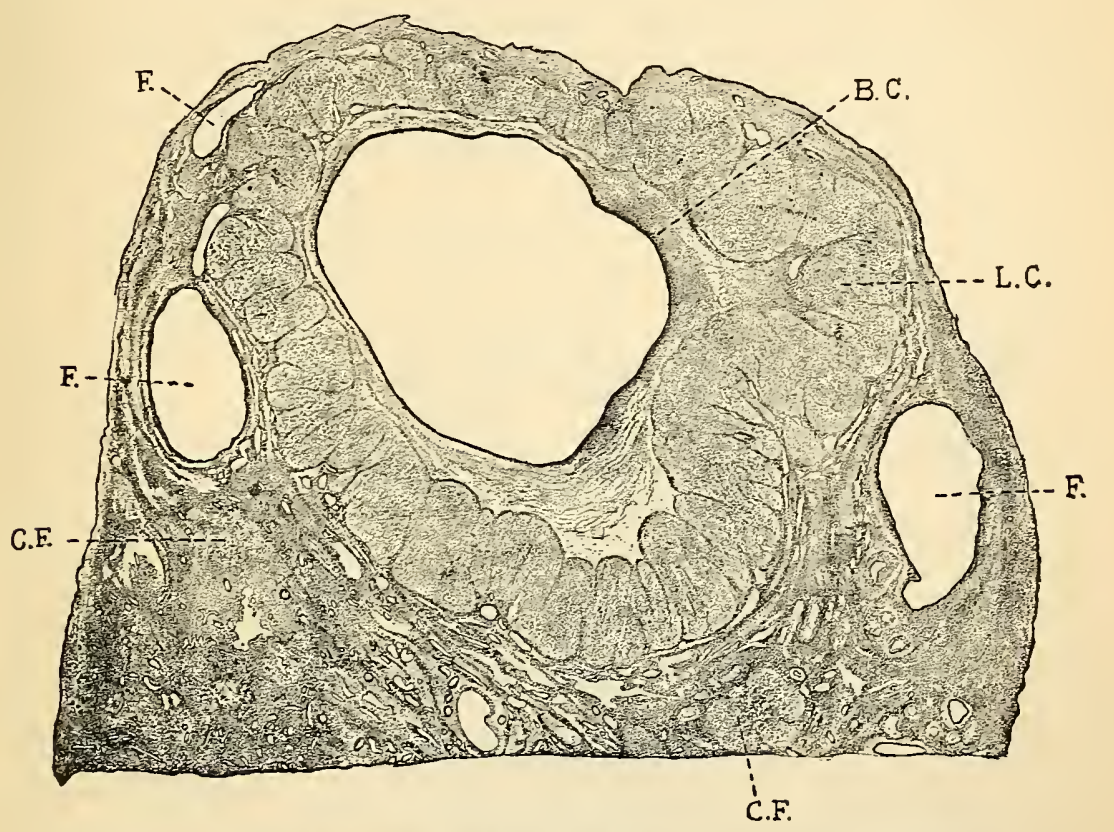

Fig. 209. - Portion of an ovary, showing a corpus luteum of pregnancy (Williams). luteum.

$B, C$, blood-clot; $C, F, C, F$, corpus petrosum; $F, F, F$, Graafian follicles; $L, C$, folds of corpus

\section{Male Organs of Generation}

The chief physiological interest attached to the anatomy of the male organs of generation relates to the testicles, which are the organs in which the male element of generation is developed. As regards the penis, it will be necessary to do little more than describe the mechanism of erection and of the ejaculation of semen.

The Testicles. - The testicles are two symmetrical organs, situated, during a certain period of intra-uterine life, in the abdominal cavity, but finally descending into the scrotum. Immediately beneath the skin of the scrotum, is a loose, reddish, contractile tissue, called the dartos, which forms two distinct sacs, one enveloping either testicle, the inner portion of these sacs fusing in the median line, to form a septum. Within these two sacs the coverings of each testicle are distinct. These organs are suspended in the scrotum by the spermatic cords, the left 
usually hanging a little lower than the right. The coverings for each testicle, in addition to those just mentioned, are the intercolumnar fascia, the cremaster muscle, the infundibuliform fascia, the tunica vaginalis and the proper fibrous coat.

The tunica vaginalis is a closed sac of serous membrane, covering the testicle and epididymis and reflected from the posterior border of the testicle to the wall of the scrotum, lining the cavity occupied by the testicle on either side and also extending over the spermatic cord. This tunic is a process of peritoneum that has been shut off from the general lining of the abdominal cavity. The spermatic cord is composed of the vas deferens, bloodvessels, lymphatics and nerves, with the coverings already described, which expand and surround the testicle.

Beneath the tunica vaginalis are the testicles, with their proper fibrous coat. These organs are ovoid and flattened laterally and posteriorly. "They are an inch and a half to two inches (38. I to 50.8 millimeters) long, about an inch and a quarter (31.8 millimeters) from the anterior to the posterior border, and nearly an inch ( 25.4 millimeters) from side to side. The weight of each varies from three-quarters of an ounce to an ounce ( $2 \mathrm{I} .2$ to $28.3 \mathrm{grams}$ ), and the left is often a little the larger of the two" (Quain). The proper fibrous coat is everywhere covered with the closely-adherent tunica vaginalis, except at the posterior border, where the vessels enter and the duct passes out. At the outer edge of this border, is the epididymis, formed of convoluted tubes, presenting a superior enlargement, called the globus major, a long mass running the length of the testicle, called the body, and a smaller, inferior enlargement, called the globus minor. This too is covered with the tunica vaginalis. Between the membrane covering the testicle and epididymis and the layer lining the scrotal cavity, is a small quantity of serum, just enough to moisten the serous surfaces. At the superior portion of the testicle are one or more small ovoid bodies, called the hydatids of Morgagni, each attached to the testicle by short constricted processes. These have no physiological importance and are supposed to be the remains of fœtal structures.

The proper fibrous coat of the testicle is called the tunica albuginea. It is white, dense, inelastic, measures about $\frac{1}{25}$ of an inch (I millimeter) in thickness, and is simply for the protection of the contained structures. Sections of the testicle, made in various directions, show an incomplete vertical process of the tunica albuginea, called the corpus Highmorianum, or the mediastinum testis. This is wedge-shaped, about $\frac{1}{6}$ of an inch (4.2 millimeters) wide at its superior and thickest portion, is pierced by a number of openings and lodges bloodvessels and the seminiferous 
tubes. From the mediastinum, delicate radiating processes of connective tissue pass to the inner surface of the tunica albuginea, dividing the substance of the testicle into imperfect lobules which lodge the seminiferous tubes. The number of these lobules has been estimated at two hundred and fifty to four hundred. Their shape is pyramidal, the larger extremities presenting toward the surface, with the pointed extremities situated at the mediastinum.

Lining the tunica albuginea and following the mediastinum and the processes which penetrate the testicle, is a tunic, composed of blood-

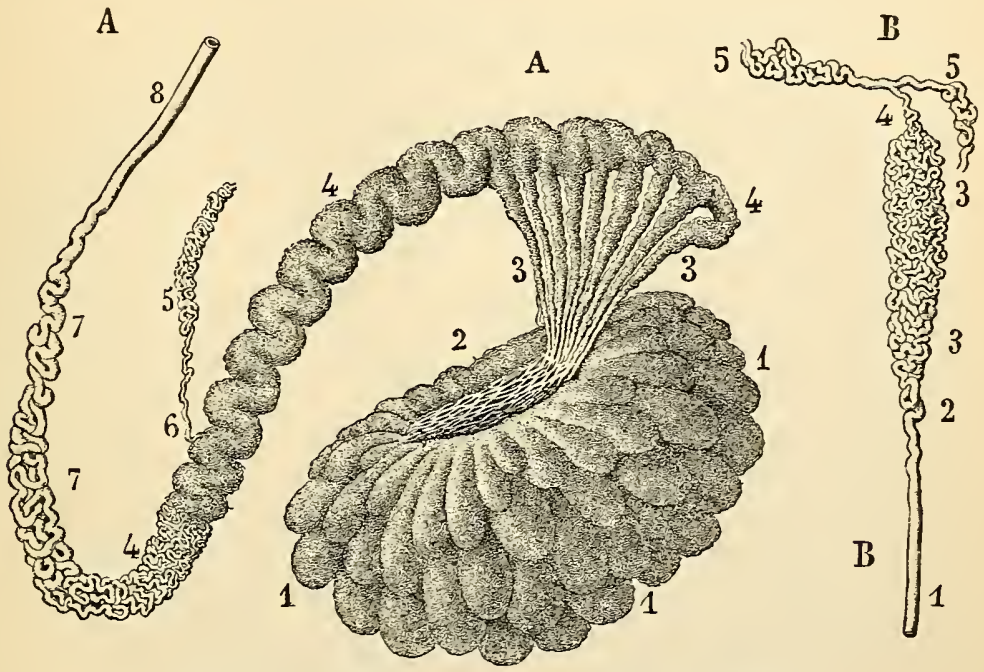

Fig. 210. - Lobes of the testicle and epididymis (Sappey).

$A, A, \mathrm{I}, \mathrm{I}, \mathrm{I}$, lobes of the testicle; 2 , rete testis; 3,3 , vasa efferentia; $4,4,4$, epididymis; 5 , vas aberrans; 6 , opening of the vas aberrans into the epididymis; 7,7 , convoluted beginning of the vas deferens; 8 , vas deferens. $B, B, \mathrm{r}$, beginning of one of the vasa efferentia; $2,3,3$, convoluted portion; 4 , opening into the epididymis; 5,5 , beginning of the epididymis.

vessels and delicate connective tissue, called the tunica vasculosa, or pia mater testis.

Lodged in the cavities formed by the trabeculæ of connective tissue, are the seminiferous tubes, in which the male elements of generation are developed (see Plate XV, Fig. 2). These tubes exist to the number of about eight hundred and forty in either testicle and constitute almost the entire substance of the lobules. The larger lobules may contain five or six tubes, the lobules of median size, three or four, and the smallest enclose sometimes but a single tube. Each tube presents a convoluted mass, which can be disentangled under water, particularly if the testicle is macerated for several months in water with a little nitric acid. The entire length of the tube when thus unravelled is about thirty inches 
( 7.6 decimeters), and its diameter is $\frac{1}{2} \frac{1}{0}$ to $\frac{1}{150}$ of an inch ( 25 to I $66 \mu$ ). It begins by two to seven short blind extremities and sometimes by anastomosing loops. The cæcal diverticula usually are found in the external half of the tube, and their. length is $\frac{1}{12}$ to $\frac{1}{8}$ of an inch (2.I to 3.2 millimeters). The anastomoses are sometimes between the tubes of different lobules, sometimes between tubes in the same lobule and sometimes between different points in the same tube. As the tubes pass toward the posterior part of the testicle, they unite into twenty to thirty straight canals, called the vasa recta, about $\frac{1}{50}$ of an inch $(0.5$ millimeter) in diameter, which penetrate the mediastinum testis. In the mediastinum the tubes form a close network, called the rete testis; and at the upper part of the posterior border they pass out of the testicle, by twelve to fifteen or twenty ducts, called vasa efferentia.

Having passed out of the testicle, the vasa efferentia form a series of small conical masses, which together constitute the globus major, or head of the epididymis. Each of these tubes when unravelled is six to eight inches ( 5 to 20 centimeters) long, gradually increasing in diameter, until they all unite into a single convoluted tube, which forms the body and the globus minor of the epididymis. This single tube of the epididymis, when unravelled, is about twenty feet ( 6 meters) in length.

The walls of the seminiferous tubes in the testicle itself are composed of connective tissue and of peculiar structures that will be fully described in connection with spermatogenesis. In the rete testis it is uncertain whether the tubes have a special fibrous coat or are simple channels in the fibrous structure. They are here lined with squamous epithelium. In the vasa efferentia and the epididymis, there is a fibrous membrane, with longitudinal and circular fibres of non-striated muscular tissue and a lining of ciliated epithelium. The movements of the cilia are toward the vas deferens. In the lower portion of the epididymis the cilia are absent. The tubular structures of the testicle, the epididymis and the beginning of the vas deferens are shown in Fig. 2 ro.

At the lower part of the epididymis, communicating with the canal, there usually is found a small mass, formed of a convoluted tube of variable length, called the vas aberrans of Haller (5, $A, A$, Fig. 210). This sometimes is wanting.

Interstitial Gland of the Testis. - Lying between the seminiferous tubes and apparently giving support to them, are collections of delicate fibres of areolar tissue with abundant bloodvessels, large cytoplasmic cells and sometimes fatty and pigmentary globules and granules. These structures are said by some anatomists to constitute an interstitial gland that influences the development of spermatozoids and may be the seat of an "internal secretion." 
Vas Deferens. - The excretory duct of the testicle extends from the epididymis to the prostatic portion of the urethra and is a continuation of the single tube that forms the body and globus minor of the epididymis. It is somewhat tortuous near its origin, and it becomes larger at the base of the bladder, just before it is joined by the duct of the seminal vesicle. Near its point of junction with this duct it becomes narrower. Its entire length is nearly two feet (about 6 decimeters).

The course of the vas deferens is in the spermatic cord to the external abdominal ring, and through the inguinal canal to the internal ring,

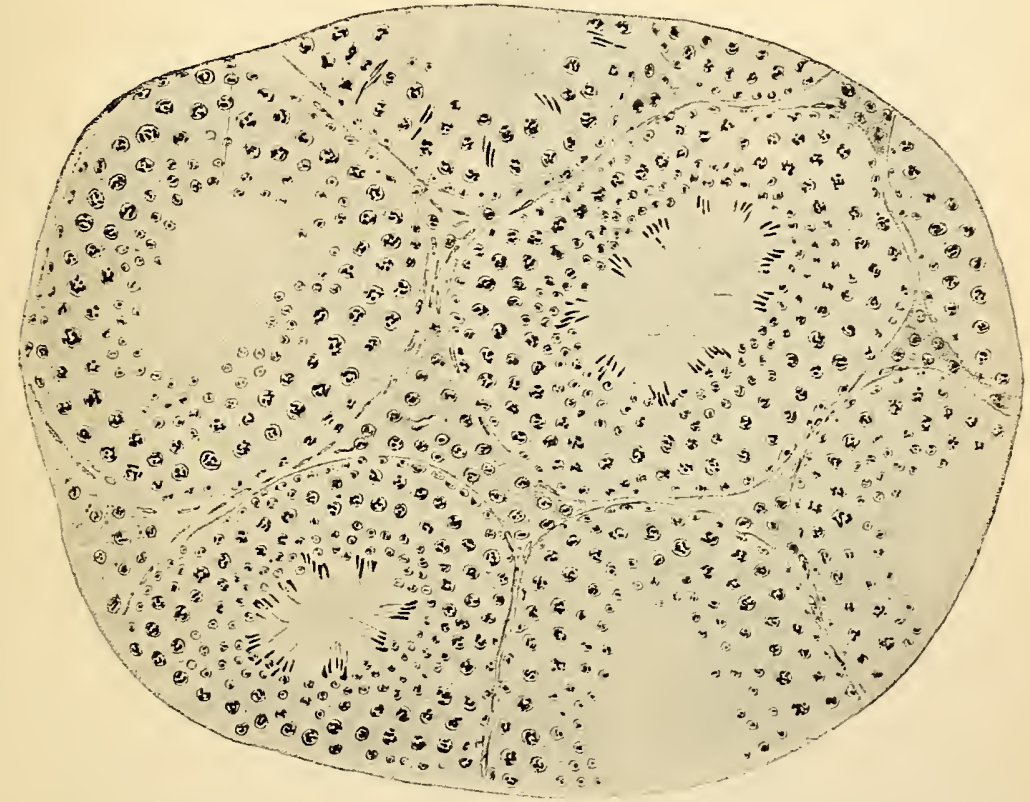

Fig. 2II. - Section of the testicle of a full-grown rabbit-Interstitial gland (Bouin and Ancel).

This figure shows the seminiferous tubes in three of which there is spermatogenesis. The darker cells between the tubes are cells of the interstitial gland.

where it leaves the bloodvessels, passes beneath the peritoneum to the side of the bladder, then along the base of the bladder by the inner side of the seminal vesicle, finally joining the duct of the seminal vesicle, the common tube forming the ejaculatory duct, which opens into the prostatic portion of the urethra.

The walls of the vas deferens are thick, abundantly supplied with vessels and nerves, and provided with an external fibrous, a middle muscular, and an internal mucous coat. The greater part of that portion of the tube which is connected with the bladder is dilated and sacculated. The fibrous coat is composed of strong connective tissue. 
The muscular coat presents three layers; an external, rather thick layer of longitudinal fibres, a thin middle layer of circular fibres, and a thin layer of longitudinal fibres, all of the non-striated variety. By the action of these fibres the vessel may be made to undergo energetic peristaltic movements, and this has followed stimulation of that portion of the spinal cord corresponding to the fourth lumbar vertebra, which is

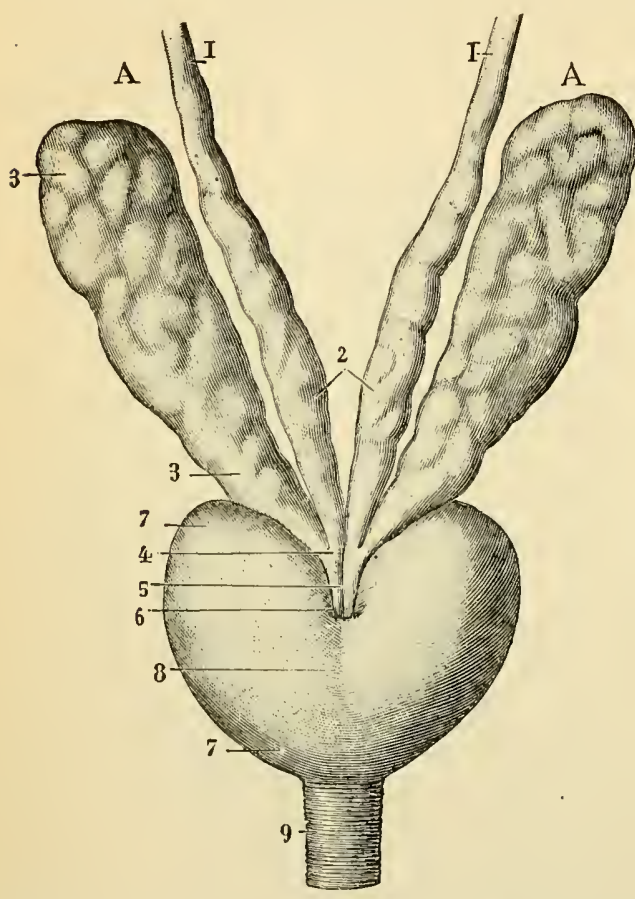

Fig. 212. - Vesicule seminales, vasa deferentia and ejaculatory ducts (Sappey).

$A, A$, vesicula seminales; I, I, 2, vasa deferentia; 3,3 right seminal vesicle; 4 , junction of the vas deferens with the duct of the seminal vesicle; 5,6 , ejaculatory ducts; 7 . left lobe of the prostate; 8 , median groove; 9 , membranous urethra. squamous epithelium, which often are filled with fatty granules. Usually the tubes present only blind extremities, but some of them occasionally communicate with the tubes of the epididymis. This part has no physiological importance. It was regarded by Giraldès as the remnant of the Wolffian body, analogous to the parovarium.

Vesicula Scminales. - Attached to the base of the bladder and situated externally to the vasa deferentia, are the two vesicula seminales. These bodies are each composed of a coiled and sacculated tube, four to six inches (IO to I 5 centimeters) in length when unravelled, and some- 
what convoluted, in the natural state, into an ovoid mass that is firmly bound to the vesical wall. The structure of the seminal vesicles is not unlike that of the sacculated portion of the vasa deferentia. They have an external fibrous coat, a middle coat of muscular fibres and a mucous lining. Muscular fibres pass over these vesicles from the bladder, both in a longitudinal and in a circular direction, and serve as compressors, by the action of which their contents may be discharged. The mucous coat is pale, finely reticulated, and covered with cells of polygonal epithelium which are nucleated and contain brownish granules. The vesicula seminales undoubtedly serve, in part at least, as receptacles for the semen, as their contents often present a greater or less number of spermatozoids. Although the membrane of the vesicles seems to produce an independent secretion, the presence of mucous glands has not been demonstrated.

The ejaculatory ducts are formed by the union of the vasa deferentia with the ducts of the vesiculæ seminales on either side, and they open into the prostatic portion of the urethra. Except that their coats are much thinner, they have essentially the same structure as the vasa deferentia.

Prostate. -- Surrounding the vesical extremity of the urethra, including what is known as its prostatic portion, is the prostate gland, or body. This organ, except as it secretes a liquid that forms a part of the ejaculated semen, has chiefly a surgical interest, so that it is unnecessary to describe minutely its form and relations. It is enveloped in a dense fibrous coat, contains many glandular structures opening into the urethra, and presents a great number of non-striated, with a few striated muscular fibres, some just beneath the fibrous coat and others penetrating its substance and surrounding the glands.

The glands of the prostate are most distinct in that portion which lies behind the urethra. In the posterior portion of this canal are found about twenty openings, which lead to tubes ramifying in the glandular substance. These tubes are formed of a structureless membrane branching as it penetrates the gland. They present hemispherical diverticula in their course and terminate in dilated extremities which are looped and coiled. In the deeper portions of the tubes, the epithelium is columnar or cuboidal, becoming tessellated near their openings, and sometimes laminated.

The prostatic secretion probably is produced only at the moment of ejaculation. Its characters will be considered in connection with the composition of the semen. According to Kraus it has an important office in maintaining the vitality of the spermatozoids. "The spermatozoa, in the absence of the prostatic fluid, can not live in the mucous 
membrane of the uterus of mammalia; but with its aid they may live for a long time in the uterine mucus, often more than thirty-six hours."

Glands of the Urethra. - In front of the prostate, opening into the bulbous portion of the urethra, are two small racemose glands, called the glands of Méry or of Cowper. These have each a single excretory duct, are lined throughout with cylindrical epithelium and secrete a viscid, mucus-like liquid which forms a part of the ejaculated semen. Sometimes there exists only a single gland, and occasionally, though rarely, both are absent. Their uses probably are not very important.

The glands of Littre, found throughout the urethra and most abundant on its anterior surface, are simple racemose glands, extending beneath the mucous membrane into the muscular structure, presenting here four or five acini. As these acini are surrounded with muscular fibres, it is easy to understand how their secretion may be pressed out during erection of the penis. They are lined throughout with columnar or conoidal epithelium, and secrete a clear and somewhat viscid mucus, which is mixed with the ejaculated semen.

\section{Male Elements of Generation}

The spermatozoids are the essential male elements of generation. They are produced in the testicle, by a process analogous to that of the development of other anatomical elements. The testicles can not be regarded strictly as glandular organs. They are analogous to the ovaries and are the only organs in which spermatozoids can be developed, as the ovaries are the only organs in which the ovum can be formed. If the testicles are absent, the power of fecundation is lost, none of the secretions of the accessory organs of generation being able to perform the office of the true fecundating elements.

In the healthy male, at the climax of a normal venereal orgasm, I I.6 to 92.6 grains ( 0.75 to 6 grams) of semen are ejaculated with considerable force from the urethra, by an involuntary muscular spasm. This liquid requires about four days for its complete restoration. It is slightly mucilaginous, grayish or whitish, streaked with lines more or less opaque, and it evidently contains various kinds of mucus. It has a faint and peculiar odor, sui generis, which is observed only in the ejaculated secretion and not in any of its constituents examined separately. It is a little heavier than water and does not mix with it or dissolve. After ejaculation it becomes jelly-like and dries into a peculiar hard mass, which may be softened by the application of appropriate liquids. The liquid is not coagulated by heat and does not contain 
albumin. Its reaction is faintly alkaline. It contains, in the human subject, IOO to I 20 parts of solid matter per IOOO.

The chemical constitution of the semen has not been thoroughly investigated and does not present the same physiological importance as do its anatomical characters. Aside from the anatomical elements derived from the testicles and the genital passages, it presents an organic substance (spermatin), which has nearly the same chemical characters as ordinary mucin. It contains also a considerable quantity of phosphates. During desiccation, elongated rhomboidal crystals make their appearance, frequently arranged in groups, which are supposed to be derived from the prostatic secretion and to consist of phosphoric acid combined with an organic base, the formula for which, united with hydrochloric acid, is $\mathrm{C}_{2} \mathrm{H}_{3} \mathrm{NHCl}$. These are sometimes called spermatic crystals.

In the dilated portion of the vasa deferentia, the mucous glands secrete a liquid which is the first that is added to the spermatozoids as they come from the testicles. This is brownish or grayish. It contains epithelium and small rounded granules, which latter are dark and strongly retractive. The liquid itself is very slightly viscid. In the vesiculæ seminales there is a more abundant secretion of grayish liquid, with epithelium, small colorless concretions of nitrogenous matter, called sympexions, and a few leucocytes. The glandular structures of the prostate produce a creamy secretion with fine granules. It is chiefly to the admixture of this liquid that the semen owes its whitish appearance. Finally, as the semen is ejaculated, it receives the viscid secretion of the glands of Cowper, a certain quantity of stringy mucus from the follicles of the urethra, with perhaps a little of the urethral epithelium.

Anatomically considered, the semen contains no important elements except the spermatozoids, the various secretions just mentioned serving simply as a vehicle for the introduction of these bodies into the generative passages of the female.

Spermatozoids. - The liquid taken from the vesicula seminales of an adult, who has died suddenly, or the ejaculated semen contains, in addition to the various accidental or unimportant anatomical elements that have been mentioned, innumerable bodies, resembling animalcules, which present a flattened, conoidal head and a long, tapering, filamentous tail. The number of spermatozoids in a single ejaculation has been estimated at $22 \mathrm{I}, 257,900$ (Lode). The tail is in active motion, and the spermatozoids move about the field of view under the microscope with considerable force, pushing aside little corpuscles or granules with which they may come in contact. Under favorable conditions, particularly in the generative passages of the female, the movements may continue for several days. It is said, indeed, that they are always present and in 


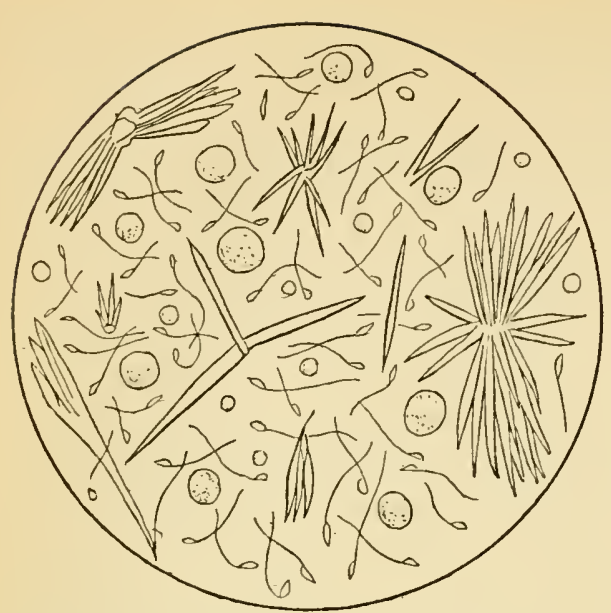

Fig. 213. - Spermatozoids, spermatic crystals and leucocytes (Peyer).

motion in the Fallopian tubes when sexual intercourse is frequent (Williams).

The head of the spermatozoid is about $\frac{1}{5000}$ of an inch $(5 \mu)$ long, $\frac{1}{800 \overline{0}}$ of an inch $(3 \mu)$ broad, and $250 \overline{0}$ of an inch ( $1 \mu$ ) in thickness. The tail (flagellum) is about $\frac{1}{500}$ of an inch $(50 \mu)$ in length. The length of the intermediate segment is about $\frac{1}{4000}$ of an inch $(6 \mu)$. At the lower end of the tail, is a short and excessively fine filament, called the terminal filament. The head contains a considerable quantity of chromatin. In the intermediate segment is the centrosome. These play an important part in the process of fertilization of the ovum.

Water soon arrests the movements of the spermatozoids, which may be restored by the addition of dense saline and other solutions. All the alkaline animal liquids of moderate viscidity favor the movements, while the action of acid or of very dilute solutions is unfavorable. The movements are suspended by extreme cold, but they return when the ordinary temperature is restored.

Before puberty the seminiferous tubes are much smaller than in the adult, and they contain small transparent cells, which in their form and arrangement resemble epithelium. As puberty approaches, however, the tubes become larger, and the contents change their character.

In the adult testicle, the seminiferous tubes are lined with two kinds of cells: I. The sustentacular cells of Sertoli, and 2, cells situated between the sustentacular cells, called spermatogonia. The spermatogonia, as they undergo development, become attached to the sustentacular cells and probably are nourished by them. The spermatogonia multiply by karyokinesis, with intervals of rest, during which the quantity of chromatin increases. The daughter-cells,

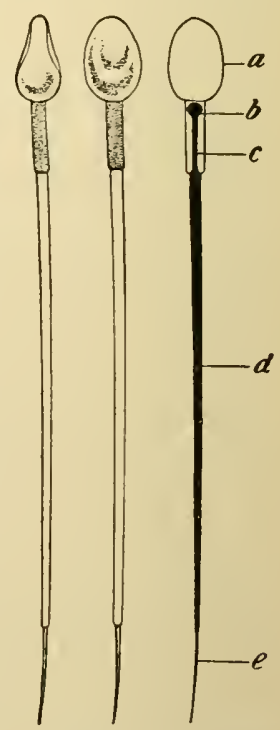

Fig. 214.-Human spermatozoids (Retzius and Jensen).

Left figure represents a side view; middle figure, flat view; $a$, head; $b$, centrosome; $c$, the intermediate segment; $d$, tail $e$, terminal filament. 
which are the result of the final division, are called spermatocytes. These divide again to form each two spermatids, the number of contained chromosomes being thereby reduced one-half, and these spermatids are developed into spermatozoids, in fan-shaped groups that project into the calibre of the seminiferous tubes.

The spermatozoids are motionless while they are within the testicle, the epididymis or the vasa deferentia, apparently on account of the density of the substance in which they are embedded; for movements are sometimes presented when the contents of the vasa deferentia are examined with the addition of water or of saline solutions. Once in the

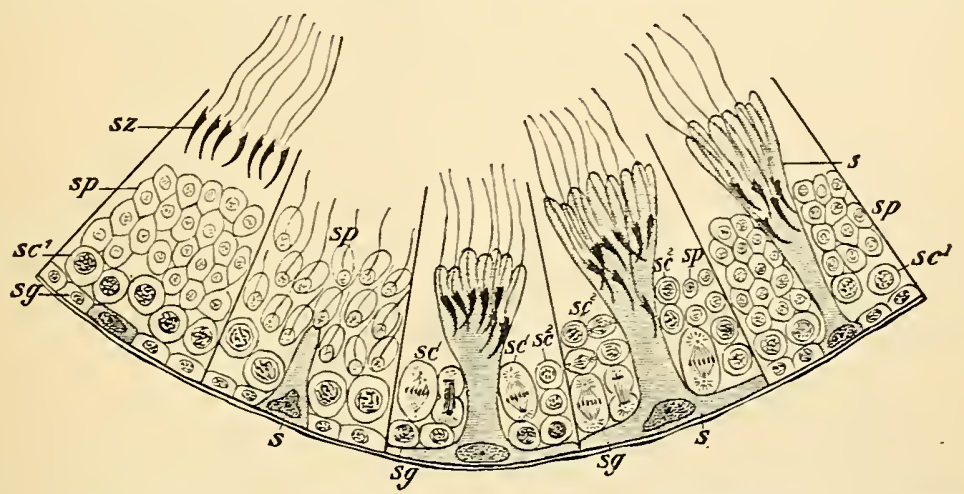

Fig. 215. - Diagram showing stages of spermatogenesis as seen in different sectors of a seminiferous tubule of a rat (MícMurrich, modified from von Lenhossek).

$s$, Sertoli cell; $s c^{1}$, spermatocyte of the first order; $s c^{2}$, spermatocyte of the second order (divided into two); $s g$, spermatogone; $s p$, spermatid; $s z$, spermatozoids.

In the first section (to the left) four different generations of cells are shown: $s g$, spermatogones; $s c^{1}$, spermatocytes; $s p$, spermatids; $s z$, spermatozoids. In the second section are spermatocytes, spermatids, and a Sertoli cell increased in size. In the third section are spermatocytes, $s c^{1}$ undivided and $s c^{2}$ divided, and a still larger Sertoli cell, with spermatozoids. In the fourth section are the two orders of spermatocytes, spermatids to the right, and a still larger Sertoli cell, with spermatozoids. In the fifth section are four spermatogones in contact with the basement membrane, spermatocytes of the first and second orders, spermatids and a Sertoli cell, with spermatozoids. $s z$, of the first section, shows spermatozoids lying in the lumen of the tubule.

vesiculæ seminales, and for a certain time after ejaculation, the spermatozoids are in active motion. When the spermatozoids have ceased their movements they are incapable of fertilizing the ovum.

The semen, thus developed and mixed with the various secretions before mentioned, is found during adult life and sometimes even in advanced age, and under physiological conditions it contains innumerable spermatozoids in active movement; but if sexual intercourse is frequently repeated at short intervals, the ejaculated liquid becomes more and more transparent, homogeneous and scanty, and it may consist of a small quantity of secretion from the vesiculæ seminales and the glands opening into the urethra, without spermatozoids and consequently deprived of fertilizing power. 
In old men the seminal vesicles may not contain spermatozoids; but this is not always the case, even in very advanced life. Instances are constantly occurring of men who have children in their old age, in which the paternity of the offspring can hardly be doubted. Duplay, in 1852 , examined the semen of a number of old men, and found, in about half the number, spermatozoids, normal in appearance and number, though in some the vesiculæ seminales contained either none or very few. Some of the persons in whom the spermatozoids were normal were between seventy-three and eighty-two years of age. These observations were confirmed by Dieu, who found spermatozoids in a man eighty-six years of age. The contents of the seminal vesicles, in these cases, were examined twenty-four hours after death. Some of the subjects died of acute, and others, of chronic diseases; but the mode of death did not present any differences in the cases classed with reference to the presence of spermatozoids. As the result of his own and of other recorded observations, Dieu concluded that the power of fecundation often persists for a considerable time after copulation has become impossible on account simply of loss of the power of erection. 


\title{
CHAPTER XXXI
}

\author{
FERTILIZATION AND KARYOKINESIS OF THE OVUM
}

Fecundation - Maturation of the ovum - Fertilization of the ovum - Mendel's laws of heredity - Superfecundation - Segmentation of the ovum - Gastrulation - Primitive streak - Formation of the membranes - Formation of the amnion - Amniotic liquid - Formation of the umbilical vesicle (yolk-sac) - Formation of the allantois and permanent chorion - Membranæ deciduæ - Formation of the placenta - Uses of the placenta.

So far as the male is concerned, coitus is rendered possible by erection of the penis. This may occur before puberty, but at this time intercourse can not be fruitful. Coitus may be impossible in old age, from absence of the power of erection; but spermatozoids may still exist in the vesiculæ seminales, and fecundation might occur if the seminal fluid could be discharged into the generative passages of the female. Coitus may take place in the female before the age of puberty or after the final cessation of the menses, but intercourse can not then be fruitful. There are many instances of conception following what would be called imperfect intercourse, as in cases of unruptured hymen, deformities of the male organs, etc., which show that the actual penetration of the male organ is not essential, and that fecundation may occur provided the seminal liquid find its way into even the lower part of the vagina. Conception also has followed intercourse when the female has been insensible or entirely passive. Unlike certain of the lower animals, the human subject presents no distinct periodicity in the development of the spermatozoids; but in reiterated connection, an orgasm may occur when the ejaculated liquid has no fecundating power.

In regard to the mechanism of erection, little remains to be said after the description that has been given of true erectile tissue in connection with the physiology of the circulation. The cavernous and spongy bodies of the penis usually are taken as the type of erectile organs. In these parts the arteries are large, contorted, provided with unusually thick muscular coats and are connected with the reins by vessels considerably larger than the true capillaries. They are supported by a strong fibrous network of trabeculæ, which contains nonstriated muscular fibres; so that when the bloodvessels are completely filled the organ becomes enlarged and rigid. Researches in regard to the nerves of erection show that the vessels of erectile tissues are dis- 
tended by an enlargement of the arterioles of supply, and that there is not simply a stasis of blood produced by constriction of the veins, except possibly for a short time during the period of greatest excitement. In experiments on dogs, Eckhard found a nerve derived from the sacral plexus, stimulation of which produced an increase in the flow of blood through the penis, attended with all the phenomena of erection. This nerve arises by two roots at the sacral plexus, from the first to the third conte sacral nerves, and is connected with the genito-spinal centre in the lower part of the lumbar region of the spinal cord. In the experiments referred to, by a comparison of the quantity of venous blood coming from the penis before and during the stimulation of the nerve, Eckhard found a great increase during erection. It is probable that in addition to the arterial dilatation, when the penis attains its maximum of rigidity, there is a certain degree of obstruction to the outflow of blood by compression of the veins, and that the rigidity is increased by contraction of the trabecular muscular fibres of the corpora cavernosa. At the climax of an orgasm, the semen is forcibly discharged from the urethra by spasmodic contractions of the vesicula seminales and the ejaculatory muscles. Although this is the physiological mechanism of a seminal discharge, friction of the parts, which usually precedes ejaculation, is not absolutely necessary, as is shown by the occurrence of orgasm during sleep, which is likely to take place in healthy men after prolonged continence.

There are some females in whom the generative function is performed, even to the extent of bearing children, who have no actual knowledge of a true venereal orgasm; but there are others who experience an orgasm fully as intense as that which accompanies ejaculation in the male. There is, therefore, the important difference in the sexes, that preliminary excitement and an orgasm are necessary to the performance of the generative act in the male but are not essential in the female. Still, there can be scarcely a doubt that venereal excitement in the female facilitates conception, other conditions being favorable. When excitement occurs in the female, there is engorgement of the true erectile tissues and possibly of the convoluted vessels surrounding the internal organs. The neck of the uterus becomes hardened and slightly elongated; and it has been observed by Litzmann and others, that there occurs a sudden opening and closing of the os, which exerts more or less suction force. These conditions, however, are not essential to fecundation, although they may exert a favorable influence on the penetration of spermatozoids and may at certain times determine the rupture of a Graafian follicle.

The spermatozoids, once within the cervix uteri, and in contact with 
the alkaline mucus, which increases the activity of their movements, may pass through the uterus into the Fallopian tubes, and even to the surface of the ovaries. Precisely how their passage is effected, it is difficult to say. It can be attributed only to the movements of the spermatozoids themselves, to capillary action, and to a possible peristaltic contraction of the muscular structures ; but these points have not as yet been subjects of positive demonstration. As regards the human female, it is impossible to give a definite idea of the time required for the passage of the spermatozoids to the ovaries or for the descent of the ovum into the uterus; and it is readily understood how these questions hardly admit of experimental investigation. It is known, however, that spermatozoids reach the ovaries, and they have been seen in motion on their surface, seven or eight days after connection.

\section{Fecundation}

The ordinary situation at which the ovum is fertilized is the dilated, or external portion of the Fallopian tube. All authorities are agreed that fertilization does not take place in the cavity of the uterus. In rabbits, when the ovum has descended into the uterus, it is surrounded with a dense albuminous coating which the spermatozoids can not penetrate. It is possible that this occurs in the human subject. Cases of abdominal pregnancy show that an ovum may be fertilized on the ovary as soon as it is discharged from the Graafian follicle.

The question of the duration of vitality of the spermatozoids, after their passage into the uterus, has an important bearing on the time when conception is most likely to follow sexual intercourse. The alkaline mucus of the internal organs actually favors their movements; the movements are not arrested by contact with menstrual blood; and, indeed, when the spermatozoids are mixed with the uterine mucus, they simply change their medium, and there is no reason to believe that they may not retain their vitality as well in the uterus as in the mucus of the vesicula seminales. It seems impossible, therefore, to fix any limit to the vitality of these anatomical elements, under physiological conditions; and it is not certain that spermatozoids may not remain either in the vagina or in the Fallopian tubes and around the ovary, when intercourse has taken place immediately after a menstrual period, until the ovulation following. Motile spermatozoids have been observed in the Fallopian tubes as long as twenty-five days after coitus (Duihrssen). The duration of life of an ovum has been estimated at about sixteen days (Issmer).

Maturation of the Ovum. - Before the formation of the polar bodies, the unripe, or immature ovum is called the resting cell. At this time, 
the nucleus, or germinal vesicle, contains a fine reticulum of linin fibres, chromatin threads, and the nucleolus, or germinal spot. By a process of karyokinesis confined to the nucleus, this divides in the same way as
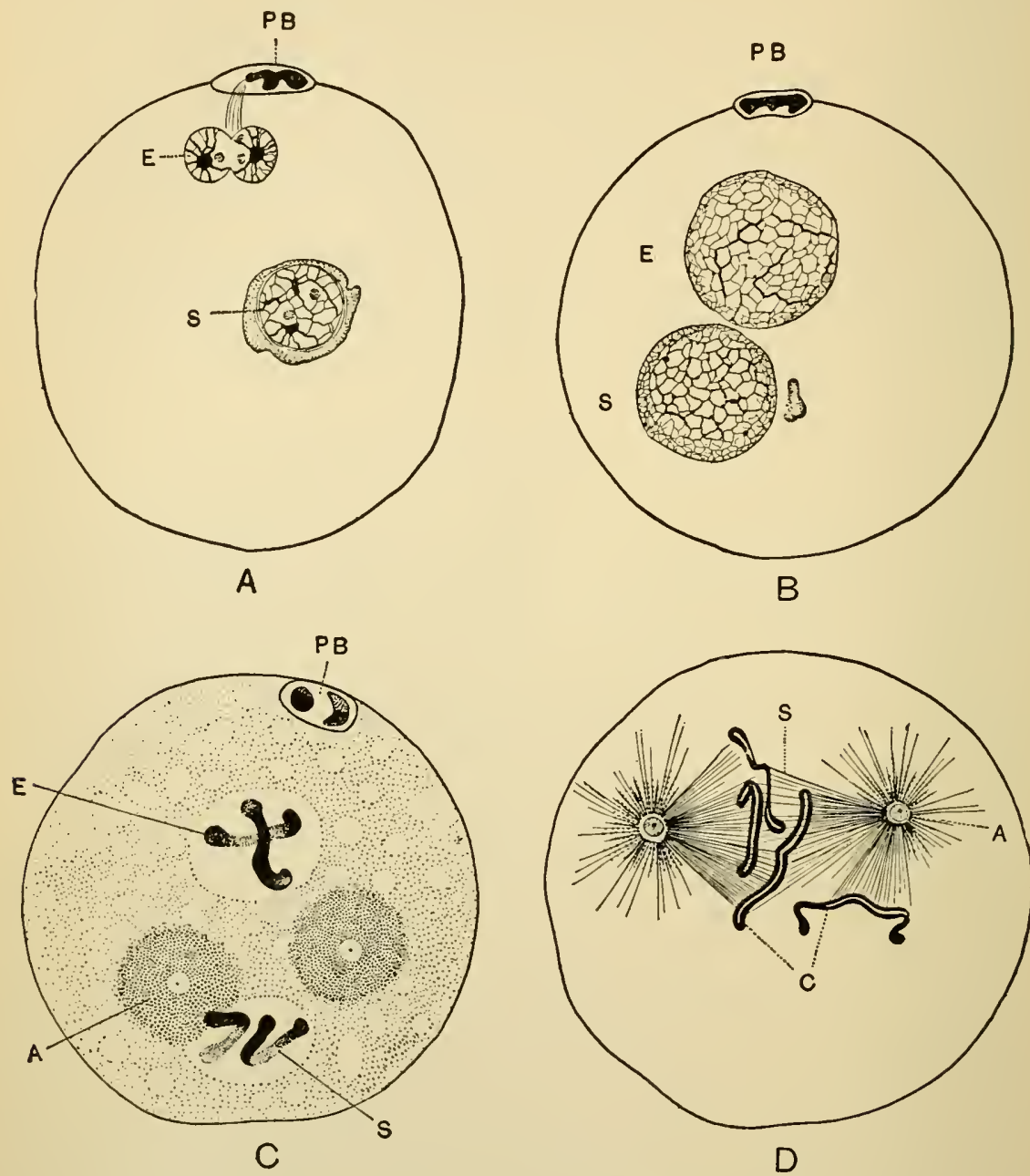

Fig. 216. - Germ-nuclei of the thread-worm - ascaris megalocephala (Boveri).

$A$, ovum immediately after the formation of the second polar body $(P B) ; E$, egg-nucleus; $S$, sperm-nucleus, derived from the head of a spermatozoid that has entered the ovum. $B$, following stage, in which $E$, the egg-nucleus, and $S$, the sperm-nucleus, have assumed the same size and structure. $C$, each nucleus has been transformed into two chromosomes, $E, S ; A$, attraction sphere containing a centrosome; $P B$, polar bodies. $D$, karyokinetic figure; $C$, chromosomes splitting lengthwise; $A$, one of the two asters; $S$, chromosomes forming a chromatic spindle.

the nucleus of an ordinary tissue-cell. After aivision into two, one of the daughter-nuclei is extruded from the ovum. This is the first polar body, and it carries with it a certain number of chromosomes. Sometimes 
the first polar body itself divides into two. A second polar body is formed in the same way by karyokinesis of the daughter-nucleus remaining in the ovum. These two polar bodies finally disappear. The

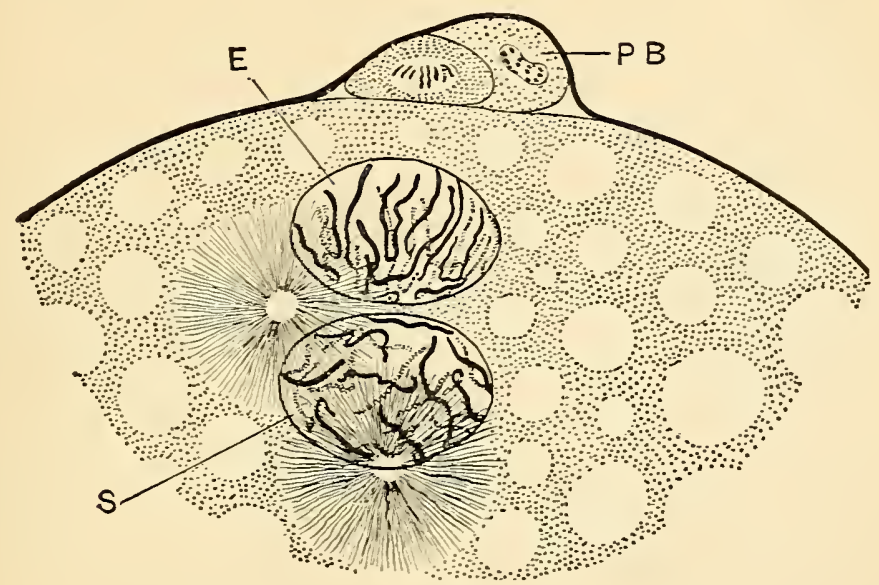

A

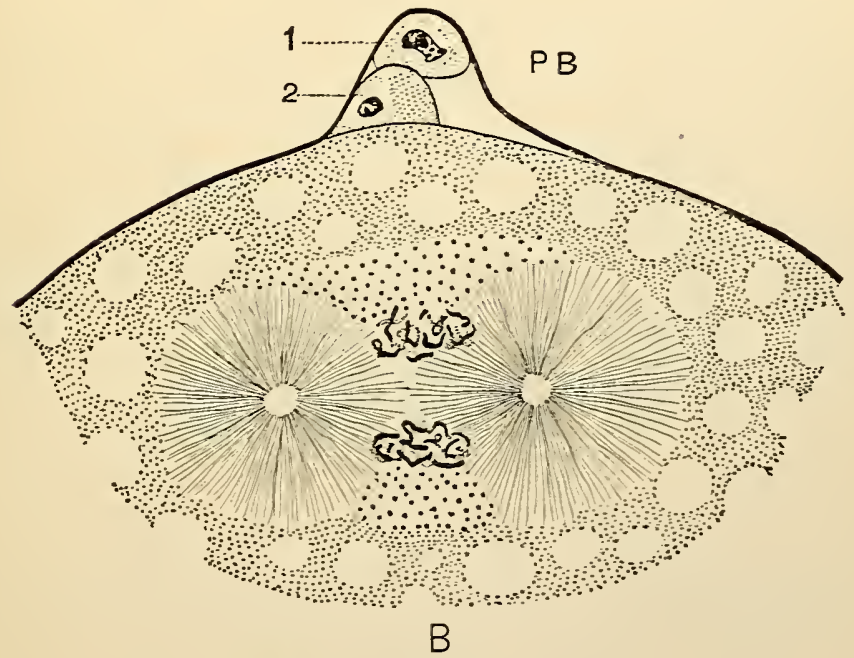

Fig. 2I7. - Karyokinesis (Boveri).

$A$, two germ-nuclei in the egg of the gastropod Pterotrachea; $E$, egg-nucleus; $S$, sperm-nucleus, each nucleus containing sixteen chromosomes; $P B$, polar bodies - the centrosome has divided into two to form an amphiaster. $B$, later stage, showing the fully-developed amphiaster; $P B$, polar bodies.

process just described is called maturation of the ovum, and it occurs in mammals before the ovum leaves the Graafian follicle.

Fertilization of the Ovum. - The head of a spermatozoid, containing chromosomes, penetrates the vitelline membrane and comes in contact 
with the vitellus. Then there forms on the surface of the vitellus what is called the entrance-cone, which receives, in a little excavation at its summit, the head of the male element. The spermatozoid afterward

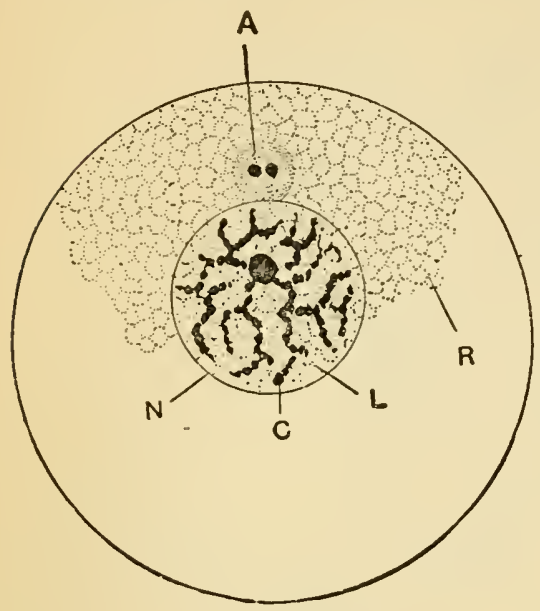

A

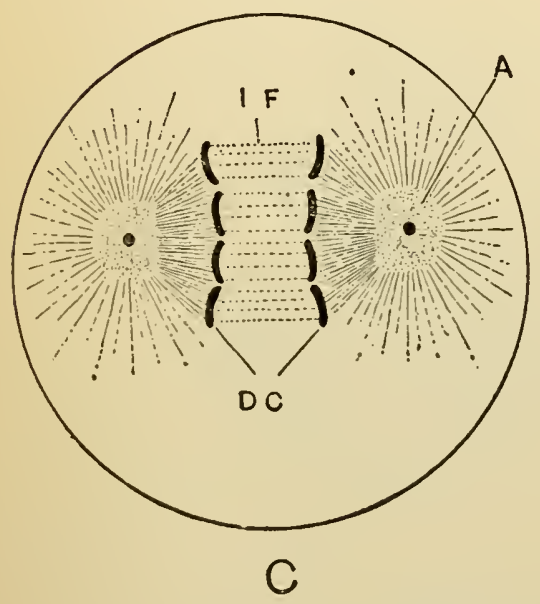

Fig. 218.-Cell-division (Wilson).

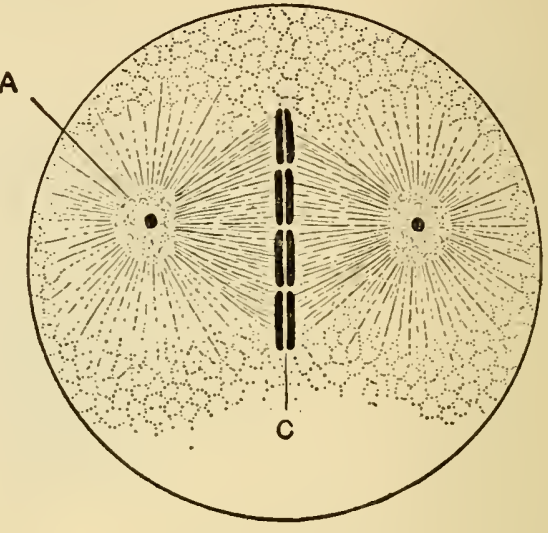

B

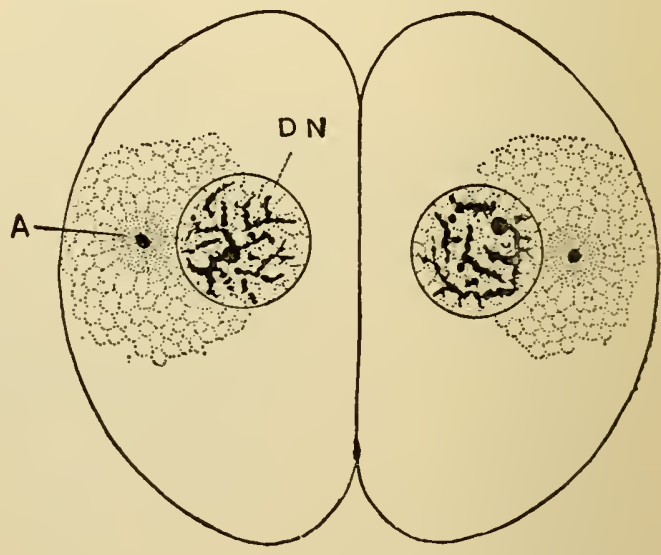

D

$A$, Resting cell, showing $A$, double centrosome; $N$, nucleus; $C$, chromatin; $L$, linin network; $B$, spongioplasm. $B$, cell in process of division, showing $A$, asters; $C$; chromosomes split lengthwise and lying in the equator of the achromatic spindle. $C$, later stage of karyokinesis, showing $D C$, daughter-chromosomes moving toward the two asters, with filaments, $I F$, between them. $D$, divided cell; $D N$, daughter-nucleus; $A$, centrosome.

penetrates the substance of the vitellus, and as it passes inward, it rotates on its short axis so that its base looks toward the centre of the ovum. The head and the middle piece of the spermatozoid now form 
the male pronucleus, or sperm-nucleus. The tail of the spermatozoid disappears.

Before the penetration of the spermatozoid, the ovum has sent off the polar bodies, and the number of chromosomes has been reduced one-half. What remains of the germinal vesicle, after the separation of the polar bodies, passes to the centre of the ovum and forms the female pronucleus, or egg-nucleus. The male pronucleus now unites with the female pronucleus to form the cleavage-nucleus, which contains the same number of chromosomes as the ordinary tissue-cells, the number in each pronucleus having been reduced one-half. The centrosome of the ovum has disappeared and the centrosome of the spermatozoid, which has been contained in the middle piece, divides into two, one passing to either side of the cleavage-nucleus. Under this view, division of the ovum can not take place without the introduction of a male centrosome. It is thought that hereditary qualities are transmitted in the chromosomes. The cleavage-nucleus thus contains chromosomes, one-half of which are from the ovum and one-half from the spermatozoid.

Mendel's Laws of Heredity.-About the year I860, Gregor Mendel, an Austrian monk and botanist, in studying hybrid forms of certain plants, observed peculiarities transmitted from parent-stocks which he formulated into what is now known to biologists as Mendel's law, or, more properly, Mendel's laws. He found that hybrids nearly always presented the characters of one parent-stock only; especially when each parent-stock was pure. The parent whose peculiarities were thus represented in the offspring presented characters that he called dominant; but the characters of the other parent, though undeveloped in the immediate offspring, remained dormant, possibly to reappear later. These latter characters he called recessive.

This result of cross-breeding, however, was not constant. In some offspring, the dominant character of one parent was not only repeated but became exaggerated; sometimes the hybrid showed individual characters, not possessed by either parent and differing from both, possibly derived from an anterior ancestral condition; but the cross always presented the same characters in case the germ-cells from the two parent-stocks were pure. The principle thus illustrated in plants was shown to exist in crossings of animals, as white and gray mice. In crossing pigmented with albino animals, the pigment character was always dominant and the albinism, recessive.

Without discussing fully the varied and frequent changes in character produced by crossing hybrids with hybrids and hybrids with either one of the pure parent-stocks, the following may be taken as the principles underlying Mendel's laws:- 
Crossing of pure germ-cells of parent-stocks presenting different characters produces a hybrid in which the characters of one parent only appear, this being the dominant.

Hybrids, however, although presenting only the dominant characters of one parent, may transmit to their offspring, characters of both parents, the recessive as well as the dominant.

In cases in which a hybrid presents individual characters of its own, differing from those of either parent, it is probable that the distinctive characters are more or less remotely ancestral in their origin.

The laws of Mendel bid fair to reduce the breeding of animals to an almost exact science. It is evident, however, that breeding in the human subject can never be conducted on scientific lines, however desirable this might appear. Still, in studying the fertilization of the ovum - as will be seen farther on - the idea has occurred to embryologists that hereditary transmission is effected through the male and the female chromosomes. It is well known, also, that the offspring of intermarriages often present intensified hereditary characters derived from one parent or the other, as is illustrated in inherited predisposition to certain diseases, such as tuberculosis and cancer.

The observations of Mendel have received but little attention at the hands of biologists. They are contemporary with what is known as the evolution-theory, which was propounded by Darwin in 1859 . Recent discoveries in biology are more favorable to the cytologists, who adopt the cell-theory of inheritance, than to the evolutionists, who knew little of the mechanism of mitosis, although Virchow's aphorism "omnis cellula e cellula" dates from i 855 .

Hereditary Transmission, Superfcundation ctc. - The first question that naturally arises relates to the conditions which determine the sex of offspring. Statistics show the proportions between male and female births; but nothing has ever been done in the way of procreating male or female children at will. According to Longet, the proportion of male to female births is about 104 to 105 , these figures presenting certain modifications under varying conditions of climate, season, nutrition etc. It has been shown, by observations on certain of the inferior animals, that the preponderance of sex in births bears a certain relation to the vigor and age of the parents; and that old and feeble females fecundated by young and vigorous males produce a greater number of males, and rice a'crsâ; but no exact laws of this kind have been found applicable to the human subject.

Reference has frequently been made to the chromosomes as the parts concerned in the transmission of inherited characters. This view, which is now - at least provisionally - adopted by most embryologists, rests 
on certain positive and indisputable facts: The nuclei of the cells of each and every species contain a definite number of chromosomes peculiar to such species and always divisible by two. In mitosis, the chromosomes of the mother-cells split and one-half goes to each daughter-cell. Maturation of the ovum involves the throwing off of one-half the chromosomes in the polar bodies. The final phase of spermatogenesis involves the division of chromosomes so that the spermatozoid has but one-half the number contained in ordinary cells. When the two cells meet, the egg is fertilized by the union of the egg-nucleus with the sperm-nucleus, the resulting cleavage-nucleus containing the full number of chromosomes; but if these two nuclei do not meet and unite, the cells die. The fertilized egg, therefore, contains chromosomes derived equally from the male and from the female. These characters and this behavior, being peculiar to and confined to chromatin and invariable in all forms of life, high or low, point almost conclusively to this substance as the medium of hereditary transmission; but they are not favorable to the theory of transmission of acquired qualities. A fuller discussion, however, of this subject would be out of place in this work.

A peculiar and, it seems to be, an inexplicable fact is that previous pregnancies have an influence on offspring. This is well known to breeders of animals. If a pure-blooded mare or bitch has been once covered by an inferior male, in subsequent fecundations the young are likely to partake of the character of the first male, even if bred afterward to males of unimpeachable pedigree. The same influence is sometimes observed in the human subject. A woman may have, by a second husband, children who resemble a former husband, and this is particularly well marked in certain instances by the color of the hair and eyes. A white woman who has had children by a negro may subsequently bear children to a white man, these children presenting some of the peculiarities of the negro race.

Superfecundation of course does not come in the category of influences just mentioned. It is not infrequent to observe twins, when two males have had access to the female, that are entirely distinct from each other in their physical characters - a fact readily explained by the assumption that two ova have been separately fecundated. This view is sustained by observation and experiment, and many illustrative cases are on record.

The following communication was received in January, 1869 , from Dr. John H. Janeway, Assistant Surgeon, U.S.A., and it illustrates superfecundation in the human subject; or at least that was the view taken by the negro father:-

"Frances Hunt, a freedwoman, aged thirty-five years, gave birth to 
twins, February 4, I867, in New Kent County, Virginia. One of these twins was black, the other was white. Frances is a mulatto. The black child is much darker than she is. Previous to the parturition, she had given birth to seven children, ail single births. She was living at the time of her impregnation in the family of a white man as house-servant, sleeping with a black man at night. She insists, however, that she never had carnal intercourse with a white man. She probably does this because
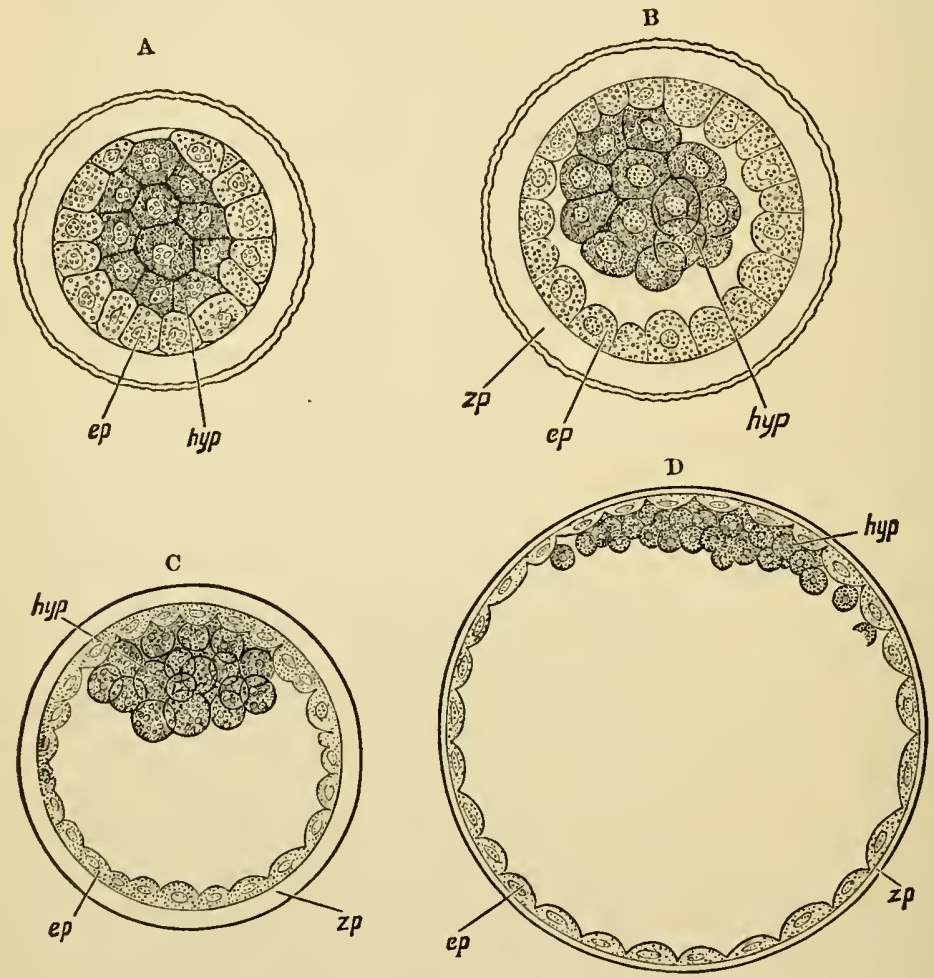

Fig. 219. - Formation of the blastodermic vesicle (van Beneden).

$A, B, C, D$, sections of ova in successive stages of development in the rabbit; $z p$, zona pellucida; $e p$, epiblastic cells; hyp, hypoblastic cells.

the black man turned her out of his house when he saw that one of the children was white. The only negro feature in the white child was its nose. There, its resemblance to its mother was perfect. Its hair was long, light, and silky. Complexion brilliant."

It is a curious fact that when a cow produces twins, one male and the other female, the female, which is called a free-martin, is sterile and presents an imperfect development of the internal organs of generation. This has led to the idea that possibly the same law may apply to the human subject, in cases of twins, one male and the other female; but 
many observations are recorded in gynecological works, showing the incorrectness of this view.

It has long been a question whether strong impressions made on the nervous system of the mother can exert an influence on the fœtus in utero. While many authors admit that violent emotions experienced by the mother may affect the nutrition and the general development of the fœtus, some writers of authority deny that the imagination can have any influence in producing deformities. The remarkable cases recorded as instances of deformity due to the influence of the maternal mind are
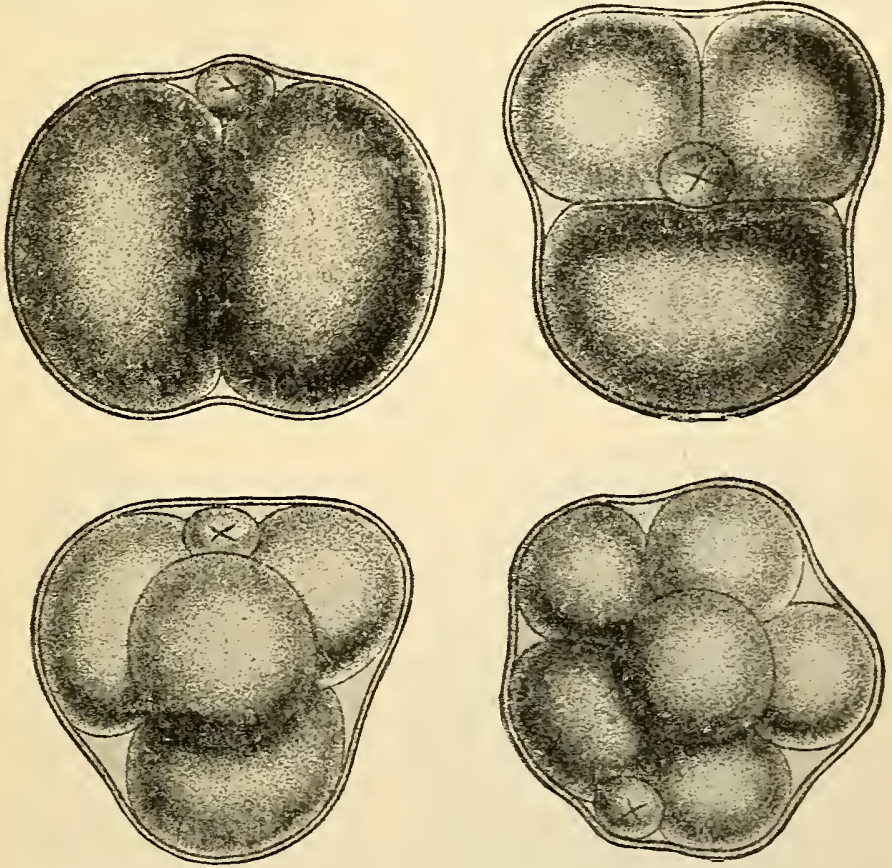

Fig. 220. - Four stages in segmentation of the ovum of a mouse (Sobotta); $X$, polar globule.

not entirely reliable; and it often happens that when a child is born with a deformity, the mother imagines she can explain it by some impression received during pregnancy, which she recalls only after she knows that the child is deformed. There is, indeed, no satisfactory evidence that the maternal mind has anything to do with the production of deformities in utero.

Segmentation of the Ovum. - Soon after fertilization and the formation of the cleavage-nucleus, a furrow appears at the point of extrusion of the polar globules. This is met by a furrow upon the opposite side, and the ovum is divided into two globes. One of the globes is slightly larger than the other and presents fewer and smaller granules. The 
larger sphere subsequently forms, by its division, the epiblastic cells, and the smaller sphere forms the hypoblastic cells. Each sphere is provided with a distinct nucleus. The two spheres resulting from the first segmentation ${ }^{1}$ are divided, each one into two, making four spheres.
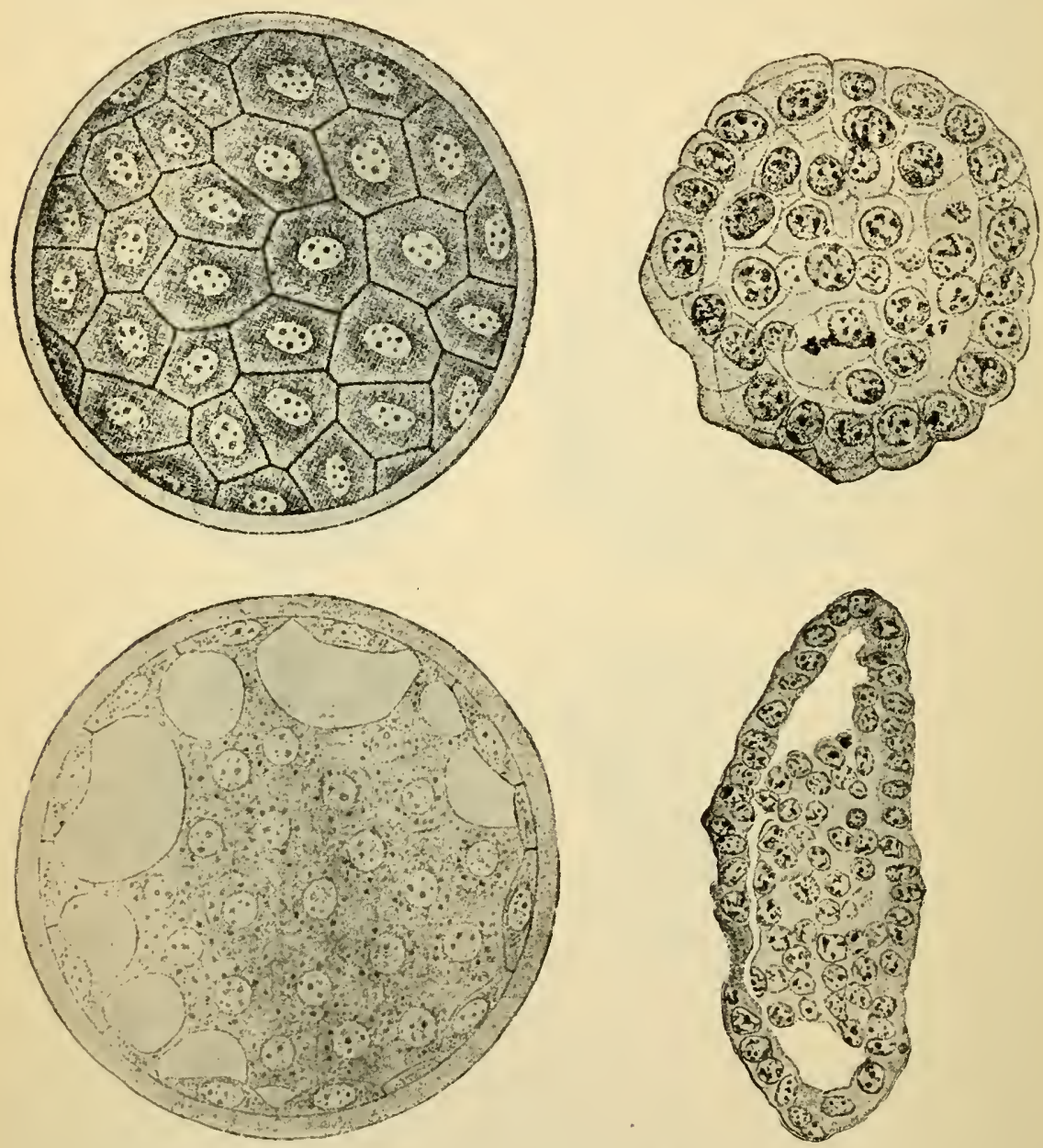

Fig. 221. - Later stiges of segmentation of the ozum of a but (van Beneden).

$A, C$ and $D$ are sections; $B$, a surface view.

These spheres are again divided into eight - four epiblastic and four hypoblastic spheres - each with a nucleus (van Beneden). One of the four hypoblastic spheres passes to the centre; and the four epiblastic

${ }^{1}$ In the mammalia, segmentation, after the first division of the ovum into two, is irregular, "three-celled, four-celled, five-celled and six-celled stages having been observed in various instances" (McMurrich). 
spheres, which are at the periphery, divide, each one into two, making eight epiblastic and four hypoblastic spheres. When this has occurred, the epiblastic spheres are smaller and clearer than the hypoblastic spheres. The four hypoblastic spheres now divide into eight. The epiblastic spheres then divide into sixteen, the hypoblastic spheres in turn divide, and this goes on until the process of segmentation is completed. In the rabbit, this occurs usually about seventy hours after impregnation. As segmentation progresses, the epiblastic cells extend over the hypoblastic cells, and become irregularly-polygonal in form. The hypoblastic cells occupy the central portion of the ovum. At first there is a circular space on the ovum where the epiblastic cells do not cover the cells of the hypoblast (see Fig. $219, A$ ); but this soon becomes closed by an extension of the cells of the epiblast (see Fig. 219, $B$ ). The hypoblastic cells, at the close of segmentation, are slightly larger than the cells of the epiblast and are darker and more rounded. The ovum now consists of a solid mass of cells and is called the morula, on account of its fancied resemblance to a mulberry. The cells of which it is composed are called collectively blastodermic cells. The ovum probably is in this condition when it passes from the Fallopian tube into the uterus.

Most of the phenomena of segmentation have been observed in the lower forms of animals; but there can be no doubt that analogous processes take place in the human ovum. In the rabbit, forty-five and a half hours after copulation, Wiel observed an ovum with sixteen segmentations, situated in the lower third of the Fallopian tube. $\mathrm{He}$ observed an ovum, ninety-four hours after copulation, with a delicate mosaic appearance, presenting a small rounded eminence on its surface. It is impossible to say how long the process of segmentation continues in the human ovum. It is stated that it is completed in rabbits in a few days, and in dogs, that it occupies more than eight days (Hermann).

Gastrulation. - After segmentation has been completed, a cavity filled with liquid appears between the hypoblastic and epiblastic cells, except at that portion which has last been covered by the epiblast. Here the cells of the hypoblast are in contact with the epiblast. The liquid in the interior of the ovum gradually increases in quantity, the ovum becomes enlarged to the diamater of $\frac{1}{50}$ to $\frac{1}{25}$ of an inch (0.5 to I millimeter), and is now called the blastula, or blastodermic vesicle. The epiblastic cells surround the blastodermic vesicle completely, forming a single layer over the greater portion; and the hypoblastic cells form a lenticular mass attached to the smaller portion of the inner surface of the layer of epiblastic cells (see Fig. 219, $C$ and $D$ ). There then occurs a gradual invagination of the hypoblastic cells, forming a double-walled sac, called the gastrula. This is the primitive gut, or archenteron. The 
process of its formation is called gastrulation. It is at this portion of the ovum that the embryonic spot, or area, afterward appears.

The albuminous covering which the ovum has received in the upper part of the Fallopian tube gradually liquefies and penetrates the vitelline membrane, furnishing, it is thought, matter for the nourishment and development of the ovum. In the Fallopian tube, indeed, the adventitious albuminous covering presents an analogy to the albuminous coverings which the eggs of oviparous animals receive in the oviducts; with the difference that this albuminous matter is almost the sole source of nourishment in the latter and exists in large quantity, while in viviparous animals the quantity is small, is usually consumed as the orum passes

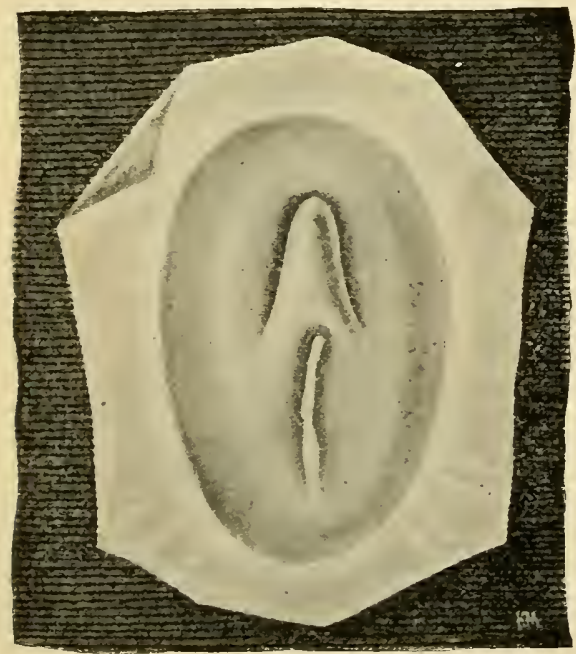

Fig. 222. - Embryonic shield of a rabbit, showing the primitive streak and the medullary groove above, $\times 28$ (Kollmann). called the area opaca. The line thus formed and surrounded by the area pellucida is called the primitive streak or trace. This primitive streak, or groove, however, is a temporary structure. After the groove is formed, there appears, in front of but not continuous with it, a new fold and a groove leading from it. This is the "head-fold," and the groove is the true medullary groove, which afterward is developed into the neural canal.

Blastodemic Layers. - The blastodermic cells, resulting originally from the segmentation of the vitellus, are first split apparently into two layers, the external, or epiblast, and the internal, or hypoblast. The epiblast is developed into the epidermis and its appendages; the glands of the skin; the enamel of the teeth; the mucous lining of the mouth, 
nasal cavities and lower part of the rectum; the brain and spinal cord; the organs of special sense, and possibly some parts of the genito-urinary apparatus. The hypoblast is developed into the epithelium lining the mucous membrane and glands of the digestive tract; the epithelium of the respiratory organs; the epithelium of the bladder and urethra. There is a thickening of both these layers at the line of development of the cerebro-spinal system, with a furrow that is finally enclosed by an elevation of the ridges and their union posteriorly, forming the canal for the spinal cord.

As the spinal canal is developed, a new layer of cells is formed between the epiblast and the hypoblast, which is called the mesoblast. The mesoblast itself afterward splits into two layers. All the parts not enumerated as developed from the epiblast or hypoblast are developed from the two layers of the mesoblast. The outer layer of the mesoblast,

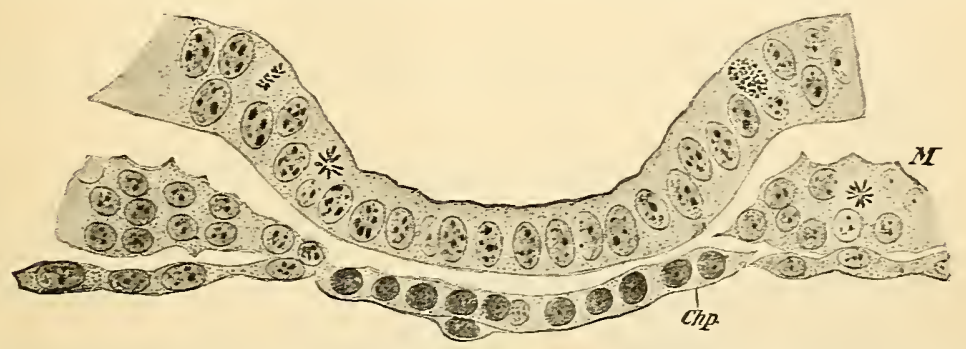

Fig. 223. - Transwerse section of the embryonic area of a dog's ovum of about fifteen days (Bonnet).

This figure is introduced especially to show the mesoblast. The section is through the head-fold (Chp.) ; $M$, mesoblast.

or the epiblastic mesoblast, unites with the epiblast, and the two membranes together form what is sometimes called the somatopleure; from which are developed the bones, muscles and external parts generally. The inner layer of the mesoblast, the hypoblastic mesoblast, unites with the hypoblast to form what is called the splanchnopleure; from which are developed the circulatory and glandular systems and the internal parts generally. The cells lining the vessels, including the lymphatics, which exist in a single layer, are called endothelial cells. This name also is applied to the cells lining the serous membranes.

\section{Formation of the Menbranes}

In the mammalia a portion of the blastoderm is developed into membranes by which a communication and union are established between the ovum and the mucous membrane of the uterus. From the ovum two membranes are developed; one non-vascular, the amnion, and another, 
the allantois, which is vascular. The two layers of decidua are formed from the mucous membrane of the uterus. At a certain part of the uterus, a vascular connection is established between the mucous membrane and the allantois, and the union of these two structures forms the placenta. The foetal portion of the placenta is connected with the foetus by the vessels of the umbilical cord, and the maternal portion is connected with the great uterine sinuses.

The external covering of the ovum, during the first stage of its development, is the vitelline membrane. As the ovum is received into the uterus, the vitelline membrane develops on its surface little villosities, which are non-vascular and formed of amorphous matter with granules. These are the first villosities of the ovum, and they assist in fixing the egg in the uterine cavity. They are not permanent, they do not become developed into the vascular villosities of the chorion, and they disappear as the true membranes of the embryo are developed from the blastodermic layers. The vitelline membrane disappears soon after the passage of the ovum into the uterus, when it is replaced by the amnion.

Formation of the Amnion. - As the ovum advances in its development, it is observed that a portion of the blastoderm becomes thickened, forming the epiblast, the two layers of the mesoblast and the hypoblast. At about the time when this thickening begins, a fold of the epiblast and of the external layer of the mesoblast makes its appearance, which surrounds the thickened portion and is most prominent at the cephalic and the caudal extremities of the furrow for the neural canal. This fold increases in extent as development advances, passes over the dorsal surface of the embryo and finally meets so as to enclose the embryo completely. At a certain period of the development of the amnion, this membrane consists of an external layer, formed of the external layer of the fold, and an internal layer; and the point of union of the two layers, or the point of meeting of the fold, is marked by a membranous septum.

The two amniotic layers are formed in the way just described, and a complete separation finally takes place, by a disappearance of the septum formed by the meeting of the folds over the back of the embryo. This process occupies four or five days in the human ovum. The point where the folds meet is called the amniotic umbilicus. When the amnion is thus completely formed, the vitelline membrane has been encroached upon by the external amniotic layer and disappears, leaving this layer of the amnion as the external covering of the ovum. At this time there is a growth of villosities on the surface of the external amniotic layer, which, like the villosities of the vitelline membrane, are not vascular. 

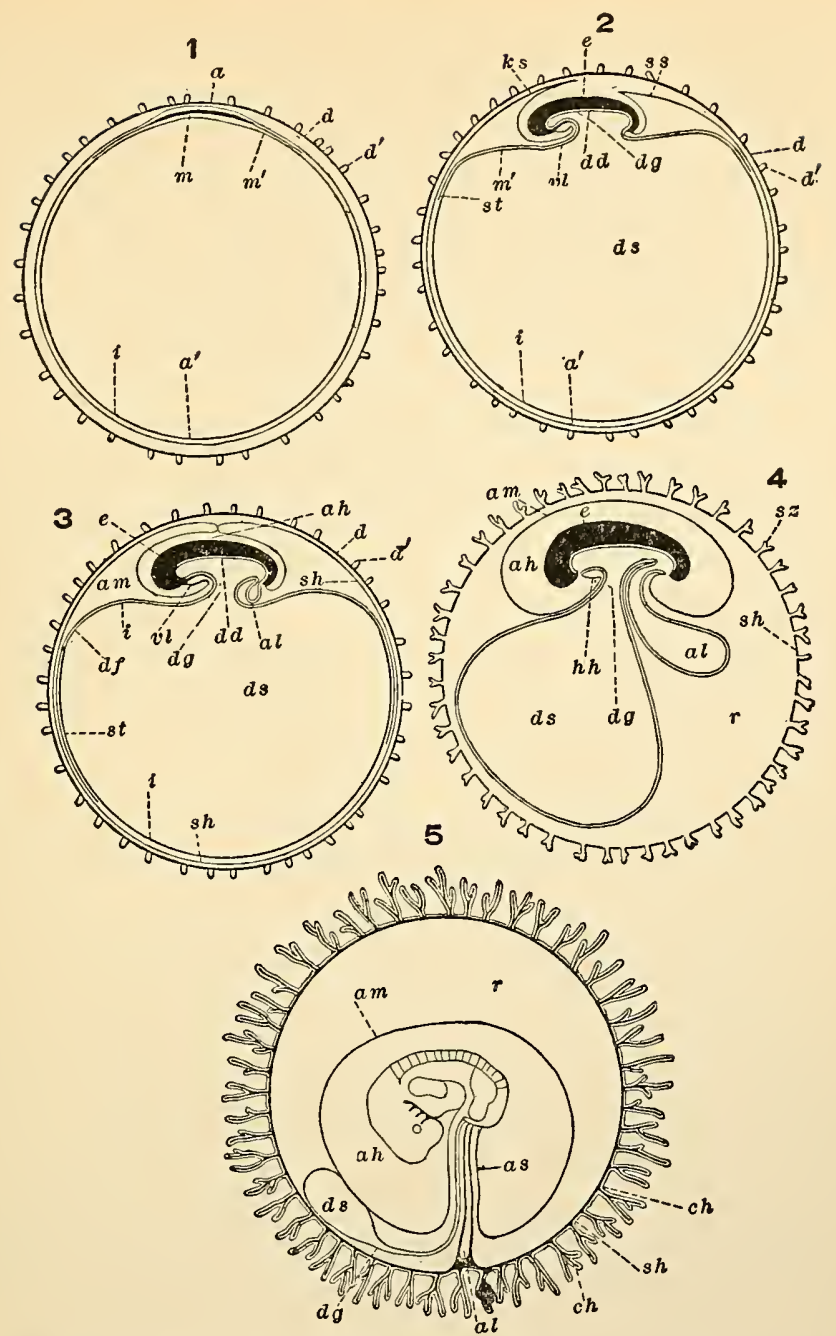

Fig. 224. - Five diagrammatic representations of the formation of the membranes in mammalia (Kölliker).

I : $a, a^{\prime}$, epiblast ; $d$, vitelline membrane; $d^{\prime}$, villi on the vitelline membrane; $i$, hypoblast; $m, m^{\prime}$, mesoblast.

2: $a^{\prime}$, external layer of the amnion; $d, d^{\prime}$, vitelline membrane; $e$, embryo; $d s$, umbilical vesicle; $v l$, $k s, s s$, folds of the amnion; $d d, m^{\prime}, s t$, lyppoblast; $d d$, connection of the embryo with the umbilical vesicle.

$3: d, d^{\prime}$, vitelline membrane; $v l$, internal amniotic layer; $e$, embryo; $a h$, amniotic cavity; $s h, s h$, external amniotic layer; $a m$, space between the two layers of the amnion; $d d$, hypoblast; $d f, s t$, $i$, walls of the umbilical vesicle; $d g$, omphalo-mesenteric canal; $d s$, cavity of the umbilical vesicle; $a l$, first appearance of the allantois.

$4: s h$, external layer of the amnion; $s z$, villi of the external layer of the amnion, which has now become the chorion, the vitelline membrane having disappeared; $h h ; a m$, internal layer of the amnion; $e$, embryo; $a h$, amniotic cavity; $d g$, omphalo-mesenteric canal; $d s$, cavity of the umbilical vesicle; $a l$, allantois; $r$, space between the two layers of the amnion..

$5: c h, s h, c h, a l$, allantois (which has now become the chorion, the external amniotic layer having disappeared), with its villi; a m, amnion; $a s$, amniotic covering of the umbilical cord; $r$, space between the amnion and the allantois; $a h$, amniotic cavity; $d s$, umbilical vesicle; $d g$, omphalo-mesenteric canal. 
Soon after the development of the amnion the allantois is formed. This membrane is vascular. It encroaches upon and takes the place of the external amniotic membrane, and is covered with hollow villi, which take the place of the villi of the amnion. Over a certain portion of the membrane the villi are permanent. The mode of development of the amnion is illustrated by the diagrammatic Fig. 224. This figure illustrates the formation of the amnion, the umbilical vesicle and the allantois. The last two structures are derived from the hypoblast and the internal layer of the mesoblast.

When the allantois has become the chorion, or the external membrane of the ovum, having taken the place of the external layer of the amnion, the structures of the ovum are the following: I. The chorion, formed of the two layers of the allantois and penetrated by bloodvessels. 2. The umbilical cord, which connects the embryo with the placental portion of the chorion, and the umbilical vesicle, formed from the same layers as the allantois. 3. The amnion, which is the internal layer of the amniotic fold, persisting throughout fœtal life. 4. The embryo itself.

During the early stages of development of the umbilical vesicle and the allantois, the internal amniotic layer, or the true amniotic membrane, is closely applied to the surface of the embryo and is continuous with the epidermis at the umbilicus. It is then separated from the allantois by a layer of gelatinous matter; and in this layer, between the amnion and the allantois, is the umbilical vesicle. At this time the umbilical cord is short and not twisted. As development advances, however, the intermembranous gelatinous matter gradually disappears; the cavity of the amnion is enlarged by the production of liquid between its internal surface and the embryo; and at about the end of the fourth month the amnion comes in contact with the internal surface of the chorion. At this time the embryo floats in the liquid contained in the amniotic cavity.

The amnion forms a lining membrane for the chorion. By its gradual enlargement it has formed a covering for the umbilical cord; and between it and the cord, is the atrophied umbilical vesicle. The amnion then resembles a serous membrane, except that it is non-vascular. It is lined with a single layer of pale delicate cells of pavement-epithelium, which contain a few fine fatty granules. At term the amnion adheres to the chorion, although it may be separated with a little care as a distinct membrane and may be stripped from the cord. From its arrangement and from the absence of bloodvessels, it is evident that this membrane is simply for the protection of the fœtus and is not directly concerned in its nutrition and development (see Plate X, Fig. 6). 
The gelatinous mass referred to above, situated, during the early periods of intra-uterine life, between the amnion and the chorion, presents a semiliquid consistence, with very delicate interlacing fibres of connective tissue and fine grayish granules scattered through its substance. These fibres are gradually developed as the quantity of gelatinous matter diminishes and the amnion approaches the chorion, until finally they form a rather soft, reticulated layer, which is sometimes called the membrana media.

Amniotic Liquid. - The process of enlargement of the amnion shows that the amniotic liquid gradually increases in quantity as the development of the fœtus progresses. At term the quantity is variable, being rarely more than two pints (about one liter) or less than one pint (about half a liter). In the early periods of utero-gestation it is clear, slightly yellowish or greenish, and perfectly liquid. Toward the sixth month its color is more pronounced and it becomes slightly mucilaginous. Its reaction usually is neutral or faintly alkaline, although sometimes it is feebly acid in the latest periods. It sometimes contains a small quantity of albumin, as determined by heat and nitric acid; and there usually is a gelatinous precipitate on the addition of acetic acid. The following table gives its chemical composition (Robin):-

\section{COMPOSITION OF THE AMNIOTIC LIQUID}

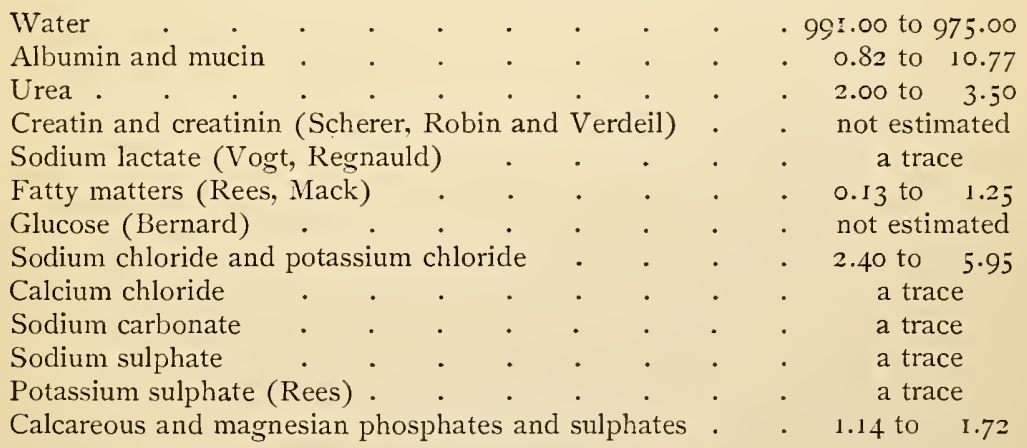

The presence of certain of the urinary constituents in the amniotic liquid has led to the view that the urine of the fœtus is discharged in greater or less quantity into the amniotic cavity. Bernard, who is cited in the table of composition of the amniotic fluid as having determined the presence of sugar, has shown that in animals with a multiple placenta, the amnion has a glycogenic action during the early part of intra-uterine existence.

In regard to the origin of the amniotic liquid, it is impossible to say 
how much of it is derived from the general surface of the foetus, how much from the urine, and how much from the amnion itself, by transudation from the vascular structures beneath this membrane. The quantity apparently is too great, especially in the early months, to be derived entirely from the urine of the fœtus; and there probably is an exudation from the general surface of the fotus and from the membranes. After the third month the sebaceous secretion from the skin of the fœtus prevents the absorption of any of the liquid. An important property of the amniotic liquid is that of resisting putrefaction and of preserving dead tissues.

Formation of the Umbilical Vesicle (Yolk-sac). - As the visceral plates, which will be described hereafter, close over the front of the embryo, that portion of the blastoderm from which the intestinal canal is developed presents a vesicle, which is cut off from the abdominal cavity but which still communicates freely with the intestine. This is the umbilical vesicle. On its surface is a rich plexus of bloodvessels; and this is an important organ in birds and in many of the lower forms of animals. In the human subject and in mammals, however, the umbilical vesicle is not so important, as nutrition is secured by means of vascular connections between the chorion and the uterus. The vesicle becomes gradually removed farther and farther from the embryo, as development advances, by the elongation of its pedicle; and it is compressed between the amnion and the chorion as the former membrane becomes distended.

When the umbilical vesicle is formed, it receives two arteries from the two aortæ, and the blood is returned to the embryo by two veins which open into the vestibule of the heart. These are called the omphalo-mesenteric vessels. At about the fortieth day one artery and one vein disappear, and soon after, all vascular connection with the embryo is lost. At first there is a canal of communication with the intestine, called the omphalo-mesenteric canal. This is gradually obliterated, and it closes between the thirtieth and the thirty-fifth day. The point of communication of the vesicle with the intestine is called the intestinal umbilicus; and early in the process of development, there is here a hernia of a loop of intestine. The umbilical vesicle remains as a rather prominent structure as late as the fourth or fifth month, but it may be cliscovered at the end of pregnancy.

The umbilical vesicle presents three coats : an external smooth membrane, formed of connective tissue, a middle layer of transparent polyhedric cells, and an internal layer of spheroidal cells. The membrane, composed of these layers, encloses a pulpy mass, composed of a liquid of ten containing cells and yellowish granules. 
Formation of the Allantois and the Permanent Chorion. - During the early stages of development of the umbilical vesicle, and as it is shut off from the intestine, there appears an elevation at the posterior

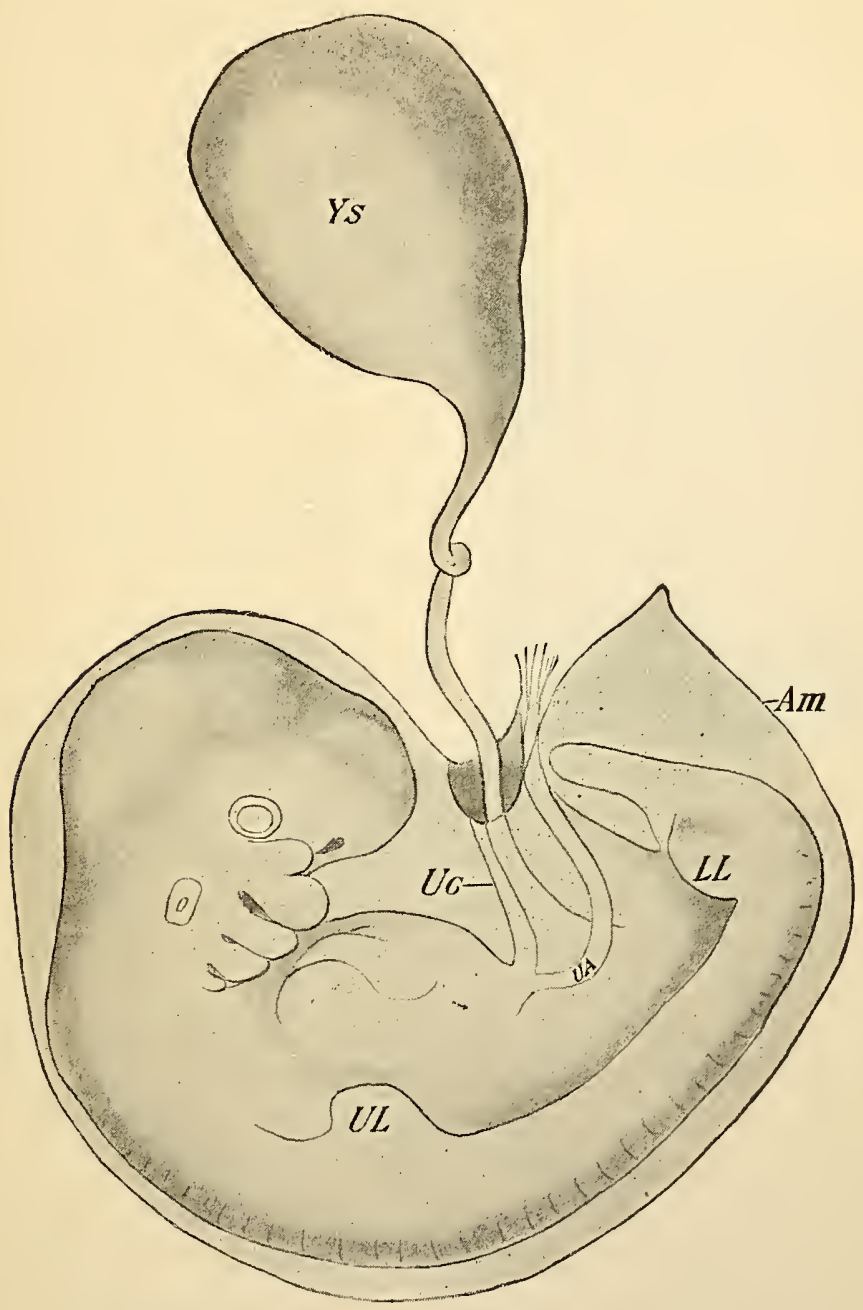

Fig. 225. - Human embryo of tweenty to twenty-five days (Coste).

$A m$, amnion; $L L$, lower limb; $U A$, umbilical artery; $U c$, umbilical cord; $U L$, upper limb; $Y s$, yolk-sac (umbilical vesicle).

portion of the intestine, which rapidly increases in extent until it forms a membrane of two layers, extending between the internal and the external layers of the amnion. This membrane, the allantois, becomes vascular early in its development, increases in size quite rapidly, and finally it completely encloses the internal layer of the amnion and the 
embryo, the gelatinous mass already described being situated between it and the internal amniotic layer before this layer becomes enlarged. While the formation of the two layers of the allantois is quite distinct in certain of the lower forms of animals, in the human subject and in mammals it is not so easily observed; still there can be no doubt as to the mechanism of its formation, even in the human ovum. Here, however, the allantois soon becomes a single membrane, the two original layers of which can not be separated from each other. The different stages of the development of the allantois are shown in the diagram-

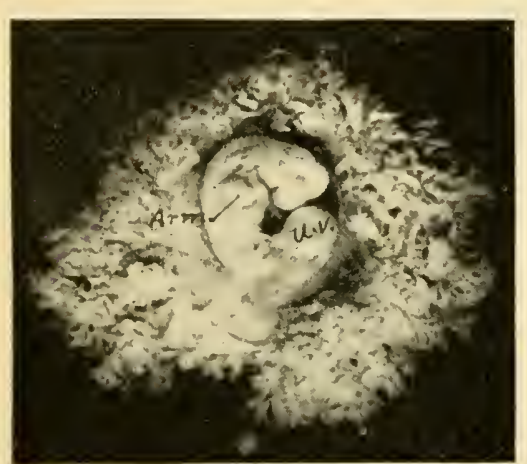

Fig. 226. - Human embryo of the fourth to the fifth week, $\times 2$-prepared by Dr. C. R. Corson, of Savannah, Ga. (Gage).

This ovum shows the shaggy chorion, which has been split open and the edges of the opening drawn aside to show the embryo. The embryo is curved as usual, and the head rests on the prominent heart and is almost in contact with the pear-shaped umbilical vesicle. The arm-bud and the branchial slits show clearly. The somites (protovertebræ) may be seen on close inspection. matic Fig. $224(3,4,5)$.

It is the vascularity of the allantois that causes the rapid development by which it invades and finally supersedes the external layer of the amnion, becoming the permanent chorion, or external membrane of the ovum. At first there are two arteries extending into this membrane from the lower portion of the aorta, and two veins. The two arteries persist and form the two arteries of the umbilical cord, coming from the internal iliac arteries of the fotus; and one vein, the umbilical vein, which returns the blood from the placenta to the fotus, is permanent. These vessels are connected with the permanent vascular tufts of the chorion.

The development of the allantois can not be well observed in human
twenty-fifth day. When the allantois ova before the fifteenth or the twenty-fifth day. When the allantois becomes the permanent chorion, it presents a large number of hollow branching villi over its entire surface, which give the ovum a shaggy appearance. As the ovum enlarges, over a certain area surrounding the point of attachment of the pedicle which connects the chorion with the embryo, the villi are developed more rapidly than over the rest of the surface. Indeed, as the ovum becomes larger and larger, the villi of the surface outside of this area become more and more scanty, lose their vascularity and finally disappear. That portion of the allantois over which the villi persist and increase in length and in the number of their branches is destined to form connections with the mucous membrane of the uterus and constitutes the fotal portion of the placenta. 
This change begins at about the end of the second month, and the placenta becomes distinctly limited at about the end of the third month.

It must be remembered that as the changes go on which result in the formation of the permanent chorion and the limitation of the fœtal portion of the placenta, the formation of the umbilical vesicle and the enlargement of the amnion also are progressing. The amnion is gradually distended by the increase in the quantity of amniotic liquid. It reaches the internal surface of the chorion at about the end of the fourth month, extends over the umbilical cord to form its external covering, including the cord of the umbilical vesicle, and the umbilical vesicle itself lies in the gelatinous matter between the two membranes.

At about the beginning of the fifth month the ovum is constituted as follows :-

The fotus floats freely in the amniotic liquid, attached to the placenta by the umbilical cord; the chorion presents a highly vascular, thickened and villous portion, the fœtal portion of the placenta; the rest of the chorion is a simple membrane, without villi and without bloodvessels; the amnion lines the internal surface of the chorion and forms, also, the external covering of the umbilical cord; the umbilical vesicle has become atrophied and has lost its vascularity; the hernia at the point of connection of the umbilical vesicle with the intestine of the fœtus has closed; and finally the fœtus has undergone considerable development.

Unbilical Cord. - From the description given of the mode of development of the chorion and the amnion, it is evident that the umbilical cord is nothing more than the pedicle which connects the embryo with that portion of the chorion which enters into the structure of the placenta. It is, indeed, a process of the allantois, in which the vessels have become the most important structures. The cord is distinct at about the end of the first month; and as development advances, the vessels consist of two arteries coming from the body of the fœtus, which are twisted usually from left to right, around the single umbilical vein. In addition to the spiral turns of the arteries around the vein, the entire cord may be more or less twisted, probably from the movements of the fœtus.

The fully-developed cord extends from the umbilicus of the fœtus to the central portion of the placenta, in which its insertion usually is oblique; although it may be inserted at other points, and even outside of the border of the placenta, its vessels penetrating this organ from the side. Its usual length, which varies considerably, is about twenty inches ( 50.8 centimeters). It has been observed as long as sixty ( I 52.4 centimeters), and as short as seven inches ( 17.8 centimeters). When the cord is very long, it sometimes presents knots or it may be wound around the 
neck, the body or any of the members of the fotus; and this can be accounted for only by the movements of the fœtus in utero.

The external covering of the cord is a process of the amnion; and as it extends over the vessels, it includes a gelatinous substance (the gelatin of Wharton) which surrounds the vessels and protects them from compression. This gelatinous substance is identical with the so-called membrana intermedia, or the substance included between the amnion and the chorion. The entire cord, covered with the gelatin of Wharton and the amnion, usually is about the size of the little finger. According to Robin, the umbilical cord will sustain a weight of about twelve pounds (5.4 kilos). As the amniotic liquid accumulates and distends the amnion, this membrane becomes more and more closely applied to the cord. The pressure extends from the placental attachment of the cord toward the fotus, and it gradually forces into the abdomen of the fotus the loop of intestine, which, in the early periods of intra-uterine life, forms an umbilical hernia.

The vessels of the cord - the arteries as well as the vein - are provided with valves. These are simple inversions of the walls of the vessels, and they do not exist in pairs nor do they seem to influence the current of blood. In the arteries these folds are situated at intervals of half an inch to two inches ( 12.7 to 58.8 millimeters), and they are more abundant where the vessels are very contorted. In the vein the folds are most abundant near the placenta. They are irregularly placed, and in a length of four inches ( Io centimeters), fifteen folds were found (Berger). It is not apparent that these valvular folds have any physiological importance.

As the allantois is developed, it presents, in the early stages of its formation, three portions: an external portion, which becomes the chorion, an internal portion, enclosed in the body of the embryo, and an intermediate portion. The intermediate portion becomes the umbilical cord. As the umbilicus of the fœetus closes around the cord, it shuts off a portion of the allantois contained in the abdominal cavity, which becomes the urinary bladder; but there is a temporary communication between the internal portion and the lower portion of the cord, called the urachus. This usually is obliterated before birth and is reduced to the condition of an impervious cord; but it may persist during intrauterine life, in the form of a narrow canal extending from the bladder to the umbilicus, which is closed soon after birth.

At this time, when the ovum measures one-eighth to one-fourth of an inch ( 3 to 6 millimeters) in diameter, the outer portion of the villi is covered, over either a part of the ovum or the entire surface, with a special layer of cells derived from the epiblast. These constitute the 
trophoblast. This membrane has been studied in the unguiculates only; but probably it exists in the human subject and in all mammals. The cells are fused into a thick mass and are supposed to destroy the tissues of the uterus with which they come in contact and secure implantation of the ovum in the mucous membrane. The trophoblast afterward disappears, and the area of implantation of the ovum finally becomes the placental area.

The entire mucous membrane of the uterus, however, has already undergone certain changes by which it is prepared for the reception of the ovum.

Membrance Deciduce. - In addition to the two membranes connected with the fœtus, there are two membranes formed from the mucous membrane of the uterus, which are derived from the mother and which serve still further to protect the ovum. The chorion is for the protection of the fœtus; but a portion of this membrane - about one-third of its surface - becomes closely united with a corresponding portion of the uterine mucous membrane, to form the placenta.

As the fecundated ovum descends into the uterus, it is invested with a villous covering, which is either the permanent chorion or one of the membranes that invests the ovum previous to the complete development of the allantois. The changes incident to menstruation have already been described. It has been seen that during an ordinary menstrual period, the membrane is increased three or four times in thickness and becomes more or less rugous. If a fertilized ovum descends into the uterus, the changes, in the mucous membrane progress. The glands enlarge and the membrane becomes thicker, so that at the end of the first month it measures about two-fifths of an inch (Io millimeters). This thickening is due chiefly to development of tissue between the glands, and the membrane becomes soft and pulpy. In the meantime the ovum has effected its lodgement between the folds, usually near the fundus and near the opening of one of the Fallopian tubes; and the adjacent parts of the mucous membrane extend over the ovum so that it is at last completely enclosed. This occurs at the twelfth or thirteenth day. The extension. of the mucous membrane which covers the ovum becomes the decidua reflexa; the changed mucous membrane which lines the uterus becomes the decidua vera; and the portion of the mucous membrane that remains at the site of the placenta becomes the decidua serotina. The vascular villosities of the chorion probably do not, as was once thought, penetrate the uterine tubules, but they become surrounded with tissues developed between the tubules.

As development advances, the decidua vera becomes extended, loses its vessels and glands and is reduced to the condition of a simple 
membrane. The cylindrical epithelium of the mucous membrane of the body of the uterus, soon after fecundation, becomes exfoliated, and its place is supplied with flattened cells. This change is effected at the sixth or the eighth week. The epithelium of the cervix retains its cylin-

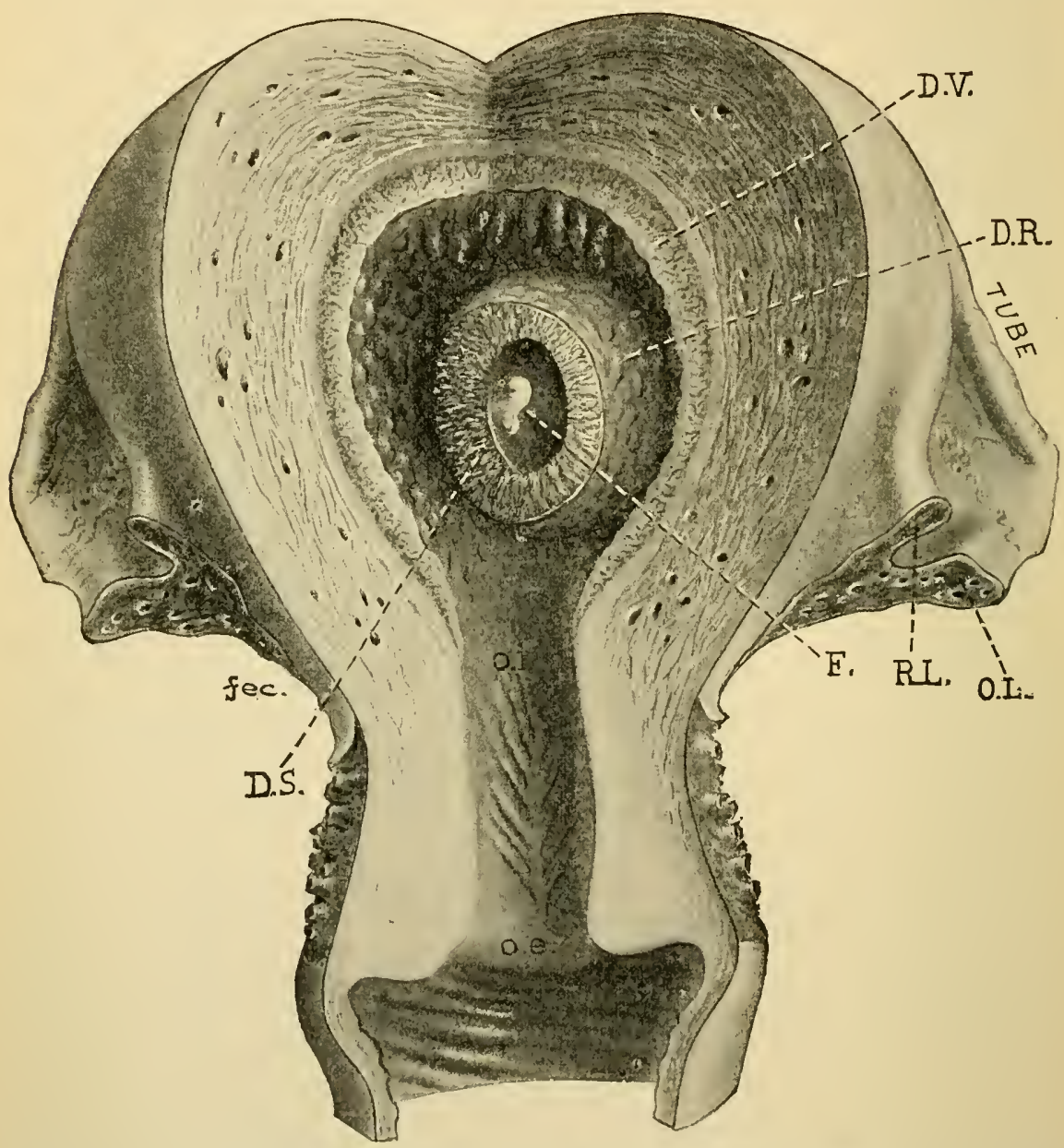

Fig. 227. - Seventeen-days' gravid uterus, $\times$ 1. - From the Anatomical Museum of Fohns Hopkins University. - Embryo drawn relatively too large (Williams).

$D . R$. , decidua reflexa; D.S., decidua serotinta; D. $V_{.}$, decidua vera; $E$, embryo; $O . L$. , ovarian ligament; R.L., round ligament.

drical character, but most of the cells lose their cilia. The decidua reflexa, which is thinner than the decidua vera, has neither bloodvessels, glands nor epithelium.

During the first periods of utero-gestation, the two layers of decidua are separated by a small quantity of an albuminous and sometimes a 
sanguinolent liquid; but this disappears at about the end of the fourth month, and the membranes then come in contact with each other. They soon become so closely adherent as to form a single membrane, which is in contact with the chorion. Sometimes, at full term, the membranes of the fœtus can be separated from the decidua; but frequently the different layers are closely adherent to each other.

The changes just described are not participated in by the mucous membrane of the neck of the uterus. The glands in this situation secrete a semisolid, transparent, viscid mucus, which closes the os and is sometimes called the uterine plug.

Toward the fourth month a very delicate, soft, homogeneous layer appears over the muscular fibres of the uterus beneath the decidua vera, which is the beginning of a new mucous membrane. This is developed very gradually, and the membrane is completely restored about two months after parturition.

Formation of the Placenta. - At about the end of the second month the villi of the chorion become enlarged and arborescent over that part which eventually forms the fotal portion of the placenta. They are then highly vascular and are embedded in the soft substance of the hypertrophied mucous membrane. This portion of the chorion is called the chorion frondosum. At the same time the villi over the rest of the chorion (chorion leve) are arrested in their growth, and they finally disappear during the third month. The bloodvessels penetrate the villi in the form of loops at about the fourth week; and the placenta is distinctly marked at about the end of the third month. The placenta then rapidly assumes the anatomical characters observed after it may be said to be fully developed (see Plate X, Fig. 6).

The fully-formed placenta occupies about one-third of the uterine mucous membrane, and usually is rounded or ovoid in form, with a distinct border connected with the decidua and the chorion. It is seven to nine inches ( 18 to 23 centimeters) in diameter, a little more than an inch (2.5 centimeters) in thickness at the point of penetration of the umbilical cord, slightly attenuated toward the border, and weighs fifteen to thirty ounces ( 425 to 850 grams). Its fœtal surface is covered with the smooth amniotic membrane, and its uterine surface, when detached, is rough, and divided into irregular lobes, or cotyledons, half an inch to an inch and a half (12.7 to 38. I millimeters) in diameter. Between these lobes are membranes, called dissepiments, which penetrate into the substance of the placenta and at its border extend as far as the fœtal surface.

On the uterine surface of the placenta, is a thin soft membrane, the decidua serotina. This is composed of amorphous matter, a large 
number of granules, and colossal cells with enlarged and multiple nuclei. A portion of this membrane is not thrown off with the placenta in parturition, but processes extend into the placenta and closely surround the fotal tufts.

The two arteries of the umbilical cord branch on the fotal surface beneath the amnion and finally penetrate the substance of the placenta. The branches of the veins, which are about sixteen in number, converge toward the cord and unite to form the umbilical vein. On the uterine surface of the placenta are oblique openings of a large number of veins which return the maternal blood to the uterine sinuses. There are, also, the small spiral arteries, which pass into the substance of the placenta to supply blood to the maternal portion. These are the "curling arteries," clescribed by John Hunter. If the umbilical arteries are injected, the liquid is returned by the umbilical vein, having passed through the vascular tufts of the foetal portion of the placenta.

According to Winkler, there are three kinds of foetal villi : I. Those which terminate just beneath the chorion, without penetrating the vascular lacunæ. 2. Longer villi, which hang free in the lacunæ. 3. Long, branching villi, which penetrate more deeply into the placenta, some extending as far as its uterine surface.

The great vascular spaces, or lacunæ of the maternal portion of the placenta, present a number of trabecula, which extend from the uterine to the foetal surface; and between these trabecula, are exceedingly delicate transverse and oblique secondary trabecular processes. The bloodvessels of the fœetal tufts are surrounded with a gelatinous connective-tissue structure, and as late as the sixth month are covered with a layer of chorionic cells.

The mode of formation of the vascular spaces in the placenta has been a subject of much discussion. The following, however, seems to be the most reasonable view in regard to this question: That portion of the uterine mucous membrane which becomes the maternal portion of the placenta extends from the decidua serotina and surrounds the villi, which are embedded in its substance. As the arborescent villi extend, they encroach on the bloodvessels of the prolongations from the serotina, which latter become much enlarged and finally form the great vascular spaces traversed by the trabeculæ mentioned above. At term, however, according to Heinz ( I888), the fœetal vessels have lost their covering of epithelium that is observed in the earlier months of pregnancy. Thus the most important parts of the placenta are formed by an interlacement of the villi of the chorion with the altered structures of the mucous membrane of the uterus.

In the human subject the maternal and fœetal portions of the pla- 
centa are so closely united that they cannot be separated from each other. In parturition the curling arteries and the veins on the uterine surface of the placenta are torn off, and the placenta then consists of the parts just described; the torn ends of the vessels attached to the uterus are closed by the contractions of the surrounding muscular fibres; and the blood that is discharged is derived mainly from the placenta itself.

Uses of the Placenta. - The placenta is the respiratory, excretory and nutritive organ of the fœtus. Its action as a respiratory organ has already been mentioned in connection with the physiology of respiration. It certainly serves as an organ for the elimination of carbon dioxide, and probably for other products of excretion. It is the only source of materials for the development and nutrition of the fœtus. It is thought that cells derived from the serotina elaborate a liquid called uterine milk, which is absorbed by the fœtal tufts. This liquid has been collected from between the fotal tufts of the placenta of the cow and has been found to contain fatty matters, albuminous matters and certain salts, but no sugar or casein. It is not probable, however, that such a liquid exists in the human placenta; although "uterine milk" of the ruminants was mentioned by Haller and has been alluded to by even earlier writers. 


\title{
CHAPTER XXXII
}

\author{
DEVELOPMENT OF THE OVUM
}

Development of the cavities and layers of the trunk in the chick - Development of the skeleton, muscular system and skin-Notochord - Vertebral column etc. - Development of the nervous system - Development of the digestive system - Development of the respiratory system-Development of the face-Developinent of the teeth-Development of the genito-urinary apparatus - Development of the urinary system-Development of the external organs of generation-Development of the circulatory system - The first, or vitelline circulation - The second, or placental circulation - Development of the heart - Peculiarities of the fotal circulation - The third, or adult circulation.

THE product of generation retains the name of ovum until the form of the body begins to be apparent, when it is called the embryo. At the fourth month, about the time of quickening, it is called the fotus, a name retained during the rest of intra-uterine life. The membranes are appendages formed for the purposes of protection and nutrition; and the embryo itself, in the mammalia, is developed from a restricted portion of the layers of cells resulting from the segmentation of the ovum.

The formation of the blastodermic cells and the appearance of the groove which is subsequently developed into the neural canal have already been described. At this portion of the ovum, there is a thickening of the blastoderm, which then presents three layers, the mesoblast - the thickest and most important - being developed between the epiblast and the hypoblast. The earliest stages of development have been studied most successfully in the chick; and it is probable that the appearances here observed nearly represent the earlier processes of development in the human subject.

Development of the Cavities and Layers of the Trunk in the CHICK

As an introduction to a description of the development of special organs in the human subject and in mammals, it will be found useful to study the first stages of development in the chick, which will give an idea of the arrangement of the different blastodermic layers and the way in which they are developed into the different parts of the trunk, with the mode of formation of the great cavities. The figures by which this 
description is illustrated in the text are those of Brücke, which were photographed on wood from drawings made by Seboth. The Plates in the atlas are photographic representations in color, of stained sections.

Figure 228 shows an early stage of development of the chick, in a transverse section near the caudal extremity. In this figure, the upper layer of dark cells $(B, B)$ represents the epiblast. The lower layer of dark cells $(D, D)$ represents the hypoblast. The middle layer of lighter cells is the mesoblast, which, toward the periphery, is split into two

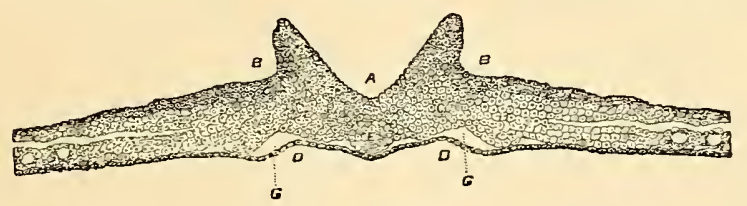

Fig. 228. - Early stage of development of the chick-about twenty-four hours (Seboth and Brücke).

layers. This figure represents a transverse section. At $A$, is a transverse section of the groove that afterward is developed into the neural canal. Beneath this groove is a section of a rounded cord $(E)$, the notochord. The openings $(G, G)$ represent the situation of the two aortæ. The other cavities are as yet indistinct in this figure (see Plate XV, Fig. 3).

Figure 229 shows the structures in a transverse section near the head. The dorsal, or vertebral plates, which bound the furrow $(A)$ in Fig. 228, are closed above, and include $(A)$ the neural canal. The notochord $(E)$ is separated from the cells surrounding it in Fig. 228. The

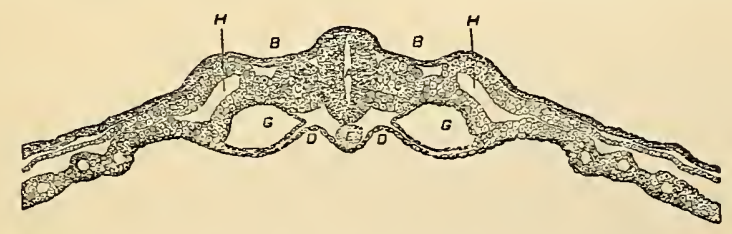

Fig. 229. - Transverse section near the head (Seboth and Brücke).

epiblast $(B, B)$ and the hypoblast $(D, D)$ present certain curves that follow the arrangement of the cells of the mesoblast. By the sides of the boundaries of the neural canal, are two distinct masses of cells $(C, C)$, which are the primitive somites. Outside of these masses of cells, are two smaller collections of cells, afterward developed into the Wolffian bodies. Beneath these two masses, are two large cavities $(G, G)$, the largest cavities shown in Fig. 229, presenting an irregular form, which are sections of the two primitive aortæ. The two openings $(H, H)$ afterward become the celom, or body-cavity (see Plate XV, Figs. 4 and 5). 
In Fig. 230 the parts are still further developed. The neural canal is represented $(A)$ nearly the same as in Fig. 229, with the notochord $(E)$ just beneath it. A groove, or gutter $(D)$ has been formed in front, which is the groove of the intestinal canal, which remains open at this time and is lined with the hypoblast. Just above $D$ is a single opening $(G)$, which is formed by the union of the two openings $(G, G)$ in Figs. 228 and 229; and this is the abdominal aorta, which has here become single. The two openings $(H, H)$ represent a section of the body-cavity. The outer wall of this cavity is the outer visceral plate, which is developed into the muscular walls of the abdomen. The lower and inner wall is the inner visceral plate, which forms the main portion of the intestinal wall. The outer wall is the outer layer of the mesoblast, and the inner wall is the inner layer of the same membrane. The two round openings (I, I) are sections of the Wolffian ducts (see Plate XV,

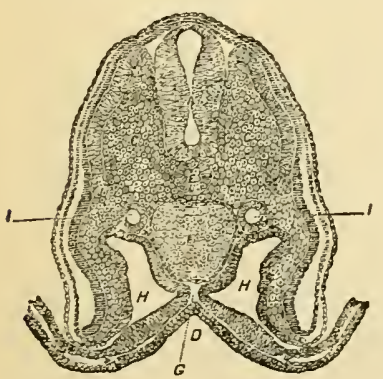

Fig. 230. - Later stage of development of the chack (Seboth and Brücke). Fig. 6).

The figures just described, it must be borne in mind, represent transverse sections of the body of the chick, made through the middle portion of the abdomen. The dorsal parts, it is seen, are developed first, the situation of the vertebral column being marked soon after the enclosure of the neural canal by the vertebral plates; and at about the same time, the two aortæ make their appearance, with the first traces of the body-cavity (celom). The enclosure of the neural canal takes place from before backward, beginning at the cephalic end. The canal remains open at the caudal end for some time after it has been enclosed above, as is shown in Figs. 228 and 229. The next organs in the order of development, after the vascular system, are the Wolffian bodies. The intestinal canal is then a simple groove, and the embryo is open in front. In the further processes of development, the visceral, or abdominal plates advance and close over the abdominal cavity, in the same way as the medullary plates have closed over the neural canal. Thus there is formed a closed tube, the intestine, which is lined with the hypoblast, the walls of the intestine being formed of the inner layer of the mesoblast. This brings the external layer of the mesoblast around the intestine, to form the muscular walls of the abdomen, the external covering being the epiblast. At this time the Wolffian bodies lie next the spinal column, between the intestine and the abdominal walls, with the single abdominal aorta situated behind the intestine. 


\section{Development of the Skeleton, Muscular System and Skin}

Notochord. - One of the earliest structures observed in the developing embryo is the chorda dorsalis, or notochord. This is situated beneath the neural canal and extends the entire length of the body. It is formed of a cord of simple cells and marks the situation of the vertebral column, though it is not itself developed into the vertebræ, which grow around it and encroach on its substance until it finally disappears. In many mammals, the notochord presents a slight enlargement at the cephalic extremity, which extends to the auditory vesicles; and it is somewhat diminished in size at the caudal extremity. By the sides of this cord are masses of cells (mesoblastic somites), which unite in front.of the neural canal and were formerly thought to be developed into the vertebræ. ${ }^{1}$ These are the so-called protovertebræ (or somites) and are shown in Fig. $233(C$, in $A$ and $B$ ). Twelve pairs of somites are shown in Fig. 233, $C$. In the chick two pairs are first formed in the upper cervical region on the second day. They rapidly increase in number from above downward, until at the fourth day there are twenty-one or twenty-two pairs. They are not formed in the region of the head

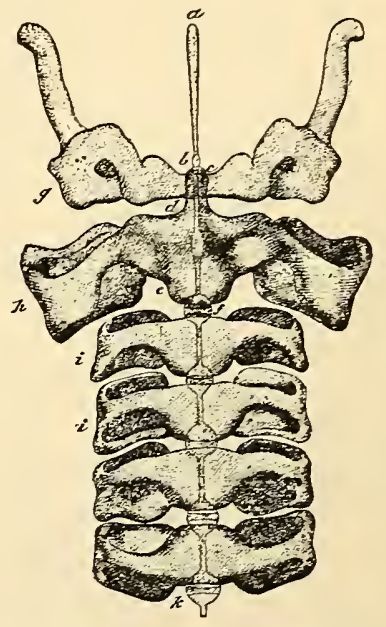

Fig. 23x. - The first six cervical vertebre of the embryo of a rabbit (Robin).

$a, b$, cephalic portion of the notochord, exposed by the removal of the cartilage; $b$, portion of the notochord slightly enlarged, which, in this embryo, was situated between the atlas and the occipital bone; $c$, odontoid process; $d$, base of the odontoid process; $e$, inferior, or second part of the body of the axis; $f, k$, enlargements of the notochord, between the vertebræ; $g$, cartilage of the lateral portion of the atlas; $h$, lateral portion of the axis; $i, i$, transverse apophyses of vertebræ. or at the lowest part of the vertebral column. The vertebræ, as they are developed, are formed of temporary cartilaginous structure, gradually extending around the notochord, which then occupies the axis of the spinal column. These carti-

1 The mesoblastic somites (protovertebræ) are sometimes called muscle-plates, or myotomes. It is thought that cells from their inner walls are concerned in the development of the vertebræ and that the other cells are developed, some into muscular structure and some into the true skin ; but their destination is somewhat obscure. The outer cells - developed into cutis - are called the skin-plates, or dermatomes. 
lages are not divided at the lines of separation of the somites, but the somites fuse together and the cartilages which are to be developed into the bodies of the vertebræ are so divided off that one cartilage occupies the place of the adjacent halves of two somites. Between the bodies of the vertebræ, the notochord presents regular enlargements surrounded by a delicate membrane. As ossification of the spinal column advances, that portion of the notochord which is surrounded by the bodies of the vertebræ disappears, leaving the enlargements between the vertebræ distinct. These enlargements, which are not permanent, are gradually invaded by fibrous tissue, their gelatinous contents disappear, and the intervertebral disks, composed of fibro-cartilaginous structure, remain. These disks are permanent between the cervical, the

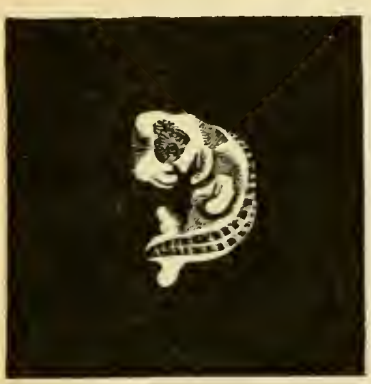

Fig. 232. - Human embryo, about one month old, showing the large size of the head and upper parts of the body, the twisted form of the spinal column, the rudimentary condition of the upper and lower extremities and the rudimentary tail at the end of the spinal column (Dalton). dorsal and the lumbar vertebræ; but they eventually disappear from between the different parts of the sacrum and coccyx, as these are consolidated, this occurring, in the human subject, between the ninth and the twelfth years.

Vertebral Colitmn, etc. - In Figs. 229 and $230(C, C)$, are seen the two masses of cells (somites) situated by the sides of the neural canal. Cells of the inner borders of these bodies extend around and encroach on the notochord and form the bodies of the vertebræ. They also extend over the neural canal, closing above, and their processes are called the medullary, or dorsal plates. From the sides of the bodies of the vertebræ, the various processes of these bones are formed. As the spinal column is developed, its lower portion presents a projection beyond the pelvis, which constitutes a temporary caudal appendage, curved toward the abdomen; but this no longer projects after the bones of the pelvis are fully developed. At the same time the entire vertebral column is curved toward the abdomen, and it is twisted on its axis from left to right, so that the anterior face of the pelvis presents a right angle to the upper part of the body; but as the inferior extremities and the pelvis are developed, the spine becomes straight. The vertebræ make their appearance first in the middle of the dorsal region, from which point they rapidly extend upward and downward until the spinal column is complete.

At the base of the skull, on either side of the superior prolongation of the notochord, are two cartilaginous processes that are developed into the so-called cranial vertebræ. In this cartilaginous mass, three 
ossific points appear, one behind another. The posterior point of ossification is for the basilar portion of the occipital bone, which is developed in the same way as one of the vertebra; the middle point is for the posterior portion of the sphenoid; and the anterior point is for the anterior portion of the sphenoid. The frontal bone, the parietal bone, the temporal bone and a portion of the occipital bone are developed from the connective tissue without the intervention of preëxisting cartilaginous structure. At the time when the vertebræ are developed, with their laminæ and their spinous and transverse processes, the ribs extend over the thorax, and the clavicle, scapula and sternum make their appearance.

At about the beginning of the second month, four papillary prominences, the first traces of arms and legs, appear on the body of the embryo. These progressively increase in length, the arms appearing near the middle of the embryo, and the legs, at the lower portion. Each extremity is divided into three portions, the arm, forearm, and hand, for the upper extremities, and the thigh, leg and foot, for the lower extremities. At the end of each extremity, there are, finally, divisions into the fingers and toes, with the various cartilages and bones of all these parts, and their articulations.

Early in intra-uterine life the skeleton begins to ossify from bony points in the cartilaginous structure. The first points appear at nearly the same time--about the beginning of the second month - in the clavicle and the upper and the lower jaw. Similar ossific points, which gradually extend, are seen also in the other parts, the head, ribs, pelvis, scapula, metacarpus and metatarsus, and the phalanges of the fingers and toes. At birth the carpus is entirely cartilaginous, and it does not begin to ossify until the second year. The same is true of the tarsus, except the calcaneum and astragalus, which ossify just before birth. The pisiform bone of the carpus is the last to take on osseous transformation, this occurring between the twelfth and the fifteenth years. As ossification progresses, the deposits in the various ossific points gradually extend until they reach the joints, which remain incrusted with the permanent articular cartilage (see Plate X, Fig. 3).

While the skeleton is thus developing, the muscles are formed from the outer layer of the mesoblast, and the visceral plates close over the thorax and abdomen in front, leaving an opening for the umbilical cord. The various tissues of the external parts, particularly the muscies, begin to be distinct at the end of the second month. The deep layers of the dorsal muscles are the first to be distinguished; then successively, the long muscles of the neck, the anterior straight muscles of the head, the straight and transverse muscles of the abdomen, the muscles of the 
extremities, the superficial muscles of the back, the oblique muscles of the abdomen and the muscles of the face.

The skin appears at about the beginning of the second month, when it is thin and transparent. At the end of the second month the epidermis may be distinguished. The sebaceous follicles are developed at the third month; and at about the fifth month the surface is covered with their secretion mixed with desquamated epithelium. This cheesy substance is the vernix caseosa. At the third month the nails make their appearance, and the hairs begin to grow at about the fifth month. The sudoriparous glands first appear at about the fifth month, by the formation of flask-like processes of the true skin, which are gradually elongated and convoluted until they are fully developed only a short time before birth.

\section{Development of the Nervous System}

It has been seen, in studying the development of the spinal column, how the dorsal, or medullary plates close over the groove for the neural canal. In the interior of this canal, the cerebro-spinal axis is developed, by cells which gradually encroach on its calibre, until there remains only the small central canal of the spinal cord, communicating with the ventricles of the brain. As the nervous tissue is developed in the interior of the neural canal, there is a separation of the histological elements at the surface to form the membranes. The dura mater and the pia mater are formed first, appearing at about the end of the second month; while the arachnoid is not distinct until the fifth month. The nerves are not produced as prolongations from the cord into the various tissues nor do they extend from the tissues to the cord; but they are developed in each tissue by a separation of histological elements from the cells of which the parts are originally constituted. The nerves of the sympathetic system are developed in the same way.

The mode of development of the spinal cord is thus sufficiently simple; but with the growth of the embryo, dilatations are observed at the superior and the inferior extremities of the neural canal. The cord is nearly uniform in size in the dorsal region, marked only by the regular enlargements at the sites of origin of the spinal nerves; but there soon appears an ovoid dilatation below, which forms the lumbar enlargement, from which the nerves are given off to the inferior extremities, and the brachial enlargement above, where the nerves of the superior extremities take their origin. At the same time there is a more marked dilatation of the canal at its cephalic extremity. Here a single enlargement appears, which is soon divided into three vesicles, 
called the anterior, middle and posterior cerebral vesicles. These become more and more distinct as development advances. The formation of these parts is shown in Fig. 233. This figure, in $C$, shows the projections, on either side, of the vesicles that are eventually developed $(o$, Fig. $233, C)$ into the nervous portions of the organ of vision.

The three cerebral vesicles now undergo further changes. The superior, or the first primitive vesicle, is soon divided into two secondary vesicles, the anterior of which becomes the cerebral hemispheres, and the posterior, the diencephalon, including the optic thalami and the third ventricle, which are eventually covered by the greater relative development of the hemispheres. The middle, or second primitive vesicle, does not undergo division and is developed into the tubercula quadrigemina. The posterior, or third primitive vesicle, is divided into two secondary vesicles, the anterior of which becomes the cerebellum, and the posterior, which is covered by the anterior, the bulb and the pons Varolii. While this division of the primitive cerebral vesicles is going on, the entire chain of encephalic ganglia becomes curved from behind forward, forming three prominent

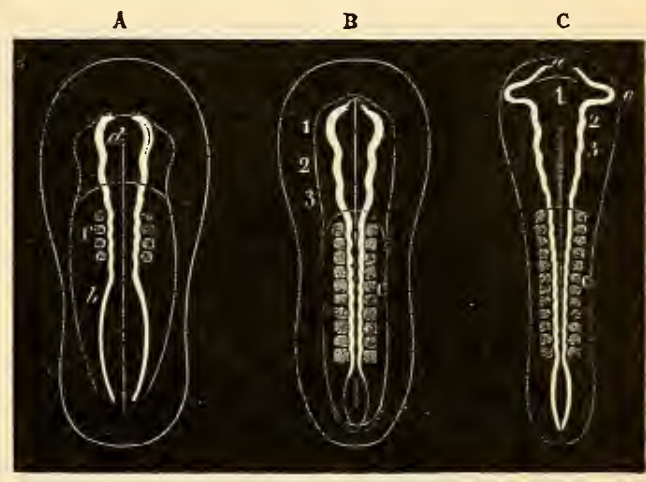

Fig. 233. - Development of the nervous system of the chick (Wagner).

$A$, the two primitive halves of the nervous system, twenty-four hours after incubation; $B$, the same, thirty-six hours after; $C$, the same, at a more advanced stage. $\dot{C}$, the somites; $b$, posterior dilatation (the lumbar enlargement) ; $d$, anterior dilatation of the neural canal; $1,2,3$, anterior, middle and inferior cerebral vesicles; $a$, slight Hattening of the anterior cerebral vesicle; $o$, formation of the ocular vesicles.

angles. The first of these angles or prominences ( $c$, Fig. 234, $A, B, C)$, counting from before backward, is formed by a projection of the tubercula quadrigemina, which at this time constitute the most projecting portion of the encephalic mass; the second prominence ( $\epsilon$, Fig. 234), situated behind the tubercula quadrigemina, is formed by the projection of the cerebellum; the third ( $d$, Fig. 234, $A, B, C$ ) is the bend of the superior portion of the spinal cord. These projections and the early formation of certain parts of the encephalon in the human subject are illustrated in Fig. 234 (see also Plate XVI, Figs. 5 and 6).

The cerebrum is developed from the anterior division of the first primitive cerebral vesicle. The development of this part is more rapid in its lateral portions than in the median line, which divides the cerebrum imperfectly into two lateral halves, forming in this way the great longi- 
tudinal fissure. At the same time, by the rapid development of the posterior portion, it extends over the optic thalami, the corpora quadrigemina and the cerebellum. Until the end of the fourth month, the hemispheres are smooth on their surface ; but they then begin to present large depressions, following folds of the pia mater, which are the first convolutions, these increasing rapidly in number and complexity, especially after the seventh month. The septum lucidum is then formed by an elevation of nervous matter from the base, which divides the lower portion of the space left between the hemispheres as they ascend, and forms the two lateral ventricles. At the base of these are developed

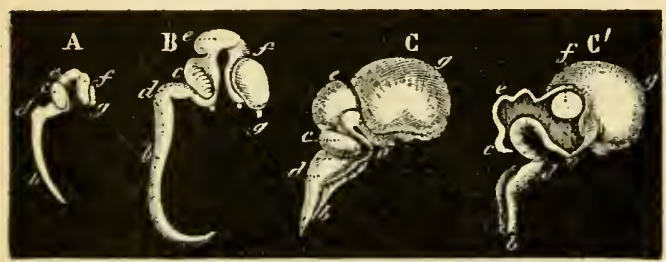

Fig. 234. - Development of the spinal cord and brain of the human subject (Longet, after Tiedemann).

$A$, brain and spinal cord of an embryo of seven weeks, lateral view.

$B$, the same, from an embryo further advanced in development $; b$, spinal cord; $d$, enlargement of the spinal cord, with its anterior curvature; $c$, cerebellum; $e$, tubercula quadrigemina; $f$, optic thalamus; $g$, cerebral hemispheres.

$C$, brain and spinal cord of an embryo of eleven weeks; $b$, spinal cord; $d$, enlargement of the spinal cord, with its anterior curvature; $c$, cerebellum; $e$, tubercula quadrigemina; $g$, cerebral hemispheres; 0 , optic nerve of the left side.

$C^{\prime}$, the same parts in a vertical section in the median line, from before backward; $b$, membrane of the spinal cord, turned backward; $d$, second curvature of the upper portion of the spinal cord, which has become thickened and constitutes the peduncles of the cerebrum; $e$, tubercula quadrigemina; $f$, optic thalami, covered by the hemispheres. the corpora striata. The septum lucidum is formed of two laminæ, with a small space between them, which is the cavity of the fifth ventricle. The posterior division of this first primitive vesicle forms the optic thalami. These become separated in front into two lateral halves, but they remain connected together at their posterior portion, which becomes the posterior commissure. The central canal of the cord is prolonged upward between the optic thalami and forms the third ventricle, which is covered by the hemispheres.

The second, or middle cerebral vesicle, becomes filled with medullary substance, extends tpward and forms the peduncles of the cerebrum, the upper portion being divided to form the tubercula quadrigemina.

The anterior portion of the third primitive vesicle is developed into the cerebellum, the convolutions of which appear at about the fifth month. Its posterior portion forms the bulb, in the substance of which is the fourth ventricle, communicating with the third ventricle by the aqueduct of Sylvius, which is left in the development of the middle vesicle. At about the fourth month there is a deposition of nervous matter in front and above, forming the pons Varolii.

In Fig. $234(C, o)$, it is seen that the vesicles for the organs of vision 
appear very early, as lateral offshoots of the anterior cerebral vesicle. These gradually increase in size and advance anteriorly as development of the other parts progresses. The eyes are situated at first at the sides of the head, gradually approaching the anterior portion. At the extremity of each of these lateral prolongations, a rounded mass appears, which becomes the globe of the eye. The superficial portions of the globe are developed into the sclerotic and the cornea, which seem to be formed of a process from the dura mater. The pedicle attached to the globe becomes the optic nerve. The iris is developed at about the seventh week and is at first a simple membrane without a central opening. As the pupil appears, it is closed by a vascular membrane which probably belongs to the capsule of the crystalline lens - called the pupillary membrane. This membrane gradually disappears by an atrophy extending from the centre to the periphery. It attains its maximum of development at the sixth month and disappears at the seventh month. The vitreous humor is formed of the liquid contents of the optic vesicle. The crystalline lens is regarded as a product of the epiblast. At the tenth week there is the beginning of the formation of the eyelids. These meet at about the fourth month and adhere together by their edges. In many mammals the eyelids remain closed for a few days after birth; but they become separated in the human subject in the later periods of fœtal life.

It is probable that the vesicle which is developed into the internal ear is formed independently; and cases have been observed in which there was congenital absence of the auditory nerves, the parts of the internal ear being perfect. Soon after the formation of the auditory vesicle, however, it communicates with the third primitive cerebral vesicle, the filament of communication being developed into the auditory nerve.

The auditory vesicle, which appears later than the organ of vision, is eventually developed into the vestibule. The next formations are the arches, or diverticula, which constitute the semicircular canals. The membranous labyrinth appears long before the osseous labyrinth; and it has been found perfectly developed at three months. The bones of the middle ear, which have no connection, in their development, with the nervous system, but which it is convenient to mention here, are remarkable for their early appearance. They appear at the beginning of the third month and are as large in the fœtus at term as in the adult. A remarkable anatomical point in relation to these structures is the existence of a cartilage attached to the malleus on either side and extending from this bone along the inner surface of the lower jaw, the two cartilages meeting and uniting in the median line to form a single cord. "This cartilage now ossifies, although, in the commencement, it 
forms most of the mass of the bone ; it disappears at the eighth month " (Meckel). This structure is known as the cartilage of Meckel.

There are no special points for description in the development of the olfactory lobes, which is very simple. These are offshoots from the first cerebral vesicle, appearing at the inferior and anterior part of the cerebral hemispheres, a little later than the parts connected with vision and audition. The vesicles themselves become filled with ganglionic matter and constitute the olfactory bulbs, their pedicles being the so-called olfactory nerves, or olfactory commissures.

So far as the action of the nervous system of the fœtus is concerned, it is probable that it is restricted mainly to reflex phenomena depending on the spinal cord, and that perception and volition hardly exist. It is probable that many reflex movements take place in utero. When a fœtus is removed from the uterus of an animal, even during the early months of pregnancy, movements of respiration occur; and it is well known that efforts of respiration sometimes take place within the uterus. These are due to the want of oxygen-carrying blood in the bulb when the placental circulation is interrupted.

\section{Develophent of the Digestive System}

The intestinal canal is the first formation of the digestive system. This is at first open in the greatest part of its extent, presenting, at either extremity of the longitudinal gutter in front of the spinal column, a rounded blind extremity, which is closed over in front for a short distance. The closure of the visceral plate then extends laterally and from the two extremities of the intestine, until only the opening remains for the passage of the umbilical cord and the pedicle of the umbilical vesicle. There is at first an open communication between the lower part of the intestinal tube and the allantois, which forms the canal known as the urachus; but that portion of this communication which remains enclosed in the abdominal cavity becomes separated from the urachus, is dilated and eventually forms the urinary bladder. When the bladder is shut off, it communicates with the lower portion of the intestine, which is called the cloaca; but it finally loses this connection and presents a special opening, the urethra.

As development advances, the intestine grows rapidly in length and becomes convoluted. It is held loosely to the spinal column by the mesentery, a fold of the peritoneum, this membrane being reflected along the walls of the abdominal cavity. In the early stages of development, a portion of the intestine protrudes at the umbilicus, where the 
first intestinal convolution appears; and sometimes there is a congenital hernia of this kind at birth, which usually disappears under the influence of gentle and continued pressure. An illustration of this is given in Fig. 235. This protrusion, in the normal process of development, is gradually returned into the abdomen, as the cavity of the pedicle of the umbilical vesicle is obliterated, at about the tenth week.

At the upper part of the abdominal cavity, the alimentary canal presents two lateral projections, or pouches. The one on the left side, as it increases in size, becomes the greater pouch of the stomach, and the one on the right side, the lesser pouch (see Plate XVI, Fig. 3).

At a short distance below the attachment of the pedicle of the umbilical vesicle to the intestine, there appears a rounded diverticulum, which is eventually developed into the cæcum. The cæcum gradually recedes from the neighborhood of the umbilicus, which is its original situation, and finally becomes fixed, by a shortening of the mesentery, in the right iliac region. As the cæcum is developed it presents a conical appendage, which is at first as large as the small intestine and is relatively longer than in the adult. During the fourth week this appendage becomes relatively smaller and more or less twisted, forming the appendix vermiformis. At the second month the cæcum is at the umbilicus, and the large intestine extends in a straight line toward the anus; at the third month it is situated at about the middle of the abdomen; and it gradually descends, until it reaches the right iliac region at about the seventh month. Thus at the

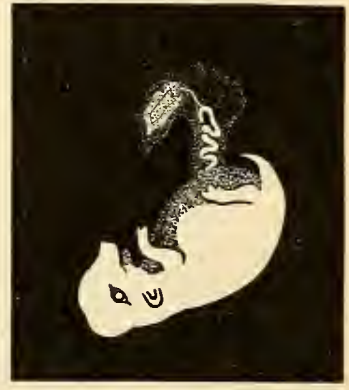

Fig. 235. - Fotal pig, showing a loop of intestine forming an umbilical hernia (Dalton).

From the convexity of the loop, a thin filament is seen passing to the umbilical vesicle, which is here flattened into a leaf-like form. second month, there is only a descending colon; the transverse colon is formed at the third month; and the ascending colon, at the fifth month. The ileo-cæcal valve appears at the third month; the rectum, at the fourth month; and the sigmoid flexure of the colon, at the fifth month. During this time the large intestine increases more rapidly in its diameter than the small intestine, while the latter develops more rapidly in its length.

In the early stages of development the internal surface of the intestines is smooth; but villi appear on its mucous membrane during the latter half of intra-uterine existence. These are found at first both in the large and the small intestine. At the fourth month they become shorter and less abundant in the large intestine, and they are lost at about the eighth month, when the projections which bound the sacculi of this 
portion of the intestinal canal make their appearance. The valvulæ conniventes appear in the form of slightly elevated transverse folds in the upper portion of the small intestine. The villi of the small intestine are permanent.

The mesentery is first formed of two perpendicular folds attached to the sides of the spinal column. As the intestine undergoes development, a portion of the peritoneal membrane extends in a quadruple fold from the stomach to the colon, to form the great omentum, which covers the small intestine in front.

As the head undergoes development a large cavity appears, which finally is bounded by the arches that are destined to form the different parts of the face. This is the pharynx. It is entirely independent, in its formation, of the intestinal canal, the latter terminating in a blind extremity at the stomach; and between the pharynx and the stomach, there is at first no channel of communication. The anterior portion of the pharynx presents, during the sixth week, a large opening, which is afterward partially closed in the formation of the face. The rest of the cavity remains closed until a communication is effected with the œsophagus. The œsophagus appears in the form of a tube, which finally opens into the pharynx above and into the stomach below. At this time there is really no thoracic cavity, the upper part of the stomach is very near the pharynx, the osophagus is short, the rudimentary lungs appear by its sides and the heart lies just in front. As the thorax is developed, however, the œsophagus becomes longer, the lungs increase in size, and finally the diaphragm shuts off its cavity from the cavity of the abdomen. The growth of the diaphragm is from its periphery to the central portion, which latter gives passage to the vessels and the œsophagus.

The development of the anus is very simple. At first the intestine terminates below in a blind extremity; but at about the seventh week a longitudinal slit appears below the external organs of generation, by which the rectum opens. This is the anus. The opening of the anus appears about a week after the opening of the mouth, at or about the seventh week.

The rudiments of the liver appear very early, and, indeed, at the end of the first month this organ has attained a large size. Two projections, or buds, appear on either side of the intestine, which form the two principal lobes of the liver. This organ is at first symmetrical, the two lobes being of nearly the same size, with a median fissure. One of these prolongations from the intestine becomes perforated and forms the excretory duct, of which the gall-bladder, with its duct, is an appendage. During the early part of fotal life the liver occupies the greatest 
part of abdominal cavity. Its weight, in proportion to the weight of the body at different ages, is as follows : At the end of the first month, I to 3 ; at term, I to I 8 ; in the adult, I to 36 . Its structure is soft during the first months. As development advances and as the relative size of the liver diminishes, its tissue becomes more solid.

The pancreas appears at the left side of the duodenum, by the formation of two ducts leading from the intestine, which branch and develop glandular structure at their extremities. The spleen is developed, about the same time, at the greater curvature of the stomach and becomes distinct during the second month.

The figures in Plate XVI show very clearly several of the important stages in the development of the nervous system, the vertebræ, the alimentary canal, liver and pancreas.

\section{Development of the Respiratory System}

On the anterior surface of the membranous tube which becomes the œsophagus, an elevation appears, which soon presents an opening into the œsophagus, the projection forming at this time a single, hollow cul-de-sac. This opening becomes the rima glottidis, and the single tube with which it is connected is developed into the trachea. At the lower extremity of this tube, a bifurcation appears, terminating first in one and afterward in several culsde-sac. The bifurcated tube constitutes, after the lungs are

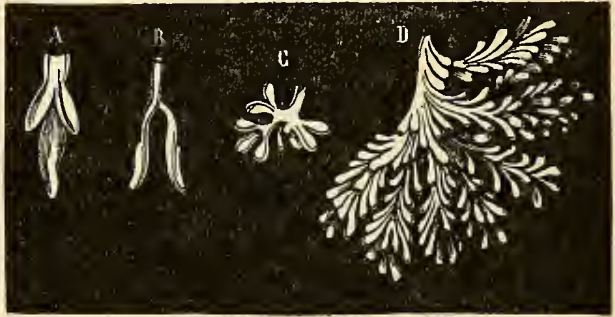

Fig. 236. - Formation of the bronchial ramifications and of the pulmonary cells. $-A, B$, development of the lungss, after Rathke; $C, D$, histological development of the lungs, after J. Miuller (Longet). developed, the primitive bronchia, at the extremities of which are the branches of the bronchial tree. As the bronchia branch and subdivide, they extend downward into what becomes afterward the cavity of the thorax. The pulmonary vesicles are developed before the trachea. The lungs contain no air during intra-uterine life and receive but a small quantity of blood; but at birth they become distended with air, are increased thereby in volume and receive all the blood from the right ventricle. This process of development is illustrated in Fig. 236. The lungs appear, in the human embryo, during the sixth week. The two portions into which the original bud is bifurcated constitute the true pulmonary structure; and the formation of the trachea and bronchial tubes occurs afterward and is secondary (see Plate XVI, Figs. I and 2). 


\section{Development of the Face}

The anterior portion of the embryo remains open in front long after the medullary plates have met at the back and enclosed the neural canal. The common cavity of the thorax and abdomen is closed by the growth of the visceral plates, which meet in front. At the time that the vis-

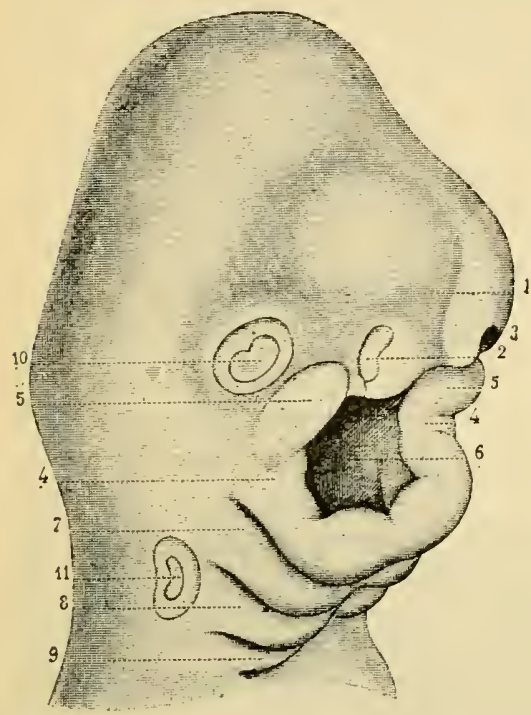

F1g. 237. - Wouth of a human embryo of twenty-five to twenty-eight day's, $\times$ I5 (Coste).

I, median, or frontal process, the inferior portion of which is considerably enlarged; 2, right nostril; 3, left nostril; 4, 4, inferior maxillary processes, already united in the median line; 5,5 , superior maxillary processes, which have become quite prominent and have descended to the level of the slope of the frontal process; 6 , mouth; 7 , first visceral arch; 8, second visceral arch; 9, third visceral arch; Io, eye; II, ear. the side, which forms, externally, the external auditory meatus, and internally, the tympanic cavity and the Eustachian tube. The other clefts become obliterated as the arches advance in their development.

From the above sketch it is seen that the face and the neck are formed by the advance and closure in front of projections from behind, in the same way as the cavities of the thorax and abdomen are closed; but the closure of the first visceral arch is complicated by the projection, from above downward, of the frontal, or intermaxillary process, and by the formation of several secondary projections, which leave certain permanent openings, forming the mouth, nose etc. 
In the very first stages of development of the head, there is no appearance of the face. The cephalic extremity consists simply of the cerebral vesicles, the surface of this enlarged portion of the embryo being covered, in front as well as behind, by the epiblast. During the sixth week, after the cavity of the pharynx has appeared, the membrane gives way in front, forming a large opening, which may be called the first opening of the mouth. At this time, however, the face is open in

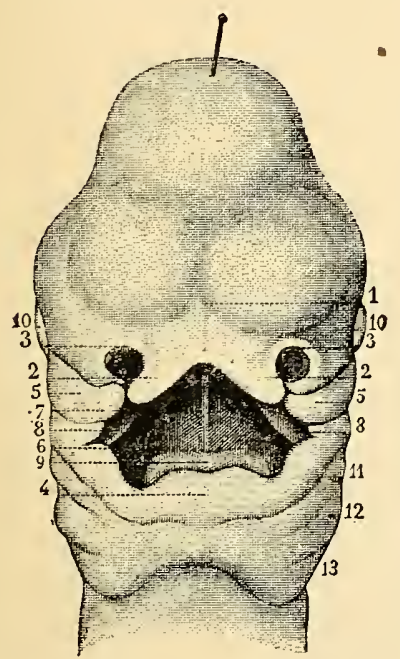

Fig. 238. - Mouth of a human embryo of thirtyfive days (Coste).

I, frontal process, widely sloped at its inferior portion; 2,2 , incisor processes produced by this sloping; 3,3 , nostrils; 4 , lower lip and maxilla, formed by the union of the inferior maxillary processes; 5, 5. superior maxillary processes, contiguous to the incisor process; 6 , mouth, still confounded with the nasal fossæ; 7 , first appearance of the closure of the nasal fossæ; 8, 8, first appearance of the two halves of the palatine arch; 9 , tongue; Io, Io, eyes; II, I2, I3, visceral arches.

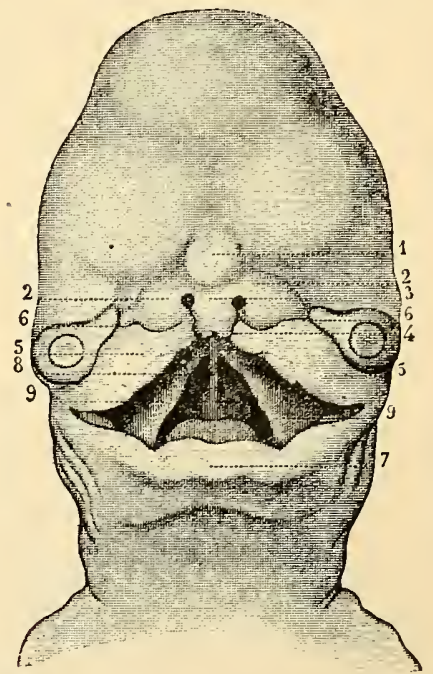

Fig. 239. - Mouth of an embryo of forty days (Coste).

I, first appearance of the nose; 2, 2, first appearance of the ala of the nose; 3 , appearance of the closure beneath the nose; 4 , middle, or median portion of the upper lip, formed by the approach and union of the two incisor processes, a little notch in the median line still indicating the primitive separation of the two processes; 5,5 , superior maxillary processes, forming the lateral portions of the upper lip; 6,6 , groove for the development of the lachrymal sac and the nasal canal; 7 , lower lip; 8 , mouth; 9,9 , the two lateral halves of the palatine arch, already nearly approximated to each other in front, but still widely separated behind.

front as far back as the ears. The first, or superior visceral arch, now appears as a projection of the mesoblast, extending forward. This is soon marked by two secondary projections, the upper projection forming the superior maxillary portion of the face, and the lower, the inferior maxilla. The two projections that form the lower jaw soon meet in the median line, and their superior margin is the lower lip. At the same time there is a projection from above, extending between the 
two superior projections, which is called the frontal, or intermaxillary process. This extends from the forehead - that portion which covers the front of the cerebrum-downward. The superior maxillary projections then advance forward, gradually passing to meet the frontal process, but leaving two small openings on either side of the median line, which are the openings of the nostrils. The upper portion of the frontal process thus forms the nose; but below, is the lower end of this . process, which is at first split in the median line, projects below the nose, and forms the incisor process, at the lower border of which are finally developed the incisor teeth. As the superior maxillary processes advance forward, the eyes are moved, as it were, from the sides of the head and present anteriorly, until finally their axes become parallel. These processes advance from the two sides, come to the sides of the incisor process, beneath the nose, unite with the incisor process on either side, and their lower margin, with the lower margin of the incisor process, forms the upper lip; but before this, the two lateral halves of the incisor process have united in the median line. At the bottom of the cavity of the mouth, a small papilla makes its appearance, which gradually elongates and forms the tongue (see Plate XVI, Fig. 5).

While this process of development of the anterior portion of the first visceral arch is going on, at its posterior portion the malleus and incus are developing, the former being at first connected with the cartilage of Meckel, which extends along the inner surface of the inferior maxilla, the cartilages from either side meeting at the chin. The cleft between the first and the second visceral arch has closed, except at its posterior portion, where an opening is left for the external auditory meatus, the cavity of the tympanum and the Eustachian tube.

At the same time the second visceral arch advances and forms the stapes, the styloid ligament and the lesser cornua of the hyoid bone. The third arch advances in the same way; and the arches from the two sides meet, become united in the median line and form the body and the greater cornua of the hyoid bone. The clefts between the second and the third and between the third and fourth arches finally are obliterated.

The fourth arch forms the sides of the neck and the larynx, the arytenoid cartilages being developed first. In front of the larynx and just behind the tongue, is a little elevation that is developed into the epiglottis. The openings of the nostrils appear during the second half of the second month. A little elevation, the nose, appears between these openings, and the nasal cavity begins to be separated from the mouth. The lips are distinct during the third month, and the tongue first appears in the course of the seventh week. 
The palatine arch is developed by two processes, which arise on either side, from the incisor process, pass backward and upward and finally meet and unite in the median line. The union of these forms the plane of separation between the mouth and the nares. At the same time a vertical process forms in the median line, between the palatine arch and the roof of the nasal cavity, which separates the two nares.

Development of the Teeth. - The first appearance of the organs for the development of the teeth is marked by the formation of a cellular projection extending the entire length of the border of either jaw, which forms a rounded band above and dips down somewhat into the subjacent structure. This band is readily separated by maceration, and the removal of the portion that dips into the maxilla leaves a groove. It extends the entire length of the jaws without interruption. Its superior surface is rounded, and that portion which dips into the subjacent mucous structure is wedge-shaped, so that its section has the form of a $V$.

So soon as this primitive band is formed - which occurs at the sixth or seventh week - a flat band projects from its internal surface, near the mucous structure, which is called the epithelial band. This also extends over the entire length of the jaw. It is thin, flattened, with its free edge curved inward and toward the jaw, and is composed at first of a central layer of polygonal cells covered with a layer of columnar epithelium.

At certain points - these points corresponding to the situation of the true dental bulbs - there appear rounded enlargements at the free margin of the epithelial band just described. Each one of these is developed into one of the structures of the perfect tooth. The mechanism of the formation of this, which is called the enamel-organ, and of the dental bulb is the following:-

A rounded enlargement appears at the margin of the epithelial band. This soun becomes directed downward - adapting the description to the lower jaw - and dips into the mucous structure, being at first connected with the epithelial band by a narrow pedicle which soon disappears, leaving the enlargements enclosed completely in a follicle. This is the dental follicle, and it has no connection with the wedge-shaped band described first. While this process is going on, a conical bulb appears at the bottom of the follicle. The enamel-organ, formed from the epithelial band, becomes excavated, or cup-shaped, at its under surface, and fits over the dental bulb, becoming united to it.

The tooth at this time consists of the dental bulb, with the enamelorgan closely fitted to its projecting surface. The enamel-organ is developed into the enamel; the dental bulb, which is provided with vessels and nerves, becomes the tooth-pulp; and on the surface 
of the dental bulb, the dentin is developed in successive layers. The cement is developed by successive layers upon that portion of the dentin which forms the root of the tooth. As these processes go on, the tooth projects more and more, the upper part of the wall of the follicle gives way, and the tooth finally appears at the surface.

The permanent teeth are developed beneath the follicles of the temporary, or milk-teeth. The first appearance is a prolongation, or diver-

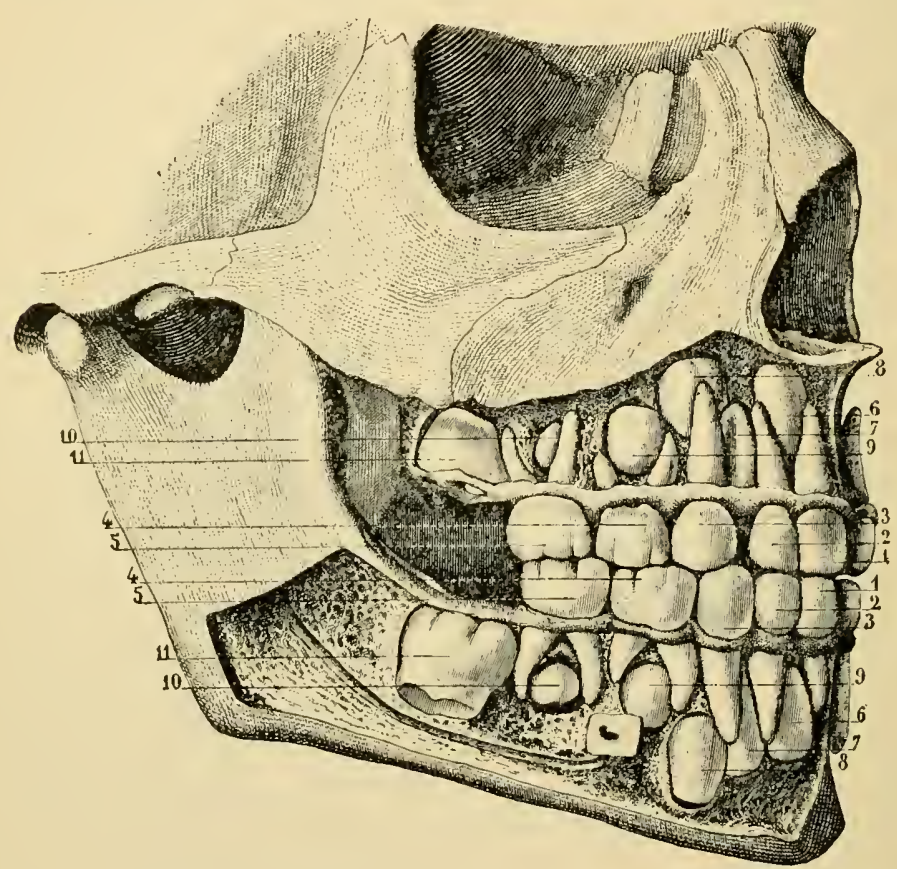

Fig. 240. - Temporary and permanent teeth (Sappey).

I, 1 , temporary central incisors; 2,2 , temporary lateral incisors; 3,3 , temporary canines ; 4,4 , temporary anterior molars; 5,5 , temporary posterior molars; 6,6 , permanent central incisors; 7,7 , permanent lateral incisors; 8,8 , permanent canines; 9 , 9, permanent first bicuspids; 10, 10, permanent second bicuspids; II, II, first molars.

ticulum, from the enamel-organ of the temporary tooth, which dips more deeply into the mucous structure. This becomes the enamel-organ of the permanent tooth; and the successive stages of development of the dental follicles and the dental pulp progress in the same way as in the temporary teeth. As the permanent teeth increase in size, they gradually encroach on the roots of the temporary teeth. The roots of the latter are absorbed, the permanent teeth advance more and more toward the surface, and the crown of each temporary tooth finally is pushed out. The number of the temporary teeth is twenty, and there are thirty-two permanent teeth. Thus there are three permanent teeth 
on either side of both jaws, which are developed de novo and are not preceded by temporary structures.

The first dental follicles appear usually in regular succession. The follicles for the internal incisors of the lower jaw appear first, this occurring at about the ninth week. All the follicles for the temporary teeth are completely formed at about the eleventh or twelf th week.

The temporary teeth appear successively, the corresponding teeth appearing a little earlier in the lower jaw. The usual order, subject to certain exceptional variations, is as follows :-

The four central incisors appear six to eight months after birth.

The four lateral incisors appear seven to twelve months after birth.

The four anterior molars appear twelve to eighteen months after birth.

The four canines appear sixteen to twenty-four months after birth.

The four posterior molars appear twenty-four to thirty-six months after birth.

The order of eruption of the permanent teeth is as follows :-

The two central incisors of the lower jaw appear between the sixth and the eighth years.

The two central incisors of the upper jaw appear between the seventh and the eighth years.

The four lateral incisors appear between the eighth and the ninth years.

The four first bicuspids appear between the ninth and the tenth years.

The four canines appear between the tenth and the eleventh years.

The four second bicuspids appear between the twelfth and the thirteenth years.

The above are the permanent teeth which replace the temporary teeth. The permanent teeth which are developed de novo appear as follows :-

The first molars appear between the sixth and the seventh years.

The second molars appear between the twelfth and the thirteenth years.

The third molars appear between the seventeenth and the twenty-first years.

\section{Development of the Genito-Urinary System}

The genital and the urinary organs are developed together, and both are preceded by the appearance of two large symmetrical structures, known as the Wolffian bodies, or the bodies of Oken. These are sometimes called the false, or primordial kidneys. They appear at about the thirtieth day, develop very rapidly on either side of the spinal column and are so large as almost to fill the cavity of the abdomen. Figure $24 \mathrm{I}$ shows how large these bodies are in the early life of the embryo, at which time their office undoubtedly is important.

Soon after the Wolffian bodies have made their appearance, there appear at their inner borders, two ovoid bodies, which finally are devel- 
oped into the testicles for the male, or the ovaries for the femalc. At their external borders are two ducts on either side, one of which, the internal, is called the duct of the Wolffian body. This finally disappears in the female, but it is developed into the vas deferens in the male. The other duct, which is external to the duct of the Wolffian body, disappears in the male, but it becomes the Fallopian tube in the female. This is known as the duct of Müler. Behind the Wolffian bodies, are developed the kidneys and the suprarenal capsules.

As the development of the Wolffian bodies attains its maximum their structure becomes somewhat complex. From their proper ducts, which are applied directly to their outer borders, tubes make their appearance at right angles, which extend into the substance of the bodies and

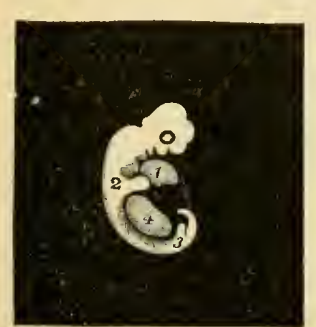

Fig. 241. - Fotal pig $\frac{5}{3}$ of an inch ( 16 millimeters) long. From a specimen prepared by Dalton.

I, heart; 2, anterior extremity; 3 , posterior extremity; 4, Wolffian body.

The abdominal walls have been cut away in order to show the position of the Wolffian bodies. become somewhat convoluted at their extremities. These tubes communicate directly with the ducts, and the ducts themselves open into the lower part of the intestinal canal opposite the point of its communication with the allantois. The tubes of the Wolffian bodies are simple, terminating in single, somewhat dilated, blind extremities, are lined with epithelium, and are penetrated at their extremities by bloodvessels which form coils or convolutions in their interior. These undoubtedly are organs of depuration for the embryo and take on the office to be afterward assumed by the kidneys; but in the female they are temporary structures, disappearing as development advances and having nothing whatever to do with the development of the true urinary organs (see Plate XV, Fig. 6).

The testicles or ovaries are developed at the internal and anterior surface of the Wolffian bodies, first appearing in the form of small ovoid masses. Beginning just above and passing along the external borclers of the Wolffian bodies, are the tubes called the ducts of Müller. These at first open into the intestine near the point of entrance of the Wolffian ducts. In the female their upper extremities remain free, except the single fimbria which is connected with the ovary. Their inferior extremities unite with each other, and at their point of union they form the uterus. The Wolffian bodies and their ducts disappear in the female at about the fiftieth day. A portion of their structure, however, persists in the form of a collection of closed tubes constituting the parovarium, or organ of Rosenmuiller.

In the female the ovaries pass down no farther than the pelvic cavity; 
but the testicles, which are at first in the abdomen of the male, finally descend into the scrotum. As the testicles descend they carry with them the Wolffian duct, that portion of the Wolffian body which is permanent constituting the head of the epididymis. At the same time a cord appears, attached to the lower extremity of the testicle and extending to the symphysis pubis. This is the gubernaculum testis. It is at first muscular, but the muscular fibres disappear during the later periods of utero-gestation. It is not known that its muscular structure takes any part, by contractile action, in the descent of the testicle in the human subject. The epididymis and the vas deferens are formed from the Wolffian duct.

At about the end of the seventh month the testicle has reached the internal abdominal ring; and at this time a double tubular process of peritoneum, covered with a few fibres from the lower portion of the internal oblique muscle of the abdomen, gradually extends into the scrotum. The testicle descends, following this process of peritoneum, which latter becomes eventually the visceral and parietal portions of the tunica vaginalis. The canal of communication between the abdominal cavity and the cavity of the scrotum is finally closed and the tunica vaginalis is separated from the peritoneum. The fibres derived from the internal oblique constitute the cremaster muscle.

At the eighth or the ninth month the testicles have reached the external abdominal ring and soon after descend into the scrotum. The vas deferens passes from the testicle, along the base of the bladder, to open into the prostatic portion of the urethra; and as development advances, two sacculated diverticula from these tubes make their appearance, which are attached to the bladder and constitute the vesiculæ seminales.

As the ovaries descend to their permanent situation in the pelvic cavity, there appears, attached to the inner extremity of each, a rounded cord, analogous to the gubernaculum testis. A portion of this, connecting the ovary with the uterus, constitutes the ligament of the ovary; and the inferior portion forms the round ligament of the uterus, which passes through the inguinal canal and is attached to the symphysis pubis.

Development of the Urinary Apparatus. - Behind the Wolffian bodies, and developed independently of them, the kidneys, suprarenal capsules and ureters make their appearance. The kidneys are developed in the form of small rounded bodies, composed of short blind tubes, all converging toward a single point, which is the hilum. These tubes increase in length, branch, become convoluted in a certain portion of their extent, and they finally assume the structure and arrangement of the renal tubules, with their Malpighian bodies, bloodvessels etc. They all open 
into the hilum. At the time that the kidneys are undergoing development, the suprarenal capsules are formed at their superior extremities. These bodies are relatively so much larger in the foetus than in the

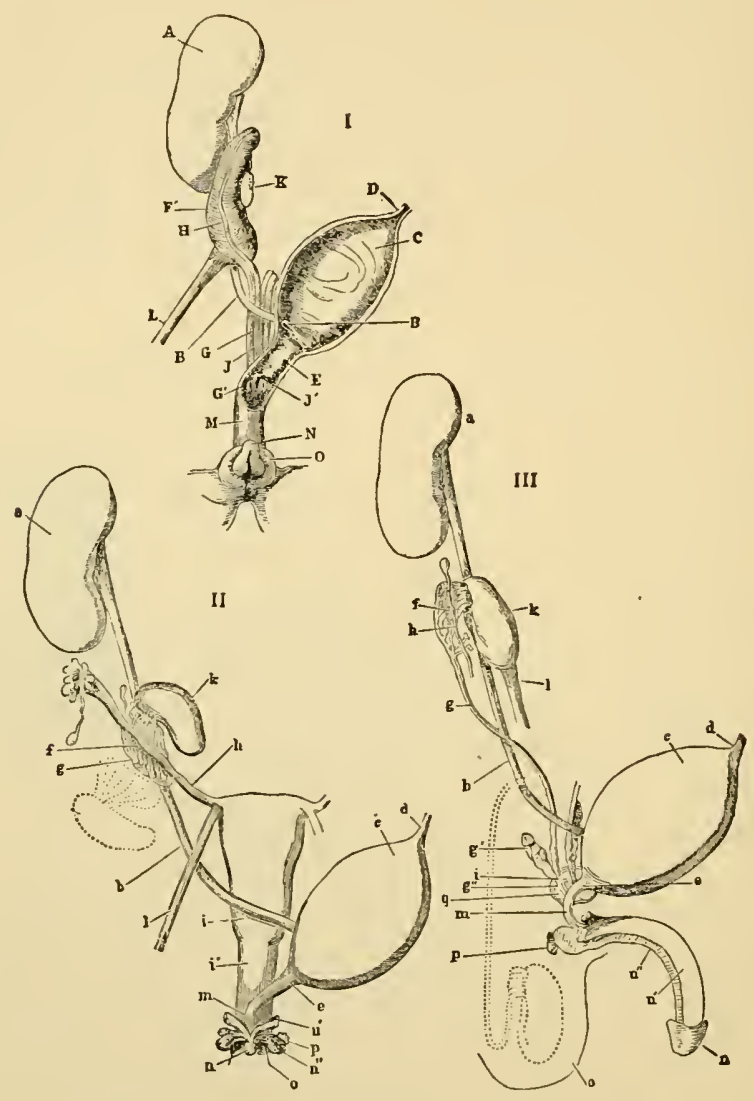

Fig. 242. - Diagrammatic representation of the genito-urinary system (Henle).

I, embryonic condition, in which there is no distinction of sex; II, female form; III, male form. The dotted lines in II and III represent the situations which the male and female genital organs assume after the descent of the ovaries and testicles. The small letters in II and III correspond to the capital letters in $\mathrm{I}$.

FIG. 242, I. - $A$, kidney; $B$, ureter; $C$, bladder; $D$, urachus, developed into the median ligament of the bladder; $E$, constriction which becomes the urethra; $F^{\prime}$, Wolffian body; $G$, Wolffian duct, with its opening below, $G^{\prime} ; H$, duct of $M$ üller, united below, from the two sides, into a single tube, $J$, which presents a single opening, $J^{\prime}$, between the openings of the Wolffian ducts; $K$; ovary or testicle; $L$, gubernaculum testis or round ligament of the uterus ; $\boldsymbol{M}$, genito-urinary sinus; $\boldsymbol{N}, O$, external genitalia.

FIG. $2 \nmid 2$, II (female). $-a$, kidney; $b$, ureter; $c$, bladder; $d$, urachus; $e$, urethra; $f$, remains of the Wolffian body (parovarium); $g$, remnant of the Wolffian duct; $h$, Fallopian tube; $i$, uterus; $i^{\prime}$, vagina; $k$, ovary; $l$, round ligament of the uterus; $m$, extremity of the urethra; $n$, clitoris; $n^{\prime}$, corpus cavernosum of the clitoris; $n^{\prime \prime}$, bulb of the vestibule; 0 , external genital opening; $p$, excretory duct of the gland of Bartholinus.

FIG. $2 \nmid 2$, III (male). $-a$, kidney; $b$, ureter; $c$, bladder; $d$, urachus; $e$, $m$, urethra ; $f$, epididymis; $g$, vas deferens; $g^{\prime}$, seminal vesicle; $g^{\prime \prime}$, ejaculatory duct; $h, i$, remains of the duct of Nüller; $k$, testicle; $l$, gubernaculum testis ; $n, n^{\prime}, n^{\prime \prime}$, wrethra and penis; 0 , scrotum; $p$, gland of Cowper; $q$, prostate. 
adult that they have been supposed to be peculiarly important in intrauterine life, although nothing definite is known on this point (see Plate XVI, Fig. 3). The kidneys are relatively very large in the fœtus. Their proportion to the weight of the body, in the fotus, is I to 80 , and in the adult, I to 240 . The ureters are developed as tubular processes from the kidneys, which finally extend to open into the bladder. The development of the genito-urinary apparatus can be readily understood, after the description just given, by a study of Fig. 3 I 2 .

Development of the External Organs of Generation. - The external organs of generation begin to be developed at about the fifth week. At the inferior extremity of the body of the embryo, a small ovoid eminence appears in the median line, at the lower portion of which there is a longitudinal slit which forms the common opening of the anus and the genital and urinary passages. This is the cloaca. There is soon developed internally a septum, which separates the rectum from the vagina, the urethra of the female opening above. In the male this. septum is developed between the rectum and the urethra, the generative and the uninary passages opening together. From this median prominence two lateral rounded bodies make their appearance. These are developed, with the median elevation, into the glans penis and corpora cavernosa of the male or into the clitoris and the labia minora of the female. In the male these two lateral prominences unite in the median line and enclose the spongy portion of the urethra. In the female there is no union in the median line, and an opening remains between the two labia minora. The scrotum in the male is analogous to the labia majora of the female; the distinction being that the two sides of the scrotum unite in the median line, while the labia majora remain permanently separated. This analogy is further illustrated by the anatomy of inguinal hernia, in which the intestine descends into the labium in the female or into the scrotum in the male. It sometimes occurs, also, that the ovaries descend, much as the testicles pass down in the male, and pass through the external abdominal ring.

\section{Development of the Circulatory System}

The blood and the bloodvessels are developed very early in the life of the ovum and make their appearance nearly as soon as the primitive streak. The mode of development of the first vessels differs from that of vessels formed later, as they appear de novo in the blastodermic layers, while afterward, vessels are formed as prolongations of preëxisting tubes. Soon after the epiblast and the hypoblast have become separated from each other, and the mesoblast has been formed at the 
thickened portion of the ovum, which is destined to be developed into the embryo, certain of the blastodermic cells undergo a transformation into blood-corpuscles. These are larger than the blood-corpuscles of the adult and usually are nucleated. At about the same time certain of

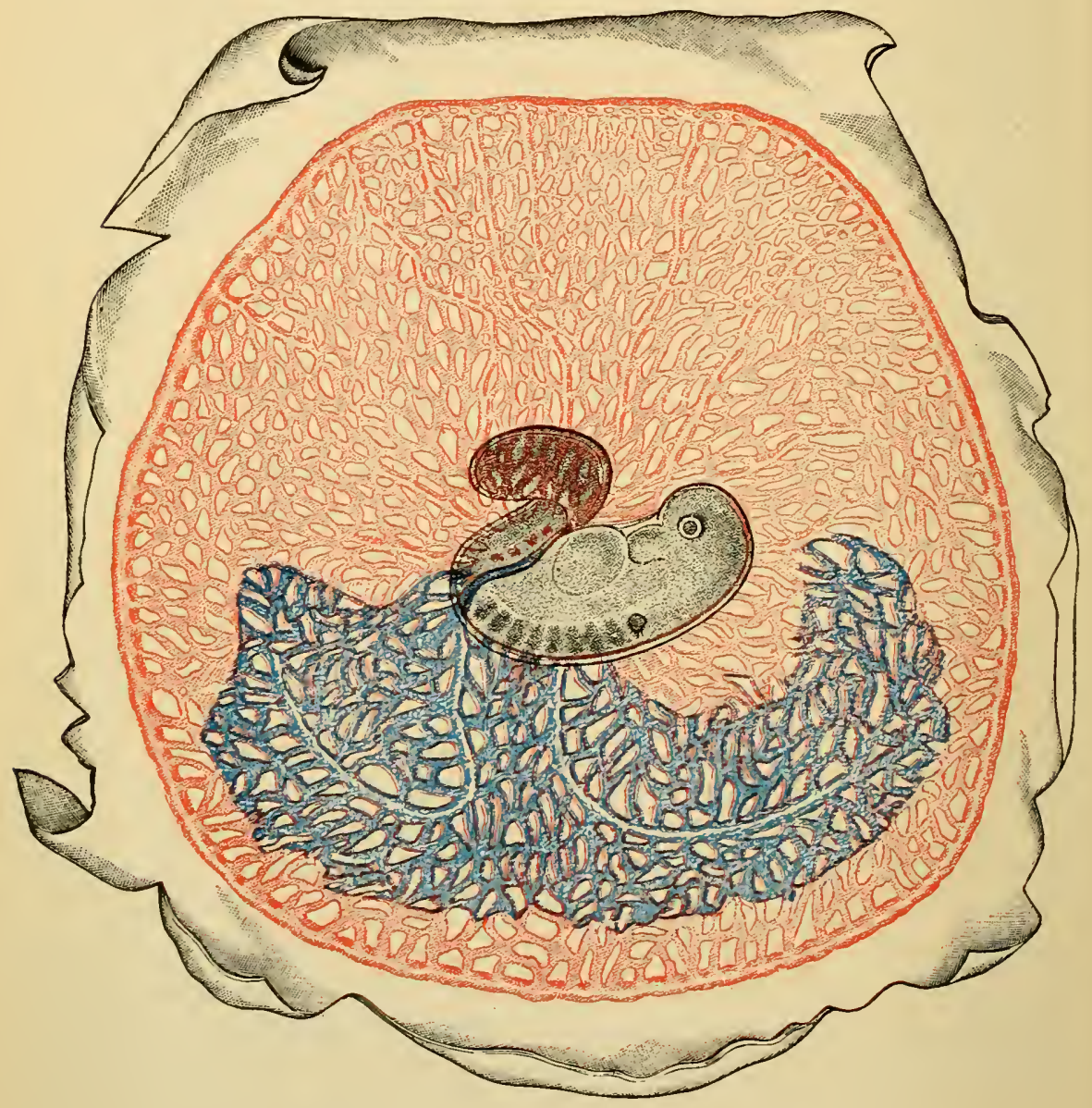

Fig. 243. - Area vasculosa of a rabbit of ten days (van Beneden and Julin).

In this figure the arteries, the arterial capillaries and the sinus terminalis (arterial) are in red, and the veins and venous capillaries are in blue.

the blastodermic cells fuse with each other and arrange themselves so as to form vessels. Leucocytes probably are developed in the same way as the red corpuscles. The vessels thus formed constitute the area vasculosa, which is the beginning of what is known as the first circulation.

The cells of the mesoblast do not take part in the formation of the blood and bloodvessels, as indicated above, but cells penetrate at the 
edges, between the epiblast and the hypoblast, and these, which are called parablastic cells, are developed into bloodvessels and blood-corpuscles. The connective tissue also is supposed to be dereloped from parablastic cells. According to this view - which, however, is not generally adopted - the parablastic cells are to be distinguished from the

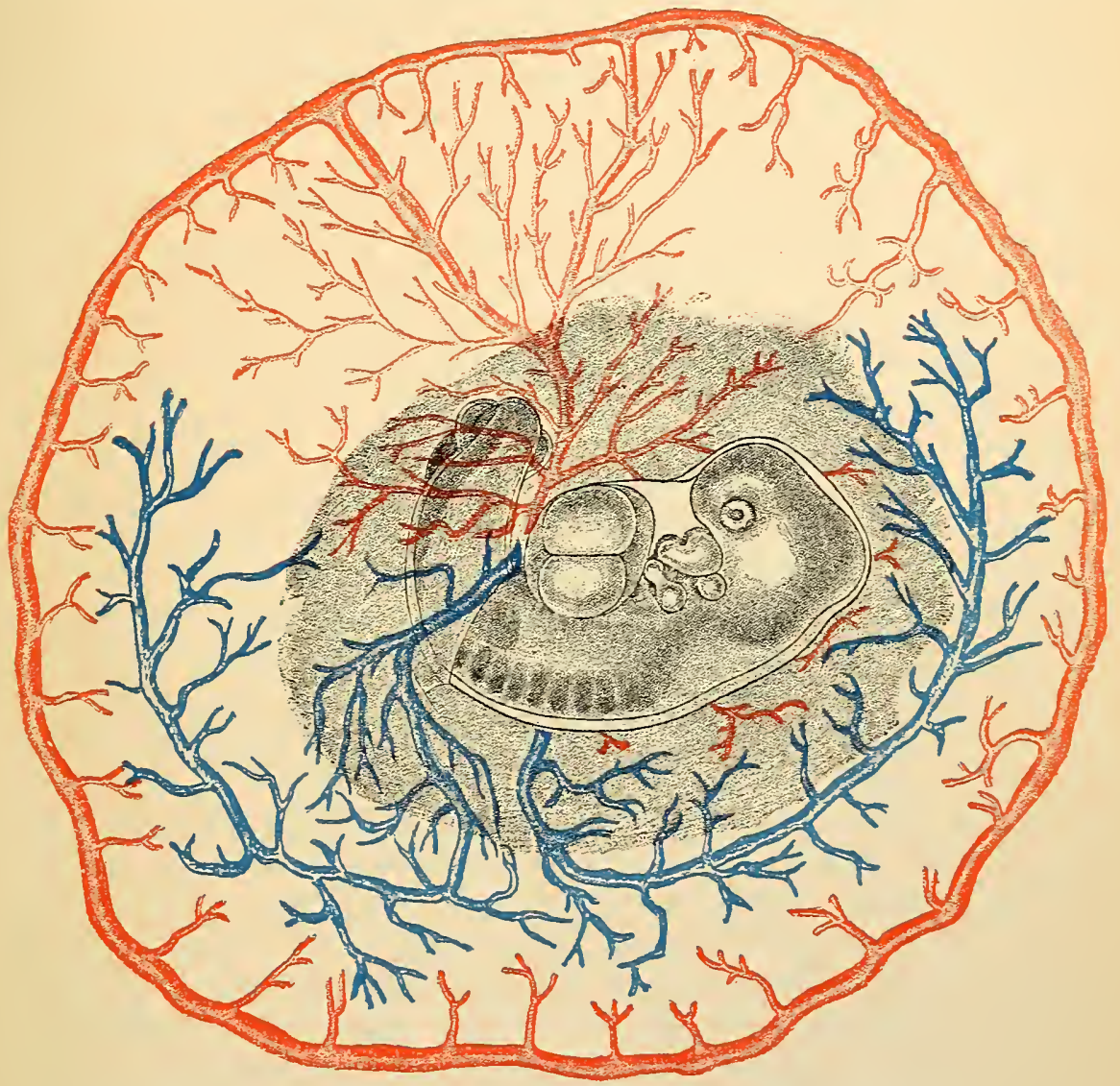

Fig. 244. - Area vasculosa of a rabbit of eleven days (van Beneden and Julin).

The capillaries are not shown in this figure.

cells of the mesoblast, which latter are called archiblastic cells. According to Rindfleisch, the so-called parablastic cells are derived from the area opaca.

The First, or Vitelline Circulation. - In the development of oviparous animals, the first, or vitelline circulation is very important ; for by these vessels the contents of the nutritive yolk are taken up and carried to the embryo, constituting the only source of material for its nutri- 
tion and growth. In mammals, however, nutritive matter is absorbed almost exclusively from the mother, by simple imbibition, before the placental circulation is established, and by the placental vessels at a later period. The vitelline circulation is therefore not important, and the vessels disappear with the atrophy of the umbilical vesicle.

The area vasculosa in mammals consists of vessels coming from the body of the embryo, forming a nearly circular plexus in the substance of the vitellus. The vessels of this plexus open into a sinus at the border of the area, called the sinus terminalis (see Figs. 243 and 244).

In examining the ovum when the area vasculosa is first formed, the embryo is seen lying in the direction of the diameter of the nearly circular plexus of bloodvessels. The plexus surrounds the embryo, except at the cephalic extremity, where the terminal sinuses of the two sides curve downward toward the head to empty into the omphalo-mesenteric veins. As the umbilical vesicle is separated from the body of the embryo, it carries the plexus of vessels of the area vasculosa with it, the vessels of communication with the embryo being the omphalo-mesenteric arteries and veins. As these processes are going on, the great central vessel of the embryo becomes enlarged and twisted on itself at a point just below the cephalic enlargement of the embryo, between the inferior extremity of the pharynx and the superior cul-dc-sac of the intestinal canal. The excavation which receives this vessel is called the fovea cardiaca. Simple undulatory movements take place in the heart of the chick at about the middle of the second day; but there is not at that time a regular circulation. At the end of the second day or the beginning of the third, the currents of the circulation are established. The time of the first appearance of the circulation in the human embryo has not been accurately determined.

In the arrangement of the vessels for the first circulation in the embryo, the heart is in the median line and gives off two arches which curve to either side and unite into a single central trunk at the spinal column below. These are the two aorta, and the single trunk formed by their union becomes the abdominal aorta. The two aortic arches only one of which is permanent-are sometimes called the inferior vertebral arteries. These vessels give off a number of branches which pass into the area vasculosa. Two of these branches, however, are larger than the others, pass to the umbilical vesicle and are called the omphalo-mesenteric arteries. In the embryo of mammals, there are at first four omphalo-mesenteric veins, two superior, which are the larger, and two inferior; but as development advances, the two inferior veins are closed, and there are then two omphalo-mesenteric arteries and two omphalo-mesenteric veins. At about the fortieth day, one artery and 
one vein disappear, leaving one omphalo-mesenteric artery and one vein. Soon after, as the circulation becomes established in the allantois, the vessels of the umbilical vesicle and the omphalo-mesenteric vessels are obliterated and the first circulation is superseded by the second.

As the septum between the two ventricles makes its appearance, that division of the right aortic arch which constitutes the vascular portion of one of the branchial arches disappears and loses its connection with the abdominal aorta; a branch, however, persists during the whole of intra-uterine life and constitutes the ductus arteriosus, and another branch is permanent, forming the pulmonary artery.

The Second, or Placental Circulation. - As the omphalo-mesenteric vessels disappear, and as the allantois is developed to form the chorion, two vessels - the hypogastric arteries - are given off, first from the abdominal aorta; but afterward, as the vessels going to the lower extremities are developed, the branching of the abdominal aorta is such that the vessels become connected with the internal iliac arteries. The hypogastric arteries pass to the chorion through the umbilical cord and constitute the two umbilical arteries. At first there are two umbilical veins; but one of them afterward disappears, and there is finally but one vein in the umbilical cord. It is in this way - the umbilical arteries carrying the blood to the tufts of the fœtal placenta, which is returned by the umbilical vein - that the placental circulation is established.

Corresponding to the four visceral arches, which have been described in connection with the derelopment of the face, are four pairs of vascular arches. These, with the two aortæ, constitute the five branchial or arterial arches, not counting an unimportant rudimentary branch connected with the fourth arch, described by Zimmermann. These arches are numbered from above downward.

In the development of the arteries distributed to the head and upper extremities, the first change observed is the disappearance of the first arch, its stem being continued forward as the temporal artery, giving off the internal maxillary and perhaps the facial (His). The second arch afterward disappears and is replaced with what is thought to form the lingual artery. The third arch remains and, with the upper part of the posterior aortic root, forms the internal carotid. The common carotid and the external carotid are formed from the anterior aortic root, the posterior and the anterior aortic roots coming from the same primitive vessel. The fourth arch on the right side becomes the subclavian artery. It joins also with the anterior aortic root to form the innominate artery. The fourth arch on the left side remains as the arch of the aorta. The fifth arch on the left side becomes the pulmo- 
nary artery and the ductus arteriosus. The fifth arch on the right side disappears. These changes occur in man and in some mammals; but in fishes the five arches are permanent, giving off branches that are distributed to the gills. In following the metamorphoses of the arches in man, it is seen that the symmetrical vascular system that exists in early embryonic life is developed gradually into an unsymmetrical system, and that two arches - the fourth and fifth-soon far exceed the others in size and importance. The destination of the five arches is well illustrated diagrammatically in Fig. 245, modified from Rathke. The aortic bulb and the five arches are represented

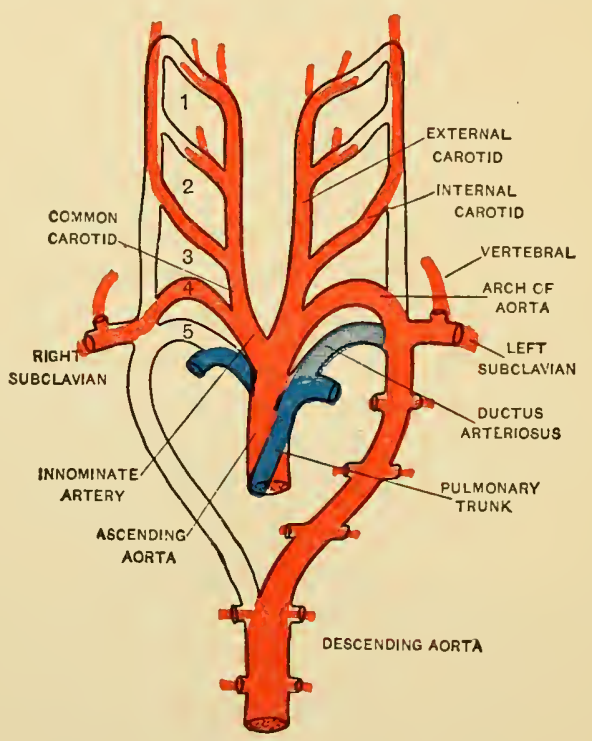

Fig. 245-Arterial arches in man and mammals (modified from Rathke).

in outline and the permanent vessels in colors. The vessels belonging to the aortic system are in red and the pulmonary vessels are in blue.

At the same time that the arteries going to the head and upper extremities are undergoing development, the vessels of the trunk and lower extremities are branching from the descending aorta and its extensions. These processes, however, are not complex and do not call for extended description in this work.

Two venous trunks make their appearance by the sides of the spinal column, called the cardinal veins. These run parallel with the superior vertebral arteries, or the two aorta, emptying finally into the auricular portion of the heart by two canals, called the ducts of Cuvier. These veins change their relations and connections as the first circulation is replaced by the second. The omphalo-mesenteric vein opens between 
the two ducts of Cuvier into the heart. As development advances, the liver is formed in the course of this vessel a short distance below the heart, and the rein ramifies in its substance; so that the blood of the omphalo-mesenteric vein passes through the liver before it goes to the heart. The omphalo-mesenteric vein is obliterated as the umbilical vein makes its appearance. The blood from the umbilical vein is at first emptied directly into the heart; but this vessel soon establishes the same relations with the liver as the omphalo-mesenteric rein, and its blood passes through the liver before it reaches the heart. As the omphalo-mesenteric vein atrophies, the mesenteric vein, bringing the blood from the intestinal canal, is developed, and this penetrates the liver, finally becoming the portal vein.

As the lower extremities are developed, the inferior vena cava makes its appearance between the two inferior cardinal veins. This ressel receives an anastomosing branch from the umbilical vein before it penetrates the liver, and this branch is the ductus venosus. As the inferior vena cava increases in size, it communicates below with the two inferior cardinal veins; and the portions of the two inferior cardinal veins which remain constitute the two iliac veins. The inferior cardinal veins, between that portion which forms the iliac veins and the heart, finally become the right and the left azygos veins.

The right duct of Cuvier, as the upper extremities are dereloped, enlarges and becomes the rena cava descendens, finally receiving the blood from the head and the superior extremities. The left duct of Cuvier diminishes in size and remains as the coronary sinus. The upper portions of the superior cardinal veins are dereloped into the jugulars and subclavians on the two sides. As the lower portion of the left cardinal vein and the left canal of Cuvier atrophy, a venous trunk appears, connecting the left subclavian with the right canal of Cuvier. This increases in size and becomes the left vena innominata, which connects the left subclavian and internal jugular with the vena cava descendens.

Development of the Heart. - The central enlargement of the vascular system in the first circulation, which becomes the heart, is twisted on itself by a single turn. The portion connected with the cephalic extremity of the embryo gives origin to the arterial system, and the portion connected with the caudal extremity receives the blood from the venous system. The walls of the arterial portion of the heart soon become thickened, while the walls of the venous portion remain comparatively thin. There then appears a constriction, which partly separates the auricular from the ventricular portion. At a certain period of development the heart presents a single auricle and a single ventricle. 
The division of the heart into two ventricles appears before the two auricles are separated. This is effected by a septum, which gradually extends from the apex of the heart upward toward the auricular portion. At the seventh week there is a large opening between the two ventricles. This gradually closes from below upward, the heart becomes more pointed and the separation of the two ventricles is complete at about the end of the second month.

At about the end of the second month, a septum begins to be formed between the auricles. This extends from the base of the heart toward the ventricles; but it leaves an opening between the two sides --the foramen ovale, or the foramen of Botal - which persists during fotal life. At the anterior edge of the opening of the vena cava ascendens into the right auricle, there is a membranous fold which projects into the auricle. This is the valve of Eustachius, and it divides the right auricle incompletely into two portions.

During the sixth week the heart is vertical and is situated in the median line, with the aorta arising from the centre of its base. At the end of the second month it is raised up by the development of the liver, and its point presents forward. During the fourth month, it is twisted slightly on its axis, and the point presents to the left. At this time the auricular portion is larger than the ventricles; but the auricles diminish in their relative capacity during the latter half of intra-uterine life. The pericardium makes its appearance during the ninth week.

Early in intra-uterine life the relative size of the heart is very great. At the second month its weight, in proportion to the weight of the body, is as I to 50. This proportion, however, gradually diminishes, until at birth the ratio is as I to I2O. The weight in the adult is about as I to I60. During about the first half of intra-uterine life the thickness of the two ventricles is nearly the same; but after that time the relative thickness of the left ventricle gradually increases.

Pcculiaritics of the Foctal Circulation. - Beginning at the abdominal aorta, the blood passes into the two primitive iliacs, and thence into the internal iliacs. From the two internal iliacs the two hypogastric arteries arise, which ascend along the sides of the bladder, to its fundus, pass to the umbilicus and go to the placenta, forming the two umbilical arteries. In this way the blood of the foetus goes to the placenta.

The umbilical vein enters the body of the foetus at the umbilicus; it passes along the margin of the suspensory ligament to the under surface of the liver; it gives off one branch of large size and one or two smaller branches to the left lobe; it sends a branch each to the lobus quadratus and the lobus Spigelii, and the vessel reaches the transverse fissure. At the transverse fissure it divides into two branches, the larger of 
which joins the portal vein and enters the liver; and the smaller, which is the ductus venosus, passes to the rena cava ascendens, at the point where it receives the left hepatic vein. Thus the greater part of the blood returned to the fœtus from the placenta passes through the liver, a relatively small quantity being emptied into the rena cara, by the ductus venosus.

The vena cava ascendens - containing the placental blood which has passed through the liver, the blood conveyed directly from the umbilical

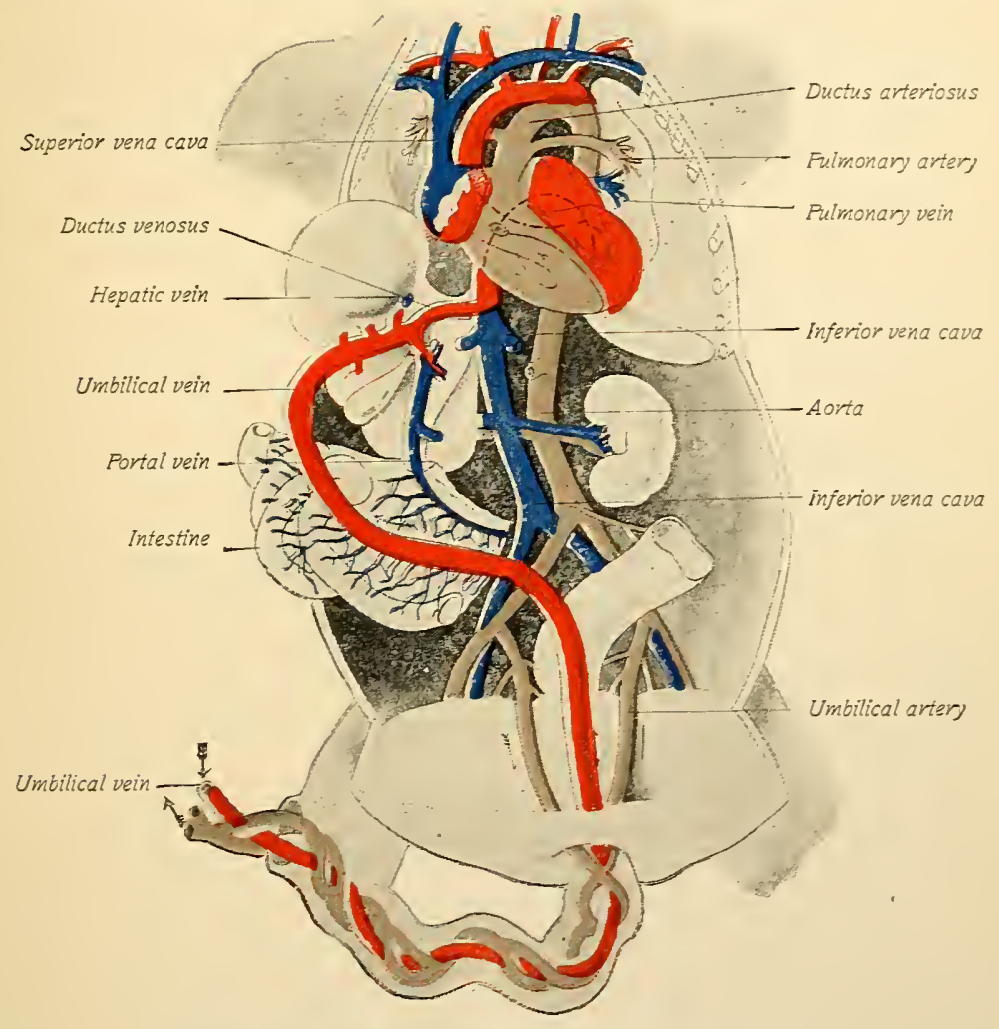

Fig. 246. - Diagram of the fretal circulation (Kollmann).

vein by the ductus venosus and the blood from the lower extremities passes to the right auricle. As the blood enters the right auricle it is directed by the Eustachian valve, passing behind the valve, through the foramen ovale, into the left auricle. At the same time the blood from the head and the superior extremities passes down, by the rena cara descendens, in front of the Eustachian valve, through the right auricle, into the right ventricle. The arrangement of the Eustachian valve is such that the right auricle simply affords a passage for the two currents 
of blood; the one, from the vena cava ascendens, through the foramen ovale, passes into the left auricle and the left ventricle; and the other, from the vena cava descendens, passes through the right auriculoventricular opening, into the right ventricle. It is probable, indeed, that there is very little admixture of these two currents of blood in the natural course of the fœtal circulation.

The blood poured into the left auricle, from the vena cava ascendens, through the foramen ovale, passes from the left auricle into the left ventricle. The left auricle and the left ventricle also receive a small quantity of blood from the lungs, by the pulmonary veins. Thus the left ventricle is filled. At the same time the right ventricle is filled with blood which has passed through the right auricle, in front of the Eustachian valve. The two ventricles, thus distended, then contract simultaneously. The blood from the right ventricle passes in small quantity to the lungs, the greater part passing through the ductus arteriosus, or ductus Botalli, into the descending portion of the arch of the aorta. This duct is half an inch (1 2.7 millimeters) in length and about the size of a goose-quill. The blood from the left ventricle passes into the aorta and goes to the system. The vessels of the head and superior extremities being given off from the aorta before it receives the blood from the ductus arteriosus, these parts receive almost exclusively the pure blood from the vena cava ascendens, the only mixture with the placental blood being the blood from the lower extremities, the blood from the portal system and the small quantity of blood received from the lungs. After the aorta has received the blood from the ductus arteriosus, however, it is mixed blood; and it is this which supplies the trunk and lower extremities.

The Third, or Adult Circulation. - When the child is born the placental circulation is suddenly arrested. After a short time the sense of want of air becomes sufficiently intense to give rise to an inspiratory effort, and the first inspiration is made. The pulmonary organs are then for the first time distended with air, the pulmonary arteries carry the greatest part of the blood from the right ventricle to the iungs and a new circulation is established. During the later periods of fatal life, the heart is gradually prepared for the new currents of blood. The foramen ovale, which is largest at the sixth month, after that time is partly occluded by the gradual growth of a valve which extends from below upward and from behind forward on the side of the left auricle. The Eustachian valve, which is also largest at the sixth month, gradually atrophies after this time, and at full term it has nearly disappeared. At birth, then, the Eustachian valve is practically absent; and after pulmonary respiration becomes established, the foraman ovale has nearly 
closed. The arrangement of the valve of the foramen ovale is such that at birth a small quantity of blood may pass from the right to the left auricle, but none can pass in the opposite direction. The situation of the Eustachian valve on the right side of the interauricular septum is marked by an oval depression, called the fossa ovalis.

When the placental circulation is arrested at birth, the hypogastric arteries, the umbilical vein and the ductus venosus contract, and they become impervious between the second and the fourth days. The hypogastric arteries remain pervious at their lower portion and constitute the superior vesical arteries. A rounded cord, which is the remnant of the umbilical vein, forms the round ligament of the liver. A slender cord, the remnant of the ductus venosus, is lodged in a fissure of the liver, called the fissure of the ductus venosus. 


\section{CHAPTER XXXIII}

FETAL LIFE-DEVELOPMENT AFTER BIRTH-DEATH

Duration of pregnancy - Size, weight and position of the foetus - Multiple pregnancy - Cause of the first contractions of the uterus in normal parturition - Involution of the uterus Meconium - Dextral preëminence - Development after birth - Ages - Death.

As the development of the ovum advances, the uterus becomes enlarged and its walls are thickened. The form of the organ, also, gradually changes, as well as its position. Immediately after birth its weight is about a pound and a half (680 grams), while the virgin uterus weighs less than two ounces (56.7 grams). The neck of the uterus, while it becomes softer and more patulous during pregnancy, does not change its length, even in the latest periods of utero-gestation. The changes in the walls of the uterus during pregnancy are important. The bloodvessels become much enlarged, and the muscular fibres increase immensely in size, so that their contractions are very powerful when the fœtus is expelled.

It is evident that on account of the progressive increase in the size of the uterus during pregnancy, it can not remain in the cavity of the pelvis during the later months. During the first three months, however, when it is not too large for the pelvis, it sinks back into the hollow of the sacrum, the fundus being directed somewhat backward, with the neck presenting downward, forward and a little to the left. After this time the increased size of the organ causes it to extend into the abdominal cavity, so that its fundus eventually reaches the epigastric region. Its axis then has the general direction of the axis of the superior strait of the pelvis.

The enlargement of the uterus and the necessity of carrying on a greatly-increased circulation in its walls during pregnancy are attended with a temporary hypertrophy of the heart. It is mainly the left ventricle that is thickened during utero-gestation, and the increase in the weight of the heart at full term amounts to more than one-fifth. After delivery, the weight of the heart soon returns nearly to the normal standard.

Duration of Pregnancy. - The duration of pregnancy, dating from a fruitful intercourse, is variable within certain limits. The method of 
calculation most in use by obstetricians is to date from the end of the last menstrual period. Taking into account, howerer, the various cases quoted by authors, in which conception has been supposed to follow a single coitus, there appears to be a range of variation in the duration of pregnancy of not less than forty days, the extremes being two hundred and sixty and three hundred days. As regards the practical applications of calculations of the probable duration of pregnancy in individual cases, the fact must be recognized that the period is variable. Dating from the end of the last menstrual flow, an average of two hundred and seventy-eight days, or a little more than nine calendar months, may be adopted.

Size, Weight and Position of the Fotus. - Estimates in regard to the size and weight of the embryo and fœtus at different stages of intrauterine life present wide variations; still, it is important to have at least an approximate idea as to these points, and the estimates by Scanzoni are given, as presenting fair averages.

At the third week the embryo is two to three lines (4.2 to 6.4 millimeters) in length. This is about the earliest period at which measurements have been taken in the normal state.

At the seventh week the embryo measures about nine lines (I9.I millimeters). Points of ossification have appeared in the clavicle and the lower jaw; the Wolffian bodies are large ; the pedicle of the umbilical vesicle is much reduced in size; the internal organs of generation have just appeared; the liver is of large size ; the lungs present several lobules.

At the eighth week the embryo is ten to fifteen lines (2I.2 to 3 r.8 millimeters) in length. The lungs begin to receive a small quantity of blood from the pulmonary arteries; the external organs of generation have appeared, but it is difficult to determine the sex; the abdominal walls have closed over in front.

At the third month the embryo is two to two and a half inches ( 50.8 to 63.5 millimeters) long and weighs about one ounce (28.3 grams). The amniotic liquid is then more abundant in proportion to the size of the embryo than at any other period; the umbilical cord begins to be twisted; the abdominal glandular organs appear; the pupillary membrane is formed; the limitation of the placenta has become distinct. At this time the upper part of the embryo is relatively much larger than the lower portion.

At the end of the fourth month the embryo is called the fœtus. ${ }^{1}$ It

${ }^{1}$ The periods of evolution are divided differently by authors. His distinguishes the three periods, as follows: First two weeks, the product is called the ovum; from the third to the fifth week, the embryo; and after the fifth week it is called the foetus. 
is then four to five inches (IO.I to I 2.7 centimeters) long and weighs about five ounces (I4I.7 grams). The muscles show contractility; the eyes, mouth and nose are closed; the gall-bladder is just developed; the fontanelles and sutures are wide; the sex is distinguishable.

At the fifth month the fœtus is nine to twelve inches (22.8 to 30.5 centimeters) long and weighs five to nine ounces (I 4 I.7 to 255 . I grams). The hairs begin to appear on the head; the liver begins to secrete bile, and the meconium appears in the intestinal canal; the amnion is in contact with the chorion.

At the sixth month the fotus is eleven to fourteen inches (27.9 to 35.5 centimeters) long and weighs one and a half to two pounds (680 to 907 grams). If the fœtus is delivered at this time, life may continue for a few moments; the bones of the head are ossified, but the fontanelles and sutures are still wide; the prepuce has appeared; the testicles have not descended.

At the seventh month the fotus is fourteen to fifteen inches $(35.5$ to 38.1 centimeters) long and weighs two to three pounds (907 to I36I grams). The hairs are longer and darker; the pupillary membrane disappears, undergoing atrophy from the centre to the periphery; the relative quantity of the amniotic fluid is diminished, and the fotus is not so free in the cavity of the uterus; the fotus is now viable.

At the eighth month, the fœtus is fifteen to sixteen inches (38.I to 40.9 centimeters) long and weighs three to four pounds (I36I to I8I4 grams). The eyelids are opened and the cornea is transparent; the pupillary membrane has disappeared; the left testicle has descended; the umbilicus is at about the middle of the body, the relative size of the lower extremities having increased.

At the ninth month the fœtus is about seventeen inches ( 43.2 centimeters long and weighs five to six pounds (2.27 to 2.72 kilograms). Both testicles usually have descended, but the tunica vaginalis still communicates with the peritoneal cavity.

At birth the infant weighs a little more than seven pounds (3.17 kilograms), the usual range being four and ten pounds (I.8I and 4.53 kilograms), although these limits are sometimes exceeded.

The position of the fœtus in the great majority of cases, excluding abnormal presentations, is with the head downward. In the early months of pregnancy the fœtus floats quite freely in the amniotic liquid; and it is probable that the natural gravitation of the head and of the upper part of the fotus is the determining cause of the ordinary position in utero.

The shape of the uterus at full term is ovoid, the lower portion being the narrower. The fœetus has the head slightly flexed upon the sternum, 
the arms flexed upon the chest and crossed, the spinal column curved forward, the thighs flexed upon the abdomen, the legs slightly flexed and usually crossed in front, and the feet flexed upon the legs, with their inner margin drawn toward the tibia. This is the position in which the fœtus is best adapted to the size of the uterine cavity, and in which the expulsive force of the uterus can be most favorably exerted, both as regards the fœetus and the generative passages of the mother.

Multiple Pregnancy. - It is not rare to observe two infants at a birth, and cases are on record where there have been four or even five, though in these latter instances they survive usually but a short time, or as is more common, abortion takes place during the first months. Examples of three at a birth have been often observed.

In cases of twins it is an interesting question to determine whether the development always takes place from two ova or whether a single ovum may be developed into two beings. In the majority of cases, twins are of the same sex, though sometimes the sexes are different. In some cases there are two full sets of membranes, each fœtus having its distinct decidua, placenta and chorion; in others there is a single chorion and a double amnion; but in some, both fœtuses are enclosed in the same amnion. As a rule the two placentæ are distinct; but sometimes there is a vascular communication between them, or what appears to be a single placenta may give origin to two umbilical cords. If there is but a single chorion and amnion and a single placenta, it has been thought that the two beings are developed from a single ovum; otherwise it would be necessary to assume that there were originally two sets of membranes which had become fused into one. The instances on record of twins, one, white and the other black, show conclusively that two ova may be developed in the uterus at the same time. While there can be no doubt of this, the question of the possibility of the development of two beings from a single ovum remains unsettled.

As pathological conditions, extra-uterine pregnancies occur, in which the fecundated ovum, forming its attachments in the Fallopian tube (Fallopian pregnancy) or within the abdominal cavity (abdominal pregnancy), undergoes a certain degree of development. The uterus usually enlarges in these instances and forms an imperfect decidua.

Cause of the First Contractions of the Uterus in Normal Parturition. The first contraction of the uterus in normal parturition is undoubtedly referable to some change in the attachment of its contents, which causes the fœtus and its membranes to act as a foreign body. When for any reason it is advisable to empty the uterus before the full term of pregnancy, the physiological method of bringing on the contractions of this organ is to separate cautiously a portion of the membranes, as is 
often done by introducing an elastic catheter between the ovum and the uterine wall. A certain time after this operation, the uterus contracts to expel the ovum, which then acts as a foreign body.

In the normal state, toward the end of pregnancy, the cells of the decidua vera and of that portion of the placenta attached to the uterus undergo fatty degeneration, and in this way there is a progressive separation of the outer membrane, so that the contents of the uterus gradually lose their anatomical connection with the mother. When this change has advanced to a certain extent, the uterus begins to contract; each contraction then separates the membranes more and more, the most depenclent part pressing on the os internum; and the subsequent contractions are due to reflex action. The first "pain" is induced by the presence of the fœtus and its membranes as a foreign body, a mechanism similar to that which obtains when premature labor has been brought on by separation of the membranes.

According to Körner, there exists in the spinal cord, at the site of the first and second lumbar vertebræ, a reflex centre for parturition. This, like other centres in the cord, is subordinate to a centre situated in the bulb.

The mechanism of parturition, although this is entirely a physiological process, is considered elaborately in works on obstetrics. The first contractions of the uterus, by pressing the bag of waters against the os internum, gradually dilate the cervix; the membranes usually rupture when the os is pretty fully dilated, and the amniotic fluid is discharged; the head then presses on the outlet; and the uterine contractions becoming more and more vigorous and efficient, the child is brought into the world, this being followed by the expulsion of the membranes and placenta. There then follows a tonic contraction of the muscular walls of the uterus, which becomes a hard globular mass, easily felt through the flaccid abdominal walls. The very contractions of the muscular fibres of the uterus which expel the fotus close the vessels ruptured by the separation of the placenta and arrest hæmorrhage from the mother. The changes which then take place in the respiration and circulation of the infant have been considered in connection with the development of the circulatory system.

Involution of the Utcrus. - At four to six days, and seldom later than eight days after parturition, the uterus has sensibly advanced in the process of involution; and it is then gradually reduced to the size and structure which it presents during the non-pregnant condition, though it never becomes quite so small as in the virgin state. The new mucous membrane, which has been developing during the latest periods of pregnancy, becomes perfect at about the end of the second month after 
delivery. It has then united, at the os internum, with the mucous membrane of the neck, which has not participated in the formation of the decidua. The muscular fibres, after parturition, present granules and globules of fat in their substance, and are gradually reduced in size as the uterus becomes smaller. Their involution is complete at about the end of the second month. During the first month, and particularly within the first two weeks after delivery, there is a sero-sanguinolent discharge from the uterus, which is due to disintegration of the blood and of the remains of the membranes in its cavity, this débris being mixed with a certain quantity of sero-mucous secretion. This discharge constitutes the lochia. It is at first red but becomes paler as it is reduced in quantity.

Meconizm. - At about the fifth month there is a certain quantity of secretion in the intestinal canal, which becomes more abundant, particularly in the large intestine, as development advances. This is rather light colored or grayish in the upper portion of the small intestine, becoming yellowish in the lower portion, and it is of a dark greenish color in the colon. The dark, pasty, adhesive mat-

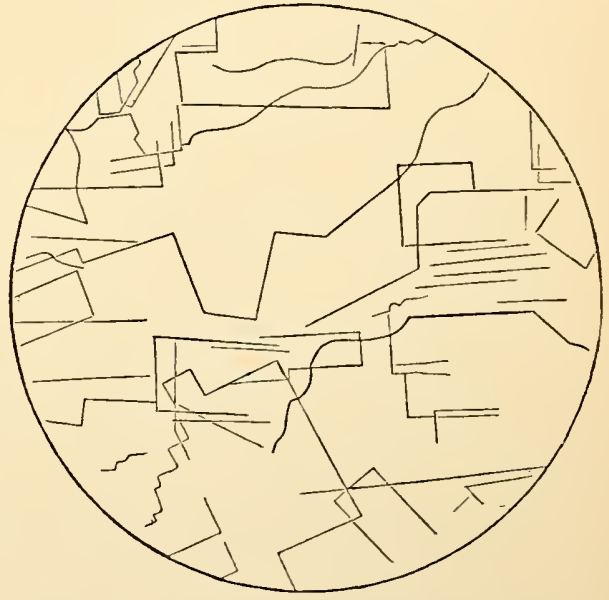

Fig. 247. - Cholesterin extracted from meconium. ter, which is discharged from the rectum soon after birth, is called meconium.

The meconium appears to consist of a thick mucous secretion, with abundant grayish granules, a few fatty granules, intestinal epithelium, and frequently crystals of cholesterin. The color seems to be due to granules of the coloring matter of the bile; but the biliary salts can not be detected in the meconium by Pettenkofer's test. The constituent of meconium that possesses the greatest physiological importance, is cholesterin. Although but few crystals of cholesterin are found on microscopical examination, the simplest processes for its extraction will reveal the presence of this substance in large quantity. In a specimen of meconium in which a quantitative examination was made, the proportion of cholesterin was 6.245 parts per 1000 (Flint). The meconium contains cholesterin and no stercorin; stercorin in the adult resulting from a transformation of cholesterin by the digestive liquids, which are not secreted during intra-uterine life. 
None of the secretions concerned in digestion appear to be produced in utero, and it is also probable that the true biliary salts are not formed at that time; but the katabolic processes and excretion are then active, and the cholesterin of the meconium is the product of the excretory action of the liver. The relations of cholesterin as a product of katabolism have already been very fully discussed, in connection with the bile and with excretion.

Dextral Prëminence. - Most persons use the right arm, leg, eye, etc., in preference to the left ; but exceptionally some use the left instead of the right. Exceptions, however, in regard to the eye, are not infrequent. There can be no doubt of the fact of a natural dextral preeminence, and also that left-handedness is congenital, difficult if not impossible to correct entirely, and not due simply to habit. It would appear that there must be some condition of organization, which produces dextral preëminence in the great majority of persons, and lefthandedness, as an exception; but what this condition is, it is very difficult to determine. An explanation offered by some anatomists is that the right subclavian artery arises nearer the heart than the left, that the right arm is therefore better supplied with arterial blood, develops more fully, and consequently is commonly used in preference to the left; but exceptional predominance of the left hand can not be explained in this way.

The most important anatomical and pathological facts bearing on the question under consideration are the following: Boyd has shown that the left side of the brain almost invariably exceeds the right in weight, by about one-eighth of an ounce ( 3.5 grams). In aphasia the lesion is almost always on the left side of the brain. These facts point to a predominance of the left side of the brain, which presides over the movements of the right side of the body. Again, a few cases of aphasia with left hemiplegia, the lesion being on the right side of the brain, have been reported as occurring in left-handed persons. Ogle gives several such instances, in which the brain-lesion was on the right side. In two left-handed individuals, the brain was examined and compared with the brain of right-handed persons. It was found that the brain was more complex on the left side in the right-handed, and on the right side, in the left-handed. Bastian found the gray matter of the brain ordinarily to be heavier on the left than on the right side. In regard to the cause of the superior development of the left side of the brain, the only explanation offered is the fact that the arteries going to the left side usually are larger than those on the right. There are no observations respecting the comparative size of the arteries on the two sides in left-handed persons. 
Reasoning from the facts just stated, Ogle has assumed that dextral preemminence depends on a natural predominance of the left side of the brain, the reverse obtaining in the left-handed. Ordinarily it is true that the members of the right side are stronger than the left, particularly the arm; but this is not always the case, even in the right-handed, although the right hand is more conveniently and easily used than the left. In many feats of strength, the left arm appears less powerful than the right, because there is less command over the muscles. As regards the cause of the superior development of the left side of the brain, it must be admitted that the anatomical explanation is not entirely satisfactory. It is a fact, however, that the two sides of the brain usually are not exactly equal in their development, the left side being superior to the right, and that the muscles of the right side of the body are used habitually in preference to those of the left side.

While it is not yet possible to explain why the left side of the brain has peculiar psychic functions not possessed by the right side, it is nevertheless true that intellectual processes take their origin mainly - and in some instances entirely - in the left half of the cerebrum. In man, sight, hearing and speech are closely connected with mental operations, at least in so far as they give rise to or express ideas. The two eyes are necessary to perfect vision; but the psychic visual centre, which receives ideas or meaning conveyed by objects seen, is on the left side of the cerebrum, except in the left-handed. The same may be said of the sense of hearing, the psychic auditory centre being on the left side, except in the left-handed. The location of the speech-centre on the left side was made in I836 (Marc Dax); and a case of aphasia with right hemiplegia was fully reported by Pourfour du Petit, in I766. Agraphia, or inability to express ideas in written language, is due to lesion of the left side of the brain. All these conditions are reversed, however, in the left-handed. When one eye is used as a means of forming a judgment or opinion, it usually is the right eye for the right-handed and the left eye for the left-handed. Curiously enough, it has lately been observed that deaf-mutes may have an aphasia that prevents the use of the right hand in the sign-language. It seems, indeed, that movements, more or less automatic, may be executed by the muscles of either side, remembering, always, that muscles of the left as well as of the right side may be educated; but in movements that involve mental operations and attention at the time they are made, the right side usually predominates. Apart from the question of education of muscles, it appears that the more automatic acts are performed indifferently by either the right or the left side; but movements more closely connected with direct mental operations are made preferably by right muscles in 
the right-handed and by left muscles in the left-handed. Still, while this may satisfactorily explain dextral preëminence, it does not explain the preëminence of the left side of the brain.

\section{Development after Birth - Ages and Death}

When the infant is born, the organs of special sense and the intelligence are dull; there is then very little muscular power; and the new being for several weeks does little more than eat and sleep. The natural food at this time is the milk of the mother; and the digestive secretions do not for some time possess the varied solvent properties that are found in the adult, though observations on the secretions of the infant are few and rather unsatisfactory. The full activity of pulmonary respiration is gradually and slowly established. Young animals appropriate a comparatively small quantity of oxygen, and just after birth they present a much greater power of resistance to asphyxia than the adult. The power of maintaining the animal temperature also is much less in the newly-born. The processes of ossification, development of the teeth etc., have already been described. The hairs are shed and replaced by a new growth a short time after birth. The fontanelles gradually diminish in size after birth and are completely closed at the age of about four years.

The period of life which dates from birth to the age of two years is called infancy. At the age of two years the transition takes place from infancy to childhood. The child is then able to walk without assistance, the food is more varied and the digestive operations are more complex. The special senses and the intelligence become more acute, and the being begins to learn to express ideas in language. The child gradually derelops, and the milk-teeth are replaced by the permanent teeth. At puberty, which begins between the fourteenth and the seventeenth years - a little earlier in the female - the development of the generative organs is attended with important physical and moral changes.

The different ages recognized by physiologists are the following: Infancy, from birth to the age of two years; childhood, to the time of puberty; adolescence, or youth, to the twenty-fifth year; adult age, to the thirty-fifth year; middle life, to the fiftieth year; old age, to the sixtieth year; and then, extreme old age. A man may be regarded at his maximum of intellectual and physical derelopment at about the age of thirtyfive, and he begins to decline after the sixtieth year, although as regards intellectual vigor, this has many exceptions.

As regards nutrition, it may be stated in general terms that the appropriation of new matter (anabolism) is a little superior to katabolism, up to about the age of twenty-five years; between twenty-five and forty-five, 
these two processes are nearly equal; and at a later period the nutrition does not completely supply the physiological waste of the tissues, the proportion of organic to inorganic matter gradually diminishes, and death follows as an inevitable consequence of life. In old age the muscular movements gradually become feeble; the bones contain an excess of inorganic matter; the ligaments become stiff; the special senses are usually somewhat obtuse; and there is a diminished capacity for mental labor, with more or less loss of memory and of intellectual rigor. It is a curious fact that remote events are more clearly and easily recalled to the mind in old age than those of recent occurrence; and, indeed, early impressions and prejudices then appear to be unusually strong.

It frequently happens in old age that some organ essential to life gives way, and that this is the immediate cause of death, or that an old person is stricken down by some disease to which his age renders him peculiarly liable. It is so infrequent to observe a perfectly physiological life continuing throughout the successive ages of man, that it is difficult to present a picture of physiological death; but it sometimes occurs that there is a gradual fading a way of vitality in old persons, who die without being affected with any special disease. It also is difficult to fix the natural period of human life. Some persons die, apparently of old age, at seventy, and it is rare that life is preserved beyond one hundred years. The tissues usually die successively and not simultaneously, nearly all being dependent on the circulating, oxygen-carrying blood for the maintenance of their physiological properties. It has been demonstrated, indeed, that the properties of tissues may be restored for a time, after apparent death, by the injection of blood into their vessels.

After death there often is a discharge of the contents of the rectum and bladder; and parturition, even, has been known to take place. The appearance which indicates growth of the beard after death is probably due to shrinking of the skin and, perhaps, contraction of the smooth muscular fibres attached to the hair-follicles. The most important phenomenon, however, observed before putrefaction begins, is a general rigidity of the muscular system, or rigor mortis, which has already been described. 

ATLAS 



\section{INTRODUCTION TO THE ATLAS}

As has been stated in the Preface, the original figures in the Atlas are reproductions of actual objects by the three-color photographic process, without retouching of the process-plates. Figures and letters are not used to designate the special histological structures. I have indicated, however, as clearly as I could without such aid, what I specially desire to illustrate in each picture. Most of the figures it has been easy to describe; but others will be found to require careful and patient study, particularly those relating to embryology. In the study of these pictures, it will be of advantage to consult first the descriptions and illustrations - which latter are more or less diagrammatic - given in the text. It should then be easy to recognize the structures as they are shown in the Atlas. Indeed, I have endeavored to supplement the text with what approaches actual laburatory study. The hours spent in such work, although without the advantage of a microscope and personal demonstration, can hardly fail of practical ralue.

It will be evident to histologists that I have been peculiarly fortunate in certain objects for reproduction. The section of the stomach, showing peptic cells and acid-cells, I believe to be unique; the platelets from human blood are seldom so clearly shown; the same may be said of branching heart-muscle; serous and mucous cells and demilunes of the submaxillary ; intestinal glands; islands of Langerhans; solitary glands; structure of adrenals, spleen, thyroid, and thymus; bone-canaliculi and elastic cartilage. The sections of the human embryo are seldom seen and will repay careful examination.

As a matter that can not fail to be of interest, I add to this introduction a note on technique, by Dr. Leaming : -

\section{NOTE ON THE TECHNIQUE OF PHOTOMICROGRAPHY IN COLORS}

As at the present time photomicrography finds one of its most useful applications in medicine, it seems but natural to expect that the future photomicrographer should be found among medical students. Bearing this in mind, a few words on the photomicrographs in color in the Atlas may prove of interest. The apparatus need not be discussed; any first-class installation will be quite equal to the demands upon it; but it is essential that the operator should be thoroughly familiar with his apparatus and its peculiarities. The most important of the accessories will be the color-filters, or screens, whereby the rays not needed are absorbed before they reach the object, and only those desired for each particular negative are allowed to pass through. It must be remembered that for our purposes the visible spectrum is divided arbitrarily into three zones; one near the red end, one near the blue end and one near the middle. From each of these zones one color is taken, and these three colors form the primary, or printing colors; they are a particular pinkish or madder-red, a canary-yellow, and a brilliant greenish blue. If we combine the above in pairs, we obtain an entirely new set of colors, and these form our taking screens, or color-filters. These new colors are termed the secondary colors. Thus, primary red plus primary yellow makes secondary red - an orange-red screen which the spectroscope will show to admit the red end of the spectrum only; primary red plus primary blue equals secondary violet - blue end only; and finally, primary blue plus primary yellow makes secondary green - middle only. It will be seen from the above, that these secondary colors are complementary to the primary. For example, an object taken through the green screen (blue and yellow) is printed in red; the violet screen (blue and red) is printed in 
yellow; the orange screen (red and yellow) is printed in blue. Having such screens, we find that they are valuable for ordinary photomicrography where the results are to be viewed in monocrome, as we are enabled to einphasize certain points much better than when dependent on the orthochromatic yellow screen alone. Now, the study of stained sections through the microscope will impress the fact upon our minds that the great majority are stained with two colors at most; and as the section before staining was white, or nearly transparent, the need of using three colors for its reproduction does not exist. It will suffice in many cases, therefore, to use but two screens - taking a negative with each - and leave the reproducer to mix his printing inks so that two impressions will give a satisfactory result. Most of the photomicrographs in this Atlas were taken in this manner, only a few in three colurs, and the remainder in one or two, as the subject seemed to demand. By eliminating one process in this way through all steps of the work, there is a great gain in every direction, - less liability to error in the original negatives, sharper impressions in the reproduction, and a saving in time and cost throughout.

An isochromatic plate was used with all colored sections, but not one specially dyed for each particular region of the spectrum that the screens allowed to pass through. Development was with glycin, the plates being allowed to stay undisturbed in the tray urtil developed, without rocking. Two negatives were taken on the same plate, usually the red and blue printing negatives, by means of a sliding plate-holder, and thus, as they were afterward developed together, each exposure had to be carefully timed to the ratio of its respective screen. The screens had the following ratio to each other, - green, 3 ; red, 5 ; violet, 9 , the electric arc being the illuminant. The exposure varied according to the objective, density of object, magnification, position of substage condenser, and length of camera draw, between $\frac{1}{30}$ of a second and five minutes, the usual time being three to ten seconds. As for illustrative purposes, flatness of field and depth of focus were important points, use was made of an objective of low power combined with a high eyepiece and sufficient camera length to obtain the desired magnification.

March, 1905. 


\section{ATLAS}

\section{LIST OF PLATES}

PLATE I

BloOd AND BONE-MARROW

(Sobotta)

PLATE II

By Three-color Photographic Process

FIG. I

Human blood

FIG. 2

Hemin crystals

Fig. 3

Heart-muscle
FIG. 4

Trachea

FIG. 5

Lung, injected

FIG. 6

Lung, stained

\section{PLATE III}

Sections of Blondtessels

(Sobotta)

\section{PLATE IV}

By Three-color Photographic Process

FIG. I

Submaxillary gland

FIG. 2

Esophagus

FIG. 3

Human stomach
FIG. 4

Duodenum - glands of Brunner

FIG. 5

Small intestine - villi and goblet-cells

FIG. 6

Small intestine - villi, etc.

\section{PLATE V}

By Three-color Photographic Process

FIG, I

Duodenum of cat, injected

FIG. 2

Small intestine of rat, injected

FIG. 3

Bloodvessels of pancreas
FIG. 4

Human pancreas

FIG. 5

Solitary gland

FIG. 6

Human breast 
PLATE VI

Section of the Human Scalp

(Sobotta)

\section{PLATE VII}

By Three-color Photographic Process

F1G. I

Sebaceous glands

FIG, 2

Sweat-glands

F1G. 3

Kidney, injected and stained
FIG. 4

Kidney, injected

FIG. 5

Liver, injected and stained

FI(;. 6

Liver, stained

\section{PLATE VIII \\ LYMPH-GLAND - SPLEEN \\ (Sobotta)}

\section{PLATE IX}

By Three-color Photographic Process

FIG. I

Adrenal

FIG. 2

Spleen, injected

FIG. 3

Spleen, stained
F1G. 4

Thyroid

F1G, 5

Thymus

Fig. 6

Human muscle

\section{PLATE X}

By Three-color Photographic Process

FIG. I

Muscle of pig, injected

F1G. 2

Bone, injected

FIG. 3

Bone-development
FIG. 4

Elastic cartilage

FIG. 5

Fallopian tube

F1G. 6

Placenta and membranes

PLATE XI

NERVE-CELLS

(Sobotta)

PLATE XII

SPINAL CORD

(Sobotta) 
ATLAS

PLATE XIII

Two Early Stages of Cleavage of the Egg (Wilson)

PLATE XIV

Two Later Stages of Cleavage of the EgG (IVilson)

\section{PLATE XV}

By Three-color Photographic Process

FIG. I

Section of the ovary

FIG, 2

Testicle

FIG. 3

Section of the chick
FIG. 4

Section of the chick Fig. 5

Section of the chick FIG. 6

Section of the chick

\section{PLATE XVI}

By Three-color Photographic Process

FIG. I

High section through thorax of human embryo

FIG. 2

Low section through thorax of human embryo

$$
\text { FIG. } 3
$$

High section through abdomen of human embryo

Fig. 4

Low section through abdomen of human embryo

FIG. 5

Sagittal section of a pig-embryo

FIG. 6

Sagittal section of a pig-embryo 



\section{PLATE I}

\section{Human Blood, $\times 700$ (Sobotta)}

solution.)

(Dry preparation. Ether-alcohol. Hematoxylin-eosin. In I, 3, and 4, Ehrlich's triacid

6 to 12 , inclusive, erythrocytes, or red corpuscles ( 9 , nucleated corpuscle).

I, small lymphocyte.

2, 3, polynuclear leucocytes, with neutrophile granulation.

$4,5,14,16,18$, ordinary polynuclear leucocytes.

15, 19, 2I, leucocytes, with eosinophile granulation.

I3, 20, large lymphocytes.

17 , mononuclear leucocyte.

\section{From the Bone-Marrow of a Mouse, $\times 700$}

22, polynuclear giant-cell.

23,28 , marrow-cells.

25,26 , eosinophile cells.

27,28 , cells with beginning karyokinesis.

29 , erythrocyte.

30, 3I, nucleated erythrocytes.

These figures can be studied to best advantage in connection with the text relating to the development of blood-corpuscles and leucocytes. 
PLATE I
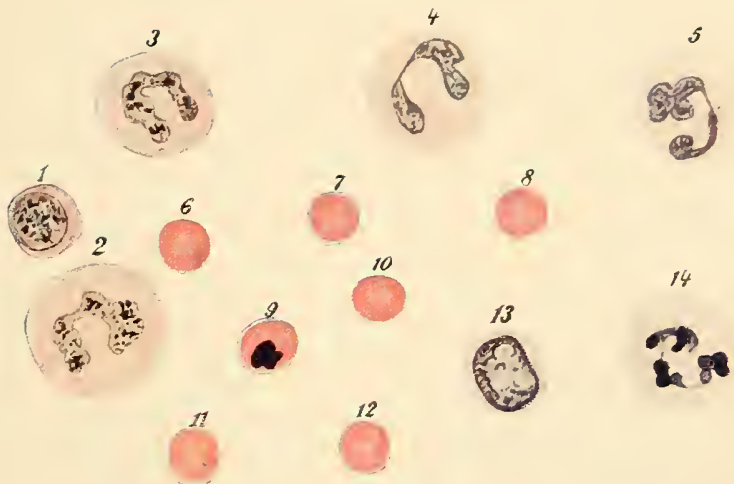

$$
\text { a }
$$
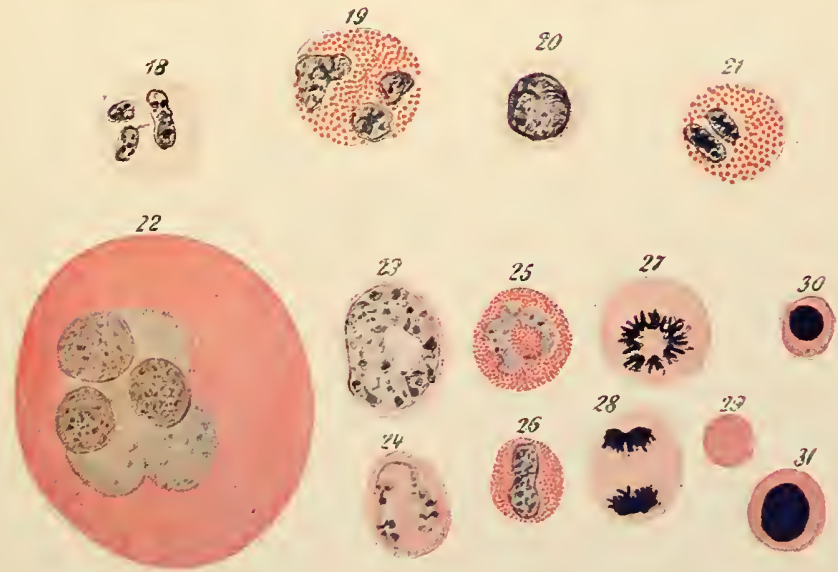



$$
\text { - }
$$
-

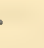

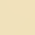


PLATE II

Fig. I. Human blood (Nocht-Romanowsky stain), $\times 335 .-$ Ewing.

This figure shows the red blood-corpuscles (erythrocytes), two clumps of platelets near the centre of the field and to the right and one large mononuclear leucocyte. The outlines of the leucocyte are faint and the nucleus is stained a dark purple.

FIG. 2. Hemin crystals, $\times 500$. - Schultze.

This specimen was prepared from a human blood-stain on muslin, $4^{8}$ hours old. The spot was extracted with a I-2000 salt-solution.

Fig. 3. Human heart-muscle (henatein and eosin), $\times$ 125.-Ferguson.

This figure shows the branching and inosculation of the fibres of the heart-muscle.

FIG. 4. Cross-section of the trachea of the dog (hematoxylin-eosin), $\times 20$. - Author's collection.

At the top of this section is the layer of epithelium, darkly stained, covering the macosa ; below is the submucosa, with three mucous glands; below, stained blue, is the cartilage, with the perichondrium next the submucosa; beneath the cartilage is the outer fibrous coat.

FIG. 5. Section of the lung of a sheep, injected, $\times 75$. - Author's collection.

This figure shows the bloodvessels, especially the capillaries. The large vessel at the lower part is a branch of the pulmonary artery; the large vessels near the upper border and to the right are branches of the pulmonary veins; some of the rounded or ovoid spaces, each bounded by a ring of capillaries seen vertically, are alveoli, one of these being nearly in the centre of the field.

FIG. 6. Section of the lung of a child (hematoxylin-eosin), $\times 125 .-$ Ewing.

The larger spaces shown in this section are the infundibula; the smaller spaces are alveoli, some opening into infundibula and some surrounded by the pulmonary tissue; at the top of the figure, part of a bronchiole, lined with epithelium, is seen. 


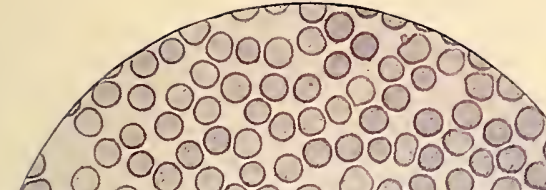

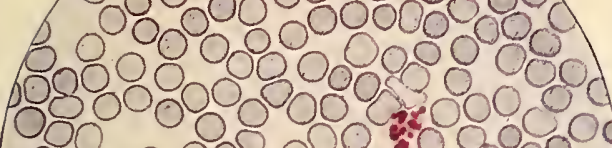

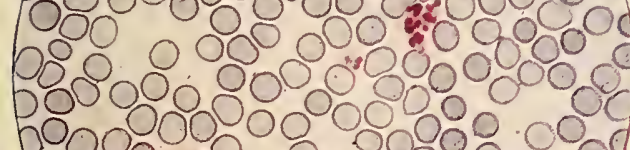

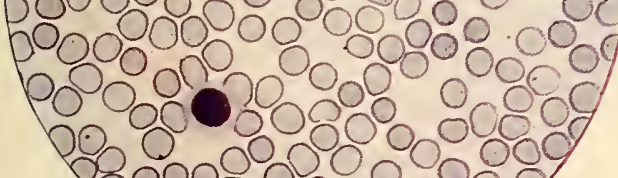
88000000000009 0.00000000
000000

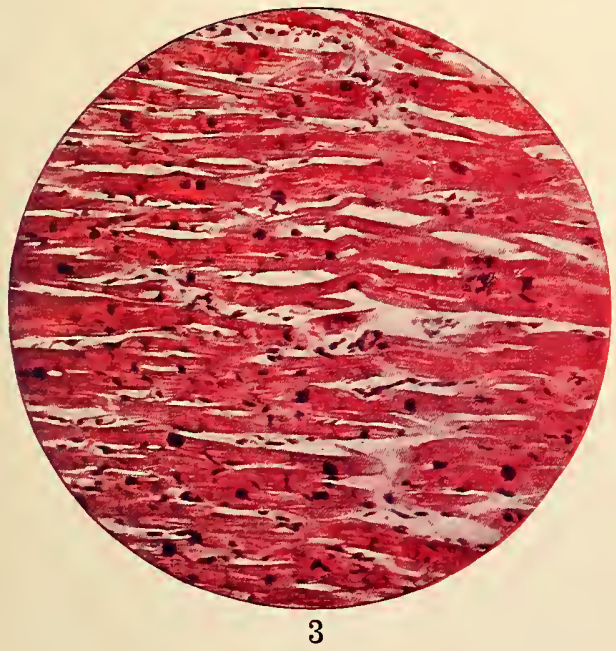

3

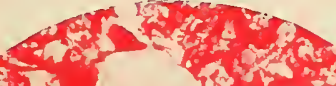

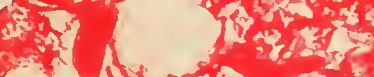

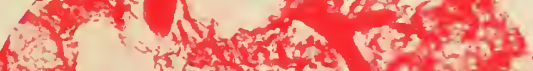

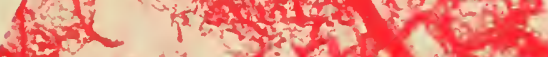

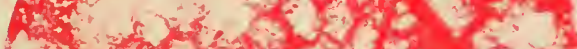

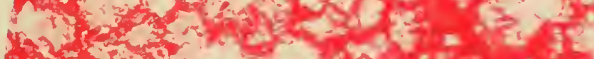
Y.r." Th

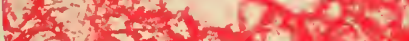

\section{i.}

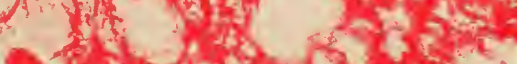

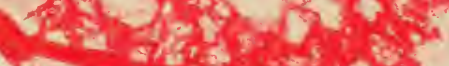
46 a

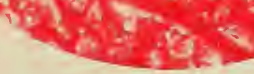
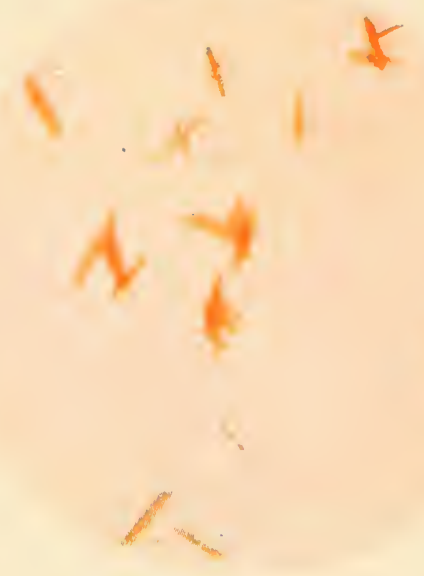

2
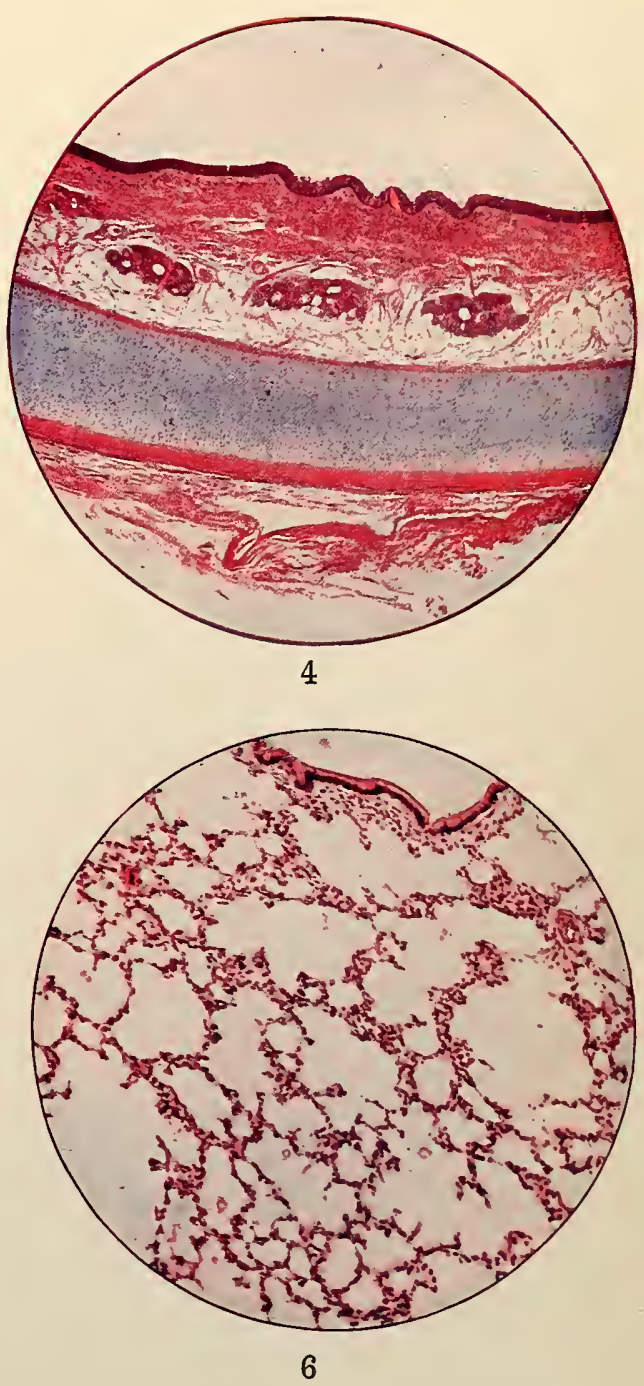



\section{PLATE III}

\section{Sections of BLOODVEsSELS (Sobotta)}

FIG. I. Section of vein - from an executed criminal (Zenker's solution; Weigert's elastictissue stain ; alum-carmin), $\times 5^{\circ}$. The elastic tissue is stained dark violet.

$A$, adventitia ; $J$, intima ; $M$, media ; $l m$, longitudinal muscle of the adventitia ; $v v$, vasa vasorum.

FIG. 2. Section of a large branch of the internal spermatic artery, $\times 80$.

$A$, adventitia ; $e i$, elastic lamella of the intina ; $e n$, endothelium; $J$, intima; $M$, media.

FIG. 3. Section of a small artery, $\times 220$.

$A$, adventitia ; $M$, media ; en, endothelium; ei, elastic lamella of the intima; ee, elastic lamella of the media.

FIG. 4. Section of a very small artery.

Lettering as in Fig. 3 . 


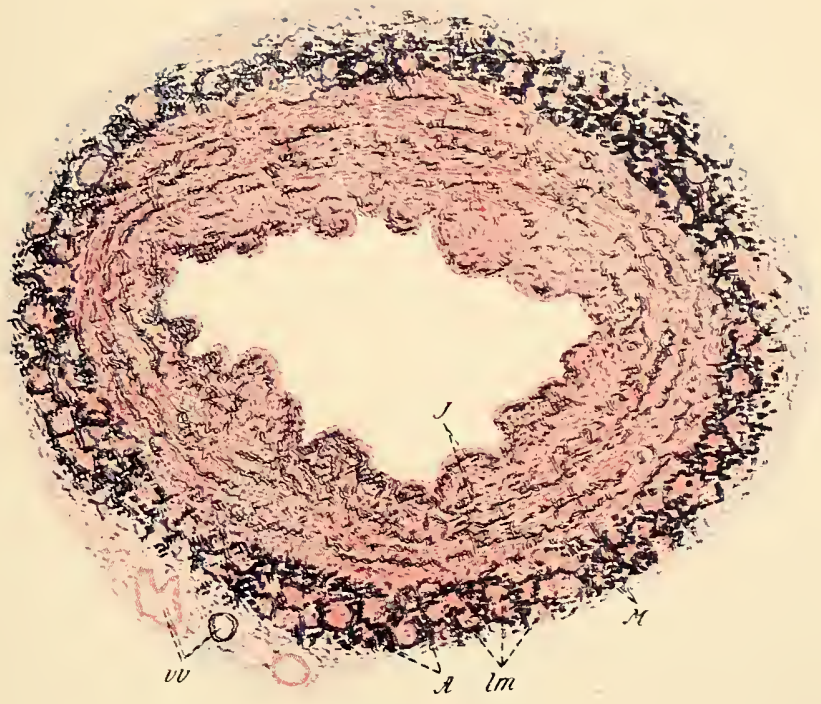

Fig.1.

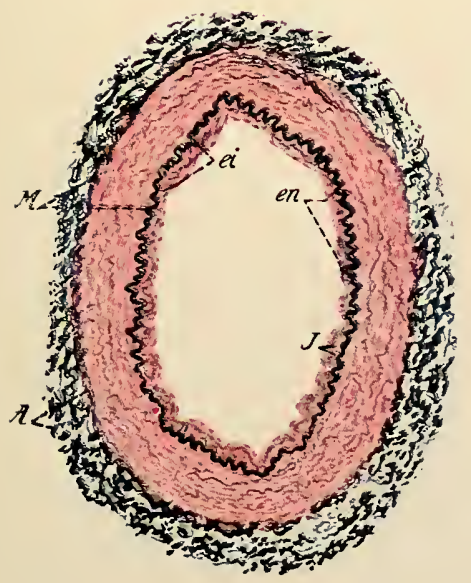

Fio. 2.
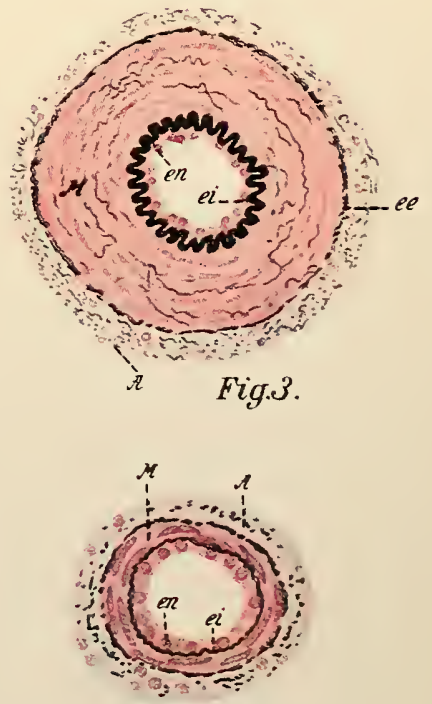

Fig.4. 



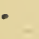




\section{PLATE IV}

FIG. 1. Section of the submaxillary gland of the human adult (hematoxylin-eosin, $\times 125$ ). - Ewing.

This figure shows the so-called serous acini, in which the cells are deeply stained; those with the cells relatively clear are mucous acini; a number of demilunes are distinctly shown; near the left lower border of the figure is a cross-section of a small duct, lined with epithelium.

FIG. 2. Section of the cesophagus of the dog (hematoxylin-eosin), $\times 10$. - Author's collection.

This section shows, at the upper border, the layer of epithelium, deeply stained, resting on a thick corium; beneath the corium is a layer of mucous glands; just beneath the glands, the fibrous tissue is plainly shown; beneath the fibrous tissue is the inner layer of the muscular coat, composed of striated and non-striated fibres, with the outer layer below, deeply stained; beneath this is the outer layer of fibrous tissue.

FIG. 3. Section of the stomach (hematoxylin-eosin), $\times$ I 25. - Author's collection.

This section is from the greater curvature, near the pylorus. The subject was executed by electricity at 6.01 A.M., April, I905, and the specimen was put into Zenker's solution two minutes after death. He was twenty-eight years of age, in perfect health and had not taken food for about twelve hours. The negatives for two colors, blue and red, were taken a few days after the section had been cut and stained.

The figure shows the tubules of the mucous membrane, the acid-cells stained red and the peptic cells, blue; it should be studied in connection with the text in the body of the book (see page 188 ).

It will be observed that although the section is near the pylorus, there are many red acidcells mixed with the blue peptic cells. There are no tubes shown in this figure that contain peptic cells only, although such tubes existed in other parts of the specimen, as well as some tubes nearly filled with acid-cells.

FIG. 4. Vertical transverse section of the duodenum of the cat (picro-carmin), $\times 35$. - Author's collection.

This figure shows above, the cæcal ends of the follicles of Lieberkühn; below, are Brunner's glands and connective tissue; beneath the connective tissue is a small portion of the muscular coat.

F1G. 5. Section of the small intestine of an infant (hematoxylin-eosin), $\times 125 .-$ Ewing.

This figure shows sections of the villi and of the tubes; the cells are red and the nuclei are blue; the great number of circular vacuoles, that are unstained, are the empty portions of goblet-cells.

Fig. 6. Lower portion of the duodenum of the dog (picro-carmin), $\times 3$ o. - Author's collection.

This figure shows above, the vili, with the epithelium deeply stained; below, are the follicles of Lieberkühn, also deeply stained; in the submucous tissue, are several glands of Brunner; the lowest part of the figure shows the muscular coat. The magnification is too low to show the cells distinctly. 

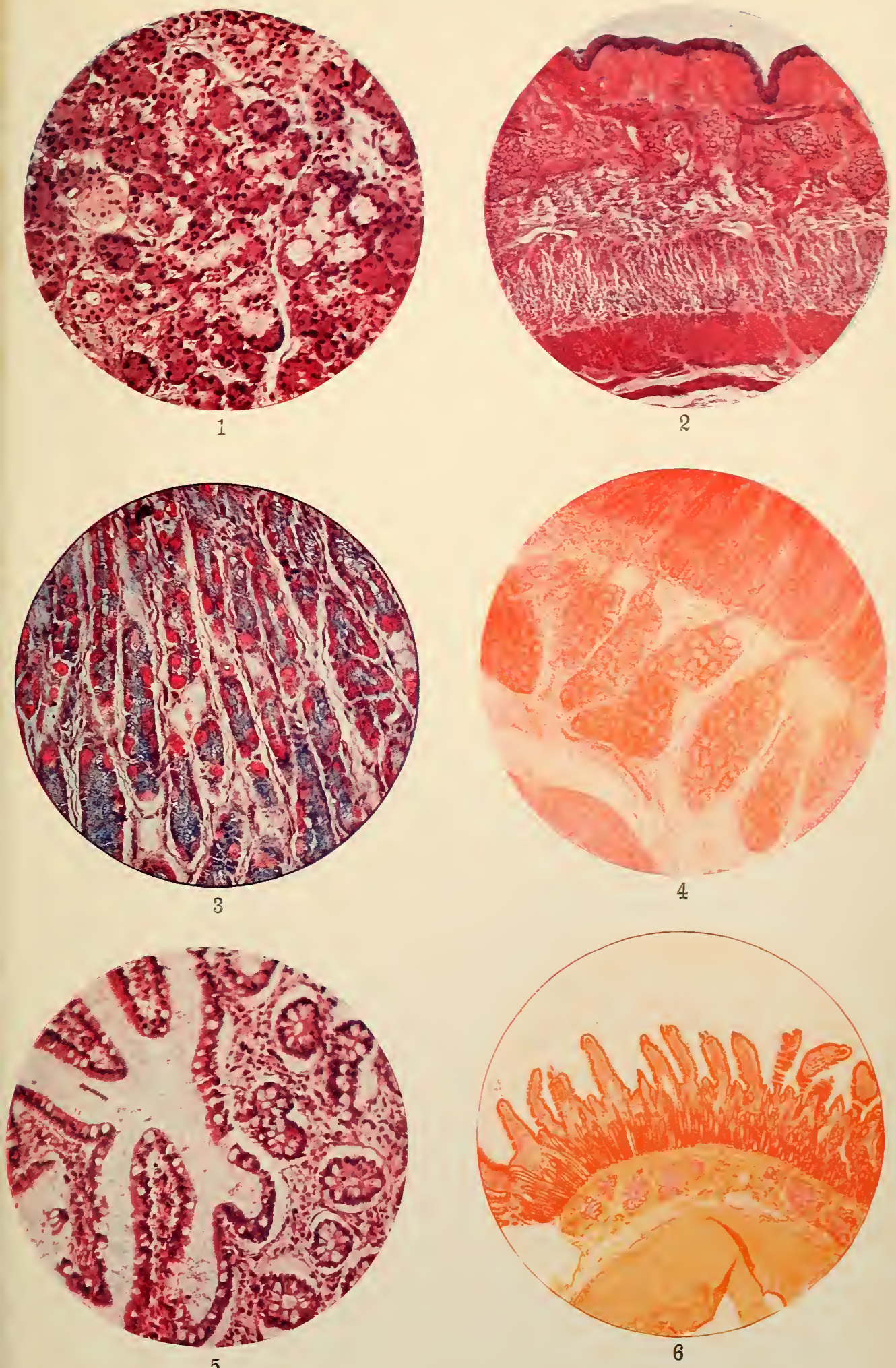




\section{PLATE V}

FIG. I. Section of the duodenum of the cat, injected, $\times 20 .-$ Author's collection.

The upper part of this section shows the distribution of the bloodvessels in the villi ; immediately below the villi are the follicles of Lieberkühn, not defined by reason of the low niagnification ; beneath these are glands of Brunner (not well defined), surrounded with plexuses of capillaries; the bottom of the figure shows the peculiar distribution of bloodvessels in the muscular coat.

FIG. 2. Section of the small intestine of the rat, injected, $\times 20 .-$ Author's collection.

This figure shows the distribution of bloodvessels in the villi of the rat, the form of the villi being quite different from the form of these structures in the cat.

FIG. 3. Bloodvessels of the pancreas of the cat, injected, $\times 30 .-$ Author's collection.

This figure shows arterioles breaking up into several plexuses of capillaries surrounding lobules of the pancreas.

FIG. 4. Section of the human pancreas (hematoxylin-eosin), $\times 125 .-$ Ewing.

Just above the centre of this figure, is a collection of centro-acinar cells forming an island of Langerhans; the rest of the figure is filled with the ordinary secreting cells of the pancreas; the outer and inner zones of the cells are shown, but not very distinctly, on account of the low magnification.

FiG. 5. Section of the large intestine of the dog (hematoxylin-eosin), $\times 20 .-$ Author's collection.

This figure shows the follicles of Lieberkühn - which are larger than in the small intestine - both in long section and in cross-section; a solitary gland is in the centre of the figure, lying in the submucous connective tissue ; the lowest part of the figure shows a section of the muscular coat, deeply stained; the connective tissue is stained red.

FIG. 6. Section of an active human breast (hematoxylin-eosin), $\times 125 .-$ Ewing.

This figure shows a portion of a lobule, the secreting cells presenting nuclei deeply stained; a portion of a duct is shown at the lower part of the figure. 

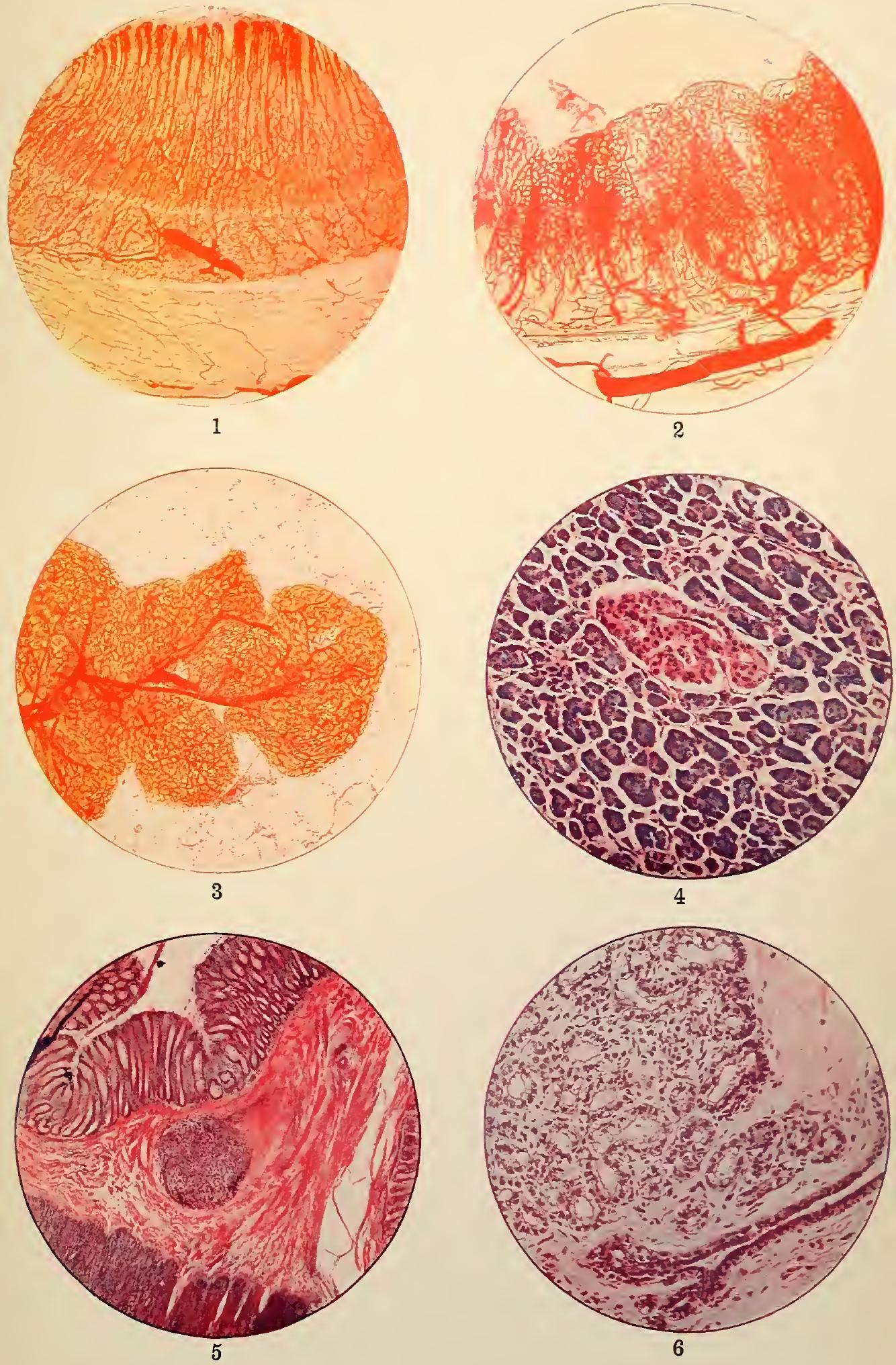




\section{PLATE VI}

Section of the Human Scalp, $\times$ 15. - (Sobotta.) (From an executed criminal. Müller's liquid; hematoxylin-eosin.)

$A p$, erector muscle ; $c$, corium ; $e p$, epidermis ; $f p$, hair-follicle ; $K H$, "club-hairs"; $p p$, papilla ; $R c$, cutis ; $R p$, root of hair; $S p$, shaft of hair ; $t$, subcutaneous layer; $x$, new formation of hair. 


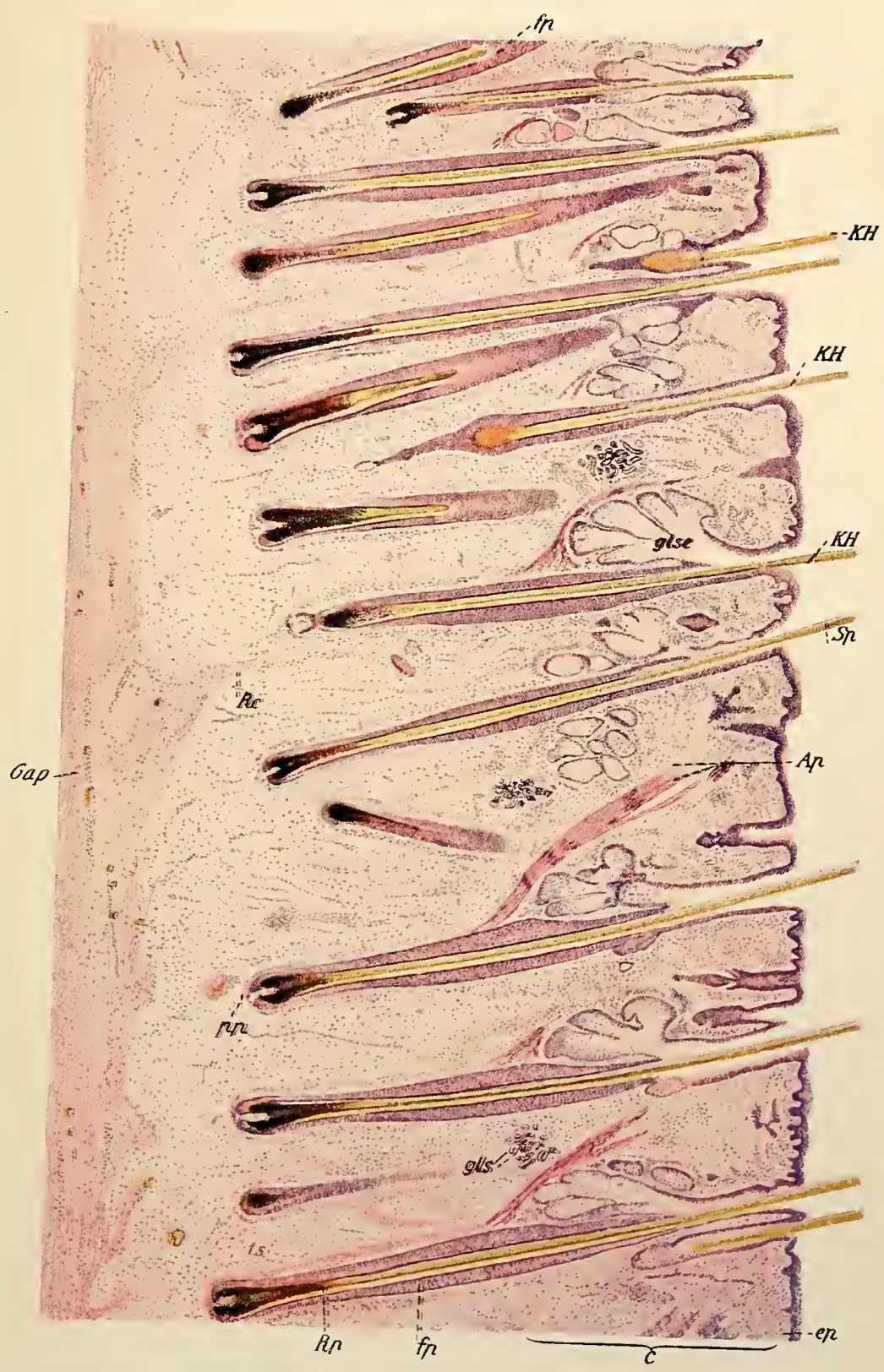



• 


\section{PLATE VII}

FIG. I. Section of the scalp of an infant (hematoxylin-eosin), $\times 35 .-$ Ewing.

This figure shows a collection of sebaceous glands near its upper portion; below the horizontal diameter is adipose tissue; a few sections of hairs are rather imperfectly shown at the left border.

FIG. 2. Section of the skin of the sole of the foot (hematoxylin-eosin), $\times 20$. -Author's collection.

This figure shows, with great distinctness, the two layers of the epidermis, with spiral sudoriferous ducts in the horny layer; the ducts take a nearly straight course through the corium, which is stained pink; the sudoriparous glands lie in the panniculus adiposus, just beneath the corium.

FIG. 3. Section of the cortical substances of the kidney of the dog, injected and stained (hematoxylin-eosin), $\times$ I 25. - Author's collection.

This figure shows three Malpighian bodies and part of fourth, each enclosed in its capsule. It also shows the general arrangement of the convoluted tubes. The magnification is too low to show distinctly the cells lining the tubes.

FIG. 4. Section of the kidney of the cat, injected, $\times$ i25. - Author's collection.

This figure shows only the glomeruli and the distribution of capillaries in the cortical substance.

FIG. 5. Cross-section of the liver of the pig, injected and stained (hematoxylin-eosin), $\times 125 .-$ Author's collection.

This figure is from a liver injected with blue through the portal vein, with the cells stained. The lobular vessels are purple; the staining shows the cells arranged in radiating columns; the central (intralobular) veins are empty. A section of a small artery appears at the lower part of the figure.

FIG. 6. Cross-section of a lobule of a pig's liver (hematein and eosin), $\times 50 .-$ Ferguson.

In this figure the empty intralobular vein is shown in the centre of the polygonal section of the lobule, three sides of which are shown to the left and above; the hepatic cells are shown in slightly wavy columns radiating from the centre toward the periphery. 
PLATE VII
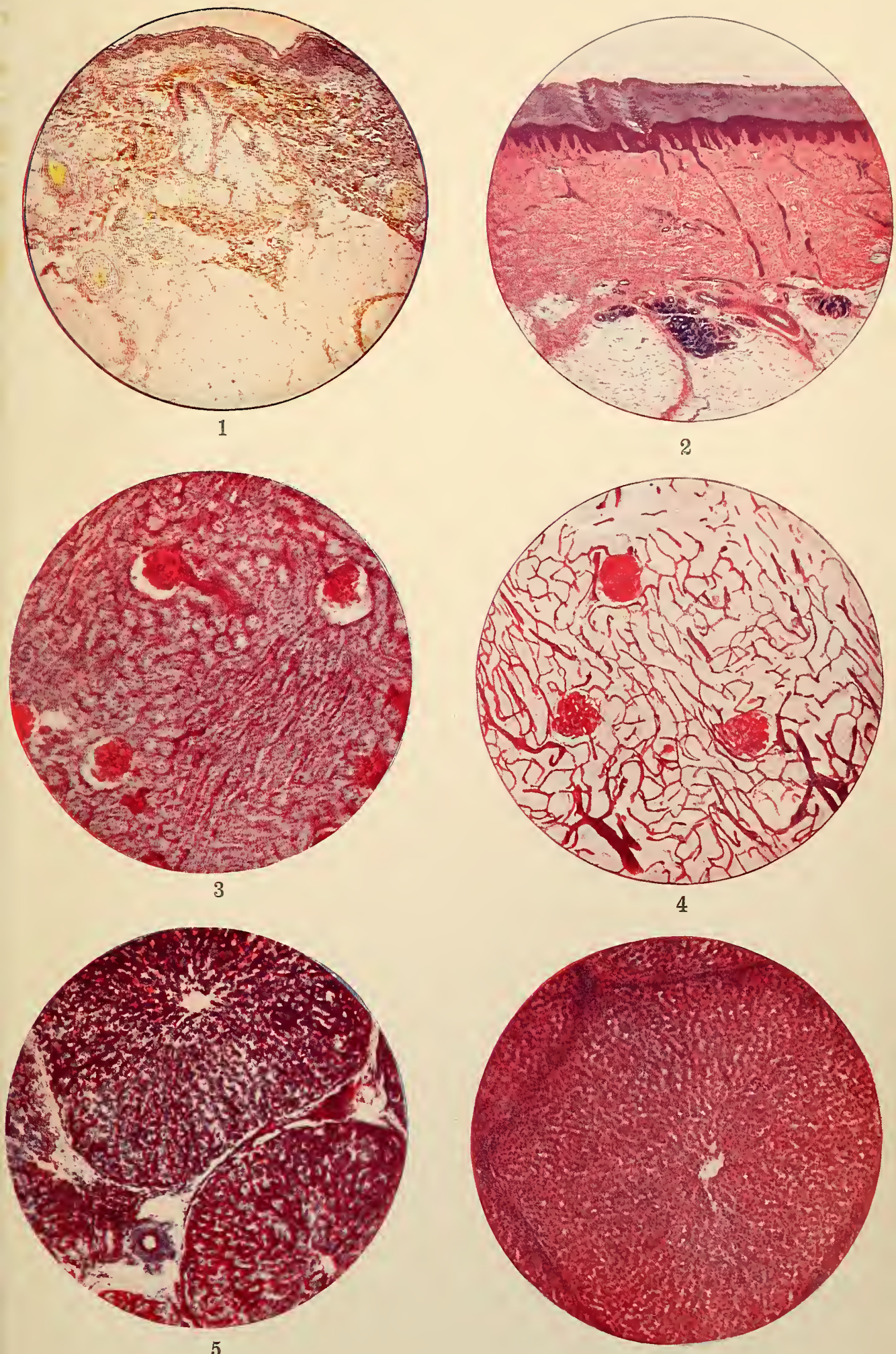


PLATE VIII

\section{LyMPH-GLAND - SpLEEN (Sobotta)}

FiG. I. Transverse section of a human cervical lymph-gland from an executed criminal (sublimate; hematoxylin-eosin), $\times \mathbf{I} 8$.

The figure shows the general structure of a lymph-gland.

$b g$, bloodvessels ; $c f$, fibrous capsule ; $H$, hilum ; $K z$, germ-centre ; $n l$, lymph-nodules ; $s c$, cortical substance; $s m$, medullary substance; $t r$, trabeculæ; $v l a$, afferent lymph-vessels; v'le, efferent lymph-vessels.

FIG. 2. Portion of an injected spleen of a rabbit, $\times 28$.

This preparation was taken from the material of the Institute for Comparative Anatorny, Würzburg.

The arterial trunks of the lymph-nodules (Malpighian corpuscles) are injected red, the veins and spleen-sinuses, blue.

$a$, artery of a Malpighian corpuscle; $M k n$, Malpighian corpuscle; $p$, spleen-pulp. 

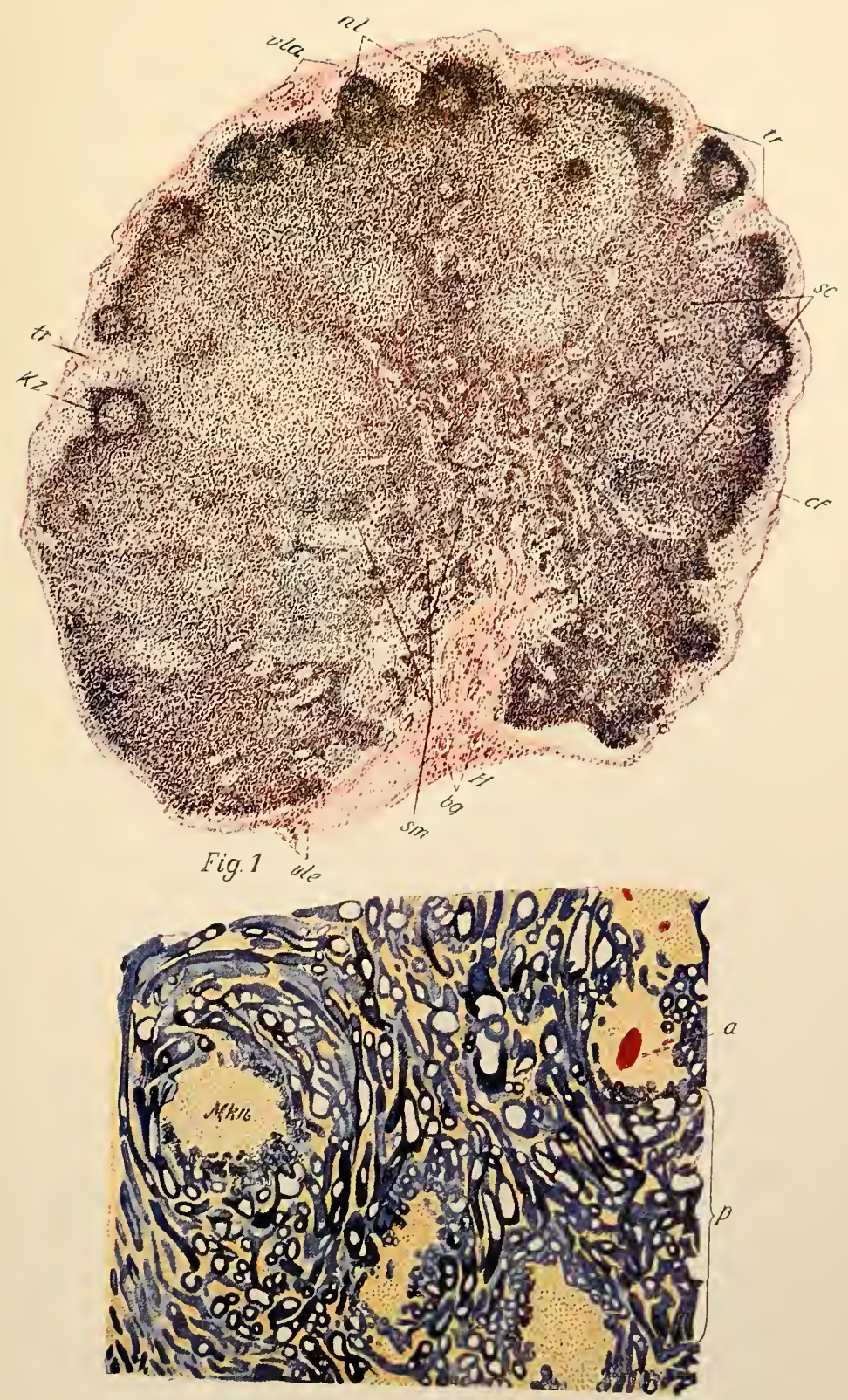

Fig. 2.

Luh. Anst F Reuchhold. München. 




\section{PLATE IX}

FIG. I. Section of the adrenal body of the rabbit (picro-carmin), $\times 20$. - Author's collection. This figure is from a section of the adrenal body taken under a magnification too low to show the cells. It shows the fibrous capsule and the cortical and medullar substances. The cortical substance has a radiated appearance, due to the arrangement of the cells in columns.

FiG. 2. Section of the spleen of the cat, injected, $\times$ i25. - Collection of Dr. A. W. Baird.

This figure shows Malpighian bodies, which are gray. The spleenic veins and sinuses are filled with a red injection.

Fig. 3. Section of the spleen of the dog (hematoxylin-eosin) $\times 30 .-$ Author's collection.

This figure shows Malpighian bodies embedded in the spleen-pulp.

FIG. 4. Section of the thyroid of the dog (hematoxylin-eosin), $\times$ I 25. - Author's collection.

This figure shows several alveoli lined with epithelial cells and filled with colloid substance. The nuclei are distinct, but the magnification is too low to bring out the contour of the cells.

FIG. 5. Section of the thymus of a hunan feetus (hematoxylin-eosin), $\times 20 .-$ Ewing.

This section shows parts of several lobules, each surrounded by the connective tissue of the trabeculæ; the cortex is more deeply stained than the medullary substance; in the medullary substance are sections of small bloodvessels and a few concentric corpuscles of Hassall, but these are very indistinct on account of the low magnification.

FIG. 6. Striated human muscle, unstained, $\times$ I25. - Author's collection.

This figure shows the structure of striated muscle; the transverse strix are apparent on close examination, especially in the fibres to the left, although the magnification is low. 




\section{PLATE $\mathrm{X}$}

Fig. I. Muscular tissue of the pig, injected, $\times$ 125. - Author's collection.

This figure shows the arrangement of the small arteries and capillaries. The magnification is too low to show the structure of the muscular fibres.

FIG. 2. Cross-section of the human radius from a young adult, injected, $\times 125 .-$ Author's collection.

Nearly in the centre of this figure is an Haversian canal surrounded with lacunæe and canaliculi, all filled with red material.

FiG. 3. Section of the finger of a child (hematoxylin-eosin), $\times$ Io. - Author's collection.

This section shows bone-development. To the left, deeply stained, is the forming bony structure invading the cartilage; to the right are the cartilaginous articulating surfaces of the phalanges; next the sides of the developing bone is periosteum; the epidermis and corium are shown at the top of the figure; the magnification is too low to show the cartilage-cells.

Fig. 4. Section of the ear of a bullock (hematoxylin-eosin), $\times 125 .-$ Author's collection.

This section shows cartilage-cells, the nuclei rather deeply stained, surrounded with a reticulum of very fine elastic fibres. These fibres are most distinct at the upper part of the figure.

FiG. 5. Transverse section of the Fallopian tube of the rabbit (hematoxylin-eosin), $\times 10$. - Author's collection.

The folds of the mucosa are shown about the horizontal diameter of the figure, the cells being rather more deeply stained than the submucosa, which is not sharply differentiated; the magnification is much too low to show the structure of the cells; the muscular layers are stained red.

FIG. 6. Section through the thorax of the foetus of the Guinea pig, showing especially the placenta and membranes (hematoxylin-eosin), $\times 4$. - Author's collection.

The placenta is to the right of the figure and occupies about one-third of the circumference of the section; internal to the lower half of the placenta is a portion of detached chorion, showing, under higher magnification, chorionic villi; the inner membrane, lining that portion of the ovum not occupied by the placenta, is the amnion; the outer layer of the section is the uterine wall; between the placenta and the uterine wall, slightly separated, is the decidua.

The section of the foetus shows the body of a vertebra and the spinal canal at the top of the figure; below this is the single aorta ; below the aorta and between the lungs is the vesophagus; by the sides of the aorta and cesophagus are the lungs, with the primitive bronchia, the one to the left more clearly shown; a portion of the liver lies below the lungs, but the liver has been broken in the section and a great part is lost; the process on the left of the foetus is the forearm.

The parts of the foetus are shown more clearly in the human sections, in Plate XVI. 

$$
\text { . }
$$ 
PLATE XI

Nerve-CELls (Sobotta)

FIG. I. Two isolated multipolar cells from the human spinal cord (isolation in weak chromic acid; carmin), $\times 160 ; x$, neurite torn away.

FIG. 2. Two multipolar cells from the lumbar enlargement of the spinal cord of a stillborn child (absolute alcohol; methyline-blue, according to Nissl), $\times 480$.

This figure shows the cell-bodies and roots of dendrites, with Nissl's granules intensely stained.

$D$, dendrites ; $K$, nucleus ; $t$, Nissl's granules.

Figs. $3,4,5$. Three cells from a human spinal ganglion from an executed criminal (Zenker's solution; hematoxylin-eosin), $\times 420$.

These figures show three spherical cells with their nucleated capsules of connective tissue. $b H$, connective-tissue capsule; $p$, pigment. 
PLATE YJ

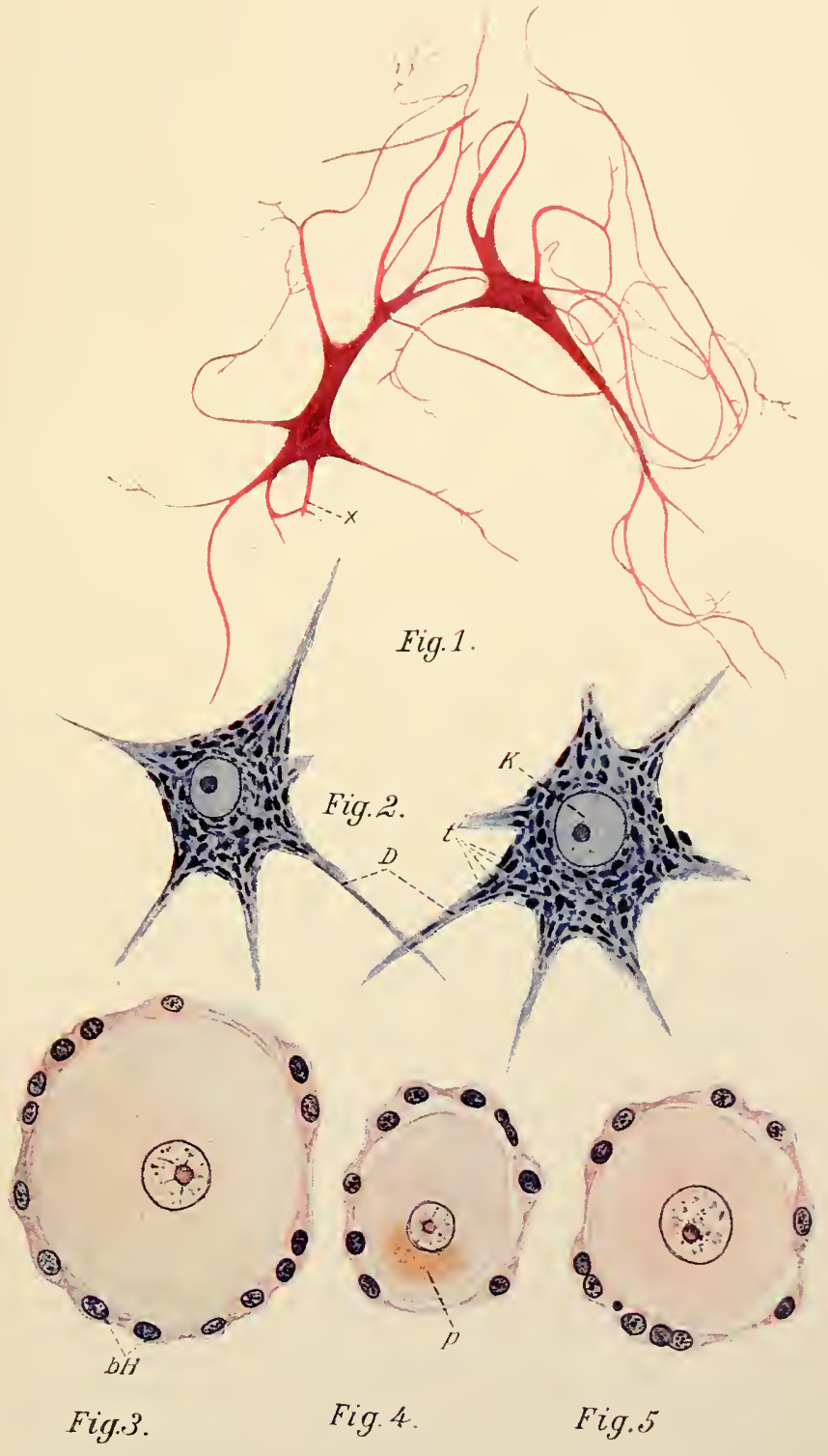






\section{PLATE XII}

\section{SPINAL CORD (Sobotta)}

FIG. 1. Cross-section of the human spinal cord at the cervical enlargement (from an adult and fixed two and one-half hours after death - Müller's liquid; sodium carminate); $\times 8$.

$b g$, sections of bloodvessels; $x$, region of the obliterated central canal; $c p$, posterior cornua; $f a$, column of Türck and anterior ground column; $c m a$, anterior median fissure; $f e$, column of Burdach; $f g$, column of Golb; $f$, lateral column; $p m$, pia mater; ra, anterior nerve-root; $r p$, posterior nerve-root; $s g$, substantia gelatinosa; $s p$, postcrior median fissure; $f r$, formatio reticularis.

This section shows quite distinctly several of the columns indicated in diagrammatic figures in the text.

FIG. 2. Cross-section of the human spinal cord at the lumbar enlargement - two-thirds of the section shown - fixed two and one-half hours after death (pia mater in red; medullated fibres in blue; nuclei in red; Müller's liquid; Weigert-Pal's stain for medullary sheaths; alumcarmin), $\times 15$.

$c a a$, anterior white commissure; $C c$, remains of the central canal; fina, anterior median fissure; $g l$, external glia sheath (neuroglia); $G z$, cells of the anterior horn; $n d$, dorsal nucleus; $p m$, pia mater; $k a$, anterior nerve-roots; $R p$, posterior nerve-roots; $s g$, gelatinous substance of Rolando; $s m p$, posterior median fissure. 
PLATE XII
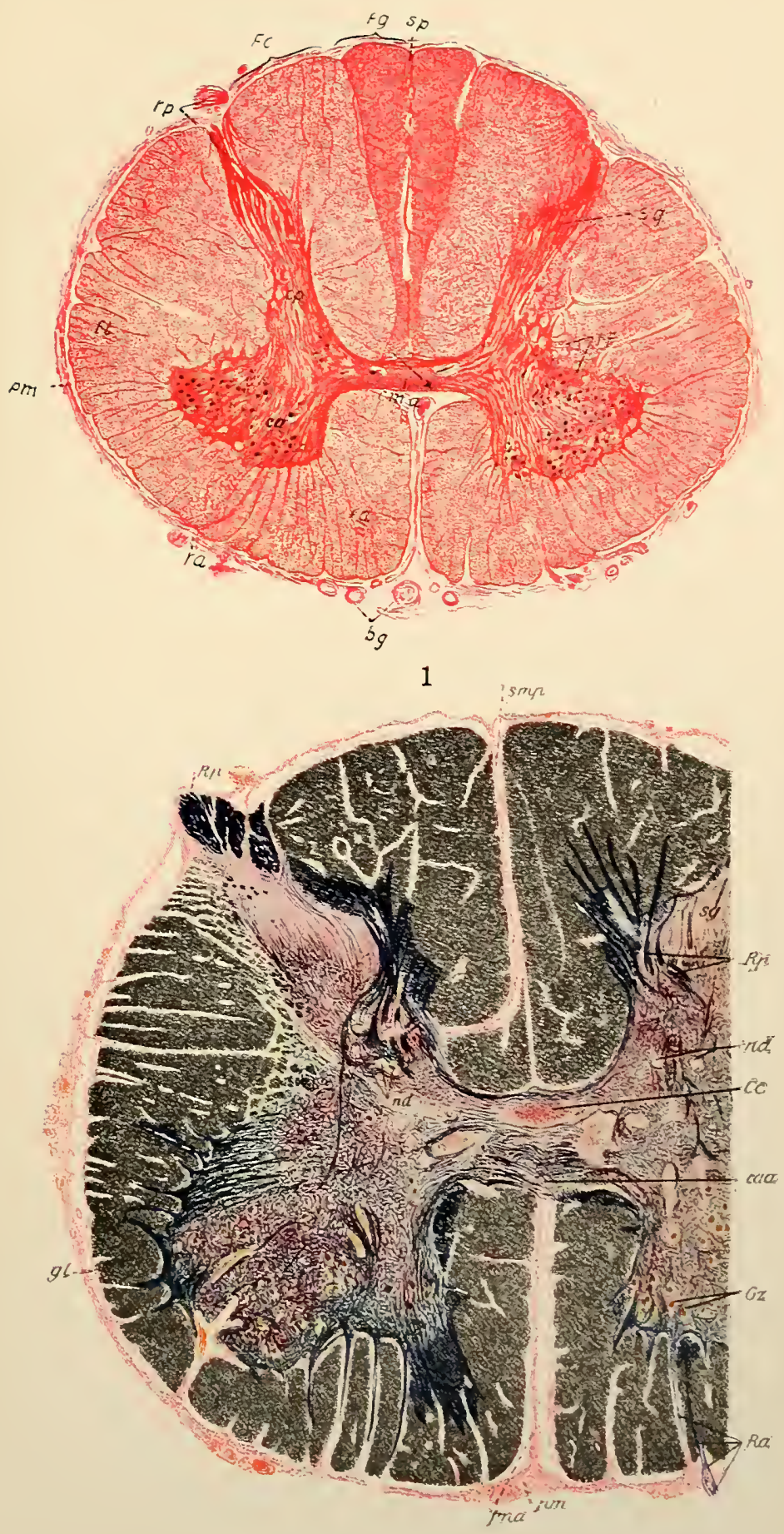



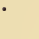




\section{PLATE XIII}

Two Early Stages of Cleavage of the Egg of the Sea-urchin, $\times 1000$ (Wilson)

FIG. I. "The section passes horizontally through the egg at the moment of division. In the centre lies the mid-body, darker and somewhat ill-defined. On either side of this is a group of 2-3 nuclear vesicles formed by the fusion of the chromosomal vesicles of the preceding stage. The remains of the centrosphere, scarcely defined, surround the nuclear vesicles."

FIG. 2. "Two-cell stage preparing for the second cleavage.

"A typical karyokinetic figure is forming in each cell at right angles to that of the first cleavage." 

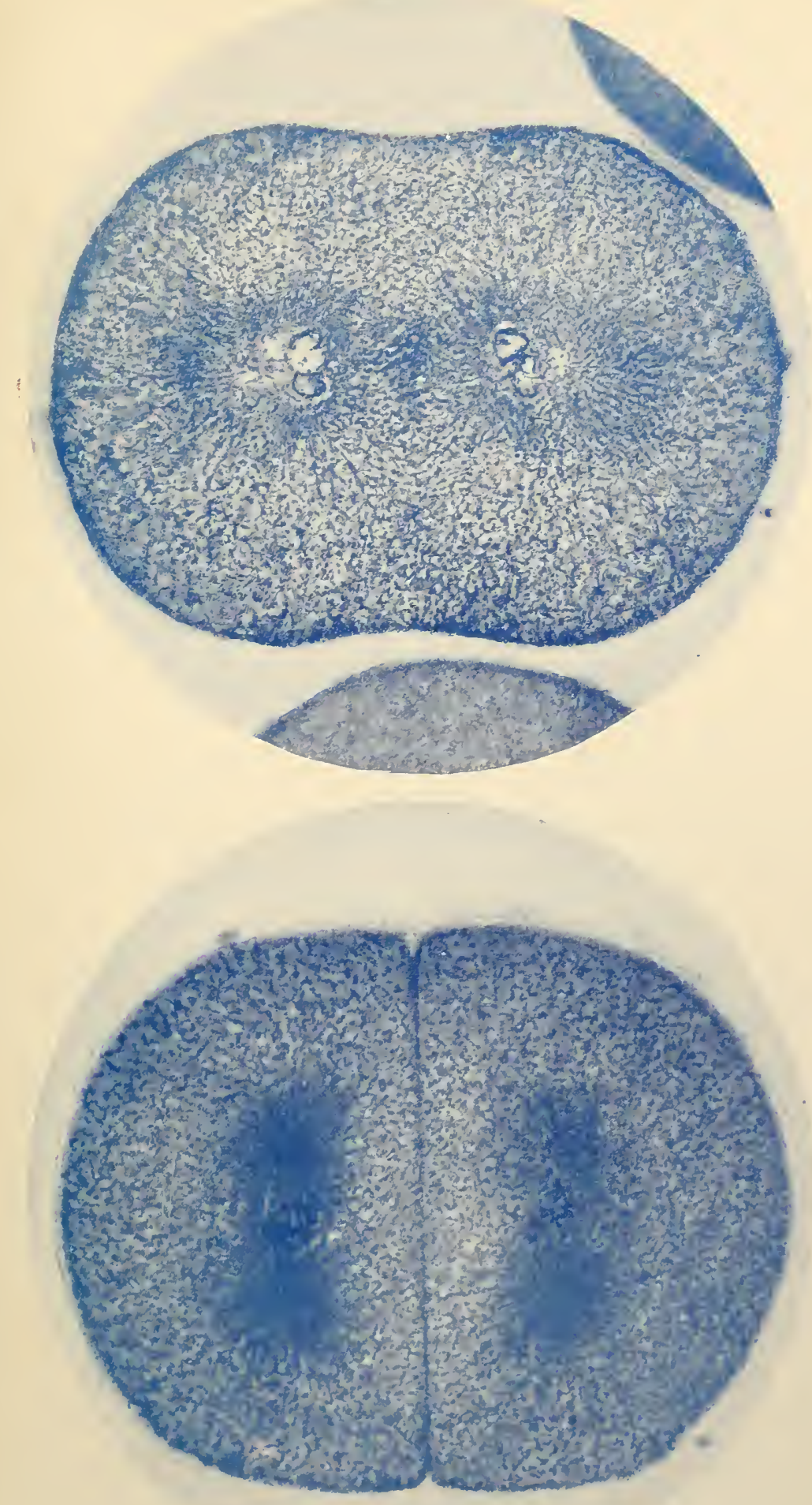



\section{PLATE XIV}

Two Later Stages of Cleavage of the Egg of the Sea-urchin, $\times$ iooo (Wilson)

FIG. 1. "The embryo is here shown in vertical section at the moment preceding the division from 8 to 16 cells. The two lower cells are dividing equally and vertically, one of the amphiasters being seen endwise, the other en face. The two upper cells are about to divide unequally to form two smaller cells or micromeres."

FIG. 2. "The blastula. Sixteen-celled stage.

"This section shows the blastomeres arranged in a hollow sphere surrounding a central blastocœl or cleavage-cavity. The nucleus is visible in each cell, and some of them show also the attraction-spheres (asters)."

The figures in Plates XIII and XIV are reproduced from the original negatives made for Wilson's Atlas. The eggs were fixed with sublimate-acetic, afterward preserved in alcohol, embedded in paraffin, sectioned and stained on the slide with Heidenhain's iron-hematoxylin for twenty-four hours, and "differentiated in a one per cent solution of iron-alum to a bright but delicate blue." The figures were printed in the Atlas in black. They are printed here in blue, but without an attempt to reproduce the exact shade of the objects. 


\section{PLATE XIV}

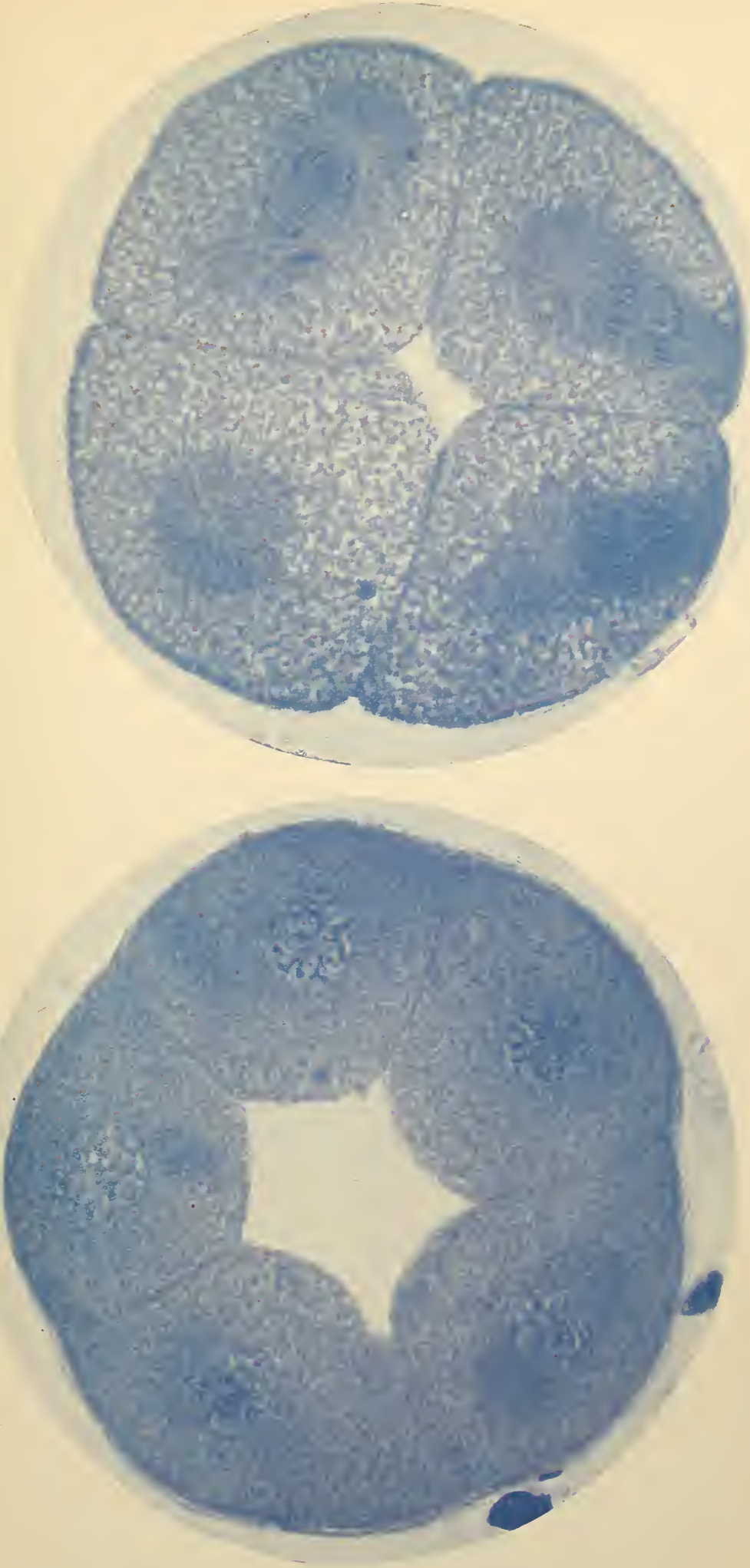





\section{PLATE XV}

FIG. 1. Section of the ovary of a kitten, injected and stained (hematoxylin-eosin), $\times 335$. - Author's collection.

This figure shows the orum, with the vitelline membrane, the protoplasm of the vitellus stained rose-color, the germinal vesicle and the germinal spot, surrounded with the cells of the corona radiata. The orum nearly fills the Graafian follicle.

FIG. 2. Section of the testicle of the rabbit (hematoxylin-eosin), $\times 35 .-$ Ferguson.

This figure shows simply the tortuous arrangement of the seminiferous tubes and the interstitial tissue. The magnification is not sufficient to show the structures within the tubes.

FIG. 3. Transrerse section of a chick of about 24 hours, near the caudal end, $\times 50$ (picrocarmin). - Strauss.

In the centre above, bounded by the rertebral plates, is the neural groove. The outer, delicate layer is the epiblast. The innermost layer is the hypoblast. The large cavity below is the enteron, finally to be closed orer by the abdominal plates. The round body below the neural groore is a section of the notochord. The masses of cells by the sides of the notochord are the primitive somites. The thick layer extending from the somites between the epiblast and the hypoblast is the mesoblast.

Fig. 4. Transverse section of same chick at about the middle of the abdomen (same technique and magnification). - Strauss.

The neural canal is nearly closed. The epiblast, hypoblast, and somites are as in Fig. 3 . The mesoblast has split into the somatopleure above and the splanchnopleure below, enclosing a large cavity, the celom.

FIG. 5. Transverse section of a chick of about 24 hours, near the head, $\times 50$ (hematoxylin). - Strauss.

The neural canal is closed orer. The epiblast and hypoblast are as in Figs. 3 and 4. $\mathrm{Br}$ the side of the primitive somite is a smaller mass of cells, the Wolfitian duct. The celom is as in Fig. 4. Between the splanchnopleure and the hypoblast is the omphalo-mesenteric vein.

FIG. 6. Transverse section of a chick of about 48 hours (same technique and magnification as Figs. 3 and 4 ). - Strauss.

The uppermost layer of cells is the external (false) amnion. The next layer below is the true amnion. The primitive somites have given off externally the muscle-plates. The two aortæ lie below the notochord. The Wolffian ducts are external to the aortæ and in the same plane. The somatopleure is thickened and curred and gives off the true amnion. The celom lies between the somatopleure and the splanchnopleure. The omphalo-mesenteric veins are better defined than in Fig. 5, especially the vein on the right side of the figure. 

FIG. I. Section through the upper part of the thorax of a human embryo (hematoxylin and orange $G), \times 4$. - Author's collection.

At the top of this figure is the spinal cord, with the body and the laminæ of a vertebra; by the side of the lamina on the left is a rib, and sections of ribs are seen in the walls of the thorax; on either side of the lower portion of the section is the arm; occupying the middle of the thorax, on either side, are the lungs; the opening to the right, just below the vertebra, is the cesophagus, on the right of what probably is the aorta; below the aorta and oesophagus are the two primary bronchia; below and between the lungs are the auricles of the heart; at the lowest part of the figure, in the median line, is a section of the sternum.

FIG. 2. Section through the lower part of the thorax of the same embryo (same technique and magnification). - Author's collection.

The left side of the figure is the right side of the embryo.

The spinal cord, vertebra, sections of ribs, lungs and sections of the arms are as in Fig. I. The round opening below the vertebra is the aorta; below the aorta and between the lungs is the oesophagus; below the lung on the left side of the figure are the ventricles of the heart, the right ventricle above the left; the large oval cavity below the lung on the right side of the figure is the abdominal cavity; between the right ventricle, the lung and the abdominal cavity is the ductus venosus.

FIG. 3. Section through the upper part of the abdomen of the same embryo (same technique and magnification). - Author's collection.

The right side of the figure is the right side of the embryo.

The spinal cord and vertebra as are in Figs. I and 2; immediately at the sides of the cord are the ganglia of the spinal nerves; in the centre of the body of the vertebra is a small spot, not seen in the other figures, which is a section of the notochord; by the sicles of the body of the vertebra are the kidneys, with the adrenal bodies below, which are larger; the immense liver nearly fills the abdomen; below the vertebra is the aorta; between the left adrenal and the stomach, on the left side, is the pancreas, extending to the median line; the opening, between the pancreas and the liver on the left side, is the stomach; to the right of the stomach and below the pancreas is intestine.

Fig. 4. Section through the lower part of the abdomen of the same embryo (same technique and magnification). - Author's collection.

The left side of the figure is the right side of the embryo.

The cord, vertebra, kidneys and aorta are as in Fig. 3; the section of the liver, being lower down, is not so large as in Fig. 3; the intestines lie between the aorta and kidneys above and the liver below.

FIG. 5. Sagittal section of a pig-embryo of $\frac{4}{5}$ inch $(20 \mathrm{~mm}$.), $\times 4$ (picro-carmin). - Strauss.

In the description of this figure, only those parts mentioned in the text are noted, although the section shows very distinctly many other structures.

At the convex border of the figure is the spinal cord, deeply stained, with the bulb at the first bend above; in front of the chord is the notochord, with the forming intervertebral disks deeply stained; the uppermost part of the figure is the head-cavity; the midbrain is in the central part of the head-cavity, above the nearly vertical projection continuous with the bulb, which forms its floor; the hindbrain and the cerebellum lie behind the midbrain; the diencephalon is in front; beneath the snout is the tongue, with the heart below; the liver is below the heart, separated from it by the diaphragm; the bronchia and lungs lie behind the heart and liver; the stomach and intestines lie below the liver, and a loop of small intestine extends into the umbilical cord, which lies in front of the intestines; the testis lies behind the intestines, close to the notochord.

FIG. 6. Sagittal section of a pig-embryo of $\frac{1}{2}$ inch $(12 \mathrm{~mm}$.) $\times 5$ (picro-carmin). - Strauss.

This figure shows an earlier stage of development than is shown in Fig. 5 . Below the hearl-cavity is the nandible - the tongue has not appeared; the diaphragm has not appeared, and the thorax and abdomen form a single cavity. 

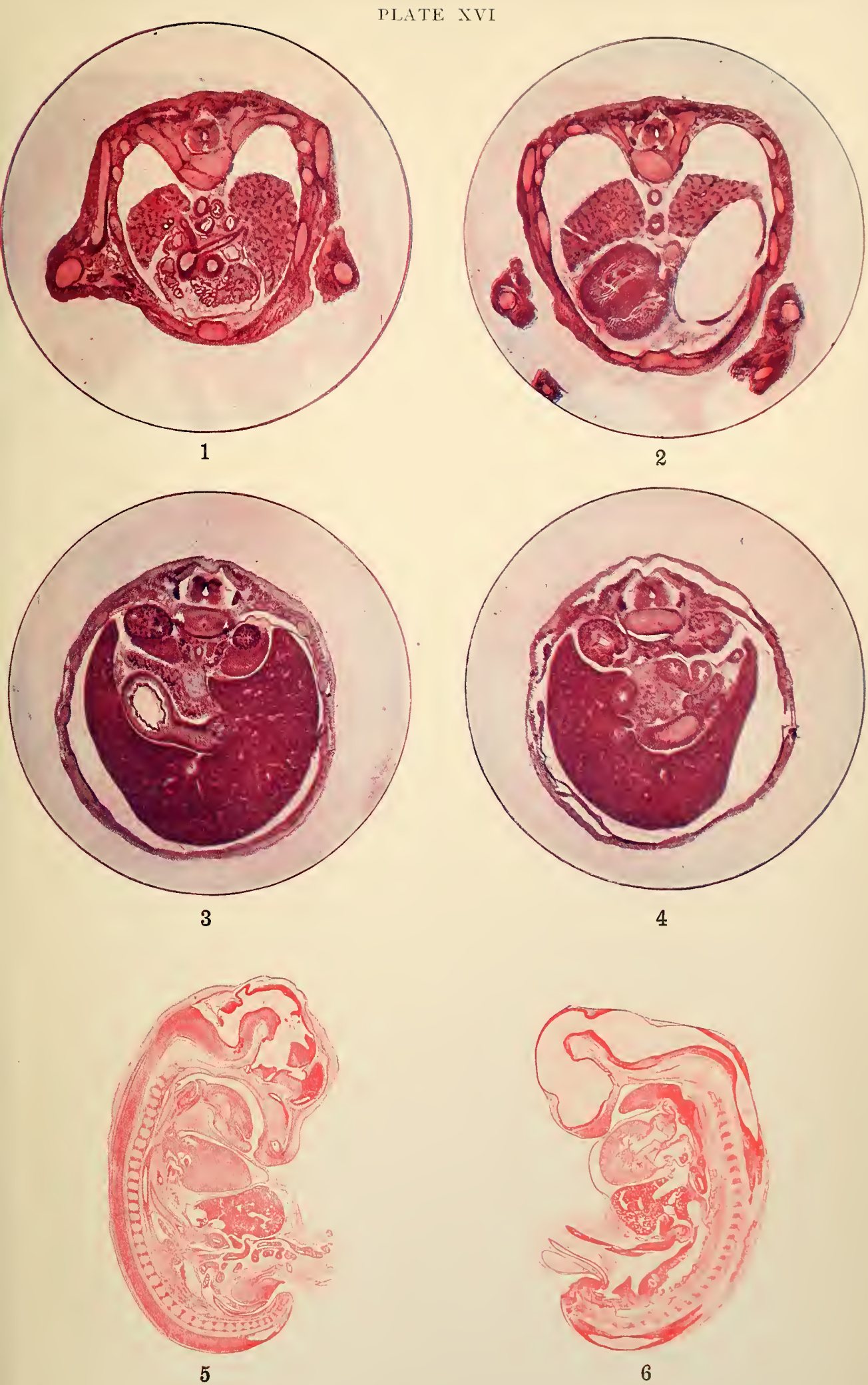



\section{INDEX}

Abdominal nerves ................ 542

Absorption....................... 240

- by closed cavities, reservoirs of glands,

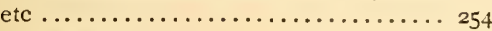

—. by lacteals................... 252

— by the respiratory surface.......... 253

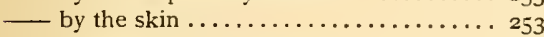

- influence of circulation........... 255

— influence of nervous system........ 255

- of fats....................... 25

Accommodation of the eye............. 686

Acid-albumin ..................... I99

Acromegaly...................... 390

Addison's disease . . . . . . . . . . . . . 378

Adolescence .................... 858

Adrenalin ....................... 378

Adrenals........................ 376 development of $\ldots \ldots \ldots \ldots \ldots \ldots \ldots 8_{38}$

Adult age....................... 858

After-images ..................... 696

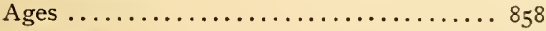

Agglutins $\ldots \ldots \ldots \ldots \ldots \ldots \ldots \ldots \ldots \ldots \ldots$ 13

Air, composition of $\ldots \ldots \ldots \ldots \ldots \ldots \ldots$ II6

Alcohol.......................... I50

_ formation of, from carbohydrates..... 393

heat-value of ................ 404

- influence of, on milk ............. 294

Alexins ......................... II

Alimentation ...................... I43

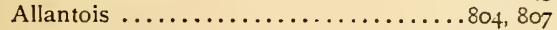

Amboceptors..................... 12

Ameba proteus.................. I

Ameboid movements $\ldots \ldots \ldots \ldots \ldots \ldots \ldots 4^{\text {I } 2}$

Amitosis $\ldots \ldots \ldots \ldots \ldots \ldots \ldots \ldots \ldots \ldots \ldots, 8$

Amnion ........................ 802

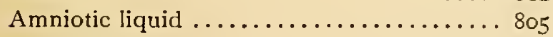

Amphiaster $\ldots \ldots \ldots \ldots \ldots \ldots \ldots \ldots \ldots \ldots .4$

Amputated members, sensation in ....... 479

Amylopsin ....................... 218

Amyloses ....................... I 45

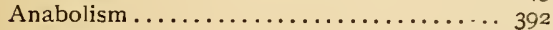

Anaphase.................... 5

Anelectrotonus ...................... 489

Angle alpha....................... 667

Antibodies...................... I2

Anticomplement................... I2

Anti-immune body ................. I2

Antitoxins ....................... Io

Antrum pylori..................... I87

Aorta, development of ............. $84^{2}$

Aphasia ......................... 59

Aqueous humor.....................6 $66_{3}$
Arachnoid ..................... 546

Archenteron ...................... 799

Archiblastic cells ................. $8+1$

Archoplasm................... 4

Area vasculosa .................. 840, 842

- appearance of blood-corpuscles in.... 2r

Arms, development of ............ 821

Arterial pressure $\ldots \ldots \ldots \ldots \ldots \ldots \ldots \ldots \ldots, 5_{5}$

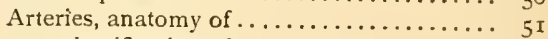

- classification of . . . . . . . . . . . . .

— development of .............. 843

- rapidity of blood-current in ........ 61

Asphyxia....................... I36

Associated movements.............. 477

Astigmatism....................68 68 I

Attraction sphere.................. 2

Audition ....................... 71

Auditory centres...................... 752

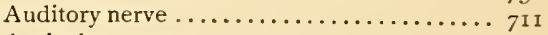

Auricular nerves.................. 536

Axillary glands...................... $3^{17}$

Beats, a cause of discord ............ 732

Bile, action of, in digestion $\ldots \ldots \ldots \ldots \ldots .221$

- composition of .................... $3^{6} 5$

- quantity of ...................... $36_{4}$

- secretion of $\ldots \ldots \ldots \ldots \ldots \ldots \ldots \ldots \ldots 3^{64}$

- tests for .......................... 368

Biliary fistula.................... 222

Biliary salts .................... 222

Bilirubin....................... 368

Binocular vision . . . . . . . . . . . . . 69 I

"Black hole" of Calcutta.............. I37

Bladder........................ 333

Blastoderm..................... 800

Blastodermic layers in the chick......... $8 \mathrm{I} 7$

"Bleeders" ....................... 29

Blood ......................... It

- coagulation of $\ldots \ldots \ldots \ldots \ldots \ldots \ldots \ldots \ldots,{ }_{26}$

- color in veins of glands ........... I6

- gases of .................... I32

- general characters of ............ I 6

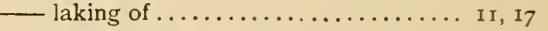

- precipitin-test for............... $2 \mathrm{r}$

- quantity of ..................... I5 $_{5}$

— table of composition of ............ 25

— test for hematin ................ 20

— transfusion of ................. 15

Blood-corpuscles ................... 18

- chemistry of .................. 20

- development of ............... 2 I 
Blood-corpuscles, structure of .......... 20

Blood-plasma...................... 24

Blood-platelets.................... 23

Blood-serum ..................... 27

Bone-formation .................. 821

Bones, anatomy of. ................. 428

Botal, foramen of. ................ 846

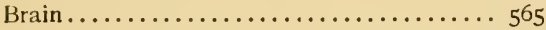

— development of ................ 823

Bread ........................ 160

Bronchia ....................... 9I

Brunner, glands of ................ 209

Bulb .............................. 598

— nerve centres in...............6 601

Butter ......................... 299

Cadaveric rigidity $\ldots \ldots \ldots \ldots \ldots \ldots \ldots 428,859$

Crecum......................... 225

Calcium oxalate ....................... 346

Calcium phosphate ............... 149

Calories ........................ 396

Calorimeter.................... 396

Capillaries, anatomy of ................ $6_{3}$ pressure of blood in............. 67

Capillary circulation................ 63

causes of..................... 68

- influence of temperature on........ 68

- relations of, to respiration.......... 67

Carbamid........................ 340

Cardiac nerves .................... 539

Cardiometer .................... 57

Cartilage, anatomy of .............. $43^{2}$

Caruncula lacrymalis ................ 707

Casein............................ 298

Catelectrotonus ..................... 489

Cell, typical animal ................. 2

Cells, death of, from old age.......... 8

- number of, in human adult.......... 3

- reproductive power of ........... 753

Cellulose ....................... $\mathbf{I}_{4} 6$

Centro-acinar cells ................ 2 I7

Centrosomes..................... 2

Cephalo-rachidian liquid. . . . . . . . . . 547

Cerebellar ataxia ................... 597

Cerebellum .................... 593

- connections of, with cerebrum....... 579

- extirpation of ................. 597

— histology of ................... 594

Cerebral convolutions............... 570

Cerebral localization................ 58 r

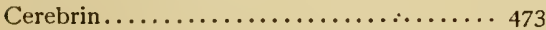

Cerebro-spinal axis.................. $5^{65}$

Cerebrum ........................ 567

- comparative anatomy of ............. $5^{87}$

- connections of, with cerebellum....... 579

- extirpation of . . . . . . . . . . . . . $5^{86}$

- fibres of..................... 579

- general uses of. .................. $5^{85}$

- histology of .................. 568

— in different races . ................ $5^{88}$

- pathology of .................. $5^{89}$

Cerumen ........................ 288

Ceruminous glands ................. 285

Chick, development of ............. 816
Childhood ...................... 858

Chocolate...................... 155

Cholesteremia.................... 37 I

Cholesterin................ I 47, 367, 369

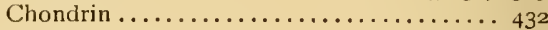

Chorda-tympani nerve..........505. 507, $63^{8}$

Chorion......................804, 807

Chorion frondosum .............. $8 \mathrm{I} 3$

Chorion leve .................. 81

Choroid ......................6. $65 \mathrm{I}$

Chromatic aberration ...............6 673

Chromatin ....................... ${ }_{2}$

Chromatolysis...................................... 470

Chromosomes.................. 5

number of, in different animals....... 5

Chyle, properties and composition of...... 262

Ciliary movements................. $4{ }^{\mathrm{I}} 3$

Ciliary muscle................... 653

Ciliary processes .................6. 652

Circulation ...................... 30

— derivative $\ldots \ldots \ldots \ldots \ldots \ldots \ldots \ldots \ldots 8_{3}$

- in erectile tissues................ 82

- in the arteries ............... 5 I

— in the capillaries ............... $6_{3}$

in the cranial cavity ............ $8 \mathrm{I}$

- in the heart-walls............... 84

- in the veins................. 69

- rapidity of................... 85

Circulatory system, conditions in, after death 88

Climacteric .................... 77 I

Cloaca .......................... 839

Cochlea, bony.................... 720

- distribution of nerves in.......... 747

- membranous ................. 743

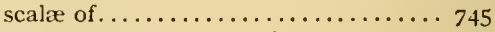

_ uses of different parts of........... 750

Coffee........................ 153

Coitus ......................... 787

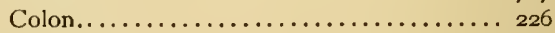

Color-blindness................... 705

Color-perception .................... 704

Colors ......................... 669

Colostrum ...................... 30

Complemental air ................. I $\mathrm{Ir}_{3}$

Complements .................. II

Condiments..................... I55

Conjunctiva...................... 707

Connective tissue .................. 420

Consonants ...................... 453

Contact, sense of $\ldots \ldots \ldots \ldots \ldots \ldots \ldots \ldots . \ldots 28$

Contraction, law of ............... 486

Coördination centre................ 597

Corium ....................... 306

Cornea ...................... 650

Corpora striata.................. 573

Corpus luteum . ................. 773

Correlation and conservation of forces..... 408

Corresponding points in the retina....... 692

Corti, ganglion and organ of $\ldots \ldots \ldots \ldots \ldots 747$

Coughing ...................... I Io

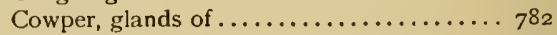

Cranial nerves...................... 494

Cream.......................... 296

Creatin and Creatinin............... 345 
Cresol.........................230, 235

Cretinism .............................. $3^{86}$

Crura cerebri .................. 577

Crystalline lens..................6. 661

Current of rest and current of action...... 427

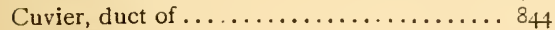

Cytoplasm ....................... ${ }_{2}$

Dacryolin...................... 7 Io

Death ........................... 859

- phenomena in the circulatory system after.

Deciduæ ...................... 8II

Decidua serotina ................ 8 8 I3

Defecation ........................ 236

Deglutition .................... I76

- duration of .................. I 84

— influence of the spinal accessory on... 5 I6

- mechanism of ................ I80

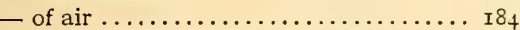

- protection of air-passages during...... I8I

Demilunes ...................... I70

Depressor nerves .................. 539

Derivative circulation............... 83

Dermatomes.................... 819

Deuteroalbumose.................. I99

Dextral preëminence.................. $85^{6}$

Dextrose.......................... I45

Diapedesis........................ 85

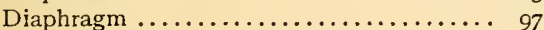

Diaster.......................... 5

Dicrotic pulse................... 55

Diencephalon .................. 823

Digestion, duration of, in the stomach..... 20I

- gastric ..................... I85

influence of various conditions on.... 202

- in the small intestine............. 206

Dilator tubæ................... 7 I9

Discords and dissonance............. 73 I

Dispirem ....................... 6

Dreams...................... 6rg

Ductless glands .................. 375

Duodenum ..................... 206

Dura mater....................... 546

Ear, development of ................. 825

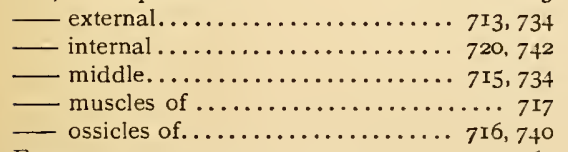

Eggs ....................... I6

Ehrlich's side-chain hypothesis ......... 9

Eighth nerve................... 7 II

Elastic cartilage .................. 433

Elastic tissue.................... 4 45

Electrodes, non-polarizable............ 485

Electrotonus ..................... 489

Eleventh nerve ................... $5^{13}$

Encephalon..................... 565

— connections of, with spinal cord...... $5^{80}$

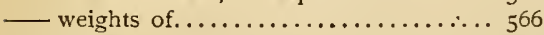

End-bulbs . . . ................... 466

Endosmosis and exosmosis............ 256
Enterokinase....................2 $21_{5}$

Epiblast ........................ 800

Epidermis ...................... 308

Epididymis ....................... 778

Epiglottis .................... 9 I

- in deglutition............... I I 182

Epinephrin ............................ $37^{8}$

Erectile tissues, circulation in......... 82

Erection, mechanism of................ $7^{87}$

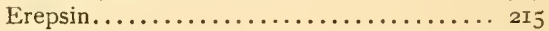

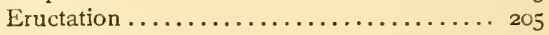

Erythroblasts $\ldots \ldots \ldots \ldots \ldots \ldots \ldots \ldots \ldots 2$ I, 43 I

Erythrocytes ...................... I8

Esophagus (see Esophagus) ............ I79

Esthesiometer.....................6 628

Estivation....................... 395

Eustachian tube................... 718

Eustachian valve ................. 846

Excretin and excretoleic acid.......... 232

Excretion, mechanism of.............. 273

Expiration .................... I02

- muscles of $\ldots \ldots \ldots \ldots \ldots \ldots \ldots \ldots$ I0 4

Expression, nerve of . . . . . . . . . . . 505

Eye, binocular vision ............... 69 I

blind spot in .................6 676

- chambers of.....................6. $66_{3}$

— development of ................ 824

— erect impressions of inverted images. . 690

- refraction in................... 679

- simple schematic .............6 68

- summary of anatomy of ........... $66_{5}$

Eyeball, anatomy of ............... 649

- muscles of ...................6 698

Eyelids ......................... 705

muscles of $\ldots \ldots \ldots \ldots \ldots \ldots \ldots \ldots, 706$

Face, development of .............. 830

Facial angle........................ 589

Facial nerve ................... 505

Fallopian tubes................... 764

Falsetto voice .................... $4^{5}$

Fatigue of muscle . . . . . . . . . . . . . . 426

Fatigue-products ................. 623

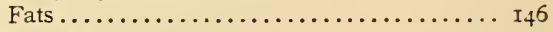

Fauces ...................... I78

Feces......................... 23I

Fecundation ..................... $7^{89}$

Fermentation in the intestines .......... 230

Fertilization of the ovum ............ 79

Fibrin ......................... 28

Fibrin-globulin ................. 28

Fibrinogen.................... 24, 28

Fibro-cartilage .................. 433

Fifth nerve, large root of $\ldots \ldots \ldots \ldots \ldots \ldots 5^{21}$

— small root of .................. 502

First nerve .................... $66_{32}$

Flavors ....................... 637

Fœtal circulation .................. 846

Fœtus, respiration by ............... I35

- weights of ................... $85 \mathrm{n}$

Foods ......................... I 43

- digestibility of ............... $20 \mathrm{x}$

— heat-value of . ............. 404,4 II necessary quantity and variety of ..... I55 
Forced movements ................ 604

Forces, correlation and conservation of.... 408

Fossa ovalıs................... 8.49

Fourth nerve..................... 499

Fovea cardiaca.................... 842

Fovea centralis ................... 656

Free-martin................. 772, 796

Fromann, striations of $\ldots \ldots \ldots \ldots \ldots \ldots+59$

Galactose...................... I 45

Gall-bladder ..................... $3^{62}$

Galton's whistle . . . . . . . . . . . . . 724

Gases in the alimentary canal ......... 237

— partial pressure of $\ldots \ldots \ldots \ldots \ldots \ldots$ 134

- tension of $\ldots \ldots \ldots \ldots \ldots \ldots \ldots \ldots \ldots \ldots \ldots \ldots \ldots$

Gasser, ganglion of ............... 522

Gastric digestion .................. 185

Gastric fistula ................... IgI

Gastric juice .................... Igo

— digestive action of $\ldots \ldots \ldots \ldots \ldots \ldots$. I95

Gastrulation ...................... 799

Gelatin, as food .................. I6I

Generation, female organs of ........... 754

- development of female organs of ..... 839

- male organs of................. 775

— development of male organs of...... 839

Geniculate ganglion................ 507

Genito-spinal centre ............. 336,780

Genito-urinary system, development of .... 835

Giannuzzi's crescents................. 170

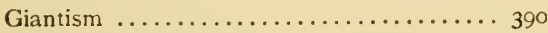

Giraldès, organ of $\ldots \ldots \ldots \ldots \ldots \ldots \ldots \ldots, 7^{80}$

Ghosts of the blood-corpuscles ........ I7, 20

Glands, classification of .............. 275

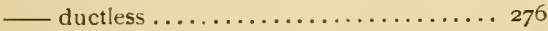

- motor nerves of............... 27t

- terminations of nerves in ........... $46_{3}$

Globin ........................ 20

Glosso-pharyngeal nerve ............. 639

Glottis......................... 90, $\$ 35$

- respiratory movements of .......... 9 I

— vocal movements of ............. 439

Glucoses........................ I 45

Glycerin ..................... I 47

Glycogen................... I 6,372

Goblet-cells.................. 210, 212

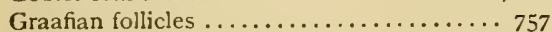

Gums ........................ I 46

Gustation ....................... $66_{37}$

Gustatory nerves ................6 $63^{8}$

Hairs.......................... 310

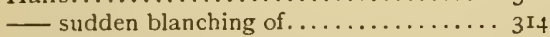

Harmony.................................. 730

Hassall, concentric corpuscles of. ......... $3^{88}$

Hauser, Kaspar, case of .............6 693

Heart ......................... 33

- accelerator nerves of $\ldots \ldots \ldots \ldots \ldots \ldots 4^{6}$

- capacity of cavities of ............. 35 cause of contractions of .......... 45 development of ................ 845 first appearance of $\ldots \ldots \ldots \ldots \ldots \ldots . \ldots \ldots+2$ frequency of action of ........... 4 I ganglia of ................ 45
Heart, influence of respiration on ........ 43

- - influence of spinal accessory on ....... 5 I6

- inhibition of ................ 47

— revolution, or cycle of ........... 39

- Stannius experiment ............. 46

— valves of $\ldots \ldots \ldots \ldots \ldots \ldots \ldots \ldots \ldots .3^{8}$

— work of .................. 49

Heart-muscle .................. 37

Heart-sounds ................... 40

Heart-walls, circulation in............. 84

Heat, animal..................... 395

— mechanism of production of ........ 403

— estimation of body-heat. .......... 396

- relations of, to force ............. 408

Heat-centres ................... +0

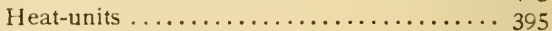

Heat-value of foods ............. 404,411

Hematin ........................ 20

Hematoidin ................... 20

Hematophorin .................. 20

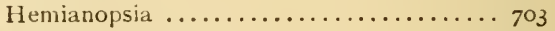

Hemin ...................... 20

Hemochromogen................. 20

Hemodrometer................... 6I

Hemodynamome:er ............... 57

Hemoglobin ...................... 20

- compounds of, with oxygen, carbon monoxide, and nitrous oxide .......... 20

Hemolvsis ..................... II

Hemophilia.................... 28

Hernia at the umbilicus of the fotus ...... ................ 806, 809. 810, 827

Heteroalbumose.................. I99

Hibernation...................... 395

— respiration in ................. I19

Hiccough ..................... I 10

Hippuric acid, etc.................. 34

Histohematins .................. I35

Horner, muscle of............. 706, 709

Horopter ........................ 692

Hunger....................... I 39

Hyaloplasm......................... 2

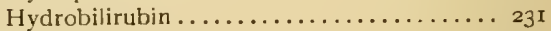

Hypermetropia ..................6. 67 I

Hypoblast .................... 801

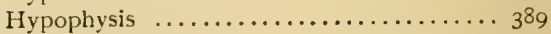

Hypoxanthin .................... 346

Ileo-crecal valve .................. 227

Ileum ....................... 208

Immune bodies $\ldots \ldots \ldots \ldots \ldots \ldots \ldots \ldots \ldots$ I2

Immunity . . . . . . . . . . . . . . . 9 hacteriolytic $\ldots \ldots \ldots \ldots \ldots \ldots \ldots \ldots$ II

Inanition $\ldots \ldots \ldots \ldots \ldots \ldots \ldots \ldots \ldots \ldots \ldots \ldots \ldots \ldots$

Indol...................... 230, 235

Induced muscular contraction ........... 488

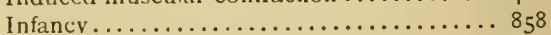

Inosite..................... I 6

Inspiration......................... 95

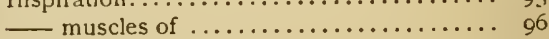

Intercostal muscles ................ 99

Internal capsule.................. 573

Internal secretion ................. 375

Intestinal digestion $\ldots \ldots \ldots \ldots \ldots \ldots \ldots .206$ 
Intestinal digestion, duration of......... 225

Intestinal fistula ................... 215

Intestinal juice ................... 215

Intestinal movements . . . . . . . . . . . . . 224

Intestinal villi ................... 2I I

Intestine, large ..................... 225

- movements of ................ 235

Intestine, smail. .................. 206

Intestines, development of. ........... 826

Invert-sugar $\ldots \ldots \ldots \ldots \ldots \ldots \ldots \ldots \ldots \ldots \ldots$

Involution of the uterus ............ 854

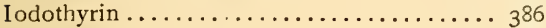

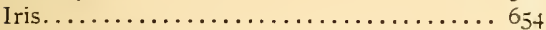

- movements of $\ldots \ldots \ldots \ldots \ldots \ldots \ldots \ldots 683$

- action of, in accommodation ........66 689

Iron....................... I49

Irradiation ....................6. 696

Isodynamic values of foods . . . . . . . 394

Jejunum ..................... 207

Jercorin .................... $3^{6} 3,3^{82}$

Karyokinesis..................... 3

duration of, in cells $\ldots \ldots \ldots \ldots \ldots \ldots \ldots,{ }_{8}$

Karyokinetic figure................. 5

Karyoplasm .................... 2

Karyosome ...................... 2

Kidneys ....................... 322

— development of ............... 837

- extirpation of................. 33 I

— internal secretion by $\ldots \ldots \ldots \ldots \ldots \ldots 353$

- work of ..................... 354

Kinase ..................... 219, 384

Kinetoplasm ................... 470

Krause, end-bulbs of $\ldots \ldots \ldots \ldots \ldots \ldots \ldots+66$

Kymograph....................... $5^{8}$

Labyrinth, bony $\ldots \ldots \ldots \ldots \ldots \ldots \ldots \ldots \ldots \ldots 720$

— distribution of nerves in ........... 746

liquids of............................ 746

membranous ................. 742

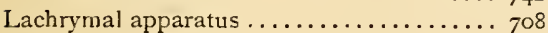

Lachrymin.................... 7 10

Lacteals .................... 24I, 247

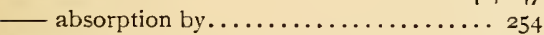

Lactose .................... I45, 299

Laking of blood................ II, I7

Langerhans, islands of ............ 2 I7

Laryngeal nerves . . . . . . . . . . . . . 537

Laryngoscope..................... 439

Larynx........................... 436

- action of, in phonation............ 442

— development of ................ 832

- respiratory movements of.......... 9 I

Laughing ....................... I Io

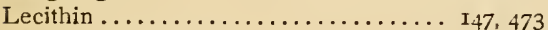

Leech-drawn blood ................ 28

Legs, development of .............. 821

Leucin ........................... $34^{6}$

Leucocytes...................... ${ }_{22}$

migration of $\ldots \ldots \ldots \ldots \ldots \ldots \ldots \ldots, 8_{4}$

Levulose....................... I 45

Lieberkühn, follicles of .............. 209

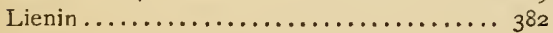

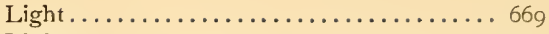

Linin ......................... 2

Lipochrome..................... 297

Littre, glands of . . . . . . . . . . . . 782

Liver....................... 355

— chemistry of .................... $3^{6} 3$

— development of ............... 828

— excretory action of...................... 368

- formation of glycogen in $\ldots \ldots \ldots \ldots \ldots 372$

Locomotor ataxia ................ 557

Lings....................... 94

— capacity of ................... III

— development of ................ 829

Luxus-consumption ................ 394

Lymph and chyle................... 258

- movements of. ................. $26_{5}$

Lymph, origin and uses of ........... $26 \mathrm{I}$

- corpuscles................... 260 glands (nodes)........... 243, 246, 250

Lymphatic duct .................... $2{ }^{4} 6$

Lymphatics...................... 241,248

— valves of . . . . . . . . . . . . . . 243,249

Lymphocytes .................... 26I

Macula acustica.................... $7 t^{2}$

Macula lutea....................... 656

Maltose ...................... I 6

Mammary glands................... 289

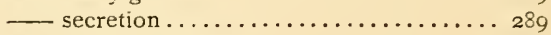

Marrow of the bones................. 430

Mastication .................... I62

- muscles of ................. I67

- nerve of...................... 502

Nastoid cells..................... 718

Meats ....................... ${ }^{56}$

Meckel, cartilage of ............... 826

Meconium ........................ 855

Meibomian glands ................. 285

- secretion .................... 289

Meissner, corpuscles of ............. 465

Membrana tympani ................. 734

Membranes of the embryo ........... 801

Mendel's laws of heredity ............. 793

Menstruation ..................... 77 I

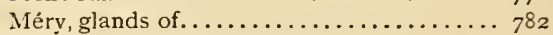

Mesenteric glands................ 247

Mesentery, development of........... 828

Mesoblast ....................... 80 I

Metabolism..................... 392

Metakinesis.................... 5

Metaphase....................

Metaplasm..................... 2

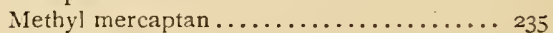

Microsomes.................... 2

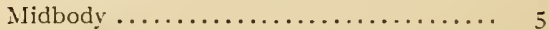

Middle age $\ldots \ldots \ldots \ldots \ldots \ldots \ldots \ldots \ldots .68_{58}^{5}$

Migration of leucocytes............. 23, 84

Milk ........................ 160

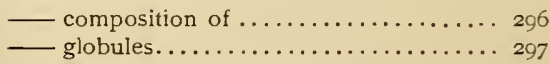

- influence of alcohol on........... 294

— in the newly-born............... 304

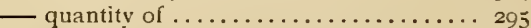

- secretion of................. 292 
Milk, uterine

Mitosis ..........................

Monaster......................... 5

Norula ........................ 799

Motor cortical zone................. $5^{82}$

Motor oculi communis................ 495

- influence of, on the iris.......... 448

Motor oculi externus ................ 501

Mouth, development of ............. 83 I

Movements.................... 412

- ameboid ...................... 412

Mucous glands..................... 279

Mucous membranes ................. 279

Mucus ........................... 280

Müller, duct of $\ldots \ldots \ldots \ldots \ldots \ldots \ldots \ldots \ldots .8_{36}$

Muscle, chemistry of ............... 421

- fatigue of . . . . . . . . . . . . . . . . 426

- involuntary ................... 417

- properties of.................. 422

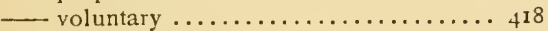

Muscle-currents ................... 427

Muscle-nerve preparation .............. 425

Muscle-plates ................... 819

Muscle-spindles................... 463,626

Muscles, development of ........... 82 I

Muscular contraction ................ 424

Muscular movements............... 4 I7

Muscular sense .................. 626

Musical scale ..................... 725

Myelocytes, of bone ............... $43^{\mathrm{I}}$

- of nerve-centres................ 473

Myeloplaxes .................... 43 I

Myo-albumin ....................... 422

Myoglobulin .................... 422

Myohematin ....................

Myopia......................6.67

Myosin ....................... 421

Myotomes ..................... 819

Myxœedema.............................. $3^{86}$

Nails....................... 309

Negative variation of muscle-current . . . . 427

- of nerve-current . .............. $49 \mathrm{r}$

Nerve-cells...................... 468

Nerve-centres . . . . . . . . . . . . . . . 468

- accessory anatomical elements of..... 472

Nerve-currents.................. 489, 49 r

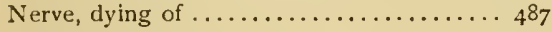

Nerve-fibres....................... 457

Nerve-muscle preparation............. 425

Nerves, accessory anatomical elements of. . 46 I

- action of electricity on..............483

- action of motor ................. 476

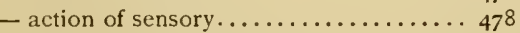

_ cranial ..................... 494

- degeneration and regeneration of..... 474

end-plates of ..................462

- motor and sensory ............... 473

negative variation in............ 49I

- sensory, terminations of........463,467 spinal. ..................... 492

Nervous conduction, rapidity of ......... 480

Nervous excitability and conductivity ..... 479

Nervous matter, composition of........ 473
Nervous system, development of. . . . . . . 822

- divisions of.................. 455

Neurilemma .................. 457

Neuroglia ..................... 473

Neuro-muscular spindles $\ldots \ldots \ldots \ldots \ldots \ldots 463,626$

Neuron...................... 47 I

Neutral point ................... 490

Ninth nerve..................... 639

Nissl's granules .................. 470

Nitrogen, elimination of............ 340

Nose, development of . .............. $83^{2}$

Notochord ................... 8 I9

Nutrition . . . . . . . . . . . . . . 394

Odors $\ldots \ldots \ldots \ldots \ldots \ldots \ldots \ldots \ldots \ldots \ldots . \ldots 635$

Esophageal nerves................. $54 \mathrm{I}$

Esophagus ....................... 179

- development of ................ 828

Oken, bodies of .................. 835

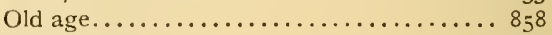

Olfaction ........................6. $66_{31}$

Oifactory cells.....................6 634

Olfactory centre ................... 636

Olfactory lobes, development of......... 826

Olfactory nerve.................. $66_{32}$

Omphalo-mesenteric vessels........... 842

Optic nerve .................... 646

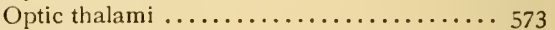

Orgasm, venereal................. 788

Osmosis .............................. 256

Osmotic pressure................... 257

Ossicles of the ear .................. 716

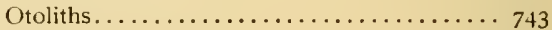

Ovaries.................................. 755

_ development of ..................... 836

- internal secretion by ........... 39I

Overtones....................... 727

Ovum ............................ 766

— development of ............... 816

— discharge of $\ldots \ldots \ldots \ldots \ldots \ldots \ldots \ldots, 769$

- fertilization of ............... 791

— maturation of................. 789

- segmentation of $\ldots \ldots \ldots \ldots \ldots \ldots \ldots \ldots 797$

Oxidation in the body .............. 405

Pacini, corpuscles of................. $46_{4}$

Palate........................ 177

Pancreas ....................... 216

- development of ................ 829

Pancreatic fistula................. 219

Pancreatic juice ................. 217

Parablastic cells.................. 84 I

Paramyosinogen ................. 42

Parapeptones ..................... I99

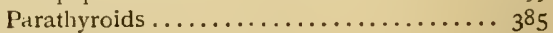

Parotid ........................ 170

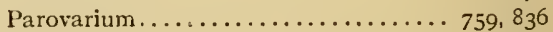

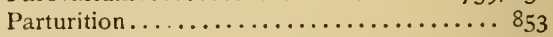

Pentre........................ 854

Patheticus ...................... 499

Pawlow's pouch ................... 192

Penis, development of............... 839

Peptones ....................... 198

Perimeter..................... $69 \mathbf{r}$ 
Periosteum

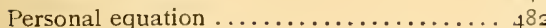

Perspiration....................... $3 \mathrm{I}_{5}$

Pettenkofer's chamber ................ II8

Peyer's patches................... 213

Pflüger's law of contraction............ 486

Pharyngeal nerves .................. 536

Pharyngeal secretion............... I72

Pharynx ...................... I 77

— development of ............... 828

Phenol ....................... 230, 235

Phonograph ...................... 454

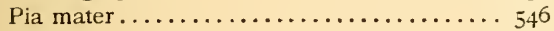

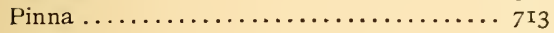

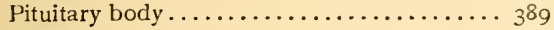

Placenta .............................. $8 \mathbf{r}_{3}$

Plastids.......................... 2

Pleura........................... 94

Pneumogastric .................. 530

- branches of.................. 532

- branches of, to abdominal viscera .... 542

- general properties of . . . . ........ 536

Polar bodies .................... 3, 790

Pons Varolii ..................... 577

Potatoes....................... I60

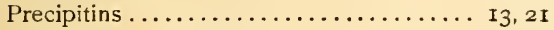

Precipitin-test for blood ............. 2 I

Pregnancy, duration of.............. 850 multiple.................... 853

Presbyopia ....................6 672

Primitive streak .................. 800

Propeptone ...................... 199

Prophases of karyokinesis............. 3

Prostate ........................... $7^{8} \mathbf{I}$

Protagon ........................ 473

Prothrombin .................... 28

Protoalbumose.................... 199

Protoplasm ...................... ${ }_{2}$

Protovertebræ.................... 8 8 ig

Pseudopods...................... 2

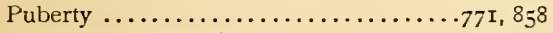

Pulmonary circulation $\ldots \ldots \ldots \ldots \ldots \ldots \ldots, 183$

Pulmonary nerves ................. 539

Pulse........................ 53

- dicrotic ..................... 55

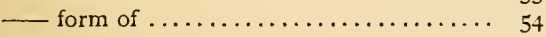

Pupil .......................... 654

Pupillary membrane.............. 655, 825

Purkinje cells .................... 596

Purposive movements............... $5^{6}$ I

Pyloric muscle .................. 187

Ranvier, nodes of.................. $45^{8}$

Ration, daily.................... I56

Reaction-time ................... 590

Receptaculum chyli................. 247

Receptors..................... 10

Rectum....................... 229

Reflex movements.................. 559

Refraction in the eye............ 667,679

Remak, fibres of.................. 459

Renin ....................... 354

Rennin ...................... 198
Reserve air .................... II

Residual air................... I12

Respiration .................... 89

— changes of the blood in.......... I3

consumption of oxygen in .......... II 7

cutaneous ................... I36

diffusion of air in the lungs......... II5

exhalation of ammonia, organic matters and nitrogen in ................ I3I

- exhalation of carbon dioxide in ..... I2I

inhalation of irrespirable or poisonuus gases ....................... II

— in hibernation ................. I I9

of pure oxygen .............. II

in the fotus ................... I35

- in the newly-born .............. II9

in the tissues ................ I35

- movements of ............. 95, I07

- organs of.................... 89

— relations between oxygen consumed and carbon dioxide exhaled ........... I27

- sources of carbon dioxide in........ I29

— temperature of expired air ......... I20

— types of .................... I06

under different degrees of atmospheric

pressure................... II6

volumes of inspired and of expired air IIt

Respiratory centres................6 602

Respiratory quotient $\ldots \ldots \ldots \ldots \ldots \ldots \ldots$ I 29

Respiratory sounds ............... I08

Retina ........................ $66_{56}$

corresponding points in .......... 692

Rhodopsin ..................... 677

Rigor mortis ................... 428, 859

Rodded cells................... 327

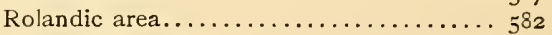

Rosenmüller, organ of . . . . . . . 759,836

Saccharoses....................... I I

St. Martin, case of................ IgI

Saliva ...................... r69

— action of, on starch. $\ldots \ldots \ldots \ldots \ldots \ldots$ I 7 7

- mechanical action of ............. I 76

Sarcolactic acid .................... 428

Savors $\ldots \ldots \ldots \ldots \ldots \ldots \ldots \ldots \ldots \ldots \ldots \ldots 66_{37}$

Schwann, sheath of $\ldots \ldots \ldots \ldots \ldots \ldots \ldots \ldots 45^{8}$

Sclerotic ...................... 650

Scorbutus....................... I59

Sebaceous glands ................. 282

Sebaceous matter .................. 286

Sebum ........................ 286

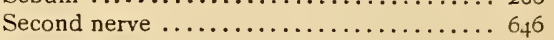

Secretion ...................... 268

- changes in the circulation in the glands during................ 27 I

— influence of the blood on ......... 273

— mechanism of . ................ 270

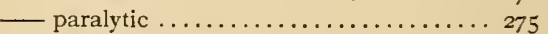

Secretions, classification of ........... 269

Secretions and excretions ............. 277

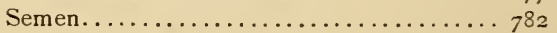

Semicircular canals, bony .................. 720

— membranous ................. 743

uses of.................... 750 
Serum-albumin.................... 24

Serum-giobulin.................. 24

Seventh nerve................... 505

Shadows of the retinal vessels .......... 675

Sighing ...................... I

Sixth nerve ...................... 50I

Skatol.......................230, 235

Skeleton, development of ............ 819

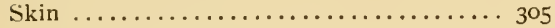

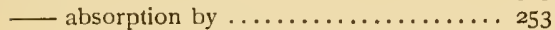

development of ............... 822

effects of covering with an impermeable coating ......................... $3^{15}$

Skin-plates..................... 819

Sleep........................618

Smegma of the prepuce and of the labia mi-

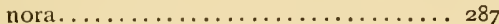

Smell (see Olfaction) .............. 63I

Sneezing........................ I

Sobbing ........................ I Io

Sodium chloride.................. I 49

Solitary glands................... 212

Somatopleure $\ldots . \ldots \ldots \ldots \ldots \ldots \ldots \ldots$. 8 . I

Somites ...................... 819

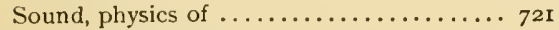
vibrations of $\ldots \ldots \ldots \ldots \ldots \ldots \ldots \ldots, 7_{22}$

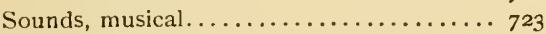

Speech, mechanisin of $\ldots \ldots \ldots \ldots \ldots \ldots .45^{\circ}$

Speech-centre ..................... 590

Spermatin ..................... $77_{3}$

Spermatogenesis .................. 784

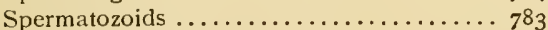

Spherical aberration ................ 672

Sphygmograph.................... 54

Sphygmomanometer ................ 60

Spinal accessory ................... $5^{13}$

Spinal cord ........................ 545

- columns of ................... $55^{\circ}$

- connections of, with the encephalon ... $5^{80}$

- direction of fibres in............ 553

- general properties of ............. 554

— motor and sensory paths in ......... 555

— nerve-centres in .............. 558, 564

_._. relations of, to muscular coördination. . 557

Spinal nerves ................... 492

Spindle in karyokinesis .............. 4

Spirem .................... 5

Splanchnopleure ................... 801

Spleen........................ 378

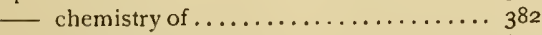

- development of ................ 829

- extirpation of . . . . . . . . . . . . . . . $3^{82}$

— relations of, to blood-corpuscles ....... $3^{8} 4$

rhythmical contractions of .......... $3^{82}$

Spongioplasm.................... 2

Stannius experiment................. 46

Stapedius....................... 7 I7

Starch ......................... I46

Starvation ....................... I39

Steapsin .................... 218

Stercobilin .................... 23 I

Stercorin................. I47, 233, 370

Stomach...................... 185

— closed follicles of ............... Igo
Stomach, development of ........... 827

— gases of..................... 205

- glands of ................... 188

- movements of ................ 203

— regurgitation from .............. 205

Stomata in lymphatics .............. 250

Sublingual gland $\ldots \ldots \ldots \ldots \ldots \ldots \ldots$ I7 I

Sublingual nerve ................. 518

Submaxillary gland................ I7 I

Sudoriparous glands................ 316

Sugars .......................... I44

Sulphocyanate in saliva........ I7 I, I73, I74

Superfecundation.................. 795

Suprarenal capsules ................ 376

- development of ................ 838

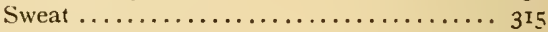

— colored $\ldots \ldots \ldots \ldots \ldots \ldots \ldots \ldots \ldots \ldots 3^{21}$

- in special parts............... $32 \mathrm{I}$

— properties and composition of ........ 320

Sweat-centres .................... 318

Sympathetic system of nerves......... 606

- direct experiments on ............612

Synapses ........................ 556

Synovia ...................... 277

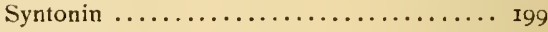

Tactile centre .................... 629

Tactile corpuscles.................. $46_{5}$

Taste (see Gustation) ............... 637

Taste-buds...................... 644

Taste-centre ...................... 645

Taurin ........................... 346

Tea......................... ${ }^{54}$

Tears .......................... 709

Teeth ...................... 162 development of............... 833

Telephone ..................... 454

Telophases, in karyokinesis . ........... 6

Temperature of the body............. 397

- equalization of. ................ 407

- sense of................... 630

— variations of, under different conditions and influences ................. 397

Tenon's capsule................... 649

Tensor tympani ................. 7 I7

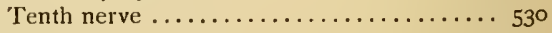

Testicles......................... 775

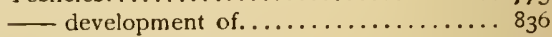

— internal secretion by ............ 390

- interstitial gland of ........... 391, 778

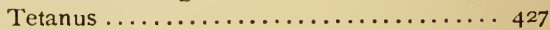

Theobromin . . . . . . . . . . . . . . . I55

Third nerve.................... 495

Thirst ...................... 140

Thoracic duct..................... 247

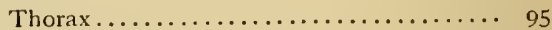

Thranin ...................... 7 10

Thrombin ........................ 28

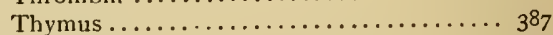

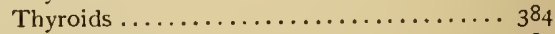

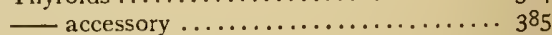

- extirpation of . . . . . . . . . . . . . $3^{86}$

Tidal air...................... II

Tigroid granules $\ldots \ldots \ldots \ldots \ldots \ldots \ldots \ldots .470$ 
Tones by influence ................ 733

Tongue ...................... 642

— development of. .............. 832

Tonsils......................... I72

Touch .................. 625, 627

Toxic molecules.................. Io

Trachea .................... gr

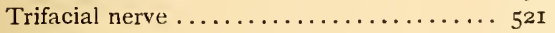

Triolein ...................... I47

Tripalmitin ................... 147

Tristearin....................... I47

Trochlearis nerve ................. 499

Trophic centres and nerves...........6 6I7

Trophoblast ................... 8 II

Trypsin ........................ 218

Tubercula quadrigemina.............. 576

Tweltth nerve.................... 5 I8

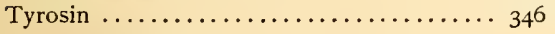

Umbilical cord..................... 809

Umbilical vesicle..................... 806

Urachus................... 810, 826

Urea ......................... 339

- elimination of ................ 340

Ureters..................... 332

Urethra......................... 334

- glands of ....................... 782

Uric acid, etc..................... 343

Urinary bladder $\ldots \ldots \ldots \ldots \ldots \ldots \ldots \ldots \ldots . \ldots \ldots \ldots \ldots \ldots \ldots \ldots \ldots$

Urinary passages.................. 332

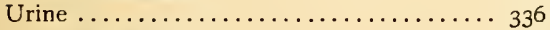

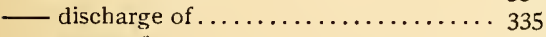

— gases of ........................ $35^{\circ}$

- inorganic salts of $\ldots \ldots \ldots \ldots \ldots \ldots \ldots 347$

- modifications of $\ldots \ldots \ldots \ldots \ldots \ldots \ldots, 340$

- production of ................. $33^{\circ}$

variations in ................... $35^{2}$

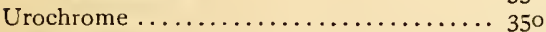

Uterine plug ................... 813

Uterus....................... 754, 759

- involution of $\ldots \ldots \ldots \ldots \ldots \ldots \ldots \ldots, 854$

Vagina ...................................... 75466

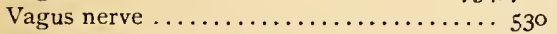

Vagus pneumonia ................. 540

Valvulæ conniventes .................. 208

Vas aberrans................... 778

Vascular arches .................... 844

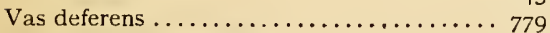

Vaso-inhibitory nerves ..............6 6I6

Vasomotor centres and nerves.........6.6 $6 r_{4}$

Vater, corpuscles of. ............................

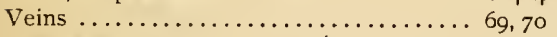

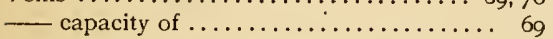

— conditions that impede circulation in .. 80

- development of ............... 844
Veins, number of .................. 69

— pressure of blood in............. 74

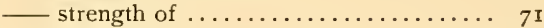

valves of ................. 72,79

Vernix caseosa................ 287,822

Vertebral column, development of ...... 820

Vesiculæ seminales................. 780

Vestibule, bony. . ................. 720

membranous ........................ 742

Villi of the small intestine ........... 2I I

Visceral arches and visceral clefts....... 830

Vision (see Eye) ....................6. 644

- binocular .................6.69

- duration of impressions in ......... 695

— field of .................. 675,69o

- perception of colors in............ 704

Visual centres.................. 703

Visual purple ................... 677

Vital capacity ................... II3

Vital point ...................6. 603

Vitreous humor $\ldots \ldots \ldots \ldots \ldots \ldots \ldots \ldots \ldots 664$

Vocal chords ........................ 90, 435

Voice, anatomy of organs of .......... 435

- action of accessory parts in ......... 443

- after castration in the male $\ldots \ldots \ldots \ldots 44$ I

— different kinds of . ............... $44^{\mathrm{I}}$

— different registers of. . . . . . . . . . 445

- in children .................. 44 I

- mechanism of production of ....... 439

— nerve of....................... $5^{15}$

- range of ................... $44^{\text {I }}$

Voltaic alternation................. 488

Vomiting ....................... 205

Vowels $\ldots \ldots \ldots \ldots \ldots \ldots \ldots \ldots \ldots, 45^{\mathrm{I}}$

Wagner, corpuscles of ...............465

Wallerian method ................. 474

Wandering cells $\ldots \ldots \ldots \ldots \ldots \ldots \ldots \ldots, 23$

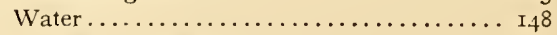

— as an excretion ............... 35 I

— production of, in the body ....... 35I, 406

Wharton, gelatin of $\ldots \ldots \ldots \ldots \ldots \ldots \ldots$ 8 10

Whispering $\ldots \ldots \ldots \ldots \ldots \ldots \ldots \ldots \ldots \ldots, 453$

Wolffian bodies.............. 818, 835

Word-blindness $\ldots \ldots \ldots \ldots \ldots \ldots \ldots \ldots \ldots, 703$

Word-deafness........................ $75^{2}$

Wrisberg, nerve of $\ldots \ldots \ldots \ldots \ldots \ldots \ldots . \ldots \ldots$

Xanthin $\ldots \ldots \ldots \ldots \ldots \ldots \ldots \ldots \ldots \ldots \ldots \ldots \ldots \ldots$

Yawning $\ldots \ldots \ldots \ldots \ldots \ldots \ldots \ldots \ldots$ I 10

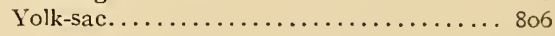

Youth ....................... $85^{8}$

Zone of Zinn.................6.6 $66_{3}$ 



\title{
Works on Medicine and Surgery
}

... PUBLISHED BY...

\section{THE MACMILLAN COMPANY}

\author{
64-66 FifTh AVEnUE, NEW YoRK
}

\section{ALLCHIN}

A Manual of Medicine. Edited by W. H. Allchin, M.D. (Lond.), F.R.C.P., F.R.S.E., Senior Physician and Lecturer on Clinical Medicine, Westminster Hospital, Examiner in Medicine in the University of London, and to the Medical Department of the Royal Navy. In five volumes.

Vol. I. General Diseases. Diseases excited by atmospheric influences, the Iniections. I2mo. Cloth. Colored Plates. pp. $\mathrm{x}+44^{2}$.

Price $\$ 2.00$ net.

Vol. II. General Diseases (continued). Diseases caused by Parasites, Diseases determined by Poisons, introduced into the Body, Primary Perversions of General Nutrition, Diseases of the Blood. I2mo. Cloth. Colored Plates and 21 other illustrations. pp. viii +380 .

Price $\$ 2.00$ net.

Vol. III. Diseases of the Nervous System. Organic Disease of the Brain and its Membranes, Diseases of the Spinal Cord, Functional Diseases of the Nervous System. I zmo. Cloth. Colored Plates and 27 other illustrations. pp. $x+417$.

Price $\$ 2.00$ net.

Vol. IV. Diseases of the Respiratory and of the Circulatory Systems. I2mo. Cloth. Colored Plates and numerous other illustrations. pp. xiv +493 .

Price $\$ 2.00$ net.

VoI. V. Diseases of the Digestive System and of the Liver, Diseases of the Peritoneum and of the Vessels of the Abdomen, Diseases of the Kidneys, Diseases of the Ductless Glands. I2mo. Cloth. Colored Plates and numerous other illustrations. pp. xii +687 .

\section{BRUNTON}

Price $\$ 2.00$ net.

On Disorders of Digestion: Their Consequences and Treatment. By Sir T. LAUDER Brunton. 8vo. Cloth. Illustrated. pp. xvi +389 .

Price $\$ 2.50$.

By the Same Author

An Introduction to Modern Therapeutics. Being the Croonian Lectures on the relationship between Chemical Structure and Physiological Actions in relation to the Prevention, Control, and Cure of Disease. Delivered before the Royal College of Physicians in London. By Sir T. Lauder Brunton. 8vo. Cloth. Illustrated. pp. vii + 195 .

Price 1.50.

Lectures on the Action of Medicines. Being the Course of Lectures on Pharmacology and Therapeutics delivered at St. Bartholomew's Hospital during the Sumizer Session of 1896. By Sir T. Lauder Brunton, M.D., D.Sc. (Edin.), LL.D. (Hon.) (Aberd.), F.R.S., etc. 8vo. Cloth. I4t Illustrations. pp. $x v+673$. Price $\$ 4.00$ net.

Sheep binding. Price $\$ 5.00$ net. Lectures on Disorders of Assimilation, Digestion, etc. By Sir T. LAUDER BRUNTON. 8vo. Cloth. pp. $\mathrm{xx}+495$.

Price $\$ 4.00$ net.

\section{DAVIS}

The Refraction of the Eye. Including a Complete Treatise on Ophthalmometry. A Clinical Text-Book for Students and Practitioners. By A. Edivard DAviS, A.M., M.D., Adjunct Professor of Diseases of the Eye in the New York Post-Graduate Medical School and Hospital, etc. 8vo. Cloth. IIg Illustrations. pp. xii $+43 \mathrm{I}$.

Price $\$ 3.00$ net. 


\section{DEFENDORF}

Clinical Psychiatry. A Text-Book for Students and Physicians. Abstracted and adapted from the Sixth German Edition of Kraepelin's "Lehrbuch der Psychiatre." By A. Ross DEFENDORF, M.D., Lecturer in Psychiatry in Yale University. Illustrated. 8vo. Cloth. pp. $x i i+420$.

Price $\$ 3.50$ net.

\section{ESMARCE and KOWALZIG}

Surgical Technic: A Text-Book on Operative Surgery. By Fr. vON EsMarch, M.D., Professor of Surgery at the University of Kiel, and Surgeon-General of the German Army, and E. Kowalzig, M.D., late First Assistant at the Surgical Clinic of the University of Kiel. Translated by Professor Ludwig H. GraU, Ph.D., formerly of Leland Stanford Junior University, and IVILLIAM N. SUllivaN, M.D., formerly Surgeon of U.S.S. " Corwin," Assistant of the Surgical Clinic at Cooper Medical College, San Francisco. Edited by Nicholas Sens, M.D., Professor of Surgery at Rush Medical College, Chicago. Wiih $I_{497}$ Illustrations and $I_{5}$ Colored Plates. 8vo. Cloth. pp. $x l+866$. Price $\$ 7.00$ net.

\section{FOSTER}

Half morocco. Price $\$ 8.00$ net.

A Text-Book of Physiology. By M. Foster, M.A., M.D., LL.D., F.R.S., Professor of Physiology in the University of Cambridge, etc. Revised and abridged from the Author's Text-Book of Physiology in Five Volumes. With an Appendix on the Chemical Basis of the Animal Body, by A. SHERIDAN LEA, M.A., D.Sc., F.R.S., University Lecturer in Physiology in the University of Cambridge. 8vo. Cloth. 234 Illustrations. pp. xlix + I35I.

Price $\$ 5.00$ net.

By the Same Author

Sheep binding. Price $\$ 6.00$ net.

A Text-Book of Physiology. In Five Volumes.

Part I. Blood; The Tissues of Movement; Vascular Mechanism. Price $\$ 2.60$ net.

Part II. 'The Tissues of Chemical Action; Nutrition. Price $\$ 2.60$ net.

Part III. The Central Nervous System. Price $\$ 2.50$ net.

Part IV. The Central Nervous System (concluded) ; The Tissues and Mechanism of Reproduction.

Part V. The Chemical Basis of the Animal Body. By LEA. Price $\$ 2.00$ net. Lectures on the History of Physiology during the Sixteenth, Seventeenth, and Eighteenth Centuries. By Sir M. Foster, K.C.B., M.P., M.D., D.C.L., Sec. R.S., Professor of Physiology in the University of Cambridge, and Fellow of Trinity College, Cambridge. 8vo. Cloth. pp. 3 ro.

Price $\$ 2.25$ net.

\section{FULLER}

Diseases of the Genito-Urinary System. A Thorough Treatise on Urinary and Sexual Surgery. By Eugene Fuller, M.D., Professor of Genito-Urinary and Venereal Diseases in the Neiv York Post-Graduate Medical School; Visiting Genito-Urinary Surgeon to the New York Post-Graduate Hospital. 8vo. Cloth. I37 Illustrations. pp. ix +774 .

\section{GIBBONS}

Price $\$ 5.00$ net.

Sheep. $\quad$ Price $\$ 6.00$ net.

Half morocco. Price $\$ 6.50$ net.

The Eye. its Refraction and Diseases. The Refraction and Functional Testing of the Eye, complete in itself, in Twenty-eight Chapters, with Numerous Explanatory Cuts and Diagrams. By EDwARD E. GIbBons, M.D., Assistant Surgeon of the Presbyterian Eye, Ear, and Throat Hospital, Demonstrator and Chief of Clinic of Eye and Ear Diseases in the University of Maryland, Baltimore. 8vo. Cloth. pp. ix +472 . Price $\$ 5.00$ net.

\section{KIMBER}

Text-Book of Anatomy and Physiology for Nurses. Compiled by DIANA Clifford KIMBER, Graduate of Bellevue Training School; Assistant Superintendent New York City Training School, Blackwell's Island, N.Y., formerly Assistant Superintendent Illinois Training School, Chicago, Ill. 8vo. Cloth. I37 lllustrations. pp. xvi +268 . 


\section{KLEMPERER}

The EIements of Clinical Diagnosis. By Professor Dr. G. KLEMPERER, Professor of Medicine at the University of Berlin. Second American from the Seventh (last) German Edition. Authorized Translation by Nathax E. Brill, A.M., M.D., Attending Physician, Mount Sinai Hospital, New York City, and Samuel M. Brickner, A.M., M.D., Assistant Gynæcologist, Mount Sinai Hospital, Out-Patient Department. I2mo. Cloth. 6I Illustrations. pp. xvii +292 .

Price $\$$ I.00 net.

\section{KOCHER}

Text-Book of Operative Surgery. By Dr. THEODOR KOCHER, Professor of Surgery and Director of the Surgical Clinic in the University of Bern. Authorized Translation from the Fourth German Edition. By HaRolD J. S'IILES, M.B., F.R.C.S. (Edin.), Surgeon to the Royal Edinburgh Hospital for Sick Children, Late Assistant Surgeon, Edinburgh Royal Infirmary, Examiner in Anatomy, Royal College of Surgeons. Edinburgh. With 255 Illustrations. pp. $x x+440$. 8vo. Cloth.

Price $\$ 5.00$ net.

Half morocco. Price $\$ 6.50$ net.

\section{LILIENTHAL}

Imperative Surgery. For the General Practitioner, the Specialist, and the Recent Graduate. By Howard Lilienthal, M.D., Attending Surgeon to Mount Sinai Hospital, New York City. 8vo. Cloth. I53 Illustrations. pp. xvi +412 . Price $\$ 4.00$ net.

Half morocco. Price $\$ 5.00$ net.

\section{MUIR and RITCHIE}

Manual of Bacteriology. By RoberT MUIR, M.A., M.D., F.R.C.P. (Edin.), Professor of Pathology, University of Glasgow, and JAMES RITchIE, M.A., M.D., B.Sc., Reader in Pathology, University of Oxford. American Edition (with Additions), Revised and Edited from the Third English Edition. By Norman MACLEOD HaRris, M.B. (Tor.), Associate in Bacteriology, the Johns Hopkins University, Baltimore. With I7o Illustrations. pp. $\mathrm{xx}+56_{5}$. 8vo. Cloth.

Price $\$ 3.75$ net.

\section{OPPENHEIM}

The Development of the Child. By Nathan Oppenheim, Attending Physician to the Children's Department of Mount Sinai Hospital Dispensary. I2mo. Cloth. pp. viii + 296.

Price \$ 1.25 net.

By the Same Author

The Care of the Child in Health. I2mo. Cloth. pp. vii +308 .

Price $\$ .25$.

The Medical Diseases of Childhood. By Nathan Oppenheim, A.B. (Harv.), M.D. (Coll. P. \& S., N.Y.). 8vo. Cloth. Ior Illustrations and I9 Charts, pp. $x x+653$.

Price $\$ 5.00$ net.

Sheep. Price $\$ 6.00$ net.

\section{SCḦ̈FER}

Half morocco. Price $\$ \epsilon .5 \mathrm{c}$ net.

Text-Book of Physiology. Edited by E. A. Schäfer, LL.D., F.R.S., Professor of Physiology, University of Edinburgh. Cloth. 8vo.

Vol. I. 27 Plates and 92 Text Illustrations. pp. xviii + Iо $_{3} 6$.

Vol. II. 499 Illustrations. pp. xxiv + 1365.

Price $\$ 8.00$ net. Price $\$$ I 0.00 net.

\section{SEDGWICK}

Principles of Sanitary Science and the Public Health. With Special Reference to the Causation and Prevention of Infectious Diseases. By William T. SEDGwick, Ph.D., Professor of Biology and Lecturer on Sanitary Science and the Public Health in the Massachusetts Institute of Technology, Boston, sometime Biologist to the State Board of Health of Massachusetts. 8vo. Cloth. pp. $x i x+368$. 
SMITH

Introduction to the Outlines of the Principles of Differential Diagnosis, with Clinical Memoranda. By FRED J. S.MITH, M.A., M.D. (Oxon.), F.R.C.P. (Lond.), Physician and Senior Pathologist to the London Hospital. I2mo. Cloth. pp. ix +353 .

SUTER

Price $\$ 2.00$ net.

Handbook of Optics. For Students of Ophthalmology. By WIILIAM NorwOon SUTER, B.A., M.D., Professor of Ophthalmology, National University, and Assistant Surgeon, Episcopal Eye, Ear, and Throat Hospital, Washington, D.C. I2mo. Cloth. 54 Illustrations. pp. viii +209 .

Price $\$ 1 . \infty$ net.

\section{VERWORN}

General Physiology: An Outline of the Science of Life. By MAX VERworN, M.D., Ph.D., A.O., Professor of Physiology in the Medical Faculty of the University of Jena. Translated from the Second German Edition and edited by FreDERIC S. LEE, Ph.D., Adjunct Professor of Physiology in Columbia University. With 285 Illustrations. 8vo. Cloth. pp. $x v i+615$.

Price $\$ 4.00$ net.

\section{WARNER}

Three Lectures on the Anatomy of Movement. A Treatise on the Action of NerveCentres and Modes of Growth. Delivered at the Royal College of Surgeons of England. By FRANCIS WARNER, M.D. I2mo. Cloth. I8 Illustrations. pp. xiv + 135 .

By the Same Author

Price 75 cents net.

The Nervous System of the Child: Its Growth and Health in Education. By FRANCIS W A RNer, M.D. (Lond.), F.R.C.P., F.R.C.S. (Eng.), Physician to and Lecturer at the London Hospital, etc. I2mo. Cloth. pp. xvii +233 .

The Study of Children and Their School Training. By FRANCIS WARNER, M.D. 12mo. Cloth. pp. $x i x+264$.

Price $\$ 1.00$ net.

\section{WILLIAMS}

The Roentgen Rays in Medicine and Surgery as an Aid in Diagnosis, and as a Therapeutic Agent. By Francis H. Williams, M.D. 39I Illustrations. 8vo. Cloth. pp.xxx+ 658.

\section{WILSON}

Price $\$ 6.00$ net. Half morocco. Price $\$ 7.00$ net.

The Cell in Development and Inheritance. By EDmund B. WiIsor, Ph.D., Professor of Zoölogy, Columbia University. Second Edition, Revised and Enlarged. 8vo. Cloth. 194 Illustrations. pp. $x x i+483$.

Price $\$ 3.50$ net.

\section{ZIEGLER}

A Text-Book of Special Pathological Anatomy. By ERnst ZIEGLER, Professor of Pathology in the Lniversity of Freiburg. Translated and edited from the Eighth German Edition by DoNAld MACAlister, M.A., M.D., Linacre Lecturer of Physic and Tutor of St. John's College, Cambridge, and Henky IV. CatTell, M.A., M.D., Demonstrator of Morbid Anatomy in the University of Pennsylvania. 8vo. 562 Illustrations.

Sections I-VIII. pp. $x i x+575+x x x i i$. Cloth.

Sections IX-XV. pp. $x v+576-122 I+x x x i$. Cloth.

Price $\$ 4.00$ net.

Sheep. Price $\$ 5.00$ net. Price $\$ 4.00$ net.

Sheep. Price $\$ 5.00$ net.

\section{THE MACMILLAN COMPANY}



COLUMBIA UNIVERSITY LIBRARIES

This book is due on the date indicated below, or at the expiration of a definite period after the date of borrowing, as provided by the library rules or by special arrangement with the Librarian in charge.

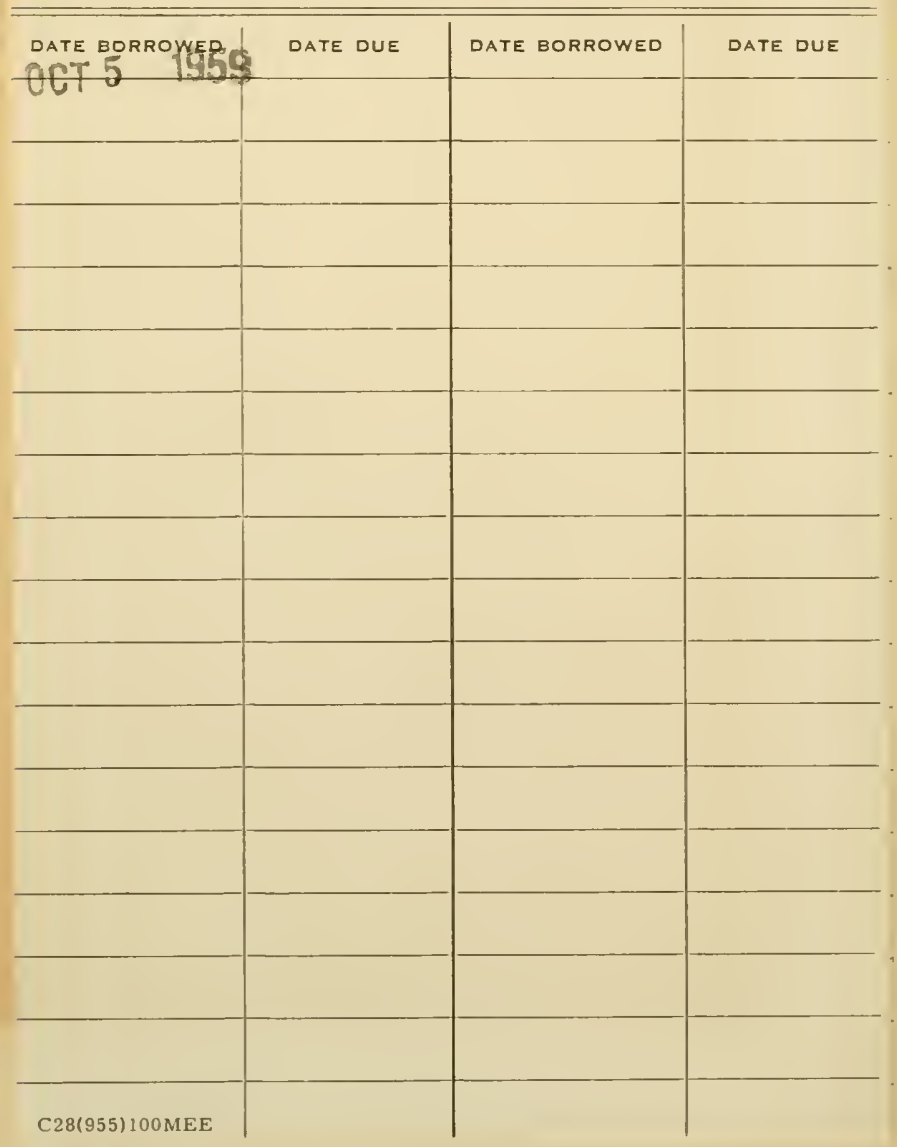




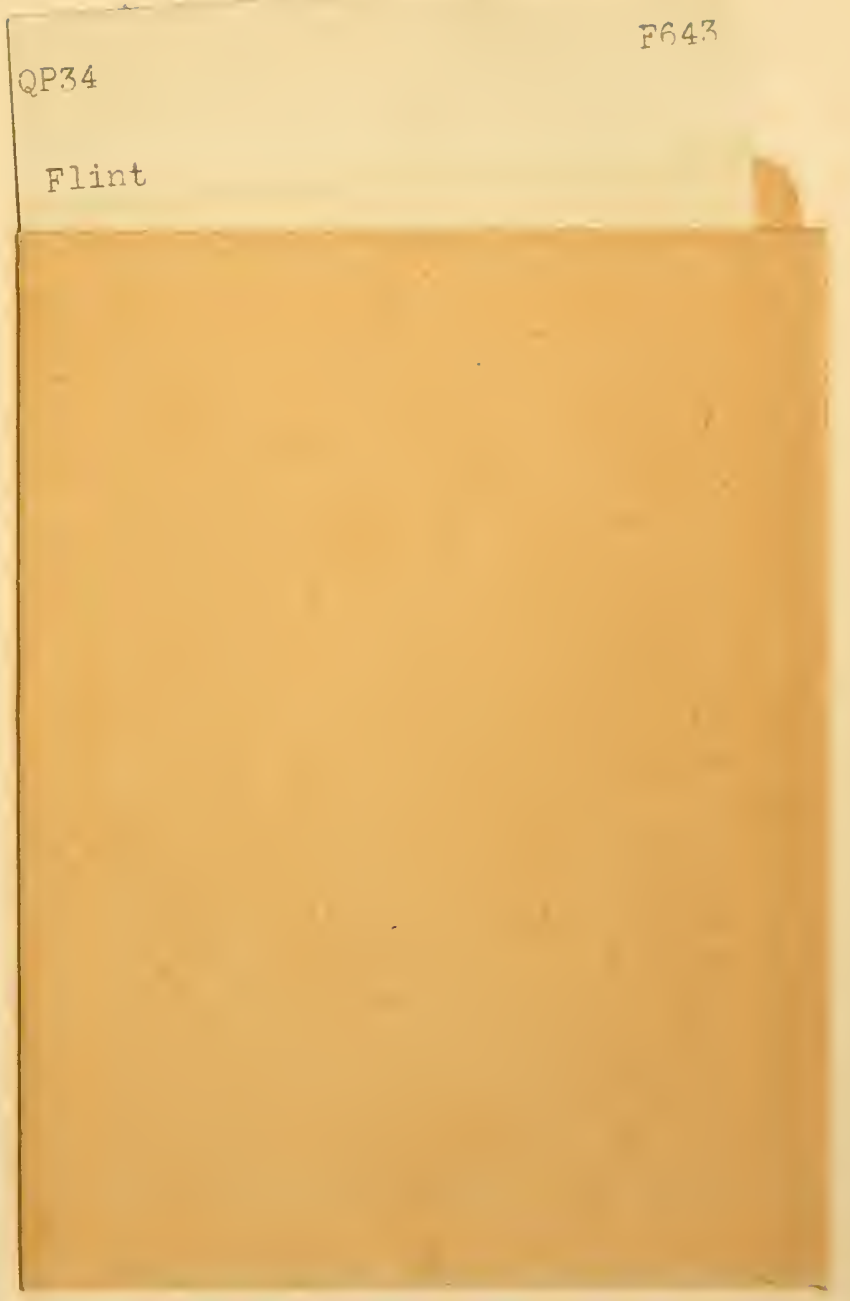


